



THE WAR LIST OF THE UNIVERSITY OF CAMBRIDGE I 914-I9I 8 
CAMBRIDGE UNIVERSITY PRESS

C. F. CLAY, MANAGER

LONDON : FETTER LANE, E.C. 4

NEW YORK : THE MACMILLAN CO. BOMBAY

CALCUTTA MACMILLAN AND CO., Ltd. MADRAS

TORONTO : THE MACMILLAN CO. OF CANADA, LTD.

TOKYO : MARUZEN-KABUSHIKI-KAISHA

ALL RIGHTS RESERVED 


\title{
Iniv
}

Cam.

\section{THE WAR LIST}

OF THE

\section{UNIVERSITY OF CAMBRIDGE I 9 I 4-I 9 I 8}

\author{
EDITED BY \\ G. V. CAREY, M.A. \\ FELLOW OF CLARE COLLEGE
}

LATE MAJOR, RIFLE BRIGADE AND ROYAL AIR FORCE
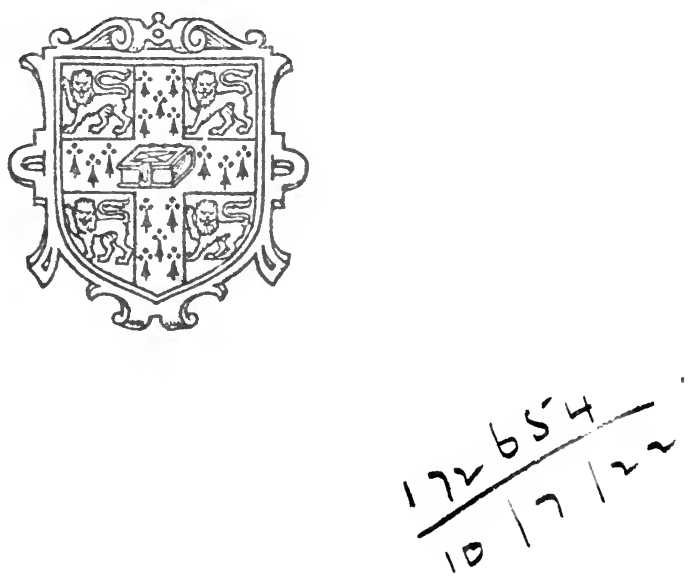

\section{CAMBRIDGE \\ AT THE UNIVERSITY PRESS}

I 92 I 
Digitized by the Internet Archive in 2007 with funding from Microsoft Corporation 


\section{CONTENTS}

INTRODUCTION LIST OF ABBREVIATIONS . • . • • xi CHRIST'S COLLEGE.$\quad$. . . . . I I CLARE COLLEGE • • • . . . . $\quad 27$ CORPUS CHRISTI COLLEGE . . . . 62 DOWNING COLLEGE • • • • • • 73 EMMANUEL COLLEGE $\quad$ • . . • • $\quad$. 85 GONVILLE AND CAIUS COLLEGE • • . II7 JESUS COLLEGE • • • • • • • $\mathrm{r}_{3}$ KING'S COLLEGE • . . . . . . . . . Igr MAGDALENE COLLEGE • • • . • . 219 PEMBROKE COLLEGE $\quad \cdot \quad \cdot \quad \cdot \quad \cdot \quad 233$ PETERHOUSE • . . . . . . . . $28 \mathrm{r}$ QUEENS' COLLEGE • . . . . . . 29r ST CATHARINE'S COLLEGE . . . . 307 ST JOHN'S COLLEGE • • • . . . . 318 SIDNEY SUSSEX COLLEGE $\quad$ • . . 347

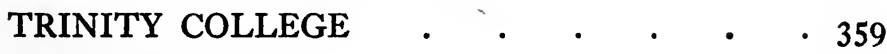
TRINITY HALL $. \quad . \quad . \quad . \quad . \quad . \quad .48 \mathrm{r}$ SELWYN COLLEGE $•$. . . . . $55^{\mathrm{II}}$ FITZWILLIAM HALL • • • • • • 527 CAVENDISH COLLEGE . . . . $\quad 536$ SUMMARY . . . . . . . after $p \cdot 536$ INDEX $•$ -.$\quad \cdot \quad \cdot \quad \cdot \quad \cdot \quad \cdot \quad 537$ 



\section{INTRODUCTION}

DURING the late war no official record was kept by the University of the services of Cambridge men with the Forces. Each College kept touch, so far as was possible, with the doings of its past, and present, members; and the various College records were collated and supplemented in a list initiated by the Executive Committee of The Cambridge Review and published by them from time to time. This list, the seventh and last edition of which appeared in April 1917, while the Battle of Arras was at its height, was the product of the patient and arduous work of $\mathrm{Mr} \mathrm{J}$. Austin Fabb, printer of The Cambridge Review; and, though no edition was published after the date mentioned above, Mr Fabb continued his efforts to keep the list up to date until, in December 1919, the publication of an official list was undertaken by the Syndics of the Press. The present War List is thus founded on the invaluable work of $\mathrm{Mr} \mathrm{Fabb}$, and it is fitting that the first words of this book should be a tribute to his labours.

But there remained much to be done. Mr Fabb had at no time had access to the Army List; to keep pace with the lists of casualties and distinctions alone was the utmost that human energy could compass during the war-to extract the promotions daily announced in The London Gazette had been quite out of the question. It was therefore obvious in December 1919 that, if anything like accuracy in the mere matter of rank was to be attained, it was necessary that every name should be checked with the official Service lists. This has duly been done, and after a prolonged and painful experience of the Army List and kindred publications the editor finds himself, if not confident of complete accuracy, at any rate conscious of having made some progress towards it.

There were also other problems to be tackled; and not the least of these were the three main questions: what, for the purposes 
of an official record, should be the definition of a Cambridge man, what should constitute "service," and what date should be regarded as marking the end of the war? Perhaps the chief difficulty in compiling a work of this nature is that of dealing with "borderline cases." No border-line can be drawn which eliminates altogether the doubtful case, nor one which, in some of its exclusions and inclusions, is wholly consistent with individual justice; the best that can be done is to make the line as clean-cut and as intelligible as possible. Before deciding the principles on which the present list should be based, the Syndics of the Press took counsel with representatives of the Colleges, and a general agreement was reached. It is hoped that the following outline, in conjunction with the List of Abbreviations, which has been drawn up with an eye to a generation unfamiliar with the naval and military common-places of to-day, will make plain to every reader both the broad principles and the details of this book.

UNIVERSITY QUALIFICATION. It was felt that, as an official record of the war services of Cambridge men, this list ought to include only those who were Cambridge men at the time of their war service. Thus the names of many hundreds who matriculated after serving in the war have been deliberately omitted, and residence prior to war service as a dividing-line has been closely adhered to, with one exception: it was decided to include those who had been admitted for the Michaelmas Term of I9I4 and were prevented from coming into residence in that term only by the fact of having joined the Forces at the outbreak of war.

SERviCE QUALIFICATION. Only those who served in some branch of His Majesty's Forces are included in this list. The Army, Navy, and Air Force Lists have formed the criterion throughout, though some latitude has been used in the case of Colonial volunteer units, which, though they do not appear in the Army List, in some instances engaged in actual fighting. This ruling necessarily excludes the names of many who performed valuable, distinguished, and often dangerous services in the war; but it was felt to be the only principle that could afford a comparatively clear dividing-line.

PERIOD OF SERvice. For the purposes of this record, the war 
closes with the armistice of November I Ith, I918. No event which took place after that date has been recorded, except deaths caused by, and honours awarded for, service prior to the armistice.

RANK. The differences between substantive, temporary, and acting rank became somewhat complicated, and the terms themselves altered in meaning, during the war. This list therefore aims at giving the highest rank held for an appreciable period, irrespective of its category-honorary rank is the only kind that is specifically stated. In cases where the service included staff appointments, the highest staff appointment attained is given.

REGIMENT, etc. The individual career of nearly every officer and man in the Army included service, if not in more than one regiment, at least in more than one battalion of the same regiment. To specify each battalion was clearly impracticable; but where transfers to different regiments, or to different arms of the service, took place, they have been recorded, so far as they are known, with the highest rank held in each regiment or arm. The semi-colon is used to separate successive stages of service.

CASUALTIES. All deaths which occurred from any cause whatsoever during service with the Forces previous to the armistice are recorded with a cross against the entry. Wherever possible, the actual date of death is given; a date given in parentheses represents the date of publication of the casualty list in which the death was announced. Deaths which have taken place since the armistice are not recorded, unless they were the result of wounds or other causes directly attributable to service prior to the armistice; in a few cases where death after the armistice may have been indirectly due to war service, a footnote has been added.

Casualties due to gas-poisoning were, for the greater part of the war, included among the "wounded" in the official casualty lists; injuries due to accident, on the other hand, were not so included. The same principle has been adopted in this list.

In cases where a "(W.)" appears against the name of one who died of wounds, it refers to an occasion other than that which resulted in death.

HoNOURS AND Awards. Distinctions gained for service in the war are shown in italics after the description of rank, regiment, etc., 
and are, approximately, in order of dignity and not in the order in which they were gained; pre-war distinctions are given in small capitals immediately after the surname and initials.

In compiling the present list I have received assistance from various quarters. My thanks are due first and foremost to my wife, whose patient help alone enabled me to carry through the task of checking each entry with the Army, Navy, or Air Force Lists; in the compilation of the Summary also she has borne a large share of the work. The Index is the work of Mr H. A. Parsons, of the University Press, and others of my colleagues on the staff of the Press have given me valuable advice and help. I am indebted to the Commanding Officer, the Adjutant, and the Headquarters staff of the Cambridge University Officers Training Corps for facilities readily granted to me at all times to consult their documents; and to the Registrar of the India Office for the loan of a copy of the Indian Army List. To the officials of several of the Colleges, whether Master, Fellow, or clerk, my acknowledgments are due for careful co-operation in compiling the portions of the book relating to their respective Colleges; and, last but not least, I wish to thank all those who, in answer to my appeals in the public press, sent me first-hand information of their services. I am only too conscious that, even now, this record must fall far short of completeness and accuracy, and I shall be grateful if those who discover mistakes or omissions will draw my attention to them. Finally, if any lover of statistics should feel tempted to test the accuracy of the Summary, I sincerely hope that the temptation may be too strong for him.

G. v. C.

University PRess,

September, r921. 


\section{LIST OF ABBREVIATIONS}

(A.) = Aeroplane officer

(A. and S.) =Aeroplane and Seaplane officer

A.A. and Q.M.G. = Assistant Adjutant and Quartermaster-General

A.A.G. = Assistant Adjutant-General

A.B. = Able Seaman

A.C.G. = Assistant Chaplain-General

(Ad.) = Administrative officer

A.D. = Assistant Director

A.D.C. = Aide-de-Camp

A.D.G.T. $=$ Assistant Director-General of Transportation

Adjt. = Adjutant

A.D.M.S. = Assistant Director of Medical Services

A.D.O.S. = Assistant Director of Ordnance Services

A.D.S. and T. $=$ Assistant Director of Supplies and Transport

A.F.C. $=$ Air Force Cross

A.I.G.T. = Assistant Inspector-General of Transportation

A.M.C. = Army Medical Corps

A.M.L.O. = Assistant Military Landing Officer

A.M.S. = Army Medical Service

A.P.M. = Assistant Provost-Marshal

A.Q.M.G. = Assistant QuartermasterGeneral

attd. $=$ attached

Bde. $=$ Brigade

Bdr. = Bombardier

Bn. = Battalion

B.S.M. = Battery Sergeant-Major

Bt. $=$ Brevet

Bty. = Battery

Capt. = Captain

C.B. = Companion of the Order of the Bath

C.B.E. = Companion of the Order of the British Empire

Cdr. = Commander

C.F. = Chaplain to the Forces

C.I.E. = Companion of the Order of the Indian Empire

cmdg. = commanding

Cmdt. $=$ Commandant

C.M.G. = Companion of the Order of St Michael and St George

Corpl. $=$ Corporal

Coy. = Company

C.P.O. = Chief Petty Officer
C.Q.M.S. = Company QuartermasterSergeant

C.R.E. $=$ Commanding Royal Engineers

C.S.I. = Companion of the Order of the Star of India

C.S.M. = Company Sergeant-Major

D. (in "D. of Wellington's," etc.) $=$ Duke

D.A. and Q.M.G. = Deputy Adjutant and Quartermaster-General

D.A.A. and Q.M.G. = Deputy Assistant Adjutant and QuartermasterGeneral

D.A.A.G. = Deputy Assistant Adjutant-General

D.A.C.G. = Deputy Assistant Chaplain-General

D.A.D. = Deputy Assistant Director

D.A.D.M.S. = Deputy Assistant Director of Medical Services

D.A.D.O.S. = Deputy Assistant Director of Ordnance Services

D.A.D.R.T. = Deputy Assistant Director of Railway Traffic

D.A.D.S.andT. $=$ Deputy Assistant Director of Supplies and Transport

D.A.D.V.S. = Deputy Assistant Director of Veterinary Services

D.A.G. = Deputy Adjutant-General

D.A.M.S. = Deputy Assistant Military Secretary

D.A.P.C. = Deputy Assistant Principal Chaplain

D.A.P.M. $=$ Deputy Assistant ProvostMarshal

D.A.Q.M.G. = Deputy Assistant Quartermaster-General

D.C.M. $=$ Distinguished Conduct Medal

D.D. $=$ Deputy Director

D.D.M.S. = Deputy Director of Medical Services

Dep. $=$ Deputy

Dept. $=$ Department

D.F.C. = Distinguished Flying Cross

D.G.T. = Director-General of Transportation

Div. $=$ Divisional

D.Q.M.G. = Deputy QuartermasterGeneral

D.S.andT. $=$ Director of Supplies and Transport 
D.S.C. $=$ Distinguished Service Cross

D.S.O. $=$ Distinguished Service Order (Companion of)

E. =East, or Eastern

empld. = employed

E.O.=Equipment Officer

Exp. = Expeditionary

F.A. = Field Artillery

Flt. $=$ Flight

Fus. $=$ Fusiliers

G.B.E. = Knight Grand Cross of the Order of the British Empire

G.C.I.E. = Knight Grand Cross of the Order of the Indian Empire

Gds.= Guards

Gen. $=$ General

Gnr. = Gunner

G.S.O. $(I, 2,3)=$ General Staff Officer (Ist, 2nd, 3 rd grade)

H.A. = Heavy Artillery

H.A.C. $=$ Honourable Artillery Company

Hdrs. $=$ Highlanders

H.Q. = Headquarters

I.A.R.O. = Indian Army Reserve of Officers

$i / c=$ in charge of

Imp. = Imperial

I.M.S. = Indian Medical Service

Infy. = Infantry

I.O.M. = Inspector of Ordnance Machinery

K. (in " $K$. Edward's Horse," etc.) =King

(K.B.) = Kite-Balloon officer

K.B.E. = Knight Commander of the Order of the British Empire

K.C.B. = Knight Commander of the Order of the Bath

K.C.M.G. = Knight Commander of the Order of St Michael and St George

K.C.S.I. = Knight Commander of the Order of the Star of India

Lce. $=$ Lance

L.I. = Light Infantry

Lieut. = Lieutenant

Lieut.-Col. $=$ Lieutenant-Colonel

L. of C. $=$ Lines of Communication

L.R.B. = London Rifle Brigade

$M$., $M_{2}$, , etc. = Mentioned in despatches once, twice, etc.

$m ., m 2$., etc. = mentioned (once, twice, etc.) in the Secretary of State's list, for valuable services in connection with the war

$M . B . E .=$ Member of the Order of the British Empire

M.C. $=$ Military Cross
Mech. = Mechanic

Med. $=$ Medical

M.G. = Machine Gun

M.G.C. = Machine Gun Corps

M.L.O. = Military Landing Officer

$\boldsymbol{M} . \boldsymbol{M} .=$ Military Medal

M.O.= Medical Officer

M.S.M. = Meritorious Service Medal

(M.T.) = Mechanical Transport

Mtd. = Mounted

M.V.O. = Member of the Royal Victorian Order

N. = North, or Northern

(O.) $=$ Observer officer

$O \cdot B \cdot E .=$ Officer of the Order of the British Empire

O.C.B. $=$ Officer Cadet Battalion

O.T.C. $=$ Officers Training Corps

(P.) = Prisoner of War

P. and B.T. = Physical and Bayonet Training

P.O. = Petty Officer

P. of W. Coy. $($ Camp $)=$ Prisoners of War Company (Camp)

Prob. $=$ Probationer

P.S.Bn. = Public Schools Battalion

Pte. $=$ Private

Q.M. = Quartermaster

Q.M.G. = Quartermaster-General

Q.M.S. = Quartermaster-Sergeant

Q.V.R. $=$ Queen Victoria's Rifles

R. = Royal

R.A. = Royal Artillery

R.A.C.D. = Royal Army Chaplains' Department

R.A.F. $=$ Royal Air Force

R.A.M.C. $=$ Royal Army Medical Corps

R.A.O.C. $=$ Royal Army Ordnance Corps

R.A.S.C. $=$ Royal Army Service Corps

R.A.V.C. $=$ Royal Army Veterinary Corps

R.E. = Royal Engineers

Regt. $=$ Regiment

Res. $=$ Reserve

ret. $=$ retired

R.F.A. = Royal Field Artillery

R.F.C. $=$ Royal Flying Corps

Rfn. = Rifleman

R.G.A. = Royal Garrison Artillery

R.H.A. $=$ Royal Horse Artillery

R.M.A. = Royal Marine Artillery

R.M.A.,Woolwich $=$ Royal Military Academy, Woolwich

R.M.C. $=$ Royal Military College

R.M.L.I. = Royal Marine Light Infantry 
R.N.= Royal Navy

R.N.A.S.= Royal Naval Air Service

R.N.D. = Royal Naval Division

R.N.R. = Royal Naval Reserve

R.N.V.R. = Royal Naval Volunteer Reserve

$R$. of $O=$ Reserve of Officers

R.Q.M.S. = Regimental Quartermaster-Sergeant

R.S.M. = Regimental Sergeant-Major

R.T.O.= Railway Traffic Officer, or Railway Transport Officer

S. = South, or Southern

(S.) = Seaplane officer

S. and T. Corps. = Supply and Transport Corps

Sergt. $=$ Sergeant
S.O. $(r, 2,3)=$ Staff Officer (rst, 2nd, 3 rd Grade)

Spec. $=$ Special

Sqdn. = Squadron

Supt. $=$ Superintendent

(T.) = Technical officer

T.D. $=$ Territorial Decoration

T.F. $=$ Territorial Force

T.M.B. = Trench Mortar Battery

Unattd. = Unattached

V.D. $=$ Volunteer Decoration

Vol. $=$ Volunteer

W. $=$ West, or Western

(W.), (W2.), etc. = Wounded once, twice, etc.

Yeo. $=$ Yeomanry

NotE. The date inserted with each entry is the year of matriculation, except in the following cases:

(i) For those who were admitted for the Michaelmas Term of 1914, but who never actually matriculated, the year is shown as [1914].

(ii) A date marked with an asterisk denotes that the person to whom the entry refers joined the College in that year by some procedure other than matriculation, e.g. by migration from another College or the non-collegiate body.

\section{ADDITIONS AND CORRECTIONS}

Page I3I After Fraser, D. H. read as follows: Capt., R.A.M.C.; attd.R.A.F. O.B.E. M.C. Médaille de la Reconnaissance Française, 2nd Class, and Médaille des Epidémies.

150 For RIx, R. G. read Rix, R. G. B.

260 For M'Lachlan, T. K. read Maclachlan, T. K.

277 After Wadham, W. F. A., v.D. read Lieut.-Col., King's Own (R. Lancaster Regt., T.F.) $m$.

406 Add HaRwood, H. M. Capt., R.A.M.C.(T.F.) 1892

42 I Add Lawson, H. B. Lieut., London Regt. (Kensington Bn.); attd. Manchester Regt.(T.F.) M.C. I9,16

446 For Price, Rt. Rev. McC. E. read Price, Rt. Rev. H. McC. E.

538 For Murdock, A. J. read Murdoch, A. J. 


\begin{abstract}
"WE PASS TO THE THOUGHT OF THE MANY WHO WILL NOT RETURN ; NOT BECAUSE OF ANY CHANGE OF PLAN, BUT BECAUSE THE OFFER WHICH ALL HAVE MADE HAS IN THEIR CASE BEEN ACCEPTED TO THE FULL....FOR THESE NO PRIVILEGE THAT WE CAN DEVISE AVAILS. YET THE UNIVERSITY BEARS THEM UPON HER HEART, AND WILL NOT, I KNOW, NEGLECT TO PERPETUATE THE MEMORY OF THEM IN SUCH SORT THAT IT MAY SPEAK TO THE YOUTH OF ENGLAND IN TIME TO COME.... MANY AND DIVERSE WERE THE HOPES AND EXPECTATIONS WE HAD FORMED FOR THEM, BUT EVERY ONE OF THESE HAS BEEN SURPASSED BY THE EVENT. THEY HAVE ALL BEEN FOUND CAPABLE OF MAKING THE GREATEST DENIAL OF SELF THAT MEN CAN MAKE.

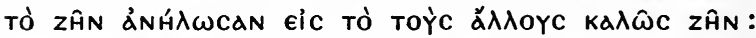
THEY PAID AWAY THEIR OWN LIFE THAT THE LIFE OF THEIR FELLOWS MIGHT BE HAPPY."
\end{abstract}

Dr M. R. JAMES, in his Address to the Senate, I October I9I5. 


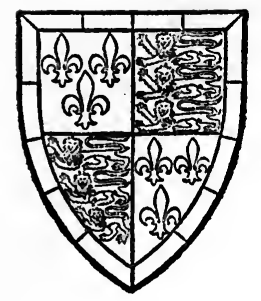

\section{CHRIST'S COLLEGE}

ЖАввотт, С. H. 2nd Lieut., Lincolnshire Regt.

Died 7 May 1917 of wounds received in action

Adami, J. G. Colonel, Canadian A.M.C.; A.D.M.S. 1880 C.B.E.

Adams, F. S. Capt., R.A.M.C. (W.)

1903

1909

Killed in action 5 fuly 1916

ADSHEAD, H. E. 2nd Lieut., R.E.

AdsheAd, M. S. Lieut., Cheshire Regt.

AgER, Rev. A. D. Chaplain, R.N.

1908

Agrus, Rev. T. A. C.F. 4 th Class, R.A.C.D. (W.) I9Io

Ainsworth Davis, J. C. Capt., Rifle Brigade; Hon. Capt. I9I4 (O.), R.A.F. (W.) $M$.

*Airey, T. A. Pte., London Regt. (London Scottish) I9I3

Killed in action I fuly I916

Akam, Rev. J. W. 2nd Lieut., R.A.S.C.

1912

AKerman, E. J. B. Major, Somerset L.I.; D.A.Q.M.G. I904

(W.) M.C. French Croix de Guerre

Alan-Williams, A. C. Capt., Warwickshire Yeo.; 1908

A.D.C. (W.) M.C.

Aldridge, C. B. M. Capt., R.A.M.C. $\quad$ I896 WAllen, J.S. Major, Northumberland Fus. (W.) M.C. I9ro

Killed in action I I April r918

Allen, R. C. Lieut.-Col., Auckland Regt., N. Zealand 1899

Force. D.S.O. and Bar. $M$.

Allen, W. H. Major, R.A.M.C.

1887

ANDERSON, H. 2nd Lieut., S. Staffs. Regt.

I9IO

Angus, W. B. G. Major, R.A.M.C. O.B.E. M.C. M. 1903 ARMOUR, J. K. C. Lieut., R.G.A.

WARmstrong, F. M. Major, R.F.A.(T.F.) $M$. $\quad 1896$

Killed in action 25 Sept. 1917

C. U.W.L. 
ARnold, W. Major, R.F.A.; empld. Ministry of Muni- $\quad 1897$ tions. (W 3.)

Arthur, W. L. Capt., Channel Islands Militia, empld. 1902 R. Jersey Garrison Bn.

AsHLey, N. D. 2nd Lieut., R.A.S.C.(M.T.)

AshwORTH, J. Lieut., R.A.S.C. $m$.

1913

1908

Astbury, Rev. H. S. C.F. $4^{\text {th }}$ Class, R.A.C.D. M.C. 1909

Astley, W. Major, R.E. (W.) 1900

Atchison, G.T. Lieut., Bedford Grammar School O.T.C. $\quad$ I896

Auden, G. A. Major, R.A.M.C.(T.F.); D.A.D.M.S. $\quad$ I890

WBaines, A. B. Capt., Oxford and Bucks. L.I. (W.) 1907

Killed in action 3 April r9I7

BAINES, D. L. Major, Uganda Vol. Regt. O.B.E. 1900

BAKER, T. Y. Instructor Cdr., R.N. Chevalier, Legion 1895 of Honour (France)

BAKEWEl, B. Lieut., R.F.A. (W 2.) $M$.

BANister, T. E. Capt., R.A.M.C.

Banks, L. 2nd Lieut., Ist Brahmans, Indian Army

HBAnyard, J. H. 2nd Lieut., Bedfordshire Regt.

Killed in action 3 Sept. 1916

Baptie, N. Pte., London Regt.

1909

1905

1909

* 1907

W. Capt, Seaforth Hdrs ; attd. Gen Staff ma.

BARBE, W. Capt., Seafth Hdrs.; attd.Gen.Staff. m2. 1903

Barclay, A. E. Capt., R.A.M.C. (2nd W. Gen. Hos- I894 pital, T.F.)

BARFF, Rev. F. R. Chaplain, R.N.

BARKER, A. Lieut., Worcestershire Regt. (W.)

BARLOW, R. G. Capt., Seaforth Hdrs. (W.)

1907

I9I 5

BARNARD, H. J. Lieut., Gen. List, empld.T.M.B. (W.) [I9I4]

BARR, G. Lieut. (Ad.), R.A.F.

BARran, P. A. Major, R.F.A.(T.F.); empld. Ministry I897 of Munitions

BARreTt, L. Lieut., R.E. (Fortress, T.F.). I9I0

BARretT, Rev. L. T. S. Chaplain, R.N. 1905

BARTLETT, A. W. Lieut., R.A.M.C. (Sanitary Service, * ${ }_{1902}$ T.F.)

Bartley, Rev. P. R. C.F. 4th Class, R.A.C.D. $\quad$ I893

BARwick, R. L. Capt., R.A.M.C. 1897

BASHFORD, P. F. R. Capt., R.N.R. 1904

Bateman, W. Lieut., King's Own (Yorkshire L.I.) [1914] (W.) (P.)

सBatty, G. G. H. Capt., Northamptonshire Regt.

Died 27 Sept. 1916 of wounds received in action $26 \mathrm{Sept}$. I9I6 
Beaumont, D. C. Capt., R.A.M.C.

Beaumont, O. A. Capt., R.A.M.C. M.C. $M$.

1912

BECK, G. R. Lieut., London Regt. (Blackheath and [1914]

Woolwich Bn.); Capt. (A.), R.A.F.

\&BecketT, V. L. S. Major, Yorkshire Regt.

Died I9 Fuly I916 of wounds received in action 5 fuly

1901

I916

BeDDOw, H. J. Capt., R.A.M.C.

Begcroft, A. E. Capt. and Adjt., R.E. M.B.E.

BeEdham, H. W. Capt., R.A.M.C.(T.F.)

AELl, Rev. C. H. C.F. 4th Class, R.A.C.D. M.C.

Killed in action 23 Aug. I918

Bell, Rev. J. A. H. C.F. $4^{\text {th Class, R.A.C.D. M.C. } M . ~} 1908$

Benbow, H. Major, E. Surrey Regt.

Bengough Clark, J. See Clark, J. B.

BennetT, E. B. Pte., R.A.S.C.(M.T.)

BennetT, F. G. Capt., R.A.M.C.(T.F.) $M$.

1897

1906

r 887

1907

BENNETT, G. L. Hon. Major, Lincolnshire Regt. and 1885

Labour Corps. $M 2$.

Benoly, H. J. Pte., R.A.M.C.; Sapper, R.E.

BetTERIDGe, B. F. 2nd Lieut., Northamptonshire Regt. I9I2

Betteridge, H. G. W. Pte., R. Fusiliers

BICKNELL, R. P. 2nd Lieut., R. Marines

* 1894 $M 2$.

Bigg-Wither, H. S. Major, R.A.O.C.; D.A.Q.M.G. 1907 O.B.E. $M$.

BILDERBECK, A. C. L. O'S. Capt., I.M.S. $M$.

Binks, B. B. Lieut., R.A.S.C.(T.F.)

Bishop, R. O. 2nd Lieut., Gen. List, empld. Ministry of

1909

1891

1909

1913

1893

1912

- Munitions. M.B.E.

WBLACK, M. A. Major, Dragoon Gds.; attd. R.F.C. (W.) 1895

Killed in action II Feb. I9I7

BlaCK, R. A. Capt., King's (Shropshire L.I.); A.M.L.O. $\quad 1896$

BlackbuRN, W. H. Surgeon Lieut., R.N.

Blackburne, Rev. E. V. C.F. 4th Class, R.A.C.D.

I9I I (P.) M.C.

Blanch, N. H. Lieut., R.F.A.(T.F.)

WBligh, E. Lieut., E. Lancs. Regt.

1907

1908

I 894

Killed in action 9 May 1915

Burss, E. W. Lieut., Labour Corps

Blyth, A. C. Capt., Norfolk Regt.

Bonnalie, F. E. Lieut., R.F.A.

1914

1913

I908

1909

1914 
WBonser, W. J. Capt., Rifle Brigade

Killed in action 25 Sept. I9I 5

Воотн, A. F. Instructor Lieut., R.N.

Booty, M. G. R. Pte., H.A.C.

1912

Воттомe, G. M. Major, R.A.S.C. (Canteens). $M 2$.

1904

Boultbee, Rev. H. T. C.F., Australian Chaplains' Dept. 1904

ABOuRnE, J. C. 2nd Lieut., Worcestershire Regt.

Killed in action 18 fuly 1915

Bousfield, H. T. W. Lieut., I.A.R.O., attd 9th Bhopal 1909 Infy. $M$.

Bowen, G. Pte., London Regt. (Artists Rifles)

I90I

Branston, R. Cadet, O.C.B.

WBrasnett, T. J. G. 2nd Lieut., E. Surrey Regt.

Killed in action at the Hohenzollern Redoubt 13 Oct. I9I 5

Brawn, J. A. Lieut., R.E.(T.F.)

Brierley, W. B. Sergt., Indian Army

Briggs, P. J. Pte., H.A.C. (W.)

Briggs, W. R. Capt., Spec. List (Staff Capt.). O.B.E. IgOI

BRIsTow, C. H. Lieut., I.A.R.O., attd. 4th Gurkha . I906 Rifles. (W.)

Brittain, A. W. 2nd Lieut., Sherwood Foresters (Notts. 1909 and Derby Regt.)

Brocklehurst, H. 2nd Lieut., Northumberland Fus.

Brode, Rev. R. T. Pte., London Regt. (Artists Rifles)

1914

I9I I

Broomfield, R. S. Lieut., Railway Bn., Indian Defence

I90I

Force

Broster, E. D. Surgeon Lieut., R.N.

Brown, A. E. Lieut., Australian A.M.C. $M$.

Brown, C. A. 2nd Lieut., R. Welsh Fus.

Brown, G. W. Lieut., Queen's Own (R.W. Kent Regt.);

Capt., Spec. List(School of Instruction). (W.) M.B.E.

Brown, L. T. Pte., R. Fusiliers

1910

1907

1893

1912

Browning, T. C. Lieut.-Col., Supernumerary List,

Indian Army

Bryceson, E. Lieut., Canadian A.M.C. ; Capt., R.A.M.C. 1876

Bunting, S. A. S. Lieut., I.A.R.O., attd. Sappers and I90I Miners. M.B.E.

Burdon, Hon. J. A., C.M.G. Major, Barbados Vols. $m$. $\mathrm{I}_{88}$

BurkitT, F. T. Capt., The Queen's (R.W. Surrey Regt.) I9I I (W 2.)

BurkitT, Rev. H. J. C.F. 2nd Class, R.A.C.D.(T.F.) I 888

Burrow, Rev. W. J. A. C.F. 4th Class, R.A.C.D. 1902

EBURTon-Fanning, N. E. E. Capt., R.M.L.I.

Killed in action at Gavrelle 28 April 1917 
ZBuszard, S. G. Lieut., Norfolk Yeo.

1908

Killed in action $8 \mathrm{Dec}$. 77

ButT, H. T. H. Capt., R.A.M.C.

ButTERWICK, J. C. 2nd Lieut., Eton College O.T.C. 1909

BUTTERWORTH, R. Lieut., R.A.M.C.

BUTTERY, H. R. Surgeon Lieut., R.N.

BygRave, W. 2nd Lieut., Blundell's School O.T.C.

Byrne, Rev. J. H. C.F. 4 th Class, N. Zealand Chaplains' * ${ }^{*} 913$ Dept.

Cadman, H. S. Capt., Denstone College O.T.C.

Caiger, Rev. S. L. C.F. 4th Class, R.A.C.D.

Canney, J. R. C. Capt., R.A.M.C.(T.F.)

I 889

1906

1901

Capon, E. O. Lieut., R. Berkshire Regt. and R. Defence

1896

1905

1901

Corps

Cardwell, H. E. 2nd Lieut. (A.), R.A.F.

I89I

Carlton-Williams, E. W. Flt. Sub-Lieut., R.N.A.S.

1898

Carmichael, Rev. D. W. W. C.F. th $^{\text {th }}$ Class, R.A.C.D.

CARR, J. D. 2nd Lieut., Worcestershire Regt.

ACARrINGton,E.A. A.B., R.N.V.R.; 2nd Lieut., Wiltshire I9I I Regt. (W.)

Killed in action at Gueudecourt 18 Oct. 1916

Carstairs, J. L. Capt., R.E.

1909

1898

1897

fCarter, G. L. L. Asst. Payma

Lieut., R.N.

Died 29 Fuly 1918 of pneumonia

CARTWRIGHT, S. H. Lieut., R.F.A. (W.)

1901

1910

Casteli, S. P. Lieut., R.A.M.C.

1915

1913

Cator, A. N. L. Lieut., Hyderabad Rifles, Indian De-

1899

fence Force

Cattell, McK. Sergt., United States Army

Chaffey, Rev. L. B. T. Lieut., Eton College O.T.C.

Challoner, J. L. Lieut., Northumberland Fus. and M.G.C. (W 2.)

Chapman, H. Corpl., R.E. (Signals); Major, D. of Corn- 1905 wall's L.I. (W 2.) $M 2$.

Charlesworth, J. B. and Lieut., R.F.A.

ChIEne, G. L. Major, R.A.M.C.(T.F.)

1912

1894

1913

CHILd, F. J. Capt., R.A.M.C.

[1914]

1891

1893

1901

ChILD, W. N. Capt., R.A.M.C.

Chivers, W. B. Lieut., Middlesex Regt.

Clark, J. B. Lieut., R. North Devon Yeo.

1912

I 877

Clarke, E. B. 2nd Lieut., Gloucestershire Regt. (P.) 1913

Clarke, W. S. Major, Gloucestershire Regt.

*1 888 
Cleeve, C. E. Major, R.A.S.C.(M.T.) O.B.E. M. 1909

Clough, J. 2nd Lieut., Yorkshire Regt.; Major, Tank 1912 Corps. (W 3.) D.S.O. M.C. M.

Clough, Rev. V. C.F. 4th Class, R.A.C.D. 1908

Cochran, G. G. Lieut., R.E. $M$. 1914

Cockton, J. C. Lieut., Westmorland and Cumberland I9Io Yeo.

*Conen, G. H. Lieut., King's (Liverpool Regt., T.F.) I897

Killed in action near La Bassée 16 May I9I 5

Coley, C. Lieut., Suffolk Regt.

Collingridge, W. Lieut.-Col., R.A.M.C.(T.F.)

1909

I 875

Collins, G. A. Capt., Rifle Brigade

ConnetT, H. Capt., United States Army

Cook, V. C. Pte., H.A.C.; Capt., R.A.S.C.(M.T.)

1907

I913

O.B.E. $M$.

Cope, J. L. Lieut., R.N.R.

1904

\Copeman, E. H. Pte., Middlesex Regt.; 2nd Lieut., 1906 Queen's Own (R. W. Kent Regt.)

Killed in action 18 March 1916

CoRfield, Rev. F. DE LA P. C.F. 4th Class, R.A.C.D. $\quad{ }^{\text {th }} 893$

CoRY, J. F. T. Trooper, Berkshire Yeo. *1906

ACOTTERILl, D. Capt., R.A.M.C.

Died 2 Dec. I9I 8 of pneumonia contracted on active service

Cox, G. L. Capt., R.A.M.C

Coxwell, C. B. 2nd Lieut., R.M.A.

1900

Crastre, Rev. 1908

aCrane, H. E. Pte., R. Fusiliers

Died 27 Oct. I 916

Crawford, G. B. Lieut., Yorkshire Regt.

Crawshaw, C. B. H. Capt., R.E. M.C. $M$.

Crawshaw, C. H. Major, R.A.M.C (T.F.) $M$.

\#CREED, Rev. A. H. G. Chaplain, R.N.

Died 21 May r917

4Cressey, G. E. L. Lieut., Yorkshire Regt.

Killed in action 26 Sept. 1915

Crook, A. H. Surgeon Lieut., R.N.V.R. $M$.

Crosby, G. J. V. 2nd Lieut., R.E.(T.F.)

CRowder, G. C. G. Lieut, Border Regt.and M.G.C. (W.) 1916

Cumberlidge, W. I. Capt., R.A.M.C.(T.F.)

Cunliffe, J. H. G. 2nd Lieut., R. Marines

1898

I 898

1903

1910

1912

1906

${ }^{*}{ }_{1} 884$

[1914]

1902

1916

I9IO

I 899

1912

DavenPort, H. Lieut., W. Yorks. Regt. (W.) (P.)

1909

Davenport, S. Capt. (Airship), R.A.F. $m$. 
Davidson, A. Major, Border Regt.

Davidson, Sir W. E., K.c.M.g. Hon. Colonel, R. New- 1878 foundland Regt.

Davies, E. D. D. Surgeon Lieut., R.N.; Surgeon, R.M.L.I.

Davies, Rev. L. C. C.F. $4^{\text {th }}$ Class, R.A.C.D.

Davies, Rev. R. E. C.F. $4^{\text {th }}$ Class, R.A.C.D.

Davis, J. C. A. See Ainsworth Davis, J. C.

Dawes, H. J. Lieut., Spec. List (Dental Surgeon) * * I908

Dawson, Rev. H. P. Chaplain, R.N. I88I

DE BaRathy, S. A. Lieut., Alberta Regt., Canadian Force. * * 896 (W.)

De la Pryme, W. H. A. Major, W. Yorks. Regt.; Staff 1899 Capt., War Office. (W.) D.S.O. M. m. Chevalier, Order of Leopold (Belgium)

WDENnes, W. Major, R.F.A. M.C. and Bar

Killed in action $2 \mathrm{I}$ March 1918

Desoer, A. Sergt., Belgian Artillery

DiBb, R. K. Major, E. Yorks. Regt. (W 2.) $M$.

Dickson, H. S. Capt., R.A.M.C. (W 2.)

Dillon, H. G. S. Lieut., R.N.V.R.

1907

1906

* 1899

DIPPIE, H. Major, Worcestershire Regt. (W.) D.S.O. * * 1906 $M$.

Dixon, A. F. W. Capt., Border Regt.(T.F.) (W.) I9II

Dixon, A. H. Capt., Norfolk Regt. (Cyclist Bn., T.F.) I9I I (W.)

Dobell, H. Sapper, R.N.D. Engineers; Capt., R. I90I

Marines; Lieut.-Cdr., R.N.V.R.; Major (A.), R.A.F.

(P.)

DoDD, A. H. 2nd Lieut., Unattd. List, T.F.

Doddrell, Rev. E. C. C.F. 4 th Class, R.A.C.D.

Dodwell, G. M. Capt., R.F.A. (W.)

1908

I9I5

I9I3

1898

1907
1906

I902

Douglas, R. O. Lieut., Queen's Own (R.W. Kent Regt.) ' [19I4]

Dove, W. B. Capt., R.A.M.C.

WDownie, J. M. Capt., R.A.M.C.

Died 29 Oct. 1918 of pneumonia

Dowson, W. J. Pte., Nairobi Defence Force

$\mathrm{r} 890$

I9II

Drumond, J. G Lieut, I A R O attd 42nd Cavalry; 1906

Capt. and Adjt., Hodson's Horse

Duigan, W. Capt., R.A.M.C.(3rd S.Gen.Hospital,T.F.) I883

Dunbar, R. Capt., R.F.A. (W 2.) M.C. M. I9I4

WDyer, C. M. 2nd Lieut., Rifle Brigade

Killed in action 9 April I9I 5

I912 
EARles, F. J. Capt., Manchester Regt. (W.) 1912

Eckenstein, T. C. Capt., S. Lancs. Regt. (W 2.) M.C. 1904

EDDIson, H. W. Surgeon Lieut., R.N.

EDE, J. C. Sergt., E. Surrey Regt. 1903

EDWARDS, D. L. P. Capt., Spec. List (Dental Surgeon) *1910

AEdwards, D. W. Capt., R.A.S.C.; attd. R.F.C. M.C. * *1910 Killed in action 6 April r9i7

EDWARDS, H. W. Capt., R. Warwickshire Regt.; Lieut.- *1907 Col., R.E.; A.D.Signals. D.S.O. M.C. M2. French

Croix de Guerre

EEDwards, Ll. A. Capt., R. Warwickshire Regt. (W.) I913

Died in German hands 21 March 1918 of wounds received in action

Edwards, W. N. Pte., R.A.M.C.(T.F.)

Elliott, A. D. Capt., R.E. (Signals)

1908

1902

Elliott, T. R. H. Pte., London Regt. 1912

EI.LIS, B. J. Lieut., Worcestershire Regt.; Staff Capt. * ${ }^{*} 899$ (W.)

Ellis, M. F. Capt., R.A.M.C.

Elliston, W. R. Major, Suffolk Regt. (Cyclist Bn., T.F.) 1887

EMBleton, D. Major, R.A.M.C. $M$. $m$.

England, W. B. 2nd Lieut., R. Berkshire Regt.; Lieut., I9I I Labour Corps. (W.)

Eschwege, F. S. See Foot, T. J.

EspIn, C. E. Lieut., Coldstream Gds. (W 2.) M.C. 1900

Evison, R. R. Capt., W. Yorks. Regt.

EwarT, G. A. Capt., R.A.M.C. (4th London Gen. Hos- 1906 pital, T.F.)

EwING, A. W. Capt., R.A.M.C.

1900

WFaIR, J. C. 2nd Lieut., Coldstream Gds.

Killed in action 25 Sept. I9I 5

FFARnham, F. J. 2nd Lieut., London Regt. and R.G.A. Accidentally killed I 5 April I9I7

Faulconbridge, F. T. Pte., Worcestershire Regt.

FAwCETT, W. F. Bt. Colonel, cmdg. Depôt, Northamp- 1876 tonshire Regt.

FFawsitt, T. R. Pte., Middlesex Regt. (P.S. Bn.); 2nd 1905 Lieut., York and Lancaster Regt.

Killed in action 16 Sept. I916

Fay, C. R. Lieut., The Buffs (E. Kent Regt.); Capt., *1909 M.G.C. and Spec. List (Asst. Instructor). $M$.

FAY, S. J. Capt., R.A.S.C.(M.T.) $M$. Ig0I

Fellows, R. B., c.B. Hon. Colonel, Bedfordshire Regt. $\quad 185^{\circ}$ 
Ferguson, A. C. W. Lieut., Loyal N. Lancs. Regt. (W.) 1896

Ferguson, S. C. Major, Northumberland Fus. (R. of O.); 1887 Embarkation S.O. O.B.E. m.

Finlayson, J. G. Sergt., R.A.M.C.

FISHER, L. G. 2nd Lieut., N. Staffs. Regt.

FISHER-Smith, E. L. Lieut., R.A.S.C.

WFITCH, A. S. 2nd Lieut., R. Sussex Regt.

Killed in action 9 April I9r7

Fiтch, E. W. Instructor Cdr., R.N.

Foot, T. J. Capt., R.A.M.C.

Forbes, J. G. Capt., R.A.M.C. $M$.

Forbes, J. W. F. Capt., Christ's Hospital O.T.C.

1904

1914

[1914]

[1914]

FORDER, Rev. F. G. Capt., Charterhouse School O.T.C. 1902

Francillon, F. E. Capt., Gloucestershire Regt. $M$.

Francillon, F. J. Lieut., R. Fusiliers and M.G.C.

Francis, J. A. Capt., R.A.S.C. $M 2$.

FRASER, F. R. Major, R.A.M.C.

FrASER, W. A. Lieut., W. Yorks. Regt.

FreEMAN, H. Lieut. (T.), R.A.F.

FreTz, W. T. S. Trooper, British S. African Police

FRYER, J. H. Capt., R.A.M.C.

Fulton, E. C. Major, S.O. 2, R.A.F.

FURSE, W. K. 2nd Lieut., R.F.A.

1895

I90I

I891

1888

1907

1899

1894

1904

I9II

1906

I910

1893

1897

1902

Gandy, E. S. Lieut., Epsom College O.T.C.

GARDINER, A. F. Major, R.F.A. M.C. $M$.

1905

1906

Gardiner, O. C. Lieut., R.E.(T.F.) (W.)

1903

\#GARDNER, H. M. Lieut.-Col., Lincolnshire Regt. (W.) I884

Died 28 Oct. 1918 of pneumonia

Gaskell, L. S. Capt., R.A.M.C.

1889

GAY, Rev. J. J. Chaplain, R.N.

1890

HGibbons, A. St H. Lieut.-Col., R. Fusiliers and King's 1878 (Liverpool Regt.) $M 2$.

Died I 5 Fuly 1916 of wounds received in action

GiBSON, I. F. 2nd Lieut., K. Edward's School, Birming- 1909 ham, O.T.C.

Gibson, Rev. R. M. C.F. 4th Class, R.A.C.D. I908

Gilbertson, W. Capt., R.A.M.C.

I 886

Gilmore, A. E. Lieut., Essex Regt. (W.)

1890

GLASER, W. H. 2nd Lieut. (T.), R.A.F.

Glen, R. A. Lieut., Middlesex Regt. (T.F.Res.)

GlenISTER, D. J. 2nd Lieut., R.G.A. (W.)

GoDDARD, F. W. 2nd Lieut., R.F.A. (W.)

1910

1894

1906

1912

GODDARD, J. Major, H.A.C. 
Gordon, F. J. Lieut., R.A.M.C.

Gower-ReEs, Rev. A. P. C.F. 2nd Class, R.A.C.D. (W.) *1903 M.C. $M$.

Grace, E. M. Capt., R.A.M.C.

Grant, F. G. Lieut., R.N.V.R.

1905

Graves, B. Capt., R.A.M.C. (W.) M.C.

Gray, W. H. Asst. Paymaster, R.N.

1902

1907

Greathead, J. M. Major, R.E. $M$.

I9II

*Greathead, J. R. 2nd Lieut., Rifle Brigade

Killed in action 23 Oct. I 916

Green, A. G. N. Lieut., Durham L.I.(T.F.) (P.)

GreEn, Gabriel. Lieut.-Col., Essex Regt. M.C.

1904

1914

Green, George. Capt., Loyal N. Lancs. Regt.; empld. Ministry of Munitions. (W.)

Greene, L. Capt., Natal and Orange Free State Regt., I90I S. African Force. (W.) D.S.O. M.C. M.

GreEnwood, C. F. 2nd Lieut., Northamptonshire Regt. 1913 and R.A.S.C.

Gribbin, Rev. J. A. C.F. 4th Class, R.A.C.D.

AGriffith, H. H. Capt. (A.), R.F.C.

Killed in flying accident 2 Nov. I917

Griffith, J. R. Capt., R.A.M.C.

Groves, J. D. Capt., Derbyshire Yeo. (W.)

Groves, W. PeER. Flt.-Cdr., R.N.V.R., attd. R.N.A.S.; 1908

* 1905

1894 Major, S.O. 2, R.A.F. Order of the Rising Sun, $4^{\text {th }}$ Class (Fapan)

GundRY, P. G. Lieut., R.N.V.R., attd. R.N.A.S.

GuTCH, J. Capt., R.A.M.C.(T.F.)

1913

1909

Gutch, W. Capt., Yorkshire Hussars; Staff Capt., War Office. $m$.

Hacking, A. Major, Gen. List. O.B.E.

Haddon, E. B. Hon. Capt., Uganda Carrier Coy.

1905

1899

I897

WHaIGH, A. G. 2nd Lieut., R.E.

Killed in action $15 \mathrm{Feb}$. 1916

Hales, G. T. 2nd Lieut., Worcestershire Regt.; empld. 1905 War Office

Hall, W. Capt., R.E. ('Tyne Electrical Engineers, T.F.); 1892 attd. R. Marines

Hamilton, A. Capt., R.A.M.C. (W 2.) Officer, Ordre 1902 de l'Etoile Noire (France)

Hance, J. B. Capt., I.M.S.

Hancock, Rev. W. H. M. C.F. 4th Class, R.A.C.D. 
HANDCOCK, W. A. S. Lieut., R.A.S.C.

HANDFORD, Rev. W. B. Chaplain, R.N.R.

HANDS, A.C. Lieut., R. Warwickshire Regt.; Lieut.(Ad.), I9r3 R.A.F.

WHANnINGTON, G. J. 2nd Lieut., R.A.S.C.(M.T.)

1903 Accidentally killed I Oct. I9I5

Hansell, G. F. Lieut., E. Yorks. Regt. and King's Own 1912 (Yorkshire L.I.) (W.)

Hanson, E. T. 2nd Lieut., R.G.A. (W.)

1901

HHARDing, Rev.W.J. Pte.,R.A.M.C.; Chaplain, R.N.V.R. $\quad$ I904 (R.N.D.) M.C.

Killed in action $3 \mathrm{I}$ Oct. 1917

HaRDingham, C. H. Lieut., R.G.A.

HARDY, E. W. D. Capt., R.A.M.C. (W.) M.C.

1896

HARE, W. T. Major, R.A.M.C.; D.A.D.M.S. M.C. 1907

Hargreaves, R. Capt., Border Regt. (T.F.)

HARPER, C. H. Major, R.F.A. M.C. and Bar

1910

HARRICKS, N. 2nd Lieut., R.A.S.C. and Spec. List

1906

HARRIS, A. A. A.B., R.N.V.R.; Lieut. (K.B.), R.A.F. $\quad 1899$

HARRIS, E. T. Major, I.M.S. D.S.O. $M_{3}$.

1896

HARRIS, H. E. Capt., R.A.M.C.(T.F.)

1878

HaRRIS, H. E. junr. Lieut., King's (Shropshire L.I.); [19I4] attd. T.M.B. (W.) M.C.

HaRrison, E. M. Capt., Lincolnshire Regt. (W 3.) I9I2

HARRISON, P. R.A.M.C.

1893

HaRveY, Rev.C.H. C.F. $4^{\text {th }}$ Class, N.Zealand Chaplains' I 898 Dept.

WHARveY, W. 2nd Lieut., King's (Liverpool Regt.)

Killed in action 25 Sept. I915

HaRveY, W. H. Capt., R.A.M.C.

I9I I

Harvie, E. F. Capt., Gordon Hdirs. (W 4.) M.C.

Killed in action 15 Fune 1918

HARVIE, J. K. Lieut., 3rd Hussars. (W 2.) I9I2

Hassard-ShORT, Rev. F. W. C.F. $4^{\text {th }}$ Class, R.A.C.D. 1892 (T.F.)

Hastings, W. Major, Egyptian A.M.C. O.B.E. $\quad$ M. $\quad{ }_{1898}$

WHatch, P. R. Capt., The Buffs (E. Kent Regt.) I9I I

Killed in action 7 Oct. I916

Hawkins, A. G. J. Lieut., Gen. List (Intelligence). M3. 1906

Hawkins, R. J. Lieut., R.F.A. (W.) I9I I

HaYton, Rev. J. D. W. C.F. 4 th Class, R.A.C.D. IgI I

HaYward, Rev. G. C.F. $4^{\text {th }}$ Class, R.A.C.D.

*I9II

Heaton, R. Capt., R.A.M.C. M. Serbian Distinguished I904

Service Medal 
Henderson, J. A. Capt., R.E.

HeNDRY, F. H. A. 2nd Lieut., Highland L.I. (W.)

Herklots, H. Lieut., R.A.S.C.

HerRing, J. H. Major (A.), R.A.F. (W.) D.S.O. M.C. 1908 $M$ 2. French Croix de Guerre.

Hetт, A. I. Lieut., Bedfordshire Regt. and The Buffs 1912 (E. Kent Regt.) (W.)

Heydon, G.A.M. Major, Australian A.M.C. (W.) M.C. 1900 WHindLe, H. B. Lieut., R.H.A. (W.)

Killed in action 27 March 1918

Hindley, Rev. W. T. C.F. 4th Class, R.A.C.D.(T.F.) $\quad 1899$

Hobson, W. W. 2nd Lieut., E. Yorks. Regt. 19r3

Hodges, C. E. Major, R.F.A.(T.F.); empld. Ministry * I903 of Munitions

Hodgson, A. E. Capt., R.A.M.C. $m$.

Hodgson, T. R. Capt., R.A.S.C.(T.F.)

Hodgson, W. Hammond. Capt., R.A.M.C.

Hodgson, W. HARRY. Lieut., King's Own (R. Lancaster Regt.) $M$.

Hoffmann, W. A. See Arnold, W.

HogarTh, T. J. Lieut., R.A.S.C.; Major, D.A.D. Labour 1902

Holland, C. C. Capt., R.A.S.C.

Holland, C. E. Lieut., R.N.R.

Holmes, H. W. H. Major, R.A.M.C. $M$

Holroyd, G. Capt., I.M.S.

Holt, R. Pte., R.A.M.C.

1903

I9OI

1899

HonY, G. B. 2nd Lieut., 4th (R. Irish) Dragoon Gds.; 1912

Lieut., Res. Regt. of Cavalry. (W.)

Hood, J. A. 2nd Lieut., R.A.S.C. $M$.

Hoon, Rev. J. C. F. C.F. $4^{\text {th }}$ Class, R.A.C.D.

Hooker, J. S. Lieut.-Col., I 2th Pioneers, Indian Army

HHorne, J. A. Pte., London Regt. (Artists Rifles); 2nd

Lieut., London Regt. (Queen's Westminster Rifles)

Killed in action 1 Fuly 1916

Horniman, J. E. 2nd Lieut., Essex Regt.

1896

1884

1913

1898

1910

1907

1902

1896

1910

Hosken, H. 2nd Lieut., Rifle Brigade; Capt., O.C.B.

Houlton, J. W. Capt., Suffolk Regt. (W.)

Hudson, N. B. Lieut.-Col., R. Berkshire Regt. (W 3.) D.S.O. and Bar. M.C. and Bar. $M 2$.

HumphreYs, W. H. Cadet, O.C.B.

Hurst, W. H. Lieut., R.A.S.C.

INCE, S. R. 2nd Lieut., K. Edward's School, Bath, 1904 O.T.C. 
InNocent, A. Trooper, Canadian Mtd. Rifles

INGles, W. H. S. Pte., Oxford and Bucks. L.I.

Iredale, H. C. Lieut., Bedfordshire Regt. (W 2.)

Jackson, A. C. D. Lieut., E. Lancs. Regt.; Capt., Gen. 1901 List, empld. Inland Waterways and Docks

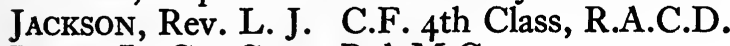

JACOB, L. G. Capt., R.A.M.C.

JAMEs, A. E. G. Sergt., M.G.C.

James, Rev. T. J. C.F. $4^{\text {th }}$ Class, R.A.C.D. M.C.

WJames, W. M. Capt., Monmouthshire Regt.

Killed in action 8 Oct. 1918

Jarvis, F. J. Pte., Australian Force

Jenkin, N. W. Capt., R.A.M.C.

Jenkins, J. Capt., Welsh Regt. (W.) $M$.

1903

1909

* 1912

1895

I890

Jessop, G. L. Capt., Manchester Regt. and Gen. List, 1896

* 1904

I901

1909 empld. Ministry of National Service

WJewitT, D. P. 2nd Lieut., Worcestershire Yeo.

Killed in action 23 April 1916

Johnson, A. V. Capt., Worcestershire Regt.

1912

JoNes, E. Capt., R.E. $M 2$.

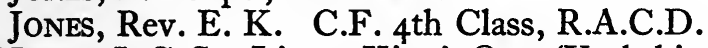

1912

I9I I

1890

सJonES, I. C.S. Lieut., King's Own (Yorkshire L.I., T.F.) I9I3

Died 21 Sept. 1916 of wounds received in action

Jones, L. T. P. 2nd Lieut., The Buffs (E. Kent Regt.) 1897

JoNes, R. T. P. Capt., R.F.A.

Jones, W. B. Lieut.-Col., Welsh Regt.

I 897

I 883

JoNEs, W. H. Surgeon Lieut., R.N.

WJOSEPH, W. F. G. 2nd Lieut., R. Berkshire Regt.

Killed in action 27 May r9 8

JupP, A. O. Lieut., Spec. List (Recruiting Staff)

1900

I9OI

I895

KaPp, E. Lieut., R. Sussex Regt.

WKaRIM KHAN, A. Major, 129th Baluchis, Indian Army Killed in action (3 1 Dec. I9r4)

I910

I 894

Kearns, H. W. L. Lieut., R.F.A.(T.F.); Staff Capt. M. 1909 Belgian Croix de Guerre

WKelleher, H. Pte., Canadian Infy.

Killed in action April 1915

Kempson, Rev. J. H. C.F. 3 rd Class, R.A.C.D. $M . \quad 1889$

HEnnedy, R. S. Capt., R.A.M.C. M.C. M.

Killed in action 17 April 1918

Kent, Rev. N. B. Chaplain, R.N.

I910

1906

1905 
KerR, J. G. 2nd Lieut., Cameronians (Scottish Rifles, 1892 T.F.)

\#KItTtermaster, A. N. C. Capt., Worcestershire Regt. $\quad$ I890 Killed in action 5 April 1916

Knight, R. Lieut., Middlesex Regt.; Capt., T.M.B. rgI I (W 2.) M.C.

LADD, L. S. Capt. and Adjt., R.A.S.C. $M$. I9I I

LAMBERT, F. 2nd Lieut., R.G.A. 1902

LANE, P. Lieut., R.E.(T.F.) 1912

LANGERMan, A. H. R. S. African Force 1903

LANGERman, E. S. S. African Force 1907

LARYMORE, H. D., c.M.g. Major, R.G.A.; Asst. Inspector, 1903 Woolwich Arsenal

LAw, R. R. Capt., R.A.M.C. $\quad \mathbf{8 8 6 6}$

LAZARUS, E.L. Lieut.,Worcestershire Regt.; attd.M.G.C. I9ro (W 2.)

LeACH, Rev. N. K. C.F. 4th Class, R.A.C.D. . 1900

LEACH, R. W. Capt., Suffolk Regt.; Brigade Major. 1903 (W.)

LEE, A. 2nd Lieut., Unattd. List, T.F. (O.T.C.) 1899

LEE, W. E. Corpl., R.E. $\quad 1909$

LEECH, E. B. Capt., R.A.M.C. (W.)

LewIs, F. H. Capt., R. Welsh Fus. (W.)

WLEwIS, R. C. Capt., R. Berkshire Regt. M.C.

Killed in action on the Somme 1 fuly 1916

LEwIS, W. B. A. Lieut., King's (Shropshire L.I.) (W.) 19 I I

Liptrot, R. N. Pte., Cheshire Regt.(T.F.); Lieut., 1908 R.F.A.

Livens, W. H. Capt., R.E.; Staff Capt. D.S.O. M.C. 1908 $M_{3}$.

Llewellyn, E. E. Surgeon Lieut., R.N.

Lloyd George, R. Capt., R. Welsh Fus.; Major, R.E. 1907

Lovibond, J. L. Major, Northumberland Fus. (T.F.); 1893 empld. P. of W. Coy. (W.) T.D. $M$.

Low, Rev. W. P. C.F. 4th Class, R.A.C.D. $M . \quad 1895$

Lowe, Rev. C. A. H. C.F. $4^{\text {th }}$ Class, R.A.C.D. $M . \quad 1909$

LuCE, Sir R. H. Major-Gen., A.M.S.(T.F.); Director of 1886 Medical Services. K.C.M.G. C.B. C.M.G. V.D. $M 3$.

Lumby, A. F. R. Capt., 69th Punjabis, Indian Army; 1909 G.S.O. 2. (W)

ELundie, R. C. Major, R.E. (W.) D.S.O. M 2. Killed in action 15 Oct. 1918 
MaAsDorP, V. H. Lieut., R. Fusiliers. (W 2.)

McAllum, J. H. Capt., R.A.M.C.

MCARThur, R. Lieut., R.F.A.(T.F.) (P.) M.C.

McCall, H. D. Capt., R.A.M.C.

MacCallan, A. F. Major, R.A.M.C., empld. Egyptian r89I Army. C.B.E. O.B.E. M.

McColl, A. M. Sergt., King's Royal Rifle Corps; Cadet, I9I3 R.A.F. M.M. and Bar

McColl, H. H. 2nd Lieut., Rifle Brigade; Capt., D. of 1912 Wellington's (W. Riding Regt.); Hon. Capt. (O.), R.A.F. (W 3.) M.C. M.

MaCDONALD, Rev. H. Chaplain, R.N.

McDougall, A. Capt., London Yeo.(Middlesex Hussars). D.S.O.

McGowan, N. S. 2nd Lieut., Sikhs, Indian Army. (W.) 1909

MCINNES, A. N. 2nd Lieut., Northumberland Fus.(T.F.) I9I4

McIntosh, J. G. H. Capt., Scottish Horse; attd. Cam- I895 eron Hdrs.

MACK, A. A. Sergt., R.A.S.C.; attd. R.A.M.C.

सMACKAY, D. R. G. Pte., R. Fusiliers (P.S. Bn.); 2nd [1914] Lieut., Argyll and Sutherland Hdrs.; Capt. (A.), R.A.F. (W.) D.F.C.

Died I I Nov. I9I 8 of wounds received in action

McLean, K. G. Lieut., R.E.

MACNAB, J. T. Surgeon Lieut., R.N.

*McVitTie, G. H. 2nd Lieut., Border Regt.(T.F.)

Died on H.M. transport 12 Mar. I9I 5 of cerebro-spinal

1904 meningitis

Malim, J. W. Capt., R.A.M.C.(T.F.). $m$.

MALONE, C. R. R. Lieut.-Col., Hampshire Regt.; Major, 1877 Worcestershire Regt. (R. of O.) $m$.

Mann, F. A. W. Pte., R.Fusiliers (P.S.Bn.); Lieut. (A.), [1914] R.A.F. (W.)

MANNERS-Smith, E. V. Cadet, R.M.A., Woolwich [I9I4] Mardon, E. J. Major, Devon Regt. and Rifle Brigade 1886 (T.F.) $m 2$.

MARKham, E. B. Lieut., Lincolnshire Regt. and Gen. I9r2 Staff, empld. British Military Mission

Marquand, C. V. B. and Lieut., Tank Corps.

Marriot, Rev. F. G. C.F. $4^{\text {th }}$ Class, R.A.C.D.

Marrot 1912

MARTiN, R. G. 2nd Lieut., Plymouth College O.T.C. $\quad$ I909

Masterman, W. S. Major, Welsh Regt. $\quad$ I897

Masters, Rev. T.H. C.F. Ist Class, R.A.C.D.; A.C.G. 1886 C.B.E. M2. 
Maufe, H. B. Lieut., Rhodesia Motor Vols.

WMaule, G. L. Capt., R.A.M.C.

Died at Baghdad 15 Nov. I918 of pneumonia

WMaule, R. Lieut., R. Scots(T.F.)

Killed in action in Gallipoli 27 May 1915

Maw, R. P. Lieut., R.G.A.

Maxwell, W. W. Major, R.G.A.

May, A. H. Lieut.-Col. R.E. O.B.E. m.

May, C. P. Pte., Middlesex Regt. (P.S. Bn.); Lieut., The 1896 Buffs (E. Kent Regt.)

MAYBREY, H. J. Lieut., Wiltshire Regt. and Worcestershire Regt.; empld. Ministry of Munitions

WMayer, F. C. Sergt., 52 me Regt., French Army. French Croix de Guerre. M2.

Killed in action in Champagne 21 Sept. 1915

Mayer, N. E. Pte., R. Fusiliers; 2nd Lieut., Spec. List. $\quad$ I 908 $M$.

Mayne, C. R. G., D.s.o. Major, Highland L.I.; Brig.- $\quad$ I893 Gen. (W 2.) C.M.G. Brevet Lieut.-Colonel. M 5. Osmanieh, $4^{\text {th }}$ Class and Medjidieh, $3^{\text {rd }}$ Class (Egypt)

MeE, J. T. M. Capt., Suffolk Yeo. $M$.

Melitus, P. N. Lieut., R. Warwickshire Regt.; Hon. Lieut. (O.), R.A.F.

Melling, J. S. Pte., Cheshire Regt.; 2nd Lieut., R. 1909 Welsh Fus.

Mercer, Ven. H. F. Capt., Australian Force; Capt.(Ad.), I890 R.A.F.

MERCER, J. Instructor Cdr., R. N.

WMere, C. L. Lieut., King's Own (R. Lancaster Regt.)

Killed in action in Gallipoli ro Aug. 1915

Midgley, W. A. L. Lieut., R.G.A.

WMilne, G. W. Lieut. and Adjt., R.F.A. (W.)

Died 22 Oct. I917 of wounds received in action

Misquith, O. G. Lieut., London Regt. (Queen's) I9I I

*1910

1907

1905

I9I 5

Mobbs, G. F. Pte., London Regt. (Artists Rifles). (W.) 1916

MoncriefF, A. Capt., R.E.; S.O. to Chief Engineer. 1912 (W 2.) M.C. M.

Montgomery, J. K. Lieut., Spec. List (Censor's Staff) I892

Moore, R. F. Capt., R.A.M.C. O.B.E. M. $*_{1} 898$

AMoore, R. T. Trooper, R. Wiltshire Yeo.

Drowned on S.S. Leinster 1o Oct. I9I8

I910

Morris, G. P. Lieut., Oxford and Bucks L.I.; Major, I9II M.G.C. 
Morrison, J. T. J. Lieut.-Col., R.A.M.C.(T.F.) * * 88 I Mosley, I. H. Lieut., H.A.C.; Capt., Spec. List (Adjt., Igor School of Instruction). M.C.

Mountarn, H. Lieut.-Col., R.F.A.

1898

Mowll, G. M. Cadet, O.C.B.

Murray, E. G. D. Capt., R.A.M.C. O.B.E.

Murray, Rev. E. T. C.F. $4^{\text {th }}$ Class, R.A.C.D. (W.) I895

Muspratt, P. K. Capt., R.A.M.C.

NAOROJI, K. A. D. Sergt., Middlesex Regt. (W.) I9I2

NAPIER, O. J. W. 2nd Lieut. (T.), R.A.F.

1908

NeEdHAM, E. Lieut., E. Surrey Regt.; Major, M.G.C. $\quad$ I907

(W.) (P.) $M$.

सNELDER, G. C. A. 2nd Lieut., Hampshire Regt. 1912

Killed in action at Suvla Bay 6 Aug. 191 5

Nelson, E. W. Lieut.-Cdr., R.N.V.R. M.

Nelson, R. D. Capt., R.F.A.

Nelson, T. B. 2nd Lieut. (Ad.), R.A.F.

Newman, E. Capt., R.A.O.C.; D.A.D.O.S.; attd., I90I

R.A.F. Cavalier, Order of the Crown of Italy

WNewman, J. S. 2nd Lieut., E. Yorks. Regt.

Killed in action at Suvla Bay 9 Aug. I9I 5

Newton, G. F. Pte., H.A.C.

Nicholas, A. J. Lieut., R. Welsh Fus.

NICKAL, G. B. 2nd Lieut., R.A.F.

Nightingale, H. P. C.Q.M.S., Queen's Own (R. W. Kent Regt.)

Nixon, Rev. W. H. C.F. and Class, R.A.C.D. m. $\quad 1883$

Noble, Rev. R. H. C.F. 4th Class, R.A.C.D. ${ }^{*}$ I9I6

NutTall, E. D. Lieut., R.G.A.

Nutrall, W. L. F. 2nd Lieut. (A.), R.A.F. D.F.C. 1916

WOdell, R.E. Pte., R. Fusiliers (P.S. Bn.); Lieut., Black r9r3

Watch

Died $20 \mathrm{Dec}$. 1916 of wounds received in action $18 \mathrm{Dec}$. I9I 6

OgILvie, J. Capt., R.A.M.C.

1886

O'ReILly, J. B. Major, Durham L.I. and Leinster Regt. $\quad 1878$ HORMESHER, H. Lieut., Lincolnshire Regt.

Killed in action 4 Oct. I9I 5

OrmSBY, M. H. Lieut., I.A.R.O., attd. S. Provinces Mtd. I896

Rifles; Major and Cmdt., Clerks Training School

OrTon, W. A. Lieut., Dorset Regt.; attd. T.M.B. (W.) 1916

OsBorne, D. R. Major, Northumberland Fus.

I90I

C. U.v.L. 
ÆOsborne, L. H. Lieut., Lancs. Fus.

Killed in action in Gallipoli 7 Aug. 1915

Otway, J. T. F. Hon. Lieut.-Col., Spec. List

Oulton, E. V. Major, R.A.M.C. $M$.

OwEN, G. Lieut., N. Zealand Rifle Brigade. (W.)

Owen, P. R. T. 2nd Lieut., The Buffs (E. Kent Regt.) (W.)

Paget, H. E. G. Capt., 46th Punjabis, Indian Army

PARKER, Rev. J. C. Chaplain, R.N. $M$.

PARRY, T. H., M.P. Lieut.-Col., R. Welsh Fus.(T.F.)

(W 2.) D.S.O. M. Order of the Nile, $4^{\text {th Class }}$ (Egypt)

Partridge, C. Lieut., Spec. List (R.T.O.)

Patrick, N. C. Capt., R.A.M.C.

\&Payton, R. S. Lieut., R. Warwickshire Regt.

Killed in action 22 Fuly I916

Peck, A. H. Lieut., Devon Regt.; Major (A.), R.A.F. 1906 D.S.O. M.C. and Bar. M.

PeCK, E. S. Lieut.-Col., I.M.S.

PeErs, E. A. 2nd Lieut., Felsted School O.T.C.

Peile, J. Lieut., E. Surrey Regt.; Capt., Gen. List

1870

1900

1902

1907

1905

1904

1900

I 892

1895

1913

Peters, A. J. Pte., Middlesex Regt. (P.S. Bn.); Capt., S.

Lancs. Regt.

Peters, M.W. Lieut., King's Royal Rifle Corps and M.G.C. (W 2.) M.C.

Pettit, J. R. Lieut., R.A.S.C.(M.T.) $M$.

Peyton-Burbery, Rev. R. J. P. Chaplain, R.N.

Phillips, E. S. Major, R.G.A. D.S.O. $M$.

Phillips, J. E. Capt., O.T.C.

Wickard-Cambridge, H. E. W. Lieut., Sussex Yeo.

Killed in action $\mathrm{I}$ Nov. I9I 7

Pigeon, H. W. Lieut., R.A.M.C. $m$.

PPigeon, J. W. Capt., I.M.S.

Pim, F. H. Capt., R.A.S.C.

PIRKIS, F. C. L. Lieut., R.F.A.; Staff Lieut., War Office. (W.)

Pither, F. E. L. 2nd Lieut., Somerset L.I.

Pope, S. B. Lieut.-Col., 58th (Vaughan's) Rifles, Indian Army; G.S.O. I. D.S.O. Brevet Lieut.-Colonel. $M_{4}$. Chevalier, Legion of Honour (France). Order of the Nile, 3 rd Class (Egypt)

PorTer, R. E. Major, R.A.S.C. M.C.

1885

1909

I 897

I9II

1907

1912

1901

I 895

1903

I9I 5

I 877

1905

I 897

I895

I9I 4

I 898

1 Killed in action in Mesopotamia after the armistice. 
Priestley, R. E. Capt., R.E. (Signals, T.F.). M.C.

I9I3

Pumphrey, C. E. Capt., Durham L.I. (W.) M.C. M 2. 1899

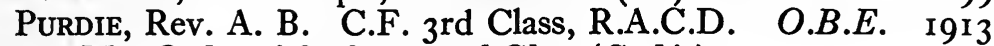
M. Order of St Sava, 3 rd Class (Serbia)

Purton, G. A. Lce.-Corpl., Training Res. Bn.

Pyman, W.H. Lieut., R.F.A.; empld. Ministry of Ship- 1905 ping

Quillet, L. A. Lieut., R.A.S.C.

I9I4

Radice, W. A. Capt. and Adjt., Calcutta Bn., Indian I90I Defence Force

RAmSAy,D. 2nd Lieut., The Queen's(R.W.Surrey Regt.) I906 WRAMSAY, L. N. G. 2nd Lieut., Gordon Hdrs. I9I I

Killed in action 21 March I91 5

Ramsay, M. G. 2nd Lieut., The Buffs (E. Kent Regt.) 1909

RANSFORD, W. M. Capt., R.E. M.C.

RAPPIS, P.A. G. 2nd Lieut., Wireless Corps, Italian Army

READ, R. S. Lieut., Suffolk Regt.; empld. Ministry of Labour. (W 2.)

AREED, B. Lieut., W. Yorks. Regt. (W 2.)

Killed in action 12 April 1918

I 909

1899

I9I 5

* 19 I 3

REED, C. H. Capt., R.E. (London Electrical Engineers, I900 T.F.)

ReEs, T. J. Lieut., Welsh Regt.(T.F.); attd. R.E. HREese, J. Wireless Telegraphist, R.N.

Killed in action on H.M. patrol boat 10 Feb. 1918

1908

1915

Reid, E. D. W. See Whitehead Reid, E. D.

Rendle, A. C. Capt., R.A.M.C.

सRice-Jones, A. T. Capt., King's (Liverpool Regt.) 1909

(W 2.)

Died 23 March 1918 of wounds received in action

Rice-Jones, B. R. Lieut., R.G.A.(T.F.) (W.)

RICHARDS, C.T. 2nd Lieut, R.G.A. . Lieut. (Ad),R. 1907

RICHARDS, W. G. Major, I.M.S.

Richardson, D. J. A. Gnr., R.G.A.

Richardson, G. P. N. Capt., R.A.M.C.

Richardson, J. H. Asst. Paymaster, Army Pay Dept.

Richardson Kuhlmann, D. See Richardson, D. J. A. WRIEU, A. Pte., 2me Regt. Etranger, French Army, and Middlesex Regt.

Killed in action 3 fuly rgi6

Rigby, Rev. P. C.F. 4th Class, R.A.C.D. $\quad 1904$

Riley, A. Air Mechanic, R.A.F. 
Roberts, F. H. Lieut., R. Sussex Regt.(T.F.)

RoberTs, Rev. Ll. C. C.F. $4^{\text {th }}$ Class, R.A.C.D.

Robertson, D. D. A.B., R.N.V.R. (Anti-Aircraft)

Robins, J. N. Capt., R.A.M.C.(T.F.). (W.)

RoBinon A C Lieut.Col.R A S.C.A A and M.G. 1895

D.S.O. M. Order of the Nile, $4^{\text {th Class (Egypt) }}$

Robson, Rev. E. I. Lieut., Spec. List (Intelligence) I892

ROGERS, J. L. Lieut., R.G.A.

Romanes, J. Capt., Border Regt.; empld. War Office. 1905 (W.)

Rose, C. A. Lieut., R.F.A. (W.) M.C.

Rouse, W. H. D. Lieut., Perse School, Cambridge,

O.T.C.

Rubinstein, L. Russian Army

Rudkins, F. P. Lieut., Emanuel School, Wandsworth, 1907 O.T.C.

Russell, J. 2nd Lieut., Gordon Hdrs. (W 2.)

I9I 5

SANER, F. D. Capt., R.A.M.C.

SAUNDERS, A. H. Instructor Lieut., R.N.

1902

1908

SAunders, J. T. Capt., Durham L.I. (W 2.)

1907

Saunders, Rev. W. D. C.F. 4 th Class, R.A.C.D. $m . \quad{ }^{*}$ I 9 I I

ScharfF, G. E. Sapper, R.E.

*Schwarz, R. O. Capt., S. African Infy.; Major, King's

Royal Rifle Corps; D.A.Q.M.G.; Asst. Controller of Salvage. (W.) M.C. M.

Died 20 Nov. 19r8 of influenza contracted on active service

ScotT, J. H. Capt., York and Lancaster Regt.

ScotT, L. B. Major, I.M.S.

\#SEABRooke, A. S. Capt., R.A.M.C.

Died in Mesopotamia I Fuly I916 of typhoid

Selby, J. S. E. Capt., R.A.M.C.

1909

1893

SeVERnE, A. DE M. 2nd Lieut., R.F.A.; Lieut. (A.), $\quad$ I915

R.A.F. (W.)

Sewell, H. W. Capt., Border Regt.

1905

1894

1903

1886

SEwell, R. B. S. Major, I.M.S. $M$.

Shanks, P. M. 2nd Lieut., St John's School, Leatherhead, O.T.C.

Sharp, D. G. Lieut., W. Yorks. Regt.; Capt., T.M.B. I9I5 M.C.

Sharpe, G. R. Lieut., Loyal N. Lancs. Regt. (W 3.) I9I2

Shell, Rev. A. Chaplain, R.N.

Shelley, J. Pte., R.A.S.C. 
Shennan, W. D. Capt., R.E. M.C. $M$.

ShePHARD, W. H. Capt., R.A.M.C.; D.A.D.M.S. (W.) 1908

SHEPPARD, W. S. Capt., R.A.M.C.

ShIELDS, T. Major, R. Berkshire Regt.(T.F.)

$\mathrm{r} 889$

I88I

WSHILCOCK, J.W. Lieut., The Queen's (R.W.Surrey Regt.) 1907

Killed in action near Baghdad 22-24 Nov. 191 5

ShInER, Rev. R. P. C.F. 4th Class, R.A.C.D.

Shufflebottom, E. Pte., Essex Regt.; 2nd Lieut., R. Fusiliers

Shurlock, F. W. Pte., R. Fusiliers (P.S. Bn.); Instructor

Lieut., R.N.

Simpson, A. J. G. Major, N.S.W. Bn., Australian Force 1906 Simpson, A. W. W. Lieut.-Col., Manchester Regt. (T.F.)

O.B.E. Brevet Lieut.-Colonel. M3. Order of the

Nile, 3 rd Class (Egypt)

WSimpson, G. B. G. Pte., Australian Infy.

Killed in action in Gallipoli 6 Aug. I9 5

Simpson, J.G. Pte., London Regt.(Artists Rifles); Lieut., 1908

R. Sussex Regt. (W 2.) M.C.

सSing, C.M. Pte., R. Fusiliers; 2nd Lieut., R. Sussex Regt. 1907

Died 7 July $19 \mathrm{I} 6$ of wounds received in action

SKYRME, C. R. Capt., R.A.M.C.

1892

Slaughter, C.E. Capt., R.A.S.C.; attd.R.G.A. (W.) $M$. I9I3

SleEman, C. M. Lieut., R.N.V.R.

SMALl, D. W. Lieut., R.E.

1902

1914

Smith, A.C.Denison. Lieut.,E. Yorks. Regt. (W.) M.C. 1906

Smith, A. E. Clarence. Capt., S. Lancs. Regt.

Smith, A. E. S. Lieut., R.A.S.C.

*Smith, C. R. B. 2nd Lieut., Bedfordshire Regt.

Killed in action 28 April I9I7

Smith, F. C. Cadet, O.C.B. (R.F.A.)

Smith, J. Capt., R.F.A. (W.)

Smith, J. T. Lieut., Sussex Yeo. (W.)

Smith, W. H. 2nd Lieut., Coldstream Gds.

1906

I 893

I9I 5

Smith-Carington, Rev.A.E.C. C.F.4th Class, R.A.C.D. 1909

Smithson, A. E. Lieut.-Col., R.A.M.C.

I9I7

1912

I9I 5

1905

Smuts, Rt Hon. J. C. Lieut.-Gen.; Cmdt.-Gen., S. $\quad$ I89I

African Defence Force; Member of Imp.War Cabinet.

Companion of Honour. Commander, Legion of Honour

(France). Grand Officer, Order of Leopold (Belgium).

Belgian Croix de Guerre

SNAPE, H. J. Capt., Repton School O.T.C.

SNowDEn, A. DE W. Hon. Major, R.A.M.C. (Red Cross 1891 Hospital, Netley). C.B.E. 
Sowels, F. Lce.-Corpl., Oxford and Bucks. L.I.; Capt., 1895 Gen. Staff (Intelligence). Chevalier, Order of the Redeemer (Greece)

Spencer, Rev. L. D. W. C.F. 3 rd Class, R.A.C.D.(T.F.) 1896 Spurrell, R. K. Capt., D. of Cornwall's L.I. (W 2.) [I9r4] M.C. $M$.

SQuire, A. M. 2nd Lieut., R.A.O.C.

Souire, S. G. Lieut., Oundle School O.T.C.

WSTANDEN, L. J. D. Lieut., Lincolnshire Regt.(T.F.)

Killed in action 18 March 1916

Stanton, H. J. C. Staff Capt., War Office. $m 2$. 1879

WStarkey, V. G. Pte., R. Fusiliers (P. S. Bn.); Lieut., 1913

King's Own (Yorkshire L.I.)

Killed in action I4 Oct. I9I 5

Stavers, W. M. Capt., R. Scots Fus. (W.) I9II

Steen, S. W. P. 2nd Lieut., Rifle Brigade. (W.) I9I8

Stephens, Rev. H. H. C.F. 4th Class, R.A.C.D. 1916

Stephenson, Rev. H. S. Senior Chaplain, Indian Army 1894

Stevens, F. B. Lieut., Sussex Yeo.; Capt. (K.B.), R.A.F. $\quad 1899$ $M$.

Stewart, C. B. Capt., R.A.M.C.(T.F.) I883

Stewart, P. M. Major, R. Fusiliers (P. S. Bn.) and 1890

Training Res. Bn.

Still, H. N. 2nd Lieut., Shropshire Yeo.; Lieut., 1912

R.G.A. $m$.

Still. Rev. W. H. C.F. $4^{\text {th }}$ Class, R.A.C.D.

Stolterforth, G. H. Lieut., Cheshire Regt. and 1897

M.G.C. (W.)

Strachan, Rev. R. H. C.F. $4^{\text {th }}$ Class, R.A.C.D. $m$. IgII

Sullivan, Rev. A. M. C.F. 4th Class, R.A.C.D.(T.F.) $\quad$ I897

Sullivan, J. H. B. Lieut., I.A.R.O. (Cavalry) I909

Sutton, Rev. F. O. C.F. 4 th Class, R.A.C.D.(T.F.) $M . \quad{ }^{*}{ }_{1883}$

Swann, C. H. Lieut., R.N.V.R.

\#TANNER, G. R. Lieut., Wiltshire Regt. (W 3.) M.C. I9r3

Died 8 April I $9 \mathrm{I} 8$ of wounds received in action 24 March I9I 8

TANNer, H. R. Capt., Somerset L.I. (W.)

TANNER, R. R. Lieut.-Col., Rifle Brigade (T.F.)

TAYLOR, C. H. Major, York and Lancaster Regt. and 1883

King's Own (Yorkshire L.I.)

TAYlOR, D. R. Capt., R.A.M.C. $\quad 1898$

TAYLOR, G. C. Major, R.A.M.C.(T.F.); D.A.D.M.S. $\quad{ }^{1887}$

O.B.E. $M_{2}$ 
Theophilus, S. C. Pte., H.A.C.; Lieut., Rifle Brigade ; 1903 Capt., T.M.B.; Major, Tank Corps. M.C.

Thomas, A. A. Lieut., Welsh Regt.

I9I I

Thomas, E. C. Capt., Suffolk Regt.

1894

Thomas, T. J. Lieut., R. Welsh Fus.; attd. T.M.B. 1902 (W.) M.C.

Thomas, W. Major, S. African Med. Corps. O.B.E. M. $\quad{ }_{1889}$

Thompson, Rev. A. E. C.F. 4th Class, R.A.C.D. $\quad 1898$

Thompson, A. P. Capt., R.A.S.C. $\quad 1912$

Thompson, G. H. M. Lieut., Spec. List, empld. R.E. I910

(Inland Water Transport)

Thompson, G. W. Capt., R.A.M.C.

Thompson, P. A. Capt., R.A.S.C.

1886

ThORNE, H.S. Lieut., R. Scots; attd. Gen. Staff. (W.) IgI I

Thurston, E. T. Lieut., I.A.R.O., attd. Garrison Arty., 1908 Indian Defence Force

Tindall, C. Lieut., R.E.

ToDD, M. Lieut., R.F.A. (W.)

\&Townroe, G. C. Capt., S. Lancs. Regt.

Killed in action 8 Sept. I9I7

Townson, B.A. Lieut., The Buffs (E. Kent Regt., T.F. I9II Res.)

ATRemearne, A. J. N. Major, Seaforth Hdrs. Killed in action in the Battle of Loos 25 Sept. 1915

ATREMEARNe, W. C. 2nd Lieut., Seaforth Hdrs.

Killed in action in the Battle of Loos 26 Sept. 191 5

Trend, J. B. Capt., R.A.S.C.; Lieut., R.G.A.; empld. 1906 War Office. $m$.

Trendell, Rev. G. J. W. C.F. 4th Class, R.A.C.D. 1883

Trestrail, A. E. Y. Lieut.-Col., Cheshire Regt. (W.) 1894 D.S.O. $M$.

Turkington, J. S. Trooper, R. Canadian Dragoons; .1912

Lieut., Gen. List, Canadian Force. (W 2.)

Turnbull, E.L. 2nd Lieut., King's Royal Rifle Corps. (W.) 1907

ATURner, E. P. Capt., R.F.A.

Killed in action 19 March 1917

Turner, H. W. Lieut., Spec. List (Intelligence) . * *906

TURNER, R. L. Lieut., I.A.R.O.; Capt. and Adjt., 3rd 1907

Gurkha Rifles (attd.) M.C. M.

Turner, W. H. Major, R.F.A.(T.F.) M.C. $M$.

ATURPIN, J. K. Lieut., R.F.A.(T.F.)

Killed in action 14 Aug. I917

Twentyman, J. M. Capt., R.A.M.C.

Twentyman, Rev. W. P. C.F. $4^{\text {th }}$ Class, R.A.C.D.

1912

I9II

I 888

1897 
Ullrich, E. H. Pte., Middlesex Regt.; Sapper, R.E. $\quad$ I9I4 (Signals)

Unwin, C. H. Major, D. of Wellington's (W. Riding 1896 Regt.); empld. O.C.B. (W.) $M$.

UPWARD, H. A. Capt., R.A.M.C.

VARLEY, G. Sergt., R. Fusiliers

Veale, H. P. Major, S. African Med. Corps.

Vint, A. W. Capt., Worcestershire Regt. M.C.

Vint, M. D. Capt., R.A.M.C. (W.) $M$.

Voelcker, H. E. 2nd Lieut., S. Lancs. Regt. (W.)

Killed in action 20 Fuly 1916

Waddy, R. A. Pte., London Regt. (Artists Rifles)

WADE, R. Capt., R.A.M.C.

Wadham, S. M. Lieut., Durham L.I.; Capt., Spec. I9ro List, empld. R.E. $M$.

Wagner, R. H. Capt., London Regt. (R. Fusiliers); attd. I9ro R.E. (Signals). M.C.

WaIAPU, Rt Rev. Bishop of. C.F. 3 rd Class, N. Zealand 1879 Chaplains' Dept.

WWainwright, G. L. Pte., Middlesex Regt. (P.S. Bn.); I9I3 2nd Lieut., R. Sussex Regt.

Killed in action 25 Sept. I9r 5

Walker, D. G. Capt., Highland L.I. (W.)

WaLkeR, F. C. Lieut., Yorkshire Regt. (W.)

Walker, K. M. Capt., Suffolk Regt. (W 2.)

WALKEY, Rev. J R. C.F and Class, R A.C D. A C. [1914] $M$.

Wallis, H. H. Lieut., Trent College O.T.C.

WARD, F. K. Lieut., I.A.R.O., attd. II6th Mahrattas

WARD, R. 2nd Lieut., R.G.A.

Warren, Rev. S. C.F. $4^{\text {th }}$ Class, R.A.C.D.

Waterworth, Rev. H. C.F. 4th Class, R.A.C.D.

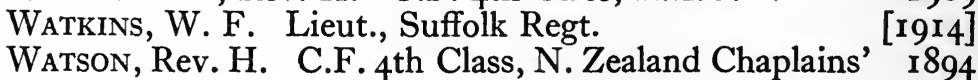
Dept.

Watson, H. S. 2nd Lieut., R.E. (Tyne Electrical Eng- 1895 ineers, T.F.)

Watson, Rev. R. C.F. 4th Class, R.A.C.D. (W.) $\quad 1889$

Watson, T. T. B. Capt., R.A.M.C.

WWebb, A. H. Pte., R. Fusiliers (P. S. Bn.); 2nd Lieut., I9I2 Leicestershire Regt.

Died 4 May r916 
Webb, E. O. Pte., London Regt. (Artists Rifles); 2nd I9I I Lieut., M.G.C.

WWebB, H. C. Capt., Border Regt.; attd. T.M.B. Killed in action 19 Sept. 1916

WedDELl, J. M. Capt., R.A.M.C. $M 2$.

WWestwood, A. H. Capt., R. Warwickshire Regt. (W 2.) Killed in action 21 Sept. 1918

WWhaley, O. S. Pte., R. Fusiliers (P.S. Bn.); 2nd Lieut., 1908 Hampshire Regt.

Killed in action in Gallipoli 10 Aug. 1915

WheAT, E. G. Capt., R.A.M.C.

WheElER, J. N. Major, R.A.M.C. $M$.

WheEler, Rev. S. M. C.F. $4^{\text {th }}$ Class, R.A.C.D.

WWhite, L. 2nd Lieut., Welsh Regt.

Killed in action 19 March 1916

WhiteheAD, H. M. Capt., Sherwood Foresters (Notts. $\quad$ I893 and Derby Regt.); A.D.C.; Lieut.-Col., Labour Corps. O.B.E. Chevalier, Ordre du Mérite Agricole (France)

WhItEHEAD, J. H. M. Capt., W. African Frontier Force

WhiteHEAD, R. B. Lieut., King's Royal Rifle Corps; attd. R.E. (Signals). $M$.

Whitehead Reid, E. D. Capt., R.A.M.C.

Whiteman, R. J. N. Major, Australian A.M.C.

WILES, H. H. Lieut., Wiltshire Regt.; Capt., Devon Regt.; Capt. and Adjt., Labour Corps. (W.)

WILES, J. W. Hon. Capt., Serbian Army. Serbian Royal *19I6 Red Cross

Wilkinson, S. J. Capt., R.A.S.C. $M$. $m$.

1892

1908

1902

1907

Wilkinson, V. Capt. and Adjt., Border Regt. M.C. I9I I $M$.

Williams, A. Major, Gen. Staff. O.B.E. M. Cavalier, 1882 Order of the Crown of Italy

Williams, Rev. C. M. C.F. $4^{\text {th }}$ Class, R.A.C.D.

*1897

Williams, D. G. Lieut., 4th (R. Irish) Dragoon Gds. $\quad$ I9I2

Williams, E. C. Capt., R.A.M.C.; D.A.D.M.S. I 885

Williams, F. L. Capt., Bedfordshire Regt. and Gen. I9IO List. (W.) $M$.

Williams, H. H. Capt., R.A.S.C. (M.T.) M.B.E. $\quad$ m. ${ }^{\cdot} \quad 1900$

Williams, Rev. J. E. C.F. 4th Class, R.A.C.D. $m$ 2. * * 1909 WWilliams, K. G. 2nd Lieut., N. Zealand M.G.C. 1906 Killed in action 9 Fune ror7

Williams, Rev. W. D. C.F. 4th Class, R.A.C.D. $\quad *^{2} 888$

Williamson, R. S. 2nd Lieut., Yorkshire Regt. I90I

Willis, A. G. F. Lieut., The Buffs (E. Kent Regt.) I9०9 
Wilson, C. J. Capt., E. African Mtd. Rifles and Spec. $\quad$ I 898 List. M.C. $M 2$.

Wilson, E. Pte., W. Yorks. Regt.

Wirson, F. A Capt., Cameronians (Scottish Rifles, 1914 T.F.); and Lieut. (O.), R.A.F. (W.)

Wilson,H.F. Major, R.A.M.C. (W.) M.C.and Bar. M. 1904 Wolfe, J. A. Lieut., R.G.A.

1915

Wood, C. F. Lieut., I.A.R.O.; Capt., Mohmand Militia 1903 Wood, S. Capt., R.A.M.C.

Wood, W. L. Major, R.E. (W.) O.B.E. $M 2$.

WOODARD, E.A. 2nd Lieut. King's Own (Yorkshire I I)

WoODHEAD H. M and Lieut D of Wellington's (W. IO

Riding Regt.); Lieut., Cheshire Regt.; attd. T.M.B.

WWoolf, W. R. M. 2nd Lieut., Border Regt.

Died 26 Sept. 1915 of wounds received in action $25 \mathrm{Sept}$. I9I 5

Woolley, E. C. 2nd Lieut., Loyal N. Lancs. Regt. (W.) * 1908 WoOlley, J. M. Lieut.-Col., I.M.S.

WorRall, L. 2nd Lieut., R.A.S.C.

Wright, C. R. Pte., R.A.S.C.(M.T.)

Wright, O. K. Lieut.-Col., R.A.M.C.(T.F.)

WrIGHT, P. J. Lieut., R.G.A.

Wrigley, A. A.B., R.N.V.R.; Lieut., R.A.S.C. $M$.

Wyatt, T. C. Major, R.A.S.C. O.B.E. M.

1904 1890 1893 1905 1900 1896 1908 1906

WYARDE, J. T. Capt., Bedfordshire Regt.(T.F.) (W 2.) I9I3 M.C. and Bar. $M 2$.

Died 21 Sept. 1918 of wounds received in action in Palestine I9 Sept. I918

Yelverton, E.E. C.F. 4th Class, R.A.C.D. O.B.E. M. 1907 Young, B. W. Lieut., R.G.A.; empld. Ministry of Muni- I9ro tions

WYoung, E. T. Lieut., Manchester Regt.

Killed in action in Gallipoli ro fune 1915

1903

Young, F. E. Lieut.-Col., Rifle Brigade. (W.) M.C. M. 1908

Young, F. F. Capt., London Regt. (Artists Rifles) [I9I4]

Young, F. P. Capt., R.A.M.C.

Young, J. G. Lieut., Somerset L.I. and M.G.C.

Young, P. C. Colonel, R.E. A.I.T. $C B E$. $O B E$ I 899 $M$.

Young, R. F. Capt., R.A.M.C. (W.) M.C. M. $\quad 1898$ Young, Rev. S. D. C.F. 3 rd Class, R.A.C.D. D.S.O. 1905 O.B.E. $M 2$.

Young, S. L. O. Capt., R.A.M.C. 


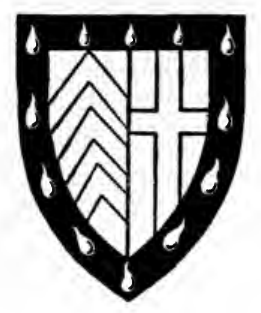

\section{CLARE COLLEGE}

Abrahamson, I. See Lubbock, I.

Adams, H. P. Corpl., H.A.C. $\quad 1880$

ADLER, F. B. Capt., S. African F.A.; Major, R.F.A. I90I (P.) M.C. $M 2$.

Arkman, K. B. Capt., R.A.M.C.; Surgeon Lieut., R.N.; 1907 Capt. (Med.), R.A.F.

Arkman, R. B. 2nd Lieut., Lanarkshire Yeo. 1902

ALCOCK, J. F. Lieut. (A.), R.A.F. 1914

AleXANDER, J. G. Lieut., R. Inniskilling Fus. $\quad 1899$

AleXANDER, J. W. Capt., R.A.S.C. 1905

AlEXANDER, R. M. Lieut., E. Yorks. Regt. and T.M.B.; 1903 empld. War Office

WALEXANDER, W. G. 2nd Lieut., King's (Liverpool Regt.) 1909 Killed in action at Festubert 15 May 1915

Allan, P. B. M. Capt., London Regt. (London Scottish); 1903 empld. War Office

Allen, W. Trooper, R. Horse Gds. 1908

Alston, W. E. Capt., R.A.M.C.(T.F.)

A AMPHLETT-Morton, J. F. 2nd Lieut., King's Royal Rifle I9II

Corps. $M$.

Killed in action 10 fan. 1915

ANDERSON, K. B. Lieut., R.G.A.(T.F.) 1900

ANDERSON, R. C. Lieut., R.N.V.R. (Coastal Motor- 1902 boat Service)

ANDERson, S. M. Brig.-Gen., R.A., Australian Force. 1898 D.S.O. Brevet Lieut.-Colonel. M2. Chevalier, Legion of Honour (France). American Distinguished Service Medal

Anderson, W. A. Capt., R.A.M.C.(T.F.) . M. French 1903 Médaille d'Honneur and Médaille des Epidémies 
WAnderton, G. E. A. Lieut., Lancs. Fus.; attd. M.G.C. [19I4] (W.) $M$.

Killed in action 22 March 1918

ANDREw, G. H. Instructor Lieut., R.N. $M$.

सAngus, R. E. Lieut., Ayrshire Yeo.; attd. R.F.C.

Killed in action 20 Nov. I9I 7

Ansell, A. E. Lieut., S. Staffs. Regt.(T.F.); Capt., 1904 R.A.S.C.

Appleton, E. L. Lieut., Border Regt. and Training Res. 1897 $\mathrm{Bn}$.

AAPPLEYARD, W. Lieut., Yorkshire Regt.

Killed in action in Gallipoli 22 Aug. 1915

ARDERN, L. Lieut., London Regt. (Surrey Rifles); attd. 1906 Durham L.I.

Armitage, W. A. Capt., York and Lancaster Regt.; 1898 Major, M.G.C. (Motor). (W.) D.S.O. M.

Armstrong, G. F. Pte., H.A.C.; Lieut., Labour Corps I9or ARNOLD, L. M. Surgeon Lieut., R.N.

ARNold, W. B. Lieut., Rifle Brigade. (W 2.) $M$.

Aspinwall, G. R. Capt., W. Yorks. Regt. (W 2.)

Atherton, Rev. E. C. C.F. ist Class, R.A.C.D.(T.F.)

Atterbury, H. H. Capt., Derbyshire Yeo.; Lieut., Gds.

M.G. Regt. (W.)

Avery, G. C. Instructor Cdr., R.N. Chevalier, Legion 1896 of Honour (France)

Aydon, J. Surgeon Lieut., R.N.

1906

BAGge, H. P. Major, Yorkshire Regt.(T.F.) (W.) M.C. $\quad{ }_{1898}$

BAIKIE, R. Capt., R.G.A.(T.F.)

Bailey, B. F. Capt., R.A.M.C. M.C. 1909

BAIley, E. B. Lieut., R.G.A. (W 2.) M.C. $M$ 2. 1899

Chevalier, Legion of Honour (France). French Croix de Guerre

Bailey, M. A. Capt., R.F.A. M.C.

WBAlLy, P. G. Major, R.F.A.

Killed in action 26 April r917.

${ }^{1}$ BaIley, T. E. G. Capt., Yorkshire Regt. (W.) M.C. 1902

BAKER, E. T. L. Lieut., Queen's Own (R.W. Kent Regt.) I9I3

(W.)

BAKEwEll, G. V. Major, R.A.M.C. O.B.E. M $2 . \quad 1906$

BALFOUR, R. N. Capt., R.A.S.C. $M$.

BANKs, W. E. H. Surgeon Sub-Lieut., R.N.V.R.

BARBER, H. W. Capt., R.A.M.C.

${ }^{1}$ Killed in action in N. Russia after the armistice.

1905 
WBarham, W. S. Capt., The Buffs (E. Kent Regt.)

Died Io Oct. I9I 5 of wounds received in action

1914

BARKER, K. E. M. Capt., Cambridgeshire Regt.(T.F.Res.) I896

BARNES, J. E. Lieut., R.A.S.C. $M$.

Barran, C. A. Capt., Oxford and Bucks. L.I.; Asst. 1912

Officer i/c R.F.C. Records. (W.)

BartletT, Rev. D. M. M. C.F. $4^{\text {th }}$ Class, R.A.C.D. $\quad 1892$

Barton, W. J. Major, Norfolk Regt.(T.F.); Asst. 1893

Officer $\mathrm{i} / \mathrm{c}$ R.A.M.C. Records

ABAss, V. A. 2nd Lieut., R. Fusiliers

Killed in action 20 fuly r916

BassetT, P. R. Lieut., Suffolk Regt.

WBastow, F. Capt., W. Yorks. Regt. (W.)

Killed in action near Rheims 27 May 1918

BATHER, E. J. Major, R.F.A. D.S.O. M 3 .

WBAwDON, R. H. 2nd Lieut., S. Wales Borderers

Died 1o fuly 1915 of illness contracted on active service

BAYLY, H. W. Major, R.A.M.C. (W.) M.C.

ABAYnes, D. L. H. Major, R.G.A. M.C. $M$.

Killed in action near Ypres 14 Oct. 1918

BazeTT, S. C. Major, Indian Army; empld. Govt. of 1893 Burma

Bealey, R. N. Capt., R. Fusiliers and Training Res. Bn. $\quad$ I880

BEARD, R. H. 2nd Lieut., R.F.A. (W.)

BEARDMORE, W. J. M. Capt., Gordon Hdrs (W)

Beck, B. R. Pte., Australian A.M.C.

BEck, E. W. T. Capt., R. Fusiliers; Staff Capt.; Major,

S.O. 2, R.A.F. (W 3.) D.S.O. M.C. $M 2$.

BeCK, F. G. M. Capt., R.G.A.(T.F.)

1907

I897

1912

1907

1913

$189 \mathrm{r}$

1905

I9I3

1898

1905

1901

ABecketT, P. A. Pte., Middlesex Regt.; 2nd Lieut., Queen's I9I I Own (R.W. Kent Regt.)

Killed in action $14 \mathrm{Feb}$. I9I 7

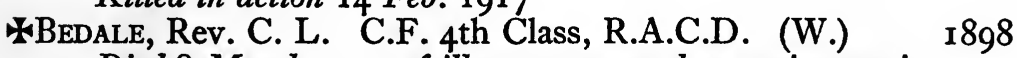

Died 8 March I9I9 of illness contracted on active service

Bedale, F. S. Capt., R.A.M.C.(T.F.) (W.) M.C. M. 1905

BELL,D.W. Lieut., Worcestershire Regt.; Major,M.G.C. $\quad$ I905 M.C.

Bell, J. H. Capt., Hampshire Regt. M.C.

WBemrose, R. H. Lieut., R.F.A. M.C.

Died 7 Nov. 1918 of wounds received in action $25 \mathrm{Sept}$.

1905

19I 5 1918

ABenjamin, J. A. Capt., D. of Wellington's (W. Riding I9I I Regt.)

Killed in action 5 fuly 1916 
Benjamin, R. N. Capt., R. Fusiliers. (W 3.) M.C.

BennetT, H. C. L. Capt., N.S.W. Bn., Australian Force

Bennett, K. L. Sergt., Australian Infy. (W.)

ABenson, C. D. Pte., R. Fusiliers

Died of wounds received in action $\mathrm{Feb}$. I9I7

BERNEY-FICkLIN, A. T. M. Major, Norfolk Regt. (W.) [19I4] M.C. M.

Berry, H. S. Capt., R.A.M.C. (W.)

Best, J. Cadet, O.C.B.

\#Bevan, C. B. Capt., Suffolk Regt. (W.)

1912

1909

1902

I9I3

Killed in action 20 fuly 1916

Beveridge, G. 2nd Lieut., Gordon Hdrs.(T.F.) (W.) I9ro

Bicket, T. B. Capt., R.F.A.(T.F.); empld. War Office. r9ro (W.) M.C.

BigGs, E. K. Capt., R.F.A.(T.F.) ; attd. R.G.A. I90I

Bignold, C. S. Pte., R.A.M.C. (W.) 1907

Billinton, H. L. Lieut., Lancs. Fus.; Lieut. (T.), R.A.F. I9I3 (W.)

Bilton, C. H. E. Capt., Highland Cyclist Bn.

Bilton, E. B. Capt., King's Own (Yorkshire L.I) M.

Killed in action at Achiet-le-Petit 14 March 1917

Birch, J. R. Capt., R.E. (London Electrical Engineers, 1902 T.F.)

Bird, S. 2nd Lieut., Dorset Regt.

BIRKBECK, M. Lieut., R.N.A.S.

Birnstingl, C. A. 2nd Lieut., R. Fusiliers

Biscoe, V. F. Capt., London Regt. (L.R.B.)

Bishop, P. F. Capt., R.A.M.C.

WBLACK, G. D. A. Lieut., R. Fusiliers

Killed in action $2 \mathrm{I}$ fune $19 \mathrm{I} 6$

*Black, J. N. Major, Somerset L.I. $M$.

Killed in action 9 April 1917

Black, R. B. 2nd Lieut., 3rd Dragoon Gds.; Capt., $\quad 1896$

Middlesex Regt. and Spec. List (Recruiting Staff)

1906

I910

I9I3

1905

I913

[1914]

1913

Blackburne, Rev. H. W. C.F. Ist Class, R.A.C.D.; $\quad$ I897

A.C.G. D.S.O. M.C. $M_{4}$.

Bladon, J. W. Pte., London Regt. (Artists Rifles)

1909

Bladon, M. W. B. Sub-Lieut., R.N.V.R.

I9I3

BlaIR, A. 2nd Lieut., R. Scots(T.F.) (W.)

1897

Blatherwick, R. Capt., R. Scots Fus.; Capt. (A.), 1908 R.A.F. (W 2.)

Blockey, H. S. Capt., S. Staffs. Regt.; Major (Ad.), 1904

R.A.F. (W.) $m$. French Croix de Guerre

BLow, A. E. Lieut., Spec. List, empld. Records 
Bodley, A. L. Capt., R.A.M.C.(T.F.). M.C. BODVEL-RoberTs, H. F. Capt., R.A.M.C. Bolland, J. F. Cadet, O.C.B. Bond, G. W. Major, S. and T. Corps, Indian Army. I89I D.S.O. $M 2$.

BonHote, Rev.E.F. Lieut., Rugby School O.T.C.; 1907 Pte., M.G.C.

ZBoston, L. Lieut., W. Yorks. Regt.; attd. M.G.C. (W.) I9I I $M$.

Accidentally killed at Aldershot 9 May 19г6

\#Botham, A. F. Gnr., H.A.C.; 2nd Lieut., R.F.A. M. 1908

Died 18 fune 1917 of wounds received in action in $E$. Africa

$\begin{array}{ll}\text { BotTomley, A. C. } \quad \text { Lieut., R.N.V.R. } & \text { r904 } \\ \text { BotTomley, R. A. A. } \quad \text { Lieut.-Col., W. Yorks. Regt. (T.F. } \quad \text { I889 }\end{array}$ Res.); empld. Ministry of National Service. $m$.

Boucher, R. E. Capt., Leicestershire Regt.; Capt. and I9r3 Adjt., Tank Corps. $M$.

Boulderson, G. Lieut., S. and T. Corps, E. African Force 1908 Boulton, H. Lieut.-Col., I.M.S.; D.A.D.M.S. Brevet I89I Lieut.-Colonel. $M_{3}$.

Boulton, R. J. Capt., Herefordshire Regt. and King's I9I I (Shropshire L.I.)

BowCHER, F. H. Lieut., R.G.A.

Bowen, T.S. Capt., Welsh Regt.; attd M.C.C; 1895 Admiralty

WBowles, R. J. A. Lieut., R. Welsh Fus. (W.) Died 20 Fuly 1916 of wounds received in action

ABRADSHAW-IsherwoOd, F. E. Lieut.-Col., York and Lan- 1887 caster Regt. Brevet Lieut.-Colonel. $M$.

Killed in action at Zillebeke 8 May r9r5.

WBrewer, C. H. 2nd Lieut., Lancs. Fus.

Killed in action 4 Oct. I9I7

\#Briggs, G. C. Capt., R. Scots Fus. $M$.

Killed in action on the Aisne 15-16 Oct. 1914

सBRodIE, H. W. Capt., The Buffs (E. Kent Regt.) M. ${ }_{1895}$ Killed in action 13 Oct. I9I 5

Brodie, P. Lieut., Highland L.I.; Capt., Spec. List 1899 (Bde. Bombing Officer). (W.)

Brogden, G. A. Capt., R.A.M.C.(T.F.)

WBROGDEN, I. R. R. Lieut., R.A.M.C.

Drowned on H.M. transport 15 April 1917

WBRomet, J. N. Lieut., R.F.A.

1903

1896

Killed in action 30 Nov. I917

1887

I910

I9I I 
Brooks, L. W. Lieut., W. Yorks. Regt.; attd. Lincoln- I9I I shire Regt.

Killed in action in the Battle of Loos 25 Sept. 191 5

WBrothers, M. Capt., E. Lancs. Regt.(T.F.)

Killed in action 28 May 1917

HBown, G. S. R. J. 2nd Lieut., R. Scots Fus.

Died 22 May 1915 of wounds received in action

I90I

1909

Brown, J. D. 2nd Lieut., Labour Corps

Browse, G. Colonel, I.M.S.; A.D.M.S. D.S.O. Brevet

Lieut.-Colonel. M 2.

Brunton, A. Corpl., R.A.S.C.(M.T.)

Beunton, A. W. Trooper, K. Edward's Horse; Lieut

R.F.A. (W.)

Killed in action 10 Aug. 1917

Burges-Bayly, A. R. Lieut., Herefordshire Regt. 1893

Burlison, J. C. Capt. and Adjt., M.G.C. (Motor). M 2. 1892

W Burnand, G. C. Lieut. (E.O.), R.F.C.

Killed in action 7 April 1917

Burn-Callender, F. Capt., R.F.A. M.C.

BURnside, B. Surgeon Lieut., R.N. Mentioned in

French General Orders for saving life from drowning.

French Silver Medal, 2nd Class

Burrell, E. M. Capt., R. Welsh Fus.

Burrell, G. P. Major, Hampshire Regt. M.C. $\quad$ M. $\quad 1899$

Burrell, H. A. Capt., Hampshire Regt.

Burrill-Robinson, W. R. Capt., Yorkshire Regt.

Burton, G. E. Surgeon Lieut., R.N.

Burton, P. M. A.B., R.N.V.R.

Buss, H. S. 2nd Lieut., Rifle Brigade

Butler, E. N. Major, R.A.M.C.(T.F.); Capt., Spec.

List. M.B.E.

ButTanshaw, C. Lieut., R.G.A.

ZByatT, H. V. B. Capt., I.M.S.

Died (I I March I9I 5) of wounds received in action near

Neuve Chapelle

Byatt, R. N. B. 2nd Lieut., R.F.A.

Byrne, M. G. M. C. Capt., Middlesex Regt.

ABrnne-Johnson, J. V. Capt. and Adjt., Rifle Brigade. $M$.

Killed in action at Delville Wood 23 Aug. 1916

I90I

1907

1909

1900

1903

I9I I

I 890

I9I3

1907

I9IO

I901

1907

1912

1912

Campbell, B. P. Capt., R.A.M.C.

Campbell, E. N. McC. Cadet, O.C.B.

Carden-Roe, W. R. Capt., R. Irish Fus.; Major, I893 G.S.O. 2. M.G. $M$. 
CAREY, F. C. S. Lce.-Corpl., R.A.M.C.(T.F.); Major, I9or R.A.O.C.; D.A.D.O.S.

CARr-Forster, E. W. Lieut., R.G.A.

1912

CARTHEW, R. J. Hon. Colonel, R.F.A. (R. of O.);Lieut.- 1882 Col., Gen. Staff. $m$.

Cartman, J. P. C. Lieut., Dorset Regt. (W.) 1894

Carver, A. E. Capt., R.A.M.C.

Carver, H., T.D. Capt., Cheshire Yeo.

CASEY, H. J. Major, R.G.A.(T.F.) M.C.

Cassidy, M. A. Capt., R.A.M.C.(T.F.)

Cawthra, J. J. Capt., Canadian A.M.C.

Cazalet, R. G. Capt., I.A.R.O., attd. I th Cavalry. $M$.

Chappell, H. S. 2nd Lieut., King s Own (Yorkshire L.I.); Lieut., Res. Regt. of Cavalry and King's African Rifles

Chaytor, A. H. Capt., London Regt. (Post Office 1888 Rifles); A.D.C.

Chaytor, D'A. Lieut.-Col., Canterbury Rifles, N. 1893 Zealand Force; Cmdt., Administrative H.Q., Egypt. C.M.G. M 2. Order of the Nile, $3^{\text {rd Class (Egypt) }}$

Chibnall, A. C. Capt., R.A.S.C.; Capt. (A.), R.A.F.

Chignell, N. J. Lieut., Charterhouse School O.T.C.

1891

1902

1903

1898

1897

1909

1906

Chinneck, S. T. E. and Lieut., Bradfield College O.T.C. I8 WClaIRMonte, G. E. 2nd Lieut., Gloucestershire Regt. [19I4] Killed in action at Hulluch 25-26 Sept. $19 \mathbf{1}_{5}$

AClark, G. R. H. 2nd Lieut., R. Fusiliers; Lieut., N. I9I4 Staffs. Regt. (W.) $M$.

Killed in action near Baku 26 Aug. 1918

Clark, H. J. Lieut., Durham L.I.; Staff Capt.; empld. IgI I War Office

Clark, Rev. S. H. C.F. $4^{\text {th }}$ Class, R.A.C.D.

Clarke, A. S. Major, R. Sussex Regt.; attd. Gen. Staff WClarke, Harold M. Lieut., London Regt. (Poplar and Stepney Rifles)

Killed in action in the Battle of Loos 26 Sept. I9I 5

Clarke, Henry M. Capt., R.A.M.C.(T.F.) $M$.

Clarke,H.T. Lieut.-Col., Worcestershire Regt. D.S.O. 1890 and Bar. $M 2$.

EClaye, G. W. Lieut., Cheshire Regt.

Died 29 March I9I7 of wounds received in action

1888

1900

1907

I 897

1912

Clayton, J. Capt., R.A.M.C. (Sanitary Service, T.F.) 1905 $M$ 2. Chevalier, Ordre du Mérite Agricole (France)

Clifford, E. C. Major, R.F.A. (W.) D.S.O. M.C. 1908 $M 2$. 
Clissold, Rev. C. H. C.F. $4^{\text {th }}$ Class, R.A.C.D.

Cocks, H. S. Lieut., R.A.S.C.

CoE, C. G. Major (T.), R.A.F. M.B.E. M.

1910

1893

*Cole, L. S. 2nd Lieut., Cheshire Regt.

Killed in action 3 Oct. 1915.

Collier, A. C. 2nd Lieut., King's Own (R. Lancaster [1914] Regt.); Lieut. (A.), R.A.F. (P.) $M$.

Colville, N. R. Lieut., Argyll and Sutherland Hdrs.; 1913 Capt., Spec. List, empld. Ministry of Labour. (W.) M.C. $M$.

Considine, H. H. Lieut., R.G.A.; empld. Board of Trade \#Constantine, H. N. Capt., Yorkshire Regt.(T.F.) (W.) M.C.

Killed in action 27 May 1918

Constantine, W. W. Major, Yorkshire Regt.; empld. Ministry of Shipping. (W 3.) M.C. M.

Conway, S. V. Lieut., R.G.A.

1905 1910

Cooper, A. L. 2nd Lieut., London Regt. (St Pancras Bn.)

Killed in action at High Wood 15 Sept. 1916

Cope, J. R. O. Lieut., R.A.S.C.

Cope, W., M.P. Major, Glamorgan Yeo.

CoRnelius, N. S. Lieut., R.G.A.(T.F.)

Cotr, A. M. Lieut. (T.), R.A.F.

Cotr, A. W. Lieut. (T.), R.A.F.

1905

1903

I9I3

1897 1888

1905

1906

1906

Cousens, R. B. Major, R.F.A.; Lieut.-Col., A.A. and Q.M.G. D.S.O. $M_{4}$.

Cow, C. S. 2nd Lieut., R.A.S.C.

Cox, R. B. 2nd Lieut., King's Own (Yorkshire L.I.); Capt., Yorkshire Regt.

Crampton, E. B. Lieut. (A.), R.F.C.

Crampton, H. P. Capt., R.A.M.C.(T.F.)

1898

CrICK, Rev.P.C.T. C.F. 2nd Class, R.A.C.D.; D.A.C.G.

CRItchett, G. M. Capt., London Regt.(Q.V.R.); empld. War Office

Crocker, G. G. Lieut., 6th (Inniskilling) Dragoons; attd.

Gen. Staff (Intelligence). M.B.E. m.

Crofton-Atkins, W. A. Lieut., R.A.S.C.

Crosfield, Rev. A. C. C.F. $4^{\text {th }}$ Class, R.A.C.D.

Croshaw, F. P. Lieut., Yorkshire Regt.; attd. S. Wales Borderers

※Cross, R. C. Trooper, Strathcona's Horse, Canadian 1898

1912

1899 1897

*1906 1903

1912 I 896 1887 1900 Force; Lieut., Dorset Yeo.; attd. S. Lancs. Regt. (W.) $M$.

Killed in action 7 Fune $\mathrm{r} 9 \mathrm{I} 8$

I9I I 
*Crossley, A. H. Lieut., Herefordshire Regt.

Died Io May 1917 of wounds received in action 9 April

I 897 1917

CrossLey, E. A. Capt., Sherwood Foresters (Notts. and 1899 Derby Regt.) (W.)

Crossley, F. M. Lieut., R.A.S.C.

Crowe, D. M. Lieut., Bedfordshire Regt. and Nigeria I9I I Regt., W. African Frontier Force. (W.) M.C.

Crowther, H. O. Pte., Queen's Own (R.W. Kent Regt., 1907 T.F.); Capt., I.A.R.O., attd. I I th Infy. M.B.E. M. CRUICKSHANK, G. Lieut., R.F.A.(T.F.); attd. R.E. (Sig- I9I3 nals). $M$.

Cumming, W. Lieut., Highland L.I. (W 2.) $M$.

Cunningham Reid, A. See Reid, A. Cunningham

1897

CuRLING, W. G. Major, R.F.A.; empld. Ministry of 1898 Munitions

ACuthBERTSON, E. H. Lieut., R. Warwickshire Regt. (W.) 1907

Died in Mesopotamia 24 fuly 1917 of sunstroke

FCuTLER, E. T. 2nd Lieut., Essex Regt.

Killed in action 9 Aug. 1917

Cutler, H. A. Capt., R.A.M.C.

1905

1893

DaIN, G. R. Lieut. and Adjt., Sherwood Rangers; 1903 Major, R.G.A.(T.F.) M.C. $M$.

D'AlBUQUERQUe, N. P. Lieut., King's (Shropshire L.I.) 1912 (W.)

WDale, A. P. Major, W. Yorks. Regt.(T.F.)

Killed in action $27 \mathrm{Feb}$. 1917

DALTRY, R. W. O. 2nd Lieut., Northamptonshire Regt. 1917 (W.)

Daniels, Rev. A. P. C.F. $4^{\text {th }}$ Class, R.A.C.D. M.C. 1908 DARBISHIRE, H. D. Major, R.F.A.(T.F.)

DARBY, A. J. L. Lieut., R.N.V.R.

DashWOOD, H. T. A. Lieut., Grenadier Gds.

1895

1896

DaveY, H. C. Lieut., Essex Regt.(T.F.); Capt., R. 1893

Sussex Regt. (T.F.)

Davey, H. N. 2nd Lieut., R.G.A.; Lieut., R.E.; Capt., 1907 G.S.O. 3. $M$.

WDAVEY, S. G. Major, Norfolk Regt.(T.F.); attd. M.G.C. I9I2 Killed in action 25 March 1918

David, M. Major, R.E.(R. Monmouth) $\quad$ D.S.O. $M . \quad 1895$

Davidson, Rev. G. M. C.F. $4^{\text {th }}$ Class, R.A.C.D. $\quad 1894$

WDavies, T. J. C. 2nd Lieut., Welsh Regt.

Killed in action in the Battle of Loos 2 Oct. 1915

[1914] 
Davis, C. E. J. Capt., Rifle Brigade

WDavis, W. J. Lieut., Northumberland Fus.(T.F.) Killed in action 30 June 1916

Dawson, C. P. Lieut.-Col. (ret.), attd. R.A.O.C.

de Courcy Ireland, G. B. See Ireland, G. B. De Courcy

De Freitas, D. A. A. 2nd Lieut., Gloucestershire Regt.; attd. E. Lancs. Regt. (W 2.)

De Freitas, J. M. Capt., Gloucestershire Regt. (W.) 1909 O.B.E.

Delius, S. St M. Capt. and Adjt., Yorkshire Dragoons. $\quad$ I 908 $M$.

WDenis Browne, W. C. Sub-Lieut., R.N.V.R. (Hood Bn., R.N.D.) (W.)

Died I4 fune I9I5 of wounds received in action in Gallipoli

DePreE, H. T. Surgeon Lieut., R.N.

Dew, J. W. Major, R.A.M.C. M.C. M 2.

ADEwar, A. Lieut., R.E. $M$.

Died 21 Dec. 1914 of wounds received in action near Ypres

Dinn, H. K. Lieut., King's Royal Rifle Corps. (W.) 1903

WDolby, H. A. Pte., Middlesex Regt. (P.S. Bn.); Lieut., I9I3 Leicestershire Regt.

Killed in action 7 May 1917

WDonahoo, M. G. Capt., King's Own (Yorkshire L.I.) 1892 M.C.

Died $3 \mathrm{I}$ Fan. I9I 7 of wounds received in action

Donnithorne, V. H. 2nd Lieut., Hampshire Regt. (W.) M.C.

Dorling, Rev. E. E. C.F. $4^{\text {th }}$ Class, R.A.C.D.

DotTridge, C. A. Capt., R.A.M.C.

Doughty, W. H. Lieut., Liverpool Institute O.T.C.

Douthwaite, A. W. S. Lieut., R.G.A.

1904

1906

1904

Downton, A. M. Capt., R.F.A.(T.F.)

WDrummond-Fraser, H. R. Capt., Cheshire Regt.; attd. I9I3

Herefordshire Regt. M.C.

Killed in action I Aug. I9I 8

Duckham, T. H. Capt., R.F.A.(T.F.) 1906

DunCAN, M. M., T.D. Lieut.-Col., R.F.A.(T.F.) C.M.G. $\quad$ I885 $M 2$.

※Dunn, P. M. Capt., R. Welsh Fus.

1908

Killed in action in India $3 \mathrm{Feb}$. 1917

Durell, C. V. Lieut., R.G.A.; Staff Capt. M. 1900

Dyer, F. N. V. Surgeon Lieut., R.N. 
※Dyson, H. A. Capt., The Buffs (E. Kent Regt.) (W.) I912 $M$.

Killed in action 18 Nov. I916

EDwards, H.S. Capt., R.A.S.C.; attd.The Queen's(R.W. I9I I Surrey Regt.) $M$.

EDwarDs, R. H. Lieut., Worcestershire Regt.; empld. 1905 Command Depôt

ELDERTON, M. B. Capt., R.G.A.

Elderton, T. H. Lieut., Bedfordshire Regt. (W.).

Elliotr, M. L. F. Capt. and Adjt., Gloucestershire $\quad$ I909 Regt. M.B.E.

Elliott, T. R. Colonel, A.M.S. C.B.E. D.S.O. $M_{3} \cdot{ }^{*}{ }_{1908}$ Ellis, B. W. Capt., R.F.A. Ellis, D. W. Lieut., Scots Gds.; Major, Spec. List I910 (Chief Instructor, Corps Reinforcement Depôt). (W.) Italian Croce di Guerra

England, F. Capt., King's Royal Rifle Corps and Gen. 1902 List (A.P.M.) (W.) $M$.

Entwisle, F. W. Capt., Manchester Regt.(T.F.) M.C. [1914] $M$.

EnYs, C. R. S. Pte., Suffolk Regt.

Evans, Rev. E. G. C.F. 4th Class, R.A.C.D.

Evans, H. J. A. Major, R.F.A. and Gen. Staff (O.C.B.) $\quad$ I898 M.B.E. $m$.

Evans, J. R. A. Capt., R.F.A.(T.F.). $M$.

Evers, Rev. M. S. C.F. 4th Class, R.A.C.D. (W.) 1906 M.C. and Bar

Ewen, J. F. B. Lieut., Gen. List, attd. Sherwood For- 1912 esters (Notts. and Derby Regt.) $M$.

Exshaw, T. S. N. Lieut., R.A.S.C.

1912

सFallowes, J.T. C. Pte., Canadian Infy.; Lieut., Suffolk rgro

Regt.

Killed in action 15 Sept. 1916

FARIE, A. J. C. Major, R.A.S.C.

FFARMER, J. I. 2nd Lieut., King's Royal Rifle Corps

Killed in action 9 May 1915

Fasson, F. H. Capt., Scottish Horse and Remount Ser- $\quad$ 1896 vice. $M 2$.

$\begin{array}{ll}\text { Faulder, T. J. Capt., R.A.M.C.(T.F.) } & 1889 \\ \text { FauQuet Lemaitre, P. A. Cuirassiers of the Guard, } & 1906\end{array}$ French Army 
AFeILden, O. H. Capt. and Adjt., Leicestershire Regt.

Died 29 Sept. I9I7 of wounds received in action 28 Sept. I9I7

Fergus, A. McF. H. 2nd Lieut., Seaforth Hdrs.

Findlay, J. Capt., R. Irish Rifles. (W.) M.C.

Fischer, F. N. 2nd Lieut., R.A.S.C.

WFisher, G. H. Pte., Canadian Mtd. Rifles; 2nd Lieut.,

Sherwood Foresters (Notts. and Derby Regt.)

Died 25 Oct. I9I 5 of wounds received in action

Fleming-Brown, G.F. Lieut., Bedfordshire Regt.;

Capt., O.C.B.

Fletcher, W. T. Capt., 97th Deccan Infy., Indian Army (P.)

Follit, H. H. B. Lieut.-Col., Australian A.M.C.; D.A.D.M.S. $M$.

Forbes, J. F. Lieut., Seaforth Hdrs.; Staff Lieut.

Ford, H. J. Pte., London Regt. (Artists Rifles)

Forde, C. L. Major, R.A.M.C.

Fowle, Sir H.W. H. Colonel, S. African Defence Force;

Provost-Marshal and Commissioner for Enemy Subjects. K.B.E. C.B.E.

Fox, R. W. Lieut.-Col. Devon Regt.(T.F.) $M$.

Killed in action in Mesopotamia 8 March I916

Franks, G. L. T. Pte., R.A.M.C.

Fraser, J. H. Lce.-Corpl., Highland L.I.

Fraser, T. Colonel; R.E.; Major-Gen. C.B. C.S.I.

C.M.G. Brevet Colonel. M 7. Order of the White

Eagle, 4th Class, with swords (Serbia)

FREEBORN, J. H. R. Lieut., York and Lancaster Regt.; Capt., Spec. List (Adjt., Vol. Bn.)

Freeman, J. E. Lieut., Essex Regt.; Capt., Spec. List 1900 (Bde. Bombing Officer)

Frost, H. K. Major, R.F.A.(T.F.)

AFrost, T. L. Capt. and Adjt., Cheshire Regt. $M$.

Killed in action near Ypres 28 March I9I 5

Fry, K. R. B. 2nd Lieut., King's Royal Rifle Corps

I9II

1913 1886

1906

1912

1902

1900

1908

1879

1900

1890

1885

1908

1897

1884

I9II

1904

1907

I90I

1910

$M$.

Killed in action at Caix 9 Aug. 1918

Fursdon, A. A. Capt., Devon Regt.(T.F.)

1914

*Gaffikin, G. H. Major, R. Irish Rifles. $M$.

Killed in action at Thiepval I Fuly I9I6

Galloway, W. D. Surgeon Lieut., R.N. $M$.
1905

1907 
Garrard, G. G. C. Pte., H.A.C.; 2nd Lieut., R.E.(T.F.) I909 AGARROD, R. P. 2nd Lieut., London Regt. (Rifles) Killed in action 22 May 1915

Garson, H. L. Capt., R.A.M.C. (W.) O.B.E. M.C. 1909 $M 4$.

Gascoigne, T. 2nd Lieut., Northamptonshire Regt. $\quad$ I900

Gaskell, J. G. Major, R.F.A.(T.F.); attd. R.G.A. 1903

GASkING, E. B. 2nd Lieut., Labour Corps I9I6

Gatehouse, H. 2nd Lieut., R.E. and R.A.S.C.; Major, I890 Spec. List (Courts-Martial Officer). O.B.E.

Gawler, A. E. J. Lieut., Somerset L.I.(T.F.)

GaY, L. H. Capt., S. Lancs. Regt.; Major, R. Defence 1889 Corps. $m$.

Gayne, A. A. A. Capt., Army Pay Dept.

Gibbs, J. W. Pte., London Regt.; 2nd Lieut., R. Sussex I90 I Regt.

Gibson, D. K. Lieut., R.G.A.(T.F.); attd. R.A.O.C. I908

Gibson, G. M. Pte., Canadian Force

Gibson, W. F. A.B., R.N.V.R.

GIFFORD, R. E. Capt., Somerset L.I.; Instructor in Musketry

GiLl, H.L. O. Trooper, Worcestershire Yeo.; and Lieut., Staffordshire Yeo.; Lieut., Gloucestershire Regt.; attd. R. Welsh Fus. (W 2.) M.C.

ZGleNNY, H. Q. Assistant Resident, N. Nigeria

Died 18 Nov. I9I4 of wounds received in action at Gazabu (Cameroons) I6 Nov. I9I4

GlynN, E. E. Capt., R.A.M.C. (Ist W. Gen. Hospital, I892 T.F.). $m$.

Glynn, E. H. Trooper, K. Edward's Horse

GlynNe, A. Lieut. (A.), R.A.F. (W.)

GoING, T. H. 2nd Lieut., R.F.A.; Lieut., R.T.O.

I899

I9I4

1903

I9I3

I 898

*Goldthorpe, A. F. 2nd Lieut., E. Yorks. Regt.

Killed in action 12 May 1917

\&Goodhart, E. J. Sergt., R.E. (Signals). D.C.M. M. I9I3

French Médaille Militaire

Died April I9I 5 of typhoid contracted on active service GorDon, E. P. Lieut., R.E.; attd. ro4th Rifles, Indian Army

Killed in action in Mesopotamia Nov. I9I 5

Gordon, W. B. Capt., R.A.M.C. (W.) M.

Gordon Browne, H. H. Lieut., Worcestershire Regt.;

Lieut.-Col., Somerset L.I.(T.F.); Administrative

I912

1910

I895

19I3

Cmdt. D.S.O. M 2 .

1909

1908

I 893 
Gould, E. 2nd Lieut., King's Own (Yorkshire L.I.); 1898 Capt. and Adjt., Lovat's Scouts; attd. I2th Lancers;

Capt., Spec. List (Intelligence Staff)

Gould, H. C. Trooper, K. Edward's Horse. (W.)

Gowans, J., D.s.o. Hon. Lieut.-Col. R.A. (R. of O.), 1890 empld. R.F.A. $M 2$.

Grace, C. R. Lieut., Lovat's Scouts; Hon. Lieut. (A.), 1905 R.A.F.

Graham, G. L. 2nd Lieut., I8th Hussars; Capt. (A.), 1914 R.A.F. D.F.C. Chevalier, Legion of Honour (France). French Croix de Guerre

Graham, G. W. Major, R.G.A.; Instructor of Artil- 1892 lery

aGraham, R. L. Lieut., R.F.A. and R.F.C. (W.) $M$. Killed in action I6 Sept. I9I7

Graham-Hodgson, H. K. Corpl., R.E. (Signals); Capt., I9ro R.A.M.C.

AGrandage, W. B. Lieut.-Col., R.F.A.(T.F.)

Died I4 May I9I 7 of wounds received in action

Granger, E. H. H. Capt., R.A.M.C. M.C.

Gray, W. Capt., Yorkshire Regt. (W.) (P.) $M$.

Green, B. B. Capt., Essex Regt.

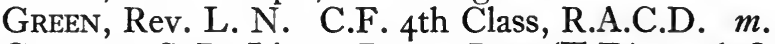

Gregory, S. B. Lieut., Devon Regt.(T.F.); attd.Queen's I9I3 Own (R. W. Kent Regt.) (P.)

Died at Mosul 3 Fune I9I6

Gregson, C. D. Major, 2oth Deccan Horse, Indian I899 Army

Greig, J. L. Major, Remount Service $\quad$ I887

GRIEVE, A. B. Instructor Lieut., R.N. 1908

Griffith, A.L.P. Major,R.F.A. (W3.) D.S.O. Brevet 1905 Major. $M 4$.

Griffith, R. Major, R. Welsh Fus.(T.F.); Lieut.-Col., $\quad 1892$ R.A.M.C. (W.) Brevet Lieut.-Colonel. M2.

Gronow, W. H. 2nd Lieut., R. Welsh Fus.

Grose, T. Capt. and Adjt., R.A.S.C.

Grose, T. A. Lieut., R.G.A. (W.)

GuILFORD, E. L. 2nd Lieut., Sherwood Foresters (Notts. and Derby Regt.)

Gush, C. R. Lieut., R.E. (W.)

Guthrie, A. G. Lieut., R.E.(T.F.)

Guthrie, N. B. Corpl., R.E. (Signals); Lieut., King's (Liverpool Regt., Liverpool Scottish, T.F.). (W.)

Gyles, W. H. K. Capt., R.E. (Railways). $M 2$. 
Habershon, Rev. E. F. C.F. $4^{\text {th }}$ Class, R.A.C.D. (W.) 1904 WHABERSHON, P. H. 2nd Lieut., King's Royal Rifle Corps 1912 Killed in action 25 Sept. 1915

WHABERSHON, S. H. 2nd Lieut., Suffolk Regt. (W.) M. 1908 Killed in action 8-13 April 1918

HabGoOd, G. Capt., R.A.M.C.

WHAINES, F. P. Lieut., Leicestershire Regt.

1909

Killed in action 15 fune 1917

Hall, A. K. D. Major, Dorset Regt.; G.S.O. $3 \quad 1904$

Hall, H. Corpl., R.E. (Signals); Lieut., R.F.A. (W.) I9I I

Hall, J. A. Lieut.-Cdr., R.N.V.R.

1905

Hall, P. A. Major, Oxford and Bucks. L.I.; Lieut.-Col.,

1910

R. Warwickshire Regt. D.S.O. M.C. M. Italian

Bronze Medal for Military Valour

Hansen, J. C. Capt., Hampshire Regt. $M$.

Hanson, C. E. B. Major, D. of Wellington's (W. Riding Regt.)

HHARBORD, J. Capt., Norfolk Yeo.; attd. Norfolk Regt. I9I I (W.) M.C.

Died ro fuly 1918 of wounds received in action

HARGREAVES, R. Q.M.S., H.A.C.

HARRIES-Jones, J. S. D. Lieut. (T.), R.A.F.

HarRis, A. F. S. Major, R.G.A.(T.F.)

I9I3

1892

Harrison, W. J. Capt., R.G.A.; Asst. Proof and Experimental Officer, Woolwich Arsenal. M.B.E. m.

HarRowing, W. W. 2nd Lieut., D. of Cornwall's L.I. I9I 5

HART, S. Major R.F.A.(T.F.) (W.) M.C. 1907

Hartcup, G. H. W. Major, R.G.A. M.C. M. 1904

HARTCUP, R. E. 2nd Lieut., R.G.A. 1907

HaWThorNe, C. B. Capt., R.A.M.C.(T.F.) I

HaY, J. A., T.D. Major, Inns of Court O.T.C. m. 1884

Hay-Robertson, J. W. Capt., Black Watch (T.F.) (W.) Igo8 M.C. and Bar

Hedderwick, A. S. Capt., Spec. List, empld. Records; 1904 Staff Capt. $M$.

HedDERwick, G. Lieut., R. Scots; Major, Tank Corps. I9I2 M.C.

Hedges, A. D. 2nd Lieut., R.F.A. (W.)
[19I4]
HeELIS, J. R. Major, Manchester Regt.; attd. Welsh ${ }_{1898}$

Regt. (W.) M.C. $M_{3}$.

Hegarty, M. B. 2 nd Lieut., R.A.S.C.
I9I5
HHele-Shaw, H. R. Pte., R. Fusiliers (P.S. Bn.); 2nd [1914]

Lieut., R.G.A.; Lieut. (A.), R.F.C. (W.)

Killed in action 19 July 1916 
WHemsworth, A. H. 2nd Lieut., King's Own (Yorkshire L.I.) Accidentally killed in motor accident 6 Fune 1915

4Henderson, D. 2nd Lieut., King's Royal Rifle Corps 1912 Killed in action I I Fan. I9I 5

WHenderson, N. C. 2nd Lieut., Black Watch

Killed in action 25 Sept. I9I 5

Henderson, R. K. 2nd Lieut., Grenadier Gds.

1913

1903

HendRICKs, C. A. C. J. Capt. and Adjt., King's Own 1902 (Yorkshire L I.) (W.) M.C.

HeRriot, J. A. Capt., Northumberland Fus.; attd. M.G.C.

Herrman, L. C. Sergt., Middlesex Regt.

Hesketh, G. M. . Major, Loyal N. Lancs. Regt.(T.F.)

Hicks, E. J. Sergt., London Regt. (Artists Rifles)

Higgins, L. G. Surgeon Lieut., R.N.

Hill, G. M. Capt., D. of Wellington's (W. Riding Regt.). (W 2.) $M$.

Hinde, S. L. Major, R.A.M.C. $M$.

Hodge, D. S. Capt., R.A.S.C.

Hodge, E. H. V. Capt., I.M.S. $M$.

Hodgson, A. J. Capt., King's (Liverpool Regt.) and Spec. List (Courts-Martial Officer). (W.) $m$.

Holland, J. E. D. Capt., 5th (Princess Charlotte of 1898

Wales's) Dragoon Gds.; Major, G.S.O. 2. (W.) D.S.O. M.C. $M_{3}$.

Holman, A. F. Capt., D. of Cornwall's L.I.

Holmes, A. K. Lieut., R. Sussex Regt.

Holt, H.D. G. Lieut., R. Fusiliers; attd. N. Staffs. Regt. and R.F.C. (W.)

Hooker, C. W. R. Lieut., R.N.V.R. O.B.E.

Hooper, F. H. Capt. and Adjt., R.F.A.; Major, R.F.C. (W.) $M . m 2$.

Hope, H. N. Lieut.. Grenadier Gds.

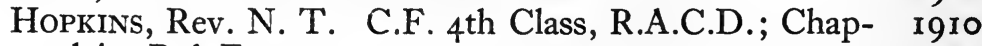
lain, R.A.F.

Horsley, G. Capt., R.G.A.(T.F.)

HorsLEY, S. Major, R.G.A.(T.F.)

I 888 1900

1913

I913

1906

1910

1912

I9II

I 88 I

1894

1902

1906

1904

1905

1902

[1914] I 901

\#Hoskyns, H. C. W. Major, Lincolnshire Regt. D.S.O. $\quad$ I893 $M$.

Killed in action 25 Sept. 191 5

Hotblack, H. S. Lieut., R.F.A.(T.F.)

Houseman, E. A. Major, Indian Defence Force Med. I89I Corps 
Howard, J. C. Lieut., Lincolnshire Regt.; Capt., Spec. 1906 List (Courts-Martial Officer)

Howell, J. H. Lieut., Pembroke Yeo.; attd. Dragoon 1899 Gds.

HowirT, A. B. Capt., R.A.M.C.

Hudson, B. Major, R.A.M.C.

Hudson, N. Pte., R. Fusiliers (Sportsman's Bn.) (P.)

Hughes, E. C. Capt., R.A.M.C. (2nd London Gen. 1898 Hospital, T.F.) O.B.E.

Hughes, E. L. Major, Northamptonshire Regt.; Lieut.- I I $89 \bar{\jmath}$ Col.,A.A. and Q.M.G.; Base Cmdt., Taranto. D.S.O. O.B.E. M 4. Officer, Order of St Maurice and St Lazarus (Italy)

Hughes, O. Capt. (A.), R.A.F.

Hughes, T. J. 2nd Lieut., Loyal N. Lancs. Regt.

1898

1896

(W.) I9II

HulBerT, M. L. Lieut., R. North Devon Yeo.; attd. M.G.C.

HumPHRYs, R.P. Capt., King's(Liverpool Regt., T.F.) M. 1909

Hunt, E. G. Lieut., Middlesex Regt. (W.)

1898

Hunter, E. J. Capt., Suffolk Regt. (Cyclist Bn., T.F.) I909

Hunter, M. J. Capt., R.F.A.(T.F.)

1908

1913

1905

HHunter, N. F. Lieut., R. Warwickshire Regt.; attd. R. 1897

Fusiliers

Died 16 Fune 1915 of wounds received in action

Hussey-MacPherson, L. F. Capt., Cameron Hdrs.; · 1908 empld. War Office and O.C.B. (W 2.) $M$.

Hutchinson, G. C. Capt., Lancs. Fus.(T.F.); (W.) I9I2 M.C. $M$.

सHutchinson, J. G. Lieut., R.F.A.(T.F.)

Killed in action ro Nov. I9r 8

सHutchinson, L. G. Lieut., E. Yorks. Regt. (W.) 1912

Killed in action ro Sept. 1918

Hutchinson, N. W. 2nd Lieut., R. Jersey Militia; Capt. 1907 and Adjt., R.A.S.C. $m$.

Hutchinson, R. H. Capt., R.A.M.C.

I901

IMBERT-TeRRY, F. B. Lieut., Devon Regt. M.C. M 2. 1906

INCHBald, Rev. C. C. E. C.F. 4th Class, R.A.C.D. $\quad$ I909

IONIDES, A. G. Lieut., R.N.V.R.

Ireland, G. B. DE Courcy. Capt. and Adjt., King's Royal

Rifle Corps. (W 2.) M.V.O. M.C.

IRELAND-BlaCKBURNE, C. G. C. M. Lieut., R.A.S.C.

IsHerwood, F. E. B. See Bradshaw-Isherwood, F. E.

I 888

1913

1909 
Jackson, A. L. Capt., R.A.M.C.

JAckson, R. W.P. Major, R.A.M.C. M.C. M. French 1909 Croix de Guerre

JAMES, A. M. T. Capt., R.F.A.(T.F.) M.C.

WJemmetr, C. W. Lieut. and Adjt., The Buffs (E. Kent 1905

Regt., T.F.) (W.)

Killed in action 15 March 1918

John, D. W. Capt., R.A.M.C. M.C. $M 2$.

Johns, S. H. M. Surgeon Lieut., R.N.

Johnson, E. S. H. Pte., H.A.C.; Capt., R.A.O.C.

Johnson, R. F. Pte., H.A.C.; Capt., R.A.O.C.;

D.A.D.O.S. $M$.

Johnstone, C. M. Major, Dragoon Gds. (R. of O.)

JoNES, H. Ll. Major, Hussars; Lieut.-Col., Welsh Regt.

(W.) D.S.O. M 2. Chevalier, Legion of Honour (France)

JONES, T. E. Warrant Schoolmaster, R.N.

Jordan, A. R. Capt., R.A.M.C.

1906

1909

I 899

I9I0

1883

1905

Keay, E. D. Capt., R. Warwickshire Regt.(T.F.) (W.) 1908

WKeAY, J. G. 2nd Lieut., R. Warwickshire Regt.(T.F.) I9I4

Died 2 Fuly I9I 6 of wounds received in action

Keigwin, W.S. 2nd Lieut., Welsh Regt.; Lieut., Train- 1907 ing Res. Bn.

\#KELseY, W. Lieut., R.F.A.

Died 23 Sept. I9I6 of wounds received in action 14 fuly I9I6

Kennington, J. Major, Lincolnshire Regt.; Lieut.-Col., 1894 R. Warwickshire Regt. (W.) D.S.O. M.C. M.

Kenworthy, C. H. Sub-Lieut., R.N.V.R.

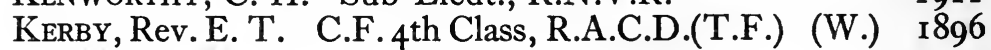
M.C. $M 2$.

Kerby, P. W. Lieut., R.F.A. (T.F.Res.); empld. Army 1898 and Navy Canteen Board

Kerr, J. F. Lieut., Seaforth Hdrs. and Gen. List (Staff 1913 Lieut.) (W.)

KerR, M. J. D. Major, R.E. (W 2.) $M$.

WKIDD, J.N. Capt., 6th Dragoon Gds. (Carabiniers). (W.) I899 Killed in action 19 Fan. 1916

KING, K. Lieut., R.A.S.C.

KING, S. Major, Middlesex Regt.(T.F.) (W.) $M^{*}$.

KIRBY, H. 2nd Lieut., The Buffs (E. Kent Regt.); Lieut. (A.), R.A.F. (W.) (P.) $M$.

KIRKE, G. G. Capt., Black Watch (T.F.)

1914 
KIsch, E. R. Capt., London Regt. (Kensington Bn.) 1905 M.C.

KitTermaster, Rev. D. B. C.F. 3 rd Class, R.A.C.D. $\quad 1896$ M.C. $M$.

KLUGH, H. Lieut., R.N.V.R. D.S.C.

Knowles, C. K. Lieut., Dorset Regt. and Tank Corps

Knox, B. Capt., R.H.A.(T.F.)

I 88 I

I9I I

1910

Ladenburg, A. L. Capt., R. Fusiliers

I 888

LANE, T. W. Lieut., R.F.A.

1912

LANGDAle, K. M. Sapper, R.E. (Signals)

1903

Laughlin, C. E. H. Major, Leinster Regt.; Brigade 1877

Major; Lieut.-Col., Hampshire Regt.(T.F.); empld.

Ministry of Munitions. Brevet Major. $m$.

LEACH, R. C. Capt., King's Own (R. Lancaster Regt.); 1908 empld. O.C.B. (W 2.) M.C. $M$.

LeathaRT, P. W. Capt., R.A.M.C.

WLEES, T. P. Major, London Regt. (Q.V.R.) M.C.

Killed in action near Ypres 21 April r915

Le Fleming, E. K. Hon. Lieut., R.A.M.C.

1895

I893

Le Fleming, J. Lieut.-Col., Kent Cyclist Bn.; Major, Queen's Own (R.W. Kent Regt., T.F.)

LEgGE-CuRrIE, J. D. Lieut., R.A.M.C.

LehmanN, J. R. 2nd Lieut., Northamptonshire Regt.

Lemon, G. T. Capt., I.A.R.O., attd. 3oth Punjabis and ro4th Wellesley's Rifles; Staff Capt.; Major, D.A.D. Military Works. (W.) O.B.E. M2.

सLeon, E. J. 2nd Lieut., London Regt. (Post Office Rifles) Killed in action 7 Oct. 19r6

Leonard, H. V. Capt. and Adjt., E. Lancs. Regt.; Capt., Cheshire Regt. (W 3.) M.C. and Bar. M. French

Croix de Guerre

Leveson, W. E. Capt., R.G.A. M.C.

LEvY, L. A. Lieut., Spec. List.; Capt., R.E. $m$.

LEwIN, H. W. Major, R.A.S.C. $m 2$.

WLEwIS, H. M. 2nd Lieut., Middlesex Regt.

Killed in action in Gallipoli 4 Nov. 1915

LEwIS, L. H. Major, E. Lancs. Regt. (W.) D.S.O. 1912 M.C. $M$.

WLinDSAY, F. H. Major, London Regt. (London Scottish). $\quad$ I895

(W.) $M$.

Killed in action I Fuly r9r6

LINFORD, W. A. M. Lieut., Northamptonshire Regt.

Lipschitz, J. Capt., R.F.A. (W.) 
Lloyd, L. W. Lieut., 2rst Lancers; Capt. and Adjt., 1912 Command Depôt. (W.)

WLloyd, T. G. Capt., Welsh Regt.

Killed in action Io May 1918

HLloyd-Williams, K. P. 2nd Lieut., Welsh Regt.

Killed in action 17 Oct. I916

LOMBARD, Rev. B. S. C.F. 4th Class, R.A.C.D.

Longstaff, F. V. Major, E. Surrey Regt.(T.F.)

I910

LongstafF, G. C. Lieut., R.N.V.R. (Coastal Motor-boat

Service)

Loring, R. W. Pte., H.A.C.

Lovell, J. S. Capt., R.G.A. (W 2.)

Low, A. R. Major (T.), R.A.F. (Aircraft Production 1900

Dept.)

Lowcock, D. R. Pte., R. Fusiliers

I9I4

I 887

1899

1903

1905

1888

1903

Lowe, F. G. Lieut., I.A.R.O., attd. I 3 th Rajputs; Adjt.,

I 7 th Infy. Depôt; Staff. Lieut. (Asst. Embarkation S.O.) $M$.

Lowe, G. B. Surgeon-Lieut., R.N.

Lowndes, Rev. R. G. C.F. $4^{\text {th }}$ Class, R.A.C.D.

LUARD, S. D'A. 2nd Lieut., Worcestershire Regt.; Lieut.,

Cheshire Regt.

Luвbоck, I. Capt., R.G.A. (W.)

LuCAS, T. C. Major, R.A.M.C.

1902

LUCY, R. S. Lieut., Worcestershire Regt.(T.F.); Major (A.), R.A.F. A.F.C.

सLumb, H. Pte., London Regt. (Artists Rifles); Lieut., 1905 R.F.A.

Died 8 Oct. I9I 5 of illness contracted on active service

Lupton, R. H. Capt., R. Sussex Regt. M.C. M 2.

Lupton, W. M. Capt., I.M.S. $M$.

Lushington, Rev. F. DE W. C.F. $4^{\text {th }}$ Class, R.A.C.D.

Macartney, M. E. Pte., R.A.S.C.(T.F.)

I9II

1901

1912

r9ro

I895

1912

1903

1907

I 887

1902

WMcClenaghan, A. B. P. 2nd Lieut., Wiltshire Regt. [19r4] Killed in action 16 Fune 1915

McClure, I. H. Capt., Spec. List; G.S.O. 3 (Intelli- I9Io gence). D.S.O. M 2. Italian Croce di Guerra

«McCosh, E. Major, Highland L.I. (W.) M.C. M 2. 1910 Died 26 Sept. 1918 of wounds received in action

McCririck, D. H. G. Capt., Somerset L.I.

McGeagh, J. P. Lieut., Sherwood Foresters (Notts. and Derby Regt.); attd. Nigeria Regt., W. African Fron1912 1912 tier Force 
McGown, T. W. M. Major, R.A.O.C.; D.A.Q.M.G. M. ${ }^{1894}$ MACHIN, B. W. Lieut., Black Watch

MacintYre, D. L. Capt. and Adjt., Cameron Hdrs. I9I2 $M 2$.

WMackenzIe, B. M. S. Capt., The Queen's (R.W. Surrey 1908 Regt.)

Killed in action 22 March 1918

Mackenzie-Kennedy, H. C. D. C. Lieut., E. African 1908 Force; attd. N. Rhodesian Police

HMcKerRow, C. K. Capt., R.A.M.C.

Died 20 Dec. I 916 of wounds received in action

Mackrill, O. W. Air Mechanic, R.A.F.

Maclaren, A. I. Lieut., R.F.A. M.C.

WMcMrchael, D. W. Pte., R. Fusiliers (P. S. Bn.); and

Lieut., Bedfordshire Regt.

Died 17 April 1916 of wounds received in action

MaCNEE, E. A. Capt., M.G.C. $M$.

McNeILl, A. H. Capt., King's Own Scottish Borderers

(T.F.) (W.) Order of Karageorge, $4^{\text {th } \text { Class, with }}$ swords (Serbia)

MacPherson, D. G. Lieut., Cameron Hdrs. (W.)

MacQueEn, R. H. Capt, R.A.S.C. O.B.E. $M 2$.

1902

1902

1907

1912

WMakanT, A. V. Capt., Loyal N. Lancs. Regt.(T.F.)

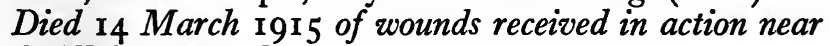
St Eloi 10 March I915

Makant, R. K. Capt., Loyal N. Lancs. Regt.(T.F.); [I9I4] A.D.C. (W.) M.C. and Bar

WMalPas, C. C. Seaman, R.N.V.R. (R.N.D.)

Died ro March I9I 5 of cerebro-spinal meningitis

1904

1902

1912

I897

1909

MARRIotT, C. E. Major, R.A.M.C.(5th N.Gen. Hospital, I 888 T.F.)

MarriotT, C. G. L. Trooper, W. Kent Yeo.; Capt., $\quad$ I912 The Queen's (R.W. Surrey Regt., T.F.)

MARriotT, C. J. B. Capt., R.A.S.C. $m$.

1880

Marsden, C. H. Major, Yorkshire Regt.; Chief In- 1894 structor, School of Musketry. $m$.

Marshall, E. A. Lieut., R.N.V.R.

WMARSLAND, S. H. Lieut., Manchester Regt.

Killed in action in Gallipoli 7 Aug. I91 5

WMARTEN, C. P. Lieut.-Col., W. Yorks. Regt. and King's 1898

Royal Rifle Corps. $M$.

Killed in action near Flers 15 Sept. 1916

Martin, Rev. E. W. L. Capt., Labour Corps $\quad 1908$

MarTin, Rev. H. C. C.F. $4^{\text {th }}$ Class, R.A.C.D.

I9I I

1910

1896 
Masser, B. R. Pte., H.A.C.

1901

MAsser, C. S. 2nd Lieut., Unattd. List, T.F.; A.D.C. I910

Mathews, J. K. Sapper, R.E.

Matravers, F. G. Eng. Lieut.-Cdr., R.N.

Matthews, J. B. Capt., R.A.M.C. M.C. M.

Maufe F. W. B. Capt., R.F.A.(T.F.) (W 2.) M.C [ and Bar. $M$.

WMaufe, S. B. Major, W. Yorks. Regt. $M$. Killed in action on the Somme 5 Fuly 1916

Mayall, G. Capt., R.H.A.(T.F.) M.

MaYo, T. A. Lieut., R.A.M.C.

MedlycotT, W. Capt., R.A.S.C.

MeERES, Rev. B. H. Chaplain, R.N.

Mellin, E. L. 2nd Lieut., Labour Corps

Mellin, G. L. Capt., London Regt. (W.)

WMellis, G. D. Capt., R.F.A.(T.F.) (W.) $M$.

Died I I Dec. I9I 7 of wounds received in action 30 Nov. I9I7

Methuen, L. H. Capt., Argyll and Sutherland Hdrs.; Staff Capt. (W 2.) O.B.E. M.C. M.

Midwood,R. Lieut., MontgomeryshireYeo.and M.G.C. I9I4 Capt., Spec. List (P. and B.T. Staff). $m$.

AMillar, A. L. Capt., Rifle Brigade

Killed in action 15 April 1918

MilleR, H. M. Lieut., Spec. List; Staff Lieut. $M 2 . \quad 1896$

MilleR, J. C. Major, Gresham's School, Holt, O.T.C. $\quad 1887$

MilleR, R. M. Capt., R.A.M.C. (W.) D.S.O. M. 1896

*Mills, C. G. 2nd Lieut., Coldstream Gds. $M$.

Killed in action near Béthune 26 Fan. I9I 5

Mills, F. A. Capt., R.A.M.C.

1912

Milne, D. D. W. Major, Highland L.I.(T.F.); empld. War Office; Major (T.), R.A.F. (Aircraft Production Dept.)

Mitchell, F. J. L. Lieut., R.G.A. (W.)

WMitchell, F. M. Capt., I 8th Hussars; attd. Worcestershire Yeo. M.C.

Killed in action 2 May 1918

Mitchell, G. W. Capt., R.A.M.C. (W.)

Mitchell, J. R. Capt., R.A.M.C. M.

1888

1906

MogGrIDGE, C. D. Lieut., Spec. List (Graves Registration Commission)

Montgomery, H. R. G. Capt., Norfolk Regt. (W 2.) 1909 M.C.

Montgomery, W. E. Lieut.-Cdr., R.N.V.R. M.B.E. I88I 
MOORE, F. R. Lieut., R.E. (R. Anglesey).

MOore, G. Capt., R.A.M.C.(T.F.) M.C. $M$.

1907

Morcom, A. F. Capt., R.A.M.C.

1904

Morgan, O. G. Capt., R.A.M.C.

1903

1907

Morgan, W. P. Capt., R.A.M.C.

1895

\#MORIsON, D. R. 2nd Lieut., Wiltshire Regt.

Killed in action 13 March 1915

Morland, D. M. T. Major, R.E.(T.F.) (W.) M.C. M. I9I I

WMORRICE, W. W. Capt., Wiltshire Regt.; attd. Labour 1900

Corps

Killed in action 30 Dec. I9I7

HMORTIMER, E. Lieut., Northumberland Fus.(T.F.)

Killed in action at Ypres 26 April I9I 5

AMORTON, E. A. Lieut., R.F.A. (R. of O.)

Died 6 Nov. I9I8

Morton, J. F. A. See Amphlett-Morton, J. F.

Mourilyan, Rev. C. A. C.F. $4^{\text {th }}$ Class, R.A.C.D.

Mumford, C. G. 2nd Lieut., Essex Regt.(T.F.)

Munday, E. Major, Lancs. Fus. (W 2.) $M$.

Munro, R. M. C. 2nd Lieut., R. Welsh Fus.; attd. King's 1893 (Liverpool Regt.)

Murnoch, A. J. Capt., Rifle Brigade. (W.) 1912

Nathan, J. Capt., R. Sussex Regt.(T.F.)

$\mathbf{1} 898$

1897

NeIld, W. C. Capt., Essex Regt.; empld. Ministry of I9ro Labour. (W 2.) M.C. and Bar. M.

NeILson, W. Capt., Highland L.I.; Major, Tank Corps. I89 I (W.) (P.)

सNess, G. S. Lieut., R. Scots Fus.

Killed in action ro Nov. 1914

Newnes, Sir F. H., Bart. Capt., Bedfordshire Regt. 1894

Newton Clare, E. T. Major (A.), R.A.F. D.S.O. I90I

O.B.E. Chevalier, Order of Leopold (Belgium)

Newton Clare, H. J. Major, S.O. 2, R.A.F. M $2 . \quad 1906$

Newton Clare, W. S. Capt. (Ad.), R.A.F. M.B.E. 1906

Nrcholson, G. B. Capt., R.A.M.C.

WNicholson, G. C. N. Capt. (A.), R.F.C.

Killed in flying accident I I March I9I6

Nicholson, R. S. Lieut., Spec. List (Recruiting Staff) $\quad$ I889

NiELSEN, E. E. M. 2nd Lieut., Norfolk Regt. (W.) I9I I

NoBlE, A. H. Lieut., King's (Liverpool Regt., Liverpool 1906

Scottish, T.F.) and M.G.C. (W.)

WNorman, C. Capt., N. Irish Horse (R. of O.) 1898 Killed in action $12 \mathrm{Feb}$. 1917

c. U.w.I. 
Norman, R.E. Lce.-Sergt., London Regt.(Artists Rifles); 1908 Lieut., Oxford and Bucks. L.I. (W.)

WNorThrop, J. E. Flt. Sub-Lieut., R.N.A.S. Killed in flying accident 2 March 1917

WNoyes, J. C. Pte., Hertfordshire Regt. Killed in action 16 Sept. 1916

Odling, F. C. Surgeon Lieut., R.N.

Ogilvie, N. Pte., H.A.C.

Oldnall, R. W. Major, R. Munster Fus.; Capt., Gen. r889 List (Town Major). (W.)

Olive, G. W. Lieut., Oundle School O.T.C.

OIIVER, W. S. V. Lieut, Lancs. Fus.; Lieut. (A), R A. F. 1904 (W.)

OpPENHEIM, W., T.D. Hon.Major, R.E. (Signals, T.F.Res.) 1890 $M$.

\#Orford, W. K. Pte., R. Fusiliers (P. S. Bn.); 2nd Lieut., I9r3 Manchester Regt. and Gen. List (T.M.B.)

Killed in action on the Somme I fuly I916

Orpen-Palmer, R. A. H. Lieut.-Col., Leinster Regt. $\quad 1896$ (W 2.) D.S.O. M.

Osborn, E. B., T.D. Major, R.F.A.(T.F.) $M 2$.

Ovens, A. B. Capt. (A.), R.A.F.

ZOwen, W. H. K. Lieut., Welsh Regt.

Died I Oct. I9r 5 of wounds received in action

Page, C. C. Capt., R.A.O.C.

PALMER, C. Major, R.A.O.C.

Palmer, N. St C. Lieut., Hertfordshire Regt.; Capt., r900 N. Staffs. Regt. (W.) (P.)

Pam, E. A. 2nd Lieut., The Queen's (R.W. Surrey Regt.) (W.)

Panter, A. E. Surgeon-Lieut., R.N.; Capt. (Med.), I907 R.A.F. (W.)

Parez, C. C. T. Lieut., Spec. List (Staff Lieut.)

PARKIN, R. C. Lieut., London Regt. (Blackheath and Woolwich Bn.)

PARkin, R. T. Lieut., Rifle Brigade; empld. War Office. Igoo. (W.)

Parnell-Smith, W. Capt., Victoria College, Jersey, 1902 O.T.C.

Parrington, N. Capt., W. Yorks. Regt. (W 3.) M. 1905 Parrington, W. F. Major, R.F.A. M.C. $M 2$. 
Parry, A. H. Capt., Rifle Brigade; Staff. Capt.; empld. I9ro Ministry of Munitions. (W.) O.B.E.

ParRy, D. B. Lieut.-Col., London Regt. (London Irish 1896 Rifles) and M.G.C. (W.) D.S.O. $M_{3}$.

Parton, E. G. Capt., London Regt. (Kensington Bn.) 1897 and Gen. Staff (Draft Conducting Officer)

Paterson, K. Lieut., Suffolk Regt. and 9th Gurkha r912 Rifles, Indian Army

APATERSON, R. D. Lieut., King's (Liverpool Regt.). (W.) I9I I Killed in action 12 Oct. I916

Paton, W. Capt., King's (Liverpool Regt.); empld. War I9I I Office. (W 2.)

Paul, R. Lieut., Cheshire Regt.; attd. T.M.B. (W.) 1915

Pavey Smith, A. B. Capt., R.A.M.C.(T.F.) M.

PeAcey, Rev. C. C. C.F. 4th Class, R.A.C.D. I898

Peacock, G. Capt., R.A.S.C.

PeAcock, G. H. Lieut., Eastbourne College O.T.C. $\quad$ I884

PeAke, E. G. Capt., R.A.S.C.; Major, R.E. M. $\quad$ I895

PEARCE, A. H. Surgeon Lieut., R.N. $M$. I9I I

PPearless, H. N. Sergt., British Columbia Regt., Can- 1903 adian Force. D.C.M.

Killed in action near Ypres 24 April I915

PEGG, J. 2nd Lieut., R.A.S.C.; Capt. (T.), R.A.F. 1908

Pellier, C. DU C. Capt., R.A.M.C. 1892

Pelling, Rev. S. B. C.F. 4th Class, R.A.C.D. $M$ 2. 1904

Pelly, Rev. R. L. C.F. 4th Class, R.A.C.D. $m . \quad 1905$

Pemberton, J. Lieut., Cheshire Regt.(T.F.) $M 2 . \quad$ I912

Penistan, Rev. E. J. C.F. 4th Class, R.A.C.D. I895

Pennington, Rev. C. G. T. S. C.F. $4^{\text {th }}$ Class, R.A.C.D. $\quad 1893$ (T.F.)

Phelps, G. I. DE B. Capt. and Adjt., R.E. $m$. I895

PILCHer, E. M., D.s.o. Colonel, R.A.M.C.; Hon. Sur- 1884 geon to the King; Professor of Military Surgery, R. Army Medical College. C.B. C.B.E. M.V.O. $M_{3} \cdot m$.

APINFIELD, G. V. Lieut., 8th (King's R. Irish) Hussars

Killed in the Irish rebellion 24 April 1916

Pinks, E. D. P. Lieut.-Cdr., R.N.V.R.

I9I3

PITT G NewTon Major R A M.C (and London Gen. 1906 Hospital, T.F.) O.B.E.

Pitt, R. B. Lieut.-Col., R.E. M.C. M3. Cavalier, 1907 Order of the Redeemer (Greece)

Player, H. Lieut., Montgomeryshire Yeo. and M.G.C. I89o (Cavalry). 
Plews, H. W. Lieut., R.N.V.R.

Plowden, H. R. Capt., I7th Lancers. $M 2$.

Pollard, H. J. A. R.A.M.C.

PotTER, W. M Lieut, Seaforth Hdrs. Capt, Lanark- 1908 shire Yeo. (W.)

Powell, H. M. Lieut., R.F.A. M.C.

WPower-Clutterbuck, J. E. Corpl., R.E. (Signals); and Lieut., R.F.A.; attd. R.F.C. (W.)

Killed in action 25 June 1917

Poynder, E. G. T. Capt., R.A.M.C.(T.F.)

Prance, Rev. E. R. C.F. 4th Class, R.A.C.D.

APRANkerd, R. P. Lieut., Warwickshire Yeo. and M.G.C. 1914 Killed in action 10 Nov. 1918

APreeston, R. S. Gnr., H.A.C.; Cadet, O.C.B.

Died April r916 of septic pneumonia

Prichard, A. A. Capt., R.A.M.C.

Prichard, R. G. Major, R.E. $M 2$.

Pringle, J. S. Capt., R. Scots (T.F.) French Croix de Guerre

Procter, J. A. Lieut., Sherwood Foresters (Notts. and 1900 Derby Regt.) and Gen. List (T.M.B.)

Procter, R. A. W. Capt., R.A.M.C. (W.) M.C.

Pryce Jones, A. W. Major, R. Welsh Fus. (T.F. Res.);

Lieut.-Col., Saskatchewan Regt., Canadian Force; attd. Gen. Staff. O.B.E. M.

APuckridge, C. F. H. Pte., R. Fusiliers (P.S. Bn.); Capt., D. of Cornwall's L.I.

Killed in action 28 March 1917

Pulman, W. P. Lieut., Somerset L.I.; A.D.C. $M . \quad 1907$

APyman, A. Lieut., Yorkshire Regt.

Killed in action 15 Fune 1915

Pyman, R. L. Gnr., R.G.A.(T.F.)

RADFORD, R. L. Capt., R.A.S.C. and R.F.A. (W.) 1908 Ramsay, J. G. Major, Cameron. Hdrs.; Lieut.-Col., 1900 A.A. and Q.M.G. (W.) D.S.O. O.B.E. M 2. Belgian Croix de Guerre

Ramsbotham, W. H. Capt., W. Yorks. Regt.

Rankin, R. Corpl., R. Fusiliers (P. S. Bn.); 2nd Lieut., 1898 S. Staffs. Regt. M.C. M.M.

Ravenshear, E. W. Major, R. Berkshire Regt. $M 2$. 
RawCliffe, D. M. Capt., Manchester Regt.(T.F.); Lieut. 1907 (A.), R.A.F.

RAWLENCE, A. R. Major, R.A.S.C. $M$.

ReaD, H. M. Lieut., R.E. (W 2.) M.C. $M$.

1907

READE, J.A. D. and Lieut, St Edmund's School, Canterbury, O.T.C.

Rebsch, R. F. W. Capt., S. Wales Borderers and Un- I90I attd. List, T.F. (W.) M.C.

ReED, H. L. Capt. and Adjt., Middlesex Regt.; Major, 1903 Gen. List (Staff Capt.)

ReEs, A. G. T. Lieut., R.F.A. M.C.

REes, G. M. T. Major, S.O.2, R.A.F. O.B.E.

ReEve, A. E. 2nd Lieut., Norfolk Regt. (W.) (P.)

I9II

1905

ReID, A. A. 2nd Lieut., R.E.

1909

1904

Reid, A. Cunningham. 2nd Lieut., R.E.; Capt. (A. and I9I3

S.) R.A.F.; S.O.3. (W.) D.F.G. A.F.C. M.

ReID, E. B. Lieut., R.F.A. (W 2.)

1914

RHodes, F. A. Lieut., D. of Wellington's (W. Riding I9I5 Regt.) (W.)

Richards, H. A. Capt., R.A.M.C.

सRICHARDSON, E. B. 2nd Lieut., R.E.

Died 28 Oct. I9I5 of wounds received in action in Gallipoli

Richardson, T. Capt., Gen. List, empld. Ministry of 1899

National Service. O.B.E.

RILEY, J. H. 2nd Lieut., Yorkshire Regt.

Rintoul, A. J. Capt., Spec. List, empld. P. of W. Camp

RoberTs, A. C. Pte., R.A.S.C.

1903

1903

सRoBerts, C.Q. Corpl., R.E. (Signals); 2nd Lieut., R.F.A. M. French Médaille Militaire

Killed in action 16 May I9I 5

RoBerTs, FF. Capt., R.A.M.C.(T.F.); attd. R.A.F.

ROBERTS, H. T. LL. Capt., R.A.M.C. $m 2$. Order of I9OI St Stanislas, 2nd Class (Russia)

WRoberts, R. B. Trooper, Denbigh Yeo.; Capt., West- 1900 morland and Cumberland Yeo.

Died I3 Fan. I91 8 of illness contracted on active service

Robertson, J. W. H. See Hay-Robertson, J. W.

Robinson, Rev. A. H. R. Chaplain, R.N.

1879

1902

1914

1912

Robinson, L. D. Lieut., R.G.A.

सRobson, Rev. E. G. U. Chaplain, R.N.

Drowned on H.M.S. Aboukir 22 Sept. 1914

Roddam, R. J. Lieut.-Col., Northumberland Fus. and 1876 Spec. List (Staff Lieut.) O.B.E. M. m. 
Roe, J. C. Lieut., Lincolnshire Yeo.

Rogers, A. L. Major, R.F.A. (W 3.) D.S.O M. 1900

ROPNER, L. Major, R.G.A. M.C.

WRoscoe, T. le B. Pte., S. African Infy.

Killed in action 18 Sept. 1917

Rowe, E. F. Capt., Suffolk Regt. (R. of O.)

ARUTHERFORD, J. D. Surgeon Lieut., R.N.

Died I3 Sept. I9I7 of tuberculosis contracted on active service

Ruthven, J. St C. 2nd Lieut., R.E.

SAdler, P. H. Lieut. (A.), R.A.F.

SALTER, M. A. Lieut., R.A.S.C.(M.T.)

1914

SAlTER, R. E. Capt., R.A.S.C.

Samuel, C. H. Capt., Lancs. Fus.

[19I4]

1897

I 884

1908

\#SASSOON, H. W. 2nd Lieut., R.E.

Died I Nov. I915 of wounds received in action in

Gallipoli

Sassoon, S. L. Capt., Montgomeryshire Yeo. and R. 1995

Welsh Fus. (W 2.) M.C.

Saul, C. L. Lieut., Tank Corps. (W 2.)

SAUNDERs, A. G. Major, Bedfordshire Regt. ; attd. Essex

Regt. (W). m. French Croix de Guerre

SAundERs, D.M. Capt. and Adjt.,Bedfordshire Regt. M.C. 1912

Saunders, F. P. Capt., R.A.M.C.

Sawtell, H. D. Lieut., R.A.O.C.; I.O.M. 3rd Class

\#Scholey, C. H. N. Capt., Rifle Brigade

Killed in action 25 Sept. r91 5

Sclater, F.A. Capt., R.E. (Signals). O.B.E. M.C. M 2.1912 Italian Croce di Guerra

ScotT, J. H. Lieut., Scottish Horse; Major, Gen. Staff. $\quad 1892$

O.B.E. M. Order of the Nile, $4^{\text {th Class }}($ Egypt)

ScotT, H. W. Surgeon, R.N.

ScotT, W. L. Lieut., R.N.V.R. D.S.G.

ScowCroft, H. D. Lieut., R.F.A.; Lieut. (O.), R.A.F.

ScutT, C. A. 2nd Lieut., Spec. List (Interpreter)

Seddon, G. N. Capt., Suffolk Regt. (W.) M.C.

SEDDON, W. D'A. 2nd Lieut., Worcestershire Regt.

SEDGwICK, H. R. Capt., R.A.M.C. $m 2$.

I9I3

I908

1904

1909

1912

I9I5

I910

1905

WSemple, W. D. Pte., R. Fusiliers (P.S. Bn.); 2nd Lieut., I9r3

King's Royal Rifle Corps

Killed in action 29 Fune 1916

WSenior, J. Lieut., W. Yorks. Regt.; attd. R.F.C.

Died 9 May 1917 of wounds received in action

1902

1910

1912

1908

I9I 2

1910

I 888

3

I9I I 
Sessions, L. F. Capt., R.A.M.C. (W.) Italian Silver I9I I Medal for Military Valour

Sessions, R. V. Pte., London Regt. (Artists Rifles); I9I4 Lieut. (A.), R.A.F.

Seth-Smith, K. J. Capt., R.F.A.; Staff Capt. O.B.E. $\quad$ I900 SewILl, J. W. 2nd Lieut., Army Cyclist Corps; Lieut., 1908

R.E. (Spec. Bde.); empld. Ministry of Munitions

Sewill, R. W. Capt., R.A.S.C.

I9I I

Shackleton, A. G. Hon. Capt. and Q.M., London Regt. I9I4

(R. Fus.) O.B.E. $M$.

ShAw, C. R. Major, Loyal N. Lancs. Regt.(T.F.) I899

SHAXBY, R. U. Major, R.A.O.C.; I.O.M. Ist Class

SHEARD, M. H. Lieut., R.N.V.R. Officer, Order of 1908 Nichan-Iftikhar (Tunis)

Whearman, V. Pte., R.Fusiliers(P.S.Bn.); Capt., R.Scots 1907 Killed in action 25 March 1918

ShEPHERDSON, J. F. 2nd Lieut., Leys School O.T.C. I9I3

SHERLOCK, E. L. Lieut., R.N.V.R.

Sholl, A. E. Lieut., Loyal N. Lancs. Regt.(T.F.). 1912 (W.)

Sidebotham, F. N. Capt., R.A.M.C.

WSidebotham, J. N. W. Capt., Manchester Regt.

Killed in action 12 Oct. 1916

SidGwICK, H. C. Lieut.-Col., R.A.M.C. O.B.E. $M$ 2. ${ }_{1896}$ ESIlverToP,W.A. Capt., 2oth Hussars. (W.) M.C. M2. 1903 Killed in action 27 Nov. 1917

Simpson-Hayward, G. H. Capt., Oxford and Bucks. 1894 L.I. (T.F.)

«Sisson, G. Lieut., R.G.A.

Died in Germany 20 Dec. 1917. of wounds received in action near Cambrai 30 Nov. r917

Sisson,H.A. Major, R.E.; D.A.D. Gas Services. O.B.E. 1904 M2.

WSkIRRow, G. Capt., W. Yorks. Regt.(T.F.) French Croix I9I4 de Guerre

Killed in action 27 Aug. 1918

सSLACKE, R. C. Major, The Buffs (E. Kent Regt.); attd. 1899 The Queen's (R.W. Surrey Regt.) $M$.

Killed in action 16 May I9I 5

Slade, E. A. Capt., R. Warwickshire Regt. and Labour [I9I4] Corps

Slade, J. G. Capt. and Adjt., R.A.M.C.

Slaney, J. N. Lieut., King's (Shropshire L.I.) and 1995 M.G.C. (W.) 
Smith, A. B. P. See Pavey Smith, A. B.

Smith, J. F. H. Capt. and Adjt., R.F.A. (W.) 1906

Smith, N. H. Capt., R.A.M.C.; Surgeon Lieut., R.N. 1908 D.S.C.

Smith, S. Pioneer, R.E. (Spec. Bde.)

\&Smith, W. G. F. Pte., R. Fusiliers (P.S. Bn.); Lieut., 1907

N. Staffs. Regt.

Died 5 Fuly I9I 5 of wounds received in action

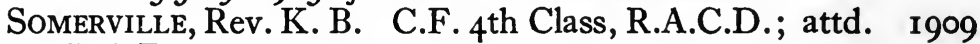
R.A.F.

Sorley, G. M. Capt., London Regt. (R. Fus.); R.T.O. 1910 (W.) O.B.E. M2.

SouTHwell, H. M. 2nd Lieut., Highland L.I.

SPENCER, R. Capt., Denbighshire Yeo. and Tank Corps. I90I

M.C. M 2. Chevalier, Legion of Honour (France)

ESpittle, T. S. Capt., Monmouthshire Regt.

Died 2 Oct. I9I7 of wounds received in action

Spurrell, H. W. Pte., R. Fusiliers (P. S. Bn.); Capt. 1913 M.G.C. (W 2.) M.C.

¿Stanfield, A. V. 2nd Lieut., The Queen's (R.W. Surrey 1903 Regt.)

Killed in action 16 Aug. I916

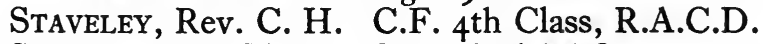

Steedman, M. T. W. Capt., R.A.M.C.

Stephen, A. F. Lieut., R.G.A. $M$.

\#Sternberg, R. O. Corpl., R.E. (Signals); 2nd Lieut.,

R.F.A. (W.)

Died I fuly I9I 6 of wounds received in action

Stirling, F. Lieut., Hampshire Regt.

WSTirling, G. S. Capt., Argyll and Sutherland Hdrs. I905

(R. of O.) and King's African Rifles. D.S.O. M.C.

$M$.

Died 26 Dec. I9I6 of wounds received in action

\#Stoodley, P. B. 2nd Lieut., Wiltshire Regt.

Died 9 Nov. I9I 6

STORER, R. S. 2nd Lieut., Hertfordshire Regt.

StOREY, C. B. C. 2nd Lieut., Lancs. Fus.(T.F.)

सStorrs, J. P. 2nd Lieut., Cheshire Regt.(T.F.)

Died 8 Aug. I9I 7 of wounds received in action

Strain, L. H. Lieut.-Col. (A.), R.A.F.; S.O. I. D.S.C. 1894

$M$ 3. Order of the Redeemer, $4^{\text {th }}$ Class (Greece)

Stringer, C. H. Capt., Suffolk Regt. (Cyclist Bn., T.F.) 1905 $m$.

Swete-Evans, W. B. Major, R.A.M.C. 
Swinstead, N. H. Lieut., R.E. (Signals); empld. War Igr I Office. M.B.E. $m$.

Syme, D. A. Lieut., K. Edward's Horse; Capt., Tank rgr3 Corps. (W.) $M$.

Symington, A. W. Lieut., King's Royal Rifle Corps; I9I I Lieut. (A.), R.A.F. (W.) M.C. M.

सTALbot, C. E. C. 2nd Lieut., Somerset L.I.

Killed in action 25 Sept. 191 5

ATANBURN, W. L. Lieut., I.A.R.O., attd. 2nd Gurkhas [1914] Killed in action 13 April 1917

Tatham, C. F. Lieut., R.A.S.C.

TAYLOR, C. P. Corpl., R.A.S.C.(M.T.)

TaYlor, J. T. 2nd Lieut., Dorset Yeo.

Telfer, Rev. W. C.F. 3 rd Class, R.A.C.D.; Cadet, 1905 O.C.B. M.C.

ETemperley, A. R. Pte., Northumberland Fus.(T.F.)

Died of injuries accidentally received while on duty 26 Aug. I9I4

Temperley, H. R. Lieut., R.F.A. (W.)

THA, R. R. H. O. Lieut., I.M.S.

ThIRkILl, H. Major, R.E. (Signals). M.C.

1906

1900

1914

1907

Thомas, H. J. Capt., King's (Liverpool Regt.) (W 2.) *1904 M.C.

Thomas-Peter, G. F. Capt., D. of Cornwall L.I.(T.F.) I90I

Thompson, S. W. Lieut., R. Scots Fus.; Capt. (A.), rgI I R.A.F.

EThorne, C. Capt., E. Surrey Regt. M.C. M.

Killed in action on the Somme 30 Sept. 1916

I9II

EThornton, A. C. Pte., Canadian Force

Died at Bailleul 22 Nov. I915 of wounds received in

1904 action

ThORNYCROFT, O. Lieut., R.N.V.R.

1903

Thursby, P. H. Capt., R.F.A. $M 2$.

I913

Timins, Rev. F. C. C.F. 3 rd Class, R.A.C.D. D.S.O. $\quad$ r884 $M$.

TIPPER, G. H. Lieut., I.A.R.O.

1900

TOLlER, T. E. 2nd Lieut., Leicestershire Regt.; Capt., 1904

R. Munster Fus. $M$.

\&Toolis, J. H. Lieut., Lincolnshire Regt.

Killed in action 1 fuly 1916

1912

Toone, C.L. Capt., Cheshire Regt. and Gen.List(A.P.M.) $\quad 1896$

Tootal, F. E. O. Lieut., R.F.A.; Capt., Spec. List. 1895 Chevalier, Military Order of Avis (Portugal) 
सTopham, H. A. C. 2nd Lieut., Indian Army, attd. Welsh I9ro Regt.

Died ${ }_{2} 5$ May 1915 of wounds received in action 24 May I9I 5

Tozer, W. Capt., York and Lancaster Regt. (T.F.); I9I3 empld. War Office. (W 4.) $M$.

Treadgold, C. H. Capt., R.A.M.C.

Trechman, O. L. Major, R.G.A. $M$.

4TRevor-Jones, E. E. 2nd Lieut., Rifle Brigade

Killed in action on the Somme I fuly I916

1899

1902

1914

WTrevor-Jones, J. E. Capt. and Adjt., Rifle Brigade. M.C.

Killed in action 22 April 1918

WTrevor-Roper, C. C. Capt., Hampshire Regt.(T.F.)

Died 3 Aug. 19I7 of wounds received in action

ETrier, N. E. Pte., London Regt. (Artists Rifles); 2nd Lieut., E. Yorks. Regt.; attd. Yorkshire Regt.

Died 6 Oct. I915 of wounds received in action at the Hohenzollern Redoubt

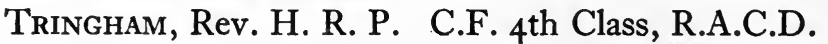

Trotter, J. Capt., Highland L.I. (W.) $M$.

Trotter, L. B. C. Capt., R.A.M.C.

Trubshaw, A. R. Lieut., R.F.A.(T.F.); Staff Lieut.

Trubshaw, C. S. Capt. and Adjt., R. Welsh Fus.

Trubshaw, H. E. Major, R.E.(T.F.). $M$.

Tufnell-Klug, M. W. T. Capt., R.A.S.C.(T.F.)

Turner, J. T. Capt., I.A.R.O., attd. 28th Light Cavalry

Tweddell, J. R. M. Lieut., Leicestershire Regt.; attd.

R. Defence Corps; Capt. (Ad.), R.A.F.

Unwin, W. N. Lieut., R.F.A.(T.F.)

1897

VAN DER ByL, C. F. Capt., I6th Lancers (R. of O.); 1894 Major, Army Cyclist Corps. $M$.

Vane, Hon. W. L. Hon. Colonel, Durham L.I.(T.F.) m. 1877

VANRENen, J. E. Lieut.-Col., R.E.; C.R.E. Brevet 1884 Colonel. $M$.

VAtcher, H. M. Lieut., R.E. and R. Jersey Militia. 1905 M.C.

Vaux, R. W. Pte., The Buffs (E. Kent Regt.)

VeSTEY, F. 2nd Lieut., Border Regt.

Vestey, P. C. Lieut., Suffolk Regt.

VeY, D. C. L. Capt., R.A.M.C. M.C. M.

VeY, F. H. Surgeon Lieut., R.N.
1910

I9II

I9II

1908

1907 
WWainwright, G. C. 2nd Lieut., Northamptonshire Regt. I9I3

Died 22 Dec. 1914 of wounds received in action

WaLkER, C. E. Major, R.G.A.(T.F.) M.C. $\quad 1904$

Walker, C. V. Cadet, O.C.B.

WalKer, G. A. C. Lieut., Inniskilling Fus. and Gen. I9I2 List, empld. R.E. (Signals). M.C. $M$.

WalkeR, J. Major, R.A.M.C.(T.F.) (W.) M.C. M 4. 1902

WalkeR, V. A. Capt., Res. Regt. of Cavalry and Spec. I901

List; A.M.L.O.; D.A.D.R.T. Brevet Major

Wallace, H. L. Pte., Worcestershire Regt.

WWalley, G.S. Lieut. King's Royal Rifle Corps. (W 2.) I9I I

Died 20 Aug. 1916 of wounds received in action

WALlINGER, E. A. Lieut.-Col., R.F.A.; G.S.O. 2. (W.) 1897

D.S.O. $M 4$. Chevalier, Legion of Honour (France).

Officer, Ordre de la Couronne (Belgium). Belgian

Croix de Guerre

WWallis, E. P. Capt., R. Sussex Regt.

Killed in action 18 Oct. I916

Wallis, W. E. Capt., R.A.M.C. (W.) 1902

WAlters, W. J. Capt., R.A.M.C. 1909

WaNe, H. B. 2nd Lieut., Labour Corps 1909

WWARD, F. W. Capt., Gloucestershire Regt.(T.F.) 1904

Killed in action 9 Oct. I9I7

WARD, H. M. A. Major, R.G.A.; A.D.C. D.S.O. T.D. 1903 $M$.

WARD, W. H. 2nd Lieut., W. Yorks. Regt.

Warlow, N. F. Pte., King's Own (Yorkshire L.I.)

Watson, A. W. H. Capt., King's Royal Rifle Corps and

Gen. List (Brigade Major). D.S.O. M.C. M 2.

Watson, D. H. Lieut., Ist Dragoons

Wauton, A. D. B. Lieut., R.A.S.C.; Capt., R.T.O. $\quad$ I903

WEAVER, F. W. H. Naval Transport Officer. O.B.E. 1898

WEBB, J. C. Major, R.A.M.C.

WEBER, D. MCR. Lieut., Middlesex Regt.

Weber, H. G. Lieut., Bombay Garrison Arty., Indian Defence Force

WWebster, J. R. Pte., H.A.C.; Capt. and Adjt., London 1899

Regt. (R. Fus.)

Killed in action 9 Sept. ror6

Weller, C. A. Capt., R.A.M.C. $M$.

Whatham, A. Capt., King's Own (R. Lancaster Regt.) 1907 and R. Defence Corps

Whitaker, G. H. 2nd Lieut., King's (Liverpool Regt.); 1908 Lieut., M.G.C.; Hon. Lieut. (T.), R.A.F. (W.) 
White, A. K. G. Major, R.F.A.; G.S.O. 2. D.S.O. 1900 $M 2$.

White, L. T. and Lieut., R.G.A.

WhITEHEAD, B. Major, R.A.M.C.; D.A.D.M.S. M.C. 1907

Whitehead, C. M. Capt., King's Own (R. Lancaster 1900 Regt.) D.S.O. M.C. M 2. Chevalier, Legion of Honour (France)

WWhitehead, P. N. Trooper, S. African Light Horse; Capt., R.E. (W 2.) M.C. M.S.M.

Killed in action $2 \mathrm{I}$ March $\mathrm{rg} 8$

Whitehead, R. H. H. Capt., R.H.A.; Courts-Martial 1899 Officer. M.C.

Whitfield, J. G. Capt., R. Warwickshire Regt.(T.F.); $\quad 1895$ Major, Spec. List (Cmdt., Reception Camp)

Wilcox, Rev. A. G. C.F. $4^{\text {th }}$ Class, R.A.C.D. $M . \quad{ }^{*} 188 \mathrm{r}$

Wiles, J. J. Capt. and Adjt., Wiltshire Regt.(T.F.) 1909

Wilkinson, C. L. G. Capt., King's Royal Rifle Corps 1914

Wilkinson, R. DU C. Lieut., R.A.S.C.

Wilkinson, S. Capt., King's Own (Yorkshire L.I.) M.C. I910

WWilliams, C. J. Lieut., Bedfordshire Regt.

Died I9 Dec. I91 5 of gas-poisoning received near Ypres

Williams, E. H. Y. Lieut., King's (Liverpool Regt.)

(W.)

Williams, Rev. H. F. F. Junior Chaplain, Indian Army

Williams, N. S. Lieut., R.A.M.C.

Williams, W. F. Lieut., R.E. (Signals). M.B.E. $M$.

Willox, A. G. Lieut., Gordon Hdrs.; empld. War Office. (W.) $M$.

Wilson, A. Capt., W. Yorks. Regt.(T.F.) (W.)

WWilson, A. E. Capt., R. Warwickshire Regt.

Died 3 Dec. 1918 of pneumonia contracted on active service

Wilson, A. E. J. Major, Somerset L.I.; Lieut.-Col., 1898 A. A. and Q.M.G. D.S.O. M 3. Medjidieh, $4^{t h}$ Class

Wilson, Rev. E. C. C.F. $4^{\text {th }}$ Class, R.A.C.D.

WILson, G. H. A. Major, Unattd. List, T.F.; G.S.O. 3, 1892 War Office. O.B.E. M.B.E. Brevet Lieut.-Colonel. $m 2$.

Wilson, Rev. K. F. C.F. 4th Class, R.A.C.D.

Wilson, W. H. Pte., London Regt. (London Scottish); 1914 Lieut., Devon Regt.(T.F.) $M$.

Wiltshire, H. W. Major, R.A.M.C. D.S.O. O.B.E. $\quad 1897$ $M$ 2. Order of St Sava, $4_{\text {th Class (Serbia) }}$ 
Winter, B. E. Lieut.-Col., Army Pay Corps; Staff 1875 Paymaster

WinTER, C. E. G. Capt., R.A.M.C.

1889

Winter, W. Pte., H.A.C.

WWinton, E. W. 2nd Lieut., R.G.A.

1915

Killed in action $15 \mathrm{Dec}$. 1917

Woon, A. R. Lieut., R.G.A.(T.F.); Capt. (T.), R.A.F. I9I4

WWood, P B. Lieut., R. Fusiliers

Killed in action 23 April I917

Wood, S. R. Lieut., R.G.A. (W.)

Wood, W. V. Lieut.-Col., R.A.M.C. M.C.

WWoodhouse, Rev. D. C. C.F. $4^{\text {th }}$ Class, R.A.C.D.

Died on active service 6 Oct. 1916

Woodhouse, G. W. Surgeon Lieut., R.N.

Wootton, H. A. Capt., Spec. List; empld. Ministry of Munitions. M.B.E. m.

Workman, R. Major, R. Irish Rifles. (W.) 1902

Worsley, Rev. F. W. C.F. $4^{\text {th Class, R.A.C.D. I9Io }}$

WWRAGG, N. J. Lieut., S. Staffs. Regt. (W.)

Died 18 July 1916 of wounds received in action 16 fuly 1916

Wright, A. J. Capt., R.A.O.C.; I.O.M., 2nd Class. 1909 O.B.E.

WRIGHT, F. T. Lieut., R.E. (W.) M.C. I909

WYER, F. F. Lieut., R.F.A. (W.) I9IO

Wynne, R. O. Capt., Bedfordshire Regt.; Lieut.-Col., 1912 King's (Liverpool Regt.). (W.) D.S.O. $M_{3}$.

Yeatman, F. D. Lieut., Middlesex Regt. (W.) 1908

Youll, G. B. Lieut., Northumberland Fus. (W.) I897

Young, C. S. Major, R.F.A.; Inspector, Woolwich 1897 Arsenal. Brevet Lieut.-Colonel. $m$.

Young, K. R. 2nd Lieut., Lancs. Fus.; Lieut., R. Welsh 1913 Fus.

WYoung, P. M. Lieut., King's (Liverpool Regt.) $M$.

Killed in action at Givenchy 10 March 1915

I9I I 


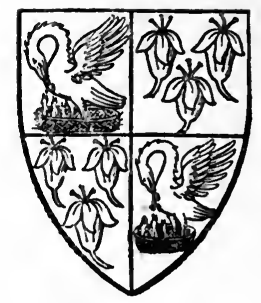

\section{CORPUS CHRISTI COLLEGE}

Adam, D. Bruce. See Bruce Adam, D.

Adams, Rev. N. P. C.F. 4th Class, R.A.C.D.

Alford, Rev. C. S. L. C.F. 4th Class, R.A.C.D.

1895

Alford, Rev. J. G., v.D. C.F. Ist Class, R.A.C.D.

1903

C.B.E. $m 2$.

ARnold, A. J., D.S.o. Bt. Colonel, Manchester Regt. and 1884 R. Welsh Fus. C.B.E. m.

ABARKer, W. Major, Worcestershire Regt.

Died ${ }_{5}$ Aug. I91 8 of wounds received in action

WBARNES, J. E. T. Capt., Gloucestershire Regt.

Killed in action $3 \mathrm{Feb}$. 1917

Bell, R. H. Capt., Oxford and Bucks. L.I. (W.) M.C. 1906 and Bar

BERNARD, D. V. 2nd Lieut., D. of Wellington's (W. Riding Regt.); Lieut., The Queen's (R.W. Surrey Regt.)

BolD, T. A. 2nd Lieut., Loyal N. Lancs. Regt.

Booth, W. R. Lieut., R.F.A.

I894

Boultbee, B. St J. 2nd Lieut., E. Yorks. Regt.; Lieut., I9I3

Northamptonshire Regt.; Capt. (A.), R.A.F. M.C. $M$.

Bournes, G. H. Corpl., R.E.

WBower, C. F. Capt., Sherwood Foresters (Notts. and

Derby Regt.)

Killed in action $\mathrm{I}_{3}$ Sept. 1917

EBray, E. F. Lieut., R.N.A.S.

Missing, presumed killed in action, 19 Fuly 1917

I9I3

r914

I9I0

1893

1910

[1914]

WBrownleE, W. M. 2nd Lieut., Dorset Regt.

Died 12 Oct. 1914 of pneumonia 
Bruce Adam, D. Lieut., Argyll and Sutherland Hdrs. I9I I and M.G.C.; attd. R.E. (Signals)

ABruce Lockhart, N. D. S. Lieut., Seaforth Hdrs.

Killed in action 25 Sept. I9I 5

Buck, G. R. Lieut., Bedfordshire Regt.(T.F.)

WBudgen, R. G. Lieut., King's (Shropshire L.I.)

Killed in action 24 Aug. I9I 5

\#Bullock, G. F. 2nd Lieut., S. Wales Borderers Killed in action $3 \mathrm{I}$ Fuly 1917

1913

1909

I9I3

1900

ABuRgess,D. Lieut.-Col.,R.A.M.C.(3rd N.Gen.Hospital, 1872 T.F.)

Died 17 Fan. I9I7

ButLeR, R. L. G. Capt., Spec. List; Town Major, Ypres. *I9I3 (W.)

CAREy, R. S. Surgeon Lieut., R.N. O.B.E. $M$.

1901

CAssidi, F. L. Surgeon Lieut., R.N.

1907

Cave, T. S. Capt., R.G.A.; empld. Ministry of Muni- rgıo tions

Chadwick, Rev. C. E. C.F. 2nd Class, R.A.C.D.; 1905 D.A.C.G.; Senior Chaplain, R.A.F. (W.) M.C. M. $m$.

Chappell, F. E. Major, R.A.S.C. $M$.

1900

Christie, R. G. Capt., R.E. $M 3$.

Churchill, G. S. Capt., E. Surrey Regt.

ChurChILl, H. E. Lieut., R.A.S.C.(M.T.)

Churchward, Rev. B. C.F. $4^{\text {th }}$ Class, R.A.C.D.

*Churchward, H. A. 2nd Lieut., London Yeo. (West- I9I I minster Dragoons); attd. R.F.C.

Killed in action I6 Aug. I9I7

Clark Kennedy, A. E. Lieut., The Queen's (R. W. Igi I Surrey Regt.); Capt., R.A.M.C.

*Clarke, J. P. D. Lieut., Worcestershire Regt. I9IO Accidentally killed I9I5

Colletr, W. G. Major, Rifle Brigade

I9I3

1898

1912

1908

Collins, E. G. W. Lieut., Wiltshire Regt. (W 2.) M.C.

Conningham, W. F. M. 2nd Lieut., R.A.S.C.(M.T.)

1888

COOPER, H. O. Lieut., R.F.A.; Staff Capt. O.B.E. 1907 $M$ 2. French Croix de Guerre

ACOOPER, H. W. F. 2nd Lieut., R. Fusiliers

Died 28 April 1917 of wounds received in action

1899

Copeman, S. A. M., T.D. Lieut.-Col., R.A.M.C. (Sanitary Service, T.F.Res.) Order of St Fohn of Feru-

I879 salem 
Coulcher, G. B. Pte., Durham L.I.; Lieut., R.A.S.C. 1901 $M$.

Craft, H. B. Sergt., R.A.M.C.(T.F.)

CRICK, G. H. Lieut., R. Scots; Asst. Instructor in Gunnery; Capt. (T.), R.A.F.

Crispin, A. E. Pte., London Regt. (Artists Rifles)

*Crosse, R. G. Lieut., Queen's Own (R.W. Kent Regt.)

Died 14 Fuly 1916 of wounds received in action

Crowther, W. C. Lieut., London Regt. (St Pancras Bn.) 1904

Cullen, W. G. 2nd Lieut. (T.), R.A.F.

1904

1913

1894

1913

Cunnington, E. C. Capt., R.A.M.C. $M$. Killed in action 23 March 1918

DARE, A. G. 2nd Lieut., St Lawrence College, Rams- 1902 gate, O.T.C.

Davies, Rev. P. M. C.F. $4^{\text {th }}$ Class, R.A.C.D.

WDavies, T. A. M. Lieut., R.F.A.(T.F.)

Killed in action I Fuly I9I 6

Dawbarn, G. R. Lce.-Corpl., R. Fusiliers

Dawes, Rev. A. W. C.F. 2nd Class, R.A.C.D.

WDAY, N. L. 2nd Lieut., King's (Liverpool Regt.)

Killed in action 14 Sept. 1916

WDevereux, H. W. Lieut., S. Staffs. Regt.(T.F.) (W.) 1913 Killed in action 26 June I916

WDixon Wright, Rev. H. D., M.v.o. Chaplain, R.N.

Died 31 May 1916 of wounds received in action in the Battle of Futland

Donaldson, C. H. Rfn., London Regt.(L.R.B.) (W.) (P.)

WDoudney, Rev. C. E. C.F. $4^{\text {th }}$ Class, R.A.C.D.

Died 16 Oct. 1915 of wounds received in action

1908

1912

1912

1891

1913

1889

Doughty, E. C. Major, Suffolk Regt.; empld. War Office 1888

DruRY, Rev. W. C.F. 2nd Class, R.A.C.D. M.C. $M_{4} . \quad 1895$

सDuckwORTH,W. C. 2nd Lieut., Welsh Regt.; attd. King's *19I0

(Shropshire L.I.)

Killed in action 8 Oct. 1918

Dunlor, C. C. Lieut., R. Scots

Durnford, Rev. F. H. C.F. 4th Class, Australian Chap- Ig0I lains' Dept. M.C.

WDyer, H. F. 2nd Lieut., D. of Wellington's (W. Riding 1904 Regt.)

Died 28 Aug. 1917 of wounds received in action 8 Aug. I9I7 
Eddowes, Rev. H. C. C.F. $4^{\text {th }}$ Class, R.A.C.D.; attd. 1899 R.A.F.

ElwiN, W. D. Capt., R.E.(T.F.)

Everett, C. E. F. Pte., R. Fusiliers

1899

[1914]

Farnsworth, Rev. C. R. C.F. 4th Class, R.A.C.D. I9Io

FARRER,E.R.B. Capt., R.A.S.C.;Major,D.A.D.Supplies. I910 M.C. $M_{4}$.

Findlay, J. G. Lieut., Bedfordshire Regt.

1902

Fison, E. T. Capt., R.A.M.C. (Sanitary Service, T.F.) 1888

Forse, Rev. L. N. C.F. 4th Class, R.A.C.D. (P.) 1903

Foulston, S. V. Corpl., R.E. (Spec. Bde.)

1912

Gaitskell, M. H. Lieut., R.E.

GALER, F. B. Capt. and Adjt., London Regt. (Queen's) 1892

GaleR, R. V. Capt., London Regt. (Queen's) I898

Galloway, A. Capt., Cameronians (Scottish Rifles); [1914] G.S.O.3. M.C. $M$.

GARDNER, J. Lieut., R.G.A.

Gates, S. B. Pte., R. Fusiliers (P. S. Bn.)

I899

Gell, Rev. E. A. S. Lieut., R. Fusiliers; Lieut.-Col., 1894 Lancs. Fus. (W 2.) (P.) D.S.O. M.C. M.

Gillibrand, A. Pte., R. Fusiliers (P. S. Bn.); Lieut., D. 1904 of Wellington's (W. Riding Regt.) (W 2.)

GledHILl, A. Lieut., Monmouthshire Regt. (W.) M.C. I9I4 \&GlegG, A. L. 2nd Lieut., King's Royal Rifle Corps I9I4 Killed in action 10 Aug. I9I 5

Goolden, D. C. 2nd Lieut., R. Fusiliers

GrabURN, G. N. Lieut., R.F.A.(T.F.) (W 2.)

[1914]

I903

Gren, T. R. Pte., R. Fusiliers; Lieut. (K.B.), R.A.F. . I9I0

GRIfriths, Rev. R. C.F. th $^{\text {th }}$ Class, R.A.C.D. $M$. 1893

Hall, A. F. Lce.-Corpl., Gloucestershire Regt.

Hall, A. L. Pte., Kent Cyclist Bn.; Lieut., Lancs. Fus. 1908

Hall, J. T. Lieut., Loyal N. Lancs. Regt. ; attd. King's 1912 Royal Rifle Corps. (W 2.) $m$.

AHamilton, J. and Lieut., Border Regt.(T.F.) Killed in action 5 Nov. 1916

WHanna, J.H. 2nd Lieut., London Regt. (St Pancras Bn.) I90 I Killed in action 20 Sept. I9I7

HARPER-Smith, S. W. Q.M.S., R. Fusiliers; 2nd Lieut., $\quad 1892$ Labour Corps

Harrison, F. E. Capt. and Adjt., R.F.A. M.C. and I9I I Bar. $M$. 
ZHarston, F. N. Capt., E. Lancs. Regt.; Brigade Major. 1909 M.C. $M 2$.

Killed in action 22 April I918

Hart, B. H. L. Capt. and Adjt., King's Own (Yorkshire r9r3 L.I.) (W 2.)

Harvey, G. T. B. Lieut., I.A.R.O., attd. Corps of r9ro Guides; Major, Calcutta Bn., Indian Defence Force

Harvey, H. M. 2nd Lieut., Northamptonshire Regt. and R.G.A.

Havers, C. R. Lieut., Hampshire Regt.; Capt. and Adjt., 1908 Tank Corps. (W.) $M$.

Heaton, R. Lieut., R.F.A. (W.)

HHewitT, Rev. F. W. C.F. $4^{\text {th }}$ Class, R.A.C.D. Killed in action near Vermelles 28 Sept. I9I 5

WHill, W. R. Lieut., Durham L.I. (W.) M.C. and 1915 Bar

Died at Stralsund, Germany, 6 Nov. I9r8 of blood poisoning

Hinderlich, A. A. W. Capt. and Adjt., R.G.A. (W.) I912 $M$.

Hodder, F. E. Lce.-Corpl., R. Munster Fus.

Holt, F. N. Capt., Queen's Own (R. W. Kent Regt.); 1912 empld. Ministry of Labour

Hooley, L. J. Corpl., R.E. (W.)

Hoskyns, Rev. E. C. C.F. $3^{\text {rd Class, R.A.C.D. (W.) * }{ }^{*} 9{ }^{1} 5}$ M.C. $M$.

Hunter, C. J. Lieut., N. Staffs. Regt. (W.) M.C.

Hutchinson, J. H. Air Mechanic, R.A.F.

1907

1912

Illingworth, O. Capt., W. Yorks. Regt. (W.) (P.) 1906 Ison, A. J. Lieut., Northumberland Fus. and Welsh I9I I Gds.; attd. Gds. M.G. Regt.; Cmdt., School of Instruction

JACKs, M. Lieut., E. Lancs. Regt.; Lieut. (Ad.) R.A.F. WJAmes, E. S. P. K. Capt., King's Royal Rifle Corps

Killed in action 17 March 1915

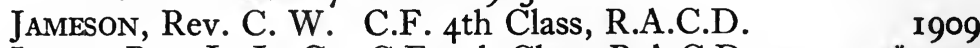

JeEves, Rev. L. L. G. C.F. 4th Class, R.A.C.D. m. *19ro

Jenkins, G. E. Lieut., E. Yorks. Regt. and The Queen's I9I3

(R.W. Surrey Regt.)

Jomaron, A. C. Capt., R.F.A. (W.)

Jones, B. C. Capt. (A.), R.A.F. (W.) $M$.

1912

JoNES, R. R. P. Instructor Lieut., R.N. 
Jordan, G. P. 2nd Lieut., Essex Regt.(T.F.); Lieut., 1908 Labour Corps

KaIN, H. G. Lieut., R.G.A.

सKeating, G. H. Pte., Middlesex Regt.(P.S. Bn.); Lieut., I9I I

Cambridgeshire Regt.; Instructor in Bombing

Killed in action 18 Sept. I918

Kelham, M. H. C. Lieut., Durham L.I.; attd. R.Defence 1907 Corps. (W.)

KEMP, K. M. Capt., I.A.R.O., attd. Baluchistan Infy. $\quad$ I903

Kempe, Rev. W. N. C.F. 4th Class, R.A.C.D. 1907

Kendall, J. M. A. Capt., Norfolk Yeo. I9I I

KIRKCALDY, G. I. Lieut., R.A.S.C.

1913

KirkPatrick, R. M. Capt., Rifle Brigade and Gen. Staff. 1909 (W.)

WKNIGHT, E. A. 2nd Lieut., M.G.C.

Killed in action 24 Sept. 1917

1905

LA Brooy, M. V. T. J. Lieut., R.G.A.; empld. Ministry I9I3 of Munitions

HLaing, A. T. Capt., Northumberland Fus.

Died 24 Fuly 1916 of wounds received in action

LAMbert, W. Capt., R. Fusiliers; Major (K.B.), R.A.F. 1909 $m$.

La Mothe, H. D. Nigeria Regt., W. African Frontier 1909 Force

ALANG, H. A. Major, Worcestershire Regt. $M$.

Killed in action in Gallipoli 9 Fune 1915

Laporte Payne, A. A. Major, R.F.A. (W.) $M 2$. 1909

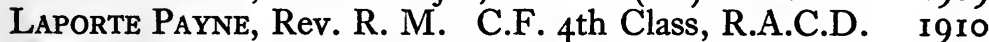

LART, C. E. Capt., Devon Regt.(T.F.) T.D. I886

LAST, F. W. 2nd Lieut., Lincolnshire Regt.

\&La Touche, D. D. 2nd Lieut., King's (Shropshire L.I.); [19I4]

Capt., Welsh Regt.

Killed in action in Gallipoli 8 Aug. I9I5

LATTEY, W. T. Lieut., R.F.A.

LAWRENCE, Rev. G. H. R.A.M.C.

LEAKEY, R. A. Capt., Indian Army; R.T.O.

WLEEKE, H. A. Lieut., R. Warwickshire Regt.

Died 29 May r9r 5 of fever

ALeeming, A. J. Capt., R. Fusiliers. $M$.

Killed in action $3 \mathrm{I}$ Fuly $\mathrm{r} 9 \mathrm{I} 7$

L'Estrange FAWCETT, A. W. Capt., Gloucestershire I9I3 Regt.; Major, M.G.C. (W.) M.C. 
${ }^{1}$ Lewis, A. M. Pte., R. Fusiliers (P.S. Bn.); Lieut., Devon

Regt.; Capt., 52nd Sikhs, Indian Army. (W 2.) M 2.

WLING, L. S. 2nd Lieut., Norfolk Regt.

Killed in action 4 May 1917

LitTle, A. H. Capt., R.A.M.C.

LoFT, Rev. E. W. B. C.F. th $^{\text {th }}$ Class, R.A.C.D.

LuCAS, Rev. R. H. C.F. $4^{\text {th }}$ Class, R.A.C.D.

Lyle, R. C. Capt. and Adjt., R.A.S.C. M.C. $M$.

I913

1915

1909

I 884

1892

1906

WMacintosh, H. M. Capt., Argyll and Sutherland Hdrs. IgI I Died 26 fuly 1918 of wounds received in action

HMACKay, C. L. 2nd Lieut., Worcestershire Regt.

Died 7 Fune 1915 of wounds received in action 28 May I9I 5

Macleod, J. D. Capt., Cameron Hdrs. and M.G.C.

MaInwaring, C. L. Lieut., R.G.A.

Mansfield, Rev. J. Sergt., R.A.M.C. (2nd S. Gen. Hospital, T.F.)

WMARRIOTT, J. F. L. 2nd Lieut., D. of Cornwall's L.I. 1907 Died 26 Fan. 19I 5 of spotted fever

Marshall, A. T. Lieut., Suffolk Regt.(T.F.)

WMarTin, E. N. M. 2nd Lieut., 5th (R. Irish) Lancers Killed in action 30 Sept. 1916

Martindale, R. G. Lce.-Corpl., R.E. (Fortress, T.F.); 2nd Lieut., R.G.A.

MASTERS, Rev.F.G. C.F.4th Class, Australian Chaplains' $\quad 1890$ Dept.

WMathews, A. Lieut., Cheshire Regt.

Died 14 April 1916 of wounds received in action

MaundRell, Rev. W. H. Chaplain, R.N.

Mawdesley, J. L. Sergt., London Regt.; Capt., R.A.O.C.

Merriman, Rev. H. S. C.F. 4 th Class, R.A.C.D.

Mrchell, Rev. E. W. C.F. $4^{\text {th }}$ Class, R.A.C.D.

Mrlls, E. Lieut., Bedfordshire Regt. and Northamptonshire Regt.; Staff Capt.; Major, Military Governor of Gaza. (W.) O.B.E. M.

Montagu, G. H. S. Lieut., London Regt. (W.)

1895

I9II

1905

1896

I9IO

MorTon, H. S. Capt., R.A.S.C.; Major, D.A.D. Quartering, War Office. O.B.E.

Mounsey, J. P. Lieut., Lancs. Fus. and King's African Rifles

Murray, D. C. L. Lieut., Middlesex Regt. M.C.

1 Killed in action against the Kurds after the armistice.

2 Died of influenza shortly after demobilisation. 
Neale, F. S. Pte., R. Fusiliers; Lieut., M.G.C.

सNELSON, E. B. 2nd Lieut., I.A.R.O., attd. Indian Infy. $\quad$ I909

Died 15 March 1916 of wounds received in action

Nevinson, G. R. G. Sergt., R. Fusiliers

1907

NisBet, A. T. 2nd Lieut., R.A.S.C.

1913

NoRRIs, W. H. H. Lieut., R.E.; empld. Ministry of 1903 Munitions. (W.)

※oke, R. W. L. Capt., R. Berkshire Regt.

Killed in action 25 Sept. 1915

Oldfield, Rev. H. D. C.F. 3rd Class, R.A.C.D. M.C. 1899 $M 3$.

Onyon, R. R. Capt., E. Surrey Regt.(T.F.)

1906

Page, C. H. W. Capt., R.A.M.C.

Palliser, W. F. Lieut., Worcestershire Regt.

1896

PALMER Rev S C.F ist Class, RA.CD. A.C.G I8 D.S.O. M.C.

Parker, Rev. H. L. C.F. 4th Class, R.A.C.D.

सPatch, N. J. S. Pte., Australian Force

Killed in action 11 Oct. I917

Patteson, C. Major (A.), R.A.F. (W.) M.C. M 2. 1909

Payne, A. A. See Laporte Payne, A. A.

Pearson, L. Pte., London Regt. (Artists Rifles); and Lieut., S. Staffs. Regt.

Penzer, N. M. 2nd Lieut., Essex Regt.

PEROWNE, J. T. W., v.D. Lieut.-Col., R.F.A.(T.F.) I882

PETTY, W. Lieut.-Col., Seaforth Hdrs. (W 2.) D.S.O. 1894 $M 2$.

Pickthorn, K. W. M. Capt., London Regt. (Civil Ser- * I9I4 vice Rifles); empld. War Office; Capt. (O.), R.A.F. (W 2.)

EPierson, L. D. Lieut., E. Yorks. Regt. $M$. Killed in action 30 Oct. 1916

Poignand, G. C. I. 2nd Lieut., Leinster Regt.; Capt. igro R.A.S.C.

Pook, J. DE C. Lieut., I.A.R.O., attd. S. and T. Corps 1906 Pryor, G. H. D. Lieut., King's Royal Rifle Corps. (W.) I9I2 Pullinger, S. R. Capt., Leicestershire Regt.(T.F.)

I910

Quentin, G. A. F. Lieut., King's Royal Rifle Corps; 1900 Asst. Officer $\mathrm{i} / \mathrm{c}$ Records. $m$.

Raven, G. E. Lieut., W. Yorks. Regt.; A.D.C. (W.) I912 M.C. 
सRead, Rev. E. O. C.F. $4^{\text {th }}$ Class, R.A.C.D.

Killed in action 3 Oct. 1918

Reckitt, F. N. Lieut., Middlesex Regt.; Capt., Labour I89I Corps

Resker, Rev. B. A. Lce.-Corpl., London Regt. (Artists 1908 Rifles)

Richardson, J. A. St C. Lieut., E. Yorks. Regt.

Rintoul, D. Colonel, Clifton College O.T.C.

Rodgers, Rev. H. N. C.F. $4^{\text {th }}$ Class, R.A.C.D.

Rowan, A. Lieut., Spec. List (Staff Lieut.)

WRoxburgh, J. H. Major, M.G.C. M.C. M.

Killed in action 2 Oct. 1918

Roylance, P. Lieut., D. of Lancaster's Own Yeo.

Ryotт, T. G. Pte., M.G.C.

1914

1882

1906

1905

[1914]

1910

1899

WSANDFord, C. R. F. Capt., King's Own (Yorkshire L.I., I9r3 T.F.) (W.) M.C.

Killed in action $22 \mathrm{Feb}$. r917

¿SAnkey, W. M. Lieut., Monmouthshire Regt. (W.) I9I4 M.C.

Died 23 March 1918 of wounds received in action

SAYERS, L. D. W. Lieut.-Col., R.A.S.C.

1897

Seddon, A. D. Capt., King's Own (R. Lancaster Regt.) 1908

(W.) (P.)

Selwyn, Rev. E. G. C.F. $4^{\text {th }}$ Class, R.A.C.D. $M$.

* 1909

Selwyn, J. Capt., R.F.A.; A.D.C.; Staff Capt.; Major, I9I I S.O. 2, R.A.F. (W.)

Sharp, Rev. G. F. C.F. $4^{\text {th }}$ Class, R.A.C.D.

WShaw, R. Capt., King's (Liverpool Regt., T.F.)

1905

Killed in action 20 Sept. 1917

Skinner, E. F. Capt., R.A.M.C.(T.F.)

Smith, Rev. F. S. C.F. $4^{\text {th }}$ Class, R.A.C.D.

¿Smith, G. R. Lieut., Canadian Infy.

Killed in action 6 May 1917

Smith, W.CAMPBell. Lieut.-Col.,London Regt.(Artists 1906

Rifles); attd. R.E. (Spec. Bde.) M.C. M2.

Sмyth, Rev. J. W. W. C.F. 4th Class, R.A.C.D. M. 1893

Stephens, A. R. Corpl., R.A.S.C.; Staff Capt. (W.) $\quad 1897$

Stephens, E. A. Corpl., Middlesex Regt. and M.G.C. I90I (W.)

Stevens, J. A. Lieut.-Col., Rangoon Bn., Indian Defence I89I Force. C.I.E. O.B.E.

Stuart Prince, D. Capt., I.A.R.O., attd. Indian Army [19I4] Clothing Dept. 
Swanston, E. R. Lieut., R.E.

I9I I

Tebis, J. A. Lieut., R.F.A.

I9ro

Telfer, R. G. Lieut., Border Regt.; Draft Conducting $\quad$ I896 Officer

WTerrell, F. W. Lieut., Gloucestershire Regt.; attd. Wor- I9I2 cestershire Regt. (W.)

Killed in action 3 Sept. 1916

Thомаs, T. G. Lieut., King's (Liverpool Regt.); Capt., 1904 Spec. List (Bombing Officer)

Thompson, J. C. Capt., Cameron Hdrs. (W.) 1899

Thomson, G. P. Capt., The Queen's (R.W. Surrey *igI4

Regt.); empld. British Military Mission; attd. R.A.F.

$m$.

Thouless, R. H. 2nd Lieut., R.E. (Signals) I9I2

Toogood, Rev. J. H. C.F. $4^{\text {th }}$ Class, R.A.C.D.(T.F.) I89o

Vaughan, J. H. Lieut., R. Inniskilling Fus.; attd. R.E. I9I I (Signals). (W.) M.C.

Viggers, Rev. S. C.F. $4^{\text {th }}$ Class, R.A.C.D. $M$. 1909

Ward, Rev. A. C.F. 4th Class, R.A.C.D. I887

WARD, D. C. L. Capt., London Regt.; Staff Capt. (W.) r9ro M. $m$.

WeATHERHEAD, R. Instructor Cdr., R.N.

1895

WWeBb, A. H. 2nd Lieut., The Buffs (E. Kent Regt., T.F.) I9I I

Killed in action 23 Fune I9I7

Wells, H. M. Lieut., R.E.; empld. Ministry of Muni- *19I2 tions

WWhitTaM, M. J. G. Lieut., D. of Wellington's (W. I9I2 Riding Regt.)

Died II Aug. I9I5 of wounds received in action in Gallipoli

WILBERFORCE;H.H. Lieut.-Col., R.A.S.C.(T.F.) D.S.O. 1900 $M$.

Williams, G. D. Pte., London Regt. (Artists Rifles); I90I Lieut., Essex Regt.

Williamson, Rev. F. L. C.F. 4th Class, R.A.C.D. 1900

Wills, A. G. P. Capt., R.A.M.C. M.C.

I910

1904

Killed in action in E. Africa I I March 1916

Wilson, Rev. T. Pte., King's Own (Yorkshire L.I.) 1912 
WWynne,E.E. Pte., R. Fusiliers(P.S.Bn.); Capt., Leicester- I913 shire Regt.(T.F.) (W.) Killed in action 8 fune 1917

YenCKen, A. F. Major, R.F.A.(T.F.) M.C. M. YeNCKEN, E. D. Capt., R.A.S.C. M. 


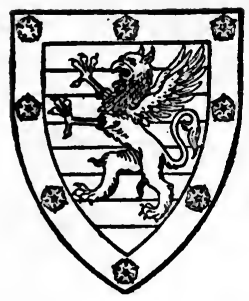

\section{DOWNING COLLEGE}

A-Beckett, A. H. Capt., Australian Light Horse $\quad 1887$ ADAMS, E. T. Hon. Lieut., R.N.V.R. 1900 Ahmad, A. M. Capt., R.A.M.C. 1910 AINSworTH, C. G. Surgeon Lieut., R.N. 1907 Allen, Rev. C. A. B. Chaplain, R.N. 1906 Allen, W. L. Lieut., King's School, Chester, O.T.C. $\quad 1909$ ANDERSON, A. C. Capt., I.M.S. 1902 ANDREws, T. E. Lieut., R.F.A. Appleton, A. B. Capt., R.A.M.C. 1907 AAston, W. D. Capt., Cambridgeshire Regt. I901

Died 2 Nov. 1917 of wounds received in action Atkinson, J. L. Capt., Quebec Regt., Canadian Force 1907

Back, N. Capt., R.F.A.(T.F.); attd. 2nd Dragoons r9ro (R. Scots Greys)

Baldwin, T. H. Lieut., Essex Regt.

BAines, W. 2nd Lieut., R.F.A. 1906

Ball, G. R. Lieut., W. Yorks. Regt.; empld. Ministry r9ro of Munitions. (W.)

BARLOW, P. Capt., Spec. List (Recruiting Staff) * *893

BARNARD, E. C. Lieut., Hertfordshire Regt. 1907

Bedworth, A. C. Capt., R. Warwickshire Regt. I9I3

Bell, J. A. Capt., Durham L.I.(T.F.); Staff Capt. (W 3.) r9r3 M.C.

WBENnETT, S. G. 2nd Iieut., Suffolk Regt.

Killed in action 20 fuly 1916

Bennett-Evans, G. L. Capt., S. Staffs. Regt. (W.) 1905

Bourne, A. W. Capt., R.A.M.C.

Bowen-Davies, J. Capt., Pembroke Yeo.; attd. R. North 1894

Devon Yeo.; Courts-Martial Officer 
Boyer, G. W. B. Lieut., R.G.A. [I9I4]

Bradbury, J. B. Lieut.-Col., R.A.M.C. (Ist E. Gen. ${ }^{*}{ }^{2} 865$ Hospital, T.F.)

Brailey, A. R. Surgeon Lieut.-Cdr., R.N.V.R. $\quad$ I896

BRAImbridge, C. V. Capt., R.A.M.C. I9II

Brewis, C. C. Capt., R.A.M.C. 1908

Brinton, R. D. Capt., R.A.M.C. $\quad 1879$

Brook, R. Lieut., Manchester Regt.; empld. Board of I9I4

Trade. (W.)

Brown, H. Lieut. (A.), R.A.F.

WBrown, I. M. Capt., R.A.M.C.

Killed in action $\mathrm{I} 5$ Nov. 1916

I916

1907

Brown, S. V. Lieut., Liverpool Institute O.T.C.

WBRyan Brown, Rev. G. S. C.F., N. Zealand Chaplains' 1904

Dept. (W.)

Killed in action 4 Oct. 1917

Bryant, V. S. Major, Wellington College O.T.C. $m$. ${ }^{1897}$

HBuchanan, A. Pte., King's (Liverpool Regt, Liverpool 19 Io Scottish, T.F.)

Killed in action near Ypres 16 Fune 1915

WBurgess, W. C. 2nd Lieut., Somerset L.I.(T.F.)

Killed in action 22 Aug. I9I7.

Byrde, E. H. Capt., Monmouthshire Regt.; Major, Gen. 1905 Staff. (W.) $m$.

Chapman, E. H. Capt., R.E. $M$.

1905

ChOPRA, R. N. Major, I.M.S.

1903

ClARK, O. A. P. Lieut., Northumberland Fus. (T.F.); I9r3 attd. R.E. (Spec. Bde.) (W.)

Clarke, E. Capt., R.A.M.C.

I879

Clarke, G. A. C. Capt., R.G.A. (W.) M.C.

Clifton, G. F. Capt., R.A.M.C. 1908

Cole, Rev. T. E. F. C.F. 4th Class, R.A.C.D. * * 884

Collingwood, C. A. Lieut., Northumberland Fus.; 1909

Capt., Spec. List (Courts-Martial Officer). (W.)

\#Corke, G. H. 2nd Lieut., Northumberland Fus. M. 1909

Killed in action I7 Sept. 1916

Cotton, C. K. Capt. and Adjt., Cheshire Regt. $\quad 1906$

Cowell, J. Pte., R.A.M.C. 1912

Crampton, G. P. Capt., Northamptonshire Regt.; attd. 1912 9th Gurkha Rifles, Indian Army

Crouch, H. A. Capt., R.A.M.C. M.C. 1909

Cunningham, L. Capt., R.A.M.C. I9II

Curl, S. W. Capt., R.A.M.C.(T.F.) 
Curzon, C. T. B. Paymaster Sub-Lieut., R.N.V.R. $\quad$ I909

DARE, A. J. Lieut., R.E. 1905

Davies, D. G. Lieut., Welsh Horse and M.G.C. (Cavalry) I9ro WDavies, F. C. Capt., R.A.M.C.

Killed in action 17 Oct. 1917

Davies, G. B. Capt. and Adjt., 48th Pioneers, Indian 1902 Army

Davies, J. B. See Bowen-Davies, J.

Davies, J. T. Bdr., R.G.A.

DAviES,LL.J. Lieut., R. Warwickshire Regt.(T.F.) (W 2.) 1913

Davies, R. L. Lieut., King's (Liverpool Regt., T.F.) 1909

(W.)

WDawe, A. H. 2nd Lieut., King's Royal Rifle Corps I9I3

Killed in action I I April I9I7

DAy, C. D. Capt., R.A.M.C.

DekKers, L. A. Lieut., R.F.A.

1908

I 896

1912

Killed in action 22 March 1918

Dick, J. R. Capt., R.A.M.C.

DrCKson, A. N. Capt., I.M.S. M.C.

DigbY-Johnson, N. Lieut., R.A.S.C. (W.)

Dixon, W. E. Surgeon Lieut., R.N. $m$.

Dodson, F. K. Capt., E. Lancs. Regt. (W.)

1903

1907

* 1907

1902

1909

Douglas-Hamilton, Rev.W.A. C.F.4th Class, R.A.C.D. $\quad 1887$

Duffield, C. A. W. Lieut., Queen's Own (R.W. Kent *1909

Regt.) $M . C$.

ADunkerley, H. Major, R.A.M.C. (W.)

Died 23 March 1918 of wounds received in action

1907

Dunscombe, C. Pte., R.A.M.C.(T.F.)

Dúnscombe, N. D. Surgeon Sub-Lieut., R.N.V.R.

1914

I9I 5

Eccles, G. T. Capt., R.A.M.C.

${ }^{*} 1885$

EDmunds,C.H. Lieut., London Regt.(Surrey Rifles). (W.) 1908

EHRHARDT, W. H. Capt., R. Warwickshire Regt. (W.) I9I I

Ellison, J. Capt., R.A.M.C.

1904

HEminson, R. A. F. 2nd Lieut., King's Royal Rifle Corps; 1909 attd. M.G.C.

Killed in action 20 fuly 1916

Evans, D. A. 2nd Lieut., Norfolk Regt. (W.)

Evans, G. Colonel, I.A.R.O. (Agricultural Directorate). ${ }^{\text {I9I }}$ I9I

C.I.E. $M$.

Evans, G. L. B. See Bennett-Evans, G. L.

Evans, G. H. Pioneer, R.E. (Spec. Bde.) 
EEveretr, W. W. Capt. and Adjt., Norfolk Regt.

Killed in action near Brancourt 8 Oct. 1918

1910

Fieldhouse, E. E. A.B., R.N.V.R.(Hawke Bn., R.N.D.); 1908

Paymaster Sub-Lieut., R.N.R. Cavalier, Order of

the Crown of Italy

FisheR, J., D.S.O. Lieut.-Col., I.M.S. $m$.

Fontaine de Mazinghen, A. Cpl., French Infy.; and

Lieut., R.G.A. (W.) French Croix de Guerre

Fordham, W. H. Capt., R.F.A.

Forsyth, A. B. Lieut., Bedfordshire Regt.; Capt., Army

Cyclist Corps

Foster, A. W. 2nd Lieut., R. Warwickshire Regt.

FreEman, P. Lieut., R.G.A. (W.)

GABB, H. S. Lieut., R.A.M.C.

Gainsborough, H. Capt., R.A.M.C.

GARDNER, C. G. Lieut., Suffolk Regt. (W 2.) MC.

Gibson, N. Lieut., E. Yorks. Regt.(T.F.) (P.) 1910

Gill, Rev. H. V. C.F. 3rd Class, R.A.C.D. D.S.O. 1907 M.C. $M 2$.

Ginsburg, H. H. See Gainsborough, H.

GIRARD, D. L. M. Liaison Officer, French Army. French

Croix de Guerre

Gordon-Vaudin, Rev. C. G. Chaplain, R.N.

Goulden, C. B. Capt., R.A.M.C. O.B.E. M.

Goulden, D. Lieut., N. Staffs. Regt. (W.)

Goulden, E. O. Lieut., Queen's Own (R.W. Kent Regt.)

(W 2.) M.C.

Gower, L. C. 2nd Lieut., S. Wales Borderers; Gnr., R.F.A. (W.)

Grant, C.V. Pte., R.A.M.C.;Lce.-Corpl., R.E.(Meteorological Section)

Grantham, J. Lce.-Corpl., Malay Vol. Rifles

AGreen, J. L. Capt., R.A.M.C.(T.F.) (W.)

V.e्C. "For most conspicuous devotion to duty. Although himself wounded, he went to the assistance of an officer who had been wounded and was hung up on the enemy's wire entanglements, and succeeded in dragging him to a shell hole, where he dressed his wounds, notwithstanding that bombs and rifle grenades were thrown at him the whole time. Captain Green then endeavoured to bring the wounded officer into safe cover, and had nearly succeeded in doing so when he was himself killed." Supplement to The London Gazette, 5 Aug. 1916.

Killed in action I Fuly 1916

1908

* 1881

1906

1914

1913

1909

1914

1909

1907 
GreEN, S. J. Lieut., R.N.V.R.

Greene, G. W. Major, R.A.M.C.(T.F.)

1908

GREGORY, C. C. L. Sapper, R.E. (Meteorological and IgI Sound-ranging Sections)

Grellier, E. F. W. Capt., R.A.M.C.

Grice, J. E. Pte., R.A.S.C.(M.T.)

Gudgin, S. H. Capt. (K.B.), R.A.F.

1905

1912

1907

Hall, R. 2nd Lieut., Loyal N. Lancs. Regt.

HaM, P.S. Capt., R.A.S.C.; attd. S. and T. Corps, r9ro Indian Army

Hamilton, J. L. Capt., R.A.M.C.(T.F.). (W.) M.C. 1908

HAMmOND, J. Capt., Norfolk Regt. (W 2.) 1907

Hardman, W. H. Pte., R.A.S.C.

WHARMER, G. 2nd Lieut., N. Staffs. Regt.

Killed in action II Aug. 1916

HarRison, F. Capt., R.G.A. M.

HART, N. B. Capt., I.A.R.O., attd. 6th Gurkha Rifles. 1909 $M$.

HaRvey, H. W. Lieut., R.N.V.R. $M$.

HaYnes, H. G. L. Lieut.-Col., R.A.M.C.(T.F.)

WHilliard, G. W. R.N.A.S.

Died Aug. 1915 of wounds received in action

Hilss, T. W. S. Capt., R.A.M.C.(T.F.) (W.)

Hirsch, F. B. 2nd Lieut., Durham L.I.

1907

1914

1912

Hone, P. F. Capt., Welsh Regt.; Staff Capt.; Lieut.-Col., Middlesex Regt. D.S.O. and Bar. M.C. and Bar. $M$.

HowletT, J. M. Capt., Norfolk Regt.(T.F.); empld. 1907 O.C.B. (W 2.) M.C.

WHuckle, H. W. 2nd Lieut., Cambridgeshire Regt.

Killed in action 5 Sept. I918

Hunt, R. V. Major, R.A.S.C. and Outram's Rifles, * 1892 Indian Army. $M 2$.

HuRst, N. V. Lieut., R.F.A.(T.F.)

HyDE, H. A. 2nd Lieut., R.G.A.

1914

1910

IsAacs, M. G. 2nd Lieut., R. Warwickshire Regt. I9I I

JARDINE, R. F. Capt., R. Warwickshire Regt. (W.) I9I3

JARVIS, H. E. G. A.B., R.N.V.R.

1909

1912

Killed in action 8 April 1916

Jenkins, W. L. Capt., R.E. $M$.

I9I5 
Johnson, N. D. See Digby-Johnson, N.

Johnson, O. G. Lieut., Middlesex Regt. (W.)

JJolly, B. O. 2nd Lieut., Yorkshire Regt.

Died 9 Feb. I917 of wounds received in action

Jones, C. McC. Capt., R. Welsh Fus.

I9II

JONES, C. S. Lieut., Suffolk Regt.

r9r 4

JONES, E. LloYD. Major, R.A.M.C. (Ist E. Gen. Hospital, I892 T.F.)

Kaminski, V. Aviation Corps, Russian Army. (W.)

KaRN, Rev. J. C. Chaplain, R.N.

\#Kearney, J. J. Trooper, Gloucestershire Yeo.

Killed in action in Gallipoli 21 Aug. I915

KeEsey, G. E. H. Capt., Rifle Brigade. (W.) $M$.

Killed in action on the Somme 24 Aug. 1916

AKeith, A. J. 2nd Lieut., Middlesex Regt.

Killed in action on the Somme 14 fuly I9I6

Kelly, D. P. J. Capt., Connaught Rangers; empld. War 1906 Office. M.C. Belgian Croix de Guerre

Kerby, W. M. Pte., Middlesex Regt.

Kirkman, R. W. Capt. Lancs. Fus.; Asst. Officer i/c 1909 Records

KNowles, R. Capt., I.M.S. (W 2.) M 2.

1914

1908

1902

1905

I9I2

1906

1902

LAKER, W. N. 2nd Lieut., Suffolk Regt. and R. Warwickshire Regt.

LAMB, Rev. P. C. C. C.F. 4 th Class, R.A.C.D.; Chaplain, 1907 R.A.F. $m$.

LAMBERT, J. Surgeon Lieut., R.N. O.B.E. M.

LANDER, P. E. Capt., R.A.M.C. (Sanitary Service, T.F.) $M$.

LaNGLEY, A. S. Lieut.-Cdr., R.N.V.R. C.M.G. M. 1905

LANSBERRY, H. G. Pte., R.A.M.C.

LAw, H. S. Lieut., R.F.A.(T.F.)

I910

1892

Layton, D. H. Capt., E. Yorks. Regt. (W 2.) M.C. 1907

Lazarus-Barlow, P. Lce.-Sergt., W. Kent Yeo.; Pte., I9r3

R.A.M.C.; 2nd Lieut., Dorset Regt. and Tank Corps

LaZARUS-Barlow, W. S. Capt., R.A.M.C.

LEADER, H. E. Lieut.. Sherwood Foresters (Notts. and Derby Regt.). (W.)

LEGGE, R. J. Lieut., R.A.S.C. and R.F.A. 1906

LEIGH, F. 2nd Lieut., S. Lancs. Regt.

LeIghtoN, Rev. J. W. C.F. $4^{\text {th }}$ Class, R.A.C.D. 
Lewis, J. B. S. Capt., R.A.M.C.

. Welsh Fus. and Gen List (Asst. Instructor, Command Depôt). M.C. and Bar

LEwIS, W. A. H. 2nd Lieut., R.G.A.

LidDlE, H. W. 2nd Lieut., King's Royal Rifle Corps. 1909 (W.) (P.)

WLINE, J. Y. A. 2nd Lieut., N. Staffs. Regt.

Died 13 March I916 of wounds received in action near Neuve Chapelle I2 March 1916

Linfoot, G. C. Gnr., R.N.

1914 .

Litrlewood, A. Lieut., St Elizabeth College, Guernsey, O.T.C.

Llewelyn, Rev. D. E. K. C.F. 4th Class, R.A.C.D.

Logan, M. J. S. Lieut., S. Lancs. Regt. and King's Own (R. Lancaster Regt.)

London, G. E. Lieut., Gloucestershire Regt. (W.) 1908 Long, R. A. Lieut., R. Fusiliers; empld. Ministry of [1914] Munitions. (W.)

Macdonald Brown, I. See Brown, I. M.

MackenzIe, J. W. Lieut., R. Fusiliers; empld. Army 1912 and Navy Canteen Board. (W 2.)

McKergow, R. W. Major, Sussex Yeo.; Lieut.-Col., $\quad 1889$ Queen's Own (R.W. Kent Regt., T.F.) O.B.E. T.D.

\#McLaren, A. D. Pte., R. Scots Killed in action 9 April $\mathrm{r} 9 \mathrm{I} 7$

MacTier, J. C. Lieut., R. Glasgow Yeo.

Mallett, F. J. Capt., R.E. M.C. and Bar

MARSH, C. J. Major, R.A.M.C.

I9I4

1908

1910

1900

AMARSH, F. Howard. Hon. Colonel, R.A.M.C.(T.F.) *

Died 24 Fune I9I 5

Mathews, E. V. D. Lieut., D. of Cornwall's L.I.; I9I3 Lieut. (A), R.A.F.

Matthews, J. C. Capt., R.A.M.C. M.C.

MILleR, D. C. Major (A.), R.A.F.

$\begin{array}{ll}\text { MilleR, D. C. Major (A.), R.A.F. } & \text { r912 } \\ \text { MilleR-Williams, E. J. Lieut., R.F.A.(T.F.) (W.) } & \text { r9Io }\end{array}$

Millward, G. D. Lieut., R.A.S.C. $M$.

1908

1899

1909

*1912

MILWARD, H.H. Lieut., Worcestershire Regt. and M.G.C. (W 2.)

MonckTon, F. H. Capt., R.E.

I 896

1906

r913

I9I I

1909

Morgan, D. A. Trooper, Welsh Horse

Morgan, D. F. Lieut., St Edward's School, Oxford, I9I2 O.T.C.

Morgan, H. A. Lieut., R.A.O.C. 
Mowlam, H. J. Capt., Durham L.I.(T.F.); attd. Sher- 1907 wood Foresters (Notts. and Derby Regt.) (W.) (P.)

Newman, F. C. Surgeon Lieut., R.N. $M$. 1902

Newman, L. F. Capt., R.A.S.C. $m$. 1906

Nicholls, G.S. 2nd Lieut., R.E.; Staff Lieut. (Draft 1909 Conducting Officer)

Nicholls, L. Capt., R.A.M.C.

Nicolson, L. G. Lieut., R.A.F.

Norman, N. F. Major, R.A.M.C.

North, J. F. A. Lieut., Northamptonshire Regt.

1904

1910

* 1905

1913

Oliver, E. G. Major, Essex Regt. and Gen. Staff. (W.) 1898

Ollard, J. W. A. Capt., Cambridgeshire Regt.; Capt., 1916 and Adjt. Northamptonshire Regt.

Ontanon, C. 2nd Lieut., R.G.A.

HO'Reilly, H. D. R. Pte., R. Fusiliers; Capt., The Queen's [1914] (R.W. Surrey Regt.)

Died 31 May I919 of wounds received in action in Mesopotamia

Ormrod, W. Capt., Lancs. Fus.; Brigade Major. M.C. 1909 $M_{3}$.

Passant, Rev. E. J. C.F. $4^{\text {th }}$ Class, R.A.C.D.

PeAD, J.H. Surgeon Cdr., R.N. M. Chevalier, Legion *1890 of Honour (France). Order of St Stanislas, 2nd Class, with swords (Russia)

Peckover, H. D. Cadet, O.C.B.

PetTy, M. J. Lieut., R.A.M.C.

Phelps, Rev. P. B. Lieut., R.N.V.R.

Phillips, D. I. W. Pte., R. Fusiliers (P.S. Bn.)

Phillips, W. D. Pte., R.A.S.C.(M.T.)

Pitt, W. J. Pte., Gold Coast Vol. Force

Port, W. N. W. Capt. Palmer's 1906

Porteous, N. W. Capt., Palmer's School, Grays, O.T.C. 1910

Portway, D. Major, R.E. (Signals)

Prevost, P. G. C.F. 4th Class, R.A.C.D.; Cadet, R.G.A. $\quad$ I902

Price, A. F. M. Pte., Malay States Vol. Force

1917 1906 1908 1909 I9II 1906 1906 1901

REA, D. Lieut., Suffolk Regt. M.B.E. 1913

REDFERN, W. A. K. Capt., R.F.A. and Spec. List (Adjt., *' ${ }^{*} 907$ Graves Registration Units); Staff Capt.

ReECE, R. J. Surgeon Lieut.-Col., R.F.A.; Hon.Surgeon- 1888 Colonel, H.A.C. C.B. $m$. 
ReEs, R. A. T. Major, Loyal N. Lancs. Regt.; attd. S. 1904 Staffs. Regt.(T.F.) (W.)

ReID, W. L. 2nd Lieut., R.A.S.C.

Robinson, A. D. Capt., R.A.S.C.

Robinson, W. H. Lieut., R.A.M.C.

I9I3

1895

1898

Rothfield, I. Lieut., King's (Liverpool Regt.) (W 2.) I9I I M.C.

ZRound, W. H. Capt., Sherwood Foresters (Notts. and I9r3 Derby Regt., T.F.)

Killed in action I Fuly 1916

ARowland, S. D. Major, R.A.M.C.

Died 6 March 1917 of illness contracted on active service

Rowse, A. A. Lieut., R.E.

Rumsey, C. F. Capt., R.A.M.C.

Rushton, W. F. Lieut., King's (Liverpool Regt.)

Ruthven, J. Capt., Monmouthshire Regt.

1889

SaInT, A. P. Major, R.A.M.C.; D.A.D.M.S. M.C. M. 1908

SAunders, H. F. Capt., Railway Bn., Indian Defence 1904 Force. (W.)

Savage, E. G. Major, Queen's Own (R.W. Kent Regt.); 1903 empld. Ministry of Munitions. (W.)

SelBy, E. J. Major, R.A.M.C.; D.A.D.M.S. O B.E. 1909 $M 3$.

Shelton, Rev. R. N. C.F. 4th Class, R.A.C.D. 1903

ShrubBS, C. A. Lieut., R.A.S.C. 1907

Simons, E. Capt., S. Wales Borderers. M.C. and Bar 1904

SiNClaIR, W. M. A.B., R.N.V.R.

Smith, D. W. Corpl., R.A.F.

Solvay, M. A. Gnr., 2me Regt. d'Artillerie, Belgian I916

Army. Belgian Croix de Guerre

SPERO, L. H. Rfn., London Regt. (L.R.B.)

SPOWART, W. C. Lieut., I.A.R.O., attd. S. and T. *1910

Corps. (W.)

SPRAKe, G. G. 2nd Lieut., R.G.A.

SPROAT, R. H. Lieut., S. Staffs. Regt.; attd. R.E.; Capt., I9I3

Spec. List (Cmdt., Anti-Gas School). Italian Croce di

Guerra

Starkey, H. S. C. Capt., R.A.M.C.(T.F.) O.B.E. M. 1904 Stenning, Rev. E. H. 'Capt., K. William's College, I. of 1906 Man, O.T.C.

¿Sterckeman, P. Cpl., French Infy. (W.) (P.) I909

Died ${ }_{5}$ Feb. 1917 of wounds received in action

c.U.w.I. 
Stevens, H. L. Lieut. (A.), R.A.F.

STEWARD, S. J. Capt., R.A.M.C. D.S.O. $M$.

ASTRATFORD, E. P. Lieut., R.A.M.C.

Died 20 April I9I 5 of wounds received in action near

Neuve Chapelle 17 March 1915

\#Stringer, G. M. 2nd Lieut., Cheshire Regt.

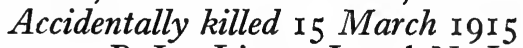

SuRRIDGe, B. J. Lieut., Loyal N. Lancs. Regt.; empld. 1912 Recruiting Staff

Surton, W. H. R. Major, S. African Med. Service. I900 $M 2$.

TAFfs, L. H. Lieut., 84th Punjabis, Indian Army

TAlPUR, Mir G. A. 2nd Lieut., Imp. Service Cavalry

Staff, Egyptian Army

TAYlor, A. E. Capt., Australian A.M.C.

TAYlOR, R. S. Major, E. African Med. Service

ThaCker, C. R. A. Lieut., R.A.M.C.

Thoday, F. A. Major, Devon Regt.(T.F.)

Thomas, G. P. Pte., Welsh Regt.

Thomas, H. H. Lieut., R.F.A.; Capt. (T.), R.A.F. M.B.E. $\quad M$ 2. Order of the Nile, $4^{\text {th }}$ Class $(E g y p t)$

Thomas, M. E. Lieut., R.E.(T.F.) (W.) M.C. and Bar

Thomas, N. L. Capt., London Regt. (R. Fus.) (W.) I9I I

Thomson, M. S. Lieut., R.A.M.C.

1910

1898

1899

1908

1907

1914

1904

I9I4
TTopham, M. Sergt., R. Fusiliers (P.S. Bn.); 2nd Lieut., [I9I4] R.F.C.

Killed in action $\mathrm{I}_{3}$ April I9I7

Treadgold, H. A. Capt., R.A.M.C.

Turner, H. J. Lieut., R. Welsh Fus.

1903

Turner, J. W. Lieut., Indian Army; Capt., Sherwood * ${ }^{1906}$ Foresters (Notts. and Derby Regt.). M.C. $M$.

Vigurs, C. C. Capt., R.A.M.C.

${ }^{*} \mathrm{I} 892$

Warte, W. F. Lieut., Lancs. Fus. and Spec. List (Re- 1904 cruiting Staff); empld. Ministry of Food. (W.) $M$.

WaLKer, B. Sub-Lieut., R.N.V.R.

Walker, R. Capt., The Buffs (E. Kent Regt., T.F.)

Wallis, R. L. M. Capt., R.A.M.C.

WaLSH, E. S. Capt., D. of Wellington's (W. Riding Regt.) and R.E. (W.)

1892

1908

1904

1913 
Watrs, A. E. Capt. and Adjt., London Regt. (Kensing- 1909 ton $\mathrm{Bn}$.)

WaUGH, G. E. Major, R.A.M.C.

WeBB, F. E. A. Lieut.-Col., R.A.M.C.(T.F.) O.B.E. $\quad$ I895 Brevet Lieut.-Colonel. $M 2$.

WEBB, F. H. Lieut., R. Warwickshire Regt. (W.) I9I2

WherRY, G. E. Lieut.-Col., R.A.M.C. (Ist E. Gen. Hos- 1878 pital, T.F.). $m$.

Whiтвy, L. E. H. Capt., Queen's Own (R.W. Kent Regt.); Major, M.G.C. (W.) M.C.

Whiting, M. H. Capt., R.A.M.C. O.B.E. M. 1904

Whitmore, C. J. R. Major, R.G.A. (W.) M.C. M. 1907

WhitTINGDALE, J. Lieut., R.A.M.C. I9I3

\#WhITwORTH, A. G. R. 2nd Lieut., Northumberland I9I4 Fus.

Died of wounds received in action 21 March 1918

WidDICOMBE, E. P. Lieut., I.A.R.O. attd. Sappers and ${ }^{*}{ }_{1} 898$ Miners; Capt., Spec. List; Major, R.E. $M$.

WILcock, J. A. 2nd Lieut., R.F.A. (W.) M.C.

WildersPIN, B. C. Lieut., Worksop College O.T.C.

I9II

1908

WILKS, E. L. 2nd Lieut., York and Lancaster Regt. I9I2 (W.)

WWILL, J. G. Lieut., Leinster Regt.; attd. R.F.C. (W 2.) I9I I Killed in action 25 March I9I7

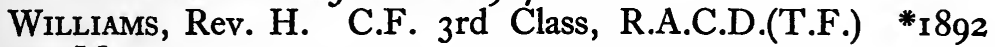
$M$.

WILliams, T.P. 2nd Lieut., Suffolk Regt.; Lieut., Welsh I9I4 Regt.

Williams, W. J. P.O. Telegraphist, R.N.V.R.

Williams, W. P. G. Capt., I.M.S.

I9I 5

Willis, R. E. Lieut.-Col., British W. Indies Regt.

\#Wilton, S. B. Capt., N. Staffs. Regt. (W 2.) M.C. I9I I Killed in action I4 March I9I7

Winfield, A. Lieut., R.A.M.C.

WINFIELD, F. B. Major, R.A.M.C.; D.A.D.M.S. (W.) 1909 O.B.E. M 2. French Croix de Guerre

Withinshaw, J. W. Lieut., R. Scots and M.G.C. Igro

Wood, F. E. Capt., Malay States Guides Med. Service $\quad 1895$

Wood, Rev. W. C.F. 4th Class, R.A.C.D. I9Io

Woodhouse, R. A. Capt., R.A.M.C. I9I I

Woods, R. S. Capt., R.A.M.C. M. I9I I

Worster-Drought, C. C. Capt., R.A.M.C.

WORTley, H. A. S. Capt., R.G.A. $M . m$. 1904 
Yorke, G. O. 2nd Lieut., Army Cyclist Corps; Staff 1908 Lieut.

YounG, V. C. Lieut., R. Irish Rifles. (W 3.) $M$. I9I4

ZAN, M. B. Subadar, Indian Army $\quad 1908$ 


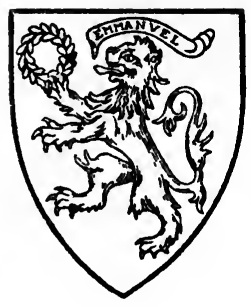

\section{EMMANUEL COLLEGE}

Abrahams, A. Major, R.A.M.C. O.B.E. $m$.

ADDIsON, N. H. Lieut., R. Fusiliers (R. of O.)

AdKIN, B. C. Capt., R.A.S.C.; attd. Suffolk Regt.

1903

1900

I9I I

AInsworTh,J.M. Corpl.,R.A.M.C. and R.E.(Spec.Bde.) 1912 Allan, F. L. Capt., Northumberland Fus. (W.) M.C. 1912 $M$.

Allen, Rev. F. B. C.F. 4th Class, R.A.C.D. $\quad 1906$ Allen, Rev. J. M. C.F. 4th Class, R.A.C.D. Allsopp, Rev. F. G. C.F. 4th Class, R.A.C.D. (W.) 1895 Almond, J. Capt. and Adjt., I.A.R.O., attd. Mohmand r9ro Militia

Altounyan, E. H. R. Capt., R.A.M.C. (W.) M.C. 1908 Anderson, G. B. Capt., Middlesex Regt.; Major, R. [1914] Berkshire Regt. (W.) M.C. M.

Anderson, L. A. P. Capt., I.M.S. (P.) $M$.

AṆRAdE, E. N. DA C. Capt., R.G.A.; empld. Ministry 1912 of Munitions. $M$.

ANDREw, G. W. M. Capt., R.A.M.C.(T.F.) $M$. 1904 ANGAS, D. T. Sub-Lieut., R.N.A.S. (W.) AAPPlegarth, T. W. Pte., R.A.S.C.; 2nd Lieut., Dur- 1912 ham L.I.

Died in Germany 9 Aug. 1918 of wounds received in action 3 June 1918

ARMitage, W. J., T.D. Major, York and Lancaster Regt. $\quad 1886$ (T.F.); Garrison Musketry S.O.

ARNOLD, E. C. 2nd Lieut., Eastbourne College O.T.C. $\quad{ }^{1887}$ ARrowsmith, R. Driver, H.A.C. Arundel, F. D. Capt., R.G.A. $M$.

AAshby, K. H. Corpl., R.E. (Signals) Missing, presumed killed in action at Mons, r Sept. 1914

1912

1908 I910 
WAshcroft, F. Lieut., King's (Liverpool Regt.)

Killed in action at Armentieres 9 April I9I7

Atkinson, J. C. Lieut., R.E. (Signals, T.F.) M.C.

WAtkinson,M.L. Pte.,M.G.C.(Motor); 2ndLieut., Tank

Corps

Killed in action near Cambrai 20 Nov. 1917

सAtkinson, R. E. Lieut., Durham L.I.(T.F.) (W.)

Killed in action at Armentières $20 \mathrm{Feb}$. 1916

1914

1907

Attrield, W. H. Lieut., Hampshire Regt.; attd. King's Royal Rifle Corps

Attree, C. K. Capt., R.A.M.C.

Attneave, A. L. Sapper, R.E.

I910

1907

1894

1914

Aylwin, W. E. Pte., R. Fusiliers (P. S. Bn.); Capt., [19I4] Bedfordshire Regt. (W 2.) M.C. M.B.E. m.

Bainbridge-Bell, L. H. Lieut., R. Munster Fus.; attd. I9I2

R.E.; Hon. Lieut. (T.), R.A.F. M.C.

Baker, Rev. A. T. B. Chaplain, R.N.

Baldwin, H. J. Capt. and Adjt., R.A.M.C. (Sanitary 1899

Service, T.F.)

BALl, Rev. W.A. R. C.F. 4th Class, Canadian Chaplains' 1904

Dept. (W.)

Ballingall,D.C.G. Major,R.A.M.C. (W.) M.C. M2. 1907

BALY, H. Sergt., Canadian Hdrs.; attd. Army Pay Corps 1893

BAmFord, A. J. Lieut. and Adjt., Armoured Motor Bty.; 1904

Capt., Gen. List, empld. R.E. (Field Survey Coy.) M.C. $M 2$.

Bampfield, L. A. 2nd Lieut., W. Yorks. Regt.; Capt., I90I

Gen. List; A.D.C. M.B.E. M 3. Italian Croce di

Guerra

Banks, E. H. Capt., Cheshire Regt.

BARK, G. M. Lieut., R. Warwickshire Regt.; empld. War Office. (W.)

Barker, F. A. Capt., I.M.S. O.B.E. M.

Barlow, Rev. C. W. Chaplain, R.N.

1903

1901

1900 1898

BARnetT, F.S. G. Trooper, Gloucestershire Yeo.; Capt. I9I0 and Adjt., R.F.A. M.C. $M$.

Barrett, F. G. Lieut., London Regt. (Surrey Rifles) 1906

BARRITT, J. L. 2nd Lieut., W. Yorks. Regt.; Capt., R.E. I9II $M$.

Barrow, H. E. Capt., King's (Liverpool Regt.) and [1914] T.M.B. M.C.

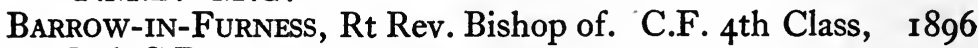
R.A.C.D. 
Bartlett, A. C. Corpl., R.E. (Signals) 1912

BatchelOR, B. W. Capt., Rifle Brigade. (W.) I9I4

Bates, Rev. H. R. C.F. $4^{\text {th }}$ Class, R.A.C.D. (W.) 1900

BathURST, C.R. Lieut., Northamptonshire Regt.; empld. $\quad 1898$

Ministry of Labour. (W.) $M$.

Bawdon, W. S. Pte., Australian Infy. I9IO

BAXTER,F.H. Capt.,Portsmouth Grammar SchoolO.T.C. $\quad 1892$

Beamish, Rev. C. N. B. C.F. 4th Class, R.A.C.D. I806

Beevor, R. B. Pte., London Regt.; 2nd Lieut., Bedford- [1914]

shire Regt. and R. Irish Fus.; Lieut. (A.), R.A.F.

Italian Croce di Guerra

BeLl, A. W. Lieut., R.F.A.(T.F.) M.C.

Bellerby, Rev. A. C. B. C.F. $4^{\text {th }}$ Class, R.A.C.D.

I9I4

1907

Bellhouse, S. Lieut., W. Yorks. Regt.(T.F.) (W 2.) I9I3 French Croix de Guerre

Bellward, G. W. F. Lieut., Essex Regt. (T.F.) and I9ro R.A.S.C.

\#Bengough, J. C. Capt., Gloucestershire Yeo.; A.D.C. 1908 $M$.

Killed in action in Egypt 26 Feb. I916

BennetT, H. H. G. Lieut., Worcestershire Regt.(T.F.); I9IO empld. O.C.B.

Bennett, W. P. Lieut., Border Regt.; Capt., R.G.A. 1909 (W.)

Bensly, Rev. W. J. Capt., Dorset Regt.; Major, British $\quad 1893$

W. India Regt. D.S.O. $M 2$.

Benson, Rev. H. W. Cadet, O.C.B.

Bentley, J. D. Lieut., R.F.A.(T.F.) (W.)

Benton, D. Lieut., Manchester Regt.

Bergheim, P. Capt., R.G.A.; empld. War Office. O.B.E. M.C.

Bescoby, A. C. Capt., R.A.M.C. (Sanitary Service, T.F.) I905 ABettington, A. F. Sqdn. Cdr., R.N.A.S.

Killed in flying accident 12 Sept. I9I7

Bevan-Brown, C. M. Sergt., N. Zealand Med. Service I 1909

Bienemann, G. A. J. and Lieut., Nigeria Regt., W. 1909

African Frontier Force

Bigger, W. K. Capt., R.A.M.C. M.C.

1910

I910

1910

1904

BIRD, A. K. Capt Suffolk Regt.; empld O.C.B. (W) ${ }^{1909}$

BIRD, M. W. K. Capt., R.A.M.C.(T.F.)

Bispham, J. W. Capt., R.E. O.B.E. M 2.

Biss, H. C. J. Major, R. Irish Regt.

BissetT, C. C. Lieut. (T.), R.A.F. (Aircraft Production I9I3

Dept.) 
WBlake, C. 2nd Lieut., Bedfordshire Regt.

Killed in action on the Somme 4 Sept. 1916

Blakiston, A. F. Trooper, K. Edward's Horse; Lieut., 1909 R.F.A. (W.) M.C.

Blanchard, J. F. J. Corpl., K. Edward's Horse; Capt., I9I I Canadian F.A. (W.)

Bland, B. S. Capt., W. Yorks. Regt.(T.F.) (W 2.) 1909

BLAND, C. Major, Leicestershire Regt.

Bloxнam, L. A. Lieut., London Regt. (Kensington Bn.) I9r4 and Gen. Staff (Intelligence). (W.)

Bluett, D. C. Pte., R. Fusiliers; Capt., R.A.M.C. $\quad 1902$

BluetT, T. L. C. Pte., R.A.M.C.

Boardman, H. Pte., Queen's Own (R.W. Kent Regt.)

ABoddington, O. W. Lieut., N. Staffs. Regt.(T.F.) 1907

Killed in action $\mathrm{I}_{3}$ Oct. I91 5

Bodpy, J. A. V. Lieut., Durham L.I.; Lieut. (A.), [1914] R.A.F. (W.)

Bolingbroke, C. B. Capt., Norfolk Regt.; attd. Man- 1909 chester Regt. (W.)

BoND, J. S. Instructor Lieut., R.N.

Bonner, C. A. J. Capt., Lancs. Fus.; empld. Ministry of 1905 Labour. (W.) $M$.

\#Boote, C. W. Capt., Cheshire Regt.

Killed in action 4-5 April 1916

¥Bostock, G. E. Capt., R. Munster Fus.

Killed in action at Loos 30 Fan. 1916

Botting, C. G. 2nd Lieut., St Paul's School O.T.C. $\quad$ I889

Bourdillon, T. E. Lieut., R. Sussex Regt. (W.) (P.) [1914]

Bower, H. J. Capt., R.A.M.C. (W.)

Bowes, G. B. Major, Cambridgeshire Regt. and Labour $\quad$ I892 Corps. T.D.

Bowker, R. C. S. 2nd Lieut., R.F.A.; Lieut. (A.), R.A.F. I9I I

Bowle-Evans, C.H. Colonel,I.M.S.; A.D.M.S. C.M.G. $\quad$ I886 C.B.E. $M_{3}$.

Boyd, Rev. H. J. C.F. $4^{\text {th }}$ Class, R.A.C.D.

Braunholtz, G. E. K. Corpl., Manchester Regt.

BRAYNE, W. F. Major, I.M.S.

1895

1906

1894

ABrearley, A. J. Capt., Devon Regt.(T.F.) and R.E. $\quad$ I909

Killed in action 20 Fune 1917

BREWER, J. Lieut., R.A.M.C.

BRIGGS, H. W. 2nd Lieut., Gloucestershire Regt.; Lieut., I9I3 R.A.F.

WBriggs, R. S. Lieut., W. Yorks. Regt.(T.F.)

Killed in action 29 Fuly r9I 5 
Brooke, J. 2nd Lieut., Dorset Regt.

1904

Brookes, H. V. Pte., Middlesex Regt.

1915

ABrough, Rev. J. S. B. C.F. 3 rd Class, R.A.C.D. $M . \quad 1897$ Died I I Nov. I918 of pneumonia

Brown, A.W. Lieut., Cameronians (Scottish Rifles, T.F.) Igr3

(W.)

Brown, H. C. Major, I.M.S. C.I.E.

I 895

Brown, H. G. Surgeon Lieut., R.N.

Brown, O. N. Pte., R.A.M.C.; attd. R.F.A.

BRowne, C. R. Lieut.-Cdr., R.N.V.R. (R.N.D. and I9I2

Coastal Motor-boat Service). $M$.

Browne, R. D. H. Cadet, O.C.B.

1887

BrutTon, J. C. Lieut., 8th Hussars (R. of O.); Capt., 1894

Res. Regt. of Cavalry

Bryant, G. W. Lieut., Malay Vol. Force

«BuckNILL, J. C. 2nd Lieut., Hampshire Regt.(T.F.) ${ }_{1898}$

M.C. $M$.

Killed in action in Mesopotamia 21 fan. 1916

Burbidge, E. D'A. Capt., R.F.A. M.

ABURCH, R. S. Lieut. (A.), R.A.F.

Killed in action 28 fune I918

BurkitT, F. Lieut., Lincolnshire Regt.; attd. R. Irish I9r3 Rifles. (W.)

Burn, J. H. Capt., R.E. (Signals)

Burrows, C. Major, R.A.M.C. (T.F.)

1891

I9II

Burton, D. F. Lieut., Suffolk Regt.; Lieut. (A.), R.A.F. 1906 (P.)

Burton, Rev. W. 2nd Lieut., Sir R. Manwood's School, 1904 Sandwich, O.T.C.

Butler, G. G. Capt., R.A.M.C. M.B.E.

Bútlin, Rev. T. H. C.F. 4th Class, R.A.C.D.

Byron, H. Pte., R. Fusiliers; 2nd Lieut., R.E.

1899

1909

1893

Carnegy, P. L. St C. Pte., R.A.M.C.

*Cass, L. F. Capt., R. Sussex Regt.

Killed in action 21 Dec. 1915

Causton, E. P. G. Surgeon Lieut., R.N.; Major, 1894

R.A.M.C. O.B.E. $m$.

Cave-Orme, G. A. R. Capt., Spec. List, empld. Ministry 1886 of National Service

Challands, R. S. 2nd Lieut., Worcestershire Yeo.; 1899

Lieut. Grenadier Gds.; attd. Gds. M.G. Regt.

AChalmers, H. S. Capt., R.F.A.(T.F.)

Died 29 Sept. I917 of wounds received in action

1913 
Chambers, E. L. Capt., Bedfordshire Regt.; Major,

Northumberland Fus.; Lieut.-Col., King's Own (Yorkshire L.I.) $M$.

AChaning-Pearce, W. T. Capt., R.A.M.C. M.C.

Killed in action I Oct. IgI7

Chapman, E. J. C. Lieut., Spec. List (R.T.O.) M.C. M. 1896

Charter, H. R. Lieut., E. Surrey Regt.; Major, R.E. 1909

(Spec. Bde.); Chemical Adviser. M.C.

Chawner, W. R. Capt., R.E.

Cheetham, C. E. Capt., R.G.A.(T.F.Res.); empld. Min- 1899 istry of National Service

ChIlds, A. E. 2nd Lieut., Somerset L.I.; Capt., R.E. 1914 (W.)

Chrystall,H.M. Lieut., R.N.V.R.(Howe Bn.,R.N.D.); Staff Capt.; Capt., R.E. (W.) $M$.

ChubB, C. Capt., R.A.S.C.

Clarke, Rev. H. S. S. C.F. th $^{\text {th }}$ Class, R.A.C.D.

Clarke, L. B. Capt., R.A.M.C.(T.F.) $M$.

AClifford, A. C. 2nd Lieut., 3rd Dragoon Gds. (R. of O.) Killed in action at Ypres 2 fune I9I 5

Cовв, Rev. F. W. C.F. $4^{\text {th }}$ Class, R.A.C.D. Cobham, Rev. E. Pte., R.A.M.C.; C.F. 4th Class, 1900 R.A.C.D. M.C.

Died I9 Sept. I917 of wounds received in action in $E$. Africa

Cohen, Rev. A. C.F. $4^{\text {th }}$ Class, R.A.C.D.

Colley, W. H. Major, Yorkshire Regt.; Lieut.-Col., Manchester Regt. (W.) O.B.E. M. Chevalier, Order of Leopold (Belgium). Belgian Croix de Guerre Collie, A. E. Lieut., R.A.M.C.

Collins, Rev. R. L. C.F. $4^{\text {th }}$ Class, R.A.C.D. $m$.

Colson, Rev. F. S. C.F. $4^{\text {th }}$ Class, R.A.C.D.

1912

1903 1895 1905 1906 1892

Coltman, R. L. 2nd Lieut., Coldstream Gds.; attd. Gds.

M.G. Regt. (W.)

Killed in action near Cambrai 27 Nov. 1917

ConACHER, H. 2nd Lieut., R.A.S.C.; Major, D.A.D. 1900 Army Printing and Stationery Services

Cooke, E. R. C. Capt., R.A.M.C. M.C.

Cooke, H. L. Lieut., R.E.

Cookson, H. A. Major, R.A.M.C.(T.F.)

1906

1907

1906

1900

1908

1914

Coombs, H. M. McC. Capt., R.A.M.C.(T.F.) $M 2$.

Cooper, A. L. Lieut., Fettes College O.T.C.

COOPER, H. Surgeon Lieut.-Cdr., R.N.; Lieut.-Col.

1905

1903

1915

I90I

1906

I 896

(Med.), R.A.F. D.S.O. Belgian Croix de Guerre 
Cooper-Hunt, Rev. C. L. C.F. $4^{\text {th }}$ Class, R.A.C.D. I908 Cooper-Hunt, D. L. Lieut., Hampshire Regt.(T.F.) I908 Coote, A. Lieut., R.A.S.C. Cornwall, G. Lieut., Suffolk Regt.; Capt., Gen. List I9I4 (T.M.B.); Lieut. (K.B.), R.A.F. (W.)

Corser, J. S. Pte., R. Fusiliers (P.S. Bn.); Lieut., Hamp- 1897 shire Regt.

Cotringham, J. W. Pte., W. Yorks. Regt. (W.) 1912

Cowell, Rev. J. B. C.F. 3rd Class, R.A.C.D. M 2. 1909

CowIE, D. H. Lieut., R.G.A. I9Io

Coxe, K. H. Capt., I.A.R.O., attd. 2nd Gurkha Rifles. 1909 M.C.

Coxon, T. Pte., R. Fusiliers (P.S. Bn.); Capt., S. Lancs. I9I3 Regt. (W.) $M$.

WCoyne, C. T. Capt., W. Yorks. Regt. (W.)

Killed in action 27 Aug. I9I7

Cozens, F. C. Surgeon Sub-Lieut., R.N.V.R.

Craig, G. W. Lieut.-Col., R.A.M.C.(T.F.) $m$.

Creighton, W. R. Capt., R.A.S.C. M.C. $M 2$.

CREW, F. D. Capt., R.A.M.C.

I913

Crisford, Rev. K. N. C.F. $4^{\text {th }}$ Class, R.A.C.D. (W.) 1906 M.C.

Crofts, J. M. Capt., E. Lancs. Regt.; Major, Spec. List I893

(Chemical Adviser). $M$.

Crompton, R. A. 2nd Lieut., K. Edward's Horse

CROOKE, R. H. A.B., R.N.V.R. (Hawke Bn., R.N.D.) 1907 (W.)

Crowther, H. N. Pte., Wiltshire Regt.

ZCRuikshank, G. L. Capt., Gordon Hdrs.; attd. R.F.C. 1908 D.S.O. M.C. $M 2$.

Killed in action 15 Sept. I9r6

सCubITT, V. M. Lieut., Norfolk Regt.

Killed in action at Suvla Bay I2 Aug. 1915

CuCKney, J. Lieut., R.E. (Spec. Bde.)

CUMBerR, B. S. Major, R A.S.C.(M.T.) O.B.E. $M 2$.

Cumming, J. E. Capt., R.F.A.(T.F.) M.C.

Cuthbertson, Rev. J. H. C.F. 4th Class, R.A.C.D.

Cutrs, Rev. F. J. Hon. Chaplain, R.N.

1906

I9I3

I910

I913

1900

1906

Daman, T. W. A. Major, R.A.M.C. (4th N. Gen. Hos- 1887 pital, T.F.)

WDANDRIDGE, W. L. Lieut., R.A.M.C.

Died ${ }_{5}$ Oct. 1918 of wounds received in action ${ }_{3}$ Oct. 1918

1912 
Daniell, J. Pte., R. Fusiliers; Capt., R.A.S.C.(T.F.) $\quad 1897$

DAvenPoRT, T. 2nd Lieut., Loyal N. Lancs. Regt. I9I3 and M.G.C.; Lieut., 40th Pathans, Indian Army. (W 2.)

Davidson, W. W. Pte., Malay Vol. Rifles; Lieut., R.E. 1903 (W.) M.C.

Davies, Rev. J. C. C.F. $4^{\text {th }}$ Class, R.A.C.D. (W.) (P.) 1904 WDavies, J. G. Capt., Welsh Regt.

Died I Feb. I9I 6 of wounds received in action

WDavies, J. Ll. Major, Essex Regt.

Died at Wesel, Germany, of wounds received in action in the Battle of Loos 26 Sept. I9I 5

Davies, J. Ll. Capt., R.A.M.C. M.

Davies, J. S. H. Pioneer, R.E. (Spec. Bde.)

1910

I901

WDavies, L. F. St J. Lieut., Norfolk Regt.; Major, M.G.C. (W 2.) M.C. M.

Died ro Nov. 1918

Davies-Colley, R. Colonel, A.M.S. G.M:G. $m 2$. WDavis, H. N. 2nd Lieut., R.E.

Died $22 \mathrm{Feb}$. I9I 5 of wounds received in action $2 \mathrm{I} F e b$. I9I 5

Dawe, L. S. Lieut., Hampshire Regt.

Dawkins, R. McG. Lieut., R.N.V.R. Cavalier, Order of the Redeemer (Greece)

WDAY, G. R. Lieut., Bedfordshire Regt.; Capt., R. War- *1912 wickshire Regt. (W.)

Killed in action on the Somme 27 Aug. 1916

DeED, N. G. Lieut., R.F.A. (W.) M.C.

DenNehy, H. G. Lieut., I.A.R.O., attd. Ioth Gurkha I909 Rifles

Denniston, J. G. Lieut., K. Edward's Horse, empld. I912 Corps Cavalry Regt.

Denton-Thompson, B. J. 2nd Lieut., Gloucestershire I9I3 Regt.; Capt., Manchester Regt. (W 2.) M.C.

Dickson, F. J. C. Trooper, Essex Yeo.; Lieut., R.A.S.C. 1908

Dobson, A. T. A. 2nd Lieut., Hampshire Regt.; Staff 1904 Capt.; Major, S.O. 2, R.A.F.

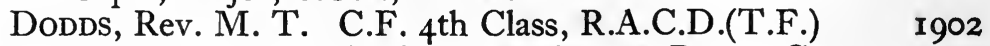

Dodwell, H. B. 2nd Lieut., R. Sussex Regt.; Capt., I9I I R.A.M.C.

Doggart, H. Pte., R.A.S.C.

Donaldson, Rev. J. T. Cadet, O.C.B.

WDorrell, H. G. H. 2nd Lieut., Durham L.I. (W 2.) I9ro

Killed in action 3 April r916 
Drew, V. Lieut., Serbian Army. Order of St Sava, $5^{\text {th }}$ I9ro Class (Serbia)

DuCKET, A. A. 2nd Lieut., R.E.

Duncanson, E. F. Lieut., R.N.V.R. (Coastal Motor-boat 1898 Service)

Duncanson, T. J. G. Lieut., R.N.V.R. (Coastal Motor- 1892 boat Service)

Durand, P. F. 2nd Lieut., I.A.R.O., attd. Sikhs. (W.) 1907 M.C. $M$.

DurIE, J. A. Capt., Black Watch; Staff Capt.; Lieut.- 1907 Col., Argyll and Sutherland Hdrs. (W.) M.C. Brevet Major. $M 2$.

Durling, J. A. Lieut., The Buffs (E. Kent Regt.) (W.) I914 Dvorkovitz, V. Driver, Armoured Cars, Russian Army r9I I DYER, A. C. Lieut., King's (Shropshire L.I.) $M$. 1907

EARDLEY-Simpson, L. E. Capt., R.F.A.(T.F.) 1898 EEAST, G. D. Capt., R.A.M.C.

Killed in action in the Third Battle of Ypres 31 Fuly

1907 I917

EDDowES, W. B. Lieut.-Col., Manchester Regt., North- 1896 umberland Fus., Yorkshire Regt., and Spec. List. $M$.

Edmonds, J. Capt., Wilson's School, Camberwell,O.T.C. * ${ }^{*}$ I90 I EDWARDS, Rev. T. G. C.F. 4th Class, R.A.C.D. 1906 ElliotT, A. F. Capt., R.A.M.C.

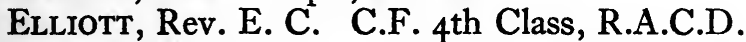

Ellis, J. M. 2nd Lieut.,.R. Warwickshire Regt. (W.) I9I4

Ellis, Rev.P.D. C.F. 4th Class, R.A.C.D.; Pte., London 1899

Regt. (Artists Rifles); Lieut., R.A.O.C.

Ellison, W. J. Capt., R.F.A.; empld. Ministry of I9ro Labour. (W 2.) $M$.

Elworthy, C. W. Capt., R.A.S.C.

England, E. M. Asst. Paymaster, R.N.V.R.

Evans, C. H. See Bowle-Evans, C. H.

Evans, J. LL. Lieut., S. Wales Borderers. (W.) I9I3

EvitT, Rev. E. U. C.F. $4^{\text {th }}$ Class, R.A.C.D.

1904

Farmery, J. W. Pioneer, R.E. (Spec. Bde.) (W.) I9I4 Farmiloe, K. M. Pte., London Regt.; 2nd Lieut., Rifle I9I3 Brigade

WFAwCETT, R. H. 2nd Lieut., Bedfordshire Regt.

Killed in action near Ypres 26 April I91 5

1897

I913

1914
I9I 3

I912

FAwCETT, T. G. Lieut., W. Yorks. Regt.(T.F.); Lieut. I9I4 (O.), R.A.F. (W.) 
WFayle, B. J. L. Capt., R.A.M.C.

Killed in action 24 Oct. I916

1908

FELL, F. J. 2nd Lieut., Black Watch. (W.)

1914

WFenN, E. G. P. 2nd Lieut., R. Welsh Fus.; attd. Essex rgr4 Regt.

Killed in action 19 Sept. 1918

Fenwick, W. Pte., R. Fusiliers (P.S. Bn.); Lieut.,

I9II

Durham L.I.; Staff Capt. $M$.

WFerguson, J. C. M. Capt., Bedfordshire Regt. (W.)

Died I9 Nov. I 918 of illness contracted on active service

1907

Fiddian, E. A. Surgeon Lieut., R.N. Bronze Medal for gallantry in saving life at sea

Fiddian, J. V. Capt., R.A.M.C.

FinCH, G. 2nd Lieut., R.G.A.

FINTER, F. B. Lieut., R.E. (W.)

FIsHer, E. G. Surgeon Lieut., R.N.

WFisher, E. H. Pte., London Regt. (Artists Rifles); 2nd Lieut., Yorkshire Regt.

Killed in action near Festubert I9 May 1915

Fisher, W. H. Major, R.A.M.C.(T.F.) O.B.E. $M$.

Fletcher, A. W. Capt., Loyal N. Lancs. Regt. (W.)

1895

\&Foley, M. J. A. Capt. and Adjt., Middlesex Regt.(T.F.)

Killed in action in Gallipoli ro Aug. 191 5

Fоoт, S. H. Capt., R.E.; Major, G.S.O. 2, empld. Tank 1906

Corps. D.S.O. Brevet Major. M2.

Fothergill, C. F. Lieut., R.A.M.C.

Frampton, H. H. C. Pte., Army Pay Corps

Frazier-Upton, Rev. G. Chaplain, R.A.F. $m$.

Freeman, H. Pte., R. Fusiliers (Sportsman's Bn.)

FRY, C. R. M. 2nd Lieut., R. Sussex Regt. (W.)

AFulford, Rev. R. H. C.F. 4th Class, R.A.C.D.

Killed in action 15 Dec. I9I6

I9II

1907

1912

1912

1902

1902

1912

1900

1898

1889

1900

1898

1914

1905

Gabriel, E. V., C.S.I., c.v.o. Lieut.-Col., Gen List., 1893

Indian Army; attd. Gen. Staff, War Office, Italian Army, and Palestine. C.M.G. C.B.E. M2. m. Officer, Order of the Crown of Italy. Officer, Order of St Maurice and St Lazarus (Italy). Italian Croce di Guerra

Gabriel, J. B. S. Capt., R.E. (London Electrical Engineers, T.F.) $m$.

GABRIEL, O. B. Major, R.A.S.C.(M.T.)

Gandy, E. W. Lieut.-Cdr., R.N.V.R. O.B.E. M.

Chevalier, Legion of Honour (France)

1907

1909

1898 
GARDNER, G. H. 2nd Lieut., Gen. List; Lieut., Army 1901 Printing and Stationery Services

GARDNER, R. Lieut.-Col., King's Own (R. Lancaster 1908 Regt., T.F.) M.C. $M 2$.

GARDNER, Rev. R. T. C.F. 4th Class, R.A.C.D. 1882

GARNETT, R. T. Lieut., R.G.A.

GARRETT, D. T. Lieut., R.N.V.R.

HGarRod, A. N. Lieut., R.A.M.C.

Killed in action 25 Fan. 1916

*Gauld, A. G. Capt., London Regt.

Killed in action on the Somme 15 Sept. I916

German, R. L. Lieut., Hampshire Regt.; attd. Tank I9I I Corps

Giвb, P. Major, R.A.S.C. M.C. $M$.

GILBERT, H. Major, York and Lancaster Regt. and 1912 M.G.C. M.C. $M 2$.

Gimson, B. L. 2nd Lieut., R.A.S.C.

Gimson, C. 2nd Lieut., I.A.R.O., attd. I8th Infy.

Girling, Rev. F. B. C.F. $4^{\text {th }}$ Class, R.A.C.D.

GLEN-BotT, C. L. Engineer Lieut., R.N.V.R.

Glenday, R. G. Lieut., King's African Rifles. (W.) 1908 M.G. $M$.

GoodAll, J. F. 2nd Lieut., E. Lancs. Regt.

Goodwin, E. Staff Lieut., Ministry of Munitions; Capt., 1896 Spec. List. O.B.E. $m 2$.

Goolden, C. E. 2nd Lieut., R. Munster Fus.; Lieut., I9I I Labour Corps

Gordon, G.A. Lieut., 2nd Dragoon Gds. (Queen's Bays). 1907 (W.)

Gossling, F. N. Major, R.E.(Postal Section). M.C. M. 1908 Gough, D. L. Capt., Somerset L.I. (W 2.) M.C. [1914] Gough, H. A. Major, W. Yorks. Regt. (W 2.) M.C. ${ }_{1907}$ $M 2$.

Gover, J. E. B. Air Mechanic, R.N.A.S.

सGow, C. H. Surgeon Lieut., R.N.V.R. (R.N.D.)

Killed in action on the Somme 13 Nov. 1916

Graham Hobson, W. J. Lieut., R.F.A.; attd. R.G.A.

Graham-Jones, J. L. Capt., R.A.M.C.

I913

1909

Grange, E. L., T.D. Hon. Colonel, R.F.A.(T.F. Res.) m. 1877

GraY, A. H. Lieut., R.G.A. $M$.

Gray, N. Capt., R.A.M.C. $M$.

Greaves, H. G. Lieut., R.A.M.C.

Green, Rev. A. G. C.F. 4th Class, R.A.C.D.

Green, C. C. Pte., Middlesex Regt. 
Green, E. A. Surgeon Lieut., R.N. $M$.

Greenberg, B. M. See Greenhill, B. M.

1910

Greene, C. W. Capt., R.A.M.C.(T.F.) (W.) $M_{3 .} .1900$

GreENHILl, B. M. 2nd Lieut., Buckinghamshire Yeo.; I9I I Lieut., R. Horse Gds. (W.) (P.)

Gregory, C. H. Capt., R.A.M.C.(T.F.)

Gregory-Jones, E. Lieut., R.F.A. (W.) M.C.

Gribble, H. W. G. Pte., London Regt.

Griptitu-Wiulaus, G. C. Lieut., R.F.A.(T.F.) (W) 1910 $M$.

Grove, A. J. Hon. Lieut., Spec. List (Entomologist) I9Io

Guinness, Rev. P. W. C.F. 2nd Class, R.A.C.D. D.S.O. 1895 M.C. $M 2$.

\&Gunton, J. W. 2nd Lieut., Somerset L.I.; attd. R.F.C. I914 Killed in action 9 Aug. 1916

Gurney, B. T. Sergt., R.E.

Gurnhill, Rev. C. J. C.F. $4^{\text {th }}$ Class, R.A.C.D.; Pte., I908 R.A.M.C.

Gwins, R. F. Lieut., Cambridgeshire Regt. ; attd.North- 1913 amptonshire Regt.; Capt., King's (Liverpool Regt.) (W.)

Gwyn, R. F. 2nd Lieut., S. Wales Borderers; Lieut., 1906 Army Cyclist Corps

HaHN, F. M. 2nd Lieut., E. Yorks. Regt. and N. Staffs.

Regt.; Capt., R.A.O.C.; I.O.M. 3rd Class. (W.)

HaINES, H. Y. Lieut., Canadian Infy.

HALE, G. D. Lieut.-Col., Loyal N. Lancs. Regt.(T.F.)

I9II

Hall, D. G. Major, R.A.M.C. (2nd E. Gen. Hospital, I894 T.F.)

Hall, G. V. 2nd Lieut., R. Welsh Fus.

Hall, J. Capt., R. Scots; empld. P. and B.T. Staff

Hanson, Rev. R. E. V. C.F. 2nd Class, R.A.C.D.; Dep.

Chaplain-in-Chief, R.A.F. O.B.E. $m$.

Harding, Rev. N. S. C.F. $4^{\text {th }}$ Class, R.A.C.D.

Harland, Rev. H. C. C.F. 3 rd Class, R.A.C.D. M.C.

Harland, R. E. C. Lieut., The Queen's (R.W. Surrey

Regt.); Liaison Officer with Portuguese Army. (W.)

Harries, A. T. Lieut., R. Welsh Fus.; Brigade Major. I9I4 $M 2$.

HarRIS, A. D. Lieut., R.F.A.

HaRRIS, Rev. H. C.F. $4^{\text {th }}$ Class, R.A.C.D. M.C. M. 1896

HHARRIS, H. A. Capt., R.A.M.C. (W.)

Killed in action $3 \mathrm{I}$ fuly I9I7 
HARRIS, T. Lieut., R.E. (Meteorological Section)

1909 HHARRISON, D. H. Lieut., R.F.A.(T.F.)

1912

Killed in action 16 Sept. 1918

Harrison, L. Hon. Lieut., Spec. List (Entomologist) I9I4

Hartman, R. T. Capt., London Regt. (R. Fus.); Asst. 1912 Supt., Ordnance Factories

HatTersley, S. M. Major, R.A.M.C.; D.A.D.M.S. (P.) 1906 M.C. M. French Croix de Guerre

Hawkins, A. G. 2nd Lieut., Worcestershire Regt.(T.F.) $\quad 1898$

Hawkins, C. L. Lieut., R.A.M.C.

HAYTER, G. K. H. Lieut., R.F.A.; Staff Capt. M. 1906

Head, Rev. F. W. C.F. 3 rd Class, R.A.C.D. M.C. and 1893 Bar

HeANY, W. P. Lieut., E. Yorks. Regt. (W.)

WHearN, J.. S. 2nd Lieut., Suffolk Regt.

Killed in action at Gueudecourt 12 Oct. 1916

Heath, C. N. Capt., R.E.

Hele, T. S. Capt., R.A.M.C.(T.F.) O.B.E. M 2.

HEMPSON, E. R. 2nd Lieut., R. Fusiliers

WHensley, W.H Pte, R. Fusiliers (P.S. Bn.); Capt, Somerset L.I. (W.)

Killed in action at Amiens 21 March 1918

HePwORTH, Rev. B. G. Lce.-Corpl., Australian Infy. 1905

HerberT, Rev. F. F. C.F. $4^{\text {th }}$ Class, R.A.C.D.

HeYwood, W. B. Capt., R.A.M.C.

Higgins, Rev. W. N. C.F. 4th Class, R.A.C.D.

HighField, A. 2nd Lieut., R.E. (Spec. Bde.)

Hill, A. Capt., R.G.A.(T.F.) $M 2$.

1912

1908

1906

1900

[9I4]

HILl, A. T. 2nd Lieut., Berkhamsted School O.T.C.

1898

1889

1898

I9I3

1897

Hill, H. L. Trooper, N. Somerset Yeo.; 2nd Lieut.,

Loyal N. Lancs. Regt. ; Lieut., N.Somerset Yeo. ; attd.

Dragoon Gds.

Hill, R. Pioneer, R.E. (Spec. Bde.)

WHillbrook, W. Capt. (M. O.), Congo Carrier Corps, I9Io

E. African Force

Died at Nairobi 22 Fuly 1916

Hind, A. M. Capt., R.A.S.C.; Major, D.A.Q.M.G. $\quad$ I899 O.B.E. $M 3$.

Hinde, Rev. B. F. C.F. $4^{\text {th }}$ Class, R.A.C.D. M.C.

HINDE, E. B. Capt., R.A.M.C.(T.F. Res.) $M$.

1905

1900

Hinde, Rev. P. M. C.F. 4th Class, R.A.C.D. M.C. 1903

Hobson, W. J. G. See Graham Hobson, W. J.

Hodges, Rev. L. N. C.F. 4th Class, R.A.C.D.

Hole, H. W. 2nd Lieut., Hampshire Regt.

1905

I9I $x$

C. U.W.L. 
सHollins, E. R. L. Capt., King's Own (R. Lancaster Regt.)

Died 3 March 1916 of wounds received in action 2 March I9I 6

Hoole, N. Lieut., W. Yorks. Regt. and Labour Corps. I9II (W.) $M$.

Hooper, A. N. Capt., R.A.M.C. (W.) $M$.

Hooper, A. W. Capt. and Adjt., R.G.A. M.C.

AHOOPER, L. J. 2nd Lieut., Dorset Regt.

Killed in action 26 Sept. I916

Hope, Rev. B. L. C.F. $4^{\text {th }}$ Class, R.A.C.D.

Hopkinson, H. C. 2nd Lieut., King's (Shropshire L.I.) and R.F.C.

Horder, C. A. Surgeon Sub-Lieut., R.N.V.R.

Horner, P. W. M. Capt., R.A.S.C.

1907

1908

I9I3

1899

I9II

Hornsby-Wright, G. J. Lieut.-Col., Essex Regt. (T.F.)

1915

D.S.O. M. m.

Horton, E. F. Capt., Dorset Regt. (W.) $M$.

Hoste, T. B. Sergt., King's Royal Rifle Corps

Howard, C.G. Lieut., Nigeria Regt., W. African Frontier Force

Howard, T. H. Major, Hampshire Regt.; Staff Capt.

Howell, E. B. Lieut.-Col., Spec. List (Censor's Dept.);

Military Governor of Baghdad. C.I.E. C.S.I. M 5 .

Howell, R. A. Lieut., R.A.S.C.

Howell, R. G. D. 2nd Lieut., R.A.S.C.

Howland, R. C. J. Gnr., R.G.A.

I9I3

I 893

1903

1914

I9I I

1902

1895

1908

1896

I9I 5

AHuddart, L. H. L. Lieut., R.E.; attd. Nigeria Regt., W. $\quad$ I898 African Frontier Force

Died I 2 Feb. I9I7

Hughes, L. W. Lieut., R.A.S.C.

I9II

Huld, H. C. Major, Gen. Staff, attd. S.W. African Field 1905 Force

Humphreys, I. H. M. Capt., Border Regt. (T.F.); empld. I9I2 Air Ministry. (W.)

Hunt, Rev. J. B. Lieut., R. Fusiliers; empld. Ministry of Igog Munitions. (W.) M.C.

Huntbach, G. W. Capt., King's (Shropshire L.I.); 1907 attd. R.F.C.; Major, Tank Corps. (W.)

AHussey, E. T. Pte., R. Fusiliers (P.S. Bn.); and Lieut., [I9I4] R. Munster Fus. (W.)

Killed in action 7 Fune 1917

Hutchings, C. E. 2nd Lieut., R. Berkshire Regt.; Capt., 1908 Army Cyclist Corps. $M$. 
Hutchings, Rev. H. W. Pte., London Regt. C.F. 4th 1906 Class, R.A.C.D. (W 2.) M.C

HIllffe, C. A. M. Pte., R. Fusiliers

Killed in action on the Somme Fuly 1916

I9I I

IMLAY, A. D. 2nd Lieut., Clifton College O.T.C.

IINGoldBY, Rev. R. H. Trooper, Alberta Dragoons, Can-

adian Force; 2nd Lieut., R. Dublin Fus.

Killed in action on the Somme I Fuly 1916

Irvine, L. C. D. Surgeon Lieut., R.N.V.R.

1905

1904

1905

WJACOT, E. Lieut. (A.), R.F.C.

Killed in action 6 June 1917

JAFFÉ,A.C. Major,London Yeo.(Westminster Dragoons); 1902

Lieut.-Col., Remount Service

JAGO, E. O. Lieut., Gloucestershire Regt.(T.F.) (W.) 1905

JAMES, L. H. Capt., Worcestershire Regt.

1909

JAMES, T. M. 2nd Lieut., Sutton Valence School O.T.C. I9IO

Jameson, F. R. W. Corpl., H.A.C.; Lieut., R.E. (Signals) 1912

D.S.O. M.C. and two Bars. M2.

Jameson, Rev. W. L. C.F. 4th Class, R.A.C.D. (W.) 1905 $M$.

Jamie, J. P. W. Capt., Leicestershire Regt.(T.F.) M.C. $\quad$ I913

JAQUET, Rev. D. A. C.F. 4th Class, R.A.C.D. 1906

JARVIE, J. M. Capt., R.A.M.C.

Jenkins, W. A., Pte., W. Yorks. Regt.

John, Rev. W. R. C.F. 4th Class, R.A.C.D.

Johns, N. A. 2nd Lieut., Queen's Own (R. W. Kent

Regt.); Major, M.G.C. M.C. and Bar

WJohns, O. LL. 2nd Lieut., R.F.A. M.C.

Killed in action 25 Fune 1916

WJohnson, D. F. G. Lieut., Manchester Regt.

Died 15 fuly 1916 of wounds rectived in action on the Somme

Jolly, Rev. R. B. C.F. $4^{\text {th }}$ Class, R.A.C.D.

1903

1912

1894

1908

I9II

I9II

JONES, B.M. Lieut.-Col.(T.), R.A.F.(Aircraft Production

Dept.) A.F.C. M. m.

Jones, C. J. Capt., R. Welsh Fus. and R.E.; Staff Capt. I9ro

Jones, E. Gregory. See Gregory-Jones, E.

Jones, Rev. F. H. Chaplain, R.N. O.B.E.

1897

JonEs, I. F. H. Lieut., Hampshire Regt.(T.F.); Capt., 1906

S. Staffs. Regt.(T.F.)

JoNES, L. W. Capt., R.A.M.C.

Jones, W. E. D. Corpl., R.E. (Spec. Bde.) 
Jones, W. N. Capt. (T.), R.A.F.

Jordan, J. H. Major, R.A.M.C. (W.) M.C.

Joyner, C. B. Capt., Essex Regt. and Gen. List, empld. Ministry of Munitions. O.B.E. M.B.E. m.

WJunor, P. B. Pte., E. African Rifles

Killed in action in $E$. Africa Sept. I9I4

1908

1901

1900

KanN, E. A. Pte., R. Sussex Regt.

1908

KeEton, G. H. . 2nd Lieut., Reading School O.T.C.

1897

Kelland, W. H. C. Capt., R.E.(T.F.)

1912

WKELLOCK, H. P. Lieut., N. Irish Horse; attd. R.F.A.

Died 6 Oct. 1918 of wounds received in action

Kellock, T. H. Capt., R.A.M.C. (3rd London Gen. 1880 Hospital, T.F., and R. Army Med. College)

Kent, A. G. Lieut., Coldstream Gds.

Kent, T. P. P. Lieut., Devon Regt.

KILNER, S. D. Surgeon Lieut., R.N.

1893

KING, H. Lieut., Seaforth Hdrs.; attd. R.E. (Signals). (W.) M.C. and Bar

Kingham, B. V. Lieut., Oundle School O.T.C.

1910

1908

1906

AKIRBY, W. E. 2nd Lieut., I.A.R.O., attd. I $5^{\text {th }}$ Sikhs

Wounded and missing, presumed killed in action in Gallipoli (Io fuly 1915)

Kirkland, Rev. A. E. C.F. 4th Class, R.A.C.D.

1908

1902

WKIRKLAND, F. W. 2nd Lieut., Rifle Brigade

Killed in action on the Somme I Fuly 1916

AKnight, W. F. Lieut., W. Yorks. Regt.

Killed in action $27 \mathrm{Feb}$. I9r7

KNights, Rev. H. J. W. C.F. $4^{\text {th }}$ Class, R.A.C.D. 1899

Knights, K. M. W. Corpl., Canadian Engineers. (W.)

1904

Lang, A. Trooper, Scottish Horse; Corpl., R.E.(Signals); 1902 2nd Lieut., Bedfordshire Regt.; attd. Cambridgeshire Regt.

Langmead, L. G. N. Major, Rifle Brigade. M.C. $M$.

LANGTRY, R. L. Lieut., Welsh Regt. (W.)

LANKESTER, Rev. R. F. 2nd Lieut., Labour Corps

1908

1914

1908

LAsbrey, Rev. E. W. C.F. $4^{\text {th }}$ Class, R.A.C.D.

Lathbury, R. J. Capt., London Regt. (Rifles). (W 2.)

LeA, Rev. H. A. H. Lieut., Labour Corps

LEE, R. O. Capt., R.A.M.C.

LeEs, A. A. Capt., R.A.M.C. M.C. $M$.

Lesley, J. W. Capt., Yorkshire Regt.(T.F.) and King's Royal Rifle Corps. (W.) (P.) M.C.

I9I I 
Levien, Rev. E. G. Lieut., R.N.V.R.

I 898

LEWIS, C. B. Lieut., R.G.A. 1900

LEWIS, J. H. 2nd Lieut., E. Surrey Regt.; Lieut., 14 Middlesex Regt.; attd. T.M.B.

ALEwIS, W. H. 2nd Lieut., R.F.A. $M$.

Died (1 5 Aug. 1917) of wounds received in action

1913

ALIDGETT, J. C. Lieut., S. Lancs. Regt.

Killed in action 23 March 1918

WLillie, F. S. Major, R. Irish Regt.

Killed in action at St Eloi 15 March 1915

Line, J. Capt., R.A.S.C.; Hon. Capt. (A.), R.A.F. I9I2 A.F.C.

Lloyd, H. I. Lieut., Northamptonshire Regt.; Capt., I9I3 King's Own (R. Lancaster Regt.) M.C.

LloYd, R. B. Capt., I.M.S. $M$.

1900

WLloyd-Jones, E. W. Capt., R. Welsh Fus.(T.F.) M. 1907 Killed in action in Gallipoli ro Aug. I9r 5 .

WLloyd-Jones, J. Pte., London Regt. (Artists Rifles); I910 Capt., Yorkshire Regt. (W.) M.C. M.

Died I I March I9I6 of septic pneumonia and pleurisy

Longden, J. M. Lieut.-Col., Durham L.I. and Gen. 1892 Staff

LonSDALE, H. Lieut., Suffolk Regt. (W 2.) $M$. $\quad$ I904

Loseby, Rev. P. J. Pte., R.A.M.C.; C.F. 4th Class, 1894 R.A.C.D. (W.)

LuCAS, E. Pte., R.A.M.C.

Lush, H. Pte., R. Warwickshire Regt.; Lieut., W. Yorks. Regt. (W.) M.B.E.

LyoN-SMITH, G. Lieut. and Adjt., R.F.A.(T.F.); Brigade 1912 Major. (W.) $M$.

McCombe, F. W. W. Corpl., Army Pay Corps; 2nd I9r3 Lieut., R.G.A.

MCCREA, E. D. Capt., R.A.M.C.

Macdonald, C. L. Lieut.-Col., Manchester Regt.; attd. 1900 Devon Regt. and King's (Liverpool Regt.). D.S.O. and Bar. M2.

MaCkenzIE, C. Major, R.A.M.C. O.B.E. M.

MACKENZIE, H. W. G. Capt., R.A.M.C. (2nd London Gen. Hospital, T.F.)

Maclean, E. W. Capt., N. Zealand M.G.C.

MaCleod, D. J Capt, King's (Shropshire L. gade Major; empld. O.C.B. (W.) $M$.

MaCLEOD, N. D. Capt., Black Watch. M.C. and Bar I9I3 
MacMaster, H. V. Capt., Spec. List

1898

MCNaIR, A. J. Capt., R.A.M.C.

Macrae, L. Capt., Cameron Hdrs.(T.F.)

Madge, Q. Capt., R.A.M.C. O.B.E. M.

1905

I9I 1

MaKePEACE, F. L. Lieut. and Adjt., Suffolk Regt.; Lieut., Labour Corps

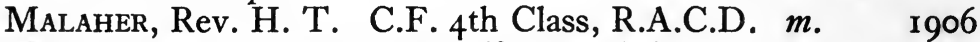
WMalcomson, L. Corpl., R.E. (Spec. Bde.)

Killed in action Nov. 1916

Malcomson, W. T. Lce.-Corpl., R. Dublin Fus.; 2nd 1908 Lieut., R.E. M.C.

Malleson, W. M. Pte., R. Fusiliers

Man, Rev. M. L. C.F. 4th Class, R.A.C.D.

WManley, J. D. 2nd Lieut., R.E.

Killed in action at Vendresse 26 Sept. 1914

ManN, R. 2nd Lieut., Lancs. Fus.; Lieut., M.G.C. 1900 (Motor)

Mann, T. C. Asst. Paymaster, Army Pay.Dept.; Capt., R.E. (London Electrical Engineers, T.F.) M.B.E. m.

Mansell, R. A. Capt., R.A.M.C.; Major, D.A.D.M.S. M.B.E. M. Order of the White Eagle, $5^{\text {th }}$ Class (Serbia)

Mansfield, H. Y. Capt., R.A.M.C.

Mansfield, W. S. Lieut., 8th (King's R. Irish) Hussars (R. of O.) Chevalier, Ordre du Mérite Agricole (France)

MarRiotT,F.W.P. Pte.,R.A.M.C.; Capt..R.G.A. M.C. 1897

MARrIS, E.D. Lieut., R. Warwickshire Regt.(T.F.); Capt. I9IC R.E. (Signals)

Marsden, W. G. Capt., R.A.M.C.

Marshall, A. R. Major, R.E. (Signals) and Spec. List (Chief Instructor, Training Centre.) D.S.O. M.C. $M 2$.

Marshall, Rev. H. G. C.F. 2nd Class, R.A.C.D. $M$.

MaRShall, Rev. N. E. 2nd Lieut., Nagpur Rifles, Indian Defence Force; Junior Chaplain, Indian Army

Martin, H. W. L. 2nd Lieut., Northumberland Fus.; Lieut., York and Lancaster Regt.; attd. S. Notts. Hussars. (W.)

Martin, J. H. B. Surgeon Lieut.-Cdr., R.N. $M$. 1905 I 905 1907 I9I 2 1908 I 896 I9IO 1907 1909 
Matthews, H. DE C. 2nd Lieut., Nigeria Regt., W. I89I African Frontier Force

Maude-Roxby, Rev. J. H. T. C.F. $4^{\text {th }}$ Class, R.A.C.D. 1902 (T.F.)

WMAYNARD, A. F. Lieut., R.N.V.R. (Howe Bn., R.N.D.) (W.)

Killed in action on the Somme 13 Nov. 1916

Maynard, H. A. Capt., Middlesex Regt. and Gen. List; I9 I I Staff Capt. (W 2.) M.C. and Bar. M.

WMelhuish, I. V. B. 2nd Lieut., Somerset L.I. Killed in action 27 Oct. 1915

Mellis-Smith, D. B. Lieut., Cameronians (Scottish 1905 Rifles) and Seaforth Hdrs.; A.D.C. (W 2.)

MenendeZ, Sir M. R. Major, Spec. List, empld. Ministry I 887 of National Service. $m$.

Metcalf, Rev. F. W. R. Chaplain and Instructor Cdr., I 894 R.N.

Metcalfe-Gibson, R. A. 2nd Lieut., R.A.S.C. 1905

WMilbuRn, W. H. 2nd Lieut., Suffolk Regt.(T.F.) r9ı Killed in action on the Somme 15 fuly 1916

MillaRd, C.S. 2nd Lieut., Gloucestershire Regt.(T.F.); 19 I I Gnr., R.G.A.

Miller, J. W. E. Capt. and Adjt., Argyll and Sutherland I9I3 Hdrs.; Staff Capt. M. French Croix de Guerre

Million, Rev. A. B. C.F. 4th Class, R.A.C.D. $\quad 1909$

Million, C. H. S. Gnr., H.A.C. I9I4

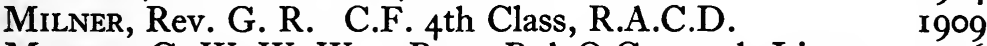

Milroy, G. W. W. W. Pte., R.A.O.C.; 2nd Lieut., 1906 Labour Corps

AMrtchell, Rev. C. W. C.F. 4th Class, R.A.C.D. M. 1902 Died 3 May 1917 of wounds received in action

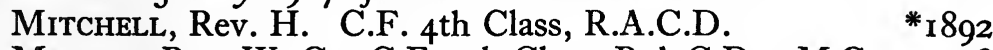

Moeran, Rev. W. G. C.F. 4th Class, R.A.C.D. M.C. 1908

Moline, R. W. H. Capt., Rifle Brigade; Major, M.G.C. $\quad$ I 909 (W 2.) (P.) M.C.

Molson, J. E. Major, R.A.M.C.(T.F.) I882

Money, Rev. W. T. C.F. 4 th Class, R.A.C.D. * * 1893

WMoorcock, F. A. Lce.-Corpl., R.A.M.C.; 2nd Lieut., I9I3

King's Own (Yorkshire L.I.)

Killed in action 3 May 1917

Moore, A. W. Lieut.-Col., R.A.M.C.(T.F.) O.B.E. $\quad 1899$

$M_{3}$. Order of the Nile, 3 rd Class (Egypt)

Morgan, E. H. Lieut., R.A.O.C.

Morgan, Rev. W. E. C.F. $4^{\text {th }}$ Class, R.A.C.D. 
Morier, C. E. Capt., D. of Wellington's (W. Riding I908 Regt.) (W 2.)

Moritz, M. Capt., R.A.M.C. (and W. Gen. Hospital, I905 T.F.)

WMORRIS, J. O. Corpl., London Regt.

Killed in action $3 \mathrm{I}$ Oct. I9I7

Morris, W. Lieut., Lancs. Fus. (W.)

Morse, C. G. H. Major, R.A.M.C.

Morton, H. T. Lieut., Mtd. Rifles and M.G.C., N. 1909 Zealand Force. (W.)

Mould, R. C. L. Capt., Leicestershire Regt.(T.F.)

Moulsdale, J. R. B. Cadet, O.C.B.

Mountfort, Rev. C. C. Capt., Uppingham School O.T.C.

Mousley, E. O. Capt., R.F.A.; A.D.C. (W.) (P.) M. 1912 Muir, J. C. Capt., R.A.M.C. (P.)

Muller, Rev. H. C. A. S. Chaplain, R.N.

1891

1896

Murray, Rev. D. W. C.F. $4^{\text {th }}$ Class, R.A.C.D. M.C. $\quad 1905$ and Bar

Musson, F. W. Capt., Loyal N. Lancs. Regt.; Capt. I9r3 (T.), R.A.F. (W.) A.F.C.

Myles, D. 2nd Lieut., Northumberland Fus.; Capt., R.E. $\quad$ I9r2 (Tyne Electrical Engineers, T.F.) $M$. French Croix de Guerre

Naisby, J. V. Capt., R.G.A. (W.) M.C.

NAISH, W. V. Capt., R.A.M.C.

Naters, Rev. C. C. T. C.F. 4th Class, R.A.C.D.(T.F.) I900

Naylor, Rev.A.T.A. C.F. 2nd Class, R.A.C.D.; A.C.G. $\quad{ }_{1908}$ (W.) O.B.E. $M 2$.

Nethersole, F. R. Lieut.-Col., Supernumerary List, $\quad$ I884 Indian Army. C.I.E.

NevetT, Rev. R. B. Cadet, O.C.B.

Neville, E. G. M. Lieut., Essex Regt.(T.F.)

Neville, H. A. D. Capt., R.E.(T.F.); empld. Ministry of Munitions. $m$.

New, F. O. W. Lieut., Leicestershire Regt. (W 2.) 1907

Newton, T. H. Lieut., R.N.V.R.; Capt. (A. and S.), 1902 R.A.F. D.S.C. $M$.

Newton, W. H. Lieut., R.A.M.C. (W.)

Nicholas, F. Capt., Worcestershire Regt.(T.F.) (W.) r9ro $M$.

Nichols, C. W. Sergt., R.E. (Spec. Bde.) 1909

Ninill, J. H. B. Capt., R. Munster Fus. (W.) M.C. I9II 
NobBs, S. W. 2nd Lieut., R.E. (W.)

Norman, D. T. Capt., R.E. M.C. Chevalier, Order of ${ }_{1908}^{\text {I9I4 }}$ Leopold (Belgium). Belgian Croix de Guerre

WNoRman, J. Sub-Lieut., R.N.V.R. (Howe Bn., R.N.D.) 1912 (W.)

Killed in action in Gallipoli 7 June 1915

Norman, N. F. Major, R.A.M.C.

NorQuoy, F. Pte., R. Fusiliers

NorthCote, T. F. Lieut., K. Edward's Horse and Res. I9I3 Regt. of Cavalry; 2nd Lieut. (T.), R.A.F. (W.)

Norton, Rev. V. E. B. C.F. 4th Class, R.A.C.D. «NoTT, H. P. Lieut., Gloucestershire Regt.(T.F.) (W.) I9I3 $M$.

Killed in action 27 April r916

※NotT, L. C. Capt. and Adjt., Gloucestershire Regt. 1912 (T.F.) M.C.

Killed in action on the Somme I8 April 1917

सNotr, T. W. Lieut.-Col., Gloucestershire Regt.(T.F.) 1907 D.S.O. $M 2$.

Killed in action on the Somme I8 April 1917

OAKES, M. W. Lieut., Nigeria Regt., W. African Frontier 1909 Force

Ogden, H. 2nd Lieut., N. Staffs. Regt. and Gen. List, I9I3 empld. Ministry of Munitions

Oldham, R. S. Capt., R.A.M.C.

Oliphant, J. Major, Canadian A.S.C. M. French 1904 Croix de Guerre

Osborne, W. M. Capt., R.A.S.C.; attd. R. Scots. (W.) 1908 Osmaston, U. E. Major, R.F.A.; Instructor in Gunnery. $\quad 1903$ M.C. M. $m$.

Osmond, T. E. Capt., R.A.M.C. (P.) $M$. 1903

Owst, G. R. Sergt., R.E. (Signals) I9I3

Padwick, H. B. Surgeon Lieut., R.N. D.S.O. M. 1908 PAGE, S. W. Capt., R.A.M.C. 1912

PAGE, W. E. 2nd Lieut., Wellington School, Somerset, I9ro O.T.C.

PaIN, B. H. Surgeon Cdr., R.N.

1897

Paine, B. W. F. Pte., Middlesex Regt.

PARES, B. Surgeon-Major, 2nd Life Gds.; A.D.M.S. $\quad$ I887 C.M.G. D.S.O. M 2 .

Parker, G. M. Capt., Australian A.M.C.

PARRY, Rev. T. J. C.F. $4^{\text {th }}$ Class, R.A.C.D. 
Paterson, A. R. Major, R.A.M.C.(T.F.)

Payne, D. N. 2nd Lieut., R.A.S.C.

1890

1902

I9II

Killed in action I4 April I9I7

Peacock, F. W., T.D. Major, Derbyshire Yeo. C.B.

Pearce, T. Lieut., I.A.R.O., attd. 48th Pioneers

Peard, C. J. Lieut.-Col., Somerset L.I. (W.) D.S.O. $M$.

Peard, J. C. N. Capt., Somerset L.I. (W 4.) M.C. M. 1909

Pearman-Smith, P. B. Pte., R. Fusiliers

1879

1904

1908

Pearsall, H. G. Capt., Tank Corps. M.C.

Died 19 March 1919 of pneumonia following gaspoisoning

Pearson, E. E. Capt., Suffolk Regt. (P.)

1907

1907

Pearson, J. W. Capt., Unattd. List, T.F.

1898

Pearson, L. H. Capt., Leicestershire Regt.(T.F.) (W3.) (P.) M. French Croix de Guerre

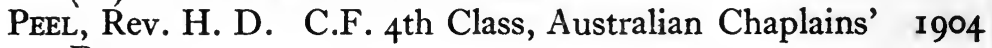
Dept.

Pelly, Rev. D.R.,v.D. C.F.3rd Class, R.A.C.D. D.S.O. 1883 $M 3$.

Perkins, F. A. Lieut., R.E.

Perkins, J. J. Capt., R.A.M.C.(T.F.)

Perry, C. G. Pte., R. Fusiliers; Lieut., Spec. List I 901

Phillips, P. Lieut., R.E. $M$.

Philp, F.E. L. 2nd Lieut., Res. Regt. of Cavalry ; Capt., R.A.S.C. $M$.

Philpot, A. J. Capt., R.E. O.B.E. M.

Phipps, W. T. Lieut., W. Yorks. Regt. (W.)

Picton-Warlow, Rev. F. T. C.F. $4^{\text {th }}$ Class, R.A.C.D.

\&Piggott, F. C. H. Capt., R.A.M.C.

Died 26 Fune 1917

Pilter, R. 2nd Lieut., Interpreter, Indian Army; Lieut., R.A.S.C.; Capt., Spec. List (Directorate of Requisitions and Hirings)

APinkerton, Rev. J. Lce.-Corpl., R. Scots

Killed in action (1 Oct. 1916)

Pitr, T. G. Trooper, Imperial Light Horse, W. Africa. 1903 (W.)

PitTs, Rev. B. T. C.F. $4^{\text {th }}$ Class, R.A.C.D.

1907

1885

1893

1904

1896

IgI4

I90I

I 898

1878

1904

1909

P905 2nd Lieut., Durham L.I.

Died 30 fuly 1916 of wounds received in action 28 fuly I916 
APlumptre, Rev. B. P. C.F. 4th Class, R.A.C.D. M.C. 1902 Killed in action 16 fuly 1917

Ponder, C. W. Capt., R.A.M.C. (Sanitary Service, T.F.) 1898

Porter, S. L. Capt., Spec. List, empld. Ministry of 1899 National Service

Postlethwaite, J. M. Capt., R.A.M.C.(T.F. Res.) $\quad$ I 898

PotTer, A. R. Capt., R.A.S.C. I90I

Powell, D. W. Major, Northamptonshire Regt.; Lieut.- $\quad 1896$

Col., R. Berkshire Regt.; Capt. (A.), R.F.C. (W.) D.S.O. $M$.

Powell, J. M. S. Pte., H.A.C.; Sapper, R.E.

Powell, R. R. Capt., R.A.M.C.(T.F.)

I9I I

1909

Power,A.G. Capt., R.Munster Fus.; Major, M.G.C. M. 1905

Prall, S. R. Surgeon Prob., R.N.V.R.; Capt., R.A.M.C. I9Io

Preston, R. W. D. Pte., Grenadier Gds.

APrince, A. L. Capt., Loyal N. Lancs. Regt. $M$.

Killed in action 8 Nov. I9I4

WPINGLE, W. R. Lieut., S. Lancs. Regt.

Killed in action on the Somme 22 fuly 1916

Prior, B. C. Lieut., Essex Regt.(T.F.) 1902

PrIOR, H. A. S. Lieut.-Col., Yorkshire Regt. and Train- 1893

ing Res. Bn. D.S.O. O.B.E. M2. m.

Punchard, C. Capt. and Adjt., R.F.A.(T.F.); A.D.C. $\quad$ I9I5

Putnam, P. W. Lieut., R.A.M.C. I9I 3

Putrock, R. Capt., R.A.M.C. (W.) 1897

Ramsbotrom, J. Lieut., Spec. List (Pathologist). O.B.E. 1905 M.B.E. $M_{3}$.

Ramsвоттом, W. H. Lieut., R.G.A. (W.) M.C. 1914 M.B.E. M.

Raven, Rev. C. E. C.F. 4th Class, R.A.C.D. * *igro

Raven, H. P. Capt., R.F.A.(T.F.) 1896

Rawlinson, H. G. Major, Madras and S. Mahratta 1899 Railway Rifles, Indian Defence Force

RAYNER, S. Capt., Durham L.I.(T.F. Res.)

WREADE, R. W. Lieut., R. Warwickshire Regt. (W.)

Killed in action at Kut 5 April 1916

READE, W. P. Major, Cheshire Regt.(T.F.) and Gen. 1893 Staff. T.D. $M$.

Reeve, G. T. 2nd Lieut., Bridlington Grammar School 1906 O.T.C.

Reid, D. M. Major, R.F.A.(T.F.) (W 2.) M.C.

I9I I

Rennie, W. B. Major, Spec. List; Lieut.-Col., A.A. 1895 and Q.M.G. D.S.O. M.G. M 3 . 
Reynolds, G. D. Corpl., Suffolk Regt. and Bedford- I9I4 shire Regt.

Reynolds, K. Lieut., Bedfordshire Regt.

Richards, C. J. Capt., Dorset Regt.; attd. D. of Welling- I9I3 ton's (W. Riding Regt.)

RichaRdSON, C. Major, Worcestershire Regt. (W.) $\quad$ I897 Brevet Lieut.-Colonel

ARICHARDSON, V. Lieut., R. Sussex Regt.(T.F.); attd. [I9I4] King's Royal Rifle Corps. M.C.

Died 9 Fune 1917 of wounds received in action 9 April I9I7

Rickman, J. 2nd Lieut., E. African Force, attd. Road I893 Corps. $M$.

WRigby, J. R. A. Lieut., Yorkshire Regt.

Killed in action in the Battle of Loos 26 Sept. I91 5

RigG, J. H. Pte., Border Regt.; Lieut., I.A.R.O., attd. 1912 93rd Burma Infy. (W.)

Roberton, J. A. W. Lieut., Gordon Hdrs. (W.) I9I2

Roberton, J. B. W. Capt., Northumberland Fus. (W 2.) [1914] D.S.O.

RoBERTS, B. F. Lieut., Oxford and Bucks. L.I.

Robertson, C. J. T. Lieut.-Col., R.A.O.C.; A.D.O.S. 1897 O.B.E. M.C. M.

Robertson, W. R. D. Trooper, K. Edward's Horse; IgII Major, R.F.A. M.C. M.

Robinson, A. S. Lieut., R.A.M.C.

Robinson, C. S. Pte., R.A.S.C.

Robinson, F. J. Corpl., S. African F.A.

RoDERICK, H. B. Lieut.-Col., R.A.M.C.(T.F.) O.B.E. * ${ }^{1} \mathrm{I} 892$ $M 2$.

Rodick, R. L. Major, R.A.S.C.

1910

Rogers, F. E. W. Major, R.A.M.C. (Sanitary Service, 1904 T.F.) M.C. $M$.

Rose, H. A. Hon. Lieut.-Col., Indian Army 1886

Rose, R. DE R. Major, R. Irish Rifles. (W.) M.C. and 1897 Bar. $M$.

\#Rothera, A. C. H. Capt., Australian A.M.C.

Died 3 Oct. I9I 5 of pneumonia

Roxby, Rev. J. H.T.M. See MAUdE-Roxby, Rev. J.H.T.

Roy, P. L. Pte., H.A.C.

Rudd, N. B. Lieut., Norfolk Regt. and I.A.R.O., attd. 48th Pioneers

Rule, Rev. G. S. C.F. 4th Class, R.A.C.D.

Rumboll, N. Capt. (Med.), R.A.F. 
Ruscoe, R. G. Lieut., R.A.S.C.(T.F.) $M$.

Rushton, E. R. Lieut., Middlesex Regt. (W.) (P.) I910

Russell, W. A. Capt., R.A.M.C. M.

1905

RYLEY, C. M. Surgeon Lieut., R.N.

1904

Salisbury, Rev. C. E. C.F. $4^{\text {th }}$ Class, R.A.C.D.

SAlmon, B. A. Capt., London Regt. (Poplar and Stepney [19r4] Rifles)

SAUNDERS, W. H. Lieut., Suffolk Regt.

Killed in action 20 fune 1915

ScholTz, C. J. Capt., R.A.M.C.

Schuddekopf, W. G. A. See Shuttleworth, W. G. A.

Sclater, Rev. J. R. P. C.F. 4th Class, R.A.C.D.(T.F.) 1895 $m$.

Scotт, J. E. Capt., R. Munster Fus. and Gen. List r9ro (O.C.B.) (W.) M.C.

ScotT, J. S. Lieut., Durham L.I.(T.F.); Interpreter. 1900 M. Cavalier, Order of the Redeemer, $5^{\text {th }}$ Class (Greece)

Scort, W. D. Pte., London Regt. (Artists Rifles); Lieut., I9I2 R.G.A. and Gen. Staff (Intelligence)

WSeAver, C. Capt., R. Inniskilling Fus. (W.)

Died 3 Oct. 1916 of wounds received in action $\mathrm{I}_{3} \mathrm{Sept}$. 1916

Sellwood, F. G. Major, R.A.S.C. O.B.E. M.C. M. 1912 Selwyn, Rev. W. M. C.F. $4^{\text {th }}$ Class, R.A.C.D.(T.F.) $\quad 1898$ Shakespeare, G. H. Capt., Norfolk Regt.(T.F.); attd. 1912 The Queen's (R.W. Surrey Regt.)

SharP, E. W. L. Capt., R.A.M.C. $M$.

Sharpe, A. G. Lieut., R.A.S.C.(T.F.) and $4^{\text {th }}$ Cavalry, Indian Army

Shaw, Rev. K. E. C.F. $4^{\text {th }}$ Class, R.A.C.D.

SheldON, T. W. Capt., R.A.M.C.

1912

Shelford, Rev. L. McN. C.F. 4th Class, R.A.C.D.

Shenstone, A. G. Capt., R.E. M.C. $M$.

SherA, A. G. Capt., R.A.M.C.

SHERLOCK, J. H. Lieut., R.E.

SHERwood, G. D. Capt., R.A.M.C.

1909

1914

Shields, C. St B. Capt., R.G.A. (W.)

1902

1907

1890

1914

1908

1912

I 906

SHILDRICK, L. R. Major, R. Munster Fus. $M$.

1908

ShIPTON, W. Capt., R.A.M.C.

Shone, H. J. Major, R.A.M.C. (W.) $M$.

1906

1902

1896

ShUCKBuRgh, R. S. 2nd Lieut., R.G.A.

I899 
Shuttleworth, W. G. A. 2nd Lieut., W. Yorks. Regt. I9ro (T.F.); Lieut., R.A.M.C.

Sibley, F. H. Lieut., R.F.A.(T.F.); Hon. Lieut. (O.), [1914] R.A.F.

Sim, P. W. Lieut., R.F.A.; Lieut. (A.), R.A.F. $M$. [1914] Sindall, R. D. Cadet, O.C.B.

Sisson, B. H. 2nd Lieut., R.G.A.; Capt. (K.B.), R.A.F. 1906 $m$.

Slater, J. A. Capt., R.F.A.

Smetham, S. J. Corpl., R.E.

Smith, C. S. Capt., R.E. (Signals, T.F.)

Smith, D. B. M. See.Mellis-Smith, D. B.

Sмiтн, H. Instructor Lieut., R.N. $m$.

Smith, Rev. L. C.F. $4^{\text {th }}$ Class, R.A.C.D.

Smith, R. E. Surgeon Lieut., R.N. O.B.E.

Smith-Rewse, H. G. Capt., Suffolk Regt.

1903

1914

I9ro

*Smyth, W. H. Lieut., Devon Regt. and Worcestershire 1897

Regt.

Killed in action 17 April r918

Smythe, G. A. Capt., R.A.M.C.

Soothill, V. F. Major, R.A.M.C. $M$.

1912

1904

I899

1907

Sopwith, S. S. 2nd Lieut., Shrewsbury School O.T.C.

Soutar, A. K. Capt. (Med.), R.A.F.

South, F. W. Lieut., Singapore Vol. Force

SPARK, D. S. Capt., Somerset L.I. M.C.

1906

1906

1905

1905

1904

I9II

\#Spearing, E. Lieut., King's Own (R. Lancaster Regt., 1908

T.F.) (W.)

Killed in action at Ginchy I I Sept. 1916

SpIlsbury, L. J. Lieut., R.E. M.C.

1905

Staley, H. S. Lieut., Unattd. List, T.F.

Stancomb, Rev.J.M.D. C.F.4th Class, R.A.C.D.; Capt., 1908

Devon Regt. (T.F.) and .Somerset L.I. (T.F.) $M$.

Staples-Browne, R. C. Capt. and Q.M., N. Zealand 1899

Med. Corps. M.B.E.

SteEl, O. W. D. Staff Capt.; Major, R. Monmouthshire

Regt.; Lieut.-Col., R.A.M.C.(T.F.) M.C. $M 2$.

\#STePhen, F. C. Lieut., Gordon Hdrs.(T.F.)

Killed in action in the Battle of Loos 25 Sept. 1915

Stevens, C. G. B. Pte., N. Bengal Mtd. Rifles, Indian 1908

Defence Force

Stimson, W. B. Sergt., R. Fusiliers; Lieut., Bedfordshire Regt. (W 2.) M.C.

Stinson, H. J. E. Major, R.G.A. M.G. and Bar 1905

Stokes, W. A. Capt., R.A.M.C.(T.F.) $m$. 
Stone, B. J. V. 2nd Lieut., Leicestershire Regt.; Lieut., 1912 Cheshire Regt. (W.)

Stone, E. R. Capt., R.A.M.C.

STORRS, K. S. Capt., R.A.M.C. (T.F. Res.)

ASTrachan, H. Lieut., Durham L.I.(T.F.) (W 2.)

Died 29 Fuly 1918 of wounds received in action

STRAHAN,W.R. Capt., London Regt.(London Irish Rifles) 1909 Streeten, Rev. A. H. C.F. $4^{\text {th }}$ Class, R.A.C.D. (W 2.) 1904 M.C.

Stringer, C. E. W. Lieut., Canadian H.A.

Strong, J. P. Lieut., Loyal N. Lancs. Regt. (W.) I9I4

StURGeS, Rev. E. L. Chaplain, R.N.

I90I

I 888

1914

Sturton, K. M. Capt. (T.), R.A.F. (Aircraft Production Dept.)

StuRTon, S. D. Surgeon Sub-Lieut., R.N.V.R. I9I5

STYle, A. H. Capt., R.A.M.C.

1893

SuFferN, C. Surgeon Sub-Lieut., R.N.V.R. IgII

Summerhayes, C. H. Capt., Durham L.I. and Glouces- [1914] tershire Regt. (W.)

Summers, F. Lieut.-Col., R.E.; C.R.E. D.S.O. M.C. ${ }^{1913}$ $M_{4}$.

SutCliffe, A. Capt., R.A.M.C.(T.F.) $M 2$. 1905

Sutcliffe, P. T. Surgeon Cdr., R.N. 1892

SYKES, W. S. Surgeon Lieut., R.N.

Tabberer, C. O. Pte., R.A.M.C.; Sergt., Leicestershire 1908 Regt.; Capt., London Regt. (L.R.B.) M.C.

Taggart, W. Q. Pte., R. Fusiliers (P. S. Bn.); Capt., I9I I King's (Liverpool Regt.) M.C.

TAlbOT, Very Rev. A. E. C.F. Ist Class, Australian 1902 Chaplains' Dept. (W.)

TANNER, Rev. E. V. C.F. $4^{\text {th }}$ Class, R.A.C.D. (W.) 1905 M.C. and Bar

Teale, Rev. K. W. P. C.F. 4th Class, R.A.C.D. $\quad 1894$

Teuten, Rev. L. M. C.F. 4th Class, R.A.C.D. 1907

Theobald, G. W. Surgeon Prob., R.N.V.R. I914

Thomas, E. F. Corpl., R.E. 1912

Thomas, E. R. Capt., R.A.O.C.; Major, D.A.Q.M.G. I9I I $M$.

Thompson, A. B. Capt., Northumberland Fus.(T.F.); 1907 Staff Capt. (W.) $m$.

Thomson, N. G. Surgeon Prob., R.N.V.R.

Thornton, C. G. Lieut., R. Inniskilling Fus.; Capt., 1907 O.C.B. 
Thornton, L. H. D. Capt., R.A.M.C. $M$.

Thornton, V. S. 2nd Lieut., Essex Regt. (W.)

Thorp, C. F. Lieut., Northumberland Fus. and King's Royal Rifle Corps

Thorp, H. C. Capt., R.A.M.C.

TIDY, T. Signalman, R.N.V.R.

Killed in explosion on H.M.S. Bulwark 28 Nov. I914

TILly, R. L. Lieut., The Queen's (R.W. Surrey Regt.); Hon. Lieut. (A.), R.A.F.

Tomlinson, A. E. Lieut., S. Staffs. Regt.; empld. War I9ı2 Office. (W.)

Tomlinson, W. A. Corpl., R.G.A.

Toovey, T. P. Capt., The Queen's (R.W. Surrey Regt.) (W.) M.C. and Bar

Toulmin-Smith, A. K. Capt. (T.), R.A.F. (Aircraft 1899 Production Dept.)

Tracy, G. D. C. Lieut., Gordon Hdrs. (T.F.); Pte., I9I2 R.A.S.C.

Trautmann, H. F. See Trewman, H. F.

Trewman, H. F. Lieut., Bedfordshire Regt. (W 2.)

I9II

Tribe, K. W. Lieut., N. China Vol. Force

TrIPP, N. F. Pte., R. Fusiliers (P. S. Bn.); Lieut., I9I I

R.A.S.C.; attd. R. Fusiliers and R. Warwickshire Regt. (W 2.) M.C.

Tunmer, E. J. E. Capt., King's (Shropshire L.I.) (W 2.) 1902

Turnbull, J. B. Lieut., Shropshire Yeo.; attd. R. Welsh Fus. (W.)

WTuRnbull, W. A. Lieut., Bedfordshire Regt.

Killed in action 13 Nov. 1916

1913

1912

Turner, A. C. Lieut., R. Fusiliers and Gen. List; In- I9I I spector, Woolwich Arsenal. M.B.E.

TurNer, H. C. Major, London Regt. (Queen's). M. $\quad$ I 898 French Croix de Guerre

\&Twining, R. W. Trooper, Devon Yeo.; 2nd Lieut., I9I3 Dragoon Gds. and Devon Regt.

Killed in action on the Somme I Fuly r916

URLING-Smith, F. M. Lieut., Nigeria Regt., W. African I897 Frontier Force

WURQuhart, J. L. Lieut., Northamptonshire Regt. Killed in action 2 Nov. 1915

Vallé-Pope, E. 2nd Lieut., King's Own (R. Lancaster 1892 Regt.) 
Verniquet, W. G. Capt., R.A.M.C. I9I I

VERRALl, F. H. Artificer, R.N.V.R. 1902

VICKeRs, V. C. W. Capt., R.A.M.C. M. 1907

VinING, Rev. L. G. C.F. $3^{\text {rd Class, R.A.C.D. } M 2 . \quad 1907}$

VISCHER, H. 2nd Lieut., Nigeria Regt., W. African I896

Frontier Force; Major, Spec. List; G.S.O. 3 (Intelligence). C.B.E. Chevalier, Legion of Honour (France). Officer, Ordre de l'Etoile Noire (France). Chevalier, Ordre de la Couronne (Belgium). Cavalier, Order of the Crown of Italy

Vischer, Rev. M. M. C.F. 3 rd Class, R.A.C.D. $M . \quad 1898$

Waddington, J. E. 2nd Lieut., R.A.S.C.

Wailes, F. G. Capt. and Adjt., R.G.A. $M$.

Waldegrave, Rev. G. T. Hon. Chaplain, R.N.

1908

Waldegrave, Rev.S. C. C.F. $4^{\text {th }}$ Class, R.A.C.D. M.C. $\quad 1907$

WALKER, A. Capt., R.A.M.C.(T.F.) D.S.O. Brevet 1897 Major. $M$.

WalKER, A. R. Lieut., Welsh Regt.

WWalker, E. B. 2nd Lieut., Dorset Regt. and Queen's

Own (R.W. Kent Regt.) $M$.

Killed in action 18 April I9I 5

WALKER, E. R. C. 2nd Lieut., Black Watch. (W.) 1916

WALKER, L. P. Lieut., R.A.O.C.; Major (Ad.), R.A.F. I900

WaLKer, Rev. L. W. L. Chaplain, R.N.

WALKER, S. W. Lieut., Nigeria Regt., W.African Frontier Force

Wallace, F.W. 2nd Lieut., Cameronians(Scottish Rifles); Lieut., R.E. (Signals). (W.)

Wallace, J. C. Capt., R.E.; Hon. Capt. (T.), R.A.F. 1909 M.C. $M$.

WalleR, N. H. Major, Gloucestershire Regt. (T.F.) $\quad$ I899 M.C. $M$.

Wallis, O. B. Capt., Herefordshire Regt.; attd. King's 1907 (Shropshire L.I.) (W 2.)

Walthall, H. D. D. Capt., R.A.S.C. O.B.E. M. $\quad 1899$

WARREN, A. C. Lieut., R.A.M.C.

Warrington, T. Capt., R.A.M.C.

I894

WATERER, C. R. Corpl., R.E. (London Electrical En- 1906 gineers, T.F.)

Watkins, C.R. Capt., Bengal Vol. Rifles, Indian Defence I90I Force. C.I.E.

WWatkins, E. L. C. Lieut., R.F.A.

Died 17 March 1917 of wounds received in action

I9IO c. U.w.L. 
Watkins, W. F. Lieut., Gloucestershire Regt. (W.) 1906 M.C. $M$.

WatTs, Rev. B. H. C.F. 4th Class, R.A.C.D.

WAYET, Rev. J. W. F. Lieut., Lincolnshire Regt. and rgor

Gen. Staff

Wayman, G. B. Pte., Devon Regt.(T.F.)

Weatherell, R. K. Lieut., Suffolk Regt.; attd. Essex I905 Regt. (W.)

Webster, V. T. P. Capt., R.A.M.C.

Weigall, G. J. V. Capt., Kent Cyclist Bn.

Welbourne, E. Major, Durham L.I.; attd. W. Yorks. I9I2 Regt. (W.) M.C. m.

Weld, C. G. Lieut., Worcestershire Regt. (W.) 1896

Wells, C.A. Major, Hampshire Regt.; Staff Capt. O.B.E. 1900 Brevet Major. $M 2$.

\#Wells, C. D. Lieut., King's Own (R. Lancaster Regt.); [1914]

Lieut. (O.), R.A.F. M.C. M.

Killed in action $\mathrm{x} 6$ May $\mathrm{r} 9 \mathrm{r} 8$

Wells, R. C. Capt., R.E.; attd. R. Irish Regt. (W.) (P.) 1904

Wernicke, W. G. See Verniquet, W. G.

Westbrook, H. W. 2nd Lieut., Cheshire Regt.

West-Watson, C. See Barrow-In-Furness, Rt Rev.

Bishop of

Whistler, F. Major, R. Scots; Staff Capt. ; empld. High- * ${ }^{*} 877$ land L.I. Depôt

Whistler, Rev. W. W. C.F. 2nd Class, R.A.C.D. $m . \quad 1888$

Whitaker, G. M. Pte., London Regt. (Artists Rifles); I9I5

Lieut., R.G.A.; attd. R.E. (Signals). $M$.

White, F. Capt., Cheshire Regt.(T.F.) and 25th Pun- 1912 jabis, Indian Army. (W.)

WhITEHEAD, R. F. Air Mechanic, R.A.F.

Whitley, N. H. P. Capt., Manchester Regt.(T.F.); Igor

G.S.O. 3. (W.) M.C. M3. Cavalier, Order of

the Crown of Italy. French Croix de Guerre

WhITwILl, T.N. Capt., Gloucestershire Regt.(T.F.); attd. I9I2

Suffolk Regt.

Wigley, W. C. S. Sergt.-Instructor, R.E. (Signals) 1909

Wilcox, Rev. A. J. C.F. 4th Class, R.A.C.D. (P.) 1909

Wilkinson, G. Major, R.A.M.C. (3rd N. Gen. Hospital, 1885 T.F.)

Willan,G.T. Lieut.-Col.,R.A.M.C.(T.F.) D.S.O. M. 1893

Willans, E. T. Capt., R.A.M.C.

Williams, G. Capt., Welsh Regt.(T.F.); Cmdt., Anti- I9r2

Gas School 
Williams, H. A. Capt., R.A.M.C.

Williams, H. P. W. B. Major, R.A.S.C. M.C.

Williams, Rev. O. St M. C.F. 4th Class, R.A.C.D. 1902

Williamson, F. Lieut., Middlesex Regt.(T.F.); Capt.,

1909

I.A.R.O., attd. Ist and IIth Gurkha Rifles. (W.) $M$. WWilliamson, G. H. Capt., King's Royal Rifle Corps. M.C. $M$.

Died I2 April I9I7 of wounds received in action

WiLlis, A. G. DE L. Lieut., R.N.V.R. (Howe Bn., R.N.D.); Capt., R.G.A. (W.)

WILLS, A. S. Capt., I 8th Hussars (R. of O.)

WILLS, J. P. Lieut., R.F.A. (W.) M.C.

WWillson, F. J. Lieut., Sappers and Miners, Indian Army

Died 10 Fan. 1917 of wounds received in action

Wilson, Rev. H. Sergt., King's Royal Rifle Corps

WILson, H. G. Lieut.-Col., Lincolnshire Regt.(T.F.) (W.) D.S.O. T.D. $M 2$.

WWILson, H. V. Lieut., Hampshire Regt.

Died 15 Dec. I9I9 of wounds received in action in the

Struma Valley 30 Sept. 1916

Wilson, L. E. Lieut., Queen's Own (R.W. Kent Regt.,

T.F.); attd. 27th Punjabis, Indian Army

\#WILSON, W. R. Lieut., R.A.M.C.

Died I 2 fuly 1916 of wounds received in action I I Fuly 1916

Wiltshire, H. G. Capt., R.A.M.C.

Wimberley, D.N. Capt., Cameron Hdrs.; Major, M.G.C. (W.) M.C.

WINDSOR, F. N. Lieut.-Col., I.M.S.

WINSER, E. F. Capt., Sherwood Foresters (Notts. and Derby Regt., T.F.); Major, N. Staffs. Regt.(T.F.) M.C. $M$.

Winter, A. W. B. Air Mechanic, R.A.F.

WWinter, J. F. Lieut., R.E. (Field Survey Coy.)

Killed in action 28 Oct. 1918

Witherington, A. S. Lieut., R.F.A.(T.F.) (P.) 1908

WOMERSLEY, W. D. 2nd Lieut., D. of Wellington's (W. 1912

Riding Regt.); Lieut., R.E. (Signals, T.F.); attd.

R.A.F. $M$.

Wood, F. E. Pte., R.A.S.C.

Wood, J. I. Pte., R. Fusiliers; Lieut., Border Regt.; I9I3 empld. War Office. (W 2.)

Woods, D. Pte., R.A.M.C.

Woolf, A. E. M. Capt., R.A.M.C. 
Wordley, E. Capt., R.A.M.C. M.C.

Worley, N. A. Lieut., Malay Vol. Force

Worthington, E. H. B. S. African Force

1910

1890

WWorthington, R. F. Capt., Gloucestershire Regt.(T.F.)

1900

Died 4 May I9I7 of wounds received in action 7 April I9I7

Wright, C. S. E. Major, R.A.M.C. M 2. French Croix 1902 de Guerre

Wright, H. L. Major, King's (Liverpool Regt.); empld. $\quad 1897$ R.A.M.C. (W 2.)

Wright, R. B. Lieut., R.F.A.(T.F.)

Wurtzburg, C. E. Capt. and Adjt., King's (Liverpool Regt.); Brigade Major. M.C.

Wyatт, H. E. Lieut., Worcestershire Regt.; empld. I914 O.C.B. (W.) M.C.

Wyer, J. F. W. Capt., R.A.M.C.(T.F.) (W.) 1902

Yeo, K. J. Capt., R.A.M.C.

Young, H. G. K. Capt., R.A.M.C.

Young, Rev. H. P. C.F. 4th Class, R.A.C.D.

1895

1910

Yusuf, Z. M. Lce.-Corpl., R.E. (Signals) 


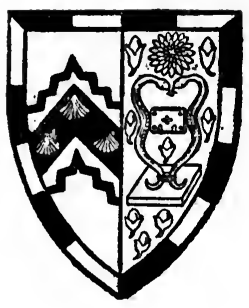

\section{GONVILLE AND CAIUS COLLEGE}

ABERCRombie, G. F. Surgeon Sub-Lieut., R.N.V.R. M. 1914 AberCROMBIE, R. G. Capt., R.A.M.C. (W.) I89I Abrahams, A. 2nd Lieut., Border Regt.; Lieut., M.G.C. [1914] ABRAM, G. S. Major, R.A.M.C.(T.F.) $m$. 1885 ACKROYD, E. Lieut., King's (Liverpool Regt.) and R. 1894 Defence Corps

\#ACKroyd, H. Capt., R.A.M.C. (W.) M.C.

V.C. " For most conspicuous bravery. During recent operations Capt. Ackroyd displayed the greatest gallantry and devotion to duty. Utterly regardless of danger, he worked continuously for many hours up and down and in front of the line tending the wounded and saving the lives of officers and men. In so doing he had to move across the open under heavy machine gun, rifle and shell fire. He carried a wounded officer to a place of safety under very heavy fire. On another occasion he went some way in front of our advanced line and brought in a wounded man under continuous rifle and machine gun fire. His heroism was the means of saving many lives, and provided a magnificent example of courage, cheerfulness and determination to the fighting men in whose midst he was carrying out his splendid work. This gallant officer has since been killed in action."-Supplement to The London Gazette, 6 Sept. 1917.

Killed in action I 1 Aug. 1917

Adams, J. W. Lieut., Singapore Field Ambulance 1903 Aeron-Thomas, G. E. Capt., R.F.A.; Staff Capt. $M_{4}$. 1904 Ainsley, A. C. Capt., R.A.M.C.(T.F.) (W.) M.C. 1909 AINSLIE, M. A. Instructor Cdr., R.N. 1888 AldERson, G. G. Capt., R.A.M.C. 1903 
WAldrich, C. P. G. 2nd Lieut.. R. Fusiliers

Killed in action 7 Oct. 1916

Alexander, E. Hon. Capt., R. Marines

Alexander, W. A. Capt., R.A.M.C.

Allbutt, Rt Hon. Sir T. C., k.c.B. Hon. Colonel, R.A.M.C.(T.F.)

Allen, F. D. C. Capt., R.G.A.; Instructor in Gunnery. I9I I (W.) $M$.

Allen, P. H. C. Lieut., E. Lancs. Regt.

Killed in action 9 May 1915

ANDERSON, A. R. S. Lieut.-Col., I.M.S.

Anderson, W. L. Capt. (S.), R.A.F. D.S.C.

Armstrong, J. C. Major, R.A.S.C.(M.T.) M.C. $M$. ARNELl, O. R. Lieut., R.E.

1905

1899

I855

1909

1879

I9I I

1906

1907

\#ARnot, D. W. Lieut., R. Warwickshire Regt. (W.)

Killed in action 3 Sept. I9I6

AAron, F. A. Lieut., S. Lancs. Regt.

Killed in action 23 Aug. 1918

Arrowsmith, Rev. W. G. C.F. 4th Class, R.A.C.D.

Ascoli, G. H. D. Lieut., 2nd Dragoon Gds. (Queen's

Bays); attd. R.E. (Signals). (W.) M.C.

Aserman, C. Lieut., R.G.A. M.C.

1903

1906

Ashcroft, A. H. Major, S. Staffs. Regt. D.S.O. M 3. 1906

Officer, Order of the Crown of Italy

AsHCROFT, J. M. Lieut., I.A.R.O., attd. rgth Lancers

Ashcroft, R. L. Capt. and Adjt., King's Own (R. Lancaster Regt.) M.C.

AAshCRoft, W. Lieut. and Adjt., King's (Liverpool Regt.)

(W.)

Killed in action 22 March 1918

Aston, R. L. Lieut., Tonbridge School O.T.C.

Atherton, T. J., C.B. Bt. Colonel, Res. Regt. of Cavalry

and Labour Corps. C.M.G. $M 2$.

Atkin, C. S. Lieut., R.A.M.C.

Atkin, E. E. Capt., R.A.M.C.

1908

1906

I9I 5

1902

I9II

1900

Atkins, B. S. Capt., I Ith Rajputs, Indian Army; attd. R.F.C. (W.) (P.) $M$.

Atkinson, E. W. Capt., R.A.M.C.

I 888

1875

1906

1899

1903

ATtwood, H. C. Lieut., R.A.M.C.

1900

AUDRA, E. Lieut., 279me Regt., French Army, and 1905 1908

French Mission with British Third Army. M.C. French Croix de Guerre Avent, Rev. E. C.F. 3 rd Class, R.A.C.D. $M$. Avent, M. Capt., R.A.M.C. (W.) 
BACHe, H. G. Pte., Lincolnshire Regt.; 2nd Lieut., Lancs. 1908 Fus.

Killed in action 15 Feb. 1916

BACK, G. A. Capt., R.A.M.C.

ABACK, H. A. W. Chaplain, R.N.

Accidentally killed on H.M.S. Vanguard 9 fuly 1917

Bagnall-Wild, R. K. Brig.-Gen., R.A.F. (Aircraft Pro- 1864 duction Dept.) C.M.G.

WBailey, C. M. Pte., R. Fusiliers; 2nd Lieut. (A.), R.F.C. I9I3

Killed in action 3 Aug. I9I7

BaIley, T. B. Capt., R.A.M.C.

BAIRD, R. F. Lieut.-Col., I.M.S.

1910

1909

BAKER, F. R. C.S.M., Transvaal Scottish and $4^{\text {th }}$ S. 1897 African Infy. (W.) (P.)

Baker, S. Lieut., The Buffs (E. Kent Regt., T.F.); 1909 Capt. (A.), R.A.F. $M$.

Balfour, A., C.M.g. Lieut.-Col., R.A.M.C. C.B. $M . \quad 1896$

BALFour-Browne, W. A. F. Capt., R.A.M.C. (Sanitary *I9I3 Service, T.F.)

HBannatyne, E. J. Lieut., Igth Hussars; Major (A.), I9ro R.F.C. D.S.O. $M$.

Died I I Sept. I9I7 of injuries received while flying 30 Aug. 19I7

BARBER, P. E. Major, R.A.M.C.

BarClaY, E. D. Lieut., Worcestershire Regt. (W.) M.C. I9I3

BARKER, L. E. H. R. Capt., R.A.M.C.(T.F.)

BARKER, R. W. Capt., Unattd. List, Indian Army

BarnetT, R. O. Capt., London Regt. (Kensington Bn.) 1896

BarnetT, S. H. Lieut., Highland L.I. (W.)

BarRaclough, J. N. Capt., King's Own (Yorkshire L.I.) Igog and Spec. List, attd. Egyptian Army

BarretT, R. S. Hon. Capt., R.E. (Signals, T.F. Res.) 1902

BARrington, F. E. P. Capt. (A.), R.A.F.

Barris, J. D. Capt., R.A.M.C.

I9II

1898

Barron, G. D. Lieut., Connaught Rangers I9Io

BARRY, Sir E., Bart. Lieut.-Col., Berkshire Yeo. (T.F. I88I Res.)

BartletT, H. S. Lieut., R.F.A.; attd. R.E. M.C. 1906

Batten, W. D. G. Capt., 3rd Gurkha Rifles, Indian 1909

Army. Order of El Nahda, 3 rd Class (Hedjaz)

Batterham, D. J. Capt., R.A.M.C.

BatTy-Smith, S. H. Capt., Loyal N. Lancs. Regt. (W.) I9I2

BAYER, S. F. Pte., R.A.S.C.(M.T.) 
Baynes, F. W. W. United Provinces Horse, Indian De- 1908 fence Force

*Beale, C. W. Pte., R. Fusiliers (P. S. Bn.); Lieut., R. I9I I Sussex Regt.; attd. T.M.B.

Killed in action 3 March r9I6

Beamish, R. DE B. Major, R.G.A. 1896

Bean, C. V. Lieut., Devon Regt.

Beattie, L. H. Capt. and Adjt., R.F.A.

1916 1898

BeCKetT, C. M. Lieut., R.E. (Signals) 1912

WBEDFoRD, S. H. 2nd Lieut., R. Berkshire Regt. (W.) 1907 Killed in action $\mathrm{r}$ Fuly $19 \mathrm{r} 6$

ZBeECH, J. Lieut., S. Staffs. Regt.; Capt., R.E. (W.) 1906 Killed in action 12 May 1918

WBEER, H. O. 2nd Lieut., Queen's Own (R.W. Kent Regt.) [1914]

Killed in action near Hulluch 26 Sept. Igr 5

BegG, M. G. Capt., Rifle Brigade; Lieut. (A.), R.A.F. 1914 (P.) M.C. and Bar

Belfield, S. St G. C. Major (K.B.), R.A.F. 1906 WBELL, J. D. Lieut., R.F.A.

Died 30 Oct. 1918 of influenza

BENEST, E. E. Sub-Lieut., R.N.V.R. 1906

BennetT, E. K. Pte., Worcestershire Regt.; 2nd Lieut., 1914 Spec. List (Intelligence)

WBennetT, H. R. Capt., King's (Liverpool Regt.) (W.) M. $\quad$ I912 Killed in action 23 March ror 8

Benson, A. H. Major, R.A.M.C.

1882

Died 24 Sept. 1916

Bentall, C. E. Capt., R.A.S.C.(T.F.) $M$. ABerry, A. J. Pte., R. Fusiliers (P. S. Bn.); and Lieut., [1914] Lancs. Fus.(T.F.) (W.)

Killed in action 21 Aug. 19r6

BERRY, H. V. Lieut., R.A.S.C.; attd. Somerset L.I. (W.) rgro

BERRY, W. L. Surgeon Lieut., R.N.

Besant, G. B. Lieut., Somerset L.I. $M$. 1913

Besly, E. M. Capt., R.E. (P.) M. Belgian Croix de 1907

Guerre

Bevan, J. M. Capt., R.F.A.; Staff Capt. M.C.

\#BEwley, E. N. 2nd Lieut., Sherwood Foresters (Notts. 1903 and Derby Regt., T.F.)

Killed in action 26 June 1917

BICKERDIKe, R. B. Major, R.F.A.(T.F.) D.S.O. M $M_{3 .} 1902$ BICKFORD-Smith, W. N. Capt., D. of Cornwall's L.I. 1900 (T.F.)

Bigland, A. D. Capt., R.A.M.C. 
Billing, E. Capt., R.A.M.C.

Billington, C. M. Capt., E. Yorks. Regt.

1903

Birks, A. H. Capt., R.A.M.C.

I9I3

Black, J. A. Pte., Cambridgeshire Regt.; Lieut., Seaforth Hdrs.; Capt., M.G.C. (W.)

Black, P. Capt., R.A.M.C.

Blackett, G. E. Capt., Durham L.I. (P.)

1903

1912

I 898

1912

BlaikIe, C. J. Capt., R.A.M.C.

1903

Blanchard, N. Pte., London Regt.; 2nd Lieut., R.A.S.C. $\quad$ r898

Blandy, R. Major, IIth Gurkha Rifles, Indian Army. 1902 (W.) M.C. and Bar. M2.

Blew, C. L. Capt., Worcestershire Yeo.; Major, Wilt- 1900 shire Regt. (W.)

WBoARDMan, J. H. Major, Oxford and Bucks. L.I.; attd. I9ro Rifle Brigade. (W.) $M 2$.

Died in German hands 25 April 1918 of wounds received in action.

Bock, E. N. Capt., S. Lancs. Regt.

WBODEY, A. R. Lieut., King's (Liverpool Regt., T.F.)

Killed in action 28 fune I9I6

Bomford, J. F. Capt., Worcestershire Regt. M.C. [I9I4]

Bond, C. E. Capt., R.A.M.C.

Bousfield, J. K. Lieut., R.E.; attd. R.F.C. (P. Escaped 1912 from Germany.) M.C. and Bar

Bousfield, R. B. Pte., 9th Bn., Australian Infy. $\quad 1905$

Bousfield, S. Hon. Major, R.A.M.C.

1889

Bousfield, W. E. Lieut. (T.), R.A.F.

Bower, C. W. 2nd Lieut., Middlesex Regt.(T.F.)

1900

Boyson, H. A. Lieut., ${ }_{5} 5$ th Hussars

Boyson, J. C. Capt., Coldstream Gds. M.C.

[1914]

1912

1906

Bracken, R. J. M. E. Capt., R. Welsh Fus.(T.F.); 1896 Lieut.-Col., M.G.C. M.C. M.

Bradbury, J. B. Lieut.-Col., R.A.M.C.(Ist E. Gen. Hos- 1862 pital, T.F.)

BRADFIELD, L. G. Lieut., R.F.A.

BRAdFIELd, R. Major, R.E. (Electric Lights, T.F.)

1905

1905

1905

BRADLEY, A. S. Capt., R.A.M.C.

I910

BraIlsford, R. W. Capt., R.F.A.(T.F.)

I9I3

Braithwarte, C. F. 2nd Lieut., R.F.A.(T.F.); empld. r898 Ministry of Labour. (W.)

WBraithwaITe, P. P. Capt., I.A.R.O., attd. 36th Cavalry 1899

(Jacob's Horse). (W.)

Killed in action in Palestine 23 Sept. 1918 
Bratton, A. B. Capt., Loyal N. Lancs. Regt. (W.) 1909 D.S.O. M.C. $M 2$.

Brett, J. H. Major, Suffolk Regt. $M 2$.

I9I I

BrewER, F. G. 2nd Lieut., R.G.A.(T.F.)

Briggs Gooderham, E. J. R. See Gooderham, Rev.

E. J.R. B.

Bright, W. A. Capt., R.A.S.C.

HBRISLEY, C. E. Major (A.), R.A.F.

Killed in flying accident 30 fuly 1918

Brittain, E. S. Capt., R.F.A.

1898

1905

Broad, A. M. Lieut., R. Fusiliers; attd. M.G.C.

Killed in action 12 fuly r916

1910

1914

ABroadbent, E. R. Major, 8th (King's R. Irish) Hussars; 1898

D.A.Q.M.G. M.C. $M$.

Died 3 I Oct. r9r 8 of influenza

Broadmead, H. H. Lieut., Somerset L.I.; Staff Capt. 1907

Brockman, E. P. Surgeon Sub-Lieut., R.N.V.R. $M$. I9I 3

Brockman, R. St L. Surgeon Lieut., R.N. 1907

Brockman,W.D. Lieut., Rifle Brigade(T.F.) and M.G.C. I910 (W.)

Bromet, E. Capt., R.A.M.C.(T.F.) $M . \quad 1885$

BROMLEY, L. Capt., R.A.M.C.

1903

Brooke, J. C. Capt. (S.), R.A.F. D.S.G.

Brooke, Z. N. Capt., E. Surrey Regt. and Gen. List * Igo8 (Intelligence)

Broughton, A. D. 2nd Lieut., Res. Regt. of Cavalry; I910 Capt. (A.), R.A.F.

ABrown, A. A. Capt., R.F.A.; Staff Capt. M.C.

Died 4 March 1918 of heart failure

WBRown, A. R. 2nd Lieut., R.F.A.; attd. R.F.C.

Killed in action 6 April r9I7

«Bown, A. W. S. Lieut., Rifle Brigade

Killed in action 18 Aug, 1916

Brown, C. B. Lieut., R. Fusiliers (P. S. Bn.) and Gen. I906 List (Staff Capt.) M.C. M.

Brown, E. B. Capt., Loyal N. Lancs. Regt.

Brown, G. D. Capt., R. Fusiliers

1912

I888

Brown, H. H. Capt., I.M.S.; D.A.D.M.S. $M$ 3. Order 1904 of the Nile, $4^{\text {th Class }}$ (Egypt)

सBrown, H. M. Trooper, Natal Light Horse; 2nd Lieut., 1906

E. Lancs. Regt.

Killed in action in Mesopotamia 9 April r916

«Brown, R. W. Capt., Wiltshire Regt. (W.)

Killed in action 9 April r917 
WBown, T. Sergt., R. Fusiliers (P. S. Bn.); 2nd Lieut., 1908 Norfolk Regt.

Killed in action near Guillemont 4 Sept. I916

सBRown, W. S. Capt., R.A.M.C.

Died while returning from Mesopotamia 27 March 1919

of illness contracted on active service

सBRowne, G. Buckston. Lieut.-Col., R.F.A.(T.F.) (W.) I895 D.S.O. M 3 .

Died 6 fan. I919 of pneumonia contracted on active service

HBrownsword, D. A. Capt., R.A.S.C.(T.F.) and King's

Royal Rifle Corps

Died 25 Dec. I9I7 of wounds received in action on

Passchendaele Ridge

Buckell, E. R. Strathcona's Horse, Canadian Force

Bull, H. C. H. Capt., King's Own (Yorkshire L.I.) (W.) M.G.

BulL, W. E. H. Major, R.A.M.C.(T.F.) M.C.

Bullock, J. C. Paymaster Sub-Lieut., R.N.V.R.

Bullough, E. Lieut., R.N.V.R., empld. Admiralty

Burgess, A. S. Capt., R.A.M.C.

I9I I

Burgess, R. Lieut.-Col., R.A.M.C.(T.F.) (W.) D.S.O. 1899 M.C. M 2. French Croix de Guerre

Burgoyne Johnson, F. W. Capt., Durham L.I.; attd. I9I I M.G.C.

ABuRN, C. J. Lieut., Leicestershire Regt. (W 2.) 1912

Killed in action I Oct. I9I7

Burnell, Rev. E. W. C.F. $4^{\text {th }}$ Class, R.A.C.D. 1898

BurNet, J. R. W. Lieut., Spec. List, empld. Ministry of 1905

National Service

BuRNETT, F. E. Lieut., R.E. (Electric Lights, T.F.)

Burton, E. T. D. Capt., R.F.A. (W.) $M$.

BURWELl,W.K. Lieut.,E.Yorks.Regt.;Capt., R.E. (W.) I IOOI

Bush, F. R. 2nd Lieut., E. Surrey Regt.; Lieut., Rifle 1895

Brigade; Major (Ad.), R.A.F.

Buswell, H. L. F. United States Signal Corps. French 1905

Croix de Guerre

ACAIgER, F. H. S. 2nd Lieut., R.F.A.

Killed in action I I Nov. I9I6

CAIger, G. H. Lieut., R.F.A.(T.F.) (W.)

I9I 5

I9I5

CALDERWOOD, J. L. Capt., King's (Liverpool Regt.) M. 1906

Caldwell, H. G. Lieut., Cameronians (Scottish Rifles). 1914

(W.) 
CAM, W..H. Capt., R.A.M.C.

CAMERON, D. W. Instructor Lieut., R.N.

CAMPBell, G. Sub-Lieut., R.N.V.R. (R.N.D.); Lieut., I9I4 R.M.A.

Campbell, W. H. Major, R.A.S.C. $M$.

CANDY, K. E. Lieut., Lancs. Fus.; Capt., Labour Corps I9I0 (P. of W. Coy.) (W.) M.C.

Cannington, A. S. Capt., R.A.S.C.

Cant, F. V. Capt., R.A.M.C.

Cardwell, C. R. Capt., Dorset Regt.(T.F.)

Cardwell, W. Lieut., Spec. List (Censor's Staff)

CAREY, G. V. Major, Rifle Brigade; Major (Ad.), R.A.F.; S.O. 3, Air Ministry. (W.) M. Belgian Croix de Guerre

CARr, G. D'R. Lieut.-Col., R.A.M.C. (W 3.) M.G. and 1908 two Bars. Brevet Major. $M$.

Carsberg, A. E. Capt., R.A.M.C.

1891

Carter, G. S. Capt., Leicestershire Regt. and R.E. 1912 (W 2.)

Carver, A. E. A. Capt., R.A.M.C.

Castellan, C. E., T.D. Major, R.F.A.(T.F.)

Cave, H. W. Lieut., R.F.A.(T.F.) M.G.

Cave, W. T. C. Capt., London Regt. (P.)

1905

1909

1907

1901

1906

Cave-Moyle, Rev. G. E. P. C.F. 4th Class, R.A.C.D.

सCECIL, C. Pte., R. Fusiliers (P. S. Bn.); 2nd Lieut., R. 1894 Berkshire Regt.(T.F.)

Killed in action on the Somme 16 fuly rar6

4Challoner, A. C. 2nd Lieut., D. of Cornwall's L.I. Killed in action 30 Fuly $19 \mathrm{r} 5$

Chamberlain, D. A. Capt., R.A.M.C.

1902

1894

1910

1901

I 894

ChancelloR, R. B. 2nd Lieut., 5th (R. Irish) Lancers [1914]

\$Chapman, G. M. Lieut., R.A.M.C. French Medal for 1907 saving life at sea

Killed in action 13 May I915

Chappel, B. H. Lieut., D. of Cornwall's L.I.; attd. [1914] ro7th Pioneers, Indian Army; Capt., Norfolk Regt. (W 2.)

Chappel, G. P. Major, R.A.M.C.(T.F.)

1886

Charles, J. R. Capt., R.A.M.C. (2nd S. Gen. Hospital, I89I T.F.)

ACheAPE, J. DE C. Lieut., R. Sussex Regt. Killed in action 3 Sept. 1916

Cheffaud, P. H. M. Capt. 295me Regt., French Army. I910 (W.) 
\#Chessex, R. E. A. Lieut., R.N.V.R.

Accidentally killed on H.M.S. Vanguard 9 Fuly 1917

1896

¥CHILD, G. J. Lieut., King's Own (Yorkshire L.I.)

I9I I

Killed in action 18 April 1915

Chisholm, D. C. H. Major, Worcestershire Regt. (R. 1897 of O.); A.P.M. $M$.

ChitTick, H. S. Capt., R.E.(T.F.) $M$.

Christie, J. F: Capt., Hertfordshire Regt. and Bedford- I9I I shire Regt. (W 2.) M.C.

Christie, S. O. K. Hon. Capt., R. Marines, empld. igi I Admiralty

Churcher, W. D. Lieut., E. Surrey Regt.(T.F.); Capt., 1912 67 th Punjabis, Indian Army

Churchill, A. R. Capt., London Regt. (Cyclist Bn.) and 1902 Middlesex Regt.(T.F.)

Churton, W. A. V. Lieut.-Col., Cheshire Regt.(T.F.) 1895 D.S.O. T.D. $M 3$.

Clarence-Smith, K. W. Lieut., R.F.A.

Clark, G. W. Pte., R. Fusiliers

Clarke, A. G. A. Lieut., R.A.S.C.

Clarke, A. J. M. See Michell-Clarke, A. J.

1909

1896

1890

Clarke, A. V. Colonel, A.M.S. (T.F. Res.); A.D.M.S. $\quad 1889$

Clarke, J. M. See Michell-Clarke, J.

Clarke, J. S. Capt., R.A.M.C.(T.F.) M.C.

Clarke, R. H. Surgeon Sub-Lieut., R.N.V.R.

Clarke, S. H. Capt., R.A.M.C.(T.F.)

Clarkson, J. F. Lieut.-Col., R.G.A. (R. of O.)

1892

1908

1897

1879

Claudet, B. J. A. Capt., R.N.V.R. (R.N.D.); Capt. [I9I4] (Ad.), R.A.F.

Claudet, F. H. B. Pte., H.A.C.; Lieut., R.F.A.(T.F.); 1908 (W.)

Clauder, R. A. O. Pte., H.A.C.; Lieut., 2nd Dragoon [I9I4] Gds. (Queen's Bays). (W.)

Claye, H. Capt., Sherwood Foresters (Notts. and Derby 1907 Regt., T.F.); Hon. Capt. (O.), R.A.F. (W 2.) (P.)

Clayton, E. B. Capt., R.A.M.C. (4th London Gen. 1900 Hospital, T.F.)

Cleminson, F. J. Capt., R.A.M.C.

1897

\#Cloudesley,H. Lieut., The Queen's(R.W.Surrey Regt.) 1902

Killed in action I fuly I916

Coast, W. Corpl., R.A.M.C.

ACoates, A. D. Lieut., London Regt. (R. Fus.)

1916

Killed in action in Gallipoli 27-28 April I9I 5

CoAtes, N. H. Lieut., R.A.S.C.

1912

1910 
Coates, V. H. M. Capt., R.A.M.C. M.C.

Cobbold, A. W. Capt., Remount Service

ACobBold, C. T. 2nd Lieut., R.F.A.

1912

Killed in action 3 Oct. 1916

Cоввоld, J. V. 2nd Lieut., Yorkshire Regt.(T.F.)

* Cochran, F. A. 2nd Lieut., Gordon Hdrs.

Killed in action $25 \mathrm{Sept}$. I915

Cockayne, A. A. Surgeon Lieut., R.N. D.S.C.

Cockin, M. S. Capt., E. Yorks. Regt.; empld. Ministry of Munitions. (W.) $M$.

Cockin, R. P. Lieut., R.A.M.C.

1900

Cocksedge, T. A. B. Major, R.A.V.C.(T.F.) $M$.

Colbeck, E. H. Major, N. Zealand Med. Corps

I 898 T.F.)

Colledge, L. Capt., R.A.M.C.(T.F.) $M$.

Collingwood, B. J. Major, R.A.M.C. O.B.E.

1902

I 884

I 887

Collinson, G. E. C. Lieut., Cameron Hdrs.; attd. [19I4]

R.F.C.

Killed in flying accident I 3 April I9I7

4Collot, T. A. 2nd Lieut., Dorset Regt. and R. Berk- I9I2

shire Regt.

Killed in action I Fuly 1916

Colquhoun, J. C. Capt., Highland L.I. and Gen. List I9r3 (P. and B.T. Staff). (W.) M.B.E. M.

Combe, E. P. Capt., R. Scots; Brigade Major. M.C. and 1909 Bar. $M 2$.

Compton, A. G. W. Capt., R.A.M.C. (W.) M.C. M. 1903

Connolly, B. B., с.в. Colonel, A.M.S.; A.D.M.S. m. i863

ConNop, H. Major, R.A.M.C.(T.F.)

Cooke, W. I. Corpl., R.E. (Signals)

1885

1906

Coombe, R. Major, R.A.M.C.(4th S. Gen.Hospital, T.F.) 1879

CoOper, H. C. Lieut., R.A.S.C.(M.T.) I9I4

CoOper, H. J. Capt., R.A.M.C.

1882

Corbet-Singleton, M. G. Lieut., R. Dublin Fus. and [19I4]

Gen. List

Corbett, R. S. Capt., R.A.M.C.

4Cory, C. W. 2nd Lieut., Suffolk Regt.(T.F.)

1912

Killed in action 12 Aug. I9I 5

Cory, R. F. P. Surgeon Lieut., R.N.

Costigan, R. H. Lieut., R. Warwickshire Regt. (W.) 1909

Costobadie, L. P. Capt., R.A.M.C.

1906

Cotтam, H. C. B. Capt., Hampshire Regt.(T.F.) M.C. 1909

Killed in action 30 Sept. 1918 
Couchman, H. J. Capt., R.A.M.C. $M$.

Coulson, R. N. Major, Cameronians (Scottish Rifles);

1904

I 898

Lieut.-Col., King's Own Scottish Borderers. D.S.O.

$M$ 2. French Croix de Guerre

Cowan-Douglas, H. Capt., Rifle Brigade

ACowie, A. G. Capt., Seaforth Hdrs. (W.)

Died 6 April 1916 of wounds received in action

I9I4

1909

Cox, D. H. Capt., Scottish Horse and R.E. (Signals) 1900

ACox, E. Fleet-Surgeon, R.N.

Accidentally killed on H.M.S. Vanguard 9 fuly r9I7

Cox, R. Capt., R.A.M.C.

1892

Craig, M. Lieut.-Col., R.A.M.C. C.B.E.

Craigie, R. C. Capt., King's (Shropshire L.I.) (W.) [19I4] M.C. and Bar

Crawhall, T. L. Lieut., R.A.M.C.

I9I 2

सCREAN, T. Capt., Northamptonshire Regt.; attd. R.F.C. $\quad$ I899 Killed in flying accident 26 Oct. I9I4

Creed, Rev. J. M. C.F. $4^{\text {th }}$ Class, R.A.C.D.

1908

CREswell, H. E. Capt., R.A.M.C. (W.) M.C. and Bar 1907

CRIMP, G. L. Lieut., R.A.M.C.

1894

Cromie, B. P. 2nd Lieut., London Regt.; Lieut., R.F.A. [19r4] M.C. $M$.

Crompton, J. Capt., roth Jats, Indian Army. O.B.E. 1905

Cross, W. E. Capt., R. Sussex Regt.; attd. Lovat's Scouts 1892

ACrosse, T. L. Capt., Border Regt. (W 2.)

Killed in action 3 fuly r916

Croysdale, J. H. 2nd Lieut., R.A.S.C.

1908

Crozier, J. E. D. 2nd Lieut., Coldstream Gds.

1897

1917

CRUMP, N. E. Lieut., Middlesex Regt. (T.F.); attd. R.E. [19I4]

(Signals)

ACRUNDWELl, A. Lieut., King's (Liverpool Regt.)

Killed in action 23 April r9r8

[1914]

Cullimore, C. Capt., R.F.A.(T.F.) (W.)

Cullimore, James. Lieut., R.F.A.(T.F.)

1909

1904

ACullimore, John. Lieut., Cheshire Regt. $M 2$.

Died 16 April 1916 of wounds received in action

1912

Cullimore, W. Lieut., Cheshire Regt.(T.F.)

1906

Cumming, J. B. 2nd Lieut., Scottish Horse; Lieut. and [19I4] Adjt., R.F.A.(T.F.)

Curme, D. E. Lieut.-Col., R.A.M.C.

Curnow. A. T. Pte., Middlesex Regt.; 2nd Lieut., E. rgr6

Riding of Yorkshire Yeo.

Cursetjee, H. J. M. Capt., I.M.S. (W.) D.S.O. M 2. 1908 Order of the White Eagle, $5_{\text {th Class (Serbia) }}$ 
Curwen, B. M. Major, Rifle Brigade

4Curwen, C. N. Lieut., King's Royal Rifle Corps Killed in action 15 Sept. 1916

DALY,I.DE B. Flt. Lieut., R.N.A.S.; Capt.(Med.), R.A.F. IgI I (W.)

Daly, U. DE B. Capt., R. Dublin Fus.; Major, Spec. List I9I I (Cmdt., Bombing School). (W.) $M$.

Darley, C. B. 2nd Lieut., R.F.A. (W.) (P.) 1906

Daukes, S. H. Capt., R.A.M.C. (Sanitary Service, T.F.) 1897 O.B.E. M.

DAVENPORT, W. H. Lieut.-Col., R. Warwickshire Regt., 1885 attd. R.A.O.C.

Davidson, G. E. Capt., R.A.M.C.

Davies, I. T and Lieut., Oxford'and Bucks. I. Killed in action 22 Fune 1915

WDavies, J. R. 2nd Lieut., London Regt.

Died 28 Nov. I9I7 of wounds received in action

1896

1913

1899

Davies, R. E. L. Lieut. (A. and S.), R.A.F. D.F.C. M. 1913

WDavis, C. J. B. Capt., R.E.(T.F.)

Died 29 Sept. 1917 of wounds received in action 25 Sept.

1913 1917

Davis, F. M. Capt., London Regt. (W 2.) (P.)

Davis, F. P. Capt., R.A.S.C. and M.G.C. (P.)

Davy, G. H. Capt., R.A.M.C. O.B.E. M.

Dawbarn, J. R. Lieut., Northamptonshire Regt. and Bedfordshire Regt.

Dawson, H. H. Major, 75th Carnatic Infy., Indian 1895 Army; D.A.A.G. $M$.

Day, E. C. Major, Remount Service

1892

DAY, G. 2nd Lieut., R.E. M.C.

DAY, W. F. L. Lieut., R.A.M.C.

Dearden, H. Hon. Capt., R.A.M.C. (W 2.)

Deballitul A Pte, French Army; Interpreter with British Exp. Force

Debenham, F. Major, Oxford and Bucks. L.I. (W.) 1913 O.B.E.

Dendy, E. H. Capt., R.A.M.C.

WDevitT, G. F. O. 2nd Lieut., Rifle Brigade 1912 Killed in action at Hooge 30 Fuly 1915

WDickson, A. F. Lieut., I.A.R.O., attd. 14th Murray's 1909 Jat Lancers and 34th Poona Horse

Killed in action in Palestine 14 Fuly 1918 
WDietrichsen, F. C. Capt., Sherwood Foresters (Notts. I90 I and Derby Regt., T.F.) $M$.

Killed in the Irish rebellion I May 1916

Dill,J.F.GoRdon. Surgeon Major, Norfolk Yeo. O.B.E. 1877 T.D. $m$.

Disney, H. A. P. Capt., Cambridgeshire Regt.; 1912 D.A.Q.M.G.; Lieut.-Col. (T.), R.A.F.; S.O. I. $M$. $m$ 2. Officer, Order of the Crown of Italy

WDixon, T. H. Capt., Manchester Regt. (W.) M.C. I9I3 Killed in action 25 Aug. 1918

DoAk, J. K. R. Lieut., Cameron Hdrs. (W 3.)

DoBIE, J. N. Major, R.A.M.C.

1909 1885

WDobIE, W. M. Lieut., Queen's Own (R. W. Kent Regt.) [1914] Killed in action 9 April rgr6

Dobson, D. R. 2nd Lieut., R.A.S.C.

Dobson, G. M. B. Capt. (T.), R.A.F. $m$.

Dobson, H. D. Lieut., R.A.S.C.

Donaldson, E. A. Chaplain, R.N.

WDonaldson, G. B. Capt., R. Warwickshire Regt.(T.F.)

Died in German hands 19 Fuly 1916 of wounds received in action

DonNell, J. H. Capt., R.A.M.C.(T.F.)

DracuP, A. H. 2nd Lieut., I.A.R.O.

DREWRY, G. H. Lieut., R.F.A.(T.F.)

Drummond, J. M. F. Lieut., Highland L.I.

DruRY, A. N. Major, R.A.M.C.(T.F.); D.A.D.M.S.

1898

Dryland, G. W. Lieut., R.A.M.C.

Duckworth, L. Capt., Loyal N. Lancs. Regt.(T.F.); I9IO

Major, King's (Liverpool Regt., T.F.) $M$.

Duddell, A. G. Lieut., R. Warwickshire Regt.; Capt., $\quad$ I912 Spec. List (Bde. Bombing Officer). (W.)

DukA, A. T., D.s.o. Major, R.A.M.C. $M$.

Duke, H. L. Capt., Spec. List, empld. E. African Force

Duncan, J. H. Major, R.A.S.C. $M$.

सDunlop, J. G. M. 2nd Lieut., R. Dublin Fus.

Killed in action at Clary 27 Aug. I914

Dunlop, W. N. U. Lieut., London Regt. (Kensington 1912 Bn.); Capt. and Adjt., M.G.C. $M$.

Dunn, T. W. N. Capt., R.A.M.C.

$\begin{array}{lll}\text { Dunn, T. W. N. Capt., R.A.M.C. } & & 1898 \\ \text { Durst, A. Capt., R.E.(T.F.); attd. Labour Corps. } & M . & \text { I894 }\end{array}$

Dyas, G. E. Capt., R.A.M.C. M.C.

Dykes, K. Major, Queen's Own (R. W. Kent Regt.); 1907

1909

1892

1912

1896

1907

1907

1900

1909

1900

1884

1902

1905

1905

I 905

I9I 3

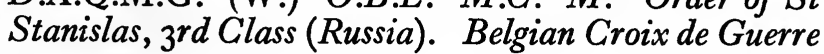
c. U.w.L. 
EALAND, V. F. Lieut., R.F.A.

EAmes, W. L'E., C.B., v.D. Bt. Colonel, R.A.M.C. C.B.E. $\quad 1882$

$M$ 2. Commander, Military Order of Avis (Portugal)

Ebden, J. W. Capt., I.A.R.O.; Staff Capt.

EBDEN,W.S. 2nd Lieut., King's (Shropshire L.I.) (W 2.). 1906 (P.)

Eccles, R. Bt. Colonel, Durham L.I. and King's (Liver- 1872 pool Regt.)

EDGEWORTH, F. H. Major, R.A.M.C. (2nd S. Gen. 1883 Hospital, T.F.)

Edwards, C. G. Capt., R.A.S.C. (W.) $m$.

I9I I

EISDELl, H. M. Lieut., R.N.V.R.

$\begin{array}{ll}\text { Elam, J. E. 2nd Lieut., Monmouthshire Regt. } & \text { I9I4 } \\ \text { Eley, H. G. Lieut., E. Surrey Regt.; Major, R.E. (W.) } & \text { I906 }\end{array}$ M.B.E.

Ellison, H. B. Capt., R.A.M.C.

Elton, H. B. Capt., R.A.M.C.

Eltringham, H. C. 2nd Lieut., D. of Wellington's (W. Riding Regt.); attd. R.F.C.

EElworthy, T. 2nd Lieut., R.E.; Lieut., King's Own I9I

(R. Lancaster Regt.) $M$.

Killed in action 3 May r9I7

Elworthy, W. R. Lieut., R.G.A.

EMrYs-Jones, M. F. Capt., R.A.M.C.

ENGland, H. Capt., R.A.M.C.(T.F.)

Evans, M. W. H. Flt. Lieut., R.N.A.S.

ZEvatr, J. M. Capt., R.A.M.C.

Killed in action $2 \mathrm{I}$ March $\mathrm{I} 9 \mathrm{I} 8$

Eve, H. F. H. Capt. and Adjt., R.A.S.C. M.C. I9II

Everett, Rev. B. C. S. C.F. $4^{\text {th }}$ Class, R.A.C.D. M. 1894

EveritT, H. L. Capt. (A.), R.A.F. A.F.C. I9Io

Evershed, Rev. F. T. P. C.F. 4th Class, R.A.C.D. $\quad$ I888

Ewens, B. C. Capt., R.A.M.C. Order of St Sava, 5th 1907 Class (Serbia)

Ewing, W. T. Lieut.-Col., R. Scots (T.F.) D.S.O. and 1906 Bar. M. French Croix de Guerre

EYrE, L. B. Lieut., E. Surrey Regt.(T.F.)

FAGAN, C. H. J. Major, R.A.M.C.(T.F.) O.B.E. $\quad 1897$

FAIRBROTHER, J. Surgeon Lieut., R.N. 1908

Fawcett, R. W. Surgeon Prob., R.N.V.R.; 2nd Lieut., r910

S. Staffs. Regt.

Died 26 Sept. 1915 of wounds received in action

Fearfield, C. J. Major, R.E. M.C. M.

I910 
Felton, D. G. W. and Lieut., Hereford School O.T.C. $\quad$ I905 Fenwick, J. S. Lieut., Oxford and Bucks. L.I. (W.) I9I2 FerRand, J. B. P. Major (S.), R.A.F. (P.) D.S.O. [19I4] Ferrers, E. B. Major, Cameronians (Scottish Rifles); 1897

Lieut.-Col., Spec. List (Cmdt., School of Instruction).

(W.) D.S.O. $M 2$.

FeUERHEERD, L. M. R. Fusiliers

FFielding, E. F. Lieut., W. African Frontier Force

Killed in action in the Cameroons 24 Oct. I9I 5

FinLaY, R. V. K. Lieut., R.N.V.R.

r 894

1898

Fing (Artists Rifles);

Devon Regt. (W.) $M$.

Died I7 May I9I6 of wounds received in action

Fitton, R. Capt., R.F.A.; Lieut. (O.), R.A.F. (W.) 1909 M.C. $M$.

FitzGerald, H. S. Lieut., Northumberland Fus.; attd. 1902

W. African Frontier Force. (W.)

Fiтzjohn, T. Lieut.-Col., Worcestershire Regt. (W.) 1898

D.S.O. and Bar. M3. French Croix de Guerre

Flemming, M. G. Corpl., R.E. (Signals); Lieut., R.G.A. I9I I

Fletcher, A. B. Capt., Ioth Jats, Indian Army $M$ 2. $\quad 1912$

Fletcher, W. Capt., R.A.M.C.

Forman, D. P. Capt., Sherwood Foresters (Notts. and 1906

Derby Regt., T.F.); attd. R.E. (Signals). $M$.

Formoy, R. R. Pte., London Regt. (Artists Rifles)

Forster, C. M. Capt., R.A.M.C. (W.) M. Order of 1905

St Sava, $5^{\text {th }}$ Class (Serbia)

FForster-Brown, J. C. Lieut., Rifle Brigade

Died 27 Aug. 1916 of wounds received in action

ForsYTH, G. Lieut., London Regt.(Q.V.R.); A.D.C. $\quad 1899$

ForsYTh, L. W. Capt., R.A.M.C.

Fowler, Sir J. K., K.C.v.o. Colonel, A.M.S. C.M.G. M. 1876

Fowler, T. G. Sub-Lieut., R.N.V.R. (Coastal Motor- I9I0 boat Service)

Fox, H. M. Capt., R.A.S.C.

Francis, G. L. B. Capt., Monmouthshire Regt.; Major, 1904 R.A.S.C.(T.F.)

Franklin, C. D. G. Lieut., Rifle Brigade and M.G.C. 1903 (Motor)

Fraser, D. H. Capt., R.A.M.C.; attd. R.A.F. M.C. $\quad{ }^{1896}$ M.B.E. French Médaille d'Honneur and Médaille des Epidémies

WFreEman, G. C. Capt., R. Berkshire Regt.

Killed in action I Oct. 1916 
French, E. N. Major, Lincolnshire Regt.; Lieut.-Col., $\quad$ I897 London Regt. (W.)

Frost, T. F. C. Lieut., I 5 th Hussars. (W.)

FRUHE-SutCliffe, R. Major, R.E.(T.F.) $m$.

WFRY, J. D. Lieut., London Regt. (R. Fus.)

Killed in action 15 Sept. 1916

Fuchs, H. M. See Fox, H. M.

Fyffe, W. K. Major, N. Zealand Med. Corps. (W.) 1882

GABB, J. D. Capt., The Queen's (R. W. Surrey Regt., I9ro T.F.); attd. 74th Punjabis, Indian Army. $M$.

Galbraith, H. G. Lieut., R.G.A. (W.)

Gamlen, R. L. Capt., R.A.M.C.

I9I3

1909

I913

Gardiner, A. L. 2nd Lieut., Norfolk Regt.; Lieut., I.A.R.O., attd. 23rd Cavalry

GaRdiner, H. H. Capt., R.F.A.; A.D.C. (W 3.) M.C. I9ro $M 2$.

Gardner, E. Capt., R.A.M.C. $M$.

GARDNER, E. A. Lieut.-Cdr., R.N.V.R. Officer, Order I880 of the Redeemer (Greece)

Gardner, R. C. B. Lieut., R.G.A.; attd. Gen.Staff. $M .1908$ Garnsey, E. Major, H.A.C. and Gen. Staff; A.P.M. M. 1898 French Croix de Guerre

Gaskell, J. F. Capt., R.A.M.C.(T.F.) $M$. 1897

GATER, B. A. R. Lieut., R. Fusiliers and Gen. List. (W.) I9I4

Gavin, N. D. I. Lieut., King's Own Scottish Borderers; I9I3 Lieut. (A.), R.A.F.

GAYe, A. W. Lieut., R.A.M.C.

Gell, W. C. C. Lieut.-Col., R. Warwickshire Regt.

(T.F.) (W.) D.S.O. and Bar. M.C. M 3. Italian Silver Medal for Military Valour

AGeorge, A. K. D. Lieut., Dorset Regt.

Died I 4 Sept. 1914 of wouna's received in action

Gerard, R. J. L. Lieut., Gordon Hdrs. (T.F.); Capt. I9I3 (Ad.), R.A.F.

\&Getty, J. H. Capt., W. Yorks. Regt. $M$.

Killed in action 3 May I9I7

Gibson, A. M.. Lieut., R.G.A. and Spec. List (Intelli- I9I4 gence). Italian Croce di Guerra

Gillies, H. D. Major, R.A.M.C. C.B.E. m. I90I

GimbletT, C. L. Surgeon Lieut., R.N.

Gimlette, C. H. M. Surgeon Lieut., R.N.

Gingell, W. C. Lieut., R.A.S.C.(M.T.) 1908

Glanville, W.J. Major,R.A.O.C.;I.O.M. IstClass. $M$. I90I 
Glendinning, H. W. Capt., Cheshire Regt.(T.F.) (W.) [I9I4] WGLOSTER, H. C. Lieut., Gordon Hdrs.(T.F.)

Killed in action at Neuve Chapelle $\mathrm{I} 3$ March $\mathrm{191} 5$

Glover, G. H. Lieut., 2oth Hussars. (W.)

Glover, H. P. McC. Major, R.F.A. (W 2.) M.C.

GLYN, J. P. and Lieut., N. Irish Horse; Lieut., R. 1905 Horse Gds.

Goodall, C. C. Hon. Capt., R.A.M.C.

WGoodchild, E. L. Pte., Norfolk Regt.

Killed in action 28 April I9r6

I9IO

AGooderham, Rev. E. J. R. B. 2nd Lieut., R. Irish Regt. 1908 and M.G.C.

Killed in action $\mathrm{I} 3 \mathrm{Dec}$. $19 \mathrm{I} 6$

GoodhuE, F.W.J. Lce.-Corpl., R. Fusiliers (Sportsman's $\quad \mathrm{I}^{8} 85$ Bn.)

Goodwin, E. St G. S. Surgeon Lieut., R.N. $M$.

Gordon, A. Capt., Border Regt. and Tank Corps. (W.) [1914]

सGoRdon, G. Lieut., I2th Lancers

Killed in action at Ypres 30 April 191 5

सGoRdon,H. Lieut., King's Own Scottish Borderers. (W.) [I9I4]

Died 19 Dec. I91 5 of wounds received in action in

Gallipoli

Gosse,A.H. Major,R.A.M.C.(T.F.) Brevet Major. M4. 1903

Gosse, R. W. Lieut., Northamptonshire Regt.(T.F.); I9IO Lieut. (A.), R.A.F.

Gosset, A. C. V. Lieut., Oxford and Bucks. L.I. (W 2.) I9I3

Gostling, E. V. Colonel, R.A.M.C.(T.F.); A.D.M.S. I89I (W.) D.S.O. M. m.

\#Gotch, D. H. Pte., London Regt. (Artists Rifles); 2nd I9ro Lieut., Worcestershire Regt.

Killed in action at Neuve Chapelle I I March I9I 5

Gould, L. McL. Sergt., 2nd Central Ontario Regt., I897

Canadian Force. Belgian Croix de Guerre

Goullet, A. S. C. Lieut., Middlesex Regt.

Graham, Rev. C. Chaplain, R.N. O.B.E.

Graham, L. A. Capt., R.A.M.C.

Graham, W. P. G. Lieut.-Col., R.A.M.C.

Grantham-Hill, C. Lieut., 3rd Hussars. (W.)

Gray, W.A. 2nd Lieut., Rangoon Bn., Indian Defence 1904

Force

GrazEBROOK,O.F. Lieut.,Worcestershire Regt.(T.F.Res.) I904

Greenberg, A. W. See Greenhill, A. W.

Greene, G. E. 2nd Lieut., Natal Carabineers. (W.) 1899

Greenhill, A. W. Pte., R.A.S.C.(M.T.) 
Greenish, F. H. S. Surgeon Lieut., R.N.

Gretton, G. F. Major, 7th Hariana Lancers, Indian 1896 Army

Griffith, C. Capt., W. Yorks. Regt. (W 2.)

GRIFFITH, F. L. 2nd Lieut., R.F.A.; empld. Ministry of 1915 Labour. (W.)

Griffith-Jones, C. Surgeon Sub-Lieut., R.N.V.R.

Griffiths, P. D. Pte., London Regt. (Artists Rifles)

Griffiths, Rev. T. T. Chaplain and Instructor Cdr., R.N.

Griffiths, W. L. Capt., R.A.M.C.(T.F.)

AGrimwade, E. E. 2nd Lieut., Lancs. Fus.

Killed in action 17 Sept. I9I6

*Growse, R. H. Major, R.A.S.C.

Died 12 Feb. 1919 of pneumonia contracted on active service

GrummitT, C. C. Capt., R.A.M.C.(T.F.)

Grylls, E. A. H. Capt., R.F.A. M.

Guinness, J. F. G. Lieut., R.F.A.; empld. Ordnance College. (W.) $M$.

FGulliland, J. H. Capt., Essex Regt. (W.)

Died 18 fuly 1916 of wounds received in action

GunTER, Rev. W. H. C.F. 4th Class, R.A.C.D.

GuRNEY, K. T. C. Lieut., R.F.A. and Spec. List (Intelligence)

Hadfield, P. H. Capt., R.A.M.C.

HaIGH, B. Capt., R.A.M.C.

Haines, R. T. M. Capt., R.G.A.

Hale, J. Surgeon Lieut., R.N.

1913

1917

1882

1890

1906

1905

Hale, R. E. V. Capt., R.A.M.C.; Capt. (Med.), R.A.F. $\quad$ I897

Hall, A. J. Lieut.-Col., R.A.M.C. (3rd N. Gen. Hos- 1884 pital, T.F.)

WHall, E. W. Capt., Lincolnshire Regt. M.B.E.

Killed in action 26 Sept. 1917

Halliwell, A. C. Surgeon Sub-Lieut., R.N.V.R.

Halliwell, C. C. 2nd Lieut., Welsh Gds. and Gds. I9I7 M.G. Regt.

Hamblin, E. C. C. Lieut., R.E.

Hammond Searle, A. C. Lieut.-Col., R.A.M.C.; D.A.D.M.S.

1888

I9I I

1908

I9II

1904

1914

1887

I897

1910

I9II

1912

1914

917

1910

I 899

Hardie, E. E. 2nd Lieut., Gordon Hdrs. (W.)

1912

HaRding, E. W. Lieut., R.N.V.R.

1902

HARDY, F. K. Major, York and Lancaster Regt.; empld. I900 Ministry of Labour. D.S.O. M 2 . 
सHARDY, J. 2nd Lieut., R.A.S.C.(M.T.)

1908

Died 2I Oct. I9I 8 of pneumonia

Hare, A. C. Lieut., R.E. (Signals, T.F.)

HaRe, Rev. S. H. C.F. $4^{\text {th }}$ Class, R.A.C.D.

I910

Hargreaves, R. Capt., R.A.M.C.(T.F.)

1904

1908

HARPER, E. R. Capt. and Adjt., Monmouthshire Regt. 1904 M.C.

HARPER-Smith, G. H. Capt., R.A.M.C.(T.F.)

HarREY, C. O. 2nd Lieut., R.A.S.C.; Lieut., Gen. List (Intelligence). M 2. French Croix de Guerre

I 896

I9I3

HarRis, Rt Hon. F. Leverton, M.P. Hon. Cdr., R.N.V.R. I88I

Harris, R. T. Lieut., R. North Devon Yeo. (T.F. Res.) 1883

HARRIS, W. J. Capt., R.A.M.C.

Harrison, C. E. Capt., R.A.S.C.

1888

I 888

Harrison, C. F. 2nd Lieut., K. Edward's Horse; Capt., I9I I Dorset Regt.

HarRison, G. A. Capt., R.A.M.C.

Harrison, L. K. Colonel, R.A.M.C. (5th N. Gen. I890 Hospital, T.F.) C.B.E. Brevet Colonel. $m 2$.

HARRISON, T. S. Surgeon Lieut., R.N.

HART, H. E. Major, R.F.A.; A.D.C. (W 3.) M.C. I9II

HarT-Smith, H. M. Capt., R.A.M.C. $M$.

WHARTRE, C. 2nd Lieut., R.G.A.

Killed in action 29 May 1918

Hartree, K. Capt., Queen's Own (R. W. Kent Regt., 1908 T.F.)

Harwood, A. H. F. Capt., Essex Regt.(T.F.) and I9I I M.G.C.

Hatch, K. 2nd Lieut., R.A.S.C.; Lieut., 34th Poona [I9I4] Horse, Indian Army

Hatch, M. L. Lieut., R.F.A.

WHATHORN, C. N. 2nd Lieut., Loyal N. Lancs. Regt. I9I3 Killed in action ro Aug. I91 5

HathoRn, W. B. Lieut., 6th Dragoon Gds. (Carabiniers). I9I2 (W.) $M$.

Hatten, G. Pte., Essex Regt. and Labour Corps 1902

Hatton, G. A. L. Capt., Queen's Own (R. W. Kent 1907 Regt.) (P.)

Haultain, W. F. T. Capt., R.A.M.C. O.B.E. M.G. M. I9I I

HAwker, G. P. D. Major, R.A.M.C.(T.F.) M. m. 1894

HAWkeR, H. L. L. Sergt.-Instructor, R.E. I9I4

Hawkins, C. H. G. W. Lieut., R.A.S.C.

Hawley, A. T. Surgeon Sub-Lieut., R.N.V.R.

1908

Haydon, A. D. Capt., R.A.M.C. 
HaYdon, T.H. Colonel, R.A.M.C.(T.F.) Brevet Colonel. 1884 $m$.

Hayward, L. G. Lieut., Suffolk Regt.(T.F.); attd. Lon- I913 don Regt.

WHayward, M. C. Capt., R.A.M.C.(T.F.) (W.) $\quad 1889$

Died 23 Aug. 1916 of septic pneumonia

HAZEldine, D. Lieut., R.E. (W.)

I910

HeAld, C. B. Lieut.-Col., R.A.M.C.; Lieut.-Col.(Med.), ' I902 R.A.F.; S.O. I, Air Ministry. C.B.E.

Heald, W. M. 2nd Lieut., Suffolk Regt.; Capt., 1904 R.A.O.C. $m$.

Heard, Rev. A. St J. C.F. 4th Class, R.A.C.D. M. 1904

HeNDley, H. J. H. Lieut., R.F.A.(T.F.) (W.)

Herbert, P. L. W. Colonel, Sherwood Foresters (Notts. Igor and Derby Regt.); Brig.-Gen., R.A.F. C.M.G. Brevet Major. M 4. m. Order of St Anne, $3^{\text {rd Class, }}$ with swords (Russia). Grand Commander, Order of St Saviour (Greece). Order of the Nile, 3 rd Class (Egypt)

HeRbERT, S. Capt., R.A.M.C.

Hereford, J. C. Lieut., R.N.V.R.

WHermann, J. Pte., London Regt. (Q.V.R.)

Killed in action 25 Sept. 1916

Herriot, D. R. Capt., Northumberland Fus. (W 2.) I9I2

Hesketh-Wright, J. H. Lieut., Spec. List, empld. Min- I892 istry of National Service

Heurtley, W. A. Capt., E. Lancs. Regt.; Major, Gen. 1902 List (Dep. Governor of Military Prison). O.B.E. $M 2$.

HewetT, F. S., M.v.o. Surgeon Lieut., R.N.V.R.

Hewitt, R. C. Capt., R.A.M.C.

Hey, C. E. M. Capt., R.A.M.C.(T.F.)

Hickman, W. C. 2nd Lieut., R.F.A.

Killed in action I fuly I9I6

Hicks, E. H. Hon. Major, R.A.M.C. M. Chevalier Order of Leopold (Belgium)

Higgins, S. J. Lieut.-Col., R.A.M.C.

Higgins, W. R. Capt., R.A.M.C.(T.F.)

1884

1905

r 884

Higginson, G. Capt., R.A.M.C.(T.F.)

1898

1909

1882

1906

Higson, G. H. 2nd Lieut., Manchester Regt. (T.F. Res.)

WHrll, P. A. Capt., S. Wales Borderers (T.F.)

Killed in action 23 April r917

Hill, R. A. P. Major, R.A.M.C.

Hills, A. H. 2nd Lieut., Hampshire Regt.(T.F.)

Killed in action I9 April I9I7 
HilPERN, W. T. H. Lieut., E. Lancs. Regt. (P.) 1909 Hinchcliffe, J. W. Pte., R. Fusiliers; Capt., North- I9I2 amptonshire Regt.; attd. Gloucestershire Regt.

Hindley, N. L. Lieut., Sherwood Foresters (Notts. and I9r3 Derby Regt.); Capt., Gen. Staff. (W 2.) $M$.

Hirst, G. G. Major, I.M.S.; D.A.D.M.S. M. $\quad$ I896

Hirst, J. W. Lieut., D. of Wellington's (W. Riding Regt., I9I4 T.F.) (W.)

Hislop, T. C. A. Capt., Wellington Regt., N. Zealand 1907 Force

HHobson, A. F. Major, R.E. D.S.O. M 2 . Killed in action 28 Aug. I916

WHobSON, L.F. 2nd Lieut., York and Lancaster Regt.(T.F.) [I9I4] Died 12 fuly 1915 of wounds received in action

HoFf, H. G. Lieut., Lincolnshire Regt.(T.F.) (W.) 1908

HOFFMEISTER, C. J. R. Lieut., R.A.M.C.

Holden, E. G. Lieut., Welsh Regt. and M.G.C. (W.) Holden, G. H. R. Capt., R.A.M.C.(T.F.) $m$. WHolland, W. D.A. Lieut., 9th Lancers; Capt., Cavalry, I9 I I Indian Army. (W.)

Died I 3 Aug. 1917 of illness contracted on the Mahsud expedition

Hollis, Rev. W. C.F. $4^{\text {th }}$ Class, R.A.C.D.

Holman, C. C. Capt., R.A.M.C.

WHolman, G. H. W. Lieut., Wiltshire Regt. (W.)

Died 6 fuly 1916 of wounds received in action near Thiepval 5 Fuly ror6

Holmes, G. Capt., R.A.M.C.(T.F.)

Holmes, T. E. Major, R.A.M.C. $m$.

WHoon, G. C. Lce.-Corpl., Middlesex Regt. (P. S. Bn.)

Died 19 Feb. 1916 of wounds received in action $\mathrm{I}_{5} \mathrm{Feb}$. I9I6

Hope, G. M. Lieut., R.N.V.R.

HOPE, S. J. C.P.O., R.N.A.S.

Horner, N. G. Capt., R.A.M.C.

AHorsey, A. M. Surgeon Prob., R.N.V.R.

Killed in action 9 Aug. 1917

HoRSLEY, C. D. Lieut.-Col., 2 rst Lancers; D.A.Q.M.G. 1894 (W.) M.C. M. French Croix de Guerre

Hotblack, G. V. Capt., Welsh Regt. and Gen. List 1909 (Brigade Major). (W.) M.C.

WHowarTH, H. V. Lieut, Devon Regt.(T.F.) (W.) I9I4

Died 2 May 1918 of wounds received in action

Howell, C. G. 2nd Lieut., R.F.A. (W.)

1913 
Howes, A. G. Capt., Remount Service $\quad$ I889

Howse, T. F. Cadet, O.C.B. $\quad 1892$

Hudson, R. J. Lieut., R. Fusiliers; Major (A.), R.A.F. 1905 M.C.

Huelin, E. S. B.S.M., H.A.C.

Hulbert, H. B. Lieut., Worcestershire Regt. (W.) I9I I

Hullah, J. Nagpur Rifles, Indian Defence Force . $\quad 1895$

Humfrey, S. H. G. Lieut., R.F.A.(T.F.) • I9I3

Humphrys, H. E. Capt., R.A.M.C. I90I

Hunkin, Rev. J. W. C.F. 2nd Class, R.A.C.D.; 1906

D.A.C.G. (W.) O.B.E. M.C. and Bar. M2.

Hunter, J. F. S. Capt., R.E. (Tyne Electrical Engineers, I9I3 T.F.) M.B.E. $M$.

Hunter, J. H. 2nd Lieut., Rangoon Bn., Indian Defence 1908 Force

Hutchence, B. L. Capt., R.A.M.C.

Hutchence, W. G. Major, Durham L.I. and S. Staffs. 1903 Regt. (W.)

Iles, J. H. Capt., R.A.M.C.

1896

HILES, J. O. Lieut., S. Staffs. Regt.; attd. R. Welsh Fus. I9I2

Killed in action 25 Sept. I915

IlotT, C. H. T. Capt., R.A.M.C. (W.)

IRVING, W. Lieut., R.A.M.C.

IRwin, A. P. B. Lieut.-Col., E. Surrey Regt. (W 3.) 1906

D.S.O. and two Bars. Brevet Major. $M_{3}$.

W Jackson, A. J. 2nd Lieut., Middlesex Regt.

Killed in action 27 April 1915

JACKSON,H.S. Lieut.-Col., R.F.A. (W 2.) D.S.O. $M_{3} . \quad 1898$

JACOB, A. C. Lieut., R.F.A.

WJAMES, C. K. Major, Border Regt.; Lieut.-Col., W.

Yorks. Regt. (W 2.) D.S.O. and Bar. Brevet Major.

M6.

Killed in action 19 May 1918

JAmES, E. H. Capt., R. North Devon Yeo.; attd. Dorset 1909

Regt. M.C. and Bar

James, Rev. J. A. C.F. $4^{\text {th }}$ Class, R.A.C.D. (W.) $M$. 1912

JaRvis, Rev. F. C.F. 4th Class, R.A.C.D. 1906

JASPER, R. F. T. Lieut., Essex Regt.; empld. Ministry of 1909 Munitions. (W.)

WJennings, F. M. Capt., 8th (King's R. Irish) Hussars 1892

Died I I Nov. I9I 8 of wounds received in action

Jернсотт, C. Capt., R.A.M.C.(T.F.) 
JePhCott, Rev. E. W. C.F. 4th Class, R.A.C.D. 1907 Jepson, Rev. G. C.F. $4^{\text {th }}$ Class, R.A.C.D. I875 JERRAM, R. M. Lieut., Hampshire Regt. and Tank Corps. [19I4] M.C.

JJEUdwine, S. H. Capt., Lincolnshire Regt. (W 2.)

[1914]

Killed in action I fuly 1916

Johnson, F. W. B. See Burgoyne Johnson, F. W.

Johnson, H. C. J. Major, R.A.O.C.; D.A.D.O.S. M. 1902 JoHnson, H. P. Major, R.A.M.C. (R. of O.) 1882

Johnson, L. O. Lieut., King's Own (Yorkshire L.I.) I913

(W 2.)

Johnson, R.E. Lieut., The Queen's (R.W.Surrey Regt.) I9I3 (W 2.)

Johnston, H. B. 2nd Lieut., Suffolk Regt. and Spec. I9I I

List, empld. Sudan Govt.

JoNEs, T. A. Capt., R.A.M.C.

1899

Wones-Bateman, F. Capt., R. Welsh Fus. (W.) $M$.

Killed in action near Englefontaine 4 Nov. 1918

JUPE, H. M. Surgeon Lieut., R.N.

I912

Kaufrmann, A. L. Ensign, United States Naval Res. 1912 Flying Corps

KaY, R. L. Capt., Cheshire Regt. M.C. M.

1913

Keating, J: H. Capt., Cambridgeshire Regt. (W 2.)

KEIR, L. 2nd Lieut., R.G.A.

Kellie, K. H. A. Capt.. R.A.M.C.

Killed in action 25 fune 1916

Kellow, W. Engineers, S. African Force.

$\begin{array}{ll}\text { KeLLOW, W. Engineers, S. African Force. } & 1912 \\ \text { KeLWAY, K. S. Corpl., R.E. (Signals); Lieut., R.A.S.C. } & 1912\end{array}$ (M.T.)

Kempson, Rev. F. C Capt,. R.A.M.C.(T.F.); Capt. $\quad 1886$ (Med.), R.A.F.

WKendall, L. F. W. A. Lieut., Norfolk Regt.; attd. I9II M.G.C. (Cavalry)

Died 22 Nov. 1917 of wounds received in action 21 Nov. 1917

KenNEDY, A. Capt.. I.M.S.

KenNedy, M. W. Lieut., R. Scots Fus.

KENT, H. H. Lieut-Col, Northumberland Fus. [1914]

HKINDER, T. H. Capt., Suffolk Regt.

Killed in action 3 fuly 1916

KING, E. F. H. Capt., R.F.A.(T.F.) (W.)

KING, F. H. Flt. Sub-Lieut., R.N.A.S.

KInG, G. C. Capt., R.A.M.C.(T.F.) $M$.

1903

1893 
WING, S. W. T. 2nd Lieut., Cheshire Regt.(T.F.) Killed in action ro Aug. 1915

KINGSFORD, G. T. Major, R.E.(T.F.) D.S.O. M. 1900

Kingsley-Smith, C. H. Lieut., R. Sussex Regt. I9I4

KNAGGS, R. L. Major, R.A.M.C. (2nd N. Gen. Hospital, I876 T.F.)

KNight, E. F. Major, R.E. (W.) M.C. and Bar

KNIGHT, H. F. Lieut., Spec. List (R.T.O.)

KNox, R. W. Lieut.-Col., I.M.S. D.S.O. M 4. Order

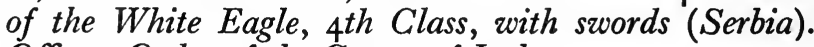
Officer, Order of the Crown of Italy

WKnox, W. Capt., Cameron Hdrs.; attd. R.F.C. Killed in action $20 \mathrm{Feb}$. 1916

ЖКонм, W.A. Pte., R. Fusiliers; 2nd Lieut., E. Lancs. Regt. Killed in action $\mathrm{I}$ fuly 1916

Kon, G. A. R. Lieut., R.A.M.C.; Capt., R.E. $m$.

Koop, G. G. Lieut., R.F.A.

1907

1905

I 890

La Fontaine, J. S. Lieut., R.N.V.R. (W.)

La Fontaine, S. H. Capt., E. African Force. D.S.O. M.C. $M$.

Lake, W. I. Sergt., The Queen's (R. W. Surrey Regt.) and Middlesex Regt.

Landau, H. Capt., R.F.A. and Spec. List. O.B.E.

LANE, H. J. Lieut., R.G.A.; attd. R.E. $M$.

WLANG, H. Lieut., N. Zealand Rifle Brigade

Killed in action 20 fuly I9I6

Langdale, A. H. Lieut., York and Lancaster Regt. M. 1909

WLangdale, H. C. Pte., H.A.C.; Capt., R. Sussex Regt. I9I2 (W 2.)

Killed in action 26 Sept. 1917

LANGdon, H.C.T. Capt.,R.A.M.C.; Lieut.-Col.(Med.), $\quad 1890$ R.A.F. O.B.E. $M$.

Langley, E. R. Lieut., Spec. List (Carrier Corps)

Langley, F. O. Capt., S. Staffs. Regt.(T.F.); Major, Asst. Military Attaché. M.C. M. Chevalier, Legion of Honour (France)

Lankester, E. A. Major, H.A.C. (W.) $M$. 1902

LAUderdale, E. M. Surgeon Lieut., R.N.

Laurie, F.G. Capt., Loyal N.Lancs. Regt. (W 2.) M.C.

LAURIE, H. C. Lieut., King's Royal Rifle Corps

1907

I910

1905

I910

LAWRENCE, J. S. G. Lieut., R.E.; Asst. Inspector of R.E. Machinery

LEA, M. Capt. and Adjt., York and Lancaster Regt. (W.) 1903 
LEACH LEWIS, W. Lieut., R.F.A.

1902

LEE, C. S. Capt., R.A.M.C.(T.F.)

I 898

LEES, K. Lieut., Manchester Regt. (W.)

I9I3

ELeEte, W. J. H. Capt., Lancs. Fus.

1905

Killed in action 21 fan. 1916

Leighton, A. F. Lieut., R.F.A. M.C. $M . \quad 1907$

LENEY, R. J. B. Capt., R.A.M.C. 1902

ALENNy, L. A. Pte., R. Fusiliers (P. S. Bn.); 2nd Lieut., S. [I9I4]

Staffs. Regt.; Lieut., R. Irish Fus.

Killed in action 20 Dec. I9I 7

LESCHER,F.G. Major,R.A.M.C. (P.) M.C.andtwoBars 1907

Lester, Rev. C. V. C.F. $4^{\text {th }}$ Class, Canadian Force 1900

LEwIS, G. A., T.D. Lieut.-Col., Sherwood Foresters I888

(Notts. and Derby Regt., T.F.) C.M.G. M. m.

LEwIS, H. H. Major, R.F.A.(T.F.); Brigade Major

HLEwIS, T. E. Capt., S. Wales Borderers

Killed in bombing accident 28 Aug. I91 5

Lewis, W. H. Lce.-Corpl., Bihar Light Horse, Indian 1907

Defence Force

Lewtas, F. G. Pte., R. Fusiliers (P. S. Bn.); Surgeon 1910 Lieut., R.N.

Leyton, A. S. F. Major, R.A.M.C.(T.F.)

Lilly, G. A. Capt., R.A.M.C. M.C. $M$.

LinleY-Howlden, R. C. Lieut., R.G.A. (W.)

LIPP, G. A. S. Lieut., M.G.C. (W.) M.C. French I9I4 Croix de Guerre

Little, J. C. Capt., Queensland and Tasmania Bn., I905 Australian Force. M.C.

Little, R. A. Lieut., R.F.A. (W.)

LitTlejohns, A. S. Major, R.A.M.C. D.S.O. M.

I9I4

1896

Lloyd, A. H. Capt., Calcutta Bn., Indian Defence Force 1902

Lloyd, I. G. Major, Rangoon Bn., Indian Defence Force 1897

WLloyd-Jones, I. T. Capt., R. Welsh Fus.(T.F.) [1914]

Killed in action 26 March I9I7

Lloyd-Jones, P. Capt., R. Welsh Fus.

WLloyd-Price, Ll. O. Rfn., King's Royal Rifle Corps

Killed in action 10 fan. I91 5

Lock, N. F. Capt., R.A.M.C.

Lock, P. G. Capt., R.A.M.C.

ALOdge, J. W. Colonel, Yorkshire Regt.

Died 23 Aug. 1917

ALong, B. A. Capt., S. Lancs. Regt. and King's Own (R. 1894 Lancaster Regt.)

Died ro Feb. 1917

1904

I 893

1874 
Long, C.W. Capt., London Regt.(L.R.B.) and Spec. List I9I I (Adjt., Vol. Bn.)

Loveband, F. R. Bt. Colonel, W. India Regt. and W. $\quad \mathbf{I} 882$ Yorks. Regt. $m$.

Loveday, G. E. Capt., R.A.M.C.(T.F.) $M$.

Lovelock, A. R. Capt., Cameron Hdrs. (W.) $M$. 1895 Low, A. J. Instructor Lieut., R.N. 1908

Capt. R.A.M. (5th London Gen. Hospital, T.F.)

WLOWRY, W.A.H. 2nd Lieut., I.A.R.O., attd. 14th Sikhs 1908 Killed in action in Gallipoli 4 Fune I9I 5

Lumb, T. F. Capt., R.A.M.C. $M$.

LunNiss, S. F. 2nd Lieut., R.A.S.C.

Mabane, W. Lieut., E. Yorks. Regt.; Capt., Spec. List. 1914 (W.) $M$.

MCBRIDE, L. G. 2nd Lieut., Seaforth Hdrs. (W.) I9I I

McCardie, W. J Capt., R.A.M.C. (Ist S. Gen. Hos- 1885 pital, T.F.)

McCaskie, H. B. Capt., R.A.M.C.

McCaskie, N. J. Lieut., R.A.M.C.

McCAw, O. C. 2nd Lieut., Middlesex Regt.

\#McClenaghan, G. M. Capt., Queen's Own (R. W. Kent [19I4] Regt.) (W.)

Died 8 Nov. 191 8 of wounds received in action

WMacCombie, W.J. Capt., King's Own Scottish Borderers. I9ro (W.) $M$.

Killed in action 17 Fuly r916

MacDonald, J. N. Major, K. Edward's Horse. (W.) 1906 $M$.

Macfarlane, C. B. Lieut., Gordon Hdrs. (W.) I905

MacFIE, R. A. S. R.Q.M.S., King's (Liverpool Regt., I886 Liverpool Scottish, T.F.)

McGeagh, G. R. D. Capt., R.A.M.C. M.C. 1906

*Macgregor, A. H. Capt., Seaforth Hdrs. (T.F.) M. 1909 Killed in action 13 Nov. I9r6

Mackenzie, A. V. Lieut., King's (Shropshire L.I.) (W.) I9I5 M.C.

McKerrow, G. Capt. (T.), R.A.F. $m$.

MacLean, H. Driver, R.G.A.

Macleod, D. N. Capt., R.A.M.C.

MacMullen, A. R. Surgeon Lieut., R.N., attd. R.N.D. 1907 D.S.C. and Bar

Died 7 Sept. 1918 of wounds received in action 
MCNaIR, W. L. Lieut., R. Warwickshire Regt.; Capt., I9I I O.C.B. (W.) $m$.

MacPherson, Rev. D. G. C.F. $4^{\text {th }}$ Class, R.A.C.D. 1898 O.B.E. $M$.

MacQuaRrie, H. Lieut., R.F.A.; empld. Ministry of I9I I Munitions

MAcRuRY, E. Capt., Spec. List (Intelligence). M 2. 1907 Cavalier, Order of the Crown of Italy. Order of the Nile $4^{\text {th }}$ Class (Egypt)

McTagGart, H. A. Hon. Capt., Gen. List, Canadian rgr3 Force

MaIRIS, E. S. Lieut.-Col., R.M.L.I.; empld. Admiralty I9I4 MAKIN, E. L. Major, Wiltshire Regt.; G.S.O. 2. D.S.O. $\quad$ I896 $M$ 3. $m$.

Malcolm, A. S. L. Capt., R.A.M.C. M. $\quad 1906$ Malden, E. C. Capt., R.A.M.C. $M$. 1909 HMalet, H. A. G. Lieut., King's Own Scottish Borderers I9IO Killed in action at Hill 60 18 April 1915

MarChaNT, W. F. Capt., London Regt. (Blackheath and 1892 Woolwich Bn.); empld. Infy. Base Depôt. O.B.E. M.

MaretT Tims, R. D. See Tims, R. D. Marett

MaRkLOVE, J. C. Major, R.A.M.C.(T.F.) $M 2$.

Marriott, G. A. Capt., King's Royal Rifle Corps and Gen. List

MARRIS, H. F. Major, R.A.M.C. Brevet Major. M. $\quad{ }_{1897}$

Marsden, Rev. T. C.F. $4^{\text {th }}$ Class, Canadian Force 1903 WMARSH, A. S. Capt., Somerset L.I. Killed in action 6 fan. 1916

MMarshall, A. R. Corpl. R.E. (Signals); Capt. and Adjt., 1909 R.G.A.

Died 2 Feb. 1918 of wounds received in action 8 Dec. 1917

Marshall, D. Capt., Manchester Regt. M.C.

WMarshall, F. Capt., King's (Liverpool Regt.)

Died 30 Sept. I914 of wounds received in action on the Aisne 20 Sept. I914

WMARTEN, H. H. 2nd Lieut., King's Royal Rifle Corps and 1912 Manchester Regt. $M$.

Killed in action 13 Aug. I91 5

MARTEN, R. H. Major, R.A.S.C. M 2. Italian Silver 1909 Medal for Military Valour

Martin, N. T. Lieut., R.F.A.

$\begin{array}{lr}\text { Martin, R. C. Pte., R. Fusiliers } & \text { I9I3 } \\ \text { Martin, W. E. Lieut., Devon Regt. and Gen. List } & \text { [1914] }\end{array}$ (Adjt., Base Depôt). (W.)

I910

I896

1914

1913

1914] 
Mason, H. B. Capt., R. Warwickshire Regt.(T.F.); I9I3 empld. Ministry of Munitions. (W.)

Mason, J. H. Capt., R.E. (Fortress, T.F.)

Mason, J. W. 2nd Lieut., R.G.A. M.G. $M$.

MAster, A. E. Lieut.-Col., R.A.M.C. $m$.

Matthews, R. W. Y. Lieut., R.F.A.(T.F.)

Mattingly, H. Pte., London Regt. (Artists Rifles)

Maturin, F.H. Lieut.-Col., Hampshire Regt. (T.F.) and I88g R.A.M.C.

Maunsell, L. B. Lieut., S. Staffs. Regt. and M.G.C.; I9I4 empld. Ministry of Labour. (W 2.) M.C.

Mawer, A. Lieut., Durham Univ. O.T.C.

Maxwell, L. B. Hon. Capt., Gen.List. O.B.E. Chevalier, Ordre de la Couronne (Belgium). Order of St Fohn of Ferusalem. French Croix de Guerre

MAY, E. Capt., R.G.A.

Mayfield, E. Hon. Lieut.-Col., R.F.A.

Maynard, H. A. V. Lieut., R. Welsh Fus. (W.)

Mayne, C. F. Surgeon Lieut., R.N. O.B.E. $M$.

Mayo, H. R. Capt., R.A.M.C.

WMeadowcroft, J. 2nd Lieut., R.E.

Died 7 Nov. 1918 of pneumonia

Medley, R. P. Capt., Felsted School O.T.C.

Meggeson, R. R. H. Lieut., R.G.A.

Melvill, L. V. Lieut., Signal Corps, S. African Force

MerCER, W. B. Lieut., R.A.M.C.(T.F.)

Messiter, C. C. Capt., R.A.M.C.(T.F. Res.)

Metcalfe, G. C. Capt., R.A.M.C.

Metcalfe-Gibson, A. E. Lieut., R.A.S.C.(M.T.)

Methyen, M. D. Capt., London Regt. (Hackney Bn.); Lieut.-Col. (T.), R.A.F. O.B.E. M. $m$.

WMichell, R. W. Capt., R.A.M.C. $M$. Died 19 fuly 1916 of wounds received in action

Michell-ClaRke, A. J. Major, Gloucestershire Regt.; Major (A.), R.A.F. M.C.

Michell-Clarke, J. Lieut.-Col., R.A.M.C. (2nd S. Gen. $\quad 1879$ Hospital, T.F.)

Miller, A. T. Capt., Sherwood Foresters (Notts. and Derby Regt.); Major, G.S.O.2. M.C. and Bar. Brevet Major. M3. French Croix de Guerre

MilleR, C. A. M. Lieut., R.H.A.

Milligan, D. W. Lieut., Cameron Hdrs.

1900

I 906

I 906

I 886

1902

1906

I 906

1909

I 880

I9I3

1879

1906

WMilner-BARRY, E. L. Lieut.-Cdr., R.N.V.R. Died 7 May 1917 of heart failure following bronchitis 
Mrskin, G. 2nd Lieut., Coldstream Gds. 1908

Mrtchell, A. L. Capt., Spec. List (Inland Water Trans- 1900 port)

Mitchell, A. W. C. Lieut., R.G.A. 1909

Mitchell, F. R.F.A. $\quad 1893$

MorR, A. Capt., R.E. I9I3

WMOIR, R. Lieut., R.E. I9I I

Died 9 Nov. I9I 5 of illness contracted on active service

Moller, N. H. Capt., Middlesex Regt. (W.) I9I I

Monckton, J. F. E. Lieut., Northamptonshire Regt. $\quad 1912$

Monro, C. G. Capt., R.A.M.C.

MonRo, H. E. Lieut., R.G.A.; empld. War Office $\quad 1898$

WMontague, P. D. Lieut., Rifle Brigade; attd. R.F.C. 1909

Killed in action 13 Nov. I9I7

Moore, C. G. H. Major, R.A.M.C. m. 1903

MOORE, J. H. E Capt., R.A.M.C. I9I3

MOORE, M. Lieut., York and Lancaster Regt.; attd. [1914]

R.F.C. (P.)

Moore, W. G. Major (S.), R.A.F. D.S.C.

MoorHouse, S. Major, Argyll and Sutherland Hdrs. $\quad$ i 897

MORE, T. 2nd Lieut., Cameron Hdrs.

Moreton, T. W. E. Capt., R.A.M.C.

Morgan, F. J. 2nd Lieut., S. Wales Borderers; Capt., 1907 Norfolk Regt. D.S.O. M.C. M.

Morgan, H. T. Lieut., Welsh Regt.(T.F.); Capt. and 1912 Adjt., Wiltshire Regt. M.C. M.

Moriarty, G. H. Lieut., R.F.A. and Spec. List (Intelli- 1912 gence). (W.)

MORRIS, A. Capt., R.A.M.C. (W 2.)

MorRIs, C. A., c.v.o. Major, R.A.M.C.(T.F.)

MorRISON, E. O. Surgeon Lieut., R.N.

MORRISON, H. Surgeon Lieut., R.N. I9I3

Mosse, C. G. T. Capt., R.A.M.C. Order of St Sava, I909 5th Class (Serbia). Serbian Royal Red Cross

Mossop, M. C. Lieut. (A.), R.A.F. A.F.C. 1906

Mossop, N. R. Capt., Suffolk Regt.; Hon. Capt. (K.B.), I9I3 R.A.F. (W.) $M$.

Motion, D. G. 2nd Lieut., R.F.A.

wMotion, S. H. Lieut., Northamptonshire Regt. (W) $M$ I917

Died I Aug. I9I7 of wounds received in action $3 \mathrm{I}$ fuly

I9I7

WMowAT, O. Lieut., roth Hussars

Died 22 April I917 of wounds received in action at

Monchy-le-Preux I Ith April I9I7

c. U.w.L. 
Mowll, C. K. Capt., R.A.M.C.

WMoysey, L. Capt., R.A.M.C.(T.F.)

Drowned on H.M. hospital ship Glenart Castle 26 Feb. 1918

Muirhead, J. A. O. Lieut., Clifton College O.T.C.

\#Murphy, J. K. Staff Surgeon, R.N.V.R.

Died I 3 Sept. I9I 6

Myers, C. S. Lieut.-Col., R.A.M.C. C.B.E. M 2.

AMyles, W.W. 2nd Lieut., Cameronians (Scottish Rifles); attd. T.M.B.

Died 20 Sept. I916 of wounds received in action

WMylREA, W. P. G. Lieut.-Col., R.F.A.(T.F.)

Died 25 Aug. I9I5 of illness contracted on active service

WNangle, E. J. Capt., R.A.M.C.

Killed in action 26 Sept. 1915

Neame, T. Lieut., Worcestershire Regt. and Gen. List. (W.) M.B.E.

NeILson, H. V. 2nd Lieut., Sherwood Foresters (Notts. and Derby Regt.) and Gen. List (Asst. Officer i/c Records). (W.)

WNelson, W. Foreign Legion, French Army; 2nd Lieut., $\quad 1889$ S. Staffs. Regt.(T.F.)

Killed in action at the Hohenzollern Redoubt $\mathrm{I} 3$ Oct. I9I 5

Newbold, C. J. Lieut.-Col., R.E. D.S.O. $M 3$.

Newington, H. A. H. Capt., London Regt. (London Scottish); Lieut.-Col., Deputy Controller of Labour. D.S.O. $M 5$.

Newman, E. A. R. Lieut.-Col., I.M.S. C.I.E.

Nichol, R. W. Major (A.), R.A.F. (P.) $M$.

Nicholls, S. H. Lieut., R.A.S.C.

1904

1909

Nicholson, C. J. Capt., R.A.M.C.

Nickels, R. N. Lieut., R.F.A.(T.F.) (W 2.)

1900

I895

Nicoll, H. M. D. Capt., R.A.M.C.

Nixon, J. A. Colonel, A.M.S. C.M.G. M 2.

*North, W. G. B. Corpl., R. Fusiliers

Killed in action ro fune I916

Norton, D. G. Major, Lancs. Fus. and Labour Corps. 1905 (W.)

Norton, E. H. P. 2nd Lieut., R.F.A.

Norton, G. P. Lieut.-Col., D. of Wellington's (W. I900 Riding Regt., T.F.) D.S.O. and Bar. M2. 
WNoyes, H. F. G. Capt., R.A.M.C.

I899 Died 5 Sept. 1916

OAts, W. 2nd Lieut., The Buffs (E. Kent Regt.) I90I O'Brien, J. C. P. Major, R. Irish Fus. (W 3.) M.C. 1903 सOke, R. W. L. Capt., R. Berkshire Regt.

Killed in action 25 Sept. I91 5

Oliver, F. R. Surgeon Sub-Lieut., R.N.V.R. I9I4

Oliver, H. G. Capt., R.A.M.C. (W.) M.C. M. 1908

Oliver, T. H. Major, R.A.M.C.

OlPherT, R. A. Capt., R.A.M.C.

OPIE, P. A. Major, R.A.M.C.; D.A.D.M.S. $M 2$. $\quad 1904$

ORCHARD, S. Lieut., R.F.A.(T.F.) (W.) I9I4

Owen, H. B. Capt., E. African Force. D.S.O. $\quad 1897$

PAGE, D. S. Capt., R.A.M.C.

Page, Rev. F. G. J. C.F. $4^{\text {th }}$ Class, R.A.C.D. I88I

PAGE, J. Capt. ('T.), R.A.F. $\quad 1903$

PAGET, O. F. Major, Australian A.M.C. I89I

PAIGE, J. F. Lieut., R.E. M.C. $M$.

PALMER, C. E. Major, I.M.S. $\quad$ I898

PaNK, P. E. D. Capt., R.A.M.C. I9ro

Parish, G. W. 2nd Lieut., Westmorland and Cumber- 1905 land Yeo. (W.)

Parker, R. D. Capt., S. African Med. Corps r89o

PARsons, H. Major, R.A.M.C. $\quad$ I902

PAterson, T. W.S. Major, R.A.M.C.(T.F.) 1893

Patrick, C. V. Surgeon Sub-Lieut., R.N.V.R.

PAUl, H. Lieut., D. of Wellington's (W. Riding Regt.); r9ro attd. R.E. (Spec. Bde.)

Pawle, H. Capt., R. Berkshire Regt.; Staff Capt. M 2. 1904 French Croix de Guerre

WPeArKes, A. M. Pte.,London Regt.(Artists Rifles); Capt., 1906 W. Yorks. Regt.

Killed in action in Gallipoli 7 Aug. 191 5

WPEARMAN, J. O'H. 2nd Lieut., R. Warwickshire Regt. IgOI

Killed in action 25 Fan. I9I7

Pears, R. Capt., Middlesex Regt.; empld. O.C.B. $\begin{array}{lll} & 1909 \\ \text { PECK, J. N. Lieut.-Col., King's (Liverpool Regt.) (W.) } & \text { r905 } \\ \text { (P.) M.C. } & & \end{array}$

Pegge, A. V. Capt., R.A.M.C. M.C.

I9I2

Pendered, J. H. Major, R.A.M.C.; D.A.D.M.S. M.C. 1906 French Médaille des Epidémies 
Pentland, G. C.C. Lieut., R. Dublin Fus.; attd. M.G.C. 1908 (Motor); Capt. (T.), R.A.F.

Percival, A. F. Lieut., R.G.A.

Perkins, B. M. N. 2nd Lieut., Spec. List (Interpreter)

Perrin, W. S. Lieut., R.A.M.C.

PERRY, E. W. Capt., I.A.R.O., attd. II4th Mahrattas, I roth Mahratta L.I., and Gwalior Imp. Service Infy.

Perth (Australia), Archbishop OF. Chaplain-General, 1874 Australian Force

Peshall, S. F. Lieut., King's Royal Rifle Corps; empld. I902 War Office. (W.) M.C.

Petch, D. B. Capt., Leicestershire Regt. (W 3.) M.C. I9I5 and Bar

Peters, E. A. Capt., R.A.M.C. $M$.

Peters, R.A. Capt., R.A.M.C.; empld.Ministry of Munitions. M.C. and Bar. $m$.

Phear, H. W. Lieut., R.F.A.; Lieut. (A)., R.A.F. (W 2.) I9I2

Phillips, L. C. P. Lieut.-Col., R.A.M.C. $M 2$.

PICkering, B. M. 2nd Lieut., The Queen's (R.W. Surrey Regt.)

PicketT, A. C. Capt., R.A.M.C. O.B.E. $m 2$.

Picton-Warlow, A. J. P.O., R.N.V.R. (P.)

1905

1907

I90I

1910

1887

1908

1889

I910

Pike, H. H. Lieut., W. Yorks. Regt.

Pinkham, C. Capt., Middlesex Regt. and Spec. List

Pinto-Leite,H.M. Capt., R.A.M.C.(T.F.);D.A.D.M.S. $m$.

Platt, A. H. Capt., R.A.M.C.(T.F.)

\&PlatTs, A. L. Capt., Suffolk Regt.

Killed in action 20 fuly 1916

Platts, S. G. Capt., R.A.M.C.

Playfair, K. Capt., R.A.M.C. $M$.

Poignand, Rev. C. W. Chaplain, R.N.

*Pollak, H. L. Lieut., R. Marines and Rifle Brigade

Killed in action 23 Oct. 19I6

Pooley, G. H. Major, R.A.M.C.(T.F.)

Pope, H. B. Major, R.A.M.C.(T.F.) M.C. M. French Croix de Guerre

PorritT, R. N. Capt., R.A.M.C. (W.)

Porter, C. E. V. Capt., Essex Regt.(T.F.); Hon. Capt. 1912 (A.), R.A.F.

APorter, G. H. Lieut., R. Welsh Fus.

Killed in action 3 Oct. I9I6

APorter, J. E. 2nd Lieut., S. Lancs. Regt.

Killed in action 23 Fuly 1916 
Porter, W. N., C.I.E., V.D. Major, R. Sussex Regt. ; Presi- 1869 dent, Central Quartering Committee. (W.) $M$.

PotTs, J. L. Lieut., R.N.V.R.

Powel Smith, L. J. Lieut., Durham L.I.

1914

Powell, G. G. Capt., Gordon Hdrs.

WPowell, L. M. 2nd Lieut., Gordon Hdrs.

Killed in action 17 Fune 1915

Prestige, A. R. Capt., R.A.S.C.(M.T.)

Priddle, A. E. Major, R. Welsh Fus.(T.F. Res.)

I9II

1907

I913

Pridham, C. F. Capt., R.A.M.C. (W.)

Priest, R. C. Capt., R.A.M.C.

Pring, J. G. Lieut., R.G.A.

Prior, H. B. Major (A.), R.A.F.

Purvis, J. H. Lieut.-Col., Highland L I D.SO. M 2.

Pye-Smith, T. E. B. Capt., Wiltshire Regt.(T.F.) M. 1905

Pyman, F. C. Capt., Yorkshire Regt.; empld. Ministry 1907 of Munitions. (W.) $M$.

Pytches, G. J. Capt., Suffolk Regt.(T.F.); attd. M.G.C. 1900

Quiggin, E. C. Lieut., Spec. List (Censor's Dept.); I893 Lieut., R.N.V.R., empld. Admiralty

RABY, G. H Lieut., Oxford and Bucks. L.I.(T.F.)

RADFORD, A. C. Lieut., R.A.S.C.(T.F.)

RAE, A. J. Capt., R.A.M.C.

I910

1889

1885

1901

I899

1905

RaIKes, W. O. Lieut., The Buffs (E. Kent Regt.); Capt.

1912

1901

1900

(A.), R.A.F.; Lieut.-Col., S.O. r, Air Ministry.

O.B.E. M.B.E

सRAIMES, Rev. L. Capt., Durham L.I.(T.F.)

Died I Fune I916 of wounds received in action $3 \mathrm{I}$ May 1916

ZRAmSAy, A. 2nd Lieut., R. Fusiliers; Lieut., R. War- 1906 wickshire Regt.

Killed in action 25 April r91 5

RatTray, I. M. 2nd Lieut., R. Inniskilling Fus.

Raven, Rev. C. E. C.F. 4th Class, R.A.C.D.

Rawling, L. B. Major, R.A.M.C. (T.F.)

RAYMOND, C. Hon. Capt., I.M.S.

RAYNER, A. E. Major, R.A.M.C. (Sanitary Service, T.F.) O.B.E. $M 2$.

Rayner, E. C. Capt., R.A.M.C.

REA, R. H. T. 2nd Lieut., R.F.A.

READ, Rev. H. C. C.F. 4th Class, R.A.C.D.

1907

1906

READ, R. I. 2nd Lieut., R.A.S.C.

1910

1904

1890

1900

1902

1904

I9I 7

1909

1907 
Reddaway, H. Lieut., R. Fusiliers; Hon. Lieut. (O.), I9Io R.A.F. (W.)

Reid, C. B. Canadian Force

WRenton, T. Pte., London Regt. (Kensington Bn.)

Killed in action at Fromelles 9 May 1915

Reynolds, E. P. Major, R.E. (Signals). (W.) M.C. 1906

Reynolds, T. W. Capt., Suffolk Regt.

Rhodes, S. H. Capt., R.F.A.(T.F.)

Rhodes, W. A. Pte., R. Berkshire Regt.; Corpl., Devon

Regt. and Labour Corps

Richards, C. S. Lieut., R.E. (W.)

$\begin{array}{lll}\text { RICHARDS, C. S. Lieut., R.E. (W.) } & 1904 \\ \text { Richards, F. S. Capt., Spec. List (Survey Coy., R.E.) } & 1905\end{array}$ $M 2$.

Richards, F. W. Major, R.E. (W 2.) D.S.O. M.C. $M$.

ARIChardson, A. S. Sergt., R.A.M.C.; 2nd Lieut., R.G.A.

Killed in action $25 \mathscr{F}$ une 1917

Richardson, C. Sub-Lieut., R.N.V.R.

Richardson, D. E. E. Capt., R.E.(T.F.) - (W.)

1908

1910

1907

1908

1902

I9I3

1912

1909

1912

1912

RICHARDSON, D. W. R. Surgeon Lieut., R.N.

$\begin{array}{ll}\text { Richardson, F. K. Capt., E. Yorks. Regt. (W 2.) M 2. } & \text { I910 } \\ \text { ERIEu, H. Pte., Middlesex Regt. (P.S. Bn.) } & 1897\end{array}$

Killed in action 30 Fan. 1916

ARigby, F. J. Capt., Seaforth Hdrs. (W.) M.C. M. 1906

Killed in action in Mesopotamia 21 Fan. 1916

Rigby, J. C. A. Major, S. African Med. Corps

RIGG, R. Major, Border Regt. O.B.E.

1890

1897

RILEY, Rev. C. L. C.F. 4th Class, Australian Chaplains'

1906

Dept.

Riley, Rt. Rev. C. O. L. See Perth, Archbishop of

RIPLEY, H. E. R. Lieut., R.A.S.C. $m$.

1902

Rix, R. G. Gnr., H.A.C.

Robathan, K. M. Capt., I.A.R.O., attd. 2nd Gurkha Rifles

Roberts, A. D. Pte., R. Fusiliers (P.S. Bn.); Capt., 1907 Welsh Regt. (W.) M.C.

Roberts, C. J. Lieut., R. Welsh Fus.

RoBerts, F. M. 2nd Lieut. (T.), R.A.F.

I9I 5

1893

I897

Died 25 Aug. I9I 5

Robertson-Shersby-HaRvie, R. Lieut., R.N.V.R.

Robinson, C. A. Capt., R.A.M.C.(T.F.)

1909

1890

Robinson, H. D. Paymaster Sub-Lieut., R.N.R.

WRogers, E. H. 2nd Lieut., R. Warwickshire Regt.

Killed in action 3 Fuly rar 6 
Rogers, G. F. Capt., R.A.M.C. (Ist E. Gen. Hospital, I 885 T.F.)

Rolston, A. C. Lieut., R.E.(T.F.)

1906

Romer, C. Capt., R.E., attd. Gen. Staff. M.C. M. m. 1902

Rooke, C. P. Major, Middlesex Regt.; Lieut.-Col., R. $\quad$ I893 Warwickshire Regt.; Lieut.-Col. (T.), R.A.F. (W.) D.S.O. $M$.

Roscoe, W. Lieut., S. Lancs. Regt.; Major, M.G.C. 1907 (W.) $M$.

Rотн, G. J. Lieut., r66me Regt., French Army; Capt., I9I I I55me Regt. (W 2.) Chevalier, Legion of Honour (France). French Croix de Guerre. M2.

Routh, L. M. Capt., R.A.M.C. (P.)

RushfoRTH, F. V. Lieut., I.A.R.O.; Controller of War 1907 Accounts

Russell, E. C. Major, London Regt. (Blackheath and 1896 Woolwich Bn., T.F. Res.); G.S.O. 3. $m$.

Russell, J. C. Major, R.F.A. M.C. Belgian Croix de 1907 Guerre

ARutherford, W. McC. Pte., R.A.M.C.; 2nd Lieut., E. 1908 Yorks. Regt.

Died I9 April I9 8 of wounds received in action

Ryan, J. Major, R.E. (Signals). (W.) M.C. M.

I9I3

Sabin, J. H. Capt., R. Welsh Fus.(T.F.) (W.) I9I I

Salomons, Sir D., Bart. Hon. Colonel, R.E. (Fortress, I870 T.F.)

¥Salomons, D. R. H. P. Capt., R.E. (Fortress, T.F.)

Drowned on H.M.S. Hythe 28 Oct. 1915

Sanctuary, A. G. E. Lieut., R.F.A.(T.F.); empld. War I9ro Office

Sanctuary, C. T. Capt., R.F.A.(T.F.); Hon. Capt. (A.), 1908 R.A.F.

WSANDERSON, F. B. 2nd Lieut., R.F.A.(T.F.)

Died Io Aug. 1916 of wounds received in action

Sanderson, R. E. R. Surgeon Sub-Lieut., R.N.V.R.

SANFORD, D. W. Capt., R.E. M. Greek Medal for 1909 Military Merit, $3^{\text {rd }}$ Class

Sargeaunt, G. M. 2nd Lieut., Marlborough College I9O2 O.T.C.

SAUndER, D. A. Lieut., N. Staffs. Regt.; attd. R.E. (Sig- I9ro nals). M.C.

SAunders, E. G. S. Capt., R.A.M.C. (4th S. Gen. Hos- $\quad 1883$ pital, T.F.) 
Saunders, H. Capt., R.A.M.C.

Savile, W. S. Capt., Dorset Yeo.

\&Schaefer, T. S. H. Lieut., Northumberland Fus.

Killed in action in the Battle of Loos 26 Sept. 19r 5

Schlesinger, G. L. Capt., Somerset L.I. (W 2.)

Scholfield, J. A. Capt. and Adjt., Manchester Regt. (T.F.) (W.) (P.)

Schonland, B. F. J. Capt., R.E. O.B.E. M.

SchurR, C. G. Capt., R.A.M.C.

ScotT, J. Capt., R.A.M.C.

ScotT, R. S. Major., R.A.M.C. $M$.

Scowcroft, H. E. Surgeon Lieut., R.N.

Scrimgeour, G. C. Major, R.F.A.(T.F.) D.S.O. M.C. and Bar. $M$.

Sedgwick, R.E. Capt., R.A.M.C.

SELl, F. W. Capt., Med. Res. Corps, United States Army 1888

*Sellars, E. F. Capt., Cheshire Regt. (W 2.) M.C. M. I9I4

Killed in action in Macedonia 18 Sept. 1918

Sephton, R. Lieut.-Col., R.E. M2. Order of the Rising

Sun, $4^{\text {th }}$ Class (Yapan)

Shann, S. E. T. Hon. Capt., R.A.M.C.

Sharp, J. E. Capt., R.A.M.C.

Sharp, L. W. Hon. Capt., R.A.M.C.

Shaw. A. G. Lieut.-Col., The Queen's (R. W. Surrey

Regt.) Brevet Colonel. $m 2$.

Shaw, R. D. Capt., Hampshire Regt. M.C.

Sheldon, H. F. Capt., R.A.M.C.

Shelley, L. W. Capt., R.A.M.C.

Sheppard, G. Capt., R.G.A. M.C.

\#Sherman, R. Capt., R.A.M.C.

Died Io Oct. I9I7 of wounds received in action

I9OI

I90I

1907

I9OI

I884

I9I3

I 890

I906

I 895

1906

Showell-Rogers, E. N. Lieut., 5th (R. Irish) Lancers; 1909 A.D.C.

ShutTe, M. W. Capt., R.A.M.C.

Sidebotham, F. L. Major, R.F.(T.F.) (W.) M.C.

1892

Sidebotham, J. B. Lieut., R.E.(T.F.); Lieut. (E.O.), I9I I R.F.C. (W.)

SilbuRN, L. Lieut., R.A.S.C.(T.F.); attd. Durham L.I. I910 «Simon, Sir R. M. Lieut.-Col., R.A.M.C.(T.F.) I870

Died 22 Dec. 1914

Simonds, C. C. B. Lieut., R.E.(T.F.)

Simpson, Rev. J. B. C.F. $4^{\text {th }}$ Class, R.A.C.D. $M$.

I910

Simpson, J. C. Capt., R.A.M.C. (Ist E. Gen. Hospital, I898 T.F.) 
Simpson,W.H. Pte., Cambridgeshire Regt.; Lce.-Corpl., 1897 Northamptonshire Regt.; Capt., R.G.A. m.

Sington, H. S. Surgeon Lieut., R.N.

Skelding, H. Major, R.A.M.C.(T.F.)

SkINNER, J. A. D. Surgeon Lieut., R.N.

Slater, F. C. Lieut., King's Own (R. Lancaster Regt.)

Smalley, S. Lieut., Cambridgeshire Regt. and I.A.R.O., attd. 69th Punjabis

Smallwood, A. McN. Lieut., Northumberland Fus.; I9I4 Capt., Spec. List (P. and B.T. Staff)

Smart, A. H. J. Surgeon Lieut., R.N.

1896

I879

1910

I9I3

I912

Smith, Rev. E. W. C.F. 4th Class, R.A.C.D. M.C. M. $\quad{ }^{1901}$

Smith, G. B., C.M.g. Colonel (Chief Paymaster), Army 1879

Pay Dept. C.B. $M 3$.

Smith, H. E. Lieut., The Queen's (R.W. Surrey Regt.) I9ro (W 2.)

SмIтH, K. P. Capt., Northamptonshire Regt. M.C. M. I9I2

Smith, S. H. Lieut., Cheshire Regt.; Major, Gen. Staff. 1907 M.C. M 2. French Croix de Guerre

«Smith, S. P. Capt., S. Staffs. Regt.(T.F.) (W.)

Killed in action $28 \mathrm{Feb}$. I9I7

Smith, T.L. Corpl., R. Fusiliers (P.S.Bn.); Lieut., Black I9 I Watch

Smith, W. E. Capt., R.A.S.C.

I9I I

SNELI, H. C. Capt., R.A.M.C. (3rd N. Gen. Hospital, rgor T.F.)

SNell, J. A. B. Surgeon Lieut., R.N.

\#Solly, A. N. Sergt., R. Fusiliers (P.S. Bn.); 2nd Lieut., I9I3

Manchester Regt.; Capt. (A.), R.F.C. (W.)

Killed in action I I Aug. I9I7

SOMERS-ClaAkE, G. Major (T.), R.A.F. O.B.E. $M . \quad 1899$

Somervell, T. H. Capt., R.A.M.C.(T.F.) $M$. 1909

WSPARENBORG, H. R. Capt., King's Own (R. Lancaster I894

Regt.)

Killed in action at Le Cateau 26 Aug. 1914

Sparks, C. E. Hon. Lieut., R.A.M.C.

1888

SPARROW, G. Surgeon Lieut., R.N.; attd. R.N.D. M.C. 1905

SPEAKMAN, L. A. Lieut., Haileybury College O.T.C. 1903

Spearman, B. Med. Officer, E. African Force 1896

Spearman, W. Lieut., Devon Regt.; Capt., Gen. List 1900

(Courts-Martial Officer)

SPENCE, R. B. Major, 96th Berar Infy., Indian Army; I90 I

D.A.A. and Q.M.G. O.B.E. M 3 .

SQuires, F. V. Lieut., R.F.A. (W.) 
Stafford, H. N. Capt., R.A.M.C. (W.) M.C. M. 1909

Stanley-Clark, C. Capt., R.A.M.C.

Stephens, J. W. W. Lieut.-Col., R.A.M.C.

Stephenson, H. M. Capt., R.A.M.C. (W.) M.C.

Stevens, G. H. Lieut., R.F.A.; attd. R.E. $M$.

Stevens, L. B. Capt., R.F.A.; Staff Capt. M.C.

StewarT, F. H. Major, I.M.S.

STEWarT, W. H. E. Capt., R.A.M.C.(T.F.) M.G.

Stewart-Savile, W. S. See Savile, W. S.

Stiff, H. H. Capt., R.A.M.C.

I 898

1884

1906

1908

1904

1900

I 893

Stirling, E. M. Capt.. London Regt. (London Scottish);

Major, R.E. (W.)

Storey, H. H. Lieut., R.E.(T.F.); Lieut. (A.), R.A.F.

Storey, L. H. T. Lieut., R.N.V.R.

Stradling, A. R. Capt., R.A.S.C. $M$.

Stratton, F. J. M. Capt., Unattd. List, T.F.; Lieut.-

Col., R.E. (Signals); A. D. Signals. D.S.O. Brevet

Lieut.-Colonel. $M$ 5. Chevalier, Legion of Honour (France)

Stretton, J. W. Capt., R.A.M.C. (Ist S. Gen. Hospital, 1906 T.F.) $m$.

Strickland, A. F. P.O., R.N.V.R. (R.N.D.); Capt., 1905 R.G.A. (W.) M.C.

Strickland, C. Capt., N. Zealand Med. Corps 1899

Stuart, A. A. P. R. Capt. and Adjt., Manchester Regt.; 1899

Major, Sherwood Foresters (Notts. and Derby Regt.)

StUarT, R. S. D. Capt., Gloucestershire Regt.(T.F.)

(W 2.)

Sturgess, J. Capt., R.F.A.(T.F.)

Suhr, A. C. H. See Hammond Searle, A. C.

Summers, G. Lieut., R.E.(T.F.); Capt., Spec. List

Sutcliffe, J. H. Lieut., Essex Regt.

Sutherland, F. B. Lieut., Durham L.I.; Major, M.G.C. Sutherland, J. F. Lieut., R.G.A.(T.F.)

Svensson, R. Lieut.-Col., R.A.M.C. D.S.O. M.C.

\section{$M$ 2. French Croix de Guerre}

Swainson, E. A. C. Capt., R.A.M.C.

Swann, M. B. R. Surgeon Lieut., R.N.

1892

I90I

1913

1907

1905

I9OI

Swift, B. H. Capt., R.A.M.C. M.C.

Swindlehurst, J. E. Lieut., R.E.(T.F.)

SwindleHuRst, T. R. Lieut., R.F.A.; attd. T.M.B. $M$.

1910

1908

1899

1910

1904

1912

1908

1900

1886

1912

I9II

1908

WSykes, E. T. Capt., D. of Wellington's (W. Riding Regt., T.F.) (W.)

Killed in action 3 May 1917 
Sykes, S. W. Capt., Spec. List (Intelligence). O.B.E. 1903 M.C. $M_{3}$.

Syme, G. W. 2nd Lieut., Gordon Hdrs.; Lieut., R.F.A.; 1908 empld. Ministry of Munitions. (W 2.)

Symns, J. LL. M. Major, R.A.M.C.(T.F.)

Symns, J. M. Major, I.A.R.O.; G.S.O. 2

1904 1898

Symonds, F. C. Capt., Cambridgeshire Regt. 1906

Sympson, E. M. Lieut.-Col., R.A.M.C. (4th N. Gen. 1879 Hospital, T.F.)

TAIT, W. H. 2nd Lieut., R.E. (W.) French Médaille [1914] Militaire

Tallerman, K. H. Capt., R.F.A. (W.) M.C.

सTAPP, T. A. Capt., Coldstream Gds. and M.G.C. (W.) I902 M.C. and Bar. $M$.

Died 21 Oct. I9I7 of wounds received in action II Oct. I9I7

Taylor, A. R. Capt., Dulwich College O.T.C.

1900

TAYlOR, C. H. S. Lieut.-Col. (Med.), R.A.F.; S.O. 2, I90I Air Ministry. $M$.

ATAYLOR, C. P. Capt., E. Yorks. Regt. $M$.

Killed in action 28 Oct. I9I6

TaYlor, C. R. Capt., R.A.M.C. O.B.E. $M$.

TAYlor, E. J. D. Major, R.A.M.C. (5th S. Gen. Hos- 1892 pital, T.F.)

TAYLOR, E. L. T. Lieut., R.G.A.

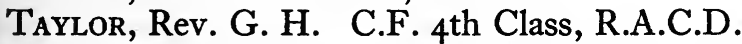

TAYLOR, S. H. S. Capt., R.A.M.C.

Teichmann, O. Capt., R.A.M.C.(T.F.) (W 3.) D.S.O. M.G. M 2. French Croix de Guerre. Italian Croce di Guerra

Telford, E. D. Capt., R.A.M.C.(T.F.)

1914

I906

I 897

1910

1897

1898

Tewson, E. G. Lieut., R.G.A.

1894

1905

1908

attd. Gordon Hdrs.

Killed in action 12 Nov. I9I4

Thomas, F. G. Major, R.A.M.C. (3rd W. Gen. Hos- 1890 pital, T.F.)

Thomas, G. E. A. See Aeron-Thomas, G. E.

Thomas, J. G. T. Capt., R.A.M.C. M.C.

Thomas, T. P. Capt., R.A.M.C.(T.F.)

I910

1893

\#Thomasset, G. T. Lieut., London Regt. (Blackheath I9I3 and Woolwich Bn.)

Killed in action 25 Sept. 1915 
Thompson, J. H. G. Cadet, O.C.B.

Thompson, O. S. Surgeon Lieut., R.N.

$\begin{array}{lll}\text { Thompson, R. C. Capt., Spec. List (Intelligence). } & M_{3} . & \text { I910 } \\ \text { Thompson, R. } & & \end{array}$

Thompson, R. M. Lieut., R.G.A.

Thompson, T. W. Capt., R.E. $M$.

I9I3

1896

*Thompstone, R. Lieut., S. Staffs. Regt. (W.)

Died 25 Fan. $192 \mathrm{I}$ of illness contracted on active service during the war

Thomson, G. D. Pte., London Regt. (Artists Rifles); Lieut., R.A.S.C.; attd. R.A.F.

WThorburn, E. F. Lieut., Manchester Regt.(T.F.)

[1914] Killed in action in Gallipoli ro fune $19 \mathrm{I} 5$

Thornton, L. H. Colonel, Rifle Brigade; A.Q.M.G. *'I9I I C.M.G. D.S.O. $M_{4}$.

Thorp, R. C. 2nd Lieut., R.F.A.

Threlfall, C. R. F. Capt., R.E. (Signals). M.C.

Threlfall, R. E. Lieut., R.E. (Signals); empld. Ministry of Munitions

Threlfall, W. B. Lieut., R.N.V.R.

Thresher, W. H. Capt., R.A.M.C.

सThurlow, A. G. 2nd Lieut., D. of Wellington's (W. Riding Regt.)

Died 29 Aug. I918 of wounds received in action in Gallipoli 21 Aug. I918

Tidman, O. P. Lieut., R.N.A.S. and Naval Transport Service

Tims, R. D. MaretT. Lieut., London Regt.; Capt., 1909 Northumberland Fus. and Training Res. Bn. (W.)

सTodD, A. F. Capt., Norfolk Regt. M.

Killed in action at Hill 6021 April 1915

Todhunter, J. R. A. D. Capt., R.A.M.C.

Tonks, J. W. Capt., R.A.M.C.

Topham, D.B. Lieut., Middlesex Regt.(T.F.) and Grenadier Gds.

सToppin, S. M. Major, R.G.A. M.C. $M$.

Died 27 Sept. I917 of wounds received in action

1892

1904

1906

1910

I 896

Torrey, J. Corpl. R.E. (Signals)

Tottenham, C. E. L. 2nd Lieut., Middlesex Regt.

Townsend, A. L. H. E. African Force

ATrafford, G. T. Lieut., Life Gds.; Capt., Tank Corps. [I9I4] (W 2.) $M$.

Killed in action 23 fuly 1918

Trapnell, H. Capt., Gloucestershire Regt.(T.F.); attd. I9r3 R.E. 
Travis-Clegg, G. R. 2nd Lieut., Loyal N. Lancs. Regt I9I3 (T.F.)

ETregelles, G. P. Capt., Devon Regt. $M$.

Killed in action I Fuly 1916

Treves, F. B. Major, R.A.M.C.(T.F.) O.B.E. $M . \quad 1898$

Treves, W.W. Major, R.A.M.C. O.B.E. $M 4$. I90I

Trollope, T. A. Lieut., R.A.S.C.(T.F.); attd. Rifle 1912 Brigade

Tucker, E. G. Pte., R. Fusiliers; Sapper, R.E. (Signals)； 1904 attd. Intelligence Staff. M.M. and Bar

TuckeTT, J. E. S., T.D. Major, Marlborough College 1889 O.T.C.

TurCan, J. S. Major, Seaforth Hdrs. (W 4.) M.C. 1909

TURNeR, Rev. H. A. C.F. $4^{\text {th }}$ Class, R.A.C.D.

Tweedie, F. I. G. Capt., Manchester Regt.(T.F.) (W.) I9I I

TweedIE,J.M. Capt.,R.A.S.C.; Major,D.A.D.Canteens, I903 War Office. $M 2$.

WTwEEDIE, L. K. 2nd Lieut., R.F.A.

Killed in action 17 fan. I916

TwEedy, O. M. Capt., Leinster Regt. and Gen. Staff. 1908 (W.) $M$.

Tweedy, R. J. Capt., Igth Lancers, Indian Army; Staff 1907 Capt.

ATween, A. S. Major, Essex Regt. D.S.O. M2.

Died 23 March 1918 of wounds received in action at Rouez

Twigg, G. W. Capt., R.A.M.C. $M$.

Tyson, W. Capt., R.A.M.C. (Ist E. Gen. Hospital, T.F. Res.)

Uhthoff, R. K. Capt., R.E. (W.) M.C.

UNDERHILl FAITHORNE, C.F. Major, Connaught Rangers;

Lieut.-Col., Spec. List (Cmdt., School of Musketry). (W.)

UsHer, C. T. Major, R.A.M.C.(T.F.)

1910

I90I

1889

1906

1898

1883

VAUdREY, W. E. Major, R.G.A. (W 2.) M.C. and Bar igr2

VERNON, R. J. Major, R.A.M.C. $M$.

1899

Vickers, S. Major, R.F.A.(T.F.) (W.) D.S.O. M. 19 Io

VIVIAN, C. St A. Capt., R.A.M.C. I90I

Vos, P. Lieut., Norfolk Regt.; empld. War Office. (W.) 1909

French Croix de Guerre

Vyvyan, M. C. Lieut., Natal Carabineers and M.G.C. Igro (W.) 
Waddy, A. C. Lieut., R.F.A.(T.F.); Staff Capt. O.B.E. I9I I $M$.

Wadson, F. P. Lce.-Corpl., Lincolnshire Regt. 1895

Wainwright, C. B. Capt., R.A.M.C.

WALFORD, H. H. Lieut., R.A.S.C.(T.F.)

WAlker, A. C. Major, R.F.A. (W.) M.C.

Walker, C. A. P. Lieut., Highland L.I.; attd. 44th Infy., 1912 Indian Army

Walker, D. Lieut., King's (Liverpool Regt.); Capt., I9I3

M.G.C. (W.) M. Belgian Croix de Guerre

Walker, K. M. Capt., R.A.M.C. O.B.E. M 3. I90I

Walker, W. J. Sergt., Army Pay Corps; attd. R.E. I913

WALKER, W. M. Lieut., King's (Liverpool Regt.). (W.) I9I] M.C.

Wallace, R. W. J. Lieut., R.F.A. (W 2.) Igo8

WANKLYN,W.McC. Capt., R.A.M.C.(T.F.);D.A.D.M.S. I 88

WARD, G. H. Surgeon Sub-Lieut., R.N.V.R. I9I]

WARDEN, A. R. S. Surgeon Lieut., R.N.

Warden, G. Pte., King's Own (Yorkshire L.I.) and I9I3

Wiltshire Regt.; Sergt., Devon Regt.

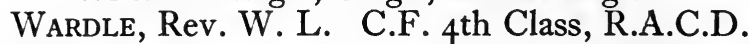

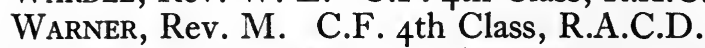

WWARNER, T. L Major, Leicestershire Regt. (W 2) 189 D.S.O. $M_{3}$.

Died 27 Dec. I9I7 of wounds received in action

Warnes, G. G. Capt., Suffolk Regt.(T.F.) (W.)

Watermeyer, E. F. Lieut., Cape Garrison Artillery

Watermeyer, H. A. Capt., R.A.M.C.

Waterworth, S. Capt., Loyal N. Lancs. Regt. (W 2.) 1912 M.C.

Watkins, D. J. G. Major, R.A.M.C. (4th N. Gen. Hos- 1886 pital, T.F.)

Watkins, W. B. Capt., R.F.A.(T.F.) (W.)

Watson, F. H. Surgeon Lieut., R.N.V.R.

Watson, W. G. Surgeon Lieut., R.N.

Watson Williams, E. Major, R.A.M.C. M.C.

Watr, G. T. C. Lieut., King's College School, Wimbledon, O.T.C.

WatTs, E. M. Capt., R.F.A. (W.) M.C.

WeAver-Adams, E. R. Lieut., R.E. (Signals). $M$.

1905

1909

WebB, T. L. Capt., Yorkshire Regt.

WWedD, E. P. W. Capt., Essex Yeo.; Staff Capt.; Capt., R.A.M.C. M.C. $M$.

Killed in action 13 Fuly 1918 
सWEDGwood, A. 2nd.Lieut., Northumberland Fus.

Killed in action I9 Aug. I9I 5

WeEKS, Ll. M. Major, R.A.M.C. (W.) M.C.

I9I I

WeEks, R. M. Major, Rifle Brigade; G.S.O.2. D.S.O. M.C. and Bar. Brevet Major. M3. French Croix de Guerre

WWegG, H. N. Capt., Middlesex Regt.

Killed in action 25 March 1918

WEGG, W. H. J. 2nd Lieut., R.G.A.

WWeLlS, N. L. Lieut., Loyal N. Lancs. Regt.

Killed in action in Gallipoli 10 Aug. 1915

1905

1909

Wells-Cole, G. C. Capt., R.A.M.C.

Welsford, A. G. Capt., R.A.M.C. $m$.

\#Welsford, G. J. L. 2nd Lieut., Middlesex Regt. and

R.F.C. (W.)

Killed in action 30 March 1916

WeSt, F. R. 2nd Lieut., King's Royal Rifle Corps

West, P. C. Capt., R.A.M.C.

Weston, G.H Capt. R. A.C. (Sanitary Service, T.F.) 1896

1900

I895

1906

1913

1883

1913

EWetenhall, W. T. Capt., Leicestershire Regt.

Killed in action 16 fuly 1916

WhELDON, E. J. 2nd Lieut., R.E.

1912

1896

1879

1907

1907

WWHITAKER, T.S. 2nd Lieut., D. of Wellington's (W. I9I I Riding Regt., T.F.)

Killed in action 7 Nov. I9I 5

WWhitby, J. H. Capt., London Regt. (St Pancras Bn.) I9I2 $M$.

Died 16 March r9r6 of illness contracted on active service

Whitcombe, E. P. Lieut., Worcestershire Regt. (W.) I9r3

Whitcombe, R. H., v.D. Major, R.A.S.C.(T.F.) D.S.O. * 1880 $M 2$.

WWhite, C. W. M. 2nd Lieut., Norfolk Regt.

Killed in action 26 Sept. I9I 5

White, G. F. Air Mechanic, R.A.F.

[1914]

I894

WWhiteheAd, A. G. 2nd Lieut., W. Yorks. Regt.(T.F.); I9I I Capt. (A.), R.F.C.

Killed in action 29 fan. 1918

WhiteheAd, C. E. Capt., R.A.M.C.(T.F.) (W 2.) I90I

Whitfield, E. O. 2nd Lieut., S. Staffs. Regt.

Whiting, E. R. S. M. Pte., R.A.M.C. I9I2

WHITMORE, A. Major, I.M.S. 1894

WhitTall, H. C. Lieut.-Cdr., R.N.V.R. O.B.E. Officer, I907

Order of the Redeemer (Greece) 
Whitty, H. N. Capt., London Regt. (Kensington Bn.) I90 I (W 2.)

WhiтwоRтн, J. Lieut., R. Ist Devon Yeo.; attd. Devon 1902 Regt.(T.F.)

WidDICOMBE, E. P. Lieut., I.A.R.O., attd. Sappers and *1899 Miners; Capt., Spec. List; Major, R.E. $M$.

WWiggin, N. H. Lieut., R.F.A. $M$.

Died I I Fan. I9I7 of wounds received in action

Wigmore, J. B. A. Lieut.-Col., R.A.M.C. $M 2$.

WWileman, G. W. B. Capt., S. Wales Borderers. (W. 1905 Killed in action 8 Sept. I9I6

WWilkinson, G. J. Sergt., Middlesex Regt.

Killed in action I Fuly I916

Wilkinson, N. Lieut., Bombay Garrison Artillery, Indian Defence Force

Wilks, J. H. Capt., R.A.M.C.

Willcocks, R. W. Capt., R.A.M.C. $M$.

Willey, H. L. Capt., King's Own (Yorkshire L.I.) (W.)

WWilley, R. H. D. Pte., R.A.M.C. and R. Fusiliers (P.S. Bn.); Capt., 13th Hussars

Died at Amadia 14 Fuly 1919 of wounds received in action

Williams, A. C. 2nd Lieut., R.E.; Capt., Corps of 1889 Military Accountants

Williams, A. D. J. B. Major, E. African Med. Service. 1902 O.B.E. $M_{4}$.

Williams, A. F. Lieut., R.E.(T.F.); empld. Ministry of I9I3 Labour

Williams, A. G. Surgeon Lieut., R.N. (W.) O.B.E. 1907 M. French Croix de Guerre

Williams, Rev. A. J. Chaplain, R.N.

Williams, Rev. C. C.F. $4^{\text {th }}$ Class, R.A.C.D.

Williams, C. E. Lieut.-Col., I.M.S.

Williams, E. K. Capt., R.A.M.C.

Williams, G. C. Capt., R.A.M.C.(T.F.)

Williams, J. C. S. Capt., R.A.S.C.

Williams, J. L. C. Capt., R. Welsh Fus. (W.) I9Io

Williams, J. S. Capt., R.F.A. and Gen. Staff. (W.) I9I4 M.C. $M$.

Williams, M. B. Major, Welsh Regt. (W.)

Williams, N. A. Lieut., M.G.C. (Motor)

WWilliams, R. B. Capt., R.E. M.C. M.

Killed in action 19 Sept. I9I6

Williams, R. F. Capt., R.A.M.C. (W.) M.C. M 2. 1894 Williams, R. G. Lieut., W. African Frontier Force 
WWilliams, S. C. Lieut., R.F.A.

1912

Killed in action 18 Fan. I917

Williams, S. R. K. Edward's Horse

1912

Williams, Rev. T. B. C.F. $4^{\text {th }}$ Class, R.A.C.D.

1905

Williams, W. P. Lieut., R. Fusiliers; empld. War Office r9I4 WWilliamson, J. M. Pte., London Regt. (Artists Rifles); 1908 2nd Lieut., Gordon Hdrs.

Killed in action 16 May 1915

WWillink, H. J. L. Pte., R. Fusiliers (P. S. Bn.); Capt., 1903 D. of Wellington's (W. Riding Regt.)

Died 5 Nov. I9I8 of wounds received in action I Nov. I9I 8

Wilson, A. G. Capt., R.A.M.C.(T.F.) $M$.

Wilson, Rev. J. V. C.F. 4th Class, R.A.C.D.

Wimbush, G. S. H.A.C.

WimbuSH, Rev. R. C.F. $4^{\text {th }}$ Class, R.A.C.D.

WIMPERIS, H. E. Lieut.-Cdr., R.N.V.R.; Major (T.), R.A.F. (Aircraft Production Dept.) O.B.E.

WindER, J. F. E. Lieut., Oxford and Bucks. I.I. $\quad 1894$

Winslow, W. Capt., R.A.M.C.

Winter, P. G. D. Capt., R. Defence Corps

1883

1892

WINTER, W. H. Capt., Herefordshire Regt. and M.G.C. IgOI

Winterbotham, F. P. Lieut., R.G.A. $M$.

WolfF, L. Soldat-Secrétaire, French Army

Wood, C. S. Lce.-Corpl., R.E.

Wood, D. E. Lieut.-Col., Remount Service; Inspector of Remounts. C.B. $M$.

Wood, E. C. Corpl., R.E. (Signals)

1904

1906

1907

1871

I913

1904

Killed in action 23 Oct. 1916

WWood, J. W. M., M.v.o. Lieut.-Col., Remount Service; 1873

D.A.D. Remounts

Died 9 Dec. 1916

Woophouse, G. F. Capt., Unattd. List, T.F.

WoodrufF, G. G. Lieut., R.F.A.; empld. Ministry of I905 Munitions. $M$.

Woodsend, P. D. Capt., R.A.S.C.

Woolf, H. M. A. 2nd Lieut., R.A.S.C.; Hon. Capt., 1907 Spec. List

Woollcombe-Boyce, K. W. Sergt., R. Fusiliers; attd. I907 Infy. Bde. H.Q. M.S.M. M.

Woolward, A. T. Lieut., R.F.A.; Capt., R.A.M.C. M. 1909 WWORDSWORTII, J. L. Lieut., $5^{\text {th }}$ (R. Irish) Lancers I90I Killed in action 4 Nov. I9I 4

c. U.W.L. 
Worsley-Worswick, C. F. 2nd Lieut., R. Welsh Fus.; 1904 Lieut., Leicestershire Regt.

Worthington, C. R. Capt., Canadian A.M.C.

Wortley, Rev. R. W. C.F. $4^{\text {th }}$ Class, R.A.C.D.

Wright, C. S. Capt., R.E.; G.S.O. 3. O.B.E. M.C. $M$ 2. Chevalier, Legion of Honour (France)

WRIGHT, H.M. Lieut., Black Watch; attd. King's African 1906 Rifles. (W.) M.C.

Wright, M.E. A. Major (T.), R.A.F. (Aircraft Produc- 1912 tion Dept.) A.F.C. M. Belgian Croix de Guerre

Wyllie, A. K., C.B. Hon. Colonel, D. of Wellington's 1874 (W. Riding Regt.)

WyMAN, B. Lieut., Cheshire Regt.(T.F.);attd.R.E. (W.) 1902

Wynne-Yorke, B. A. Y. Lieut., E. Yorks. Regt.; empld. 1907 P. and B.T. Staff

Yeats, B. E. 2nd Lieut., R. Scots (T.F.)

Yeomans, F. A. Capt., N. Staffs. Regt.(T.F.)

Yolland, R. H. Capt., R.A.M.C. $M$.

Young, M. L. 2nd Lieut., R. Sussex Regt. (W.)

Ziegler, G. G. Capt., R. Fusiliers. (W 2.) M.C. M. I9I3 


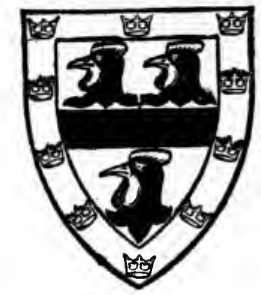

\section{JESUS COLLEGE}

Adami, J. G. Colonel, Canadian A.M.C.; A.D.M.S. * ${ }^{*} 89$ I C.B.E.

AdDy, R. Capt., E. Yorks. Regt.

AinleY, J. A. G. Lieut., Durham L.I. and M.G.C. I9I I

AAINley, Rev. W. P. C.F. $4^{\text {th }}$ Class, R.A.C.D.

Died I 2 Oct. I9I 5 of cerebro-spinal meningitis

AkHuRst, A. F. Instructor Lieut., R.N.

AAlderson, A. E. Lieut., The Queen's (R. W. Surrey 1902 Regt.); Capt., King's Own (Yorkshire L.I.)

Accidentally drowned in March I9I 8

HAldous, A. E. Trooper, I2th Lancers; 2nd Lieut., Bor- I9I I der Regt. (W.)

Killed in action 3 Fuly r9r6

Aldous, F. C. Major, Manchester Regt. (T.F.); Lieut.- $\quad$ I 898 Col., M.G.C. (W.) D.S.O. M 2 .

\#Allen, J. H. Lieut., Worcestershire Regt.

Killed in action in Gallipoli $2 \mathrm{I}$ Fune $19 \mathrm{I} 5$

1907

AAllen, R. G. R. 2nd Lieut., W. Yorks. Regt.(T.F.); 1908 attd. R.F.C.

Killed in action 16 Nov. 1916

AAllison, H. S. Major, R. Irish Rifles. $M$.

Killed in action 7 Aug. I9I7

Allison, W. W. Lieut., R. Sussex Regt., M.G.C., and 1914 R.G.A. $M$.

Alston, C. R. 2nd Lieut., S. Lancs. Regt.; Capt. (A.), 1912 R.A.F. (W.)

ANDERson, Rev. F. I. C.F. Ist Class, R.A.C.D. C.M.G. $\quad \mathrm{I} 893$ $M$ 5. Officer, Order of the Crown of Italy

ANDREw, R. H. Lce.-Sergt., R.H.A.; Capt., Suffolk 1903

Regt.; Lieut.-Col., Spec. List (D.A.M.S.) O.B.E.

M.C. M. Order of the Nile, 3 rd Class (Egypt) 
Anstey, A. Major, Devon Regt. T.D.

AARmstrong, C. 2nd Lieut., R. Fusiliers; attd. Loyal N. 1907 Lancs. Regt. $M$.

Killed in action in Mesopotamia 9 April 1916

ARNold, B. M. Major, R.G.A.(T.F.) D.S.O. M.

AARoN, E. M. Sub-Iieut., R.N.V.R. (R.N.D.)

Killed in action I3 Nov. I9I6

Astbury, W. T. Lce.-Corpl., R.A.M.C.

AAtkin, K. Capt., R.A.M.C. (P.)

Died 6 June 1918 of wounds received in action

AAyre, B. P. Capt., Norfolk Regt.

Killed in action I Fuly 1916

1903

1910

I9I6

I9II

I9II

Baillieu, H. L. Lieut., R.G.A. (W.) M.C.

BALDWIN, F. E. W. Major, I 2 Ist Pioneers, Indian Army;

D.A.A.G. M. Order of the Nile, $4^{\text {th }}$ Class (Egypt)

WBanister, C. W. 2nd Lieut., R. Fusiliers

Killed in action 16 June 1915

Banister, J. B. Capt., R.A.M.C.

Banning, H. B. S. 2nd Lieut., R.G.A.(T.F.)

Bardwell, T. G. N. Capt., E. Riding of Yorkshire Yeo. and Spec. List, empld. Egyptian Army. T.D.

WBarnard, H. D. 2nd Lieut., Rifle Brigade

Killed in action 21 Aug. 1916

Barton, C. H. Pte., S. Staffs. Regt.

Batty, W. R. Capt., Manchester Regt. M.C.

Baynham, C. T. Major, R.H.A. D.S.O. $M 3$.

BAYNHAM, Rev.J.H. C.F. 2nd Class, R.A.C.D.; D.A.C.G. $M$.

Beasley, H. O. C. 2nd Lieut., R. Welsh Fus.; Capt., 1896 Cameronians (Scottish Rifles); Major, Labour Corps; Asst. Controller of Labour. O.B.E. M.

Beck, A. C. T., M.P. Hon. Lieut., R.N.V.R.

«Bескh, R. H. Pte., R. Fusiliers (P.S. Bn.); 2nd Lieut., I9I3 E. Yorks. Regt.

Killed in action 16 Aug. rgr6

Bell, A. C. Capt., R.F.A. (W 2.) M.C. French Croix I9I I de Guerre

Bell, H. A. Capt., R.A.M.C.(T.F.)

I9II

1898

I9I I

1898

1898

1903

I9II

I9I6

1907

1908

I893

1895

1906

BeLl, W. B. Capt., I2th Lancers; Major, G.S.O. 2. 1900 (W.) Brevet Major. $m$.

Bellamy, J. Sergt., London Regt. (London Scottish); 1903 Instructor, School of Musketry 
Bellhouse, A. P. Capt., Welsh Regt.; Lieut., 8th Raj- I9I3 puts, Indian Army

BeNEy, C. C. Lieut., I.M.S.; Surgeon Lieut., R.N. I9I I

Benham, J. H. F. Trooper, E. African Mtd. Rifles 1903

BERNEY-FiCkLIN, H. P. M. Lieut., Norfolk Regt.; Capt., I9I I Spec. List. (W 2.) (P.) M.C. Brevet Major on promotion to Captain. M2. Chevalier, Legion of Honour (France)

BetTERIDGE, C. D. Lieut., R.E.

BetTeridge, J. E. H. Pte., R.A.M.C.

Bevan, Rev. J. C.F. 4th Class, R.A.C.D.

1910

1909

I9II

Bevan, W. H. 2nd Lieut., King's Royal Rifle Corps; I9I4

Capt., Gen. List (R.T.O.) (W.)

BickNel, C. H., T.D. Capt., Unattd. List, T.F. $\quad 1882$

Bindloss, W. Capt., King's (Liverpool Regt.) and R. $\quad 1887$

Defence Corps

Blaker, R. N. R. Lce.-Corpl., Queen's Own (R. W. ${ }_{1898}$ Kent Regt.); Lieut., Rifle Brigade. M.C.

Bland, E. C. 2nd Lieut., Essex Regt. $M$.

BoDEN, Rev. J. F. W. Lieut., I.A.R.O., attd. S. and T. ${ }^{[1914]}$

Corps. $M$.

Boot, J. C. Capt., Sherwood Foresters (Notts. and 1907 Derby Regt.), and Gen. Staff

BouQueT, Rev. A. C. C.F. $4^{\text {th }}$ Class, R.A.C.D. m. * * 1905 Bowe, J. H. Major, Border Regt. and Gen. List. (W.) I900 $M$.

Bowles, H. F. Hon. Colonel, Middlesex Regt.

ÆBoyton, H. J. Capt., R. Fusiliers; Lieut., Grenadier Gds. I9IO

(W.)

Killed in action 14 Dec. 1916

BraceCAMP, F. W. Lieut., R.F.A. M.C.

I910

Bradley, E. J. Major, R.A.M.C. (W.) M.C. and Bar. 1908 $M$.

ZBradley, G. M. Lieut., Rifle Brigade; attd. Welsh Regt. I9 I I $M$.

Killed in action near Festubert 21 Dec. 19I4

Bradley, V. M. Capt., R.F.A.; Staff Capt.

Bradley, W. DE W. H. Capt., Wiltshire Regt. (W.) 1907

BRADSTOCK, G. Major, R.F.A. (W.) D.S.O. M.G. and 1907

Bar. $M$.

BREed, F. G. 2nd Lieut., Suffolk Regt.

BRIGGS, G. R. Major, R.F.A. (W 2.) $M$.

Brook, A. K. Lieut., R.A.S.C.

Brooks, Rev. D. G. Chaplain, R.N.

I9I3

1908

1887

1893 
Brown, G. L. Pte., R.A.M.C.; Lieut., R.A.S.C.; Capt., I9ro Connaught Rangers

ABrown, G. M. Lieut., I2th Lancers. M.C. M. Killed in action 27 Nov. I9I7

WBRown, H. Major, Yorkshire Regt.(T.F.) (W 3.) D.S.O. 1899 M.C. Brevet Major. M 2. French Croix de Guerre Killed in action 23 March 1918

सBrown, K. A. Capt., Cameronians (Scottish Rifles). 1905 (W.) $M$.

Killed in action 14 April 1917

Brown, M. L. C. Capt., M.G.C.

Brown, V. S. Major (T.), R.A.F. (Aircraft Production Dept.) m. French Croix de Guerre

WBrunwin-Hales, G. O. Capt., Essex Regt.; Capt. and 1907 Flt. Cdr., R.F.C.

Killed in action 24 March 1917

Brunwin-Hales, Rev. G. T. C.F. $4^{\text {th }}$ Class, R.A.C.D. $\quad 1878$ $m$.

Bruty, W. G. Capt., Spec. List (A.P.M.) M.B.E. $\quad$ m2. 1888

BRYDONE, P. 2nd Lieut., R.A.S.C. 1895

\#Bullen, R. E. Capt., King's Royal Rifle Corps. (W 2.) I9I I $M$.

Died I9 April I916 of wounds received in action

WBullough, J. L. Lieut., Argyll and Sutherland Hdrs. 1912 Killed in action 25 Sept. I9I 5

BulmER,A.C. Lieut.,E.Yorks. Regt.; attd.R.E.(Signals). 1909 $M$.

BuRNARD, C. F., D.S.o. Major, R. Warwickshire Regt. and I894 Labour Corps

Burrough, Rev. J. C.F. 3 rd Class, R.A.C.D.

Burton, D. C. F. Lieut., Northumberland Fus. ; empld. 1906 Foreign Office

EBut, H. A. Capt., Gloucestershire Regt.

Killed in action 8 fune rgr 6

Calthorpe, Hon. F. S. G. See Gough-Calthorpe, Hon. F. S.

Calvert-Jones, H. F. Capt., R.F.A. and Gen. Staff. $M .1912$

Cameron, A. G. Capt., Argyll and Sutherland Hdrs. and I9I3 Tank Corps. M.C.

Campbell, F. R. M. Capt., 91st Punjabis, Indian Army 1902 CANE, E. G. S. Capt., R.A.M.C. (P.) D.S.O. M. 1904 CARD, F. W. F. Lieut., Ist Dragoon Gds. M.C. 
ECarmichael, D. Capt., Rifle Brigade

Killed in action in the Ypres Salient 25 Sept. 1915

I9II

Carroll, F. H. Capt., Devon Regt.

1907

Carter, J. L. Pte., King's (Liverpool Regt.); Lieut., I9I3 Manchester Regt. (W.)

ACARTWRIGHT, E. Lce.-Corpl., Canadian Infy.

Died II Oct. I9I 6 of wounds received in action

1904

ACARver, L. H. L. 2nd Lieut., Irish Gds.

Killed in action 26 May 1918

1902

Cazalet, G. L. Capt., R. Fusiliers; Brigade Major. 1909 (W 2.) D.S.O. M.C. $M 2$.

Champion, R. E. Cadet, O.C.B.

1910

ACHANDLER, E. S. 2nd Lieut., Sherwood Foresters (Notts. I9I4 and Derby Regt.)

Killed in action near Ypres 14 Feb. 1916

Chandler, F. G. Capt., R.A.M.C.

Chandler, K. S. Capt., Cheshire Regt.(T.F.) (W.) [19I4]

Chandler, R. A. Major, King's (Liverpool Regt., T.F.); 1910 empld. War Office

Charlesworth, M. P. 2nd Lieut., Labour Corps 1914

Chivers, J. S. Lieut., R.F.A.(T.F.) I9I I

Chudleigh, C. A. E. Capt. and Adjt., Leicestershire 1908 Regt. and R. Defence Corps. (W.)

Clark, A. G. Lieut., Highland L.I.(T.F.) (W 2.) 1909

Clark, G. L. Capt., W. Yorks. Regt. (Leeds Rifles, T.F.) I9ro (W.)

Clark, N. M. Lieut., Highland L.I.

Clarke, A. B. Lieut., I.A.R.O., attd. Indian Labour 1896 Corps

Clayden, H. K. Capt., R.A.S.C.

CLEGG, M. T Capt, R.A.C.(T.F) M.

Coaks, H. C. Pte., R. Fusiliers (P. S. Bn.); Lieut., E. 1909 Lancs. Regt. (W.)

WColeman, H. N. 2nd Lieut., N. Zealand Infy. I903

Killed in action 13 April I918

¿Coles, C. 2nd Lieut., E. Lancs. Regt.(T.F.)

Killed in action in Gallipoli 4 Fune I9I 5

Coles, W. T. Lieut., R.A.S.C.

Collier, Rev. A. G. C.F. 4 th Class, R.A.C.D.

Coode, A. T. Cdr., R.N.V.R. Order of St Anne (Russia)

COOPER, J. S. Capt., R.A.M.C.(T.F. Res.)

1907

1914

1903

I 895

1896

ECOOPER-Marsdin, Rev. A. C. C.F. $4^{\text {th }}$ Class, R.A.C.D. 1887

Died Aug. I9I8

Coplestone, W. D. Capt., R.A.M.C.

I 898 
Corfield, B. C. Lieut., R.F.A. $M 2$.

Cox, F. B. H. Lieut., Fife and Forfar Yeo.

$\begin{array}{lr}\text { Cox, F. B. H. Lieut., Fife and Forfar Yeo. } & 1906 \\ \text { Cox, P.H. Pte., R. Fusiliers (P. S. Bn.); Lieut., Durham [1914] }\end{array}$ L.I. and Grenadier Gds. (W.) (P.)

¥Coy, J. C. 2nd Lieut., Durham L.I.; Capt., North- 1909 umberland Fus.

Killed in action 27 Sept. 1918

Craven, A. E. L. Lieut., Wiltshire Regt. and Labour I9I2 Corps. (W.)

Craven, W. L. Pte., R.A.M.C.; Corpl., Labour Corps; 1906 Cadet, O.C.B.

HCreyke, E. R. Capt., King's Own (Yorkshire L.I., T.F.) 1905 $M$.

Killed in action 5 fuly 1916

Cronk, H. L. Capt., R.A.M.C.

ACrookham, H. A. R. Lieut., Cambridgeshire Regt.

1908

Died 4 Aug. 1915 of meningitis following wounds received in action

WCROWE, T.M. Sub-Lieut., R.N.V.R.,(Anson Bn., R.N.D.) 1905 Killed in action in Gallipoli I I June 1915

Cuffe, G. E. Capt., R.E. (Signals). $M$.

CURRIE, J. H. Instructor Lieut., R.N.

I9I I

CutTer, R. C. Capt. and Adjt., R. Fusiliers and Gen. 1905 List (Courts-Martial Officer). (W.)

David, R. S. R. Lieut., Welsh Regt.(T.F.) (W.) 1915

Davidson, W. E. F. Major, E. Yorks. Regt.; Major (A.), I9I I R.A.F. (W.)

Davies, C. E. H. See Hughes Davies, C. E.

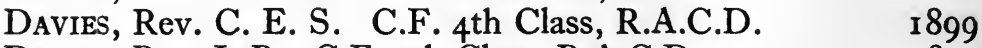

Davies, Rev. J. B. C.F. 4th Class, R.A.C.D. 1897

Davies, J. P. H. Hon. Capt., R.A.M.C.(T.F.) 1902

WDavies, K. G. Lieut., R.E. 1914

Killed in action 19 May 1917

Davies, W. E. Lieut., R.E.

Davis, E. M. Lieut., Middlesex Regt.(T.F.) I9I I

Davy, G. Pte., R. Fusiliers (P. S. Bn.); Major, R.E.; 1908 D.A.D. Roads. M.C. M.

Day, E. C. Capt., King's (Shropshire L.I.) M.C. and 1909 Bar. $M$.

DeEDES, J. Capt., King's (Shropshire L.I.) (P.) I912

DEEDES, W. 2nd Lieut., Spec. List (Asst. Officer i/c 1875 Records)

Delafield, M. E. C. Capt., R.A.M.C. M.C. 
WDENT, A. C. Sergt., R.A.M.C.

Killed in action in Gallipoli 19 July 1915

1910

DE VINE, Rev. C. N. C.F. 4th Class, R.A.C.D. (W.) 1902 M.G. $M$.

सDE VINe, Rev. H. B. St J. C.F. 4th Class, R.A.C.D. I9io Killed in action 27 April 1916

Dexter, E. N. Trooper, W. Kent Yeo.; Lieut., R.F.A. I9I I

Dexter, J. E. Trooper, W. Kent Yeo.; Capt., R. Fusiliers 1909 and Training Res. Bn. (W.)

DeXTER, R. M. Major, R.F.A. M.C.

DigBY-Johnson, N. Lieut., R.A.S.C. (W.) r

Dixon, J. G. Pte., R. Fusiliers (P.S. Bn.); Capt., Wor- [19I4] cestershire Regt.(T.F.) M.C.

Donaldson, A. Capt., 34th Sikh Pioneers, Indian Army 1905 Dore, A. S. W. Major, Worcestershire Regt.(T.F.); I90I

Lieut.-Col. (A.), R.A.F. (W.) D.S.O. M2.

Dower, E. L. Flt. Sub-Lieut., R.N.A.S.

Down I9I3

Drysdale, J. E. Major, R.A.S.C. M.C.

Drysdale, T. Capt., R.A.M.C.

DuckworTh, W. L. H. Capt., R.A.M.C.(T.F.)

Dutfield, D. Capt., London Regt. (R. Fus.) $M$.

WDwyer, C. H. Capt., Worcestershire Regt. $M$.

Killed in action 17 Nov. I916

Drson, E. A. Capt., R.A.M.C.

1905

1906

r 898

1889

I9I I

[1914]

1903

Eason, E. K. Lieut., R. Dublin Fus.

1903

EDGE, A. S. 2nd Lieut., S. Lancs. Regt.; Lieut., R.F.A. I9I I

(T.F.) $M$.

Edmunds, Rev. H. V. C.F. 4th Class, R.A.C.D.

Elliot, R. H. 2nd Lieut., R. Scots

EElliot, C. A. B. 2nd Lieut., Norfolk Regt. and Somerset L.I. (W.)

Killed in action in the Battle of Arras 12 April I917

Ellis RoberTs, R. Cadet, O.C.B.

Elmslie, G. F. Capt., E. Surrey Regt.(T.F.)

EElverson, R. W. Lieut., E. Surrey Regt.

Killed in action 25 Sept. 1915

Ensell, Rev. C. S. C.F. 2nd Class, R.A.C.D. $M . \quad 1889$

EEvers, B. S. Capt., W. Yorks. Regt. (W.) I9Io

Killed in action 14 Sept. 1916

Evors, C. A., T.D. Capt., Middlesex Regt.(T.F.) and 1879

Highgate School O.T.C.

Ewing, J. R. Capt., R. Sussex Regt.

1880 
FABER, L. E. Lieut., R. Fusiliers; Major, M.G.C. (P.) 1896 M.C. $M$.

FaIrbairn, C. O. Capt., Loyal N. Lancs. Regt.; Major 1912 (A.), R.A.F. (W.) A.F.C.

FaIrbairn, C. P. Lieut., Scots Gds. 1905

Fairbairn, G. A. Lieut., I8th Hussars; Capt., Spec. I9II List, empld. Egyptian Army. (W.)

WFairbairn, G. E. 2nd Lieut., Durham L.I.

Killed in action 20 June 1915

Farrell, W. J. Lieut., R.F.A.; Capt., Gen. Staff (In- I90I telligence). M.C.

Faunce-De-Laune, E. 2nd Lieut., R. East Kent Yeo. $\quad 1894$ WFELL, D. M. 2nd Lieut., R.F.A.

Killed in action 17 Fuly r9r6

Finch, A. Capt., Norfolk Regt., R.E., and Gen. List 1890 (Military Port Assistant). (W.)

WFindlay, I. C. 2nd Lieut., York and Lancaster Regt. $\quad 1914$ Died Io Aug. I9I 5 of wounds received in action

Finlow, L. W. Capt., Loyal N. Lancs. Regt. and I9II M.G.C. (W.) M.

FIRTh, M. M. Lieut., R. Wiltshire Yeo. and R.F.A. I9I4

Fisher, B. O. F. DE C. Capt., Norfolk Regt.

Fisher, G. A. Lieut., King's Royal Rifle Corps. (W 2.) I9ro M.C. M.

WFisher, G. W. 2nd Lieut., Suffolk Regt.

Killed in action 18 Nov. I917

FIтch, C. E. Major, Worcestershire Regt.; Lieut.-Col., $\quad$ I889 R. Welsh Fus.(T.F.)

Fletcher, E. Lieut., R.A.S.C.(T.F.) (W.)

Flood, C. B. Capt., Derbyshire Yeo. O.B.E. $M 2$.

WFlower, O.S. Brigade Major; Lieut.-Col., R. Welsh 1890

Fus. $M$.

Died 24 Fuly 1916 of wounds received in action at Mametz Wood

Forman, G. E. G. Capt., Black Watch

Forster, Rev. G. W. C.F. $4^{\text {th }}$ Class, R.A.C.D.

Foster, J. D. Capt., Australian A.S.C.

Foster, J. H. Pte., Middlesex Regt. (P. S. Bn.); Capt.,

R. Fusiliers; Major, King's Own (R. Lancaster Regt.); Lieut.-Col., Highland L.I. and Northamptonshire Regt. (W 3.) $M 2$.

Fraser, J. H. P. Lieut.-Col., R.A.M.C.(T.F.) D.S.O. I89I M.C. and Bar. M3. French Croix de Guerre 
Galloway, Rev. S. J. C.F. $4^{\text {th }}$ Class, R.A.C.D.

GARNE, T. Lieut., Connaught Rangers; attd. R.F.C.

GARRETT, T. R. H. Lieut.-Cdr., R.N.V.R. O.B.E.

Gibson, R. W. B. Capt., R.A.M.C.

$\begin{array}{ll}\text { GiBson, R. W. B. Capt., R.A.M.C. } & \text { I903 } \\ \text { Giffard, J. S. 2nd Lieut., Manchester Regt.; Capt. } & \text { I908 }\end{array}$ (K.B.), R.A.F. M. Belgian Croix de Guerre

Gillson, G. Brig.-Gen., R.A. (W.) G.M.G. D.S.O. 1886 Brevet Colonel. M 6 . Order of Danilo, $3^{\text {rd Class }}$ (Montenegro)

Gilman, J. Major, R.A.S.C. $M$.

WGoldsmith, H. M. Lieut., Devon Regt.; attd. Lincoln- 1904 shire Regt.

Killed in action at Fromelles 9 May 1915

Goodacre, Rev. J. C.F. 3 rd Class, R.A.C.D.(T.F.) 1894 M.C. $M$.

Goodchind, H. N. Lieut., R.F.A.; Lieut. (Ad.), R.A.F. 1908

Goodman, E. L. Lieut., R.N.A.S.

Goodwin, H. A. C. Capt., S. Lancs. Regt. and Gen. List 1907

(T.F. Res.); Instructor, School of Musketry

WGoodwin, H. J. 2nd Lieut., R.G.A.

Killed in action 24 April I9I7

Goss, J. Capt., R.A.M.C.

1905

1902

1899

I 898

Gough Calthorpe, Hon. F. S. Lieut., Staffordshire

Yeo.; Capt., Spec. List (P. and B.T. Staff)

Gould, R. Gnr., H.A.C.; Capt., R.F.A. $M$.

Graham, H. E. Capt., R.A.M.C.

1905

1896

I9I I

I9II

1896

AGraham-Montgomery, G. J. E. Capt., Hampshire Regt. I9I2 Killed in action 24 April I917

WGrasetT, E. B. Lieut., 28th, attd. 33rd, Punjabis, Indian * I908 Army

Killed in action 25 Sept. I9I 5

Gray, A. K. Capt., Spec. List (Graves Registration 1907 Commission)

Gray, Rev. R. H. C.F. 4th Class, R.A.C.D.

GrAY, S. T. Capt., I.A.R.O., attd. 58th Vaughan's Rifles. 1909 (W.)

GreEn, W. R. C. Capt., The Queen's (R. W. Surrey I9I3 Regt.) (P.)

Greenham, R. G. H. Lieut., Army Pay Dept. 1905

Grenfell, E. B. Capt., D. of Cornwall's L.I. (T.F.); I9II Capt. (A.), R.A.F. 
Grigg, G. H. Trooper, R. North Devon Yeo.; Lieut., [1914] N. Irish Horse and R.F.A.

Grigg, J. H. Trooper, R. North Devon Yeo.; Lieut., [1914] N. Irish Horse

GripPer, A. G. Sergt., R.E. 1913

Grogan, E.S. Major, E. African Field Force. D.S.O. 1893 $M$ 2. Officer, Order of Leopold (Belgium)

Gross, W. S. Sergt., Middlesex Regt.; 2nd Lieut., Wor- r9 I I cestershire Regt.; Lieut., R.A.M.C. (W.)

GrubB, H. C. S. Pte., R. Fusiliers (P. S. Bn.); Lieut., R. 1912 Irish Fus. and R.E. (Signals). M.C. and Bar

Gullick, C. D. Capt., The Buffs (E. Kent Regt.) (W 4.) I9I I M.C. and Bar

Guy, O. V. Capt., W. Yorks. Regt.; Major, Tank Corps. 1910 (W 2.) D.S.O. M.C. and Bar. M. Chevalier, Legion of Honour (France)

HabGOOD, A. H. Major, R.A.M.C. D.S.O. $M$.

HACKWORTh, A. Gnr., H.A.C.; Lieut., R.F.A.; attd. 1909 T.M.B. (W.) $M$.

WHaldane, J. O. 2nd Lieut., Rifle Brigade

Killed in action 18 Aug. I9r6

Haldane, M. M. Major, R. Scots (R. of O.); G.S.O. 2, 1896

War Office. Brevet Lieut.-Colonel. $m$.

Hales, Rev. J. P. C.F. 2nd Class, R.A.C.D.; D.A.C.G. 1888 D.S.O. $M 2$.

WHamilton, N. C. Pte., London Regt. (Artists Rifles); 1912 and Lieut., Northamptonshire Regt. (W.)

Killed in action 14 Fuly I9I6

HANDS, W. J. G. Major, R.A.S.C.

HANSARD, L. A. S. Sub-Lieut., R.N.V.R.

Harcourt, Rev. G. H. Chaplain, R.N.

Hardwick, F. 2nd Lieut., London Regt. (Post Office Rifles); Major, R.E. (Postal Section). M.B.E. $M$.

Hardy, F. W. Colonel, R.A.M.C. $M 2$.

I9II

1913

1903

I910

HARRIS, A. L. S. 2nd Lieut., Oxford and Bucks. L.I. (T.F.); Lieut., R. Marines

Harrison, W. P. 2nd Lieut., W. Kent Yeo.; Lieut., Life 1904 Gds.

Harry, N. G. Capt., Worcestershire Regt.(T.F.) and 1896 R.A.M.C.

Hartley, B. C. Lieut., Hertfordshire Regt.; empld. War 1897 Office. (W.) 
WHARVEST, G. L. Lieut., London Regt. (L.R.B.) M.C. [1914] Killed in action 20 fune 1917

HawDON, H. W. Lieut., Durham L.I. (W.)

1909 HAwDon, Rev. N. E. C.F. $4^{\text {th }}$ Class, R.A.C.D.

Died 16 Nov. 1918 of pneumonia contracted on active 1905 service

HawkINS, O. C. Major, R.E. (T.F.); Staff Capt., War 1908 Office. (W.) $m$.

Hawkins, O. L. 2nd Lieut., E. Yorks. Regt.

Died 26 April 1915 of wounds received in action

Hay, A. C. Capt., Loyal N. Lancs. Regt. (W.) 1907

HEAD, H.G. Capt., London Regt. and Tank Corps. M.C. 1907

HEARFIELD, J. 2nd Lieut., R.A.S.C.

Hearn, R. J. Capt., R.A.M.C.; Capt. (Med.), R.A.F. 1908 (W.) $m$.

HeARSEY, G. A. C. Lieut., I.A.R.O. (Cavalry) 1905 HeAthcote, G. S. Capt., Sherwood Foresters (Notts. 1902 and Derby Regt.); Major, Camp Cmdt. M.V.O. $M 2$.

HENDERSON, J. A. Major, 8th (King's R. Irish) Hussars 1883 (R. of O.); A.P.M. D.S.O. O.B.E. M 4 .

HenTy, E. C. Capt., R.A.S.C.; Lieut., R.F.A. (W.) M. 1906 HiggiNs, W. G. Capt., 2nd Dragoon Gds.(Queen's Bays). 1913 $M$.

Higson, F. Lieut.-Col., Norfolk Regt. O.B.E. $M . \quad 1899$

HILL, H. P. Major, Hampshire Regt.(T.F.) 1907

HobBs, C. R. Trooper, K. Edward's Horse; Major, I9Io R.F.A. (W.) $M$.

Hobson, F. W. E. 2nd Lieut., Labour Corps 1902 Hobson, R. I. Lieut., London Regt. (Civil Service 1890 Rifles); empld. Admiralty,

Hodgsón, C. L. Major, King's Own (R. Lancaster Regt.) $\quad 1898$ and Gen. Staff (O.C.B.) (W.) $m$

HodGSON, E. J. Lieut., S. Staffs. Regt.; attd. Leicester- 1913 shire Regt.; Lieut., M.G.C. (W.)

Holland, Rev. P. F. Pte., R.A.M.C.

1905

1907

Killed in action 27 Sept. 1915

Holloway, N. J. Lieut., R. Fusiliers (P.S. Bn.); Capt., 1909 R.E.

Holman, A. H. Lieut., R.N.V.R. 1908

Holman, P. Pte., H.A.C.

Killed in action 17 Feb. 1915

HoLMDEN, F. A. A., D.s.o. Major, R.A.M.C.

1910

1889 
Holme, H. R. Lieut., King's Royal Rifle Corps.; Capt., 1908 M.G.C. (W.)

Holmes, J. C. Capt., R.E. M.C. M.

Holt, E. Lieut., Oxford and Bucks. L.I.; Capt., R. [I9I4] Warwickshire Regt. (W 2.) M.C.

Hood, H. M. Capt., I.A.R.O., attd. 28th Light Cavalry 1903 HHopcraft, E. G. DE L. Lieut., Middlesex Regt.

Killed in action 27 Sept. I918

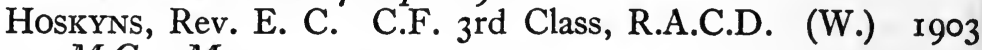
M.C. $M$.

Hovil, R. Major, R.F.A. D.S.O. M 2.

HowARD, J. Capt., R.E.(T.F.)

Hoyte, W. N. Lieut., Sherwood Foresters (Notts. and Derby Regt.) M.C. and Bar

Hudson, H. C. H. Major, IIth Hussars; Lieut.-Col., 1906 A.Q.M.G. (W.) M.V.O. Brevet Major. M2. Chevalier, Legion of Honour (France). French Croix de Guerre. Chevalier, Ordre de la Couronne (Belgium). Belgian Croix de Guerre

Hughes, J. W. C. Paymaster Sub-Lieut., R.N.V.R.

Hughes Davies, C. E. Capt., R.F.A.; Staff Lieut. (W.) I909 M.C.

Hughes-Hallett, N. M. 2nd Lieut., Worcestershire [I914] Regt.; Capt., King's (Shropshire L.I.) (W.)

Hulton-Sams, K. A. Pte., Oxford and Bucks. L.I. $\quad$ I903 Hutchinson, G. Capt., R. Inniskilling Fus. 1908

INGLes, Ven. C. W. C. Chaplain of the Fleet, R.N.

IremoNGer, E. A. Lieut.-Col., Suffolk Regt.; Colonel r88I i/c Records; empld. Ministry of National Service. C.B.E. $m$.

IRONS, W. H. Sergt., R.E.

JACKson, A. Lieut., E. Yorks. Regt.

J JACkson, D. F. Lieut., Lanarkshire Yeo. (W.) M 2. 1906 Killed in action I I Oct. I9I8

JACOBY, A. H. M. 2nd Lieut.(T.), R.A.F. 1903 JAMES, H. M. Capt. and Adjt., Middlesex Regt. (W.) 1903 M.C.

JAQueT, Rev. E. G. C.F. 4th Class, R.A.C.D.

WJEFFCOCK, H. C. F. Lieut., Sherwood Foresters (Notts. 1904 and Derby Regt.)

Died 30 May I9I 7 of wounds received in action 28 May r9I7 
JefFCOCK, P. E. Lieut. (T.), R.A.F.

JEFFCOCK, W. H. C. 2nd Lieut., York and Lancaster 1907 Regt; Lieut., W. Yorks. Regt. (W.)

JENkin, R. T. Lieut., I.A.R.O., attd. S. and T. Corps 1906 Jenkins, Rev. C. E. C.F. $4^{\text {th }}$ Class, R.A.C.D.(T.F.) $\quad{ }^{*}{ }^{2} 896$ Jenkins, R. E. Capt., Hampshire Regt.(T.F.)

JENNINGS, A. R. Capt., R.A.M.C. M. Order of St Sava, 1904 $4^{\text {th }}$ Class (Serbia)

Jennings, R. W. Lieut., Worcestershire Regt. $M$.

Died 3 Fuly I9I6 of wounds received in action at La Boisselle

JeRwOOD, Rev. F. H. Capt., Oakham School O.T.C.

1905

JJerwood, J. H. Pte., London Regt. (Artists Rifles); 1909

Capt., Durham L.I.; Major, Somerset L.I. (W 2.) M.C.

Killed in action 21 March 1918

WJohnson, G. B. Capt., Norfolk Regt.

Killed in action 22 Nov. 1915

Johnson, N. D. See DigBY-Johnson, N.

Johnstone, C. C. G. Capt., Argyll and Sutherland Hdrs.; 1908 Major, R. Scots. (W.)

JoHNSTONE, W. Lieut., Coldstream Gds. (W.) 1907

JorceY, J. Major, N. Somerset Yeo.

JoICEY, Hon.J.A. Major, Northumberland Fus.(T.F.) $m$. $\quad$ I 898

JoNES, C. E. Lieut., I.A.R.O. I9IO

JONES, E. W. and Lieut., Welsh Regt.

Died 9 Nov. I915 of dysentery contracted on active service

JONES,H.A. Lieut.-Col.,R.A.S.C.;Brig.-Gen.,D.Q.M.G. $\quad$ I898 Brevet Lieut.-Colonel. m. Chevalier, Legion of Honour (France)

Jones, J. W. B. 2nd Lieut., Labour Corps

1900

Keats, J. R. Capt., Suffolk Yeo. (W 2.) M.C.

KeBLE, T. H. Lieut., The Buffs (E. Kent Regt.); Capt., [1914] G.S.O.3. $M$.

Kemp, Sir K. H., Bart. Colonel, Norfolk Regt. C.B.E. I87I $m$.

KENNEDY, Rev. D. J. C.F. 3 rd Class, R.A.C.D.

KIDD, W.A. T. Lieut., R. Sussex Regt.; Capt., R.E. M. 1907 KING, W. B. R. Capt., R. Welsh Fus.(T.F.); S.O. to 1908 Engineer-in-Chief. O.B.E. $M 2$.

KIRKPATRICK, J. B. Capt., Rifle Brigade; empld. O.C.B. $\quad$ I9I2 (W.) 
Krtching, A. E. E. African Field Force

Kitching, G. C. Capt., London Regt.(L.R.B.) (P.)

Knight, C. E. Capt., R.F.A.

WKoettgen, E. A. Pte., London Regt. (London Scottish) I 892 Killed in action 30 fune 1917

Krause, E. H. Lieut., Durham L.I. and Gen. List. I9I I (W 2.)

KutNow, H. S. Lieut., R.A.O.C.

Laistner, M. L. W. Pte., Middlesex Regt.

LANG, R. Pte., R. Fusiliers(P.S.Bn.); Lieut.(A.), R.A.F. [I9I4] (W.)

ELAUDER, J. C. Capt., Argyll and Sutherland Hdrs. (W 3.) I9ro Killed in action 29 Dec. I9I6

LEA, D. H. Pte., M.G.C. (W.)

Leale, R. J. Capt., R. Guernsey Militia

WLeary, G. G. W. Lieut., Gloucestershire Regt.

Killed in action 25 Sept. I915

LeEs, J. Capt., Queen's Own (R. W. Kent Regt.) and I878 Spec. List (Adjt., Rest Camp). $M$.

LE Gros, F. G. 2nd Lieut., R. Jersey Militia; Lieut., 1912 R.G.A.

Le Neve Foster, B. A. J. C. Sergt., R. Fusiliers

1912

LetTs, C. F. C. Major, Rifle Brigade. (W 3.)

1900

1910

1910

ALevick, P. Capt., R.A.M.C.

Accidentally killed on active service 21 March I9I8

Lias, W. J. Capt., Lancs. Fus. and R.E. $M$.

1907

1892

Light, P. Capt., Cheshire Regt. M.C.

1886

Lilly, C. O. Capt., Dorset Regt.; G.S.O. 3 ; Hon. Capt. 1908 (A.), R.A.F. (W 2.) D.S.O. $M$.

Livingston, P. C. Surgeon Prob., R.N.V.R.; Capt. 1912 (Med.), R.A.F.

Lloyd, B. S. Lieut., R.G.A.

Lloyd, H. W. C. Capt., Wiltshire Regt.; G.S.O. 3. (P.) I910 D.S.O. M.C. M. French Croix de Guerre

Lloyd-Barrow, R. A. Capt., R.F.A.

Lloyd George, G. Lieut., R. Welsh Fus.; A.D.C.; I9I3 Major, R.G.A. $M$.

Lockhart, J. H. B. 2nd Lieut., Seaforth Hdrs. and Gen. 1908 Staff. $M$.

LoEwE, L. L. Lieut., Oxford and Bucks. L.I. (T.F.) and I9I I R. Sussex Regt. (W.)

Loveband, G. Y. Capt., R. Dublin Fus. $M$. 
Low, A. Capt., R. Irish Fus.; Major, M.G.C. (W.) 1912 M.C. $M$.

Lowe, P. R. Capt., R.A.M.C. $M$.

1887

LuCE, H. W. Capt., R.F.A.(T.F.)

1913

WLunN, H. C. Sergt., R. Fusiliers (P. S. Bn.); Lieut., I908 Northumberland Fus. and R. Scots. (W.)

Killed in action 22 March 1917

Macalpine, J. L. Lieut., 9th Lancers. (W.) M.C. 1904

McBarn, N. S. Capt., R.F.A.(T.F.)

WMacBryan, E. C. Lieut., Somerset L.I. (W.)

1913

Killed in action I Fuly 1916

1912

McCAughey, S. Lieut., R.F.A.

McConnel, A. E. Capt., R. Irish Rifles. $M$.

McCorquodale, K. Capt., Lovat's Scouts. M.C.

McGowan, I. A. W. Capt., R.F.A.(T.F.) M.C.

McIlroy, H. D. Surgeon Prob., R.N.V.R.; Capt., R.A.M.C. (W.)

WMack, I. A. Capt., Suffolk Regt.; attd. T.M.B. Killed in action I fuly 1916

Mackay, A. S. Major, 7 th Gurkha Rifles, Indian Army. 1909 M.C. $M$.

Mackie, E. D. Pte., R. Fusiliers (Sportsman's Bn.)

1888

AMcLAughlin, A. Lieut., R. Irish Rifles

Killed in action 9 May 1915

Maclean, F. S. Trooper, K. Edward's Horse; Lieut., I9I I R.F.A. (W.)

McNeIL, J. Sub-Lieut., R.N.V.R.

MacnutT, Rev. A. C. C.F. $3^{\text {rd }}$ Class, R.A.C.D.

1907

1898

MaInPRICE, H. Capt., King's Royal Rifle Corps and Gen. 1902 Staff. $m$.

ZMartland, J. D. Pte., R. Fusiliers (P.S. Bn.); 2nd Lieut., I9ro D. of Wellington's (W. Riding Regt.)

Killed in action 22 Feb. 1916

Manderson, J. R. 2nd Lieut., Devon Regt.(T.F.); Capt., 1890 Argyll and Sutherland Hdrs.

Manduell, M. D. Capt., R.F.A. M.C. French Croix 1896 de Guerre

Manifold, E. W. Lieut., R.F.A. M.C.

MaNifold, J. Lieut., R.F.A.

WManN, R. L. Lieut., 7 th Dragoon Gds. Killed in action at Festubert 23 Dec. I9I4

Manning, R. Capt., Buckinghamshire Yeo.and M.G.C. 1908 M.C. M. French Croix de Guerre

I9I I

1907

I9I0

C. U. W.L. 
Manning, T. E. Major, Northamptonshire Yeo. $M . \quad 1902$

Marnham, A. E. Lieut.-Col., R.G.A. M.C. $M . \quad$ I909 Chevalier, Order of Leopold (Belgium). Belgian Croix de Guerre

Marshall, G. T. Sergt., R. Welsh Fus.

Marshall, Rev.H.A. C.F.3rd Class, R.A.C.D. (W.) M. ${ }_{1902}^{1913}$

Martin, B.S. Lieut., M.G.C. (Motor) and Tank Corps. I9Io (W.)

Masefield, Rev. W. B. Chaplain, R.N.

WMASON, K. R. 2nd Lieut., Suffolk Regt.(T.F.) Killed in action $2 \mathrm{I}$ Fune I9I 5

*Mason, R. S. 2nd Lieut., Rifle Brigade Killed in action 14 March $19 \mathrm{I} 5$

WMassey, J. H. Capt., R.F.A. (W.) M.C. French Croix 1912 de Guerre

Killed in action 27 May 1918

Matthews, N. H., T.D. Major, R. Defence Corps $\quad 1870$

Meakin, F. G. Lieut., R.F.A.(T.F.) 1903

HMeeres, H. W. H. Lce.-Corpl., R. Fusiliers Killed in action 19 March 1915

Mellor, J. E. Capt., Leys School O.T.C.; empld. O.C.B. 1903

WMEREDith, O. W. W. H. 2nd Lieut. (A.), R.F.C. I9I4 Killed in action near Cambrai 20 Nov. 1917

Milburn, B. Capt., Hertfordshire Regt. and Coldstream Gds. (W 2.) D.S.O. M.C. M 3 .

Milburn, W. H. Capt., Yorkshire Regt.; Major, Em- 1902 barkation S.O.

Miller, C. P. Lieut., Yorkshire Regt.

Mills, H. J. F. Capt., King's Royal Rifle Corps. (W.) I913 (P.)

WMrtchell, A. C. O. 2nd Lieut., S. Wales Borderers. M. 1905 Killed in action in Mesopotamia 30 April I9I7

Mitchell, S. Major, Fife and Forfar Yeo.(T.F. Res.) 1903 and Gen. Staff. $m$.

Mitchelson, Rev. J. K. C.F. 4th Class, R.A.C.D. (P.) 1907

Mitford, A. H. Capt., York and Lancaster Regt. and 1884 Gen. List. (W.)

Mitford, C. W. 2nd Lieut., Shrewsbury School O.T.C. 1904

WMoffitT, J. P. Capt., Durham L.I. M.C. I9I I Killed in action 3 Dec. 1917

Monks, G. Lieut., R. Scots. (W.)

MONTGOMERY, H. K. Lieut., Black Watch. (W.) I9I4

Moore, H. M. M. Lieut., R.G.A. 1906

Moore, N. Lieut., Intelligence Dept., Uganda Force 1907 
Morgan, R. T. Lieut., R.F.A.(T.F.); attd. Ordnance I897 College

Morgan, T. D. Capt., R.A.M.C.

Morley, G. C. Trooper, Gloucestershire Yeo.

AMorRIs, A. C. Trooper, I2th Lancers; Pte., R. Munster 1912 Fus.

Killed in action 27 Nov. I9I 5

Morris, G. G. 2nd Lieut., Sherborne School O.T.C. * *I9I I

MorRIson, M. J. Capt., Durham L.I.(T.F.) (W.) M.C. 1912

Mortlock, Rev. C. B. C.F. 4th Class, R.A.C.D. *igi I

Morum, S. D. Capt., Rifle Brigade. (W.) I9I4

WMoss, F. W. Pte., Suffolk Regt.; 2nd Lieut., Leicester- I9I3 shire Regt.; attd. R.E.

Killed in action 28 May 1918

Mosse, Rev. C. H. C.F. 4th Class, R.A.C.D.

Muir-Mackenzie, K. J. Pte., H.A.C.; Lieut., R. Munster I9OI Fus.

WMuir-Mackenzie, Sir R. C., Bart. Lieut., Durham L.I. I9ro M.C.

Killed in action 12 April 1918

MUUmFoRD, P. S. Capt., London Yeo. (Westminster 1912 Dragoons); Hon. Capt. (Ad.), R.A.F.

WMurray, M. A. Pte., Middlesex Regt. (P.S. Bn.); Lieut., [19I4] Essex Regt.

Killed in action in the Battle of Loos 25 Sept. r9r 5

WMurRaY, R. L. Capt., Northamptonshire Regt.(T.F.) I9r2 (W.) $M 2$.

Killed in action 19 April I917

Musson, T. M. B. Pte., King's (Liverpool Regt., Liver- I9ro pool Scottish, T.F.); Lieut., R. Welsh Fus. and Essex Regt. (W.)

AMyddelton, E. G. 2nd Lieut., Suffolk Regt.

Killed in action at Le Cateau 26 Aug. 1914

NALDER, F. W. Lieut., Somerset L.I.

NALDRETT, H. C. Lieut., Essex Regt. (T.F.); Lieut. (A.) I9r3 R.A.F. (W.)

NapIER, Sir J. W. L., Bart. Pte., London Regt. (London 1913 Scottish); Lieut., S. Wales Borderers. (W 2.) (P.)

WNAPIER, Sir W. L., Bart. Major, S. Wales Borderers 1886 Killed in action in Gallipoli 13 Aug. 1915.

Nathan, A. A. Capt., Hampshire Yeo.; Major, R.A.F.; 1905 S.O. 2, Air Ministry. $M$. $m$.

Neale, J. B. Capt., R.A.S.C. $M$. 
WNevill, W.P. Capt., E. Surrey Regt.; attd.E. Yorks. Regt. Killed in action I fuly I9I6

Neville, G. J. E. P.O., R.N.V.R.; 2nd Lieut., R.A.S.C. 1904 (M.T.)

Newham, C. E. Capt., Army Cyclist Corps. (W.)

Newling, A. J. Lieut., London Regt. (Finsbury Rifles); Hon. Lieut. (T.), R.A.F.

Newman, F. H. C. Capt., R.A.S.C.

Nicholas, F. P. Surgeon Sub-Lieut., R.N.V.R.

सNichols, W. H. Major, Somerset L.I.

Died in Germany 15 Oct. I9I 5 of wounds received in action in the Battle of Loos 26 Sept. I9I 5

Norman, T.V. 2nd Lieut., Northamptonshire Regt. (W.) I9II

North, G. D. Lieut., Life Gds. (W.) 1912

Nowell-Usticke, G. W: Capt., R.F.A. M.C. I9I3

Nutman, B. K. Lieut., R.A.M.C. 1900

Oldham, E. A. S. Gnr., H.A.C.; Capt. and Adjt., Sea- 1912 forth Hdrs.(T.F.) (W.)

OlIVER, H. C. Instructor Lieut., R.N.

OPPENHEIMER, K. M. 2nd Lieut., Durham L.I.; Lieut., I9I I I.A.R.O.

OrR, H. R. Pte., Canadian Force, empld. Records

Osborne, E. C. Lieut., Black Watch. (W.)

OwEN, T. Surgeon Lieut., R.N.

Owston, C. S. 2nd Lieut., King's Royal Rifle Corps; 1908 Lieut., R.F.A.; empld. Records

Palmer, A. S. M. Capt., R.A.M.C.

Palmer, F. W. M. Capt., R.A.M.C.

सPARKe, A. Capt., Lancs. Fus. (W 2.) M.C. I9II

Killed in action 27 Sept. I918

Parker, H. V. Capt. and Adjt., R.F.A. (W.) M.C. I9Io

PARKes, E. B. H. Capt., R.A.S.C. (W.) I886

Parmenter, Rev. G. E. P. Chaplain, R.N. I9I I

APassingham, E. G. Pte., London Regt. (Artists Rifles); 1913

Capt., Northumberland Fus. (W.) M.C.

Killed in action at Monchy-le-Preux 3 May 1917

Paterson, G. A. R. Capt., Gordon Hdrs.(T.F.) M.C. I9I I

WPatterson, R. A. Capt., Rifle Brigade. (W.) I9I4 Killed in action 12 April 1917

Pattinson, L.A. Lieut., Durham L.I.(T.F.) and R. Fusi- 1909 liers; Lieut.-Col.(A.), R.A.F. D.S.O. M.C. D.F.C. $M$. 
WPaYne, G. H. Lieut., Suffolk Regt.

I9I I

Killed in action at Le Cateau 26 Aug. 1914

Peacock, W. M. Capt., Leicestershire Regt.(T.F.) (W.) 1909

PEACOCK, W. T. Lieut., R.A.S.C.(M.T..)

APEASE, M. R. Lieut., E. Yorks. Regt.

Killed in action 20 Oct. 1914

Pedley, C. F. Lieut., R.A.M.C.

Pegg, H. G. Pte., London Regt. (Artists Rifles); Capt., 1909

Argyll and Sutherland Hdrs. and Gen. List. (W.)

Pennell, K. E. L. Capt., R.E. M.C. M 2. 1908

PenNy, C. J. Surgeon Prob., R.N.V.R.; Capt., R.A.M.C. I9I I

O.B.E. M.

Penny, G. S. Capt., Spec. List (Intelligence) 1904

Penny, W. M. Capt., R.A.M.C. (W.) M.C. 1903

Peters, B. A. I. Lieut., R.A.M.C.

1Peters, J. H. G. Lieut., The Buffs (E. Kent Regt., T.F.); I9I3

Capt., 5 Ist Sikhs, attd. I 52nd Punjabis, Indian Army.

(W.)

Petro, J. A. W. Capt., R.F.A.(T.F.); Cmdt., P. of W. ${ }^{1913}$

Camp; empld. War Office

Phayre, Sir A. Lieut.-Gen., Indian Army. K.C.B. $\quad{ }_{1876}$

Phelps, M. N. Capt., R. Warwickshire Regt.(T.F.); 1889

A.P.M. M. Officer, Ordre de l'Étoile Noire (France)

Phipps, C.E. Lieut.-Col., R.G.A.; Dep. Chief Inspector, 1884 Woolwich Arsenal. C.B. $m$.

सPIGG, B. W. Sergt., H.A.C.; 2nd Lieut., Worcestershire 1907 Regt.

Killed in action at La Boisselle 3 July 1916

Pigg, C. H. Capt., Worcestershire Regt. and Gen. List; 1906 Brigade Major. (W.) M.C. $m$.

Pink, H. S. A.B., R.N.V.R.(R.N.D.); Capt., Sherwood 1906 Foresters (Notts. and Derby Regt.)

Plummer, W. F. 2nd Lieut., London Regt. (London Irish I9I4

Rifles)

Killed in action ${ }_{5} 5$ Sept. r916

Polmill, S. F.P. Corpl., R.E.; Lieut. (A.), R.A.F. M2. I9ro

WPREEDy, A. Capt., Devon Regt.

Killed in action I I Fuly 1916

Prior, Rev. C. B. C.F. $4^{\text {th }}$ Class, R.A.C.D. M.C.

Pryce, E. O. Trooper, K. Edward's Horse; Capt., R.F.A. I9I3

(W.) M.C.

Pryce-Jones, Sir E., Bart. Hon. Colonel, R. Welsh Fus. $\quad 1880$ Pryce-Jones,P.V. Capt.,Welsh Horse ; attd.Cheshire Yeo. 1906

1 Killed in action on the N.W. Indian frontier after the armistice. 
Pryor, J. W. Lieut., S. Lancs. Regt. (W.) (P.) [1914]

Purdy, T.W. Major, Norfolk Regt.(T.F.) (W.) T.D. m. ${ }^{1893}$

APyman, R. L. Lieut., Middlesex Regt.

Killed in action 3 May 1917

PyPer, J. R. Capt., London Regt. (R. Fus.); attd.M.G.C. I9II (W.) M.C. and Bar. M.

Quiller-Couch, Sir A. T. Capt., D. of Cornwall's L.I. *'1912

RaImes, E.A. Lieut., Staffordshire Yeo. and W. Yorks. I9I I Regt.; empld. War Office

Raimes, G. H. Lieut., Yorkshire Dragoons; attd. 21st 1914 Lancers and 14 th Hussars

RatclifF, R. F., M.P., v.D. Lieut.-Col., N. Staffs. Regt. 1886 (T.F. Res.) C.M.G. $m 2$.

Ravenscroft, P. D. Corpl., R. Fusiliers; Capt., King's 1908 Royal Rifle Corps. (W.) M.C. $M$.

Rawes, P. L. Lieut., R.A.S.C. M. Chevalier, Military 1902 Order of Avis (Portugal)

Rawstorn, J. O. Capt., R. Welsh Fus.(T.F.) (W.) I910

ReA, J. G. G. Capt., Northumberland Yeo. and Labour 1904

Corps. (W.) D.S.O. M 2. Officer, Ordre du Mérite Agricole (France)

READ, C. F. 2nd Lieut., R. Sussex Regt.

Read, J. W. Lieut., R. Sussex Regt.

READ,W. R. Capt., Ist Dragoon Gds.; Major (A.), R.A.F.

(W.) M.C. D.F.C. A.F.C. M.

ReED, D. H. Capt., Devon Regt.(T.F.) (W.)

ReID, C. H. Lieut., 5th Dragoon Gds.; Capt., M.G.C. (Cavalry). M.C. $M$.

Reid, J. S. C. Lieut., R. Scots; Major, M.G.C.

Rendell, W. R. Capt., Cambridge Univ. O.T.C., attd. Gen. Staff, War Office Ricardo, A. ST Q., D.s.o. Lieut.-Col., R. Inniskilling 1886

Fus.; Brig.-Gen.; Colonel, Gen. Staff (Base Cmdt.)

C.M.G. C.B.E. Brevet Lieut.-Colonel. M 7 .

Richardson, A. Gnr., R.H.A.; Lieut., R.F.A. M.C. $\quad$ I9I5

Richardson, A. J. M. Capt., N. Somerset Yeo. I9I3

Richardson, P. J. Corpl., Calcutta Vol. Artillery; Capt., I9Io King's African Rifles. $M$.

Ridley, A. H. Lieut., Northumberland Yeo.

ARIDleY, C. N. Capt., Northumberland Yeo.

Died 7 Oct. I91 5 of wounds received in action 30 Sept. 1913 1909 1908 1887 1915 
WRIDLEY, L. E. Lieut., R. Berkshire Regt.

1910 Killed in action 18 Aug. I916

Ridout, P. M. Capt., Oxford and Bucks. L.I. (W.) r9r3

RoberTs, A. H. Lieut., Middlesex Regt. and Gen. List, I 909 empld. Inland Waterways and Docks. (W.)

RoberTs, A. J. R. Lieut., Mill Hill School O.T.C. 1900

WRoBerts, F. B. Capt., Rifle Brigade I90I

Killed in action $8 \mathrm{Feb}$. 1916

RoberTs, R. E. Major, Manchester Regt. $\quad 1900$

Robinson, G. H. Pte., R. Fusiliers (Sportsman's Bn.) I887

RoDERICK, D. B. 2nd Lieut., Rifle Brigade 1916

Rolfe, W. J. L. Lieut., R.E. (Signals) I9I 5

Roscoe, G. T. Lieut., R.A.S.C.(T.F.); attd. Worcester- I913 shire Regt.

Ross, O. F. Capt., N. Staffs. Regt.

WRowe, H. P. Capt., R.E. (Signals)

Died 6 Nov. I91 8 of pneumonia

Rowe, W. H. C. Lieut.-Col., R.A.S.C. C.B.E. O.B.E 1902 $M 5$.

WRowsell, H. G. Pte., R. Fusiliers (P. S. Bn.); Capt., 1907 Hampshire Regt.

Killed in action I I Sept. r916

WRoyle, J. B. Major, S. Wales Borderers

Killed in action 15 Fan. I917

RubIE, T. A. C. Lieut., Uganda Protectorate Force and Unattd. List, T.F.

ARuck-KeENe, R. E. Lieut., R. Welsh Fus.

Accidentally killed 24 Fan. I9I 6

I913

1908

RRudD, K. S. Pte., King's (Shropshire L.I.); Capt. and

Adjt., W. Yorks. Regt. (W.)

Killed in action ro Oct. 1918

Russell, A. Major, R.G.A. (W.) M.C.

RUTHERFORD, G. L. Capt., Durham M.C. $M$.

Ryan, C. M., D.s.o. Colonel, R.A.S.C.; Brig.-Gen., I885 Director of Supplies and Transport. C.M.G. C.B.E. $M$ 2. Officer, Legion of Honour (France)

SADLER, H. Major, 6th Dragoon Gds. (Carabiniers); 1909 G.S.O. 2. French Croix de Guerre

SAlE, H. G. Lieut., Northumberland Fus. (W.) 1912

Salmon, H. B. 2nd Lieut., R.G.A.; Staff Lieut. I9ro

Salmon, L. H. Sergt., King's Royal Rifle Corps; Capt., Igor Worcestershire Regt. 
\#SAnderson, G. E. Pte., R. Fusiliers (P.S. Bn.); Lieut., I907

R. Irish Rifles; attd. M.G.C.

Killed in action 15 Fuly I9r6

SANER, J. D. J. Capt., R.E. (Signals, T.F.) M.C. M. ${ }_{1913}$

*SARSBY, R. A. 2nd Lieut., Norfolk Regt.

Killed in action 31 Dec. I9I 5

WSAunders, C. P. G. Capt., Loyal N. Lancs. Regt.

Killed in action at Eaucourt l'Abbaye 28 Sept. 1916

1912

I9I4

4SAVORY,H.L.S. 2nd Lieut., Durham L.I.and SuffolkRegt. 1916 Killed in action 26 April 1918

सSchif, M. E. H. Capt., Suffolk Regt.

Killed in action at Gouzeaucourt 25 Sept. 1917

ScotT, D. A. 2nd Lieut., King's Royal Rifle Corps

Scott, Rev. F. E. C.F. $4^{\text {th }}$ Class, R.A.C.D.

Scotr, Rev. H. P. F. Chaplain, R.N.

Scotr, S. C. Pte., R. Fusiliers (P. S. Bn.); Capt., Essex

Regt. and I.A.R.O., attd. Iogth Infy. (W.) M.C.

ScotT, W. Capt., R.A.S.C. $m 2$.

1907

Sendell, C. H. Pte., London Regt. (Artists Rifles); Lieut., R.F.A. (W.)

Senior, F. G. Capt., E. Yorks. Regt.; A.M.L.O. M. I891

Seymour, Rev. A. G. Chaplain, R.N.

Shackle, S. A. Capt., Spec. List (Remount Service)

Sharp, Rev. J. S. C.F. 4th Class, R.A.C.D.

Sharpe, Rev. E. C.F. 4 th Class, R.A.C.D.

Shelmerdine, Rev. T. G. C.F. 4th Class, R.A.C.D.

Shelton, O. W. M. Lieut., R.A.S.C.; attd. King's Own

(Yorkshire L.I.)

Shenton, Rev. J. S. C.F. $4^{\text {th }}$ Class, R.A.C.D.

Shera, F. H. Capt., Malvern College O.T.C.

WShIELDS, H. J. S. Lieut., R.A.M.C. $M$.

Killed in action 25 Oct. I9I4

Shurlock, A. G. Capt., R.A.M.C.

SHUTER, A. E. Major, R.F.A.(T.F.) (W.)

Shuter, C. H. Major, H.A.C. and R.F.A.(T.F.)

1905

1904

1902

1912

I913

19I3

1905

1896

1901

1906

1905

1909

SIDNEY, P. Capt., Northumberland Fus.; Lieut.-Col., I900 S.O. I, R.A.F. $m$.

\#Srmson, J. Lieut., Australian F.A. Killed in action $3 \mathrm{I}$ fuly 1917

«Skinner, H. S. Pte., London Regt.

Died 13 Sept. 1916 of wounds received in action

1909

I90I

1906

I913

I89I

1893

I910

I9I4

Sleigh, G. P. Capt., D. of Wellington's (W. Riding I9I2 Regt.); empld. O.C.B.; attd. Embarkation Staff, Indian Army 
Smith, A. S. D. 2nd Lieut., Forest School, Waltham- 1902 stow, O.T.C.

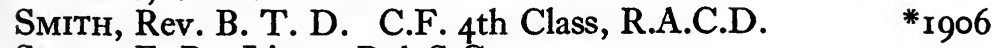

Smith, F. B. Lieut., R.A.S.C.

Smith, Rev. G. A. C. C.F. 4th Class, R.A.C.D. $\quad$ I899

Smith, J. P. F. H. 2nd Lieut., R.A.S.C. (W.) 1905

Smith, W. H. Capt., King's Own (Yorkshire L.I.) (W.) I898

Smith-Carington, M. C. H. Capt., R.F.A.(T.F.) M. $\quad 1905$

SMYTH, S. S. Lieut., King's (Liverpool Regt.) I9I I

Smythe, D. Pte., Black Watch; Lieut., Seaforth Hdrs. I9Io

(W.)

SNELl, Rev. C. C. C.F. 4th Class, R.A.C.D. M. 1893

Sole, B. J. B. Pte., Middlesex Regt. (P. S. Bn.); 2nd r9r3

Lieut., King's Royal Rifle Corps

«Solomons, M. Cadet, O.C.B.

Died I 6 fuly I916 of appendicitis

Somerset, A. P. F. C. Lieut., R. Fusiliers $\quad 1908$

SOPER, F. P. P. Major, R.A.S.C.(T.F. Res.) 1894

SPENCER, T. B. W. Lieut. (Ad.), R.A.F. 1902

SPICER, H. W. Lieut., R.E. (Signals) 1909

StafFord, G. B. Capt. and Adjt., Durham L.I. (W.) I908

StAFFoRD, R.S.H. Lieut.-Col., King's Royal Rifle Corps; 1909

attd. Middlesex Regt. and Gen. Staff. D.S.O. and

Bar. M.C. $M_{4}$.

Stamper, E. P. F. Capt., R. Welsh Fus. $M$.

Stansfeld, C. A. Lieut., R.A.S.C. and IIth Bengai I905 Lancers, Indian Army

Starling, E. C. W. Major, R.A.M.C. (W.) M.C. 1907

AStaveley, H. S. Lieut., E. Yorks. Regt. 1907

Killed in action 3 May 1917

Stedall, G. St G. Capt., London Yeo. (Sharpshooters); I9I I

Hon. Capt. (A.), R.A.F.

Stewart, D. H. Lieut., S. African Force

WStobarT, W. Lieut., Durham L.I.; attd. R.F.C. (W 2.)

Killed in action 24 Aug. 1916

Stoddard, J. W. Lieut., W. Yorks. Regt. (T.F.); Capt., 1902

Spec. List (School of Instruction). M.B.E.

StorRs, B. St J. Lieut., R.F.A.

WSTORRS, F. E. Lieut., R.N.V.R.

Died ro Nov. 1918 of pneumonia

EStrachan, E. S. 2nd Lieut., Sherwood Foresters (Notts. $\quad 1898$ and Derby Regt., T.F.)

Killed in action 14 Oct. I9I 5

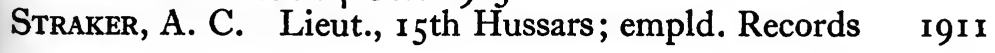


Straker, G. H. Capt., I5th Hussars; A.D.C. D.S.O. 1910 M.C. Brevet Major. M 5. Chevalier, Legion of Honour (France). French Croix de Guerre. Order of St Anne, $4^{\text {th Class (Russia) }}$

Straker, L. S. Lieut., Irish Gds.; Capt., Gds. M.G. Igro Regt. and Gen. Staff. (W.) $M$.

Stroyan, J. R. A. Major, R.F.A.

Sutton, Rev. F. O. C.F. $4^{\text {th }}$ Class, R.A.C.D.(T.F.) M. ${ }^{*} 1883$ Swainson, F. E. Lieut.-Col., Middlesex Regt. (W.) $m$. $\quad$ I89I Swanston, H. E. Capt., R.F.A.(T.F.) 1906

Swayne, P. C. Lieut., D. of Wellington's (W. Riding 1896 Regt.); Capt., W. Yorks. Regt.

\#Swears, H. M. Sergt., R. Fusiliers (P. S. Bn.); Lieut., I9I2 M.G.C.

Killed in action II April I9I7

Syer, H. B. Major, R. Irish Rifles

Sykes, A. H. C. 2nd Lieut.,D. of Wellington's(W.Riding I9I I Regt.); Lieut., R.A.S.C.(M.T.)

Sykes, Sir M., Bart., M.P. Hon. Colonel, E. Yorks. 1897 Regt. and Gen. Staff. $m$. Order of St Stanislas, 2nd Class (Russia). Commander, Order of the Star of Roumania

Symons, H. J. Capt., E.Yorks. Regt.; Major,Tank Corps. $\quad$ I897 $M$.

Tanner, H. O'S. F. Major, I 9 th Hussars and Gen. Staff. 1894 (W.) $M$.

Tate, J. E. Lieut., R.F.A. $M$.

WTAYLOR, E. F. H. 2nd Lieut., R. Fusiliers

Killed in action 27 Fuly 1916

TAYlor, R. H. H. Pte., R.A.S.C. I9I6

TAYlOR, W. R. Lieut., The Buffs (E. Kent Regt.) r910 (W 2.) (P.)

Telfer, Rev. W. C.F. 3rd Class, R.A.C.D.; Cadet, * ${ }^{*} 1908$ O.C.B. M.C.

Thomas, A. L. Capt., R.G.A.

1887

सThomas, O. V. 2nd Lieut., R. Welsh Fus.; Capt. (A.), I9I2 R.A.F. $M 2$.

Died 29 Fuly 1918 of injuries received in flying accident

Thompson, W. Capt., Durham L.I.

Thorburn, K. D. S. M. Pte., R. Fusiliers (P. S. Bn.); I 895 Capt., Northumberland Fus.

Thorne, F. J. Major, R.A.M.C. M.B.E. M. 
Thornton, W. H. J. Major, R.F.A.; Lieut.-Col., 1907 A.Q.M.G. M.C. Brevet Major. Brevet Lieut.Colonel on promotion to substantive Major. $M_{3}$. Order of the Nile, $4^{\text {th Class (Egypt) }}$

Tillyard, E. M. W. Capt., King's Own (R. Lancaster 1908 Regt.) and Gen. Staff (Intelligence). O.B.E. $M_{3}$. Greek Military Cross

सTodD, J. G. Pte., R. Fusiliers (P.S. Bn.); Capt., North- Igor umberland Fus.

Killed in action I fuly 1916

ÆTomlin, C. G. 2nd Lieut., London Regt. (W.) I9ro Died 9 fuly 1916 of wounds received in action

WTown, C.A. Capt., W. Yorks. Regt. (W 2.) M.C. M. 1903 Killed in action 20 Sept. I9r7

Tracy, S. J. Capt., R. Marines. (W.) I9I3

TREE, H. B. LE D. Capt., I I2th Infy., Indian Army; 1900 Brigade Major. M2. Order of the Nile, $4^{\text {th }}$ Class (Egypt)

TURNER, H. F. A. Capt., Loyal N. Lancs. Regt. $M$. I9I3

WTURNER, R. B. 2nd Lieut., Cheshire Regt.(T.F.) I914 Killed in action 9 April 1916

TwYFoRD, H. Major, Sherwood Foresters (Notts. and 1893 Derby Regt.) and Gen. List (A.P.M.)

Tyndale-Biscoe, C. J. Lieut., R.F.A.; Staff Lieut. (W.) [1914] M.C.

Tyndale-Biscoe, H. L'E. Capt. (A.), R.A.F.

ATYRRELl-GrEeN, D. N. Capt., R. Sussex Regt.(T.F.) I9I3 Killed in action 26 March 1917

UbSDELL, T.R. Major,R.F.A.(T.F.); Staff Capt. D.S.O. I89I $M$ 4. Officer, Legion of Honour (France)

Upton, R. Lieut., London Regt. (Cyclist Bn.) 1899

WVan Schaick, J. B. M.G. Bn., American Army

Died 9 Dec. 1918 of influenza contracted on active service

ZVANn, A. H. A. Capt. and Adjt., W. Yorks. Regt. Killed in action 25 Sept. 1915

WVANN, Rev. B. W. Lieut.-Col., Sherwood Foresters $\mathrm{I} 884$

1909

(Notts. and Derby Regt.) (W 5.) M.C. and Bar. $M$ 2. French Croix de Guerre

V.(T. "For most conspicuous bravery, devotion to duty and fine leadership during the attack at Bellenglise and Lehaucourt on September Igth, 1918. He led his battalion with great skill across the Canal du Nord 
through a very thick fog and under heavy fire from field and machine guns. On reaching the high ground above Bellenglise the whole attack was held up by fire of all descriptions from the front and right flank. Realising that everything depended on the advance going forward with the barrage, Col. Vann rushed up to the firing line and with the greatest gallantry led the line forward. By his prompt action and absolute contempt for danger the whole situation was changed, the men were encouraged and the line swept forward. Later, he rushed a field gun single-handed and knocked out three of the detachment. The success of the day was in no small degree due to the splendid gallantry and fine leadership displayed by this officer. Lieut.-Col. Vann, who had on all occasions set the highest example of valour, was killed near Ramicourt on $3^{\text {rd }}$ October, 1918, when leading his battalion in attack."-Supplement to The London Gazette of ${ }_{4} 4$ Dec. I9I8.

Killed in action 3 Oct. 1918

Verdon, P. Capt., R.A.M.C.; Lieut., I.M.S.

VICK, G. R. Capt., Durham L.I. and Gen. List (O.C.B. VICK, R. M. Major, R.A.M.C.(T.F.) O.B.E. M 3 . R.E. (Signals)

Wadsworth, S. Lieut., Madras Gds., Indian Defence 1908 Force

Wailes-Fairbairn, N. W. F. Capt., Yorkshire Hussars; IgI I Lieut., R.E.(T.F.)

Wailes-Fairbairn, W. F. Major, S. Notts. Hussars. 1879 (W.)

WaIt, R. J. Corpl., R.E. (Signals); Lieut., R.G.A. (W.) 1912

Wakefield, W. V. Capt., R. Warwickshire Regt.(T.F. I9ro Res.); empld. War Office

Walker, A. E. J. 2nd Lieut., Labour Corps; Lieut., 1908 Gen. List (Claims Commission)

Walker, H. Capt., R.A.M.C. M.C. M. 1905

Walker, H. C. Capt., Canadian F.A.

WARE, B. O. Lieut., Loyal N. Lancs. Regt. (W.) I9I4

WARren, C. K. W. 2nd Lieut., Monkton Combe School 1912 O.T.C.; Air Mechanic, R.A.F.

WARren, L. A. 2nd Lieut., S. Lancs. Regt.; Lieut., 1906 Cheshire Regt.

Waterall, L. S. Lieut., Suffolk Regt. and Rifle Brigade. 1904 (W.) 
Watson, J.A. Lieut., W. Yorks. Regt.; attd.Durham L.I. I9I0 Watson, Hon. T. H. Capt., R.A.S.C. WatT, A. F. Lieut.-Col., Yorkshire Hussars; A.D.C. 1890 D.S.O. T.D. Brevet Lieut.-Colonel. M. Chevalier, Legion of Honour (France)

WAYLEN,D.C. Capt.(K.B.), R.A.F.; S.O.3,Air Ministry. I9II M.B.E. M 2.

WWebB, J. C. Pte., R. Fusiliers (P.S. Bn.); 2nd Lieut., 1912 Leicestershire Regt.

Killed in action 15 Sept. 1916

Welch, D. 2nd Lieut., Cameronians (Scottish Rifles); 1905 Lieut. (A), R.A.F.

WWelinKaR, S. C. Lieut. (A.), R.A.F.

Died in German hands 30 June 1918 of wounds received

I9r 5 in action

WENLEY, J. A. S. 2nd Lieut., R.F.A.

Whale, G. H. L. Capt., R.A.M.C. (T.F.)

WhELPTON, L. G. Lieut., R. Berkshire Regt.(T.F. Res.); attd. King's Own (Yorkshire L.I.)

WhITE, G. G. Capt., E. Surrey Regt.; attd. Gen. Staff. I9I I O.B.E. $m$.

WhITE, J. Lieut., Northamptonshire Regt.(T.F.)

WWhiTE, M. Trooper, S. African Horse

Died May 1916

WILD,J.A.P. Capt., Yorkshire Regt.; empld.O.C.B. (W.) rgor

WILDE, C. A. G. 2nd Lieut., London Regt. (Kensington I9II Bn.) M.C.

Wilkinson,F.C. 2nd Lieut., Argylland Sutherland Hdrs.; 1906 Capt. (T.), R.A.F. (Aircraft Production Dept.) (W.)

Williams, A. L. Pte., R.A.S.C.; Capt., R. Fusiliers

Williams, C. S., v.D. Hon. Colonel, Queen's Own (R.W. 1868 Kent Regt., T.F.) $m$.

Williams, Rev.F.F.S. Lieut.,Eastbourne College O.T.C. $\quad 1889$

Williams, Rev. H. F. F. Junior Chaplain, Indian Army ${ }^{*} 1907$

Williams, L. Capt., Welsh Regt. 1913

WWILsON, D. R. Lieut., The Queen's (R.W. Surrey Regt.) I910 Killed in action near Ypres 30 Oct. 1914

WWILSON-BARKWORTH,K.A. Capt.,E. Yorks.Regt. (W 2.) 1902 M.C.

Killed in action 25 Oct. 1917

WiLToN, J. P. Capt., Spec. List; Bdr., R.G.A. 1902

WILton, T. R. Capt., R.E. (Fortress, T.F.) M. 1897

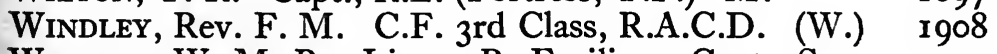

WINGATE, W. M. R. Lieut., R. Fusiliers; Capt., Spec. 1905 List (Recruiting Staff). $m$. 
Winnicott, D. W. Surgeon Sub-Lieut., R.N.V.R.

WWiseman, P. H. F. Pte., R. Fusiliers (P.S. Bn.); Lieut., 1906 Loyal N. Lancs. Regt. (W.)

Died 27 Oct. 1917 of wounds received in action

Wiseman, Sir W. G. E., Bart. Lieut.-Col., Gen. List. 1904 C.B. $m$.

WWolryche-Whitmore, P. M. 2nd Lieut., Lincolnshire 1910 Yeo.; Lieut., R.F.A.

Killed in action $\mathrm{I}$ Aug. $19 \mathrm{I} 8$

Wood, C. J. Lieut., The Buffs (E. Kent Regt.); Capt. I913 and Adjt., Queen's Own (R. W. Kent Regt.) M.C.

Wood, E. W. Capt., S. Staffs. Regt. (W.) M.C. and rgr I Bar

Wood, Franklin G. Surgeon Lieut., R.N.

Wood, F. Gordon. Leading Telegraphist, R.N.V.R.

Wood, G. H. A. Pte., R. Fusiliers (P. S. Bn.); Lieut.,

Border Regt.; attd. Wiltshire Regt.; empld. Ministry of Munitions. (W 2.)

Wood, J. Cadet, R.A.F.

Wood, W. B. Capt., R.A.M.C. $M$.

1910

1907

1913

WOODARD, E. H. J. Lieut., R. Warwickshire Regt.; Capt., Gen. List (R.T.O.) (W.)

Woodard, Rev. F. A. Capt., Forest School, Waltham- 1908 stow, O.T.C.

Woodroffe, L. W. Capt., R. Marines; M.L.O.

Woods, S. M. J. Lieut., Somerset L.I., R. Warwickshire Regt., and Devon Regt.; Capt., Labour Corps

WWray, K. C. G. Capt., S. Lancs. Regt.(T.F.)

Killed in action 9 Aug. 19r6

WWRENFORD, A. L. Major, Worcestershire Regt.; attd. R.

Inniskilling Fus.; Lieut.-Col., E. Lancs. Regt. (W.) $M$.

Killed in action 21 March 1918

Wright, A. J. Capt. and Adjt., Northamptonshire Regt. 1908 (T.F.); Brigade Major. D.S.O. M.C. $M 3$.

Wyld, E. J. M. Capt., Wiltshire Regt.(T.F.)

1900

Wyley, D. H. F. Capt., R.F.A.; Staff Capt. O.B.E. 1906 M.C.

Wynn, Rev. H. E. C.F. 3rd Class, R.A.C.D. M. *igro Italian Croce di Guerra

Wynne, H. F. D. Pte., R. Fusiliers (P. S. Bn.) and I913 R.A.M.C.

WYates, H. B. Lieut.-Col., Canadian A.M.C. Died 22 Fan. 1916 of illness contracted on active service 


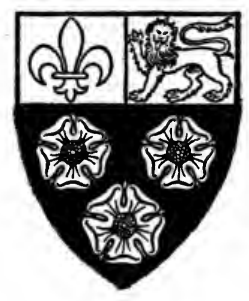

\section{KING'S COLLEGE}

Acland, T. W. G. Capt., R.E. (London Electrical En- 1909 gineers, T.F.); empld. Ministry of Munitions

Adam, C. G. Forbes. See Forbes Adam, C. G.

AdaMs, W. T. Lieut., E. Yorks. Regt. M.C. $\begin{array}{ll}\text { ADAMS, W. T. Lieut., E. Yorks. Regt. M.C. } & \text { I9I3 } \\ \text { ADCOCK, F.E. Lieut.-Cdr., R.N.V.R., empld. Admiralty. } & \text { I905 } \\ \text { O.B.E. } & \end{array}$

Agar, W. E. Capt., Highland L.I. (T.F.) I900

AGNEw, G. C. 2nd Lieut., Gen. List (Intelligence) I90I

HAINGER, T. E. 2nd Lieut., Berkshire Yeo.

Killed in action in Gallipoli 21 Aug. I915

Alexander, R. D. Flt. Cadet, R.A.F.

1903

Alford, E. J. G. Lieut., Spec. List (R.T.O.)

Allbook, W. Pte., Middlesex Regt.

1917

1909

I9I 5

Allen, H. S. Capt., King's (Liverpool Regt., Liverpool I914 Scottish, T.F.) (W.)

Amps, L. W. Lieut., R.E.; empld. Ministry of Munitions. I9ro (W.) $M$.

AnNan, J. G. Capt., R.G.A.; empld. Foreign Office. 1905

Chevalier, Legion of Honour (France)

AArbuthnot, M. A. Capt., I6th Lancers; A.D.C.; Staff 1908

Capt. M.G. M2. French Croix de Guerre

Died 14 Oct. I9r 8 of septic pneumonia

ArCHER, J. F. Major, King's Own (Yorkshire L.I., T.F. 1900 Res.)

ArIs, H. Major, Winchester College O.T.C. $m$. $\quad 1887$ Armbruster, C. H. Major, Gen. Staff. O.B.E. $M_{4}$. 1893 Order of the Nile, 3 rd Class (Egypt)

सArmstrong, E. W. 2nd Lieut., Rifle Brigade

Died I I Fuly I9I 5 of wounds received in action ro Fuly I9II I9I 5 
EBacon, D. F. C. 2nd Lieut., Durham L.I.; attd. North- [1914] umberland Fus.

Died I Nov. I91 5 of wounds received in action 2 Oct. 1915 at the Hohenzollern Redoubt

Bagenal, N. B. Lieut., Irish Gds.; empld. Ministry of I9ro Labour. (W 4.)

BagGe, Sir A. W. F., Bart. Lieut., Norfolk Regt. (T.F.); 1894 Capt., King's (Liverpool Regt.)

Bagshawe, H. V. Lieut.-Col., R.A.M.C.; A.D.M.S. 1893

C.B.E. D.S.O. Brevet Lieut.-Colonel. M 3. Order of the Nile, 3 rd Class (Egypt)

BaILEY, R. F. 2nd Lieut., Shrewsbury School O.T.C.

Bainbridge, O. J. Lieut., Sherwood Rangers

Ball, R. G. Capt., R. Inniskilling Fus.; Major, M.G.C. 1900 (W.)

Ball, W. V. A.B., R.N.V.R. (Anti-Aircraft). O.B.E. 1893 Banham, Rev. V. G. C.F. $4^{\text {th }}$ Class, R.A.C.D. M.C. 1900 and Bar

Bannatyne, A. G. Capt., R.A.S.C. M.B.E. $m$.

BARKer, G. P. Major, Suffolk Yeo. (W.)

Barker, R. I. P. Capt., R.A.S.C.(T.F.); Capt. (A.), 1908 R.A.F.

Barnard, F. A. B. Capt., R. Fusiliers; attd. T.M.B. (W.) I9I I $M$.

BARNES, J. S. 2nd Lieut., R. Horse Gds.; Major (A. and I913 S.), R.A.F. (W.)

Barraclough, N. E. Capt. (A.), R.A.F. M.C.

BARROW, R. M. Cadet, O.C.B.

Bartholomew, Rev. G. T. C.F. 4th Class, R.A.C.D. Ig00 (T.F.)

Bate, A. G. Capt., R.A.M.C. (2nd E. Gen. Hospital, 1894 T.F.)

Bateman, R. J. S. Lieut. Essex Regt.(T.F.)

1901

Bather, Rev. A. G. Capt., Winchester College O.T.C. I887

BAXTER, T. H. 2nd Lieut. (T.), R.A.F.

WBAYley, C. H. Capt., S. Lancs. Regt.

Died 7 Aug. I9I 7 of wounds received in action

Bayliss, C. W. Capt., Welsh Regt. (Cyclist Bn., T.F.); 1913 attd. R.E. (Sound-ranging Section)

Beale, E. V. Capt., R.A.M.C.

BECK, H. M. Lieut., Aldenham School O.T.C. 
Beddington, F. Lieut., King's Royal Rifle Corps [1914] Benson, C. T. V. Major, R.A.M.C. I90I

Benthall, E. C. Capt., Devon Regt.; empld. War Office. 1912 (W.)

BERESFORD, J. B. Lieut., R.A.S.C. M.B.E.

BERRY, A. 2nd Lieut., Spec. List (Intelligence). O.B.E. $\quad$ I882 ABERRY, P. H. Lieut., R.A.M.C.

Drowned I7 March I916 on the Egyptian coast in the attempt to rescue a comrade

ABERRY, W. Lieut., R.A.S.C.

Died 5 fuly 1917 of gas poisoning and shell shock

1907

Bevan, A. E. Major, M.G.C. (W.)

ABevan, F. H. V. Capt., S. Wales Borderers; attd. R.F.C. Killed in action April 1917

Bevan-Brown, R. E. Sergt., R.A.M.C. M.S.M. IgI I

Bevan-Petman, B. A. Trooper, Punjab Light Horse, In- $\quad 1889$ dian Defence Force

Bevan-Petman, B.H. Lieut., 2ist Cavalry, Indian Army [I914]

BEVERIDGE,E.W. Lieut., R. Dublin Fus.; attd. R.E.(Sig- [1914] nals). $M$.

BIRCH, F. L. Lieut.-Cdr., R.N.V.R.; empld. Admiralty. 1909 O.B.E.

BIRCH, W. C. Sapper, R.E. (Signals)

BIRD, C. K. Lieut., R.E.

1904

I9I I

EBlaIR, P. C. B. 2nd Lieut., Rifle Brigade Killed in action 6 fuly 1915

I910

Blake, A. G. Capt., Essex Regt.; attd. 79th Punjabis, [1914] Indian Army. (W.)

WBLECH, E. L. 2nd Lieut., E. Surrey Regt. and M.G.C. [1914] Killed in action near Guillemont 18 Aug. 1916

सBliss, F. K. Pte., London Regt. (Artists Rifles); 2nd I9I I Lieut., R.F.A.

Killed in action at Thiepval 28 Sept. 1916

Blunt, Rev. A. S. V. C.F. 4 th Class, R.A.C.D. O.B.E. $\quad 1889$

BlunT, D. L. Lieut., R.A.S.C.

Bolton, P. Lieut., Oundle School O.T.C.

1909

I908

Bоoтн, C. H. B. Capt., R.A.M.C. $M$.

Bosanouet, W. S. B. Capt., Coldstream Gds. (W. I905 D.S.O. $M$.

सBottomley, E. W. Capt., London Regt. (R. Fus.)

Killed in action 15 Fune 1917

Boulenger, C. L. Hon. Lieut., Spec. List

Bowen, A. G. W. Fleet-Surgeon, R.N.

Bowring, H. Capt., R.A.M.C.

[19I4]

c. U.W. L. 
Bradbury, J. F. Major, R.A.S.C.(T.F.) $m$.

Braithwaite, J. G. Lieut., R.N.V.R.

[1914] 1909

The Queen's (R. W. Surrey Regt.), and Middlesex

Regt.

Brewster, G. W. 2nd Lieut., Oundle School O.T.C. $\quad 1899$

Bristed, G. T. Capt., R.E. $M$.

WBooke, R.C. Sub-Lieut., R.N.V.R. (Howe Bn.,R.N.D.) 1906

Died at Scyros 23 April r91 5 of blood-poisoning following sunstroke

WBRooke, W. A. C. 2nd Lieut., London Regt. (Post Office

Rifles)

Killed in action 14 Fune 191 5

Brooks, C. D. Capt. and Adjt., R.G.A. M. 1905

Brooks, D. C. M. Capt. (A.), R.A.F. M.C. A.F.C. I9I4

Brown, A. C. Lieut., R.F.A. (W.) 1902

Brown, G. B. 2nd Lieut., Tank Corps. (W.) 1905

Brown, G. M. Capt. and Adjt., Suffolk Regt.; Staff I9ro

Capt. O.B.E. M. m.

Brown, W. B. Lieut., Northumberland Fus. (W.) 1912

Brundrit, J. C. Capt., R. Welsh Fus.(T.F.) $\quad 1897$

BRUndRIT, P. W. Cadet, O.C.B.

Brunwin, A. H. Lieut., King's Own (R. Lancaster [1914]

Regt.) (W 2.) M.C.

Bryan, F. Capt., R.A.M.C.; Asst. Embarkation S.O. 1895

BuCKLE, C. L. Capt., R.A.S.C. 1904

Buckley, Hon. B. B. Lieut., London Regt. (Rangers, I909

T.F. Res.); empld. Admiralty

ÆBurgoyne Johnson, L. V. Capt., Durham L.I.(T.F.) 1909

Killed in action 26 April 1915

Burke, H. F. Capt., R.F.A.; Staff Capt. M.C. 1902

Burke, M. L. Lieut., K. Edward's Horse; Capt., Tank 1907

Corps

Burr, Rev. A. L. C.F. $4^{\text {th }}$ Class, R.A.C.D.

Burton, A. Capt., R.A.M.C.

Bury, E. B. Lieut., Suffolk Regt.; Staff Lieut. (W.) I909

Bushell, W.F. Capt., Unattd. List, T.F.; Lieut., Here- 1903 fordshire Regt.

\#Uusk, E. T. 2nd Lieut., R.E.

Killed in flying accident 5 Nov. I9I4

ABusk, H. A. Flt. Cdr., R.N.A.S.

Killed in action over Gallipoli 6 Fan. I916

\#Bythway, M. H. P.O., R.N.A.S.

Died 9 Dec. I9I 5 of wounds received in action 
\#Campbell, R. C. C. Capt., King's Own Scottish Bor- 1907 derers

Died 20 May I9I 5 of wounds received in action

CANE, A. S. Major, R.A.M.C.; D.A.D.M.S.

D.S.O. O.B.E. $M_{4}$.

Cane, L. B. Capt., R.A.M.C.

CANE, M. H. Major, R.A.M.C. O.B.E.

Carlisle, H. B. Capt., Lancs. Fus.(T.F.); Major, M.G.C.

(P.) 1903

CARNEGIE, J. D. Lieut., Hampshire Yeo. and Hampshire Regt.; attd. 6th (Inniskilling) Dragoons. (W.)

Carrow, R. B. Lieut., The Buffs (E. Kent Regt.) (W.) IgI I (P.)

सCARTER, J. S. Capt., Grenadier Gds.

Killed in action at Hesquières 27 Sept. 1918

1900

1906

1910

I913

Cartwright, J. L. 2nd Lieut., Labour Corps; attd. R. I9I5 Sussex Regt.

CARVER, N. C. Surgeon Lieut., R.N.

1895

Chambers, H. T. Major, R.A.S.C.; D.A.D.S. and T. I9I3 O.B.E. $M 2$.

Champion, H. H. Capt., Uppingham School O.T.C. $\quad 1892$

\#Chester, H. K. Capt., Essex Regt.

Died 28 March I9I 7 of wounds received in action at Gaza

ChItTy, J. W. Capt., Essex Regt.(T.F.)

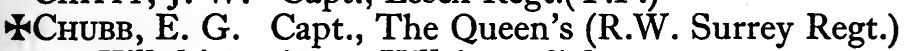
Killed in action at Hill 6012 Fuly 1915

ЖСнuвв, F. J. M. Lieut., King's Own (Yorkshire L.I.) 1912 Killed in action near Ypres 18 April I9I 5

Clark, A. J. Hon. Capt., R.A.M.C. M.C. M.

Clark, Rev. P. N. Capt., Spec. List.

I9I3

Clark, W. M. Lieut., King's Own Scottish Borderers. I903 (W.)

Clarke, R.S. Capt., Sussex Yeo.; attd. R. Sussex Regt.; I9I I A.D.C. (W.)

\#Claughton, I. D. Lieut., Suffolk Regt.

1 Killed in action 4 March 1916

Clifford, F. A. Capt., R.E. (W.)

Clout, C. W. Capt., London Regt. (Blackheath and I9I3 Woolwich Bn.); Staff Capt., War Office. (W.) M.B.E. M.

Cohen, A. M. Major, Queen's Own (R. W. Kent Regt., $\quad$ I895 T.F.)

CoHen, Sir H. B., Bart. Major, Queen's Own (R. W. 1892 Kent Regt., T.F.); Embarkation S.O. O.B.E. m. 
\#Colbeck, L. G. 2nd Lieut., R.F.A. M.C.

Died at sea 3 Fan. 1918

Cole, G. H. 2nd Lieut., Somerset L.I.

Cole, Rev. H. B. C.F. $4^{\text {th }}$ Class, R.A.C.D. M.C.

COLE, L. B. 2nd Lieut., R.F.A.

Collin, E. P. C. Lieut., I.A.R.O., attd. 18th Lancers

Collingham, D. H. Capt., R.A.M.C. $M$.

Comeau, E. A. Lieut. (T.), R.A.F.

1915

1894

1916

1909

1897

1912

1907

Killed in action 14 Fuly 1916

Comyn, H. F. Capt., R.A.M.C.

*Concanon, G. L. B. Capt., Australian Infy. $M$.

Killed in action in Gallipoli 25 March 1915

Conybeare, A. E. Capt., Eton College O.T.C.

\Cooke, A. W. H. Capt., Durham L.I. (W.) $M$.

Killed in action 24 March 1918

Cooke, C. H. Capt., R. Berkshire Regt.; Brigade Major. M.C. $M 2$.

Cooke, S. R. Capt., London Regt. (Post Office Rifles); G.S.O. 3, War Office. (W.) $m$.

Copeman, C. E. F. Lieut.-Col., Cambridgeshire Regt. * 1906 and Northamptonshire Regt.(T.F.) C.M.G. T.D.

$m 3$.

Corbett, G. H. U. Capt., R.A.M.C.

1903

1900

1894

1906

1902

I9II 1898

Corrie, G. T. A.B., R.N.V.R. (R.N.D.); Lieut., R. Warwickshire Regt.; empld. Ministry of Munitions. (W.)

Coryton, W. A. Lieut., Rifle Brigade; Hon. Lieut. (A.), R.A.F. (W.)

Courtauld, J. S. Major, Gen. List; D.A.A.G. M.C. 1899 $M$ 3. French Croix de Guerre

Courtauld, S. L. Capt., Worcestershire Regt.; Major, 1901 M.G.C. M.C. M2.

Cowan, J. M. Colonel, A.M.S. $M$.

* Coward, H. Lieut., Border Regt.

Died 20 April 1917 of wounds received in action

Crace, J. F. Lieut., Eton College O.T.C.

ACracroft, R. B. Lieut., E. Yorks. Regt. (W.)

Killed in action near Mametz Wood 10 fuly 1916

1888 1906

*Craigmile, A. M. Capt., Rifle Brigade. (W.) M.C. 1914 Killed in action 29 March 1918

\#CREASY, R. L. Major, R.F.A. (W 2.) M.C. M.

Died 22 Oct. 1918 of wounds received in action at Cambrai 
Crewdson, B. F. 2nd Lieut., Rifle Brigade; Major, 1905 Border Regt.; Capt., Irish Gds. (W.) G.B.E. m.

Crewdson, R. B. Capt., R.F.A. $M 2$.

CROMPton, N. G. Lieut., R.E. (W.) $M$.

Killed in action near Armentières 5 Nov. I9I 5

WCROWE, H. A. 2nd Lieut., London Regt. (R. Fus.)

Died 30 May 1915 of wounds received in action

Cushion, E. J. Corpl., R.A.M.C.

1912

1907

[1914]

1905

Dalrymple, C. M. Lieut., University College School 1895 O.T.C.

Dalton, C. H. C. 2nd Lieut., Northamptonshire Regt.; I9I4 Lieut., Manchester Regt.

Dalton, E. H. J. N. Lieut., R.G.A. Italian Bronze 1906 Medal for Military Valour

WDARLEY, D. J. 2nd Lieut., Suffolk Regt.

Killed in action at La Boisselle $\mathrm{r}$ Fuly 1916

Davies, D. Lieut.-Col., R. Welsh Fus.(T.F.) Order of 1899

St Stanislas, 2nd Class, with swords (Russia)

Davies, G. F. Capt., Gloucestershire Regt.(T.F.); 1894

Major,Spec. List (Asst. Cmdt., Reinforcement Camp)

Davies, R. G. R. Lieut., I6th Lancers; Major, M.G.C. 1909 (Cavalry). (W.) M.C. $M_{4}$.

Davies, T. M. Lieut., R. Sussex Regt.(T.F.); Capt., 1899 R.G.A. (W.)

Davis, G. F. S. Lieut., R.A.S.C.; attd. R.G.A. (W.) 1905

Davis, K. J. A. Capt., R.A.M.C.

1902

WDawswell, G. A. 2nd Lieut., London Regt. (St Pancras 1912 Bn.)

Killed in action 20 March 1916

DE HAMEL, H. G. Sergt., London Regt. (London Scot- $1899^{\circ}$ tish). (W.)

\&Deighton, G. W. Capt., Suffolk Regt. M.C. M. I9I I Chevalier, Legion of Honour (France)

Killed in action at Ovillers 3 fuly 1916

DE LINDE, C. A. Corpl., London Regt. (London Scot- [1914] tish); 2nd Lieut., R.E. (W.)

Deuchar, J. L. Lieut., Tank Corps. (W.)

Dexter, J. Pte., N. Staffs. Regt. (W.)

1905

1916

Dickinson, P. P. Paymaster Sub-Lieut., R.N.V.R. $\quad$ I902

DILKE, C. W. 2nd Lieut., Surrey Yeo.

DobBs, A. F. Major, R.G.A. $M$.

1896

Dobson, E. L. Capt., H.A.C. and R.A.M.C.

1895

1906

DogGarT, J. H. Surgeon Sub-Lieut., R.N.V.R.

1917 
DoherTy, W. D. Capt., R.A.S.C

Douglas, C. K. M. Lieut., R. Scots; Capt. (A.), R.A.F. 1912 (W.) A.F.C.

WDowson, H. Pte., H.A.C.; Capt., King's Royal Rifle 1908

Corps. M.C. $M$.

Killed in action 15 Sept. I916

Drinkwater, J. R. Major, R.F.A. (W.) 1906

Dunn, Rev. C. S. C.F. $4^{\text {th }}$ Class, R.A.C.D. (W.) 1892

DuRHAM, H.E. Hon.Lieut., R.A.M.C.; Major,Spec. List 1884

Durnford, H. G. E. Capt., R.F.A. (P. Escaped from 1905 Germany.) M.C.

WDurnford, R. C. Capt., Hampshire Regt.(T.F.) (W.) [1914] D.S.O. M.

Killed in action in Mesopotamia 21 Fune 1918

WDuRnFord, R. S. Capt., King's Royal Rifle Corps

Killed in action at Hooge 30 fuly 1915

WDuval, M. R. Capt., 66me Regt., French Army. French 1887 Croix de Guerre

Killed in action 5 May 1916

Dyne, H. E. L. Lieut., The Queen's (R.W. Surrey Regt.) 1904

DYNe, J. B. Cadet, O.C.B. 1894

WDyson, W.H. and Lieut., London Regt. (Queen's West- I9ro minster Rifles)

Died in German hands 14 Fuly 1916 of wounds received in action

Eaton, J. E. C. Sergt., London Regt. (Artists Rifles); 1892 2nd Lieut., Spec. List (Intelligence)

EckhaRD, O.P. Capt., Manchester Regt.; empld. O.C.B. 1907 (W.)

EDE, C. Lieut., R.A.M.C.

EDE, M. C. Lieut., S. Wales Borderers. (W.)

1905

EDmunds, P. M. L. Lieut., 12th Lancers; Capt. (A.), R.A.F. (W.) $M$.

Edwards, C. J. Capt., Northumberland Fus. and Gen. 1905 Staff (O.C.B.) (W.) M.B.E. $m$

EEDwards, G. T. Capt., R. Fusiliers

Killed in action at Delville Wood $3 \mathrm{r}$ Fuly r9r6

सEıcke, O. M. Lieut., R.F.A. M.C.

Killed in action 5 Nov. 1916

Ellington, N. B. Major, Cheshire Regt.(T.F.) (W.) 1899 M.C. $M$.

Elliott, C. A. B. Capt., Leicestershire Regt. (W 2.) r913

ElliotT-COOPER, M. Lieut., R.E. 
Ellis, C. D. B. Lieut., R.H.A (T.F.) (W.) M.C. M. I913 EElmslie, K. W. Lieut., 4th (R. Irish) Dragoon Gds. 1906 Killed in action near Messines 4 Nov. 1914

WElwin, F. H. Pte., King's (Shropshire L.I.); 2nd Lieut., [I9I4] Wiltshire Regt.

Killed in action at Neuve Chapelle $\mathrm{I}_{4}$ March 1915

Emrys-Evans,P.V. Lieut., Suffolk Regt.; empld. Foreign Office. (W.)

ENGLISH, B. A. Lieut., Garrison Artillery,Indian Defence 1904 Force

ERrera, A. J. J. H. Lieut., Artillery and Field Survey, I904 Belgian Army

Evans, C. L. Capt., Dover College O.T.C.

Evans, D. D. Capt., R.A.M.C. M.C.

I900 1908

ZEvans, D. L. Capt., Northamptonshire Regt.

Died 26 Sept. I9I 6 of wounds received in action

Evans, E., T.D. Surgeon Lieut.-Col., Welsh Regt.(T.F.) * I88r

Evans, G. F. F. Capt., R.E.; Staff Capt., War Office; 1907 Major, R.A.F.; S.O. 2, Air Ministry. (W.) $m$.

Evans, U. R. Pte., London Regt. (Artists Rifles); Lieut., 1907 E. Surrey Regt.; attd. R.E. (Signals)

FALK, H. Major, I.M.S. M. Order of the White Eagle, 1897

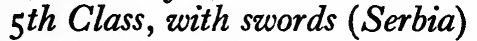

FARNELl, H. L. Lieut., Bedfordshire Regt. and Gen. List 1907 (Intelligence). $M$.

FaulKNeR, A. K. Lieut., Hampshire Regt. and Nigeria 1906 Regt., W. African Frontier Force

FAY, C. R. Lieut., The Buffs (E. Kent Regt.); Capt., 1902 M.G.C. and Spec. List (Asst. Instructor). $M$.

Felkin, A. E. Lieut., Spec. List (Interpreter)

FEw, J. E. Capt., Suffolk Regt. and Spec. List, empld. I896 British Military Mission

Fiddian, C. M. 2nd Lieut., S. Staffs. Regt.

Fiddian, W. M. Lieut., Suffolk Regt.

Filon, L. N. G. Major, London Regt. (R. Fus., T.F. $\quad 1898$ Res.); Hon. Major (T.), R.A.F. $m 2$.

WFinlay, E. L. 2nd Lieut., London Regt. (Post Office r9ro Rifles) and Devon Regt.(T.F.)

Died 20 March 1916 of wounds received in action in Mesopotamia

FFIRTH, R. B. 2nd Lieut., London Regt. (L.R.B.)

Died 26 Sept. 1917 of wounds received in action near 1906 St Fulien 21 Sept. I9I7 
Fishwick, J. F. Lieut., R. Sussex Regt.

FFitzGerald, G. T. Capt., Durham L.I. (W.)

Killed in action near Armentières 30 Dec. 1915

Fleming, G. B. Capt., R.A.M.C.(T.F.) (W.) M.B.E. 1900 $m$.

Flitch, J. E. C. Capt., R.F.A.; attd. T.M.B. (W.) M.C. 1900 $M$.

Forbes, B. C. Major, I.A.R.O.; Divisional Recruiting 1900 Officer

Forbes AdAm, C. G. Lieut., I.A.R.O., attd. 34th Sikh 1908 Pioneers

Foster, H. P. R. Lieut., D. of Cornwall's L.I

Foster, R. L. V. Lieut.-Col., R.A.M.C. $M$.

Foster, V. le N. Lieut., Eton College O.T.C.

Foulkes Jones, W. Surgeon Lieut., R.N.

Fox, C. L. Major, R.E.(T.F.) M.C.

FRANKLIN, G. D. Major, I.M.S. O.B.E. M.

सFRY, A. H. 2nd Lieut., London Regt. (Queen's)

Died 30 Oct. 1916 of wounds received in action 10 Oct. I9I 6

FRY, L. S. Surgeon Lieut., R.N.

FRY, T. P. Capt., Durham L.I.(T.F.) (W.)

Funduklian, A.A. Corpl., 22nd Infy., United States Army

1905

I 893

1894

I 901

1903

1895

1904

*Gallimore, H. B. Capt., R.F.A.(T.F.)

Killed in action 26 May r9I7.

GARDNER, A. Paymaster Sub-Lieut., R.N.V.R.

GARDNER, C. Lieut., R.M.A.

GARDNER, H. Lieut., R.M.A. $M$.

Garrod, L. P. Surgeon Sub-Lieut., R.N.V.R.

*Gaselee, A. M. 2nd Lieut., I 5 th Hussars

Killed in action near Ypres 24 May 1915

Geary, A. B. Capt., Spec. List (Intelligence)

GeldaRT, H. R. Lieut., Northamptonshire Regt.; attd.

Norfolk Regt. (W.) $M$.

Gemmell, A. A. 2nd Lieut., King's (Liverpool Regt.,

Liverpool Scottish, T.F.); Capt. and Adjt., Cameron

Hdrs. (W.) M.C. M. Greek Military Cross

\#Gent, F. E. Lieut., W. Yorks. Regt.

Killed in action in Gallipoli 7 Aug. 1915

\&Gibbins, R. B. Capt., R. Warwickshire Regt.

Killed in action 4 Dec. I9I7

1908

1910

I9I I

1904

1897

1899

I9II

1914

1912

1897

1914

I9II

Bbin, L. F. Major, 4oth (Tasmania) Bn., Australian

Force. (W 3.) D.S.O. M.C. M. 
Giles, G. C. T. 2nd Lieut., King's (Liverpool Regt.); r9ro Capt., Army Cyclist Corps

Gill, C. I. C. Capt., Manchester Regt.(T.F.)

Gillum, S. J. Trooper, Bombay Light Horse, Indian 1895 Defence Force

Goodden, C. P. Capt., R. Marines

Gordon, C. A. Lieut., Grenadier Gds.; Capt., T.M.B. 1904

(W.) M.C. Belgian Croix de Guerre

Grant-Ives, J. C. (formerly Grant). Capt., Rifle Brigade. 1908 $M$.

Gray, J. Capt., The Queen's (R.W. Surrey Regt., T.F.) 1909

M.C. French Croix de Guerre

Gray, J. M. Capt., Sherwood Foresters (Notts. and 1908

Derby Regt., T.F.); empld. Recruiting Staff. (W.)

GreEN, J. R. E. 2nd Lieut., R.F.A.

Greenwood, J. E. Pte., London Regt. (Artists Rifles);

Capt., E. Surrey Regt.(T.F.); Lieut., Grenadier Gds.

(W.) $M$.

GrICE, J. W. Capt., R.A.M.C.

GRIFFIN, F. W. W. Capt., R.A.M.C.

Griffith, H. C. Lieut., R.G.A.

Griffiths, J., T.D. Colonel, R.A.M.C. (Ist E. Gen. Hos- * 1890 pital, T. F.) C.M.G. $m 3$.

GritToN, H. J. 2nd Lieut., R.A.S.C.

\#GuThRIE, R. F. Capt., King's (Liverpool Regt.)

Killed in action 9 Aug. 1916

GutTeridge, H. C. Capt., R.A.O.C. $M$.

1910

1900

I 895

1899

I910

I895

19I3

Died in Germany 12 Aug. I917 of wounds received in action

GwatkIn, Sir W. G., c.B. Major-Gen., Chief of Gen. Staff in Canada. K.C.M.G. m. Commander, Legion of Honour (France). Commander, Ordre de la Couronne (Belgium). Order of St Sawa, 2nd Class (Serbia)

Hadley, O. H. Capt., Loyal N. Lancs. Regt. (W 2.) [1914] HaDOw, R. H. Capt., Argyll and Sutherland Hdrs.; attd. [I9I4] Seaforth Hdrs. (W 2.) M.C. M.

Haggard, A. J. R. Lieut., The Queen's (R. W. Surrey 1908 Regt.) (W 2.)

Hall, F. G. Lieut., Dorset Regt.; Capt., Spec. List 1907 (Asst. Base Cmdt.) $M$.

WHALL, Rev. W. Chaplain and Instructor, R.N.

Died I Nov. 1916

I 886 
WHalliday, C. W. A. 2nd Lieut., R.F.A.

Died 17 Nov. I9I 6 of wounds received in action 8 Nov. 1917

Hamilton, G. C. H. Lieut., Manchester Regt.(T.F.) 1906 (W.)

Hamilton, J. A. DE C. Capt., Hampshire Regt.(T.F.) I9I4 (W.) M.C.

Hamilton, J. L. Lieut., R.F.A.; Staff Lieut. $M$.

Hammersley, S. S. Lieut., E. Lancs. Regt.; Capt., Tank Corps. (W.)

WHammond-Chambers, H. B. B. Capt., King s Own (R. 1905 Lancaster Regt.)

Killed in action on the Somme 20 fuly 1916

Happell, A. C. Capt., Suffolk Regt.

HARBORD, V. Capt., R.E.(Fortress, T.F.); empld. Min- I9I3 istry of Munitions. (W.)

HHARDMAN, F. M. 2nd Lieut., R. Fusiliers

Killed in action at Neuve Chapelle 29 Oct. 1914

Harman, C. E. Lieut., Middlesex Regt. (W.) (P.) I9I3

HARMER, W. D. Capt., R.A.M.C. (Ist London Gen. 1892 Hospital, T.F.)

HARPER, F. A. Capt. (T.), R.A.F.

HARRISON, E. P. Lieut., R.F.A.

Hartley, L. B. Surgeon Lieut., R.N.

1907

1903

1912

HARTRIDGE, H. Lieut., R.N.V.R.

1905

Haslam,W.H. Hon.Lieut., R.N.V.R. O.B.E. Cavalier, I908 Order of the Crown of Italy

Haviland, W. P. Capt., Argyll and Sutherland Hdrs. 1899 (T.F.); G.S.O. 3. M.B.E. $m 2$.

Haward, T. W. Lieut. and Adjt., Inns of Court O.T.C. $\quad$ I903 $m 2$.

Hawes, E. L. Capt., E. Surrey Regt.(T.F.)

Haynes, G. S. Capt., R.A.M.C. (Ist E. Gen. Hospital, I899 T.F.)

Healey, F. G. 2nd Lieut., R.E. (Signals). (W.) I9II

Heap, F. G. Corpl., R.E. (Signals); Lieut., Tank Corps. I9II M.C.

Hearson, H. F. P. and Lieut., Bombay Light Horse, 1904 Indian Defence Force

WHebblethwaite, A. R. Trooper, 18th Hussars; 2nd 1913 Lieut., R.F.A.

Killed in action 3 Oct. I9I 5

Hedley, E. W. Capt., R.A.M.C. (5th London Gen. 1892 Hospital, T.F.) M.B.E. 
Hedley, I. M. Lieut., I7th Lancers. $M$. I9ro

Hedley, J. P. Capt., R.A.M.C. (5th London Gen. Hos- 1895 pital, T.F.)

HedLEY, W. Major, R.G.A. D.S.O. M. $\quad 1898$

HeElas, R. J. Capt., R.G.A. (W.) $M$. 1902

Helm, C. Lieut.-Col., R.A.M.C. D.S.O. M.C. M. 1907

Henderson, I. M. Capt., London Regt. (London Scot- 1896 tish). (W.) (P.) M.B.E. Brevet Major

Hensley, E. H. V. Capt., R.A.M.C.

HERBERT, Hon. E. J. B attd. M.G.C. Order of the White Eagle, 5th Class (Serbia)

Killed in action 12 Nov. 1917

Herman, A. E. Surgeon Lieut., R.N. O.B.E.

Herman, G. L. Capt., Queen's Own (R. W. Kent Regt.); 1905 Staff Capt.

Heycock, C. T., v.D. Lieut.-Col., Cambridgeshire Regt. $\quad 1877$ (T.F. Res.) $m$.

HeYworth, E. L. Lieut., Manchester Regt.; Lieut.(Ad.), I9ro R.A.F. (W.) (P.)

HibberT, J. P. M. Major, R.F.A.(T.F.) M.C.

WHickman, T. Pte., H.A.C.; Lieut., Leinster Regt. (W.) ${ }_{1907}$

Killed in action near Armentières 26-27 Fune 1916

Hicks, R. J. B. Pte., Gloucestershire Regt.

Higson, F. S. Capt., Welsh Regt. M.C.

Killed in action 31 Aug. I9I7

Hill, A. V. Capt., Cambridgeshire Regt.; empld. *I9ı6 Ministry of Munitions. O.B.E. Brevet Major. $m 2$.

Hill, A. W. Hon. Capt., Spec. List (Graves Registration 1894 Commission). $m 2$.

Hine, T. G. M. Hon. Major, R.A.M.C. C.B.E. O.B.E. 1892 $m 2$.

HoAre, C. G. Lieut., Essex Yeo., R. Horse Gds., and I90I Gds. M.G. Regt. M.C.

HoAre, L. G. Capt., Durham L.I.; Staff Capt. $M . \quad 1898$

Hoвbs, F. D. Lieut., Life Gds.; Lieut. (A.), R.A.F. I9I I

HodDER,A.E. Lieut.-Col.,R.A.M.C.(T.F.) (W.) D.S.O. 1895 $M 2$.

Hodges, A. G. A. Capt., Northamptonshire Regt.(T.F.); 1912 Hon. Capt. (K.B. and Ad.), R.A.F. (W.)

Holland, F. Capt. and Adjt., King's (Liverpool Regt., 1900 Liverpool Scottish, T.F.) M.B.E.

Hollins, E. M. Capt., R.A.S.C.(M.T.)

Hollond, R. C. Capt., Rifle Brigade. (W.) $M$. 
WHolmes, T. S. 2nd Lieut., The Queen's (R.W. Surrey 1912 Regt.); attd. The Buffs (E. Kent Regt.)

Killed in action 12 Nov. I9I4

WHolT, H. W. 2nd Lieut., R.E.

Killed in action 23 Aug. 1914

Homfray, S. G. Lieut., R.N.V.R.

1906

Hope-Johnstone, C. J. 2nd Lieut., Gen. List(T.F. Res.) 1903

HoPe-Jones, W. Lieut., R.G.A.; attd., R.E. 1903

WHopkinson, B., F.R.S. Major, Unattd.List,T.F.; Colonel, *I914 D.A.D., Air Ministry. C.M.G. $m 2$.

Killed in flying accident 26 Aug. 1918

HoRne, H. F. Major, R.A.M.C. (Sanitary Section, T.F.); 1897

Lieut.-Col. (Med.), R.A.F. T.D. Brevet Lieut.-

Colonel. Brevet Major

WHornung, A. O. 2nd Lieut., Essex Regt.

[1914]

Killed in action at Ypres 6 fuly r9I 5

WHorsfall, C. F. Capt., D. of Wellington's (W. Riding 1908 Regt.) (W.)

Killed in action 18 Sept. I916

WHorsfall, R. E. Capt., King's (Liverpool Regt.) (W.) 1912

Killed in action at La Vacquerie 20 Nov. 1917

WHoward, A. J. Lieut., Bedfordshire Regt.

Killed in action 4 Sept. I9r6

Howarth, W. G. Capt., R.A.M.C. (2nd London Gen. 1897 Hospital, T.F.)

Howell, G. F. Lieut., The Buffs (E. Kent Regt.) 1908

Howson, H. E. E. Lieut., Shrewsbury School O.T.C. 1907 and Eton College O.T.C.

Huntington, Sir C. P., Bart. Lieut., R. Irish Regt. (W.) 1907

Hurst, G. H. J. Capt., R.G.A.; Major, Gen. Staff 1890

INFELD, H. Capt., London Regt. (Rangers); Major, Spec. 1912 List (cmdg. Spec. Coy., R.E.) (W 2.) M.C.

INGLE, L. M. Capt., R.A.M.C. (W.)

INGLIS, A. E. J. Lieut., Army Printing and Stationery 1906 Services

INGLIS, C. E. Major, R.E.; Staff Capt., War Office. 1894 O.B.E. Brevet Major. $m$.

INGLIS, G. S. Lieut., Connaught Rangers and R.F.C.; I9I3 Staff Lieut. M.C.

Ingram, E. M. B. Capt., Spec. List; G.S.O. 3, War 1909 Office. O.B.E. m. Order of the White Eagle, $5^{\text {th }}$ Class (Serbia)

INSKIP, J. H. Lieut. (K.B.), R.A.F. 
FIRving, A. G. 2nd Lieut., R.E. and R.F.C.

Killed in flying accident 15 March 1915

1908

JAFFÉ, A. D. Capt., London Regt. (London Irish Rifles) 1899 JARDINE, J. W. Major (K.B.), R.A.F. (W.) M. 1899 JebB, R. D. Capt., R. Sussex Regt.(T.F.) M.C. M. 1903 Jenkin, C. O. F. 2nd Lieut., Suffolk Regt.; Lieut., Gen. 1909 List, empld. Ministry of Munitions. (W.)

WJenkins, E. E. Capt., R. Warwickshire Regt.; attd. 1906 Lancs. Fus.(T.F.) (W 2.) M.C.

Killed in action 25 March 1918

JoHN, J. C. Capt., I.M.S. O.B.E. M.

Johnson, C. Capt., Cheshire Regt. (T.F.); empld. Ministry of Munitions. (W.) M.B.E. Brevet Major. $m_{3}$.

Johnson, E. H. Capt., R.F.A.(T.F.) (W 2.) M.C. M. Johnson, G. F. Major, R.F.A.(T.F.) (W.) $M 2$.

Johnson, G. G. F. Capt., Essex Regt. and King's African Rifles

WJohnson, R. L. Capt., R.F.A. (W.) Killed in action 28 May 1917

Johnston, F. B. Pte., King's (Liverpool Regt., Liver- 1912 pool Scottish, T.F.); Capt., Northumberland Fus. (T.F.); attd. Highland L.I.

Johnstone, G. G. Capt., R.A.M.C. (Sanitary Service, 1905 T.F.) M.C. $M$.

Jones, A. P. Capt., King's (Liverpool Regt., T.F.) I9Io WJones, A. V. Pte., H.A.C.

Killed in action 25 Nov. 1914

Jones, C. E. M. Capt., R.A.M.C.(T.F.) (P.) 1900

WJONES, R. A. Major, R. Warwickshire Regt. I90I

Killed in action 21 May 1916

Jones, R. L. A.B., R.N.V.R. (Anti-Aircraft); Lieut., $\quad 1896$ R.G.A.

JosePh, H. H. Capt., Durham L.I.(T.F.) (P.) 1903 Joyce, J. L. Major, R.A.M.C.(T.F.) Brevet Major. m. $\quad$ I90I

KarPELES, A. R. Lieut., Gen. List (T.M.B.) (W.) [19I4]

KEDDIE, C. M. Lieut., I.A.R.O., attd. 36th Sikhs and 1909 82nd Punjabis

HKene, C. A. Capt., Rifle Brigade

Killed in action in the Ypres Salient 12 Fuly 1916

KeMPSEY, F. Lieut., R.G.A.

WKemp-Welch, M. 2nd Lieut., Yorkshire Regt.

Killed in action I I April I9I7

1913

1910

I899 
Kendall, E. A. Capt., King's Own (R. Lancaster Regt.) 1906 (W 2.) M.C. M.

KenNedy, J. S. Surgeon Lieut., R.N.

KING, H. H. Capt., London Regt. (Post Office Rifles); 1908 attd. R.E. (Signals, T.F.) (W.)

KinghoRn, E. C. Lieut., Border Regt.; attd. Oxford and r9ro Bucks. L.I.; Lieut. (O.), R.A.F. (W 2.)

KIRK, J.W. C. Lieut.-Col., D. of Cornwall's L.I. D.S.O. $\quad 1896$ $M 2$.

Knox, A. D. Lieut., R.N.V.R., empld. Admiralty

1903

LACEY, A. T. Major, M.G.C. (W.) $M 2$.

LAmbarT, J. H. L. Capt., R.F.A.; A.D.C.; Staff Capt. 1912 (W.) $M$ 2. French Croix de Guerre

Lance, H. W. Lieut., Norfolk Yeo.; Capt., R.A.M.C. 1889 $M$.

Lanchester, W. F. Capt., R.A.M.C. (Sanitary Service, r893 T.F.)

Langdon, Rev. A. G. C.F. 4th Class, R.A.C.D.

LAPAGE, F. C. 'Capt., R.A.M.C.

HLarking, R. G. Capt., R.E. (Signals). M.C. and Bar I9ro Accidentally killed on active service I April r918

Larkworthy, F. G. C. Lieut., R. Welsh Fus.

LAYARD, A. H. Lieut., R. Sussex Regt.; attd. E. Surrey [1913 Regt.

LAZARUS, G. M. Lieut., R.G.A.

LEE, E. O. Lieut., I.A.R.O., attd. 34th Sikh Pioneers

WLEGG, H. G. Pte., H.A.C.; Lieut., Durham L.I. (W 2.) Killed in action on the Somme 25 March rgr8

WLegGate, L. C. 2nd Lieut., Rifle Brigade and Cold- 1913 stream Gds.

Killed in action at Pilkam Ridge $3 \mathrm{I}$ fuly r917

LEwIN, E. O. Lieut.-Col., R.F.A.; G.S.O. r. C.B. ${ }_{1897}$ C.M.G. D.S.O. Brevet Lieut.-Colonel. $M 6$. Chevalier, Legion of Honour (France). French Croix de Guerre. Commander, Military Order of Avis (Portugal)

LEwIS, A. D. Capt., R.E.; Major, D.A.D. Inland Water 1902 Transport

LIAS, A. G. Capt., D. of Wellington's (W. Riding Regt.) 1907 and Gen. List, empld. R.M.C., Sandhurst

LiNG, G. A. Capt., Suffolk Regt. (W.)

Livesey, T. R. M. Lieut., I.A.R.O., attd. Cavalry

LLOYD, J. R. Lieut., R.A.F. 
Lов, H. Lieut., E. Lancs. Regt. and R.E. (Field Survey 1905 $\mathrm{Bn}$.

LocH, D. H. Capt., Spec. List. Order of the Redeemer, 1902

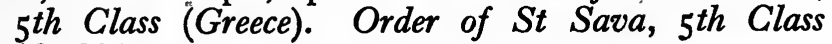
(Serbia)

WLongworth, E. C. Pte., R. Fusiliers (P.S. Bn.); Capt., 1908 Lancs. Fus.

Killed in action at Thiepval 26 Sept. 1916

WLoRING, W. Capt., Scottish Horse. $M$.

Died 24 Oct. 1915 of wounds received in action in Gallipoli

ZLupton, G. A. Lce.-Corpl., R. Fusiliers

Killed in action $17 \mathrm{Feb}$. 1917

LuPTON, Rev. J. M. Capt., Marlborough College O.T.C. 1886

Lyon, Rev. W. T. C.F. 4th Class, R.A.C.D.(T.F.) 1905

McArthur, A. G. F. Capt., R.A.M.C.

1885

WMCDonnell, J. Major, D.A.Q.M.G.; Lieut.-Col., Lein- 1897 ster Regt.

Killed in action 29 Sept. 1918

सMcDougall, S. Lieut., Manchester Regt.(T.F.) 1895

Killed in action in Gallipoli 7 Aug. 1915

WMAchell, H. G. 2nd Lieut., Border Regt.

Died 12 Fune 1918 of wounds received in action

MCINTYRE, J. Capt., R.A.M.C. M.C.

1912

MAcray, R F B Capt, Essex Regt.(T.F) m.

MACKAY, R. F. B. Capt., Essex Regt.(T.F.) m. I9Io O.B.E. M. $m$.

Mackeson, G. P. Lieut., London Regt. (R. Fus.)

McLean, C. F. Surgeon Lieut., R.N.V.R.

Macmillan, W. E. F. Capt., Cameronians (Scottish I9I4 Rifles, T.F. Res.) and Gen. Staff

Macmorran, K. M. Lieut., E. Surrey Regt. (T.F.); attd. 1902 R. Sussex Regt.; Capt., Spec. List (Judge-AdvocateGeneral): $M$.

AMAdDRELl, J. D. H. Lieut., D. of Cornwall's L.I.

Died 13 Dec. 1916 of wounds received in action 22 Nov. 1916

Mahaffy, R. P. Capt., Devon Regt. and Gen. List, I89o empld. Egyptian Army. (W.)

Malden, Rev. R. H. Chaplain, R.N.

Mansell-Moullin, O. Lieut. (A.), R.A.F. (P.)

Marett Tims, H. W. See Tims, H. W. MaretT

I 898

1906

Marsden, A. T. Major, Loyal N. Lancs. Regt. M.C. M. ${ }^{1913}$ 
Marseille, R. K. G. Capt., Loyal N. Lancs. Regt.; I9I3 empld. Ministry of Pensions. (W.) (P.)

*Marsh, F. Howard. Hon. Colonel, R.A.M.C.(T.F.) * * 1903 Died 24 June 1915

Marshall, H.G. Lieut., Border Regt.(T.F.); Staff Capt., 1902 Indian Army

MaRTIN, O. L. Capt., York and Lancaster Regt.; I9I I G.S.O.3. (W 2.) m. French Croix de Guerre

Martin, R. E. Lieut.-Col., Leicestershire Regt.(T.F.) 1893 (W 2.) G.M.G. M.

WMartin, S. S. Corpl., Middlesex Regt.

Killed in action I3 Aug. I9I7.

Mason, F.L. L. Lieut., Rifle Brigade; empld. War Office 1907

MASSEY, B. W. A. Lieut., R.F.A.; Interpreter. (W.) 1903

Masson, K. Surgeon Prob., R.N.V.R.; Capt., R.A.M.C. 1909

Mathias, H. H. Capt., R.A.M.C.

Mawe, E. S. Capt., R.A.M.C.

MAWE, Rev. M. D. Major, R.F.A. and R.G.A.

Mayor, H. B. Capt., Clifton College O.T.C.

Meister, G. C. Lieut., Sedbergh School O.T.C.

Meister, G. E.W. Pte., R. Fusiliers; Lieut., R. Defence 1890 Corps

MerCER, J.L.C. Capt., The Queen's (R. W. Surrey Regt.) 1909

MEREDITH, H. O. Lieut., Belfast Univ. O.T.C.; empld. 1897 Ministry of Munitions

Milne Home, C. A. Lieut., King's Own Scottish Bor- 1909 derers. (W.)

Milner, G. Capt., R.E.; empld. Egyptian Army 1912

Milner-White, Rev. E. C.F. 3rd Class, R.A.C.D. $\quad 1903$ D.S.O. M.

Moberly, A. H. Capt., London Regt. (Surrey Rifles) and 1903 Gen. Staff (O.C.B.) (W.) $m$.

MoIr, K. M. Lieut., E. Surrey Regt.(T.F.); Capt. and [1914] Adjt., M.G.C. M.C. $M$.

\#Mond, F. L. Lieut., R.F.A.(T.F.); Staff Lieut.; Lieut. 1912 (A.), R.A.F.

Killed in action at Bouzencourt ${ }_{5}$ May 1918

MonRo, K. N. Major, R.E.

4MOORE, M. E. J. Lieut., R. Irish Rifles. (W 2.) M.C. I9I3

Died in German hands 27 March 1918 of wounds received in action 24 March 1918

Moorsom, J. Lieut., Life Gds. and Gds. M.G. Regt. 1899

Moorsom, R. S. Capt., Westmorland and Cumberland I9II Yeo.; attd. Border Regt. (W.) 
Morrison, Rev. A. C.F. $4^{\text {th }}$ Class, R.A.C.D. $\quad$ I894

Morse, F. A. V. Capt., R.E. (London Electrical Engin- 1897 eers, T.F.)

Morse, L. G. E. Capt., R.E. (London Electrical Engin- 1904 eers, T.F.) M.B.E.

${ }^{1}$ Morton, H. S. Capt., R.A.S.C.; Major, D.A.D. Quar- * ${ }^{*}$ I9I5 tering, War Office. O.B.E.

Morton,J.A.F. Capt., Northamptonshire Regt.; empld. 1912 Ministry of Labour

Moulton, H. F. 2nd Lieut., R.F.A. (W.) 1895

\#Moulton, W. R. O. 2nd Lieut., Manchester Regt. I9IO

Killed in action 5 Aug. 1916

Mowls, Rev. H. W. K. C.F. 4th Class, R.A.C.D. 1909

Mozley, B.C. Capt., Dorset Regt.; empld.O.C.B. (W2.) I912 D.S.O. M.

MozLEY, J. H. Capt., R.F.A. (W.)

Mudie, R. F. Capt., I.A.R.O.; Asst. Instructor, School 1908 of Musketry. O.B.E.

MummerY, R. T. Lieut., R.G.A. $\quad 1914$

WNANCARROW, J. V. Capt., Yorkshire Regt.(T.F.) 1903 Killed in action near Ypres 25 April 1915

NAPIER, L. P. Lieut., Canadian Hdrs.; Capt., Canadian 1909 F.A.; Staff Capt. M.C.

Nathan, E. J. Capt., Spec. List (Directorate of Railways 1907 and Roads, War Office). O.B.E. m.

NaumanN, J.H. Capt., Rifle Brigade and Nigeria Regt., 1912 W. African Frontier Force; Staff Capt. (W.)

Neal, A. W. Lieut., R.F.A. M.C.

NeAL, J. Major, R.G.A. M.C.

WNEWBERRY, J.D. Flt. Cdr., R.N.A.S. French Croix de I9I3

Guerre

Killed in flying accident 28 Sept. 1917

Newton, H. W. G. 2nd Lieut., Somerset L.I. (W.) (P.) 1902

NobLe, H. B. Capt., Northumberland Yeo.; G.S.O. 3. I9I I M.C. M 2. Chevalier, Order of Leopold (Belgium). Belgian Croix de Guerre

Norton, R. H. Lieut., Coldstream Gds.

I 896

Oatfield, W. J. Capt., King's (Liverpool Regt.,T.F.) I9ro (W 2.)

WO'Callaghan, T. F. 2nd Lieut., Leicestershire Regt. I9I3 (T.F.)

Killed in action 13 Oct. I9I 5

1 Died of influenza shortly after demobilisation. 
Ogilvie, H. Lieut., R.E. (W.) 1900

OgILvy, L. W. Trooper, Calcutta Light Horse, Indian 1896 Defence Force

\#ORD, O. R. Lieut., Rifle Brigade

Killed in action at Lesboufs I6 Sept. I916

Osmaston, D. F. Capt., R.E. $M$.

1914

I9I I

WPage, R. B. Colonel, Lancs. Fus.

Died I I Nov. I9I4 of injuries accidentally received on active service

Parker Smith, A. C. H. Capt., Argyll and Sutherland Hdrs.

*PARKer Smith, W. B. Lieut., Scottish Horse

Died I I Sept. I9I5 of wounds received in action in Gallipoli 2 Sept. I9I 5

Partridge, H. W. Lieut., Gresham's School, Holt, 1906 O.T.C.

Pass, A. D. Capt., Dorset Yeo. (W.) (P.)

APaterson, I. R. 2nd Lieut., Cameron Hdrs.

Killed in action $\mathrm{I} 2$ Oct. I9I7

Peacey, H. M. Lieut., R. Sussex Regt.

Pearsall, R. H. Capt. and Adjt., R.E.(T.F.) $M$.

Perrin, M. Capt., Loyal N. Lancs. Regt. M.C.

I 877

Pilkington, L.E. Lieut.-Col., S. Lancs. Regt.(T.F. Res.) C.M.G. M. $m 2$.

सPilter, C. Lieut., I8th Hussars. (W.)

Died 30 May 1915 of wounds received in action

WPlayfarr, P. L. Capt., Black Watch(T.F.) (W.)

Died I I April I9I8 of wounds received in action at Lestrem

Plowden-Wardlaw, Rev. J. T. C.F. $4^{\text {th }}$ Class, R.A.C.D. 1892 सPochin, A. C. 2nd Lieut., Essex Regt. (W.) I9I4 Killed in action 26 Sept. 1916

Pocock, S. E. Major, R.A.S.C. O.B.E. $m$.

WPolack, B. J. Pte., R. Fusiliers; 2nd Lieut., Worcester- 1909 shire Regt. $M$.

Killed in action 9 April I916

Pollak, L. A. Capt., London Regt. (St Pancras Bn.); 1908 Major, M.G.C. (W 2.) M.C. and Bar. M. French Croix de Guerre

Popham, A. E. Capt. (S.), R.A.F. M. French Croix de 1908 Guerre

सPowel, K. Pte., H.A.C.

Killed in action $18 \mathrm{Feb}$. I9I 5 
WPowell, P. 2nd Lieut., Rifle Brigade

Killed in action at Ypres 2 Aug. 1915

PretTy, K. Capt., R.A.M.C. M.

1898

PRIOR, H.C. Lieut., I.A.R.O., attd. Ist Gurkha Rifles, $M$.

Puckle, F. H. Capt., I.A.R.O., attd. N. Waziristan Mil- r908 itia

Purves, C. L. Capt., E. Lancs. Regt

1909

QuIRK, Rev. R. C.F. $4^{\text {th }}$ Class, R.A.C.D.

I90I

RaImes, A. L. Major,Durham L.I.(T.F.) (W3.) D.S.O. 1904 $M 2$.

Rand, H. M. Capt., R.E. (Signals, T.F.) $M$.

RANSFORD, F. B. Capt., London Regt. (Cyclist Bn.)

RAwlins, A. Lieut., 5th (R. Irish) Lancers; Lieut. (Ad.), R.A.F. (W.)

RAYMOND, E. L. Lieut., Rossall School O.T.C.

Reading, E. W. Capt., N. Staffs. Regt.; D.A.A.G. $M . \quad$ IgI I

ReckItT, A. Capt., E. Yorks. Regt.; Capt. and Adjt., 1892 Durham L.I. $m$.

REDDEN, F. A. C. 2nd Lieut., Worcestershire Regt.

REECE, R. H. Surgeon Lieut., R.N.

ReED, Rev. L. G. C.F. 2nd Class, R.A.C.D.; D.A.C.G. 1902 M.C.

REES, J. R. Capt., R.A.M.C. Chevalier, Ordre de la 1908 Couronne (Belgium)

REID, K. G. Lieut., R.N.V.R.

Reinert, E. L. See Raymond, E. L.

ReNwick, J. E. Lieut., Cheshire Regt.

WRenwick, T. B. Lieut., Rifle Brigade; attd. Middlesex

Regt.

Killed in action 29 April 1915

Revillon, J. W. 2nd Lieut., R.E. O.B.E. M. $\quad 1905$

RHOdes, H. E. Capt., R.A.M.C.

1887

1913

I9I I

1904

1908

I9II

808

I899

1904

1912

1910

1906

Killed in action near Ypres 25 April r91 5

Rice, Rev. C. M. C.F. 4th Class, R.A.C.D.

Richardson, P. W., v.D. Bt. Lieut.-Col., T.F.Res.; Chief 88 Instructor, School of Musketry. O.B.E. m.

Richmond, O. L. 2nd Lieut., London Regt.; Capt., 1900 Spec. List. m. Cavalier, Order of the Crown of Italy

WRICKEARD, C. W. Lieut., London Regt. (St Pancras Bn.) 1913 Killed in action 25 Sept. I9I 5

RICKETT, G.R. Lieut.-Col.,R.A.M.C.(T.F.) O.B.E. M3. ${ }_{1} 896$ 
Riddiough, S. Lieut., R.A.M.C. (W.) 19 ro

Riggall, H. B. Capt., Lincolnshire Regt.(T.F.) (W 2.) I9I3

Ripman, C. H. Capt., R.A.M.C.

1898

${ }^{1}$ Ritchie, S. E. Capt. (A.), R.A.F.

सRoberts, C. H. Pte., R. Fusiliers

Killed in action at Thiepval Sept. 1916

1914

I9I 5

Robertson Smith, N. M. Capt., Cameronians (Scottish 1905 Rifles, T.F.)

Robinson, R. H. O. B. Surgeon Lieut., R.N.

WRolleston, F. L. Lieut., London Regt. (R. Fus.)

Killed in action at Armentières 26 April 191 5

Rooth, A. V. Capt., I.A.R.O. (W.) M.C. M 2. 1905

सRoscoe, A. 2nd Lieut., Queen's Own (R.W. Kent Regt.) 1909 M.G.

Died 5 Sept. 1916 of wounds received in action

Rose, G. K. Major, Oxford and Bucks. L.I. (W.) M.C. 1908 and Bar. $M 2$.

Ross, C. M. Cadet, O.C.B.

RowlatT, A. Capt., D. of Cornwall's L.I.

Howntree, L. E. 2nd Lieut., R.F.A. (W.)

Killed in action 25 Nov. I917

Rowntree, M. 2nd Lieut., R.E.

Rubens, H. V. Pte., R. Fusiliers

1914

[1914]

Rumsey, Rev. H. St J. 2nd Lieut., Epsom College O.T.C. $\quad$ I906

Russell, G. G. Lieut.-Col., K. Edward's Horse. D.S.O. 1900 $M_{3}$.

Russell, P. D. Lieut., N. Zealand M.G.C.

Russell, S. C. Lieut., Cameron Hdrs. (W 2.)

I901

1913

Sainsbury, W. T. Pte., R. Fusiliers (P. S. Bn.); Capt. I9I I and Adjt., W. Yorks. Regt. (W.) M.G. and Bar

SAMBrook, H. F. Major, R.E. (Postal Section)

SAXTON, W. I. Instructor Lieut., R.N.

1905 I9II

※Scholes, W. P. 2nd Lieut., Leicestershire Regt.(T.F.) I9I3

Killed in action $\mathrm{I}_{3}$ Oct. I9I 5

Scholfield, A. F. Pte., Calcutta Port Defence, attd. 1903 Sappers and Miners, Indian Army

Schwann, H. See Swann, H.

ScotT, W. M. Major, R.E.(T.F.)

¥Scrutton, H. U. Corpl., R.E. (Signals); Capt., North- I9I3 umberland Fus. (W.) M.C. $M_{3}$.

Died in a Bulgarian hospital ro Sept. 1916 of wounds received in action on the Struma

1 Killed in flying accident after the armistice. 
Scrutton, Rev. T. B. C.F. $4^{\text {th }}$ Class, R.A.C.D. (W.) 1906 SECondé, E. C. Capt., r6th Rajputs, Indian Army 1902 Seddon, A. E. Capt., Suffolk Regt. (W.) Selwyn, Rev. E. G. C.F. $4^{\text {th }}$ Class, R.A.C.D. $M$. ¥Seyrig, J. R. Pte., ire Régt. Etranger, French Army

Killed in action in the Champagne 25 Sept. 1915

Shand, P. M. Gnr., R.F.A.

SharPe, W. H. S. Capt. (K.B.), R.A.F.

1910

1904

I913

SHAw, E. A. Surgeon Cdr., R.N. O.B.E.

1906

1903

Shaw, G. R. D. 2nd Lieut., E. Riding of Yorkshire Yeo.; [I9I4] attd. Essex Regt.(T.F.)

\#Shingleton-Smith, F. Capt., I.M.S.

Killed in action at Ctesiphon 22-24 Nov. I91 5

Simey, A. I. Lieut., R.A.M.C.

¿Simon, H. H. Major, R.F.A.(T.F.)

Died 8 Sept. I9I7 of wounds received in action I Sept. I9I7

Simpkinson, F. V. Capt., R.E. (W 2.)

Sinclair, G. W. Lieut., Manchester Regt.(T.F.); empld. 1908 Ministry of Labour

SMarT, F. W. B. Major, Charterhouse School O.T.C. 1892 T.D. Brevet Lieut.-Colonel. $m$.

सSmith, A. F. Corpl., Suffolk Regt. and R. Irish Rifles 1915

Missing, presumed killed in action, 16-17 Aug. 1916

Smith, C. E. G. Capt., Army Cyclist Corps and M.G.C.

Smith, E. F. Lieut., E. Lancs. Regt.; empld. Command Depôt. (W.)

Sмiтh, E. M. Capt., R.E. M.C. M M. Military Order 1909 of Avis, 3 rd Class (Portugal)

SмIтн, F. B. Capt., R.A.M.C.(T.F.) M.C. Chevalier, 1906 Military Order of Avis (Portugal)

SмIтH, W.W. Lieut., R.A.S.C. and R.E. (Sound-ranging I9I I Section)

ASNell, F. S. Pte., R. Fusiliers (P.S. Bn.); 2nd Lieut., 1906

R. Berkshire Regt.

Killed in action I I fuly r916

Somervell, A. C. Major, R.A.S.C.; D.A. D. Supplies. 1902 O.B.E. $M 2$.

Somervell, L. C. Capt., R.A.M.C. $M$.

ASomerville, M. A. 2nd Lieut., Rifle Brigade; attd. Lon- 1906 don Regt.

Died $2 \mathrm{I}$ Sept. 1918 of wounds received in action at Wadi Kanah

Southern, N. Major, R.F.A. (W.) (P.) M.C. 
WSPencer, J. M. J. Gnr., R.F.A.(T.F.); Lieut., North- [1914] umberland Fus.; attd. R.F.C.

Killed in action 3 Nov. 1916

Spens, H. B. Lieut.-Col., Cameronians (Scottish Rifles, 1904 T.F.) (W.) D.S.O. and Bar. Brevet Major. $M_{4}$. French Croix de Guerre

Spielman, C. M. Major, R.E. M.C. Brevet Major. 1907 French Médaille d'Honneur

STACKARD, S.F.C. Corpl., R.E.(Signals); Capt., R.A.S.C. I9I I $M$.

Stanley, E. S. Lieut., R.G.A. (W.)

STEAd, J. Lieut., R.E.

STEPHEN, A. M. Major, R.G.A. (P.) M.C. $M$

\#Stephen, J. H. F. Lieut., Highland L.I. (W.)

Killed in action in Mesopotamia I I Fan. I917

Stocks, P. Lieut., R.A.M.C.

I910

1903

I9I I

1913

Stockwell, G. E. St C. Major, W. Yorks. Regt.(T.F.) and R.A.M.C.(T.F.) $M$.

Stokes, E. F. Capt., Northamptonshire Regt. (W.) I9I I

WStone, A. Lieut.-Col., Lancs. Fus. D.S.O. M.

Killed in action 2 Oct. 1918

WSTONE, H. B. Lieut., R.E.(T.F.)

Killed in action $18 \mathrm{Feb}$. I9I 5

Storrs, B. St J. Lieut., R.F.A.

Streatreild, H. G. C. Capt., Hampshire Regt.(T.F.)

Streeten, Rev. E. R. C.F. $4^{\text {th }}$ Class, R.A.C.D.

सStroud, H. C. Capt., R.E.(T.F.); attd. R.F.C. (W.)

Killed 7 March 1918 in defence of London during an air raid

STURDY, A. E. Lieut., Igth Hussars; Capt., Leicester-

shire Regt.; G.S.O.3. (W.) M.B.E. M. Order of St Stanislas, 2nd Class (Russia). Order of St Anne, $3^{\text {rd Class (Russia) }}$

\Swainson, J. L. Lieut.-Col., D. of Cornwall's L.I. and I896 King's Own (R. Lancaster Regt.) (W.) D.S.O. $M 2$.

Died 9 Aug. 1916 of wounds received in action 8 Aug. I9I 6

SwanN, H. Major, K. Edward's Horse

SwIFT, C. T. Capt., Grenadier Gds. (W.) $m 2$.

\#Symington, P. G. Lieut., Highland L.I.

Killed in action I fuly I9I6

Symonds, W. P. Cadet, O.C.B.

1904

1905

[1914]

I9I8 
TAIt, G.B. Capt., Middlesex Regt.(T.F.); Major,M.G.C. (W.) $M 3$.

TAYLOR, A.J. Major, R.A.S.C.; D.A.D.S.and T. O.B.E. I912 $M$ 2. Officer, Ordre de l'Etoile Noire (France)

TAYlOR, E. S. Capt., R.A.M.C.(T.F.) O.B.E. M 2. 1907 French Médaille des Epidémies

सTebbutr, R. J. Capt., Cambridgeshire Regt.; attd. Essex 1912 Regt. (W 2.)

Killed in action 23 Aug. 1918

Temperley, H.W.V. Capt., Fife and Forfar Yeo.; Major, $\quad 1898$ G.S.O. 2; Asst. Military Attaché, Belgrade. O.B.E. $m$ 2. Officer, Order of the Crown of Roumania, with swords. Order of the White Eagle, 5th Class, with swords (Serbia)

सThomas, H. W. Lieut., Rifle Brigade

Killed in action 3 Sept. I916

Thompson, B. W. Surgeon Sub-Lieut., R.N.V.R.

Thompson, H. Lieut., R.N.V.R.

Thompson, J. Lieut., R.E. $M$.

WThompson, W. F. Lieut., R.A.M.C.

\section{Died I Fan. I9I 6 of wounds received in action}

Thornhill, N. Lieut., Grenadier Gds. (W.) M.C. Igor

EThorp, R. O. V. Lce.-Corpl., R. Fusiliers (P. S. Bn.); 1897 Lieut., Northumberland Fus.; attd. T.M.B. (W 2.) M.C.

Killed in action 22 March 1918

WTILlEY, J. Capt., Norfolk Regt. (W.)

Killed in action near Arras 28 Nov. 1916

1909

I9I I

1908

1913

1906

Tims, H. W. MARETT. Lieut.-Col., R.A.M.C. O.B.E. 1899 m. Order of St Sava, $3^{\text {rd Class (Czecho-Slozakia) }}$

WTIPPET, A. A. 2nd Lieut., King's (Shropshire L.I.) Died 19 Aug. I9I 5 of wounds received in action

ToDd, L. Major, Eton College O.T.C.

Tomkinson, G. S. Capt., Worcestershire Regt.; Lieut.Col., R.E.; C.R.E. (W 2.) O.B.E. M.C. M.

Trapnell, F. C. Capt., R.A.M.C.

TrotTer J Y Tieut, A African Force; Cap

[1914]

1894

1902

1898

List (O.C.B.)

Trower, G. S. Capt., R.A.M.C. (W.)

TruscotT, R. F Capt, Sherwood Foresters (Nott and Derby Regt.) and Gen. List; Staff Capt. O.B.E. $M 2$.

Tuck, D. A. Capt., French Flying Corps; Capt. (A.), 1907 R.A.F. (W.) French Croix de Guerre 
Tuck, G. L. J. Lieut.-Col., Suffolk Regt.(T.F.) (W 2.) 1909 C.M.G. D.S.O. and Bar. M 4. Chevalier, Legion of Honour (France)

Tudsbery, F. C. T. Lieut., Middlesex Regt.; Major, 1906 D.A.D. Lands, War Office. C.B.E. O.B.E. $m 3$. \&TuRnbull, W. E. Lieut., R. Scots

Killed in action in Gallipoli 28 April 1915

Turner, J. R. Capt., Rangoon Bn., Indian Defence Force rgor Turner, L. B. Capt., Spec. List. $m$. 1904 Tyer, A. A. Capt. and Adjt., R.F.A. M.V.O. M 3. 1906

VAchell, E. T. Capt., R.E. (R. Monmouth). $M$. 1912 van Grutten,W.N.C. Capt., R.F.A.; Staff Capt. O.B.E. 1914 M.C. M 5. French Croix de Guerre

Vulliamy,E.O. Capt., Cambridge Univ.O.T.C.; empld. 1895 O.C.B.; Lieut., Spec. List (Intelligence)

Waley, F. R. Capt., S. Lancs. Regt. M.C. WWalker, B. S. 2nd Lieut., Cheshire Regt.

Killed in action ro May I9I 5

WALKER, E. A. Lieut., R.A.S.C.

Walker, Rev. H. C. C.F. $4^{\text {th }}$ Class, R.A.C.D.

Walker, H. R. Capt., King's Own (R. Lancaster Regt.) (W.)

WALKER, J. 2nd Lieut., R.F.A.

WWALKER, T. C. 2nd Lieut., Manchester Regt.(T.F.) Killed in action in Gallipoli 5 Fune 1915

WWallace, W. M. 2nd Lieut., Rifle Brigade; attd. R.F.C. 1912 Killed in action 30 Aug. I91 5

WALLER, J. C. 2nd Lieut., E. Surrey Regt. I9Io

Wallice, P. Capt., R.A.M.C. 1908

Walls, F. R. Capt., R.E. (W.) M.C. M. Chevalier, r9ro Ordre de la Couronne (Belgium). Belgian Croix de Guerre

Walton, J. H. Lieut., I.A.R.O., attd. 7th Gurkha Rifles 1909 WWARRE-CORNISH, G. W. Major, Somerset L.I. $\quad 1894$ Killed in action at Flers 16 Sept. 1916

WARRE-CoRNish, W.H. Lieut., N.Somerset Yeo.; empld. $\quad 1892$ War Office

WWatkins, I. E. M. Capt., Monmouthshire Regt.

1908

Killed in action near Ypres 7 May I9I5

Watson, A. P. Major, R.A.M.C.(T.F.) O.B.E. $M . \quad 1898$

WWatson, C. C. Lieut., R.F.A.(T.F.) $M$.

Died I Fune 1917 of wounds received in action near Lens 3I May I9I7 
Watson,F.W. Capt.andAdjt.,R.F.A.(T.F.) (W.) M.G. I9I I and Bar

WWatson, G. C. Capt., Devon Regt.

Killed in action in Mesopotamia 8 March 1916

\#Watson, R. W. Lieut., King's Royal Rifle Corps

Killed in action at Hooge $3 \circ$ fuly 1915

Waylen, G. H. H. Capt., R.A.M.C.(T.F.) M.C. M. $\quad 1899$

WebsTER, C. K. Capt., Spec. List; G.S.O. 3, War Office 1904 WWEINBERG, P. D. Lieut., Black Watch (T.F.) [1914] Killed in action 9 May 1915

WWeLCH,J.S. L. Lieut., King's Own (Yorkshire L.I.) M. [1914] Killed in action on the Somme I Fuly 1916

Wenham, R. A. Capt.; R.A.S.C. (Canteens). $M . \quad 1892$ *WeRnER, C. A. Capt., Rifle Brigade 1896

Killed in action at the Aubers Ridge 9 May 1915

West, R. R. F. Capt., Spec. List (Intelligence). D.S.O. 1910 $M 2$.

WWeston, J. C. and Lieut., London Regt. (Queen's West- rgor minster Rifles)

Died 6 Fune 1917 of wounds received in action 21 May I917

WhelON, C. E. Lieut., R.E. (Signals, T.F. Res.) 1906

WhitcomBE, R. C. P. Surgeon Lieut., R.N.

White, A. H. Capt., S.O. 3 , R.A.F.

WhiTE, A. S. Lieut., Manchester Regt.

WWhiTE, M. G. Lieut., Rifle Brigade

Killed in action I fuly r916

WhIte, R. HALE. Lieut., R.A.S.C.; attd. King's Own rgr3 (Yorkshire L.I., T.F.) (W.) M.C.

WhITTING, R. E. Capt., R.A.M.C. M.C.

WIDGERY, G. H. Lieut., R.F.A. M.C.

WigGans, J. T.V. Capt., Northumberland Fus. (W.) 1914 (P.)

WWIGLESWORTH, G. 2nd Lieut. (A.), R.F.C. I9I3

Killed in flying accident 8 fuly 1916

WILDE, E. H. N. Gnr., R.F.A.

WILkIN, A. Capt., R.A.M.C. M 2.

1898

1898

WILlCocKs, R. H. Sergt., H.A.C.

WilleY, H. Major, R.G.A.

Williams, H. G. E. Capt., R.A.M.C.

Williams, I. A. Lieut., Yorkshire Regt.(T.F.) and Gen. I9I0 Staff (Directorate of Requisitions and Hirings)

*WILLIAMS, R. A. W. Lieut., King's Own (Yorkshire L.I.) 1912 Killed in action at Ypres 18 April 1915 
Williamson, R. H. W. Capt., R.A.S.C.

Willink, A. J. W. Lieut., Border Regt.

WWillock, G. C. B. Capt., London Regt. (London Irish igro Rifles)

Killed in action in the Battle of Loos 25 Sept. 1915

Wills, A. G. Pte., Transvaal Scottish, S. African Force; rgro Capt., Cambridgeshire Regt. and M.G.C. (W 2.) M.C.

WWilson, H. S. Lieut., Worcestershire Regt.

Killed in action 15 Sept. I9I 5

Wilson, J. S. Capt., King's Royal Rifle Corps. (W 2.) 1908 Cavalier, Order of the Crown of Italy

WWilson, T. I. W. Capt., Manchester Regt. (W.) M.C. I90r Killed in action near Beaumont-Hamel 28 Nov. 1916

WiLson, W. G. Lieut., R.N.V.R.; Major, Tank Corps. 1894 C.M.G. $m 2$.

WingField-STRatFord, E. C. Capt., Queen's Own (R.W. I900 Kent Regt., T.F.)

WiNkFIELD, W. B. Lieut.-Col., R.A.M.C. I89I

WinterbothaM, W. Lieut., R.G.A. 1897

Wollaston, A. F. R. Surgeon Lieut., R.N. D.S.G. 1893 $M 2$.

WWomersley, S. P. 2nd Lieut., M.G.C.

Killed in action 15 April $19 \mathrm{r} 8$

WWood, P. L. 2nd Lieut. (A.), R.F.C.

Killed in action 4 March 1917

WoodHEAD, Sir G. S., v.D. Colonel, R.A.M.C. (Sanitary * *1899

Service, T.F.) K.B.E. O.B.E. Brevet Colonel. $m 2$.

Woolley, V. J. Capt., R.A.M.C.

WWright, E. M. Capt., E. Lancs. Regt.(T.F.)

Killed in action at Monchy-le-Preux Io April I9I7

Wright, F. W. Capt. (T.), R.A.F.

1910

I914

$\mathbf{1 8 9 6}$

1910

I90I

1Yorke, F. Trooper, Cheshire Yeo.; Lieut., Cheshire 1912 Regt.(T.F.); Lieut. (A.), R.A.F. (W 2.)

Young, M. A. Capt., Rifle Brigade. (W 2.) (P.) 1905

Young, R. A. Capt., Yorkshire Regt. and Northumber- I904 land Fus.

1 Killed in flying accident after the armistice. 


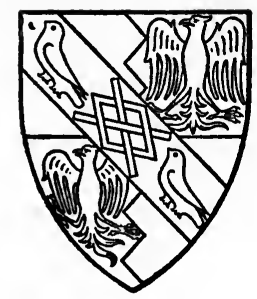

\section{MAGDALENE COLLEGE}

Ackerlex, J. R. Capt., E. Surrey Regt. (W.) (P.) [1914] Agar-Robartes, Hon. C. E. Lieut., Rifle Brigade; Capt., I9I I Tank Corps WAllin, H. W. Lieut., King's (Shropshire L.I.) 1908

Died 29 Dec. 1917 of wounds received in action

Allsopp, R. Capt., E. African Mtd. Infy. 1894 ARCHDALE, R. M. Lieut., Igth Hussars. (W.) M.C. I9II ARmstrong, H. M. Pte., Canadian Infy.; Gnr., Cana- 1909 dian F.A.

WARNold, A. C. P. 2nd Lieut., R. Fusiliers (P.S. Bn.) IgI I Killed in action 7 fuly 1916

Atkinson, Rev. A. V. Chaplain, R.N. 1905

BaGNall, R. O. G. 2nd Lieut., R. Berkshire Regt. 1912 Bagot-Chester, H. A. Capt., Staffordshire Yeo. (T.F. 1890 Res.); A.D.C.

WBaillie, A. H. Major, Norfolk Regt. 1886

Died 18 Oct. 1918

BaIN, P. W. Capt. and Adjt., Connaught Rangers; 1905 Capt., R. Munster Fus. (W 2.)

Barber, Rev. R. W. C.F. $4^{\text {th }}$ Class, R.A.C.D.(T.F.) $\quad 1872$

Barber-STARKEY, C. C. Air Mechanic, R.A.F. 1909

Barkworth, R. C. Capt., R. Fusiliers. (W 3.) M.C. 1914 and Bar. $M$.

BARNES, F. P. Lieut.-Col., R.A.S.C.(M.T.) D.S.O. 1899 O.B.E. M 2.

WBarrow, A. J. Capt., Lancs. Fus. (W.) M.C.

Died in Germany 24 Fune 1918 of wounds received in action

Barstow, J.N. Capt., R.F.A. (T.F.); A.D.C.; Brigade 1909 Major. D.S.O. M.C. M 3 . 
Basset, A. F. Lieut., Gen. List (T.F. Res.); Lieut.(Ad.), I89I R.A.F.

Bathurst, P. L. Capt., E. Lancs. Regt.; empld. War I9r I Office. (W.) $M$.

Bayliss, B. H. Pte., London Regt. (Queen's Westminster 1907 Rifles); 2nd Lieut. (T.), R.A.F.

Beaumont, H. R. Capt., Gen. List (T.F. Res.) I885

Bellars, A. E. Lieut., Rangoon Bn., Indian Defence I899

Force

WBERLEIN, L. H. Lieut., R. Berkshire Regt.

Killed in action 25 Sept. r91 5

Bevir, W. 2nd Lieut., Roysse's School, Abingdon, O.T.C. $\quad$ I898

Biss, J. C. DE V. Capt., I.A.R.O. (Cavalry); attd. Gen. IgI I Staff. (W.)

Blakiston, Rev. B. R. Chaplain, R.N.

Blakiston, Rev. J. N. C.F. 4th Class, R.A.C.D.

Blamire-Brown, C. Lieut., Christ's Hospital O.T.C.

Blaxter, K. W. Lieut., R.A.S.C. and R.F.A. (W.)

BoND, D. Lieut., R.N.V.R.

Booker, G. H. Lieut. Spec. List, empld. War Office

Borrett, P. R. Capt., Scots Gds. (W.)

Boyne, Viscount. Hon. Colonel, Durham L.I.(T.F.)

BROAdBENT, F. M. Instructor Cdr., R.N.

Brophy, G. M. Capt., R.G.A. M.

Buckley, H. H. C. Lieut., Coldstream Gds.

\&Bull, G. J. O. Lieut., R.E.(T.F.) (W.)

Killed in action in Gallipoli 8 fuly 1915

Bundy, H. P. Lieut., Worcestershire Regt.(T.F.) M.C. I9I4 Burton, Rev. H. J. C. C.F. 4 th Class, R.A.C.D. M.B.E. $\quad 1897$

Butler, H. M. 2nd Lieut., Denstone College O.T.C. 1908

Canham, W.D. 2nd Lieut., Norfolk Regt.; Lieut., M.G.C. 1914 (W.) M.C.

Carlile, C. Capt., R. Scots

Carlile, E. Lieut., R.G.A.

*CARrack, C. J. R. Fusiliers

Killed in action $17 \mathrm{Feb}$. I917

I 895

1898

1904

I9I4

1899

1912

1908

I883

1886

I910

1907

1908

Carter, H. G. Major, R. Welsh Fus. (W 2.) M.C.

* Cave, W. H. C. 2nd Lieut., Dorset Regt.

Killed in action 16 March 1915

Chai Chan, M. R. Interpreter, Siamese Exp. Force

ACharlesworth, W. H. Major, King's Own (Yorkshire 1912

L.I.) (W.)

Killed in action 15 Sept. 1916 
Childe, C. P. Lieut.-Col., R.A.M.C. (5th S. Gen. Hos- 1877 pital, T.F.) $m 2$.

\#Clapton, A. 2nd Lieut., R. Fusiliers

Killed in action 5 Sept. I916

I912

Clarke, J. S. 2nd Lieut., W. Yorks. Regt.

Clarke, W. K. C.S.M., R. Fusiliers (P.S. Bn.); Lieut., Bedfordshire Regt.; empld. O.C.B. (W.)

Clements, W. G. Capt. and Adjt., R.F.A.(T.F.) M.C. I913

Coates, A. S. Capt., London Regt. (St Pancras Bn.); I909 attd. Rifle Brigade

AColes, E. R. Capt., 3rd Dragoon Gds.

Killed in action 12 May 1915

Collier, A. E. Capt., R.E. M.C.

1907

Collins, A. J. 2nd Lieut., London Regt. (L.R.B.); Capt. 1912 and Adjt., R. North Devon Yeo.

Colonna, Don Mario. Italian Army

Coryton, A. F. Capt., Hampshire Yeo.; attd. Hampshire Regt.; Lieut., I8th Hussars

1907

1913

Tron, V. E. Major, R.F.A.(T.F.) and Spec. List

(T.M.B.) O.B.E. $M_{3}$. French Croix de Guerre. Italian Croce di Guerra

Couch, A. W. Lieut., "Hampshire Regt.(T.F.); attd. 1904 W. Yorks. Regt.

Courthope, R. Pte., R.A.M.C.

Coxon, A. C. M. Capt., Norfolk Regt.(T.F.) (W.) (P.) 1907

ACRAwFord, C. N. 2nd Lieut., Northamptonshire Regt. 1914

Killed in action 8 April r916

Crichton-Browne, H. W. A. F. Lieut.-Col., King's Own 1884 Scottish Borderers. $m$.

CriçK, L. C. Capt., Lincolnshire Regt. (W.) M.C. M. Igro

CrisP, J. F. Capt., Suffolk Yeo.

Crosbie-Oates, E. C. Pte., R. Fusiliers (P. S. Bn.); I9I2 Lieut., M.G.C.; attd. R.E., Signals

Cullinan, M. W. F. Capt. and Adjt., King's Royal Rifle I9I2 Corps; empld. O.C.B. M.C. $M$.

Cuninghame, R. J. Lieut., Gen. List. M.C.

CurTIS, E. D. Lieut., United States Arty.; attd. Intelligence Dept.

DaIsh, T. Capt., R.E. M.C. $M$.

DashwOOD, C. E. Lieut., R:A.M.C.

1890

1914

DASHWOOD, Rev. R. C. C.F. 3 rd Class, R.A.C.D. m. $\quad 1887$

Davidson, A. H. G. 2nd Lieut., Highland L.I.; empld. 1916 P. of W. Camp 
Davidson, A. J. Capt., Gordon Hdrs.; empld Command 1908 Depôt

Davidson, J. C. F. Paymaster Sub-Lieut., R.N.V.R.; I9I I empld. Admiralty

Davies, T. H. Lieut., King's (Shropshire L.I., T.F.) 1896 and Labour Corps

Davis, J. O. Major (K.B.), R.A.F. M.C. M. French 1908 Croix de Guerre

Dawes, E. S. Capt. R. East Kent Yeo.

De Hoxar, C. F. M. Capt., Spec. List

1912

1883

De las Casas, M. Lieut., R. North Devon Yeo. (T.F. $\quad 1889$ Res.); A.D.C.

DE LeDESMA, A. F. Lieut., R.F.A.

WDenNis, J.N. Lieut., N.Staffs. Regt.; attd. M.G.C. M.C. 1912 Died ${ }_{1} 5$ Oct. 1917 of wounds received in action

DE Prosperi, F. C. Italian Army

DE WindT, H. W. D. Capt., Spec. List (cmdg. P. of W. 1875 Camp)

Dibble, T.E. 2nd Lieut., Wellington School, Somerset, I9I I O.T.C.

Dickins, B. 2nd Lieut., Lincolnshire Regt., King's Own 1909 (Yorkshire L.I.), and Hampshire Regt.

Dove, C. K. Lieut., R.G.A.; Asst. Officer i/c Records 1905

Doyne, R. W. Capt., Westmorland and Cumberland Yeo. $\quad 1887$

Druce, A. F. Lieut., Surrey Yeo. M.C. M. French I9r3

Croix de Guerre

Duke, M. 2nd Lieut., R.E.; empld. Ministry of Labour 1909

DuleEP-Singh, H.H. Prince F. V., M.V.o. Major, Norfolk 1887 Yeo. and Gen. Staff

Duncan, C. W. Lieut., Welsh Gds. and Gds. M.G. Regt. 1912

WDurrant, W. B. W. 2nd Lieut., Rifle Brigade I9I3

Killed in action 9 May r9r 5

Eccles, A. G. Capt., King's (Liverpool Regt.,T.F.) I9ro (W.) M.C.

EDwards, Rev. A. L. C.F. $4^{\text {th }}$ Class, R.A.C.D.

Elliott, A. G. Lieut., Grenadier Gds. (W 2.) M.G. 1898 Ellis, V. L. R.A.M.C.

ENGLAND, J. A. Lieut., Bedfordshire Regt.; attd. Durham L.I. (W.)

AEstcourt, A. C. Sotheron. Lieut., Wiltshire Regt. and Gloucestershire Regt.; Capt., T.M.B.; Lieut. (O.), R.A.F. (W 2.) M.C.

Killed in action $8 \mathrm{Aug}$. 1918 
Estcourt, W. B. Sotheron. Lieut., E. African Force 1909 Eustace, W. R. G. Capt., R.G.A. I9ro Evans, O. L. J. Lieut.-Col., R. Welsh Fus. $m$. ExETER, Marquess of. Lieut.-Col., R.F.A.(T.F.) $M$ 2. $\quad{ }_{1897}$

Fairbourn, A. N. Lieut., R.E. (W.) I908 Fairley, W. Capt. and Adjt., E. Surrey Regt.(T.F.); I9Io Capt., King's African Rifles. (W.) $M$.

Fergusson, N. M. Major, R.A.M.C.(T.F.) $M 2$. 1901

Fixsen, B. A. Lieut., Rifle Brigade

Fletcher, A. M. T. Capt., R.A.S.C.

I912

I 899

Fletcher, E. T. D. Lieut., London Yeo. (Middlesex 1908 Hussars); Surgeon Lieut., R.N.

Fowke, L. A. $\quad$ Lieut., Leicestershire Regt. $\quad$ (W.) $\quad M . \quad$ I906 FrEAKe, F. M. Capt., R.F.A.; A.D.C. M. I894 Frend, J. P. N. Zealand Rifle Brigade

FreweN, S. Lieut.-Col., A.A. and Q.M.G. 1896

* 1877

Friederichs, L. H. T. Capt., Cheshire Regt. and Spec. $\quad$ I893 List

FurNess, W. Capt., Loyal N. Lancs. Regt. (W.) 1906

\#Garnetr, I.W. 2nd Lieut., King's (Shropshire L.I.) 1912 (W.)

Killed in action 12 Feb. 1916

GrbBS, A. R. Pte., Hampshire Regt.; Lieut., Worcester- 1904 shire Regt.

Gibson, F. A. S. Capt., N. Staffs. Regt. (W 3.) M.C. 1912

Grles, C. C. T. 2nd Lieut., Devon Regt.; Lieut., Gren- 1913 adier Gds. (W.)

Gissing, A. C. Lieut., R.G.A.; attd. Indian Army [I9I4] GlasBrOOK, J. H.L. Lieut., Welsh Regt.; attd. R. Welsh I9I4 Fus. (W.) $M$.

Glasspool, R. T. B. Capt., Durham L.I.(T.F.) M.C. 1899 $M 2$.

ZGoodFoRD, C. J. H. Lieut., Hampshire Regt. M.C. [rgr4] $M$.

Killed in action I Fuly 1916

Goodwin, A. C. Pte., Suffolk Regt.; Lce-Sergt., Suffolk I9I4 Yeo.; Lieut., Labour Corps

GorDON, E. B. 2nd Lieut., Lancing College O.T.C. $\quad$ I912 Gore, I. St J. Capt., Gloucestershire Regt. (W.) I900 Grant, D. W. Lieut., Lovat's Scouts; attd. Cameron I9r3 Hdrs. 
*Gray, A. F. Lieut., Wiltshire Regt. and Cambridgeshire I9I I Regt. (W.)

Killed in action 26 Aug. 1918

Gray, Rev. P. C.F. $4^{\text {th Class, R.A.C.D. }}$

Grear, A.T.L. Capt., Gloucestershire Regt.(T.F.) (W 2.) 1906

GreEN, F. N. Lieut., R. Welsh Fus. $m$.

Greenwood, Rev. F. B. C.F. 4th Class, R.A.C.D. 1903

GroOM, H. L. Lieut. (A.), R.A.F.

Groom, Rev. R. W. C.F. $4^{\text {th }}$ Class, R.A.C.D.

Gubbins, C. F. R. Major, London Yeo. (Middlesex 1898 Hussars)

Gwyer, Rev. H. L. C.F. $4^{\text {th }}$ Class, R.A.C.D.

1902

HAIG, D. P. Lieut.-Col., Cameron Hdrs. and Cameron- $\quad$ I 886 ians (Scottish Rifles). O.B.E. $m$.

WHall, H. 2nd Lieut., Lincolnshire Regt.

Killed in action $\mathrm{I}_{5} \mathrm{Feb}$. 1916

Hall, V. C. Lieut.-Col., R.E. (Postal Section). O.B.E. 1900 $M 2$.

Hallows, R. W. Major, R.G.A.

Hammond, F. W. Lieut., King's Own Scottish Borderers. (W.)

Hancock, D. M. Lieut., Gen. List (T.F. Res.), empld. $\quad$ I893 Ministry of National Service

Hankin, G. T. Capt., Oxford and Bucks L.I.(T.F.) I 896 and Gen. Staff. $M$. $m$.

Hanson, Sir G. S., Bart. Capt., R.F.A and Suffolk Regt. 1886 Hardcastle, A. E. L. Capt., The Buffs (E. Kent Regt., I9I3 T.F.) (W.)

Harding, A. M. Capt. (K.B.), R.A.F.

1908

Harley, R. G. G. Capt., R. Fusiliers. and Gen. List 1900 (A.P.M.)

WHarrison, G.H. 2nd Lieut., Loyal N.Lancs. Regt. M.C. Died 21 Aug. 1916 of wounds received in action

Harrison-Broadley, J. Capt., E. Yorks. Regt.

Harrisson, A. E. Capt., R.A.M.C. $M$.

WHARTER, J. C. F. 2nd Lieut., Res. Regt. of Cavalry, attd. I908 Nottinghamshire Yeo.

Killed in action 28 Nov. I917

WHarvey, R. V. Capt., British Columbia Regt., Canadian I89r Force

Died in Germany 8 May 1915 of wounds received in action near Ypres 24 April I9I5 
Henn, W. F. Capt., R. Munster Fus.; attd. R. Irish IgI I Regt. and Gen. Staff (Intelligence). (W.)

Henryson-Caird, A. J. Capt., King's Own Scottish 1902 Borderers. (W.) M.C. $M$.

AHepbuRN, M. A. 2nd Lieut., Seaforth Hdrs.

Killed in action 30 Nov. I9I 4

AHepburn, R. P. 2nd Lieut., R.E. (Signals). (W.) M.C. I9I I

Died 3 Aug. I9I7 of wounds received in action

Higgs, H. J. Lieut.-Col., R.E.; A. D. Labour.

O.B.E. Albert Medal, ist Class. $M_{3}$.

WHILl, B. W. Lieut., Rifle Brigade and R.F.C. (W.) I9I I

Killed in action 4 March I9I7.

WHILl, M. C. Lieut., Leicestershire Regt. (W.)

Killed in action 17 fuly 1916

Hindle, E. Capt., R.E.(T.F.)

Hindley-Smith, J. D. Lieut. and Adjt., R.F.A.

Hollings, J. H. B. Lieut., 2 Ist Lancers. (W.)

I910

Hopewell, E. R. Capt., Worcestershire Regt. (T.F.) I908 (W.) M.C.

Hore, C. W. C. R.A.F.

HoRnsBy, J. A. Capt., 5th Lancers; A.D.C. (W.) 1909

Hughes, H. C. Lieut., R.E.

Hunt, R. Major, London Yeo.(T.F. Res.)

Hunter, G. J. Capt., Worcestershire Yeo.

Hunter-MusketT, R. G. Major, Middlesex Regt

Hyde Parker, W. S. See Parker, W. S. H.

1913

I910

I9I2

1906

1908
1909
1911
1879

I879

1907

I893

IsHERWOod, W. Lieut., R.G.A. (W.)

I9I5

JaCKson, C. R. Capt., Rifle Brigade; empld. O.C.B.; I9I2 Major, M.G.C. (W.)

JePson, R. W. Lieut., Cheshire Regt.; Capt.(T.), R.A.F. $\quad$ I907

WJoHns, B. C. 2nd Lieut., R.G.A.

Died 22 Oct. I9I8 of pneumonia

Johnson, C. R. I. Lieut., Northumberland Fus.(T.F.) I90I (W.)

Johnstone, C. A. Lieut., King's (Shropshire L.I.) (W.) I905

Jolly, A. F. Lieut., R. Fusiliers. (W.) M.C. [1914]

Jonsson, A. T. Capt., R. Irish Rifles. (P.) 1899

JourdaIn, R. O. Capt., R. Fusiliers; Staff Capt., War 1889 Office. (W.) Brevet Major. m. Cavalier, Order of the Crown of Italy

Joynson, R. Capt., King's Own Scottish Borderers. 1906 (P.) $m$.

C.U.W.I. 
Judd, L. A. Capt., Highland L.I. and R. Scots. (W.)

KAY, Rev. A. I. 2nd Lieut., I.A.R.O.

Keable, Rev. R. Chaplain, S. African Native Labour 1905 Contingent

WKelK, A. F. H. 2nd Lieut., Welsh Regt. (W.) M.C. I9ro Killed in action 9 March 1917

Kelsey, A. R. Lieut., Coldstream Gds.; Capt., Spec. 1909 List (School of Instruction). M.C. Italian Croce di Guerra

WKeMP, N. 2nd Lieut., Lancs. Fus.(T.F.) (W.) Killed in action 9 Sept. 1916

Kindersley, C. E. Surgeon Lieut., R.N.

Kindersley, G. W. Capt., Cameronians (Scottish Rifles); [19I4] attd. Suffolk Regt. (W 2.) $M$.

KIng, Rev. F. J. C.F. 3rd Class, R.A.C.D.; D.A.C.G. I891 (W.) $M 2$.

Kirkbride, G. Capt., R.A.S.C.; Lieut., Imp. Service r9r3 Cavalry and 27 th Punjabis, Indian Army

KnAPPETT, P. G. Lieut., R.F.A.; Staff Lieut. $M$.

KnotT, P. G. Capt. and Adjt., R.G.A.

Knubley, C. Corpl., Australian A.M.C.

HKnubley, R. L. Capt., Wiltshire Regt. (W.) M.C. M. 1906 Died 9 fuly 1916 of wounds received in action

Lambe, J. L. P. Sub-Lieut., R.N.V.R.

Lambton, J. F. Capt., Northumberland Fus.(T.F.)

1913 1905

WLANe, J. E. 2nd Lieut., The Buffs (E. Kent Regt., T.F.)

Killed in action 3 May r917

Lawson-Johnston, E. A. Lieut., 2nd Dragoons (R. 1897 Scots Greys). (W 3.) $M$.

WLeACH, G. K. Lieut., Border Regt.

Killed in action in Gallipoli ro Aug. 1915

LeE-Williams, O. Ll. Lce.-Corpl., Gloucestershire Regt. I9 Io

Le Fleming, G. F. A. H. Hon. Colonel, Spec. List 1878 (Draft Conducting Officer)

Le Fleming, M. R. Lieut., Queen's Own (R. W. Kent I9r3 Regt., T.F.); Capt. and Adjt., M.G.C. (W.) $M$.

Leigh-Mallory, G. H. Lieut., R.G.A.

Leigh-Mallory, T. Lieut., Lancs. Fus.; Major (A.), I9I I R.A.F. (W.) D.S.O. $M$.

Le Rougetel, I. H. Lieut., Northamptonshire Regt.; I9I3 Capt., M.G.C. M.C. and Bar

LiNTHORNE, E. L. R. Seaman, R.N.V.R. 
WLloyd, M. E. Capt., R. Welsh Fus.

Killed in action 23 Oct. I9I4

WLong, F. E. Pte., Hampshire Regt.; Capt., King's r9r I (Liverpool Regt.) M.C.

Killed in action at Hooge 24 Aug. 1917

LOVEDAY, D. G. 2nd Lieut., Oxford and Bucks. L.I. I9I5 Lowry, G. C. Capt., Cheshire Regt. and Gen. List I9I3 (School of Instruction). (W 2.)

LOWTHER, Hon. L. E. Capt., Gen. Staff. O.B.E. Order $\mathrm{r}^{885}$ of the Nile, 4th Class (Egypt)

LuCAS, W. R. Major, R.A.S.C. D.S.O. M 2.

1903

LumbY, C. D. R. Lieut., Manchester Regt. (W 2.) 1907 Luson, T. G. L. Capt. and Adjt., R.A.S.C. O.B.E. M. I9r3 Lyon, R. C. G. Capt., R.F.A.(T.F.); Staff Capt. 1909

McConnell, W. E. Capt. (T.), R.A.F. MackenzIe, C. Lieut., King's Own (Yorkshire L.I.) I9r4 MaCkenZIE, L. H. M. Capt., Northamptonshire Regt. $M$. I9r3 MacMrchael, H. A. Capt., Spec. List, empld. Egyptian r90 I Army. D.S.O. M.

MacMichael, H. C. Capt., Border Regt. (W.) 1909

MACNAB, C. Lieut., Highland L.I. (W.) $m$. 1914

MacPherson, A. S. 2nd Lieut., Labour Corps 1915

McPhillamy, J. M. 2nd Lieut., R.G.A.

McWilliam, J. Lieut., King's (Liverpool Regt.) (W.) r9i I M.C.

Mallory, G. H. L. See Leigh-Mallory, G. H.

Mallory, T. L. See Leigh-Mallory, T.

Margesson, H. D. R. Capt. and Adjt., Irth Hussars. 1908 M.C. $M$.

Matthews, A. S. Lieut., 8th (King's R. Irish) Hussars 1906 Maule, W. H. F. Capt., Loyal N. Lancs. Regt. (W 3.) 1908 D.S.O. M.B.E. M.

Mayo, R. H. Capt. (A.), R.F.C.; Major (T.), R.A.F. 1909 (Aircraft Production Dept.) O.B.E.

Methven, C. M. Lieut., Black Watch (T.F.) (W.) 1908

Monson, C. S. Lieut., Lovat's Scouts; Capt., Cameron 1902 Hdrs. (W.)

WMORGAN, H. R. Lieut., D. of Lancaster's Own Yeo.; attd. 1905 R.F.C.

Killed in action 8 Nov. I9r7

Morley, J. Lieut., R. Wiltshire Yeo.

WMORLEY, M. R. H. Lieut King's Own (Yorkshire L.I. 1907 Killed in action at Ovillers I Fuly 1916 
*Morris, J. C. Lieut., Welsh Regt. Killed in action in Gallipoli 8 Aug. 1915

MorSHEAD, O. F. Capt., R.E.; Major, G.S.O. 2. D.S.O. M.C. Brevet Major. M ${ }_{5}$. Italian Croce di Guerra I9II

MorTimer, H. S. Capt., King's Royal Rifle Corps and Gen. List (A.P.M.) M 2. French Croix de Guerre

Mountbatten, Lord L. A. L., g.c.v.o. Capt., King's 1907 Royal Rifle Corps

Murray, K. R. 2nd Lieut., Cameron Hdrs.; Capt., Wilt- 1907 shire Regt.(T.F.); attd. Oxford and Bucks L.I.; A.D.C.; Staff Capt.

Murray Smith, J. E. Major, R. Horse Gds. (W.) 1909 WMusgrave, T. Lieut., Irish Gds. $M$. Killed in action $6 \mathrm{Feb}$. 1915

WNASH, G. 2nd Lieut., Worcestershire Regt.

Died 20 June 1915 of wounds received in action

Neville, A. G. Major, R.H.A.; A.D.C. M.C. $M$.

Neville, H. S. Capt. (A.), R.A.F.; S.O. 3, Air Ministry

Newman, J. C. Lieut., Res. Regt. of Cavalry; A.D.C. French Croix de Guerre

Newton, H. A. A.B., R.N.V.R.

Nicholson, O. W. Lieut., R.E. (Signals). $m$.

1908

Oldroyd, F. N. Lieut., R.A.S.C.(M.T.)

Oliver, G. Y. Hon. Major, R.F.A.(T.F. Res.)

1909

1909

1914

1899

1880

1910

Ollerenshaw, F. 2nd Lieut., Cheshire Regt.(T.F.); I9IO Asst. Paymaster, R.N.V.R.

Orlebar, J. A. A. Capt., R.A.M.C.

1897

I9II

Killed in action 7 Nov. I9I4

Otter, W. W. Capt. and Adjt., Sussex Yeo.

I897

Packe, E. C. Major, R. Fusiliers; Lieut.-Col., A.A. and $\quad$ I896 Q.M.G. D.S.O. O.B.E. $M_{3}$.

Palmer, Sir E. G. B., Bart. Major, Leicestershire Regt. $\quad$ I882

PAPE, A. G. 2nd Lieut., Worcestershire Regt. (W.) I9I2

PARker, W. D. Hon. Lieut., A.S.C. (T.F. Res.) I898

Parker, W. S. H. Lieut., Ist Dragoon Gds. I9Io

PARKer-Jervis, G. Lieut., N. Staffs. Regt.(T.F.); empld. I9I4 O.C.B. $M$.

Parkinson, G. R. 2nd Lieut., King's School, Rochester, $\quad$ I895 O.T.C. 
Paterson, W. H. Lieut., Gordon Hdrs. M.C. M. 1912 PeEk, B. M. Lieut., Hampshire Regt.

PeEL, W. H. G. 2nd Lieut., R. Guernsey Militia

I910

Perks, J. N. R. Lieut., R.E.

1892

Perrins, C. F. D. Capt., R.F.A.(T.F.)

I9I I

I9II

सPhillipPs, R. W. and Lieut., Grenadier Gds.

Killed in action 26 Oct. I9I 5

Plant, Rev. A. W. Chaplain, R.N.

1914

Pollok A B Major, 7 th Hussars and Gen Staff 1878 (cmdg. Cadet Sqdn.) O.B.E. $m 2$.

Pope, P. M. Capt. and Adjt., King's Royal Rifle Corps I9ro (W.) (P.) $M$.

Poyser, A. V. Major, R.A.M.C. $M$.

I90I

PRICHARD, H. C. Major, Glamorgan Yeo.; Lieut.-Col., $\quad 1883$ Spec. List (Cmdt., P. of W. Camp). C.B.E. $m 2$.

Pryde, A. M. Pte., Norfolk Regt. (W 2.) r9r4

Ransom, P. L. Capt., Hertfordshire Regt. I9I I

REECE, C. M. Lieut., Cheshire Regt.; Lieut. (A.), R.A.F. I9I3 (W.) (P.)

ZREeves, V. C. M. Major, Dorset Yeo. (W.) $M$. 1906 Killed in action in Egypt 26 Feb. 1916

RidLEY, G. A. Lieut., E. Yorks. Regt.; attd. 3oth Lan- [1914] cers, Indian Army

सRitson, F. Capt., Dorset Regt.

Killed in action 17 Fune 1917

RoBerTs, J. H. Lieut., Welsh Regt. ; Capt., King's (Liver- 1903 pool Regt.) and King's African Rifles

RoxBURGH, T. J. Y. Capt., I.A.R.O.

I910

Salter, F. R. Lieut., Rifle Brigade; Capt. G.S.O. 3, *19ro Irish Command. (W.)

Sandilands, R. B. Capt., The Buffs (E. Kent Regt.) 1912 M.C.

SARTORIS, G.U.L. 2ndLieut., R.A.S.C.; Lieut., R.N.V.R. I9r3

Savill, E. H. Capt., Devon Regt. (W.) M.C. I9I3

ScarletT, C. H. Lieut. R.G.A.

Scholfield, J. L. Capt., R. Fusiliers; R.T.O.

WScudamore, R. C. Capt., R. Fusiliers. M.C.

Killed in action I I March 1918

«Shaw, J. R. Lieut.-Col., King's Own (Yorkshire L.I.) 1886 Died 5 Nov. 1916

Shaw-Yates, E. B. 2nd Lieut., R.E. (Signals, T.F. Res.) 1889

ShePleY, R. G. S. Lieut., 3rd Dragoon Gds.

I9I 2 
Sheppard-Jones, J. E. Capt., R.A.M.C.

Sidney, R. J. H. Capt., King's (Liverpool Regt.)

1902

SifFKen, B. C. DE W. 2nd Lieut., R.A.S.C. (W.) $M$.

Slade, P. C. A. Capt. and Adjt., R. Defence Corps

* Smith, A. G. Capt., Loyal N. Lancs. Regt. (W.) M.C.

Killed in action 18 April I9r8

Smith, Rev. J. J. Chaplain and Instructor Cdr., R.N.

Smith, S. C. KaInes. Major, Gen. Staff (Censor's Dept.)

M.B.E. M. Officer, Order of the Redeemer (Greece).

Greek Medal for Military Merit

Sмith, W. A. N. Lieut., R.E.(T.F.)

SNaIth, E. G. Capt., Leicestershire Regt.(T.F.) (W.) I9I I M.C. $M$.

SNEYd, R. Hon. Lieut.-Col., Spec. List (A.P.M.) I884

Stedall, C. P. Major, London Yeo. (Rough Riders) and 1892 M.G.C.

\#STERN, L. H. 2nd Lieut., London Regt.(Kensington Bn.) I9Io

Killed in action 9 May 1915

STOPFORD, R. J. Lieut., R.A.S.C. $M$.

Symons, S. J. Major, Bedfordshire Regt.(T.F.)

[1914] I 887

TANQueray, T. Capt., The Queen's(R.W. Surrey Regt.); empld. O.C.B. (W.)

TAYlOR, G. C. R. Capt., Essex Regt.(T.F.); attd. Devon 1899 Regt. (W.)

Tedder, A. W. Capt., Dorset Regt.; Major (A.), R.A.F. 1909 $M$ 2. Italian Silver Medal for Military Valour

Terry, C. E. Lieut.-Col., E. Yorks. Regt.(T.F.) $188_{5}$

Thompson, D. H. 2nd Lieut., R.A.S.C.; Lieut., Gen. 1905 List, attd. R.E. (P.)

Tickell, G. W. Lieut., Argyll and Sutherland Hdrs. I9I4 (T.F.); attd. 36 th Sikhs, Indian Army

Tinker, B. Capt., Yorkshire Dragoons

WTollemache, J. E. Lieut., The Queen's (R. W. Surrey I9I I Regt.)

Killed in action 21 Aug. 1916

सTonking, D. W. Pte., R. Fusiliers (P.S. Bn.); and Lieut., 1909 D. of Cornwall's L.I.; Capt., R. Warwickshire Regt. Died 29 May I 917 of wounds received in action

Tringham, H. G. 2nd Lieut., Wiltshire Regt.; Lieut., 1886 R.A.S.C.

Tyrwhitt, Rev. the Hon. L. F., M.v.o. C.F. 2nd Class, 1882 R.A.C.D. (W.) O.B.E. $M 2$. 
Valentine, W.H. and Lieut., King's Own Scottish I9I4 Borderers; Lieut. (A.), R.A.F. (W.)

Varvill, J. K. Capt., E. Lancs. Regt. (W.) M.C. M. I9Io

VERNER, G. W. H. Lieut., R. Marines I900

Waithman, J. C. Capt., R.A.M.C.

WWALKer, G. S. Lieut., R.F.A.

Died 20 Nov. 1917 of wounds received in action

WWAlmesley, R. Lieut., Yorkshire Regt.

Killed in action 23 Oct. I9I4

Walpole, R. H. Lieut., Rifle Brigade. (W 2.)

Walter, G. L. Pte., Rhodesian Regt., S. African Force r 1909 WWatson, C. B. 2nd Lieut., S. Staffs. Regt.; Lieut., I9I3

M.G.C. M.C.

Died 12 Fuly 1918 of wounds received in action

WWatson, K. F. Flt. Lieut., R.N.A.S.

Missing, presumed drowned, 3 Aug. I9I 5

Waugh, A. D. Lieut., R.E. $M$.

Wayne, Rev. St J. C.F. $4^{\text {th }}$ Class, R.A.C.D.

Westall, D. C. Lieut., R. Sussex Regt. (W.)

Weston, G. E. Capt. and Adjt., R.E.

Wharton, W. H. A., v.D. Lieut.-Col., Yorkshire Regt. I 878 (T.F. Res.) O.B.E. m.

Whitchurch, R. H. S. G. Major, Military Accounts 1897 Dept., Indian Army

Whitefield, C. G. Lieut., Suffolk Regt.; Capt., Gen. I9ıo Staff

Whitтome, L. Capt., Hampshire Regt. (W.) [I9I4]

Wilkinson, W. R. Pte., London Regt. (Artists Rifles); 1912 and Lieut., King's Own (Yorkshire L.I.)

Williams, G. A. Capt., Gen. List (T.F. Res.) I875

WWilliams, R. 2nd Lieut., Grenadier Gds.

Killed in action 8 Oct. I9I 5

Williamson, C. G. 2nd Lieut., R.A.S.C. (Canteens) 1908

Wilson, A. S., M.P. Capt., E. Riding of Yorkshire Yeo. $\quad$ I887

(T.F. Res.) and Gen. Staff. (P.)

Wilson, R. Capt., Lancs. Fus. M.C.

Winterbotham, G. L. Rangoon Bn., Indian Defence 1908 Force

WWinton, H. B. Pte., Middlesex Regt. (P.S. Bn.); Lieut. I9I2 (A.), R.A.F. (W.)

Killed in flying accident 21 April 1918

WoMERSLEY, F. G. Lieut., R. Welsh Fus.; attd. R. Irish I9I4

Fus.; Capt., Gen. List (Asst. Embarkation S.O.) 
Woodhouse, C. H. Capt., Dorset Regt. (P.) M.C. M. 1909

Woodhouse, W. E. 2nd Lieut., Worcestershire Regt. I9IO (T.F.)

WWyndham, G.H. 2nd Lieut., Devon Regt.; attd. North- 1912 umberland Fus.

Killed in action 24 March 1915

YerbURGH, R. E. R. 2nd Lieut., Norfolk Regt. I9Io

YerburGH, R. G. C. Capt., Irish Gds.; Major, I9I I D.A.Q.M.G. O.B.E. M. Belgian Croix de Guerre. Italian Croce di Guerra

Young, R. K. Lieut., Queen's Own (R. W. Kent Regt.) rgro (W.)

Young, W. Sergt., R. Fusiliers; Capt., W. Yorks. Regt. $\quad$ I890 (W.) M.C. 


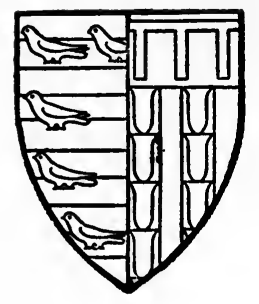

\section{PEMBROKE COLLEGE}

Abraham, L. M. Lieut., R.F.A. (W.) I912 AINSLIE, Rev. R. M., T.D. C.F. Ist Class, R.A.C.D.(T.F.) 1877 AleXANDER, D. L. 2nd Lieut., Gds. M.G. Regt. I9I7 AAlison, L. H. 2nd Lieut., R. Berkshire Regt. I909

Killed in action ${ }_{5} 5$ May 1915

ALLEN, H. R. Instructor Lieut., R.N.

Allen, S. S. Lieut.-Col., Auckland Regt., N. Zealand Force. (W 2.) C.M.G. D.S.O. and Bar. $M 3$.

AlLEN,W.S. Lieut., R.E.; Hon.Lieut.(T.), R.A.F. (W.) I9I3 AAMBLER, G. Lieut., W. Yorks. Regt.(T.F.) (W.) r912

Died 3 Aug. I9I7 of wounds received in action Fuly I917 AmEs, Rev. E. F. W. C.F. 4th Class, R.A.C.D.(T.F.) 1905 AANDERSon, C. A. K. and Lieut., R. Scots Fus. and I9II

King's Royal Rifle Corps.

Killed in action ro Nov. I9r4

Anderson, D. C. Capt., Rifle Brigade. (W.) I9I3

ANDERson, K. Major, R.F.A. M.C. M2. 1906

AANDREws, C. N. 2nd Lieut., Loyal N. Lancs. Regt. I913

Killed in action 24 March ror 5

ANNAND, A. W. Capt., Gordon Hdrs. (W 2.) M.C. 1901

APPLEBY, D. Major, Welsh Regt. $M . m$. Appleton, A. J. Capt. and Adjt., R.A.S.C. M.C. M 2. 1899 AARMITAGE, D. W. R. Fusiliers (P. S. Bn.); 2nd Lieut., I9I2

R. Sussex Regt.

Killed in action in the Battle of Loos 25 Sept. I9r 5

Armitage, E. G. H. Lieut., King's Royal Rifle Corps; I9I4

Capt., Spec. List, empld. Ministry of National Service. (W.)

AARMITAGE,F.R. Capt., R.A.M.C.(T.F.) (W.) D.S.O. M. 1902 Killed in action 30 fuly r917 
Armitage, V. H. Capt., Sherwood Foresters (Notts. and Derby Regt., T.F.) (W.) M.C. Chevalier, Ordre de la Couronne (Belgium). Belgian Croix de Guerre 1907 Armstrong, M. D. Lieut., Middlesex Regt. Armstrong, V. Pte., R.A.S.C.(M.T.) Ash, H. A. Capt., R.A.M.C.

\#Aston, F. M. Capt., D. of Cornwall's L.I.

Killed in action 30 fuly 1915

WAtkey, F. A. H. Capt., Yorkshire Regt.

Killed in action 5 fuly rgr6

AtkeY, J. F. H. Capt., R.F.A.(T.F.) (W.)

Atterbury, F. W. Lieut., R.N.V R.; empld. Admiralty. $m$.

Attwater, A. L. Capt. and Adjt., R. Welsh Fus. (W.) I9I I Attwater, G. L. Surgeon Lieut., R.N. Attwater, H. L. Capt., R.A.M.C.

1902 1904 1902 I886

1901

1895

1909

1907 1903

WAtTwater, H. St J. Capt., Northamptonshire Regt. (W.) 1912

Killed in action 26 Fune 1916

Attwater, W. F. Surgeon Sub-Lieut., R.N.V.R.

1913 1890 1912 Austin, S. P. Lieut., Northumberland Yeo.

Averill, A. S. Lieut., R.F.A.

AyKROYD, H. H. Capt. and Adjt., D. of Wellington's [I9I4] (W. Riding Regt.) M.C.

BADCoCK, G. E. Lieut.-Col., R.A.S.C.; A.D.Transport. I90I C.B.E. D.S.O. Brevet Lieut.-Colonel. $M_{4}$.

ABAgley, A. B. Lieut. (A.), R.F.C.; Capt., R. Dublin Fus. 1909 (W 2.) M.C.

Died 29 Oct. 1918 of wounds received in action

ABAGNall, G. B. and Lieut., Rifle Brigade

Killed in action 23 April r9I7

Bagshaw, W. H., T.D. Major, E. Surrey Regt.(T.F.)

BaILy, R. E. H. Capt., Herefordshire Regt. $M$. Bainbridge-Bell, Rev. W. D. C.F. $4^{\text {th }}$ Class, R.A.C.D. I88I Banks, D. J. Capt., Oxford and Bucks. L.I. (P.) 1895 Banks, R. G. Capt., R.A.S.C. BARCLAY, J. A. Capt., London Regt. (Queen's West- I9I I minster Rifles)

Barkworth, H. B. 2nd Lieut., Labour Corps 1897 Barlow, C. N. Capt., King's Royal Rifle Corps. (W 2.) I9I2 (P.)

BarLow, E. M. Lieut., R. Fusiliers; Major, Gen. Staff I9I I (Instructor, British Military Mission). (W.) $M$. 
Barlow, P. Capt., Spec. List (Recruiting Staff)

WBARNETT, R. W. Capt., King's Royal Rifle Corps; Major, I9II G.S.O. 2. (W.) M.C. and Bar

Killed in action 12 Aug. 1918

BARNICOT, H. 2nd Lieut., Labour Corps

BARNINGHAM, V. Lieut., R.A.S.C.

I 901

I9II

BARRAN, H. B. Capt., R.F.A.(T.F.) (W.) M.C. M. 1908

BARRELl, F. M. Lieut., Gloucestershire Regt.(T.F.) I9I I

BARRELl, K. C. Lieut., R.E.(T.F.) (P.) I9I4

BarretT, Rev. W. E. C. C.F. 4th Class, R.A.C.D. $\quad$ r898

BARROW, P. L. Capt., Worcestershire Regt. and Gen. I9I I

Staff (Intelligence). $M$.

BARROw, R. L. Lieut., I.A.R.O., attd. 4Ist Cavalry 1907

BARRY, A. G. Capt., Manchester Regt.; Lieut.-Col., 1905

M.G.C. D.S.O. M.C. $M_{3}$.

BARRY, C. B. Capt., I.A.R.O., attd. I7th Cavalry I905

${ }^{1}$ Barstow, J. E. J. Capt., N. Somerset Yeo.; A.D.C.; [I9I4] Hon. Capt. (A.), R.A.F. (W.)

सBarton, C. G. Capt., R. Inniskilling Fus. M.C. M 2. 1909

Killed in action 17 Oct. I9I8

WBarWell, F. L. Capt., London Regt. (Queen's West- [1914] minster Rifles); attd. R.F.C. (W.)

Killed in action 29 April r917

BateS, J. V. Major, R.A.M.C. M.C. m. I9Io

Bathurst, H. A. 2nd Lieut., R.G.A. I90I

WBAXTER,W.H.B. Capt., R.Warwickshire Regt.(T.F.) (W.) I9I I

Killed in action 27 Aug. 1917

Beaumont, J. W. F. Lieut., R.G.A.

ABECK, C. B. H. 2nd Lieut., Cheshire Regt.(T.F.)

Died 18 Aug. 1915 of wounds received in action in

Gallipoli 17 Aug. I91 5

BECKER, W. T. L. 2nd Lieut., Manchester Regt.; Major, I9Io York and Lancaster Regt.

WBell, A. F. Pte., London Regt. (L.R.B.); 2nd Lieut., [I9I4]

S. Wales Borderers

Killed in action 12 Aug. 1915

Bell, A. T. Pte., R.A.S.C.

1896

1910

Bellwood, K. B. Surgeon Lieut., R.N. O.B.E.

ABENNETT, S. L. R.N.V.R. (Armoured Car Section); Flt. I910 Sub-Lieut., R.N.A.S.

Killed in action 29 April r917

Bennion, C. F. Capt., R.H.A.(T.F.)

Bennit, F. W. Pte., R.A.M.C.

1 Killed in flying accident after the armistice. 
Bensted-Smith, W. F. Capt., R.A.M.C.(T.F.) $M$. 1906

ABerkeley, M. K. F. Canadian F.A.

Died I May I9I 5 of wounds received in action

Berrington, K. C. Pte., R.A.S.C.(M.T.)

ABEveridge, D. A. 2nd Lieut., R.F.A.

Died I3 Sept. I915 of dysentery contracted on active service in Gallipoli

\#Evir, C. E. F. Lieut., R.F.A. (W.)

Killed in action 29 Sept. 1915

Beyts, C. A. Capt., Poona Bn., Indian Defence Force 1895 सBibBy, R. E. Sergt., R.F.A.

Died in Egypt 23 Oct. I918 of pneumonia contracted on active service

Bickerton, J. M. Surgeon Sub-Lieut., R.N.V.R.

Bickford-Smith, W. N. V. Capt. and Adjt., S. Wales Borderers. (W.) M.C.

WBickley, G.H. Lieut., Devon Regt.; Capt.,M.G.C. (W.) I9I I

Killed in action 4 Oct. I9I7

Bicknell, R. A. W. Capt., Grenadier Gds. M.C. [1914]

BIRD, C. B. Capt., King's Own Scottish Borderers. (W.) I9I2 M.C.

सBishop, C. G. Major, R.E.(T.F.) D.S.O. M. $\quad$ 1898

Killed in action 30 Oct. 19I7

BLACK, F. G. Lieut., Middlesex Regt. M.C. I909

ABlackburne-Daniell, G. F. 2nd Lieut., R. Fusiliers $\quad$ I897

Killed in action 24 April r917

Blaine, H. D. Lieut., S. African Mtd. Bde.

ABlaIR, G. Y. 2nd Lieut., R.F.A.

Killed in action 24 Fuly I9I 5

1904

I9I3

Blair-Imrie, H. F. Major, Black Watch; D.A.Q.M.G., I89I N. Command. C.M.G. O.B.E. M. m.

Blake, A. L. Capt., Somerset L.I.(T.F.); attd. R.E. [rgr4] (Signals). O.B.E. M.

Blake, P. J. 2nd Lieut., R.G.A.

Blake, T. R. H. Capt., R.A.M.C. M.C. $M$.

BLANDFORD, J. J. G. Lieut.-Col., R.A.M.C. $m 2$.

Blathwayt, F. W. 2nd Lieut., R.G.A.

WBLEw, K. Pte., N. Zealand Force; 2nd Lieut., Queen's 1912 Own (R. W. Kent Regt.)

Killed in action 12 April I9r8

\&Bligh, E. H. S. Lieut. R.N.V.R. (Blake Bn., R.N.D.) 1902 Killed in action in Gallipoli ro Sept. 1915

BuIss, A. E. D. Capt., R. Fusiliers; Lieut., Grenadier I9ro Gds. (W 2.) $M$. 
Bodley, D. H. Lieut., N. Staffs. Regt.(T.F.) and Sherwood Foresters (Notts. and Derby Regt., T.F.)

I9I I

ABolton, M. B. Capt., Manchester Regt. and E. Lancs. 1912 Regt.(T.F.) M.C.

Died in German hands 26 March 1918 of wounds received in action 21 March 1918

Bompas, H. S. Capt. (K.B.), R.A.F.

ABoswell, D. St G. K. Capt., D. of Cornwall's L.I.; Major, M.G.C. (W.) $M 2$.

Died 28 Sept. 1918

Bousfield, L. Capt., R.A.M.C. (T.F. Res.) Medjidieh, 1894 $4^{\text {th Class (Egypt) }}$

\#Bovill, E. H. 2nd Lieut., London Regt. (Queen's West- 1906 minster Rifles)

Killed in action I Fuly 1916

सBovill, J.E. 2nd Lieut., 6th Dragoon Gds. (Carabiniers) I9I3 Killed in action 23 fan. 1916

सBowDEN, E. R. Lieut., Northumberland Fus.(T.F.)

Died 29 April r915 of wounds received in action

BradFord, E. C. Capt., R.A.M.C. (Ist S. Gen. Hospital, 1907 T.F.) $m$.

WBRAdLEY, E. J. Rfn., London Regt. (L.R.B.)

Killed in action 5 Dec. I9I4

Bradley, H. E. 2nd Lieut., E. Lancs. Regt.

Bradshaw, T. B. Capt., R. Defence Corps

Bramall, E. H. Major, R.F.A.; empld. War Office

BRAYNE, F.L. Lieut.-Col., Spec. List(Embarkation Staff).

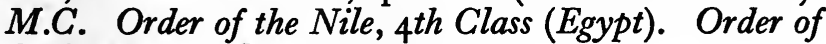
St Sava, $5^{\text {th } \text { Class (Serbia) }}$

BRISCOE, F. E. T. Major, R.A.S.C.

I9I I

I9I 2

1884

1908

1909

BROAD, C. N. F. Major, R.F.A.; Lieut.-Col., G.S.O. I. D.S.O. Brevet Lieut.-Colonel. $M_{3}$. Chevalier,

Legion of Honour (France). Belgian Croix de Guerre

Brock, A. G. Lieut., The Buffs (E. Kent Regt.) M.C. 1903 $M$.

WBrölemann, P. W. A. Sous-Lieut., I2me Cuirassiers à

Pied, French Army. (W.) French Croix de Guerre.

$M 2$.

Killed in action near Moreuil 5 April 1918

Brooke, F. N. Lieut., King's Own (Yorkshire L.I.) I9I2

Brooke TaYlor, G. P. Capt. and Adjt., R.F.A. M.C. [19I4]

Brooker, R. H. G. Capt. and Adjt., R.A.S.C.(M.T.) I9IO $M 2$.

Brown, E. M. Capt., R.A.M.C. 
\&Brown, J. C. D. 2nd Lieut., Durham L.I.(T.F.)

Died 29 April 1915 of wounds received in action near

Ypres 25 April 1915

सBrown, O. H. Rfn., Rifle Brigade; Capt., Suffolk Regt. I9ro

(W.) D.S.O. M.C. M.

Killed in action I Nov. I916

Brown, T. H. Lieut., R.A.M.C.

Browne, G. H. S. Bt. Colonel, Gen. Staff; Deputy 1884

Director-Gen., Ministry of Munitions. C.B. $m$.

Browne, R. C. S. See Seymour-Browne, R. C.

\#Buchanan, F. C. 2nd Lieut., R. Scots. (W.)

Killed in action 9 April 1917

Buckton, W. W. Capt., R.E.

Bullock, Rev. G. G. A. C.F. $4^{\text {th }}$ Class, R.A.C.D.

Bulstrode, Rev. R. C.F. 2nd Class, R.A.C.D. $M 2$.

Bulwer, E. A. E. Lieut.-Col., S. Staffs. Regt.

${ }^{1}$ Burbury, F. W. Lieut.-Col., Queen's Own (R.W. Kent Regt.) and Rifle Brigade

WBurdetT, H. G. Capt., London Yeo. (Westminster

Dragoons); A.P.M.

Died 3 March rgr6

Burges-ShORT, H. G. R. Major, Somerset L.I.; Lieut.-

Col., D. of Cornwall's L.I. (W.) (P.) D S.O. M.

1912

1894

1896

(W.) 1897

1883

I884

1895

1893

Burn, D. C. Lieut., Oxford and Bucks. L.I.; Lieut. (A.), 1914 R.A.F. (W.)

\#Burton, A. H. W. Capt., Lincolnshire Regt. (W.) I9I I

Killed in action 23 Oct. 1916

Burton, G. J. L. Capt., King's Royal Rifle Corps. [1914] (W 2.) M.C. and Bar

Buss, L. C. Madras Artillery, Indian Defence Force 1908

Butler, E P. Capt., Gloucestershire Yeo.

Butson, H. S. G: See Gould Butson, H. S.

1905

Buxton, W. L. Lieut., York and Lancaster Regt.; Capt., I913

Gen. List. (W.) $M$.

BYERS, J. 2nd Lieut., R. Fusiliers

1899

Caley, F. G. Capt., R.A.M.C. (Sanitary Service, T.F.) I902 $M . m$.

WCaley, H. W. Capt., R.A.S.C. $M$.

Died I6 Sept. I918

Calvert, L. M. Capt., London Regt.

1904

1912

1 Died on service in India since the armistice. 
Campbell, K. J. Major, Black Watch. (W.) 1897 \#CAMPBell, R. W. F. Capt., R. Fusiliers

Died I I Aug. I9I6 of wounds received in action 15 July 1906 I9I6

Capron, N. H. Lieut., London Regt. (Post Office Rifles) 1903

Carden, Rev. S. R. C.F. $4^{\text {th }}$ Class, R.A.C.D.

सCAREY, A. J. E. 2nd Lieut., Gordon Hdrs. (W.)

1892

[19I4]

Killed in action 22 Aug. I9I7

Carlile, H. G. Lieut., R.A.O.C.

1900

CARLILE, R. C. Lieut., R.A.S.C. and Spec. List, empld. $\quad$ I903 Directorate of Requisitions and Hirings

Carlisle, F. M. M. Capt., Highland L.I.; Brigade I908 Major. (W 2.) M.C. $M$.

Carlyon, E. T. R. Capt., Sherwood Foresters (Notts. 1912 and Derby Regt.) (W.) (P.)

Carpmael, A. Lieut., R.G.A.

CARr, F. R. 2nd Lieut., R.G.A.

CARR, P. R. Pte., H.A.C.; Capt., R.F.A.(T.F.) (W.) [19I4]

CARR, R. N. Major, Border Regt. and S. Staffs. Regt. I9I3 (W 2.) M.C. $M 2$.

Carrick, A. D. Capt., Northumberland Fus. (W.) I9r3

Carslake, W. B. Capt. and Adjt., The Queen's (R.W. I9I2 Surrey Regt.); empld. Ministry of Pensions. (W 4.) M.C.

Carter, Rev. J. F. Capt., Rifle Brigade and Gen. List 1896 (O.C.B.) $m 2$.

Carter, P. Capt., Worcestershire Regt.(T.F.) (W 2.) I9I3 M.C. Italian Croce di Guerra

Carter, W. R. Hon. Capt., R.A.M.C.

Cartwright, Rev. G. F. C.F. 4th Class, R.A.C.D. $\quad 1895$

Carver, E. T. Major, R.A.S.C. $M$.

ACASE, G. R. A. 2nd Lieut., S. Lancs. Regt.

Killed in action 25 Sept. I9I 5

Casey, S. N. Lieut., R.F.A.

*Casswell, E. D. S. Lieut., Rifle Brigade; attd. Army [1914]

Cyclist Corps; attd. R.F.C. (W 2.)

Killed in action 7 Nov. 1917

Causton, Rev. L. J. C.F. 4th Class, R.A.C.D. 1892

Cavendish-Butler, H. H. Capt., R. Inniskilling Fus.; 1903 empld. Ministry of Munitions

Cawston, E.P. Lieut.-Col., London Regt.(Q.V.R.) (W.) I90 I $M 2$.

Cayley, N. Lieut., R.G.A.; A.D.C.

1893

Cazalet, M. H. Capt., R.A. (R. of O.); R.T.O.

I890 
Chadwick, C. R. Lieut., Queen's Own (R. W. Kent 1906 Regt.) and Gen. Staff

ChaDwICK, J. Instructor Lieut., R.N.

Champion, C. C. Lieut.-Col., S. Lancs. Regt. (W 2.) 1906 D.S.O. $M 3$.

Charles, R. D.S. Capt., London Regt.(L.R.B.); empld. 1908 War Office. (W 2.)

Chase, Rev. F. A. C.F. $4^{\text {th }}$ Class, R.A.C.D.

1898

Chester-Master, A. G. 2nd Lieut., R.A.S.C. M.B.E. 1909 $M$.

Chichester, S. R. 2nd Lieut., R. Marines

ChILD, S. Capt., R.A.M.C.(T.F.)

\#CHIldE, C. M. Capt., Gloucestershire Regt.

Died 22 March I9I 6 of wounds received in action

1902

1894

1913

IChristmas, D. V. Lieut., Suffolk Regt.; Staff Capt.

Accidentally killed while on duty 25 Oct. I91 5

Christopher, C. M. DE A. Lieut., R.F.A.

WChurchman, C. H. Capt., Suffolk Regt. (Cyclist Bn.,

T.F.); attd. W. Yorks. Regt.

Killed in action 3 May r9I7

Clark, C. S. Capt., Suffolk Regt.; R.T.O.

1905

1907

I913

Clarke, A. P. 2nd Lieut., London Regt.

Died 24 Fuly I9I 5 of wounds received in action 22 Fuly I9I 5

Clarke, E. Russell. Capt., Spec. List (Expert Adviser I89o to Naval Staff on Wireless Telegraphy). C.B.E.

Clarke, G. T. K. Capt., R.A.S.C.

Clarke-Williams, F. Lieut., R.F.A.

1898

1913

Clayton, Rev. G. H. C.F. 4th Class, R.A.C.D. $M . \quad 1903$

Clayton, L. J. $\quad$ Lieut., Gloucestershire Regt.(T.F.); $\quad$ I898 empld. Recruiting Staff. (W.)

Clayton, T. W. Lieut., R.F.A.(T.F.)

Clements, Rev. W. D. Chaplain, R.N.

1896

Coates, R.A. Lieut., R.F.A.(T.F.) (W.)

1899

Coвbold, F. A. W. Major, R.G.A. (W.) D.S.O. M. I901

Coвbold, G. W. N. 2nd Lieut., R.E.

I9II

Cochrane, R. D. Lieut., London Regt. (Post Office I9I I Rifles). (W.)

Coke, R. A. S. Capt., Lancs. Fus. (W 2.) M.C. 1909

Colam, H. N. Lieut., R.N.V.R.

1900

COLE, C. L. 2nd Lieut., R.A.S.C.

Cole, Rev. G. L. C.F. 4th Class, R.A.C.D.

1904

1906

HColeman, E. C. Lieut., R.F.A.(T.F.)

Killed in action 2 April I9I7

1910 
Collett, G. F. Lieut.-Col., Gloucestershire Regt.(T.F.) $\quad$ I898 D.S.O. $M 3$.

Collett, H. A. A. Lieut., Irish Gds. M.C.

I9I6

Collier, Rev. F. H. S. C.F. 4th Class, R.A.C.D.

Collins, E. D. Capt., King's (Shropshire L.I.); empld. I9I2 Ministry of National Service. (W.)

ACollins, V. ST B. Lieut. (O.), R.A.F.

Missing, presumed killed in action, I8 Sept. I918

Colman, D. M. Capt. and Adjt., R.G.A.

Colman, L. M. Major, R.F.A.(T.F.)

Colville, D. Capt., Fife and Forfar Yeo. $M$.

COMBER, H. G. Major, Unattd. List, T.F.; G.S.O. 3 r89o

(Intelligence). D.S.O. M3. Chevalier, Legion of

Honour (France). Chevalier, Order of Leopold

(Belgium). Order of St Stanislas, 2nd Class, with crossed swords (Russia). Officer, Order of the Crown of Roumania, with swords

Comyn, A. F. Capt., R.A.M.C.(T.F.)

Connolly, J. S. D'A. 2nd Lieut., Cheshire Regt. (W.) I9I3

Cook, J. W. 2nd Lieut., R.G.A.

1912

1904

1904

r 909

Cooke, F. F. Capt., Essex Regt. (W 2.)

Cooke, H. D'A. M. Capt., The Queen's (R. W. Surrey I 895 Regt., T.F. Res.) and Gen. List (R. of O.)

CoоmвS, P. G. Capt., Northamptonshire Regt.(T.F.); r9ro attd. M.G.C.; Lieut., R.G.A.

Copeland, A. J. Surgeon Lieut., R.N.

CORDEUX, E. H. N. Lieut., Sherwood Foresters (Notts. [I9I4] and Derby Regt., T.F.)

Killed in action I Oct. r9I 5

Cornah, J. R. Lieut., Baluchistan Coy., Indian Defence 1888 Force

Cossar, J. M. Capt., Middlesex Regt.(T.F.); empld. 1907 Ministry of Munitions. Brevet Major. $m$.

Coulson, N. Lieut., 8th (King's R. Irish) Hussars $\quad$ I910

CourTis, A. O. Surgeon Lieut., R.N.

Courtney, G. B. Capt., R.A.M.C. $m$.

1909

1880

Cousins, B. D. Cadet Sergt.-Instructor, R.E. (Signals) I9I5

WCowan, C. J. A. Capt., R. Scots

Died 5 March $19 \mathrm{r} 8$ of wounds received in action

Cowan, R. C. 2nd Lieut., R. Scots

1912

Killed in action 24 Oct. 1914

Cowley, R. B. Capt., York and Lancaster Regt. (W.) 1907

Cresswell, C. E. Lieut., Herefordshire Regt. $m$. 1907

Crick, Rev.P.C.T. C.F.2nd Class, R.A.C.D.; D.A.C.G. Igor

c. U.w.L. 
Crockford, L. C. Capt., R. Warwickshire Regt.; Major, 1906 D.A.Q.M.G. M.C. Brevet Major. M2. Italian Croce di Guerra

Crofton, J. H. Capt., R.A.M.C.

Croghan, E. H. Lieut., R.F.A. M.C. M2.

Crosbie, R. E. H. Capt. and Adjt., R.A.S.C.

CROSBy, J. C. P. 2nd Lieut., King's (Liverpool Regt.)

Died 21 Fan. I9I 8 of wounds received in action

Crosse, S. S. Capt., R.A.M.C. M.C.

Crow, P. Major, R.F.A.(T.F.) (W 4.) $\quad$ D.S.O. M.C. 1909 $M$.

Crum-Ewing, N. R. Lieut., R. Fusiliers and M.G.C.; 1893 attd. Section Sanitaire, French Army. French Croix de Guerre

Crump, H. C. Lieut., Gloucestershire Regt.(T.F.) (W.) 1902 I9I I 1905 I900 Cumberland, R. B. L. Capt., R.E.

Cumming, J. H. Capt., R.A.M.C.(T.F.)

CuRnOCK, G. A. Lieut., Rifle Brigade

Killed in action at Langemarck I4 Aug. I9I7

Cuthbertson, M. Major, R. Fusiliers and Gen. List 1900 (Staff Capt.) M.C. $M 2$.

Cutlack, W. P. Lieut.-Col., Cambridgeshire Regt.

1900

Dale, G. F. 2nd Lieut., 2nd Dragoon Gds. (Queen's I9I7 Bays)

Dalgleish, J. P. Lieut., R.G.A. $M$.

1913

1903

1903

1912

DALlEY, R.P. Lieut., I.A.R.O., attd. Sappers and Miners

Dalton, R. F. Corpl., R.E.

DAMMERS, B. F. H. A.B., R.N.V.R.

Dammers, E. F. H. Capt., Dorset Regt.(T.F.)

DANiEl, T. W. Lieut.-Col., Sherwood Foresters (Notts. and Derby Regt.) (W.) D.S.O. and Bar. M.C. M 2.

DARBYShiRe, N. A. Lieut., R.E.(T.F.)

DASHwOoD, C. B. L. Major, Northumberland Fus.

Died 26 April 1916 of wounds received in action 24 April I9I6

David, L.W. Capt., Glamorgan Yeo.; Capt.(Ad.), R.A.F. $\quad$ I896

Davidson, J. Major, I.A.R.O.; Director of Blockade, 1897

Mescpotamia. O.B.E.

Davies, H. B. Capt., Manchester Regt. (W.) I9I0

Davies, R. G. M. Capt., Norfolk Regt. and Labour 1906 Corps. (W.) M.C.

Davies, T. H. Lieut. (A.), R.A.F.

DAw, W. F. B. 2nd Lieut., R.G.A. 
Dawes, E. 2nd Lieut., Norfolk Yeo.

DAWES, M. S. Cadet Sergt.-Instructor, R.E. (Signals) 1908

Dawson, Rev. E. C. Chaplain, R.N.

1908

Dawson, F. G. T. Flt. Lieut., R.N.A.S.

I9I I

Dawson, J. H. T. Lieut., Worcestershire Yeo. (P.) 1905

DAY, G. W. L. Lieut., R.E.; Hon. Lieut. (O.), R.A.F. [19r4]

DAY, M. F. Lieut.-Col., King's Own (Yorkshire L.I.) and 1897

Gen. Staff. M.C. Brevet Lieut.-Colonel. M 4. m.

WDean, J. H. E. Capt., Cheshire Regt. (W.) M.G. and r9r3

Bar

Killed in action 27 May 1918

Deas, P. B. Major, R.A.S.C.(T.F.) O.B.E. M2. 1893

DE Chazal, Vicomte P. E. Lieut., R.A.S.C. and King's r9r4 African Rifles

DE JERSEY, Rev. N. S. Hon. Chaplain, R.N.V.R.

1886

\#DE LA Mothe, C. D. F. Lieut., R.N.V.R. (R.N.D.) M. 1907 Killed in action at Beaumont Hamel 13 Nov. 1916

DE PASS, E. A. Capt., London Yeo. (Sharpshooters); I9II Capt. (A.), R.A.F. (W.)

DE Slubicki, J. M. M.G. Bty., Canadian Force; 2nd igr I Lieut., R.G.A.

Dewey, H. G. Major, R.F.A. (W 3.) M.C. $M$. I9r3 WDickinson, A. P. Capt., King's (Liverpool Regt., r9ro Liverpool Scottish, T.F.) M.C.

Died $\mathrm{r}$ Fune $19 \mathrm{r} 8$ of wounds received in action

Drckinson, G. F. Major, King's (Liverpool Regt., 1904 Liverpool Scottish, T.F.); attd. Rifle Brigade. (W.)

Dickson, F. P. Capt., R.A.S.C.

Dircks, L. R. A. Capt., London Regt. (London Irish [19r4] Rifles); Hon. Capt. (K.B.), R.A.F. (W 2.)

Dixon, H. H. Lieut., Dulwich College O.T.C.

Dовв, G. C. Capt., R.F.A. (W.)

Doвb, H. R. Major, R.A.S.C. O.B.E. $M$.

1906

I9II

1909

ADoвb, R. A. 2nd Lieut., R.H.A.; A.D.C.; Capt. and I9r2 Adjt., R.F.A. (W.)

Died at Baghdad 22 Dec. 1917 of dysentery

Dodson, A. D. H. Capt., Queen's Own (R. W. Kent rgr3 Regt.) (W.)

Doresa, B. S. Capt., R.F.A.(T.F.) (W 2.)

WDougall, E. S. Capt., R.F.A. (W.) M.C.

F. $(\mathfrak{C}$. "For most conspicuous bravery and skilful leadership in the field when in command of his battery. Captain Dougall maintained his guns in action from early morning throughout a heavy concentration of gas and high-ex- 
plosive shell. Finding that he could not clear the crest owing to the withdrawal of our line, Captain Dougall ran his guns on to the top of the ridge to fire over open sights. By this time our infantry had been pressed back in line with the guns. Captain Dougall at once assumed command of the situation, rallied and organised the infantry, supplied them with Lewis guns, and armed as many gunners as he could spare with rifles. With these he formed a line in front of his battery, which during this period was harassing the advancing enemy with a rapid rate of fire. Although exposed to both rifle and machine gun fire, this officer fearlessly walked about as though on parade, calmly giving orders and encouraging everybody. He inspired the infantry with his assurance that "So long as you stick to your trenches I will keep my guns here." This line was maintained throughout the day, thereby delaying the enemy's advance for over twelve hours. In the evening, having expended all its ammunition, the battery received orders to withdraw. This was done by manhandling the guns over a distance of about 800 yards of shell-cratered country, an almost impossible feat considering the ground and the intense machine gun fire. Owing to Captain Dougall's personality and skilful leadership throughout this trying day there is no doubt that a serious breach in our line was averted. This gallant officer was killed four days later whilst directing the fire of his battery."-Supplement to The London Gazette, 4 June 1918.

Killed in action 14 April 1918

Douglas, C.H. Capt., R.E.(T.F.); Major, R.A.F. (Tech- 1909 nical Res.)

WDouglas, H. K. Lieut.-Cdr., R.N.V.R. (Anson Bn., 1904 R.N.D.) (W.) $m$.

Died 2I May I9I9 of illness contracted on active service

Douglas, Rev. R. H. C.F. 4th Class, R.A.C.D.

WDouglass-James, W. Lieut., R.G.A.

Died 25 Sept. I9I 5 of wounds received in action

Downing, C. C. R. Surgeon Lieut., R.N.

Drake, A. W. C. Capt., R.A.M.C.

Drake, F. 2nd Lieut., Devon Regt.

Drew, R. S. Capt., R.A.M.C.

1900

I9I I

I9I3

1900

I896

1895

WDrummond, N. F. Capt., King's Royal Rifle Corps. M. I9I4

Accidentally killed 20 Dec. I9I6

Du BERN, G. E. Lieut., R.G.A.

Duke, Rev. E. H. C.F. 4th Class, R.A.C.D. (W.)

1915

Dukes, C. Sergt.-Major, Army Pay Corps 
Dunbar, Sir A. E., Bart. Major, W. Yorks. Regt. M.C. 1907 $M$.

Duncen, G. B. Major, I 58 th Bn., attd. 7th Bn., Cana- 1899 dian Infy. (W.)

Duncan, J. G. Capt., I.A.R.O., attd. 5oth Kumaon Rifles r9or

Dunkley, G. W. Capt., R. Marines; empld. Ordnance I9I I Committee. O.B.E.

WDunsmure, C. H. T. 2nd Lieut., Cameron Hdrs. Killed in action in the Battle of Loos 25 Sept. 1915

Dunstan, V. J. 2nd Lieut., R.G.A.

Durrant, H. B. See Lahore, Bishop of

Durst, C. S. Capt., R.E. (Fortress, T.F.)

I9I3

I9I7

1907

EADE, C. Lieut., Res. Regt. of Cavalry and M.G.C. (Cav- 1909 alry). M.C.

WEdDison, J. R. 2nd Lieut., Sherwood Foresters (Notts. 1908 and Derby Regt., T.F.)

Killed in action 22 April r9r 5

Eden, J. R. Lieut., S. Wales Borderers; Capt. R. Welsh I9I I Fus.

Edwards, J. S. Lieut., Fettes College O.T.C.

Elder, A. L. Pte., London Regt. (W.)

1899

1909

Eliot, E. F. Lieut.-Col., R.A.M.C. Cavalier, Order of I880 the Crown of Italy

Elischer, M. H. 2nd Lieut., Unattd. List, T.F.

Elliotr, C. P. Capt., Sherwood Foresters (Notts. and Derby Regt.) (W 2.) M.C. M.

Elllis, T.P. Capt., R.A.S.C.

Elphinston, A. Lieut.-Col., Argyll and Sutherland Hdrs.

ElRington-Bisset, Rev. M. C.F. 4th Class, R.A.C.D.

Elrington-Bisset, W. F. 2nd Lieut., Gordon Hdrs.

Killed in action 25 Sept. I9I5

Elverson, J. H. Major, R.F.A.; empld. Ministry of 1900 Munitions

WErskine, F. A. 2nd Lieut., R.M.L.I. $M$.

Killed in action in Gallipoli 1o May 1915

1904

1914

I910

1898

1878

1907

[1914]

WEvans, C. H. 2nd Lieut., Border Regt.

Died in German hands 26 Oct. I914 of wounds received in action

Evans, F. R. Instructor Lieut., R.N.

Evans, P.D. Lieut., Northumberland Fus.; Capt., Labour Corps

Evans, R. Du B. Capt., King's (Shropshire L.I.) (W.) Igro (P.) 
Eve, A. S. Colonel, Quebec Regt., Canadian Force. I88I C.B.E.

Every-Halsted, C. E. Major, King's Own (R. Lancaster $\quad 1877$ Regt.) and Spec. List (Recruiting Staff)

EErre, C. H. Capt., King's Royal Rifle Corps

Killed in action 25 Sept. 1915

FFaGan, N. 2nd Lieut., Rifle Brigade

Died 20 Fuly 1916 of wounds received in action 1 fuly I9I6

FAIR, C. H. Lieut.-Col., London Regt. (St Pancras Bn.); empld. O.C.B. D.S.O. M.

FALCON, J. H. Capt., R.F.A.(T.F.) $M$.

FALCON, M. Major, R.F.A.(T.F.) $M$.

1902

FAlle, Sir B. G., Bart., M.P. Major, R.F.A. and Spec. List; Staff Lieut.

Fargus, Rev. A. H. C. Chaplain, R.N.

FARRAR, Rev. E. M. C.F. $4^{\text {th }}$ Class, R.A.C.D.

FARRAR, F. P. Foreign Legion, French Army

Favell, N. B. Capt., Rifle Brigade; attd. R.E. $M$.

FAwEll, C. L. Lieut., R.N.V.R. (Coastal Motor-boat Service). D.S.C.

Fawell, S. H. 2nd Lieut., R.G.A.

FeIling, A. Capt., R.A.M.C. $M$.

Fernie, Rev.E.H. C.F. 4th Class, Australian Chaplains' Dept. $M 2$.

FFRENCH, W. K. P. Lieut.-Col., S. Wales Borderers. M.C. Brevet Major. $M 3$.

FFielding-Johnson, H. G. Corpl., R.E. (Signals)

Missing, presumed killed in action, 23 Aug. I914

AFIENnEs, J. E. Capt., Gordon Hdrs. (W.)

Died 18 June 1917 of wounds received in action 17 Fune I9I7

Figgis, B. E. Capt., R.A.S.C.

Figgis, J. M. Capt., Labour Corps. $M$.

I9I4

1904

I9II

1907

1879

1898

I885

1894

1905

I901

1904

1903

I901

r 898

I913

[1914]

Findlay, C. W. McD. Major and Adjt., Gordon Hdrs. (R. of O.) $m$.

FIsk, A. A. Capt., Cheshire Regt. $M$.

Fleming, A. L. Lieut., D. of Cornwall's L.I.; Capt. (A.), I9ro R.A.F. (W 2.) M.C.

FletcheR, W. G. Major, R.F.A. (W.)

Flin, O.S. Lce.-Corpl., R. Fusiliers (P.S. Bn.); Capt., The Queen's (R. W. Surrey Regt.) and Gen. Staff I 888 1896 1891 (Intelligence). (W.) 
WFooRD, G. H. 2nd Lieut., R.A.S.C.

Died I3 Oct. I9I 5 of wounds received in action in

1903

Gallipoli 12 Oct. I9I 5

Forman, H. Lieut., S. Wales Borderers. (W.) (P.) 1907

Forman, Rev. T. P. G. C.F. $4^{\text {th }}$ Class, R.A.C.D. 1904

\&FosDick, J. H. Lieut., Rifle Brigade

Died $3 \mathrm{I}$ Fuly $\mathrm{I} 9 \mathrm{I} 5$ of wounds received in action at Hooge

1913 30 Fuly r9I 5

Foulger, H. C. Capt., R. Fusiliers; attd. King's African I9ro Rifles

WFowlie, J. L. Lieut., Highland L.I.

Killed in action 23 April 1917

Fox, A. S. 2nd Lieut., N. Staffs. Regt.(T.F.)

[19I4]

Killed in action $\mathrm{I}_{3}$ Oct. I9I 5

Fox, D. S. Capt., Sherwood Foresters (Notts. and [19I4]

Derby Regt.); Hon. Lieut. (O.), R.A.F. (W 2.) (P.)

Fox-Strangways, W. A. Suez Local Rifles

1912

Francis, J. C. W. Major, I9th Hussars. (W 2.) $M_{3} .1907$ Belgian Croix de Guerre

Francis, K. Capt., King's Royal Rifle Corps

1903

Francis, R. Cadet Sergt.-Instructor, R.E. (Signals) 1902

FrankLIN, C. R. Instructor, Australian Navy 1907

FrankLIN, F. B. Lieut., R.G.A.; empld. Admiralty $\quad 1892$

Franklin, R. P. 2nd Lieut., Australian F.A.

Fraser, A. A. Capt. and Adjt., R.A.S.C. M.C.

AFrASER, A. L. 2nd Lieut., R.G.A.

Died I fuly r 917 of wounds received in action

FraSER, D. P. Capt., Canterbury Regt., N. Zealand 1896 Force. $M$.

WFrASER, R. Capt., Rifle Brigade

Killed in action I Fuly r916

Frecheville, G. Capt. (T.), R.A.F.

1904

I9I3

1907

Freeman, C. R. Major, Northumberland Fus. (W 2.) 1912 D.S.O. M.C. $M 3$.

Freeman, H. Capt., W. Yorks. Regt. (W.)

FrEeman, W. H. Major, W. Yorks. Regt. M.C. and Bar FRERE, J. E. Capt., R.A.M.C. $M$.

Frith, Rev. J. B. C.F. $4^{\text {th }}$ Class, R.A.C.D.

Frost, Rev. N. P. C.F. $4^{\text {th }}$ Class, R.A.C.D.

Fullalove, A. L. Sub-Lieut., R.N.V.R.

1908

1908

I9I2

I910

I895

1902

I898

rgog

ZGabain, W. G. Corpl., R.E. (Signals); Capt., Spec. List 1909 (Intelligence); attd. Rifle Brigade. M.C. $M 2$.

Killed in action 24 March 1918 
GAMmell, J. A. H. Major, R.F.A.; G.S.O. 2, War I9II Office; Lieut.-Col., S.O. I, R.A.F. (W 2.) D.S.O. M.C. M 7. Cavalier, Order of the Crown of Italy. Order of Karageorge, 4th Class, with swords (Serbia)

Gammell, S. J. Capt., R.G.A. 1886 Gardiner-Hill, C. Surgeon Lieut., R.N.

GARDINER-Hill, H. Capt., R.A.M.C.; attd. R.A.F. $\quad 1909$ O.B.E. M.

Gardner, J. H. Capt., Loyal N. Lancs. Regt.; Major, 1907 M.G.C. (W.)

Gardner-Brown, Rev. F.S. G. C.F. $4^{\text {th }}$ Class, R.A.C.D. 1900 (W.)

\&GARDNER-Brown, J. G. G. Indian Defence Force

Died I8 May r9i 7 of small-pox

GaRnETT, C. 2nd Lieut., Labour Corps

Garnett, D. G. Surgeon Prob., R.N.V.R.

1895 1888

GARNETT, J. N. Lieut., R.F.A.(T.F.); Lieut.(A.), R.A.F. 1909 (P.)

*Garnett, W. P. Lieut., R. Berkshire Regt.; attd. R.F.C.

Killed in action 30 March 1917

GaRrard, N. Capt., R.A.M.C.

GARRETT, G. D Lieut, RN.R (W) Chevalier, 1906 Military Order of Avis (Portugal)

1913

RETT, H. F. Capt., E. Yorks. Regt.

Killed in action at Suvla Bay 22 Aug. 1915

*GARRETT-SMITH, L. 2nd Lieut., R.E.

Died 31 Fuly 1915 of wounds received in action in the

Ypres Salient $30 \mathcal{F u l y}$ I9r 5

Gaskell, C. H. Capt., Wiltshire Regt. (W.) M.C. 1905 Chevalier, Ordre du Mérite Agricole (France)

Gates, C. Lieut., R.F.A.

Geare, J.W. A. Capt., London Yeo.(Middlesex Hussars); A.D.C. $M$.

Geaves, W. L. Cadet, O.C.B.

Gedge, Rev. A. A. L. C.F. Ist Class, R.A.C.D. M 2. Chevalier, Legion of Honour (France)

Gething, J. E. Lieut., R.A.M.C.

Ginan, J. L 1903 (W.)

4GibBs, B. 2nd Lieut., Rifle Brigade. M.C. $M$.

Killed in action 6 Fuly r91 5

Giberne, H. B. Capt., R.A.S.C. 
FGibson, J. G. 2nd Lieut., Cameron Hdrs. (W.)

Died in Germany 12 Sept. I917 of wounds received in

I9II action 3I Fuly 1917

Gibson, R. J. T. Lieut., Devon Regt.(T.F.)

Gibson, T. G. Major, W. Yorks. Regt. (W.) $M$.

Gidlow-Jackson, C. W. Capt., R.E. (W.) M. Order of St Anne, $3^{\text {rd Class, with swords (Russia) }}$

WGielgud, H. L. F. A. Lieut.-Col., Norfolk Regt. (W.) 1900 M.C.

Killed in action 30 Nov. 1917

GiLdEA, Sir J., K.c.v.O., C.B. Hon. Colonel, R. Warwick- 1855 shire Regt. G.B.E.

*Giles, E. Capt. and Adjt., London Regt. (R. Fus.)

Died 16 Fuly 1916 of wounds received in action

Gillan, G. V. B. Major, 9th Gurkha Rifles, Indian Army. Brevet Major. $M$.

※Gillies, C. P. Lieut., Loyal N. Lancs. Regt.; Capt., I9I I M.G.C. M.C.

Died 5 May 1916 of wounds received in action 25 April I9I6

Gilligan, A. E. R. Capt., Lancs. Fus. and Gen. List I9I4 (P. and B.T. Staff)

Gisborne, L. G. Lieut.-Col., R.F.A.(T.F.) C.M.G. $\quad \mathrm{I}^{8} 85$ M2.

GodleE, S. Capt., R.G.A. (W 2.)

Godson, F. P. Lieut., E. Yorks. Regt.; Capt., Spec. List (cmdg. Tunnelling Coy., R.E.) M.C.

GoldING, H. C. Capt., D. of Wellington's (W. Riding

Regt., T.F.); attd. T.M.B.; Capt., Spec. List (cmdg. Sound-ranging Section, R.E.) (W.) M.C. $M_{3}$.

Goldschmidt, W. N. Lieut., R.A.M.C.

Goldsmith, E. O. Capt., R.A.M.C.

Gooch, C. T. 2nd Lieut., R.E.

Gooch, K. T. Staff Capt.; Major, R.F.A. M.C.

Goodall, H. M. Lieut., Worcestershire Regt.

Goodman, B. M. 2nd Lieut., S. African Force

ZGordon, A. J. M. Capt., London Regt. (Queen's West-

1902

1906

I9II minster Rifles)

Killed in action 27 Nov. I9I7

Gordon, A. S. Capt., R.A.S.C.(M.T.) $M 2$.

Gordon, R. E. C. Lieut., Gordon Hdrs.; attd. Cameron 1908 Hdrs. (W.)

Gordon, S. Capt., I.M.S. M.C. M. 
WGorell-Barnes, C. R. Capt. and Adjt., Rifle Brigade; [1914] G.S.O. 3. D.S.O. M.C. $M 2$.

Died 21 April I918 of wounds received in action 5 April I9I 8

Gould, H. F. Lieut., R.F.A.

Gould, K. L. Capt., R.A.S.C.

Gould Butson, H. S. Capt., R.A.S.C.

Gover, Rev. C. E. J. C.F. $4^{\text {th }}$ Class, R.A.C.D.

Gow, L. H. Capt., R. Glasgow Yeo.; A.D.C.; Staff r9ro Lieut. $M$.

AGraham, M. H. Lieut., Yorkshire Regt.

Killed in action at Givenchy 15 Fune I9I 5

Graham, W. J. Capt., Gordon Hdrs.; Staff Capt.;

Deputy Asst. Controller of Salvage. (W.) M.C. M2.

Chevalier, Ordre de la Couronne (Belgium)

Grant, G. N. Lieut., R.A.S.C.

Grant, R. M. 2nd Lieut., R.F.A.

1906

1896

1902

1909

I9I3

1909

I9I5

1908

WGrant, W. St C. Capt., Cameron Hdrs. (W.) M.C. I9I3

Belgian Croix de Guerre

Killed in action 26 Sept. 1918

Gray, R. E. G. Capt., R.A.M.C. Greek Medal for ${ }_{1897}$ Military Merit, $4^{\text {th }}$ Class

GraY, W. S. Capt., R.F.A.(T.F.) $M$.

1910

Grayson, D. H. H. Lieut., Irish Gds. and Gds. M.G. 1912 Regt.

AGreENHILl,T.W. Lieut., 4th (R. Irish) Dragoon Gds. $M$.

Killed in action I $\mathrm{Feb}$. 1916

GreER, H. Lieut., R.A.S.C.

Gregson, P. Lieut., R.G.A. $M$.

GRENSIDE, C. F. Capt., R.A.S.C.

Grier, S. M. Lieut., Nigerian Land Contingent

Grierson, H. Capt., Northamptonshire Regt. (W.)

Griffin, Rev. T. N. R. C.F. $4^{\text {th }}$ Class, R.A.C.D.

*Grose-Hodge, D. E. 2nd Lieut., Suffolk Regt.

Killed in action near Ypres 24 April I91 5

Grose-Hodge, H. Lieut., I.A.R.O., attd. Cavalry

I9II

1901

1910

1903

1897

1910

1894

I9II

1910

I9II

Guggenheim, H. F. Lieut., United States Naval Res.

\&Guillebaud, E. C. 2nd Lieut., Worcestershire Regt.

Died I9I 5 of illness contracted on active service

GunN, J. D. Major, R.A.M.C.

GunNING, C. S. 2nd Lieut., I.A.R.O.

1912

1902

1908

Hacking, E. M. Capt., Sherwood Foresters (Notts. and I90I

Derby Regt.); attd. Rifle Brigade (T.F.) (W.) 
*Hadley, P. S. Capt., Northamptonshire Regt. (W 2.) [1914] M.C.

Died ${ }_{25}$ Oct. 1918 of septic pneumonia following influenza

WHaig-Brown, A. R. Lieut.-Col., Middlesex Regt. (W 2.) 1896 D.S.O. $M 2$.

Killed in action 25 March 1918

HALE, E. N. Capt., Black Watch (T.F.); G.S.O. 3. (W.) $\quad 1889$ M. French Croix de Guerre

HALES, H. W. Surgeon Lieut., R.N.

HALL, G. E. Capt., R. Scots; Major, G.S.O. 2. M.C. $\stackrel{\text { I909 }}{1909}$ Brevet Major. M $M_{4}$. Greek Medal for Military Merit, 3 rd Class

HALl, H. S. Lieut., R.A.M.C.

Died I4 March 19I 5 of pneumonia

HaLl, J. B. Capt., R.Á.M.C.(T.F.)

I 899

HaLL, R. Capt., Irth Bn., Australian Infy.; Staff Capt. 1896 M.C. $M 2$.

HALl-SMith, P. Capt., R.A.M.C.

1899

Haмmick, H. A. Capt., Manchester Regt.(T.F.); Major, I900 Spec. List. (W.) M.C. M2.

Hampshire, C. D. Capt., R. Berkshire Regt.(T.F.) (W.) 1896 M.C.

HanharT, A. A. Capt., R.G.A.

HANKEY, Rev. B. C.F. $4^{\text {th }}$ Class, R.A.C.D.

1898

1896

Hannington, Rev. J. E. M. C.F. 4th Class, R.A.C.D. 1896

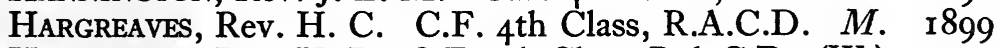

Hargreaves, Rev. H. P. C.F. $4^{\text {th }}$ Class, R.A.C.D. (W.) 1909 M.C.

HARGREAVES, Rev. W. F. C.F. $4^{\text {th }}$ Class, R.A.C.D. $\quad 1902$

HARKE, S. L. Surgeon Lieut., R.N.

1895

HARKER, G. C. W. Capt., London Regt. (Rangers). (W.) rgro M.C.

Died I Dec. 19I7 of wounds received in action 27 Nov. I9I7

HaRkNesS, H. L. Capt., S. and T. Corps, Indian Army; 1897 attd. R.F.C.

Harling, R. W. Capt., Oxford and Bucks. L.I. (T.F.); 1890 G.S.O. 3 .

HARPER, K. J. Lieut., I.A.R.O., attd. I2th Cavalry 1900

HARRIS, C. R. Lieut., Middlesex Regt.(T.F.) IgII

HARRIS, J. C. N. Capt., R.A.M.C.

HARRIS, W. B. Lieut., Gen. List, attd. R.E. (Signals) 1908

HARRIS, W. E. Lieut., Northumberland Fus.(T.F.) 1909

(W 2.) 
Harrison, G. R. D. Major, Montgomeryshire Yeo. $\quad$ I 896

Hartcup, J. A. Capt. and Adjt., E. Yorks. Regt.; Major, 1908 S.O. 2, R.A.F. (W.)

HarveY, G. H. Lieut.-Col., R.A.S.C. D.S.O. $M_{5} . \quad 1896$ French Croix de Guerre

Harvey, N. R. Capt., Rifle Brigade

Haslam, W. A. 2nd Lieut. (T.), R.A.F. Miners. $M$.

सHatch, L. C. Lieut., Durham L.I.

Killed in action 27 Sept. 1915

НатT-CoOK, G. Major, Cheshire Regt.(T.F.)

1914 I 899 1910

I9I2

Havell, C. C. W. Lieut., Suffolk Regt.(T.F.); Capt., [1914] Spec. List (cmdg. T.M.B.) M.C. M2.

WHAYWARD, C. O. Lieut., Lincolnshire Regt.; attd. R.F.C. 1912 Killed in action near Ledeghem 17 fan. 1916

Healing, J. A. Lieut., R. Warwickshire Regt.; Major, ${ }_{9} 892$ - Manchester Regt. (W.) M.C.

HEARD, N. Major, R.E.

Hearfield, T. Pte., King's Own (R. Lancaster Regt.)

Heath, A. F. Lieut., Sherwood Foresters (Notts. and 1912 Derby Regt.) (W 2.)

Heath, R. J. Lieut., R.A.S.C. M.B.E.

HHeathcock, T. Capt., E. Yorks. Regt.

Killed in action ro fuly r9r6

Heesom, K. E. 2nd Lieut., R.E. (W.)

WHemmant, M. Capt., Rifle Brigade

Killed in action I4 Aug. I9I7

Hemus, W. L. Capt., R. Warwickshire Regt. and Worcestershire Regt. (W 2.)

Henkel, W. E. G. Capt., Middlesex Regt. (W.) I9ro

Herdman-Newton, C. Capt., R.A.M.C. 1909

Herdman-Newton, R. H. Surgeon Lieut., R.N. M. 1905

WHeriz-Smith, A. J. C. Lieut., Devon Regt.(T.F.) 1897

Killed in action in Mesopotamia 8 March 1916

I9II

1912

1916

1906

1913

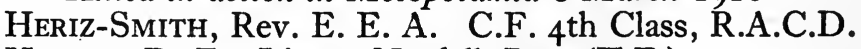

WHERVEY, D. F. Lieut., Norfolk Regt.(T.F.)

Died I 7 May 1917 of wounds received in action 19 April I9I7

HHerveY, G. A. Lieut., R.G.A.(T.F.)

1907

1914

Killed in action 8 Aug. I9I7

Hesketh, G. E. Lieut., R.G.A.

1900

Heycock, M. S. Capt., Rifle Brigade and R.E. (Signals). (W 2.) M.C. 
Heyland, H. M. Major, King's Royal Rifle Corps; 1909 Lieut.-Col., M.G.C. (W 2.) D.S.O. M2.

Heyn, R. G. Major (A.), R.A.F. O.B.E. $m$.

WHEYwood, L. J. 2nd Lieut., R. Fusiliers

Killed in action 20 fuly r 916

Higgrns, C. A. Lieut., Rifle Brigade; Capt. (T.), R.A.F. 1905 (Aircraft Production Dept.) (W.)

Hiles, M. Capt., Wiltshire Regt.(T.F.) and Spec. List. 1902 O.B.E. $\mathrm{m} 2$.

HILL, G. Lieut., R.F.A. (W.)

\begin{tabular}{lll} 
HILl, G. B. Major, R.A.S.C.(M.T.) O.B.E. $M 2$. & 1909 \\
\hline
\end{tabular}

Hill, R. N. 2nd Lieut., R.F.A. (W.) I9I6

Hills, W. H. Capt., R.A.M.C.

1907

1906

WHIND, C. R. Lieut., S. Staffs. Regt. $M$.

Killed in action 30 May r916

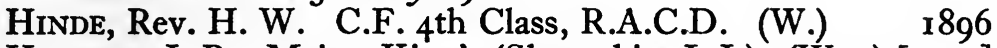

Hinmers, J. R. Major, King's (Shropshire L.I.) (W 3.) [1914] M.C. French Croix de Guerre

HHINNELl, T. S. 2nd Lieut., Suffolk Regt.(T.F.)

Killed in action 12 Aug. 1915

HIRD, F. L. Lieut., E. Surrey Regt.(T.F.); Lieut. (A.), ${ }^{915}$ R.A.F. M.C.

HiscotT, G. S. Lieut., R.G.A. (W.)

HiscotT, L. S. Pte., H.A.C.; Lieut., R.G.A.; Lieut.(Ad.) I9I5 R.A.F. (W.)

Hiтchсоск, W. H. L. Lieut., S. Wales Borderers (T.F.) 1904

WHOARE, A. B. Capt., Loyal N. Lancs. Regt.(T.F.) (W.) I90I Killed in action near Poelcapelle 26 Oct. 1917

HoвBs, E. N. B. Lieut., R.A.S.C.

1903

Hodge, J. D. V. Bengal Light Horse, Indian Defence 1906 Force

Hodson, G. S. Lieut., R.E. (Signals, T.F.)

Hodson, R. Capt., R.A.M.C. M.C.

1914

How 1905 $M$.

Holder, N. F. Lieut., R. Warwickshire Regt. and Tank 1903 Corps. (W.)

Holman, Rev. J.H.T. Pte., Canadian A.M.C. ; Chaplain, 1896

Canadian Force. M.M.

Home, C. F. M. Capt., Welsh Regt.

Hooper, R. G., D.s.o. Major, E. Riding of Yorkshire Yeo. $\quad \begin{aligned} & 1992 \\ & 1892\end{aligned}$

HoPLEY, F. J.V. Capt., Grenadier Gds.; empld. R.M.C., 1902

Sandhurst. (W.) D.S.O. M.

HORNE, J. W. Lieut., R.F.A. 
Horner, M. S. Pte., R.A.S.C.(M.T.)

Hornidge, D. G. P. Capt., R.A.S.C.

Hotblack, G. F. 2nd Lieut., R.G.A. $M$.

Hотнам, J. C. Major, 4th Cavalry, Indian Army; Supt. 1900 of Remounts

Housley, C. 2nd Lieut., R. Defence Corps

Woward, C. R. Capt., R.A.M.C. O.B.E. M 2.

Killed in action in E. Africa 6 Sept. I918

Howe, J. C. Lieut., R.G.A.; empld. War Office

WHoyLE, H. K. 2nd Lieut., Lancs Fus.(T.F.)

Killed in action in Gallipoli I May I9I 5

WHoyle, J. B. Lieut., S. Lancs. Regt. M.C.

Killed in action I Fuly I916

HubBuck, R.E. Lieut., R.F.A.; empld. Ministry of Muni- $\quad$ I906 tions

Hudson, L. C. A. Lieut., E. Surrey Regt. and Gen. List 1898

Hudson, Rev. R. C.F. 2nd Class, R.A.C.D.(T.F.) 1882

Hudson-Kinahan, G. F. Lieut.-Col., Durham L.I.; 1898 attd. Egyptian Army. C.B.E. $M 2$.

Huggan, G. H. Capt., Queen's Own (R.W. Kent Regt.) [I9I4]

Hughes, M. B. Paymaster Sub-Lieut., R.N.V.R.

Hughes, N. W. Lieut., Rifle Brigade and R.F.A.

Hughes, T. W. G. J. Capt., N. Irish Horse. $M$.

(W.) 1904

Hulbert, C. G. K. Capt., R.E.; R.T.O.

Hume, W. E. Colonel, A.M.S. C.M.G. M 2.

舗Humphreys, D. F. Pte., London Regt. (Artists Rifles); 2nd Lieut., The Queen's (R. W. Surrey Regt.)

Died I6 May I9I 5 of wounds received in action near Ypres

Hunter, J. DE G. Trooper, United Provinces Horse, I900 Indian Defence Force; Capt., Mesopotamia Survey Party

Hutchinson, E. H. P. Capt., R. Guernsey Militia

WHutchison, D. H. 2nd Lieut., London Regt. (Queen's [I9I4]

Westminster Rifles)

Killed in action 9-10 Aug. I9I5

Iвbotson, A. W. Capt., I.A.R.O., attd. Indian Defence 1905 Force; Staff Capt. M.C. M.B.E.

Igglesden, R. S. Capt. and Adjt., The Buffs (E. Kent 1908

Regt.); Capt., Labour Corps

INGPEN, W. F. Lieut., R.F.A. (R. of O.) I896

INNES, W. K. Capt., King's Own Scottish Borderers; 1912 Brigade Major. (W 2.) D.S.O. $M$. 
Ironside, R. W. Capt., R.A.M.C.

IIsAac, D. C. Lieut., N. Staffs. Regt.; Capt., M.G.C. (W.) $M$.

Killed in action ro April r917

IzARD, K. H. 2nd Lieut., R. Sussex Regt. and Norfolk 1906 Regt.

Jackson, C. B. A. Capt., Suffolk Yeo.; A.D.C.; Staff I900 Capt. M.C. $M$.

JaCkson, I. Capt. and Adjt., E. Yorks. Regt.; Staff 1904 Capt. (W 2.) M.C.

JaCkson, W. Capt., Sherwood Foresters (Notts. and I889 Derby Regt.)

JAmes, J. N. A. Capt., S. Wales Borderers and Gen. List rgro (R.T.O.)

Jameson, G. D. Capt., R.A.M.C.

WJAQUES, A. Capt., W. Yorks. Regt.

Killed in action 27 Sept. r91 5

Jenkinson, C. H. Capt., R.G.A.

1907

1907

I901

Jennings, C. Capt., Leicestershire Regt. and Gen. List. 1900 (P.)

Jephson, P. H. R. Capt., E. Surrey Regt.; Major, I906 M.G.C. and Gen. Staff. (W.) O.B.E. m.

Jepson, A. C. Capt., R.A.M.C.

1904

Johnson, G. S. Capt., 6ist Pioneers, Indian Army. I9ro M.C. $M$.

WJohnson, M. R. W. Lieut., I.A.R.O., attd. R.G.A.

Killed in action in Mesopotamia 28 fune 1918

Johnson, W. L. Major, R.A.M.C.

WJohnston, B. 2nd Lieut., Rifle Brigade Killed in action 3 Sept. 1916

Johnstone, C. P. Lieut., Highland L.I. (W.)

[19I4]

Johnstone, R. O.S. Pte., H.A.C.; Lieut., R.G.A. (W.) I9I3

Jones, C. R. Selous. Corpl., R.E.; Lieut., R.G.A. I9I3

JoNEs, H. K. Gnr., R.G.A.

JoNES, J. S. Trooper, Strathcona's Horse, Canadian Force

Jordan, H. R. Major, Devon Regt.; attd. King's Own

(Yorkshire L.I.) $M$.

Juckes, R. Capt., R.E. (W.) M.C. $M$.

1902

1907

1902

I9II

KanN, P. W. G. Lieut., The Buffs (E. Kent Regt.); I9I3 Capt., Tank Corps. (W.)

WKeats, F. T. Lieut., Suffolk Regt. Killed in action 25 May 1916 
Kelsey, L. R. Capt., S. African Horse Artillery

Kemble, H. M. Major, R.G.A. D.S.O. M.

Kendail, H. B. Capt. and Adjt., R.F.A.(T.F.) $M$.

KENDALl, Rev. H. E. Chaplain, R.N. O.B.E.

Kennedy, G. H. Sergt., Signal Corps, United States Army

Kensington, H. Le G. Lieut., Rifle Brigade. (W.)

KenYoN, H. G. Major, R.F.A. (W 2.) $M$.

Keown, R. W. Capt. and Adjt., The Buffs (E. Kent Regt.) and Gen. List (Staff Capt.) M.C. and Bar. M.

Kerr, P. W. Capt. and Adjt., R.F.A. (W.)

WKewney, Rev. G. S. Chaplain and Instructor, R.N.

Killed in action in the Battle of Futland 31 May 1916

Keymer, Rev. B. N. C.F. 3rd Class, R.A.C.D. $M 2$.

KeYmer, Rev. B. W. C.F. $4^{\text {th }}$ Class, R.A.C.D. O.B.E. $M$.

Keynes, G. L. Major, R.A.M.C. $M$.

KIDD, E. L. Capt., R.F.A.

KIDD, L. S. Lieut., R.E.

Kidston, R. A. P. R. Capt., R.F.A. $M$.

KING, H. H. Lieut., R.N.V.R.; attd. R.N.A.S.

KIng, R. H. Gnr., H.A.C.; Capt. and Adjt., R.F.A. $M$.

KINLOCH, Rev. M. W. C.F. $M . m$.

WKInNaCH, S. J. 2nd Lieut., Yorkshire Regt. (W.) Killed in action 5 Fuly I916

KirBY, H. R. Lieut., M.G.C.

KIRKBY, N.W. Lieut., Yorkshire Regt.(T.F.); Lieut.(O.), 1908 R.A.F.

Knight, C. M. 2nd Lieut., The Buffs (E. Kent Regt.); Capt., M.G.C. and Tank Corps. (W.) M.C.

Knight, P. D. Capt., Spec. List (Asst. Supt., Remount 1899 Sqdns.)

सKnighton, G. G. Major, Oxford and Bucks. L.I. M. 1906 Died 30 April 1917 of wounds received in action near Arras 28 April I9I7

Knowles, F. Capt., R.F.A. M.C. M. Cavalier, I9Io Order of the Crown of Italy

LAgDen, L. A. Lieut., Rifle Brigade

LAGDEN, R. B. Major, Rifle Brigade. (W.) M.C.

AHORE, Bishop of Cor. IgI C.B.E. $M 2$.

LAKIN, E. L. Lieut., R.G.A. 
LAmbert, J. E. H. Capt., King's African Rifles. M.C. 1909 LAMBERTON, A. R. Lieut., Highland L.I. (W.) M.C. I9I4 LANCASTER, H. Lieut., London Regt. (Finsbury Rifles) I898 LANChester, Rev. H. C. O. C.F. $4^{\text {th }}$ Class, R.A.C.D. $\quad 1896$ LANE, S. F. B. Lieut., R.A.S.C. 1902 LANe-Claypon, J. C. 2nd Lieut., Lincolnshire Regt.; 1900 Lieut., Gen. List. (W.)

Langton, Rev. H. B. C.F. 4th Class, R.A.C.D. $\quad 1895$

LARNER, Rev. H. M. C.F. 4th Class, R.A.C.D. LARPENT, J. P. G. DE H. Capt. and Adjt., R. Fusiliers; 1906 Capt., Gen. List

LATHAM,P.H. Major, Haileybury College O.T.C. Brevet I89I Major. $m$.

LA Touche, W.F.D. Capt. and Adjt., Border Regt.(T.F.) [1914] (W.) $m$.

LATTEY, H. P. T. Lieut., I.A.R.O., attd. 7th Gurkha 1902 Rifles. (W 2.)

Laurance, J. B. Capt., Huntingdonshire Cyclist Bn. $\quad 1908$

LAW, C. Lieut., Devon Regt.; attd. Worcestershire Regt. 1909

WLaw, H. 2nd Lieut., Rifle Brigade; Lieut., R. Welsh I9II Fus. $M 2$.

Died 21 Fuly 1915 of wounds received in action ro fune I9I 5

LAWRENCE, H. R. Major, 84th Punjabis and Super- 1897 numerary List, Indian Army. $M$.

HLawrence, J. R. M. Pte., R. Fusiliers (P. S. Bn.); and I9r3 Lieut., E. Surrey Regt.

Killed in action 16 Aug. 1916

LAWRENCE, T. Capt., R.A.S.C. $M$.

Lawson, C. G. Lieut., R.N.V.R. $M$.

Lawson, J. C. Lieut.-Cdr., R.N.V.R. O.B.E. M. ${ }_{1893}$

Cavalier, Order of the Redeemer, Greece. Greek Medal for Military Merit, 3 rd Class

LAWSON-Walton, A. 2nd Lieut., Rifle Brigade. (W.) I9I3

LEA, E. I. Major, R. Warwickshire Regt. (T.F.); Lieut.- $\quad$ I897 Col., A.Q.M.G. M.C. T.D. M 3. Chevalier, Legion of Honour (France)

LEACH, C. DE L. Capt., Rifle Brigade; A.D.C. $M .1914$ Order of the Redeemer, 5 th Class (Greece)

LEACH LewIS, A. F. Major, R.East Kent Yeo.; D.A.A.G. I90I LEACROFT, J. Capt. (A.), R.A.F. M.C. and Bar 1907 LE BrasseUR, J. H. Capt., R.F.A. (W.) I9Io LE BrocQ, C. N. Surgeon Capt., R. Jersey Med. Corps; 1897 attd. R.A.M.C.

c. U.W.L. 
LEDWARD, C. H. Lieut., R.G.A.

LEDWARD, E. F. Capt., Suffolk Regt.; Major, Tank Corps. (W 2.)

LeE, H. F. Capt., Epsom College O.T.C.

LeE-Norman, F. T. Capt. and Adjt., R. E.

LEEs, A. Lieut., Queen's Own (R. W. Kent Regt.); [19I4] Lieut. (O.), R.A.F. (W.) (P.) $M$.

WLEGGETT, H. A. Major, Sherwood Foresters (Notts. and 1892 Derby Regt.) and Gen. List. (W.)

Died 3 March 1920 of the effects of trench fever

WLeigh, E. H. Lieut., Rifle Brigade. $M$.

Killed in action at Fromelles 9 May 1915

Leslie, L. D. A. Cadet, O.C.B.

Letchworth, G. H. S. Capt., R.A.M.C.

WLevinstein, G.E. Lieut., Manchester Regt.

Killed in action 12 Oct. I916

Levy, L. Lieut., R.N.V.R.

WLEY, C. F. A. Capt., S. Notts. Hussars; attd. R.F.C. (W.)

Killed in flying accident 10 March 1918

LEY, R. L. Capt., R.A.M.C.

Light, D. O. Capt., R.A.S.C. (W.) (P.) $M$.

Ling, H. W. Lieut., R. Fusiliers. (W.) M.B.E.

LinsLey, A. R. Lieut., R.A.S.C.(M.T.)

1907

1918

1904

1905

Lister, A. V. Lieut., London Regt.(R. Fus.); attd. Wiltshire Regt. (W.)

Lister, W. K. Capt., R.G.A.(T.F.) (W.)

LITCHFIELD, Rev. W. G. Chaplain and Instructor Cdr R.N.

Littlejohn, H. A. Major, R.F.A.; attd. Egyptian Army. $\quad 1912$ (W.) M.C. Order of the Nile, 4th Class (Egypt)

WLivesey, A. G. H. Lieut., Loyal N. Lancs. Regt.

Killed in action 25 Sept. 1915

Lloyd, M. A. Lieut., R.N.V.R.

WLock, W. A. Lieut., Wiltshire Regt.

Killed in action in the Battle of Loos 25 Sept. 1915

WLongman, F. Lieut., R. Fusiliers. (W.)

Killed in action 18 Oct. I9I4

Longridge, R. B. Lieut., I6th Lancers; A.P.M.; Capt., 1905 S.O. 3, R.A.F.

LongrigG, G. E. Capt., N. Somerset Yeo. (W.) 1896

Loveless, M. L. Capt., R.A.M.C. $\quad 1908$

Loveless, W. B. Capt., R.A.M.C. (W 2.) M.C. 1907

Lowe, C. N. Capt., R.A.S.C.; Capt. (A.), R.A.F. (W.) I9II M.C. D.F.C. 
Lowe, W. D. Major, W. Yorks, Regt.; Lieut.-Col., $\quad 1898$ E. Lancs. Regt. and Durham L.I. D.S.O. M.C. $M_{4}$.

Lowe, W. W Lieut., Malvern College O.T.C.

Lowndes, W. P. Lieut., Loyal N. Lancs. Regt.; Lce.Corpl., H.A.C. (W 2.)

HLowry, S. H. Capt., Hertfordshire Regt. M.C.

Killed in action 31 fuly 1917

LuCAS, G. F. Capt., Yorkshire Regt.; Hon. Capt. (T.), 1897 R.A.F. $M$.

LuCAS, R. B. Lieut., Res. Regt. of Cavalry; A.D.C.; Staff 1893 Capt. $M$.

LuKER, R. Lieut.-Col., Lancs. Fus.; A.A.G. C.M.G. ${ }_{1} 897$ M.C. Brevet Lieut.-Colonel. $M 6$.

LUKER, S. G. Major, R.A.M.C. $M$.

Lumsden, H. B. Lieut., R. Scots; Capt., Spec. List 1900 M.C.

LuMSDEN, R. Capt., Gordon Hdrs. (W.) M.C. French 1912 Croix de Guerre

HLYLE, T. B. 2nd Lieut., Black Watch Killed in action 9 May I9I5

[1914]

LysteR, A. St G. Lieut., I.A.R.O., attd. Sappers and 1906 Miners; Cmdt., Labour Corps. $M$.

WMcAfee, L. A. Capt., Rifle Brigade. (W.)

Killed in action at Hooge $30 \mathcal{F u l y}$ 1915

MACARTNEY, M. H. H. Lieut., Spec. List (R.T.O.) 1899

MAcBRaYNe, L. Cdr., R.N.V.R. O.B.E. 1885

McCleland, N. P. K. J. O'N. Capt., Queen's Own 1906 (R.W. Kent Regt.); Chemical Adviser. (W.) $M$.

Macdonald, G. K. Lieut., Sherwood Foresters (Notts. 1908 and Derby Regt.); Capt. (A.), R.A.F.; Major, S.O.2. (W.)

Macdonald, N. J. Surgeon Sub-Lieut., R.N.V.R.

Macdonald, S. H. Capt., Cameron Hdrs. (W.)

I9I3

I9II

1908

Killed in action 20 Oct. r914

McGrigor, A. M. Capt., Gloucestershire Yeo.; A.D.C. 1906 O.B.E. M 2. Chevalier, Military Order of Avis (Portugal)

MacIlwaine, G. W. Lieut., Res. Regt. of Cavalry. (W.) r9ז3

WMAckenzIE, G. M. Capt., Seaforth Hdrs. (W.) I908 Killed in action 21 April I916

MACKENZIE, Sir J. K. D., Bart. Lieut., R.N.V.R 
Mackenzie, R. S. Capt., R. Warwickshire Regt.(T.F.) 1906 $m$.

Mackenzie, R. T. H. Capt., Bombay Bn., Indian De- 1906 fence Force

Mackern, G. T. G. Capt., R. Irish Fus.; empld. War IgII Office

M'Lachlan, T. K. Capt., Argyll and Sutherland Hdrs. [1914] (T.F.) (W.)

Maclaren, J. F. P. Lieut., Ayrshire Yeo.; attd. R. Scots I9I4 Fus.; Lieut., (A.), R.A.F. $M$.

Macleod, K. G. Capt., Gordon Hdrs. (W.) m. 1905

McNaughton, H. L. Lieut., R. Scots Fus.; Capt. (Ad.), 1906 R.A.F. $m$.

McNeile, Rev. A. H. C.F. 4th Class, R.A.C.D.

McQuade, W. F. Gnr., R.G.A.

1889

MaDDEN, J. G. Capt., Manchester Regt. (W.) D.S.O. $M$. $m$.

Magnay, Sir C. B. W., Bart. Capt., 2nd Dragoon Gds. 1903 (Queen's Bays). M.C.

MaIle, W. C. D. Capt., R.A.M.C.(T.F.) (W.)

Makeig-Jones, T. G. R. 2nd Lieut., Oxford and Bucks. [19I4] L.I.; Lieut., R.E. M.C.

WMalcomson,H. Lieut. and Adjt., R. Irish Regt. (W.) M. 1909 Died 16 Sept. 1916 of wounds received in action

Malcomson, W. D. Corpl., London Regt. (London r9ro Scottish). (W.)

WManfield, N. P. Lieut., Northamptonshire Regt.(T.F.); 1912 attd. R.F.C.

Killed in action 9 Sept. I916

\#ManN, C. J. Lieut., 2oth Hussars. (W.)

I9II

Killed in action 3 Oct. 1918

MaNN, E. J. Capt., Norfolk Regt.(T.F.) (W.) I90I

Mann, F. T. Capt., Scots Gds. (W 3.) 1907

Mannering, Rev. L. G. C.F. $4^{\text {th }}$ Class, R.A.C.D. M.C. ${ }^{*} 1912$

Mansfield, J. H. Capt., Coldstream Gds.

MarburG, C. L. H. Capt., R. Scots. (W.) I909

'Marsh, B. C. Capt., Cheshire Regt.; attd. 69th Punjabis, I908 Indian Army

Marsh, O. DE B. Capt., R.A.M.C. O.B.E. M. French 1904 Médaille des Epidémies

AMARShall, R. C. Lieut., R.F.A.(T.F.) (W.) Died 7 Fan. 1918 of wounds received in action

1 Killed in action in Waziristan after the armistice. 
WMarshall, W. Lieut., Durham L.I. Accidentally killed 27 April I915

Marshall, W. H. Capt., R.A.M.C.

1907

Martin, C. F. Lieut., R.G.A.

Martin, Rev. E. W. C.F. 3 rd Class, R.A.C.D. $m$.

EMARTIN, F. H. 2nd Lieut., R.E.

Killed in action 24 Nov. 1917

MarTin, J. A. Lieut., R.A.M.C.

MarTindale, J. R. Lieut., R.G.A.(T.F.) (W.) $M$.

Martyn, R. V. Capt., Coldstream Gds. (W.) $M$.

Martyn-Linnington, A. L. Capt., Suffolk Yeo. and I9I I Suffolk Regt. (W.)

MarzetTI, C. Lieut., The Queen's(R. W. Surrey Regt.); 1908 empld. War Office

MarzetTi, L. Lieut., The Queen's (R. W. Surrey Regt.) I9Io

MATHER, E. L. Sub-Lieut., R.N.V.R.

1907

1905

1908

1907

1906

I9I4

I9I2

Mather,

Mathews, A. G. Major, R.G.A.

Matthews, C. Capt., London Regt. (Queen's)

Matrhews, M. C. Major, Spec. List; Hon. Lieut.-Col., I I88I attd. Gen. Staff

Matthews, M. H. Lieut., Nigeria Regt., W. African 1909 Frontier Force

Matthey, G. A. Trooper, Strathcona's Horse, Canadian I906 Force

Maw, F. G. Cadet, O.C.B.

WMaw, G. O. Capt., R.A.M.C.

Died ro July 1916 of wounds received in action on the Somme

Maxwell, Rev. M. L. C.F. $4^{\text {th }}$ Class, R.A.C.D.

1918

I906

WMAYER, G. M. Capt., London Regt. $M 2$.

Died 16 Feb. 1917 of wounds received in action 1 Fan. I9I7

Mead, G. C. F. Capt., Aldenham School O.T.C.

I906

I9I I

MEADE-KIng,W.T.P. Major, R.A.M.C.(T.F.) (P.) $M$.

MeERS, R. H. Capt. (T.), R.A.F.

Melhuish, T. W. Capt., R.A.M.C.

Mellersh, F. G. 2nd Lieut., London Regt. (Surrey I9I4 Rifles); Capt., T.M.B.

Mellor, P. H. L. Capt., W. Yorks. Regt.; P. and B.T. 1906 Staff. $m$.

Metcalfe, C. H. F. Major, Bedfordshire Regt.(T.F.); 1906 G.S.O.2. D.S.O. $M 2$. 
Metcalfe, P. K. Capt., R.A.S.C.

MetCAlfe-Gibson, A. 2nd Lieut., R.A.S.C.

Middlemas, P. Lieut., E. Lancs. Regt.; attd. M.G.C.; 1912 Capt. (T.), R.A.F. M.B.E. M.

Miles, B. L. Capt., London Regt. (Queen's West- 1903 minster Rifles). M.C. M.

\#Miles, C. V. 2nd Lieut., S. Wales Borderers; Capt., I9I I Welsh Regt.

Killed in action in the Battle of Loos 25 Sept. 19i 5

AMiles, H. R. Pte., R. Fusiliers (Sportsman's Bn.); 2nd 1885 Lieut., Connaught Rangers

Killed in action 18 fuly 1916

Miles, W. H. Capt. and Adjt., Somerset L.I. (W.) M. 1906 WMillar, G. H. Lieut., R.N.V.R. (R.N.D.); Capt. (A.), 1903 R.A.F. (P. Escaped from Germany)

Killed in flying accident 29 April 1918

WMilleR, H. T. Lieut., E. Yorks. Regt.; attd. D. of Well- I9I3 ington's (W. Riding Regt.). (W.)

Died 6 May I9I 5 of gas poisoning

Miller, P. T. Capt., R. Welsh Fus. (W.)

Miller,W.M. Capt.,London Yeo.(Rough Riders); Hon. I9I2 Capt. (A.), R.A.F. (W.)

Milligan, W. Capt., R.F.A.(T.F.); attd. R.G.A.; Staff igio Capt. $M$.

Milliken, H. E. Capt., King's Royal Rifle Corps. (W 2.) 1907

Mills, A. E. 2nd Lieut. (T.), R.A.F.

Mills, T. G. Capt. and Adjt., R.G.A.(T.F.)

Mills, W. G. Capt., D. of Cornwall's L.I. (W 3.)

Miln,C.J. Capt.(T.),R.A.F. M. Greek Military Cross

Milne Home, A. C. Capt., Northumberland Fus. and Nigeria Regt., 'W. African Frontier Force; Brigade Major. M.C. M.

MilNeR-White, R. 2nd Lieut., I.A.R.O.

MolonY, A. W. Lieut., King's Royal Rifle Corps

WMolson, E. E. Lieut., R. Scots

Killed in action 2 April I9I 5

Molson, H. E. Capt., King's Royal Rifle Corps. (W.) igr I

Monks, F. R. Major, S. Lancs. Regt.(T.F.) 1903

Montagu, G. W. Lieut., R.A.S.C.

MonteIth, H. G. Lieut.-Col., R.A.M.C. D.S.O. 1902

O.B.E. $M 2$.

WMONTFORD, D. R. Capt., 98th Infy., Indian Army; 1908 A.D.C. (W 2.) $M$.

Missing, presumed killed in action (12 April 1918) 
\#Moon, L. J. Lieut., Devon Regt.

Died 23 Nov. 1916 of wounds received in action

1906

WMoor, C. 2nd Lieut., Hampshire Regt. (W.)

I9ro

Killed in action in Gallipoli 6 Aug. 1915

Moore, C. A. ST G. Lieut., R.E.(T.F.) M.B.E. 1900

MOORE, J. W. Lieut., R.A.S.C.(M.T.) $M$. 1914

WMORGaN, Rev. G. W. F. Chaplain, R.N.

Killed in action in the Battle of Futland 31 May 1916

MORLEY, B. C. Lieut., R.N.V.R.

MORLEY, C. Major, Manchester Regt. (W.) (P.) 1897

Morrice, W. Capt., R. Glasgow Yeo.; A.D.C.; Hon. 1912 Capt. (A.), R.A.F.

सMorRIS, C. A. S. Major, Bedfordshire Regt.; attd. R. I9I3

Irish Fus. (W 2.)

Died in German hands 7 May 1917 of wounds received in action in the Battle of Arras 28 April 1917

MorRIs, F. B. Major, S. Staffs. Regt. $M$.

MMent C. Lieut. The Buffs (E. Kent Regt) and R.E. (Tunnelling Coy.)

Killed in action 7 Dec. 19r7

सMorse, E. V. Capt., The Buffs (E. Kent Regt.) M.C. I9Io

Killed in action 23 Oct. 1918

Morton, Rev. C. J. C.F. th $^{\text {th }}$ Class, R.A.C.D.

Morton, H. J. S. Capt., R.A.M.C.

*Morum, J. P. 2nd Lieut., Rifle Brigade

Killed in action $\mathrm{I}$ Fuly 1916

Moss, H. K. Staff Sergt.-Major, R.A.S.C.; Lieut., 1894 Labour Corps (Cmdt., P. of W. Camp)

Mosseri, F. N. $35^{\circ}$ Regt. Fanteria, Italian Army

Mossop, A. G. Capt., R.A.M.C.

1912

1904

Mountain, S. W. Capt., R. Fusiliers. Cavalier, Order I9I I of the Redeemer (Greece)

Moxey, E. R. Corpl., R.E.; Capt. (T.), R.A.F. M. I9ro

Moxey, J. L. Capt., R. Fusiliers. (W 2.)

MugGeridge, G. D. Instructor Lieut., R.N.

Mugliston, F. H. Lieut., D. of Cornwall's L.I.; empld.

Home Office. (W.) O.B.E. Chevalier, Ordre de la

Couronne (Belgium)

MuIRHEAD, M. Major, D.A.A.G.; Lieut.-Col., R.F.A. $\quad$ I897 D.S.O. and Bar. Brevet Lieut.-Colonel. M 5. Chevalier, Legion of Honour (France). Belgian Croix de Guerre

AMurLy-GotTo, J. Lieut., R.E. $M$.

Died 20 Aug. 1916 of wounds received in action

1908

1896

1904

1908 
Murray, A. R. Capt., Seaforth Hdrs.; Staff Capt. I9Io

Murray, C. M. Lieut.-Col., S. African Med. Corps. 1895 D.S.O. M 2 .

WNadin, T. Capt. and Adjt., Șherwood Foresters (Notts and Derby Regt., T.F.)

Died 8 Fune 1918 of pneumonia following wounds received in action

WNAPIER, G. G. Capt., 35th Sikhs, Indian Army

Died 25 Sept. I91 5 of wounds received in action

NARAYAN, Maharaj K. H. Hon. Lieut., Indian Army. M. 1908

Nash-Wortham, F. L. D. Capt., R.A.M.C. $M$.

I 896

Naylor, P. A. E. Capt., Rifle Brigade; A.D.C.; Hon. I9I3 Capt. (A.), R.A.F. (W.)

WNeate, N. R. Capt., R. Fusiliers; attd. H.A.C. (W2.) [I9I4] M.C.

Killed in action 3 May I9I7

New, T. G. Sergt., R.E. M.S.M.

Newmarch, J. G. Surgeon Lieut., R.N.

Newton, C. H. See Herdman-Newton, C.

Newton, R. H. H. See Herdman-Newton, R. H.

Nicholl, V. Lieut.-Col. (A. and S.), R.A.F. D.S.O. I9II D.S.C.

Nicolls, Rev. G. E. C.F. 4th Class, R.A.C.D. I88I

WNiven, E. O. Lce.-Sergt., Bedfordshire Regt. 1909

Died I9 April I9I7 of wounds received in action

WIx, P. K. Fleet Surgeon, R.N.

Killed in explosion on H.M.S. Bulwark 26 Nov. I9I4

Ogilvie, P. G. Capt., I.A.R.O.; Inspector of Munitions I908

Oglethorpe, H. C. Capt., King's College School, I904 Wimbledon, O.T.C.

Oldfield, G. E. B. Major, 74th Punjabis, Indian Army; $\quad$ I893 Lieut.-Col., 89th Punjabis

Oldfield, R. W. Capt., R.F.A.; Major, D.A.A.G. (W 2.) I9ro D.S.O. M.C. and two Bars. Brevet Lieut.-Colonel on promotion to substantive Major. Brevet Major. $M$ 6. Italian Bronze Medal for Military Valour. Italian Croce di Guerra

WOliver, R. E. C. 2nd Lieut., Rifle Brigade Missing, presumed killed in action, 25 Aug. 1916

Oliver-JoNes, W. S. Lieut., Bedfordshire Regt. (W 3.) I9I3 M.C. 
Orde, S. E. H. Capt. (T.), R.A.F. $M$.

OrLebAR, R. A. B. Capt., Bedfordshire Regt. and R. Irish

1912

Fus.; attd. King's Own (R. Lancaster Regt.); empld.

War Office; S.O. 3, R.A.F. (W 2.)

ORMOND, W. P.S. Cadet, R.F.A.

OrPen, H. F. Lieut., The Queen's (R. W. Surrey Regt.) I908 (W.)

OrPen-Palmer, H. B. H. Lieut.-Col., R. Irish Fus.; 1895 Brig.-Gen. C.M.G. D.S.O. Brevet Lieut.-Colonel.

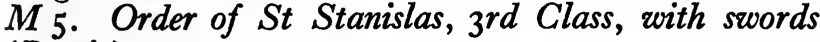
(Russia)

FORR, E. F. B. Corpl., R.E. (Signals); Lieut., R.F.A.

Killed in action 23 March 1918

ORR-EwING, A. Surgeon Lieut. R.N.

Owen, S. H. E. G. Lieut., Pembroke Yeo. (W.)

1913

I9I7

1 Page, C. F. G. Lieut.-Col., R.G.A. C.M.G. D.S.O. M 3. m. Italian Croce di Guerra. Italian Silver Medal for Military Valour

PAGET, C. W. Lieut.-Col., R.E.; D.A.D. R.T. C.M.G. D.S.O. M 4. Officer, Legion of Honour (France). Officer, Ordre de la Couronne (Belgium)

सPaIn, E. D. Pte., R. Fusiliers; Capt., Somerset L.I. Killed in action 18 Oct. I9I6

PaIN, J.W. Capt., Suffolk Regt.; Capt.(A.), R.A.F. (W.) 1905

Palmer, B. H. Capt., R.A.M.C.

I9I3

I910

I 898

1898

I90I

EPARAdISE, J. R. T. 2nd Lieut., R. Warwickshire Regt. Missing, presumed killed in action, 30 Oct. I9I7

Parker, C. L. Y. Capt., R.E.; Staff Capt., War Office. Igro (W.)

Parker, H. E. Capt. (Airship), R.A.F.

Parker, J. D. Lieut., R.N.V.R. (Coastal Motor-boat I906 Service)

Parmiter, C. L. Major, The Buffs (E. Kent Regt.) and 1886 King's (Liverpool Regt., R. of O.). O.B.E.

ParRY-JoneS, P.E. H. Capt., S. Wales Borderers; empld. I9I I O.C.B. (W.)

Parsons, F. P. N. Surgeon Lieut., R.N.

APartington, J. B. Capt., Devon Regt.(T.F.)

Killed in action $3 \mathrm{Feb}$. 1917

WPARTRIDGE, R. H. Capt., Norfolk Regt.(T.F.) Accidentally killed on active service 4 Sept. I9I7 
Paterson, G. McL. Lieut.-Cdr. (Asst. Constructor), 1909 Naval Construction Dept., Admiralty

Paton, E. R. Lieut., Gen. List (T.F. Res.), empld. Min- 1907 istry of National Service

Paton, F. A. R. Capt., King's (Liverpool Regt.); Lieut., 1914 Gen. List. (W.)

सPaton, J. E. 2nd Lieut., Monmouthshire Regt. $M$. [I9I4] Killed in action $31 \mathrm{Dec}$. 1914

Patteson, C. Lieut., Marlborough College O.T.C.

WPAul, J. W. E. 2nd Lieut., King's Royal Rifle Corps. (W.) I9I I Killed in action 27 Fuly. I916

Pead, J. H. Surgeon Cdr., R.N. M. Chevalier, Legion 1887 of Honour (France). Order of St Stanislas, 2nd Class, with swords (Russia)

Pearson, C. McM. Lieut., I.A.R.O., attd.S. and T.Corps I90I

Pearson, D. G. Capt., R.A.M.C.

WPEED, S. W. N. Zealand Force

Died in Egypt 22 March I9r6 of pneumonia

Pelly, E. Lieut., R.A.S.C. M.B.E.

Pemberton, W. H. Capt., R.A.S.C. $M$.

Pendlebury, H. S. Capt., R.A.M.C.(T.F.)

Pennell, V. C. Capt., R.A.M.C.

PePin, A. R. Sub-Lieut., R.N.V.R.

PERkINs, Rev. F. L C I9i2

1 Perrin, M. N. Major, R.A.M.C.; attd. R.A.F.

Peshal Rev. C. J.E Chaplain R N D SO

Peters, A. N. G. 2nd Lieut., Yorkshire Regt.(T.F.); 1915 attd. Manchester Regt.

Phillips, D. M. P. See Scott-Phillips, D. M. P.

WPhillips, E. S. Lieut., Monmouthshire Regt. (W.) I90 Killed in action 8 May 1915

Phillips, P. R. O'R. Capt., R.A.M.C.

Phillips, T. Capt., Cambridge Univ. O.T.C.

WPiggot, A. A. Lieut., Northumberland Fus.

Killed in action in the Battle of Loos 26 Sept. 1915

PiggotT, J. I. Capt., Gen. List; A.D.C.; G.S.O. 31907 (Intelligence). M.C. $M$.

Pigou, H. LA T. Lieut., Herefordshire Regt.; Draft 1890 Conducting Officer

Pilcher, W. H. ' Lieut., Black Watch; Capt., Cameron 1904 Hdrs. (W.)

Pilditch, P. H. Major, R.F.A.(T.F.) $M$.

1 Killed in flying accident after the armistice. 
Pilkington, D. H. 2nd Lieut., Suffolk Regt.(T.F.) (W.) I912

PIPER, J. H. Lieut.-Col., Northamptonshire Regt.; Capt., I900 Gen. Staff (O.C.B.) (W.) M.C. and Bar. $m$.

Pirkis, G. C. L. M. Capt., York and Lancaster Regt. $\quad 1896$ and M.G.C. (W.)

Playne, B.A. Surgeon Lieut., R.N.;Major(Med.), R.A.F. 1904 D.S.O.

Playne, H. F. 2nd Lieut., Upper Burma Bn., Indian 1904 Defence Force; Major, I.A.R.O., attd. Military Accounts Dept. $M$.

Plumptre, E. A. W. Lieut., Northumberland Fus.; attd. 1907 R.E. (W 2.) French Croix de Guerre

Pocock, W. A. Surgeon Lieut., R.N.

APonsonby, C. T. Lieut., King's Royal Rifle Corps Killed in action 23 Aug. 1916

सPoole, R. E. S. Lieut., King's Royal Rifle Corps

Killed in action 4 Nov. I9I8

Pooley, E. H. Capt., R.G.A.(T.F.)

PPorter, A. G. Capt., R. Irish Fus. M.C.

Died 29 Oct. 1918 of wounds received in action 25 Oct. 1918

Potter, Rev. W. N. C.F. $4^{\text {th }}$ Class, R.A.C.D.

WPound, M. S. 2nd Lieut., The Queen's (R. W. Surrey

Regt.)

Died 7 Nov. I9I4 of wounds received in action

$\begin{array}{ll}\text { Powell, M. B. H.A.C. } & \text { I909 } \\ \text { Prest, H. E. W. Capt., R. Berkshire Regt. and Spec. } & 1908\end{array}$

List (Chief Instructor, School of Instruction). $M$.

French Croix de Guerre

Preston, E. W. Lieut., The Qúeen's (R. W. Surrey 1905 Regt., T.F.); Capt., Spec. List

Priestley, D. R. O. Capt., R.E. (Signals). M.C. 1912

PRIOR, A. V. Paymaster Lieut.-Cdr., R.N.V.R. (R.N.D.) I9I2

PrIor, Rev. C. E. C.F. 4th Class, R.A.C.D. 1899

Pritchard, F. C. Capt., R.F.A.(T.F.) M.C. Italian I9I3

Bronze Medal for Military Valour

Procter, R. F. Pte., R.A.M.C.

PRYOR, G. D. Major, Cambridgeshire Regt and R A.F I898

S.O. 2, Air Ministry

Pusinelli, S. J. Capt., R.F.A.

Pyne, F. S. Major, R.F.A. (W 2.) D.S.O. M.

1908

1904

\&QuINCEY, T. E. DE Q. 2nd Lieut., Rifle Brigade

Killed in action 9 May I9I 5

I9I2 
WRamsay, A. H. Pte., Foreign Legion, French Army; and

Lieut., Oxford and Bucks. L.I.

Killed in action $\mathrm{I}_{3}$ Oct. I9I 5

Ranger, Rev. A. S. B. Chaplain, R.N.

RaNking, G. L. Lieut., R.A.M.C.

Ranking, R. M. Major, R.A.M.C. (R. of O.)

Ransom, J. Capt., R. Berkshire Regt.; empld. Records.

(W.) M.C. French Médaille d'Honneur

Ransom, P. W. Capt., R.A.M.C. (W.)

Rattray, R. C. Capt., R.E. (Signals). $M$.

Rawlinson, A. R. Lieut., York and Lancaster Regt. 1912 and M.G.C.; empld. War Office. M.B.E. $m$.

WRAYNER, E. Surgeon Lieut., R.N.

Killed in explosion on H.M.S. Vanguard 9 Fuly r917

Readman, C. H. B. Lieut., Durham L.I.; Capt. (A.), R.A.F. (W.)

Readman, J. F. A. Major, R.E. (W.) M.C.

Redman, C. E. Capt., R.A.M.C. (P.) $m$.

WReID, G. M. Capt., London Regt. (Finsbury Rifles)

Killed in action 9 May 1918

Reid, M. B. Major, R.E. (W 2.) M.C. $M$.

REINER, N. A. Lieut., I.A.R.O., attd. 6th Gurkha Rifles

Renshaw, H. W. Pte., Middlesex Regt.; Lieut., Hampshire Regt. (W.) (P.)

WRenwick, H. A. Capt., S. Wales Borderers; Capt. (A.),

R.A.F. (W.)

Killed in flying accident 10 Aug. 1918

Rhys Jones, I. Lieut., Suffolk Regt.

Richard, W. R. Capt., R. Scots (T.F.) (W 2.)

WRichardson, R. S. Pte., Middlesex Regt. (P. S. Bn.); 2nd Lieut., Cameronians (Scottish Rifles); Lieut., M.G.C. M.C.

Died I Sept. I916 of wounds received in action 31 Aug. I9I6

WRickerby, J. H. E. Capt., Gloucestershire Regt.(T.F.) [I9I4] M.C. Italian Silver Medal for Military Valour

Killed in action near St Quentin 22 March 1918

Riley, C. J. M. Capt., Cold̂stream Gds. (W.) M.C. M. 1912

Riley, H. C. C. 2nd Lieut., Coldstream Gds.

WRIley, Rev. T. Capt., R.F.A.

Died 5 Aug. 19I6 of wounds received in action 3 Aug. I9I 6

सRItchie, T. P. A. 2nd Lieut., Rifle Brigade

Killed in action ${ }_{5} 5$ March 1915

I9I6

I 903

I9I5

I9I 4

I 9 I I 
Robbins, F. H. Major, R.A.M.C.(T.F.) M.C. M. 1906

Roberts, Rev. B. C. C.F. 4th Class, R.A.C.D.

RoBerts, F. W. Capt., Northamptonshire Regt.; Major, I904 Gen. List (O.C.B.) $m$.

RoberTs, H. E. F. Lieut., Imperial Service College, I902 Windsor, O.T.C. $m$.

सRoberTs, J. R. B. 2nd Lieut., Northumberland Fus. Killed in action near Hill 60 I Feb. 1916

I910

RobERTS, S. C. Lieut., Suffolk Regt.(T.F.); empld. 1906 O.C.B. (W.)

Roberts, S. R. P. Lieut., R. Sussex Regt.; Interpreter; I9I3 R.T.O. (W.)

Robinson, Rev. E. V. C.F. 4th Class, R.A.C.D. M.C. I903

RobInson, G. 2nd Lieut., R.F.A.(T.F.); Lieut., R.H.A., [1914] Indian Army. (W.)

ARobINson, Rev. G. B. Chaplain, R.N.

Drowned on H.M.S. Formidable I Fan. I9I 5

Robinson, H. M. Capt. and Adjt., N. Staffs. Regt. 1908 (W 2.) $M$.

Rocke, E. M. Gnr., R.G.A.

RoE, S. V. Lieut., R.A.M.C.

1890 ROPNER, W. G. Capt., R.G.A.(T.F.); Instructor in [1914] Gunnery. (W.) Rosher, Rev. H. G. C.F. $4^{\text {th }}$ Class, R.A.C.D. Order of $\mathrm{I} 890$ St Sava, 5th Class (Serbia)

सRoss, W. M. Lieut., Gordon Hdrs.

Killed in action II March I9I 5

Rowe, B. W. Capt., Cambridgeshire Regt.; attd. R.E. I9I3 (Signals). M.G. $M$.

ARoyle, A. L. Pte., R. Fusiliers

Killed in action 3 Aug. I916

Russell, P. Major, R.F.A. M.C. and Bar
Russell, W. S. K. Capt., R. Sussex Regt. and Gen. Staff. [1914] O.B.E. M.

Rust, N. A. Lieut., I.A.R.O.

Rust, P. J. 2nd Lieut., I.A.R.O.

Ruston, V. F. M. Capt., R.G.A. I9II

1902 1891

I894

1903

1899

1901

1916

Salt, C. E. F. Lieut., R.A.M.C.

Salusbury, J. T. Lieut., R.G.A. $M$.

1899

1900

¿SAnderson, A. K. Pte., R. Fusiliers (P.S. Bn.); 2nd Lieut., I9I3

Middlesex Regt.; attd. London Regt.

Killed in action 25 Sept. I9I 5

Satterthwarte, M. E. Sergt.-Major, Remount Service 1900 
Scholes, W. N. Lieut., Manchester Regt.; Staff Capt.; 1908 Capt., R.E. (Spec. Bde.) O.B.E. M2.

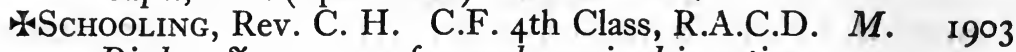

Died $2 \mathrm{I}$ Fune $19 \mathrm{I} 7$ of wounds received in action

WSchwalm, C. E. Lieut., Gloucestershire Regt.(T.F.)

Accidentally killed on active service 22 Nov. I9r7

«SCOTT, J. G. 2nd Lieut., Black Watch

Killed in action at Rue du Bois 9 May r9r 5

ScotT, J. G. C. Trooper, Indore Mtd. Infy., Indian 1907 Defence Force

ScotT-Phillips, D. M. P. Lieut., Middlesex Regt.

*Sealy, C. F. N. P. Pte., London Regt. (Kensington Bn.); 1912 and Lieut., R. Fusiliers

Killed in action near Ypres 24 May 19r 5

Searle, C. F. Major, R.A.M.C. (W.) M.C. M 2.

Searle, W. C. Capt., Bedfordshire Regt.; attd. R. Inniskilling Fus.; empld. O.C.B.

*Seaton, A. A. Capt., Cambridgeshire Regt.

Died 4 Sept. I9I 5 of wounds received in action

I9II

1910

r9ro

1901

1908

1903

Sebag-Montefiore, J. Pte., R. Fusiliers (P.S. Bn.); and r9ro Lieut., R.F.A. (W.)

Seth-Smith, G. Lieut., R.A.S.C.

Seth-Smith, L. M. Lieut., E. African Transport Corps. 1898 M.C.

Sewell, A. P. Capt. and Adjt., R.G.A. $M$.

1898

SEwEll, E. P. Colonel, R.A.M.C.; D.D.M.S. C.M.G. 1892 D.S.O. $M 3$.

Seymour-Browne, R. C. Lieut., R. Fusiliers. $M$. [I9r4]

Sharp, H. W. Capt., Leicestershire Regt.

WSharP, S. O. Lieut., York and Lancaster Regt.

Killed in action I fuly r9I6

Sharpe, H. Major, R.A.M.C.(T.F.) Brevet Major 1902

SHEEPSHANKS, E. Lieut., I.A.R.O. (Cavalry) 1903

SHEPPARD, J. H. D. Major, R.A.O.C.; D.A.D.O.S. M 2. 1907

Sherston, W. M., D.s.o. Hon. Colonel, N. Somerset 1877

Yeo.; Lieut.-Col., Gen. List (T.F. Res.); Camp

Cmdt.

Shuter, L. R. W. A. Capt., R.E. (Fortress, T.F.) 1906

Simcox, J. L. Capt., R. Warwickshire Regt.(T.F.) (W.) 1906

Simcox, W. M. 2nd Lieut., R. Warwickshire Regt. 1907

Simpson, J. H. 2nd Lieut., Grenadier Gds. 1902

SiNG, L. M. Major, R.F.A.(T.F.) (W 2.) 1906

WSingh, K. I. Capt., I.M.S. M.C.

Killed in action I Dec. 1914

1902 
Singi, K. S. Major, I.M.S.

Singleton, J. E. Capt. and Adjt., R.F.A. $M$. 1902

SlaCK, E. Capt., R.A.M.C.

1893

Slingsby, W. E. Capt. (T.), R.A.F.

Smale, G. F. P. Lieut., Lancs. Fus.; attd. Gloucester- I9I3 shire Regt. (W 3.)

SMEdLey, R. D. Capt., R.A.M.C.

Smith, A. K. 2nd Lieut., Bombay Light Horse, Indian 1895 Defence Force; Lieut., I.A.R.O., attd. 12th Cavalry; A.D.C.

Smith, H. H. M. Capt., Loyal N. Lancs. Regt. (W.) I9Io M.C.

«Smith, J. A. H. 2nd Lieut., R. Scots Killed in action 14 Aug. I9I5

Smith, Rev. N. C. C.F. $4^{\text {th }}$ Class, R.A.C.D.

«SMiтh, W. A. Major, King's (Liverpool Regt.); Lieut.- 1899 Col., Manchester Regt. $M$.

Died 9 Fuly I916 of wounds received in action

Smyly, A. F. Capt., S. Staffs. Regt. (W.)

SмYтH, W. J. D. Capt., R.A.M.C. $M$.

1909

SмYTH, W. R. B. Lieut., R.A.S.C.

1897

SNeath, R. E. F. Lieut.-Col., London Regt. (R. Fus.); I9I I Capt., M.G.C. M.C. $M$.

Solomon, B. A. Pte., S. African Force

Somers, J. P. Major, Gen. List (Instructor, School of Musketry). O.B.E. Brevet Major. m.

सSomers-Cocks, R. Capt., Somerset L.I. M.C. $M$. Killed in action 24 April I9I8

WSOUTHERN, H.' 2nd Lieut., I.A.R.O., attd. Infy.

Missing, presumed killed in action, 18 April 1916

SpeIR, G. T. Lieut.-Col., S. Staffs. Regt. and Northern 1893 Cyclist Bn.

SpeIR, K. R.N. Lieut.-Col., R.E. D.S.O. M2. Chev- 1902 alier, Legion of Honour (France)

¿SPENCE, C. B. Lieut. (A.), R.F.C. $M$.

Killed in action 9 May 1915

WSpielmanN, H. L. I. Capt., Manchester Regt.(T.F.)

Killed in action in Gallipoli 13 Aug. I91 5

1905

1903

1912

1904

1892

1912

1905

1907

I9II

SpitTle, J. T. Major (T.), R.A.F. O.B.E. $m$.

1905

STACK, E. H. E. Capt., R.A.M.C. (2nd S. Gen. Hospital, I884 T.F.)

Stallard, Rev. H. K. Chaplain, R.N.

Stapleton, E. P. Lieut., R. Fusiliers; Major, S.O. 2, 1908 R.A.F. (W.) O.B.E. 
Steavenson, A. G. Lieut., R.A.S.C.; empld. Records

Stedman, J. A. 2nd Lieut., E. Surrey Regt. and Connaught Rangers; Lieut. (A. and S.), R.A.F.

Stenhouse, R. H. R.N.V.R. (R.N.D.)

Stephen, J. G. Capt., Highland L.I. (W.) M.C.

Stephens, Rev. J. F. D. C.F. $4^{\text {th }}$ Class, R.A.C.D.

Stevenson, R. W. Capt., Worcestershire Regt. (T.F.); Major, D.A.A.G. M.C. M.

Stewart, N. B. Lieut., R.N.V.R. (Coastal Motor-boat I9Io Service). $M$.

Stickland, J. R. Capt., Cambridgeshire Regt. and 1906 R.G.A. (W.) $M$.

Stidston, Rev. P. H. C.F. 4th Class, R.A.C.D. (W.) 1905 WSTIleman, C. H. 2nd Lieut., R. Fusiliers; attd. London 1912 Regt. (Civil Service Rifles); attd. R.F.C.

Killed in action $2 \mathrm{Feb}$. 1916

WStileman, F. W. C. Pte., H.A.C.; Capt., Gloucester- 1905 shire Regt.

Killed in action 23 Fuly 1916

Stileman, G. R. 2nd Lieut., Devon Regt. (T.F.) and Labour Corps

\#Stobart, J. G. 2nd Lieut., Rifle Brigade

Killed in action 15 March 1915

\#Stone, W. N. Capt., R. Fusiliers

1917

1910

Y.C. "For most conspicuous bravery when in command of a company in an isolated position 1000 yards in front of the main line and overlooking the enemy's position. He observed the enemy massing for an attack, and afforded invaluable information to Battalion Headquarters. He was ordered to withdraw his company, leaving a rearguard to cover the withdrawal. The attack developing with unexpected speed, Capt. Stone sent three platoons back and remained with the rearguard himself. He stood on the parapet with the telephone under a tremendous bombardment, observing the enemy, and continued to send back valuable information until the wire was cut by his orders. The rearguard was eventually surrounded and cut to pieces, and Capt. Stone was seen fighting to the last, till he was shot through the head. The extraordinary coolness of this heroic officer and the accuracy of his information enabled dispositions to be made just in time to save the line and avert disaster."-Supplement to The London Gazette, 13 Feb. I9I8.

Killed in action 30 Nov. 1917 
«Stoney, T. R. 2nd Lieut., King's Own Scottish Borderers Killed in action ro April r918

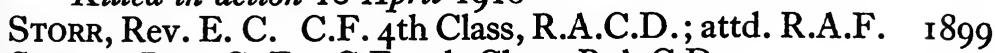

StorRs, Rev. C. E. C.F. $4^{\text {th Class, R.A.C.D. }} 1907$

StorRs, R. H. A. Brig.-Gen., attd. Egyptian Army. I900

C.M.G. C.B.E. M 2. Commander, Order of the

Crown of Italy. Commander, Order of the Redeemer

(Greece)

Straker, H. G. Major, R.F.A. (W.) $M$ 2. $m$.

Strange, H. St J. B. Lieut., Black Watch. (W.)

Streatfeild, A. H. O. Capt., R. Sussex Regt.

Street, H. Capt., 2oth Hussars (R. of O.); Staff Capt.

\#Sturdy, A. C. Capt., R.A.M.C. M.C.

Died I May I9I9 of dysentery contracted on active service

Sturdy, E. C. 2nd Lieut., I.A.R.O., attd. 28th Light 1903

Cavalry

StURT, Rev. H. C.F. $4^{\text {th }}$ Class, R.A.C.D.

WSugDEN, G. H. Lieut., D. of Wellington's (W. Riding rgo7

Regt.)

Killed in action $\mathrm{I} 2$ Oct. I9r 6

SurRage, H. J. R. Surgeon Lieut., R.N.

SurRage, J. L. Lieut., Hampshire Regt.

Susskind, M. J. Lieut., Transvaal Scottish, S. African

Force; 2nd Lieut., R.G.A. (W.)

Sutron, K. H. M. Lieut., Grenadier Gds. and Gds. I9ro

M.G. Regt. (W.)

\#Sutton, W. M. Lieut., York and Lancaster Regt.

Died 17 Sept. 1916 of wounds received in action 16 Sept. 1916

Swan, F. G. Capt., Tonbridge School O.T.C.

Swan, H. N. Capt., R.A.S.C.

SweET, R. McM. Cadet, O.C.B. (R.E.)

Symonds, S. L. Capt. and Adjt., Oxford and Bucks. L.I.

(W.) $M . C$.

TaIt, M. W. Capt., London Regt. (London Scottish); Major, M.G.C. (W.) M.C. M. Belgian Croix de Guerre

Talbot, Rev. A. T. S. C.F. $4^{\text {th }}$ Class, R.A.C.D.(T.F.) $\quad$ I897

TANner, L. E. Hon. Lieut., Spec. List (Navy and Army 1909

Canteen Board)

Tate, J. F. F. Major, King's Royal Rifle Corps; I895 Colonel, A.A.G., War Office. $m$.

1906

1908

I9I 8

1914

1913

895

I 8 
TAYlOR, F. C. Lieut., R.N.V.R.; empld. British Military $\quad$ I895

Mission

Taylor, G. P. B. See Brooke Taylor, G. P.

ATAYloR, H. H. Capt., R.A.M.C.

Died 3 April 1918 of wounds received in action

1900

Taylor, J. Holman. See Holman, Rev. J. H. T.

TAYLOR, Rev. J. R. S. C.F. 4th Class, R.A.C.D.

TAYLOR, L. M. Major, King's Own (Yorkshire L.I.,

T.F.); G.S.O. 2. D.S.O. M.G. $M_{5}$. French

Croix de Guerre

WTAYLOR, M. L. 2nd Lieut., Rifle Brigade

Killed in action 26 Aug. 1916

TAYlor, R. E. Lieut., Middlesex Regt.; Staff Capt. 1899 M.C. $M$.

WTAYLOR, R. F. 2nd Lieut., King's (Shropshire L.I.)

Killed in action 8 Aug. I9I 5

TAYLOR, S. S. Lieut.-Col., S. African F.A. C.M.G. 1894

D.S.O. $M_{3}$. Order of St Stanislas, 3rd Class, with swords (Russia). French Croix de Guerre

Templer, W. F. Lieut.-Col., Army Pay Dept.; Command Paymaster. C.B.E. M.

Tennant, B. V. A. Lieut., Hertfordshire Regt.

Tennant, N. R. D. Capt., London Regt. (St Pancras Bn.) (W.)

Tetley, R. F. Capt., W. Yorks. Regt. (T.F.); empld. War Office

Thackeray, J. M. Capt., Suffolk Regt. and Labour 1907 Corps. O.B.E. M.

AThicknesse, R. S. 2nd Lieut., Lancs. Fus.

Killed in action 10 Oct. 1917

Thomas, A. G. I. Capt., The Queen's (R. W. Surrey [I9I4] Regt., T.F.) (W.)

Thomas, B. H. Major, R.A.S.C. O.B.E. M 2. 1902

Thomas, E. M. See Rocke, E. M.

Thom-Postlethwaite, A. C. S. 2nd Lieut., Bedford- 1902 shire Regt.

Thompson, A. G. G. Major, R.A.M.C. (Sanitary Service, 1908 T.F.) $M$.

Thompson, A. H. J. Major, Spec. List empld. War Office. $\quad$ I 898 O.B.E.

Thompson, B. C. Major, Lincolnshire Regt.(T.F.Res.) 1895 T.D.

Thompson, E. Lieut.-Col., R.E. O.B.E. $M . \quad 1899$

Thompson, J. B. London Regt. (W.) 
AThompson, J. C. C. 2nd Lieut., Scots Gds.

1900

Killed in action 25 Fan. 1915

Thompson, N. F. P.O., Calcutta Port Artillery, Indian 1902 Defence Force

Thompson, R. G. Major, The Queen's (R. W. Surrey 1910 Regt.) (W.)

Thompson, W. F. Major, R.A.M.C.

Thomson, D. G. Capt., R.E. (Fortress, T.F.)

1905 1908 1907

Killed in action near Ypres I 3 May 1915

Tномson, W. L. H. Lieut., Highland L.I.

ThorburN, M. M. Capt., Black Watch. (W.) M.C. 1907

Thorman, J. L. Lieut., Durham L.I. and R.E.; empld. I9I I British Military Mission

Thorneycroft, T. H. Capt., Argyll and Sutherland [1914] Hdrs.(T.F.) (W.)

ThorNTon, A. R. Lieut., R.G.A. $M$.

Thynne, G. A. C. Major, R. North Devon Yeo.; A.D.C.; $\quad$ r888

Capt., Labour Corps

TillaARD, E. R. Major, Suffolk Regt.(T.F.)

Tillard, P. S. Capt., R.F.A.(T.F.)

सTIlly, J. Capt., Yorkshire Regt. (W 2.) M.C.

Died 6 June 1918 of wounds received in action

Tindall, C. G. Capt., Queen's Own (R.W. Kent Regt.) Igr2 (W.) M.G.

ATINDALl, H. S. Lieut., R. Berkshire Regt. Killed in action $3 \mathrm{I}$ fuly 1917

ETindall, R. F. 2nd Lieut., Lincolnshire Regt.

Killed in action $25 \mathrm{Sept}$. 1915

Titley, Rev. L. G. C.F. 4th Class, R.A.C.D.

1892

I894

1905

ToD, A. K. Pte., R. Scots; Capt., Cameron Hdrs.; attd. Bedfordshire Regt.

Tonge, R. D. Capt., Cheshire Regt. (W 2.) M.C. I9ro

TonkIN, R. S. Lieut., E. Yorks. Regt. and I I Ith Ma- IgI I hars, Indian Army

ToppInG, A. R. Gnr., R.G.A.

Townsend, Rev. J. H. C.F. $4^{\text {th }}$ Class, R.A.C.D.

I9I I

1898

Townsend, J. M. Midshipman, R.N.V.R.

1905

1909

1895

1909

TTREE, C. J. Lieut., Worcestershire Regt.

Died 20 Fuly 1915 of wounds received in action in Gallipoli

Trench, M. C. Lieut., R.E.; Hon. Lieut. (O.), R.A.F. [I9I4] *Trevor, F. P. and Lieut., D. of Cornwall's L.I.

Killed in action near Ypres 8 May 1915 
Trew, M. F. Capt., Coldstream Gds.

ATREWBy, A. Lieut., R.E.

Died 17 May 1915 of wounds received in action

I9I I

1899

Trewhella, C. B. Capt., R.G.A.(T.F.) (W.)

WTryon, G. A. Lieut.-Col., King's Royal Rifle Corps. 1905

(W.) M.C. $M$.

Killed in action 7 Nov. 1918

सTuck, G. B. O. Lieut., Australian Force

Killed in action 1917

Tunnicliffe, G. H. Hon. Major, N. Staffs. Regt. $m$. 1878

Turner, D. P. R. Fusiliers; 2nd Lieut., Spec. List 1895

Turnly, J. E. A. L. Capt., R.A.M.C.

1889

Twisleton-Wykeham-Fiennes, N. I. E. Major, R.F.A. 1894 (W.) D.S.O. $M 2$.

Tyers, F. G. Capt., S. African Infy. (W.) 1899

Tylor, G. C. Lieut., R.F.A.; Staff Capt. O.B.E. 1908 $M$.

Tyrwhitt-Drake, Rev. B. H. C.F. $4^{\text {th }}$ Class, R.A.C.D. 1901

Uloth, A. W. Major, R.A.M.C. M.C. 1908

URE, C. McG. Capt., R.E. (P.) I9I I

UrQuhart, W. L. A. W. Capt., Black Watch 1893

Vallance, H. I. A. Capt., R. East Kent Yeo. [I9I4]

VAN DER BYL, A. H. Imperial Light Horse, S. African 1905 Force

VAN DER Byl, A. M. Capt., H.A.C.; Capt. (K.B. and A.), 1900 R.A.F.

VAN DER ByL, P. B. Capt., R.H.A. (T.F. Res.) 1886

VAN DER BYL, P. V. G. Capt., S. African Defence Force; 1907

G.S.O. 3; Capt., S.O. 3, R.A.F. M.C. $M$ 2. Chevalier, Legion of Honour (France)

van Someren, H. A. A. Capt., R.F.A. (W.)

WVARDY, A. T. Pte., Middlesex Regt. (P. S. Bn.); 2nd 1907 Lieut., R. Warwickshire Regt.

Killed in action 4 July r9r6

Vigo, J. D. Capt., Rifle Brigade and Spec. List (cmdg. I9I2 T.M.B.)

WVillar, R. P. Major, King's (Liverpool Regt.)

Killed in action 22 March $19 \mathrm{I} 8$

WVincent, C. A. 2nd Lieut., Rifle Brigade

Killed in action 13 April 1915

WVipond, H. J. Pte., Coldstream Gds.

Killed in action Feb. 1917

1904

1913

1904 
Vyvyan, E. C. F. Capt., King's Royal Rifle Corps. (W.) I905

WWace, H. E. Capt., King's (Shropshire L.I., T.F.) 1908 Killed in action 14 April 1918

WWADDY, J. R. Lieut., R.A.M.C. (W.) M.C. M. 1906 Killed in action 17 March 1915

WADE, H. B. Capt., W. Somerset Yeo. M. 1902

WadHAM, W. F. A., v.D. Lieut.-Col., King's Own (R. 1883 Lancaster Regt., T.F. Res.) $m$.

WAgGeTt, E. B. Major, R.A.M.C.(T.F.) D.S.O. V.D. ${ }^{1884}$ $M_{3}$.

WAKELAM, H. B. T. Capt., R.F.A. (W.) $M$. I9I I

WWalden-Vincent, A. C. Capt., Dorset Regt.

Killed in action 26 Sept. I916

Walker, A. Capt., R.A.M.C.

WWalker, D. Pte., H.A.C:; 2nd Lieut., E. Lancs. Regt. 1907 (T.F.); attd. M.G.C.

Died I9 Sept. I916 of wounds received in action

WWALKER, M.J.L. 2nd Lieut., Queen's Own (R. W. Kent I9I I

Regt.)

Killed in action 3 May 1917

WWallis, D. B. Lieut., Connaught Rangers; attd. R. Mun- r9ro ster Fus.

Died 23 Fuly I9I7 of wounds received in action

Wallis, G. D. 2nd Lieut., Scots Gds.

WALlis, P. B. Surgeon Lieut.-Cdr., R.N.

WWANKLYN, K. Lieut., R.F.A.

Died ${ }_{5} 5$ Nov. I918 of pneumonia contracted on active service

WARD, O. W. Lieut., R. Sussex Regt. and Gen. Staff Igor

WARD, Rev. R. S. C.F. 4th Class, R.A.C.D. $\quad$ I899

WARDEN, H. F. W. Capt., R.A.M.C. 1906

WARNER, C. Surgeon Lieut., R.N. 1906

Warren, G. M. Capt., Wiltshire Regt.(T.F.) I9I I

WARRY, H. E. Capt., Devon Regt.; Lieut., Iogth Infy., [1914] Indian Army. $M$.

Waters, J. B. Capt., King's Own Scottish Borderers; I90I Major, Spec. List (School of Instruction)

Watson, A. H. Lieut., Remount Service and Border I9I I Regt. (W.)

Watson, Rev. T. H. C.F. 4th Class, R.A.C.D. $\quad 1900$

Watson-ScotT, C. H. Capt., R.A.S.C. $M$. 1909

WWaugh, A. J. Capt., R.A.M.C.

Killed in action 17 Aug. I916

1905 
Webi, J. H. J. Capt., R.A.S.C.

WebB, M. E. Major, R.E. (Signals). D.S.O. M.C. $\quad{ }^{1898}$ $M 3$.

Webb-Peploe, Rev. H. M. C.F. 2nd Class, R.A.C.D. 1889

O.B.E. $M_{3}$. Order of St Sava, $4^{\text {th Class (Serbia) }}$

Weber, R. E. Major, R.F.A.(T.F.) (W 2.) M.C.

Wedderburn, C. C. Cadet, O.C.B.

Wells, Rev. E. C.F. $4^{\text {th }}$ Class, R.A.C.D.

Wells, J. P. 2nd Lieut., London Univ. O.T.C.

WWenden, G. Lieut., Border Regt.; Capt. (A.), R.F.C.

Killed in action 16 March 1917

WWhale, A. Lce.-Corpl., R. Fusiliers

Killed in action 3 Aug. I916

Whale, G. Major, S.O. 2, R.A.F. $m$.

WHEELER, F. O. Lieut., R.F.A.(T.F.)

1902

1900

1899

19I3

I9II

1907

Whelpton, M. Lieut., Cheshire Regt. and Gen. List, attd. R.E., Signals

Whitaker, F. Capt., The Buffs (E. Kent Regt.); Major, D.A.A.G. (W.) M.C. M. French Croix de Guerre

White, A. E. Lieut., R.E.

White, B. Capt., Highland L.I.; attd. R.F.C. (Balloon Officer). (W.)

White, Rev. R. A. R. C.F. 3rd Class, R.A.C.D.(T.F.) 1872

Whitelegge, M. H. Capt. (K.B.), R.A.F.

WhitField, Rev. J. O. Capt., Shrewsbury School O.T.C. 1904

Wilkin, W. H. Capt., Sherwood Foresters (Notts. and 1895 Derby Regt.) (P.)

Wilkinson, H. A. Lieut., Dorset Yeo.

1905

1914

1912

I9II

[1914]

1907

Wilkinson, K. Capt. and Adjt., Middlesex Regt. (W.) I9Io

Williams, E. A. Capt. and Adjt., E. Surrey Regt. [1914] (T.F.) (W.)

Williams, R. B. Capt., London Regt. (W.)

Williams, T. R. Capt., R. Welsh Fus. M.C. M.

WIuLIAMS, W. C. B. Major, R. Welsh Fus. M.C. 1909

Williams, W. C. B. Major, R. Welsh Fus. M.C. M. 1904

Williams, W. E. Cadet, O.C.B.

WWilliamson, A. J. N. 2nd Lieut., Seaforth Hdrs.

Killed in action 14 Sept. 1914

Williamson, J. N. 2nd Lieut., Life Gds.; Lieut., Gds. 1915 M.G. Regt.

Willink, A. H. Capt., Border Regt. (T.F. Res.)

WWilson, A. H. 2nd Lieut., Rifle Brigade

Killed in action 17 March 1915

Wilson, A. L. Pte., R. Sussex Regt.

Wilson, A. P. Capt., Border Regt.(T.F.) (W.)

1878

I9I I

1899

1908 
WWILson, E. Sergt., Rand Rifles; Lieut., S. African Infy. 1894 Killed in action 3 May 1916

Wilson, G. H. B. Capt., R.A.S.C.; Capt. (A.), R.A.F. 1914 M.C. A.F.C.

Wilson, Rev. G. M. C.F. $4^{\text {th }}$ Class, R.A.C.D.

Wilson, H. B. Major, R.A.M.C. O.B.E. M2.

WILson, H. W. Lieut., R.N.V.R.

1899

1902 1896

Wilson, J. P. Major (S.), R.A.F. D.S.C. A.F.C. 1902

Chevalier, Ordre de la Couronne (Belgium)

Wilson, J. R. M. Capt., R.E. O.B.E. m.

Wilson, P. M. Pte., London Regt. (Artists Rifles); 2nd Lieut., R.G.A. (W.)

$\begin{array}{lr}\text { Wilson, R. N. P. Lieut., W. Yorks. Regt. (W 2.) } & \text { I913 } \\ \text { Wilson, R. S. Capt., London Regt. (Surrey Rifles). [1914] }\end{array}$ (W 2.)

WWilson, T. B. Pte., Middlesex Regt. (P. S. Bn.); 2nd I9I I Lieut., Irish Gds.

Killed in action 18 fuly 1917

Wilson, W. A. Capt., Rifle Brigade; empld. O.C.B. (W.) 1912

Wilson, W. E. Pte., R. Fusiliers

Wimbush, H. G. Lieut., Devon Regt.; Major, M.G.C. I9I2 $M 2$.

WWinch, E. M. 2nd Lieut., Rifle Brigade

Died 25 March 1915 of wounds received in action 7 March I9I5

Wingate-Gray,W.S. Major,R.H.A. (W3.) M.C. M2. 1909 WWINK, J. E. 2nd Lieut., Seaforth Hdrs.

Died 21 Sept. I916 of appendicitis

Wolffsohn, A. W. Capt., R. Welsh Fus. WolffsoHn, A. W. Capt., R. Welsh Fus.
Woon, G. E. C. Capt., Gloucestershire Regt.; Staff Capt. ${ }^{1912}$
(W.) M.C. M.

Woop, R. McK. Sapper, R.E.; 2nd Lieut., R.A.F.(Tech- I9I I nical Res.)

WWoodrofre, K. H. C. Lieut., Rifle Brigade. $M$.

Killed in action 9 May 1915

WWoodroffe, W. G. Capt., Middlesex Regt.(T.F.) French Croix de Guerre

Killed in action 16 Sept. 1916

Woods, G. C. Lieut., R.G.A.

Woons, Rev. S. C. C.F. $4^{\text {th }}$ Class, R.A.C.D.

WWoolston, J. H. Lce.-Corpl., S. African Infy.

Died 28 Oct. 1918 of wounds received in action 1o Oct. I9I 8

Worthington, G. V. Lieut., R.A.M.C.
1910

1909

1913

I9I 5

I9I2

I9I2

1904

I 886

I 893

I887 
WWreford, B. W. H. Capt., Devon Regt. (W.)

Killed in action 23 April r917

Wright, A. G. Lieut., R.A.S.C.

Wright, A. G. W. Lieut.-Col., R.F.A.(T.F.) T.D.

Wright, C. C. G. Capt. and Adjt., Durham L.I.

Wright, J. M. M. Surgeon Sub-Lieut., R.N.V.R.

WWright, W. E. B. Lieut., King's Own (Yorkshire L.I.)

Killed in action 22 Sept. I9I 5

*Wright-Ingle, C. H. 2nd Lieut., R. Fusiliers; attd. 1903

Leinster Regt.

Killed in action 30 April r9r6

Wroth, E. C. Lieut., R. Warwickshire Regt. (W.) 1902

Wylie, A. Capt., R.A.M.C. (W.)

WWylie, H. M. Pte., London Regt. (London Scottish) 2nd Lieut., Seaforth Hdrs.

Killed in action on the Tigris 7 Fan. 1916

Wyndham, J. Capt., Welsh Regt. (R. of O.); attd. W. 1898 African Frontier Force

Yeatman, H. M. Capt., Wiltshire Regt.; Capt. (A.), [I9I4] R.A.F. (W 2.)

Yeld, G. G. 2nd Lieut., Labour Corps

YETTS, L. M. Capt., The Queen's (R.W. Surrey Regt., 1905 T.F.); A.P.M. M.C. M.

Young, G. P. Lieut., Loyal N. Lancs. Regt. I9I6

Young, J. D. Lieut., R.G.A.(T.F.) $m$.

WYung, M. H. Lieut., Lancs. Fus.(T.F.)

Died in German hands 29 Fune 1916 of wounds received in action

Younghusband, L. N. Major, R.F.A. M.C. $M$. 1902 I9I3

ZaIman, B. A. Lieut., I.A.R.O., attd. 7oth Burma Rifles

1912

I9I I 


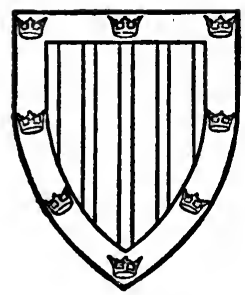

\section{PETERHOUSE}

Abraham, L. A. Lieut., Gen. List (T.F. Res.); empld. I9I3 Ministry of National Service

AABrams, L. G. Lieut., R.A.S.C.

Died 3 Nov. 1918 of pneumonia.

1902

सAdams, E. G. Pte., Cambridgeshire Regt.; 2nd Lieut., I9I5 Norfolk Regt. (W.)

Died 26 Fune 1918 of wounds received in action

Abcock, W. R. C. 2nd Lieut., R.E. (Signals); Lieut., 1902

Nigeria Regt., W. African Frontier Force. $m$.

AdsheAd, A. R. Sergt., Manchester Regt.(T.F.) I9I I

Alcock, J. H. Lieut., Lincolnshire Regt. (P.) I9I3

Alderson, M. J. G. Major, R.A.S.C.(T.F.) I886

ANDREws, E. A. Trooper, Assam Valley Light Horse, 1908 Indian Defence Force

ANDREws, J. L. Capt., Hampshire Regt.(T.F.) (W.) 1912

ANSELl, E. 2nd Lieut., King's (Liverpool Regt.) (W.) 1916

Ashron, A. A. G. Transport Officer, E. African Force I909

सAtkinson, G.H. Lieut. and Adjt., Rattray's Sikhs, Ind- I909 ian Army. $M$.

Died I Feb. I9I7 of wounds received in action

Austin, Rev. T. Chaplain, R.N.

${ }^{*} 1878$

Baker, F. B. Capt., York and Lancaster Regt.; Asst. I9I I Officer i/c R.E. Records. (W.) M.B.E. m.

Bales, P. G. Capt., D. of Wellington's (W. Riding Regt., 1906 T.F.) M.C. $M$.

Barker, Rev. G. D. C.F. 4th Class, R.A.C.D.(T.F.) * * 1903 BBEALE, E. Sergt., R. Fusiliers (P.S. Bn.) I9I I

Killed in action 2 Fan. 1916

Bell, R. L. Capt., R.A.M.C. $M$. 


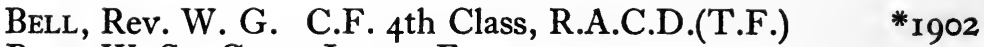

Bell, W. S. Capt., Lancs. Fus.

BerRy, Rev. B. A. C.F. 3rd Class, R.A.C.D.(T.F.) I889

Best, R. W. G. Pte., Army Cyclist Corps; 2nd Lieut., I9I I R.G.A.

Bhatia, S. L. Lieut., I.M.S. M.C.

BIRDwOOD, G. T. Lieut.-Col., I.M.S.

I910

I 886

Birdwood, H. B. Colonel, 27th Light Cavalry, Indian 1889 Army; Brig.-Gen.

WBirdwood, H. F. 2nd Lieut., London Regt. (Blackheath 1913 and Woolwich Bn.); Lieut. (A.), R.F.C.

Killed in action 2 March 1916

Bishop, G. S. 2nd Lieut., R.G.A.

I 898

Blackie, A. Pte., London Regt. (Artists Rifles); Capt., I I I I R.E. (London Electrical Engineers, T.F.)

Blakeney, J. E. C. Major, Essex Regt.; Lieut.-Col., ${ }^{2885}$ Gen. Staff.

WBoardman, T. H. Major, R. Fusiliers; Lieut.-Col., R. * I896 Inniskilling Fus. D.S.O. M.

Died 5 Aug. I9I 7 of wounds received in action

WBodington, C. H. Capt., R. Horse Gds. and Household 1899 Bn.

Killed in action I I April 1917

Boyle, G. L. Capt., Welsh Regt. (W.)

[1914]

BRICKWOOD, H. Lieut., R.N.V.R. $m$.

1895

Burne, S. A. H. Lieut., King's (Shropshire L.I., T.F.) 1898

ButTerworTh, A. B. Major, R.A.S.C. O.B.E. M. 1906

Greek Medal for Military Merit

Callis, M. C. Capt., R.E.(T.F.) (W.)

CAMP, J. Instructor Cdr., R.N.

1912

1895

Chaffer, C. Instructor Lieut., R.N.

1903

\Chalmers, R. Lieut., London Regt.(Civil Service Rifles). I9I I $M$.

Died 25 May I9I 5 of wounds received in action

Clark, Rev. P. N. Capt., Spec. List

1888

Clarke, Rev. F. W. C.F. 4th Class, R.A.C.D. 1896

Clayton, Rev. G. H. C.F. $4^{\text {th }}$ Class, R.A.C.D. M. * * 1908

Clemmow, C. A. Lieut., R.G.A.; empld. Ministry of 1907

Munitions

Clemmow, E. P. Lieut., R.G.A.; empld. R.E.

Collins, E. R., D.s.o. Major, E. Lancs. Regt. (W.) 1889

(P.)

Colman, C. J. Capt., King's (Liverpool Regt.) (W.) I9II 
ÆColson, A. F. D. Lieut., R.F.A. M.C.

I9II

Killed in action ro Nov. I917

Colville, R. N. K. Lieut., N.Staffs. Regt.; empld. Com- I90 I mand Depôt

Cooper, L. G. 2nd Lieut., R.A.S.C.(M.T.)

I 897

Cosgrove, W. O. Lieut., R.F.A.(T.F.)

Costello, L. W. J. Capt., R.A.S.C.

I9I4

* 1900

I 887

CRAIG, N. C., M.P. Lieut.-Cdr., R.N.V.R.

1905

Cranston, H. N. Lieut., R.N.V.R. $M$.

Crocombe, F. R. Lieut., 76th Punjabis, Indian Army * *1912

Cruickshank, R. S. Capt., R.A.S.C. O.B.E. M 3. $\quad 1898$

CURTIS, I. Instructor Capt., R.N.; empld. R.A.F.

1893

Darbyshire, Rev. H. S. C.F. $4^{\text {th }}$ Class, R.A.C.D. $m$. 1895

Davies, V.P. Capt., M.G.C. (W.)

WDawkins, C. J. R. 2nd Lieut., Welsh Regt.

Killed in action 25 Sept. I9I 5

WDay, G. R. Lieut., Bedfordshire Regt.(T.F.); Capt. R. 1907 Warwickshire Regt. (W.)

Killed in action on the Somme 27 Aug. 1916

DE HAHN, C. P. Lieut., Imperial Russian Mtd. Grenadier 1912

Gds. (W.) Order of St Anne, with swords (Russia).

Cross of St Stanislas (Russia)

Deighton, F. Lieut.-Col., R.A.M.C.(T.F.)

Dent, G. J. C. Pte., H.A.C.

Drew, Rev. J. A. Lieut., Black Watch (T.F.)

[1914]

I910

DRY, E. F. K. Lieut., R.N.V.R. (R.N.D.)

1873

1897

1899

1901

Drysdale, J. M. Capt. (A.), R.A.F. (W.) $m 2$. 1905

WDRYSDAle, R. G. 2nd Lieut., R. Warwickshire Regt. I908

Died I 5 April I9I5

DU VAlloN, G. C. D. DE J. Lieut., R.F.A. (W.) I897

Dymond, G. W. Lieut., Cheshire Regt. M.C. I9I3

Edgington, H. Major, R.A.S.C. O.B.E. $M 2 . \quad \mathrm{I} 89 \mathrm{I}$

EDwards, H. J., C.B., T.D. Hon. Colonel, Unattd. List, * ${ }^{*} 899$ T.F.; cmdg. O.C.B. C.B.E. O.B.E. $m 3$.

EElkington, T. G. Pte., R. Fusiliers (P.S. Bn.); 2nd I9I2 Lieut., Suffolk Regt.

Died 4 March 1916 of wounds received in action 3 March 1916

EEllis, E. W. Pte., R. Fusiliers (P. S. Bn.); Lieut.,

I9II

R.N.V.R. (R.N.D.)

Killed in action 4 Feb. 1917 
English, D. A. Capt., London Regt. (Surrey Rifles); 1890 Capt. (T.), R.A.F.

Evans, F. H. F., D.s.o. Major, King's Own (R. Lancaster 1886

Regt.); Adjt., Loyal N. Lancs. Regt. Depôt; empld.

Board of Trade. O.B.E.

Evans, T. M. Lieut., R. Defence Corps. (W.) 1898

ExhaM, R. K. S. Lieut., King's (Shropshire L.I., T.F.) I913 (W.)

FAlKner, A. H. Major, R.A.M.C.(T.F.) (W 2.) $M$ 2. $\quad 1894$ Fisher, A. R. C. Lieut., R.G.A. 1910

Fleming, N. Capt., King's (Liverpool Regt.); attd. E. 1907 Surrey Regt. $M$.

Fletcher, P. Pte., Canadian A.M.C.

WFormby, T. H. Capt., Cambridgeshire Regt. (W.)

Killed in action $\mathrm{I}_{3}$ Oct. 1916

Franklin, W. Lieut., Dorset Regt.; Lieut. (A.), R.A.F. [I9I4] (W 2.)

French, C. H. A. Capt., Lincolnshire Regt.; Major, [I9I4] Gen. List (R.E., Signals). (W.) M.C. $M 2$.

Frink, F. C. B. Capt., S. Staffs. Regt. and Labour Corps 1887

Gaskin, E. A. L. Lieut., Spec. List (R.T.O.)

Gavin, A. G. D. 2nd Lieut., Black Watch; attd. R.F.C. I9I I (Balloon Section). M.C.

GibB, J. H. O. Lieut., attd. 4th (R. Irish) Dragoon Gds. I9I0 (W.)

Giles, L. T. Capt., R.A.M.C.

Gilham, C. W. Corpl., R.E.; 2nd Lieut., R.G.A. (W 2.) I9ro

Green, J. E. S. Capt., Rifle Brigade. (W 2.) 1905

GREEN, R. G. 2nd Lieut., M.G.C.

GreENwood, J. Lieut., S. Lancs. Regt. and Spec. List 1905 (Loyal N. Lancs. Regt. Depôt). (W 2.)

GreENwOOD, O. Lieut., Yorkshire Regt. (W.) (P.) 1908

GrIFFIN, E. H. Capt., R.A.M.C. (W 4.) (P.) D.S.O. 1895 M.C. and Bar. $M 3$.

GunDRY, Rev. R. H. C.F. 3rd Class, R.A.C.D.(T.F.) * * 1887

Gurney, J. C. Capt., Northamptonshire Regt.; G.S.O.3. 1912 O.B.E. M 2. Belgian Croix de Guerre

Guy, P. C. Lieut., Worcestershire Regt.; Capt., Spec. 1899 List, empld. Ministry of National Service

EHamilton, C. F. P. Capt., Scots Gds. $M$.

Died 27 Oct. I914 of wounds received in action 
Happold, F. C. Lieut., Loyal N. Lancs. Regt.; Capt., I9I2 Gen. List (School of Instruction). (W.) D.S.O. M.

Hargrove, C. R. Capt., R.A.S.C.

HaRT, Rev. P. H. Sergt., R.G.A.

WHARTLEY, W. J. Capt., R. Irish Fus.

Killed in action in Gallipoli 16 Aug. 1915

WHeatley, L. Capt., King's (Liverpool Regt.) (W.)

Died 17 Aug. 1917 of wounds received in action

1900

*I 908

1907

I9I I

HHenderson, W. L. 2nd Lieut., Northumberland Fus.; 1902 Capt., W. Yorks. Regt. (W.) $M$.

Died 3 May I916 of wounds received in action 3 April 1916

Henman, W. W. Lce.-Corpl., Military Police

Hils, V. St C. Capt., M.G.C. M.C. M.

HodgetTs, W. J. Driver, H.A.C.

Holman, B. W. Trooper, Otago Mtd. Rifles, N. Zealand

Force; Pte., N. Zealand Ordnance Corps; Capt.,

Labour Corps; attd. R.A.O.C.

HoneYbURNe, W. R. Capt., R.A.M.C.

Housden, E. F. Major, R.F.A. (W.) M.C.

1898

1913

1917

1895

Housden, E. J. T. Major, R.F.A. (W 2.) M.C. $M_{4}$.

Hughes, H. C. Lieut., R.F.A.(T.F.) (W.)

Hurst, E. E. 2nd Lieut., Northamptonshire Regt. $M$.

HydE, G. A. 2nd Lieut., King's Royal Rifle Corps; Capt. I9I3 (A.), R.A.F. M.C.

Hylton Stewart, B. D. Lieut., Haileybury College rgio O.T.C.

JefFCOCK, W. P. Capt., Yorkshire Dragoons (T.F. Res.) 1887 Jervols, R. C. W. 2nd Lieut., R.G.A.; Lieut. (O.), I9I5 R.A.F.

Jones, W. H. See Macnaughten-Jones, Rev. W. H.

Kaufmann, H. P. Lieut., Norfolk Regt.(T.F.); attd. I9I I Gloucestershire Regt. (W.)

Keigwin, A. L. Capt., Lancs. Fus. (W.)

Weigwin, H. D. 2nd Lieut., Lancs. Fus.

Killed in action 20 Sept. 1916

Keigwin, R. P. Lieut., R.N.V.R. Chevalier, Order of 1902 Leopold (Belgium)

KenRICK, C. H. W. 2nd Lieut., The Buffs (E. Kent Regt.) I9I4

WKershaw, M. 2nd Lieut., Gloucestershire Regt.

Killed in action 7 Nov. I9I4

KItson, Rev. B. M. C.F. $4^{\text {th }}$ Class, R.A.C.D.

1908

1900

I905

1905 
Laurie, M., M.v.o. Capt., R.G.A.(T.F.); empld. War 1887 Office

ELAwson, H. S. Lieut., R.F.A.(T.F.)

Killed in action $5 \mathrm{Feb}$. 1918

ALeigh, B. H. 2nd Lieut., R. Berkshire Regt.

Killed in action 18 Aug. I9I7

Lewis, V. Lieut, R.F.A.

Lias, E. T. M. Sapper, R.E.

1895

1908

1913

I9II

Liddle, G. E. Capt., K. Alfred's School, Wantage, 1904 O.T.C.

LightFoot, B. Major, R.E. M.C. $M$.

Lillie, W. H. 2nd Lieut., United States Air Service

LindSAY, R. L. G. Lieut., R.E. (Signals)

ZLivingstone, F. D. Capt., R.A.S.C.

Died 22 March 1918 of wounds received in action

Lloyd, G. W. D. B. Major, R. Welsh Fus.; empld. ${ }^{8} 885$

Ministry of National Service. O.B.E.

Lloyd, K. Capt., London Regt.(Q.V.R.); empld. O.C.B. $\quad$ I9r3 (W.)

WLloyd, R. A. 2nd Lieut., King's (Liverpool Regt.)

Killed in action near Ypres 27 April I915

Lloyd, Rev. W. R. Lieut., Haileybury College O.T.C. * *1907

Long, F. W. C. Sapper, R.E. (Signals). (W.)

WLORD, C. E. Major, S. Staffs. Regt.

Died 23 Fune I9I 5 of wounds received in action

LöwY, A. E. Flt. Sub-Lieut., R.N.A.S.

LutTMan, R. L. Sub-Lieut., R.N.V.R.

1913

1914

1887

1907

r894

McBain, W. R. B. Lieut., R.F.A.(T.F.); Major (A.), 1910

R.A.F. M.C. A.F.C. M 3. French Croix de Guerre

McCarthy, Rev. J. D. Chaplain, R.N.

McCarthy, J. M. Lieut.-Col., R.A.M.C.

I894

1891

McKeown, W. W. Lieut., R.F.A. M.C. 1914

Maclean, J. E. B. B. Major (S.), R.A.F. (W.) D.S.G. I9I3 $M 2$.

Macnamara, E. D. Capt., R.A.M.C.

Macnaughten-Jones, Rev. W. H. C.F. $4^{\text {th }}$ Class, 1888 R.A.C.D.(T.F.)

Manser, F. B. Major, R.A.M.C. $\quad$ I894

Marchant, T. H. S. Major, I3th Hussars; Brig.-Gen. 1893 D.S.O. $M 3$.

Marillier, H. C. Lieut., R.N.V.R.

MARLEY, A. E. Lieut., Spec. List (Interpreter)

Marriott, G. E. J. Capt., Lancs. Fus.(T.F.) 
Marshall, F. W. D. Pte., R.A.M.C.

I 908

Marshall, P. T. Capt., R.A.S.C.

1898

WMARTIN, T. 2nd Lieut., The Queen's(R.W.Surrey Regt.) 1904 Killed in action 7 fune 1917

MarTYN, R. F. Lieut., R.A.S.C. $M$.

I912

Mason, A. S. Major, Devon Regt.(T.F.); D.A.Q.M.G. [I9I4] (W.)

Mason, R. F. Capt., R.F.A.; Staff Capt. (W.) M.C. M. I9r3

MAY, Rev. C. J. C.F. $4^{\text {th Class, R.A.C.D. }}$

Maynard, C. B. Lieut., S. Lancs. Regt. (W.)

1906

Meadows, R. Capt., R. Warwickshire Regt.; attd. King's I9I4 Own (Yorkshire L.I.) (W.)

Middleton, H. S. Lieut., Suffolk Regt.(T.F.)

Miller, A. G. S. Capt. and Adjt., Fife and Forfar Yeo. 1909

MILNER, P. R. Capt., Leicestershire Regt. (W 2.) M.C. I9II and $\mathrm{Bar}$

सMond, F. L. Lieut., R.F.A.(T.F.); Staff Lieut.; Lieut. *1914 (A.), R.A.F.

Killed in action at Bouzencourt ${ }_{5} 5$ May 1918

Morgans, G. E. Lieut.-Col., R.E. O.B.E. m.

MorRIS, P. E. 2nd Lieut., R.A.S.C.

I 901

1899

Murray, E. Lieut.-Col., King's Royal Rifle Corps and 1904 Gen. List (Staff Lieut.) (W.)

Neville, M. R. Lieut., R.G.A. M.C.

1913

NorTh, J. B. 2nd Lieut., 2nd Dragoons (R. Scots Greys) I9I5 and Labour Corps

Northfield, H. D. Pte., R. Scots

Norton, L. M. Capt., R.G.A.(T.F.)

I9I 5

* 1908

OAKDEN, W. M. Capt., R.A.M.C.

${ }^{1}$ Oon Israsena, M. L. Interpreter, Siamese Exp. Force I9I4

Packard, H. N. Capt., Spec. List (O.C.B.)

PARISH, A. J., C.B. Instructor Cdr., R.N.; Dep. Adviser I88I on Education. C.B.E.

Parish, Rev. W. O., T.D. C.F. ist Class, R.A.C.D.(T.F.) 1878

Parkinson, W. Lieut., R.E. (Signals). M.C. M. 1906

PAYNe, F. R. Lieut., R.A.S.C. $M$. 1899

Pearce, Ven. E. H., T.D. C.F. Ist Class, R.A.C.D.; 1884 A.C.G. C.B.E.

Perrin, E. C. Capt., Cheshire Regt.(T.F.); Major, I905 S.O. 2, R.A.F. O.B.E.

1 Accidentally killed on service after the armistice. 
Phillipson, E. Sergt., R.A.M.C.; 2nd Lieut., Lancs. I9I I Fus.(T.F.)

Pinfold, E. S. Pte., Upper Burma Bn., Indian Defence 1907 Force

Plaskitt, G. M. Lieut., Middlesex Regt.

सPuCKLE, J., D.s.o. Lieut.-Col., R.A.S.C. $M$.

Drowned on H.M. transport ${ }_{5} 5$ April I9I7

1913 1889

Rawlins, F. H. Major, R.E.

READ, E. Capt., R.F.A.(T.F.) Belgian Croix de Guerre [1914] Reynolds, H. E. K. 2nd Lieut., York and Lancaster I9I3 Regt.

Rhodes, W. E. Lieut., R.E. (W.)

Ritchie, A. G. Capt., R.E. (Signals). $M 2$.

WRobinson, J. C. C. H. Capt., E. Lancs. Regt.(T.F.)

1912

I9II

Killed in action 3 Fune 1917

सRobinson, W. DE H. Pte., R. Fusiliers (P.S. Bn.); Capt., 1907

Border Regt. (W 2.) M.C.

Killed in action 27 fan. I9I7

RotTNER-SMITH, R. Cadet, O.C.B. I9I7

Routh, A. L. Major, R.G.A.

Routh, H. V. Capt., R.F.A. $M$.

RyFFEL, J. H. Capt., R.A.M.C.(T.F.)

\#SAnderson, C. O. St J. Lieut., R.E. (Signals). M.C. I9ro Killed in action 27 April 1918

ScotT, G. M. Capt., R.A.M.C.; Lieut.-Col., N. Zealand *1884 Med. Service

WScrace, J. Lieut., The Buffs (E. Kent Regt.); Lieut.(A.), I9I I R.A.F.

Killed in flying accident 24 Aug. 1918

SEALY, P. T. Major, R.A.S.C. O.B.E. $M$.

SeAly, W. H. Capt., R.A.S.C. $M$.

\#SEwell, H. V. 2nd Lieut., R.F.A.

Killed in action 13 Nov. I9I6

WShipley, A. H. B. Pte., R. Fusiliers (P. S. Bn.); 2nd [1914] Lieut., Yorkshire Regt.

Killed in action 27 Sept. 1916

Smith, Rev. E. F. H. Chaplain and Instructor Cdr., $\quad$ I894 R.N.

Smith, J. Y. Pte., R.A.S.C.(M.T.)

SPRING, A. Lieut. (T.), R.A.F. (Aircraft Production 1906 Dept.)

Springfield, C. O. Capt., R.E. 
WSprott, M. W. C. Capt. and Adjt., Norfolk Regt. (W.) 1906

' M.C. M.

Killed in action 21 March 1918

«Sproxton, C. Capt., Yorkshire Regt.(T.F.) (W 2.) 1909 M.C. $M$.

Killed in action 19 fuly 1917

Stacknouse, J. H. Lieut., Sherwood Foresters (Notts. 1908 and Derby Regt.); Capt., R.A.S.C. (W.)

StEPHen, L. P. Lieut., R.A.S.C.

SteWART, A. C. Capt., Welsh Regt. and R.E. (Field I9I I Survey Coy.)

Stimson, J. P. Sub-Lieut., R.N.V.R.(R.N.D.) (W.) 1909

Stoneham, R. T. Corpl., Rangoon Bn., Indian Defence 1906 Force

TAIt, J. G. Lieut.-Col., Bangalore Rifle Vols.

WTAYLOR, C. T. Lieut., I 8th Hussars

Killed in action 24 Aug. I914

WTAYLOR, F. Major, York and Lancaster Regt.

Killed in action 13 March 1916

TEBB, B. M. 2nd Lieut., Suffolk Regt.

TebB, R. N. S. Capt., Spec. List (O.C.B.) and Hamp- I913 shire Regt.

Temperley, H.W.V. Capt., Fife and Forfar Yeo.; Major, * ${ }^{*} 904$ G.S.O. 2; Asst. Military Attaché, Belgrade. O.B.E. $m$ 2. Officer, Order of the Crown of Roumania, with swords. Order of the White Eagle, 5th Class, with swords (Serbia)

Temple, R. J. G. Trooper, Dorset Yeo.; Lieut., R.G.A.; Capt. (T.), R.A.F.; Instructor, School of Aeronautics, Toronto

Thomas, K. G. Capt., E. Lancs. Regt.(T.F.); attd. Man- 1907 chester Regt. $M$.

\#Tindall, J. H. Gnr., R.G.A.

Died 15 Feb. I9I7 of malaria

1880

1909

1874

1917

I9I3

1908

1913

Regt.) (W.)

Killed in action $8 \mathrm{Aug}$. I916

Tomson,D.V. Lieut., R.F.A.(T.F.) (W.) M.C.andBar 1902

UtTing, H. A. Lieut., R.F.A.(T.F.)

1914

Vellacott, P. C. Major, S. Lancs. Regt. and Gen. List r9ro (Brigade Major). (W.) (P.) D.S.O. M 2.

c.u.w.L. 
WWakerley, A. J. Capt., Leicestershire Regt.(T.F.)

Killed in action 8 fune 1917

WALKER, E. M. Lieut., I.A.R.O., attd. S. and T. Corps. I912

WALTER, H. C. A.B., R.N.V.R.

Watson, G. T. Lieut., R.A.M.C.

WatTs, N. H. Major, Sherwood Foresters (Notts. and Derby Regt.); empld. O.C.B.

WEIR, N. Lieut., Gloucestershire Regt. (W.) 1908

Whitehead, E. T. Capt., Yorkshire Hussars; G.S.O. 3, 1892 War Office

Whitehouse, H. E. Lieut., R.G.A. M.C.

WhitTAker, Rev. G. Chaplain, R.N.

Willey, B. Lieut., W. Yorks. Regt. (P.)

Williams, Rev. A. J. C.F. 4th Class, R.A.C.D.

I9II

1890

1904

Williams, G. V. Capt., Loyal N. Lancs. Regt.(T.F.)

WILIIAMS, Rev. R. C. L. C.F. Ist Class R.A.C.; 1912 A.C.G. D.S.O. $M 2$.

Williams, T. Capt., Welsh Regt.; Major, D.A.D. Docks * ${ }^{*} 1905$

WILlis, R. E. B. Lieut., R. Warwickshire Regt.

Wilson, T. M. Capt. (T.), R.A.F.

[1914]

I9I 5

1908

1916

1893

WisEMAN, C. L. Instructor Lieut., R.N.

WWood, H. G. W. Capt., Worcestershire Regt.(T.F.)

(W.) D.S.O. M 2 .

Killed in action 3 Aug. 1918

WWood, T. P. Lieut., I.A.R.O., attd. Gurkha Rifles

Killed in action 25 Sept. I915

. Yeld, P. H. Capt., Spec. List (R.T.O.)

1901

1903 


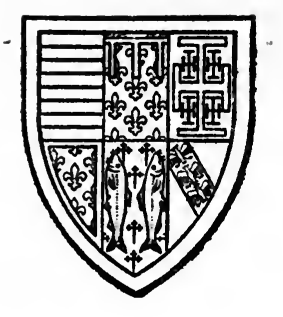

\section{QUEENS' COLLEGE}

WAdAM, H. W. Lieut. (A.), R.A.F.

Killed in flying accident 4 fuly 1918

WAdAMs, L. H. Lieut., Rifle Brigade

Killed in action 22 April 1918

Artchison, G. C. Lieut.-Col., Sherwood Foresters I88I

(Notts. and Derby Regt.) $m$.

Ambrose, W. J. L. Lieut., Res. Regt. of Cavalry and 1897

Nigeria Regt., W.African Frontier Force. (W.) Chevalier, Ordre de l'Etoile Noire (France)

ANDREws, Rev. L. M. C.F. 4th Class, R.A.C.D. (W.) 1906 M.C.

Anthony, H. D. Lieut., Loyal N. Lancs. Regt. and IgI I

R.E. (Sound-ranging Section)

AARDEN, H. W. 2nd Lieut., R.G.A.

Died 6 fune 1917 of wounds received in action

Atkinson, R. H. Lieut., Durham L.I.; Capt., R.E. 1909

(Spec. Bde.); empld. Ministry of Munitions. (W.) $m$.

Aưstin, Rev. H. W. C.F. $4^{\text {th }}$ Class, R.A.C.D. M. 1910

*Bailey, W. G. W. Capt., Hampshire Regt.

Killed in action 15 Sept. 1916

BaLL, A. E. Lieut., Middlesex Regt.(T.F.) (W.) 1909

WBARKER, Rev. E. W. C.F. 4 th Class, R.A.C.D.

Died 18 March 1918 of wounds received in action 9

March 1918

BARKER, H. A. K. Capt., Loyal N. Lancs. Regt. (W.) I9I I

BARLTROP, E. A. Lieut., R.E.(T.F.); attd. R.F.C. 1909

Killed in action 23 April I917

BARLTROP, I. C. Major, R.E.(T.F.) I9I I

Barton, J. F. Trooper, Calcutta Light Horse, Indian 1904 Defence Force 
Bentley, R. C. Lieut., Yorkshire Regt. (W 2.) (P.) 1910

Bentley, W. W. Major, R.G.A.(T.F.) (W.) 1903

Best, Rev. J. K. C.F. 4th Class, R.A.C.D.(T.F.) M.C. 1907 $M$.

Best, O. H. Lieut., R.E. (Signals)

Beverley, R. Capt. and Adjt., R.F.A.(T.F.)

Bigger ,W. G. Surgeon Lieut., R.N. D.S.C. Order of St Anne, 3rd Class(Russia). Belgian Croix de Guerre

1912 1908 1907

Winks, B. H. 2nd Lieut., King's Own (R. Lancaster Regt.) I906 Killed in action 23 Oct. I916

BlaCK, L. L. D. Lieut., R.G.A.

Blackden, S. C. 2nd Lieut., Oxfordshire Yeo.

Blacker, H. A. C. Capt., I.A.R.O., attd. $3^{\text {rd Punjab }}$ Rifles; Asst. Recruiting Officer

Blackwall, Rev. W. A. C.F. $4^{\text {th }}$ Class, R.A.C.D.

BLEE, E. L. Lieut., York and Lancaster Regt.; attd. Manchester Regt.; empld. Ministry of Munitions. (W.)

सBone, H. Corpl., R.E. (Spec. Bde.) D.C.M.

Died 30 Aug. I9I7 of gas poisoning caused by an explosion

Boswell, P. R. Capt., R.A.M.C. (W.) M.C.

«Botwood, Rev. E. K. C.F. $4^{\text {th }}$ Class, R.A.C.D.(T.F.) * * 1892 Died 28 fuly 191 6 of illness contracted on active service BoumPHREY, D. Lieut., King's (Shropshire L.I.); Major, M.G.C. M.C. M.

Bourchier, Rev. B. G. C.F. 4th Class, R.A.C.D.(T.F.) 1899

Brailey, W. H. Major, R.A.M.C. (2nd E. Gen. Hos- 1893 pital, T.F.)

BRISCOE, F. E. Major, Yorkshire Regt.; attd. Norfolk 1912 Regt.(T.F.) D.S.O. M.

Broadbent, H. G. 2nd Lieut., St Bees School O.T.C. I9I I ABRodie, M. M. 2nd Lieut., London Regt. (London [1914] Scottish); attd. Seaforth Hdrs.

Killed in action 7 Fan. 1916

Browne, A. D. Capt., R.G.A.

1908

WBuckley, E. J. K. Flt. Sub-Lieut., R.N.A.S. Belgian I9I4 Croix de Guerre

Killed in flying accident 28 Sept. 1917

BudgetT, S. G. Lieut., King's (Shropshire L.I.); Lieut. [1914] (A.), R.A.F. (W.)

Bull, G. G. C. 2nd Lieut., R.E.(T.F.); Lieut., Bedford- [1914] shire Regt.; attd. 98th Infy., Indian Army

Bullard, R. W. Capt., Spec. List. C.I.E. 
Burton, G. D. Capt., R.E. $M 2$.

Bussey, A. Corpl., Suffolk Yeo.

BuTCHER, T. A. 2nd Lieut., King's Royal Rifle Corps;

Capt., R.A.M.C. (W 2.) O.B.E. M.

Butler, W. G. 2nd Lieut., Christ's Hospital O.T.C.

1912

1905

WCallinan, T. W. 2nd Lieut., Durham L.I.(T.F.)

1902

Killed in action near Ypres 25 April I9I 5

Carnley, W. B. 2nd Lieut., R.A.O.C.; Capt. (T.), $\quad 1903$ R.A.F. $M . C$.

ACARr, D. N. 2nd Lieut., R. Fusiliers; Capt., S. Persian I9I3 Rifles

Died 26 Nov. I9I8 of pneumonia contracted on active service

Case, H. A. Lieut.-Col., Dorset Regt. (W.) C.M.G. ${ }_{1} 898$ D.S.O. Brevet Lieut.-Colonel. M 3 .

Cassels, W. C. Capt., Worcestershire Regt. (W 2.) M.C. I9r3 ACatmur, H. A. F.V. Pte., R. Fusiliers (P.S. Bn.); Lieut., I9I I

R. Sussex Regt.; attd. M.G.C. M.

Killed in action I Fuly r. 6

Challenor, B. H. Capt., Lincolnshire Regt.(T.F.) (P.) I9r3

Champion, Rev. A. C. C.F. 4th Class, R.A.C.D.

Chandler, H. E. Capt., The Queen's (R. W. Surrey Regt.); Staff Capt., War Office. (W.) M.B.E. $m 2$. Chevalier, Ordre de la Couronne (Belgium)

Channing, C. E. Lieut., R.A.S.C.(T.F.); Lieut. (O.), I9I4 R.A.F. A.F.C.

Chase, Rev. G. A. C.F. 4th Class, R.A.C.D. M.C. 1905

Clarke, C. M. S. Corpl., R.A.M.C. I9I4

Clayton, N. W. Capt., M.G.C. and Gen. List, attd. 1897 Indian Political Dept. M.C. M.B.E. M2.

Clear-Davidson, F. R. 2nd Lieut., R.G.A.

Crewert Rev. T H C F th

ClewORTH, Rev. T. H. C.F. 4th Class, R.A.C.D. M.C. 1906

CoAst, J. P. C. Lieut., R. Warwickshire Regt.; Capt., I90 I The Queen's (R. W. Surrey Regt.)

ZCoATES, B. M. 2nd Lieut., Rifle Brigade Killed in action 7 Sept. 1915

सCohen, E. 2nd Lieut., R. Fusiliers. M.C. Killed in action 31 fuly r917

ACohen, J. I. Lieut., E. Lancs. Regt.; Capt., Labour Corps I9I I Died 24 Aug. 1917 of wounds received in action

Colchester, G. V. Capt., R.E. (W.) M.C.

Colenutr, F. A. Lieut., Spec. List (Interpreter)

Collins, B. S. 2nd Lieut., Herefordshire Regt.

1912

1914

* 1908 1906

[1914] 
Collis, F. S. 2nd Lieut., W. Kent Yeo.; attd. Corps of I9I4 Guides, Indian Army

Conder, A. C. Lieut., Black Watch (T.F.) (W.) I9I I

Cooke, Rev. H. R. C.F. $4^{\text {th }}$ Class, R.A.C.D. (W.) 1904 M.C. $M 2$.

Cookson, R. T. C. Pte., London Regt. (Artists Rifles); 1894 Lieut., R.F.A.(T.F.)

Cope, H. E. Cadet, O.C.B.

Cortazzi, F. E. M. Major, R. Scots. (W.)

Cotron, H. W. S. Capt., Cheshire Regt. (W.) $M$.

Couch, C.J. 2nd Lieut., M.G.C. and Tank Corps; Capt., I9I2

Cheshire Regt

Couchman, Rev. M. L. C.F. 4th Class, R.A.C.D. 1910

*Coultas, T. B. Lieut., E. Yorks. Regt. (W.) 1912

Killed in action on the Somme 26 Sept. 1916

Cowell, S. J. Capt., R.A.M.C. (W.)

Craigs, W.N. Capt., Northumberland Fus.(T.F.) (W2.) I9ro M.C.

Crow, A. D. Capt., E. Surrey Regt.; Director of Ball- 1913 istics, Woolwich Arsenal. (W.) $m$.

Crozier, Rev. P. H. C.F. 3rd Class, R.A.C.D. M. $\quad 1892$

Crump, G. H. Capt. and Adjt., Essex Regt. 1909

Cullen, Rev. A. H. C.F. 4th Class, R.A.C.D. I912

Culverwell, C. T. Lieut., R.A.S.C.(M.T.) I913

CURrYer, W. H. S. Lieut., R.F.A.(T.F.) (W.) I9I3

Curtors, P. A. Lieut., Suffolk Regt.; attd. The Queen's I9I I

(R.W. Surrey Regt.) (W 2.)

WDAlley, J. P. 2nd Lieut., I.A.R.O.; attd. R.F.C.

Killed in action 9 Nov. 1917

Daltroff, E. M. 2nd Lieut., R. Fusiliers; empld. War 1909 Office

Davidson, G. S. Lieut., I.A.R.O., attd. S. and T. Corps I90I

Davies, D. H. S. 2nd Lieut., S. Wales Borderers ; Lieut., I9I4

R. Warwickshire Regt.; Capt. (A.), R.A.F. (W.)

DenYeR, S. E., C.M.g. Capt., R.A.M.C. $m 2$.

1891

Devey, J. H. J. C. Lieut., Monmouthshire Regt.; Capt., 1914 Tank Corps. (W.)

Dewé, C. D. E. Lieut., Gloucestershire Regt. (W.) $\quad 1898$

WDick, J. McN. Pte., Cameron Hdrs. and M.G.C.

Died 2 Oct. 1918 of wounds received in action 1 Oct. 1918

Dickson, Rev. R. J. C.F. 4th Class, R.A.C.D.

1915

Dixon, H. J. Capt. and Adjt., R. Warwickshire Regt. 198 M.C 
Dodson, C. S. Capt., R.A.M.C. $M$.

WDoggetT, G. P. Corpl., Cambridgeshire Regt.; 2nd [1914]

Lieut., D. of Wellington's (W. Riding Regt.); attd.

T.M.B. $M$.

Died 4 Fuly 1917 of wounds received in action 7 June

I9I7

Down, R. A. Capt.; E. Surrey Regt.

DREYFUS, B. E. Lieut., I.A.R.O., attd. 32nd Lancers I9I I

Duffield, C. A. W. Lieut., Queen's Own (R. W. Kent 1906 Regt.) M.C.

Duke-Baker, C. A. Lieut., R.G.A.(T.F.); Staff Capt. $M$. I9I I

WDuncan, J. F. Capt. and Adjt., Cameronians (Scottish I9I3 Rifles). $M$.

Killed in action 25 Sept. r9r 5

Durrant, C. E. Capt., Norfolk Regt.(T.F.)

Dyson, W. L. Capt. and Adjt., Border Regt.

सEAdIE, R. A. 2nd Lieut., Lincolnshire Regt.

Killed in action 6 Aug. 19r6

EEagle, G. C. 2nd Lieut., Suffolk Regt. (W.)

Killed in action 12 Oct. 1916

Eatherley, W. Capt., R.G.A. O.B.E. M. Italian 1900 Croce di Guerra

EDWARDS,F.M. Lieut.,Egyptian Camel Transport Corps 1904

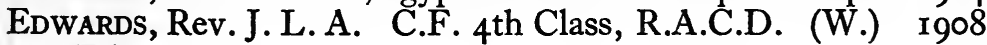
(P.)

EDWARDS, R. G. 2nd Lieut., Somerset L.I.; Major, Re- I910 mount Service

ELEY, H. J. Lieut., Sherwood Foresters (Notts. and Derby Regt.) (W.)

Ellison, A. D. Trooper, K. Edward's Horse; Capt., 1912 R.F.A. (W.) M.G.

Ellison, H. S. Trooper, K. Edward's Horse; Major, I914

R.F.A. (W 3.) M.C. M. French Croix de Guerre

Emtage, W. L. 2nd Lieut., Middlesex Regt.; Lieut., I9I I British W. Indies Regt.

Evans, A. Ll. Capt., Monmouthshire Regt.; Lieut., 9th r9ro Bhopal Infy., Indian Army. (W 2.) $M$.

Evans, G. S. W. Lieut., S. Wales Borderers. $M$.

Evans, H. G. Lieut., R.A.S.C.; attd. Rifle Brigade. (W.) I9I2

Evelyn-White, Rev. K. V. C.F. 4th Class, R.A.C.D. 1905

Ewbank, A. L. J. Capt., R. Dublin Fus. and Gen. Staff r9Io (O.C.B.)

EwING, A. G. 2nd Lieut., K. Edward's Horse

1912 
Failes, Rev. B. J. Chaplain, R.N. $M 2$.

Falloon, Rev. W. M. C.F. 4th Class, R.A.C.D. $M$.

FARNFIELD, A. J. Capt., King's Royal Rifle Corps

FARNFIELD, Rev. H. V. C.F. $4^{\text {th }}$ Class, R.A.C.D.

FARNFIELD, P. H. Lieut., Sidcup Hall School O.T.C. 1908

WFARRAR, H. R. 2nd Lieut., Leicestershire Regt.; attd. 1906

Manchester Regt.

Killed in action 24 Dec. 1914

Fawkes, R. B. Capt., Northamptonshire Regt. (W 2.) I9r3 D.S.O. M.C. M.

FFearnLey-Whittingstall, G. H. 2nd Lieut., R.A.S.C.; I9ro Lieut., Northumberland Fus. (W.)

Killed in action 3 Aug. 1916

Fenton, V.N. Pte., R.A.M.C.(Ist E.Gen.Hospital,T.F.) I914 WFenwick, M. E. E. 2nd Lieut., Devon Regt.

Killed in action 2 April r917

Ferguson, M. G. Lieut., R.G.A.

1913

Frauson, W. H. Major, R.A.C. (W) M.C. and 1907 Bar. $M$.

Field, H. T.C. Capt., Oxford and Bucks. L.I. (W.) M. 1909

Finch, E. G. Major, King's (Liverpool Regt., T.F. Res.); 1889 empld. Records

FIson, C. H. Lieut., The Queen's (R.W. Surrey Regt.) [19r4] (W.)

Ford, J. C. Pte., H.A.C.

FRY, D. G. Pte., London Regt. : Lieut. Gloucestershire Regt.; Capt., Spec. List (Education Officer). M.B.E.

Galloway, W. Lieut., King's Own Scottish Borderers 1904 (T.F.) (W.)

GARDNER, F. W. Major (T.), R.A.F.

GARDNER, H. W. Q.M.S., Suffolk Regt.

GARDNER, Rev. R. L. C.F. $4^{\text {th }}$ Class, R.A.C.D.

HGarret, H. F. Lieut. and Adjt., E. Yorks. Regt. Killed in action 4 Fune 1915

Garrod, W. E. E. Capt., Yorkshire Regt.(T.F.); attd. 1912 King's Own (Yorkshire L.I.) (W.) M.C. and Bar

AGeare, Rev. W. D. C.F. $4^{\text {th }}$ Class, R.A.C.D.

Killed in action in the Third Battle of Ypres 31 Fuly

1909 I9I7

Gentle, F. W. Lieut., Life Gds. and Gds. M.G. Regt. 1912

GibBs, W. J. R. Capt. and Adjt., R.A.S.C. $M$.

1909

1912

1904

1913

Gibson, S. R. Pte., London Regt. (Artists Rifles); 1909 Lieut., R.G.A. (W.) 
Glover, J. G. Lieut., King's (Liverpool Regt.); attd. I9I I Cheshire Regt. (W.)

Goddard, E. T. Capt., R.E. (Signals). M.C. 1914

Good, C. W. Capt. and Adjt., Sherwood Foresters I9I I (Notts. and Derby Regt.) $M$.

Goodwyn, P. W. Pte., Cameronians (Scottish Rifles). I9I I (W.) (P.)

\&Grace, H. C. Pte., R. Fusiliers (P. S. Bn.); Capt., Nor- 1910 thamptonshire Regt.

Died in German hands 2 Sept. 1917 of wounds received in action

Grace, Rev. H. M. Chaplain, E. African Force

\#Grant, G. L. Pte., London Regt. (London Scottish); 1908 Capt., R.A.M.C. $m$.

Killed in action II Oct. I9I 5

Gray, E. C. G. Pte., Middlesex Regt.; Capt. and Adjt., $\quad$ I 895 R.A.S.C. M.B.E. $m$.

GraY, G. Capt., Lancs. Fus. (W 2.) (P.)

GreEN, Rev. J. Senior Chaplain, Australian Force

Green, Rev. H. T. Pte., R.A.M.C. (W.)

GreEN, R. J. Lieut., Norfolk Regt.

Greenwood, A. W. Pte., R.A.M.C.

GrEgorY, A. I. 2nd Lieut., Labour Corps

I90I

1907

1905

I910

1898

I9I 5

1908

and Gen. List. (W.)

Gulldford, Rev. E. M. C.F. 3 rd Class, R.A.C.D. M.C. 1914

Gurney, P. S. Lieut., R.G.A. (P.)

HaKe, H. D. Capt., Hampshire Regt.(T.F.) $M$. [1914]

Hallet, H. J. Capt., King's (Liverpool Regt., T.F.); I9I I attd. Manchester Regt.

WHALSE, L. W. 2nd Lieut., Gloucestershire Regt.

Died ${ }_{17}$ Oct. 1918 of wounds received in action 21 Aug.

1912 1918

WHamer, A. D. Capt., Northern Cyclist Bn.; attd. Man- r9r3 chester Regt.

Killed in action 6 Nov. 1918

Hampson, S.H. Capt. and Adjt., Lancs. Fus. (W.) 1912 M.C.

HANDFORD, J. R. Lieut., York and Lancaster Regt.(T.F.) I9I I

Hargreaves, R. Capt., S. Lancs. Regt.(T.F.) M.C. I9I4

Harrison, G. B. Capt., The Queen's (R. W. Surrey I9I3 Regt., T.F.); Staff Capt. 
Harrison, G. L. Major, Middlesex Regt.; Lieut.-Col., 1903 The Queen's (R.W. Surrey Regt.) M.C.

WHarvey, S. Pte., Devon Regt. Killed in action 25 Sept. I91 5

HHaughon, A. J. Lieut., Durham L.I.(T.F.) 1913 Killed in action 23 Fune I9I 5

Hayward, A. D. Pte., London Regt. (Artists Rifles); I9ro Lieut., W. Yorks. Regt.

Heath, W.E. Surgeon Lieut., R.N. Order of St Stanislas, 1912 $3^{\text {rd Class, with swords (Russia) }}$

Hemsworth, E. P. D. Capt., Norfolk Regt.

Herapath, C. A. 2nd Lieut., King's (Shropshire L.I.) [1914]

AHerapath, N. F. 2nd Lieut., Somerset L.I.

Killed in action I I April I9I7

HERIZ-SMITH, D.M. 2nd Lieut., Northamptonshire Regt. [1914] Died 17 Feb. I9I7 of wounds received in action

Hewitt, L. McN. See McNeIll-Hewitt, L.

Hitchcock, Rev. A. E. N. C.F. 4th Class, R.A.C.D. M. 1906

HOARE, F. O. Lieut., R.F.A.(T.F.); Staff Lieut. (W.) I9I3 M.C.

Hobson, C. M. Lieut., S. Lancs. Regt. and M.G.C. 1912

Hodges, A. N. Capt., R.A.M.C. $M$.

1902

Holbech, L. Capt., Grenadier Gds.; A.D.C. (W.) 1907 D.S.O. M.C.

HHolcroft, G. C. 2nd Lieut., Durham L.I.

Killed in action 9 Aug. I9I 5

Holden, A. Lieut., Sheffield Univ. O.T.C.

Hollis, D. Pte., Nigeria Land Contingent and Somerset L.I.; Lieut., R.F.A.(T.F.)

XHolme, B. L. Lieut., R. Welsh Fus.; Staff Capt.

Died 25 April 1916 of wounds received in action ro April 1916

Hoole, B. 2nd Lieut., Bloxham School O.T.C.

1913

1900

1897

I906

Hoole, Rev. D. Lieut., R. Fusiliers; C.F. $4^{\text {th }}$ Class, R.A.C.D.

Hooper, K. A. Lieut., R.G.A.

AHope, R. A. Lieut., N. Staffs. Regt. (W.)

Killed in action $3 \mathrm{I}$ Fuly 1917

Hopewell, A. F. J. Lieut., D. of Wellington's (W. Rid- IgrI ing Regt.) (W.)

Houseman, Rev. F. O. C.F. 4th Class, R.A.C.D. 1895

Hubbard, J. W. 2nd Lieut., Gloucestershire Regt.; I9I3 Lieut., R.A.S.C.(M.T.) $M$.

Hughes, H. H. R.N. 
Hughes, Rev. L. A. C.F. $3^{\text {rd Class, R.A.C.D. }}$

HHughes-Games, J. B. Major, Durham L.I. (W2.) M.C. 1907 Died 17 Oct. 1918 of wounds received in action

Humby, S. R. Capt., R.E. (T.F.) (W.) M.C.

Hunt, F. R. W. Capt., The Queen's (R.W. Surrey Regt.) 1910 (W.)

Hutchinson, L. C. Major, E. Yorks. Regt.; attd. R. I9I4 Inniskilling Fus.; empld. O.C.B. (W 2.)

AIngle, R. G. 2nd Lieut., Lincolnshire Regt.

Killed in action I Fuly 1916

Ingleson, P. Lieut., R. Fusiliers (P.S. Bn.); Capt., Gen. I9I I List (Staff Capt.) $M$.

INGLIS, W. M. Lieut., Sherwood Foresters (Notts. and I912 Derby Regt.) (W.)

IsaAcson, Rev. C. H. C.F. $4^{\text {th }}$ Class, N. Zealand Chap- 1890 lain's Dept.

JAMES, J. H. Lieut., E. Surrey Regt.

Jameson, T. B. Major, Durham L.I.(T.F.) (W 3.) I9I3 M.C. $M$.

WJohnson, T. P. Lieut.-Col., R.A.S.C. D.S.O. $M_{3}$. $\quad 1890$ Died 12 fune 1918

Johnston, C.McA. Lieut., Seaforth Hdrs.; attd. London 1899 Regt.

WJoycE, G. E. Lieut., Leicestershire Regt.

Killed in action 20 Sept. 1916

Kafka, E. J. Pte., Middlesex Regt.

KeANE, R. D. Lieut., R.E. $M$.

Kelly, I. G.' Capt. (K.B.), R.A.F. A.F.C.

KeLly, P. J. Gnr., R.G.A.

${ }^{*}$ I907

Kelton, P. St G. Capt., The Buffs (E. Kent Regt.);

Major, Gold Coast Regt., W. African Frontier Force; attd. Gen. Staff. (W 2.) M.C. M 2. Officer, Military Order of Avis (Portugal)

Kennedy, F. R. Pte., Middlesex Regt.; Lieut., M.G.C. (W.) $M$.

Kennet, B. L. A. Lieut., Border Regt. and Gen. List, igro empld. Egyptian Govt. (W.)

KennetT, Rev. E. J. B. M. C.F. $4^{\text {th }}$ Class, R.A.C.D. 1909

KHAN, T. H. Interpreter, Indian Army

WKIDson, C. W. Lieut., R. Dublin Fus. (W.)

Killed in action 17 Oct. 1918 
KIDson, N. S. Capt., London Regt. (Rifles). (W.) M.C. 1913

WKIng, E. H. 2nd Lieut., R. Sussex Regt.

Killed in action fuly 1917

KingDon, G. H. Capt., Sherwood Foresters (Notts. and 1912 Derby Regt.) (W.)

Kirkland, W. N. Capt., London Regt. (Post Office 1912 Rifles); Lieut., R.A.S.C.

Knight, F. H. Lieut., The Queen's (R.W. Surrey Regt.) 1900

Laffan, Rev. R. G. D. C.F. $4^{\text {th }}$ Class, R.A.C.D. Order * 1912 of St Sava, $5^{\text {th }}$ Class (Serbia)

LAMBERT, H. E. Lieut., Gloucestershire Regt. and King's 1912 African Rifles. (W.)

WLAmbert, M. B. Lieut., Yorkshire Regt.

Killed in action in Gallipoli 7 Aug. 1915

1913

Lancaster, E. T. 2nd Lieut., St Paul's School O.T.C. 1906

LANGLEY-SMITH, N.H. Lieut., Gloucestershire Regt. (W.) I9 I I

Leigh-Clare, H. J. L. Lieut., I.A.R.O., attd. 34th rgro

Poona Horse and 6th Cavalry. M.C.

LEwIS, M. B. Lieut., Tank Corps

1910

Lhoyd-Owen, E. E. 2nd Lieut., Loyal N. Lancs. Regt. 1916

Lilley, T. G. Lieut., D. of Cornwall's L.I. I914

Locke, C. E. L. Capt., R. Welsh Fus.

LoEwe, H. M. J. 2nd Lieut., Lincolnshire Regt.; Lieut., 1901 Gen. List

WLomax, E. H. Capt., S. Lancs. Regt.

Killed in action 13 Aug. I9I7

LunN, H. F. Lieut., R.G.A.

Lyon, A. J. Hon. Colonel, Cambridgeshire Regt. and *1879 Spec. List (cmdg. School of Instruction)

Macdonald, R. Lieut., King's (Liverpool Regt., T.F.); I9I4 Lieut. (Ad.), R.A.F. (W.) M.B.E.

MACGREGOR, A. M. Lieut., R.G.A. (W.)

WMcKenzie, K. N. 2nd Lieut., E. Yorks. Regt.

Killed in action 4 Fune $19 \mathrm{r} 5$

McNeIll-Hewitt, L. Capt. and Adjt., R.A.S.C.

Malden, H. R. Lieut., Rifle Brigade; empld. Intelli- I903 gence Staff

Mansell, R. Lieut., R.F.A.; Major, Tank Corps. (W.) I9r2

MARLEY, F. L. Lieut., R. Warwickshire Regt.(T.F.) (W.) 1903

MAster, Rev. C. H. C.F. 4 th Class, R.A.C.D. M. ${ }^{*} \mathrm{r} 886$

Maxwell, E. W. Lieut., London Regt. (Civil Service 1915 Rifles). (W.) 
Maynard, Rev. A. C. M. C.F. $4^{\text {th }}$ Class, R.A.C.D. ${ }^{*} 1904$ (W.)

Molesworth, G. E. N. Capt., Devon Regt.; attd. 69th I9I3 Punjabis, Indian Army. (W.)

MOORE-ANDERSON, M. E. 2nd Lieut., R.A.S.C. 1899

MoyLE, T. Lieut., R. Fusiliers; attd. Gloucestershire [1914] Regt. (W.)

WNason, J. W. W. Capt., R. Sussex Regt. and R.F.C. 1908 Killed in action 26 Dec. 1916

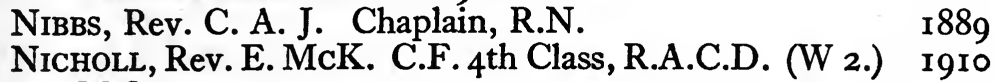
M.C.

Nicholl, J. W. McK. Surgeon Lieut., R.N.

1912

Nicholls, G. B. Capt., Middlesex Regt. (W.)

1913

Nightingale, C. L. Lieut. (S.), R.A.F.

1910

Noble, A. H. Capt., R.A.S.C. $M$.

NobLE, Rev. R. H. C.F. $4^{\text {th }}$ Class, R.A.C.D.

1905

NORDEN, F. L. 2nd Lieut., R. Scots; 2nd Lieut. (O.) R.A.F. (W 4.)

NoRTH, J. Capt., Worcestershire Regt.

WNosworthy, P. C. 2nd Lieut., Cheshire Regt. Killed in action II May 1915

NunN, V. W. H. 2nd Lieut., The Queen's (R.W. Surrey Regt.); attd. Middlesex Regt.

O'FlynN, D. R. C. D. Major, King's (Liverpool Regt.); 1899 attd. W. African Regt.; empld. O.C.B.

Oliphant, G. R. Capt., S. Lancs. Regt.; empld. War 1908 Office. (W.)

ORME, E. S. Surgeon Lieut., R.N.

I9I I

Page, Rev. C. A. C.F. $4^{\text {th }}$ Class, R.A.C.D.

1913
[1914]

1917

PAGE, Rev. F. A. C.F. $4^{\text {th }}$ Class, R.A.C.D.

1907

PaIN, K. W. Lieut., R.F.A.; Asst. Officer i/c R.A.M.C. I9IO Records. (W.) $M$.

PARKER,P.H. Lieut., King's(Liverpool Regt., T.F.) (W 2.) I9I I

PARKER, T. W. L. Lieut., King's (Liverpool Regt., T.F.); I9I I attd. Middlesex Regt.

Parnell, H. and Lieut., Tank Corps

Partridge, G. J. Capt., Sherwood Foresters (Notts. and

1914

Derby Regt.) and Gen. Staff

Partridge, Rev. J. W. C.F. th $^{\text {th }}$ Class, R.A.C.D.

Partridge, Rev. W. W. C.F. $4^{\text {th }}$ Class, R.A.C.D. 
Paterson, A. G. Hon. Major, R.A.M.C. $M . \quad$ I88I

PeAce, L. R. Lieut., R.F.A.(T.F.) 1908

Pearson, A. H. Capt., Loyal N. Lancs. Regt.; Major, 1904 M.G.C. M.C.

Pearson, C. H. M. Capt. and Adjt., R.F.A. M. 1906

Perry, G. A. Lieut., R.A.S.C.

Perry, Rev. H. C. C.F. 4th Class, R.A.C.D.

Perry, L. B. Capt., R.A.M.C.

Pettman, H. C.F. $4^{\text {th }}$ Class, R.A.C.D. I893

Phillp, J. DE R. Capt., R. Warwickshire Regt.(T.F.) 1907 (W 2.)

Pickard, W. B. Corpl., London Yeo. (Middlesex 1908 Hussars); attd. T.M.B. (W.) (P.)

WPinder, A. H. Lieut., Leicestershire Regt. M. 1906

Killed in action 15 Sept. I916

Poole, E. F. Lieut., Northamptonshire Regt. and Spec. [1914] List (R.T.O.)

Price, Rev. C. L., T.D. C.F. Ist Class, R.A.C.D.(T.F.) 1889 $m$.

APrichard, A. I. Pte., London Regt. (Civil Service Rifles) 1899 Killed in action at Vimy Ridge 23 May 1916

Proctor, S. H. A. R. See Rathbone-Proctor, S. H.

Pugh, C. D. O. Capt., R.G.A. $M$.

Pye, A. N. W. Sub-Lieut., R.N.V.R.

Rankin, J. K. 2nd Lieut., Rifle Brigade

Rathbone-Proctor, S. H. Capt., R.E. (W.)

Rayner, E. B. A. 2nd Lieut., The Buffs (E. Kent Regt.); I9I3

Capt., S.O. 3, R.A.F. (W.) $M$.

Redwood, Rev.F.A. C.F. 4th Class, R.A.C.D.; 2nd 1909 Lieut., King's (Shropshire L.I.)

Riddell, G.B. Capt., Northumberland Fus. (W2.) M.G. 1910

Robbins, C. Paymaster Sub-Lieut., R.N.V.R. 1909

सRobinson, J. H. 2nd Lieut., Somerset L.I.(T.F.); attd. 1907

N. Staffs. Regt.(T.F.)

Killed in action 30 Nov. I9I7

Roche, A. R Capt., R.A.M.C. (W.) M.C. M. 1894

Rodway, A. R. 2nd Lieut., E. Surrey Regt.; Capt., 1913

M.G.C. (W 2.)

Rogers, Rev. T. G. C.F. 4 th Class, R.A.C.D.

Rogers, W. D. Capt. and Adjt., S. Lancs. Regt.

1905

Rus

RUSHMER, H. F. Lieut., Norfolk Regt. (W 2.)

ARutherfurd, H. G. G. Pte., R. Fusiliers.

Killed in action near Pozières (1 5 Fuly 1916) 
Samson, Rev. A. M. C.F. 4th Class, R.A.C.D. ${ }^{*}$ Igi I

SANDBERG, Rev. W. B. C.F. 4th Class, R.A.C.D.(T.F.) I893

WSANDERSON, R. B. 2nd Lieut., R.G.A. I908

Died 17 April 1918 of wounds received in action

Savage, K. Sapper, R.E.

I9II

Savill, S. C. Capt. and Adjt., W. Yorks. Regt.(T.F.); I9I3 Hon. Capt. (A.), R.A.F. (W 2.)

※Saxon, H. Lieut., King's Own (R. Lancaster Regt.) M. I9r3 Killed in action 30 Nov. 1916

ScotT, J. T. 2nd Lieut., R. Berkshire Regt. 1909 Scotr, M. H. B. Lieut., R.N.V.R. I9IO Selby-Lowndes, Rev. G. N. C.F. $4^{\text {th }}$ Class, R.A.C.D. 1905 Shelton, A. T. Capt., King's Own (Yorkshire L.I.); [19I4] empld. Ministry of Munitions. (W.)

Simey, P. A. T. Rhodesian Volunteers

I9II

Simmons, C.E. Lieut., I.A.R.O., attd. S. and T. Corps. 1908 $M$.

\#'smms, W. Capt., R. Warwickshire Regt.(T.F.) I9ı

Killed in action 19 Fuly 1917

«Simpson, H. D. Lieut., King's Royal Rifle Corps. (W.) 1914 Killed in action 24 Aug. I9I7

Skelton, N. A. Lieut., The Queen's (R. W. Surrey 1908 Regt.) (W.)

Skene, Rev. A. P. C.F. $4^{\text {th }}$ Class, R.A.C.D.

WSkey, C. H. Lieut., R. Fusiliers; Capt., Black Watch.

(W.) M.C.

Killed in action 18 Aug. I916

Skey, C. O. Major, R. Fusiliers. (W.) D.S.O. M.G. M. I9I I

SleEman, C. M. Lieut., R.N.V.R.

*1912

Sleigh, G. B. Capt., King's Own (R. Lancaster Regt.) I908 (W.)

Sloman, A. E. P. 2nd Lieut., R.A.S.C.; Lieut., R.F.A. 1909

Smales, R. Lieut., Northumberland Fus.

Smellie, G. H. Capt., King's Own (R. Lancaster [r9I4]

Regt.); attd. Cheshire Regt.

Smith, Rev. C. M. C.F. $4^{\text {th Class, R.A.C.D.(T.F.) }} 1905$

Smith, F. W. Capt., Life Gds.

1912

«Smith, H. L. C. Lieut., Sherwood Foresters (Notts. and r910

Derby Regt.)

Killed in action 20 Oct. I9I4

Smith, H. P. Major, R.F.A.(T.F.)

SMITH, Rev. M CF th Class, R A C D (W) M 19

SMITH, Rev. M. C.F. 4th Class, R.A.C.D. (W.) M. 1898

Smith, S. Lieut., Middlesex Regt.; attd. T.M.B. (W 2.) 1908 $M$. 
«SMITH, W. W. 2nd Lieut., R.A.S.C. (W.)

Killed in action on the Somme 9 Fuly 1916

SNAPE, W. R. C. Lieut., Leicestershire Regt.; attd. 1909 T.M.B. (W.) M.C.

Somerset, R. H. E. H. Lieut., R.A.S.C.; Capt., Spec. 1904

List, empld. War Office

\#Sowell, A. D. Lieut., D. of Cornwall's L.I.

Killed in action 24 Aug. 1916

Spackman, E. D. Capt., R.A.M.C.

Spafford, H. H. Pte., Welsh Regt.; 2nd Lieut., R. Welsh 1916

Fus.

SPARLING, H. P. Lieut., R.E.

Sparling, W. H. Major, Cheshire Regt. (W.) M.C. I9ro

Spear, G. F. Lieut., Spec. List (Recruiting Staff) 1898

SPENCER-SMIth,P. Lieut., London Regt. (Queen's West- I90I minster Rifles). (W.) (P.)

SpowarT, W. C. Lieut., I.A.R.O., attd. S. and T. Corps. 1908 (W.)

Stearn, Rev. C. H. C.F. 4th Class, R.A.C.D.

Stebing, T. H. L. Capt., Sherwood Foresters (Notts. and Derby Regt., T.F.) (W.) M.C.

Stephens, Rev. E. C. Chaplain, R.N.

Stileman, C. E. C. Lieut., E. Surrey Regt.; Capt., Gen.

List, Indian Army, attd. 55th Coke's Rifles. (W.)

Stileman, D. C. G. Capt., King's Own (R. Lancaster 1908 Regt.) (W.) $M$.

Stileman, D. F. Lieut., R. Berkshire Regt. (W 2.) I9r3

Stubis, Rev. N. H. C.F. $4^{\text {th }}$ Class, R.A.C.D.

सStuckey, R. A. Pte., R. Welsh Fus.

Died 27 July 1917 of heat stroke while on active service

Swatridge, C. J. Lieut., Sherwood Foresters (Notts. and Derby Regt.) and M.G.C.; Hon. Lieut. (A.), R.A.F. (W.) $M$.

Symonds, E. T. Corpl., R.E. (Signals); Lieut., Bedfordshire Regt. (W.)

Taylor, A. A. Pte., Queen's Own (R. W. Kent Regt.) 1902

TAYLOR, R. Lieut., R.E.; empld. Ministry of Munitions. 1909 (W.)

Temperley, A. C. Major, Northumberland Fus.; Lieut.- $\quad 1896$ Col., Norfolk Regt.; G.S.O. I. C.M.G. D.S.O. Brevet Lieut.-Colonel. $M 8$.

TharP, P. A. Capt., Queen's Own (R.W. Kent Regt.) 1909 (W.) 
Thompson, G. Major, R.A.S.C.

सThompson, H. B. 2nd Lieut., R. Berkshire Regt. M.C. $\quad$ I909 Killed in action 24 April I9I7

Thompson, H. J. Lieut., R. Fusiliers

Thompson, M. Capt., R.F.A. (W 3.)

I910

Thompson, R. D. 2nd Lieut., Somerset L.I. (W.)

I910

Thompson, W. S. Lieut., I.A.R.O., attd. 2nd Gurkha

I9I3 Rifles

Thorman, Rev. F. P. Canadian A.M.C.

I910

Thursby, W. Capt., R.F.A.; A.D.C.

1906

Tindall, C. R. W. Lieut., R.A.S.C.

1909

Tinsley, R. P. Lieut., Middlesex Regt.; attd. T.M.B. I9I I (W.)

TownEND, H. D. Lieut., Bombay Bn., Indian Defence I9ıo Force

Treglown, C. J. H. Lieut., Norfolk Regt.; Capt., I9I I S. Wales Borderers. (W.) M.C.

Tulloch, H. M. 2nd Lieut., K. Edward's Horse; I9I I

Lieut., 33rd Light Cavalry, Indian Army; Lieut. (A.),

R.A.F.

Turnbult, Rev. P. H. C.F. 4th Class, R.A.C.D. $\quad 1908$

TURNER, Rev. A. Chaplain, R.N.

Turner, J. W. C. Capt., R.F.A. M.C. 1906

ETurner, R. Pte., London Regt. (Artists Rifles); 2nd 1904 Lieut., Essex Regt.(T.F.)

Killed in action at Suvla Bay 15 Aug. I9I 5

UPWard, L. V. Capt., R. Fusiliers and Gen. List. (W 2.) 1909

सVeItch, A. G. Lieut., R.F.A.(T.F.)

1907

Killed in action 23 April I9I7

Vertch, J. L. Major, R.H.A.(T.F.) (W.) Chevalier, I9I4 Ordre de la Couronne (Belgium). Belgian Croix de Guerre

WAGG, F. J. Lieut., Seaforth Hdrs.

WAINWRIGHT, L. A. Lieut., Spec. List (Inland Water- I909 ways and Docks)

WWALKER, A. N. Lieut.-Col., R.A.M.C.

Killed in action 24 Sept. I9I6

WWARNER, A. 2nd Lieut., London Regt. (R. Fus.)

Killed in action I Fuly r9I7

Watson, N. W. Lieut., E. Yorks. Regt.; Hon. Lieut. I9I I (T.), R.A.F. (W 3.)

C. U.W.L. 
WWatts, R. W. A. Pte., Sherwood Foresters (Notts. and

Derby Regt.); 2nd Lieut., Worcestershire Regt. M.C.

1912

Died 12 Nov. 1916 of wounds received in action 5 Nov. I9I6

WWells, L. H. E. 2nd Lieut., Lancs. Fus.

Died 4 May 1915 from the effects of gas poisoning at Ypres

Westall, B. C. Capt., Essex Regt.(T.F.)

Westcotr, G. F. 2nd Lieut., R.A.S.C.(T.F.); Lieut. 1912 (A.), R.A.F. (P.)

WWhEELER, H. L. 2nd Lieut., The Buffs (E. Kent Regt.); attd. Queen's Own (R.W. Kent Regt.)

Died 26 Dec. I9I 5 of wounds received in action

WheEler, Rev. H. W. C.F. 4 th Class, R.A.C.D.

WWhitfield, J. B. Lieut., R.E.

Died 20 Fan. I916 of wounds received in action

1904

Whitty, L. W. Capt., Lancs. Fus. (W.)

Whyte Venables, H. A. Capt., R.A.M.C.

Wilkinson, Rev. C. F. W. Chaplain, R.N.

1914

Wilkinson, Rev. G. A. W. C.F. 4th Class, R.A.C.D. 1906 (W.) M.C.

Wilkinson, Rev. J. R. C.F. $4^{\text {th }}$ Class, R.A.C.D.

WWilkinson, M. L. 2nd Lieut., Northumberland Fus.

Died 8 Fuly 1917 of wounds received in action

Williams, J. G. 2nd Lieut., King's (Liverpool Regt.) 1914

Williams, O. H. 2nd Lieut., Army Cyclist Corps I9I I

Williamson, F. A. Surgeon Lieut., R.N. 1908

Williamson, T. R. Lieut., R. Fusiliers. (W.) M.C. 1912

Wilson, Rev. B. C. C.F. 4th Class, Australian Chaplains' Igor

Dept. $M$.

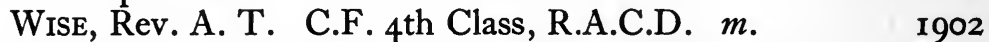

Wright, E. G. D. Pte., Rand Rifles, S. Africa Force 1903

YATES, H. G. Major, R.F.A.(T.F.) M.C. $\quad 1905$ 


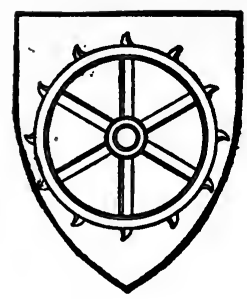

\section{ST CATHARINE'S COLLEGE}

Alexander, J. H. R. Lieut., Devon Regt.

AlleN, Rev. H. C.F. $4^{\text {th }}$ Class, R.A.C.D.

ANABlE, A. Lieut., Norfolk Regt. (W.)

ARMSTRONG, H. R. Major, R.E.(T.F.) T.D.

ArmstronG, J. L. Lieut., Yorkshire Regt. (W.)

Astley, B. A. F. 2nd Lieut., Devon Regt.; Lieut., 1892

Training Res. Bn. and Labour Corps

Atkins, E. M. Lieut., R.E. (W.)

Austin, Rev. S. Lieut., R.F.A.

BaKer, E. C. Capt., London Regt. (R. Fus.); Major (Ad.), I9I I R.A.F. (W.)

BAKER, Rev. P. C.F. 4th Class, Australian Chaplains' *Igog Dept.

WBarker, R. A. Capt., Sherwood Foresters (Notts. and 1914 Derby Regt.); empld. Recruiting Staff. (W.) M.C.

Died 13 Oct. 1918 of wounds received in action

BarTLETt, A. G. Capt., King's (Liverpool Regt., T.F.) I9I3 and Labour Corps

WBARTLETT, L. A. Lieut., Queen's Own (R.W. Kent Regt.) 1903 Killed in action 22 fuly 1916

BarWell, H. B. 2nd Lieut., W. Yorks. Regt.; Capt., 1912 Gen. List (Recruiting Staff). (W.)

Bates, H. S. Capt., Cambridgeshire Regt.; Lieut. I9I2 I.A.R.O., attd. Igth Punjabis. (W 2.)

BelCHER, G. Capt., R. Berkshire Regt. M.C. M. 1904 Killed in action $\mathrm{I} 5-\mathrm{I} 7$ May $\mathrm{I} 9 \mathrm{I} 5$

BENNETT, J. C.S. Sub-Lieut., R.N.V.R.(R.N.D.) (W.) [1914] M.C. 
Benstead, C. R. Lieut., R.G.A. M.C. $M$.

Berwick, E. B. H., T.D. Major, Rossall School O.T.C. m. *1892

BIDgood, Rev. G. J. B. C.F. $4^{\text {th }}$ Class, R.A.C.D.

BIRD, M. B. Lieut., Norfolk Regt.(T.F.)

ABIRD, P. C. H. 2nd Lieut., Norfolk Regt.; attd. Loyal I9I I

N. Lancs. Regt. (W.)

Killed in action in Mesopotamia 5 April 1916

Birmingham, Rt. Rev. Bishop of. C.F. Ist Class, I9Io R.A.C.D.(T.F.)

BlackbuRn, Rev. B. R. C.F. $4^{\text {th }}$ Class, R.A.C.D. 1907 M.B.E. $m 2$.

BluCke, R. S. Lieut., Dorset Regt.; Hon. Lieut. (O.), [I9I4] R.A.F.

Booth, Rev. H. F. C.F. 4th Class, R.A.C.D.

Boothman, J. H. E. D. 2nd Lieut., The Buffs (E. Kent I9I 5 Regt.); Pte., R. Fusiliers and M.G.C. (W.)

EBoultbeE, A. E. Lieut., Northamptonshire Regt. and I9I5

R.F.C.

Killed in action 17 March 1917

Bower, F. Sergt., King's Own (R. Lancaster Regt.); 2nd

Lieut., I.A.R.O., attd. 4th Rajputs. (W.)

Boyce, A. H. Sergt., E. Surrey Regt.; attd. Middlesex 1914 Regt.

Boyd, C. W. Capt., King's Own Scottish Borderers. (W.)

Boyd-Carpenter, Rt. Rev. Bishop, k.c.v.o. Hon. C.F., 1860 R.A.C.D.(T.F.)

Boyd-Carpenter, J. P. Capt., R. Scots Fus. (W.) 1892

BRACHI, C. C. Capt., King's Own (R. Lancaster Regt.); 1900 attd. Gen. Staff. $M$.

BragG, W. H. Capt., R.F.A.(T.F.); Capt. (Ad.), R.A.F. 1913 (W.)

Brameld, A. J. M. Capt., R. Warwickshire Regt.(T.F.) $\quad$ I903 and Labour Corps. (W.)

Brigley, C. G. Lieut., D. of Wellington's (W. Riding 1914 Regt., T.F.); attd. R.E. (W.)

Brown, C. L. M. Telegraphist, R.N.V.R.

Bruce, A. A. Capt., R.G.A. (W 2.) M.C. M.

1914

1913

EBuckley, E. Capt., York and Lancaster Regt. (W.) M. 1905

Killed in action at Lens 30 Sept. I917

Buls, J. C. Major, D. of Wellington's (W. Riding Regt.) 1900 (W.) M.C. M 3 .

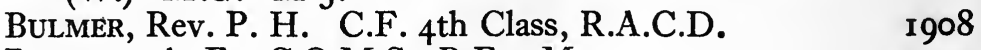

BuRdeTt, A. F. C.Q.M.S., R.E. $M$. 
${ }^{1}$ CaIN, R. C. Capt. (A.), R.A.F. (W.) D.F.C.

Calthorpe, Hon. F.S. G. See Gough Calthorpe, Hon.

I9ro F. S.

WCARTER, B. 2nd Lieut., Cambridgeshire Regt. Killed in action 18 Sept. 1918

CARTER, Rev. J. C.F. 4th Class, R.A.C.D. Order of St I9I I

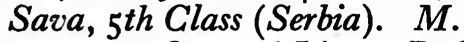

ACARTwright, C. 2nd Lieut., Bedfordshire Regt. Killed in action I9 April I916

1904

Cattley, R. Major, W. Yorks. Regt.(T.F.) and Training 1890 Res. Bn. (W 2.)

\#CAyley, F. D. E. 2nd Lieut., King's Royal Rifle Corps 1913 Killed in action 29 Sept. I9I 5

Chesterman, H. Capt., R.A.S.C.

Circuit, Rev. E. F. C.F. $4^{\text {th }}$ Class, R.A.C.D.

1902

1908

सClark, G. M. Major, Northamptonshire Regt. M. $\quad 1899$ Killed in action 14 Fuly 1916

Cllarke, M. T. Pte., Ioth Bn., Canadian Infy.

Killed in action (3 May r9I 5 )

Cockell, B. W. Capt., Suffolk Regt.(T.F.); A.D.C. I913 French Croix de Guerre

Collins, Rev. H. E. Chaplain, R.N.

Compton, F. 2nd Lieut., Cambridgeshire Regt. (P.)

WConan-Davies, B. I. Lieut., Worcestershire Regt.; attd.

I9I 5

York and Lancaster Regt. (W.)

Died 23 Nov. I9I 8 of pneumonia contracted on active service

Cooney, A. B. Capt., Denbighshire Yeo. and R. Welsh rgro Fus.

Cooper, Rev. F. T. C.F. $4^{\text {th }}$ Class, R.A.C.D.

सCoRbeTt, H. V. Capt., Cambridgeshire Regt.; attd. I9I2 Essex Regt. (W.)

Killed in action 17 Oct. 1918

Corfield, C. L. Capt., Cambridgeshire Regt.; empld. I9I2 O.C.B. (W 2.) M.C.

CrisP, Rev. H. Capt., D. of Wellington's (W. Riding 1909 Regt.); empld. Ministry of Pensions. (W.)

Culverwell, J. S. Capt., I.A.R.O., attd. 53 rd Sikhs. 1908 (W 2.) $M$.

Cumming, G.E. 2nd Lieut., Gordon Hdrs.; empld. I9I I Command Depôt

CurTis, S. W. Lieut., R.F.A.

1 Killed in flying accident after the armistice. 
Davenport, S. F. Lieut., R.G.A.

Davidson, M. C. 2nd Lieut., Oxford and Bucks. L.I.; [I9I4] Lieut., M.G.C. (W 3.)

Davies, A. L. Seaman, R.N.V.R.

Davies, D. Ll. E. Capt., W. Yorks. Regt.(T.F.) (W 2.) 1912

Davies, J. O. Corpl., Worcestershire Regt. 1917

Davies, K.C. J. Capt., R.A.S.C. and Ioth Gurkha Rifles, I9I2 Indian Army

Davis, J. W. F. McN. See McNaught-Davis, J. W. F.

Davison, G. Lieut., R.A.S.C. $M$.

DAY, Rev. G. P. J. C.F. $4^{\text {th }}$ Class, R.A.C.D.

DelPh, L. W. Lieut., R.E. (W.) M.C.

1912

1907

1910

Dempsey, G. B. Capt. and Adjt., Manchester Regt. I9I2 M.C. and Bar. $M 3$.

DIER, C. V. Cadet, O.C.B.

Dingad-Davies, F. Ll. Lieut., R.F.A.; Capt., R.A.O.C.

DodD, Rev. J. M. C.F. $4^{\text {th }}$ Class, R.A.C.D.

Dracup, A. H. 2nd Lieut., I.A.R.O.

WDriffield, H. G. 2nd Lieut., Queen's Own (R. W. Kent IgoI Regt.)

Died I Aug. 1917 of wounds received in action 22 April 1917

WDrifField, L. T. Capt., St John's School, Leatherhead, 1899 O.T.C.

Died 9 Oct. I917

Duke, A. C. H. Major, R.G.A. (R. of O.); Lieut.-Col., $\quad \mathbf{I} 898$ A.A. and Q.M.G. C.M.G. D.S.O. $M_{4}$.

EAster, Rev. A. J. T. 2nd Lieut., R.A.S.C.(T.F.); empld. Recruiting Staff

EAstwell, M. M. Major, Cambridgeshire Regt. and 1908 Spec. List (Cmdt., Reception Camp)

EdE, E. E. Sub-Lieut., R.N.V.R. (W.)

ELLIS, R. Major, R.A.M.C.(T.F.) $M$.

I9II

1903

Elliston, G. S. Capt., R.A.M.C. (Sanitary Service, *1896 T.F.) M.C. Order of St Fohn of Jerusalem

Elwood, A. G. F. Capt., Sherwood Foresters (Notts. 1908 and Derby Regt.) (P.)

Evans, A. O. Lieut., Sappers and Miners, Indian Army 1906

Evans, I. Lieut., R.G.A.

Evans, L. G. Lieut., R. Marines. (W.)

EvERETT, H. F. Major, R.A.M.C.(T.F.) $m 2$.

Everington, G. F. Instructor Lieut., R.N.
1912

1913

* 1896

1910 
FARR, H. F. Instructor Lieut., R.N. $M$.

FARROW, W. P. 2nd Lieut. (T.), R.A.F. (W 2.)

1904

I9I I

WFlaxman, W. J. and Lieut., R.A.S.C.

Died on active service in Mesopotamia 27 May 191 7

Francis, B. A. Capt., R.G.A. (W.)

*I909

I 897

Garmonsway, G. N. 2nd Lieut., R.G.A.

GiBB, Rev. J. C.F. $4^{\text {th }}$ Class, R.A.C.D.

I9I6

Gibson, H. H. Lieut., Rossall School O.T.C.

1905

GilberT, Rev. L. A. Chaplain, R.N.

I 897

$\begin{array}{ll}\text { GILBERT, Rev. L. A. Chaplain, R.N. } & \text { I903 } \\ \text { AGIll, K. C. Capt., Cambridgeshire Regt.; Capt. (A.), } & \text { I9I2 }\end{array}$

R.A.F. (W.) M.C. $M 2$.

Died 22 Oct. 1918 of wounds received in action

Gill, Rev. W. Chaplain, R.N.

Goldberg, S. V. See Goldhurst, S. V.

Goldhurst, S. V. Lieut., R.A.M.C.

Gough Calthorpe, Hon. F. S. Lieut., Staffordshire

Yeo; Capt., Spec. List (P. and B.T. Staff)

Graham-Brown, G. F. Capt. and Adjt., King's Own rgio

Scottish Borderers. (W.)

Grand, G. C. Cadet, O.C.B.

AGRAY, E. T. 2nd Lieut., Durham L.I.

Killed in action 22 Oct. I9I 5

Greenhough, H. 2nd Lieut., R. Fusiliers; Lieut., Gen. I9ro List, attd. R.E. (Signals)

*GrIFFITHS, C. R. 2nd Lieut., R. Fusiliers

Died I May I9I7 of wounds received in action

Grindon, Rev. A. W. H. C.F. 4th Class, R.A.C.D.

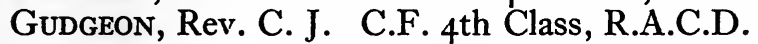

AGullick, A. L. Lieut., The Buffs (E. Kent Regt.)

Killed in action 3 Oct. I9I 5

Gullick, L. B. Lieut., R.A.S.C.

GuY, B. M. E. 2nd Lieut., S. Lancs. Regt. $M$.

*1907

1910

*I9I3

I9I7

I9I4

1910

I9I 5

* 1894

1905

1904

1906

I9I5

Hacking, Rev. H. C.F. $4^{\text {th }}$ Class, R.A.C.D.

Hamilton, J. W. Capt., Highland L.I.(T.F.)

HARE, S. G. Pte., R.A.S.C.(M.T.)

$\begin{array}{lr}\text { Haslewood, B. T. Major, R.F.A. } & \\ \text { Hayes, J. C. } & \text { 2nd Lieut., Middlesex Regt.; Capt., } \\ \text { [I9I4] }\end{array}$ M.G.C. and Spec. List

Headley, H. H. Capt., R. Fusiliers

* I906

HELDER, L. B. Lieut., R. Fusiliers; Lieut. (O.), R.A.F. [I9I4] (P.)

Hellins, Rev. E. W. J. C.F. $4^{\text {th }}$ Class, R.A.C.D.(T.F.) $\quad$ I 894 
Hepworth, L. D. 2nd Lieut., R.G.A. (W.) [1914]

Herd, J. G. M. Lieut., Trinity College, Glenalmond, 1896 O.T.C.

Hitchсоск, J. V. Capt., The Buffs (E. Kent Regt.) 1908

HodDER, H. G. Lieut., R.E. (W.) D.C.M. M. .

Hodgson, F. W. 2nd Lieut., Lancs. Fus. (T.F.); Lieut., I9I 5

R. Warwickshire Regt. (T.F.); empld. O.C.B.

Hoffmeister, C. E. See Hough, C. E.

Hollingworth, L. Pioneer, R.E. (Spec. Bde.)

Hollis, A. J. 2nd Lieut., Bedfordshire Regt.(T.F.)

1913

Hopkin, D. Capt., E. Yorks. Regt.; Major, R. Fusiliers. r9ro M.C.

Hosband, E. A. Lieut., S. Staffs. Regt. (W.)

Hough, C. E. Capt., S. Wales Borderers. (W 2.)

Howard, Rev. G. W. A. C.F. 4th Class, R.A.C.D.

Howard, Rev. P. C.F. 4th Class, R.A.C.D.

HowDEN, E. R. Lieut., R A.S.C.

Howe, R. G. Lieut., Sherwood Foresters (Notts. and Derby Regt.) and R. Dublin Fus. (W.) (P.) $m 2$.

Hudson, G. H. Capt., York and Lancaster Regt.; attd. T.M.B. (W.) $M$.

WHughes, Harold. 2nd Lieut., R.F.A.

Died 23 April 1917 of wounds received in action

Hughes, HorACE. Sergt., Welsh Regt.; 2nd Lieut., Norfolk Regt. (W.) M.C.

Hughes, J. B. W. Lce.-Corpl., London Regt.; 2nd Lieut., Gloucestershire Regt. (W.) (P.)

Humphreys, G. N. Hon. Capt. (A.), R.A.F. (P.) $M$. IgoI

Hunt, H. N. Lieut., D. of Wellington's (W. Riding r9r3 Regt.) and Manchester Regt. (T.F.); Capt., Spec. List (Asst. Officer i/c R.E. Records). $m$.

Hurst, E.S. Lieut., R.F.A.(T.F.); attd. T.M.B. (W 2.) 1913

JaY, C. D. Capt., R. Sussex Regt.; Major, M.G.C. *'I9r I D.S.O. $M 3$.

Jeeves, Rev. F. W. C.F. $4^{\text {th }}$ Class, R.A.C.D.

I9I 5

1905

I909

1894

1914

1912

1910

1913

I9I5

1914

Johnson, Rev. W. H. C.F. $4^{\text {th }}$ Class, R.A.C.D.

*I9I I

rgor

WJolley, J. A. B. Sergt., R. Fusiliers (P. S. Bn.); 2nd [1914] Lieut., Lincolnshire Regt.

Killed in action I I Oct. I9I 5

Jones, A. M. Lieut., St Paul's School O.T.C.

Jones, W. H. S. 2nd Lieut., Perse School O.T.C.

Kendall, Rev. W. A. C.F. $4^{\text {th }}$ Class, R.A.C.D. 
Key, Rev. S. W. C.F. $4^{\text {th }}$ Class, R.A.C.D.(T.F.)

KIlBuRN, D. and Lieut., Durham L.I. I9II

WKIRkus, C. H. Capt., R.G.A.

Killed in action $3 \mathrm{I}$ fuly 1917

KNAPP, K. K. Brig.-Gen., R.A. C.B. C.M.G. D.S.O. ${ }^{1885}$

Brevet Colonel. $M$ 7. Order of St Stanislas, 3rd Class

(Russia). American Distinguished Service Medal

KneEN, C. W. Lieut., R. Welsh Fus. and M.G.C.

KNight, J. E. H. Corpl., R.A.V.C.

KöBLICH, G. R. Lieut., Norfolk Regt.(T.F.); empld. [1914]

Ministry of Munitions

LASBREY, Rev. B. Chaplain, R.N.

WLEE, H. V. 2nd Lieut., Suffolk Regt.

Died 17 Nov. 1916 of wounds received in action

सLemmey, F. G. Lieut., R. Scots. (W.)

Killed in action $14 \mathscr{F}_{4}$ ly $19 \mathrm{I} 6$

LewIS, J. H. Corpl., R. Fusiliers

LEWIS, J. M. C. Lieut., Welsh Regt. (W.)

1899

Lloyd, R. T. Pte., Suffolk Regt. (W.)

1912

1907

LOEwE, H. M. J. 2nd Lieut., Lincolnshire Regt.; Lieut., *I9I I

Gen. List

Lowndes, A. G. 2nd Lieut., K. Edward's School, Can- I9ı terbury, O.T.C.

Luscombe, B. P. Lieut., R.F.A.; attd. T.M.B. (P.) 1912

LUTTER, R. B. Lieut., R.G.A. (W 2.)

1900

* I909

1893

$\mathbf{I} 908$

1913

1913

McArthur, R. Lieut., R.F.A.(T.F.) (P.) M.C.

McGowan, Rev. H. C.F. $4^{\text {th }}$ Class, R.A.C.D.

1916

McMichael, G. W. Pte., Worcestershire Regt.(T.F.); I9IO 2nd Corpl., R.E. (Field Survey Coy.)

McMurtrie, Rev. S. G. C.F. 4th Class, R.A.C.D. $\quad 1896$

WMcNaught-Davis, J. W. F. Lieut., S. Wales Borderers 1912 Killed in action 17 Fan. I9I 5

WManN, J. W. 2nd Lieut., The Buffs (E. Kent Regt., T.F.) 1907

Died 22 Aug. 1918 of wounds received in action

MaRCH, Rev. W. W. C.F. 4th Class, R.A.C.D. I9ro

Marsh, A. W. 2nd Lieut., Hampshire Regt.; Lieut., *'1914

Gen. List (attd. R.E., Signals)

WMayo, W. C. Lieut., Sherwood Foresters (Notts. and 1905

Derby Regt.)

Killed in action in Gallipoli 7-I I Aug. 1915

${ }^{1}$ Milner, Rev. D. R. Chaplain, R.N.

1 Accidentally killed at Archangel after the armistice. 
Mrtchell, W. G. Lieut., R.A.S.C.

सMOORE, G. 2nd Lieut., R. Sussex Regt.

1912

Killed in action in the First Battle of Ypres 7 Nov. 1914

Morris, W. F. Lieut., Norfolk Regt.; Major, Army

Cyclist Corps. M.C. M.

Mountford, Rev. A. W. C.F. $4^{\text {th }}$ Class, R.A.C.D.

Mousley, Rev. T. H. C.F. $4^{\text {th }}$ Class, R.A.C.D.

Mullins, A. F. Capt., Saskatchewan Regt., Canadian Force; Staff Capt. (W.)

Newell, A. 2nd Lieut., S. Staffs. Regt.

Nichols, F. P. Lieut.-Col., R.A.M.C.

Norby, Rev. R. H. C.F. $4^{\text {th }}$ Class, R.A.C.D.

I9I I

Norris, C. G. Pte., R. Fusiliers (P.S. Bn.)

* I910

* I 908

1909

O'Connor, Rev. J. C.F. $4^{\text {th }}$ Class, R.A.C.D.

1892

1875

1912

1910

Oliver, F. J. Capt., Gen. List (T.F. Res.); Instructor *I9I4 in Musketry

OtTy, H. R. Lieut., R.E. $M$.

1908

Palmer, E. de S. Capt., Norfolk Regt.

1907

Parish, W. O. Lieut., Norfolk Regt.; attd. R. Dublin I9I2 Fus.

Parker, C. 2nd Lieut., D. of Wellington's (W. Riding I9I3 Regt.) M.C.

Parker, H. N. Lieut., Oxford and Bucks. L.I.; attd. 1909 Hampshire Regt.(T.F.) (W.)

Parker Smith, R. Capt., Perse School O.T.C. 1900

PERKins, F. H. Lieut., R.A.S.C. (W.)

Phillips, C. K. Lieut., R. Irish Rifles; Major, Gen. List I 1899 (D.A.A.G.) O.B.E. M.

Phillips, I. A. Cadet, O.C.B.

Powell-Price, E. Capt., R. Welsh Fus.; A.D.C.; Staff Capt. (W.) M.C. M. Order of the Nile, $4^{\text {th }}$ Class (Egypt)

APReddy, E. F. S. Capt., R.G.A.

Killed in action $8 \mathrm{Dec}$. I9I7

APRITCHARD, R. 2nd Lieut., Devon Regt.; attd. Gloucester-

1916

1912 shire Regt. (W.)

Died 22 Aug. 1916 of wounds received in action

APudumjee, N. S. Pte., R. Fusiliers (P. S. Bn.)

I9I I

Drowned on H.M.S. Persia 30 Dec. I9I 5

Pulliblank, Rev. J. C.F. 4th Class, R.A.C.D. $^{\text {I } 894}$

Purvis, J. S. Lieut., Yorkshire Regt.(T.F.) (W.) $m . \quad 1909$ 
RAMSEY, H. L. 2nd Lieut., R.A.S.C.

Ranking, G. S. A. Lieut.-Col., R.A.M.C. (3rd S. Gen. 1869 Hospital, T.F.) C.M.G. $m 2$.

WRAwES, J. H. R. Lieut., Bedfordshire Regt.

Killed in action I fuly ror6

Rayner, C. G. Cadet, R.F.C.; Lce.-Corpl., London I9I7 Regt. (St Pancras Bn.)

ARigBY, G. Lieut., York and Lancaster Regt. M.C.

Killed in action 7 Fune 1916

RILEY, W. N. Capt., Leicestershire Regt.(T.F.) (W.) I9 I I

Rivers-Smith, S. Capt., King's African Rifles. $M . \quad 1898$

Robinson, Rev. B. C. C.F. $4^{\text {th }}$ Class, R.A.C.D. 1908

Robinson, L. R. C. 2nd Lieut., W. Yorks. Regt.; Lieut., 1909 I.A.R.O. (Cavalry.) (W.)

Robinson, Rev. L. S. C.F. $4^{\text {th }}$ Class, R.A.C.D. I883

Rogers, A. D. S. Major, Northumberland Fus. (W.) 1902

Rose, Rev. C. P. G. Chaplain, R.N.

सRoss, W. S. Pte., R. Fusiliers (P.S. Bn.); Lieut., Border I9 I I Regt. (W.)

Killed in action 23 fuly 1917

Rowley, Rev. F. B: Chaplain, R.N.

Rushmore, F. M., T.D. Lieut.-Col., Cambridge Univ. 1895 O.T.C.

WRyan, A. C. T. K. Lieut., Middlesex Regt. (W.)

Killed in action 24 Oct. 1918

Ryan, D. P. K. Lieut., King's Royal Rifle Corps

1912

I9I3

ScotT, A. D. B. Capt., R. Irish Rifles and Gen. Iist, 1898 empld. Ministry of National Service

सScotT, W. F. Pte., London Regt. (Q.V.R.); 2nd Lieut., 1909 Somerset L.I.

Killed in action I fuly r916

Sewell, F. A. S. Capt., Bedford Grammar School O.T.C. $\quad 1899$

Shaw, G. E. Flt. Cadet, R.A.F.

ShePPEY-Greene, Rev. R. F. Lieut., R.A.S.C.

Skrimshire, C. V. S. Major, R.G.A.

Slater,A. Lieut., I.A.R.O., attd. Io6th Hazara Pioneers. 1906 $M$.

WSmart, W. E. Sergt., King's Royal Rifle Corps; 2nd I913 Lieut., Yorkshire Regt.; attd. W. Yorks. Regt.

Killed in action near Cambrai I I Oct. I91 8

«Smith, D. G. Lieut., King's(Shropshire L.I.) (W 2.) I9I4 M.C.

Died 16 Aug. I9I7 of wounds received in action 
Smith, R. C. S. See Standring-Smith, R. C.

Soole, Rev. W. B. Gnr., R.G.A.

\#Southwell, F. E. G. Lieut., E. Yorks. Regt.(T.F.)

Died ro April r917 of wounds received in action

SQUiRES,A.P.H. Capt., Lincolnshire Regt.; attd.T.M.B. [1914] (W.) M.C.

StandRING-SMith, R. C. Major, R.A.M.C.(T.F.)

Starling, G. Flt. Cadet, R.A.F.

Stearn, G. F. 2nd Lieut., R. Fusiliers.

STEEL, D. M. Lieut., R.G.A. (W.)

I913

SteERS, J. A. 2nd Lieut., Labour Corps

I9I 5

Stevenson, W. F. J. F. 2nd Lieut., Seaforth Hdrs. 1916 1913

※Stokes, R. J. Pte., R. Fusiliers (P.S. Bn.); 2nd Lieut., 1907

King's Royal Rifle Corps. (W.)

Killed in action 20 Aug. 1916

Stuart, J. A. G. Lieut., R.G.A. (W.) (P. Escaped I9I4 from Germany)

Swann-Mason, Rev. R. S. Chaplain, R.N. O.B.E. * *1895 WSwIFT, W. 2nd Lieut., Lincolnshire Regt.

Killed in action $\mathrm{I}-3$ fuly 1916

WTALl, J. J. 2nd Lieut., Devonshire Regt.

Killed in action 17 Feb. 1918

Tavener, F. E. 2nd Lieut., R.G.A.

TAYlOR, Rev. B. W. C.F. 4th Class, R.A.C.D.

TAYlor, C. C. Lieut., London Regt. (R. Fus.) (W.)

WTheobald, R. Lieut., Suffolk Regt. (W.) M.C.

Killed in action ro April r91 8

Thomas, A. H. Major, Lancs. Fus. $M 2$.

Thomas, E. A. Capt., R.G.A. (T.F.); Hon. Capt. (A.), I9I I R.A.F. (W.)

Thomas, J. O. 2nd Lieut., D. of Wellington's (W. Rid- I9r4 ing Regt.) (W.)

Thomas, W. E. Leading Telegraphist, R.N.V.R.

Thompson, W. B. Seaman, R.N.V.R.

TRIST,L.H. Capt., Lincolnshire Regt.; Major, E. Lancs.

Regt.; Lieut.-Col., Welsh Regt. (W 2.) D.S.O. M.C. $M 2$.

Tuck, C. H. Lieut., Lancaster Grammar School I9II O.T.C.

Turner, E. M. Sergt., Gloucestershire Regt.(T.F.) 1908 M.M.

Turner, G. McD. Capt., W. Yorks. Regt.(T.F.); Capt. 1909 (A.), R.A.F.; S.O.3. 
WUnwin, E. F. Major (A.), R.F.C. (W.) $M$.

1900 Died 22 March I916 of injuries received in flying accident 31 Fan. 1916

Wakefield, Rev. A. H. C.F. 4th Class, R.A.C.D.

WalbaNk, C. F. Writer, R.N.R.

I912

1916

WWARD-PrICE, L. S. Corpl., K. Edward's Horse; Lieut., 1912 Life Gds.; Capt. (A.), R.F.C. (W.) $M$.

Killed in action 25 March 1917

$\begin{array}{ll}\text { Waters, Rev. G. M. Chaplain, R.N. } & \text { 1889 } \\ \text { Watson, R. E. 2nd Lieut., Seaforth Hdrs.; attd. R. } & \text { I9I6 }\end{array}$ Scots Fus. (W.)

Watson, Rev. W. N. C.F. 4th Class, R.A.C.D. I89I WWatTs, H. L. Capt., Essex Regt.

Killed in action 20 Oct. I91 5

Webster, H. Capt., Norfolk Regt.

WWhITE, F. R. 2nd Lieut., R.E.; attd. R.F.C.

Died 23 fan. I9I7 of wounds received in action

White, G. E. Lieut., R.G.A. (W.)

Whitham, A. and Lieut., The Queen's (R. W. Surrey Regt.) and Labour Corps

WilKinson, E. Lieut., R.F.A. (W.)

1903

1912

I9I I

1907

1910

1912

WWilliams, H. G. 2nd Lieut., King's Royal Rifle Corps Died 5 Fan. I917 of injuries received on active service

Williams, W. S. 2nd Lieut., Welsh Regt. (W.)

Wolfe, B. T. 2nd Lieut., Cheshire Regt.

I9II

Wood, A. J. and Lieut., Monmouthshire Regt.; Lieut., S. Lancs. Regt.

Woon, E. R. Capt., Cambridgeshire Regt. (W 4.) M.C. 1912 and $\mathrm{Bar}$

Woon, L. S. Capt., R.A.S.C.; Major, D.A.D. S. and T. $\quad$ I897 M.B.E. M.

Woodcock, G. 2nd Lieut., S. Lancs. Regt.; Lieut., 1912 King's Own (R. Lancaster Regt.); empld. Intelligence and Education Staff

WWormald, O. E. Capt., Suffolk Regt.(T.F.)

Died $2 \mathrm{Feb}$. 1917 of appendicitis

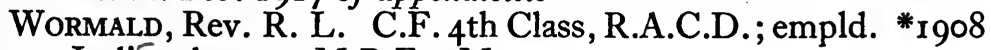
Indian Army. M.B.E. M. m.

Wormell, R. L. See Wormald, Rev. R. L.

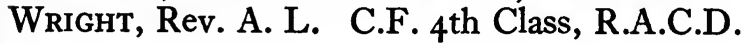




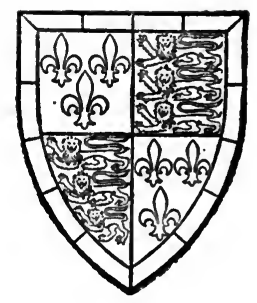

\section{ST JOHN'S COLLEGE}

Acton, H. Lieut., S. Staffs. Regt. (W.)

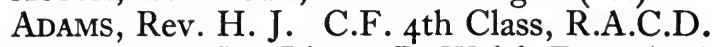

1909 1876 WAdams, J. B. P. Lieut., R. Welsh Fus. (W.) Died $27 \mathrm{Feb}$. $19 \mathrm{I} 7$ of wounds received in action

1909

HAdamson, F. D. Lieut., Border Regt.

Killed in action $\mathrm{I} 6$ Nov. I9I 5

AdLeR, H. M. Capt. and Adjt., R.A.S.C. M.B.E. m. $\quad 1894$ *Ainley, K. E. D. 2nd Lieut., R.E.(T.F.)

Died 9 Fune I9I 5 of wounds received in action in Gallipoli I I May I9I 5

WAlexander, Rev. P. G. Chaplain, R.N.

Drowned on H.M.S. Hampshire 5 Fune 1916

Alexander, Rev. R. C. Chaplain, R.N.

1913

ALLDRD, R. A Lieut. Loyal N. Lancs. Regt.; R.T. 1905 (W.)

Allen, F. Lieut., I.A.R.O., attd. 28th Punjabis. (W.) 1912

WAllen, G. A. Pte., London Regt. (Artists Rifles); 2nd I905 Lieut., Essex Regt.

Killed in action on the Somme I fuly I916

Allott, P. B. Capt., Northamptonshire Regt.

ANDERson, L. R. D Capt and Adjt. R F A. I899

ANDERSON, L. R. D. Capt. and Adjt., R.F.A. M.C. 1905

ANDREws, J. A. Capt., R.A.M.C.(T.F.) (W.) 1895

Andrews, J. C. Capt. and Adjt., London Regt.(Q.V.R.); 1909

Staff Capt. M.C. M2.

ANThony, A. L. Capt., R.A.M.C. (W.) 1908

ANTROBUS, H. Lieut., R.E.

ApPleton, E. V. Capt., R.E. I9II

ARCHER-HIND, L. 2nd Lieut., Lincolnshire Regt. $\quad$ I9I5

ARmitage, B. W. F. Lieut., R.A.M.C. I909 
Arnold, J. C. Capt., Northumberland Fus.; empld. 1900 Ministry of Pensions. (W.)

ARNOT, E. W. Major, R.F.A.(T.F.) O.B.E. M. 1902 AshbURner, W. Capt., Gloucestershire Regt.(T.F.); 1885 Major, Labour Corps

Ashby, Rev. N. Lce.-Corpl., R.A.M.C.

Askey, Rev. A. H. C.F. 4th Class, R.A.C.D.

Askey, S. G. Capt., R.A.M.C.(T.F.)

Atkinson, G. Pioneer, R.E. (Spec. Bde.) $M$.

1902

* 1879

1907

I9II

AAtkinson, H. N. and Lieut., Cheshire Regt. D.S.O. 1908

Missing, presumed killed in action, 22 Oct. 1914

AttleE, W. H. W. Major, R.A.M.C. $M$.

Aubry, C. P. Lieut., R.G.A.

Averill, T. H. Lieut., N. Staffs. Regt. (W.)

1894

1908

1909

WBADCOCK, A. L. 2nd Lieut., Northamptonshire Regt. I9I3 and King's Own (Yorkshire L.I.)

Killed in action $\mathrm{I} 3$ Oct. 1915

BaILY, G. G. Capt., Sherwood Foresters (Notts. and 1892

Derby Regt., T.F.) and Labour Corps

Baker, M. W. Capt., R.A.M.C.

Bannerman, Rev. W. E. C.F. $4^{\text {th }}$ Class, R.A.C.D.

Barbour, G. B. Pte., H.A.C.; 2nd Lieut., R.F.A.

BARKER, P. T. 2nd Lieut., R.F.A.

Barlow, P. S. Capt., R. Sussex Regt. (T.F.); Capt. ${ }_{1902}$ and Adjt., R.E.

Barnes, G. G. Major, London Regt. (Post Office Rifles). 1905 (W 2.) $m$.

ZBARneTt, B. L. T. Capt., R.A.S.C.

Died I8 April r91 5

BARRETT, H. S. Capt., King's (Liverpool Regt., T.F.) 1906 O.B.E. M 2.

BARRETt GreEne, A. H. Lieut., N. Staffs. Regt.(T.F.) I9I3 and Training Res. Bn.

BarritT, W. V. Capt., R.A.M.C.(T.F.)

ZBARTLETT,W.H. Lieut., Fort Garry Horse, Canadian Force 1906 Killed in action 14 Sept. I916

Barton, F. S. Lieut. (T.), R.A.F.

BEALE, C. E Capt., R. Berkshire Regt,; attd Devon I9I4 Regt. (W.) $m$.

Beard, A. J. Capt., Essex Regt.; empld. O.C.B. (W 2.) I9I2 M.C.

EBEARD, E. C. Lieut., Essex Regt.(T.F.) Killed in action at Gaza 26 March 1917 
\#Beaumont-Checkland, M. B. Lieut., W. Somerset Yeo.; 1902 attd. Somerset L.I.

Killed in action at Langemarck 17 Aug. 1917

BECKLEY, V. A. Lieut., R.G.A. M.C.

ABeechey, C. R. Pte., R. Fusiliers

Killed in action in E. Africa 18 Oct. 1917

Beith, I. H. Capt., Argyll and Sutherland Hdrs.; Hon. 1895

Major, M.G.C.; Major, Spec. List, empld. British

Military Mission. C.B.E. M.C. M.

Bell, T. O. Capt., Essex Regt.

Bellman, Rev. A. F. C.F. $4^{\text {th }}$ Class, R.A.C.D.

BennetT, C. W. Capt., R.A.S.C.

Bennion, J. M. Lieut., R.A.M.C.

Benoy, Rev. J. C.F. Ist Class, R.A.C.D.; A.C.G.

1914

I897

Benoy, J. F. Lieut., S. Staffs. Regt.; Capt., Spec. List

(Bde. Bombing Officer). (W 2.) $M$.

ABenson, G. E. Rfn., Rifle Brigade

Killed in action near Fromelles 9 May 1915

Benstead, A. S. Lieut., Lincolnshire Regt.; Major, Gen.

List (Cmdt., Reception Camp)

Bentley, A. J. Capt., Border Regt. (W.) M.C. and Bar

Bentley, Rev. J. H. Chaplain, R.A.F.

BERESFORD, G. A. Lieut., R.F.A.

ABERNARD, H. C. 2nd Lieut., Gloucestershire Regt.; attd.

Worcestershire Regt. (W.)

Killed in action 3 Sept. I9I6

Bevan, E. J. 2nd Lieut., King's Own (Yorkshire L.I.) I9I4

Bevan, G. T. M. Major, R.E. $M$.

1905

1909

1898

1895

1882

1913

1913

1914

1907

1903

1907

1912

Bevan, Ven. H. E. J. C.F. $3^{\text {rd Class, R.A.C.D.(T.F.) }}$

1909

1873

I9I I

ABILlinger, H. F. 2nd Lieut., E. Lancs. Regt.

Killed in action 23 Nov. 1916

Billinghurst, W. B. Capt., Gen. List (T.F. Res.) $m . \quad 1872$

Bilsland, A. S. Capt., Cameronians (Scottish Rifles, I9I0 T.F.); Staff Capt. M.C.

Bindloss, A. H. Major, R.A.M.C.

Binns, A. L. Capt., Lincolnshire Regt.; empld. R.E. I9I I (W.) M.C.

BisdeE, J. S. M. Trooper, K. Edward's Horse; Lieut., I913 R.F.A.

BLACK, S. G. Air Mechanic, R.N.A.S.

I910

Bladwell, E. W. Corpl., R.E.

I9I3

WBlakeley, F. R. 2nd Lieut., Somerset L.I.; attd. Indian I914 Army

Killed in action $22 \mathrm{Feb}$. 1917 
Blaxter, A. P. LL. Lieut., Northamptonshire Regt.

Blumhardt, E. H. F. See Mills, E. H. F.

\#Boddington, Rev. V. C. C.F. 4th Class, R.A.C.D.(T.F.) 1905

Died I 3 March ${ }_{1} 917$ of tuberculosis contracted on active service

Bond, B. W. Capt., Connaught Rangers. (W.) M.C. I913 ABonser, G. A. G. Capt., R.A.M.C.(T.F.) (W.)

Killed in action near Armentières 29 Sept. 1918

BonsEY, Rev. W. H. C.F. 4th Class, R.A.C.D.(T.F.) $\quad 1892$

Bоoтн, E. Lieut., Middlesex Regt. and M.G.C. I9I3

Bowdon, Rev. W. S. C.F. $4^{\text {th }}$ Class, R.A.C.D.

$1899^{\circ}$

WBowen, L. H. Corpl., London Regt. (Q.V.R.); Lieut., 1907

Lincolnshire Regt.

Killed in action 22 Dec. 1915

BracketT, A. W. K. Lieut., Queen's Own (R. W. Kent I9I3 Regt.) (W.)

BRASH, E. J. Y. Major, R.A.M.C.(T.F.) Order of St 1907 Sava, $4^{\text {th Class (Serbia) }}$

BraunholTZ, H. J. Lce.-Corpl., R.A.M.C. 1908

BRIAN, F. R. H. Lieut., R.G.A.

BRICE-Smith, H. F. Capt., R.A.M.C. (W 2.) M.C. 1908 $M$.

ABRICE-Smith, J. K. 2nd Lieut., Lincolnshire Regt.

Died I I Sept. I9I 5 of wounds received in action in the

Ypres Salient 6 Sept. 1915

BRIGGS, G. E. Sergt.-Instructor, R.E. (Signals)

Briggs, Rev. W. A. Chaplain, R.N.

\#BRock, E. G. Capt., King's (Liverpool Regt., T.F.) M.C. Killed in action 31 Fuly r917

Brooke, Z. N. Capt., E. Surrey Regt. and Gen. List 1902 (Intelligence)

WBrown, C. W. Capt., R. Scots Fus. (W 2.)

Killed in action I May 1916

सBRown, E. M. Lieut., Tank Corps. (W.)

[1914]

Killed in action 29 Sept. 1917

BRown, S. R. Lieut., R.F.A.(T.F.) M.C. $\quad 1899$

Brown, W.L. Capt., R.A.M.C. (Ist London Gen. Hos- 1889 pital, T.F.)

BrowNE, B. 2nd Lieut., R.A.S.C.

WBRowning, Rev. G. A. Chaplain, R.N.

Killed in action in the Battle of Futland 31 May 1916

$\begin{array}{ll}\text { BRownING, H. A. Surgeon Lieut.-Cdr., R.N. } & \text { I898 } \\ \text { BRowning, K. C. Lieut., R.E.; empld.Ministry of Muni- } & \mathbf{1 8 9 4}\end{array}$ tions

1910

1896

1898

1894

C. U.W.L. 
WBrownson, R. D. D. D. Capt., R.A.M.C. (W.)

Died at Peshawar $2 \mathrm{I}$ Oct. I918

$\begin{array}{lr}\text { BuchanaN, G. B. Major, R.A.M.C.(T.F.) } & \text { I887 } \\ \text { Buckley, W. H. 2nd Lieut., 6th (Inniskilling) Dragoons. [19I4] }\end{array}$

1902 (W.)

Bullen, F.J. Lieut., R.G.A.; empld. Ministry of Muni- I9I I tions

Bunt, A. P. Capt. and Adjt., D. of Cornwall's L.I.

Burdon, R., v.D. Hon. Colonel, Durham L.I.(T.F.) 1849 C.B.E.

- Burling, E. J. P. Capt. (A.), R.A.F. D.S.C. D.F.C. 1912 $M$. French Croix de Guerre

ABurr, F. G. Capt., R. Scots Fus.

Killed in action in the Battle of Loos 26 Sept. 191 5

Burrell, J. H. Capt. and Adjt., Durham L.I. (P.) $M$.

Burton, Rev. H. P. W. C.F. $4^{\text {th }}$ Class, R.A.C.D.

Burton-Fanning, F. W. Major, R.A.M.C.(T.F. Res.)

Butler, A. G. Colonel, Australian A.M.C. D.S.O. $M 2$.

Button. A. E. Air Mechanic, R.A.F.

1908

Cadle, H. S. Lieut., E. Surrey Regt.(T.F.); Capt., 1882 Labour Corps.; empld. Ministry of Labour. $M$.

*Callender, R. H. 2nd Lieut., Durham L.I.

Accidentally killed near Armentières 5 Oct. 1915

Calvert, E. Capt., I.M.S.

I9I3

Campbell, Rev. A.J. C.F.3rd Class, R.A.C.D.(T.F.) $m$.

Campbell, C. G. H. Capt., R.A.M.C.

Cardwell, A. G. Lieut. (T.), R.A.F.

Carlill, H. B. Surgeon Lieut., R.N. $M$.

Carnegy, Rev. F. W. C.F. 4 th Class, R.A.C.D.

CARTER, W. H. Lieut., I.A.R.O., attd. 6th Gurkha Rifles and $4^{\text {th }}$ Assam Rifles; Capt., Spec. List. $m$.

ACassels, W. G. Capt., Border Regt. $M$.

Killed in action near Bouzencourt 13 fuly 1916

Casson, R. Capt., I.A.R.O.

ACASTle, C. W. Lieut., S. Lancs. Regt.

Killed in action 3 Aug. r9r7

Chadwick, B. Ll. 2nd Lieut., R.G.A.; empld. Ministry of Labour

Chadwick, M. Capt., R.A.M.C.

1907

1894

1905

1908

1900

1889

1908

1912

1897

1914

1910

1908

*Chapman, A. R. B Lieut., Loyal N. Lancs. Regt.(T.F.) ' I9I3 (W 2.)

Killed in action 6 fune 1916 
Chapple, A. Lieut. (T.), R.A.F.

1895

Chapple, H. Capt., R.A.M.C.

1901

Chasteney, H. E. 2nd Lieut., R.G.A. (W.)

1907

Checkland, M. Beaumont. See Beaumont-Checkland, M. B.

ACheese, Rev. W. G. C.F. 4th Class, R.A.C.D.

Died 7 Nov. I918 of pneumonia contracted on active service

Cheeseman, A. L. Capt., 9th Infy. Bn., S. African Force 1896 Cheetham, E. M. Corpl., R.E.

CHELl, H. Lieut., R. Fusiliers

Died ro Aug. I915 of wounds received in action 9 Aug. I9I 5

Cheshire, F. M. Lieut., and Nagpur Rifles, Indian De- 1906 fence Force

※Chidson, L. D. Capt., King's Royal Rifle Corps. M.C. [1914] $M$.

Killed in action 24 April I9I7

Churchward, Rev. A. C. Pte., R.A.S.C.

1912

1908

Churchward, Rev. M. W. C.F. Ist Class, R.A.C.D.; * 1879 A.C.G. C.B.E. $m$.

AClaRK, H. R. E. 2nd Lieut., London Regt. (Civil Ser- I9Io vice Rifles)

Died 3 Fune 1915 of wounds received in action 25 May I9I 5

AClarke, D. Pte., H.A.C.; 2nd Lieut.(O.), R.F.C. Killed in action 26 Aug. I9r6

Clarke, J. H. Capt., D. of Cornwall's L.I. (W.) I9I2

Clarke, J. S. Lieut.-Col., Wiltshire Regt.; G.S.O. 2, 1882 War Office. Brevet Lieut.-Colonel. $m$.

ZClARKE, R. S. Capt., King's (Shropshire L.I.) 1909

Killed in action near Hooge 25 Sept. I9I 5

Clay, W. K. 2nd Lieut., R.A.S.C.

Cleland, J. R. Capt., R.F.A.

Clements, T. H. 2nd Lieut., R.A.S.C.(M.T.)

Clough, T. Capt., I.A.R.O., attd. 2 Ist Cavalry

CoAD, C. N. Capt., R.A.M.C. (W.) M.C. and Bar. M. 1902

ACobbold, R. H. W. Lieut., Rifle Brigade

Killed in action 9 Sept. I9I 5

Coleman, Rev. N. D. C.F. $4^{\text {th }}$ Class, R.A.C.D.

1912

Constable, W. G. Major, Sherwood Foresters (Notts. 1906 and Derby Regt.) and Lancs. Fus. $M$.

Coomвs, A. G. Major, R.G.A. D.S.O. M. Belgian 1903

Croix de Guerre 
※Coop, W. Pte., King's (Liverpool Regt., Liverpool 1902 Scottish, T.F.)

Died 24 Fune I9I 5 of wounds received in action in the Ypres Salient 16 fune I9I 5

Cooper, H. Capt., Unattd. List T.F. (O.T.C.) I908

COOPER, M. C. Lieut., Oxford and Bucks. L.I.; Major, 1898 M.G.C. M.C. and Bar. M 2.

CorT, J. L. P. Lieut., R.A.S.C.; Hon. Lieut. (Ad.), 1903 R.A.F.

*Cotton, Rev. R. H. A. 2nd Lieut., R.A.S.C.

Died at Taranto r.2 Oct. I91 8 of pneumonia

Cox, H. B. Lieut.-Col., R.G.A.(T.F.) O.B.E.

Crauford, L. G. Sapper, R.E. (London Electrical Engineers, T.F.)

CRICK, L. G. M. Lieut., Cheshire Regt.(T.F.)

ACroggon, J. F. S. Capt., Sherwood Foresters (Notts. and

Derby Regt.) (W.)

Died 18 Nov. I918 of influenza contracted on active service

Crole-Rees, Rev. H. S. Chaplain, R.N.

Crowther, C. R. Capt., R.A.M.C.(T.F.) (P.)

ACruickshank, D. E. Pte., R. Fusiliers (P. S. Bn.); and

Lieut., Border Regt.; attd. Wiltshire Regt.

Killed in action in Mesopotamia 9 April 1916

Cuff, A. W. Major, R.A.M.C.(T.F. Res.) $M$.

1908

1901

1904

1910

1899

Cullis, L. Lieut., I.A.R.O., attd. 23rd Sikh Pioneers

Cummings, R. R. Instructor Cdr., R.N.

Cummins, F. J. Capt., Dorset Regt. (W.)

Curzon-Siggers, W. A. Air Mechanic, R.F.C.

Cushing, W. E. W. 2nd Lieut., Norfolk Regt.; Lieut. (Ad.), R.A.F.

Dale, F. Pte., H.A.C.; 2nd Lieut., King's Own Scottish Borderers

Darlington, W. A. C. Capt. and Adjt., Northumber- 1909 land Fus.(T.F.); empld. War Office. (W.)

WDavenport, A. Lieut., Rifle Brigade; attd. Tank Corps. 1915 (W.)

Killed in action near Boyelles 23 Aug. 1918

Davies, E. Capt., Sherwood Rangers; empld. Egyptian 1908 Govt. (W.)

Davies, R. M. Lieut., R.F.A. (W 2.)

Davis, H. Lieut., Somerset L.I.(T.F.); attd. 3 rd Gurkha Rifles, Indian Army 
Davis, H. J. Sergt., R. Welsh Fus

Davy, C. L. Capt., M.G.C.; attd. Tank Corps. (W 3.) 1914 M.C.

Dawson, A. M. Capt., Hampshire Regt.(T.F.) (W.) 1905 M.C.

Dawson, R. T. Lieut., Edinburgh Academy O.T.C.

WDAy, D. I. 2nd Lieut., R.F.A.

Died 7 Oct. I9I5 of wounds received in action at

Vermelles 25 Sept. 1915

DAY, G. L. Major, Huntingdonshire Cyclist Bn.; attd. r9ro Gloucestershire Regt. $M$.

DenhaM, H. A. Major, R.G.A.(T.F.) (W.) D.S.O. M. ${ }_{1898}$

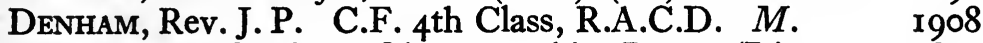

Desmond, G. G. Pte., Gloucestershire Regt. (P.) I890

Digges La Touche, H. N. See La Touche, H. N. D:

Dixon, C. Capt., R. Scots.; empld. O.C.B. (W.) 1906

DodD, Rev. R.P. C.F. 3rd Class, R.A.C.D. (W.) 1905 M.C. $M$.

DodD, W. P. Lieut., R. Welsh Fus. (W.) M.C. 1908

Donovan, E. L. 2nd Lieut., E. Yorks. Regt. (Cyclist 1907 Bn., T.F.); Lieut., King's African Rifles

Douglas, J. 2nd Lieut., R.E.; Lieut. (A.), R.A.F. (W.) I913

Douglas, S. M. 2nd Lieut., R. Fusiliers. (W.) I 898

DRUMmond, J. B. Sapper, R.E. I9I4

Drysdale, J. H. Lieut.-Col., R.A.M.C. (Ist London I88I Gen. Hospital, T.F.)

DufField, H. W. 2nd Lieut., D. of Cornwall's L.I.; [19I4] Capt., M.G.C. (W.)

Dumas, A. B. Capt., R. Warwickshire Regt. ' (W.) I913

Dundas, A. C. Major, Middlesex Regt.; D.A.A.G. $\quad$ I899 (W 2.) O.B.E. M 2. $m$.

Dunkerley, C. L. Capt., Queen's Own (R. W. Kent Igi I Regt.) (W.) M.C.

DunloP, J. K. Capt., London Regt. (Rangers); Major, I9Io

M.G.C. and Gen. Staff, empld. British Military

Mission. (W.) M.C. M 2. m. Order of St Anne, $4^{\text {th Class (Russia) }}$

DutToN, H. Lieut., N. Staffs. Regt. and M.G.C.

Dyke-Marsh, H. St. G. Sapper, R.E. (Meteorological Section)

EARLE, G. F. Lieut., R.A.S.C.

EASTON, J. W. Lieut., R.G.A.

EbERLI, W. F. Surgeon Lieut., R.N.

1907

r9I 4

1908

1906

I910 
Edwardes, F. E. 2nd Lieut., Harrow School O.T.C. $\quad 1893$ AEDwardes, H. F. E. 2nd Lieut., D. of Cornwall's L.I. $\quad 1893$ Killed in action $6 \mathrm{Feb}$. 1917

EDWARDS, A. T. Major, R.A.M.C. 1908

EDwards, G. R. Lieut. (A.), R.A.F. (W.) (P.) EDwards, Rev. N. W. A. C.F. 3rd Class, R.A.C.D. $\quad 1896$ O.B.E. M.C. M2.

$\begin{array}{ll}\text { Ellis, A. I. Capt., R. Fusiliers } & \\ \text { Engledow, F. L. Lieut.-Col., Queen's Own (R.W. } & 1903 \\ 1910\end{array}$

Kent Regt. T.F.) $M$. French Croix de Guerre

ENGLEFIELD, F. R. H. Lieut., Hampshire Regt. I9ro

ENGLISH, F. H. 2nd Lieut., Aldenham School O.T.C. I9II

Evans, E. D. Lieut., Middlesex Regt., Labour Corps, I901 and R.G.A.

\#Evans, H.C. Lieut.-Cdr., R.N.V.R.(Nelson Bn., R.N.D.) * I909 $M$.

Killed in action in Gallipoli 5 June 1915

Evans, R. D. 2nd Lieut., King's Royal Rifle Corps. (P.) 1912

Evans, W. E. Lieut., R.G.A.

WEvat, G. R. K. Capt., Middlesex Regt.

Killed in action near Armentières 14 Nov. 1914

I9II

1900

Fairbank, J. Pte., R.A.S.C.(M.T.)

FAyERMAN, A. P. G. Major, R. Warwickshire Regt.(T.F.) 1904

Fergusson, A. Major, Middlesex Regt. (W.) 1900

Fergusson, J. N. F. Capt., R.A.M.C.

Fergusson, L. R. Lieut., R.F.A.; Capt., Spec. List

WFerris, S. B. C. 2nd Lieut., ioth Hussars

Accidentally killed 6 April 1915

Fewings, J. A. 2nd Lieut., R.G.A.

Filmer, W. G. H. Capt. and Adjt., The Buffs (E. Kent Regt.) M.B.E. M.

FisheR, F. B. Lieut., I.A.R.O., attd. 5th Gurkha Rifles. 1907 (W.)

. Fison, A. K. Capt., Essex Regt. (W 3.) M.C. Chevalier, I9ro

Legion of Honour (France). French Croix de Guerre

FleEt, Rev. C. S. C.F. 4th Class, R.A.C.D. M.C. M. 1906 AFletcher, J. H. B. Lieut., London Regt.

Died ${ }_{1} 3$ May I9I 5 of wounds received in action 12 May I9I 5

Foden, W. B. Gnr., R.G.A.

Forbes, A., D.s.o. Colonel, R.A.O.C.; Major-Gen., I871 Principal Ordnance Officer. C.B. C.M.G. M. 
ForD, F. C. 2nd Lieut., D. of Cornwall's L.I. $\quad$ I886

Forster, M. Pte., R.A.O.C. 1894

Foster, Rev. J. R. C.F. 4th Class, R.A.C.D. $\quad 1894$

FFoster, R. D. Lieut., Lincolnshire Regt. I9IO

Wounded and missing, presumed killed in action, in

Gallipoli 7 Aug. 1915

Fox, T. S. W. Capt., Oxford and Bucks. L.I.(T.F. Res.) $\quad 1897$

Franklin, C. S. P. Instructor Cdr., R.N. 1895

Franklin, H. W. Air Mechanic, R.N.A.S. I9I5

Franklin, J. H. Lieut., R.N.V.R. Order of the White 1898

Eagle (Serbia). French Croix de Guerre

Frankin, T. B. Capt., Fettes College O.T.C. $m$ 2. Igor

FraSER, D. S. 2nd Lieut., I.A.R.O. (Cavalry) 1906

Frean, H. G. Capt., R.A.M.C. I90I

EFREDERICK, T. Major, Norfolk Regt. (W.) M.C. M. 1912

Died 14 Dec. 1917 of wounds received in action near

Cambrai 3 Dec. 19I7

Gale, C. C. Capt., R.A.S.C.

1908

GaLt, R. B. 2nd Lieut., King's (Liverpool Regt.) (W.) I9I3

FGARDINER, K. J. R. Lieut., R.E. (Tunnelling Coy.); I909

Capt., King's African Rifles

Died on H.M. hospital ship I Feb. I9I7 of dysentery

GaRDNER, J.M.S. Capt., 124th Baluchistan Infy., In- 1913 dian Army

GARNER, H. M. Sub.-Lieut., R.N.V.R.

GARRETT, H. L. O. Capt., I.A.R.O.; Recruiting Officer

Garrood, J. R. Capt., R.A.M.C.(T.F.)

Gaussen, J. M. Major, R. Warwickshire Regt.(T.F.); I9I2 empld. O.C.B. (W.) $M$.

WGaze, G. A. Capt., London Regt. (Civil Service Rifles) 1900 Killed in action 15 Sept. 1916

George, J. T. Capt., Monmouthshire Regt.; empld. [19I4] Ministry of Pensions. (W.) M.C.

Gill, C. G. Hope. Capt., R.E. (R. Monmouth) [I914]

GILL, G. A. Lieut., R.G.A. M.C. and Bar I9I4

GiLl, R. G. Sapper, R.E.

1903

GillesPIE, J. J., T.D. Colonel, Northumberland Fus. I889 (T.F.) Brevet Colonel. $m$.

Gillespie, T. Capt., R.A.M.C.

Gilling, H. T., T.D. Lieut.-Col., R.F.A.(T.F.) O.B.E.

Gillson, A. H. S. Instructor Lieut., R.N.

Gleave, Rev. J. W. C.F. 4th Class, R.A.C.D.

1908

1909 
HGleave, T. R. Lieut., S. Lancs. Regt.(T.F.)

Killed in action I I Oct. 1916

Gledhill, W. G. Capt., Norfolk Regt. (T.F.); Capt. 1899 (T.), R.A.F. (Aircraft Production Dept.) $M$.

Glover, J.A. Capt., R.A.M.C. O.B.E.

*GLYN, C. R. 2nd Lieut., Hodson's Horse, Indian Army 1913 Killed in action in Mesopotamia 17 Fan. I917

GobвitT, R. H. S. Paymaster Lieut., R.N.R.

Gold, E. Lieut.-Col., R.E. (Meteorological Section). I900 D.S.O. O.B.E. $M_{4}$.

GoldIE, A. H. R. Major, R.E. (Meteorological Section). I9ro $M 2$.

Goldwater, H. G. 2nd Lieut., R.G.A.

Goode, R. H. Lieut., M.G.C.; empld. Ministry of La- 1908 bour. (W.)

Gooding, S. Capt., R.A.M.C.

Goolden, H. J. Rfn., King's Royal Rifle Corps. (W.) I9I2

GorDon, E. F. S. Surgeon Lieut., R.N.

Goyder, F. W. Hon. Capt., R.A.M.C.

GrabhaM, G. W. Lieut., R.A.S.C.(M.T.)

AGrail, C. G. Capt., N. Staffs. Regt.

Died 23 Fuly I9I5 of wounds received in action in Gallipoli

Grear, E. J. L. Lieut., Middlesex Regt. and M.G.C. I9ro (W 2.)

GreEN, N. Lieut., Sherwood Foresters (Notts. and 1906 Derby Regt.); Capt., Gen. List. (W.)

GreEN, S. M. Capt., London Regt. (Kensington Bn.) 1907 (W.) $M$.

GreENLEES, J. R. C. Lieut.-Col., R.A.M.C. D.S.O. and $\mathrm{I} 898$ Bar. $M_{3}$. Chevalier, Legion of Honour (France)

Greenstreet, N. B. DE M. 2nd Lieut., Norfolk Regt. 1912 (T.F.)

Gregory, A. R. Capt., Border Regt.(T.F.) (W.)

Gregory, H. L. Major, R.A.M.C.(T.F.)

1909 I89I

\#Gregory, R. P. Capt., Cambridge Univ. O.T.C., empld. 1898 O.C.B.; 2nd Lieut., Gloucestershire Regt. (W.)

Died 24 Nov. 1918 of pneumonia following influenza contracted on active service

Grice, N. Capt., W. Yorks. Regt.(T.F.) (W 3.) I9I2

Griffiths, Rev. G. A. M. C.F. 4th Class, R.A.C.D. $\quad 1908$ GRIGG, P. J. Lieut., R.G.A.

Grigson, P. St J. B. Capt., I.A.R.O., attd. 7oth Burma r90I Rifles 
Guest-Williams, W. K. Capt., Spec. List (R.T.O.) I906 Gwatkin-Graves, E. A. Lieut., M.G.C.

Hagger, N. W. Lieut., R. Sussex Regt.; empld. O.C.B. I9I2 HAIGH, P. B. Lieut., I.A.R.O., attd. I I4th Mahrattas; 1897 empld. School of Musketry; Major, Poona Bn., Indian Defence Force

Hall, Rev. H. A. C.F. $4^{\text {th }}$ Class, R.A.C.D. $M$. I88I Hall, Rev. S. H. C.F. Ist Class, R.A.C.D.(T.F.) $\quad 1869$ HHalliwell, W. N. 2nd Lieut., Yorkshire Regt.

Died 21 Sept. 1916 of wounds received in action

Halsey, R. T. Lieut., Cheshire Regt.

I909

1907

WHamilton, A.S. Lieut.-Col., Sherwood Foresters (Notts. $\quad 1883$ and Derby Regt.) and Durham L.I.

Died I 3 Oct. I9I 5 of wounds received in action 26 Sept. 1915

Hanson, J. Lieut., Coldstream Gds. and Gen. Staff

Harding, Rev. W. H. C.F. $4^{\text {th }}$ Class, R.A.C.D.

HaRdMAN, W. H. Major, R.E.(T.F.) (W 2.) M.C. $M$.

HaRnetT, W. L. Lieut.-Col., I.M.S. $M$.

Harris, H. Pte, R. Fusiliers (P. S. Bn.); Lieut., M.G.C. $\quad 1899$ M.C.

HARTREe, D. R. Lieut., R.N.V.R.

WHaRvey, A. W. Capt., R.A.M.C.

Died 7 Sept. I916 of wounds received in action

Haslam, V. K. Lieut., R.G.A.

WHAWCRIDge, R. S. Corpl., R. Fusiliers

Killed in action near Delville Wood 28 Fuly r 916

1909

1906

I913

1896

I $9 \div 5$

I894

1906

1905

Hayes, J. H Capt., Rifle Brigade and Gen. List (O.C.B.) 1894 (W.) $m$.

HaYwaRD, A. W. Major, R.A.M.C.(T.F.)

HAZleRIgG, G. 2nd Lieut., Sherwood Foresters (Notts. 1897 and Derby Regt., T.F. Res.)

WHeald, W. M. Lieut., R.A.M.C.

Died 8 Sept. I91 8 of wounds received in action on the

I9I3

Somme 22 Aug. 1918

HHearn, R. C. Capt., London Regt. (Blackheath and I9II Woolwich Bn.) M.C.

Killed in action near ferusalem 30 April 1918

Heimann, H. P. Pte., Essex Regt.

Hellings, G. S. Lieut., D. of Cornwall's L.I.

Henderson, P. Capt., R.E. 
Henry, W. D. M. Capt., R.A.S.C. $M$.

HePworth, F. A. Major, R.A.M.C.(T.F.) O.B.E.

Herzl, H. Pte., Middlesex Regt.

Hewit, J. T. Major, R.E. and Spec. List, empld. Min-

1910 istry of Munitions)

Hibberd, A. S. Lieut., Dorset Regt.; Capt. and Adjt., 1912 25th Punjabis, Indian Army

Hicks, Rev. F. W. Chaplain, R.N.

Higgins, F. E. Lieut., R.A.M.C.

Highfield-Jones, P. H. Capt., S. Staffs. Regt. (W.) I9I2 M.C.

Higson, L. A. Capt., Middlesex Regt. (W 2.)

Hilary, R. J. Lieut., The Buffs (E. Kent Regt.) (W.) 1912

WHILl, J. R. Pte., W. Yorks. Regt.; 2nd Lieut., R.E. 1902 (Spec. Bde.)

Killed in action 6 May r9r7

WHiller, A. M. 2nd Lieut., The Queen's (R.W. Surrey 1913 Regt.) $M$.

Killed in action 16 May 1915

Hillier, T. L. Surgeon Prob., R.N.V.R.

Hitching, W. W. Lieut., R.G.A.; attd. R.E.

HHobis, A. V. Pte., Queen's Own (R. W. Kent Regt.); and Lieut., R. Sussex Regt. and R.F.C.

Killed in action over Valenciennes ${ }_{5} 5$ Dec. I9I 5

सHoвbs, V. W. J. Lieut., The Buffs (E. Kent Regt., T.F.) Killed in action at Morlancourt 9 Aug. 1918

Hogan, R. V. J. S. Lieut., E. Lancs. Regt.; Capt., 1904 R.A.F.; S.O. 3, Air Ministry. (W 2.)

Holden, H. F. Lieut., S. Staffs. Regt. (W.)

Holden, J. R. R. Fusiliers (P.S. Bn.); 2nd Lieut., R.E. (T.F.); Lieut. (A.), R.A.F.

WHoldEN, N. V. Lieut., Lancs. Fus.(T.F.)

Died 4 June I9I 5 of wounds received in action in Gallipoli

Holthouse, Rev. C. L. C.F. $4^{\text {th }}$ Class, R.A.C.D. 1913

1915

1913

1905

1914

I9II

1909

Holtzapffel, J. G. H. Capt., London Regt. (R. Fus.)

Honeybourne, H. C. Capt. and Adjt., London Regt. (Blackheath and Woolwich Bn.)

Honeybourne, V. C. Major, R.A.M.C. $M$.

Hook, C. W. T. Lieut., The Buffs (E. Kent Regt., T.F.);

Capt., Spec. List (T.M.B.)

Horlington, F. Lieut., R.F.A.(T.F.); Staff Lieut.

Horton-Smith, L. G. H. Pte., London Regt. (London Scottish) 
Horton-Smith-HaRtley, Sir P., c.v.o. Major, R.A.M.C. $\quad 1886$ (Ist London Gen. Hospital, T.F.)

How, Rev. J. C. H. C.F. 4th Class, R.A.C.D. $\quad$ I900

Howe, G. A. Capt., Lancs. Fus. (W.) I9II

WHowell, M. I. B. 2nd Lieut., The Queen's (R. W. [I9I4] Surrey Regt.)

Killed in action 25 Sept. I9I 5

WHughes, J. L. Lieut., Welsh Regt.; attd. R.F.C. Killed in action I Oct. I9I7

$\begin{array}{lr}\text { Hunter, J. B. Capt., R.A.M.C. M.C. M. } & 1909 \\ \text { Hunter, W. Colonel, A.M.S. C.B. Order of St Sava, }+1887\end{array}$

I910 2nd Class (Serbia)

HuRRY, A. G. Lieut., Gloucestershire Regt.; attd. R. 1912 Irish Regt. (W.)

Hutchinson, R. W. 2nd Lieut., Labour Corps I9I4

HydE, R. W. Capt., Lincolnshire Regt.; attd. Gen. Staff, 1907 Indian Army; A.D.C.

INChLEY, O. Lieut., R.A.M.C. (Ist E. Gen. Hospital, 1892 T.F.)

INGRAM, A. C. Major, I.M.S.

AIremonger, E. V. Pte., R. Fusiliers

Died in German hands at Le Quesnoy $\mathrm{I}_{3}$ Sept. 1918

Irving, J. B. Capt., R.A.M.C.

IRVING, J. C. 2nd Lieut., R.G.A.

1895

1905

IRVING, P. A. L 1907 (Spec. Bde.) (W.)

IsAAC, C. L. Major, R.A.M.C.(T.F.) 1896

JACKLIN, J. V. Capt., Essex Regt. (W 3.) I9I I

Jackson, G. E. Pte., Queen's Own (R. W. Kent Regt.); 1908 2nd Lieut., Northamptonshire Regt.

Jackson, Rev. J. E. N. C.F. 4th Class, R.A.C.D. 1905

सJACOB, A. R. 2nd Lieut., Durham L.I. 1912

Died 18 Sept. 1916 of wounds received in action I7 Sept. 1916

JACOBSOHN, A. 2nd Lieut., R.A.S.C.

JJACQuest, S. P. Gnr., Canadian F.A.

Killed in action 18 Oct. I9I 6

WJAMES, F. A. Capt., Manchester Regt.(T.F.) (W.) $\quad 1905$

Died 18 Sept. I9I5 of wounds received in action in Gallipoli

James, G. Capt., R.A.M.C. 
James, R. W. Capt., R.E. $M$.

JARCHOW, C. J. F. 2nd Lieut., Sherwood Foresters 1898 (Notts. and Derby Regt., T.F.)

Jeans, F. A. G. Hon. Major, R.A.M.C.

JocE, J. B. D. Lieut., R.N.V.R.

Johnson, E. F. Lieut., Berkshire Regt. (P.)

Johnson, Rev. V. Y. C.F. $4^{\text {th }}$ Class, R.A.C.D.

WJohnston, F. Major, King's (Shropshire L.I.)

Died 3 I May I9I8 of wounds received in action

Johnston, M. C. Capt., R.A.S.C. $M$.

Jones, I. E. Major, R. Welsh Fus. (W.)

1896

1901

1914

1910

1904

Jones, R. F. Capt., R.A.M.C.

Jones, R. M. Lieut., Northumberland Fus. (W.)

JosePh, F. A. Driver, H.A.C.; attd. R.E.

[1914]

1913

1904

1905

1914

KeEBLE, C.F.A. Lieut., Border Regt.; attd. R. Scots Fus. $\quad$ I903 \&Kemp, P. V. Pte., R. Fusiliers (P.S. Bn.); Capt., Dur- I9ro ham L.I.

Died 31 May I9I8 of gas poisoning

Kempthorne, G. A. Lieut.-Col., R.A.M.C. (W.) (P.) 1895 D.S.O. $M 2$.

Kendall, G. M. Capt., R.A.M.C.

Kennett, W. H. Major, Rifle Brigade. M.C. M.

Kenny, H. T. Colonel, Gen. Staff

KERR, J. Major, R.A.M.C.

KeY, Rev. S. W. C.F. $4^{\text {th }}$ Class, R.A.C.D.(T.F.)

KING, L. A. L. Lieut., R.F.A.(T.F.)

I9I I

1899

1876

$188 \mathrm{I}$

1892

1898

Kingdom, W. A. Lieut., S. Staffs. Regt. and M.G.C. I9I I (W.)

Kingsfold, Rev. P. A. C.F. 4th Class, R.A.C.D.

Kinman, G. W. Major, Hertford School O.T.C. T.D. 1884 KIRK, J. H. District Officer, Nigeria. (W.)

Kirkness, L. H. Lieut.-Col., Spec. List, attd. R.E.;

A. D. Railways, Salonika Force. D.S.O. O.B.E.

$M_{4}$. Order of the White Eagle, 4th Class (CzechoSlovakia). Order of the White Eagle, ${ }_{5}$ th Class (Serbia).

Greek Medal for Military Merit

Knowles, J. A. Capt., Cheshire Regt. (W 2.) M.C.

Knox, R. U. E. Capt., Suffolk Regt.; empld. War Office. (W.) D.S.O. $M$.

ALaidlaw, C. G. P. Pte., London Regt. (London Scottish) 1907

Died 2 April I9I5 of wounds received in action near Neuve Chapelle 
WLAIDLAW, W. S. Lieut., R.E. (W.)

Killed in action near Ypres 23 Nov. 1917

*LANe, H. C. H. 2nd Lieut., Border Regt.

Killed in action 10 Fuly 1917

LASBREY, Rev. P. U. C.F. $4^{\text {th }}$ Class, R.A.C.D. m. 1900

LA Touche, H. N. D. Lieut., 63rd Palamcottah L.I., I9I0 Indian Army

LATTEY, H. Capt. Gen. List(T.F. Res.) $\quad 1875$

WLaughlin, P. H. Lieut., The Queen's (R. W. Surrey I9I4 Regt.) (W.)

Died 21 Dec. I9I7 of wounds received in action near Ferusalem

Lawe, F. W. Capt., E. Yorks. Regt. (W.) (P.)

HLEAKey, Rev. H. N. C.F. $4^{\text {th }}$ Class, R.A.C.D.

Died at Dar-es-Salaam 23 Dec. 1917 of sunstroke

LEDGARD, W. H. Capt., Hampshire Regt.(T.F.) (W.) 1893 M.C.

WLEE, E. H. Lieut., King's (Shropshire L.I.)

Died ig Sept. 1916 of wounds received in action

LEE, H. Capt., R.A.M.C.(T.F.)

I9I3

1909

Lee Warner, R. P. Pte., The Queen's (R. W. Surrey Regt.)

LEES, S. Engineer Lieut., R.N.

LEONARD, P. J. Corpl., R.A.S.C.; Lieut., R.G.A.

LEwIS, J. M. Lieut., R.G.A.

1914

1901

I9II

I906

1905

1914

LEwIS, P. J. Capt., Herefordshire Regt.; empld. O.C.B. $\quad$ I903 (W 2.)

LINCOLN, N. Lieut., I.A.R.O., attd. Mahrattas and Rail- 1904 way Bn., Indian Defence Force

LINDSELl, J. Capt. and Adjt., Loyal N. Lancs. Regt. I9I I M.C. $M$.

LINNELL, J. W. Major, R.A.M.C. (W.) M.C. $\quad 1899$

WLINNELl, R. McC. Capt., R.A.M.C.

Died 16 March I9I 5 of meningitis

Lloyd, E. LL. Capt., S. Wales Borderers. (W 2.) 1912

LlOYD, Rev. W. R. Lieut., Haileybury College O.T.C. 1906

ALloyd-Jones, P. A. Major, R.A.M.C.; D.A.D.M.S. 1895 D.S.O. M 2.

Died 22 Dec. I916 of wounds received in action near Saulty

Long, A. E. Pte., Training Res. Bn.

LorD, Rev. A. E. C.F. 4th Class, R.A.C.D.

I9I I

LORD, G. F. Calcutta Vol. Artillery

1892

Low, B. B. Gnr., H.A.C.; and Lieut., M.G.C.

1909

I914 
Lumb, W. Capt., R.A.S.C. $M$.

LUND, G. S. 2nd Lieut., Manchester Regt.(T.F.) Corps

ELusk, J. Staff Capt.; Capt. and Adjt., Cameronians 1902 (Scottish Rifles, T.F.) M. Chevalier, Legion of Honour (France)

Died 28 Dec. I91 5 of wounds received in action 25 Dec. I9I 5

Lymbery, A. W. Pte., Canadian Force

I897

Macalister, G. H. K. Capt., I.M.S.

WMcAulay, F. W. Capt., R.F.A. Killed in action 21 May 1916

McCormick, G. D. Major, 72nd Punjabis, Indian 1897 Army; attd. I i2th Infy. $M$.

McCormick, Very Rev. W. P. G. C.F. ist Class, 1896 R.A.C.D.; D.A.C.G. D.S.O. $M_{4}$.

MacDonald, S. G. Capt., R.A.M.C.

McDonnell, T. F. R. 2nd Lieut., Rangoon Bn., Indian Defence Force

McDougall, W. Major, R.A.M.C.

McFadyen, W. A. Capt., The Buffs (E. Kent Regt., 1912 T.F.); empld. Ministry of Munitions. (W.) M.C. $M$.

McGrady, S. H. Bdr., R.G.A.

McKenzie, R. P. Lieut.-Col., S. African Med. Corps. 1897 C.M.G.

Mackinlay, D.M. Capt., King's Royal Rifle Corps. (W.) I912 WMACLAY, E. Capt., Cameronians (Scottish Rifles); Lieut., 1909 Scots Gds.

Died I I April rgI 8 of wounds received in action

MacMulleN, W. A. Lieut., R.A.S.C.(M.T.) M.B.E. 1909

MCNeIle, Rev. A. P. C.F. 4th Class, R.A.C.D. $m$. I 1892

Mansbridge, E. Capt. (T.), R.A.F. (Aircraft Production I9I4 Dept.)

Marchand, G. I. C. Capt., R.F.A.(T.F.); Brigade Major. 1907 $M$.

Marlow, C. C. 2nd Lieut., R. Warwickshire Regt. I9I3

MARr, F. A. Capt., Cambridgeshire Regt.; Brigade I9I3 Major. D.S.O. M.C. $M 2$.

Marrack, J. R. Capt., R.A.M.C. (W.) D.S.O. M.C. 1905 $M$.

MARRS, F. W. 2nd Lieut., Worcestershire Regt. 
WMarshall, W. Lieut., Leicestershire Regt. Killed in action in Gallipoli 4 Fune 1915

Marshall, W. B. Major, R.A.M.C.(T.F.)

MART, W. T. D. Major, R.A.M.C.(T.F.)

1912

Mason, E. W. Capt., Northumberland Fus. (W.)

WMason, P. 2nd Lieut., King's Royal Rifle Corps

Killed in action at Miraumont $17 \mathrm{Feb}$. 1917

WMay, F. S. Pte., R. Fusiliers

Killed in action 12 Aug. 1916

1899

1895

1909

1915

1895

MaY, H. R. D. Lieut., Warwickshire Regt.; Capt., Spec. $\quad 1897$ List. (W.) M.C.

WMaY, P. L. 2nd Lieut., 2nd Dragoons (R. Scots Greys) 1894 Killed in action at Hulluch 13 Feb. 1916

MeNENDEZ, F. T. S. Lieut., York and Lancaster Regt.; 1914 Lieut. (A.), R.A.F. (W.) M.C.

Merivale, B. Capt., London Regt. (Post Office Rifles); 1900 empld. Ministry of Food. O.B.E.

MetCALF, H. K. 2nd Lieut., R.G.A.

MiLlER, F. Major, 108th Infy., Indian Army; attd. 1900 $95^{\text {th }}$ Infy.

MiLls, E. H. F. Lieut., Northumberland Fus.(T.F.) I910

Mills, E. J. Lieut., Cheshire Regt.(T.F.); Capt. and 1904 Adjt., M.G.C. O.B.E. M.

Millyard, T. Capt., Herefordshire Regt.; attd. King's 1912 (Shropshire L.I.) (W.)

WMirfin, J. C. 2nd Lieut., York and Lancaster Regt.

Died 17 Aug. 1917 of wounds received in action 7 Dec.

1913

I916

Mitchell, A. H. MCN. Lieut.-Col., R.A.M.C. $M$. I890

MonRo, A. E. Instructor, Cdr., R.N. I886

Moody, B. Lieut., I.A.R.O.; Major, D.A.D. Transport- 1908 ation

MOORE, Rev. C. Chaplain and Instructor Cdr., R.N. $\quad 1889$

MOORE, R. M. Lieut., R.A.M.C. $\quad 1902$

WMorley, G. H. Lieut., King's (Shropshire L.I., T.F.) 1912 $M$.

Killed in action 30 Dec. 1917

MoRrIs, P. E. Pte., Suffolk Regt.

MorRIson, D. C. A. Major, Wiltshire Regt.(T.F.) $\quad$ I898

Morton, F. D. Capt., Highland L.I.; empld. War 1906

Office. M.C.

Morton, W. B. Lieut., Belfast Univ. O.T.C.

Mosely, F. M. Surgeon Lieut., R.N.

1889

1906

Mowton, W. E. Pte., Suffolk Regt.

I9I I 
Mulholland, W. Lieut., Manchester Regt. (W 2.) I9ro M.C. $M$.

WMuller, Rev. J. S. Pte., Norfolk Regt.(T.F.) Died 27 Fune 1918 of illness contracted on active service

MurPhy, W. L. Capt., R.A.M.C.(T.F.) M. Chevalier, 1896 Legion of Honour (France). Order of St Sava, $4^{\text {th }}$ Class (Serbia). Cavalier, Order of the Redeemer (Greece). French Croix de Guerre

Murray-Aynsley, C. M. Lieut., King's Royal Rifle 1913 Corps; attd. Army Cyclist Corps. (W.)

NeEd, G. S. Sergt., R.A.M.C.(T.F.)

NeIll, N. C. Sub-Lieut., R.N.R.; Lieut., R.N.V.R.; 1902 empld. Admiralty

Ness WalkeR, J. Lieut., R.F.A.(T.F. Res.) 1913

Newbery, R. E. Lieut., M.G.C. M.C.

Newling, S. W. Pioneer, R.E.

WNewton, H. G. T. Capt., I $3^{\text {th Hussars }}$

Drowned 25 April I9I7 on active service

1903

1891

1904

Nicholls, A. C. Lieut., Leicestershire Regt. and Gen. List. (W 2.)

Nicholson, J. E. Lieut.-Col., R.A.M.C. $m$.

Nicklin, G. N. Capt., I.A.R.O., attd. 97th Deccan 1908 Infy.

WNorbury, F. C. Capt., King's Royal Rifle Corps

Killed in action near Béthune 10 fan. I9r 5

Norman, A. C. Lieut.-Col., 5th Cavalry, Indian Army 1897

Norman-LeE, Rev. F. B. N. C.F. Ist Class, R.A.C.D. $\quad 1874$

NorregaArd, Rev. A. H. Chaplain, R.N.

NoRTHCOTT, J. F. Surgeon Lieut., R.N.

NoRTHORP, Rev. F. C.F. $4^{\text {th }}$ Class, R.A.C.D. $m$.

Nowell-Rostron, Rev. S. C.F. $4^{\text {th }}$ Class, R.A.C.D.

NuRSE, H. H. Major, Spec. List. (Recruiting Staff)

1890

1892

1906

1902

1882

Odgers, L. N. B. Lieut., Middlesex Regt.; Capt., R.E. I9I I (W 3.) M.C.

AOdgers, R. B. Capt., R.A.S.C.(T.F.)

Died $3 \mathrm{I}$ Aug. I9I7 on active service

Okell, C. C. Capt., R.A.M.C. M.C.

Ormerod, G. S. Lieut.-Col., Training Res. Bn.; Major, 1876 Spec. List (Asst. Cmdt., P. of W. Camp). m.

Owen, D. H. Lieut., Welsh Horse and R. Welsh Fus. 1912 
Owens, F. H, Pte., London Regt. (Artists Rifles)

Palmer, T. N. P. 2nd Lieut., Coldstream Gds. $\quad$ I899 Palmer, W. E. Capt., Dorset Regt. and Training Res. 1912 Bn. $M$.

PARKER, G. Major, R.A.M.C. (2nd S. Gen. Hospital,T.F.) 1873

PARRY, J. H. Capt., I.M.S.

Parsons, Hon. Sir C. A., K.c.B. Hon. Colonel, North- 1873 umberland Fus.

Pascoe, E. H. Lieut., I.A.R.O., attd. Sikh Pioneers $\quad 1897$

Pascoe, F. J. Lieut., D. of Cornwall's L.I. and M.G.C. $\quad$ I9I2

PASkIn, J. J. Capt., Worcestershire Regt.; Major, M.G.C. $\quad$ I9I2 M.C. French Croix de Guerre

Pass, Rev. H. L. C.F. 4th Class, R.A.C.D.

Paterson, M. W. Major, R.A.M.C.; D.A.D.M.S.

O.B.E. M.C. M 2. French Médaille d'Honneur

Patterson, R. F. Lieut., R. Irish Rifles

Paulley, H. Capt., Norfolk Regt.

Pearson, C. E. Lieut., Durham L.I.

Pellow, J. E. Major, R.A.M.C.

1894

1905

Penfold, H. L. Capt., R.E.; empld. Ministry of Labour I906

Percy, J. R. Lieut., Border Regt.; attd.R.E.(Signals).(W.) [I9I4]

PERKins, A. B. Lieut.-Col., Hampshire Regt. $m$. I890

Perkins, J. S. S. See Steele-Perkins, J. S. S.

Perry, J. C. Pte., London Regt. (Q.V.R.); Capt. and 1907 Adjt., R.G.A. (W.)

Peters, A. G. Sergt., Middlesex Regt.

Phillips, H. E. Lieut., Welsh Regt.

Phillips, R. S. Lieut., 39th Garhwal Rifles, Indian Army

Phillips, W. R. Lieut., Lancaster Grammar School O.T.C.

सPhilp, C. H. G. Capt., R.A.M.C.

Killed in action 28 March 1918

Philpot, F. H. Warrant Schoolmaster, R.N. 1914

Plowright, C. T. M. Capt., R.A.M.C.

Polack, A. I. Lieut., R.E.(T.F.)

APolack, E. E. Lieut., Gloucestershire Regt.(T.F.)

I9II

I9I3

1.912

1908 Killed in action 17 Fuly 1916

APollaRD, W. M. N. 2nd Lieut., N. Staffs. Regt.(T.F.) ' 1909 Killed in action I I April I9I7

Poole, Rev. J. T. Chaplain, R.N.

1904

1914
1897

I9II

1912

PotTER,C. G. Bdr., R.F.A.;Lieut.,Hampshire Regt.(T.F.) I895

Killed in action 15 Sept. I9 6

Powell, E. C. Capt., R. Welsh Fus. (W.) 
Pralle, E. L. R. Capt. (S.), R.A.F. A.F.C.

Pratt, G. W. Surgeon Lieut., R.N.

Precious, C. M. Pte., Middlesex Regt. (W.)

Prichard, R. M. 2nd Lieut., Cheshire Regt.

I9I 5

Prideaux, H. S. Lieut., D. of Cornwall's L.I.

1913

\&Puddicombe, D. R. 2nd Lieut., E. Yorks. Regt.

Died 24 Fuly 1916 of wounds received in action on the

I90I

Somme 20 fuly rigr

सPullin, J. H. Lieut., Loyal N. Lancs. Regt.

Died 21 Fan. 1916 of wounds received in action near Armentières 19 Jan. I916

Purser, Rev. W. C. B. Pte., Rangoon Bn., Indian De- 1897 fence Force

Quick, Rev. E. K. C.F. $4^{\text {th }}$ Class, R.A.C.D. $m$.

Quin, B. G. Capt., Cambridgeshire Regt. and Suffolk Regt.(T.F.) M.C.

Raffle, W. Capt., R.A.M.C. $M$.

Raven, Rev. E. E. C.F. $4^{\text {th }}$ Class, R.A.C.D.

READ, A. J. Lieut., D. of Cornwall's L.I.; empld. Ministry of Munitions. (W.)

ReAD, G. D. Capt., R.A.M.C.; D.A.D.M.S.

Reade, G. L. Capt., Rifle Brigade. M.C.

Redlich, S. Capt. and Adjt., R.G.A. M.B.E. M.

AREES, F. E. 2nd Lieut. (A.), R.A.F.

Missing, presumed killed in action, 22 Aug. 1918

ReEves, J.H. Lieut., R.G.A.; empld. Ministry of Labour. $\quad 1887$ (W.) M.B.E.

WRENNIE, D. W. 2nd Lieut., R. Fusiliers; attd. R. War- 1904 wickshire Regt.

Killed in action I I Nov. I9I4

Reynolds, Rev. W. H. R. C.F. 4th Class, R.A.C.D. $\quad$ I9ro

RicE, Rev. C. M. C.F. 4th Class, R.A.C.D.

Rice, H. G. Capt., R.A.M.C. (W.)

Rice, L. C. Capt., Loyal N. Lancs. Regt. (W 3.)

Richardson, A. H. Surgeon Lieut., R.N. O.B.E.

Killed in action 25 Sept. I9I5

Ritchie, Rev. C. H. Chaplain, R.N.

Ritcule G. L. Capt R. Scots Fus. (W 2.) M.C. M. 1907

Witchie, J. N. 2nd Lieut., Seaforth Hdrs.

Killed in action in Mesopotamia 21 April 1916

Rivers, W. H. R. Capt., R.A.M.C. 
Roвb, A. A. Service Sanitaire, French Army. French 1894 Croix de Guerre

RoberTs, Rev. A. C. C.F. $4^{\text {th }}$ Class, R.A.C.D. $\quad 1882$

Robinson, E. H. Major, King's (Shropshire L.I.) (W 3.) * I9ro D.S.O. M.C. and Bar. M.

सRobinson, L. F. W. Lieut., R.E.

1912

Killed in action 25 May I9I7

सRobinson, M. H. Instructor Lieut., R.N.

1897

Died I 5 Fune I9I7

Rolleston, Sir H. D., c.B. Surgeon Rear-Admiral, R.N. $\quad 1883$ K.C.B

Ronaldson, J. B. Surgeon Lieut., R.N.V.R.

Rose, F. A. Capt., R.A.M.C.(T.F.)

1903

1892

Rose, H. A. Lieut., R. Scots (Cyclist Bn., T.F.) (W.) 1905

Rose, H. C. Major, R.A.S.C. $M$.

I902

WRoseveare, H. W. 2nd Lieut., Wiltshire Regt.

Died 20 Sept. IgI4 of wounds received in action in the

[19I4]

Battle of the Aisne

Ross, Rev. J. E. C. C.F. $4^{\text {th }}$ Class, R.A.C.D.

Rostron, S. See Nowell-Rostron, Rev. S.

Rowell, A. H. Corpl., R.E.

Rowetr, F. E. Flt. Lieut., R.N.A.S.

RudD, W. A. Lieut., R. Berkshire Regt. $m$.

Russell-Smith, A. Pte., H.A.C. (W.)

\&Russell-Smith, H. F. Capt., Rifle Brigade

Died 5 Fuly I916 of wounds received in action on the Somme I Fuly r9i6

ARYLeY, D. A. G. B. 2nd Lieut., Manchester Regt.;

Lieut., N. Staffs. Regt.

Killed in action at Hulluch 2 Feb. 1917

SADDLER, W. Lieut., R.G.A. $M$.

SaInt, P. J. Lieut., Pioneers, Indian Army. (W.) 1904

*Salmond, W. G. Trooper, 9th Lancers; 2nd Lieut., I912

N. Somerset Yeo.; Capt. and Adjt., Wellington Regt.,

N. Zealand Force. (W 2.)

Killed in action at Rossignol Wood 9 fuly r9r 8

SAmpson, M. T. Major, King's Royal Rifle Corps. I9I4

(W.) M.C. and Bar

Sanceau, R. J. Capt. (A.), R.A.F. $M$.

Sandall, Rev. H. C. C.F. 4th Class, R.A.C.D. $\quad 1899$

SANDALl, T. E. Colonel, Lincolnshire Regt.(T.F.) (W.) 1888

C.M.G. M.

Sargent, E. L. K. Capt., R.A.M.C. 
Sargent, P. W. G. Lieut.-Col., R.A.M.C. C.M.G. I89I D.S.O. $M 2$.

Scarborough, O. L. Capt., R.A.M.C.(T.F.) $\quad 1896$

SCARTH, R.E. 2nd Lieut., R.F.A.(T.F.)

\#Scholfield, R. D. 2nd Lieut., King's Own (R. Lan- I910 caster Regt.)

Killed in action in Gallipoli ro Aug. 1915

Schroeder, A. E. See Long, A. E.

Scoular, A. C. Major, Border Regt.; attd. Cheshire Regt. $\quad$ I893 T.D.

Scoular, J. G. Major, R.G.A. (W 2.) $M$.

ScutT, J. A. H. Pte., H.A.C.; Lieut., Hampshire Regt. (T.F.) M.C.

Seccombe, P. J. A. Major, R.A.M.C. Order of St Sava, $\quad 1887$ $5_{\text {th Class (Serbia) }}$

Sewell, S. E. Capt., Madras Garrison Artillery, Indian 1906 Defence Force; Asst. Proof Officer, Ordnance Inspection Staff

Shanly, H. Capt., London Regt. (St Pancras Bn.); 1912 Major, M.G.C. (W.) $M$.

Sharp, C. G. 2nd Lieut., The Queen's (R. W. Surrey 1903 Regt.) (W.)

ShEPHERD, Rev. E. H. C.F. $4^{\text {th }}$ Class, R.A.C.D.

Shillito, N. W. Lieut., R.E. M.C.

1909

Shimield, W. S. Lieut., D. of Cornwall's L.I.; Capt, Labour Corps

Shore, L. R. Capt., R.A.M.C. (W 2.) M.G. M. 1908

SHORE, T. H. G. Capt., R.A.M.C.

Short, Rev. J. M. C.F. $4^{\text {th }}$ Class, R.A.C.D.

Sibly, T. M. Capt., Gloucestershire Regt. $M$.

Silk, G. W. Lieut., E. Yorks. Regt. and Gen. List. I9I4 (W.) M.C.

Simpson, G. C. E. Lieut.-Col., R.A.M.C.(T.F.) O.B.E. 1899 $M 2$.

Skene, Rev. C. M. B. C.F. 4th Class, R.A.C.D.

Skene, Rev. F. N. C.F. 4th Class, R.A.C.D.

Slater, S. B. Capt., King's Royal Rifle Corps and Spec. List (T.M.B.) M.C. $M$.

Sleight, A. H. Pte., R. Fusiliers (P. S. Bn.); Sergt., 1908 attd. Div. H.Q. (Intelligence Branch)

SmeE, C. W. Capt., R. Fusiliers

1903

1896

1913

1906

1906

1904

14

Smith, A. F. 2nd Lieut., Middlesex Regt.; attd. London

Regt. (W.)

Missing, presumed killed in action, 9 Sept I916. 
Smith, Rev. B. T. D. C.F. $4^{\text {th }}$ Class, R.A.C.D.

SmITh, O. C. 2nd Lieut., R.G.A.(T.F.)

*1915 1906

SmrTh, V. S. Lieut., D. of Wellington's (W. Riding I9r3 Regt.) and I.A.R.O., attd. S. and T. Corps. $M$.

Smithson, A. E. Lieut.-Col., R.A.M.C.

1883

SNow, Sir T. D'O., K.C.B. Lieut.-Gen. K.C.M.G. 1877

$M$ 3. Commander, Legion of Honour (France). Grand

Officer, Order of Leopold (Belgium). Belgian Croix de Guerre

Soden, W. S. Capt., R.A.M.C.(T.F.) $M$.

SoTHERS, E. D. 2nd Lieut., London Regt. (L.R.B.)

ASOuPER, N. B. 2nd Lieut., R. Berkshire Regt.

Killed in action $\mathrm{r}$ Fuly $\mathrm{I} 9 \mathrm{I} 6$

Southam, Rev. J. F. S. C.F. $4^{\text {th }}$ Class, R.A.C.D. $\quad 1898$

SPACKMan, H. M. Capt., R.F.A.; attd. T.M.B. (W.) I9I I

Spargo, F. W. Lieut., I.A.R.O., attd. 70th Burma Rifles 1907

SPARKS, C. H. Capt., R.F.A.

SPENCER, G. W. Capt., R.A.M.C.

1907

I9I I

1899

Stanford, H. C. Capt., Suffolk Regt. and Gen. List (Intelligence). M.C.

Stanham, C. T. Lieut., The Buffs (E. Kent Regt., T.F.) rgro and King's African Rifles

Stansfeld, R. Capt., R.A.M.C.

Steele-Perkins, J. S. S. Capt., R.A.M.C.

Steen, F. D. Capt. and Adjt., King's Royal Rifle Corps.

(W 3.) M.C.

Stephenson, F. Lieut., Loyal N. Lancs. Regt.; Capt., r9r5 Spec. List (Bde. Signal Officer). (W.)

Sterndale-Bennetr, J. Major, IO7th Pioneers, Indian 1897 Army; Cmdt., School of Musketry. O.B.E.

Sterndale-BennetT, J. B. Pte., H.A.C.; Capt., S. Wales 1907 Borderers. (P.) M.C. m.

SteRndale-BenNETT, R. Major, Uppingham School I90I O.T.C.

Stevens, J. K. 2nd Lieut., R. Fusiliers; Lieut., Spec. I9I I List (R.T.O.) (W.)

Stevenson, C. M. Capt., R.A.M.C.

1898

StewarT, D. M. Lieut., Welsh Regt.

Stimpson, R. Pte., London Regt. (Rangers)

[1914]

Stockwood, I. H. Lieut., S. Wales Borderers; Capt., I9I I

Tank Corps; Hon. Capt. (O.), R.A.F.

Stokes, J. W. G. Lieut., R.G.A.

Stopford, J. Lieut., Lancs. Fus.(T.F.) and M.G.C. I9ro

STrEeT, R. O. 2nd Lieut. (T.), R.A.F. 
AStreeten, Rev. B. R. C.F. 4th Class, R.A.C.D.

Died in France I Nov. 1918 of pneumonia

STRONG, S. D. Lieut., R.F.A.(T.F.) (W.)

Struthers, J. A. Lieut., Durham L.I.; empld. Ministry

1914 of Labour. (W.)

Stuart, C. E. Lieut., N. Staffs. Regt.; attd. Hampshire Regt. (W.)

Swift, H. W. Capt., London Regt. (Q.V.R.) (W.) $M . \quad$ I9I4

Swift, Rev. J. M. C.F. 4th Class, R.A.C.D.

Sykes, M. G. Lieut. (O.), R.A.F.

1905

1902

Tanner, L. E. Capt., Gloucestershire Regt.(T.F.); empld. Ministry of Munitions. (W.)

1910

TAte, Sir R. W. Major, Dublin Univ. O.T.C. K.B.E. $189 \mathrm{I}$ C.B.E.

TAYLOR, E. C. Major, I.M.S.

TAYLOR, F. L. Capt., R. Fusiliers.; empld. Ministry of Labour. (W.) M.C.

सTAYlor, H. C. N. Capt., London Regt. (Blackheath I9I I and Woolwich Bn.)

Killed in action on Vimy Ridge 21 May 1916

TaYlOR, J. N. Lieut., Calcutta Light Horse, Indian 1902 Defence Force

Taylor, G. M. C. Lieut., R. Marines; Capt., R.E. 1904 M.C. $M$.

Teakle, Rev. S. G. C.F. 4th Class, R.A.C.D.(T.F.) 1899

Teall, G. H. Major, Lincolnshire Regt.; D.A.A.G. I900 (W.) D.S.O. M 5. French Croix de Guerre

Templeman, W. H. Capt., R.A.O.C.

Thomas, R. B. H. 2nd Lieut., R.G.A.

Thomas, R. Ll. and Lieut., Welsh Regt.

Thомas, T. Lieut., Haileybury College O.T.C.

Thomas, W. W. Lieut., S. Wales Borderers; Capt., I0I Spec. List (T.M.B.)

Thompson, A. R. Lieut., Berkhamsted School O.T.C. 1906

Thompson, C. N. Capt., Rifle Brigade; empld. O.C.B. I9I I (W 2.)

AThompson, E. E. 2nd Lieut., R.G.A.

Died $\mathrm{I} 6$ Oct. I9I 8 of wounds received in action

Thompson, S. L. Capt., I I 3 th Infy., Indian Army

1903

«Thomson, K. S. Lieut., 2ist Cavalry, Indian Army; 1906 attd. I6th Cavalry

Killed in action near the Persian Gulf 3 March rgr 5

Thorne-Waite, A. Lieut., King's (Shropshire L.I.) 
Thursfield, Rev. G. A. R. Junior Chaplain, Indian 1905 Army; C.F. $4^{\text {th }}$ Class, R.A.C.D. $M$.

WThwaItes, G. Major, R.A.S.C. D.S.O.

Accidentally drowned on the White Nile 29 May 1917

TicenURST, C. B. Capt., R.A.M.C.

TICEHURST, G. A. Capt., R.A.M.C.

Tillard, L. B. Capt., London Regt. (Rifles)

Topd, Rev. H. W. C.F.4th Class, R.A.C.D M.C. M. 1908 TоOтH, H. H., c.M.g. Colonel, A.M.S. (T.F. Res.) C.B. 1873 $M$ 2. $m$.

Topley, W. W. C. Capt., R.A.M.C. Order of St Sava, 1904 $4^{\text {th Class (Serbia) }}$

WTORRY, A. J. D. Lieut., R.G.A.; attd. R.F.C. (W.) 1905 M.C.

Killed in action 9 Oct. 1917

Towle, J. H. Lieut., United Provinces Horse, Indian 1897 Defence Force

Townsend, R. W. Capt., Devon Regt. M.C. M 2. I910 Tozer, Rev. E. F. C.F. $4^{\text {th }}$ Class, R.A.C.D

ATozer, S. P. Lieut., Devon Regt.

Killed in action near St Quentin 8 Oct. 1918

\#TRACHTENBERG, M. I. Lce.-Corpl., R. Fusiliers

Died at ferusalem 12 Oct. 1918 of malaria

1905

1914

I901

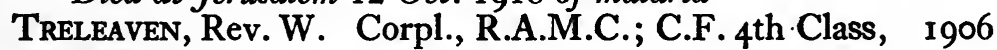
R.A.C.D.

Trotman, S. R. Capt., Nottingham Univ. College O.T.C. 1890 $m$.

Trotr, A. C. Lieut., Devon Regt.; Capt., Spec. List. M. I913

TrotT, F. W. Capt., Devon Regt.; Major, S.O. 2, R.A.F. 1912

(W 2.) O.B.E. M.C. M 2.

Trought, T. Capt., Queen's Own (R. W. Kent Regt., I9ro T.F.)

Trumper, J. H. W. 2nd Lieut., Monmouthshire Regt. 1904 (W.)

TUCKER, D. H. M. Lieut., Manchester Regt.

TURNER, R. Lieut., York and Lancaster Regt. M.C. [1914]

\#Twentyman, D. C. T. Capt., York and Lancaster Regt. 1909 (W.)

Killed in action I fuly $\mathrm{g} 9 \mathrm{I} 6$

URIE, R. W. Capt., R.F.A. (W.) $M$.

1913

Vale, H. E. T. Capt., Gen. List, attd. R.E. (Signals) 1909 van Druten, H. J. Capt., Middlesex Regt. (W.) 
VARwell, R. P. Capt., R. Irish Rifles; Major, D.A.A.G. I90I (W.) M.C. M3. French Croix de Guerre

WVAuse, T. C. 2nd Lieut., W. Yorks. Regt.(T.F.)

Killed in action near Albert 3 Sept. 1916

VEEVERS, W. 2nd Lieut., R.G.A.

1904

Vernon, C. H. Lieut., Hampshire Regt. (T.F.); Capt. roI I (Med.), R.A.F. (W.) $m$.

VINT, J. Lieut., R.G.A. (W.) M.C.

Vyvyan, P. H. N. N. Major, R.A.S.C. D.A.Q.M.G I9I4 O.B.E. M.C. M 2. m. Italian Croce di Guerra

WaIt, J. A. Lieut.-Col., R.G.A.(T.F.) $M$. 1883 WWales, H. R. 2nd Lieut., E. Yorks. Regt. (W.) I9I4 Killed in action 14 fuly r9r6

Walker, J. Ness. See Ness Walker, J.

Waller, B. P. Lieut., Hertfordshire Regt. $\quad 1898$

Walmsley, Rev. A. M. 2nd Lieut., I.A.R.O., attd. S. *1904 and T. Corps

WWarren, J. L. E. Capt., Welsh Regt. (W.)

Killed in action at the Hohenzollern Redoubt 2 Oct.

1913 I9I 5

WATERHOUSE, G. Lieut., R.N.V.R.

Waterhouse, H. Capt., Lancs. Fus. M.C.

WWaters, K. S. 2nd Lieut., Garrison Artillery, Indian 1909 Army

Killed in action at Bara Gali 30 May 1917

Watson, B.L. Capt., Gen. List, attd. R.E. (Signals). M. 1908

Weatherhead, E. Capt., R.A.M.C.

Wells, F. A. Capt., Hampshire Regt.(T.F.)

Wells, W. D. Lieut., Chigwell School O.T.C.

Weston, E. A. Lieut.-Col., R.E.; A. D. Movement and I899 Railways, War Office. C.M.G.

Weston, T. A. Capt., R.A.M.C.

Wharton, J. Capt., R.A.M.C. (2nd W. Gen. Hospital, 1895 T.F.)

Wheldon, W. P. Major, R. Welsh Fus. (W.) D.S.O. 1900 $M$.

Whiddington, R. Major (T.), R.A.F. m3. 1905

Whiteley, G. T. Capt., Gen. List (T.F. Res.) and 1892 Cheshire Regt.(T.F.)

WWhitfield, E. H. D. 2nd Lieut., York and Lancaster :910 Regt.

Killed in action in Gallipoli 7 Aug. 1915 
WhYE, J. W. Lieut., Leicestershire Regt. (W.) M.C. ${ }^{*}{ }^{1903}$ WWickham, B. W. T. Lieut., S. Staffs. Regt. (W.) M.C. I9I3 Killed in action near Ypres 14 April 1917

WilleTt, E. W. 2nd Lieut., R.A.S.C.(M.T.)

1905

WWilletT, J. A. Pte., Gloucestershire Regt.; 2nd Lieut., r9I3 Somerset L.I.; Lieut., R. Fusiliers

Killed in action in Gallipoli 2 Fuly I9I 5

Williams, G. W. Major, R.E. D.S.O. M.C. M. ${ }_{1898}$ WWilliams, H. B. 2nd Lieut., King's (Liverpool Regt.) I913 M.C.

Killed in action in the Battle of Arras 3 May 1917

Williams, R. Lieut., R. Warwickshire Regt.; Capt., *1899 Rifle Brigade(T.F.)

Williams, W. H. Capt. and Adjt., R.A.S.C. M.C. I9I3

Williamson, H. Capt., R.A.M.C. (Ist London Gen. I890 Hospital, T.F.)

Williamson, K. B. Capt., R.A.M.C. (Sanitary Service, I894 T.F.) $M 2$.

WILls, R. G. Capt., R.A.M.C.(T.F. Res.) * * 898

WILLS, W. K. Surgeon Lieut.-Cdr., R.N.V.R. O.B.E. I891 WWILson, A. S. Lieut., S. Lancs. Regt.; attd. M.G.C. I9I3

(W.)

Killed in action in the Scarpe Valley 23 April I917

सWILsON, A. W. 2nd Lieut., Scots Gds.; attd. M.G.C. $\quad$ I9I5 Killed in action 30 Fuly 1917

Wilson, G. Lieut., Norfolk Regt.

WINDER, R. McD. Lieut., R. Marines 1908

Winfield, P. H. Lieut., Cambridgeshire Regt.; empld. 1896 War Office. (W.)

Wood, N. W. Pte., Cheshire Regt.

Wood, T. A. V. Capt., D. of Cornwall's L.I. (W.) I9I2

WoOdall, F. E. Lieut., Gds. M.G. Regt.

1908

Woodmansey, Rev. G. E. C.F. 4th Class, R.A.C.D. I9ro

Woods, B. F. Capt., R. Fusiliers

सWooler, C. A. 2nd Lieut., W. Yorks. Regt. (W.) [1914]

Died 20 Fuly I916 of wounds received in action I Fuly 1916

WWooler, H. S. 2nd Lieut., W. Yorks. Regt. $M$.

Died 28 March 1916 of wounds received in action at

I9I I

St Eloi

Woolrich, W. G. Capt., R.A.M.C.

WORDIE, J. M. Lieut., R.F.A.(T.F.) (W.)

1912

1910

WWORSTENHOLM, J. 2nd Lieut. (O.), R.F.C.

Killed in action 25 Sept. I917

1916 
Worthington, F. Lieut.-Col., R.A.M.C. (W.) D.S.O. $\quad 1898$ and Bar. O.B.E. M 2. French Croix de Guerre

Wren, T. L. Lieut., R.A.S.C.; empld. Ministry of Muni- 1908 tions

Wright, J. C. Major, R.A.M.C.

WRIGHT, T. 2nd Lieut., King's Own (Yorkshire L.I.) I9I2

Wyeth, F. J. S. Major, Essex Regt. and Gen. List, 1897 empld. Ministry of Munitions. M.C. M 2 .

Wynne-Willson, Very Rev. St J. B. C.F. 4th Class, 1887 R.A.C.D.

Yeats, G. F. W. Pte., Middlesex Regt. 


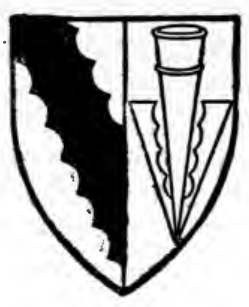

\section{SIDNEY SUSSEX COLLEGE}

AbERCRombie, D. M. 2nd Lieut., R.A.S.C. $\quad 1904$

Armstrong, W. E. Pte., R.A.M.C. (W.) I9I4

AstBuRY, S. J. 2nd Lieut., Labour Corps and Gen. List, 1916 empld. Ministry of Munitions

Atkins, G. R. Lieut., R.E.(T.F.)

Atkinson, H. G. Lieut.-Cdr., R.N.V.R.; Major (T.), 1906 R.A.F. (W.) O.B.E.

Austin, L. J. Major, R.A.M.C. 1899

Baboneau, C. A. Lieut., R. Sussex Regt.

Bacchus, J. G. Gnr., R.F.A. I913

BBaillie, A. La T. Capt., Cameronians (Scottish Rifles) 1913

Killed in action 29 Oct. 1915

BAKER, S. E. Capt., D. of Wellington's(W. Riding Regt.); 1909 Major, Gen. List (O.C.B.) (W 2.)

BaldwIN, C. W. Capt., Durham L.I.; Capt. (A.), R.A.F. 1910

BALL, H. F. Lieut., Gen. List(T.F. Res.) M.C. 1909

Ballance, G. 2nd Lieut. (A.), R.A.F. (P.) 1917

BARKER, R. A. Capt., Loyal N. Lancs. Regt.; Lieut., I9IO

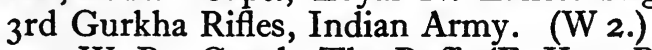

Barker, W. B. Corpl., The Buffs (E. Kent Regt.) I9I5

Bartlett, G. B. Capt., R.A.M.C.

Barton, R. A. E. Lieut., Cambridge Univ. O.T.C. I9I3

Batten, L. W. Capt., R.A.M.C. $\quad 1908$

ABell, W. A. Corpl., R. Fusiliers (P. S. Bn.); 2nd Lieut., 1913 Border Regt.

Killed in action 14 fune 1917

BENNETT, G. S. Lieut., Sherwood Foresters (Notts. and [19I4] Derby Regt.); Lieut. (O.), R.A.F.

BENNETT, W. E. 2nd Lieut., Norfolk Regt. (W.) I9II

BERENS, H. A. Sapper, R.E. $\quad 1908$

Bindon, W. F. V. Lieut., R.A.S.C.

I897 
Blackmore, J. K. Gnr., R.G.A.

WBlair, H. S. P. 2nd Lieut., D. of Cornwall's L.I.

Died 31 Oct. I9I 6 of wounds received in action

1890

1907

Blumhardt, R. A. See Mills, R.A.

Borthwick, C. H. Capt., R.F.A. (W.) M.C. m.

BorTHWick, L. C. Lieut., E. Yorks. Regt.; Major, M.G.C. I9Io $M$.

Bourne, L. P. S. Lieut., Manchester Regt.

Bowdler, A. P. Capt., R.A.M.C.

I913

1895

Boyd, E. J. Surgeon Lieut., R.N.; Capt. (Med.), R.A.F. 1907

Braby, H. W. Pte., R.A.M.C.

BREND, W. A. Major, R.A.M.C.

1907

1892

1906

I912

Killed in action 2 Aug. 1916

\#Brown, J. C. B. Lieut., Middlesex Regt.

Killed in action 29 Sept. 1918

BRUNDRETT, F. Lieut., R.N.V.R.

Buddin, W. Capt., R.A.M.C. (Sanitary Service, T.F.)

[1914]

I9I3

1909

1903

Killed in action 13 March 1916

4Cane, L. D. Capt. and Adjt., R. Fusiliers

Killed in action 24 Fan. I9I6

Carmichael, A. M. Lieut., R.F.A.(T.F.)

Cassidy, C. Capt., R.A.M.C. and Spec. List, empld.

Egyptian Army; Major, A.A.G. M.C. M 2. Order

of the Nile, $4^{\text {th }}$ Class (Egypt). Medjidieh, $4^{\text {th Class }}$ (Egypt)

Chapman, H. 2nd Lieut., S. Staffs. Regt.

Chapman, P. D. H. Capt., R.A.M.C.

1910

I9I I

Chase, C. D. Capt., R. Irish Rifles and Gen. List. M.C. $\quad 1897$ $M$.

Clark-Turner, F. Lieut., R.A.S.C.(M.T.)

Clarke, E. R. Capt., Connaught Rangers. M.C.

1912

I9II

Clarke, Rev. E. T. C.F. $4^{\text {th }}$ Class, R.A.C.D.

ClaArke, Rev. S. J. C. Chaplain, R.N.

Clayton, F. Capt., Cambridgeshire Regt. and Suffolk Regt.(T.F.) (W.)

Clement, L. Corpl., R.E.

Colt, G. H. Major, R.A.M.C. $M$.

Cook, S. G. Major, Huntingdonshire Cyclist Bn.; I89I

G.S.O. 2. O.B.E.

Cooke, J. G. Capt., R.A.M.C.(T.F.)

Corner, E. M. Major, R.A.M.C.(T.F.) $M$.

1891

1891 
Cornish, Rev. A. C.F. $4^{\text {th }}$ Class, R.A.C.D.

Coules, St V. F. 2nd Lieut., Labour Corps

1893

CoYte, S. E. Lieut., R.E.

1914

1912

Creswick, F. N. Capt., R. Defence Corps $\quad$ I893

CRISP, E. J. Flt. Lieut., R.N.A.S.; Hon. Capt., R.A.F. I9I4

Daniels, M. 2nd Lieut., R.G.A.

1910

WDaNiels, T. H. R. 2nd Lieut., King's Own (R. Lan- I9I4 caster Regt.)

Killed in action 9 April r916

DavenPoRT, A. H. Major, R.E. (W.) M.C. M. 1907

DEAN, L. T. Capt., R.A.M.C. I90I

De la Bere, I. Capt., Dorset Regt.; attd. Connaught I9I I

Rangers; Capt., Gen. Staff (Intelligence). (W.)

Denton, H. S. Lieut., Gloucestershire Regt.(T.F.)

DERBYSHIRE, H. Major, R.G.A. M.C.

WDick, N. B. 2nd Lieut., Middlesex Regt.

Killed in action 28 April ror7

WDickson, C. G. Lieut., Loyal N. Lancs. Regt.

Killed in action in $E$. Africa 4 Nov. I9I4

Donald, A. G. Capt., R.F.A. (W 2.)

Donaldson, C. E. Cadet, O.C.B.

19I5

1905

I90I

1909

I9II

1917

Donovan, S. J. Lieut.-Col., R.A.S.C. D.S.O. $M_{3} \cdot m$. ${ }^{1898}$

Downing, A. B. Lieut., E. Yorks. Regt.(T.F.)

DruitT, C. E. H. Capt., The Buffs (E. Kent Regt.) and M.G.C. (W.) M.C.

Dunlop, J. B. Lieut., R.A.M.C.

सEDmonds, S. F. Trooper, E. African Mtd. Rifles

Killed in action on the Magadi River 25 Sept. 1914

Elliotr, H. D. E. 2nd Lieut., King's (Shropshire L.I.) 1905

WEmbreY, C. S. 2nd Lieut., S. Staffs. Regt.(T.F.) M.C. 1916

Killed in action 12 Oct. 1918

Eustace, E. M. 2nd Lieut., R.G.A.

Evans, E, T.D. Surgeon Lieut.-Col., Welsh Regt.(T.F.)

Evans, K. R. Lieut., Lancs. Fus.; attd. King's Own (Yorkshire L.I.) (W.)

WEwen, G. C. Lieut., Nigeria Regt., W. African Frontier 1908

Force

Killed in action 24 Fan. 1917

FaIRChILd, G. C. Surgeon Lieut., R.N.

1906

FARR, T. H. 2nd Lieut., Res. Regt. of Cavalry 1916

FAWCETT, W. L. Capt., W. Yorks. Regt.(T.F.); Lieut., [1914]

9th Gurkha Rifles, Indian Army. (W.) M.C.

FerRar, H. T. N. Zealand Force 
Fisher, Rev. L. N. C.F. $4^{\text {th }}$ Class, R.A.C.D. 1900

FISHER, R. Capt., R.A.M.C.

Fison, W. J. Surgeon Lieut., R.N.

\#Fitzherbert, G. C. 2nd Lieut., Suffolk Regt.; Lieut., [1914]

York and Lancaster Regt. M.C.

Killed in action 18 Sept. 1918

AFletcher, R. R. R. Lieut., S. Lancs. Regt. (W.)

Died 29 Oct. I9I9 of dysentery contracted on active service

Foulds, J. G. Capt., S. African Military Labour Corps Fox, C. Gnr., R.G.A.

Fox, J. T. Capt., R.A.M.C.

WFyson, G. Lieut., R. Scots. (W.)

Killed in action in Macedonia 4 Sept. 1918

1905

Garns, S. G. 2nd Lieut., R.G.A.

1909

1915

1903

1904

*Garstin, D. N. Lieut., roth Hussars; Capt., M.G.C. D.S.O. M.C.

Killed in action 15 Aug. 1918

Garstin, E. J. L. Capt., Middlesex Regt. (W.) M.C. IgI I $M 2$.

Gates, C. E. 2nd Lieut., R.G.A. (P.)

Gathergood, G. W. Capt. (A.), R.A.F. A.F.C.

Gathergood, L. S. Capt., R.A.M.C.

1907

[1914]

I9II

Gaussen, E. A. Capt., Merchiston Castle School O.T.C. I89I

*Gawan-Taylor, N. 2nd Lieut., York and Lancaster I9I3

Regt.

Killed in action 24 April I9I 7

WGawan-TAYLOR, T. F. 2nd Lieut., York and Lancaster 1912

Regt.

Killed in action at Hooge 9 Aug. I9I 5

Gluckstein, I. M. Capt., London Regt. (Hackney Bn.) (W.) $M$.

Gooch, G. W. 2nd Lieut., Marlborough College O.T.C. I9I I

Goodall, C. E. G. Capt., Lincolnshire Regt.(T.F.) [I9I4] (W.) M.C. $M$.

GoodCHILD, G. F. 2nd Lieut., London Univ. O.T.C. $\quad 1895$

सGORDON, W. H. E. Lieut., Gordon Hdrs. 1912

Died 30 Sept. I9I 5 of wounds received in action at the Hohenzollern Redoubt

सGough, H. P. B. Major, Welsh Regt. M.C. and Bar

Died 22 April 1918 of wounds received in action 13 April I9I8

*Gould, W. J. Lieut., Northamptonshire Regt.

Killed in action near Ypres 23 Aug. 1915 
Green, H. F. Capt., N. Staffs. Regt.(T.F.); Major, Asst. 1912 Chemical Adviser. $M$.

GreEN, R. C. 2nd Lieut., S. Wales Borderers; Lieut., I9I4 M.G.C.

Greenwood, E. T. Capt., Devon Regt.(T.F.) (W.) 1912

GrIfFITH, A. W. 2nd Lieut., R. Inniskilling Fus. 1912

GrINBERG, S. Russian Army

Grogan, J. D. Lieut., R.E.

1913

1908

Groom, S. H. Sergt., Devon Regt.(T.F.); 2nd Lieut., 1906

R. Fusiliers; Lieut., Rifle Brigade. (W.)

Ground, E. G. Lieut., London Regt. (Finsbury Rifles) 1904

Hackforth, R. Pte., London Regt. (Artists Rifles) * *19

Hague, T. H. Major, Spec. List, empld. Army Trade I910 Test Centre

Hainworth, A. D. 2nd Lieut., King's School, Warwick, 1896 O.T.C.

WHall, B. Lieut., S. Staffs. Regt.

Killed in action in the Battle of Loos 25 Sept. 191 5

[I9I4]

Hall, J. Pte., London Regt. (Artists Rifles); Lieut., I9I I R.F.A.

WHall, J. S. Lieut., Rifle Brigade. (W.)

1904 Killed in action 21 March 1918

WHall, W. Lieut., S. Staffs. Regt. (W 2.)

Died I Fuly 1916 of wounds received in action

[1914]

Hamilton, C. J. Pte., London Regt. (Artists Rifles) I9ro

Hamilton, G. H. Lieut., R.G.A.(T.F.)

1908

Hammond, N. W. Lieut., St Edward's School, Oxford, 1897 O.T.C.

Hanitsch, K. V. Capt., R.F.A. $M$.

I9I I

HHARris, L. G. Pte., H.A.C.

Killed in action near Ypres 28 April I9I 5

Harrison, A. M. 2nd Lieut., R.F.A.

HARVEY, R. P. Lieut., Norfolk Regt.; attd. R.F.C.

Haswell, P. 2nd Lieut., R.G.A.

I9I3

Hatley, A. J. Lieut., R.N.V.R.

HaworTh, C. W. B. Lieut., Manchester Regt. (W.)

HaYgarTh, C. H. S. Lieut., R.E.(T.F.) (P.)

1908

[1914]

1907

1912

I9II

1914

HHaYthoRnthwaIte, R. M. 2nd Lieut., The Buffs (E. I9I3

Kent Regt.)

Killed in action 24 May 1915

Heads, J. E. B. Capt., Durham L.I.(T.F.)

HeaP, E. F. G. T. Capt., R.A.M.C.

HERMAN, W. S. Lieut., R.A.M.C.

HewitT, N. S. Surgeon Lieut., R.N. 
Heyer, G. Capt., Spec. List (Directorate of Requisi- 1888 tions and Hirings). M.B.E. M.

Hoffman, C. D. Corpl., W. Yorks. Regt.

Holland, H. H. Instructor Capt., R.N.

Hollingsworth, J. H. Capt., R. Marines. D.S.C. 1894 Belgian Croix de Guerre

WHolmes, B. S. Pte., R.A.S.C.; 2nd Lieut., King's Royal 1903 Rifle Corps and M.G.C.

Accidentally killed 24 Oct. 1916

HolT, J. L. Instructor Cdr., R.N.

Horan, A. K. Pte., H.A.C.; Lieut., R.F.A.(T.F.)

Housden, R. J. Lce.-Corpl., 6th Dragoon Gds. (Carabiniers); 2nd Lieut., R. Fusiliers; Lieut., Northamptonshire Regt.; Lieut. (A.), R.A.F.

Hughes, F. A. 2nd Lieut., Wellington College O.T.C. 1906

Jackson, A. H. Pte., Foreign Legion, French Army; Major, R. Fusiliers. (W.)

JaCkson, R. W. Lieut., R.F.A.; Hon. Lieut. (O.), R.A.F. 1912

WJAMESON, A. B. 2nd Lieut., Cambridgeshire Regt. I9I3

Killed in action 21 fuly 1916

Jerwood, B. E. Capt., R.A.M.C.

I9II

Jobson, J. S. Surgeon, R.N.

Johnson, F. H. Pte., King's Own (R. Lancaster Regt.) I9I3

JJohnson, O. B. G. 2nd Lieut., Norfolk Regt. and 1912 Suffolk Regt.

Killed in action 9 April 1917

JonAS, G. J. Lieut., R.G.A.(T.F.)

JoNES, E. W. Capt., R. Warwickshire Regt.; attd. Loyal

N. Lancs. Regt. (W 2.) Belgian Croix de Guerre

JoNES, G. B. H. Lieut., Welsh Horse and M.G.C. M.C. 1915 JONES, J. M. See MORRIS-JONES, J.

JoNEs, R. L. Surgeon Cdr., R.N.

Karran, T. W. Lieut., R. Welsh Fus.

KERNICK, J. W. Lieut., R.E.

1912

1913

KING, C. B. R. Lieut., Middlesex Regt.; Capt., M.G.C. (W.) M.C.

WKING, F. C. Rfn., King's Royal Rifle Corps

Killed in action $2 \mathrm{Feb}$. 1917

KING, H. B. Capt. and Adjt., Northamptonshire Regt.; I9I I Staff Capt. M.C. $M 2$.

King, N. Major, E. African Force

WKING, W. O. R. Lieut., Spec. List (Medical Research) 1906 Died Igrg of illness contracted during the war 
KNIPE, R. C. Sergt., London Regt. (R. Fus.); Capt., 1909 R.A.S.C.; attd. Gen. Staff

Knox-SHAw, P. Lieut., Sherwood Foresters (Notts. and 1912 Derby Regt.) (P.)

Knox-Shaw, T. 2nd Lieut., S. Lancs. Regt.; Capt. and 1905 Adjt., York and Lancaster Regt.; Brigade Major. M.G. M3. Belgian Croix de Guerre

LaIrd-Clowes, G. S. R.E.; Lieut., Spec. List. $M$.

LANDER, A. J. M. Capt., Sherwood Foresters (Notts. and

Derby Regt.); empld. O.C.B. (W.) M.C.

Landon, J. W. Capt., R.E.; Asst. Instructor, School 1898 of Military Engineering. (W.) M.B.E.

Langton, G. B. Pte., R. Fusiliers; Capt., S. Staffs. Regt. I9I I WLawson, R. H. Lieut., Rifle Brigade. (W.) M.C. M. 1912 Killed in action on the Somme 24 Aug. 1916

LEE, N. Lieut., King's Own Scottish Borderers. (W.) 1912

LEIGH, S. P. 2nd Lieut., Suffolk Regt.(T.F.) (W.) I9I3

Lindesay, J. H. C. Capt., Army Cyclist Corps. M.C. I9I2

LiNDSAY, W. J. Lieut., R.A.M.C.

LitTle, H. L. Air Mechanic, R.A.F.

1890

I9I I

LlOYD, J. D. 2nd Lieut., Labour Corps

1903

LOCKSPEISER, B. Pte., R.A.M.C.; empld. Ministry of I9IO Munitions

सLong, L.P. Lieut., London Regt. (Blackheath and Wool- 1912 wich Bn.)

Killed in action 25 Sept. I9r 5

Longrigg, J. H. Trooper, S. Provinces Mtd. Rifles, 1909 Indian Defence Force

Low, A. H. 2nd Lieut., R.E. (London Electrical En- I910 gineers, T.F.)

LuDLOw, F. Lieut., I.A.R.O., attd. 97th Deccan Infy. 1905

McArThur, G. K. 2nd Lieut., Norfolk Regt.; Lieut.(O.), I9IO R.A.F.

McFarland, J. B. Capt., R.A.M.C. M.C. French 1908 Croix de Guerre

MaCKIE, J. B. Capt., Somerset L.I.(T.F.) $M$.

McLeod, E. R. Capt., Lancs. Fus. (P.)

MACRAE, R. D. Capt., Gen. List (Intelligence)

WMaIR, E. M. 2nd Lieut., Cameron Hdrs.

Killed in action 3 Sept. I916

Mannering, Rev. L. G. C.F. $4^{\text {th }}$ Class, R.A.C.D. M.C. $\quad 1902$

Manning, C. R. U. Lieut., Northamptonshire Regt. 1909 (T.F.)

c.U.W.L. 
Marshall, A. G. Lieut., W. Yorks. Regt.

Marshall, Rev. H. C.F. 2nd Class, R.A.C.D. $M$ 2. 1903 Montenegrin Silver Medal for Bravery

Marshall, L. P. Capt., W. Yorks. Regt. (W 2.) M.C. I9I3

WMartin, S. T. Lieut., R. Inniskilling Fus. (W.) 1909 Killed in action I fuly rgr6

Martyn, J. V. 2nd Lieut., R.A.F.

Mason, N. Capt., Punjab Rifles, Indian Defence Force

Mather, F. H. Lieut., Lincolnshire Regt.; empld. O.C.B. (W.)

Mathieson, W. Major, R.A.M.C. O.B.E. $M_{3}$. I90I

MAY, A. J. Capt R.A.M.C 1902

Mayall, Rev. J. B. C.F. $4^{\text {th Class, R.A.C.D. (W 2.) I910 }}$ M.C. $M$.

Mayall, R. C. Capt. and Adjt., Northumberland Fus. D.S.O. M.C. and Bar. M. Italian Bronze Medal for Military Valour

Mayes, Rev. R. M. C.F. 4th Class, R.A.C.D.

Megson, M. Lieut.-Cdr., R.N.V.R.

Metcalfe, F. W. Capt., Rifle Brigade; empld. Home Office

Michell, A. H. Lieut., Warwickshire Yeo. and M.G.C. IgoI

Mills, R. A. Lieut., R.A.S.C.(M.T.)

Milman, L. C. P. Major, R.F.A.; Brig.-Gen., Asst. 1896 Director, Ministry of Munitions. C.M.G. M. m.

Mitchell, A. G. Lieut., Northumberland Fus. and R.E. 1904 Molony, A. C. B. Lieut., Border Regt. and M.G.C. (W.) I9 I I

Molony, Rev. J. A. C.F. 4th Class, R.A.C.D. 1909

WMorris, J. W. G. Capt., Welsh Regt.

Killed in action $\mathrm{r}$ April r916

MORRIS-Jones, J. 2nd Lieut., Welsh Horse; Lieut., 4Ist I9I5 Cavalry, Indian Army. $M$.

Mountain, B. Capt., R.A.M.C.

Mullins, C. DE C. C. and Lieut., Ioth Hussars

1902

I9I0

Naish, F. C. P. Capt., R.E. (W.) M.B.E.

Neighbour, P. M. Capt., R.A.M.C.

Noble, E. R. Pioneer, R.E. (Spec. Bde.)

1900

1912

1905

OAten, E. F. Lieut., I.A.R.O., attd. I Ith Lancers I903

OAten, W.S. Lieut., R.F.A.; Capt., Spec. List (Chemical rgII Adviser). (W.) $M$.

O’NeILl, F. R. Capt., R. Inniskilling Fus.; Major, I898

R. Sussex Regt.(T.F.) Brevet Major. $M$.

Oscroft, E. P. Lieut., R.E. (Signals) 
WPaine, W. L. Pte., Grenadier Gds.; Capt. and Adjt., I900 King's Own (R. Lancaster Regt.); attd. Lancs. Fus.

Killed in action in Gallipoli 4 June 1915

Pakeman, S. A. Capt., Wiltshire Regt. (W 2.) M.C. I9ro

PALMER, W. H. Lieut., R.A.M.C.

PARgeter, H. A. A. Surgeon Sub-Lieut., R.N.V.R.

Paterson, H. Lieut., R.F.A.

1914

I916

Peake, S. C. 2nd Lieut., Norfolk Regt.; Capt., Labour 1914 Corps. (W.)

Pearson, A. C. Major, R.A.M.C.(T.F.) M.C.

Pearson, B. L. Capt., R.A.S.C.(M.T.) O.B.E. $M$.

1893

1911

PECK, S. C. Lieut.-Col., R.G.A.; G.S.O.2. D.S.O. 1890 Brevet Lieut.-Colonel. $M 3$.

WPemberton, V.T. 2nd Lieut., R. Munster Fus.; Major, I9r3 R.G.A. M.C.

Killed in action at Bélicourt 7 Oct. 1918

Pinches, H. I. Capt., R.A.M.C.

PoINTER, E. H. Gnr., R.G.A.

I897

1907

I90I

Poole, F. S. Capt., R.A.M.C.

Poole, H. Lieut., The Buffs (E. Kent Regt.) and R.G.A.(T.F.)

WPope, L. K. Trooper, Otago Mtd. Rifles, N. Zealand Force

Killed in action in Gallipoli 7 Aug. 1915

Purchase, W. B. Major, R.A.M.C.; D.A.D.M.S. (W.) 1908 Brevet Major. M.C. M. Order of the White Eagle, 5th Class (Czecho-Slovakia)

RADFord, V. N. Capt. and Adjt., R.A.S.C. $M 2$.

19I3

RADFORD, W. N. Lieut., R.A.S.C.

1909

Ramsden, G. C. F. Capt., R. Sussex Regt.

WRAPP, R. 2nd Lieut., D. of Wellington's (W. Riding I9I3

Regt., T.F.)

Killed in action 18 fune 1915

RAPP, T. C. Capt., D. of Wellington's (W. Riding I9 I I Regt., T.F.); Major (A.), R.A.F. (W.) M.C. $M$.

WRATCliffe, A. V. Lieut., W. Yorks. Regt.

Killed in action I fuly 19r6

WRAYNER, O. C. Lieut., Manchester Regt.

Killed in action 18 Nov. 1916

READ, R. S. 2nd Lieut., R.E.

1907

I9I I

1908

1914

Killed in action at Gueudecourt 18 Oct. 1916

WReES-MogG, L. L. Lieut., R.E.

1908

Killed in action in Gallipoli I I Aug. I91 5 
WReynolds, J. W. 2nd Lieut., York and Lancaster Regt. *igro (T.F.)

Killed in action near Ypres 7 Aug. I9I 5

Richards, C. W. Capt., R.A.S.C.

1912

Richardson, C. A. 2nd Lieut., St Bees School O.T.C. I910

Robinson, J. H. Gnr., R.G.A.

ARobinson, J. S. Instructor Lieut., R.N.

Died on active service I3 Nov. I9I8 of meningitis following influenza

RoBSON, A. Instructor Lieut., R.N.

Rogerson, S. Capt., W. Yorks. Regt.

Roper, H. Capt., Devon Regt. (W.) M.C.

\&Rosier, J. E. R. Lieut., R.F.A.(T.F.)

Died 20 Sept. I9r 6 of wounds received in action

1899

1907

*RouQuette, D. G. 2nd Lieut., R.A.S.C.; 2nd Lieut. (A), I9ro

R.F.C.

Killed in action 26 Sept. 1917

Roy, D. W. Surgeon, R.N.V.R.; Major, R.A.M.C. $\quad 1899$

Russell, H. B. G. Major, R.A.M.C.; D.A.D.M.S. M. 1905

French Croix de Guerre

Russell, Rev. W. B. C.F. $4^{\text {th }}$ Class, R.A.C.D. 1908

SARra, E. R. Surgeon Sub-Lieut., R.N.V.R. I913

SAunders, C. Lieut. (A.), R.A.F. (W.) r9r4

Schiller, L. C. T. Capt., Lincolnshire Regt.(T.F.) [1914] (W.) M.C.

Schooling, A. J. Pte., R.A.M.C.; 2nd Lieut., R.A.S.C. $\quad$ I900

Scorgie, N. G. Lieut.-Col., Deputy Director, Army 1903

Printing and Stationery Services. O.B.E. M 2.

Scotr, D. A. Lieut., King's Own (Yorkshire L.I.) 1912

Sedgwick, C. H. Capt. and Q.M., R.A.M.C. (2nd N. 1895

Gen. Hospital, T.F.)

Sells, A. L. Pte., H.A.C.

WShankster, G. Lieut., Northamptonshire Regt. (W 2.) 1912

Killed in action 9 Oct. 1916

Sherwood, H. P. Capt., R. Warwickshire Regt.(T.F.); 1912 attd. R.E. (Signals). M.C. M.

ShorTer, R. C. 2nd Lieut., R.E.; Capt., Spec. List (Area 1904 Gas Officer)

Silley, P. G. Lieut., R.N.V.R.

1892

Simpson, S. G. Major, R.A.S.C.; G.S.O. 3 (Education). 1900 O.B.E. $M 2$.

Sims, Rev. F. A. Chaplain, R.N.

Sleight, K. R. Lieut., London Regt. (Blackheath and I9ro Woolwich Bn.) 
Smith, C. W. W. See Winwood-Smith, C. W.

Smith, J. L. Lieut., Denstone College O.T.C.

सSmith, W. H. Major, R.F.A. $M 2$.

Killed in action 12 April r917

SPERO, L. Lieut., King's Royal Rifle Corps. (W.)

«SPINK, C. C. 2nd Lieut., E. Yorks. Regt.

Killed in action 4 Fune 1916

Spring, E. Capt., Somerset L.I.(T.F.) (W.)

Staffurth, A. E. Capt., R.A.M.C.

1908

1904

Stanhope, R. M. 2nd Lieut., R.G.A.

ÆSTenhouse, J. M. Capt., R.A.M.C. M.C.

Died 25 Aug. I916 of wounds received in action

1907

1910

I910

1906

1905

1897

Stevenson, A. W. 2nd Lieut., N. Staffs. Regt. and

Sherwood Foresters (Notts. and Derby Regt.); Lieut.

1914

(A.), R.A.F. D.F.G.

Stevenson, F. P. Capt., Radley College O.T.C.

Stewart, E. W. H. Capt., Canadian A.S.C.

Strickland, N. Capt., Repton School O.T.C.

Stokes, F. L. B. 2nd Lieut., Sherwood Foresters(Notts. and Derby Regt.)

StubBS, S. S. Lieut., R.G.A.

SutCliffe, A. L. Surgeon Lieut., R.N. $M$.

Sylvester, H. A. Rhodesia Field Force

Symons, W. J. F. Capt., R.A.M.C.

1902

1905

1899

1913

I913

1906

I9I I

1907

सTAYlor, G. S. Pte., R.A.M.C.

Missing, presumed killed in action, 18 Sept. 1916

I9II

Telfer, L. P. Pte., Bengal Bn., Indian Defence Force 1909

Temperley,E.E.V. Major, R.E. M.C. and Bar. Belgian 1908

Croix de Guerre

Terry, H. V. Capt. (Airship), R.A.F. $M$.

Thomas, D. E. Lieut., R.G.A. Medal for Military Valour

Thomson, F. G. Capt., R.A.M.C.

1904

Tobias, Rev. G. W. R. C.F. $4^{\text {th }}$ Class, R.A.C.D. M.C.

Tresawna, W. S. Capt., R.A.M.C.(T.F.) $M$.

1892

1904

1898

Vogel, E. P. See Oscroft, E. P.

Waldram, H. G. Capt., Devon Regt.(T.F.) (P.) $M . \quad 1908$

Wales, H. Capt., R.A.M.C. $M$.

1896

Walker, J. E. Capt., R. Fusiliers and Labour Corps $\quad 1896$

Walker, L. H. T. Capt., Welsh Regt. (W.) M.C. I9I3

Walker, S. L. Capt., Canadian A.M.C. $M$. I900

Wallace, C. R. P. 2nd Lieut., E. Yorks. Regt.

1912 
WALleR, F. 2nd Lieut., Border Regt. (W.)

WALTER, C. Lieut., R.G.A.

Ward, Rev. E. H. C.F. th $^{\text {th }}$ Class, R.A.C.D.

1903

1896

$\begin{array}{ll}\text { WARD, Rev. E. H. C.F. 4th Class, R.A.C.D. } & 1907 \\ \text { WWARD, W. D. Sergt., London Regt. (Cyclist Bn.); Capt., } & \text { I90I }\end{array}$

Hampshire Regt. (Cyclist Bn.); attd. Indian Army

Died 4 Sept. 1918 of injuries accidentally received

Watkins, E. V. Lieut., Welsh Regt.

WWatson, T. P. Capt. and Adjt., E. Lancs. Regt. (W.) I9Io

M.C. $M 2$.

Killed in action 7 March 1917

Webb, G. C. N. Capt., Dorset Regt. $M$.

Webi-Benton, J. American Army

WeLIFR, H. J Jnd Lieut. Bedfordshire Regt.; Iieut, I9I I M.G.C. (W 2.)

Westhead, W. H. Lieut., R.G.A. (W.) M. Chevalier, I9I4

Ordre de la Couronne (Belgium). Belgian Croix de Guerre

WHEELER, A. M. Lieut., R.G.A.(T.F.)

White, K. H. Major, R.A.S.C. $M$.

WhItMORE, H. S. 2nd Lieut., King's (Shropshire L.I.)

Wurus. 1909 Spec. List. M.B.E. Cavalier, Order of the Crown of Italy

Wilkinson, F. Lieut., S. Staffs. Regt.(T.F.); Lieut. (A.) 1913 and Capt. (Ad.), R.A.F. (W 2.) $M$.

Wilkinson, F. C. Hon. Capt., R.A.M.C.

Williams, C. H. 2nd Lieut., Welsh Regt.

Williams-Ellis, R. G. Lieut., R. Welsh Fus.(T.F.); 1896 Capt., R.A.S.C.

Wilson, B. Capt., Manchester Regt.; attd. T.M.B. $\quad 1908$

Wilson, H. O. S. Lieut., R.A.S.C.

Winwood-Smith, C. W. Lieut., Devon Regt. and Gen. 1912 List (T.M.B.); empld. War Office

Wood, B. R. Trooper, United Provinces Horse, Indian 1909

Defence Force

Wood, N. P. 2nd Lieut., Cheshire Regt.

Woolf, E. S. Capt., R.A.S.C.; Major, D.A.D. Transport 1902

Woolf, P. S. Lieut., 2oth Hussars. (W.) $M$. 1908

Wright, J. Aldren. Major, R.A.M.C. (Ist E. Gen. Hos- * I902 pital, T.F.)

WyCHE, C. K. H. Lieut., Norfolk Regt.

Wynne-Edwards, J. C. Capt., R. Welsh Fus.

Young, J. G. C. Lieut., R.G.A. 


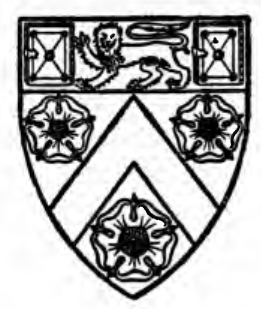

\section{TRINITY COLLEGE}

Аввот, H. Capt., R.F.A. (W.) $M$.

1889

Acheson-Gray, C. G. A. Lieut., Dorset Regt.(T.F.) 1907

AdaIr, J. V. Capt., N. Irish Horse. M.C.

Adams, C. W. Lieut., R.G.A.(T.F.) (W.)

1907

Adams, E. P. Capt., R.E. O.B.E. M.

1901

ADAMS, W. McM. 2nd Lieut., R.E.; Lieut. (K.B.), United 1902

States Air Service. (W.) M.C.

1914

AAdamson, W. Capt., Loyal N. Lancs. Regt.

Killed in action 24 April rgı 6

AAdDy, J. C. Capt., E. Yorks. Regt. M.C.

Killed in action 3 May 1917

Adie, C. J. M. Lieut., Eton College O.T.C.

Adrian, E. D. Capt., R.A.M.C.

AgAR, C. T: Lieut., R.G.A.

AgAR, H. E. T. Major, R.E.(T.F.) $m 2$.

1904

I910

I895

1908

1891

1894

Agrus, E. E. G. Lieut., London Regt. (R. Fus.); Capt., 1907

Spec. List (Adjt., Reception Camp). (W.)

AGNew, C. G. Capt., Manchester Regt. and Gen. List I90I (O.C.B.) $M$.

Agnew, J. S. Capt., Suffolk Yeo.; D.A.A.G. M. Cav- 1898 alier, Order of the Crown of Italy

Agnew, V. C. W. Capt., Suffolk Yeo. and Suffolk Regt. 1906 (T.F.)

AInsworTh, T. 2nd Lieut., I Ith Hussars

A ITKEN, D. B . Lieut., Seaforth Hdrs ; Lieut (A), R.A.F. (W.) D.F.C.

AAlbRight, M. C. Major, Worcestershire Yeo.

Died 8 Nov. I9I 7 of wounds received in action

I9I3

1905

HAldersey, H. Capt., Cheshire Yeo.

Killed in action 10 March 1918 
Aldridge, E. A. Capt., R.A.M.C. (W.) M.C. Brevet 1895 Major. $M$.

Alexander, F. D. Capt., I9th Hussars; Major, D.A.D. $\quad$ I897

Remounts. (W.) M2. Belgian Croix de Guerre

Alexander, J. F. Lieut., R.A.M.C.

Allan, A. C. 2nd Lieut., Middlesex Regt. (W.)

I895

AllCARD, R. Capt., R.E. O.B.E. M.

Allchin, G.C. Lieut., Queen's Own (R.W. Kent Regt.); I9I4

Capt., R.E. M.C.

Allen, Rev. G. K. C.F. $4^{\text {th }}$ Class, R.A.C.D.

Allen, J. Lieut., R.G.A.

\#Allen, J. E. R. Lieut., I6th Lancers. (W.) $M 2$.

Died 8 April 1918 of wounds received in action

Allen, Rev. L. J. Chaplain, R.N.

AAlleN, M. R. H. A. Lieut. (A.), R.F.C.

Killed in flying accident $2 \mathrm{I}$ March $\mathrm{I} 9 \mathrm{I} 7$

AlleN, R. C. Lieut., R.F.A.(T.F.); Lieut.(A. and Ad.), I9I4 R.A.F. (W.)

Allen, W. H. Hon. Major, R.G.A.

Allfrey, F. H. Capt., R.A.M.C. $M$.

1892

1891

Allgood, G.H. Capt., Northumberland Fus. (W.) M2. I9I I

Allhusen, O. Major, R.F.A.(T.F.)

Allmusen, R. Major, R.F.A. (W.)

1907

1912

Allix, C. I. L. Lieut., Coldstream Gds.

1891

HAllom, C. C. G. Capt., R.F.A.

Died 20 Oct. I9I7 of wounds received in action 9 Oct. 1917

AlthoRP, Viscount. Capt., Life Guards; A.D.C. (W.) I910 ANCASTER, Earl of, T.D. Lieut.-Col., Lincolnshire Yeo. 1886 (T.F. Res.) $m$.

Anderson, A. E. D. Capt., King's Own Scottish Borderers; Brigade Major; Lieut.-Col., Spec. List (School of Instruction). (W.) D.S.O. M.C. M 3 . Anderson, A. P. M. See Moore-Anderson, A. P.

Anderson, C. A. Surgeon Lieut., R.N.

Anderson, G. B. Capt., R.F.A. M.C.

ANDERson, N. L. Capt., S. Staffs. Regt.; empld. O.C.B. (W 2.)

ANDRAS, J. B. Capt. and Adjt., E. Surrey Regt.(T.F.) 1910 HANDREwes, C. N. Lieut., Labour Corps

Died 29 Nov. I9I 8 of influenza contracted on active service

ANDREWS, K. T. Major, R.A.S.C.

Ansbacher, S. S. See Ansley, S. S.

1905

1914

1913

1895

1912 
Ansdell, T. A. Australian A.M.C.

1909

सANsell, A. G. 2nd Lieut., R.E. (Field Survey Coy.) I9r2

Died 25 April 1918 of gas poisoning

Ansley, S. S. Capt., R.H.A.(T.F.) (W.) M.C.

Anson, G. F. V. Surgeon Lieut., R.N. (W.)

1912

Anstey, T. C. R. Capt., Sherwood Rangers. (W.)

I9IO

ANTrobus, M.

(W 2.)

सAPPERLEY, B. L. M. 2nd Lieut., Queen's Own (R.W. I9I I Kent Regt.) (W.)

Died I9 April I9I7 of wounds received in action

Arbuthnot, L. G. Capt., Lancs. Fus.; A.D.C. (W.) 1886 M.B.E. Order of the White Eagle, 5th Class, with swords (Serbia)

Arbuthnot, M. A. Capt., Seaforth Hdrs.; D.A.M.S., 1897

War Office. (W.) O.B.E. Brevet Major

Arbuthnot, R. W. M. Capt., R.F.A.; Staff Capt. (W.) 1908

M.C. French Croix de Guerre

ArCher, C. W. Surgeon Lieut., R.N.

ARKwRIGHT, J. A. Capt., R A M.C. Order of the 1905

White Eagle, 5 th Class, with swords (Serbia)

Arkwright, R. O. W. Lieut., 4th Hussars. (W.)

Armitage, N. C. Lieut., R.G.A.

Armstrong, J. R. B. Lieut., N. Irish Horse and 8th I9II

(King's R. Irish) Hussars; Capt., Tank Corps

WArmstrong, M. R. L. 2nd Lieut., R.F.A. and R.E. $\quad$ I908

Killed in action 23 April r916

Armstrong, R. R. Capt., R.A.M.C.

Arnallt-Jones, G. C. 2nd Lieut., Suffolk Regt.

ARNotT, R. J. Lieut., King's Royal Rifle Corps

Ash, F. H. Lieut., 4th Hussars. French Croix de Guerre 1912

Ast, G. B. Capt., Spec. List (Dental Surgeon)

Ash, O. A. Lieut., R.G.A.

Askew, E. J.P. Capt., R.F.A. ; empld. Ministry of Labour Aspinall, G. Lieut., Lancs. Hussars

Assheton, R. T. Capt., Cambridgeshire Regt.; Major,

M.G.C. (W.)

Athill, C. R. W. 2nd Lieut., Middlesex Regt.

1903

I9I6

[1914]

I90I

1914

1905

1903

1913

Athill, F. R. I. Lieut.-Col., Northumberland Fus.; 1899

D.A.A.G., War Office. (W.) C.M.G. M2. m.

Atkinson, A. G. Capt., R.A.M.C. (Sanitary Service, I900 T.F.) M.B.E.

Atrinson, H. B. Capt., E. African Force. O.B.E. M 2. $\quad 1898$ Atkinson, P. Y. Lieut., 5th Dragoon Gds. M.C.

1905 
Attenborough, C. R. W. Capt., London Regt.(R. Fus.) 1906 (W.)

*Austen-Cartmell, A. J. Lieut., King's Royal Rifle Corps 1912 Killed in action I Fune 1916

Austen-Smith, H., C.I.E. Lieut.-Col., I.M.S.

Aylmer, E. K. G., C.B. Bt. Colonel, Res. Regt. of Cavalry 1877

Ayton, W. A. Lieut., R.G.A.

I 884

1899

BABER, J. B. Capt., London Regt. (Queen's Westminster I9I I Rifles) and M.G.C. M.C. M.

BaCK, H. C. Lieut., Spec. List. (R.T.O.)

1882

सBackhouse, H. E. Capt., Sherwood Foresters (Notts. I90I and Derby Regt.)

Killed in action 15 Oct. I916

Backus, A. R. Capt., Rifle Brigade. (W 2.) M.C.

Accidentally killed near Neuve Eglise 22 Sept. I917

Bacon, F. Lieut., R.N.V.R.; empld. Admiralty

Bacon, F. R. Lieut., Cameronians (Scottish Rifles)

Bacon, R. C. Capt., R. Defence Corps

BAERLEIN, H.P. D. Service Sanitaire, French Army

Baerlein, O. F. Capt., R.A.S.C. M.C.

I9I3

I 899

I9ro

1885

1893

I9II

BagGallay, M. E. C. Lieut., I I th Hussars; A.D.C. M. 1907

BAHR, P. H. Capt., R.A.M.C. D.S.O. Brevet Major. 1900 $M$.

BaIley, F. G. G. Lieut.-Col., R.F.A. (W.) Brevet 1898 Lieut.-Colonel

BAILEY, W. N. Instructor Lieut., R.N.

Bainbridge, F. A. Capt., R.A.M.C.

1912

1893

ABarnbrigge, P. G. 2nd Lieut., Lancs. Fus.; attd. Welsh 1909

Regt.

Killed in action 18 Sept. 1918

Baines, J. T. Capt., Welsh Regt.; empld. O.C.B. (W.) I9I3

Balfour, C. M. Lieut., R.E.(T.F.); Capt., Tank Corps. 1909 $M$.

HBalfour, J. Capt., Scots Gds.; attd. R.E.(Signals). M.C. I9I3 $M$.

Killed in action 21 March 1918

Balfour, M. Major, R.F.A. M.C.

I897

Ball, O. J. H. Hon. Brig.-Gen. (ret.); Staff Lieut. m. 1871

ABallamy, H. W. Lieut., R.F.A.(T.F.) (W 2.)

Killed in action 15 Aug. I917

WBallance, L. A. 2nd Lieut., London Regt. (Civil Service 1907 Rifles); Capt., King's Royal Rifle Corps

Killed in action 28 Sept. 1916 
Balston, F. W. Capt., R.A.S.C.

1899

Balzarotti, G. P. 2nd Lieut., Italian Army. m. 1909

BANBURY, F. E. 2nd Lieut., Coldstream Gds. I9I2

Banbury, R. E. Capt., R. Fusiliers. (W.)

1898

Banister, C. G. Lieut., King's (Shropshire L.I.); Capt., 1908

R.E.; Hon. Capt. (T.), R.A.F.

Banks, P. Capt., R.F.A.

BARBER, J. R. Capt., Gen. List (T.F. Res.) M.C. $\quad 1896$

BARBER, T. P. Major, S. Notts. Hussars. (W.) D.S.O. 1894 $M 3$.

Barber-Starkey, F. W. G. Capt., Canadian M.G.C. 1903 (W.)

Barber-Starkey, R. J. K. 2nd Lieut., Shropshire Yeo. 1902 ABARBER-STARKEY, W. H. J. Capt., R.F.A.

Died in German hands Io Sept. I9I4 of wounds received in action at Le Cateau 26 Aug. 1914

Barbour, R., T.D. Major, Cheshire Yeo.

Barclay, A. V. Corpl., E. African Mtd. Rifles; Capt., 1906 King's African Rifles. (W 2.)

Barclay, C. Major and Adjt., Res. Regt. of Cavalry; 1885 attd. Ioth Hussars. $m$.

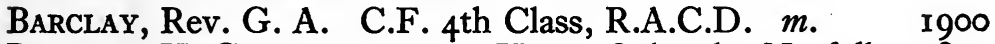

Barclay, H. G., M.v.o., v.D. Hon. Colonel, Norfolk I870 Regt.(T.F. Res.)

Barclay, R. L. Capt., Norfolk Yeo.; Major, Q.M.G.'s I886 Dept., War Office. C.B.E. O.B.E.

Barclay, R. W. Lieut.-Col., Surrey Yeo.; Capt., 2nd Life 1900 Gds.

सBarclay, T. H. Major, Surrey Yeo. Silver Medal for 1902 gallantry in saving life at sea

Drowned on H.M. transport Transylvania 4 May I9I7

Baring, Hon. M. Major, S.O. 2, R.A.F. O.B.E. M 2. 1893 Chevalier, Legion of Honour (France)

BARKER, A. Capt., R.A.M.C.

BARKer, Rev. A. L. C.F. 4th Class, R.A.C.D.

BARKer, F. W. Capt., London Yeo. (Westminster Dra- 1907 goons). $M$.

Barker-Hahlo, H. Capt., N. Somerset Yeo. and Gen. I89I Staff

Barlow, A. W. L. 2nd Lieut., King's Royal Rifle Corps I9Io BARNARD, Lord. Major, Westmorland and Cumberland 1907 Yeo.; attd. Border Regt. (W 2.) M.C.

BARNes, R. S. Capt., Suffolk Regt.(T.F.); A.P.M. M. $\quad$ I898

BARNES, T. H. Lieut., R.F.A. (W.) 
Barnes-Gorell, T. A. R. Lieut., Sherwood Foresters I9I I (Notts. and Derby Regt.) (W.)

BARNETT, B. L. Lieut., R.E.(T.F.) M.C. $M 2$.

Barningham, E. Capt., Loyal N. Lancs. Regt.

I9I3

BarNINCHAM, H. Lieut., Loyal N. Lancs. Regt.; Lieut. [ I9I I (A.), R.A.F.

BARNSLEY, D. G. Major, Gloucestershire Regt.; Lieut.- 1902 Col., R. Warwickshire Regt. (W.) M.C. m.

BARNSLEY, R. E. Major, R.A.M.C.; D.A.D.M.S. (W.) 1905 M.C. Brevet Major

WBarnsley, T. K. Capt., R. Warwickshire Regt. and Cold- I9I I stream Gds. (W.)

Killed in action 31 fuly 1917

Barraclough, R. F. Lieut., R.A.S.C. $m$.

BARRAN, C. R. Lieut., R.G.A. $M$.

BARRAN, H. V. F. 2nd Lieut., R.F.A. (W.) $M$.

1903

1904

BarratT, W. D. Capt., King's Own (R. Lancaster Regt., T.F. Res.)

BARRETt, R. C. Capt., D. of Wellington's (W. Riding rgr3 Regt., T.F.); A.D.C. $M$.

BarRon, E. A. W. Lieut., Res. Regt. of Cavalry; attd. rgor I2th Lancers. M.C.

BARROW, E. B. P. 2nd Lieut., R.F.C.

BarRowClOUGH, S. Lieut., R.F.A.

Barry, E. G. Wolfe. See Wolfe Barry, E. G. 1909 r9r3

Barry, K. A. Wolfe. See Wolfe Barry, K. A.

BARRY, T. Lieut., Cameron Hdrs. (W 2.)

ABARThropp, S. A. N. S. 2nd Lieut., R. Sussex Regt. Killed in action at Cuinchy 29 Fan. 1915

Bartlett, E. G. Lieut., King's Own (Yorkshire L.I.); 1913 Staff Capt. (W.) O.B.E. M.C. and Bar. M 2.

Bartram, L. H. Surgeon Sub-Lieut., R.N.V.R.

BARWELI N. F. Major, Oxford and Bucks. L I. L ieut 1913

Col., Gloucestershire Regt. (W.) M.C. M.

Batchelder, G. L. Lieut., United States Naval Res.

Bates, F. A. Capt., Denbigh Yeo.; Major (A.), R.A.F. 1903

(W.) M.C. A.F.C. M4 Greek Military Cross

Bateson, Hon. E. DE Y. Capt., R.A.S.C.(T.F.)

WBatley, R. C., T.D. Major, Dorset Yeo. I88I

Died 23 Oct. 1917

BatTiscombe, Rev. G. C. C.F. $4^{\text {th }}$ Class, R.A.C.D.(T.F.) 1879

Baxter, C. W. Capt., S. Lancs. Regt.(T.F.) (W.) M.C. I9I3

Baynes, G. Capt., 3rd Hussars. (W.) 1899

Baynes, H. G. Capt., R.A.M.C. $M$. 1904 
Baynes, W. E. C. Capt., Coldstream Gds. and Gen. 1894 Staff. (W 3.) M.C. M.

Beale, H. L. 2nd Lieut., Cambridgeshire Regt.; Capt., $\quad$ I897 R.E. (Inland Water Transport). $m$.

ABealey, A. C. Capt., Somerset L.I. M.C. M.

Died 22 Nov. 1917 of wounds received in action in

Palestine

Beasley-Robinson, A. C. Lieut., R.F.A. (W.) [I9I4]

Beauchamp, Rev. Sir M. H., Bart. C.F. 4th Class, $\quad 4^{\text {th }} 879$ R.A.C.D. $m$.

Beaufoy, H. M. Lieut., Spec. List, empld. Ministry of 1906 National Service

Beaumont, Hon. W.H. C. Capt., 2nd Life Gds.; Major, 1909 Gds. M.G. Regt. M.C.

Beaumont-Nesbitt, W. H. Capt., Grenadier Gds. (W.) I9I3 M.C.

Killed in action at Bourlon Wood 27 Nov. 1917

Beazley, C. M. Capt., Rifle Brigade. (W 2.)

1909

BeCher, G. G. Lieut., Marlborough College O.T.C. I905

ABECHER, M. A. N. Capt., King's Own Scottish Borderers I903

Killed in action in Gallipoli 26 April I9I 5

Beddington, C. Lieut.-Col., Westmorland and Cumber- $\quad{ }^{8} 88_{5}$ land Yeo. (T.F. Res.) (W.) $M$.

HBEDELl-Sivright, D. R. Surgeon, R.N.

Died in Gallipoli 5 Sept. I91 5

BEDFORD, D. J. Capt., R.A.M.C. $m$.

ABEER, A. H. Lieut., R.F.A.(T.F.) M.C. $M$.

Died 21 April I918 of wounds received in action at Béthune 19 April 1918

Beeton, A. E. Capt., R.E. M.C. M. French Croix 1898 de Guerre

Bell, C. W. D. Lieut., Ioth Hussars; Capt. (A.), R.A.F. IgI I WBell, G. M. Major, Hampshire Regt. D.S.O. M. Killed in action in the Third Battle of Ypres $3 \mathrm{I}$ Fuly 1917 EBell, J. J. Major, Ayrshire Yeo. Died 2 March 1915

WELL, W.H.D. Capt., N.Zealand Force and K. Edward's I902 Horse. $M$.

Killed in action 31 Fuly 1917

BelPER, Lord. Capt., Life Gds.; Major, Tank Corps 1902 Belville, G. E. Capt., I6th Lancers and Gen. Staff 1897 (O.C.B.) (W.) (P.)

Benjamin, C. M. 2nd Lieut., King's Own (Yorkshire [19I4] L.I.); Lieut. (T.), R.A.F. 
Bennett, E. Capt., R.A.S.C.(M.T.) $M 2$.

Bentinck, A. W. D. Capt., Coldstream Gds.; attd. 1906 Egyptian Army. (W.)

WBentinck, H. D. A. Major, Coldstream Gds. Brevet 1899 Major. $M$.

Died 2 Oct. 1916 of wounds received in action ${ }_{5} 5$ Sept. 19I6

Benturf, H. D. Lieut., Essex Regt.(T.F.); attd. Middle- I9ro sex Regt.(T.F.) (W.)

Bentwich, N. DE M. Major, Spec. List, attd. Egyptian rgor Camel Corps. (W.) O.B.E. M.C. M 2.

Berg, A. W. Lieut., Welsh Gds.; Capt., Spec. List

BERgER, S. H. 2nd Lieut., S. Wales Borderers; empld. I9I I Recruiting Staff

Bessborough, Earl of. Hon. Capt., Suffolk Yeo. (T.F. Res.); empld. War Office. C.M.G. m. Chevalier, Legion of Honour (France). Cavalier, Order of St Maurice and St Lazarus (Italy). Officer, Order of the Redeemer (Greece). Order of St Anne, 3rd Class, with swords (Russia)

Best, T. W. Major, Leicestershire Yeo.; Major (Ad.), R.A.F. (W.)

\&Bethell, C. Pte., Coldstream Gds.; Capt., King's Own 1904 (Yorkshire L.I.) $M$.

Killed in action $20 \mathrm{Feb}$. 1916

Bethell, D. J. Lieut., King's Own (Yorkshire L.I.); r9I I Capt., Scots Gds. M.C. and Bar. M.

Bethway, Rev. W. S. C.F. 4th Class, R.A.C.D.

Bevan, T. R. Capt., Hertfordshire Regt. (T.F. Res.); 1909 A.D.C.

Beveridge, H. Capt., Dorset Regt. (P.)

Beves, C. H. Paymaster Lieut., R.N.V.R. $M$.

Bewicke-Copley, R. G. W. Lieut., King's Royal Rifle Corps; Major, M.G.C. (W.) M.C. M. Italian Bronze Medal for Military Valour

Bickersteth, R. A. Major, R.A.M.C. (Ist W. Gen. Hos- I88I pital, T.F.) $m$.

BiCKnell, P. W. Capt., R.A.S.C.

BidDER, H. F. Major, R. Sussex Regt.; Lieut.-Col., 1894 M.G.C. D.S.O. M.

Biddle, F. A. Capt., Gen. List (T.F. Res.), empld.School 1896 of Musketry

Bigge, H. J. Lieut., Igth Hussars

BilNEY, A. A. H. Lieut., R.A.S.C. M.C. 
WBinning, Lord, c.B., M.v.o. Lieut.-Col., Lothian and 1876 Border Horse; Brig.-Gen.

Died 12 Fan. 1917 of pneumonia

BinYon, B. Major (A.), R.A.F. O.B.E.

1904

\Bon, R. E. Lieut., 2oth Hussars; Lieut. (A.), R.A.F. 1910

Killed in action 9 April 1918

BrRCH, A. G. Major, R.E.(T.F.); D. A. D. Light Rail- 1900 ways. (W.) D.S.O. and Bar. O.B.E. $M 4$.

Bird, G.F. Major, R.A.M.C.; D.A.D.M.S. M.C. M3. 1897

BIRD, L. W. Major, R. Berkshire Regt.; empld. School 1901 of Instruction. (W 2.) D.S.O. O.B.E. M2.

ABirkBECK, G. Lieut., Norfolk Yeo.

Died I9 Feb. 1916

WBirkbeCK, G. W. Capt., Norfolk Regt.(T.F.) $M$. Killed in action 19 April 1917

BirkBECK, H. A. Major, Norfolk Yeo. and Norfolk Regt. 1904 (T.F.) (W.) M.C.

BirLey, O. H. J. Capt., R. Fusiliers and Gen. Staff (In- 1898 telligence). M.C.

Bishop, J. H. Lieut., R.F.A.

ABLACK, D. C. 2nd Lieut. (A.), R.A.F.

Killed in flying accident 23 April 1918

Black, J. C. Capt. and Adjt., R.E. $M$.

I913

1905

BlaCK, R. P. Lieut., R.G.A. M.C.

I9II

1916

Blackburn, A. Capt., Rifle Brigade (T.F.)

BlackBuRN-MAZE, C. I. Lieut., Queen's Own (R.W. 1908 Kent Regt.)

BlackeTt, B. J. Lieut., N.S.W. Bn., Australian Force 1905

BlaCkLOCK, G. H. Lieut., Shropshire Yeo.; Lieut.-Col., 1896 A.D. Docks. $M$.

Blair, H. M. Capt., R. Welsh Fus. (W 2.) 1896

ZBlake, G. P. Capt. R. Welsh Fus.

Killed in action 20 Fuly 1916

Blakeney, Rev. E. P. C.F. $4^{\text {th }}$ Class, R.A.C.D. $\quad 1889$

Blanco-White, G. R. Capt., R.G.A. 1902

BLAND, A. J. T. Lieut., R.F.A. (W.) M.C.

Blom, A. H. Capt., Irish Gds. (W 2.) 1909

HBodenham, H. E. C. H. 2nd Lieut., Black Watch; I9r4 Lieut., M.G.C.

Killed in action on the Somme 7 Sept. 1916

\&olitho, G. R. 2nd Lieut., Devon Regt. and R.F.C. I9I I (W.)

Died 25 Oct. 1916 of wounds received in action

Bolitho, T. G. G. Capt. (K.B.), R.A.F. M.G. D.F.G. 1908 
Bolton, D. C. Capt., Manchester Regt.

WBolton, W. S. Sergt., R. Fusiliers (Sportsman's Bn.) 1904

(W.)

Died 7 Feb. r919 of pneumonia contracted on active service

Bond, A. C. 2nd Lieut., King's Own (R. Lancaster Regt.) ABond, C. G. Capt., Wiltshire Regt.

Killed in action 25 Nov. I9I 5

BonHam, E. H., M.v.o. Major, 2nd Dragoons (R. Scots $\quad 1892$ Greys). $M . \quad m$. Chevalier, Legion of Honour (France)

Wonham Carter, A. T. S. African Defence Force; Capt., 1887 Hampshire Regt. $m$.

Killed in action 1 Fuly 1916

Bonham Carter, F. H. Capt., R.G.A.

Bonvalot, A. C. Capt., Coldstream Gds.

WBonvalot, E. St L. 2nd Lieut., Coldstream Gds.

Killed in action 9 Oct. 1915

Воотн, C. Z. M. A.B., R.N.V.R.(R.N.D.); Lieut., Leic- 1906 estershire Yeo.

Booth, T. M. Major, Gordon Hdrs.; Brigade Major; 1892 Lieut.-Col., Asst. Military Attaché, the Hague. (W.) D.S.O. and Bar. $M 2$.

Bosanquet, A. R. Capt., King's Own (R. Lancaster 1909 Regt.) (W.) M.C.

Boscawen, Hon. M. T. Major, Rifle Brigade; Lieut.- I9ı Col., London Regt. D.S.O. M.C. M2.

HBoscawen, Hon. V. D. 2nd Lieut., Coldstream Gds.

Killed in action near Ypres 29 Oct. I914

Bostock, S. C. Lieut., Life Gds. (W.)

Bostock-Hill, A. J. Lieut., R.N.V.R.

Boughey, C. L. F. Lieut., Grenadier Gds. (W.)

BouQuet, Rev. A. C. C.F. $4^{\text {th }}$ Class, R.A.C.D. $m$.

Bourchier, Rev. LE G. C. C.F. $4^{\text {th }}$ Class, R.A.C.D.

Bouwens, B. G. Lieut., R.A.S.C.(M.T.) (W.)

1909

1898
[1914]

I910

Bovill, E. W. Lieut., Ioth Hussars; attd. Nigeria Regt.,

W. African Frontier Force. (W.)

Bowen, J. B. Capt., Pembroke Yeo.; Lieut.-Col. ('T.), 1902 R.A.F. O.B.E. $m$.

WBowen Colthurst, R. M. Capt., Leinster Regt.

Killed in action $\mathrm{I}_{5}$ March I9I 5

Bower, H. M. Capt., W. Yorks. Regt.(T.F.); empld. 1873 Records

WBowes-Lyon, G. P. Lieut., Grenadier Gds.

Killed in action 27 Nov. I9I7 
Bowes-Lyon, Hon. P. Major, Essex Regt.

1882

Bowlby, R. F. Lieut., London Regt.

Bowle, C. W. Lieut.-Col., R.A.M.C.

1909

1898

Bowman, F. Instructor Lieut., R.N.

I9II

Bowman, G. H. Capt., R. Warwickshire Regt.; Major 1910

(A.), R.A.F. (W.) D.S.O. M.C. and Bar. D.F.C.

M. Belgian Croix de Guerre

Bowman Vaughan, E. W. See Vaughan, E. W. B.

Bowring, J. F. E. Capt. and Adjt., Lancs. Fus.; Major, 1885

Labour Corps

\#Boyd, H. A. 2nd Lieut., R. Inniskilling Fus.

Killed in action 7 Sept 1914

Boyd, J. Lieut., United States Ambulance Service

I9I3

Boyd Rochfort, G. A. See Rochfort, G. A.B.

Boyle, G. F. Capt., R. Scots Fus.

Boys, G. V. Corpl., R.E. (Signals).

I910

Bozman, E.F. Capt., Queen's Own (R. W. Kent Regt.); [I9I4]

attd. The Buffs (E. Kent Regt.) (W.) M.C.

BRADFORD, Earl of. Lieut.-Col., R. Scots. $M$. 1893

BRADley, M. G. Major, Middlesex Regt. and Labour rgor Corps

Bradney, J. A., C.B., T.D. Hon. Colonel, London Regt. 1877 (Q.V.R., T.F. Res.); Lieut.-Col., Labour Corps. $m$.

WBradshaw, P.C. 2nd Lieut., King's Own Scottish I9I4 Borderers

Killed in action I May 1916

WBRadshaw, R. E. K. Lieut., London Regt. (Rangers) I9I4 Killed in action I fuly 1916

WBradshaw, W. D. 2nd Lieut., R.F.A.

Killed in action $3 \mathrm{I}$ Oct. I9I6

ABRAGG, R. C. 2nd Lieut., R.F.A.

Died 2 Sept. I915 of wounds received in action in

Gallipoli I Sept. I9I 5

BraGG, W. L. Major, R.H.A.(T.F.) O.B.E. M.C. M. 1909

BRAMwEll, B. S. Capt., R.F.A.(T.F.)

Bramwell, J. C. Capt., R.A.M.C.

1896

Branson, F. H. E. Lieut., R.A.O.C.; Major, D.A.D. ${ }^{1896}$

Equipment and Ordnance Stores, War Office. O.B.E.

M.B.E. $m$.

Branson, J. R. B. Major, R.A.O.C.; D.A.D.O.S.

Branson, W. P. S. Colonel, A.M.S. C.B.E. M.

Branston, C. A. Lieut., R.E. and R.A.S.C.(T.F.)

Brass, W. Capt., Surrey Yeo.; Capt. (T.), R.A.F.

Braun, G. C. P. 2nd Lieut., R.A.S.C.

I9I 5

1912

$$
\text { c.U.w.L. }
$$


Braunholtz, W. T. K. Sapper, R.E. (Signals)

Bray, Sir E. H. Brig.-Gen., Q.M.G.'s Dept., Indian 1893 Army. C.S.I.

Bray, F. E. Major, The Queen's (R. W. Surrey Regt., I90r T.F.) M.C. M 2. Italian Silver Medal for Military Valour

Bray, J. Capt., Surrey Yeo.(T.F. Res.) and Gds. M.G. $\quad 1897$ Regt.

WBREESE, W. L. 2nd Lieut., R. Horse Gds. Killed in action 14 March 1915

Bremner, F. D. H. Capt. (T.), R.A.F.

Brenan, A. R. M. Capt., R.A.M.C.

Brereton, J. L. and Lieut., R.G.A.; empld. Ministry of Munitions

Bretherton, Rev. H. Pte., London Regt. (Artists Rifles)

WBReul, O. G. F. J. Lieut., R.E. (Signals). M.C.

Died on active service 16 Oct. I9I7

Bridgeman, R. O. Capt., Rifle Brigade; Staff Lieut., 1908 War Office. (W.) Brevet Major

Brightman, E. W. Lieut.-Col., R.A.S.C. (Canteens). 1906 O.B.E. M.

BRINDLE, W. S. Lieut.-Col., Worcestershire Regt. $m . \quad 1888$ ZBRoAdBENT, C. H. 2nd Lieut., King's Own (Yorkshire 1900 L.I., T.F.)

Died I March 1916 of injuries received in bombing accident

BroadBENT, W. Major, R.A.M.C.(T.F.)

BRocklebaNk, J. J., D.s.o. Major, Scottish Horse; attd. Northamptonshire Regt.(T.F.) $m$.

HBodie, E. J. Capt., Cameron Hdrs.

Killed in action I I Nov. I9I4

Brodsky, G. A. 2nd Lieut., 18th Hussars; Capt., Gen. 1906 List

Brooke, B. W. D. Hon. Capt., R.H.A.(T.F. Res.)

Brooke, H. K. Capt., Gen. List, attd. Egyptian Army

Brooks, H. R. G. Major, R.G.A. (W.) M.C.

Brown, A. O. Lieut., R.E.(T.F.)

Brown, C. Clifton. Lieut., Sussex Yeo. and Grenadier Gds.

Brown, D. Clifton. Capt., Ist Dragoon Gds.; attd. $\quad$ I898 Army Cyclist Corps; Brigade Major

Brown, F. C. 2nd Lieut., Sherwood Foresters (Notts. 1900 and Derby Regt.) (W.) 
Brown, H. C. B. Capt., Worcestershire Regt.(T.F.) and I9I I Gen. Staff. (W.)

Brown, H. G. Capt., London Regt. (Finsbury Rifles). 1894 (W.)

Brown, J. L. C. Capt., R.G.A. (W.)

BRown, J. T. Trooper, Scottish Horse 1903 I880

BROwN, Rev. R. P. C.F. $4^{\text {th }}$ Class, R.A.C.D. 1902

Brown, Rev. T. B. Pte., London Regt. (Artists Rifles)

1912 of Labour. (W 2.) M.C. M.

Brown Douglas, F. C. Lieut., Rifle Brigade; Capt. (A.), I9I2 R.A.F.

सBrowne, C. P. Capt., Corps of Guides, Indian Army 1905 Killed in action I I April I915

\#Browne, M. B. 2nd Lieut., Sherwood Foresters (Notts. 1894 and Derby Regt., T.F.)

Died 30 April I916 of wounds received in the Irish rebellion

Browne, M. G. Capt., E. Lancs. Regt. (W 3.) (P.) 1907

Browne, O. L. Lieut., Cheshire Regt.(T.F.); Major, I9I3 M.G.C. (W.) $M$.

Browning, Rev. P. T. C.F. $4^{\text {th }}$ Class, R.A.C.D. I90I

BRUCE Clarke, W. R. Capt., London Regt. (London 1906 Scottish); Capt. (T.), R.A.F. M.B.E. $M 2$.

ABRUdENELl-BruCE, J. E. J. Lieut., Northamptonshire 1897 Yeo.

Died I I April I9I7 of wounds received in action

BRUNT, D. Capt., R.E. (Meteorological Section); attd. 1908 R.A.F. $M$.

\#BRunton, E. H. P. Lieut,, R.A.M.C.

Killed in action 8 Oct. I9I 5

Brunwin, A. D. Hon. Capt., R.A.M.C.

1907

1897

Brutton, H. L. Capt., Worcestershire Yeo.; attd. Wor- 1899 cestershire Regt.

Bryce, W. T. P. Lieut., R.G.A. $M$.

WBuchanan, A. N. Lieut. (T.), R.A.F.

Died 14 Oct. 1918 of pneumonia

Buchanan, B. R. 2nd Lieut., Labour Corps I9I4

Buchanan, J. N. Capt., Grenadier Gds.; Brigade Major. 1905 D.S.O. M.C. M.

Buckland, D. H. Lieut., King's Royal Rifle Corps. $M$. I9I4 ABuCKLaND, T. A. Lieut., Norfolk Regt. $M$.

Died 18 Oct. 1915 of wounds received in action $\mathrm{I}_{3}$ Oct. I9I I I9I5 
Buckle, G. W. Lieut., York and Lancaster Regt.; Capt., 1904 Army Cyclist Corps. $M 2$.

Buckley, J. P. Capt., R.A.M.C.(T.F.)

Buckley, R. M. Capt., King's Royal Rifle Corps; I9I3 Major, R.E. M.C.

Buckmaster, W. S. Service Sanitaire, French Army I89r

Buckston, G. M. Capt., Derbyshire Yeo.

Budenberg, C. F. Capt., R.E.(T.F.) M.C.

ABudenberg, D. H. Capt., Manchester Regt.

Killed in action near Voormezeele 25 April 1918

Budget, , H. M. Lieut., Spec. List

Bull, H. Capt., R.G.A. M.C.

Buller, M. L. Capt., King's Royal Rifle Corps. M.C. 1912

WBullivant, R. P. Capt., London Yeo. (Middlesex Hus- 1902 sars). (W.) M.C.

Killed in action 24 Sept. 1918

Bullock, C. Ll. Capt., Rifle Brigade; Capt.(A.), R.A.F.; 1910 Major, S.O. 2, Air Ministry. (W.) O.B.E. M.

Bullock, H. M. Capt., Scots Gds.; A.D.C. (W.) 1909

M.B.E. Chevalier, Legion of Honour (France)

Bullough, E. Lieut., R.N.V.R., empld. Admiralty 1899

Bulstrode, C. V. Major, R.F.A.(T.F.); Lieut.-Col., 1894 R.A.M.C. D.S.O. M.

Bunbury, C. H. N. Lieut., Wiltshire Yeo. and Cold- 1905 stream Gds.

Bunbury, Hon. T. L. McC. See McCinntock Bunbury, Hon. T. L.

Burbidge, P. W. Sergt.-Major, N. Zealand Force

Burchell, J. M. Capt., London Regt. (Q.V.R.); Staff Capt. (W.) O.B.E. M.

Burder, G. E. L. Lieut., R.G.A. (W 2.)

BurdetT, Sir F., Bart. Capt., I7th Lancers (R. of O.); 1888 Brigade Major

Burges, W. E. P. Hon. Colonel, Gloucestershire Regt.; 1879

Lieut.-Col., Spec. List, empld. Ministry of National

Service. O.B.E. $m$.

Burgess, O. I. 2nd Lieut., Monmouthshire Regt.; 1912

Capt., R.E. (Sound-ranging Section). $M$.

Burgoyne, L. S. Lieut., King's Royal Rifle Corps. M. I9ro

BurN, Rev. A. E. C.F. 2nd Class, R.A.C.D.(T.F.) I882

BuRN, J. S. Hon. Capt., R.A.M.C.

Burn-Murdoch, A. Lieut., R. Scots(T.F.) I905

BurN-Murdoch, H. Major, Cameron Hdrs. (R. of O.) 1899 and Argyll and Sutherland Hdrs. 
ABuRnaby, G. Lieut., London Regt. (R. Fus.)

Died ${ }_{23}$ Oct. $19 \mathrm{I} 6$ of wounds received in action

I913

Burnaby, Rev. H. B. F. C.F. $4^{\text {th }}$ Class, R.A.C.D. M.C. $\quad 1896$

Burnaby, J. Capt., London Regt. (R. Fus.) (W.) I9Io

WBURNAND, C. F. 2nd Lieut., Grenadier Gds.

1910

Killed in action I I March I9I 5

BurnetT, A. E. Major, King's Own Scottish Borderers. I900 (W.) O.B.E.

BurNs, J. W. Major, Res. Regt. of Cavalry I88I

BurReLl, L. S. T. Major, R.A.M.C. I90I

\#BURrell, R. F. T. 2nd Lieut., Queen's Own (R.W. I908

Kent Regt.)

Killed in action $26 \mathrm{Sept}$. I91 5

Burroughes, H. N. Lieut.-Col., R.A.M.C.(T.F.) I896

D.S.O. M 2. French Médaille des Epidémies

Burton, B. L.E. Capt., R.A.S.C.; Major, D.A.D. Trans- 1909 port. $M$.

Burton, M. G. W. Capt., R.A.S.C. (Canteens)

BURY, J. Lieut., I7th Lancers; A.D.C.

1912

Bury, L. E. Colonel, R.E. C.B.E. O.B.E. M 2.

[1914]

Bury, R. F. Capt., Essex Regt.; Major, Gen. List 1895

(D.A.A.G.) (W.) $M$.

Bushby, H. N. G. Capt., R. Defence Corps

*Butcher, W. G. D. Capt., London Regt. (L.R.B.)

Killed in action at Glencorse Wood I6 Aug. I917

Butler, Rev. A. J. A. Capt., Leicestershire Regt.

AButler, G. K. M. Lieut., Scottish Horse. (W.)

Died in Egypt 17 Fuly 1916

ButLER, H. C. 2nd Lieut., R. Inniskilling Fus.; Lieut., I9ro

R.E. (W.) M.C.

Butler, H. G. St P. Lieut., R.G.A.

Butler, J. R. M. Capt. and Adjt., Scottish Horse; 1907

Major, G.S.O. 2 (Intelligence). O.B.E. $M 2$.

Butler, N. M. 2nd Lieut., Scottish Horse and House- 1912

hold Bn.; Capt., Gen. Staff (Intelligence)

Butler, R. L. G. Capt., Spec. List; Town Major, Ypres. 1902

(W.)

${ }^{1}$ ButLER, W. M. Major, R.E.(T.F.)

Butler-Stoney, C. K. Major, Hampshire Aircraft Parks 1896

(T.F. Res.); Hon. Major, R.A.F. $m$.

WButlin, Sir H. G. T., Bart. Capt., Cambridgeshire Regt. I9 I I $M$.

Killed in action 16 Sept. 1916

1 Died on service after the armistice. 
ButT, G. M. Capt., R.A.S.C.

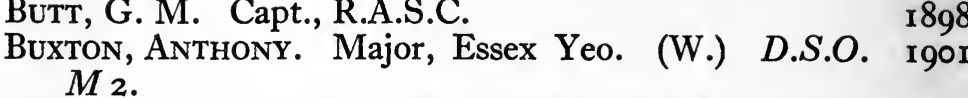

Buxton, Rev. Arthur. C.F. $4^{\text {th }}$ Class, R.A.C.D.

Buxton, Аввот R. Major, Norfolk Yeo.(T.F. Res.)

WBuxton, Andrew R. Capt., Rifle Brigade

Killed in action 7 Fune 1917

Buxton, B. G. Capt., D. of Wellington's (W. Riding I913 Regt., T.F.) (W.) M.C. and Bar

Buxton, C. E. V. Major, R.F.A.; A.D.C.; G.S.O. 3; 19 II Cmdt., Trench Mortar School. M.C. $M_{4}$.

Buxton, E. N. Capt., R.H.A.(T.F.) M.C. M.

Buxton, G. C. Capt., Coldstream Gds.; Major, King's 1897 African Rifles

Buxton, H. F. Capt., Suffolk Regt.(T.F.)

Buxton, H. G. Capt., Norfolk Regt.

Buxton, I. Major, Norfolk Yeo.; Brigade Major. (W.) 1903 D.S.O. $M_{3}$.

Buxton, L. G., M.v.o. Major, R.A. (R. of O.); D.A.A. $\quad$ I895 and Q.M.G. M.G. M. Chevalier, Legion of Honour (France)

Buxton, M. B. Capt., Norfolk Regt.(T.F.) (W.) M.C. 1908

Buxton, P. A. Capt., R.A.M.C.

Buxton, R. G. Capt., Norfolk Yeo.

Buxton, T. F. Lieut., Essex Yeo.; A.D.C. $M$.

ByrNe, L. W. Hon. Lieut.-Cdr., R.N.V.R.

I9II

1905

1908

1894

Cadbury, E. Major (A. and S.), R.A.F. D.S.C. D.F.C.

Cadman, P. S. Major, E. Riding of Yorkshire Yeo.(T.F. $\quad$ I890 Res.)

Caldwell Smith, E. L. Surgeon Lieut., R.N.

Caledon, Earl of. Capt., Ist Life Gds. and M.G.C. (W.)

Callingham,L.F. Lieut., R.N.V.R.(Hood Bn., R.N.D.) 1906 (W.)

Calverley, E. L. Major, Essex Regt.(T.F. Res.) and 1883 Hampshire Regt.; Staff Capt. O.B.E. $M 2$.

Calvert, H. Lieut., Loyal N. Lancs. Regt.(T.F. Res.) 1895 Calvert, H. H. Lieut.-Col., Gloucestershire Yeo.(T.F. 1878 Res.)

Calvert, W. J. R. Capt., Harrow School O.T.C.

Camden, Marquis. Major, W. Kent Yeo.(T.F. Res.) 
Campbell, A. D. Lieut., 9th Lancers

Campbell, A. D. P. Lieut., Denbigh Yeo. and Remount 1899 Service

Campbell, A. Y. G., C.I.E. Major, S. Provinces Mtd. I89I Rifles, Indian Defence Force. C.B.E. M.

Camprell, D. S. Capt., W. Kent Yeo. and The Buffs rgio (E. Kent Regt., T.F.) M.C.

Campbell, E. M. Lieut., R. Scots(T.F. Res.)

Campbell, E. R. Capt., R. Sussex Regt.(T.F.) and Egyp- 1906 tian Camel Corps. (W.) $M$. Order of the Nile, $4^{\text {th Class }(E g y p t)}$

\&Campbell, I. M. Lieut., Sussex Yeo.; attd. R. Sussex [1914] Regt.

Died 4 April 1918 of wounds received in action

Campbell, Hon. I. M. Major, Lovat's Scouts; Lieut.- 1902 Col., Argyll and Sutherland Hdrs. D.S.O. M.

\&CAMPBell, J. A. Lieut., 6th (Inniskilling) Dragoons

Died in Germany 2 Dec. 1917 of wounds received in action

Campbell, K. A. Lieut., Grenadier Gds. (W 2.) D.S.O. $M$.

Campbell, R. C. Lieut., Somerset L.I.; Capt. (Ad.), I9I4 R.A.F.

CAMPBell, Sir W., C.B., D.S.o. Major-Gen.; D.Q.M.G. 1882 K.C.B. K.C.M.G. . M II. Commander, Legion of Honour (France). Order of the White Eagle, 2nd Class, with swords (Serbia). Grand Commander, Order of the Redeemer (Greece). Order of the Nile, 2nd Class (Egypt). Order of El Nahda, 2nd Class (Hedjaz)

Campbell, W. G. Lieut., Sussex Yeo.; attd. R. Sussex I9Io Regt.

Campbell-Douglas, Rev. Hon. L. C. H. C.F. $4^{\text {th }}$ Class, I899 R.A.C.D.

\&Campbell-Johnston, P.S. Lieut., R.F.A.; A.D.C. (W.) I9I3 M.C. Belgian Croix de Guerre

Died 30 Aug. 1919 of wounds received in action 21 May 1918

Campling, Rev. W. C. C.F. $4^{\text {th }}$ Class, R.A.C.D.

Cantrell-Hubbersty, G. A. J. Major, S. Notts. Hussars and M.G.C. (W.) D.S.O. M.

CAPpel, N. L. Lieut., R.N.V.R.

Capstick, J. W. Service Sanitaire, French Army

CAREw, P. G. Capt., R. North Devon Yeo. and Remount Service. (W.) 
*Carlile, E. H. H. Capt., Hertfordshire Yeo.; attd. Hert- $\quad$ I899 fordshire Regt.

Killed in action 22 March 1918

Carlisle, J. C. D. Capt., London Regt. (Civil Service 1906 Rifles); Major, G.S.O. 2. D.S.O. M.C. $M_{3}$.

CARmichael, A. D. Major, Lovat's Scouts and Black 1897 Watch. $M$.

CARR, A. L. Lieut., R.E. $M$.

CARr, C. T. Capt., Wiltshire Regt.(T.F.); Major, $\quad$ I897 D.A.A.G. $M$.

Carr-Ellison, H. G. C. Capt., Northumberland Yeo.; 1893 attd. 2ist Lancers

CARroll, H. E. Lieut., R.E. (Fortress, T.F.) . 1902

Carson, T. Major, R. Irish Regt.

CARTER, C. N. Surgeon Lieut., R.N.

CARTER, W. E. Lieut., R.G.A.; Draft Conducting Officer 1904

Carus-Wilson, C. C. Capt., R.M.A. M.C.

Carus-Wilson, E. Capt., R.E. (Signals). M.C. M 2. 1908

Carver, G. A. Major, R.F.A.(T.F.) M. $\quad 1906$

4CARVER, O. A. Capt., R.E.(T.F.)

Died 7 Fune 1915 of wounds received in action in Gallipoli

CASEY, R. G. Major, Gen. List, Australian Force; I9ro G.S.O. 2. D.S.O. M.C. $M 2$.

Cassillis, Earl of. Major, R. Scots Fus. and Gen. Staff 1890

(Town Major of Bailleul). $M$.

$\begin{array}{llll}\text { Castellain, J. G. Capt., R.A.M.C. } & & \text { 1894 } \\ \text { Castellan, V. E. Major, R.F.A.(T.F.) } & \text { O.B.E. } & \text { T.D. } & \text { I89I }\end{array}$ $M$.

¥CAstle, T. R. Pte., R. Fusiliers (P. S. Bn.); 2nd Lieut., I90I The Queen's (R. W. Surrey Regt.)

Killed in action $3 \mathrm{I}$ Aug. I9I6

Castle, W. F. R. Surgeon Lieut., R.N.V.R. D.S.C. I9ro

Castleman, E. W. F. Major, Dorset Yeo. (W.) 1889

Castlerosse, Viscount. Capt., Irish Gds.; G.S.O. 3; 1909 empld. Ministry of Information. (W.)

Cator, C. G. L. Lieut., R.N.V.R.

Catterall, E. C. 2nd Lieut., S. Staffs. Regt.; Lieut., I9I3 M.G.C. (W.)

Cave, C. J. P. Capt., R.E. (Meteorological Section). $m$. $\quad$ I889 CAve, L. C. H. Lieut. (T.), R.A.F.

Cavendish, A.E. J. Colonel, A.A.G.; Brig.-Gen. C.M.G. 1877

CAVENDISH, Lord J. S., D.s.o. Major, Ist Life Gds. 1893

Killed in action 20 Oct. I914 
Cavendish, Lord R. F. Hon. Colonel, King's Own 1889 (R. Lancaster Regt., T.F.) C.B. C.M.G. M.

सCAY, A. J. Lieut., Worcestershire Yeo.

Killed in action 23 April 1916

Cayley, D. C. Capt., King's Own Scottish Borderers; [I9I4] Major, M.G.C.

Cazalet, C. H. L., D.s.o. Major, Spec. List

FCazalet, E. 2nd Lieut., The Buffs. (E. Kent Regt.) and I9I3 Welsh Gds.

Killed in action ro Sept. 19r6

$\begin{array}{ll}\text { CECIL, A. W. J. Capt., Grenadier Gds. } & \text { I895 } \\ \text { CECIL, R. E. Major, 2 Ist Lancers; Lieut.-Col., G.S.O. I. } & \mathbf{1 8 9 7}\end{array}$ D.S.O. M.

Chadwick, H. Capt., R.A.M.C.

Chadwick, J. Pte., R.A.M.C.

CHaDwick, J. F. Capt, R.E. (Signals) M.C. ${ }^{\text {I9I4 }}$

सChadwick, J. H. Pte., R. Fusiliers (P. S. Bn.); Lieut.- 1907

Col., Manchester Regt. D.S.O.

Killed in action 4 May 1917

Challinor, J. and Lieut., R.G.A.

Chaulinor,W F. 1913

ChallinOR, W.F. Hon.Lieut.-Col.,R.F.A. D:S.O. M. r900

Chalmers, I. P. H. Capt., Seaforth Hdrs. (W 2.) 1906

Chaloner, T. W. P. L. Capt., Yorkshire Regt.(T.F.); 1908 Capt. (A.), R.A.F. (P.)

AChamberlain, E. D. 2nd Lieut., Loyal N. Lancs. Regt. 1912 Killed in action 30 Nov. I917

Chamberlain, W. B. Lieut., Worcestershire Yeo. (W.) rgor (P.) M.C.

Chambers, B. F. Capt., R.A.S.C.

Chambers, C. E., v.D. Bt. Colonel, R.F.A.(T.F. Res.) $m$. I88I

Chambers, W. F. A. Lieut., King's Royal Rifle Corps. I9r3 (W 2.)

\&Chance, E. S. Capt., R. Welsh Fus.; Lieut.-Col., 2nd 1900

Dragoon Gds. (Queen's Bays); D.A.A. and Q.M.G.

(W.) Brevet Major. $M 2$.

Killed in action 29 May 1918

Chance, R. C. 2nd Lieut., Spec. List

Chance, R. J. F. Capt. and Adjt., 4th Dragoon Gds.; I9I2

Capt., Rifle Brigade. (W 2.) M.C. $M 2$.

Chandless, C. T. C. Lieut., R.A.S.C.(M.T.)

Channell, H. M. T. 2nd Lieut., R.A.S.C.

Chaplin, Rev. A. C.F. $4^{\text {th }}$ Class, R.A.C.D.

1906

1905

1895

Chapman, A. E. Lieut., Gen. List (T.F. Res.); Capt., 1904

Spec. List, empld. Ministry of National Service 
Chapman, T. M. G. Lieut., R.A.S.C.

WChapman, W. H. Capt., Yorkshire Regt.

Killed in action in Gallipoli 7 Aug. 1915

4Charles, J. A. M. 2nd Lieut., King's (Shropshire L.I.) 1908 Died ro Feb. I91 5 of wounds received in action 23 Oct. 1914

Charles, L. B. Lieut., The Queen's (R. W. Surrey Regt., T.F.); Staff Capt. (W.) M.C. M.

Chart, H. J. Lieut., R.F.A.(T.F.); empld. Ministry of $\quad$ r889 Munitions. $m$.

Cheales, R. D. Major, E. Lancs. Regt.; Lieut.-Col., 1888 R. Scots and Gen. List. (W.) O.B.E. $M 2$.

Chenevix-Trench, A. S. Major, R.E. (W.) M.C. M. 1905

Chepmell, C. H. Major, R.G.A. (R. of O.) I883

Chester, R. C. Cadet, O.C.B. (Household Bde.) 1914

Chevassut, F. G. 2nd Lieut., R.F.A. (W.) 1909

Cheyne, W. H. W. Surgeon Lieut., R.N. 1907

Chichester, A. O'N. C. Capt. Surrey Yeo.; Major, The rgo8 Queen's (R.W. Surrey Regt.) (W.) M.C.

Chichester, Earl of. Major, R. Sussex Regt.(T.F.); 1899 Lieut.-Col:, A.A.G., War Office. O.B.E. Brevet

Lieut.-Colonel. Brevet Major. $m$.

*Chichester, W. G. C. Lieut., London Regt. (R. Fus.) I9I I Killed in action 15 Sept. 1916

Chidson, L. H. Lieut., E. Surrey Regt.; Major,M.G.C. [I914] M.B.E. $M_{3}$.

Child, J. F. 2nd Lieut., Northumberland Fus.

CHILdERS, R. E. Lieut.-Cdr., R.N.V.R.; Major, S.O. 2, 1889 R.A.F. D.S.C. M.

Chisholm, C. J. Major, gth Lancers. (W 2.) $M$.

Chitrock, C. Lieut., Felsted School O.T.C.

I9II

C M.C. $M$.

Christie, L. D. Capt., R. Sussex Regt.; attd. Gen. Staff 1909 (Intelligence)

Churchill, A. Lieut., R.N.V.R. (Armoured Car Section) 1903 ¥CHurchill, W. M. Major, I2th Cavalry, Indian Army. I90I

(W.) M. Order of St Anne, 3 rd Class (Russia)

Died 4 Nov. 1918 of pneumonia

Clark, A. S. Major, S. and T. Corps, Indian Army

Clark, E. D. Lieut., Yorkshire Regt. (W.)

I 897

1906

AClARK, E. F. Lieut., The Buffs (E. Kent Regt.); attd. [1914] R.F.C. (W.)

Killed in action I Fan. I9I7 
Clark, E. K., T.D. Lieut.-Col., W. Yorks. Regt. (T.F. $\quad{ }^{8885}$ Res.)

ClaRk, H. D. Capt., Argyll and Sutherland Hdrs.(T.F.) 1906 and M.G.C.; attd. Inland Water Transport. (W.)

Clark, J. H. M. Lieut., R.N.V.R.

Clark, S. B. Pioneer, R.E. (Signals)

Clark, S. H. Major, Worcestershire Regt.(T.F.)

Cllark-Kennedy, A. K. Capt., King's Own Scottish 1902 Borderers (T.F.)

Killed in action 19 April 1917

Clarke, C. S., v.D. Lieut.-Col., R. Sussex Regt.(T.F.) $\quad$ I892

Clarke, E. J. Major, W. Yorks. Regt. and Army Cyclist 1897 Corps. M 2. French Croix de Guerre

Clarke,E.S. 2nd Lieut., R. Sussex Regt.(T.F.); Lieut., I9I2 Scots Gds. (W.) $M$.

Clarke, H. A. Lieut., R.A.S.C.(M.T.)

Clarke, H. H. Capt., R.A.M.C.

I910

1896 1886

Clarke, J. H. P.O., R.N.V.R. (R.N.D.); Capt., R.E. 1909 (Field Survey Coy.)

Clarke, S. Lieut., Hampshire Regt.

I9I I

Clarkson, W. B. Pte., R. Fusiliers (P. S. Bn.); Major, 1909 R.G.A. D.S.O. $M$.

Clay, B. A. Lieut., Res. Regt. of Cavalry; Capt., S.O. 3, 1904 R.A.F. $m$.

ClaY, F. H. Lieut., N. Somerset Yeo. and R.A.S.C. $\quad 1895$

Clay, J. H. Lieut., R.A.S.C.

Clayton, F. Major, R.A.M.C.(T.F.) $M 2$.

Clegg, J. A. Lieut, King's (Shropshire LI T.F.) Capt., D.A. D. Labour

Cleghorn, W. A. Pte., R.A.M.C.; 2nd Lieut., Scottish I9r3 Horse

Clifton, E. N. Capt., Coldstream Gds.; Major (A.), 1909 R.A.F. (W.)

AClifton, H. E. 2nd Lieut., Devon Regt. (W.) M.C. I910 Died ${ }_{4}$ Oct. 1916 of wounds received in action 23 Sept. I9I 6

\#Clissold, H. Major, R.E.(T.F.) (W.) $\quad$ D.S.O. M $M 2 . \quad{ }^{1889}$ Killed in action 28 Sept. 1917

AClose-Brooks, A. B. Capt., Manchester Regt. M.C. M. 1903 Died 10 Yan. I9I7 of wounds received in action

¥Close-Brooks, J. C. Lieut., Life Gds.

Killed in action 30 Oct. I9I4

AClough, A. Capt., W. Yorks. Regt.

Killed in action I Fuly I916 
Clutton, B. Lieut., Bedfordshire Regt. (W.) m. 1902

Cobbold, C. J. F. Lieut.-Col., Suffolk Regt. (Cyclist 1900 Bn., T.F.) $M . m$.

CоввоLd, G. F. Major, York and Lancaster Regt. (W.) 1903 M.C.

Cobbold, P. W. Capt., Suffolk Regt. $\quad 1893$

Cochrane, A. C. Major, R.A.S.C. 1894

Cochrane, A. K. O. Capt., Yorkshire Regt.; Hon. Capt. 1909 (A.), R.A.F.

Cochrane, G. D. Lieut., Yorkshire Regt.; empld. W. 1904 African Frontier Force

Cock, T. A. Capt., R.A.S.C.

Cockburn, A. F. Capt., R.E. (Fortress, T.F.) M. $\quad 1909$

4Cockerell, S. P. 2nd Lieut., R.F.C.

Died at Ismailia 20 March I9I 5 of smallpox

4 Cocks, W. F. Lieut., Lincolnshire Regt.

Died 9 April 1917 of wounds received in action

Cockshotт, F. G. Pte., Saskatchewan Regt., Canadian 1899 Force. (W.)

Coggin, H. F. F. Pte., H.A.C.; Lieut., R. Berkshire 1902 Regt. (W.)

Cohen, C. B. Capt., R.A.M.C.

CoHen, D. H. Capt., Sherwood Foresters (Notts. and Derby Regt.) and Gen. List, empld. Ministry of Munitions. (W.)

Cohen, W. S. Lieut., Hertfordshire Yeo.; Capt., Labour 1890

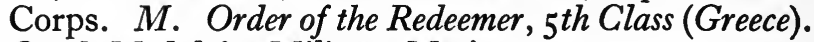
Greek Medal for Military Merit

Coke, Hon. Richard. Major, Scots Gds. (W 2.) 1895

Coleman, C. J. Capt., R.A.M.C.(T.F. Res.)

Coleman, P. G. Lieut., N. Staffs. Regt.(T.F.) and Welsh Gds. (W.) D.S.O. M.

Coles, E. J. Major, R.A.O.C.

Collet, G. G. Lieut.-Col., R.A.M.C.

Collett, R. L. Capt., R.A.M.C. (Sanitary Service, 1905 T.F.)

Collier-Johnstone, N. S. Lieut., 5th Dragoon Gds. 1910 Chevalier, Ordre de la Couronne (Belgium). Belgian Croix de Guerre

Collingwood, E. F. Midshipman, R.N.

Collins, B. K. T. Capt., R.A.M.C.(T.F.)

Collins, D. C. Lieut., Ruahine Regt., N. Zealand Force 1907 Collins, R. F. Capt., R. Fusiliers. (W.) D.S.O. M.C. 1909 $M 2$. 
Collinson, J. W. Major (T.), R.A.F.

1903

Colman, J. Capt. and Adjt., London Regt. (Surrey 1905 Rifles). $m$.

$\begin{array}{lll}\text { Colquhoun, G. R. E. Capt., R.A.M.C. } & 1907 \\ \text { Colthurst, G. O. Capt., S. Irish Horse; A.D.C. } & 1901\end{array}$ French Croix de Guerre

Colthurst, R. St J. J. Capt., London Regt. (L.R.B.) 1906

Coltman, W. H. Major, Gordon Hdrs.(T.F.) (W.) 1882 T.D. $m$.

Colville, D. J. Capt., Cameronians (Scottish Rifles, I912 T.F.) (W 3.)

Colville, Hon. J. G. Lieut., R.N.V.R.

Colville, T. R. Capt., D. of Cornwall's L.I.; empld. I90I Egyptian Army. $M$.

Colvin, C. H., D.s.o. Hon. Colonel, Essex Regt. and 1877 Spec. List (Cmdt., P. of W. Camp). C.B. m.

Colvin, R. B., C.B., T.D. Colonel, Spec. List; Brig.-Gen. 1874 $m 2$.

Conolly, C. G. Lieut., R.A.S.C.

1896

Coode, A. P. Major, D. of Cornwall's L.I.(T.F.) $M$. $\quad 1890$

HCoOK, F. R. Lieut., E. Yorks. Regt. and R.F.C. (W.) ${ }_{1915}$ Killed in flying accident $22 \mathrm{Feb}$. 1918

Cooke, A. Major, R.A.M.C. (Ist E. Gen. Hospital, T.F.) $\quad 1898$ Cooke-Hurle, J. See Hurle, J. C.

COOPER, J. G. Capt , R.A.M.C.

Cooper, P. H. Lieut., R. Fusiliers; Capt., M.G.C.

COOPER, W. G. D. Capt., R.A.S.C.

Cope, T. G. Capt., R. Fusiliers; Lieut.-Col., The Buffs (E. Kent Regt.); Brig.-Gen. (W 2.) C.M.G. D.S.O. and Bar. Brevet Lieut.-Colonel. 'Brevet Major. $M_{4}$. Chevalier, Legion of Honour (France).

Copeland, W. B. Capt., N. Staffs. Regt.; empld. Min- 1909 istry of Munitions. (W.) $M$.

Copland Griffiths, F. A. V. Lieut., Welsh Gds.; Capt., 1912 O.C.B. (W.) M.C. M. $m$

Corbin, J. L. Lieut., Northamptonshire Yeo. and Gen. I90r Staff

CoRnford, F. M. Capt., Gen. List (School of Musketry) I893

\&CoRnish, C. L. Lieut., Highland L.I.

Killed in action 13 Nov. I9I4

CORNISH, H. D. Lieut., R.G.A.

Cornwall, J. W. Lieut.-Col., I.M.S.

1893

I 897

1896

1902

Corrie, O. C. K. Lieut., R.F.A.(T.F.); Capt., Spec. I90 I List (T.M.B.) M.G. M. 
Corry, F. R. H. L. See Lowry-Corry, F. R. H.

CORRY, J. P. I. M. Sub-Lieut., R.N.V.R.

CORRY, W. M. F. Lieut., R.F.A.(T.F.) M.C.

CORSER, E. E. Lieut., King's (Shropshire L.I.)

Cotterill, G. H. Major, Lancs. Fus.

CotTERILl, H. E. Major, Gen. List ('T.

Conducting Officer

CotTerill, L. Major, R.A.M.C.

Cotron, B. Major, King's (Shropshire L.I.) and Gen 1880 List (Cmdt., Detention Barracks)

Couper, J. D.C. Lieut.-Col., R.E. ; Colonel, D.D. Docks. 1894 C.B.E. $M 2$.

Courtauld, L. Capt., R.A.M.C.(T.F.) (W.) m. 1895

CoutTs, H. A. T. Lieut., R.A.S.C. 1912

Coventon, A. W. D. Capt., R.A.M.C. French Médaille 1897 des Epidémies

Cowell, W. J. R. See Rooke-Cowell, W. J.

Cowie, H. C. Capt., R.E. (W.)

Cowley, J. N. Capt. and Adjt., R.H.A. $M$.

COWPer, G. M. Capt., R.A.M.C. $M$.

Died 3 Oct. I91 8 of wounds received in action

ACox, D. P. 2nd Lieut., R.F.C.

Killed in action 21 Aug. I9I7

Cox, W. A. M. Lieut., Black Watch; Lieut. (A. and [rgr4] Ad.), R.A.F. (W.)

Coxon, A. W. Major, Army Pay Dept.; Staff Pay- 1890 master. O.B.E. in.

Crackanthorpe, O. M. Capt., R. Scots and Border Regt. $\quad$ I894 $M$.

Cradock, N. Capt., R.F.A.(T.F.)

Crane, L. F. N. Capt., Loyal N. Lancs. Regt. and Gen. 1898 List. O.B.E. M. $m$.

Cranmer Byng,L.A. Capt., Gen.List(T.F.Res.); Cmdt., I89I P. of W. Camp

Cranworth, Lord. 2nd Lieut., Norfolk Yeo.; Capt., $\quad 1896$ R.F.A.(R. of O.) M.C. M 2. French Croix de Guerre

Crawford, D. Sqdn. Sergt.-Major, K. Edward's Horse; 1909 Lieut., R.F.A. (W.)

Crawford, K. A. Lieut., R.G.A.

Crawhall, Rev. T. E. C.F. 2nd Class, R.A.C.D.(T.F.) D.S.O. T.D. $M 2$.

*Crawley, E. Major, I2th Lancers. $M$.

Killed in action 2 Nov. 1914 
Crawshay, J. W. L. 2nd Lieut., Scottish Horse; Capt., [1914] Welsh Gds. M.C.

Creed, P. R. Capt., Rifle Brigade and Gen. List $\quad 1892$

ACRewdson, T. W. Capt., Manchester Regt.; A.D.C. 1914

Died 6 Nov. 1916 of wounds received in action 28 Oct. 1916

Crewdson, W. D. Lieut.-Col., Border Regt.

CRIPPS, W. L. Surgeon, R.N.

1898

1896

CRISPIN, H. T. Lieut.-Col., Northumberland Fus. and I887

R. Sussex Regt. $M$.

Killed in action 30 Oct. 1914

Crocker, J. A. Capt., London Yeo. (Sharpshooters); 1893

Capt. and Adjt., Tank Corps

WCROFT, J. A. C. 2nd Lieut., R. Warwickshire Regt.; 1907 attd. D. of Wellington's (W. Riding Regt.)

Killed in action at Hill 60 18 April 1915

ZCROPPER, JoHN. Lieut., R.A.M.C.

Drowned on H.M.S. Britannic 2 I Nov. I9I6

1883

Cropper, J. W. Major, Westmorland and Cumberland I898 Yeo. (T.F. Res.); empld. War Office

Cross, E. K. 2nd Lieut., Rifle Brigade. (W.) 1916

ZCRossley, B. Lieut., Highland L.I. $M$. . 1904

Killed in action 18 May 1915

Crossley, E. Major, IIth Hussars. O.B.E. $m$.

Crow, C. A. Capt. (T.), R.A.F. (Aircraft Production

Dept.)

Crowder, W. I. R. Capt., Border Regt.(T.F.) $m$.

ACROWE, H. B. Lieut., R. Fusiliers

Drowned on H.M. transport off Gallipoli 28 Oct. I9I 5

Cundell, J. Capt., R.A.S.C.

Cunliffe, N. Capt., Spec. List (Inspection Staff, Woolwich Arsenal). M.B.E. m.

Cunningham, A. J. W. Capt., R.A.M.C. $M$.

1897

1914

1904

I912

1900

1908

Cunningham, Rev. B. K. C.F. $4^{\text {th }}$ Class, R.A.C.D.

O.B.E. $M$.

\#Cunningham, J. M. Capt., Suffolk Regt.

Died 28 March 1918 of wounds received in action

1901

1889

1897

ZCunningham, J. S. Sergt., R. Highlanders, Canadian 1904

Force

Killed in action 31 Oct. 1916

Cunningham, T. E. Capt., King's (Liverpool Regt., 1905 T.F.)

Cunningham, W. A. Capt., R. Welsh Fus.; attd. The rgor Queen's (R.W. Surrey Regt.) (W.) 
\#CurRIE, R. F. I. Lce.-Corpl., R. Fusiliers

Killed in action 15 fuly 1916

CURRY, P. A. Capt., Gen. List, attd. R.E.; Major, Asst. 1902 Embarkation S.O. O.B.E. M2. American Distinguished Service Medal

CurTIS, A. R. W. Capt., I Ith Hussars; attd. R.F.C. (W.) 1908 M.C. $M$.

Curtis, T. L. C. Capt., Coldstream Gds.; Major, Gds. 1907 M.G. Regt.

CURTIS-BENNETT, H. H. Lieut., R.N.V.R.

Cuthbert, C. A. Capt. (T.), R.A.F.

1897

1910

\#affarn, M. 2nd Lieut., N. Rhodesian Police

1906

Killed in action in Rhodesia 24 April I915

Dale, F. R. Capt., R. Welsh Fus. and Welsh Regt. 1902 D.S.O. M.C. M.

Dalton, J. O. C. 2nd Lieut., R.F.A.

DaLton, T. E. Canadian A.M.C. M.M.

DaniEll, H. E. B. Capt., Durham L.I.(T.F.); attd. R.E. 1903

(W 2.) M.C.

DARBY, W. S. Lieut., R.A.M.C.

DARLEY, H. R., D.S.o. Major, 4th Dragoon Gds. (R. of 1884 O.); Staff Capt. O.B.E. $m 2$.

Darmady, E.S. Hon. Capt. and Q.M., R.A.M.C.(T.F.) 1886

DARNTON, R. E. Capt. (A.), R.A.F. D.F.C. I9I3

DARROCH, A. R. Hon. Capt., Spec. List (Navy and Army 1899 Canteen Board)

WDart, H. Pte., Middlesex Regt. (P.S. Bn.); Capt. and 1900 Adjt., York and Lancaster Regt.

Died 2 fuly 1916 of wounds received in action

DARWIN, B. R. M. Major, R.A.O.C.; D.A.D.O.S. $M . \quad 1894$

DarwIN, C. G. Capt., R.E. (Field Survey Bn.); attd. I906 R.A.F. M.C.

WDarwIN, E. 2nd Lieut., Yorkshire Regt.(T.F.) I90I

Killed in action 25 April 1915

Darwin, W. R. Capt., R.E. (W.)

DAUKES, A. H. Capt., King's Royal Rifle Corps; Staff 1908

Capt., War Office. Brevet Major. $m$.

Daun, E. 2nd Lieut., Devon Regt.

DAvid, W. T. Major, Spec. List, empld. Ministry of 1910 Munitions. $m$.

Davidson, D. F. Lieut.-Col., cmdg. Depôt, Cameron 1879 Hdrs.

Davidson, L. S. P. Capt., Gordon Hdrs.(T.F.) (W.) 1913 
Davidson, M. G. Capt., Cameron Hdrs. (W.) I9I2

WDavidson, N. R. Lieut.-Col., R.H.A.; G.S.O. (W.) 1897

D.S.O. and Bar. Brevet Lieut.-Colonel. M 3 .

Died 5 Oct. 1917 of wounds received in action

WDavies, A. C. Capt., R. Welsh Fus.

Killed in action ro Aug. 1915

Davies, Rev. A. R. C.F. 4th Class, R.A.C.D. (W.) 1908

WDavies, G. Ll. 2nd Lieut., King's Royal Rifle Corps; 1912 attd. Rifle Brigade.

Killed in action 15 March 1915

Davies, H. M. Capt., R.A.M.C.

Davies, H. R. Section Sanitaire, French Army

Davies, L. G. Capt., R.A.M.C.

Davies, P. Ll. See Llewelyn-Davies, P.

Davies, R. A. Ll. See Llewelyn-Davies, R. A.

Davies, R. R. Capt. and Adjt., r6th Lancers; A.D.C.; I9ro

Staff Capt. (W.) $M$.

Davies-Colley, H. Capt., R.A.M.C.

Davies-Colley, T. H. Lieut.-Col. Manchester 1894

(T.F. Res.)

Davis, J. R. A. Major, Spec. List

Davis, R. G. Lieut., Buckinghamshire Yeo.

I 896

1908
1912

1897

I898

Davison, J. F. Capt., Spec. List, empld. Records; Hon. I90I

Capt. (O.), R.A.F. M.C.

Dawnay, C. H. Capt. and Adjt., Yorkshire Regt. M.C. I9 Io

Dawson, J. Capt., R.E. (Fortress, T.F.) M.C. M. 1904

WDAY, M. C. Lieut., I3th Rajputs, Indian Army 19 ro

Killed in action in E. Africa 4 Nov. I9I4

Deakin, H. V. Surgeon, R.N.; Capt., R.A.M.C. $\quad 1907$

Deane, A. P. W. Pte., R.A.S.C.(M.T.) 1907

DEANE, C. H. Major, R.A.O.C.; D.A.D.O.S. M.C. m. 1902

Deane, R. W. Bt. Colonel, Lancs. Fus., empld. Gen. 1878

Staff. C.B.E. $m 2$.

Debenham, F. J. Section Sanitaire, French Army $\quad 1888$

DE CANDolle, R. Major-Gen., Director-General of 1883

Transportation, Mesopotamia. C.B. M. Grand

Officer, Order of the Crown of Roumania

DE CERJAT, C. S. Lieut., Grenadier Gds.

$\begin{array}{lll}\text { DeEPING, G. W. Major, R.A.M.C.(T.F.) } M . & \text { I895 } \\ \text { DeErhurst, Viscount. Lieut.-Col., Gen. List, T.F. } m . & \text { I884 }\end{array}$ DE Geijer, E. N. Lieut., Grenadier Gds. (W 2.) M.C. I9I2 DE GeIJeR, W. A. W. G. Capt., Hampshire Regt. 1907 Deighton, F. M. Lieut., R.F.A.; attd. R.E. (W 2.) 1905 M.C. 
\#Deighton, J. Capt., R.A.M.C.

Died 20 Sept. 1916 of wounds received in action

DE JANASZ, G. K. A. Lieut., R.A.S.C.

De la Pryme, Ven. A. G. Pte., N. Rhodesian Rifles, 1889

S. African Force; C.F. 4th Class, R.A.C.D.

DE Navarro, J. M. United States Army

Denis de Vitré, D. F. See de Vitré, D. F. D.

WDenman, R. C. Pte., H.A.C.; Lieut., Grenadier Gds.

Killed in action I Dec. I9I 7

Denman, R. P. G. Lieut., R.E. (Signals). $M$.

Denning, W. F. Capt., D. of Wellington's (W. Riding Regt., T.F.); empld. O.C.B.

WDennistoun, J. R. Lieut., Fort Garry Horse, Canadian

Force; attd. R.F.C. $M$.

Killed in action 4 May I916

Dent, G. Lieut., 4th (R. Irish) Dragoon Gds.; Major, I9Io M.G.C. (Cavalry). M.C.

Dent, L. M. E. Capt., Oxford and Bucks. L.I.; Major,

G.S.O.2. (W.) D.S.O. $M_{3}$. Chevalier, Legion of Honour (France)

Dent, R. A. W. Lieut., King's Royal Rifle Corps; A.D.C.

(W.) $M$.

DENT, W. E. Lieut., 8th (King's R. Irish) Hussars; empld. Foreign Office

Dent-Brocklehurst, G. E. Lieut., Warwickshire Yeo. 1904

DERHAM, J. A. T. 2nd Lieut., London Regt. (L.R.B.) 1905 (W.) (P.)

D'ERlanger, H. R. C. A.D.C.; Lieut., M.G.C.

DE Rothschild, A. G. Capt., Buckinghamshire Yeo.; 1906 empld. War Office. (W.) $M$.

\#DE Rothschild, E. A. Major, Buckinghamshire Yeo. M. 1904 Died I 7 Nov. I9I 7 of wounds received in action

DE Rothschild, J. E. A. Lieut., R. Canadian Dragoons; 1897

Major, R. Fusiliers

DE Rothschild, L. N. Major, Buckinghamshire Yeo. 1900 (T.F. Res.); empld. Recruiting Staff. O.B.E. m.

Desborough, G. Lieut., Bedfordshire Regt.

DE Sibour, Vicomte L. Lieut., 94me Regt., French Army.

(P.) French Médaille Militaire. French Croix de Guerre

Deterding, H. Lieut., Montgomeryshire Yeo.

DE Trafford, R. E. F. Capt., Spec. List (G.S.O.3). 1912 O.B.E. M 2. Chevalier, Order of the Crown of Roumania, with swords 
DE VITRÉ, D. F. D. Capt., Spec. List (Recruiting Staff). $\quad{ }_{1886}$ $m$.

Devonshire, Duke of, K.G. Hon. Colonel, Sherwood 1887 Foresters (Notts. and Derby Regt., T.F.); Commander-in-Chief, Dominion of Canada

Dewar, M. B. U. Lieut., R.E. (Fortress, T.F.); Major, 1905 Spec. List, empld. Ministry of Munitions. O.B.E. $m 2$.

Dewhurst. C. Major, Lancs. Hussars; empld. Navy and 1892 Army Canteen Board. (W.)

Dewhurst, C. Sergt., N. Rhodesian Rifles, S. African 1907 Force

WDewhurst, G. C. L. Lieut., Rifle Brigade. (W.) Killed in action I fuly 1916

Dewhurst, G. P. Hon. Capt., Cheshire Yeo. (T.F. Res.) 1890 $m$.

de Zoete, H. W. Capt., Essex Yeo.

Diamond, A. S. Driver, R.H.A. (W.) M.M.

Dick, H. P. Capt., R.G.A.; attd. T.M.B. (W 2.) $M 2$. 1895 1916 Dickey, E. M. O'R. Pte., The Queen's (R.W. Surrey Regt.); 2nd Lieut., R.G.A.

Dickinson, A. H. Capt., Coldstream Gds. (W.) M.C. 1890 $M$.

Dickinson, G. N. 2nd Lieut., R. Marines

WDickinson, R. S. Capt., London Regt. (Queen's West- 1912 minster Rifles)

Killed in action 2 Oct. 1915

Dickinson, W. H. Capt., R.A.M.C.(T.F.)

Dickson, E. C. S. Lieut., Highland L.I.(T.F.)

Dickson, J. W. E Capt, Seaforth Hdrs. (W 3) M.C.

Dickson, K. B. Lieut.-Col., R.A.M.C. (W.) 1906

Digges LA Touche, G. G. Lieut., R.F.A.(T.F.) (W 3.) 1907

Dill, J. M. Gordon. Capt., 5th (R. Irish) Lancers and 1908 M.G.C.; empld. O.C.B. (W.)

Dillon, T. A. Capt., Connaught Rangers. $M$.

Dingwall, C. F. Capt., E. Surrey Regt.; Major, M.G.C. I9I I (W.)

Dixon, E. T. Major, R.F.A.; empld. Ministry of Muni- $\quad$ I899 tions. O.B.E. $m$.

Drxon, G. Lieut.-Col., Border Regt.(T.F. Res.) and 1890 King's Own (R. Lancaster Regt.); Courts-Martial Officer. T.D.

Dixon, H. Capt., D. of Wellington's (W. Riding Regt.); $\quad 1893$ Major, Essex Regt. $M 2$. 
WDixon, J. E. B. Capt., R. Warwickshire Regt.(T.F.)

Killed in action I fuly rg16

Dixon, O. Capt., Lincolnshire Regt.(T.F.) (W.) rgor

Dixson, H. F. Lieut., 7th Hussars; attd. R.E. (Signals) I9Io

DobBs, F. W. Lieut., Eton College O.T.C.

WDoвbs, W. C. Capt., Middlesex Regt.; attd. R. Fusiliers. 1889

(W 2.)

Killed in action $3 \mathrm{r}$ Fuly r9r7

Doвbs, W. E. J. Lieut., I.A.R.O.; Major, D.A.A.G. $\quad 1899$

Dobson, J. F. 2nd Lieut., Bristol Univ. O.T.C. 1894

Dockray, J. V. 2nd Lieut., The Buffs (E. Kent Regt.) I9I7

WDodgshon, A. J. C. Lieut., Gloucestershire Regt.(T.F.) [I9I4] Killed in action ro Nov. I917

WDodgson, F. Capt., Yorkshire Regt.

Killed in action ro fuly r916

Dodgson, P. H. Capt., R.F.A.(T.F.) (W.) M.C. 1910

Doll, M. H. C. Lieut., I 3 th Hussars; Staff Capt. (W.) 1907 $M$.

WDon, A. W. R. Lieut., Black Watch

Died in Macedonia II Sept. I916 of dysentery

Don, F. P. Capt., Scottish Horse; Capt. (A.), R.A.F.; 1904

Major, S.O. 2. (W.) (P.) $m$.

Donald, M. H. 2nd Lieut., Border Regt.; Lieut., R.G.A. $\quad$ r913

Donald, W. G. C. 2nd Lieut., Norfolk Regt.

Donaldson, E. Capt., R.A.M.C.(T.F.) 1907

WDonaldson, Sir H. F., K.c.B. Brig.-Gen., empld. Min- 1877 istry of Munitions

Drowned on H.M.S. Hampshire 6 June I9r6

Donaldson, M. Capt., R.A.M.C. (P.) M. 1902

*Donaldson, N. Lieut., R.F.A. M. 1897

Killed in action 10 March 1915

Donaldson, S. H. M. Lieut., King's Royal Rifle Corps; 1905

Capt., Spec. List; Hon. Capt. (Ad.), R.A.F.

DonNER, J. D. 2nd Lieut., R.F.A.

Dorman, Sir A. J. Hon. Major, Yorkshire Regt.(T.F. Res.) $M$.

Dorman, B. L. Capt., R.A.O.C. and Spec. List (Staff 1897 Capt., War Office). O.B.E. m.

Douglas, F. C. B. See Brown Douglas, F. C.

Doune, Lord. Capt., Scottish Horse; Capt. (A.), R.A.F.; I9II S.O. 3. (W.) M.C.

Dove, Rev. F. J. C.F. $4^{\text {th }}$ Class, R.A.C.D.

I9IO

WDowling, G. C. W. Capt., King's Royal Rifle Corps I9ro

Killed in action at Hooge 30 fuly 1915 
Dowson, E. M. 2nd Lieut., Labour Corps

Drake, C. W. T. See Tyrwhitt Drake, C. W.

I899

Drake, H. R. O. Lieut., R.E. (Signals). $M$.

I912

Drew, A. L. Capt., R.A.S.C. $m$.

ADREwE, A. Capt., R.G.A. $M$.

Killed in action 12 fuly $19 \mathrm{1} 7$

DreWE, C. Lieut., R.F.A. (W.)

Drinkwater, R. C. Lieut., Cambridge Univ. O.T.C.; I912 empld. O.C.B.

Droop, A. H. 2nd Lieut., Northamptonshire Regt. and $\quad$ I899 Gen. List, empld. Ministry of National Service

Droop, C. E. Capt., R.A.M.C.

DrucE, V. P. Lieut.-Col., Bedfordshire Regt.; empld. 1897 P. of W. Camp

Druce, W. G. Capt., Dorset Yeo.

1891

DRUMmond, G. H. Lieut., R.H.A.(T.F.) and Res. Regt. I90I of Life Gds. (W.)

WDrysdale, D. R. Lieut., Dorset Regt. (W.)

Died 25 Sept. 1916 of wounds received in action

I913

Dubs, C. I. A. Capt. and Adjt., Ayrshire Yeo. and R. 1909 Scots Fus.

Ducane, C. G. Lieut.-Col., R.E. O.B.E. M $M$. 1897

Duckworth, A. C. Major, 2nd Dragoons (R. Scots 1889

Greys, R. of O.); D.A.Q.M.G. Brevet Major. M 2.

Dudfield, S. R. O. Capt., R.A.M.C. (Sanitary Service, $\quad 1879$

T.F.); empld. War Office. O.B.E. $M$.

Duff, A. G. Lieut., R.A.O.C.

WDufr, B. P. 2nd Lieut., Cameron Hdrs.

Killed in action 25 Sept. 1915

DufF, D. G. Capt., R.A.M.C. M.C. M. 1906

Duffield, K. L. 2nd Lieut., R. North Devon Yeo.; 1903

Lieut., R. Warwickshire Regt.(T.F.)

DufField, W. G. Lieut. (T.), R.A.F.

Duffin, C. G. Major, R.F.A. (W.) M.C. M. 1908

Dufton, A. F. Lieut., R.F.A.(T.F.); attd. Gen. Staff. I913

(W.)

Dufton, J. T. Capt., W. Yorks. Regt.

Duke, D. St J. Lieut., R.N.V.R.

Duke, R. E. Lieut. (A.), R.A.F. (P.)

1902

I9II

Dukes, E. H. Lieut., Lincolnshire Regt. (W.)

I9I I

1895

19I3

Duncan, Rev. G. S. C.F. 4th Class, R.A.C.D. $M 2$.

Duncan-Hughes, J. G. Capt., R.F.A. M.C. Belgian 1902 Croix de Guerre 
Duncannon, Viscount. See Bessborough, Earl of

Dunkels, E. Lieut., R.A.S.C. $M$.

Dunkels, W. Capt., King's Royal Rifle Corps and Gen. 1904 Staff. (W.)

Dunlop, J. S. Major, R.A.S.C. $M 3$.

Dunning, G. K. Capt., Hampshire Regt.(T.F.)

Dunville, J. Lieut.-Col. (K.B.), R.A.F. C.B.E.

Wunville, J. S. 2nd Lieut., ist Dragoons

V.CE. "For most conspicuous bravery. When in charge of a party consisting of scouts and Royal Engineers engaged in the demolition of the enemy's wire, this officer displayed great gallantry and disregard of all personal danger. In order to ensure the absolute success of the work entrusted to him, 2nd Lt. Dunville placed himself between an N.C.O. of the Royal Engineers and the enemy's fire, and, thus protected, the N.C.O. was enabled to complete a work of great importance. 2nd Lt. Dunville, although severely wounded, continued to direct his men in the wire-cutting and general operations until the raid was successfully completed, thereby setting a magnificent example of courage, determination and devotion to duty to all ranks under his command. This gallant officer has since succumbed to his wounds."-Supplement to The London Gazette, 2 Aug. I9I7.

Died 26 Fune 1917 of wounds received in action 25 Fune I917

Dupree, E. Capt., Hampshire Regt. $M$.

Dupuis, C. E. Lieut.-Col., R.E.

Durack, J. J.E. Lieut., I.A.R.O., attd. 34th Poona Horse; 1900 Capt., R.E. (attd.)

DuthiE, W. L. Capt., R.F.A. (T.F.); empld. Ministry I9I I of Munitions. (W.) $M$.

Dyson, C. B. Capt., R.A.M.C.

Dyson, W. Major, R.A.M.C.(T.F.) O.B.E. M. 1895

WEADE, A. 2nd Lieut., Yorkshire Regt.

Killed in action 9 Oct. 1917

EADEN, J. Lieut., R.F.A. $M$.

EAdie, P. Lieut., R.E. (Fortress, T.F.); Hon. Lieut.(T.), R.A.F.

EAmes, E. J. H. Capt., R.A.S.C.(T.F.) $M 2$. 1907

EARLE, L. M. Lieut., R.A.S.C.

1907
1900

EAsterling, H. G. Air Mechanic, R.A.F.

EAston, G. L. E. Major, R.F.A. M.C. and Bar

I910

I 900

1914

I906

I9IO 
EAstwood, H. E. Major, R.A.O.C. M.C. Chevalier, 1907 Legion of Honour (France)

Eastwood, J. F. Lieut., Grenadier Gds.; Capt., Spec. 1906 List (Courts-Martial Officer). $m$.

Eastwood, N. W. Lieut., 3rd Hussars; attd. Egyptian 1909 Army. (W.)

Eaton, Hon. F. O. H. Capt., Grenadier Gds.; Major I9I3 (Ad.), R.A.F. D.S.O. M.

EBDEN, C. H. M. Lieut., R.N.V.R.

ECCLES, W.E. L. Lieut., Res. Regt. of Cavalry and Spec. $\quad 1898$

List; A.D.C. $M$.

ECKERSLEY, T. L. Lieut., R.E. (Signals) $M 2 . \quad 1909$

EDdison, Rev. F. W. C.F. 4 th Class, R.A.C.D.(T.F.) M. $\quad 1894$

Edge Partington, Rev. E. F. See Partington, Rev. E. F.E.

Edwards, G. B. Capt., King's (Liverpool Regt., T.F.) I910 and R.E. (Signals). (P.) M.C. M.

EDwards, H. J., C.B., T.D. Hon. Colonel, Unattd. List, 1888 T.F.; cmdg. O.C.B. C.B.E. O.B.E. $m 3$.

EDWARDS, H. V. Surgeon Lieut., R.N.

Edwards, L. P. L. See Firman-Edwards, L. P. L.

EDwards-Moss, J. Capt., R.G.A.(T.F.)

1914

EEgerton, P. DE M. W. Capt., I9th Hussars

Killed in action 8 Oct. 1918

EgGaR, A. T. E. Lieut., R.G.A.; Capt., S.O. 3, R.A.F. 1895 $M$.

EgGaR, J. Sub-Lieut., R.N.V.R. C.B.E. O.B.E. 1899 EEILOART, H. A. Major, London Regt. (R. Fus.) (W 2.) 1908 D.S.O. M.C. and Bar. $M 3$.

Died Fune 1920 from the effect of wounds received in action 28 May 1918

Elborne, J. 2nd Lieut., Huntingdonshire Cyclist Bn. I9r2 and R.G.A.

Eliot, Rev. E. F. W. C.F. 4th Class, R.A.C.D.(T.F.) 1882

Ellice-Clark, S. T. Capt., Suffolk Regt. and Tank 1905

Corps; empld. Ministry of Munitions. (W.)

EEllicotT, F. A. J. 2nd Lieut., King's Own Scottish I9I I

Borderers

Killed in action 8 fuly r916

WElliot, Hon. G. W. E. Lieut., Scots Gds.

Died 6 Aug. I9I7 of wounds received in action

Elliot, H. W. A. Capt., Wiltshire Regt.(T.F.)

सElliot, W. E. 2nd Lieut., Dorset Regt.

Killed in action 26 Sept. I916 
ElliotT, E. C. B. Capt., R.H.A.(T.F.)

ElliotT, G. L. L. Lieut., R.F.A. (W 2.)

ElliotT, T. C. J. Lieut., R.A.O.C. (W.)

1912

Elliot, T. R. Colonel, A.M.S. C.B.E. D.S.O. M 3. 1896

Ellis, J. W. H. Capt., Canadian Infy. (W.) (P.) 1897

Ellis, Rev. W. F. P. Chaplain, R.N.

1907

Ellison, A. J. Capt., York and Lancaster Regt. (W.) I9I4

ElpHICK, H. C. Lieut., R. Berkshire Regt.; Capt., I $5^{\text {th }} \quad 1912$

Sikhs, Indian Army; attd. 47th and 54th Sikhs

Elphinstone, K. V. Lieut., W. African Frontier Force 1897

Elphinstone, L. H. Major, Inns of Court O.T.C. and I898

British Honduras Defence Force

Elveden, Viscount, c.B., c.M.G. Capt., R.N.V.R.

Elwell, R. G. Surgeon Lieut., R.N. D.S.C. M.

I896

Emmett, R. H. Capt., R.E. (Fortress, T.F.)

England, E. T. Capt., Exeter School O.T.C.

ENGLAND, F. DE F. Lieut., I6th Lancers

EsHeR, Viscount, G.C.B., G.C.v.o. Colonel, empld. War

Office

Estridge, C. L. Capt., E. Yorks. Regt. (R. of O.); 1896

Major, Spec. List (Chief Instructor, School of In-

struction). (W.) D.S.O. $M_{3}$.

Evans, A. G. Surgeon Lieut., R.N.

EEvans, B. Lieut., Middlesex Regt. and R.F.C. $M$.

Died 8 April 1917

Evans, E. W. Lieut., Welsh Regt.

Evans, F. D. Sergt., R. Fusiliers

Evans, H. S. 'Trooper, London Yeo.; Capt., R.A.M.C.

(W.)

Evans, J. H. Lieut., R. Welsh Fus. (W 3.)

1895

1912

I896

1907

I 870

Evans, J. W. D. Capt., 2 Ist Lancers. (W.) $M$.

EEvans, R. A. Sergt., H.A.C.; 2nd Lieut., W. Yorks.

Regt.

Accidentally killed 25 Fan. 1916

Evans, R. C. A.B., R.N.V.R. (Anti-Aircraft)

Evans, V. L. Lieut., R.A.S.C.

Evans-Lombe, E. H. Capt., R. Defence Corps; Major,

Labour Corps

Everard, W.L. Lieut., Ist Life Gds. and Gds. M.G.Regt. 1908

EverY, Sir E. O., Bart. Capt., Norfolk Yeo.; Major, R.E. 1905 (Signals). Brevet Major. $M$.

EEwing, A. H. Capt., E. Yorks. Regt. (W.) M.C. and [19I4] Bar. $M$.

Died 8 Sept. 1918 of wounds received in action 
Exmouth, Viscount. Lieut., R.A.F.

1909

WEzRA, D. Lieut., R.G.A.

Killed in action 6 Aug. 1918

1902

Falmouth, Viscount. Capt., Coldstream Gds.

FANE, W. V. R. Colonel, Lincolnshire Regt. Brevet 1886

Colonel. $m$.

FARLEY, C. F. Capt., $4^{\text {th }}$ (R. Irish) Dragoon Gds. M.C. I9I I

FARLEY, R. L. Major (K.B.), R.A.F. (W.) O.B.E.

1909

WFARMER, H. C. M. 2nd Lieut., King's Royal Rifle Corps I9I I

Killed in action ro May 1915

WFarQuhar, H. B. Capt., London Regt. (Civil Service 1892 Rifles)

Killed in action near Vimy Ridge 21 May 1916

FARRANT, M. Major, R.F.A.

FARREN, W. S. Capt. (T.), R.A.F. M.B.E. $m$.

FARROW, E. P. Lieut., Manchester Regt.

FaulKner, R. Capt., S. Lancs. Regt. and Gen. List, I9 I I empld. Ministry of Munitions. $M$.

FawCetT, H. H. J. Lieut.-Col., R.A.M.C. D.S.O. $\quad$ M. $\quad$ I897

Fawcus, J. G. Pte., R. Defence Corps 1867

Fawcus, L. R. Lieut., I.A.R.O. (Cavalry) 1906

Fawkes, Rev. W. H. C.F. $4^{\text {th }}$ Class, R.A.C.D. M.C. $\quad 1894$

FAYRER, F. D. S. Lieut.-Col., I.M.S.

Featherstone, H. W. Capt., R.A.M.C. I9I I

Feetham, Rev. F. G. C.F. 4th Class, R.A.C.D. 1905

FeILding, Hon. F. E. H. J. Lieut., R.N.V.R. O.B.E. ${ }_{1} 887$

Order of the Nile, 4 th Class (Egypt). Order of $E l$ Nahda (Hedjaz)

WFEILDING, Hon. H. S. Lieut., K. Edward's Horse; 1912

A.D.C.; Capt., Coldstream Gds.

Died 9 Oct. 1917 of wounds received in action

FeltoN, L. B. Lieut., Somerset L.I. and Gen. List I9I I

WFENwICK, A. L. Capt., Lincolnshire Regt. (W.) $M . \quad$ I9I3

Killed in action 16 Feb. I9I8

Fenwick, B. A. Capt., E. Surrey Regt. (W.) (P.) $m$. 1908

Fenwick, J. C. Lieut., Northumberland Yeo. 1892

FENwICK, M. C. Capt., Suffolk Regt.(T.F.); attd. Man- 1908 chester Regt.

Ferguson, C. L. Lieut., King's Royal Rifle Corps; Capt., I9I4 O.C.B. (W.)

Ferguson, D. F. 2nd Lieut., R.M.A.

WFerguson, H. M. Capt., S. Staffs. Regt.

Killed in action I I Fune 1917 
Fergusson, N. M. Lieut., Scots Gds. (W.)

1901

Fermor-Hesketh, T. Major, Lancs. Hussars

1904

Ferrers Guy, A. W. Pte., R. Fusiliers (P. S. Bn.); and Lieut., R.G.A.

Fewtrell, A. H. H. Lieut., R. Sussex Regt.; Capt. and Adjt., 27th Light Cavalry, Indian Army. (W.)

FIELD, M. Lieut., United States F.A.

Fildes, F. L. V. Lieut., Coldstream Gds.; Major, P. and B.T. Staff

Fildes, G. P. A. Lieut., Coldstream Gds.

Fildes, P. G. Hon. Surgeon Lieut.-Cdr., R.N.V.R.

WFinch, H. A. I. Pte., 2nd Bn., Canadian Infy.; attd. T.M.B.

Died 28 April 1916 of wounds received in action

Finnis, C. R. Lieut., Scots Gds. (W.) Belgian Croix I891 de Guerre

Firman-Edwards, L. P. L. Surgeon Lieut., R.N

AFirTh, ARNold. 2nd Lieut., R.F.A.(T.F.)

Killed in action 15 April r917

FirTh, A. C. D. Capt., R.A.M.C.

FIRTH, A. M. B. Capt., York and Lancaster Regt.

FirTh, L. G. Capt., R.F.A. (W.)

FFIRTH, R. C. D. Lieut., S. Lancs. Regt.

Died 21 Dec. I9I4

FFisher, J. W. Capt., Sherwood Foresters (Notts. and Derby Regt.) (W 3.) D.S.O. M.

Died 8 Fuly 1916 of wounds received in action

WFISKE, C.H. 2nd Lieut., I I I th Regt., United States Army Killed in action near Fismes (Aisne) I2 Aug. I918

FitzGerald, D. Lieut., Remount Service and Spec. List

Fitzgerald, E. A. Major, 6th (Inniskilling) Dragoons; empld. War Office

Fitzgerald, G. M. Capt., London Yeo. (Sharpshooters) 1902 and M.G.C. (W.)

FitzGerald, J. F. Staff Sergt.-Major, R.A.S.C.

1912

I 901

1898

1908

1906

1897

1910

1914

1910

1890

I 891

FitzGerald, M. P. Capt. and Adjt., R.F.A. (W 2.) M. 1906

FitzHerberT, J. A. Lieut., R.G.A.; Lieut. (O.), R.A.F. I9I3 (W.) M.C.

FitzPatRick, E. R. Lieut.-Col., Loyal N. Lancs. Regt.; 1896

Brig.-Gen., D.A.G. C.B.E. D.S.O. Brevet Lieut.-

Colonel. Brevet Major. M 3. Chevalier, Legion of Honour (France)

Fitzwilliam, Earl, k.c.v.o., D.s.o. Major, Oxford and I891 Bucks. L.I.; Lieut.-Col., R.H.A.(T.F.) C.B.E. M. 
Fletcher, F. C. Hon. Capt. and Q.M., R.A.M.C.(T.F.) 1908 FleTCHER, HERBERT M. Major, R.A.M.C. (Ist London I884 Gen. Hospital, T.F.)

Fleuret, F. S. Capt., The Buffs (E. Kent Regt., T.F.) 1904 (W.)

Flint, A. H. Capt., R.G.A. (W.) (P.)

Flook, H.'S. Major, S. African Med. Corps

Flower, N. A. C. Lieut., Grenadier Gds. (W.)

FlynN, A. J. Capt., R.A.S.C. and Gen. Staff

Forbes, Rev. E. A. C.F. $4^{\text {th }}$ Class, R.A.C.D.

Forbes-TwEenIE, D. Capt., London Yeo. (Rough . [I9I4] Riders); A.D.C. (W.)

FORDHAM, E. K. Lieut., R.A.S.C.

I901

1899

1902

I9I I

I888

ForDHAM, H. A. Lieut., Northumberland Fus.; Capt. I9II (A.), R.A.F.; Major, S.O.2. (W.)

FORDHAM, O. 2nd Lieut., Oxford and Bucks. L.I.(T.F.) 1907

ForDYCE, A. Dingwall. Major, R.A.M.C.

1893

Fordyce, R. Dingwall. Major, 2nd Dragoons(R. Scots 1894 Greys)

Forester,Lord. Capt., R. Horse Gds.(R. of O.); Lieut.- $\quad 1887$ Col., Shropshire Yeo.

Forester, O.St M.W. See Weld-Forester, Rev.O.St M.

ForRest, H. Capt., S. Staffs. Regt.; attd. T.M.B. (W.) I9I3 M.C.

FForrest, J. W. 2nd Lieut., Seaforth Hdrs.

Killed in action 27 Sept. 19I5

Forshaw, J. H. Capt., R. Berkshire Regt.; Major, attd. $\quad$ I893 R.E. (Signals). $M$.

HForster, F. A. Capt., R. Fusiliers

Died 23 Sept. I9I4 of wounds received in action at Mons

Forster, T. Lieut., R.E.(T.F.)

FForster, W. Pte., R. Fusiliers

Killed in action 7 Oct. 1916

(W 2.) M.C. I910

I910

ForTescue, Earl, K.C.B., T.D. Hon. Colonel, R. North 1872

Devon Yeo.; A.D.C. to the King

Forwood, P. L. Lieut., R.E. (W.)

WFoster, B. LA T. Lieut., Manchester Regt.

Killed in action 24 fuly 1916

Foster, H. E. Pte., R.A.S.C.

1898

Foster, Rev. K. G. Chaplain, Indian Army.

1909

I912

Foster, M. G. Major, R.A.M.C.(T.F.) O.B.E. M 2.

Foster, M. R. Capt., I.A.R.O.

1907

I 886

I 88 I

I9I I

Foster, M. R. W. Major, Hampshire Regt. (T.F.); 1888 G.S.O. 2, E. Command. $m$. 
Foster, O. B. Major, Northumberland Fus.; D.A.Q.M.G. $\quad$ I899 M.C. M 5. Chevalier, Ordre du Mérite Agricole (France)

Foster, P. LA T. Lieut., Manchester Regt.; Lieut. (A.), 1910 R.A.F. (P.)

Fowle, T. E. Colonel, A.A. and Q. M. G., S. Africa. I88I C.B.E.

Fowler, C. H. Lieut., R.F.A.(T.F.); Staff Capt., Min- 1905 istry of Munitions. M.B.E.

FFowler, D. D. 2nd Lieut. (A.), R.F.C. (W.) Killed in action 16 March I9I7

AFOWLER, J. D. Lieut., 5 th (R. Irish) Lancers

Killed in action 30 Nov. I9I4

Fowler, R. H. Capt., R.M.A.; empld. Ministry of Munitions. (W.) O.B.E.

Fox, Rev. H. W. C.F. 3 rd Class, R.A.C.D. D.S.O. M2. $\quad$ r891

Fox, J. C. Capt., R.A.M.C.

Fox, W. S. Capt., R.A.M.C.

FFoyster, P. T. Capt., R.E.

Died I I Dec. I916 of wounds received in action $6 \mathrm{Dec}$. I9I6

Francis, E. G. 2nd Lieut., Cambridgeshire Regt. (W.) 1903

Franklin, W. B. 2nd Lieut., King's Royal Rifle Corps; Capt., Spec. List, empld. Ministry of National Service

Franklin-Smith, N. C. Lieut., Res. Regt. of Cavalry, attd. 7 th Hussars

Franklyn, A. H. Lieut., Res. Regt. of Cavalry; Traffic 1905 Control Officer

Fraser, C. S. Lieut., R. Fusiliers; Capt., Gen. List 1907 (Staff). (W.)

Fraser, G. T. Lieut., R.E.

Fraser-MackenZIE, E. R. L. Major, R.H.A.(T.F.) (W.) IgII D.S.O. M.C. M.

Fraser-MackenzIE, J. A. O. 2nd Lieut., Lovat's Scouts ; 1909 Lieut., E. African Force (M.T.)

FreEMAN, P. B. 2nd Lieut., Worcestershire Regt.

Freeman Mitford, Hon. C. B. O. See Mitford, Hon. C. B. O.F.

FreER, G. H. Lieut., Res. Regt. of Cavalry, attd. I2th 1905 Lancers

Freeston, B. D. Lieut., R.H.A.(T.F.)

WFrenCH, Hon. E. A. Capt., S. Wales Borderers

Died 16 Aug. I9I7 of wounds received in action

Frend, Rev. J. P. C.F. $4^{\text {th }}$ Class, R.A.C.D.

1913

1913

I887 
EFREND, W. R. Capt., Sherwood Foresters (Notts. and I893 Derby Regt.)

Killed in action $20 \mathrm{Sept}$. 1914

Frere, J. G. Capt., Norfolk Yeo.; attd. Norfolk Regt. [1914] FrEweN, H. M. Lieut., R.N.V.R. (W.)

FrEWEN, S. Lieut.-Col., A.A. and Q.M.G. $\quad$ I875

FreYeR, D. J. Major, London Regt. (London Irish I9OI Rifles); cmdg. Depôt

AFrost, E. L. Lieut., S. Lancs. Regt.(T.F.)

Killed in action 16 Fune 1915

FRY, C. R. Lieut. (T.), R.A.F.

FRY, F. McG. Lieut., R.F.A. (W 2.)

FRY, J. A.B., R.N.V.R. (Anti-Aircraft)

Furlonger, R. C. Capt., R.A.S.C.(T.F.)

1909

FursDon, G. E. S. Lieut., London Regt. and Spec. List (Recruiting Staff). (W.)

\#Gaddum, R. C. S. 2nd Lieut., R. Fusiliers

Killed in action ro Sept. ror6

Gaddum, W. F. Capt., Westmorland and Cumberland Yeo. and Border Regt. (W.)

AGalbraith, A. N. Capt., Ceylon Rifles

Accidentally killed in Egypt (20 Feb. 19r6)

Game, H. A. A.B., R.N.V.R. (Anti-Aircraft)

GARDINER, J. Capt. (T.), R.A.F.

GARDNER, H. D. Capt., R.A.M.C.

1907

1912

I 896

1899

1912

r899

GARDNER, J. C. Lieut., R. Marines and Gen. List, empld.

Ministry of Munitions. O.B.E. M.

Gardner, R. G. Capt., 25th Punjabis, Indian Army

Gardner-Medwin, F. M. Capt., R.A.M.C.

AGarFIT, T. N. C. Lieut., Durham L.I.

Killed in action in Gallipoli 30 April r91 5

ZGARNETT, K. G. Seaman, R.N.R.; Lieut., R.F.A. M.C. I9I I French Croix de Guerre

Died 22 Aug. 1917 of wounds received in action 21 Aug. 1916

HGARNETT, W. H. S. Lieut.-Cdr., R.N.R.; Lieut., R.F.C. I900 Killed in flying accident 21 Sept. 1916

*GarnetT-Botfield, A. C. F. 2nd Lieut., Rifle Brigade; I9I2 Lieut., S. Wales Borderers

Killed in action 9 May 1915

GarnetT-Botfield, W. McL. Lieut., R.A.S.C.

1901

GARRATT, G. H. Major, Border Regt. and R. Sussex Regt. $\quad$ I895

GARRETT, L. C. 2nd Lieut., R.G.A. 
*Garretr, S. Capt., Suffolk Regt.(T.F.)

Killed in action 12 March 1915

Garrick, G. C. Major, Surrey Yeo.

1897

Garside, G. C. Pte., R.A.S.C.

I9I 5

Gavin, W. Lieut., R.N.V.R.

Gay, C. D. Capt., E. Lancs. Regt. (W 2.) M.C.

1907

GAYE, A. S. Major, R.A.S.C.; Lieut.-Col., empld. Min- I900 istry of Munitions. O.B.E. $M$ 2. $m$.

GEARD, D.A.A. Lieut., Life Gds. and Gds. M.G. Regt. 1912

\&GEDGE, C. B. 2nd Lieut., London Regt. (R. Fus.) 1885

Killed in action 25 Sept. I915

*GEE, R. F. McL. 2nd Lieut., Wiltshire Regt.

Died 27 Oct. I9I4 of wounds received in action

[1914]

Geldard, N. Capt., D. of Wellington's (W. Riding Regt., 1908

T.F.) and Tank Corps. (W 6.) D.S.O. M.C. M.

GGLDERD-SOMERVELl, R. F. C. 2nd Lieut., Grenadier 1904 Gds.

Died I 3 March I9I 5 of wounds received in action

Gerard, Lord. Capt., R. Horse Gds. (W 2.) M.C.

Gervis, H. Capt., R.A.M.C.(T.F.)

\#Gething, H. B. 2nd Lieut., Gloucestershire Yeo.

Killed in action in Gallipoli 21 Aug. 1915

GibBon, E. L. L. Lieut., R.F.A.

GibBon, J. H. Major, R.F.A. (W.) D.S.O Brevet 1897

Lieut.-Colonel. $M 3 . m$.

सGibbons, E. I. Pte., R. Fusiliers; Lieut., Lancs. Fus.

Killed in action 29 April 1917.

GibBs, W. D. Capt., Hertfordshire Yeo. $M 2$.

Gibson, C. M. Lieut., E. Surrey Regt.

Gibson, G. M. Capt., R.A.S.C.(M.T.)

1902

I 881

1902

I 898

G 1897

Killed in action 13-17 Sept. I914

Gibson-Watt, J. M. Major, S. Wales Borderers

Gidlow-Jackson, G. H. Lieut., R.A.M.C.

1895

I9II

GILES, E. Capt., King's Own Scottish Borderers; attd. I913

W. Yorks. Regt.; empld. O.C.B. (W 5.) M.C.

Gilliland, G. F. Lieut., R.N.V.R.

1902

EGilliland, V. K. Capt., R. Irish Rifles. (W.)

1907

Killed in action 8 May 1915

*GILmour, A. S. 2nd Lieut., Argyll and Sutherland Hdrs. 1906 Killed in action 15 Sept. 1916

※Gilson, R. Q. Lieut., Suffolk Regt.

Killed in action 1 Fuly 1916

Girdlestone, C. M. Sergt., Norfolk Regt. 
*Gjers, L. Capt., Seaforth Hdrs.

Killed in action 4 Oct. 1917

GlazebrooK,A. R. Capt., W. Yorks. Regt.(T.F.); empld. I9ro Ministry of Munitions. (W.) M.G. $M$.

GleED, R. W. A. Lieut., S. Staffs. Regt. (W 2.) M.C. 1908

GLEN, R. R. Lieut., R. Glasgow Yeo.

I9I I

Godber, H. T. Lieut., Connaught Rangers; Capt., Spec. 1909

List (Cmdt., Bde. Signal School)

सGoddard, A. S. Capt., Canadian Infy.

Killed in action 26 Sept. 1916

FGoDDARD-Jackson, N. W. 2nd Lieut., Northampton- I914 shire Regt.

Killed in action at High Wood 9 Sept. 1916

Godsal, Herbert. Capt., Hampshire Yeo.

1886

Godsal, Hugh. Capt., R.F.A. (W.) $M$.

Godsell, R. T. Lieut., R.A.S.C.

GoFf, Sir H. W. D. Hon. Capt., R.A.S.C.

1905

GoF, Sir H. W. D. Hon. Capt., R.A.S.C. 1890

Goldie, C. J. D. Capt., R.F.A.; Staff Capt. M.C. M. 1896

Goldie, N. B. Lieut., R.G.A.; Staff Capt. $M$. I90I

GoldNEY, H. H. Lieut., R.E. M.C.

GoldschmidT, J. P. Capt., Manchester Regt.

Gomme, A. W. Pte., R.A.S.C.; Lieut., Spec. List

1905 .

1912

Gomme, G. J. L. 2nd Lieut., Cheshire Regt.; Lieut., 1905 R.G.A.

Goodhart, G. W. Capt., R.A.M.C. (2nd London Gen. $\quad 1899$ Hospital, T.F.) $m$.

GooDHART-RENDEL, H. S. Lieut., Grenadier Gds. $\quad 1905$

Goodwin, A. D. Lieut. (T.), R.A.F. (Aircraft Production I904 Dept.)

\&Goodwin, H. D. Lieut., Middlesex Regt.

Killed in action $\mathrm{I}$ Fuly 1916

${ }^{1}$ Goolden, A. W. Lieut., E. Surrey Regt.; empld. O.C.B. $\quad$ I908 (W.)

Gordon, A. R. Lieut., Northamptonshire Regt. (W.) 1912

Gordon, C. T. Hon. Lieut.-Col., R. Scots (T.F. Res.) 1877

Gordon, Rev. J.G. C.F. 2nd Class, R.A.C.D.; D.A.C.G. 1900

M. Italian Bronze Medal 'della Salute Publica'

Gordon Cumming, A. P. Capt., Cameron Hdrs.; Brigade 1912 Major. (W 2.) M.C. M.

*Gore Browne, H. T. T. Pte., Canadian Mtd. Rifles; 1904 2nd Lieut., King's Royal Rifle Corps

Died 23 Aug. 1916 of wounds received in action 19 Aug. 1916

1 Killed in action in Afghanistan after the armistice. 
Gorringe, W. H. Major, R. Sussex Regt.(T.F.) 1886

Gorst, G. T. Capt., E. Lancs. Regt. (W.) I9I3

Gosling, T. S. Lieut., R.G.A. 1897

Gosnell, R. P. Capt., H.A.C. 1899

Gossage, A. F. W. Lieut., I7th Lancers. M.C. 1909

Gossage, E. L. 2nd Lieut., R.F.A.; Lieut.-Col. (A.), 1909

R.A.F. D.S.O. M.C. $M_{4}$.

Goтch, M. S. Capt., Northamptonshire Regt. (W.) IgIo

Gourlay, W. B. Capt., R.A.M.C. M.C.

1899

Gow, A. S. F. 2nd Lieut., Eton College O.T.C.

Gow, I. B. 2nd Lieut., Black Watch

Grace, C. L. P. Lieut., Highland Cyclist Bn.

1905

[1914]

I910

I9II

Killed in action at Le Transloy 18 Oct. I916

Gracey, R. L. Major, R.E.(T.F.) D.S.O. M2. Order 1910 of the White Eagle, 5 th Class, with swords (Serbia)

Graham, G. Capt., R.A.M.C. $M$.

1901

Graham, H. A. R. Capt., Grenadier Gds.; A.D.C.; I9I I

Capt., Spec. List (School of Instruction). (W.)

Graham, H. H. C. Sub-Lieut., R.N.V.R.

1914

Graham, J. C. W. Capt., R.A.M.C. (Ist E. Gen. Hos- 1890 pital, T.F.)

Graham, J. R. N. Capt., Argyll and Sutherland Hdrs.; I9I I Major, M.G.C. (W.)

Y. $\mathfrak{C}$. "For most conspicuous bravery, coolness and resource when in command of a Machine Gun Section. Lt. Graham accompanied the guns across open ground, under very heavy rifle and machine gun fire, and when his men became casualties, he assisted in carrying the ammunition. Although twice wounded, he continued during the advance to control his guns and was able, with one gun, to open an accurate fire on the enemy, who were massing for a counter-attack. This gun was put out of action by the enemy's rifle fire, and he was again wounded. The advancing enemy forced him to retire, but before doing so he further disabled his gun, rendering it useless. He then brought a Lewis gun into action with excellent effect till all the ammunition was expended. He was again severely wounded, and forced through loss of blood to retire. His valour and skilful handling of his guns held up a strong counter-attack which threatened to roll up the left flank of the Brigade, and thus averted what might have been a very critical situation."-Supplement to The London Gazette, 14 Sept. 1917. 
Graham, M. W. A. P. Lieut., Life Gds.; Capt. and I9I3 Adjt., Gds. M.G. Regt. (W 2.) M.C. $M 2$.

Graham, R. P. Lieut., King's Royal Rifle Corps. (W.) I9I4 (P.) M.C.

Graham Campbell, J. Lieut., Argyll and Sutherland 1906 Hdrs. (W.)

\#Grant, A. F. M. 2nd Lieut., The Queen's (R. W. Surrey I9I I Regt.)

Died 18 June 1916 of gas poisoning

Grant, B. D. 2nd Lieut., King's Own Scottish Borderers I9Io

Grant, Rev. H. S. C.F. 4th Class, R.A.C.D. I895

Grant, M. F. Lieut.-Col., R.A.M.C.

Grant-Watson, E. L. 2nd Lieut., Hampshire Yeo. Igo6 (T.F. Res.)

*Grantham, E. R. H. 2nd Lieut., Northumberland Fus. I9I4 Died $3 \mathrm{I}$ March I9I 7 of wounds received in action

EGrantham, F. W. Capt., R. Munster Fus.

Killed in action 9 May 1915

Grantham, W. W., v.D. Major, R. Sussex Regt.(T.F.) $\quad$ i 886

Grasemann, C. Capt., R.E. 1908

*Grattan-Bellew,W. A. 2nd Lieut., Connaught Rangers; I9I3

Major, R.F.C. $M$.

Died 24 March I9I7 of wounds received in action

GraY, F. L. Capt., R.A.S.C. 1905

FGray, M. Lieut., 2nd Dragoon Gds. (Queen's Bays); I908

Capt., M.G.C.

Killed in action 8 Aug. I918

Greaves, G. M. Lieut., Leicestershire Yeo.; Capt., 1907 R. Horse Gds.; Major, Gds. M.G. Regt.

Greaves, L. B. Pte., R. Fusiliers (P. S. Bn.); Lieut., [19I4]

S. Wales Borderers. (W.) M.C. Order of the White

Eagle, 5th Class, with swords (Serbia)

Greaves, R. C. J. Lieut., R. Welsh Fus.

GREEN, B. Capt., Oxford and Bucks. L.I.(T.F.); Major, 1907

M.G.C. (W 2.) M.C. M.

GreEN, D. Lieut., Suffolk Regt. M.C.

Green, H. Pte., London Regt. (Artists Rifles)

AGreEN, H. S. Major, London Regt. $M$.

Killed in action 20 Sept. 1917

GreEn, R. D. Capt., Durham L.I.; attd. T.M.B.; empld. I9I4 British Military Mission. M.C.

Greene, W.P. C. Capt., I 3 th Hussars

Greenwell, B. E. Major, Hampshire Yeo.(T.F. Res.) I893 M.B.E.

1912

1903

1902

1903 
Greenwood, J. A. Section Sanitaire, French Army $\quad 1883$

GreG, A. H. Capt., R.A.M.C. O.B.E. M 2. French $189 \mathrm{I}$ Médaille des Epidémies

Gregory, G. M. Capt., I.A.R.O., attd. Sappers and I908 Miners; Recruiting Officer. $M$.

\&GRegory, J. S. Capt., R.A.S.C. and R.F.C. $M$.

Killed in action 19 Feb. 1918

1908

Gregson, H. G. Lieut., R. Wiltshire Yeo.; attd. Wilt- I9r2 shire Regt. (W.)

GRENVILLE-GREY, G. Cdr.,R.N.V.R.(Anti-Aircraft). m. $\quad$ I878

Greville Smith, S. H. Lieut., Middlesex Regt. (T.F. 1906 Res.) and Northamptonshire Regt.

GreY, Earl. Major, Northumberland Fus.; G.S.O.2. 1898 $M 2$.

*Gribble, C. H. Lieut., The Buffs (E. Kent Regt., T.F.) 1907 Killed in action 30 Nov. 1917

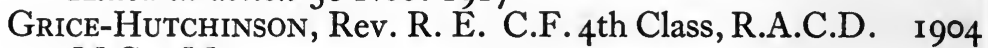
M.C. $M$.

Grieve, F. C. L. Major, R.F.A.

Grieve, W. R. Capt,, R.F.A.

1898

Griffin, A. W. M. S. Capt., N. Rhodesian Rifles. M.C.

Griffin, H. J. S. Major, R.F.A. (W.)

*Griffith, G. F. Capt., London Regt. (Q.V.R.) M. I9IO Killed in action 26 Sept. 1917

Griffith, H. K. Capt., R.A.M.C.(T.F.)

Griffith, H. L. W. 2nd Lieut., R.H.A.; Capt., Spec. I909 List, empld. Directorate of Requisitions and Hirings.

Chevalier, Ordre du Mérite Agricole (France)

Griffith, J. Major, N. Staffs. Regt.(T.F.) T.D.

Griffiths, Rev. C. C. C.F. $4^{\text {th }}$ Class, R.A.C.D. M.C. IgIo *Griffiths, R. E. Pte., Australian Force Killed in action in Gallipoli I9I5

Grinling, A. G. Capt., Hertfordshire Regt. ; attd. Bed- [19r4] fordshire Regt. (W.) M.C.

Grissell, T. DE LA G. Major, Suffolk Yeo.; Lieut.-Col., 1897 Suffolk Regt. (W.) M.C. M.

Groner, R. E. E. Lieut., Sherwood Foresters (Notts. 1903 and Derby Regt.) (W 2.) (P.)

Groves, K. G. Capt., London Regt. (Poplar and Stepney 1906 Rifles). $M$.

Guest, Hon. L. G. W. Major, R.A.F.; S.O. 2, Air Min- 1899 istry. O.B.E. M.

Guest, Hon. O. M. Major (A.), R.A.F.; S.O. 2. (W.) 1906 GuinNess, Hon. R. See ElvedEN, Viscount 
GuINNESS, R. S. Lieut., R.N.V.R. (W.)

Gunston, D. W. Capt., Irish Gds. M.C.

GuRney, C. R. Capt., R.E. $M$.

Gurney, E. H. Capt., Suffolk Yeo.(T.F. Res.)

GuRNEY, Q. E. Major, Norfolk Yeo.

GuRnEY-Dixon, S. Capt., R.A.M.C. $M 2$.

Gueterbock, P. G. J. Capt., Gloucestershire Regt.(T.F.); 1905

Brigade Major. (W.) D.S.O. M.C. $M_{3}$.

Guthrie, P. S. Lieut., Life Gds.; A.D.C.

\#GWYNNE, R. T. S. 2nd Lieut., King's Own (Yorkshire 1912 L.I., T.F.)

Died 23 May I9I 5 of wounds received in action

Gwyther, G. H. Lieut.-Col., R. Welsh Fus.; empld. I89o War Office. (W.) D.S.O. M.

WHabershon, L. O. Capt., E. Yorks. Regt.

Killed in action 13 Nov. 1916

Hackforth, R. Pte., London Regt. (Artists Rifles)

1912

Hadrill, E. W. 2nd Lieut., Queen's Own (R.W. Kent

Regt.); Lieut. (A. and Ad.), R.A.F. (W.)

Hahlo, H. B. See Barker-Hahlo, H.

Hake, H. M. Lieut., Cambridgeshire Regt. and Gen. I9I0 Staff. French Croix de Guerre

Hale, D. B. Major, R.F.A. $M$.

Hales, J. B. Lieut., Oxford and Bucks. L.I.(T.F.); Capt., G.S.O. 3. M.C. $M 2$.

Hall, A. A. Lieut., R.E. (Signals)

HaLl, A. C. Major, Suffolk Regt.

1910

EHall, F. G. Capt., Cheshire Regt.

Killed in action 7 Fuly 1916

Hall, R. S. Lieut., R.N.V.R.
WHallam, H. G.S. Lieut., R.A.S.C.(T.F.); attd.Egyptian [19I4]

Camel Corps

Killed in action near Faffa I Dec. 1917

Haller, B. C. Capt., R.A.M.C.

Hallett, H. G. Capt., R. Fusiliers

1907

I913

I 888

1909

1904

Halley, K. B. Lieut., Middlesex Regt. (W.)

I901 I 895

HALLIDAY, D. R. J. Lieut, IIth Hussars and R.E. (Sig- 1914 nals)

WHalliday, J. A. Capt., i ith Hussars

Died I3 Nov. I9I4 of wounds received in action at Messines 31 Oct. I9I4

Halliday, M. A. C. Lieut., Bedfordshire Yeo. and 1905 M.G.C. (Cavalry) 
Halnan, E. T. Capt., R.G.A. (W.) $M$. 1908

Hambro, R. O. Capt., Coldstream Gds.; G.S.O. 3. M. 1905 Belgian Croix de Guerre

Hamill, J. M. Major, R.A.M.C.(T.F.); D.A.D.M.S. $\quad 1898$ O.B.E. $M 2$.

Hamill, P. Major, R.A.M.C.

HAMILTON, E. R. Instructor Lieut., R.N.

Hamilton, E. W. Capt., London Regt.; empld. Ministry 1912 of Munitions. (W.)

Hamilton, G. Capt., Scottish Horse and Black Watch Igr I (T.F.) M.C.

WHamilton, H. O. Lieut., Northumberland Fus. Killed in action in the Battle of Loos 25 Sept. 191 5

*Hamilton, K. Lieut., W. African Frontier Force

Died in Nigeria 15 Nov. 1918 of influenza contracted on active service

Hammond, C. E. Lieut., Irish Gds. M.C. 1902

Hamond-Graeme, E. H. M. Major, Hampshire Yeo.

I9II

1905

HAMPDEN, Viscount. Major, Ioth Hussars (R. of O.);

Colonel, Hertfordshire Regt.; Brig.-Gen. C.B.

C.M.G. M 7. Officer, Legion of Honour (France)

WHANDFORD, E. F.S. 2nd Lieut., Sherwood Foresters [1914] (Notts. and Derby Regt., T.F.)

Killed in action 15 Oct. I9I 5

WHANDFORD, H. B. S. Capt., Sherwood Foresters (Notts. 1912 and Derby Regt., T.F.)

Killed in action 15 Oct. 1915

Hanna, W. B. Lieut., 92nd Infy. Bn.; Capt., Gen. List, I9I I Canadian Force

HanNen, L. G. 2nd Lieut., R.F.A. (W.)

Hanson, H. J. Lieut., R.N.V.R. O.B.E.

HHanson, O. H. Lieut.-Cdr., R.N.V.R.

Killed while prisoner in German hands 5 Nov. I9I4

Hanson, R. J. E. Surgeon Cdr., R.N.V.R. O.B.E.

1916

1893

1891

HARCOURT, R. V., M.P. Lieut., R.N.A.S. (Anti-Aircraft) 1897

HARDIE, M. Capt., Spec. List (Censor's Staff). M. $\quad 1895$

Harding, E. Capt., R.A.M.C. M.C. M.

Hardinge, Hon. A. H. L. Capt., Grenadier Gds. (W.) I9II M.C.

HaRDY, C. Capt., London Regt. (Rangers); Major, 1906 King's (Liverpool Regt.) (W.) D.S.O. M 2.

Hare, F. Capt., Northumberland Fus.(T.F.) and R.E. 1912 (Field Survey Bn.)

Harford, C. F. Capt., R.A.M.C. 
Hargreaves, A. R. Capt., R.A.M.C. (W.)

1903

Hargreaves, J. A. Lieut., King's (Liverpool Regt., T.F.) ${ }_{1898}$ (W.)

HARMENS, W. Capt., R.A.M.C. (W.)

WHARMSWORTh, Hon. V.S.T. Lieut., R.N.V.R.(R.N.D.) [19I4] Killed in action 13 Nov. 1916

Harraton, J. R. Lieut., Durham L.I.

HARRILD, W. C. Major, Yorkshire Regt.

HARRIS, E. R. Lieut., Wiltshire Regt.(T.F.) and M.G.C. [I9I4] M.C.

HarRis, H. B. Capt., Essex Regt.

Harrison, A. P. B. Capt., Rifle Brigade; Lieut.-Col., 1893 Spec. List

HARRISON, ERNEST. Lieut., R.N.V.R.

WHarRison, EvERARD. Capt., R.A.M.C.(T.F.)

Killed in action 18 April 19I7

HaRrison, E. F. Lieut.-Col., Gen. List, Australian Force; $\quad 1898$ G.S.O. I. m. Officer, Legion of Honour (France). Order of St Maurice and St Lazarus (Italy)

HARRISON, F. E. H. Major, R.F.A.(T.F.)

I 896

I90I

Harrison, H. E. Capt., The Queen's (R.W. Surrey Regt.) M.C.

HarRison, T. E., D.s.o., T.D. Lieut.-Col., Hertfordshire Yeo. (T.F. Res.) and Gen. Staff. $m$.

WHARRowing, J.S. Capt., R.A.S.C. and R. Warwickshire Regt. M.C. Chevalier, Legion of Honour (France)

Killed in action at Bullecourt 4 May I9I7

HarT, R. J. D'A. Pte., H.A.C.; Lieut., R.G.A.

Hartington, Marquis of. Hon. Colonel, Sherwood Foresters (Notts. and Derby Regt.); Capt., Derbyshire Yeo. M.B.E. M. m. Chevalier, Legion of Honour (France)

Hartley, C. R. Capt., D. of Lancaster's Own Yeo. M. $\quad$ I905 HHARTLEY, W. E. Instructor, R.N.

Accidentally killed on H.M.S. Vanguard 9 fuly 1917

Harvey, Rev. C. Pte., R.A.M.C.; C.F. 4th Class, 1903 R.A.C.D. (W.)

Harvey, Rev. E. J. W. C.F. 4th Class, R.A.C.D.

HHARveY, D. L. 2nd Lieut., 9th Lancers

I896

1898

r88I

1907

1913

I9I3

Killed in action 2 Nov. 1914

HHARVEY, F. L. Lieut., 9th Lancers

Killed in action 30 Oct. I914

Harvey, G. R. M. Pte., R. Fusiliers (Sportsman's Bn.) I89o and Middlesex Regt. 
Harvey, J. E. Capt., Irish Gds.

Harvey, O. C. Capt., Norfolk Regt. and Gen. List (In- 1912 telligence). $M$.

WHaskins, F. W. Pte., Cheshire Regt.

Died fuly 1916

HHaslam, W. K. S. Capt., R.F.A.(T.F.)

Killed in action 27 April I9I7

Hastings, W. H. Surgeon Lieut.-Cdr., R.N.

Hathorn, K. H. Lieut., 8th S. African Horse

1908

Hausburg, E. F. Major, Suffolk Regt.; G.S.O. 3, War Office. $M$. $m$.

Havelock-Allan, Sir H. S. M., Bart. Major, Lancs. I89I Fus. and Gen. List. (W.)

Hawker, C. A. S. Capt., Somerset L.I. (W 2.)

Hawkes, C. P. Major, Northumberland Fus.

I9I3

1894

Hawkins, H. D. Major, R.G.A. (W.) D.S.O. M 2. I90I

Hawkins, H. H. B. Capt., R.G.A. $M$.

1895

Hazlehurst, C. A. C. Capt., Westmorland and Cumberland Yeo.; attd. Border Regt. and Durham L.I. (W.)

Hazlerigg, Sir A. G., Bart. Capt., Spec. List; Hon. 1898 Capt. (Ad.), R.A.F.

Head, H. Capt., R.A.M.C. (2nd London Gen. Hospital, I880 T.F.)

Headlam, T. A. Lieut.-Col., E. Yorks. Regt.; attd. Welsh 1894 Regt.(T.F.) $M$.

Heald, W. H. A. Lieut., R. Fusiliers; empld. War Office I9I2

Heale,. R J. W Major, Indian Army, empld. Political 1896 Dept. O.B.E. M.

Heap, J. M. Capt. (A.), R.A.F.

WHeAPE, B. R. Capt., R.F.A.

Killed in action 16 May 1917

Hearn, Rev. E. H. C.F. 4th Class, R.A.C.D. $\quad 1896$

Heath, J. R. Capt., R.A.M.C.

Heath, L. C. Capt., Surrey Yeo.

Heath, O. Capt., R.A.M.C.

HeatucoTe, $\mathrm{H}$. Major, Sherwood Foresters (Notts. and Derby Regt., T.F.)

HeathCote-DRummond-Willoughby,Hon.C.S. Major, I 888 Scots Gds. (R. of O.); Colonel, T.F.; Brig.-Gen. C.B. C.M.G. $M 5$.

Heaton, D. R. Capt., The Queen's (R.W. Surrey Regt.); 1912 Staff Capt. (W 2.) D.S.O. M.

Heaton, J. B. Lieut., King's Royal Rifle Corps. (W 2.) 1914 
Heaton-Armstrong, W. D. F. Major, Lancs. Fus. and 1904 Labour Corps. $M$.

Heaton-Ellis, C. H. B. Major, Bedfordshire Regt.; 1882 Lieut.-Col., Spec. List. C.B.E.

\#Hebblethwaite, C. J. Lieut., Nigeria Regt., W. African 1903 Frontier Force

Killed in action near the Nigerian frontier 7 April I9I 5

Hedges, K. M. F. Capt., R.A.S.C.; Major, D.A.D. 1908 Transport. D.S.O. $M_{3}$.

Hedley, O. W. E. Lieut., R.E. (Tyne Electrical Engin- 1902 eers, T.F.)

*Hedley, W. A. C. Lieut., The Buffs (E. Kent Regt.) [I9I4] $M$.

Died 19 Fuly 1918 of wounds received in action

HeinemanN, A. B. Lieut., R.F.A.(T.F.)

Hellaby, F. A. Lieut., N. Zealand Force; Major, [I9I4] M.G.C. M.C. $M$.

Hellyer, F. E. Capt., Hampshire Regt.; S.O. 3 and 1907 Major (Ad.), R.A.F. (W 2.) O.B.E. M.

WHelm, H. P. D. Capt., Border Regt.; Capt. (O. and Ad.), I9I2 R.A.F. $M$.

Died 6 Nov. 1918

HeLme, J. Lieut., R.A.O.C.

Helme, J. M. Capt., King's Own (R. Lancaster Regt.) I907 (W.)

Helme, R. E. Capt., R.F.A.; empld. War Office I9ro

Helme, R. M., v.D. Hon. Lieut.-Col., R. Sussex Regt. I88I (T.F. Res.)

Helme, T. W. Lieut., R.A.O.C.

Helps, Rev. A. L. C.F. 4th Class, R.A.C.D. (W.) $M$ 2. 1890

Henderson, A. I. Capt., Highland L.I.(T.F.) (W.) I912

WHenderson, A. S. Capt., London Regt. (R. Fus.)

Died 25 April rgI 5 of wounds received in action

Henderson, J. K. 2nd Lieut. (Ad.), R.A.F.

Heneage, Hon. G. E. Lieut.-Col., Lincolnshire Regt. I885 (R. of O.); empld. Ministry of National Service. O.B.E.

WHenn, E. H. L. 2nd Lieut., Rifle Brigade; attd. King's I9Io Royal Rifle Corps

Killed in action 25 Sept. I9I 5

WHenRI, F. Capt., Northumberland Fus. (W.)

I912

Killed in action 15 Fune 1918

Henry, C. J. Capt., Leicestershire Yeo. $M$.

Henry, D. C. Capt., R.E. (Signals). (W.) M.C. 
Henry, S. A. Hon. Capt., R.A.M.C. (W.) Chevalier, 1898 Ordre de la Couronne (Belgium)

Hepburne-ScotT, Hon. C. F. Lieut., Scottish Horse

Hepburne-Scott, Hon. W. G. See Polwarth, Lord

1902

HePBuRne-Scott, Hon. W. T. Capt., Lothian and Border 1909 Horse

Herbert, A. G. Lieut., The Buffs (E. Kent Regt.). 1897

Herbert, E. Capt., The Queen's (R.W. Surrey Regt., r9r3 T.F.) M.C. $M$.

Herbert, E. A. F. W., T.D. Major, Yorkshire Hussars

HerberT-Smith, G. M. Lieut., R. Welsh Fus.; Major, rgro M.G.C.; empld. British Military Mission. $m$.

Hercus, E. O. Lieut., R.N.V.R.

WHerdman, G. A. 2nd Lieut., King's (Liverpool Regt.)

Killed in action I fuly rgr6

WHerman, G. A. Lieut., Cambridgeshire Regt.

Killed in action near Givenchy 20 July 1916

I9II

Heron-Maxwell, Sir I. W., Bart. Capt., Spec. List, I89o empld. British Mission to Russia

EHerries, A. D. Y. Capt., King's Own Scottish Borderers IgI I

Killed in action on the Somme 23 Fuly $19 \mathrm{r} 6$

Hervey, D. E. F. C. 2nd Lieut., Seaforth Hdrs.

1914

Hervey, E. S. Lieut., Surrey Yeo.; Capt., D.A.P.M. m. 1898

Herzog, F. J. Major, R.F.A. M.C.

Heseltine, N. E. Lieut., Essex Regt.(T.F.)

1908

H. Capt, D. of Lancaster's Own Yeo; attd 1904 1904
1898 King's Own (R. Lancaster Regt.)

WHeslop, G. H. Capt., Middlesex Regt. (W.)

Killed in action $\mathrm{I}$ Fuly 1916

WHess, H. Pte., R. Fusiliers (P. S. Bn.); 2nd Lieut., [19r4] Middlesex Regt. $M$.

Died 28 Oct. 1916 of wounds received in action

Hetherington, A. L. 2nd Lieut., Spec. List, empld. 1900 War Office

Hetherington, R. G. Lieut., R.E. (Inspection Staff). 1894 O.B.E. $m$.

Hetherington, T. R. Lieut., R.F.A.(T.F.) M.C. [1914] WHetherington, T. W. Lieut., Durham L.I.

Killed in action 17 Fuly 1916

HeTt, H. A. Capt., R.A.S.C.

HewletT, J. H. Instructor Cdr., R.N.

Hext, T. M. 2nd Lieut., King's Royal Rifle Corps

Killed in action 29 April 1917

Heycock, J. H. Major, Labour Corps

IgOI

1894

1915

I 880 
सHeywood, A. G. P. Major, Manchester Regt.; G.S.O.3. 1904 (W.)

Died 12 Sept. 1918 of wounds received in action 28 Aug. 1918

HHeywood; B. C. P., T.D. Colonel, Manchester Regt. 1882 (T.F. Res.)

Died 28 Oct. I9I 4

Heywood, C. C. Lieut.-Col., Manchester Regt.(T.F. ${ }^{1883}$ Res.); Major, R.A.M.C. $m$.

Heywood, Sir G. P., Bart. Lieut.-Col., Staffordshire Yeo. $\quad$ I897 (W.) D.S.O. T.D. M 2 .

Heyworth, G. A. F. Capt., R.A.M.C.

HHeyworth, H. P. L. Capt. and Adjt., N. Staffs. Regt. $\quad$ I896 Killed in action in Gallipoli 6 Aug. I9I 5

Hickman, C. E. Major, R.F.A.(T.F.) (W.) $M$.

WHicks, B. P. Lieut., R. Berkshire Regt.

Killed in action 25 Sept. I9I 5

Hicks, E.P. Surgeon Prob., R.N.V.R.; Capt. (A.), R.A.F. I9Io $M 2$.

Hicks, R. S. Hon. Capt., Suffolk Yeo.(T.F. Res.) I89o

Hicks, W.F. Pte., R.A.O.C.; Lce.-Corpl., Wiltshire Regt. I9I4

Higham, C. S. S. Capt., Manchester Regt.

Hilary, H. J. and Lieut., R.F.A.

Died 3 Fune $\mathrm{I} 9 \mathrm{I} 7$ of wounds received in action

Hill, A. G. E. Capt., Cameron Hdrs.(T.F.) (W.)

Hill, A. V. Capt., Cambridgeshire Regt.; empld. I905

Ministry of Munitions. O.B.E. Brevet Major. $m 2$.

*HILl, C. E. C. Lieut. and Adjt., Highland L.I. $M$.

Killed in action in Mesopotamia I7 April I916

Hill, C. L. Capt., Sherwood Foresters (Notts. and [19I4] Derby Regt., T.F.) $M$.

Hill, H. O. Engineer Lieut.-Cdr., R.N.

HILl, R. G. Lieut., R.F.A.(T.F.)

Hill, T. A. M. Capt. and Adjt., R.E. (Signals). M. $m$.

HILl, W. J. M. Major, Scots Gds. (R. of O.) and Loyal

1909

1897

N. Lancs. Regt.; Lieut.-Col., London Regt. (Hackney Bn.) D.S.O. M 2. French Croix de Guerre

Hilleary, E. L. Major, Lovat's Scouts. O.B.E. $M 2 . \quad 1890$ $m$. Order of the White Eagle, 4th Class, with swords (Serbia). Greek Medal for Military Merit, $3^{\text {rd Class }}$ WHills, W. F. W. Lieut., R.F.A. and R.F.C. Killed in action 6 March I9I7

WHilton, H. D. 2nd Lieut., Middlesex Regt. Killed in action I9 Dec. I9I4

I906

I910

1908

I895

I9I2

1902 
WHilton, M. V. Colonel, E. Lancs. Regt.

Killed in action 20 Oct. I9I 5

Hindlip, Lord. Capt., Spec. List. O.B.E. M $2 . \quad 1895$

Hingston, A. A. Major, R.A.M.C.(T.F.) I890

HipPisley, E. T. Sergt., R.E.; Lieut., R.G.A.; Capt., 1912 R.E. (Field Survey Coy.)

HIRST, G. S. Lieut., R.A.S.C.(T.F.)

HIRST, J. Hon. Major and Q.M., I.M.S. $m$.

1906

HisseY, J. B. R.N.A.S. (Anti-Aircraft)

Hoare, Rev. A. R. C.F. 4th Class, R.A.C.D. $M$.

HoAre, B. S. Lieut., Grenadier Gds.

HoARE, E. G. Capt., King's Own (Yorkshire L.I.); 1898

Lieut.-Col., King's Own (R. Lancaster Regt.) D.S.O. $M 2$.

Hoare, E. R. D. Lieut., Grenadier Gds. (W.)

HoARE, F. R. G. Major, R.A.O.C.; Lieut.-Col. (T.), $\quad$ I 898 R.A.F. C.B.E. M. $m$.

HoAre, G. DE M. G. Capt. and Adjt., London Regt. 1889 (Post Office Rifles). T.D. $m$.

HoAre, H. Hon. Major, Suffolk Yeo.(T.F. Res.) $m . \quad 1884$ WHoare, H. C. A. Capt., Dorset Yeo. (W.)

Died I9 Dec. I9I7 of wounds received in action Nov. I917

HoARE, J. E. A. Capt. (S.), R.A.F. D.S.C.

HoAre, R. B. Capt., Northumberland Yeo.

1903

1903

1890

1910

HoAre, W. R. Capt., Hampshire Regt.(T.F.) and R.F.C.

Hobhouse, A. L. Capt., Spec. List

Hobson, A. C. 2nd Lieut., R. Fusiliers

Hodge, H. S. Vere. Capt., Tonbridge School O.T.C. Brevet Major. $m 2$.

Hodges, Rev. E. C. C.F. $4^{\text {th }}$ Class, R.A.C.D.

Hodges, W. V. A. Lieut., R.G.A. M.C.

Hodgson, B. T., v.D. Lieut.-Col., R. Sussex Regt.(T.F.) C.M.G. $m 2$.

HHodgson, C. A. G. Capt., R. North Devon Yeo.

Died 20 March 1918 of pneumonia following malaria contracted on active service

Hodgson, F. W. K. Capt., Spec. List 1907

HHodgson, G. W. H. Lieut., Border Regt.

Died 6 Nov. 1914 of wounds received in action 2 Nov. I9I4

Hodgson, H. E. A. Lieut., The Queen's (R.W. Surrey Regt.); empld. O.C.B.; Capt., Norfolk Regt. 
Hodson, E. A. Capt., Rifle Brigade; Major, M.G.C.; 1912 empld. British Military Mission. D.S.O. $M 2$. m.

HoffmanN, G. S. Capt., R.A.M.C. (Sanitary Service, 1894 T.F.)

Holdgate,W.W. Lieut., Sutton Valence School O.T.C. I89I $m$.

Holdsworth, G. L. Bt. Colonel, 7th Hussars (R. of O.); 1882 Brig.-Gen., Director of Remounts. C.B. C.M.G. m 3. Order of the White Eagle, $3^{\text {rd Class (Serbia) }}$

Holland-HibBerT, T. 2nd Lieut., Hertfordshire Yeo.; 1907 Lieut., 2nd Dragoons (R. Scots Greys)

Holland-HibberT, W. Capt.,Hertfordshire Yeo.; A.D.C. I9I4 Hollond, E. R. Capt., R.A.S.C.

Hollond, G. E. Lieut., Suffolk Regt.; A.D.C. $M$. 1914

Hollond, H. A. Lieut., R.G.A.; Major, D.A.A.G. I903 D.S.O. O.B.E. $M_{3}$.

Hollond, S. E. Colonel, Rifle Brigade; Brig.-Gen. 1892 C.B. C.M.G. D.S.O. Brevet Colonel. Brevet Lieut.-Colonel. M 7. Chevalier, Legion of Honour (France)

Holman, A. McA. Lieut., 6th Dragoon Gds. (Carabin- 1908 iers). (W.)

WHolman, D. Lieut., Middlesex Regt.; attd. The Queen's [1914] (R. W. Surrey Regt.)

Killed in action 8 Aug. 19r 8

Holroyde, D. Capt., R.A.M.C.

Homiakoff, A. N. Russian Army

HoNy, H. C. Capt., Spec. List (Interpreter). M.B.E. 1907 $M$.

Hope, J. H. 2nd Lieut., R.E.

WHopgOOD, J. L. Pte., Middlesex Regt. (P.S. Bn.); 2nd [I9I4] Lieut., The Queen's (R.W. Surrey Regt.)

Died 17 Aug. 1916 of wounds received in action 13 Aug. r916

WHopkinson, B.,F.R.s. Major, Unattd. List, T.F.; Colonel, 1892 D.A.D., Air Ministry. C.M.G. $m 2$.

Killed in flying accident 26 Aug. I918

WHopkinson, E. H. Lieut., Cambridgeshire Regt. M.C. I9r3 $M 2$.

Died in German hands 2 Fune I915 of wounds received in action

HHopkinson, R. C. Lieut., R.E. (Signals). $M$.

Died 9 Feb. I9I 7 of wounds received in action 24 Nov. I9I 5 
WHopley, G. W. V. 2nd Lieut., Grenadier Gds. I910

Died 12 May I9I5 of wounds received in action Feb. I9I 5

Horne, M. Capt., R.A.M.C. (2nd London Gen. Hos- $\quad 1889$ pital, T.F.)

WHoRnsby, R. L. W. 2nd Lieut., Lincolnshire Regt. Killed in action 9 Oct. I9I 5

Hornung, G. Capt., R.F.A. (W.)

4Hornung, J. P. 2nd Lieut., R.F.A. (W.) M.C.

Died $20 \mathrm{Feb}$. I916 of wounds received in action

I9I I

1908

1913

Horsfield, R. B. Capt., Worcestershire Regt.; D.A.A. 1905 and Q.M.G. (W.) $M$.

Horton, C. E. Sub-Lieut., R.N.V.R.

1915

Horton, Le G. G. W. Capt. and Adjt., King's Royal r9r2 Rifle Corps; Major, R.A.F.

Hoskin, T. J. H. Capt., R.A.M.C. $M$.

Houghton, R. L. Lieut., R.F.A.; empld. Ministry of Labour

HoulT, J. M. Major, R.F.A.

Houstoun-Boswall, T. R. Capt., R. Scots

How, Rev. J. C. H. C.F. 4th Class, R.A.C.D.

I9II

1908

1900

Howard, Hon. B. E. Capt., Lovat's Scouts

* 1907

1903

Howard, B. H. E. Lieut., Manchester Regt.; Capt. (A.), I900 R.A.F. M.C.

Howard, C. F. Pte., London Regt. (Artists Rifles) I9ro

Howard, Hon. D. S. P. Capt., 3rd Hussars. Belgian 1910 Croix de Guerre

Howard, Hon. G., M.P. Capt., R.N.V.R. (R.N.D.) M. 1895 Howard, G. W. Capt. and Adjt., King's Royal Rifle rgI I Corps. M.C.

HowarD, H. S. Lieut., R. Warwickshire Regt.(T.F. Res.) 1899 WHoward, J. B. Capt., R. Welsh Fus.(T.F.) (W.)

Died 6 Sept. 1918 of wounds received in action

HowARD, J. P. 2nd Lieut., Hampshire Regt.

HowARD, Hon. M. F. S. Lieut., r8th Hussars. $M$.

Howard, N. M. C. Capt., R.F.A. (W.) M.C. $M$.

Howard, Rev. R. W. C.F. $4^{\text {th }}$ Class, R.A.C.D.

Howard, W. S. Pte., Army Pay Corps

[19I4]

Howard-McLean, J. R. Hon. Colonel, King's (Shrop-

shire L.I., T.F. Res.) and Gen. Staff. $m$.

WHoward Smith, G. Lieut., S. Staffs. Regt.(T.F.) M.C. 1899 $M$.

Killed in action 29 March 1916

Howden, P. F. 2nd Lieut., R.F.A.(T.F. Res.)

1907

1897

1908

1906

1906

1879 
Howick, Viscount. See Grey, Earl

HowIT, F. D. Lieut., R.A.S.C.; Hon. Lieut., R.A.F. I9I3 (W.)

HHowkins, G. A. 2nd Lieut., Northumberland Fus.

Killed in action 25-27 Sept. I91 5

HoworTH, H. Major, Sherwood Foresters (Notts. and I893 Derby Regt.); R.T.O.

Howson, Rev. J. F. C.F. 3rd Class, R.A.C.D. m. 1875

Howson, R. S. Sergt., ro7th Infy. Bn., United States I90I Army

Hoyland, C. E. Capt., Gen. List (T.F. Res.)

I 888

Hoyle, L. R. 2nd Lieut., E. Lancs. Regt.(T.F.); Lieut., [1914] R.G.A.

WHubback, F. W. 2nd Lieut., London Regt. (Rifles)

Died $12 \mathrm{Feb}$. I917 of wounds received in action

HubBard, L. E. Lieut., R.A.S.C.

Hubbard, M. E. Cadet, R.F.A.

Hubbard, P. W. Capt., E. Surrey Regt. (W 3.)

Hubbuck, G. M. Capt., Hampshire Regt. $M 3$.

WHudson, A. C. Major, R. Fusiliers

Died 2 Oct. $19 \mathrm{I} 6$ of wounds received in action

Hudson, Rev. E. C. C.F. $4^{\text {th }}$ Class, R.A.C.D. 1902

Hudson, F. A. 2nd Lieut., R.E.(T.F.)

ZHudson, R. P. M. Capt., D. of Wellington's (W. Riding I9ro

Regt.)

Died ${ }_{25}$ March 1920 from the effect of wounds received in action

Huggins, H. W. Major, R.H.A. (W.) D.S.O. M.C. I9ro Brevet Major. $M 6$.

Hughes, A. W. Lieut., R.F.A.; Lieut. (T.), R.A.F. (W.) I9I3

Hughes, G. E. Lieut., E. Surrey Regt.(T.F.); empld. 1903 Inland Water Transport

Hughes, G. R. Lieut., Dorset Regt.; Capt., Gen. List, 1906 empld. War Office

WHughes, N. A. Pte., R. Fusiliers (P.S. Bn.); Capt., Welsh 1907 Regt.

Killed in action 18 Sept. 1918

Hughes, R. Major, Berkshire Yeo. (T.F. Res.)

Hughes, R. J. Capt., R. Warwickshire Regt.(T.F.)

Hughes, T. G. Lieut., Gen. Staff (Intelligence)

*Hughes, T. McK. Pte., London Regt. (Artists Rifles);

Lieut., King's Royal Rifle Corps and Gen. Staff (Intelligence), attd. R.F.C. $M$.

Killed in action near Polderhoek 5 Feb. 1918

I 885

I90I

1905

1902 
WHughes-GibB, H. F. Lieut., R.F.A.

Killed in action 18 April $\mathrm{I} 9 \mathrm{I} 7$

HulberT, H. L. P. Capt., R.A.M.C.

Hulbert, J. G. Lieut.-Col., I.M.S.

1910

Hull, Sir C. P. A. Colonel, Middlesex Regt.; Major-

Gen. K.C.B. G.B. Brevet Colonel. M 8. Order

of St Vladimir, 4th Class, with swords (Russia).

French Croix de Guerre

Hulton, A. H. Capt., R.F.A.(T.F.); Capt. (Ad.), R.A.F. 1892 WHulton-Sams, Rev. F. E. B. Lce.-Corpl., Bedfordshire 1900 Regt.; Lieut., D. of Cornwall's L.I.

Killed in action at Hooge 30 fuly r 915

HumpheRY, H. M. Major, Hampshire Regt. (W.) 1894

HumphrEYs, W. A. Lieut., 2nd Dragoon Gds. (Queen's 1905 Bays)

Humphreys-Owen, A. E. O. Major, R. Welsh Fus.; attd. $\quad$ I 898 Dorset Regt. (W.)

Humphry, A. M. Major, R.A.M.C. $m$.

Humphry, L. Lieut.-Col., R.A.M.C.(T.F.)

1907

1873

Hunt, C. A. 2nd Lieut., Labour Corps; attd. R. Sussex 1895

Regt.

Hunt, E. R. Lieut.-Col., R.A.M.C. $M$.

HuNTER, W. E. Lieut., R.A.S.C. and R.F.A

1891

Huntington, L. W. Lieut., R.N.V.R. (W.)

I9I3

1903

WHuntsman, B. C. Capt., Sherwood Foresters (Notts. [I9I4] and Derby Regt., T.F.)

Killed in action 7 April 1917

Hurford, C. C. Capt., R.E.

1896

Hurle, J. C. Capt., W. Somerset Yeo.

Hurrell, J. N. Lieut., Devon Regt.; attd. Worcestershire Regt. and T.M.B. (P.)

Hutchinson, F. A. J. Capt., R.A.M.C.(T.F.)

Hutchinson, L. T. R. Lieut.-Col., I.M.S.

Huxley, G. A. Capt., R.E.

Huxley, M. H. 2nd Lieut., Grenadier Gds.

1906

I916

Ingleby, R. A. O. Hon. Capt., Army Pay Corps $\quad 1897$

INGRAM, H. F. 2nd Lieut., R.G.A.

INGRAM, R. S. S. Capt. (A.), R.A.F. (P.) [I9I4]

EIngram, T. L. Capt., R.A.M.C. D.S.O. M.C. M 2. ${ }^{1894}$

Killed in action 16 Sept. 1916

Inman, R. T. Paymaster Sub-Lieut., R.N.V.R. $\quad 1902$

INNES, A. C. W. Capt., Irish Gds. and R. Irish Fus. 1906 (W.) M.C. M. 
Insole, A. V. Lieut., R.F.A. (W.)

INSOLE, E. R. Lieut., Gen. List (T.F. Res.) $m$.

I913

1908

WInsole, G. C. L. Capt., Welsh Gds. (W.) M.C.

Killed in action 12 April 1918

1907

WIPswich, Viscount. Lieut., Coldstream Gds.; Lieut. (A.), 1903 R.A.F.

Killed in flying accident 23 April $\mathrm{r91} 8$

IrELAND, J. F. Capt., R.F.A. (W.) M.C. $M$.

IRwIN, C. Major, Cheshire Regt.(T.F.) M.C. M 2.

1907

1907

WJackson, B. R. Capt., Coldstream Gds.

Killed in action 15 Sept. I9I6

I906

WJACKSON, E. P. 2nd Lieut., R. Warwickshire Regt.; attd. I9I2 S. Wales Borderers

Killed in action 9 May I9I 5

JACKSON, Hon. F. S., M.P. Major, R.F.A.(T.F.); Lieut.Col., W. Yorks. Regt.(T.F. Res.); empld. Ministry of Munitions

Jackson, G. C. Lieut., Warwickshire Yeo. and 2nd Life Gds.; Capt., Gds. M.G. Regt.

JaCksON, H. A. Capt. and Adjt., King's Royal Rifle 1904 Corps. (W 2.) (P.)

JaCkson, H. C. Lieut.-Col., Bedfordshire Regt.; MajorGen. (W 2.) C.B. C.M.G. D.S.O. Brevet Colonel. Brevet Lieut.-Colonel. M 8. Officer, Legion of Honour (France)

JACKSON, J. 2nd Lieut., Rifle Brigade

Jackson, R. W. See Ward-Jackson, R.

Jackson, W. E. Pte., Northumberland Fus., Yorkshire 1900 Regt., and York and Lancaster Regt.

James, A. I. Capt., R.F.A. (W.) M.C. French Croix 1909 de Guerre

James, A. L. Capt., R.E. M.C.

1908

JAMES, A. W.H. Lieut., 3 rd Hussars; Major (A.), R.A.F.; 1912

Lieut.-Col., Dep. Inspector of Training. M.C. M.

James, Rev. S. R., v.D. C.F. Ist Class, R.A.C.D.(T.F.) 1874 C.B.E. $m$.

James, T. C. Lieut., Aberystwith Univ. College O.T.C. $\quad$ I899

JAmeson, G. J. Capt., S. Irish Horse

JAmieson,E.A.O.A. Lieut.-Col.(K.B.),R.A.F. A.F.C. 1899 $M 2$.

Janasz, G. K. A. See DE Janasz, G. K. A.

JármaY, I. B. Capt., Cheshire Yeo.; A.D.C. $M$.

JARVIS, L. K. Major, London Yeo.(T.F. Res.) T.D. 
JAY, C. D. Capt., R. Sussex Regt.; Major, M.G.C. 1908 D.S.O. $M 3$.

*Jeakes, J. W. Lieut., R. Berkshire Regt. (W.)

Died 12 Oct. 1917 of wounds received in action

1899

Jefrreys, H. G. G. Capt., R.A.M.C.

JefFreYs, J. G. Capt., Welsh Regt. and Spec. List 1904 (A.M.L.O.)

JefFreys, W. M. Capt., R.A.M.C.

JefFries, W. F. C. Capt., R. Dublin Fus.; Major, Spec. 1909

List (Chief Instructor, Reinforcement Camp). (W 2.)

D.S.O. $M 2$.

JemmetT, F. R. Capt., R.E. (Fortress, T.F.)

WJenkin, L. F. 2nd Lieut., Loyal N. Lancs. Regt.; Capt., [I9I4] R.F.C. (W.) M.C. and Bar

Killed in action I I Sept. 1917

Jenkyn, Rev. C. W. O. C.F. 3 rd Class, R.A.C.D. 1892 M.C. $M$.

Jewell, B. S. Capt., Spec. List (Graves Registration I899 Commission). $M$.

Jewell, C. J. S. Capt., R.F.A.(T.F.)

John, H. G. 2nd Lieut., York and Lancaster Regt.; I9I4 attd. Northumberland Fus.(T.F.)

Wounded and missing, presumed killed in action, at

Hooge 16 June 1915

Johnson, Rev. A. D. C.F. 4th Class, R.A.C.D. M.C. 1903 Johnson, E. S. See Darmady, E. S.

WJohnson, G. A. M. T. 2nd Lieut., London Regt. (St [19I4] Pancras Bn.)

Killed in action 21 May 1917

Johnson, P. R. Major, Devon Regt.(T.F.); empld. War 1898 Office. (W.) Brevet Major. $m$.

Johnson, R. T. Capt., N. Staffs. Regt.(T.F.)

Killed in action near Hulluch 13 Oct. I9I 5

Wohnson, W. M. Capt., Manchester Regt.

Killed in action at Montauban 2 fuly 1916

Johnson-FERguson, E. A. J. Major, Lanarkshire Yeo. 1893 and M.G.C. (W.) T.D. $M$.

Johnston, Rev. H. L. C.F. 4th Class, R.A.C.D. I893

Johnstone, G. H. Capt., R. Ist Devon Yeo. and Devon 1900 Regt. (T.F.); Staff Capt. $M$.

Johnstone, J. J. Capt. and Adjt., D. of Cornwall's L.I. 1908 (W.)

Jorcey, E. Hon. Colonel, Northumberland Fus. (T.F. 1884 Res.) $m$. 
WJorCEY, Hon. S. J. D. Capt. and Adjt., Northumberland I 1903 Fus.

Killed in action 20 March 1916

Jolley, L. B. W. Capt. (T.), R.A.F. (Aircraft Produc- 1904 tion Dept.)

Jolly, H. L. P. Lieut., R.E.

I906

Jolowicz, H. F. Lieut., Bedfordshire Regt. and Gen. 1909 List, empld. War Office

Jones, Rev. Bertram. C.F. $4^{\text {th }}$ Class, R.A.C.D.

Jones, C. H. Capt., R. Welsh Fus. (W 2.)

Killed in action 18 Sept. I918

Jones, C. W. Capt., R. Welsh Fus.; G.S.O.2, War Office. 1899 C.B.

\#Jones, E. D. 2nd Lieut., R. Fusiliers; Lieut.(A.), R.A.F. I9I I Killed in action 2 April 1918

WJones, F.J.J.R. Trooper, 27th Regt. of Dragoons, French I9I3 Army

Died $23 \mathrm{Feb}$. I9I 5 of wounds received in action

Jones, Ven. H. G. C.F. $4^{\text {th }}$ Class, R.A.C.D.

I901

1906

Jones, Rev. I. K. C.F. $4^{\text {th }}$ Class, R.A.C.D.

JoNes, O. T. Hon. Lieut., Spec. List

JoNES, T. B. Lieut., R. Fusiliers.

JoNES, W. D. Lieut.-Col., Spec. List

JoNes, W. P. M. Capt., D. of Lancaster's Own Yeo.

JosePH, H. M. Capt., R.A.M.C. (W.) M.C.

JowetT, A. C. Capt. (A.), R.A.F.

WJowetT, E. C. Lieut., Northumberland Fus.; Lieut. [1914] (A.), R.F.C.

Died in German hands 9 Fuly I916 of wounds received in action 8 fuly I916

Joy, N. H. Capt., E. Yorks. Regt.(T.F.) and Spec. List 1908 (Officer i/c Anti-Gas Schools).

Juler, F. A. Capt., R.A.M.C.

JUMP, H. Capt., Ist Dragoons. (P.)

JuMP, R. L. Air Mechanic, R.A.F.

Just, T. H. Capt., R.A.M.C. $M$.

I 899

1909

I900

1907

I873

I90I

1897

I9II

KaRney, Rev. A. B. Chaplain, R.N.; C.F. 4th Class, I893 R.A.C.D. (P.)

KaYE, G. W. C. Capt., R.E. (London Electrical En- I905 gineers, T.F.); Major (T.), R.A.F. (Aircraft Production Dept.) O.B.E. T.D. M. m.

KeELING, B.F.E. Lieut.-Col., R.E. (W.) O.B.E. M.C. ${ }_{1898}$ M2.

c. U.W.L. 
WKeeling, F. H. C.S.M., D. of Cornwall's L.I. (W.) Killed in action 18 Aug. 1916

Keeling, O. H. Capt., R.E.(T.F.) (W.) $M . m$. WEen, A. W. Major (A.), R.A.F. M.C.

Died 2 Sept. 1918 of wounds received in action ${ }_{1} 5$ Aug. I9I 8

Keenan, J. B. Capt. and Adjt., Irish Gds. (W 2.)

AKelsey, A. E. Fleet-Surgeon, R.N.; Capt., R.A.M.C.

Drowned on H.M. hospital ship Glenart Castle 26 Feb. I9I8

Kemp-Welch, H. A. Lieut., Hampshire Regt.(T.F.) and M.G.C.

Kempe, Rev. E. C. C.F. 3rd Class, R.A.C.D.

Kempson, E. W.E. Major, R.E. and R.A.S.C. M.C. $M 2$.

Kendall, F. R. N. 2nd Lieut., R.F.A.

Kennedy, Rev. E. H. C.F. $4^{\text {th }}$ Class, R.A.C.D.

KenNedy, Lord H. Lieut., Coldstream Gds. M.C. M.

WKennedy, H. T. 2nd Lieut., N. Staffs. Regt.; Lieut., 1908 R. Scots Fus.; attd. R.E. (Field Survey Coy.) (W.) Killed in action 6 Fune I9I7

WENNEDy, J. M. S. Lieut., Seaforth Hdrs.

Killed in action $10 \mathrm{Aug}$. 191 5

Kennedy, M. S. N. Capt., Border Regt.; empld. Infy. 1908 Base Depôt. (W.) $M$.

Kennedy, S. D. 2nd Lieut., Spec. List

Kennedy, W. T. Capt., Rifle Brigade and King's Liverpool Regt.)

Kennedy-Cochran-Patrick, N. J. Capt., R. Scots Fus.; 1884 empld. Ministry of National Service. M.B.E.

Kennedy-Cochran-Patrick, W. J. C. Capt., Rifle Bri- [I9I4] gade; Major (A.), R.A.F.; S.O. 2, Air Ministry. D.S.O. M.C. and Bar. M.

KenYon, M. N. Lieut., D. of Lancaster's Own Yeo.; 1905 attd. S. Lancs. Regt.

Kerby, C. C. Capt., R.A.M.C.

KERR, W. H. Lieut., Cheshire Yeo. and I Ith Hussars 1904 1913

WKerrison, R. O. Lieut.-Col., Res. Regt. of Cavalry; attd. I89I Australian F.A.

Died I8 Sept. I9I7 of dysentery contracted on active service

KERSEY, R. H. Capt., R.A.S.C.

Kershaw, J. 2nd Lieut., R.G.A. 
Keswick, H. Capt., King's Own Scottish Borderers

Keswick, H. G. 2nd Lieut., I3th Hussars

1889

1906

KIDD, C. B. Lieut., Surrey Yeo. and The Queen's 1898

(R.W. Surrey Regt.)

KIDD, F. S. Capt., R.A.M.C.

KIDD, H. L. Lieut., R.A.S.C. (Camel Corps)

KIDD, R. H. 2nd Lieut., R.E. (Signals)

KILliCK, C. Major, R.A.M.C.(T.F. Res.)

Kinahan, A. E. Lieut., R.F.A.; Supt., Remount Depôt Kindersley, Rev. C. E. C.F. 3 rd Class, R.A.C.D.(T.F.)

KindersLey, H.R. Major, Dorset Yeo. and Labour Corps

Kindersley-Porcher, C. P. W. Capt., Coldstream Gds.

(R. of O.), empld. Irish Gds.; Lieut.-Col., King's Royal Rifle Corps

WKING, A. M. Major, Rifle Brigade

Killed in action 15 March 1915

KING, B. N. 2nd Lieut., R.A.S.C.(M.T.)

King, C. Capt., R.A.M.C. O.B.E.

KInG, C. M. Capt., Coldstream Gds.; empld. Gds. Depôt

King-Webster, H. C. R. Fusiliers

Kingham, W. R. Gnr., H.A.C.

1896

1898

1915

1893

1890

1884

1882

1875

KINLOCH, Sir G., Bart. Lieut., Gen. List (T.F. Res.); empld. Ministry of National Service

HKinnaird, Hon. D. A. Capt., Scots Gds.

Killed in action 24 Oct. I9I4

$\begin{array}{lll}\text { KinnaIRd, Hon. K. F. Capt., Scottish Horse. } M . & \text { 1899 } \\ \text { KIRKe Smith, A. Lieut., I.A.R.O., attd. I Ith Bengal } & 1896\end{array}$ Lancers and I2th Cavalry

KIRKPATRICK, Rev. A. P. Chaplain, R.N.

KITson, Hon. R. D. Capt., W. Yorks. Regt.; Brigade $\quad \begin{aligned} & 1904 \\ & \text { I90I }\end{aligned}$ Major. D.S.O. M.C. $M 2$.

KITson, S. D. Capt., Yorkshire Hussars; Major, A.P.M. $\quad 1889$ $m$.

KNEESE, Rev. R. H. W. Capt., King's School, Rochester, 1907 O.T.C.; C.F. $4^{\text {th }}$ Class, R.A.C.D.

WKNIGHT, J. O.C. 2nd Lieut., The Queen's (R.W. I9r6 Surrey Regt.)

Killed in action 30 Nov. 1917

WKnight, P. C. Pte., H.A.C.; 2nd Lieut., Somerset L.I. I9I I (W.)

Killed in action I fuly r916

Knight Bruce, R. E. C. Capt., R. Ist Devon Yeo.; 1909 Major (Ad.), R.A.F.

Knobel, W. B. Capt., R.A.M.C. 
Knowles, A. J. Major, London Regt. (Queen's West- 1882 minster Rifles)

Knowles, D. Capt. (Airship), R.A.F. D.S.C.

Knowles, G. J. F. Capt., R.G.A. M 2. French Croix 1898 de Guerre. Greek Military Cross

Knowles, Sir L., Bart., c.v.o. Lieut.-Col., Lancs. Fus. 1875 O.B.E. T.D.

KNowles, R. K. Capt., Manchester Regt. $M$.

Knox, Rev. C. W. Pte., R.A.M.C. (Sanitary Service, I 884 T.F.)

Knox, G. G. Lieut., R.N.V.R. and Spec. List (Intelli- 1906 gence)

Kohan, C. M. Capt., R.F.A.(T.F.) O.B.E.

LACE, L. E. C. D. 2nd Lieut., I Ith Hussars; A.D.C. [I9I4]

LACEY, F. A. Capt., I.A.R.O., attd. 53rd Silladar Camel 1906 Corps

Lacy Thompson, T. A. Lieut., Northumberland Fus. 1913 (W.) D.S.O. M.C. M 2 .

ELafone, E. W. Capt., Durham L.I. (W.) M.C. M. [1914] French Croix de Guerre

Killed in action 15 Fune 1918

LaIdlaw, F. F. Capt., R.A.M.C.

LAING, B. Capt., Sherwood Rangers; empld. Admiralty $\quad 1896$

LaING, C. M. Capt., Northumberland Yeo.; A.D.C. 1904

(W 2.) M.C. M 2. Belgian Croix de Guerre

LaInG, J. C. Lieut.. Black Watch; empld. Ministry of [1914] Munitions

LAmB, C. H. Major, 44th (W. Australia) Bn., Australian 1906 Infy. M.C.

Lamb, G. J. Lieut., Cranleigh School O.T.C.

Lamb, R. E. Capt., R.A.S.C. $M$.

LAMB, R. P. Lieut. (K.B.) and Capt. (Ad.), R.A.F. (W.)

LAMBERT, E. T. Major, Bombay Vol. Artillery

LAMBERT, H. Lieut., Bengal Nagpur Railway Bn., Indian Defence Force

WLAmbert, H. M. Capt., ist Dragoons

Killed in action near Ypres 13 May I9I 5

LAMBERT, R. E. Lieut., R.H.A.(T.F.); Asst. Inspector, I90I Woolwich Arsenal

LAmberT, St J. M. Capt., R.H.A.(T.F.) $M$.

1909 I 898 1900 1897 1899

1897

LANCASTER, S. Capt., Dorset Regt.; empld. O.C.B. (W.) IgII

WLandale, D. B. Lieut., Rifle Brigade. $M$. Killed in action 23 Oct. I9I4 
Lander, T. E. Capt., Highland L.I.; Hon. Capt. (A.), I9I3 R.A.F. (W.) (P.) M.C.

WLANG, A. H. 2nd Lieut., Grenadier Gds.

Killed in action at Cuinchy 25-26 fan. 1915

LANG, B. T. Capt., R.A.M.C.

LANG, Rev. L. H. C.F. 4th Class, R.A.C.D.

LANGLANDS, N. M. S. Instructor Lieut., R.N.

Larmour, Rev. A. C. Capt., Wellington College O.T.C. ${ }_{\text {I }} 905$

La Touche, G. G. D. See Digges La Touche, G. G.

Laurence, C. Capt., London Yeo. (Rough Riders); attd. 1900

London Regt. (St Pancras Bn.) (W.)

Laurence, C. H. Gnr., R.F.A.; attd. Gen. Staff (Intelli- I899 gence)

Law, R. A. Lieut., Yorkshire Regt.; empld. War Office. I9I I (W.)

LAWLEY, Sir A., G.C.s.I., G.C.I.E., K.C.M.G. Colonel, Spec. 1879

List. $m$ 4. Chevalier, Order of Leopold (Belgium)

Lawrence, A. C. C. Capt., R.A.M.C.(T.F.)

LAWRENCE, B. L. Lieut., Grenadier Gds. (W.)

HLawrence, C. H. 2nd Lieut., King's Royal Rifle Corps

Killed in action $\mathrm{I}_{3}$ Oct. $19 \mathrm{r} 4$

WLawrence, M. C. Capt., Coldstream Gds. (W.)

Died 16 Sept. 1916 of wounds received in action 15

Sept. 1916

FLawrence, O. J. 2nd Lieut., London Regt. (Post Office

Rifles).- $M$.

Killed in action at Festubert 26 May r9r 5

Lawrence, W. H. A. Lieut., Essex Regt.; attd. Rifle I9r2 Brigade (T.F.)

Lawrie, C. V. E., c.B., D.s.o. Hon. Colonel, King's Own 1874

Scottish Borderers (T.F. Res.)

LAWRIE, J. M. 2nd Lieut., Spec. List (Intelligence)

Lawson, G. Major, Westmorland and Cumberland Yeo.

Lawson, R. C. Capt., Saskatchewan Regt., Canadian Force

WLawson-Johnston, A. McW. Lieut., Buckinghamshire

Yeo. and Grenadier Gds. M.C.

Died $22 \mathrm{Feb}$. 1917 of wounds received in action

fLayman, F. H. Major, R. Defence Corps

Died 3 Oct. 1917

LeA Wilson, B. H. C. Major, R.A.M.C.(T.F.); empld. 1904

Egyptian Army

WLEADER, B. E. Capt., The Queen's (R.W. Surrey Regt.) 1896

Killed in action 12 Oct. I916

LEADER, E. E. Lieut., R.N.V.R.

I90r 
Leaf, C. S. Lieut., The Buffs (E. Kent Regt.) and [1914] M.G.C.

LEAF, E. H. Lieut.-Col., R.E.(T.F.)

LEAF, F. A. Lieut., R.E. (Signals); empld. War Office 1886

LEAF, H. M. Lieut.-Col., R.E. (London Electrical Engineers, T.F.) (W.) D.S.O. M.

LEaK, E. A. Surgeon Sub-Lieut., R.N.V.R.; Lieut., 1909 R.A.M.C.

LEAK, W. N. Lieut., R.A.M.C.

Leatham, C. B. Major, King's Own (Yorkshire L.I.); 1912 attd. London Regt. (W 2.) M.C. and Bar. M.

Leatham, H. W. Capt., R.A.M.C.

LEATHER, K.J.W. Lieut.-Col.,Durham L.I. (W.) C.B.E. ALe Blanc Smith, C. R. P.O., R.N.R.; Lieut., Rifle Brigade. (W.)

Killed in action in the Ypres Salient 27 Nov. I9r 5

Le Blanc Smith, T. E. Major, R.F.A. (W 2.) M.C.

ALe Blond, R. C. G. DU P. Capt., Rifle Brigade Died I6 May I9I 5

LEDWARD, H. D. Capt., R.A.M.C.

LEE, A. N. Major, Sherwood Foresters (Notts. and Derby Regt., T.F.); Lieut.-Col., G.S.O. I. D.S.O. O.B.E. T.D. M3. Officer, Ordre de la Couronne (Belgium). Belgian Croix de Guerre. Order of the Sacred Treasure, $4^{\text {th }}$ Class (Fapan). Order of the Crown of Siam, 3 rd Class. Order of the White Eagle, 4th Class, with swords (Czecho-Slovakia)

1910

1896

1909

1913

1906

1896

1896

LEE, C. B. Major, R.F.A.(T.F.)

LEE, W. E. Major, R.A.M.C.

WLeEke, Charles. Lieut., Grenadier Gds.

Died II April I9I6 of wounds received in action

LEEKe, Rev. Christopher. C.F. 4th Class, R.A.C.D.

LEEKE, Rev. E. J. C.F. 4th Class, R.A.C.D.

LEEKE, J. A. Capt., Norfolk Regt. (W.) M.C.

LEFroy, E. J. Capt., Wiltshire Regt. and R.E.; empld. I9I I War Office. (W.)

HLeFroy, F. P. 2nd Lieut., R.E.

Killed in action 28 April 1916

1903

I894

1906

1901

1903

1904

[1914]

LEGG, Sir G. E. W., M.v.o. Capt., S. Staffs. Regt. (R. of 1887

O.) K.B.E. Chevalier, Legion of Honour (France)

Lehmann, F. H. See Layman, F. H.

Leith, H. G. Colonel, Northumberland Yeo.; Dep. 1898 Controller of Salvage, War Office. C.B. C.B.E. $M$ 2. Officer, Order of the Crown of Italy 
LE LACHEUR, W. J. Lieut., R.N.V.R.

LE RAY, H. G. 2nd Lieut., Worcestershire Regt.; Lieut., R.F.A. (P.)

LE Roy-LewIS, H., c.B., D.s.o., T.D. Colonel, General Staff; Military Attaché, Paris. C.M.G. M. Commander, Legion of Honour (France). French Croix de Guerre. Order of St Stanislas, Ist Class (Russia).

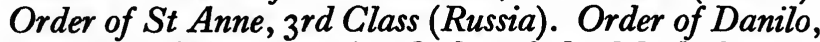
2nd Class (Montenegro). Order of the Black Star of Benin, ist Class. Order of the Crown of Roumania, $3^{\text {rd Class. Order of the White Eagle, } 4 \text { th Class (Serbia) }}$

LesLIE, A. Lieut., R.F.A.; empld. Ministry of Munitions ; Capt., Spec. List (Courts-Martial Officer). (W.)

Leslie-Melville, A. B. Capt. and Adjt., Sherwood Foresters (Notts. and Derby Regt., T.F.) (W.) $M$. LEVER, D. Instructor Lieut., R.N.

LeVETT, A. R. 2nd Lieut., King's Royal Rifle Corps and Suffolk Regt.

LevetT, E. C. Capt., R.E. (Fortress, T.F.)

LEvi, W. H. Lce.-Corpl., Essex Regt.

1895

1914

1879

1908

I $88 \mathrm{I}$

1906

1914

1907

1914

WLevinge, H. G. Lieut.-Col., Norfolk Regt. and Loyal I883 N. Lancs. Regt. $M$.

Killed in action in Gallipoli 1o Aug. 1915

Levita, C. E. Capt., Spec. List (A.P.M.) $M$.

Levita, H. P. Hon. Lieut.-Col., Spec. List; Draft Conducting Officer; Cmdt., Command Depôt. $m$. Order of St Stanislas, 2nd Class (Russia)

ELEwIN, K. R. Lieut., D. of Cornwall's L.I. Killed in action 9 March 1916

WLewThwaite, C. G. Lieut., R.F.A.(T.F.) (W.) M.C. 1903 $M$.

Killed in action 29 Fuly 1917

LEWTHWAITE, W. 2nd Lieut., R.F.A.

1886

1879

1906

ILiAs, R. J. M. Pte., Middlesex Regt. (P.S. Bn.); Lieut., R. Sussex Regt.

Killed in action $23 \mathrm{Feb}$. 1916

LichFiELD, Earl of. Capt., London Regt.(L.R.B.); A.D.C. 1902 $M$.

Lichtenberg, W. A. Lieut., R.G.A.

LidDell, C. F. J. Lieut., King's Royal Rifle Corps; I9I I

Capt., Spec. List (cmdg. T.M.B.) M.C. Chevalier, I90I 1909 Ordre de la Couronne (Belgium). Belgian Croix de Guerre

1908

Lidderdale, F. J. Capt., R.A.M.C. M.C. $M$. 
※Lightbody, W. P. Lieut., Norfolk Regt. Killed in action 26 Sept. I9I 5

LiNDEMERE, V. Capt., R.A.S.C. $M$.

LindLEY, W.M. Capt., R.E.(Signals). M.C. M. Che- 1900 valier, Legion of Honour (France)

LinDSAY, J. H. Lieut.-Col., London Regt. (London 1889 Scottish). (W.) D.S.O. M 2.

Lindsay, Hon. L. Pte., R. Fusiliers (P.S. Bn.); Capt., 1897 King's Royal Rifle Corps; empld. O.C.B. and Foreign Office. M.C. Chevalier, Legion of Honour (France)

HLingard, J. R. Lieut., Manchester Regt.; attd. Lancs. 1903 Fus.

Killed in action in Gallipoli 21 Aug. I9I 5

WLister, A. H. Lieut.-Col., R.A.M.C.(T.F.) C.M.G. ${ }_{1883}$ $M$.

Died I7 Fuly 1916 of tuberculosis

Lister, A. R. Capt., R.F.A.; Staff Capt. M.C. $M$.

Lister, Sir W. T. Colonel, A.M.S. K.C.M.G. C.M.G. 1886 M. $m$.

Littleboy, C. N. Capt., Durham L.I.(T.F.) and Sherwood Foresters (Notts. and Derby Regt., T.F.) (W 2.) M.C. and Bar

LitTlEwood, J. E. 2nd Lieut., R.G.A.(T.F.); empld. 1903 Ministry of Munitions

LleEwelyn-Davies, P. Lieut., King's Royal Rifle Corps. [1914] M.C.

WLlewelyn-Davies, R. A. Lieut., R. Fusiliers. Serbian I9I I Distinguished Service Medal

Killed in action 4 Oct. 1918

ALloyd, A. S. Lieut., R.F.A. M.C.

Killed in action 4 Aug. I9I6

Lloyd, C. G. Capt., E. Riding of Yorkshire Yeo. M. 1905

Lloyd, E. A. Lieut., London Regt. (R. Fus.); Major, 1904 D.A.D. Docks. (W.) $M$.

Lloyd, E. I. Surgeon Lieut., R.N.

Lloyd, Sir G. A., M.P. Capt., Warwickshire Yeo. and Gen. Staff. G.C.I.E. C.I.E. D.S.O. M 3. Order of St Anne, 3 rd Class, with swords (Russia)

Lloyd, H. C. Capt., Yorkshire Regt. I9I I 1898

LLOYD, H. G. Lieut., D. of Cornwall's L.I. and Gen. Staff. (W.) M.C.

WLloyd, J. F. S. Major, N. Staffs. Regt.(T.F.)

Killed in action 18 fune r9I 5 
Lloyd, L. S. Capt., R.F.A.(T.F.)

LLOYD, R. LL. 2nd Lieut., R.F.A.

I9I3

Lloyd Greame, Y. Lieut., R.F.A.

1890

Lloyd-Jones, I. G. Hon. Major, Gen. List (T.F. Res.) I 888 T.D.

Lloyd-Williams, A. R. C. Lieut., Lancs. Fus.

1899

Locker-Lampson, G. L. T. 2nd Lieut., Wiltshire Yeo. 1894 (T.F. Res.)

LockeR-Lampson, O. S. Lieut.-Cdr., R.N.V.R. (Armoured Car Section). C.M.G. D.S.O. Officer, Order 1900 of Leopold (Belgium). Order of St Vladimir, $3^{\text {rd Class, }}$ with swords (Russia)

LOCKETT, J. Lieut., Lancs. Hussars ; attd. King's (Liverpool Regt.)

LOCKETT, V.N. Capt., I 7 th Lancers; Major, I 9 th Hussars 1899

LOCKYER, W. J. S. Major (T.), R.A.F. I887

LODER, J. DE V. Capt., R. Sussex Regt.(T.F.) and Gen. [I9I4] Staff (Intelligence). $M$.

LODER, N. W. Lieut., R.A.S.C.

HLoder, R. E. Lieut., R. Sussex Regt.(T.F.); Staff Capt. Ig06 $M 2$.

Killed in action 29 March 1917

Long, W. G. 2nd Lieut., Tank Corps

WLongbotrom, H. 2nd Lieut., S. Lancs. Regt.

Killed in action in Gallipoli 9 Aug. I9I 5

LoNGhurst, A. L. Lieut.-Col.,7th Gurkha Rifles, Indian Army. (W 2.) Brevet Lieut.-Colonel. M 4.

Longhurst, H. B. 2nd Lieut., London Regt. (Rangers) 1909

LONGMAN, H. K. Capt., Gordon Hdrs. (R. of O.); Major, I900 D.A.Q.M.G. D.S.O. M.C. $M_{3}$.

Longman, R. G. Capt., Gordon Hdrs.; empld. O.C.B. 1902 (W.) $m$.

WLONGridge, Rev.A.O.C. C.F. 4th Class, R.A.C.D. I902 (W.)

Died 12 Oct. 1918 of pneumonia following influenza and gas-poisoning

LoNGRIDGE, Rev. M. Chaplain, R.N.

LongstafF, C. C. Lieut., Durham L.I. (W.)

HLonsdale, A. C. G. Lieut., King's Royal Rifle Corps; attd. R. Scots Fus.

Killed in action 10 March $19 \mathrm{I} 5$

WLovetr, Rev. R. D. Pte., Middlesex Regt.

Killed in action fuly r 916

Lowe, H. St A. Capt., Radley College O.T.C. 
WLowry CorRy, F. R. H. Lieut., R.F.A.

Died 30 Sept. 191 5 of wounds received in action 25 Sept. I9I 5

LowTHER, C. W. Capt., Westmorland and Cumberland 1905 Yeo.; Major, A.P.M. (W.) $M$.

Loyd, E. N. F. Capt., Warwickshire Yeo.; Lieut., La- 1899 bour Corps

सLoyd, L. F. I. Capt., Lovat's Scouts; Major, Worcester- $\quad$ I 898 shire Yeo.

Died 21 Sept. I918 of pneumonia

Loyd, R. A. Capt., Remount Service

LOYD, W. H. Lieut., R.E. and Spec. List (R.T.O.)

1904

1913

WLubBock, Hon. H. F. P. Lieut. and Adjt., W. Kent I906

Yeo.; Lieut., Grenadier Gds.

Killed in action 4 April 1918

LuCAs, A. R. F. Major, R.F.A. (W.) M.C. M.

LuCAS, C. E. Capt., R. Fusiliers; Staff Capt. $m 2$.

Lucas, D. Pte., H.A.C.; Lieut., Spec. List. $m$.

LuCAS, E. M. Major, Remount Service

LuCAS, F. L. Lieut., Queen's Own (R.W. Kent Regt.) and Gen. Staff (Intelligence). (W 2.) $M$.

Lucas, G. M. E. Pte., R. Fusiliers (P. S. Bn.); Capt., R. 1906 Inniskilling Fus. $M 2$.

$\begin{array}{ccc}\text { LucAs, H. A. Major, R.A.M.C.(T.F.) } M 2 . & 1904 \\ \text { ALucas, K. } & \text { Capt., R.F.C. (Hampshire Aircraft Parks, } & 1898\end{array}$ T.F.)

Killed in flying accident 5 Oct. 1916

LuCAS, W.G. Major, R. Fusiliers; attd. R.N.A.S.; Lieut.Col., R.G.A.

Lucas, W. L. Major, R.F.A. (W.)

Luскоск, E. H. M. Capt., S. Wales Borderers (T.F.); 1890 empld. Musketry Staff. $m 2$.

Luckock, R. M. Lieut.-Col., King's Own (R. Lancaster 1896 Regt.); G.S.O. I. C.M.G. D.S.O. Brevet Lieut.Colonel. M 8. Officer, Legion of Honour (France). French Croix de Guerre

Lumley, C. H. Capt., S. Lancs. Regt.; empld. Ministry of Munitions. (W.) M.B.E. m. Chevalier, Legion of Honour (France)

Lumley-Smith, T. G. Capt., 2ist Lancers; Major, $\quad{ }_{1898}$ A.P.M. D.S.O. $M 2$.

LuMSDEN, J. A. 2nd Lieut., R.G.A.

LuMSDEN, W.F. Major, R.G.A.; Lieut.-Col., A.Q.M.G. $\quad 1898$ D.S.O. $M 2$. 
Lund,H. Capt., R.A.M.C. (2nd W. Gen. Hospital, T.F.) $\quad$ I878

LuPton, A. C. Capt., Yorkshire Hussars and Remount 1893 Service

Lupton, B. C. Capt., D. of Wellington's (W. Riding I9r3 Regt., T.F.) (W 2.) M.C. and Bar

WLuPTON, F. A. Major, W. Yorks. Regt.(T.F.)

Killed in action 19 Feb. 1917

LuptoN, H. R. Capt., W. Yorks. Regt.; attd. North- 1912 umberland Fus. (W 4.) M.C. $M$.

\&Lupton, L. M. Lieut., R.F.A.(T.F.) (W.) M2. I910 Killed in action 16 Fuly 1916

WLupton, M. Capt., W. Yorks. Regt.

Killed in action 19 . Fune 1915

Lupton, N. D. Major, W. Yorks. Regt.(T.F.) and ' 1894 London Regt. (Rifles); Major, Spec. List (Cmdt., Reception Camp)

LyCETT, C. V. L. Capt., R.E. (Signals). (W.) M. 1912

LYELL, G.D. Lieut., I.A.R.O., attd. 7 th Hariana Lancers 1905

LYMan, T. Major, Engineers, United States Army I90I

WLyon, E. L. Major, I8th Hussars; attd. Somerset L.I. 1896 (W 2.) $M$.

Died I7 Sept. I91 6 of wounds received in action

Lyon, R. E., v.D. Hon. Colonel, R.F.A.(T.F. Res.) C.B. $\quad 1884$

LYSTER, L.F. Lieut., R.A.O.C. $\quad$ I909

LYThGOE, R. J. 2nd Lieut., R.G.A. I9I 5

LYTTELTON, A. G. Major, Welsh Regt.; Lieut.-Col., 1903

M.G.C. D.S.O. Brevet Major. M4.

Lytrelton, Rev. Hon. C. F. C.F. $4^{\text {th }}$ Class, R.A.C.D. 1905 M.C.

LytTelton, Hon. G. W. Lieut., Eton College O.T.C. 1902

LyTteltoN, O. Capt., Grenadier Gds.; Brigade Major. 1912 (W.) D.S.O. M.C. M2.

LytTelton, Hon. R. G. Major, R.F.A.(T.F.) (W.) 1913 French Croix de Guerre

MacAndrew, C. G. Capt., Ayrshire Yeo.; Major,M.G.C. 1906 (Cavalry)

Macarthur-Onslow, J. W. Colonel, Australian Oversea $\quad 1887$ Transport Service

MaCARTNEY, C. A. 2nd Lieut., Hampshire Regt.; Lieut., 1914 R.F.A.; attd. T.M.B. (W.)

AMacartney, H. B. G. Capt., R. Fusiliers Killed in action 24 Fune 1915 
Macbeth, A. H. Capt., D. of Cornwall's L.I.

McCall, H. W. Lieut.-Col., Yorkshire Regt.; A.A. and Q.M.G. (W.) C.M.G. D.S.O. Brevet Lieut.Colonel. M 2. Chevalier, Legion of Honour (France). Order of the Nile, $4^{\text {th Class (Egypt) }}$

McCall, H. W. L. Capt., R.A.S.C. $M$.

McCalman, J. A. C. Capt., E. Surrey Regt.

McCandlish, P. D. Major, Argyll and Sutherland Hdrs. (R. of O.); Lieut.-Col., A.Q.M.G. C.B.E. D.S.O. Brevet Lieut.-Colonel. Brevet Major. $M_{3}$. Order of the White Eagle, $5^{\text {th Class (Serbia). }}$

McClintock, Rev. E. L. L. C.F. 4th Class, R.A.C.D. 1905

McClintock Bunbury, Hon. T. L. Lieut., Spec. List. 1898 M.B.E. M. Cavalier, Order of the Crown of Italy. Italian Croce di Guerra

McClure, K. A. J. Capt., Middlesex Regt. and Spec. IgI I List, empld. British Military Mission

McCormick, E. H. Lieut., United States Naval Air 1908 Service

McCormick, L. J. Capt., 343rd Infy. Bn., United States 1907 Army

McCosh, R. Capt.,Lanarkshire Yeo.; Major,D.A.Q.M.G. 1904 O.B.E. M.C. M 2. Order of the Nile, $4^{\text {th }}$ Class (Egypt)

MCCRAITH, K. Y. Capt., Sherwood Foresters (Notts. 1909 and Derby Regt., T.F.) and Gen. Staff. (W.)

Macdona, C. L. Major, Spec.List (D.A.D.R.T.) O.B.E. 1906 $M$.

Macdonald, A. D. Capt., R.F.A.

MacDonald, Rev. A. J. M. C.F. $4^{\text {th }}$ Class, R.A.C.D.

McDougal, E. T. M. Lieut., Scots Gds. M.C. M.

MacEwen, D. L., c.B. Lieut.-Col., Cameron Hdrs.; 1886 Brig.-Gen.; D.Q.M.G., E. Command. C.M.G. M. Commander, Legion of Honour (France)

McEwen, J. H. F. Capt., Cameron Hdrs.; Hon. Capt. 1912 (O.), R.A.F. (P.)

WMcEwen, J. R. D. Lieut., R. Scots Fus.; A.D.C.

Killed in action 12 Oct. 1916

MACGREgOR, D. H. Lieut., R.E. (Signals, T.F.); Capt., $\quad$ I 898 G.S.O. 3. M.C. M.

Macgregor, M. E. Capt., Spec. List

McIntyre, E. Capt., Cameron Hdrs. and Labour Corps McIntyre, N. Capt., R.A.S.C.

MAcIver, C. R. Capt., R.E. (Light Railways). (W.) I9ro 
WMackay, E. R. Capt., Argyll and Sutherland Hdrs. Killed in action in Gallipoli 13 June 1915

Mackay, G. R. Pte., H.A.C.; Lieut., London Regt. I9I I (Q.V.R.)

MACKAy, Hon. K. Capt., I2th Lancers

MACKAY, R. D. Lieut., London Regt. (Rangers)

Mackennall, Rev. W. L. C.F. $4^{\text {th }}$ Class, R.A.C.D.

1906

1908

1903

WMACKENZIE, C. R. Flt.-Cdr., R.N.A.S. D.S.O. M. I9IO French Croix de Guerre

Killed in action 24 Fan. I9I7

WMcKenzIE, J. 2nd Lieut., Seaforth Hdrs.

Killed in action 30 Oct. I9I 5

MACKenzIe, L. H. L. Major, I.M.S. (W.) Brevet 1899 Major. $M$.

MACKenzie, S. M. Capt., R.A.M.C.

1898

MCKerrell Brown, J. Capt., Seaforth Hdrs.; Capt. and 1907 Adjt., Highland L.I.; attd. Gen. Staff. $m$.

Mackinlay, A. B. Pte., R. Fusiliers

MackWorth-Praed, C. W. Lieut., Scots Gds.

I9II

Mack 1911

MaClaren, D. Lieut., King's Own Scottish Borderers 1896

McLaren, D. B. Lieut., Canadian F.A.

Maclaren, N. Major, R.A.M.C.(T.F.) T.D.

1892

1894

I 897

Died 13 Aug. I916 of wounds received in action

McLean, A. Major and Adjt., Inns of Court O.T.C. $\quad$ I895 M.B.E. Brevet Major. $m 2$.

McLean, C. Capt., R.G.A. (W.)

WMacmaster, D. C. D. Lieut., Cameron Hdrs.

Killed in action 26 Sept. I9I 5

WMacmichael, M. W. A. 2nd Lieut., Devon Regt.; Capt., I9I3 Essex Regt.

Died 16 Sept. 1916 of wounds received in action

*Macmicking, G. T. G. 2nd Lieut., Cambridgeshire Regt. I9I3 Died in Holland I I Nov. 1918

Macmillan, D. 2nd Lieut., S. Notts. Hussars 1909

Macmillan, J. M. Capt., R. Defence Corps 1896

WMacmullen, E. R. Capt., E. African Force; A.D.C. M. 1903 Killed in action 30 Fune 1916

WMacNaghteN, A. C. R. S. Lieut., Black Watch 1906 Killed in action 29 Oct. I9I4

WMacnaghten, Sir A. D., Bart. 2nd Lieut., Rifle Brigade I9I4 Killed in action 15 Sept. 1916

Macnaghten, C. M. Lieut.-Col., $4^{\text {th }}$ (N.S.W.) Bn., 1898 Australian Force. (W 2.) C.M.G. $M$. 
WMcNeILe, J. Lieut.-Col., King's Own Scottish Borderers Wounded and prisoner, presumed killed in action, $12 \mathcal{F} u l y$ I9I 5

McNeIle, J. H. Lieut., Coldstream Gds. (W.) (P.) WMacNeill, A. D. Capt., R.G.A.

I 88 I Killed in action 29 Fuly r917

Macnutt, Rev. F. B. C.F. $4^{\text {th }}$ Class, R.A.C.D.(T.F.) $\quad$ I894 MACRobert, T. M. 2nd Lieut., R.G.A.

Madan, A. G. Lieut., R. Fusiliers. (W.)

WMaddox, J. M. 2nd Lieut., Lancs. Fus.

Killed in action 12 Aug. I916

Maillet, A. L. Battery Cdr., Belgian Artillery

MainPrize, S. L. 2nd Lieut., Army Cyclist Corps; Lieut., Tank Corps and R.E. (Signals)

MaIr, R. P. Capt., Sussex Yeo.

Wartland, Hon. A. H. Major, Cameron Hdrs. Killed in action I9 Sept. I9I4

${ }^{1}$ Maitland, E. M. Lieut.-Col., Essex Regt.; Wing Cdr., $\quad$ I898 R.N.A.S.; Brig.-Gen., R.A.F. G.M.G. D.S.O. A.F.C. American Distinguished Service Medal

WMatlland, G. McD. 2nd Lieut., Irish Gds.

Killed in action at Klein Zillebeke I Nov. I9I4

Maitland, J. Lieut., R.G.A. $M 2$.

HMaitland-Makgill-Crichton, C. J. Lieut., Seaforth Hdrs.; Major, Gordon Hdrs.

Killed in action $25 \mathrm{Sept}$. 1915

Makins, F. K. Trooper, Chota Nagpur Light Horse, 1908 Indian Defence Force

Makins, Sir P. A., Bart. Major, Remount Service $\quad$ I890

MaKower, W. Lieut., R.N.V.R.

Malcolm-Dickinson, W. 2nd Lieut., R. Scots Fus.; I9I I Capt., Highland L.I.; empld. O.C.B. (W 2.)

WMalden, W. Capt., R.A.M.C.(Ist E. Gen.Hospital,T.F.) 1877

Died Nov. I9I8

Malim, F. B. Capt., Haileybury College O.T.C.

1891

Mallaby-DeEley, G. M. Lieut., 5th Dragoon Gds. $\quad$ I9I5

Mallalieu, W. Capt., R.F.A. (W.)

Mallison, H. V. Pte., Durham L.I.

MANCHESTER, Duke of. Lieut., R.N.V.R. 1894

MandER, C. A. Major, Staffordshire Yeo. (W.) T.D. 1903

HMANDER, D'A. W. Major, Durham L.I. I888

Killed in action 20 Sept. 1914

MANDER, F. W. 2nd Lieut., R.A.S.C.

1891

\footnotetext{
${ }^{1}$ Killed in the wreck of airship 'R. 38,' Sept. 1921
} 
MANDER, G. LE M. Lieut., Spec. List, empld. Ministry I900 of National Service; Hon. Lieut. (T.), R.A.F.

Mandleberg, J. H. and Lieut., S. Lancs. Regt. (T.F. 1904 Res.); attd. R.N.A.S.

MANDleberg, L. C. Major, Lancs. Fus. (W.) D.S.O. I9I I M.C. and Bar. $M 2$.

MaNley, R. S. Capt., Staffordshire Yeo.

ManN,E.W. Gnr., R.F.A.; attd.BritishEmbassy, Copen- I901 hagen

WManN, I. A. Lieut., Cameronians (Scottish Rifles, T.F.); I9I3 attd. R.F.C. M.C.

Killed in action 9 Aug. 1916

ManNERs, Hon. F. H. Lieut., Grenadier Gds. (W.) 1914 M.C.

MANNERS, Sir G. Major, A.P.M., London District; attd. 1879 Cyclist Bde. $M$.

WMANSEl-Pleydell, J. M. 2nd Lieut., R.F.A.; A.D.C. 1903 Died 22 Sept. I916 of wounds received in action

Mansfield, R. S. Capt., R.E. (Signals); Staff Capt. I9ro O.B.E. $M$.

Manson, C. M. Capt., The Queen's (R. W. Surrey [I914] Regt., T.F.); Hon. Capt. (O.), R.A.F.

AMAPPlEbECK, G. W. Capt., N. Staffs. Regt. $M$. Died 30 Fuly 1917 of wounds received in action

I 898

Marc, G. J. A. Lieut., R.F.A.(T.F.); Staff Capt. M. 1908

Mardon, H. A. Pte., R. Fusiliers

WMARgerison, C. W. Lieut., Border Regt. $M$. Died 6 Fuly 1916 of wounds received in action $\mathrm{I}$ fuly I9I 6

MARIGOld, J. E. 2nd Lieut., R. Warwickshire Regt.

I9I I

MARKS, J. D. 2nd Lieut., 5th Dragoon Gds.; Lieut., R.F.A.

Marlborough, Duke of, T.D. Hon. Colonel, Oxford and 1890 Bucks. L.I. and Gen. Staff

Marling, W. J. P. Major, Gloucestershire Regt. $\quad$ I884

MARSDEN, C. A. Instructor Lieut., R.N. I9I3

Marsden, Rev. E. McL. Pte., R.A.M.C. 1888

WMarsh, A. S. Capt., Somerset L.I. 1909 Killed in action 6 fan. 1916

Marsh, F. D. Major, R.A.M.C.(T.F.) M.C.

Marshall, A. P. Lieut., Cameron Hdrs.(T.F.); Capt., 1895 O.C.B.

WMarshall, A. S. F. Section Sanitaire, French Army 1897 Died 25 Fuly 1918 from the effects of war service 
Marshall, W. S. D. Lieut., R.F.A.(T.F.) I893

Marsham, Rev. A. F. C.F. $4^{\text {th Class, R.A.C.D. }} 1904$

Marson, A. A. Major, R.F.A. M.C. M. 1909

MARTEN, A. J. Major, R.F.A.; empld. Ministry of Muni- $\quad$ I892 tions

MarTin, S. P. Capt. (Motor-boat), R.A.F.

Martin, T. L. Indian Defence Force

\#Martin, W. F. Major, Leicestershire Yeo. $M$.

Killed in action $\mathrm{I} 3$ May I9I 5

Martin, W. M. Corpl., Scots Gds. (W.)

Martin-Tomson, W. J. Lieut. (A.), R.A.F. (P.)

MaRTINET, M. Trooper, 29th Dragoons, French Army

Maryon Wilson, A. G. See Wilson, A. G. Maryon

MASSINGBERD, S. Major, Lincolnshire Regt. and Gen. $\quad$ I888 Staff. $M$.

WMaster, G. G. O. Lieut., Gloucestershire Regt.(T.F.) Killed in action 25 fuly 1916

Master, L. C. H. Lieut., R.F.A.; A.D.C. (W.)

I9I3

1903

I 894

1904

1907

I9I I

MASTERTON, H. W. Instructor Lieut., R.N.

Mather, A. Lieut., Highland L.I. (T.F.)

WMather, A. L. 2nd Lieut., York and Lancaster Regt.

Killed in action 7 Fan. I9I7

Mather, E. G. Capt., King's (Liverpool Regt.) $M$.

Mather, Rev. H. Section Sanitaire, French Army

Mather, L. E. Capt., R.E.(T.F.)

Matheson, I. McL. A. Capt., Lothians and Border

Horse. O.B.E. French Croix de Guerre. Order of the White Eagle, $5^{\text {th Class (Serbia) }}$

Mathew, F. A. H. Capt., R.E. (W.) O.B.E. M.G. I9II $M$ 2. French Croix de Guerre

Mathias, C. D. Capt., R.A.M.C.(T.F.)

MatThews, E. A. C. Major, I.M.S. D.S.O. M. 1895

Matruews, E. C Lieut., Middlesex Regt; Capt. Gen. List (P. and B.T. Staff)

Maude, E. A. W. Capt., N. Irish Horse; A.D.C.; Capt., I9r3 Tank Corps

Maunsell, S. A. W. Capt., R.A.S.C.

Maxwell, I. Constable. Capt., Cameron Hdrs. (W.) I9Io

Maxwell, M. T. Lieut., R.F.A. (W.) M.C.

Maxwell, R. Constable. Major, Spec. List, attd. I9IO Egyptian Army and R.N.A.S. $M$.

WMaxwell, W. F. J. Lieut., King's Own Scottish Bor- 1905 derers (T.F.)

Killed in action in Gallipoli 13 Aug. I9I 5 
Maxwell, W. H. Major, King's (Liverpool Regt., T.F.) I893 T.D.

May, G. H. Capt., Canadian A.S.C.

WMaYbrook, W. R. Pte., London Regt. (Artists Rifles); I9I3 2nd Lieut., Wiltshire Regt.

Killed in action 24 April 1916

Mayer, P. G. Capt., Rifle Brigade. (W 2.)

MAYHEWE, K. G. 2nd Lieut., Grenadier Gds.

I9I 2

Mayne, Rev. W. C. C.F. $3^{\text {rd }}$ Class, R.A.C.D.

1903

1896

Meakin, B. Capt., R.A.S.C.

1903

MeAKIN, L. Major, R.A.M.C.; D.A.D.M.S. (W.) $M$. I90I

Meakin, W. Lieut., N. Staffs. Regt.(T.F.)

1895

Medcalf, H. Capt., Hampshire Aircraft Parks (T.F.) 1905

Medlycott, H. M. Major, Dorset Yeo.(T.F. Res.)

1893

MeEK, J. 2nd Lieut., Unattd. List, T.F.

1906

MeIER, F. A. Capt., Rugby School O.T.C.

MelleR, R. W. Surgeon Lieut., R.N.; attd. R.A.F.

1907

Meller, S. A. Capt. (K.B.), R.A.F. $M$.

1905

1904

Melville, H. C. 2nd Lieut., Sherwood Foresters (Notts. I905 and Derby Regt.)

Killed in action in the Ypres Salient 14 Feb. 1916

Melville-Smith, H. McL. 2nd Lieut., R.E. (Fortress, I9I2 T.F. Res.)

\&MERCER, E. D. 2nd Lieut., Lancs. Fus.

Died 2 May 1917 of wounds received in action

Mercer, H. Bt. Colonel, 3rd Dragoon Gds. (R. of O.); 1880 cmdg. Res. Regt. of Cavalry

MERCER, J. Instructor Cdr., R.N.

WMerRyweather, C. W. Major, Lancs. Fus. (W.) M. I900 Killed in action 23 Nov. I9I6

Merton, G. Major (A.), R.A.F. (W.) M.C. $M$ 2. I9I I

Methuen, A. P. Lieut., Bedfordshire Regt. (W 2.) 1903

WMews, J. K. Capt., London Regt. (R. Fus.) (W.) [1914] Died 24 Aug. 1918 of wounds received in action

WMeYrick, E. E. Lce.-Sergt., Cambridgeshire Regt.

Died 30 Fuly 1916 of sickness contracted on active

1912 service

Meysey-Thompson, A. DE C. C. Lieut., R.F.A.; empld. 1905 Ministry of Munitions

Meysey-Thompson, E. C., M.P. Lieut.-Col., R.F.A. $\quad m$. $\quad 1877$

Meysey-Thompson, H. C. Capt., King's Royal Rifle I902 Corps. (W.)

WMichell, N. B. Capt., R. Fusiliers. (W.) $M$. 1905

Killed in action 22 March 1918

C.U.W.L. 
Middleton, R. C. G. Capt. and Adjt., R. Sussex Regt. 1909 (W.) M.C. M 2. French Croix de Guerre. Belgian Croix de Guerre

Midgley, E.C. Capt., Spec. List (Asst. Area Gas Officer) 1900

Milburn, A. Lieut., 2oth Hussars; attd. Staffordshire I9ro Yeo.

Milburn, A. W. Capt., Northumberland Yeo. M. $\quad 1905$

MilbuRn, J. D. Lieut., Black Watch

Milburn, Sir L. J., Bart. Lieut., Res. Regt. of R. Horse 1902 Gds.; A.P.M. (W.)

AMilburn, R. G. 2nd Lieut., E. Surrey Regt. Killed in action $9 \mathrm{Feb}$. 1915

Mildmay, Rt. Hon. F. B. Hon. Lieut.-Col., W. Kent I879 Yeo.; A.D.C.; attd. Gen. Staff. T.D. $M_{4}$.

WMILEY, M. Lieut., R.F.A.(T.F.)

Died 30 Dec. I9I 5 of wounds received in action

Millais, J. G. Lt.-Cdr., R.N.V.R. (Intelligence)

Miller, C. D. Capt., Sherwood Foresters (Notts. and Derby Regt., T.F. Res.)

Miller, C. H. Colonel, A.M.S. C.B.E. M. m. Jap- 1894 anese Order of Merit

HMiller, E. C. Capt., Loyal N. Lancs. Regt. Killed in action 23 Oct. r9I4

MilleR, E. D., D.S.o. Capt., I7th Lancers (R. of O.); 1883 Lieut.-Col., Pembroke Yeo.; C.B.E. $M 2$.

Miller, G. W. M. Capt., R. Scots (T.F.); Major, [I9I4] Durham L.I. M.C. $M$.

MilleR, R. B. Major, R.F.A

MILlER, R.W.R. Lieut., R.E.; empld. Ministry of Muni- r9ro tions

Mills, F. R. Lieut., R. Fusiliers; attd. R.E. (W.) 1897

WMILls, T. R. Lieut., Manchester Regt.(T.F.) 1906 Killed in action in Gallipoli 4 Fune 1915

Milne, A. A. Lieut., R. Warwickshire Regt.; empld. 1900 War Office. (W.)

WMilne, A. R. Capt. and Adjt., Hertfordshire Regt. Killed in action $3 \mathrm{I}$ Fuly 1917

Milne, E. A. Lieut., R.N.V.R.; empld. Ministry of I9I4 Munitions. M.B.E.

Milne, E. O. Capt., Somerset L.I.(T.F.) (W.) I9I3

MILNER, M. H., M.v.o., D.S.o. Major, Remount Service 1883 and Spec. List; A.D.C. M. Belgian Croix de Guerre

Milnes-Gaskell, E. Major, Yorkshire Dragoons; Asst. ${ }_{1896}$ Special Salvage Officer. $M 2$. 
Milsom, C. F. Major, R.A.S.C. D.S.O. M 3. I90I

Milsom, H. L. Capt., Somerset L.I. (W.) 1907

MirRLEES, A. J. Major, Sherwood Rangers. O.B.E. M. $\quad{ }_{1895}$ Mitchell, E. S. Capt., Worcestershire Regt. (W.) I9I3 M.C.

Mitchell, Rev.P. R. C.F. 2nd Class, R.A.C.D. O.B.E. 1895 $m$.

WMitford, Hon. C. B. O. F. Major, roth Hussars. (W.) 1896 D.S.O.

Killed in action 13 May 1915

Molony, B. C. Capt., Hertfordshire Regt.; Staff Capt. I9I I (W 2.) O.B.E. M.

Molteno, D. J. Cadet, R.M.C., Sandhurst

Momber, R. M. S. T. Capt., R.A.S.C.

MonckTon, T.A. Lieut.-Col. (T.), R.A.F. O.B.E. M. 1905

MonCRIEFF, D. C. Capt., R.F.A.(T.F.) (W 2.) M.G. 1909

MonKswell, Lord. 2nd Lieut., Spec. List (Interpreter) 1894 and R.F.A.

Montagu, J. G. E. Major, R.F.A. (W 2.)

Moor, F. Lieut., R. Berkshire Regt. (W.) M.C.

Moore, A. H. Surgeon Lieut., R.N.

I9II

r9I I

Moore, A. H.-G. C. Lieut., Sherwood Foresters (Notts. 1914 and Derby Regt., T.F.)

Moore, E. S. Capt., R.F.A.(T.F.) (W.)

WMOORE, G. A. C. Lieut., Cameronians (Scottish Rifles)

Died I I Fuly I915 of wounds received in action in Gallipoli 28 June I9I5

Moore-Anderson, A. P. Capt., S. African Med. Corps. 1892 $M 2$.

Moore-Brabazon, J. T. C. Lieut.-Col., R.A.F.; S.O. I, I90I Air Ministry. M.C. M 2. Chevalier, Legion of Honour (France)

WMoorsom, A. E. Pte., R.A.M.C.(T.F.); Lieut., Suffolk 1912 Regt.(T.F.)

Died 3 Aug. I916 of wounds received in action 15 Fuly 1916

Moorsom, C. W. M. Capt., Harrow School O.T.C.

Morcom, R. K. Lieut.-Col., R.E. C.B.E. M.B.E. 1896 $M 2$.

Moreing, A. C. Capt., London Regt. (R. Fus.)

Moreing, A. H., M.P. Capt., R.F.A.(T.F.) $M$.

HMorgan, A. C. O. Lieut., R.F.A.(T.F.) $M$.

Killed in action at the Hohenzollern Redoubt $\mathrm{I}_{3}$ Oct. 1903 I9I 5 
Morgan, S. C. Major, S. Wales Borderers and Gen. 1906 Staff (O.C.B.) (W.) M.B.E. $m 2$.

Morison, Sir T., K.C.I.E. Lieut., Cambridgeshire Regt.; 1882 Lieut.-Col., G.S.O. I, War Office. K.C.S.I. C.B.E.

M. Officer, Order of Leopold (Belgium)

Morley, C., jun. Lieut., R.F.A.

Morley, C. C. Lieut., R.N.V.R. O.B.E.

Morley, Hon. C. H. Capt., Grenadier Gds. (W.) M. 1906 Morley, F. W. Lieut., Somerset L.I.(T.F.); empld. 1900

Ministry of National Service

Morrice, G. W. Capt., R.A.S.C. $M$.

MorRIS, G. G. 2nd Lieut., Sherborne School O.T.C.

MorRIS, T.S. Capt., Rifle Brigade; Major, M.G.C. M.C. $M$.

MorRIs-Eyton, R. E. • Lieut., R.F.A.(T.F.) (W.)

MORRISON, H. N. Instructor Lieut., R.N.

MorRison, J. S. F. Major (A. and S.), R.A.F. D.F.C. and Bar. M. Italian Silver Medal for Military Valour. Italian Bronze Medal for Military Valour

Morrison, R. G. Lieut., 3rd Hussars; Hon. Lieut. (T.), R.A.F.

Morse, G. G. Capt., E. Surrey Regt. (W.) M.C.

Morshead, R. S. Capt., R.A.M.C. M.C. $M$.

MorTon, R. F. S. Major (A.), R.A.F. (W.) $M$.

Moss-Blundell, F. B. Colonel, R.F.A.(T.F.)

C.M.G. D.S.O. T.D. M 5 .

1902

1907

1914

I9II

I9I3

I9II

Muggeridge, C. E. Major, King's African Rifles; 1894 G.S.O. 2. M.G.

4Murr, B. L. Capt., R.A.S.C. and Spec. List (Courts- 1909 Martial Officer)

Died 4 Nov. 1918 of pneumonia following influenza

MuIr, D. M. Surgeon Lieut., R.N.

MuIR, J. Capt., R.A.M.C.(T.F.) O.B.E.

Muir, J. B. Major, Black Watch

I9I3

19I4

1905

1905

Muir, M. W. Capt., Gloucestershire Yeo.; Bde. M.G. Officer

Mules, F. J. Capt., Wellingborough Grammar School 1892 O.T.C. T.D.

Mulholland, Hon. G. J. A. M. L. Capt. and Adjt., Igr I R.A.S.C. M.C. $M$.

Mulholland, Hon. H. G. H. 2nd Lieut., Res. Regt. 1909 of Cavalry; Lieut. (T.), R.A.F.

WMullens, C. J. A. Flt. Sub-Lieut., R.N.A.S. Drowned in action off the coast of Flanders 5 May 1916 
Muntz, A. I. Capt., W. Somerset Yeo.; empld. Ministry 1888 of Food

${ }^{1}$ Murray, A. G. W. Capt., Spec. List (Adjt., Staff School). 1903 M.B.E.

MurRaY, C. W. Sub-Lieut., R.N.V.R.

I9I3

AMurRay, G. A. Major, R.F.A. M.C.

Died 4 April I9I8 of wounds received in action

MurRay, G. R. Colonel, A.M.S.

Murray, J. C. Lieut., Ist Life Gds.

MurRay, J. S. Australian Force

1912

I883

I912

1905

1907

Died 16 Nov. I918 from the effects of war service

Murray, P. J. Capt., Wiltshire Regt. and Gen. List. 1900 $M$.

Murray, R. B. Capt., London Regt. (Q.V.R.) and R.E. 1909 $M$.

MurRay, T. H. E. Trooper, Natal Light Horse; Lieut., I9I0 R.F.A.

MurRay-Johnson, F. K. Lieut., Ist Dragoon Gds.; Staff 1902 Capt. (W.)

AMurray Smith, A. G. Lieut., 2nd Life Gds.

Died 2 Nov. I9I4 of wounds received in action

Murton, E. Lieut., R.N.V.R.

1905

I885

NaISH, A. E. Major, R.A.M.C.(T.F.)

NAPIER-Clavering, Rev. H. P. C.F. $4^{\text {th }}$ Class, R.A.C.D. 1879

NASH, J. V. Lieut. (E.O.), R.F.C.

Nathan, S. H. Capt., R.A.M.C.

NawANAGAR, Jam of. Lieut.-Col., Spec. List; A.D.C. I889 K.C.S.I. $M$.

NAYLER, W. A. 2nd Lieut., Charterhouse School O.T.C. $\quad$ I904

NaYloR, J. M. Capt., London Regt.(L.R.B.) (W.) 1907

NaYLOR, T. H. Capt., Montgomeryshire Yeo.; attd. R. 1909 Welsh Fus. $M$.

Neale, A. K.H. Capt., King's (Liverpool Regt.); empld. I9I I Ministry of Munitions

WNegroponte, J. J. 2nd Lieut., S. Lancs. Regt. $M$.

Died 29 Oct. 1916 of wounds received in action

NeIlson, D. F. A. Capt., R.A.M.C.

NeIlson, G. E. Capt., W. Somerset Yeo. and Spec. List 1909 (Bde. Signal Officer)

1 Died after the armistice of influenza contracted on military service. 
NeIlson, H. J. T. 2nd Lieut., Life Gds.; Lieut., I9I I R.A.M.C. and R.A.S.C.(T.F.)

Nesham, R. A. Surgeon-Major, R.F.A.(T.F.) I887

Nettlefold, E. J. Capt., 5th Dragoon Gds.; Major, 1904 Spec. List. (W.) $M$.

Nettlefold, J. H. Lieut., 5th Dragoon Gds.; empld. 1909 Ministry of Munitions. (W 2.)

\#Nevile, B. P. Capt., Lincolnshire Regt.

Killed in action I I Feb. I916

WNevile, H. G. Lieut., S. Wales Borderers (W.) 1898 Killed in action 21 Aug. I9I 5

Neville, R. A.B., R.N.V.R. (Anti-Aircraft) I891

WNewall, J. H. M. Sub-Lieut., R.N.V.R. (R.N.D.) 1914 Killed in action 13 Nov. I916

Newcomb, W. D. Capt., R.A.M.C.

Newcombe, H. W. Sapper, R.E.; 2nd Lieut., R.G.A.; 1910 empld. Admiralty. (W.)

Newcombe, W. A. Lieut., D. of Cornwall's L.I.

Newman, J. B. Lieut., Middlesex Regt.(T.F.); empld. Ministry of Munitions. (W.)

Newman, J. C. Capt., R.A.M.C.(T.F.) O.B.E. M 2. I891

Newman, J. R. B. Major, Middlesex Regt. I890

NEWPORT, Viscount. Major, R. Scots

WNewson, W. A. Major, London Regt.

Died I 5 April I9I 7

WNicholas, H. C. Lce.-Corpl., Australian Light Horse 1900

Killed in action 4-6 Aug. 1916

Nicholas, T. C. Major, R.E. O.B.E. M.C. M 2. 1907

Nicholson, A. F. Lieut., Shropshire Yeo.; attd. R.E. $\quad 1899$

Nicholson, B. D. Lieut., R.N.V.R.

Nicholson, C. O. E. Capt., Scottish Horse. Brevet 1893

Major. M 2. Order of the Redeemer, $4^{\text {th }}$ Class (Greece)

Nicholson, H. J. Lieut., 6th Dragoon Gds.(Carabiniers). 1912 M.C.

Nicholson, P. C. Staff-Sergt., R.F.A.

Nicholson, R., T.D. Major, Hampshire Yeo.

Nicholson, W. N. Lieut.-Col., Suffolk Regt.; A.Q.M.G. $\quad 1896$

C.M.G. D.S.O. Brevet Lieut.-Colonel. $M 7$.

Nicol, J. Capt., R.A.M.C.(T.F.)

Nightingale, A. D. Major, Oundle School O.T.C.

Nightingale, D. A. 2nd Lieut., R. Defence Corps

1908

I 886

Nix, C. G. A. Hon. Major, Gen. List (T.F. Res.); In- 1892 spector, Woolwich Arsenal. O.B.E. 
Nixon, B. H. Lieut., Devon Regt. and Gen. List

1903

WNoel, Hon. R. E. T. M. Capt., R. Fusiliers, attd. Nigeria 1906 Regt., W. African Frontier Force

Died in E. Africa 2 Feb. 1918 of dysentery and malaria

Norbury, C. G. Capt., Rifle Brigade; empld. War [I9I4] Office. (W.) $M$. $m$.

Norman, C. L. Capt., Queen's Own (R.W. Kent Regt., I890 T.F.)

Norman, C. W. Capt., 9th Lancers. (W.) (P.) $M$. I9ro WNorRIs, W. F. Lieut., Norfolk Regt.(T.F.); attd. Div. 1912 Cyclist Coy.

Killed in action in Gallipoli 25 Aug. 191 5

NorthCote, D. S. Lieut., Oxford and Bucks. L.I. (W.) 1909

NoRTнсотт, W. W. Capt., Spec. List (Recruiting Staff); 1877 Major, S. Staffs. Regt. and Labour Corps

Northen, E. Major, I9th Hussars; Brigade Major. 1897 (W.)

Northen, F. Capt., R. Defence Corps. O.B.E. m. 1894

NoRTON, H. E. Major, S. Irish Horse; Brigade Major; 1895 Lieut.-Col., R. Irish Regt. and Tank Corps. (W.)

OAkey, J. M. Pte., London Regt. (Artists Rifles); 2nd Lieut., Rifle Brigade; Major, R.E.(Spec. Bde.) M.C. $M$.

OAKshotr, T. A. Capt., Northumberland Fus. (W.) I914 OATES, B. W. G. Lieut., R.N.V.R.

OAtes, J. S. C. Capt., Sherwood Foresters (Notts. and Derby Regt.) (W.) D.S.O. M.C. $M_{3}$.

OATs, G. Major, R.G.A.(T.F.) T.D.

AO'Connor, A. C. Capt., Norfolk Regt. (W.) M.C. 1910 Killed in action 27 Fuly 1916

Odgers, F. W. Lieut., Rugby School O.T.C.

O'Driscoll, P. F. Lieut., Irish Gds. (W 2.)

O'Ferrall, C. L. Capt., Sherwood Foresters (Notts. I9IO and Derby Regt.); Lieut., R.E. (Signals)

Ogilvie, A. Lieut.-Col. (T.), R.A.F. (Aircraft Produc- I90I tion Dept.) (W.) C.B.E. O.B.E. $M 2$.

Ogilvie, G. L. Lieut., Quebec Regt., Canadian Force I90I O'HAGAN, Lord. Major, R.F.A.(T.F.)

Oliver, M. W. B. Major, R.A.M.C. O.B.E. M. 1900 1900 HOliver, T. F. 2nd Lieut., Sherwood Foresters (Notts. 1905 and Derby Regt.) (W.)

Died 26 Oct. 1918 of heart failure following pneumonia 
Ollivant, R. C. Lieut., R.F.A.(T.F.); Staff Capt. Igor O.B.E. M 2. French Croix de Guerre

Onslow, J. W. M. See Macarthur-Onslow, J. W.

Openshaw, C. G. Capt., The Buffs (E. Kent Regt.) and I9OI Gen. List (Transport Officer)

OPPENHeim, L. C. F. Major, Highland L.I.; Lieut.-Col., $\quad$ I889 Military Attaché, the Hague. C.M.G. Brevet Lieut.-

Colonel. Brevet Major. $m 2$.

HORde-Powlett, W. P. 2nd Lieut., Yorkshire Regt.

Killed in action $\mathrm{I} 7$ May $\mathrm{I} 9 \mathrm{I} 5$

OrgILl, T. C. Lieut., I.A.R.O., attd. 125th Napier's Rifles

ORIEL, T. H. B. Major, R.G.A. M.C.

ORMroD, L. A. Lieut., I $3^{\text {th }}$ Hussars

OrR, G. P. L. Capt., Bedfordshire Regt.; attd. Rifle Brigade (T.F.)

OrR-EwING, A. I. Lieut., Berkshire Yeo.; Lieut. (A.), 1902 R.A.F. (P.)

Osborn, A. P. Colonel, United States Infy.

OTtER. A. Lieut., R.N.V.R.

Overton, I. G. Cadet, O.C.B.

1903

I9II

1913 1902

Owen, A. R. B. Lieut., E. Surrey Regt. and Spec. List

(Recruiting Staff). (W.)

OweN, D. C. Lieut.-Col., Middlesex Regt. D.S.O. M 3. 1899

Owen, H. Capt., R.F.A. M.C.

Owen, S. A. Capt., R.A.M.C.

OXLEY, A. E. Major (T.), R.A.F.

PAget, J. B. Major, W. Yorks. Regt. (R. of O.); R.T.O. 1888

Paine, H. H. Capt., R. Welsh Fus. and R.E. M.C. 1905

Paine, W. A. Capt., R. Welsh Fus. (W 3.) M.C. 1909

Pallis, A. A. Lieut., Spec. List (Interpreter). O.B.E. 1907 $M$.

Palmer, G. F. N. Capt., Coldstream Gds. (W.) 1912

Palmer, H. G. Lieut., R.E.; empld. Admiralty (W.) 1906

Palmer, K. R. Lieut., Life Gds. (P.)

Parbury, F. C. S. Lieut., Surrey Yeo.

PARKer, F. A. Lieut., King's (Shropshire L.I.) (W.) I9I3

1 PARker, F. B. Capt., Yorkshire Regt. (W 2.)

Parker, G. D. S. Capt., Rifle Brigade. (W.)

PARKer, G. W. T. Lieut., R.G.A.

Parker, H. E. Pte, Ist Rhodesia Regt, S. African Force; Lieut., R.G.A. 
Parker, Hon. J. H. 2nd Lieut., R.E.

PARKER, R. H. Capt. and Adjt., 5th Dragoon Gds. (W.) I908 M.C.

Parker, W. H. Lieut., Suffolk Regt. and Gen. List. $M . \quad 1908$

Parkin, J. Major, Border Regt.(T.F.)

Parkins, F. D. Pte., United States Army

APARRY, F. A. Major, R. Warwickshire Regt. (W.) M.C. ${ }_{\text {I9OI }}^{[\mathrm{I} 914]}$ Killed in action 27 Sept. 1918

WPARRY, N. C. Lieut., York and Lancaster Regt. (W.) 1905 Killed in action 27 July I9I 5

Parry, N. E. Lieut., I.A.R.O., attd. 7th Gurkha Rifles 1906

Parry-Jones, M. M. Capt., R. Fusiliers; A.D.C.; Major, 1907 G.S.O. 2. M.G. M2.

WParsons, D. C. Capt., Irish Gds. (W.) $M$.

Killed in action 15 Sept. I9I6

Parsons, J. R. Lieut., R.N.V.R.

Parsons, R. E. Lieut.-Col., R.E.(T.F.) $m$.

1909

PART 1907

PART, G. M. Capt., R.A.F.; S.O. 3, Air Ministry. $m$. I9I I

Partington, Rev. E. F. E. C.F. 3 rd Class, R.A.C.D. 1904 M.C. and Bar

Paschkoff, A. A. Chevaliers-Gardes, Russian Army $\quad$ I908

Paschkoff, A. B. Chevaliers-Gardes, Russian Army 1908

Pasteur, F. M. Capt., King's Royal Rifle Corps; Major, [19I4] M.G.C. M.C. and Bar. $M$.

Paterson, J. J. Lieut., Lanarkshire Yeo. $M$.

Paterson, R. J. Lieut., London Yeo. (Westminster Dra- 1896 goons), Coldstream Gds., and Gds. M.G. Regt. $M$.

Paton, J. D. Lieut., 8th (King's R. Irish) Hussars. $M$. I9ro

Paton, R. Y. Surgeon Sub-Lieut., R.N.V.R. I915

Paton Smith, E. A. Pte., 9th Kieff Hussars, Russian 1909 Army

Patron, J. A. Capt., London Yeo. (Rough Riders); [I9I4] Major, M.G.C. (W.) M.C.

Patterson, Rev. L. C.F. $4^{\text {th }}$ Class, R.A.C.D. APatteson, J. D. 2nd Lieut., 5th Dragoon Gds.

Killed in action 13 Oct. I9I4

Paul, Sir R. J. Capt. (A.), R.A.F. (P.) M. French 1902 Croix de Guerre

Pauncefort Duncombe, Sir E. P. D. Major, Bucking- 1904 hamshire Yeo.; Brigade Major. D.S.O. $M_{3}$. French Croix de Guerre

Pawle, J. Capt., Hertfordshire Regt. (W.) 1903

PaYne, M. W. Lieut., R. Fusiliers; Staff Capt.; attd. 1903 Egyptian Army 
Payne Gallwey, L. P. Lieut., 7th Hussars; attd. Life 1910 Gds.; Capt., Spec. List. M.C.

Payton, W. H. Capt., I.A.R.O., attd. 6th Gurkha Rifles I9I I

Peabody, Rev. M. E. Chaplain, United States Army I9Io

PeAche, R. C. Capt., R.E. (W.) 1908

Peacock, J. Pte., Princess Patricia's Canadian L.I. 1903

Peacock, Rev. M. R. Major, Suffolk Regt.(T.F.); attd. 1906 London Regt.

APearce, R. S. 2nd Lieut., Rifle Brigade

Killed in action 9 May $19 \mathrm{I} 5$

Pearce-Serocold, O., v.D. Lieut.-Col., R. Berkshire 1883 Regt.(T.F. Res.) C.M.G. M. m.

Pearson, A. G. Capt., R. Berkshire Regt.; G.S.O. 3; 1907 Major, Tank Corps. D.S.O. M.

Pearson, Hon. B. C. Capt., Sussex Yeo.

Pearson, J. S. Major and Adjt., D. of 'Wellington's 1895 (W. Riding Regt.)

Pearson, R. S. Major, Yorkshire Hussars; Brigade 1892 Major. O.B.E. $m$.

Pearson, T. A. Lieut., M.G.C.

Pease, C. E. Lieut., Yorkshire Regt.(T.F. Res.)

Pease, E. 2nd Lieut., Spec. List (Interpreter). (W.)

Pease, N. A. Capt., E. Surrey Regt. (W.) M.C. and [1914] Bar

Pease, R. A. Capt., Northumberland Yeo.; empld. War 1909 Office. (W 2.) $M$.

Peckнam, W. D. Lieut., Spec. List (Interpreter). M. 1906

Peek, Sir W., Bart. Capt., R. Ist Devon Yeo.; Camp 1903 Cmdt., Indian Army. D.S.O. $M_{4}$.

Peel, W. R. Lieut.-Col., Yorkshire Regt.; attd. Man- 1905 chester Regt.(T.F.) (W 3.) D.S.O. and two Bars. $M_{5}$.

Pelham, Hon. M. H. Lieut., Life Gds. and Gds. M.G. 1912 Regt.

Pelham, Hon. S. G. Capt., I Ith Hussars. (W.) M.C. 1908

Pelham, Rev. W. H. C.F. 4th Class, R.A.C.D. 1905

Pelham-Clinton, G. E. Capt., R.E. M.C.

Pelly, A. R. Capt., Norfolk Regt.(T.F.) and Spec. List. 1914 (W.)

Pelly, E. G. Lieut.-Col., R.A.S.C. D.S.O. M.C. 1908 $M 5$.

Pelly, E. P. L. 2nd Lieut., Essex Yeo. (W.)

Pelly, F. B. Lieut. (O.), R.A.F. A.F.C.

Pelly, Rev. R. A. C.F. Ist Class, R.A.C.D.(T.F.) T.D. 1877 
APemberton, F. P. C. Capt., Life Gds.

Killed in action near Roulers 19 Oct. 1914

1903

PenN, A. H. Capt., Grenadier Gds. (W.) M.C. M. 1905 French Croix de Guerre

WPenn, E. F. 2nd Lieut., Norfolk Yeo.; Capt., Grenadier 1897 Gds. $M$.

Killed in action 18 Oct. 1915

सPENN, G.M. 2nd Lieut., Rifle Brigade; attd.Somerset L.I. 1905 Killed in action II Feb. 1915

Pennell, V. H. Capt., R.A.S.C.

1894

Pennyman, J. B. W. Capt., King's Own Scottish Bor- 1902 derers; Major, M.G.C. (W.)

WPenrose, E. J. McN. Capt., R. Irish Fus. (W.) M. 1907 Killed in action near St fulien 25 April I91 5

APenrose Fitzgerald, M. J. Lieut., The Queen's (R.W. I9I3 Surrey Regt.)

Died 26 Fuly I9I6 of wounds received in action

Penton; C. F. Capt., Queen's Own (R.W. Kent Regt.) 1905 and Gen. List. (W.)

APePloe, K. Capt., Oxford and Bucks. L.I.

Killed in action 9 Nov. 1916

Percrval, H. F. Surgeon Lieut., R.N. O.B.E. I90I

PERkins, C. H. Lieut., Buckinghamshire Yeo. M.C. [I9I4]

Perrens, C. N. T. Capt., R.A.S.C.

PERrins, M. D. Lieut., Lincolnshire Regt.

WPERRY, E. W. C. 2nd Lieut., R.F.C.

Killed in flying accident I6 Aug. I914

PerRY, W. A. C. Capt., R.E.; Staff Capt. $M 2$.

1913

1910

1908

APERSSE, C. DE B. G. 2nd Lieut., 7th Dragoon Gds.; attd. I895 Irish Gds.

Died 19 Fuly I9I 5 of wounds received in action

Peters, A. Capt., Leicestershire Regt. M.C. $M$.

Peters, W. J. Lieut., Devon Regt. and Spec. List (T.M.B.) M.C.

APetersen, W. S. 2nd Lieut., Life Gds.

Killed in action 6 Nov. I9I4

Petherick, G. G. 2nd Lieut., Life Gds.; Capt., R. Ist Devon Yeo.; empld. Admiralty

Petherick, J. C. Capt., 3rd Hussars; Major, M.G.C. (Cavalry). (W.) M.C. M. Italian Silver Medal for Military Valour

Petherick, M. 2nd Lieut., 2nd Dragoons (R. Scots 1912 Greys)

Petrie, A. Capt., R. Scots Fus. (W.) M.C.

1908

1903 
Pharazyn, H. H. Capt., Worcestershire Regt. $\quad 1896$

Phear, A. G. Colonel, A.M.S. C.B. M 2.

Phelps, H. M. P. 2nd Lieut., R. Fusiliers. (W.) (P.) 1916

Phelps, T. T. Capt., London Yeo. (Middlesex Hussars) I89I

Philipson, R. Capt., Coldstream Gds.

1914

Philipson-Stow, R. M. P. Lieut., King's Own (R. r9r3

Lancaster Regt.); attd. King's African Rifles

Phillipps, F. A. Lieut., Seaforth Hdrs. (W.) rgro

Phillirs, G. W. Major, R.E.; D.A.D. Light Railways. 1909 $M$.

Phillips, L. C. W. Capt., Surrey Yeo.(T.F. Res.)

WPhillips, R. N. Capt., R. Welsh Fus.

Died 27 Dec. I9I4 of wounds received in action

Philpot, W. Lieut., R.F.A.(T.F.)

PICKles, A. Capt., Suffolk Regt.; Staff Capt.

Pickthorn, K. W. M. Capt., London Regt. (Civil Service Rifles); empld. War Office; Capt. (O.), R.A.F. (W 2.)

Pigeon, H. R. Lieut., R.A.S.C.

Piggott, J. C. C. Lieut., D. of Cornwall's L.I.; Lieut. [19I4] (A.), R.A.F. (W.)

Pike, G. Capt., R.A.S.C. $M$.

Pike, S. A. Lieut.-Col., R. Berkshire Regt. M.C. M 2. 1908 Order of the White Eagle, $5^{\text {th Class (Serbia) }}$

Pilcher, G. ST C. Lieut., Spec. List (Intelligence). 1909 M.C. $M$.

Pilkington, D. F. Lieut., R.A.S.C.(M.T.) M.B.E. M. 1912

Pilkington, E. F. Major, Manchester Regt.(T.F. Res.) 1904

Pilkington, F. M. F. Capt., R.A.S.C. 1906

Pilkington, G. R. Major, S. Lancs. Regt.(T.F.) (W 2.) 1900 D.S.O. $M$.

सPilkington, H. B. Capt., Manchester Regt.(T.F.) M. 1905 Killed in action in Gallipoli 4 Fune I91 5

Pilkington, W.N. Major, S. Lancs. Regt.(T.F.) D.S.O. $\quad 1896$ and Bar. $M 3$.

Pinsent, G. H. S. Lieut., R.G.A. $M$.

Pite, A. G. Capt. and Adjt., R.F.A. M.C.

PITE, I. B. Capt., R.E.

PITT, P. S. Capt., R.A.S.C.(M.T.)

PitTs-Tucker, G. S. Lieut., Cheshire Regt. and King's 1905 African Rifles. (W 2.)

Plaister, A. J. Lieut., R.F.A.

Platt, E. A. Lieut., Army Printing and Stationery 1907 Services 
HPlatT, M. C. Sub-Lieut., R.N.V.R. (R.N.D.) $m$.

Died 26 Nov. 1918 of illness contracted during intern-

1912

ment

Plate, R. C. Capt., E. Surrey Regt. (R. of O.)

Platt Higgins, F. M. Capt., Cambridgeshire Regt. and 1893 Remount Service. M.C. $M$.

Pochin, V. R. 2nd Lieut., Res. Regt. of Life Gds. and 1898 Gds. M.G. Regt.

Polmill, C. C. Lieut., Dorset Yeo.

Pollard, A. R. Lieut., I.A.R.O., attd. Ist Sappers and Miners. (W.) M.B.E. M 2.

Pollard, S. 2nd Lieut., R.F.A.; empld. Ministry of 1912 Munitions

PollitT, H. C. Lce.-Corpl., R.A.M.C.

1908

1900

1889

सPollock, C. T. A. Capt., Inns of Court O.T.C.; attd. 1906

E. Yorks. Regt. $M 2$.

Killed in action $3 \mathrm{I}$ March 1918

Pollock, H. H. Major, R.F.A.(T.F.) M.C. M $2 . \quad 1895$

Pollock, Hamilton R. Cadet, R.A.F.

Pollock, Humphrey R. Capt., R.A.M.C.

Pollock, H. W. Lieut. (T.), R.A.F.

सPollock, M. V. Lieut., S. Wales Borderers

Killed in action 9 May 1915

Pollock, R. C. G. Major, Corps of Guides, Indian Army； 1900 attd. 5 Ist Sikhs. (W.)

Polwarth, Lord. Hon. Colonel, R. Scots and Gen. Staff. $\quad$ I 882 C.B.E. $m 2$.

Ponsonby, Hon. B. B. Lieut., Grenadier Gds.; empld. 1904 War Office. (W.)

Ponsonby, Rev. S. G., v.D. C.F. rst Class, R.A.C.D. 1876 (T.F.)

Pooley, J. S. Capt., R.A.M.C.

Poore, W. G. Major, R.F.A.(T.F.) (W 2.) M.C.

Pope, A. R., T.D. Major, Dorset Regt.

Pope, C. Capt., Bedfordshire Regt. (P.)

«Pope, C. A. W. Capt., R.A.M.C.

Drowned on H.M. transport Transylvania 4 May I9I7

PorTer, A. R. Z. Lieut., R.E. (London Electrical Engineers, T.F.)

WPORTER, E. J. Lieut., London Regt. (Queen's)

Died in German hands 22 Sept. I916 of wounds received in action

1908

1909

I 890

1909

1896

I899

1909

Powell, A. T., T.D. Lieut.-Col., R.F.A.(T.F.); attd. 1902 R.G.A. M. Order of the Nile, $3^{\text {rd Class (Egypt) }}$ 
Powell, E. W. Lieut.-Col. (A.), R.A.F. O.B.E. $M_{4}$. $\quad 1905$

WPowell, R. C. FF. 2nd Lieut., Highland L.I.

Killed in action at Verneuil $\mathrm{I}_{3}$ Sept. 1914

WPowell, R. H. 2nd Lieut., R. Sussex Regt.(T.F.)

Killed in action at Richebourg l'Avoué 9 May I9I 5

1910

1902

Powell, R. V. Lieut., Scots Gds. (W 3.) M.C.

Powell, T. F. Capt., Coldstream Gds. French Croix de Guerre

Powell, Rev. V. P. C.F. 3 rd Class, R.A.C.D. $M$.

WPOWER, J. W. 2nd Lieut., Somerset L.I.; attd. D. of

Cornwall's L.I.; Lieut., Welsh Gds.

Killed in action ro Sept. 1916

Powers, C. Capt., St Edmund's School, Canterbury, 1903 O.T.C.

Pownall, J. C. G. Lieut., R.F.A.(T.F.)

Powney, C. DU P. P. Lieut.-Col., Hampshire Regt. M.B.E. $m$.

PreEce, C. T. Lieut., R.G.A. M.C.

Preece, P. J., T.D. Lieut.-Col., London Regt. (Post Office Rifles, T.F. Res.); empld. Ministry of Labour APretor-Pinney, C. F., D.S.O. Lieut.-Col., Rifle Brigade. (W.) $M$.

Died 28 April I9I7 of wounds received in action 24 April I917

PrICE, J. D. 2nd Lieut., King's (Liverpool Regt.); 1900 Lieut., Training Res. Bn.

Price, Rt. Rev. MCC. E. C.F. Ist Class, R.A.C.D. $\quad 1882$

$M$. Order of the White Eagle, 2nd Class (Serbia).

Price, W. R. Gnr., R.F.A.

Prichard, W. A. Capt., R.F.A. M.C.

Priestley, H. W. Major, London Regt. (Post Office 1906 Rifles). (W.) M.C.

*Pring, B. C. Pte., Middlesex Regt.; Lieut., Worcester- 1906 shire Regt. and M.G.C.

Killed in action I fuly 1916

WPringle, A. S. Capt., Cameronians (Scottish Rifles)

Killed in action in the Battle of Loos 25 Sept. 191 5

PrinseP, A. L. Lieut., R.N.V.R.

Prior-Wandesforde, R. H. Capt., R.F.A.

1896

1909

I 880

$\mathbf{1} 898$

1889

$\mathbf{1} 883$

Pritchard, J. E. M. Major, S.O. 2., R.A.F. O.B.E. 1909 A.F.C.

Pritchard, N. P. Capt., R.A.M.C. M.C. Pritchard, N. P. Capt., R.A.M.C. M.C.
Pritrie, Hon. H. C. O'C. Major, Rifle Brigade. (W.) 1903
D.S.O. 
Procter, R. Lieut., R.N.V.R. D.S.G. $M 2$. 1897

Procter, R. G. Capt., K. Edward VII School, Sheffield, 1897 O.T.C.

Pryce-Jones, H. M. Major, Coldstream Gds.; Lieut.- 1897 Col., A.A.G. M.V.O. D.S.O. M.C. Brevet Lieut.-Colonel. $M 7$. Chevalier, Legion of Honour (France). Chevalier, Ordre du Mérite Agricole (France) Pryor, N. S. Capt. and Adjt., R.F.A.(T.F.) $M$. WPrYoR, R. S. 2nd Lieut., King's Own (R. Lancaster Regt.)

Killed in action at Ypres $\mathrm{I}$ May $\mathrm{rg} 5$

Pryor, W. M. Capt., Hertfordshire Regt.; Lieut.-Col., $\quad 1898$ R. Warwickshire Regt. D.S.O. and Bar. Brevet Major. M4. Italian Bronze Medal for Military Valour Pugh, F. H. Lieut., R.A.S.C.; empld. British Military Mission

Pughe, E. B. Capt., Rifle Brigade; empld. O.C.B. $M$. I9I I

Pulling, C. R. D. Lieut., King's Royal Rifle Corps 1912

Pullinger, A. F. 2nd Lieut., Essex Regt.; Lieut., r9r4

Suffolk Regt.; empld. Ministry of Munitions. (W.)

APuRSER, F. D. Lieut., R.N.V.R. (R.N.D.)

Killed in action 27 Dec. r917

Pye, D. R. Capt. (T.), R.A.F.

Pye, E. W. Pte., Nagpur Rifles, Indian Defence Force *PYM, C. J. Pte., Canadian Force; Lieut., Irish Gds. Died 27 March 1917 of injuries accidentally received 24 March r9r7

Pyм, R. M. Sub-Lieut., R.N.V.R. (Hood Bn., R.N.D.) (W.)

Pym, Rev. T. W. C.F. Ist Class, R.A.C.D.; A.C.G. 1905 D.S.O. $M 3$.

Pym, W. St J. Lieut., R.G.A.(T.F.); empld. War Cab- 1908 inet

Quilter, Sir W. E. C., Bart., M.P. Capt., Suffolk Yeo. $\quad{ }^{1892}$ (T.F. Res.); R.T.O. $m$.

RADCLyfFe, E. J. D. 2nd Lieut., Huntingdonshire Cyclist 1902 $\mathrm{Bn}$.

RADNOR, Earl of. Bt. Colonel, Wiltshire Regt.(T.F. Res.); 1886 Brig.-Gen., Director of Agricultural Production. C.I.E. C.B.E. M2. Officer, Legion of Honour (France)

WRAIKES, F. S. W. 2nd Lieut., Rifle Brigade

Killed in action 9 May 1915 
Ramsay, J. D. Capt., Scottish Horse; attd. Argyll and 1896 Sutherland Hdrs. M.C.

Ramsden, Sir J. F. Capt., Norfolk Yeo.(T.F. Res.) I895

Randall, B. E. Capt. and Adjt., R.A.S.C.(T.F.) 1906

RANDAll, G. F. Lieut., Hampshire Regt.(T.F.); Lieut. I9I I (Ad.), R.A.F.

Rankin, G. C. Lieut., R.G.A.

Rankin, R. Capt., Irish Gds. (W.)

1897

Rathbone, C. G. Pte., R. Fusiliers (P.S. Bn.); Major, I9Io R.E. (Spec. Bde.) M.C. M.

$\begin{array}{ll}\text { Rathbone, M. P. Major, S. Lancs. Regt. } & \text { I } 884 \\ \text { Rathbone, R. R. Capt., King's (Liverpool Regt.) (W 2.) } & \text { I9ro }\end{array}$ M.C. and Bar

WRattigan, C. S. Capt., R. Fusiliers

Killed in action $\mathrm{I} 3$ Nov. 1916

WRaw, R. 2nd Lieut., Lancs. Fus.

Killed in action 7 Aug. I9I 5

${ }^{1}$ Rawlings, P. T. Capt. (T.), R.A.F. D.S.C. M. 1906

Rawlins, F. McC. Lieut., Gloucestershire Regt. (W.) I9II

Rawlins, H. G. Lieut., R.N.V.R.

Rawlins, J. D. Capt., R.E. M.C. $M . \quad 1913$

Rawson, H. F. R. Instructor Lieut., R.N. I9I0

Rawson, R. R. Capt., R.E. M.C. and two Bars. M. 1912

RAYMENT, G. V. Instructor Cdr., R.N. C.B.E. Order 1897

of the Sacred Treasure, 3 rd Class (Fapan). Order of

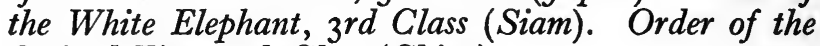

Striped Tiger, 4 th Class (China)

REARDEN, T. R. Capt., I.A.R.O.; R.T.O.

Redfern, A. S. Capt., The Queen's (R. W. Surrey Regt., T.F.); Major (A.), R.A.F.

RedFern, W. A. K. Capt., R.F.A. and Spec. List (Adjt., 1900 Graves Registration Units); Staff Capt.

Redmayne, T. Capt., R.A.M.C.

1902

1914

I $88 \mathrm{I}$

1907

Killed in action at Messines 7 fune 1917

ReED, H. S. Capt. and Adjt., Devon Regt.(T.F.); Staff Capt.

WREED, H. W. T. 2nd Lieut., Monmouthshire Regt.

Killed in action 2 May I9I 5

ReED, J. Lieut., R.N.V.R.

WREID, J. Capt., Highland L.I. $M$.

Killed in action 25 Sept. I91 5

ReID, J. S. Trooper, K. Edward's Horse; Capt., R.F.A. 1907

${ }^{1}$ Killed in flying accident after the armistice. 
REID, P. J. Lce.-Corpl., I9th Hussars; Lieut., Leicester- 1885 shire Yeo.; empld. Traffic Control

Rendall, F. G. Pte., London Regt. (Artists Rifles); Staff Lieut.; Lieut., R. Fusiliers; Capt., Spec. List 1909 (cmdg. T.M.B.) (W.)

Rendall, P. S. Lieut., King's (Shropshire L.I.) and [19I4] Gen. List (Draft Conducting Officer); empld. Air Ministry

Rennoldson, H. F. Capt., R.F.A.(T.F.) (W.) M. $\quad 1906$ Renshaw, A. H. Lieut., Lanarkshire Yeo. and R. Scots 1907 Fus.; Capt., Spec. List (cmdg. T.M.B.)

Renshaw, J. A. K. Capt., Manchester Regt. (T.F. Res.) I889

Renshaw, Sir S. C. B., Bart. Capt., Ayrshire Yeo. I903

Reunert, C. Gnr., S. African Force (Armoured Train) 1906

Revillon, A. J. Pte., Middlesex Regt. (P. S. Bn.); 2nd 1907

Lieut., King's Royal Rifle Corps

Reynard, C. E. Major, E. Riding of Yorkshire Yeo. $M . \quad 1899$

ReYnolds, C. H. Capt., R.G.A.(T.F.) Belgian Croix de 1904 Guerre

Reynolds, G. W. M. 2nd Lieut., Malvern College I9I3 O.T.C.

AREYnolds, J. W. 2nd Lieut., York and Lancaster Regt. 1905 (T.F.)

Killed in action near Ypres 7 Aug. 1915

REYNolds, L. Major, I.M.S.

ReYNolds, N. C. W. Capt., Irish Gds. (W.) O.B.E. m. 1908

RHODES, E. C. Instructor Lieut., R.N.

I9I I

RICARDO, F. Lieut., Gloucestershire Regt. and M.G.C. 1906 (W.)

RICE, G. C. D. Major, R. Fusiliers and Rifle Brigade; $\quad$ I889 Courts-Martial Officer. T.D.

RICE, G. E. Capt., R. Wiltshire Yeo.; empld. War Office. I89I $m$.

RICH, N. J. 2nd Lieut., R.E.; Lieut. (K.B.), R.A.F. I9I I

RICHARDS, R. C. Lieut., R.E.

Richardson, C. L. Capt., R.A.S.C. $M$.

ARICHARDSON, D. S. Lieut., Border Regt.

Killed in action 16 May 1915

RIChaRdson, J. P. A. Lieut., R.N.V.R. D.S.C. Italian 1904 Silver Medal for Military Valour

WRicharDson, J. S. 2nd Lieut., Northumberland Fus. 1895

Killed in action 9 April 1917

WRichardson, J. W. Major, York and Lancaster Regt. $\quad$ I900 Killed in action 3 May 1917

c. U.W.L. 
Richardson, M. E. Lieut.-Col., 2oth Hussars and 1897 Northumberland Fus.; Brig.-Gen. (W.) D.S.O.

$M_{3}$. Chevalier, Legion of Honour (France)

RIDDELL, J. R. 2nd Lieut., Unattd. List, T.F.

WRidley, H. L. Capt., R. Dublin Fus. (W.) M.C.

Killed in action 15 Fuly 1917

ARIDLEY, H. Q. Lieut., Australian Infy.

Killed in action 12 Oct. I9I7

Rigden, C. Major, R. East Kent Yeo.

WRILeY, A. C. Capt., London Regt. (St Pancras Bn.)

Killed in action 25 Sept. I91 5

Riley-SmITH, W. 2nd Lieut., Ist Dragoon Gds.; Lieut., I $3^{\text {th Hussars }}$

Ripley, H. W. G. Lieut., King's (Shropshire L.I.) (W.) 1905

Ritchie, Rev. C. S. Chaplain, R.N.

Ritchie, R. B. Capt., Cameronians (Scottish Rifles). I912 M.C.

Killed in action 20 fuly r9r6

*Ritson, A. S. Gnr., R.F.A.; 2nd Lieut., Durham L.I. I9ro Killed in action 5 Nov. 1916

Ritson, J. A. Capt., S. Lancs. Regt.

Killed in action 23 fuly 1916

Ritson, R. Capt., N. Staffs Regt. and King's African 1906 Rifles. (W.)

Rirtner, G. H. Capt. and Adjt., N. Staffs. Regt.(T.F.) 1894

Rivington, R. T. 2nd Lieut., R.A.S.C.

WRIx, J. C. Capt., R.A.M.C.

Killed in action 6 Fuly 1916

Roberts, C. C. G. Capt., London Regt. (Queen's West- 1899 minster Rifles); Major, Camp Cmdt. O.B.E. M 2.

Roberts, P. M. Pte., R. Fusiliers; Capt., Spec. List I9I4 (R.T.O., attd. H.Q., D.G.T.)

Roberts Buchanan, B. See Buchanan, B. R.

Robertson, D. H. Lieut., London Regt. (Finsbury 1908 Rifles). M.C.

RoberTson, D. S. Major, R.A.S.C. $M 2$.

Robertson, H. J. Capt., R.A.S.C.(M.T.) M.C.

Robertson, K F. Capt and Adjt, Durban LI, 1904

African Force, and Rifle Brigade. $M$.

Killed in action 27 Aug. 1916

RoberTson, M. K. Capt., R.A.M.C.(T.F.)

1910

सRobertson, R. Capt., Highland L.I.

Died I3 Sept. I9I7

Robinson, Rev. F. T. Chaplain, R.N. 
Robinson, H. Capt., R.A.M.C. (W.) I896

Robinson, J. B. Lieut. (A.), R.A.F. 1908

Robinson, J. G. Capt., London Regt. (L.R.B.); Capt. 1906 (Ad.), R.A.F.

WRobinson, R. F. 2nd Lieut., King's Royal Rifle Corps $\quad 1898$

Killed in action at Hooge 30 Fuly 1915

Robinson, W. H. Capt., R.F.A.

RoBson, E. Lieut., Durham L.I.(T.F.) and Northumberland Fus.; attd. 2nd Rajput L.I., Indian Army

WRobson, G. D. Lieut., King's Royal Rifle Corps. (W.) I912

Killed in action 24 Aug. I917

RochDAle, Lord. Lieut.-Col., Lancs. Fus.(T.F. Res.) 1884

Rochfort, G. A. Boyd. Lieut., Scots Gds.

Y.C. " "For most conspicuous bravery in the trenches

between Cambrai and La Bassée on 3rd August, 1915.

At 2 a.m. a German trench mortar bomb landed on the side of the parapet of the communication trench in which he stood, close to a small working party of his Battalion. He might easily have stepped back a few yards round the corner into perfect safety, but, shouting to his men to look out, he rushed at the bomb, seized it and hurled it over the parapet, where it at once exploded. There is no doubt that his splendid combination of presence of mind and courage saved the lives of many of the working party."-Supplement to The London Gazette, I Sept. I9I5.

RogET, S. R. Lieut., R.N.V.R.

I 894

Rollo, W. H. Lieut., 2nd Dragoons (R. Scots Greys). 1908 M.C.

RomanIS, W. H. C. Lieut., R.A.M.C.

RoNAlDSHAY, Earl of. Major, Yorkshire Regt. G.C.I.E. 1894 Rooke-Cowell, W. J. Capt., London Yeo. (Rough I887

Riders); empld. Ministry of Munitions

RoPER, F. A. Major, R.A.M.C.(T.F.) $M$.

सRose, Sir F. S., Bart. Capt., roth Hussars

Killed in action 26 Oct. I9I4

Rose, Sir H. A. Lieut.-Col., R. Scots. D.S.O. M. $\quad 1894$

Rose, L. Capt., S. Wales Borderers. (W 2.) M.C. I9I3

Rosher, J. B. Major, Durham L.I.; Lieut.-Col., M.G.C. 1907

(W 2.) D.S.O. and Bar. M.C. $M_{4}$.

Ross, R. D. Major, N. Irish Horse; Brigade Major. 1907

M.C. French Croix de Guerre

Ross, W. G. 2nd Lieut., R.F.A.; Lieut., R.A.S.C.(M.T.) 1907

Rossi, R. 2nd Lieut., Engineers, Italian Army

Died I9 March 1920 from the effects of active service

I912 during the war 
Rothband, P. L. Capt., S. Lancs. Regt. $M$.

Rouse, P. G. Lieut., R.F.A.; Lieut.-Cdr., R.N.V.R. M. 1902

Rowlands, R. B. J. and Lieut., R. Welsh Fus.; In- I9I3 structor Lieut., R.N.

Rowley, C. S. Capt., Grenadier Gds. (W.) (P.) I9ro

Rowley, G. R. F. Lieut., R. Horse Gds. (W.) 1907

RoXBurgh, A. C. Surgeon Lieut., R.N.

Roxburgh, J. F. Lieut., R.E. (Signals). $M$.

Royce, F. C. Capt., King's Own Scottish Borderers and R.E. (Sound-ranging Section). (W.) $M$.

Royston-PigotT, W. M. Major, R.A.S.C.; D.A.D.S. 1899 and T., N. Command. D.S.O. M. Chevalier, Legion of Honour (France)

RüCKer, A. N. Lieut., Suffolk Regt. (W 3.)

Ruffhead, A. C. Pte., Northamptonshire Regt.

Ruggles-Brise, E. A. Major, Essex Yeo. (W.) M.C. M. Rushton, H. L. Capt., D. of Lancaster's Own Yeo.; I90I attd. S. Lancs. Regt.

Rushworth, A. N. Surgeon Lieut., R.N.

Russell, E. N. Capt., R.A.M.C.

Russell, J. D. Capt., R.E. (Fortress, T.F.)

Ruston, W. Capt., R. North Devon Yeo. and Lincolnshire Regt.

Ruthyen-Stuart, A. W. Capt., Gordon Hdrs.(T.F.) and 1902 R.A.S.C.(T.F.)

RutTer, H. F. P. Lieut., Cheshire Regt.

Rycroft, Sir R. N., Bart. Major, London Regt. (Cyclist Bn., T.F. Res.)

Ryder, A. F. R. D. Capt., R.F.A.(T.F.) (W 4.) M.C. I9I0 and Bar

Ryder, F. J. Bt. Colonel, Gen. List; Officer i/c Cavalry ${ }_{1885}$ Records. C.M.G. $M 2$.

Sagar-Muggrave, C. L. Capt., W. Yorks. Regt.(T.F.) I9I4 (W.) M.C.

St Aubyn, E. G. Lieut.-Col., King's Royal Rifle Corps; 1899 Brig.-Gen. (W 2.) D.S.O. M. French Croix de Guerre

St Aubyn, Hon. L. M. Capt., King's Royal Rifle Corps 1897 and Gen. Staff. $M$.

¿St Aubyn, M. J. Major, King's Royal Rifle Corps. I9ro (W 2.) M.C. M.

Killed in action near St Quentin 22 March 1918 
St ClaIR, A. H. Lieut., Argyll and Sutherland Hdrs.; I9I3 Lieut. (K.B.), R.A.F. (W.) M.C.

St Johnston, A. Capt., R.A.M.C.

St Levan, Lord, c.B., c.v.o. Colonel, Grenadier Gds.; 1876 Brig.-Gen. $M$.

ASalaman, L. H. Seaman, R.N.V.R.(R.N.D.)

Killed in action in Gallipoli (Dec. 1915)

Salisbury, T. H. L. Lieut. (T.), R.A.F. (Aircraft Pro- 1902 duction Dept.)

Salter, F. R. Lieut., Rifle Brigade; Capt., G.S.O. 3, 1905 Irish Command. (W.)

Sampson, F. A. Capt., R. Fusiliers. (W.) (P.) Ig09

Sampson, N. C. Lieut., 2nd Dragoon Gds. (Queen's I902 Bays); Major (A. and Ad.), R.A.F.

Sampson, R. H. Capt., London Regt. (Q.V.R.) and [r9I4] M.G.C.

Sampson, S. J. M. Major, London Regt. (Q.V.R.) (W.) 1902 M.C. T.D. M.

Samuelson, F. H. B. Capt., Yorkshire Hussars 1908

SANDARS, E. T. Major, R.A.S.C. and Spec. List 1895

(D.A.D.R.T.) O.B.E. M. Chevalier, Military Order of Avis (Portugal)

सSANDERS, L. Y. 2nd Lieut., R.G.A. (W.) 1912

Killed in action 1o March 1917

SANDERSon, Rev. C. P. C.F. 4th Class, R.A.C.D. $\quad 1872$

SANDERson, Rev. G. P. M. C.F. 4th Class, R.A.C.D. $\quad$ I897

SANDERson, I. C. Capt. and Adjt., Black Watch; Major, 1905 Argyll and Sutherland Hdrs. M.C. M 2. French Croix de Guerre. Greek Military Cross

SANDERSON, J. R. Lieut., Spec. List (Staff Lieut.) $M . \quad 1907$ WSANDERson, R. H. Lieut.-Col., R.F.A. M. Chevalier, I895 Legion of Honour (France).

Killed in action 17 April 1918

Sanderson, T. S. 2nd Lieut., Life Gds.; Lieut., 12th 1909 Lancers and Gds. M.G. Regt.

SANDILANDS, H. R. Major, Northumberland Fus.; 1894 Lieut.-Col., G.S.O. I. (W 2.) C.M.G. D.S.O.

Brevet Lieut.-Colonel. $M 5$. Officer, Legion of Honour (France)

Sandiland, J. E. Capt., R.A.M.C. M.C. M $2 . \quad 1890$ SANDISOn, A. Capt., R.A.M.C. French Médaille d'Honneur 1904 SANGer, P. M. Lieut., R. Marines. (W.) I903 Sanger-Davies, F. M. Lieut., R. Sussex Regt.(T.F.) [I9I4] (W.) $M$. 
\#SAnger-Davies, Ll. H. Capt., Durham L.I.

Killed in action I Fuly 1916

SANKEY, H. B. Lieut., R.F.A.(T.F.) M.C.

Sassoon, E. V. Capt. (Ad.), R.A.F.

Sassoon, H. W. Lieut., Grenadier Gds. and Gds. M.G. 1906 Regt.

Saumarez, Hon. J. St V. B. Capt., Scots Gds. (W.) 1908 WSAunder, G. B. 2nd Lieut., The Buffs (E. Kent Regt.) I9I2

Killed in action 15 April 1917

Saville, S. H. Capt., Essex Regt.

Savory, C. H. Surgeon Lieut., R.N.

I910

1908
1907 M.C. $M 2$.

Scarlett, J. A. Lieut.-Col., R.F.A. D.S.O. Brevet 1896 Lieut.-Colonel. $M 3$.

Schacht, A. G. Pte., London Regt.

Schloss, W. F. R. See Castle, W. F. R.

[19I4]

Scholfield, C. N. Lieut., R.G.A.

1906

Scholfield, E. P. Lieut., E. Riding of Yorkshire Yeo. and R.G.A.

SchoN, B. C.S.M. (Instructor), School of Musketry

SCHREINER, O. D. Capt., Northamptonshire Regt. and S. Wales Borderers. (W.) M.C.

Schuster, L. F. Capt. and Adjt., R.G.A.(T.F.) (W.) M. 1907

Schwann, F. H. See Swann, F. H.

Scorgie, N. G. Lieut.-Col., Deputy Director, Army *igo6

Printing and Stationery Services. O.B.E. M 2.

Scotr, Hon. C. F. H. See HePburne-Scott, Hon. C. F.

ScotT, E. Sergt.-Major, R.E.

ScotT, G. E. 2nd Lieut., R.A.S.C.

ScotT, Rev. R. B. C.F. 3rd Class, R.A.C.D.(T.F.)

Scotr, Hon. W. G. H. See Polwarth, Lord

ScotT, W. L. Lieut., S. Staffs. Regt. (W.)

Scott, Hon. W. T. H. See HePburne-Scott, Hon. W. T. Scott-Davidson, W. W. Capt. (T.), R.A.F. $M$.

Scott-Elliot, G. F. Capt., King's Own Scottish Borderers (T.F. Res.) Order of the Nile, $4_{\text {th Class (Egypt) }}$

SCOTT-KERR, R., C.B., M.v.o., D.s.o. Brig.-Gen. (W.) 1877 C.M.G. $M 2$.

Scott-Murray, A. E. Capt., Norfolk Regt.; Staff Capt. 1900 M.C. $M 2$.

ScotT-Murray, R. C. Capt., R. Warwickshire Regt.; 1897 Major, Spec. List (Chief Instructor, School of Instruction). $M$. 
Sealy, H. N. Capt., R.A.M.C.

1905

SEARIGHT, T. P. Capt., R.A.S.C.; Major, S.O. 2, R.A.F. I900 $M . m$.

Searles, P. A. Capt., The Queen's (R.W. Surrey Regt.); I88I Major, R.F.A.(T.F.)

\&Seely, C. G. Capt. and Adjt., Hampshire Regt.(T.F.) I9I3 $M$.

Killed in action in the Second Battle of Gaza 19 April I9I7

SeEly, F. E., T.D. Lieut.-Col., S. Notts. Hussars (T.F. ${ }^{1883}$ Res.); A.D.C.

SeELy, Rt. Hon. J. E. B., D.S.o., T.D. Lieut.-Col., Hamp- 1887 shire Yeo.; Major-Gen. C.B. C.M.G. $M_{4}$. Commander, Legion of Honour (France). Commander, Ordre dela Couronne (Belgium). Belgian Croix de Guerre \#Segnitz, H. F. Corpl., H.A.C.; 2nd Lieut., London Regt. (St Pancras Bn.)

Accidentally killed 25 Sept. 1915

Severne, E. C. W. Capt., Warwickshire Yeo.; attd. 9th 1906 Lancers; A.D.C.; Lieut., Spec. List. (W.) $M$.

Sevestre, R. Major, R.A.M.C. (5th N. Gen. Hospital, 1886 T.F.)

SEwell, H. S. Lieut.-Col., 4th (R. Irish) Dragoon Gds.; Brig.-Gen. (W.) C.M.G. D.S.O. and Bar. Brevet Lieut.-Colonel. $M_{4}$. Chevalier, Legion of Honour (France)

SEwEll, J. P. C. Major (A.), R.A.F. O.B.E. M. Che- 1907 valier, Legion of Honour (France)

ASeymour, F. Lieut., King's Royal Rifle Corps

Killed in action at Hooge 30 Fuly 1915

Seymour Taylor, E. M. Capt., R.F.A. M.G. M. 1914 Seys-Phillips, H. Capt., Bedfordshire Regt. French I9I I

Croix de Guerre

Shackel, G. M. Capt., R. Sussex Regt. (W 2.) M.G. I9I2 $M$.

SHACKLE, R. J. Lieut., R.A.S.C.

Shakespear, G. A. Capt., R.A.S.C.

Shanks, E. B. 2nd Lieut., S. Lancs. Regt.; empld. War Office

SharP, H. Pte., R.A.M.C. and London Regt. (Queen's) I9I4 Sharp, L. E. S. Capt., Spec. List (Uganda Med. Service) I I 8

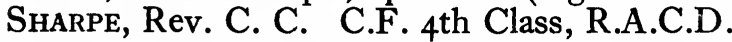
Sharpe, G. W. Lieut.-Col., King's Own (R. Lancaster 1897 Regt.) (W.) O.B.E. M.

1914 I 897

I910 I 888 
WSHAw, A. 2nd Lieut., Northamptonshire Regt. and Nor1910 folk Regt.

Killed in action 12 Oct. 1916

Shaw, E. B. Lieut., London Regt. (London Irish Rifles) r9io

Shaw, G. S. 2nd Lieut., R.F.A.(T.F.)

Shaw, J. A. P. Surgeon Sub-Lieut., R.N.V.R.

WShaw, R. P. Capt., R. Fusiliers

Killed in action in Gallipoli 28 Nov. I9I 5

Shearme, J. S. Capt., Repton School O.T.C.

Shebbeare, H. V. Lieut., R.G.A. and Labour Corps; 1895 Draft Conducting Officer

Sheepshanks, A. C. Major, Rifle Brigade; Lieut.-Col., King's Royal Rifle Corps; empld. School of Instruction. (W 2.) D.S.O. $M 2$.

whreepshanks, C. J. H. Capt., Devon Regt. Killed in action 17 March 1916

1914

1905

1892

1903

Sheepshanks, R. H. Capt., I2th Cavalry, Indian Army. D.S.O. $M$.

Sheldon, Rev. F. H. C.F. $4^{\text {th }}$ Class, R.A.C.D.

Sheldon, Rev. L. G. M. C.F. $4^{\text {th }}$ Class, R.A.C.D.

1905

SHeLter, G. E Capt. and Adjt Grenadier Gds (W)

Igog

Shelley, J. F. Capt., R. Ist Devon Yeo. and Devon 1905 Regt.

\#Shennan, D. F. F. Lieut., King's Royal Rifle Corps

Killed in action near Ypres 8 May I9I 5

Shepherd, F. McA. Capt., Surrey Yeo.

SHePHeRD, J. C. Lieut., R.F.A. (W 3.) M.C.

I9II

1910

1914

WShepherd, J. M. E. 2nd Lieut., Rifle Brigade; Capt., I9I4 R.F.C.

Killed in action $15 \mathrm{Feb}$. I9I7

SHEPPARD, B. F. Sapper, R.E. (W.)

ShePPARD, G. A. Sub-Lieut., R.N.R.; Lieut., Hertford- 1986 shire Yeo.

SherrifF, C. B. 2nd Lieut., Argyll and Sutherland 19I4 Hdrs.; Capt., R.A.S.C.

Shewell, H. W. B. Surgeon Cdr., R.N. O.B.E. M. I8gr Shipman, G. A. C. Major, R.A.M.C. (4th N. Gen. Hos- 1894 pital, T.F.) $m$.

ShIRREFF, C. R. Capt., Durham L.I.

SHORT, H. S. Lieut., R.G.A.(T.F.); empld. Admiralty. 1907 (W.)

SHORT, P. S. Lieut., R.G.A.(T.F.)

SHove, R. S. Major, R.F.A. (W.) $m$.

ShutT, J. E. Air Mechanic, R.A.F. 
\#Silvertop, F. S. J. Lieut., Oxfordshire Yeo.

Killed in action 20 May r917

Simeon, L. S. B. Lieut., R. Fusiliers and Gen. Staff. 1909 (W.) M.C.

WSimpson, C. S. Capt. and Adjt., Yorkshire Regt.

Killed in action ro fuly 1916

Simpson, Rev. F. A. C.F. 4th Class, R.A.C.D. $m$. I9ro

Simpson, L. S. Colonel, R.E.; Chief Mechanical En- 1892 gineer. C.B.E. D.S.O. $M_{4}$.

Simpson, N. D. 2nd Lieut., R.A.S.C.(M.T.)

${ }^{1}$ Simpson, R. C. Major, W. Kent Yeo.

Simpson, R. G. L. G. Lieut., R.A.F.

Simson, C. C. Major, Australian A.M.C. M.C.

SinClaIR, D. J. O. Lieut., R. Irish Fus. and Tank Corps

Singer, C. M. Lieut., Devon Regt. and M.G.C. (Motor). M.C.

Sington, A. J. C. Capt. and Adjt., Manchester Regt. 1905 (T.F.)

Sington, E. C. Capt. and Adjt., Lancs. Fus.(T.F.) rgr I

SkIPWITH, Sir G. H. D’E., Bart. Capt., London Regt. ${ }_{1903}$ (T.F. Res.)

Slade, G. O. Lieut., Essex Regt. (W.)

SlATER, B. H. Major, R.A.M.C.

1909

1897

SMART, W. M. Instructor Lieut., R.N.

Smiley, H. S. Capt., R. Fusiliers, attd. Egyptian Army

Sмrth, A. C. S. Lieut., R.A.M.C. M.C. $M 2$.

I9II

I901

SmITH, A. J.H. Capt., Coldstream Gds.; Major, G.S.O. 2. 1899

M.C. M. French Croix de Guerre

Smith, B. A. Lieut., R.G.A.; attd. R.E.

SMith, B. ABEL. Major, S. Notts. Hussars; Lieut.-Col., 1897

Middlesex Regt.

SmITH, C. H. C. Major (S.), R.A.F. D.S.C.

WSMrth, C. J. Dudley. 2nd Lieut., Grenadier Gds.

Killed in action 16 fune I9I 5

Smith, D. Abel. Lieut., Grenadier Gds.; Capt. and IgI I Adjt., Gds. M.G. Regt. (W 2.) M.C. M.

Smith, G. Gnr., R.G.A.

Smith, G. Dudley. Capt., Remount Service

Smith, G. M. Herbert. See Herbert Smith, G. M.

Smith, H. Capt., Hampshire Regt.(T.F. Res.)

Smith, H. Austen. See Austen-Smith, H.

SmITh, H. V. Sergt., R.A.M.C.

1916

1885

$\mathbf{1 8 8 5}$

r899

1 Died of pneumonia shortly after the armistice. 
सSmith,J.H.Martin. 2nd Lieut., Spec.List (Intelligence) 1906 Died ro Sept. 1914

WSmith, J. H. MichaEl. 2nd Lieut., Manchester Regt. 1908 Died I 7 Sept. I9I4 of wounds received in action

Smith, N. B. Capt., R.F.A. (T.F.); empld. War Office. ${ }^{1884}$ (W.)

Smith, O. Martin. Capt., Grenadier Gds. (W.) 1904

WSmith, PeTER. Lieut., R.E.; attd. R.F.C. $M$. 1912

Killed in action 28 April I9I7

Smith, Prince. 2nd Lieut., R.F.A. (W.) M.C. 1916

Smith, R. Lce.-Corpl., Mtd.Infy., Indian Defence Force 1900

SmITH, R. A. Lieut., R.F.A.(T.F.)

Smith, R. Abel. Capt., Hertfordshire Yeo. M.C. M. ${ }_{1908}$

Smith, R. S. S. Surgeon Sub-Lieut., R.N.V.R. Albert 1909 Medal, 2nd Class

SмIтн, R. W. Lieut., R.A.S.C. (W.) 1902

Smith, S. B. 2nd Lieut., S. Staffs. Regt.; Capt. (A.), 1909 R.A.F. M.C.

\#Smith, S. H. 2nd Lieut., S. Staffs. Regt.

Killed in action 24 Nov. I91 5

Smith, W. O. Major, Lancs. Fus.

Smith-BARRY, R. R. Colonel (A.), R.A.F. A.F.C. 1904

Brevet Major. Officer, Order of Leopold (Belgium)

Sмyтн, H. W. Lieut., R.N.V.R. C.M.G. M.

1910

\SNELgrove, S. H. Lieut., King's Royal Rifle Corps

Killed in action at Hooge 30 fuly 1915

SoAmes, A. L. Lieut., R.A.S.C.

SOAMES, J. B. 2nd Lieut., Res. Regt. of Cavalry; Lieut.-

Cdr., R.N.A.S. Order of St Anne (Russia). Order of St Vladimir, with swords (Russia)

Soames, M. H. Major, London Regt. (L.R.B.) $\quad 1897$

SOAMES, R. M. Capt., R.A.M.C. (P.) IgOI

WSOOLE, S. W. Gnr., R.F.A.

Died 3 Feb. I91 7 of cerebro-spinal meningitis

Soper, R. G. Lieut., Northumberland Fus.(T.F.) (W.) 1906

Sotham, F. A. Lieut., Cheshire Regt. (W.) $M$. I9I4

SoutheY, E. Capt., S. Wales Borderers (R. of O.); 1894

Major, R. Welsh Fus.; Supt., Military Detention

Barracks. $m$.

Southwell, R. V. Lieut., R.A.S.C.(M.T.); Lieut.-Cdr.,

R.N.V.R.; Major (T.), R.A.F. (Aircraft Production Dept.)

Sparkes, W. L. Capt., Devon Regt.(T.F.) 1913

SpARrow, E. C. Capt., R.A.M.C.

1907 
WSPartali, C. 2nd Lieut., R. Berkshire Regt.

Killed in action at Loos 13 Oct. I9I 5

Spedding, J. A. Lieut., London Regt. (Queen's, Cadet I897 Bn.)

ASpeer, A. H. T. L. Pte., R. Fusiliers (P.S. Bn.); Lieut., I9I3

R.F.A.(T.F.); attd. R.F.C.

Killed in action 9 Fuly 1916

SPEER, F. A. Major, Herefordshire Regt. and Gen. Staff I 888 (Area Cmdt.)

SPENCER, G.T.L. Capt., R. Warwickshire Regt. M.B.E. 1902

Spencer, H. V. Lieut., Bedfordshire Yeo.; Capt., Tank 1897 Corps

Spencer, T. D. Capt., Coldstream Gds.

सSPENs, A. W. Lce.-Corpl., Essex Regt.

Died 7 Aug. 1917

Spens, T. P. Lieut., Cameronians (Scottish Rifles, T.F.) and Gen. Staff. (W.) M.C. $m$.

WSPICER, E. E. Capt., London Regt. (R. Fus.)

Killed in action at Oppy Wood 28 March 1918

SPICER, G. E. Capt., R.A.M.C. (W.) M.C. $M$.

SPICER, L. D. Capt. and Adjt., King's Own (Yorkshire

L.I.); Brigade Major. (W.) D.S.O. M.C. and Bar. $M_{3}$.

SPICER, R. Capt., H.A.C. (W.) M.C.

'SPIERS, A. L. C. Lieut., King's (Shropshire L.I.)

Killed in action 26 Sept. I917

WSPRAGG, C. E. W. Capt., E. Yorks. Regt.(T.F.) (W.) I9I I

Killed in action 10 Sept. 1918

Spragge, F. B. B. Capt., R.F.A. (W.) M.C. $M$ 2. I9II

Spranger, J. A. Lieut., R.E.; Capt., Spec. List. $m . \quad 1908$

WSPrigg,H.A.G. Pte., Middlesex Regt.(P.S. Bn.); Capt., I90I

Hampshire Regt.(T.F.) (W.)

Killed in action in Palestine 9.April 1918

Springman, J. B. Capt., Denbigh Yeo. and R. Welsh igro

Fus. M.C.

SPRING Rice, C. Capt., R.A.S.C.

Sprot, Sir A., Bart., M.P. Bt. Colonel, Gen. Staff (Administrative Cmdt.) C.M.G. M2. French Croix de Guerre

SeuIre, C. E. Major, Rifle Brigade. (W 2.) M.C.

Stanbury Taylor, G. R. M. See Taylor, G. R. M. S. Stancliffe, R. S. Lieut., Life Gds.; Capt., R.E. (Signals). $M 3$.

Stanley, A. S. W. Lieut.-Col., Suffolk Regt. $M$.

1906

1871

1908

I9I4

1880 
Stanley, A. W. W. Lieut., R. Horse Gds.; Capt., Gds. 1909 M.G. Regt. M.C.

Stanley, C. S. B. W. Lieut., W. Kent Yeo.; Capt. and Igro Adjt., The Buffs (E. Kent Regt.)

Stanley, C. W. Hon. Major, Cambridgeshire Regt. $\quad 1879$

Stanning, J. Lieut., D. of Lancaster's Own Yeo. and 1896 Manchester Regt.

Starkie, R. P. A. Capt., R.A.M.C. $M$. 1908

Stathers, G. N. Surgeon Lieut., R.N.

Steane, S. W. 2nd Lieut., R.F.A.; A.D.C.; Capt., 1904 I.A.R.O.

Stedman, G. F. Capt. and Adjt., York and Lancaster I9I3 Regt. M.C. $M 2$.

Steel, S. S. Major, Lothians and Border Horse

Steinthal, R. E. Lieut., King's Own (R. Lancaster [1914] Regt.)

\#Stephenson, D. G. Pte., H.A.C.; Lieut., Scots Gds. IgOI

Killed in action 16 May 1915

Stevens, A. L. W. Capt., Middlesex Regt. and Spec. [I9I4]

List, empld. Ministry of Munitions

Stevens, C. J. Duff. 2nd Lieut., Somerset L.I. I9I I

Stevenson, S. D. Major, Black Watch 1905

Stewart, C. A. Lieut., R.E. $\quad$ I909

Stewart, C. T. Lieut., S. Irish Horse and R. Irish Regt. $\quad$ I896

Stewart, F. W. Lieut., I.A.R.O., attd. Igth Punjabis. 1904 (W.) M.C.

STEWART, H. Colonel, Canterbury Regt., N. Zealand 1904

Force. (W.) C.M.G. D.S.O. and Bar. M.C. M 5 .

French Croix de Guerre

AStewarT, J. A. L. Lieut., Rifle Brigade

Killed in action at Wieltje 13 May I91 5

WSTEwarT, R. J. Lieut., Seaforth Hdrs. M.C. $M$.

Died 28 Fan. 1916 of wounds received in action in Mesopotamia I 3 Fan. I9I 6

Stewart-Brown, R. Capt. and Adjt., D. of Lancaster's I89I Own Yeo.

4Stewart-Jones, T. A. Capt., R. Sussex Regt.(T.F.) I892 Killed in action 9 May 1915

Stigand, I. A. Lieut., Queen's Own (R.W. Kent Regt., 1899 T.F.)

Stirling, A. Lieut.-Col., Lovat's Scouts (T.F. Res.); 1885 Brig.-Gen. $M$. $m$.

Stirling-Maxwell, Sir J. Hon. Colonel, Cameronians 1884 (Scottish Rifles, T.F.) 
Stiven, H. E. S. Capt., R.A.M.C. $M$.

1902

StOcker, A. H. Capt., Hampshire Yeo.

STOCKER, C. J. Major, I.M.S. M.C. M2. Order of 1894 the Lion and Sun, 3rd Class (Persia)

Stogdon, J. H. Air Mechanic, R.A.F.

StOKes, F.F. Pte., Hampshire Regt.; Lieut., Cambridge- 1899 shire Regt.

StOREY, K. L. Lieut., 2oth Hussars; Capt., Res. Regt. 1905 of Cavalry

StOREY, R. A. Capt., R.A.S.C. $M$.

Stotr, A. W. Major, R.A.M.C.(T.F.)

Stotr, M. D. Capt., Border Regt. M.C.

Stott, R. Lieut., Lancs. Fus.

Strachan Carnegie, A. B. Capt., R.G.A.

1907

1904

1905

1908

1893

STRADBROKE, Earl of, c.B., c.v.o., v.D. Hon. Colonel, 1880 R.F.A.(T.F.); A.D.C. to the King. C.B.E.

\#Strain, J. L. Capt., R.G.A. $M$.

Killed in action $3 \mathrm{I}$ fuly I9I7

Straker, I. A. Capt., 9th Lancers. (W.)

STREET, H. B. Lieut., R.G.A.

I9I 5

STUART, Viscount. 2nd Lieut., R. Berkshire Regt.; Capt., Gen. List; Major, M.G.C. and Spec. List (Cmdt., School of Instruction). M.C.

WStuart, C. E. Capt., Suffolk Regt. (Cyclist Bn., T.F.); attd. York and Lancaster Regt.

Died $\mathrm{I}_{5}$ March $\mathrm{I}_{\mathrm{I}} 7$ of wounds received in action 12 March I9I7

StuarT, W. R. Capt., Gordon Hdrs.(T.F.) (W.)

STUdD, H. W., D.S.o. Colonel, Coldstream Gds.; Brig.Gen. (W.) C.B. C.M.G. Brevet Colonel. M 5 . Commander, Legion of Honour (France). Commander, Order of the Crown of Italy. Italian Croce di Guerra. Officer, Order of Leopold (Belgium). Grand Officer, Military Order of Avis (Portugal). American Distinguished Service Medal

WSTudD, Rev. L. F. Capt., London Regt. (Rangers)

Killed in action 14 Feb. I9I 5

Studd, V. M. Lieut., Rifle Brigade; attd. Sierra Leone 1909

Bn., W. African Frontier Force

«Sulivan, E. G. Capt., E. Surrey Regt.

1908 I887. 1908

1902

1902

I 889

Killed in action 8 May 1917

WSummers, A. S. M. Capt., I9th Hussars; attd. R.F.C. 1905 $M$.

Killed in action 15 Sept. 1916 
Surtees, A. A. Capt., Northumberland Fus. and Gen. $\quad{ }^{1884}$ List (Army Canteen Committee)

WSutherland, A. G. Pte., Gordon Hdrs.

Missing, presumed killed in action, 23 March 1916

\#Sutton, H. J. Lieut., Welsh Gds.

Killed in action 27 Sept. r9r 5

Sutton, L. N. Capt., Berkshire Yeo.

Swaine, J. K. Capt., R.A.S.C.

SwaNn,F.H. Lieut.-Cdr., R.N.V.R.; Major.(O.), R.A.F. $\quad 1892$ $M$.

Swanston, C. B. Lieut., King's Own Scottish Border- I90I ers. (W.)

Swanwick, F. B. Capt., Derbyshire Yeo.

¿Swanwick, R. K. Lieut., Gloucestershire Regt.

Killed in action 15 Sept. 1914

Sydney, H. Major, R.A.S.C. $M 2$.

Symes, Rev. R. C.F. $4^{\text {th }}$ Class, R.A.C.D.

Symons, Rev. C. D. Gnr., R.F.A.(T.F.); C.F. $4^{\text {th }}$ Class, 1905 R.A.C.D. M.G.

Sympson, T. M. Lieut., E. Riding of Yorkshire Yeo. 1908

TABOR, J. C. Colonel, Essex Regt.; attd. Queen's Own I896

(R. W. Kent Regt.) (W.)

TACON, R. C. Lieut., R.A.S.C.

TAGaRT, Sir H. A. L., C.B., D.S.O. Colonel, I 5 th Hussars; Major-Gen., D.A. and Q.M.G. K.C.M.G. $M_{4}$. Commander, Legion of Honour (France)

Talbot, J. Major, Newcastle-on-Tyne Grammar School O.T.C.

Tangye, R. T. G. Major, Gen. List. O.B.E. $M$ 2. 1894 Chevalier, Order of Leopold (Belgium). French Croix de Guerre

Tansley, L. B. Capt., R.F.A.(T.F.); Staff Capt. (W.) 1912 M.C.

Tatchell, E., D.S.o. Lieut.-Col., Lincolnshire Regt. $\quad$ m. $\quad$ I 888

TAte, A. W. 2nd Lieut., Cameronians (Scottish Rifles); 1906

Capt., Black Watch; Lieut.-Col., M.G.C. (W.) D.S.O. $M 2$.

TAthaM, C. K. Major, London Regt. (R. Fus.); empld. 1900 O.C.B.

\#Tatham, G. B. Capt., Rifle Brigade; Brigade Major. M.C. 1902 Killed in action 30 March 1918

WTatham, L. C. S. 2nd Lieut., Devon Regt. and R.F.C. I913 Killed in action ro fan. 1918 
TAYlOR, A. L. Capt., Norfolk Regt.(T.F.) I900

TAYLOR, C. W. H. Major, Queen's Own (R. W. Kent I899

Regt.); A.P.M. D.S.O. Brevet Major. $M_{4}$.

Officer, Order of the Redeemer (Greece). Order of the

White Eagle, 4th Class, with swords (Serbia)

Taylor, E. M. S. See Seymour Taylor, E. M.

TAYLOR, G. I. Major, Spec. List (Meteorological Service); 1905 attd. R.A.F. $m$.

WTAYLOR, G. R. M. S. Lieut., R.F.A.(T.F.)

Died 30 Sept. I91 7 of wounds received in action

WTAYLOR, G. W. Lieut., R.F.A. (W.)

Died 9 Nov. I9I7 of gas poisoning

TAYLOR, H. D. 2nd Lieut., R.G.A.

TAYlOR, H. G. Surgeon Lieut., R.N.

I9I4

I9IO

TAYLOR, H. M. Capt., Norfolk Regt.(T.F.); Major, M.G.C. M.C. M.

TAYlOR, Rev. H. M. S. C.F. $4^{\text {th }}$ Class, R.A.C.D. O.B.E. 1909 $M$.

WTAYLOR, L. E. Capt., Madras Gds., Indian Defence Force r9or Died 3 Dec. I917 of enteric fever

TAYLOR, Rev. S. C.F. 4th Class, R.A.C.D. (W.) 1903

Temperley, C. E. Capt. and Adjt., Rifle Brigade. (W.) I9I2 O.B.E. M.C.

TeMPLE, M. Lieut., R.A.S.C. $m$.

Templer, J. F. H. Major, R. Fusiliers. $M$.

सTennant, C. G. 2nd Lieut., Seaforth Hdrs.(T.F.) Killed in action 9 May I9I 5

Tennant, H. V. Lieut., R.E.

सTennant, W. G. Lieut., Strathcona's Horse, Canadian 1897 Force

Killed in action 25 May r9I 5

«Tennyson, Hon. A. A. Capt., Rifle Brigade; G.S.O. 3. I9Io Killed in action 21 March 1918

Tennyson, Hon. L. H. Major, Rifle Brigade. (W 3.) 1908 $M 2$.

ATERRY, R. J. A., M.v.o. Major, R. Sussex Regt.; Brigade 1888

Major. D.S.O. $M$.

Killed in action 3 Oct. 1915

TetLEy, C.H. Lieut.-Col., W.Yorks. Regt.(T.F.) D.S.O. $\quad 1895$ T.D. $M 3$.

TetLey, M. H. Lieut., W. Yorks. Regt.(T.F.) (W.) 1906

Theobald, A. C. L. Major, R.F.A.; attd. R.M.A., Wool- $\quad$ I896 wich. D.S.O. M.

Theobald, W. G. 2 nd Lieut., R.G.A.; attd. R.A.F. $\quad$ I898 
Thoday, D. Lieut., Manchester Univ. O.T.C.

Thomas, J. 2nd Lieut., R. Scots Fus.(T.F.) (W 2.) 1908 M.C.

Thomas, O. V. Major (A.), R.A.F. O.B.E. M 2.

Thomas, W. E. Capt., Labour Corps

Thompson, C. H. F. Major, London Regt. (L.R.B.); I90I

D.A.Q.M.G. D.S.O. O.B.E. $M_{3}$. French Croix de Guerre

\&Thompson, F. C. Lieut., R.F.A.

Died 3 Oct. I9I7 of wounds received in action 2 Oct. I9I7

Thompson, H. S. Lieut., R.F.A.(T.F.) (W.) (P.) M. 1903

Thompson, J. D. Capt. and Adjt., Loyal N. Lancs. Regt. I902

Thompson, T. A. L. See Lacey Thompson, T. A.

Thompson, W. J. Major, Worcestershire Regt.

Thompson, W. W. Hon. Capt. and Q.M., Lancs. Fus. O.B.E.

Thomson, G. P. Capt., The Queen's (R. W. Surrey 1910 Regt.); empld. British Military Mission; attd. R.A.F. $m$.

Thomson, J. B. Capt., R.A.S.C. $M$.

Thomson, J. O. Capt., King's Own (Yorkshire L.I.) IgII and Gen. Staff (Intelligence). O.B.E.

Thoresby, M. (late Thoresby-Jones). Pte., London I909 Regt. (Artists Rifles); Lieut., R.F.A. (W.)

EThornhill, G. R. Lieut., The Buffs (E. Kent Regt.) I9ro Killed in action 22 Oct. I9I4

Thornton, Rev. C. C. Sergt., R.A.S.C.(M.T.)

Thornton, E. 2nd Lieut., R. Fusiliers

Thornton, F. Capt., I6th Lancers. (W.) $M$.

1904

I 876

Thornton, F. R. Lieut.-Col., R.A.M.C. (W.) M.C. 1902 and Bar. $M 2$.

Thornton, G. L. Capt., R.A.M.C. M.C. and Bar $\quad \mathrm{r} 890$

Thornton, Rev. G. R. C.F. 4th Class, R.A.C.D. I90I

Thornton, Rev. J. G. C.F. $4^{\text {th }}$ Class, R.A.C.D. M. I904

*Thornton, N. S. Sergt., R. Fusiliers (P.S. Bn.); Major, I902

Rifle Brigade. D.S.O. M.C. $M 2$.

Died ro April 1918 of wounds received in action 3 April I918

Thorp, B. L. Capt., R.A.S.C. and Tank Corps. M.C. $\quad$ I900 $M$.

Thorp, E. B. 2nd Lieut., N. Staffs. Regt. (W.)

Tiarks, J. G. E. Lieut., Ist Dragoon Gds.

Trckell, E. J., D.s.o. Lieut., I4th Hussars (R. of O.) $m . \quad$ I880 
સTillard, T. A. Lieut., Norfolk Yeo.; Capt. (A.), R.F.C. 1902 (W.)

Killed in action 6 Dec. 1916

Times, W. O. Major, Bedfordshire Regt.; D.A.A.G. ' 1907 (W.) M.C. M 2.

Timmis, R. B. Capt., R.G.A. (W 2.) $M 2$.

TINkER, H. W. C. Lieut., R.N.V.R. O.B.E.

Tinsley, R. L. Capt., W. Yorks. Regt.; A.D.C.

TIRARD, N. S. Capt., R.A.M.C.

WTisdall, A. W. St C. Sub-Lieut., R.N.V.R. (Anson Bn., R.N.D.)

V.C. "During the landing from the S.S. "River Clyde" at V Beach in the Gallipoli Peninsula on the 25th April, I9I 5 , Sub-Lieutenant Tisdall, hearing wounded men on the beach calling for assistance, jumped into the water and, pushing a boat in front of him, went to their rescue. He was, however, obliged to obtain help, and took with him on two trips Leading Seaman Malia and on other trips Chief Petty Officer Perring and Leading Seamen Curtiss and Parkinson. In all Sub-Lieutenant Tisdall made four or five trips between the ship and the shore, and was thus responsible for rescuing several wounded men under heavy and accurate fire."-Supplement to The London Gazette, 3I March, r9I6.

Killed in action in Gallipoli 6 May 1915

TodD, E. W. Surgeon Lieut., R.N.

ToDD, J. Lieut., R.F.A. (P.)

Todhunter, H. W. Lieut.-Col., King's Own Scottish

Borderers; Experimental Officer, School of Musketry; 1892 empld. Ministry of Munitions. C.M.G. Brevet Lieut.-Colonel. $m 3$.

WTollemache, A. H. W. 2nd Lieut., R.E.; attd. R.F.C. Killed in action 19 fuly 1916

Tollemache, Hon. M. G. Capt., Suffolk Regt. (T.F. 1890 Res.); empld. O.C.B. $M$.

WTOLLER, G. R. Lieut., Lincolnshire Regt. Died 27 fuly I9I7

WTomlinson, F. R. J. 2nd Lieut., S. Staffs. Regt. Killed in action near Ypres 26 Oct. 1914

Tomlinson, T. S. Capt., R. Munster Fus.; attd. Leinster Regt.

FTompson, A. H. 2nd Lieut., E. African Mtd. Rifles and Grenadier Gds.

Killed in action 27 Sept. 191 5

Tomson, W. J. M. See Maktin-Tomson, W. J.

c.U.W.L. 
Toplis, J. Hon. Colonel, Spec. List (Financial Adviser). 1895 $M 2$.

Torr, H. J. Capt., R. Defence Corps

TorR, J. H. G. Capt., Lincolnshire Regt. (W.)

Torrey, C. E. Capt., King's (Liverpool Regt.) (W 2.) 1908 $M$.

Torrey, G. E. F. Capt., King's (Liverpool Regt.); 1902 D.A.Q.M.G., E. Command. (W.) M.C.

Tosswill, C. G. Major, R. Defence Corps; attd. 1894 R.A.F. $m$.

Tower, F. F. Lieut.-Cdr., R.N.R.; Cdr., R.N.V.R. $\quad 1878$ O.B.E. Italian Bronze Medal for Military Valour

Tower, G. E. Capt., R.F.A. M.C.

Townley, C. E. Capt., Suffolk Regt. and Gen. List 1906 (Intelligence). $M 2$.

Townley, M. G. Major, Remount Service $\quad 1882$

Townsend, J. S. E. A.B., R.N.V.R.; Major, Spec. List. 1895 Chevalier, Legion of Honour (France)

Townshend, H. Lieut., R.E. (Sound-ranging Section). 1909 (W.)

Towse, H. B. Lieut.-Col., E. African Force. $M 2$. $\quad 1885$

Toye, J. F. Lieut., R.N.V.R. 1904

Trafford, C. E. J. Lieut., Scots Gds.; S.O. 4, R.A.F. I901 M.C. $M$.

Traill, R. R Capt., R.A.M.C.

Trasenster, W. A. Major, R. Fusiliers. M.C. M 2. 1909

TREDCROFT, J. L. Capt., R.A.S.C.

Treeby, F. W. 2nd Lieut., R.F.A.

Tregoning, E. A. Capt., Northumberland Fus.; Staff Capt., E. Indies

Tregoning, G. N. Capt., Welsh Regt. and Spec. List, 1900 empld. Ministry of National Service. $m$.

TrenCH, F. C. Lieut., R.G.A.

Trench, R. H. Major, Garrison Artillery, Indian 1899 Defence Force

Trench, W. L. Major, W. Yorks. Regt. M.C.

Tritton, H. L. M. Major, Essex Yeo.

WTRotTer, C. L. Lieut., King's African Rifles

Died 22 Fan. 1918

Trotter, C. W., T.D. Hon. Colonel, S. Notts. Hussars I 1883 (T.F. Res.) and Gen. Staff. C.B. $M$ 2. $m$.

WTrotter, K. S. 2nd Lieut., Rifle Brigade Killed in action 26 April 1915

Trotter, R. D. Capt., Rifle Brigade 
\#Trouton, E. A. Lieut., R. Inniskilling Fus.

I9ro

Killed in action 1 fuly 1916

ETrouton, F. T. Capt., Cameronians (Scottish Rifles) rgro Killed in action 25 Sept. 1915

¿Truscotr, F. G. Lieut., Suffolk Regt. (Cyclist Bn., I9I2 T.F.); attd. R.F.C. M.C. $M$.

Killed in action 6 April I9I7

Tuck, N. J. Capt., Norfolk Regt. (W 2.)

I9I I

Tufnell, N. C. Capt., Grenadier Gds.

WTuke, Rev. F. H. C.F. $4^{\text {th } \text { Class, R.A.C.D. }}$

Killed in action 20 fuly rgr6

Turnbull, R. W. Lieut., R.F.A.(T.F.); Staff Capt. I90I M.C. $M 2$.

सTurner, A.C. Pte., R. Fusiliers; 2nd Lieut., Rifle Brigade 1900 Killed in action 16 fan. 1918

Turner, A. W. Capt., D. of Cornwall's L.I. (W 3.) 1908 M.C.

Turner, F. G. Capt., Gen. List (Brigade Major). O.B.E. 1908 M.C. $M$.

TURNER, H. E. M. Major, Norfolk Regt. (W.)

TURNER, H. H. Lieut.-Col., R.E.

TURNER, W. A. S. Major, R.F.A. M.C. M.

1907

1886

TweEdIE,H.A. Capt.(A.), R.A.F.; Major,S.O.2. A.F.C. 1907

TwEEDY,W.R. Q.M.S., Canadian Hdrs.; Major, R.A.S.C. $\quad 1907$ (W.) $M$.

Twomey, R. A. Instructor Lieut., R.N.

Twopeny, R. E. N. Lieut., K. Edward's Horse. (W.) I9r3 M.C. and Bar

Tyrwhitt Drake, C. W. Lieut., R. Defence Corps. (W.) $\quad 1898$

Ullman, R. B. Capt., R.F.A.(T.F.) (W 3.) M.C.

Ulyat, Rev. E. S. Chaplain, R.N.

1893

1891

1908

I9I3

UngoED, G. T. 2nd Lieut., Middlesex Regt.(T.F.); empld. War Office

UnNa, P. J. H. Lieut.-Cdr., R.N.V.R.

\#UpJohn, W. M. Capt., Welsh Gds.

Killed in action 24 Aug. 1918

URwiCk, R. H. Capt., R.A.M.C.

Usher, T. C. Capt., R.F.A.; Staff Capt. $M 2$.

Uthwatt, Ven. W. A. C.F. $4^{\text {th }}$ Class, R.A.C.D.

1913

1906

1900

1896

1903

I894

1898

1900

UzIELlI, C. F. Lieut., King's Own (R. Lancaster Regt.) I902 and Tank Corps. (W 2.) M.C.

Valentine, Rev. H. T. C.F. 4th Class, R.A.C.D.

1875 
Van Duzer, F. C. Lieut., R.F.A.(T.F.) French Croix [1914] de Guerre

VAN DuZER, S. R. Capt., York and Lancaster Regt.; 1899 empld. War Office

WVan PraAgh, R. B. 2nd Lieut., King's Royal Rifle [1914] Corps. (W.)

Killed in action 9 April I9I7

Van RaAlte, N. M. Lieut., R.N.V.R.

Vane, Hon. C. W. See Barnard, Lord

$\begin{array}{ll}\text { Vane, Hon. R. F. Capt., Durham L.I. } & 1909 \\ \text { VANe-TemPEST, E. C.W. Lieut., R.N.V.R. (W.) D.S.C. } & \text { I } 1212\end{array}$ $M$.

WVARLEY, L. Lieut., D. of Wellington's (W. Riding Regt., I9I I T.F.)

Killed in action 12 Nov. I9I 5

Vaughan, E. W. B. Lieut., E. Surrey Regt.; empld. 1907 O.C.B.

Vaughan, R. B. 2nd Lieut., R.A.S.C.

Vaughan-Williams, R. 2nd Lieut., R.G.A.

1904

1892

VeATER, A. W. Instructor Cdr., R.N.; empld. Admiralty. 1894

Chevalier, Legion of Honour (France)

Venn, J. A. Capt., Cambridgeshire Regt.

Venning, J. A. Capt., R.A.M.C.

Vere Hodge, H. S. See Hodge, H. S. Vere

Vereker, G. G. M. Capt., Grenadier Gds. M.C. M. 1907

VereKER, Hon. S. R. Lieut., R.H.A.(T.F.) (W.) M.C. 1907 $M$.

Verey, H.E. Lieut.-Col., Spec. List (A.D.G.T.) D.S.O. 1896 $M$.

Vernon, J. C. Sergt., H.A.C. (W.)

ZVERnON, W. H. Lieut., London Regt. (R. Fus.)

Killed in action near Les Bœufs 7 Oct. 1916

WVerrall, C. F. Lieut., R. Sussex Regt. $M$.

Killed in action 22 Dec. I9I4

Verrall, P. J. Capt., R.A.M.C.

1902

I 899

VICKers, R. 2nd Lieut., R.F.A.(T.F.)

Died Io Dec. IgI7 of wounds received in action

1904

1914

1907

1902

1912

Vincent, R. B. Capt., Gordon Hdrs.(T.F.); Major, 1906 D.A.D.R.T. Brevet Major

Vinter, P. J. Lieut., Devon Regt.(T.F.); Major, A.P.M. $\quad$ I 889

Vivian, Hon. O. R., T.D. Major, Glamorgan Yeo.; 1893 Lieut.-Col., Cameron Hdrs. M.V.O. D.S.O. M.

Vizard, W. G. Lieut., Dorset Regt.(T.F.); Staff Capt. [1914] $M$. 
VoN SchröDER, Baron W. H. Capt., Remount Service $\quad{ }_{1886}$ Vos, S. Lieut., Devon Regt.(T.F.)

1907

WADE, E. B. H. Lieut., R.N.V.R. 1891

WADE, H. O. Lieut.-Col., W. Yorks. Regt.(T.F.) and I887 Gen. Staff. (W.) D.S.O. $M 2$.

WADSWORTH, J. Corpl., R.E. (Meteorological Section) I9I3

WaEchter, Sir H., Bart. Hon. Colonel, R.F.A.(T.F.) I889 and Gen. Staff. C.M.G. Italian Croce di Guerra

WagGetT, Rev. P. N. C.F. $4^{\text {th }}$ Class, R.A.C.D. $M$ 2. * 19 Io

WAINWRIGHT, B. M. Lieut. (A.), R.A.F. (P.) [I9I4]

WaINwRIGHT, Rev. R. C. C.F. 4th Class, R.A.C.D. I908 $M$.

WAKEFIELD, A. W. Lieut. (M.O.), Newfoundland Regt.; 1895 Lieut., R.A.M.C.; Capt., Canadian A.M.C. $M$.

WAKefield, E. W. Capt., Cheshire Regt.; Major, Border 1880 Regt. (T.F.); Capt., Labour Corps

Wakefield, J. H. Lieut., W. Somerset Yeo.

WWAKEFORD, E. K. Lieut., Leicestershire Regt. (W.)

Killed in action 16 fuly 1916

Wale, E. H. Capt., R.E. M.C. $M$.

WaLKER, E. E. Lieut., R.E. (London Electrical En- 1902 gineers, T.F.); empld. Ministry of Munitions

WalKer, F. G. Major, R.A.S.C. $M 3$.

WWalker, G. F. Pte., R. Fusiliers (P.S. Bn.); 2nd Lieut., I895 York and Lancaster Regt.(T.F.)

Killed in action 7 Dec. I9I6

WALKER, J.P.E. Lieut., 2nd Dragoons (R. Scots Greys). I9I I (W 2.) $M$. French Croix de Guerre

WALKER, K. P. Capt., York and Lancaster Regt.; Major, 1905 W. Yorks. Regt. (W.) M.C. M.

WALKER, M. A. Lieut., Madras and S. Mahratta Railway 1903 Rifles, Indian Defence Force

WALKER, N. O. Hon. Major, Spec. List. O.B.E. 1895

WalkeR, Sir R. J. M., Bart. Capt., Coldstream Gds.; I908 empld. Gen. Staff, N. Zealand Force

WALKER, R. W. S. Lieut., R.A.M.C.

WALKER, T. A. Lieut., R.N.V.R.

Walker, W.E. Major, Durham L.I.(T.F.)

I901

I 896

1896

Wall, A.H. Lieut.-Col., Marlborough College O.T.C. 1892 $m$.

Wall, T. Capt., R.G.A. (W.) M.C.

Wallace, Rev. A. W. W. C.F. 4th Class, R.A.C.D.

Wallace, G. L'E. Major, Dorset Regt. 
Wallace, J. Capt., R.A.M.C.(T.F.) O.B.E.

Wallace, J. A. V. 2nd Lieut., R.F.A.

WWallace, W. E. Lieut., R. Scots (T.F.)

Killed in action 17 April r917

Waller, H. K. Capt., R.A.M.C

WWalrond, V. Major, R.F.A. (W.) $M 2$.

Killed in action 26 April r9I7

1910

1907

1900

1908

Walsh, A. D. Pte., London Regt. (Artists Rifles); 2nd

Lieut., Loyal N. Lancs. Regt.

WWalsh, P. Lieut., Loyal N. Lancs. Regt. (W.) $M$.

Died 8 fuly 1916 of wounds received in action

1909

1914

Walters, A. M. Lieut., Res. Regt. of Cavalry

WWalters, G. Y. L. Lieut., Irish Gds.

Died ${ }_{1} 5$ Sept. 1916 of wounds received in action

WALTERS, W. L. 2nd Lieut., R.F.A.

Wanliss, D. S. Lieut.-Col., 5th Infy. Bn. and Gen. List, 1884

Australian Force. C.M.G. M.

Warburg,O.E. Capt.,R.G.A. and Gen.Staff. O.B.E. m. $\quad 1895$

Ward, C. B. Capt., R.F.A.(T.F. Res.)

WWard, R. O. C.. Capt., The Buffs (E. Kent Regt.); 1900

Major, Tank Corps. (W 2.) $M 2$.

Killed in action 20 Nov. I9I 7

Ward, W. Dudley, M.P. Lieut.-Cdr., R.N.V.R. $M$.

WARD, W. J. Lieut., I.A.R.O., attd. Rangoon Bn., Indian

Defence Force

WaRD-Jackson, R. Capt., Leicestershire Regt.(T.F.) (W.)

Warde-Aldam, W. St A. Capt., Coldstream Gds.;

Lieut.-Col., London Regt. (Blackheath and Woolwich

Bn.) (W.) D.S.O. Brevet Major. Brevet Lieut.Colonel. M 4. Chevalier, Legion of Honour (France)

Wardell, J. M. Lieut., S. Irish Horse; Capt., R. Irish Regt. (W.) (P.) $M$.

WARDELl-YerbURGH, G. B. Flt. Sub-Lieut., R.N.A.S. Igr2 WWARDLEY, G. C. N. Lieut., R.G.A.

Died 24 Fuly 1916 of wounds received in action

WWare, F. H. Capt., London Regt. (Kensington Bn.) I891 Killed in action I Fuly I916

Ware, J. G. W. Capt., R.G.A.(T.F.) (W.)

Ware, Rev. M. S. C.F. th $^{\text {th }}$ Class, R.A.C.D.

1902

1891

Warington Smyth, H. See Smyth, H. W.

Warmington, E. S. Capt., Labour Corps

Warre Dymond, G. W. Capt., E. Surrey Regt. M.C. M. 
Warrington, J. C. Capt., R.E. M 2. French Croix de 1905 Guerre

WarRINGTON, T. Capt., R.A.M.C.

WaRWICK, H. B. Lieut.-Col., Northumberland Fus. 1894 (R. of O.) (W.) $M$.

WWAsbrough, W. L. 2nd Lieut., Loyal N. Lancs. Regt. I9Io Killed in action 25 Sept. 191 5

Waterlow, G. W. Lieut., R.A.S.C.; Capt., Spec. List 1902 (R.T.O.) $M$.

WWatKYN-Thomas, A. Capt., Highland L.I.

Missing, presumed killed in action, 13 Nov. 1916

Watkyn-Thomas, F. W. Capt., R.A.M.C.

1910

WATNEY, C. N., T.D. Lieut.-Col., Queen's Own (R.W. 1887 Kent Regt., T.F.)

Watney, G. N. Capt., King's Own (Yorkshire L.I.) I897

WATNEY, M. H. Capt., R.A.M.C.

WWatney, W. H. Lieut., Rifle Brigade

Killed in action ro May I9I 5

Watson, B. B. Lieut., Irish Gds. O.B.E.

Watson, D. P. Lieut.-Col., R.A.M.C. D.S.O. M.

Watson, F. B. Capt., London Regt. (R. Fus.)

Watson, H. Sapper, R:E. (Meteorological Section)

Watson, J. B. Capt., R.F.A.(T.F.) and Gen. Staff

Watson, R. A. Lieut., Border Regt. and M.G.C. (W.)

Watson, Hon. R. B. W. Lieut., R. Scots (T.F.) (W.)

Watson, R. H. Lindsay. Lieut., Gordon Hdrs.

Watson-Armstrong, Hon. W. J. M. Capt., Northumberland Fus.(T.F.) (W.) $M$.

WWatson-TAYLOR, A. S. 2nd Lieut., London Regt. 1902

Killed in action 14 Sept. 1917

Watson-Taylor, C. A. Lieut., R.N.V.R.

WatT, W. O. Capt. (Aviation), Foreign Legion, French Army; Lieut.-Col. (A.), Australian Flying Corps.

O.B.E. M4. m. Chevalier, Legion of Honour (France).

French Croix de Guerre

Watts, A. E. Lce.-Corpl., Middlesex Regt.; 2nd Lieut., 1907 Indian Army

Wauton, E. A. Capt., R.A.S.C. $M$. Igro

WeATHERHEAD, E. Capt., R.A.M.C. $\quad 1892$

WeAver, A. B. Lieut., E. Yorks. Regt.(T.F.); attd. Rifle 1893 Brigade (T.F.)

WWeBB, J. B. Lieut., N. Staffs. Regt.; attd. Bedfordshire I9r3

Regt.

Killed in action near Ypres 21 April I915 
Webb-Peploe, M. H. Capt., R.G.A. M.C. $M$.

WWebster, J. F. 2nd Lieut., Black Watch; attd. Gordon Hdrs. $M$.

Killed in action 30 Oct. I9I4

Webster, R. G. Capt., Spec. List (Recruiting Staff) and R.F.A.

WWedgwood, A. F. Capt., N. Staffs. Regt.(T.F.) (W.) 1895 Killed in action 14 March 1917

Wedgwood, F. H. Capt., Spec. List (Recruiting Staff). $\quad 1886$ $m$.

Wedgwood, R. L. Lieut.-Col., R.E.; Brig.-Gen., Director of Docks. C.B. C.M.G. M4. Officer, Legion of Honour (France). French Croix de Guerre. Commander, Ordre de la Couronne (Belgium). Officer, Order of St Maurice and St Lazarus (Italy)

WeEkes, C. R. H. Lieut., R. Sussex Regt. (W.)

WWegG-Prosser, C. F. 2nd Lieut., R. Sussex Regt. and Rifle Brigade

Killed in action 3 Sept. 1916

Weguelin, T. N. Capt., Hampshire Regt.; Capt. (T.), 1906 R.A.F.

WeIR, G. A. Colonel, 3rd Dragoon Gds.; Brig.-Gen. (W.) C.M.G. D.S.O. Brevet Colonel. Brevet Lieut.-Colonel. $M_{4}$. Officer, Order of St Maurice and St Lazarus (Italy). French Croix de Guerre

WeIr, H. B. Capt., R.A.M.C. (5th London Gen. Hos- 1899 pital, T.F.)

WeIR, H. H. Capt., R.A.M.C.

Welch, J. Capt., King's Own (R. Lancaster Regt., T.F.) and R.E. (Sound-ranging Section). (W.)

WeLCH, J. J. Lieut., R.G.A.

Weld-Forester, Hon. G. C. B. See Forester, Lord

Weld-Forester, Rev.O.St M. C.F. $4^{\text {th }}$ Class, R.A.C.D. WWeldon, Sir A. A., Bart., c.v.o., D.s.o. Colonel, Leinster Regt. Brevet Colonel

Died 28 fune 1917

WWelsh, A. R. Lieut., Yorkshire Regt.(T.F.) (W 2.) M. 1902 Died 19 Feb. 1916 of wounds received in action

Wendell, J. 2nd Lieut., United States F.A.

Wenham, C. H. Capt., Rifle Brigade. $M$.

Wenham, E. H. Capt., R.F.A. (W 2.)

1910

Wenham, J. H. Lieut., Suffolk Yeo.

1912

Wentworth-Fitzwilliam, G. J. C. 2nd Lieut., North1909 amptonshire Yeo. 
WWest,C.S. Cdr.,R.N.V.R.(R.N.D.) (W.) D.S.O. M3. 1908 Killed in action 30 Dec. I9I 7

West, E. B. Lieut., Shropshire Yeo. and R.E. (Field rgir Survey Coy.)

West, E. E. Capt., 5th Lancers (R. of O.); Major, cmdg. $\quad 1886$ Cavalry Depôt

WWestby, P. St G. C. Capt., R.F.A.(T.F.) Killed in action 23 Sept. I9I7.

WesterN, O. Sergt., H.A.C.; Lieut., R.G.A. (W.) 1907

Westmacotr, P. G. Capt., 3 Ist Punjabis, Indian Army I9I3

Westoll, J. Capt., Durham L.I.(T.F.) and Spec. List 1908 (Asst. Instructor in Musketry)

WeTHERED, H. E. Capt., Welsh Gds.; G.S.O. 3. M. 1907

Whadcoat, W. H. Capt., Spec. List, empld. Ministry 1889 of National Service

Wharton, J. R. Capt., R. Warwickshire Regt.(T.F.) 1900 (W.)

WWhatford, G. L. Capt., 66th Punjabis, Indian Army $\quad$ I 896 Killed in action at the Persian Gulf 22 Nov. I91 5

$\begin{array}{ll}\text { Whatley, W. H. Major, R.A.S.C. } M \text {. } & 1894 \\ \text { Whatman,W. D. Bt. Colonel, Remount Service; Cmdt., } & 1878\end{array}$ Remount Depôt. C.M.G. $M 3$.

Whatton, Rev. A. B. W. C.F. $4^{\text {th }}$ Class, R.A.C.D. I88I

WWhEATCROFT, G. H. 2nd Lieut., R.G.A.

Killed in action 13 Aug. 1915

WheEleR, E. V. V., v.D. Hon. Colonel, Worcestershire 1877 Regt.(T.F.) O.B.E.

WHEELER, W. R. Capt., London Regt. (Queen's). M.C. [1914]

Whidborne, B. S. Lieut., R.F.A.(T.F.) M.C. French 1912 Croix de Guerre

WWhidBORNe, G.F. Lieut., Coldstream Gds. (W.) M.C. 1909 $M$.

Died 24 Oct. I9I 5 of wounds received in action

WWHITAKeR, F. Lieut., R.A.M.C.

Died 28 Oct. 1916

WhITAKER, V. Lieut., R.F.A. (W.) M.C. M. $\quad 1905$

White, Sir A. W., Bart. Lieut.-Col., R.H.A. 1896

White, C. F. O. Capt., R.A.M.C. 1896

White, E. A. S. Lieut., Bedfordshire Regt.; empld. [19r4] O.C.B. (W.)

White, F. B. Howard. Capt., R.E. M.C. $M_{3}$. $\quad 1914$

White, H. E. Trooper, Calcutta Light Horse, Indian 1904

Defence Force. (W.)

White, J. D. C. Capt., R.A.M.C. (W.) 1890 
WWhite, L. W. Lieut., ist Dragoon Gds.; attd. 2nd 1905 Dragoon Gds. (Queen's Bays)

Died 4 Sept. I9I4 of wounds received in action at Nery I Sept. I9I4

White, R. D. Major, Hampshire Yeo. and Hampshire 1906 Regt.(T.F.)

WWhite, R. E. 2nd Lieut., R.E.(T.F.)

Died 5 March 1915 of wounds received in action at 1909

Ypres 4 March I9I 5

Whitehead, F. Lieut., D. of Lancaster's Own Yeo.; 1908 attd. R.F.C. (W.)

WWHITEHEAD, J. R. G. 2nd Lieut., R.F.C.

Killed in flying accident 3 Aug. 1916

WhitehEAD, R. W. Lieut., R.F.A.(T.F.) (W.) M.C. 1907

Whitehead, T. N. Capt., R.A.S.C.(M.T.); empld.Min- I9IO istry of Munitions

Whitehorn, R. D. Lieut., Calcutta Bn., Indian Defence r9io Force. M.B.E. M.

Whitelaw, J. B. Capt., R. Defence Corps: $m$.

Whitfield, S.P. Trooper, London Yeo.; Air Mechanic, R.A.F.

WhiтhaM, J. W. Lieut., Worcestershire Regt.; R.T.O. I910

Whitlark, J. H. Major, R.A.S.C. Brevet Major. M. 1908

Whitley, E. N. Lieut.-Col., R.F.A.('T.F.); Brig.-Gen. $\quad 1892$

C.B. C.M.G. D.S.O. T.D. M7.

WhitTington, T. Capt., R.A.S.C.

Whittle, D. Lieut., S. Staffs. Regt. (W 2.) M.C.

1909 1908

Whitworth, E. E. A. Capt., S. Wales Borderers. (W.) 1908 M.C. M 2. French Croix de Guerre

Whitworth, Rev. G. E. A. 2nd Lieut., R.G.A. 1906

Whitworth, W. H. A. Lieut., Dorset Regt. (T.F.); 1905 Capt. (A.), R.F.C. (W.) M.C.

Widdowson, F. J. Capt., Durham L.I. (W.) 1888

Wiggin, Sir C.R.H., Bart. Major, Staffordshire Yeo. M. 1903

\#Wiggin, G. R. Lieut., Worcestershire Yeo. 1907

Killed in action in Egypt 23 April 1916

Wiggin, W. H. Major, Worcestershire Yeo.; Lieut.-Col., 1906

Sherwood Rangers. (W 2.) D.S.O. and Bar. $M_{4}$.

Wigram, C. K. 2nd Lieut., R.A.S.C.(M.T.)

Wigram, Rev. H. F. E. C.F. 4th Class, R.A.C.D. $\quad 1892$

Wilde, R.W. Capt., Manchester Regt.; empld.Command I880 Depôt

WWilding, A. F. Capt., R. Marines

Killed in action in Gallipoli 9 May 1915 
WILENKIN, C. Lieut., R.F.A. (W.)

WILKES, J. F. Capt., R.A.S.C.(T.F.)

I9I4 I 898

WILkIN, W. R. Lieut., London Regt. (L.R.B.); attd. 1905 Lancs. Fus. (W.)

Wilkinson, Rev. G. R. C.F. 4 th Class, R.A.C.D. M. $\quad$ I894

Wilkinson, Rev. H. R. Chaplain, R.N. O.B.E. I89I

Wilkinson, R. J., C.M.G. Commander-in-Chief, Sierra 1886 Leone

WWillans, R. St J. Lieut., Northumberland Fus. Killed in action 9 Nov. 1914

WilletT, W. L. Lieut., London Regt.(L.R.B.) (W 2.) M. 1909

Williams, A. K. and Lieut., R. Welsh Fus.

Williams, C. Lieut., R.N.V.R.

WWilliams, C. E. 2nd Lieut., R.A.S.C.

Killed in action 17 Oct. I9I7.

WILliaMS, E. Capt., Yorkshire Regt.(T.F.); attd. 1909 R.A.O.C. (W.)

WWilliams, E. G. Lieut., Grenadier Gds.

Killed in action 12 Aug. I9I 5

WWilliams, G. Capt., Welsh Regt. (W.) $M$. Died 15 Nov. 1918 of pneumonia following influenza contracted on active service

Williams, G. H. 2nd Lieut., R.A.S.C.

WWilliams, G. T. Lieut., R.F.A. Died 19 April I91 8 of injuries accidentally received

1896

I913

1905

1900

1907

1908

WWilliams, N. D. Lieut., S. Lancs. Regt. and Gen. Staff I9I0 (Intelligence). $M$.

Killed in action 22 Oct. 1918

WILliams, P. Lieut., R.E. (W.)

WWildiams, R D Garnons. Lieut Col, R. Fusiliers Killed in action 25 Sept. I9I 5

Williams, S. C. Capt., Leicestershire Regt. and S. 1897 and T. Corps, Indian Army; Major, D.A.Q.M.G.

Williams-Ellis, B. C. Capt., Welsh Gds. and Gen. I902 Staff. M.C. $M$.

Williams-Freeman, A. P. Major, D. of Cornwall's L.I.; $\quad$ I884 Lieut.-Col., R.A.O.C.; Ordnance Officer, 2nd Class; A.D.O.S. D.S.O. O.B.E. $M 5$.

Williams-Green, W. T. Capt., Manchester Regt. (W 2.) I9I I M.C.

Williams-Thomas, F. S. Major, Worcestershire Yeo. $\quad$ I897 (P.) D.S.O. $M$.

Williams WynN, W. Lieut., Ist Dragoons. (W.) 1909

Williamson, D. A. Lieut., I Ith Hussars

1912 
Williamson, G. E. Capt., E. Surrey Regt.(T.F. Res.) 1905

Williamson, J. B. Capt., R.A.M.C. $M$.

Williamson, S. K. G. Capt., London Yeo. (West- 1906 minster Dragoons); Major, M.G.C. $M$.

Willink, H. U. Major, R.F.A.(T.F.) M.C. $M . \quad 1912$ French Croix de Guerre

Willoughby, Hon. C. S. H. D. See Heathcote- 1888 Drummond-Willoughby, Hon. C. S.

*Willoughby, F. G. G. Capt., Rifle Brigade Killed in action 9 Aug. I9I 5

WWilloughby, Sir J. C., Bart. Major, R.A.S.C. D.S.O. ${ }^{1884}$ $M 2$.

Died I6 April 1918 of illness contracted on active service in $E$. Africa

WWills, O. B. W. Lieut. (A.), R.A.F. M.C.

Died Io Nov. I9I 8 of wounds received in action

WWills, R. B. M. Capt., R.E.(T.F.) $M$.

Killed in action 15 Feb. I9I 5

Wills, R. D. Capt., Somerset L.I.(T.F.); Hon. Capt. I9ro (T.), R.A.F.

Wills, S. W. Capt., E. Surrey Regt.; empld. O.C.B. 1907

Wills, W. D. M. Capt., N. Somerset Yeo. Chevalier, 1906 Ordre du Mérite Agricole (France)

Wilson, A. C. Lieut., I2th Lancers; Major (A.), R.A.F. I913 WWilson, A. G. MARYon. Trooper, Australian Light 1900 Horse

Killed in action 15 May I9I 5

Wilson, A. H. R. Lieut., Black Watch (T.F.) (W.) 1908

Wilson, B. FitzG. Capt., King's Royal Rifle Corps and 1907 Gen. List (Staff Capt.) M.C.

Wilson, C. Capt., Lincolnshire Yeo. and M.G.C. M. [I9I4]

Wilson, C. E. Lieut., Ist Dragoon Gds.

Wilson, C. T. Lieut., R.F.A.(T.F.)

Wilson, E. R. Lieut., Rifle Brigade; Staff Lieut. (In- 1898 telligence)

Wilson, F. B. Lieut., R. Fusiliers. (W.)

Wilson, G. Lieut., R.M.A. $M$.

Wilson, G. B. 2nd Lieut., I.A.R.O., attd. 45th Rattray's 1906 Sikhs

Wilson, H. N. S. Major, Worcestershire Yeo. 1903

Wilson, J. C. Lieut., Worcestershire Regt. (W.) $M$. I9I0 WWILson, J. S. Lieut., S. Staffs. Regt. (W.) $M$. I9I4

Died 12 Oct. I9I7 of wounds received in action

Wilson, K. H. Lieut., R.E. M.C. 
*Wirson, L. C. 2nd Lieut., Norfolk Regt.

Died 12 Aug. 1915 of wounds received in action 7 fuly

1914 1915

Wirson, L. G. Capt., Gen. List (R.T.O.)

Wilson, R. E. Lieut., R. Scots. (W.)

Died 28 Sept. 1915 of wounds received in action $25 \mathrm{Sept}$.

I9II I9I 5

WimboRne, Rt. Hon. Lord. Lieut.-Gen., Lord Lieu- 1890 tenant of Ireland

WiNBY, I. S. Lieut., R.E.

WinBY, L. P. Major, London Yeo. (Westminster Dragoons); Lieut.-Col., Labour Corps. M 2. Belgian Croix de Guerre

Winch, A. B. Capt., Queen's Own (R. W. Kent Regt.); 1893 attd. R.E. M.B.E.

Winch, GeORge B. Hon. Lieut.-Col., R. East Kent Yeo. 1886 .(T.F. Res.) $m 2$.

WWINCH, GoRDON B. Major, R.F.A.(T.F.) D.S.O. M. 1895 Died 10 April 1918 of wounds received in action 9 April 1918

\#WINCH, R. B. 2nd Lieut., R. East Kent Yeo. Accidentally killed 16 April 1915

WINCH, S. B. Major, R.A.O.C.; D.A.D.O.S. O.B.E. 1903 $M 2$.

WINDSOR, Viscount. Capt., Worcestershire Yeo. and Gen. 1907 Staff (Intelligence). $M$.

WWindsoR-Clive, Hon. A. Lieut., Coldstream Gds.

Killed in action 25 Aug. 1914

Wingate, R. C. Lieut., R.A.O.C.

WINGFIELD, C. T. 2nd Lieut., Bedfordshire Regt.; 1904 Lieut., R. Dublin Fus.

WingrieLD, J. M., D.s.o. Major, Coldstream Gds. (R. of I88I O.); Lieut.-Col., Spec. List (R.T.O.) O.B.E. M.

WINKWORTH, H. S. Lieut., 2nd Dragoon Gds. (Queen's [19I4] Bays); Hon. Lieut. (O.), R.A.F.

WINTER, CECIL E. 2nd Lieut. (Ad.), R.A.F. 1898

Winter, Claud E. Pte., R.A.O.C and Queen's Own I9OI (R. W. Kent Regt.); 2nd Lieut., E. Surrey Regt.

WINTER, Rev. E. E. C.F. $4^{\text {th Class, R.A.C.D. }} 1903$

WINTER, W. DE L. Capt., Cambridge Univ. O.T.C.; 1889 empld. O.C.B.

WINTERBOTHAM, E. M. Lieut., R.G.A. M.C. I9OI

WWInTHROP-Smith, B. R. Lieut., Scots Gds.

Died 15 Nov. I9I4 of wounds received in action

1900 
Wolfe Barry, E. G. Lieut., R.N.V.R.(R.N.D.) (W 2.) 1904 Wolfe Barry, K. A. Capt., R.G.A. and Spec. List, 1897 empld. Ministry of Munitions. O.B.E. $m 2$.

Wollaston, G. W., M.v.o. A.B., R.N.V.R.

Wollaston, H. C. Capt., Sherwood Foresters (Notts. 1908 and Derby Regt., T.F.) and M.G.C.

Wolryche-Whitmore, J. E. A. Lieut., King's (Shrop- 1902 shire L.I.); empld. Directorate of Docks. (W.)

Wood, A. R. Capt., Lancs. Hussars. M.C.

Wood, Rev. C. T. T. C.F. 4th Class, R.A.C.D.(T.F.) 1903 M.C.

Wood, E. R. Lieut., W. Kent Yeo. and Spec. List (In- 1904 telligence)

Wood, F. Capt. and Adjt., Lancs. Fus.(T.F.); Major, 1908 E. Lancs. Regt.(T.F.) (P.) M.C.

Wood, J. L. Major, R.A.M.C. O.B.E. M 3. Chevalier, 1899 Military Order of Avis (Portugal)

Woop, T. L. C. Lieut., London Regt. (L.R.B.) and 1898 Labour Corps

Woodall, J. C. Lieut., Surrey Yeo. $M$.

WWoodhouse, E. J. Lieut., I.A.R.O., attd. Cavalry

Died I 8 Dec. I9I 7 of wounds received in action I Dec.

I917

Woodhouse, R. Major, Essex Yeo.(T.F. Res.)

WWoodland, C. A. Asst. Paymaster, R.N.V.R.; Lieut., 1903

N. Staffs. Regt.; attd. King's Own (Yorkshire L.I.)

(W.)

Died I April r918 of cerebro-spinal meningitis

wWoodroffe, N. L. Lieut., Irish Gds. $M$.

Killed in action 6 Nov. r9r 4

Woods, Rev. E. S. C.F. 4th Class, R.A.C.D.

1902

1903

Woons, Rt. Rev. F. T. C.F. $4^{\text {th }}$ Class, R.A.C.D.(T.F.) 1892

Woods, J. M. Lieut., Loyal N. Lancs. Regt. 1906

Woodsend, H. D. Capt., R.F.A.; Staff Capt. (W.) I9I4 Italian Silver Medal for Military Valour

జWoolf, C. N. S. 2nd Lieut., 2oth Hussars

Died 30 Nov. 1917 of wounds received in action 27 Nov. I917

Woolley, E. C. 2nd Lieut., Loyal N. Lancs. Regt. 1907 (W.)

Woosnam, Ven. C. M. C.F. $4^{\text {th }}$ Class, R.A.C.D.(T.F.) 1875

Woosnam, M. Lieut., Montgomeryshire Yeo.

\#Wootron, J. W. Capt., Suffolk Regt. (W.) m.

Died I I Oct. 1917 of wounds received in action 
WWordsworth, O. B. 2nd Lieut., Oxford and Bucks. 1906 L.I.; Lieut., M.G.C.

Killed in action 2 April I9I7

WWorkman, E. Lieut., R. Irish Rifles. M.C. $M$.

Died 26 Jan. 1916 of wounds received in action

WORKMAN, E. W. Intelligence Section, French Army 1908

WWormald, D. F. P. Capt., R.G.A.(T.F.) and Gen. Staff 1904

Died 4 Nov. 1918 of septic pneumonia

Wormald, F. W., D.s.o. Lieut.-Col., Ist Dragoons. $M$ 2. $\quad$ I888 WWormald, G. Capt., Lancs. Fus.

Killed in action I4 Sept. 1916

Wormald, H. Lieut., King's (Liverpool Regt.) and 1903

S. Lancs. Regt.

WORRALL, P. Lieut., R.A.S.C.(T.F.)

1899

Worsdell, G. B. Major, Yorkshire Regt. Brevet Major. 1900 O.B.E. $M$.

Worthington, R. T. Major, R.A.M.C. (W.) 1893

Wraith, H. D. Lieut., D. of Wellington's (W. Riding 1890 Regt., T.F.)

Wright, Rev. A. B. C.F. 3rd Class, R.A.C.D. (W 2.) 1899 M.C. $M$.

Wright, Sir A. E. Colonel, A.M.S. K.B.E. C.B. M $M_{3} \quad 1886$ Officer, Ordre de la Couronne (Belgium)

Wright, H. FitzH. Capt., R.F.A.(T.F. Res.) I889

Wright, H. G. 2nd Lieut., R.F.A.

Wright, R. M. Capt., Coldstream Gds.; Major, Gds. 1909 M.G. Regt. (W 3.) M.C. and Bar. $M$.

Wrightson, R. G. Major, London Regt. (Post Office I888 Rifles). $m$.

WRIGHTSON, T. G. Major, Durham L.I.(T.F.) $m$. $\quad 1889$

Wrightson, W. I. Lieut., Durham L.I.(T.F.) 1894

*WRigley, C. J. O. Trooper, K. Edward's Horse I9I3

Killed in action 26 May 1915

Wyкeham, P. H. Capt., R.F.A.(T.F.); Staff Capt. M.C. 1899 $M 2$.

Wynne, A. M. Capt. (A.), R.A.F. (W.) A.F.C. I9ro

WynNe, J. B. Lieut., Hampshire Regt. 1895

«WynNE-Jones, M. Lieut., R.E. 1905

Killed in action 29 Oct. I9I4

WynYard Wright, A. T. 2nd Lieut., E. Surrey Regt.; I9I3 Lieut. (A.) and Capt. (T.), R.A.F.

WYarRow,E.F. 2nd Lieut., Argyll and Sutherland Hdrs. $M$. I9I3 Killed in action near Ypres 8 May 1915 
Yates, J. M. St J. Lieut., R.N.V.R.

Yeo, H. E. Capt., King's Own (Yorkshire L.I.); Major,D.A.Q.M.G. (W.) M.C. Brevet Major. M2. \&YeO, L. F. Lieut., S. Staffs. Regt.

Died 1o March I9I 5 of wounds received in action

Yool, G. A. Major, S. Staffs. Regt.; Lieut.-Col., Lincolnshire Regt.(T.F.) (W 2.) D.S.O. M 2 .

Young, C. F. Pte., R.A.S.C.

Young, E. Hilton, M.P. Lieut.-Cdr., R.N.V.R. (W.)

D.S.O. D.S.C. M. Order of Karageorge, 4 th Class

(Serbia). Serbian Medal for Valour. French Croix de Guerre

Young, F. H. Capt., R.A.M.C. O.B.E. $M$.

Young, H A Lieut Cameron Hdrs and Gen List. (W)

Young, H. T. L. Capt., R.A.S.C.

YounG, T. Lieut., King's (Liverpool Regt.) and Tank Corps

Young, W. A. Capt., R.E. O.B.E. $M 2$.

Younger, W. J. Lieut.-Col., R. Scots. $m$.

Zabriskie, G. G. Lieut., United States Naval Air Service Zambra, N. Capt., R.F.A. M.C. 


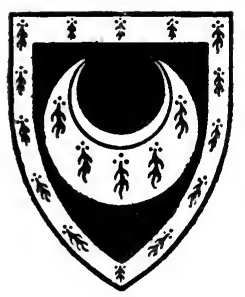

\section{TRINITY HALL}

ABDy, J. R. Capt., Sherwood Rangers. (W.) $M$ 2. I913 French Croix de Guerre. Italian Silver Medal for Military Valour

Acland Troyte, G. J. Major, King's Royal Rifle Corps.; 1895 Lieut.-Col., A.A. and Q.M.G. C.M.G. C.B.E. D.S.O. $M$ 5. m. French Croix de Guerre

Adams, N. P. Capt., K. Edward's Horse HAdAMs, R. S. Capt., R.F.A. $M 2$.

Killed in action 5 Oct. I917

FAdamson, W. C. Capt. (A.), R.F.C. $M$. Killed in action 5 Sept. I9I 5

AGnew, A. G. Major, R.F.A.(T.F.) $M . \quad 1906$

AKroyd, R. Lieut., 9th Lancers

WAlbu, W. G. Capt. (A.), R.A.F. Order of St Stanislas, 2nd Class, with swords (Russia). French Croix de Guerre

Died 29 May 1920 from illness contracted on active service

Alcock, W. B. Capt., R.A.M.C. $M$.

AlFORD, C. E. Capt., London Regt. (Finsbury Rifles) 1904

Allen, H. C. G. Capt., R.E. $\quad 1896$

Allen, J. W., C.M.G. Lieut.-Col., King's (Liverpool 1883 Regt.) $M$.

Allen, S. E. Major, R.A.O.C. $M$.

Anderson, J. Major, Canterbury Regt., N.Zealand Force. $M$.

AANNESLEY, Earl. Sub-Lieut., R.N.V.R.

Killed in action 5 Nov. I914

Ansdell, R. C. Capt., 7th Dragoon Gds. (W.)

I90I

1908

1905

1905

I910

1903

I90I

1904

1907

c.U.w.I. 
WArbuthnot, W. J. 2nd Lieut., I.A.R.O., attd. Infy. Killed in action 9 Fan. 1917

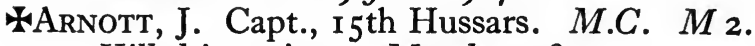

Killed in action 30 March 1918

Ashby, M. W. Lieut., R.G.A.(T.F.)

Aspinall, J. R. Major, Lancs. Hussars (T.F. Res.)

Atkey, S. C. Capt., Hampshire Regt.(T.F.)

Atkinson, G. B. 2nd Lieut., Durham L.I.

Aubry, J. F. A. Asst. Paymaster, French Navy

Austin, Sir W. M. B., Bart. Capt., R.A.M.C.

Avory, D. H. Major, R. Berkshire Regt. (W.) $M$.

Ayliff, J. Cadet, R.A.F.

1904

1904

1900

I 896

1892

I908

[19I4]

1892

1905

1909

BACK, I. G. Capt., R.A.M.C.(T.F.)

BACKHouse, M. R. C. Major, Northumberland Yeo ; 897

Lieut.-Col., Yorkshire Regt. D.S.O. and Bar. $M_{3}$.

BAILEY, P. J., D.S.o. Major, I2th Lancers

Bailey-Hawkins, A. G. Major, Durham L.I. $\boldsymbol{m} 2$.

Baines, M. T. Capt., R. Wiltshire Yeo.; Capt. (A. and Ad.), R.A.F. (W.)

WBAKER, A. F. W. Lieut., D. of Cornwall's L.I.; Capt.(A.), 1909 R.F.C. $M$.

Killed in action I I April r9r7

Baker, R. L. Capt., R.F.A.; empld. Ministry of Muni- $\quad$ I897 tions. (W.) O.B.E. m.

Balfour, A. M. Lieut.-Col., R.F.A.(T.F.Res.) D.S.O. $\quad 1889$ $M 2$.

WBarclay, G. W. Major, Rifle Brigade. (W.) M.C. M. I9ro Killed in action 28 fuly 1916

Barclay, H. F. Lieut.-Col., Bedfordshire Regt.; I 1888 D.A.A.G. $m 2$.

Barclay, Rev. H. G. C.F. $4^{\text {th }}$ Class, R.A.C.D. M.C. $\quad$ I900 $M$.

Barclay, M. E. Major, Norfolk Yeo. and Norfolk Regt. 1905 (T.F.) (W 2.) M 2. Order of the Nile, $4^{\text {th }}$ Class (Egypt)

Barclay-Milne, J. Major, R.F.A. (W.) M.C. M. 1904 French Croix de Guerre

ZBarnato, J. H. W. Pte., R. Fusiliers (P.S. Bn.); Capt. I9I2 (A.), R.A.F. $M$.

Died 26 Oct. I918 of pneumonia

Barnato, W. J. Capt., R.F.A.

Barnsdale, J. D. Major, Lancs. Fus.(T.F.) (W.)

BARR, A. G. 2nd Lieut., Spec. List (Interpreter) 
BARRETt, J. H. Lieut., King's Own (R. Lancaster Regt.) I9I0 and Gold Coast Regt., W. African Frontier Force

BARRINGER, R. E. Lieut., Sherwood Foresters (Notts. . I9I I and Derby Regt.) and Labour Corps.

Barrington, W. R. S. Capt., Oxford and Bucks. L.I. $\quad 1892$ WBarton, B. B. Lieut., King's Royal Rifle Corps

Killed in action 30 Nov. 1917

सBarton, G. R. Capt., Cheshire Regt. (W.)

Killed in action 9 April $19 \mathrm{I} 8$

WBarTon, T. E. 2nd Lieut., R. Irish Rifles

Killed in action 16 fuly 1916

Bartram, H. Lieut., Gen. List, Australian Force

BassetT, H. Ll. Lieut., Welsh Regt.; Capt., R.E. (Spec.

Bde.); Chemical Adviser. M. French Croix de Guerre

Bates, Rev. G. L. C.F. 4th Class, R.A.C.D.

Batley, J. Lieut., Worcestershire Yeo. (W.)

1905

I 893

I906

1903

1908

1890

I9II

BatTen, H. C. C. Major, Dorset Regt.; D.A.A.G. (W.) 1903

D.S.O. M 5. Chevalier, Ordre du Mérite Agricole

(France)

Batten, H. C. G. Hon. Colonel, Dorset Regt. $m$.

1866 .

\#BATten, J. H. S. Capt., King's (Liverpool Regt.)

Killed in action 25 Oct. 19I4

Bell, A. S. Capt., Derbyshire Yeo.

1894

1893

Bell, W. A. J. Lieut., London Yeo.(T.F. Res.); Major, 1902 R.A.S.C.

Bellew, B. B. Lieut., S. Irish Horse and R. Irish Regt.; 1909 attd. T.M.B.; A.D.C. (W.) M.C. $M 2$.

Bellew, E. H. Capt. (Ad.), R.A.F. M.B.E. 1908

BenNetT, D. Pte., H.A.C. 1905

BenNetT, G. M. Lieut.-Col., S. African H.A. D.S.O. $\quad{ }_{1896}$

BenNeTt, G. N. Lieut., R.A.S.C.

BENNETt, R. Lieut., Hertfordshire Regt.

BenYon, H. A. Capt., Berkshire Yeo. and Remount 1903

Service

Beresford, C. V. Major, Worcestershire Regt.; Major $\quad$ I898 (Ad.), R.A.F.

WBERESFORD, C. W. Major, R. Defence Corps

Died 9 Oct. 1917

BERESFORD, M. DE LA P. 2nd Lieut., R.F.A. I899

BERESFORD, T. DE LA P. Capt. (T.), R.A.F. 1906

BERNEY, R. G. G. Aír Mechanic, R.A.F. I9I5

BERNEY, Sir T. R., Bart. Major, Norfolk Regt.(T.F.) I9I2 M.C.

Beswick, W. T. Surgeon Lieut., R.N.

I9II 
Bewes, C. T. A. Capt., Devon Regt.(T.F.)

Bickerton, H. R. Surgeon Lieut., R.N.

BiñNey, J. Capt., Gen. List (T.F. Res.)

Birchenough, R. P. Major, Derbyshire Yeo. M. Greek 1904 Military Cross

Birkbeck, J. Lieut.-Col., D. of Wellington's (W. Riding I890 Regt., T.F. Res.) and Gen. Staff

BiRKETT,G.E. Surgeon-Prob., R.N.V.R.; Capt.,R.A.M.C. 1912 (W.) M.C.

Bispham, C. Lieut., R.E. (W.)

BLACK, L. P. Lieut., R.A.M.C.

WBlackburn, G. G. Capt., W. Yorks. Regt.

Killed in action I fuly 1916

Blackburn, L. O. G. Major, R.A.S.C. O.B.E. M. 1904

Blaine, F. H. Lieut., Manchester Regt.; Capt., R.E. $\quad 1892$

Blake, F. E. C. Capt., Northumberland Yeo.

Bland, J. C. See Cooper Bland, J.

Bloomfield, E. A. R. R. 2nd Lieut., Hauraki Regt., 1889

N. Zealand Force

Bonham-Christie, R. A. Capt., N. Somerset Yeo.; I9II Lieut., R.A.S.C.

Bonomi, J. I. Colonel, King's Own (R. Lancaster Regt.) I874

C.B.E. Brevet Colonel. $m$.

Bookless, J. S. Capt., R.A.M.C.

\#Bотт, J. A. Capt., R. Fusiliers. (W.)

Died 5 Aug. I9I7 of heart-failure

Boulter, C. S. C. Capt., R.F.A.

Bovey, F. H. W. Major, Quebec Regt., Canadian Force;

D.A.A.G. O.B.E. Médaille de la Reconnaissance

1908

1890

1907

Francaise, $3^{\text {rd }}$ Class

Bowes Lyon, Hon. M. Capt., Life Gds. (R. of O.) and 1893 M.G.C.; A.D.C. (W.) $M$.

\&oyd, G. J. 2nd Lieut., Rifle Brigade

Died 3 Nov. I918 of pneumonia

WBoyle, Hon. J. Capt., R. Scots Fus.; A.D.C. $M$.

Killed in action near La Bassée 18 Oct. 1914

Boyle, R. F. R. P. Capt., Oxford and Bucks. L.I.(T.F.) 1906

Brabazon, Hon. C. M.P. Major, Irish Gds.; Lieut.- 1894 Col. (Airship), R.A.F. O.B.E.

WBrain, F. S. Lieut., R. Berkshire Regt.; Capt., Dorset 1912 Regt. $M$.

Killed in action 3 Oct. I9I 8

Brandon, A. DE B. Major (A.), R.A.F. D.S.O. M.C. 1902 $M$. 
Bridge, E. A. Capt., Gen. List (T.F. Res.) I890

BRIDgwater, H. N. Major, Norfolk Regt.(T.F.); 1896

D.A.A.G. D.S.O. O.B.E. $M_{3}$.

Brocklehurst, E. H. Capt., R.A.S.C. $M$.

1895

Brocklehurst, Sir P. L., Bart. Major, Derbyshire Yeo. 1904 and Spec. List, empld. Egyptian Army. (W.)

WBrocklehurst, T. P. Capt., The Queen's (R.W. Surrey 1905

Regt.)

Killed in action I Fuly r916

Bromley-DavenPort, H. R. Lieut., Spec. List (Inland I890

Waterways and Docks); Capt., P. and B.T. Staff.

O.B.E. $M$.

\#BRooksbank, S. Lieut., Yorkshire Regt.

Killed in action 26 Sept. I9I 5

Brooman-White, C. J. Major, Rifle Brigade and Gen. 1904 List. C.B.E.

Brown, A. Lieut., R.A.S.C. $M$.

Brown, H. F. Capt., R.A.S.C. $M$.

Brown, H. N. Capt., Yorkshire Regt. (W.)

I9II

I9II

1912

Brown, N. A. S. Lieut., Hampshire Regt.(T.F.); In- 1913 structor, School of Musketry

Browne, A. W. Capt., W. Yorks. Regt. (W.)

BRUCE, S. M. Capt., Worcestershire Regt. and R. I903

Fusiliers. (W 2.) M.G. M. French Croix de

Guerre

BrUCE-KerR, J. 2nd Lieut., Argyll and Sutherland Hdrs. 1900

Buchanan, H. 2nd Lieut., Cameron Hdrs. (W.) r9ro

BUCKLER, F. W. Lieut., I.A.R.O., attd. 37th Lancers I9ro and 23rd Cavalry

Buckley, C. M. Staff Capt., Australian Force $\quad$ I889

Buckley, P. N. Major, R.E.; attd. Staff of High Com- 1887 missioner for Australia

BuCKNAll, L. C. Major, Northamptonshire Yeo.; A.P.M. $\quad 1892$ D.S.O. M 2. Italian Croce di Guerra

Bullard, F. R. Lieut., Derbyshire Yeo.; attd. R.E. $\quad$ I900

Bullard, G. T. Lieut.-Col., Norfolk Yeo. $m$. 1894

Bunbury, H. W. Capt., Suffolk Regt. and Labour 1907

Corps; Capt. (Ad.), R.A.F.

Burdon, W. W. Capt., Northumberland Yeo. (W.) 1909

Burrell, R. E. Capt., Army Cyclist Corps 1908

WBury, E. W. Capt., King's Royal Rifle Corps 1904

Killed in action 5 Dec. I9I 5

Bush, J. R. 2nd Lieut., The Buffs (E. Kent Regt.); [I9I4] Lieut., M.G.C. 
Busk, E. W. Major, R.F.A.(T.F.) M.C. M 2. French I9II Croix de Guerre

Caffin, E. G. Lieut.-Col., Yorkshire Regt.; attd. North- $\quad 1889$ umberland Fus.; cmdg. Depôt. $M$.

Cahusac, S. D. N. Capt., Ioth Lancers (Hodson's 1905 Horse), Indian Army. $M$.

CaIn, E. Lieut., R.A.S.C.

4CaIrnes, A. B. Major, R. Irish Rifles

Killed in action 9 Sept. I916

Caithness, Earl of. Capt., Gordon Hdrs.(T.F.); Lieut.- I88I Col., Inspector of Q.M.G.'s Services, N. Command. $m$.

Caldwell, K. F. T. Capt. and Adjt., R.F.A. (W.) m. 1906 Italian Silver Medal for Military Valour

Campbell, B. A. Capt. and Adjt., Scottish Horse; 1908 Brigade Major. $m$.

CAMPBEll, D. N. Lieut., R.A.S.C.

Campbell, J. A. Major, Argyll and Sutherland Hdrs. 1868 $M$.

सCAmpbell, K. G. Lieut. and Adjt., Highland L.I.

Killed in action in the Battle of Loos 25 Sept. 1915

Campbell-Muir, D. E. Capt., Res. Regt. of Cavalry 1895 and Remount Service

\#Cantle, L. H. Lieut., Surrey Yeo.; attd. R.F.C.

Killed in action 8 April 1917

Caporn, A. C. Capt., R.F.A.(T.F.) and Gen. Staff

CAPron, G. Capt., York and Lancaster Regt. (R. of O.); Major, N. Staffs. Regt.(T.F.); Lieut.-Col., Cheshire Regt.

Cardiff, R. H. W. Colonel, Durham L.I. Brevet Col- 1890 onel. $m$.

Carey, Rev. D. F. C.F. Ist Class, R.A.C.D.; A.C.G. 1895 D.S.O. M 2 .

CARPMAel, E. V. 2nd Lieut., King's Royal Rifle Corps 1900 CaSlaW, J. M. Lce.-Sergt., London Regt. (W.) M.M. I9I I

Cave, A. L. Capt. and Adjt., Welsh Horse and Res. 1892 Regt. of Cavalry; Capt., Tank Corps

Chandler, W. K. Lieut.-Col., Manitoba Regt., Canadian 1902 Force. D.S.O. French Croix de Guerre

Charlesworth, C. B. Lieut.-Col., King's Own (York- 1896 shire L.I.) (W.)

Chase, Rev. G. A. C.F. $4^{\text {th }}$ Class, R.A.C.D. M.C. 
Chasemore, P. A. Capt., I8th Hussars. (W.) $M$.

1903

Chirnside, R. G. Capt., Gen. List, Australian Force;

A.D.C. (W.) O.B.E. M. Chevalier, Legion of 1902 Honour (France). Chevalier, Military Order of Avis (Portugal)

Cholmley, H. A. Lieut., Yorkshire Hussars

Christie, H. A. H. Capt., W. Kent Yeo.; Major, M.G.C. 1903 (W.)

Christie, R. A. B. See Bonham-Christie, R. A.

Clark, R. Lieut., R.A.S.C.(T.F.)

Clarke, E. P. Lieut., R.N.V.R.

1913 1890

Clarke, W. G. Lieut., Life Gds.; Capt., Gds. M.G. 1912 Regt.

Clarke-Williams, A. R. Lieut., R.F.A.; attd. R.H.A. 1913 (W.)

ZClayhills, G., D.s.o. Capt., E. Lancs. Regt. Killed in action 2 Nov. 19I4

*ClegG, J. Capt., King's Own (Yorkshire L.I.) Killed in action 16 Sept. I916

Clifford, A. W. Capt., Gloucestershire Regt. and Gen. 1897. List (O.C.B.)

Coates, C. A. Pte., R. Fusiliers

Coham-Fleming, B. B. Pte., London Regt.; 2nd Lieut., 1903 R.G.A.

Coke, Hon. Reginald. Major, Scots Gds.; Courts- 1882 Martial Officer

Cole, T. G. O. Capt., Denbigh Yeo.; A.P.M. $M$.

Collings-Wells, L. C. Lieut., King's African Rifles. (W.)

Combe, H. A. B. 2nd Lieut., Res. Regt. of Cavalry; Capt., Spec. List (A.P.M.) M.B.E. $M$ 2. French Croix de Guerre

Compston, G. D. Capt., R.A.M.C.

\COOKE, C. P. 2nd Lieut., King's (Shropshire L.I.)

Killed in action 22 Aug. 1917

※Cooper, H. A. Sergt., Canadian Infy.

1895

1898

Killed in action 19 Aug. 1916

Cooper, J. R. Capt. and Adjt., R.F.A.(T.F.) (W.) $M$. I9io

Cooper, P. A. Capt., R.F.A.(T.F.); empld. Ministry 1906 of Munitions. (W.) $m 2$.

Cooper Bland, J. Lieut., 2oth Hussars

Copeman, G. W. B. Capt., R.E.

ZCorrie, L. Paymaster Sub-Lieut., R.N.R.

Died 23 Oct. 1918 of pneumonia following influenza 
\&Cotron, B. G. H. Capt., London Regt. (Poplar and Step- 1908 ney Rifles)

Died 8 Nov. I9I 7 of wounds received in action

Courthope-Munroe, C. H. Capt., I.A.R.O., attd. 32nd 1908 Lancers; attd. R.F.C. (P.)

\&COURThope-Munroe, J.W. 2nd Lieut., R.A.S.C.(M.T.) IgI I

Died at Alexandria 24 Fan. 19r6 of enteric

Cousins, N. A. C. Capt., London Regt. (Queen's). (W.) I9io

Cox, B. C. Lieut., R.N.V.R.

Cox, E. H. M. Lieut., R. Marines

1896

Cox, H. P. Lieut., R.N.V.R.

Craig, A. D. E. Capt., London Regt. (Queen's)

Crawford, C. G. Capt., I.A.R.O., attd. 3oth Punjabis I909

Crawley, W. H. T. E. Capt., R. Warwickshire Regt. I9Io and Gen. Staff. $M$.

Crocker, R. W. Capt., R.A.S.C.

Crocker, R. W. Capt. R.A.S. Brig.-Gen. C.M.G. T.D. $M 2$.

Croft, R. P. Lieut.-Col., Bedfordshire Regt. $m$. I89I

Custance, C. V. H. Lieut., R.G.A.

Custance, E. C. N. Lieut., Norfolk Regt.; Capt., Tank Corps

Darwin, G. A. M. Capt., W. Yorks. Regt.

Davidson, H. G. L. Hon. Major, King's Own R. Lan- 1890 caster Regt. (R. of O.)

D'Avigdor-Goldsmid, O. E. Lieut.-Col., R.A.S.C. M. 1895

Davy, P. L. Lieut., R.G.A. (W.) I90I

WDavy, W. J. Pte., Ceylon Planters Rifle Corps; 2nd I907 Lieut., Somerset L.I.

Killed in action 18 Aug. I916

DE LA Rue, R. W. Capt., R.A.S.C.

WDenham-Cookes, A. B. Capt., London Regt. (Queen's) I9Io Died 5 Nov. 1918

\&DE Pass, C. A. 2nd Lieut., Res. Regt. of Cavalry; attd. I912 Tank Corps

Killed in action 22 March 1918

DE Villiers, D. I. Lieut., M.G.C.

De WaAL, D. Lieut.-Col., S. African Force. D.S.O. M. 1894 WDICK, C. W. Lieut., R.N.V.R.

Died 7 Nov. 1918

WDickens, C. C. Major, London Regt. (Kensington Bn.) 1907 (W.) $M$.

Killed in action ro Sept. 1916 
Dickens, P. C. Lieut., Welsh Gds. (W 2.) 1906

DigBY, T. H. Lieut., 4 th (R. Irish) Dragoon Gds. and I9IO M.G.C. (Motor). $M$.

Dilke, Sir F. W., Bart. Capt. and Adjt., R. Berkshire 1895 Regt. (T.F.); empld. War Office

DOHERTY, H. L. 2nd Lieut. (T.), R.A.F.

Dolbey, R. C. Lieut., King's Own Scottish Borderers. 1907 (W 2.) $M$.

Doll, W. A. M. B.S.M., R.G.A.

Donisthorpe, E. R. Capt., R. Defence Corps

1904

I 884

Douro, Marquis of. 2nd Lieut., Grenadier Gds.; Lieut., 1895

Spec. List, empld. Inland Waterways and Docks

Dove, G. V. Capt., Lincolnshire Regt.; empld. Ministry 1897 of National Service

WDRAKe, G. E. 2nd Lieut., Worcestershire Regt.

Died 28 Fan. 1918 of wounds received in action

Drysdale, M. B. Lieut., Sherwood Foresters (Notts. I9I I and Derby Regt., T.F.) (W.) M.C.

Drysdale, S. A. Lieut., Dorset Regt. and Gen. List 1895 (T.M.B.)

DUBERLy, E. H. J. Lieut., Grenadier Gds.; Capt., 1905 G.S.O. 3 , N. Command. M.C. $m$.

Duff, I. A. J. Capt., Dorset Regt.(T.F.); Capt. (A.), [I9I4] R.A.F. (W 2.) M.C. M.

Duff, K. D. J. 2nd Lieut., R.G.A. $\quad 1908$

Duff, W. S. B. Capt., R.N.V.R. $\quad 1896$

DumaresQ, R. G. F. 2nd Lieut., Rifle Brigade; Major, 1899 M.G.C. (P.) M.C. $M$.

Dunbar, A. R. Air Mechanic, R.A.F.

DunCAN, H. A. Lieut.-Col., Argyll and Sutherland Hdrs. $\quad{ }^{\text {I914 }} 893$ D.S.O. $M$.

Dunlop, F. L. A.B., R.N.V.R.; 2nd Lieut., R.G.A.; 1895 Capt., Tank Corps

Edgar, W. H. A. Capt., Labour Corps

1906

WEDwards, W. A. Lieut., Glamorgan Yeo.; attd. Welsh 1909

Regt. (W.)

Died I Nov. I9I7 of wounds received in action

Egerton, Hon. F. W. G. Capt., D. of Lancaster's Own 1894 Yeo. $m$.

Ellis, W. H. M. Lieut., R.N.V.R.

Elton, W. See Marwood-Elton, W.

Elworthy, P. A. Lieut., Life Gds. 
Escombe, F. J. Sub-Lieut., R.N.V.R.

Evans, E. Capt., R.A.S.C.

Evans, Rev. H. R. Pte., R.A.M.C.; Lieut., King's Own 1908 (R. Lancaster Regt.) (W.) M.C.

Evans, W. V. E. Gnr., R.G.A.

WFarmiloe, G. F. 2nd Lieut., H.A.C. M.C.

Killed in action 26 June 1917

Farmiloe, T. H. Capt., London Regt. (Q.V.R.) M. 1908

FARQUHARSON, H. F. W. Lieut. (Ad.), R.A.F.

Fawkes, F. H. Major, Yorkshire Hussars (T.F. Res.) $\quad$ I890

FeArnsides, E. G. Hon. Major (Med.), R.A.F. 1902

Fenwick, G. Lieut.-Col., R.F.A.(T.F.); Area Cmdt. 1888

FERNIE, W. J. Lieut.-Col. (T.), R.A.F. O.B.E. 1892

Fetherstonhaugh, R. G. Capt., $4^{\text {th }}$ (R. Irish) Dragoon 1908 Gds. (W.) $M$.

Firebrace, R. C. 2nd Lieut., R.F.A.

FIRTH, E. L. Capt., S. Irish Horse and R. Irish Regt. (W 2.)

Fisher, O. Capt., Glamorgan Yeo. and Welsh Regt. 1904 (W.) M. French Croix de Guerre

Fitzhugh, A. E. L. Lieut., R. Sussex Regt.

Fitzroy, Rev. H. S. Instructor, R.N.

FlanNery, H. Fortescue. Capt., R.H.A.(T.F.); empld.

Ministry of Munitions. M.B.E.

Flawn, N. G. 2nd Lieut., R.G.A.

FogG-Elliot, C. T. Capt., Durham L.I. 1917 1905 1907 1899 1890

Foley, C. P. Lieut.-Col., E. Lancs. Regt. and Spec. 1887 List. $M$.

ForD, R. M. Lieut., S. Wales Borderers. (W.) French 1902 Croix de Guerre

Fordham, H. J. Lieut., Hertfordshire Yeo., Res. Regt. 1900 of R. Horse Gds., and Gds. M.G. Regt.

Forshaw, H. P. Capt., King's Own (R. Lancaster Regt.) 1895 M.C. and Bar

Foster, C. W. Capt., R. Marines

Foster, F. E. Lieut., R.N.V.R.

1905 1896

Foster, W. M. Major, Dorset Regt.(T.F.) (W.) T.D. $\quad$ I905 Fowke, G. H. S. Capt., Gordon Hdrs. (W.) (P.) 1899 Fox, J. St V. Major, Lincolnshire Regt.

Fox-ANDREws, N. R. Lieut., D. of Cornwall's L.I. and 1898 M.G.C.

Fraenkl, E. G. H. See Fremantle, E. G. H.

FrancIs, W. H. Major, R. Defence Corps 
Fraser, M. H. Capt., Seaforth Hdrs. and King's 1908 African Rifles

Fraser-TytLER, W. T. Major, Lovat's Scouts I88I WFRAM, W. Lieut., Gloucestershire Regt.(T.F.) (W.) I9I I Killed in action 21 Fuly 1916

FremantLe, E. G. H. Capt., R.A.S.C.

1899

Gabriel, A. Pte., R. Fusiliers (Sportsman's Bn.) I89I

GARRETT, N. L. Major, King's Royal Rifle Corps 1884

GERARD, Lord. Capt., R. Horse Gds. (W 2.) M.C. $\quad{ }^{*}$ I904

GibBoNs, S. A. 2nd Lieut., London Regt. (W.) 1909

GIBBS, A. H., T.D. Lieut.-Col., N. Somerset Yeo.; In- 1894 spector, Q.M.G.'s Services, S. Command

GilbERT, R. T. E. Capt., Norfolk Regt. (T.F. Res.) I890

GILBERT, T. 2nd Lieut., I.A.R.O.; Lieut. (A.), R.A.F. 1907

GILES, H. O'H. Major, R.F.A. M. French Croix de 1908 Guerre

GILMOUR, H. Lieut., I6th Lancers (R. of O.) $\quad$ I898

GILMOUR, J., M.P., T.D. Lieut.-Col., Fife and Forfar 1895

Yeo.; attd. Black Watch. (W.) D.S.O. and Bar. M2.

GinN, D. B. Capt., Suffolk Yeo. and Suffolk Regt.(T.F.) 1898

GLADSTONE, H. S. Capt., King's Own Scottish Borderers 1896

(T.F. Res.); empld. War Office

Gladstone, K. S. M. Capt., Rifle Brigade; Staff Capt. I9I3 M.C. $M 2$.

GoldBLATT,D. Lieut., King's Own (YorkshireL.I.,T.F.) I9I4 Goldsworthy, E. W. Capt., London Yeo. (Rough 1913 Riders); Hon. Capt. (A.), R.A.F. (W.)

AGomme, E. E. C. Pte., R. Fusiliers (P. S. Bn.); Capt., 1904 Suffolk Regt. (W 2.)

Killed in action 18 fune 1917

Gordon, A. F. L. Lieut.-Col., Irish Gds. (W.) D.S.O. I9II M.G. $M$.

GosLING, W. S. Major, Scots Gds. (R. of O.) $m . \quad 1889$

GotT, W. W. M. Capt., Rifle Brigade; attd. King's 1893

Royal Rifle Corps. $m$.

GotTo, H. S. Air Mechanic, R.N.A.S.

GouldSMith, C. C. Major, R.F.A.(T.F.)

Gower, J. R. Capt., 6th Dragoon Gds. (Carabiniers) 1894

(W.)

GraHAM, J. D. Lieut., R. Warwickshire Regt. I89I

GRANBY, Marquis of. Capt., Leicestershire Regt.(T.F.); 1906 A.D.C. 
\&Gray-Cheape, H. A. Lieut.-Col., Worcestershire Yeo. 1897 D.S.O. and Bar. $M 2$.

Drowned on H.M. transport Leasowe Castle 27 April I9I8

Green, J. E. S. Capt., Rifle Brigade. (W 2.)

GREER, T. M. Major, Manchester Regt.(T.F.)

Gregory, G. M. A. Lieut.-Col., R.F.A. Brevet Lieut.- 1896 Colonel. $M 3$.

Griffith-Jones, J. S. Capt., S. Wales Borderers and 1898 Gen. List

Griffith-Jones, M. P. Major, Gen. List (Staff Capt., 1895 War Office). O.B.E. m.

Guinness, K. E. L. Lieut., R.N.V.R.

Gunning, J. E. Major, R. Irish Rifles

\&Gunther, C. E. Lieut., Life Gds.; attd. Gds. M.G. I9IO

Regt. $M$.

Killed in action 24 Sept. I918

Gwynne, R. V. Capt., Sussex Yeo.; Lieut.-Col., The 1900 Queen's (R.W. Surrey Regt.) (W 2.) D.S.O.

WHaggard, M. Capt., Welsh Regt.

Died I 5 Sept. I9I4 of wounds received in action

Haggard, T. B. A. Capt., R.A.M.C.

1906

1899

HAGGIe, F. R. Lieut., Life Gds.

1894

HAGON, A. C. 2nd Lieut., R. Warwickshire Regt.; r910 Major (A.), R.A.F.

HaIG, O. Hon. Lieut.-Col., London Yeo.(T.F. Res.) I894 WHAINeS, W. R. Sub-Lieut., R.N.V.R. (R.N.D.)

Killed in action $17 \mathrm{Feb}$. I9I7

Hall, A. H. Capt., Essex Regt.(T.F.)

1903

Hall, G. D. Capt., Queen's Own (R. W. Kent Regt.); 1896 empld. Ministry of Munitions

Hall, R. H. E. Capt., Oxfordshire Yeo.(T.F. Res.) I901

Hampson, O. D. Capt., E. Lancs. Regt. (W.) 1897

Hannen, L. C. D. Capt., R.E. (Signals). $M$.

HanNen, N. M. B. 2nd Lieut., R.F.A. I9I7

Harbaugh, G. W. F. Pte., London Regt. (Artists Rifles) I9I5

Harber, E. W. Lieut. (K.B.), R.A.F.

Hargreaves, H. Major, Cheshire Regt.

Harmsworth, V. G. Capt. and Adjt., R.A.S.C.

Harris, H. S. Lieut.-Col., Kimberley Regt., S. African

Defence Force; Capt., R. Fusiliers; attd. Gold Coast Regt., W. African Frontier Force. (W.) O.B.E. $M$. 
HaRrison, E. J. Lieut.-Col., King's (Liverpool Regt., I895 T.F.) (W.) T.D. $M$.

Haselden, R. B. Capt., Lancs. Fus.; Lieut., Nigeria 1899 Regt., W. African Frontier Force

Hatton-Hall, H. C. Lieut., King's Own Scottish Bor- I9I I derers; Capt., Tank Corps. (W.) M.C.

WHaviland, J. D. Lieut., R. Fusiliers. (W.)

Died 16 fuly 1916 of wounds received in action 15 fuly

I901 I9I6

Heaton-Armstrong, J. D. 2nd Lieut., Spec. List (Interpreter); Capt., I.A.R.O., attd. 2oth Deccan Horse. (W.)

Helm, J. H. Capt., Manchester Regt.(T.F.). (W.) 1899

Henn-Gennys, E. C. Lieut., Remount Service 1899

Hermon, J. V. Capt., Cheshire Yeo.; attd. 6th Dragoon 1893 Gds. (Carabiniers). (W 3.) D.S.O. M.

Heseltine, C. Major, R. Fusiliers; Lieut.-Col., Gen. 1890 Staff. O.B.E. $M 2$.

Heseltine, G. Capt., 6th Dragoon Gds. (Carabiniers) $\quad \mathbf{1 8 8 9}$

Hickling, A. R. Lieut., W. Yorks. Regt. and R.E. 1906

Hickman, J. O. Lieut., Bedfordshire Regt. and Gen. 1888 Staff

Hilton-Green, C. C. H. Lieut., ist Dragoons. (W.) [1914] 4HIND, L. A. Lieut.-Col., Sherwood Foresters (Notts. 1896 and Derby Regt., T.F.) (W.) M.C. M2.

Killed in action I fuly r916

Hind, O. W. Capt., Sherwood Foresters (Notts. and I89I Derby Regt., T.F. Res.)

WHissEY, T. B. Lieut., R.A.S.C. Died Fuly 1919 from the effects of active service during the war

Hodges, W. C. Major, R.A.M.C.(T.F.) $M$.

Hodgrinson, G. A. 2nd Lieut., Rifle Brigade; Capt., 1898 M.G.C.

Hodson, J. Capt. (S.), R.A.F.

Holden, H. C. Capt., Lancs. Fus.(T.F.); empld. Re- 1895 cruiting Staff

Holden, N. E. Major, Gen. List. O.B.E. m. $\quad 1897$ HHoldsworth, A. M. Lieut.-Col., R. Berkshire Regt. 1894 $M$.

Died 7 Fuly 1916 of wounds received in action

Hole, C. H. Lieut., R.A.S.C.

Holland, J. D. C. 2nd Lieut., Oxford and Bucks. L.I. IgI I Killed in action 13 Nov. I916 
Holland, S. C., D.s.o. Major, Ist Dragoon Gds. (R. I89? of O.); empld. War Office. Brevet Major. Chevalier, Legion of Honour (France)

Holland, V. B. Lieut., R.F.A.; Staff Capt. O.B.E. $M_{3}$. I90 Holland, W. G. C. 2nd Lieut., Gordon Hdrs.

Hollins, P. L. Lieut., R.A.S.C.(M.T.)

WHollist, A. M. C. Capt., The Buffs (E. Kent Regt.)

Killed in action in the Battle of Loos 2.5-27 Sept. 1915

Holmes, T. V. Air Mechanic, R.N.A.S.

Holmes-Tarn, H. Major, King's Royal Rifle Corps

Hope, A. T. 2nd Lieut., Suffolk Regt.; Capt. (A.), R.A.F.

Hope, L. N. Capt., R. North Devon Yeo. and Devon Regt.(T.F.); attd. R.E.

WHope-Wallace, J. Lieut., Northumberland Fus.(T.F.) I89 $M$.

Killed in action ${ }_{5} 5$ Sept. 1916

Hopkins, W. D. Lieut., R.A.M.C.

Horan, Rev. F. S. C.F. $4^{\text {th }}$ Class, R.A.C.D.(T.F.) $m$. 1892 HoRn, D'A. Capt., R.A.S.C. O.B.E. $M$.

Hornidge, E. S. Capt., R.A.S.C. O.B.E. M.

HowetT, F. Lieut., R.A.S.C.(M.T.) Serbian GoldMedal

WHudson, G. Lieut., Queen's Own (R. W. Kent Regt.);

Major, M.G.C. (W.) M.C. M.

Killed in action 12 April I91 8

WHuDson, R. B. 2nd Lieut., Oxford and Bucks. L.I.(T.F.) I9Ic

Killed in action 19 fuly r 916

Hughes, D. 2nd Lieut., S. Wales Borderers; Lieut., 1906 M.G.C.

WHulton-Harrop, H. DE L. Lieut., Life Gds. (W.)

1899

Killed in action 12 May 1915

Hume, A. Hon. Colonel, King's Own Scottish Borderers

Hume-Kelly, F. V. Major, N. Staffs. Regt. $M$.

Hume-Williams, R. E. Capt., R.A.S.C.

Humphries, G. N. P. Lieut., Somerset L.I.; attd. R.E. I9I

(Spec. Bde.)

HumpHries, R.P. 2nd Lieut., Somerset L.I., attd. I9I2 Devon Regt.

WHunTER, G. J. 2nd Lieut., 5th (R. Irish) Lancers Killed in the Irish rebellion 26 April 1916

Huntriss, C. G. 2nd Lieut., 2 Ist Lancers; Lieut., Res. 1899 Regt. of Cavalry

Huntriss, E. M. Lieut.-Col., D. of Wellington's (W. 1899 Riding Regt.) (W.) M.C. $M$ 
Hurrell, H. W. Major, Gen. List (T.F. Res.) I882

HurRell, W. C. Capt., Spec. List

$188 \mathrm{I}$

AHutchinson, A. C. C. Capt., R. Fusiliers; Major, M.G.C. 1902

Died 18 Nov. 1918 of pneumonia contracted on active service

Hutchinson, G. C. Lieut., R.F.A.

1903

IngPen, D. L. Capt., W. Yorks. Regt. and Gen. Staff 1903

JACKSON, E. W. 2nd Lieut., Labour Corps

WJAFFray, Sir J. H., Bart. 2nd Lieut., Worcestershire Yeo.

Killed in action 23 April 19r6

JEFFCOCK, W. P. Lieut., S .Notts. Hussars and Res. Regt. of Life Gds.

Wunnings, G. M. Pte., Australian Infy.

Died Aug. 1916 of wounds received in action on the Somme

JEPPE, O. R. Lieut., Cape Corps, S. African Force. (W.) 1905

Jesse, W. J. Lieut., R.G.A.(T.F.)

Jessell, R. P. Hon. Major, Spec. List (Recruiting Staff) I886

JoEL, W. S. Lieut. (A.), R.A.F. I9I I

Johns, N. S. C. 2nd Lieut., Yorkshire Regt.

Johnson, H. A. Colonel, Manchester Regt. (W.) M 2. $\quad$ I884

Johnson, J. G. T. Major, Derbyshire Yeo. D.S.O. 1904 $M 2$.

Johnson, G. B. B. Capt., Army Printing and Stationery 1906 Services. $M$.

WJorCEY, C. M. Capt., Northumberland Fus.(T.F.) (W 2.) I9I I Killed in action 5 June 1917

Jorcey, E. R. 2nd Lieut., Northumberland Yeo.; Lieut., I9ro 9th Lancers. (P.) M.C.

Jones, T. C. Lieut., Pembroke Yeo. and R.F.A.(T.F.) 1893

KAPLAN, I. 2nd Lieut., York and Lancaster Regt.; 1907 Lieut., M.G.C.

KeIGwIN, C. H. S. 2nd Lieut., R.F.A.

KemP, W. R. Capt., R.A.M.C.; Capt. (Med.), R.A.F. $\quad \begin{array}{r}1909 \\ 1890\end{array}$

WKendall, P. D. 2nd Lieut., King's Liverpool Regt. 1896 (Liverpool Scottish, T.F.)

Killed in action near Ypres 25 Fan. I9I 5

Kerr, J. Bruce. See Bruce-KerR, J.

AKERRICH, H. L. Lieut., Sherwood Foresters (Notts. and [1914] Derby Regt.)

Died 27 Sept. 1917 of wounds received in action 
KERR-Smiley, P. K., M.P. Lieut., 2 Ist Lancers (R. of O.); 1899 A.D.C.; Major, R. Irish Rifles

AKILlen, E. O. B. Lieut., R.E.

Killed in action in Mesopotamia 15 Fan. I917

KING, H. R. Capt., Durham L.I.; Hon. Capt. (Ad.), I90; R.A.F.

KING, P. B. Lieut., R.A.S.C.; attd. S. Wales Borderers. I9I (W.)

KipPING, C. H. S. Lieut., London Regt. (Q.V.R.)

KItching, H. E. Capt., Durham L.I.(T.F.) M.B.E. M. Knowles, E. A. Pte., R. Fusiliers

Knowles, R. M. Capt., Norfolk Regt.; Hon. Capt.(T.), I9I3 R.A.F. (W.) M.C.

Knox, P. F. Lieut., R. Fusiliers. (W.)

Knox, W. D. C. Lieut., R. Scots and M.G.C.

Laidlay, J. C. 2nd Lieut., Black Watch; Lieut., Seaforth Hdrs.

WLandale, C. Capt., King's Royal Rifle Corps. (W.) M. 1899 Killed in action 21 Aug. 1918

Landale, D. G. Lieut., Rifle Brigade; Capt., Spec. 1903 List (P. and B. T. Staff) and Gen. Staff, empld. British Military Mission

WLangham, C. R. Capt., R. Sussex Regt.(T.F.)

Killed in action 16 Aug. 1917

Langham, F. G., C.M.G., V.D. Lieut.-Col., R. Sussex Regt. (T.F.) and Labour Corps. $M$.

Langton, S. J. Capt., London Regt. (Post Office Rifles, $\quad 1889$ T.F. Res.)

Larman, G. E. 2nd Lieut., R. Fusiliers

Lawson, Sir D., Bart. Capt., Yorkshire Hussars; attd. rgth Hussars; Capt., W. Yorks. Regt. $M$.

Lawson, D. A. Capt., R.F.A. $M$.

LAYE, P. A. W. Major, King's Royal Rifle Corps; attd. 1908 Gen. Staff. (W 2.)

WLEA, M. B. 2nd Lieut., Northamptonshire Regt.

Killed in action 18 Aug. 1916

Leake, C. F. Capt., King's (Shropshire L.I., T.F.) 1902

Leatham, C. G. Lieut., King's Royal Rifle Corps; 1905 Capt., The Queen's (R. W. Surrey Regt.) (W 2.) M.C. and Bar

WLeE, J. M. 2nd Lieut., Border Regt.

Killed in action 27 Sept. 1915

LEE-Booker, R. Lieut., S. Lancs. Regt. (W.) 
LeICester, C. B. W. Lieut., Ist Dragoon Gds.; attd. [I9I4] Cheshire Yeo.

HLeigh, C., D.S.O. Major, King's Own Scottish Borderers I89I Died 29 Aug. 1918 of wounds received in action at Mons

Leighton, F. M. 2nd Lieut., Bedfordshire Regt.; Capt. I914 and Adjt., Welsh Regt. M.C.

LetTs, E. M. Capt., Oxford and Bucks. L.I.(T.F.); 1910 Lieut. (Ad.), R.A.F. (Aircraft Production Dept.) (W.)

Lewes, W. Lieut., Welsh Regt.(T.F.) and Gen. List 1898 (T.F. Res.), empld. Ministry of National Service

Lewey, A. W. Capt., Middlesex Regt.(T.F.) (W 2.) I9I3

LEwIN, A. C., D.s.o. Hon. Colonel, Connaught Rangers; Lieut.-Col., Wiltshire Regt.; Brig.-Gen.; A.D.C. to the King. C.B. C.M.G. Brevet Colonel. M 6 . Order of St Anne, 2nd Class, with swords (Russia)

WLEwIS, B. R. Major, R.F.A.

Killed in action 2 April I9I7

LEwIS, F. G., C.M.G., T.D. Lieut.-Col., London Regt. 1892 (Kensington Bn.); Brig.-Gen. (W.) C.B. $M_{3}$. Belgian Croix de Guerre

LINDLEY, Hon. J. E. Colonel, Ist Dragoons; Major-Gen. 1878 $m$.

LitTle, T. H. Capt., Worcestershire Regt. (W.) $\quad 1885$

Litton, W. R. U. Capt., Spec. List (Adjt., P. of W. 1905 Camp)

Livingston, C. P. 2nd Lieut., King's Royal Rifle Corps. I9 I (W.)

LleWEllyn, O. J. Capt., R.A.S.C.

1888

LockwooD, E. M. Lieut.-Cdr., R.N.V.R. (Hawke Bn., 1909 R.N.D.) (W 3.) D.S.O. M 2.

HLogaN, H. Lieut., Leicestershire Yeo.; attd. R.E. Died Nov. 1918 of pneumonia

Logan, J. M. Capt., Leicestershire Yeo.; empld. War 1902 Office

Long, J. A. E. Capt., Hampshire Yeo.; attd. Hampshire 1908 Regt.

LoNG, W. E. Capt., 4th Hussars (R. of O.); Major, Re- $\quad$ I890 mount Service. O.B.E.

LongstafFe, V. C. H. Lieut.-Cdr., R.N.V.R.; Major 1904 (Ad.), R.A.F.

LORD, H. D. Capt., Hampshire Regt.

\&LORING, W. L. Lieut.-Col., R. Warwickshire Regt. 1886

(W.) $M$.

Killed in action 23 Oct. 1914

c. U.w.I. 
Love, E. W. P. Major, 2oth Hussars; Adjt., D. of Lan- 1897 caster's Own Yeo.

Low, H. F. Capt., Durham L.I. and Labour Corps 1894

LuCAS, L. W. Lieut.-Col., The Buffs (E. Kent Regt.) 1897 and Spec. List. D.S.O. M.C. $M 2$.

Lyon, C. G. Major, E. Riding of Yorkshire Yeo. and 1900 M.G.C. (W.) $M$.

Lyon, W. A. Lieut., Cambridgeshire Regt.

Lysley, W. L. Hon. Major, R. Wiltshire Yeo.(T.F. Res.) 1894

McCleary, G. F. Capt., R.A.M.C.(T.F.)

1890

McKelvie, J. Lieut., R.E.(T.F.); Major (A.), R.A.F. 1899 A.F.C. Belgian Croix de Guerre

MacLaren, T. G. Lieut.-Col., Seaforth Hdrs.(T.F. 1880 Res.) $m$.

Macnamara, C. R. Capt., R. Dublin Fus.

MAdDEN, J. C. W. Lieut.-Col., R. Irish Fus. $m$.

WMadDison, G. Lce.-Corpl., Essex Regt.; 2nd Lieut., 1905 Norfolk Regt.

Killed in action 28 Aug. 1918

MaIne, H. C. S. Lieut., Grenadier Gds.; empld. War Office. (W.)

Mainwaring, C. F. K. Capt., King's (Shropshire L.I.) I89r and Tank Corps

Malden, Rev. E. E. C.F. 4th Class, N. Zealand Chap- 1898 lains' Dept. M.B.E.

Manisty, E. A. Major, Lincolnshire Yeo.(T.F. Res.) 1892

Margetts, F. B. H. Capt., Hampshire Regt.; Lieut., 1899 Spec. List (Recruiting Staff)

Marrow, E. A. Capt. and Adjt., King's Own Scottish 187 I Borderers

Marshall, W. S. Capt., Sherwood Foresters (Notts. I895 and Derby Regt., T.F.)

Martineau, B. G. Capt., R. Warwickshire Regt.(T.F.) [1914] (W 2.)

Martineau, E., C.M.G., v.D. Hon. Colonel, R. Warwick- 1879 shire Regt.(T.F.) $M$. $m$.

Martineau, W. Capt., R. Warwickshire Regt.(T.F.); 1908 Major, R.E. (Signals). M.C. M.

MaRwOOD-EltoN, W. Lieut.-Col., Welsh Regt. 1885

Mason, A. D. C. Lieut., Cheshire Regt.(T.F.) and 1908 R.F.A.

Mason, G. H. Capt., W. Yorks. Regt.; Major, Middlesex 1897 Regt. (W 2.) 
Matheson, I. M. Capt., Seaforth Hdrs.; Capt. (A.), I9r2 R.A.F. (W.)

MaXwell, S. W. Air Mechanic, R.A.F.

MAYER, A. Capt., London Regt. (Queen's)

I9I3

1890

MeigGS, J. C. Capt., R.A.S.C.

1889

*MeLLES, G. F. Lieut., R.F.A.(T.F.)

Died at Alexandria 6 Nov. 191 5 of septic poisoning

1903

MelloR, A. 2nd Lieut., Durham L.I.; Lieut., Labour 1908 Corps. (W.)

MelloR, J. E. P. Capt., Hampshire Regt.(T.F.) M. 1900

MelloR, J. F. S. Capt. and Adjt., The Queen's (R.W. 1897 Surrey Regt.) and R.A.S.C.

MelloR, J. G. S. Brig.-Gen., R. of O.; Dep. Judge- 1890 Advocate-General. C.B. M 2. Chevalier, Legion of Honour (France)

Menzies,V. M. G. 2nd Lieut., R. Scots; Capt., Scots Gds. WMerriman, G. Pte., Nyasaland Vol. Res.

Died 9 Sept. 19I4 of wounds received in action in Nyasaland

WMetcalfe-Smith, B. C. Pte., London Regt. (Queen's Westminster Rifles); Lieut., W. Yorks. Regt.

Died 22 April 1918 of wounds received in action 18 April I918

MeYrick, F. C., C.B. Colonel, Res. Regt. of Cavalry; 1880 Brig.-Gen.; Colonel, Remount Service; D.D. Remounts. C.M.G. M2. $m$.

Miller, D.O.DE E. Lieut., R.F.A.; Staff Capt. M.C. I9ro $M$.

WMilLER,J.H. Capt.,Loyal N.Lancs. Regt.; attd.Egyptian 1902 Army

Died 25 Aug. 1917 of blackwater fever

MILNER-GIBSON, W. A. Major, R.A.S.C.; A.D.C. $m$. $\quad 1897$

Milton, H. A. Major, London Regt. M.C. M. French 1900 Croix de Guerre

\#Milvarn, Sir T., c.B., K.c. Judge Advocate-General, War $\quad$ I863 Office

Died 1916

Mitchell, G. D. Sapper, R.E.

MoffatT, C. H. Lieut., King's (Shropshire L.I.) 1902

MonTAGU, J. F. Lieut., 15 th Hussars; A.D.C. 1906

MonTAGU Douglas ScotT, Lord H. A., D.s.o. Major, 1892 Irish Gds. (R. of O.); Lieut.-Col. London Regt. (T.F. Res.); D.A.M.S. C.M.G. Officer, Legion of Honour (France) 
Monteuuis, L. E. Lieut., Berkshire Yeo. and M.G.C. Ig0I ZMONTGOMERY, H. 2nd Lieut., I2th Lancers (R. of O.); 1899 Lieut., Irish Gds.

Killed in action 13 Sept. I916

Moore, C. D.H. Colonel, R. Warwickshire Regt.; Brig.- 1895

Gen. C.M.G. D.S.O. Brevet Colonel. M5.

Chevalier, Legion of Honour (France). Order of the Nile, 3 rd Class (Egypt)

Moorhouse, E. R. Bdr., R.F.A.

Moorhouse, W. B. R. See Rhodes-Moorhouse, W. B.

Morgan, H. R., v.D. Major, Norfolk Regt.(T.F. Res.)

I913

WMorgan, W. W. Lieut., R.N.V.R. (Hawke Bn., R.N.D.) 1905

Killed in action in Gallipoli 19 June 1915

Morley, Earl of. Capt., R. Ist Devon Yeo.(T.F. Res.) 1896 and Gen. Staff

Morris, J. W. Capt., R. Welsh Fus. M.C.

Morrison, R. J. A. Pte., London Regt.

Mortimer, F. G. C. Major, R.A.O.C.; D.A.D.O.S. I90I O.B.E. M.

Mostyn-Owen, G. C. Lieut., Shropshire Yeo. and 12th I9ro Lancers; attd. Egyptian Army. $M$.

Moy, E. T. 2nd Lieut., Suffolk Regt:

Muncey, R. W. L. Pte., Norfolk Regt.

\#Murray Smith, G. Lieut., R. Fusiliers

Killed in action 29 Sept. I9I 5

NAInby-Luxmore, C. C. 2nd Lieut., R.F.A.; Lieut., I9ro Scots Gds. (W.)

NaIRn, R. S. Major, Fife and Forfar Yeo.; Staff Capt., 1900 Scottish Command. T.D. m.

WNathan, L.C. 2nd Lieut., D. of Wellington's (W. Riding I9r3 Regt.)

Killed in action 14 Sept. I916

Neave, Sir T. L. H., Bart. Major, Gen. List (T.F. Res.) I 893 and R. Defence Corps. $m$.

WNesham, C. F. Major, H.A.C.

Died 24 April 1919 of illness contracted on active service

NewBERry, E. E. Corpl., R.E. (Signals)

I 892

WNewborough, Lord. Lieut., Denbigh Yeo., Durham 1893 L.I., and Welsh Gds.

Died I9 Fuly I9I6 of illness contracted on active service

WNewman, C. C. Capt., Hampshire Regt.; attd. Hampshire Yeo. (W.) M.C.

Killed in action 4 Sept. I918 
Newman, E. D. Major, Lincolnshire Yeo. and M.G.C. 1906 (W.)

Nicholson, M. 2nd Lieut., Army Cyclist Corps; Capt., 1907 R.F.A.; Lieut.-Col., S.O. I, R.A.F. M.B.E.

NutTing, A. R. S. Capt., Irish Gds. (W.) M.C.

1907

Oliver, L. G. Bt. Colonel, Middlesex Regt. and North- $\quad$ I877 umberland Fus.(T.F.) C.M.G. $M$.

Olivier, E. Capt., S. African Defence Force

O'Neal, T. W. B. Pte., The Queen's (R. W. Surrey

1907

Regt.); and Lieut., R. Defence Corps and Spec. List (Interpreter)

Ormrod, J. Capt., R. Welsh Fus.

I886

Osborn, Sir A. K. B., Bart. Capt., Bedfordshire Regt. I89I and Labour Corps

Owen, G. C. Lieut., R. Scots Fus.; Capt., Gen. List 1900

Page, C. G. Major, Border Regt. (W.) M.C.

1904

Page Croft, H. See Croft, H. P.

Page Croft, R. See Croft, R. P.

PAGET, G. N. Lieut., Norfolk Regt. (P.)

Paget Tomlinson, T. R. Major, Westmorland and 1894

Cumberland Yeo.(T.F. Res.) and Labour Corps. $m$.

Paget Tomlinson, W. G. Major, 7th Hussars. (W.) 1896

D.S.O. M 3. Officer, Order of the Star of Roumania

Parham, H. J. Capt., R.E.(T.F.) $M$.

PARKER, H. C. T. Capt., R.F.A.

I9I I

1892

PARKER, L. H. Capt., R.E.; empld. Ministry of Munitions 1909

WPARRY, D. G. DE C. Major, R.F.A.

Killed in action 5 April $\mathrm{rg} 8$

Parry, R. B. Lieut., Army Cyclist Corps and S. Wales 1912 Borderers. (W.)

Paterson, E. W. Capt., Berkshire Yeo. (W.)

APaYne, J. H. A. Lieut., S. African Force

Died 29 Fuly 1917 of malaria

WPEAKe, C. G. W. Capt., Lincolnshire Regt. $M$.

Killed in action at Neuve Chapelle 10 March 1915

Peake, G. H. Major, Sherwood Rangers (T.F. Res.)

[1914]

1907

* 1896

I910

I 880

WPearson, Hon. F. G. Warrant Officer, R.A.S.C.(M.T.) 1909 Killed in action 6 Sept. I9I4

Pease, E. H. Capt., Yorkshire Regt.(T.F.) and Spec. I89o List (School of Instruction). O.B.E. m.

Pease, R. 2nd Lieut., W. Yorks. Regt.(T.F.); Lieut., I9I6 Tank Corps 
Penny, A. O. V., T.D. Capt., Kelly College, Tavistock, 1879 O.T.C.

Penrose, J. D. Capt., R.A.S.C.(T.F.) $M$.

Perkin, A. L. D. Lieut., Middlesex Regt.; Capt., R. I9I I

Marines

Pershouse, F. S. Major, R.F.A.

Philipps, G. W. F. Capt., Welsh Gds. (W.)

Philipps, H. E. E. Capt., Spec. List (A.D.C.)

Phillips, N. McG. Lieut., London Regt. (Q.V.R.) and 1890

R.E. (Signals, T.F.)

Phillips, W. M. Capt., Lincolnshire Regt.(T.F.);Major, 1903

Loyal N. Lancs. Regt.(T.F.) (W.)

PhIPps, C. B. H. 2nd Lieut., King's (Shropshire L.I.); 1909 Lieut., Res. Regt. of Life Gds. (W.)

Pigott, G. G. C. 2nd Lieut., R. Berkshire Regt. and 1908 R.A.F.

Pilkington, F. C., D.s.o. Lieut.-Col., r 5 th Hussars. $M$ 2. 1890 सPilleau, A. L. Major, Hampshire Regt.

Killed in action in Gallipoli ro Aug. I9r 5

Pine-Coffin, J. E., D.s.o. Major, Loyal N. Lancs. Regt. $\quad 1887$ (R. of O.) and Sherwood Foresters (Notts. and Derby Regt.)

Potrs, F. A. Lieut., D. of Wellington's (W. Riding I90I Regt.); Capt., R.E. (Spec. Bde.)

WPouletT, Earl. Capt., R.H.A.(T.F.)

Died I I Fuly I918 of pneumonia following influenza

Pratt, G. C. Lieut., W. Yorks. Regt. (W.)

Prince, G. W. Lieut., R. Sussex Regt. (W.) M.C. I88I

Protheroe, A. H. Major, R.A.S.C. O.B.E. M.C. M 3. I90I

\&Quilter, J. A. C. Lieut.-Cdr., R.N.V.R. (Hood Bn., 1894 R.N.D.) $M$.

Killed in action in Gallipoli (12 May 1915 )

Quitzow, A. Lieut., R. Fusiliers

सRAM, G. E. Capt., N. Staffs. Regt.; attd. S. Staffs. Regt. 1900 Died 25 March 1916 of pneumonia

RAMSDEN, G. T. Lieut., R.F.A.(T.F.)

Ramsey, W. J. Capt., R.A.S.C.(T.F.) and King's African I903 Rifles

RAPHAEL, Sir H. H., Bart., M.P. Major, King's Royal 1877 Rifle Corps and Gen. List (A.P.M.)

Rawdon, C. H. Capt., King's Own (Yorkshire L.I.) (P.) 1900 
Rawlins, Rev. G. C. Lieut., R.N.V.R.

19I3

Rawnsley, G. T. 2nd Lieut., Rifle Brigade

1903

Redman, G. A. Capt., London Regt. (L.R.B., T.F. Res.) 1900

ReNDell, W. R. Capt., Cambridge Univ. O.T.C., attd. * I904 Gen. Staff, War Office

AReynard, C. F. P. Capt., E. Yorks. Regt.

Died 16 Fune I 918

WRHOdes Moorhouse, W. B. 2nd Lieut. (A.), R.F.C. M. 1908

Y. C. " For most conspicuous bravery on 26 April, I9I 5, in flying to Courtrai and dropping bombs on the railway line near that station. On starting the return journey he was mortally wounded, but succeeded in flying for 35 miles to his destination, at a very low altitude, and reported the successful accomplishment of his object. He has since died of his wounds."-Supplement to The London Gazette, 21 May I9I5.

Died 26 April I9I 5 of wounds receiced in astion

Richardson, C.W. Lieut.-Col., R.G.A.(R.of O.); Cmdt., I89I L. of C. O.B.E. M.

WRichardson, J. M. Hon. Lieut.-Col., R.G.A.(T.F.) and 1869 Gen. Staff (Agriculture)

Died 30 March 1918 of wounds received in action 21 March 1918

RichaRdSON, T. D. Lieut., R.A.S.C.

RIDEAL,E.K. Lieut., R.A.M.C.; Capt., R.E. M.B.E. $M$.

Riesle, W. B. Lieut., S. African Garrison Artillery

1905

I908

1905

RIx, A. H. Lieut., E. Riding of Yorkshire Yeo.; attd. I900 E. Yorks. Regt.

RoвB, Sir F. S., K.C.B., K.c.v.o. Major-Gen., Military $\quad 1876$

Secretary, War Office; Major-Gen. i/c Administration, E. Command. K.G.M.G. M.

RoBERTS, C. H. Lieut.-Cdr., R.N.A.S. D.S.G. French I912

Croix de Guerre

RoBerts, J. C. Lieut., S. Wales Borderers. (W.)

Robertson, H. G. Capt., Connaught Rangers

RoBerTson, W.P. Capt., Hertfordshire Yeo. and R.F.A.;

Staff Capt.

Rodney, Hon. J. H. B. Capt., Rifle Brigade; Capt. (A.), I9I I R.A.F.; Major, S.O. 2. (W 3.) M.C. $M$.

Rodocanachi, P. Sub-Lieut., R.N.A.S.

WRogerson, W. E. Capt. and Adjt., Durham L.I.

Died I 3 Nov. I9I4 of heart failure

WRomer, M. L. R. Capt., King's Royal Rifle Corps

Died 20 Sept. 1916 of wounds received in action

I9I3

1892

1898

1909

I89I

I913 
Roskill, W. G. Pte., R. Fusiliers (P.S. Bn.); 2nd Lieut., 1908 R.A.S.C.; Lieut., Gen. Staff (Intelligence)

Rossdale, S. J. Lieut., R.F.A.

Rotch, C. D. Capt., R.A.S.C.

\&Rowlandson, T.S. Capt., Yorkshire Regt.(T.F.) M.C. I90I $M$.

Killed in action 15 Sept. I9I6

RowlatT, J. F. C.P.O., R.N.V.R.

ARussell, J. 2nd Lieut., The Buffs (E. Kent Regt.)

Killed in action 9 Aug. I917

ARutTer, E. F. Major, E. Lancs. Regt. $M$.

Killed in action 13 May 1915

Salaman, R. N. Capt., R.A.M.C. $M$.

Sandeman, B. S. Capt., R.G.A.

SANDERS, H. R. Lieut., London Regt. (Cyclist Bn.)

SAurin, W. M. Major, W. Yorks. Regt.; R.T.O.

Officer, Order of Leopold (Belgium)

SAvorY, K. S. Major (A.), R.A.F. D.S.O. and Bar [1914] ScotT, B. G. A. Lieut.-Cdr., R.N.V.R.

Scott, Lord H. A. M. D. See Montagu Douglas Scott,

Lord H. A.

Scratton, E. W. H. B. Capt., R. ist Devon Yeo.; Major, 1902 R.A.O.C. O.B.E. $m$.

Seligman, G. A. Lieut., R.A.O.C.

«Sharp, J. S. Pte., R. Fusiliers (P. S. Bn.); Major, R. I9II

Berkshire Regt. $M$.

Killed in action 17 March 1917

Shaw, E. H. Capt., R.A.M.C. Chevalier, Ordre de la I9I3

Couronne (Belgium)

Shaw, S. Lieut., R.A.M.C. (Sanitary Service, T.F.) I9I I

*Shaw-Hellier, A. J. B. Lieut., S. Staffs. Regt.

Killed in action in Gallipoli 9 Aug. 1915

Sheppard, G. A. Capt., Worcestershire Regt.; empld. I910 O.C.B. (W 2.)

ShePpARD, Rev. H. R. L. C.F. 4th Class, R.A.C.D. I90I

Sherburn, J. C. Capt., E. Yorks. Regt. and Gen. List 1906 (A.D.C.)

Shillington, J. M. Lieut., R.N.V.R.

Shimwel $H$ Capt London Regt. (R Fus.) m.

SHIMWELL, H. Capt., London Regt. (R. Fus.) $m . \quad 1903$

ShImwell, O. Capt., London Regt. (R. Fus.) 1904

Shrager, E. H. Lieut., Middlesex Regt. (W.) M.C. 1912 Sillem, C. F. Hon. Lieut., Spec. List., empld. British 1885 Red Cross 
¿Simpson, G. B. Capt., York and Lancaster Regt. Diea $12 /$ Nov. 1915 of wounds received in action

1909

Simpson, G. H. Sub-Lieut., R.N.V.R.; Capt. (Ad.), 1912 R.A.F.

*Simpson, W. A. Trooper, Canadian Mtd. Rifles Killed in action 1 Dec. I9I 5

Sinclair, R. C. H. Lieut., R.F.A.; A.D.C.

Skene, Rev. R. E. C.F. 4th Class, R.A.C.D.

1904

1897

* I90I

Slaughter, A. Capt., Manchester Regt.; empld. P. of W. 1904 Camp

Sloley, R. Lieut., R.A.S.C.

SmIth, A. W. E. Lieut., R. Sussex Regt.(T.F.)

I9I 2

$\mathrm{r} 898$

SmIth, E. S. Lieut., Labour Corps

Smith, F. A. Pte., Black Watch

Smith, J. T. See Tilden SMITH, J.

Smith, M. V. Capt., Herefordshire Regt. and Labour 1900 Corps

Smith, R. Lieut., R.F.A. $M$.

Smith, W. F. Lieut., Cambridge Univ. O.T.C.

SMITH.

Smith-Sligo, R. W. M. G. J. Capt., Highland L.I.; I9I I attd. Egyptian Army

ASOAMES, W. N. Lieut., Cheshire Yeo.

Died I9 May 1916 of heart failure

Solomon, C. G. R. Lieut., S. African H.A.

SpenCER, L. D. Major, King's Own Scottish Borderers;

1907

Lieut.-Col., Spec. List (Military Governor). (W 2.)

$m$ 2. Medjidieh, 3 rd Class. Osmanieh, 4 th Class (Egypt)

SPICER, M. Colonel, R.A.F.; S.O. (Equipment). M. 1902

SPICER, N. 2nd Lieut., Bedfordshire Regt. 1899

सSPRINGFIEld, G.P. O. Capt., 2nd Dragoon Gds.(Queen's I89I

Bays)

Killed in action 12 Sept. I914

Spurrell, R. J. Lieut.-Col., R. Sussex Regt.; Cmdt., 1874 L. of C. $M$.

\#Statham, N. H. Lieut., E. Surrey Regt.(T.F.); attd. I912

Devon Regt.

Killed in action $3 \mathrm{Feb}$. I917

SteEle, A. R. Major, $4_{\text {th Hussars. (W.) }}$

Steele, C. M. Capt., R.G.A.(T.F.)

1895

1896

Stenham, B. B. Chief Yeoman, Mine Force, United 19ro States Navy

ESTERnBerg, E. A. J. 2nd Lieut., King's Own (R. Lan- 1908 caster Regt.)

Killed in action 16 Oct. 1916 
Steward, R. D. Capt. and Adjt., R.F.A. M.C. M 2. 1899 StewarT, F. C. Capt., R.A.O.C. $m$.

Stewart, F. D. L. 2nd Lieut., R.G.A.

$\begin{array}{ll}\text { STEWART, F. D. L. 2nd Lieut., R.G.A. } & 1914 \\ \text { StewarT, J. C. Major, Scottish Horse (T.F. Res.); Bri- } \quad 1876\end{array}$ gade Major

\#STIEBEL, C. Lieut., I.M.S.

Killed in action in Mesopotamia 3 Feb. 1917

I 894

Stringer, H. W. Pte., R. Fusiliers (Sportsman's Bn.) $188_{5}$

Stuart, C. M. Lieut., R.N.V.R.

Stuart, D. C. R. Lieut., Border Regt.; Capt., Spec. List 1905 (Courts-Martial Officer). (W.) $M$.

Stubber, R. H. Major, S. Irish Horse; attd. Life Gds. 1897 D.S.O.

Swann, A. Lieut., R.N.V.R. (Coastal Motor-boat Ser- I9I I vice). D.S.C.

Swann, L. H. 2nd Lieut., Suffolk Yeo.

Swann, Rev. S. E. C.F. 4th Class, R.A.C.D. (W.)

4SweEt, J. L. L. Pte., Canadian Hdrs.; 2nd Lieut., R. 1906 Scots Fus. $M$.

Killed in action near Givenchy 16 June 1915

SweEting, H.C. Capt., King's Royal Rifle Corps; empld. 1904

Ministry of National Service. (W.) O.B.E. m.

Syme, H. R. Lieut., R.A.S.C. (W.)

\#Symonds-Tayler, F. K. Capt., King's (Shropshire L.I.) I9I3 (W 2.)

Died 17 April 1917 of wounds received in action

Symonds-Tayler, R. H. Hon. Lieut.-Col., Hereford- 1886 shire Regt. (T.F. Res.)

Symington, R. E. Lieut., S. Lancs. Regt.; attd. T.M.B. [I9I4] (W.)

Tacon, D. G. T. Capt., R.F.A. $M$. 1907

TAYleur, W. Capt., Shropshire Yeo.(T.F. Res.); Lieut.- 1890 Col., Labour Corps. O.B.E. T.D. M 2 .

WTAYLOR, W. E. Lieut., R.A.S.C.

Drowned 2 Fune 19I7

Thomas, J. C. C. Major, N. Staffs. Regt.

Thomas, K. D. P. 2nd Lieut., R.E. (Signals) 1899

Thomas, L. D. C. 2nd Lieut., Worcestershire Regt.

4Thomas, O. C. Lieut., Grenadier Gds.; attd. Gds. M.G. 1907

Regt.

Killed in action 1 Dec. I917

Thомas, Rev. R. A. Capt., Cheshire Regt.(T.F. Res.); 1892 C.F. $3^{\text {rd Class, R.A.C.D. }}$ 
Thompson, A. C. Capt., Middlesex Regt.; attd. Oxford I9I4 and Bucks. L.I. (W 2.) M.C.

$\begin{array}{ll}\text { Thompson, H. C. S. Capt., S. Lancs. Regt. } & 1879 \\ \text { Thomson, G. L. Lieut.-Col. (A. and S.), R.A.F. D.S.C. } & 1906\end{array}$ D.F.C. $M 2$.

Thomson, J. Lieut., London Regt. (Rifles)

Thomson, J. G. O. Capt., Ayrshire Yeo.; A.D.C.

THornes R R Sub-Lieut R NV R (Armoured Car

Section); Capt. (A.), R.A.F. (W.) D.S.C. $M$.

Thornewill, C. C. Capt., Lincolnshire Yeo.and M.G.C. $\quad 1904$

ThorP, D. B. Lieut. (T.), R.A.F.

Thynne, L. W. Lieut., Rifle Brigade

TILDEN SMIth, J. 2nd Lieut., R. Fusiliers

TuL W. S. Capt, R. Sussex Regt.; empld O.C.B. (W.) 1914

TiLl, W. S. Capt., R. Sussex Regt., empld. O.C.B. (W.) 1912

Tingey, W. R. H. Capt., R.E. $M$.

TINLINE, G. C. M. 2nd Lieut., Cameron Hdrs.; Lieut., I9I3

Highland L.I.

ToleR-AyLWARD, H. J. Trooper, Res. Regt. of Cavalry I9I4

Toulmin, H. W. Lieut., Suffolk Yeo.

TRACY, G. C. Major, D. of Cornwall's L.I.; Lieut.-Col., 1895

Spec. List (Cmdt., School of Instruction). (W.) D.S.O. $M 2$.

ETRaILl, J. M. Major, Bedfordshire Regt.

Killed in action 30 Oct. I9I4

Traill, J. W. Major, S. Lancs. Regt. and Spec. List

Tredennick, J. N. E. Lieut., R. Warwickshire Regt. and

Gen. List, empld. Ministry of National Service. (W.)

ETrenchard, F. A. Lieut., R.F.A. (W.) $M$.

Killed in action 24 May r9 5

Tristram, U. H. Major, S. Notts. Hussars (T.F. Res.) 1892 and Remount Service; Lieut.-Col., Res. Regt. of Cavalry

Trollope, T. C. S. Pte., London Regt. (Artists Rifles); I9I2 and Lieut., Labour Corps

TrotTer, J. F. A. Major, R.F.A. O.B.E. $M$.

Troyte, G. J. Acland. See Acland Troyte, G. J.

1884

1875

I9II

1906

Trusted, H. H. Lieut., D. of Cornwall's L.I.; Staff Capt., 1909 E. Indies

Tudor Owen, F. H. G. Lieut., Rifle Brigade; Staff Capt., “ I904 S. Command. (W.) $m$.

Turrall, R. G. Capt., R.E. M 2.

Tylden-Wright, W. R. Capt., 3rd Hussars; Major, I899 D.A.A. and Q.M.G. D.S.O. $M_{3}$.

Tytler, W. T. F. See Fraser-Tytler, W. T. 
Usher, H. B. Capt., Middlesex Regt.; attd. E. Surrey 1912 Regt.; empld. War Office. (W.)

UsHer, T. Capt., Suffolk Regt. $M$.

UtLey, B. T. Lieut., Connaught Rangers. (W.)

VACHELl, F. T. Trooper, K. Edward's Horse; Major, 1908 R.F.A. M.C.

WVaulkhard, J. V. 2nd Lieut., Bedfordshire Regt. Killed in action 15 Sept. 1916

WaLKer, C. C. Capt., Argyll and Sutherland Hdrs. $\quad 1894$ WWalker, J. A. Capt., R. Welsh Fus.

Killed in action $19 \mathrm{Feb}$. 1916

WWALKER, O. R. Capt., Worcestershire Regt.; attd. R. 1902 Fusiliers

Killed in action in Gallipoli 4 fune 1915

WaLLER, Rev. W. H. Chaplain, R.N.

WALSH, A. St G. Capt. and Adjt., Manchester Regt. M. 1912

WALTER, C. E. H. L. Lieut., R.A.S.C.

WARD, H. L. 2nd Lieut., R.A.S.C.

WARD, J.S.M. and Lieut., Rangoon Bn. Inding 1900 Force

WARD, Hon. R. A. Capt., Spec. List. M $2 . \quad 1890$

WARE, B. G. Lieut., Spec. List 1899

WASBROUGH, H. C. S. Lieut., Oxford and Bucks. L.I. $\quad 1897$

WATERHOUSE, C. Lieut., Life Gds.; Capt., Tank Corps. I9I3 (W.) M.C.

Watson, A. B. 2nd Lieut., Scottish Horse; Lieut., Black 1890 Watch

WWatson, A. T. Capt., Remount Service; D.A.D. Re- * ${ }_{1} 890$ mounts; Major, King's Royal Rifle Corps. (W.)

Died 5 Aug. 1917 of wounds received in action

Watson, H. N. Lieut.-Cdr., R.N.V.R.

Watson, T. H. Capt., R.A.S.C.(M.T.) 1900

WAUCHOPE, D. A., D.S.O., T.D. Major, Lothians and 1889 Border Horse; attd. Staffordshire Yeo.

WEBSTER, R. B. Capt., Northumberland Fus.(T.F.) 1908

WeBsTER, S. W. Lieut.-Col., 6th Dragoon Gds. (Cara- 1893 biniers). $m$.

WEBSTER, W. G. Trooper, K. Edward's Horse; Lieut., 1909 R.F.A.

WWelsby, S. W. H. Pte., R. Fusiliers (P. S. Bn.); Lieut., I910 Cheshire Regt. (W.)

Killed in action 30 April 1917 
Wessels, C. H. Staff Lieut., S. African Force

WWharton, F. H. Lieut., Loyal N. Lancs. Regt. Killed in action 25 Sept. I9I 5

Whatman, A. D. Major, The Buffs (E. Kent Regt., 1892 T.F.)

WheEleR, M. Capt., R. Berkshire Regt.(T.F.)

Whiten T.W. 1895

WHIFFEN, T. W. Capt., Recruiting Staff, London District

WhITAKER, J. L. Lieut. (A.), R.A.F.

White, R. J. Capt. and Adjt., R. Warwickshire Regt.; A.P.M. M. Chevalier, Military Order of Aris (Portugal)

WhiteHEAD, J., T.D. Major, Lancs. Fus.(T.F.) $M . \quad 1892$ WWhitfeld, N. B. Lieut., R. North Devon Yeo.; attd. 1909 Devon Regt.; Lieut. (A.), R.A.F.

Killed in flying accident $7 \mathcal{F u l y}_{\mathrm{1} 9 \mathrm{1} 8}$

WWickhaM, W. J. Capt., Scots Gds.

Killed in action 31 Oct. r9I4

Wilkinson, H. E. T. 2nd Lieut., Yorkshire Hussars $\quad 1893$

Williams, F. J. Capt., R. Inniskilling Fus. (W.) I890

Wills-SandFORD, T. G. Lieut., Remount Service 1899

Wilson, E. R. Capt., R. Irish Fus.; attd. Tank Corps; 1907 empld. War Office. (W 2.)

Wilson, H. M. Capt., N. Zealand Med. Corps $\quad 1895$

Wilson, R. K. 2nd Lieut., R.F.A. (W 2.)

WILsON-TodD, J. H. Lieut., Scots Gds. I9I I

WingField, C. R. B. Major, King's (Shropshire L.I.) $\quad$ I894

WingField DigbY, F. J. B. Major, Dorset Yeo. D.S.O. 1905 $M$.

WWinterbotTom, D. D. Capt., Manchester Regt.(T.F.) I9io Killed in action 7 Aug. I9I 5

Winterbottom, O. D. Capt., Cameron Hdrs. and R.E. 1909 $M$.

WoDEHOUSE, Lord. Lieut., 16th Lancers; A.D.C. (W.) 1902 M.C. $M 2$.

Wodehouse, Hon. P. Lieut., Spec. List

Woop, E. B. Capt., R.A.S.C.; Inspector of M.T. $\quad 1905$

Wood, J. C. Capt., Rifle Brigade

WoodHEAD, Sir G. S., v.D. Colonel, R.A.M.C. (Sani- ${ }^{1896}$ tary Service, T.F.) K.B.E. O.B.E. Brevet Colonel. $m 2$.

Woodhouse, G. H. Major, E. Riding of Yorkshire Yeo.; 1908 Staff Capt.

WoodrufF, J. S. Pte., Australian Force

Woolf, H. G. Major, S. Staffs. Regt. $m$. 
Wright, A. Capt., Cheshire Regt. and R. Welsh Fus. $\quad 1879$ Wright, H. T. Lieut., Scots Gds.; Staff Capt., London 1896 District

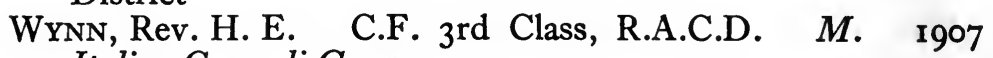
Italian Croce di Guerra

YARROW, K. G. 2nd Lieut., Black Watch (T.F.); Lieut., 1905 R.A.S.C.

Young-HerRies, W. D. Lieut.-Col., King's Own Scottish 1885 Borderers. $m$. 


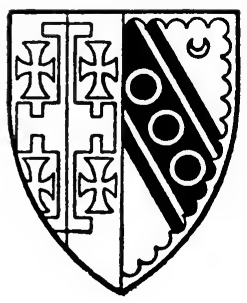

\section{SELWYN COLLEGE}

WAdam, A. G. A. Capt., The Buffs (E. Kent Regt.) M. 1904 Killed in action in Mesopotamia 21 Fan. 1916

Adams, J. K. Lieut., Devon Regt.(T.F.); attd. Rifle igi I Brigade

Adams, Rev. W. S. C.F. $4^{\text {th }}$ Class, R.A.C.D.

1896

AAdamson, H. B. 2nd Lieut., Suffolk Regt.; Capt., W. I90 I Yorks. Regt.

Died 30 Oct. I91 6 of wounds received in action 22 Oct. I916

Alderson, J. H. Trooper, United Provinces Horse, I893 Indian Defence Force

EAlderson, R. Capt., Lancs. Fus. (W.) M.C. M.

Died 25 March 1918 of wounds received in action

1913

AAlderton, C. F. Pte., H.A.C.; 2nd Lieut., Manchester 1908

Regt.

Killed in action 7 Fuly 1916

«Alston, G. K. 2nd Lieut., Suffolk Regt.(T.F.)

Killed in action in Gallipoli 3 I Aug. I9I 5

Archer, Rev. G. D. C.F. $4^{\text {th Class, R.A.C.D. }}$

1912

Ancher, R. G. D. C.F. 4th, 1907

सAverns, R. F. J. Pte., Queen's Own (R. W. Kent Regt.) I9I4

Died in German hands Oct. I918

Bacon, J.N.H. Sergt., The Queen's (R. W. Surrey Regt.) I9I4 BARKER, F. Sapper, R.E. (Signals)

BARKer, Rev. T.W. C.F. $4^{\text {th }}$ Class, R.A.C.D.

1912

BARNES, F. Capt. and Adjt., Rifle Brigade. (W.) m. I905

Barnicoat, Rev. G. H. C.F. 4th Class, R.A.C.D. 1906

BARR, L. B. Lieut., R.G.A.(T.F.); empld. Ministry of I9I4

Labour. (W.)

Barrett, Rev. C. B. G. C.F. $4^{\text {th }}$ Class, R.A.C.D.

1905 
Baxandall, A. Pte., R.A.M.C.

Beament, W. O. 2nd Lieut., Dorchester School O.T.C.; 1912 attd. Highland L.I.

BEAN, J. W. B. Major, Australian A.M.C. (W.)

ZBeanland, J. W. Lieut., R. Welsh Fus.

Killed in action in Gallipoli 28 Aug. 1915

Beaven, V. Pte., London Regt.

Bell, J. H. Pte., E. Surrey Regt.; Capt. and Adjt., I 889 R. Defence Corps

Bell, L. B. Pte., H.A.C.; Capt., London Regt. (Fins- 1913 bury Rifles)

Bendall, F. W. D. Lieut.-Col., London Regt. (R. Fus.) and Middlesex Regt. (W.) C.M.G. M2.

Bengough, G. D. Capt., R.G.A.(T.F.)

BenNetT, T. C. 2nd Lieut., Somerset L.I.; Lieut., I9I I M.G.C. (W.) $M$.

Bethune, Rev. F.P. Capt., Australian M.G.C. M.C. Ig0I

Bethune, Rev. J. W. C.F. $4^{\text {th }}$ Class, R.A.C.D.

Betton, H. R. B. 2nd Lieut., Norfolk Regt. (W.)

Bilsborough, J. H. 2nd Lieut., Exeter School O.T.C.

Birks, C. T. E. Pte., E. Surrey Regt.; 2nd Lieut., Northamptonshire Regt.

Blackwall, J. E., T.D. Lieut.-Col., Sherwood Foresters 1892

(Notts. and Derby Regt., T.F.) D.S.O. M.

Chevalier, Legion of Honour (France)

WBlakeston, B. M. 2nd Lieut., I.A.R.O., attd. Infy.

Killed in action 25 March 1917

WBlaxland, J. B. Capt., S. Wales Borderers

Killed in action 24 Fan. I917

Bleasdell, J. T. Capt., R.A.M.C. $M 2$.

Blundell, Rev. E. K. C.F. $4^{\text {th }}$ Class, R.A.C.D.

Blyth, M. M. Pte., R. Fusiliers (P. S. Bn.)

1907

1915

Bode, Rev. J. E. V. Chaplain, R.N.

\#Borton, C. E. Major, Malay States Guides. $M$.

Killed in action 2 Aug. 1917

\&Botт, W. E. Capt., R. Fusiliers

Killed in action 18 Sept. I91 8

I910

1910

1908

1905

1894

1907

1898

1912

Bourdillon, Rev. G. L. Chaplain, R.N.

ABourne, A. S. Pte., R. Fusiliers (P.S. Bn.); 2nd Lieut., I9IO S. Staffs. Regt. (W.)

Died 25 April I9I7 of wounds received in action

WBourne, C. 2nd Lieut., The Queen's (R. W. Surrey 1907

Regt.)

Died I I April I9I7 of wounds received in action 
BoycotT, A. G. Lieut., R.A.S.C.; empld. War Office 1907 Boyle, Rev. G. E. C.F. 4th Class, R.A.C.D. 1903 BREE, Rev. H. R. S. C.F. 4th Class, R.A.C.D. I904 BRIDGeS, L. W. 2nd Lieut., Queen's Own (R.W. Kent 1909 Regt.)

BrISCOE, J. R. Capt., R.A.M.C.

BRISCOE, W. T. Capt., R.A.M.C.(T.F.)

BRown, G. Lieut., R.G.A. (W.)

Brown, R. C. Capt., R.A.M.C.

I 896

1897

1895

1884

BRown, W. R. R. Lieut., E. Yorks. Regt.(T.F.) (W.) I9Io

BRownING, H. Lieut., Hampshire Regt. and R.E. I913

(Signals). M.B.E. M 2. French Croix de Guerre

Browning, Rev. W. F. Pte., R.A.M.C.

BunN, F. A. Pte., R.A.M.C.

Burfield, S. T. Lieut., King's (Liverpool Regt.) (W.) I908

Burrowes, R. V. Capt., Suffolk Regt. (W 3.) M.C. 1912

Butler, E. H. Lieut., Wiltshire Regt. (W.) I9I I

Butler, F. H. C. Lieut., Hampshire Regt.; Capt., Gen. I9I3 Staff (A.P.M.) $M$.

Calderbank, Rev. J. C.F. $4^{\text {th }}$ Class, R.A.C.D.

Cameron, Rev. C. C.F. $4^{\text {th }}$ Class, R.A.C.D.

Candler, G. Capt., R.A.M.C.(T.F.)

ACARR, B. A. 2nd Lieut., R.G.A. M.

Killed in action 25 Fuly I9I7

Carver, Rev. B. N. Chaplain, R.N.

Castlehow, Rev. J. A. S. C.F. 4th Class, R.A.C.D. 1909

ACHARLEwOod, W. H. Capt., Northumberland Fus.

Died 22 fuly 1916 of wounds received in action

Charlton, G. D. Capt., N. Staffs. Regt.(T.F.)

Chew, Rev. J. E. C.F. $4^{\text {th }}$ Class, R.A.C.D.

Chubb, R. N. Capt., Army Cyclist Corps. $M$.

I910

1906

1888

1898

Crvil, H. G. V. Major, Wellington College O.T.C. , Igo6

Clark-Kennedy, Rev. A. C. C.F. 4th Class, R.A.C.D. 1899

Clarkson, Rev. E. R. T. Major, R.A.M.C.

Clemo, F. A. Capt. and Adjt., D. of Cornwall's L.I.; I9ro Staff Capt. M.C. $M 2$.

Close, A. V. Pte., R. Fusiliers (P. S. Bn.); Lieut., Tank I9I I Corps. (W 2.)

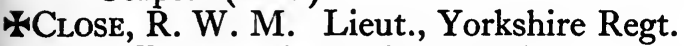

Killed in action 27 May I9I8

Collier, Rev. G. H. Chaplain, R.N.; attd. R.A.F. m. 1907

Collis, A. J. Lieut.-Col., R.A.M.C.(T.F.)

Comyn, K. Capt., I.M.S. (W.) 
Conran, Rev. M. W. T. C.F. $4^{\text {th }}$ Class, R.A.C.D. ${ }^{*}{ }^{2} 885$ M.C. $M$.

Copeman, C. E. F., Lieut.-Col., Cambridgeshire Regt. $\quad$ I886 and Northamptonshire Regt.(T.F.) C.M.G. T.D. $m 3$.

Coupe, Rev. T. O. 2nd Lieut., R.A.S.C.

Cowham, A. G. Pte., London Regt.; 2nd Lieut., W. I9I4 Yorks. Regt. (W.)

Crawley, Rev. J. L. C.F. 3rd Class, R.A.C.D. (W.) r9ro O.B.E. $M$.

Crocombe, F. R. Lieut., 76th Punjabis, Indian Army I9I I Crosthwaite, W. H. Capt., Spec. List (Map Section). 1902 O.B.E. $M 2$.

Currey, R. G. T. Major, Leinster Regt.; Lieut.-Col., 1894 Lancs. Fus. (W.)

Danvers, Rev. G. C. C.F. $4^{\text {th }}$ Class, R.A.C.D. (W.) 1900 M.C.

WDaubeney, G. R. Lieut., Queen's Own (R. W. Kent I9I3 Regt.)

Killed in action near Ypres 23 April I9I 5

Davies, C. 2nd Lieut., Essex Regt.

*Davies, G. B. Capt., Essex Regt.

Killed in action 26 Sept. I9I 5

Davis, E. B. Lieut., W. Yorks. Regt.

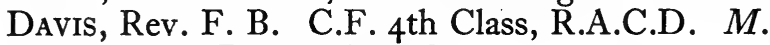

Dawson, S. Pte., R.A.M.C.

Dealtry, F. H. B. Lieut., empld. with Cadet unit DE Chaumont, Rev. T. S. B. F. C.F. 4th Class, R.A.C.D. DE Coetlogon, Rev. C. E. C. Lieut., Spec. List (Re- 1885 cruiting Staff)

DE Mowbray, L. St J. Capt., R. Berkshire Regt.

DE RIDDER, L. E. Lieut., Gloucestershire Regt.(T.F.)

Devereux, N. Capt., R.A.M.C.

Devereux, W. C Major, R.A.M.C.

[19I4]

1914 1885 1883

1912 attd. R. Irish Rifles

Died ro May $19 \mathrm{I} 5$ of wounds received in action at Ypres Dickenson, Rev. L. G. C.F. $3^{\text {rd }}$ Class, R.A.C.D. D.S.O. $m$.

Douglas, H. A. Capt., R.A.M.C.

Douglas, J. Lieut., Middlesex Regt.

Dowell LeE, R. W. Capt., Dorset Regt.; empld. O.C.B. (W.) M2. Order of the White Eagle, $5_{\text {th Class (Serbia) }}$

1908 I9I2

[1914] 1907 1913 1897 1903 .

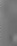


Downes, G. S. J. Lieut., W. Yorks. Regt.; Major, M.G.C. 1914 (W.) M.C. M.

WDUCKWORTH,W.C. 2nd Lieut., Welsh Regt.; attd.King's 1909 (Shropshire L.I.)

Killed in action 8 Oct. r918

WDUNWELl, F. L. 2nd Lieut., R. Fusiliers

Killed in action 6 fan. 1916

Dutron, P. E. Pte., Grenadier Gds.

EDuvall, Rev. J. R. C.F. $4^{\text {th }}$ Class, R.A.C.D. $M$.

Died 6 Oct. I9I7 of wounds received in action in Macedonia

Dyer, Rev. B. S. Junior Chaplain, Indian Army

1907

1894

1908

सEDmunds, C. V. Capt. and Adjt., Essex Regt.(T.F.)

Killed in action 26 March 1917

1898

1902

EDWARDS, E. D. Lieut., Lancs. Fus.; Hon. Lieut. (Ad.), I9I3 R.A.F. (W.)

Edwards, H. J., C.B., T.D. Hon. Colonel, Unattd. List, ${ }^{*} 1895$

T.F.; cmdg. O.C.B.; C.B.E. O.B.E. $m 3$.

EHLVERS, F. W. V. Lieut., R.F.A.

Euliotr, Rev. J. S. C.F. 4th Class, R A.C.D.

ELLIOT, Rev. J. S. C.F. 4th Class, R.A.C.D. I90I

Eltis, J. 2nd Lieut., E. Yorks. Regt. 1909

ELSEY, J. W. R.N.

ETHERIDGe, F. Major, 7 th Rajputs, Indian Army; $\quad 1899$

D.A.A.G. D.S.O. $M 3$.

Evans, A. G. Capt., Devon Regt.

r89o

FABER, V. V. Lieut., R.F.A.

FAGAN, Rev. H. W. F. Senior Chaplain, Indian Army

1913

1890

FISHER, C. E. (formerly FISCHER). Sergt.-Major, R.A.F. $\quad$ I9I2

(W 2.)

Fitzmaurice, Rev. G. L. C.F. $4^{\text {th }}$ Class, R.A.C.D.

FitzMAurice, W. H. Lieut., Gloucestershire Regt. (W) 1895

Fr

Fletcher, Rev. B. F. C.F. 4th Class, R.A.C.D. I885

FFletcher, M. Pte., R. Fusiliers (P. S. Bn.); Capt., R. 1904

Munster Fus. M.C.

Killed in action 9 Sept. I916

Fletcher, P. Pte., Canadian A.M.C.

Fletcher, W. T. Capt., 97th Deccan Infy., Indian *1904 Army. (P.)

Forbes, Rev. A. G. C.F. $4^{\text {th }}$ Class, R.A.C.D.

Ford, Rev. F. T. C.F. $4^{\text {th }}$ Class, R.A.C.D. $M$.

WFord, F. W. Capt., Cambridgeshire Regt. M.C. $M$ Killed in action 26 Sept. I917 
Foster, Rev. D. B. L. C.F. $4^{\text {th }}$ Class, R.A.C.D.

Fowler, H. R. R. Major, R.A.M.C.(T.F.)

Francis, E. C. Lieut. and Adjt., Norfolk Regt.

Frend, E. C. Pte., Queen's Own (R.W. Kent Regt.)

WFurze, A. Capt. and Adjt., King's Own (Yorkshire L.I.) I9I I Killed in action 16 Sept. 1916

Gale, A. J. V. Corpl., R.E. (Spec. Bde.); Cadet, R.G.A. I9I3

Gall, A. Pte., London Regt. (Artists Rifles)

Gardner, Rev. J. L. C.F. 4th Class, R.A.C.D.

Garrett, A. H. Lieut., R.E. $M$.

GaWNE, H. D. 2nd Lieut., Worcestershire Regt.

सGedge, Rev. B. J. C.F. $4^{\text {th }}$ Class, R.A.C.D. $M$.

Died 25 April r917 of wounds received in action

*Gedge, P. Lieut., Suffolk Regt.

Killed in action 13 Oct. 1915

\&Gibson, P. C. F. Capt., R. Fusiliers

Killed in action 10 April 1917

Gibson, T. B. Lieut., Calcutta Bn., Indian Defence Force

Gidney, F. Capt., Rifle Brigade; attd. R.E. (Signals); G.S.O. 3.

Gilbert, G. G. T. 2nd Lieut., Devon Regt.

Gilroy, P. K. Capt., I.M.S. M.C. M 2.

1909

1899

1913

I910

1901

1909

1898

Girling, E. A. Capt., Hampshire Regt. (W.) M.C.

*Goatcher, F. Lieut., Suffolk Regt. (W.)

Died 3 I Oct. I9I 7 of wounds received in action 20 Oct. 1917

Golding, R. H. C. 2nd Lieut., R.G.A.

Goldsmith, Rev. S. W. C.F. 4th Class, R.A.C.D.

Gosling, W. R. Major, S. Wales Borderers and Welsh Regt. O.B.E. $M 2$.

Grant, D. P. Lieut., King's Own (Yorkshire L.I., T.F.); 1914 Capt. and Adjt., York and Lancaster Regt.(T.F.) (W.) M.C.

Gray, Rev. F. H. A. Major, King's Own Scottish Bor- 1896 derers and R. Scots Fus. M.C.

\#Gregson, A. H. 2nd Lieut., Queen's Own (R. W. Kent [1914] Regt.)

Killed in action 19 April r917

WHailstone, G. R. Capt., R. Welsh Fus.(T.F.)

1912

Killed in action in Palestine 6 Nov. I9I7

Hall, A. F. W. Lieut., Essex Regt. and Nigeria Regt., 1914 W. African Frontier Force 
Hall, C. S. Lieut., Dorset Regt. (W.)

Hammond, Rev. J. V. C.F. $4^{\text {th }}$ Class, R.A.C.D.

1904

1889

Hanson, Rev. E. C. C.F. $4^{\text {th }}$ Class, R.A.C.D.

HaRding, Rev. C. R. C.F. $4^{\text {th }}$ Class, R.A.C.D.

1912

1889

Hardy, T. L. Capt., R.A.M.C. M.

Hargreaves, C. R. Pte., R. Fusiliers; 2nd Lieut., King's I9I 5

Own (R. Lancaster Regt.)

HarPER, Rev. W. E. C.F. 4th Class, R.A.C.D.

Harris, J. B. Capt., S. Wales Borderers. M.C. M. I9Io

Hatten, Rev. J. C. LE P. C.F. 4th Class, R.A.C.D. I896

WHEaley, R.E.H. Lieut., Queen's Own (R.W. Kent Regt.) 1904

Killed in action on the Somme 23 fuly ror6

HHeaton-Ellis, C. E. R. Lieut., King's Own (Yorkshire 1912

L.I.)

Killed in action 19 March rgr6

Hedges, Rev. P. D. C.F. 4th Class, R.A.C.D.

Hemsworth, N. E. C. 2nd Lieut., Norfolk Regt.; attd.

Worcestershire Regt.; Lieut., 29th Punjabis, Indian Army

Hendrie, H. A. Capt., Manchester Regt.; attd. T.M.B. I9ro (P.)

HensLow, T. G. W. Lieut., Argyll and Sutherland Hdrs. $\quad$ I897 HILl, W. A. Major, Indian Army

Hodges, Rev. R. A. C.F. 4th Class, R.A.C.D.

Hodgson, Rev. A. D. C.F. 4th Class, R.A.C.D.

1886

I9II

1908

Hogben, E. O'N. 2nd Lieut., N. Staffs. Regt.; Capt., I9I2 Army Cyclist Corps. (W 2.) M.C.

Holmes, Rev. A. Lieut., Sherwood Foresters (Notts. I89r and Derby Regt.)

Honeyball, F. R. 2nd Lieut., R. Fusiliers; attd. London roro Regt.; Capt., 124th Baluchistan Infy., Indian Army. (W.)

HHood, J. W. Lieut., R.G.A. $M 2$.

Died at Valenciennes 15 Nov. I918 of influenza

Horne, Rev. C. W. E. Chaplain, Canadian Force

Houghton, Rev. J. C. C.F. 4th Class, R.A.C.D.

HowARD, W. E. W. Major, R.A.S.C.

Howlett, Rev. C. E. C.F. $4^{\text {th }}$ Class, R.A.C.D.

HHubble, H. R. 2nd Lieut., King's (Liverpool Regt.)

Killed in action 20 May 1917

Huck, J. Major, Border Regt.(T.F.) O.B.E. M 2.

Hudson, Rev. R. C.F. 2nd Class, R.A.C.D.(T.F.)

wHudson, T. H. Capt., R. Berkshire Regt.

Killed in action 13 Oct. 191 5

1909

1896

r898

1906

1907

I9I I

I9OI

*I 889

1908 
Hughes, B. Major, R.A.M.C.(T.F.) D.S.O. $\quad M$ 2. $\quad 1898$ Order of St Sava, $4^{\text {th Class (Serbia). }}$

Hughes, Rev. S. J. C.F. $4^{\text {th }}$ Class, R.A.C.D. 1903

Hunter, L. A. W. Lieut., Seaforth Hdrs.

HuRLy, M. R. Lieut.-Col., Indian Army

Hybart, Rev. A. J. F. Pte., R. Welsh Fus. 1905

AHyde, J. C. Pte., London Regt. (London Scottish); [I9I4] 2nd Lieut., Sherwood Foresters (Notts. and Derby Regt.)

Killed in action I fuly r916

ICELy, Rev. F. Chaplain, R.N.

Ingham, W. Pte., R.A.S.C. (British E. Africa) and Tank Igo8 Corps

सIRving, T. H. 2nd Lieut., King's (Liverpool Regt.)

Killed in action I9 Aug. I916

IRving, W. R. Lieut., King's (Liverpool Regt.) (W.) I9II

IRwIN, H. M. Lieut., Nigeria Regt., W. African Frontier 1904 Force

JAGG, Rev. V. T. S. C.F. 4th Class, R.A.C.D. (W.) 1906

JAMES, H. M. 2nd Lieut., R.F.A.(T.F.)

1914

JEFFERY, E. J. B. Lieut., Devon Regt.

I9II

WJervis, A. C. 2nd Lieut., King's (Liverpool Regt.); 1906

Capt., King's African Rifles

Killed in action in Portuguese E. Africa 3 Fuly 1918

Jervis, Rev. E. O. C.F. 2nd Class, R.A.C.D. $M$.

Jesse, W. Lieut., I.A.R.O., attd. S. and T. Corps

Jeudwine, W. W. Major, I.M.S. C.M.G. M.

Jobling, J. S. Lieut., R. Fusiliers; empld. Admiralty

Joels, W. A. Lieut., E. Surrey Regt. $M$.

Johnson, J. B. Lieut., Uppingham School O.T.C.

JONES, J. D. Capt., R.A.M.C. $M$.

Jones, W. H. S. 2nd Lieut., Perse School O.T.C.

Jones-Bateman, Ven. W. Chaplain, Canadian Force

Joscelyne, C. H. 2nd Lieut., Leicestershire Regt.

1892

1888

1895

[I9I4]

I9I4

1909

1906

I894

I 883

I910

Keatch, F. H. Lieut., R.F.A.; A.D.C.; Staff Capt. I9I3 (W.) M.C. Belgian Croix de Guerre.

KENNEDY, D.D. A.B., R.N.V.R. (Anti-Aircraft); Lieut., I9I3 E. Surrey Regt.

Ker, R. A. Lieut., Malvern College O.T.C.

WKesteven Balshaw, N. Capt., King's Royal Rifle Corps I9I I Killed in action 13 April 1918 
Kettlewell, Rev. H. H. C.F. 4th Class, R.A.C.D.

KetTlewell,L. Major, Wiltshire Regt. (W.) D.S.O. M. I9I2

Wewley, W. C. Pte., Canadian Infy.

Killed in action at St Eloi 13 April 1916

KeYworth, W. D. Capt., I.M.S. $M$.

KING, P.E. Pte., R.A.S.C.; Lieut., R.F.A.; attd. R.G.A.

KIRBY, Rev. J. B. C.F. 4th Class, R.A.C.D.

KirTland, J. Lieut., R.F.A.; Capt., Spec. List (School of Instruction). (W.)

LABEY, T. H. 2nd Lieut., R.G.A.

WLangdon, Rev. C. C.F. $4^{\text {th }}$ Class, R.A.C.D.

Killed in action $3 \mathrm{I}$ Oct. I9I7

Langdon, Rev. C. G. C.F. 4th Class, R.A.C.D. $\quad 1895$

HLANGDON, L. Lieut., Hampshire Regt.

Died I4 March I916 of wounds received in action

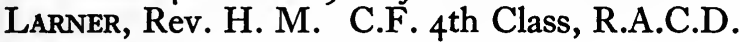

WLAwrence, T. E. Pte., R.A.S.C.

Died 6 Nov. I9I 6 of fever

Lawson, Rev. W. R. C.F. 4th Class, R.A.C.D. M. 1902

LewarNe, Rev. F. T. C.F. $4^{\text {th }}$ Class, R.A.C.D.

Lightburne, Rev. H. R. H. Chaplain, R.N.

ALine, E. A. T. 2nd Lieut., R.A.S.C.

Died in Macedonia 16 Dec. I916

LISTER, J. B. 2nd Lieut., E. Yorks. Regt.

LitTle, Rev. C.S. C.F. $4^{\text {th }}$ Class, R.A.C.D.

1912

I9OI

1895
I 893

* 1894

I9I4

Lound, Rev. R.S. C.F. $4^{\text {th }}$ Class, R.A.C.D.

Lowndes, Rev. J. C.F. 4th Class, R.A.C.D.

ELUCAS, A. 2nd Lieut., K. Edward's Horse. (W.)

Killed in the Irish rebellion 28 April 1916

Lumlex, D. O. Capt., Wiltshire Regt. and Gen. List I9I3

(Recruiting Staff). (W.) O.B.E. M.B.E. m.

Lush, E. J. Capt., King's (Liverpool Regt.) 1907

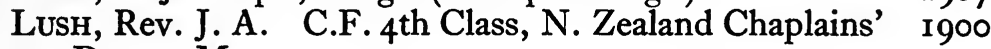

Dept. $M$.

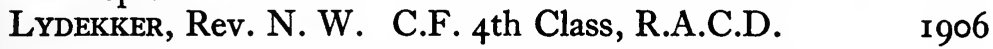

McCall, T.H. Capt., W. Yorks. Regt.; empld. Ministry of Labour. (W 2.)

WMcColl, W. L. Sergt., London Regt.; 2nd Lieut., I9I2 Northamptonshire Regt.

Died 18 Fuly 1916 of wounds received in action

WMcKIEver, V. C. 2nd Lieut., Manchester Regt.

Died I8 May I9I 5 of wounds received in action 
\#MAckworth, F. J. A. Major, R.F.A.; Brigade Major $\quad 1895$ Killed in action I Nov. I9I4

Macleod, Rev. W. A. C.F. 4th Class, R.A.C.D. $\quad$ I888

McMichael, J. F. Capt., Indian Army 1905

McMullan, H. Lieut., Lancs. Fus.; Capt., King's I9I3 (Liverpool Regt.) (W.)

MacPherson, C. E. H. C. Lieut., R. Dublin Fus.; 1909 Major (A.), R.A.F.

MacSwiney, Rev. A. J. E. Chaplain, R.N.

Madge, R.E. Capt., Lincolnshire Regt. $M$.

\#Maitland, H. M. Capt., Spec. List(Intelligence). M.C. 1907 $M 2$.

Died ro Nov. 1918 of pneumonia

Malden, Rev. R. H. Chaplain, R.N.

Maples, A. C. Capt., R. Sussex Regt.; Major, Cheshire $\quad 1897$ Regt. $\boldsymbol{m} 2$.

Maples, E. W. Major, R. Welsh Fus. and Gen. List 1889 (Staff Capt.) O.B.E. m.

Marriotr, A. E. C. 2nd Lieut., Sherwood Foresters I9I4 (Notts. and Derby Regt.)

Marsh, Rev. F. S. C.F. 4th Class, R.A.C.D.

Marshall, G. J. P. 2nd Lieut., W. Yorks. Regt.

Martin, F. J. 2nd Lieut., Leicestershire Regt.; Capt. I9Io (A.), R.A.F.

Masters, P. G. Pte., R.A S.C.(T.F.); Corpl., R.E.; 1904 2nd Lieut., London Regt. (R. Fus.)

WMatrhews, N. A. 2nd Lieut., London Regt. (Finsbury I9I I Rifles)

Killed in action 15 Sept. 1916

May, C. A. C. Capt., Cheshire Regt.; Brigade Major. I9I I (W 2.) M.C. M. Greek Military Cross

Meade, Rev. L. G. C.F. 4th Class, R.A.C.D.

Mellersh, E. L. Capt. and Adjt., R.G.A. $M$.

Melville, Rev. W. G. C.F. 2nd Class, R.A.C.D. (T.F.)

Mercer, Rev. G. H. Cadet, O.C.B.

MeYler, Rev. E. M. Junior Chaplain, Indian Army

Miller, H. C. Gnr., R.F.A.; Cadet, O.C.B. (W.)

Milles, G. H. Pte., S. African Force.

1902

1909

I 885

I910

1895

I9I3

1896

1905

Killed in action at Hooge 30 fuly I9I 5

Mitchell, J. C. Sqdn. Sergt.-Major, R. North Devon IgI I Yeo.; Lieut., Devon Regt. M.C.

Montague, W. J. Lieut., R.G.A. 
Montgomery, D. S. Capt., British Columbia Regt., 1905 Canadian Force; Staff Capt.

Montgomery, J. C. 2nd Lieut., Cameronians (Scottish rgr4 Rifles).

WMONTGOMERy, N. Lieut., British Columbia Regt., Cana- 1905 dian Force

Killed in action 21 Aug. r917

Moore, Rev. C. A. G. 2nd Lieut., R.A.S.C.

Moore, G. G. 2nd Lieut., R.F.A. 1907

MOORE, Rev. J. W. B. Chaplain, R.N. $\quad 1905$

MORRISON, H. H. Lieut., R.N.V.R.

Motт, C. C. Major, Spec. List $\quad 1884$

सMurray, R. H. Capt., Yorkshire Regt. $M$. Killed in action 7 Fuly r9r6

Nash, Rev. W. W. H. C.F. $4^{\text {th }}$ Class, R.A.C.D.

Newcombe, W. L. Major, Worcestershire Regt.; Brigade 1896 Major

Newman, J. C. F. Lieut., R. Sussex Regt. (W 2.)

NewPort, Rev. H. C.F. $4^{\text {th }}$ Class, R.A.C.D. (W.)

Nicholson, H. H. Pte., R. Fusiliers (P. S. Bn.); Capt., Durham L.I. (W 2.)

Norris, Rev. E. W. M. C.F. ist Class, R.A.C.D.

Norton, L. M. Capt., R.G.A.(T.F.)

Nosworthy, J. L. Lieut., R.F.A.(T.F.)

I9I3

1908

I9I I

1887

1906

I912

Ogle, K. W. S. 2nd Lieut., King's Own (R. Lancaster [1914] Regt.)

Oliver, L. W. Lieut., R.A.M.C.

ORME, B. C. Lieut., King's Own (Yorkshire L.I.) and I9I I M.G.C. (W 2.)

सORPEN,W.S. 2nd Lieut., N.Staffs. Regt.; attd.Lancs. Fus. I9I2 Killed in action on the Somme 6 fuly r916

Painter, H. S. and Lieut., Northamptonshire Regt.; I9I I Lieut., Manchester Regt. (W.) M.C.

Parsons Smith, S. H. Major, S. and T. Corps, Indian 1896 Army

Payne, Rev. M. G. J. C.F. 4th Class, R.A.C.D. $\quad 1906$

WPEAK, N. 2nd Lieut., Manchester Regt. Killed in action I fuly 1916

सPerks, W. L. Pte., H.A.C.; 2nd Lieut., Worcestershire I9I3 Regt.

Killed in action 24 Aug. 1916 
Perrott, G. F. D. Cadet, R.A.F.

Peskett, J. C. Lieut., R. Sussex Regt. M.C.

EPettinger, J. W. Capt., R.A.M.C.

Died 6 Oct. I9I 7 of pneumonia

Piercy, Rev. H. M. M. Pte., R.A.M.C.

WPinches, E. L. Lieut., Loyal N. Lancs. Regt.

Died 28 Nov. I9I 7 of wounds received in action

WPlowman, C. H. Lieut., Wiltshire Regt.

Killed in action 24 April I9I7

Pochin, H. Capt., S. Staffs. Regt. and M.G.C. (W.) 1902 M.C.

Poole, A. L. Lieut., Gloucestershire Regt. (W.) *I9I2

Poole, C. A. Lieut., R.F.A.(T.F.)

Powell, A. G. C. Corpl., R.G.A.

1913

I9I4

Powell Price, J. C. Major, I.A.R.O. $M$.

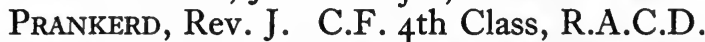

Prentice, Rev. T. N. R. C.F. $4^{\text {th }}$ Class, R.A.C.D.

Priestland, J. F. E. Lieut., Sherwood Foresters (Notts. and Derby Regt.) and M.G.C.

Prissick, C. Lieut.-Col., 56th Punjabi Rifles, Indian 1890

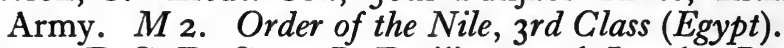

APrynne, E. G. F. Capt., R. Fusiliers; attd. London Regt. 1910 Killed in action on the Somme I6 Sept. 1916

WPrynne, N. F. 2nd Lieut., Devon Regt.

Killed in action 24-5 April 1917

Raine, R. T. Capt., R.A.M.C. (W.) M.C. and Bar.M. 1909

Ratcliffe, C. St A. 2nd Lieut., London Regt. (Surrey 1910

Rifles); attd. Durham L.I.

RawCliffe, W. B. 2nd Lieut., Hampshire Regt.; attd. I9I0 R.F.C.

WRay, T. S. Pte., R. Fusiliers (P.S. Bn.); Lieut., D. of I9I3 Cornwall's L.I.

Died I March I9I8 of wounds received in action $28 \mathrm{Feb}$. I91 8

ReED, L. H. B. 2nd Lieut., R.F.A.; Lieut., T.M.B. (W.) I9I I

ReEve, Rev. F. P. Pte., R.A.M.C.

ReEves, Rev. R. F. Pte., R.A.M.C.

ReYnolds, Rev. J. W. V. Chaplain, R.N.

Rhodes, E. L. N. Capt., R.A.M.C. $M$.

RHodes, W. F. Capt., R.A.M.C.

RICE, Rev. M. N. C.F. $4^{\text {th }}$ Class, R.A.C.D.

Richards, E. I. G. Capt. and Adjt., Welsh Regt. (W) Igo

Ridley, D. F. Capt., Border Regt. (W 2.) M.C. M. ${ }_{1912}$ 
Ricketts, G. D. See Bengough, G. D.

Roвbs, F. W. Lieut., W. African Frontier Force; R.T.O. $\quad 1893$

Roe, R. G. Pte., Canadian Scottish; Lieut., Gordon 1907 Hdrs.; Major, R.E. (Signals). (W.)

Rogerson, Rev. T. S. C.F. 4th Class, R.A.C.D. 1905 WROPER, O. S. Capt., King's Own (Yorkshire L.I.) I9I3

Killed in action 27 Nov. I917

Rose, Rev. A. L. Capt., Sherwood Foresters (Notts. and 1908 Derby Regt., T.F.)

Ross, R. W. Capt., Seaforth Hdrs. (W 2.) M.C. $\quad$ m. $\quad 1899$ Rowland, E. W. S. Capt., R.A.M.C.(T.F.) $m$. 1890 RubIe, Rev. G. O. Pte., London Regt. (Artists Rifles) 1906

SAdGrove, K.H.O'R. Lieut., S. Lancs. Regt. (W 2.) (P.) I9I2 Samson, Rev. A. M. C.F. 4 th Class, R.A.C.D. I9Io SANDERson, P. M. Capt., Worcestershire Regt.; empld. 1904 War Office

ScobIe, J. Capt., Loyal N. Lancs. Regt.

Scotr, Rev. C. W. 2nd Lieut., Labour Corps

Scruby, F. S. Capt., Cambridgeshire Regt.; attd. R.E. 1899 O.B.E. $M$.

Staman, Rev. A. J. C.F. 4th Class, R.A.C.D. $\quad 1906$

Sellwood, H. E. Air Mechanic, R.A.F. 1907

Selwyn, Rev. H. E. C.F. 4th Class, R.A.C.D. $m$. 1885

Sewell, F. C. Pte., R. Fusiliers, Army Pay Corps, and 1914 Labour Corps

Sharman, Rev. G. H. Pte., S. African Infy.

Shearwood, Rev. G. F. F. C.F. 4th Class, R.A.C.D.

Shepheard, E. P. W. Pte., London Regt.; 2nd Lieut., I9OI

King's Royal Rifle Corps. (W.) (P.)

Shillaker, E. C. H. Lieut., W. Yorks. Regt. and Tank I9I3

Corps. (W 2.) M.C.

Simeon, Rev. J. P. Lieut., R.A.S.C.(M.T.) M. $\quad$ I896

SmrTh, H. A. Lieut., R.A.M.C. 1897

Smith, Rev. S. T. 2nd Lieut., Labour Corps 1904

Smith, W. G. Capt., Cranbrook School O.T.C. 1894

SPENCE, D. L. Capt., R.A.M.C.

SPINNEY, Rev. M. H. C.F. 4th Class, R.A.C.D. I906

Standidge, C. Pte., W. Yorks. Regt. and Cameronians 1898

(Scottish Rifles); Air Mechanic, R.A.F.

Stanley, C. M. Capt., Army Cyclist Corps. 'M. 1904

WSTANTON, R. G. O. Lieut., R.M.L.I. I9I4

Died 28 April 1918 of wounds received in action at Zeebrugge 23 April 1918 
EStaunton, Rev. H. C.F. $4^{\text {th }}$ Class, R.A.C.D.

Died in Mesopotamia I 4 Fan. I9I8

Stealey, E. T. Lieut., Monmouthshire Regt. (P.)

Stockdale, R.J. Capt., Durham L.I.(T.F.) (W 3.) M.C. 1914

Stokes, Rev. L. C.F. $4^{\text {th Class, R.A.C.D. }}$

Stoneman, W. E. Lieut., R.G.A. (W.)

AStraughan, T. A. Lieut., Northumberland Fus.

Died 17 Feb. 191 8 of wounds received in action in Galli-

1914

1910

poli 19 Aug. 1915

Strong, P. N. W. 2nd Lieut., R.E.

I917 R.F.C. (P.) $M$.

ZSunderland, G. Sergt., K. Edward's Horse; Capt., R. I9ro Sussex Regt. (W.)

Killed in action at Gricourt 24 Sept. 1918

SutTon, L. J. Lieut., R.G.A.

Swiney, A. J. E. See MacSwiney, Rev. A. J. E.

Sykes, Rev.F.M. C.F.3rd Class, R.A.C.D. O.B.E. M2. 1897

Tadman, G. R. Major, R.A.S.C. (Camel Transport Igor Corps). M.C. M.

Tancock, E. O. 2nd Lieut., R.G.A. (W.) 1905

TAYlOR, J. F. Capt., R.A.M.C.

TAYLOR, Rev. L. F. M. Chaplain, R.N.

1905

1908

TelfER, A. C. 2nd Lieut., Cambridgeshire Regt.; Lieut., 1912

The Queen's (R.W. Surrey Regt.) (W 2.)

Tempest, F. L. Capt., Suffolk Regt. and Gen. List 1912 (Staff Capt.) M.C.

Thomas, H. E. E. Lieut., R.A.S.C. and R.F.A.

Thorburn, E. C. Lieut., M.G.C. M.C.

Thorneycroft, K. H Lieut, The Queen's (R. W [

Surrey Regt.); Capt., Spec. List (cmdg. P. of W.

Camp). (W.) $m$.

${ }^{1}$ Thursfield, R. M. R. . Surgeon Lieut., R.N.

Tilbury, Rev. H. F. C.F. $4^{\text {th }}$ Class, R.A.C.D.

Tilston, Rev. H. E. C.F. 4th Class, R.A.C.D. Ig0I

Tisdall, E. G. St. C. Capt., Monmouthshire Regt. I9I4 (W.) M.C.

Tomblings, D. G. Capt., E. African Med. Service. M. 1908 Tomlinson, H. Pte., R. Fusiliers

*Tovex, D. 2nd Lieut., London Regt. (London Scottish) $\quad 1892$ and Gen. List

Died 5 May 1918

1 Killed at sea after the armistice. 
Trench, Rev. G. F. C.F. $4^{\text {th }}$ Class, R.A.C.D. $M . \quad 1899$

Truman, Rev. M. G. C.F. $4^{\text {th }}$ Class, R.A.C.D. 1909

Tudor, O. C. O. Lieut., R.A.S.C.

1905

Tudor, R. G. Capt., King's (Liverpool Regt.) (W 2.) I9IO M.C. $M$.

Turner, J. P. Pte., R.A.S.C.(M.T.)

TurNer, J. S. Sub-Lieut., R.N.V.R.

TYleR, H. H. F. M. Capt., I.A.R.O., attd. 9th Ghurkas. 1896 (W.) C.S.I. M.

Unwin, H. A. R. E. Capt., R.A.M.C. $m$.

Unwin, S. R. Capt., Unattd. List, T.F., attd. Devon

1899

Regt.; Instructor, School of Musketry. $m$.

Vivian, G. L. Capt., I.A.R.O. (Cavalry)

1906

WWadeson, E. Y. Lieut., Loyal N. Lancs. Regt. (W.) I9I4 Killed in action 22 March 1918

Wadley, H. W. A. Pte., Canadian A.M.C.

WWaIt, C. F. W. Lieut., King's Own (Yorkshire L.I.) [1914]

Died I 5 Fuly 1916 of wounds received in action

WARD, J. G. Major, R.G.A.(T.F.) $M$. 1908

WARDley, D. J. Capt., R. Fusiliers; Capt. and Adjt., 1912 London Regt.; Staff Capt. M.C. and Bar

Weber, W. E. Lieut., Spec. List (R.T.O.)

Webster, J. F. Capt., Norfolk Regt.; empld. Agricultural I9IO Directorate, Mediterranean Exp. Force

WeLCh, J. J. Lieut., R.G.A.

WelleR, Rev. C. H. C.F. $4^{\text {th }}$ Class, R.A.C.D. (W.) $M . C$.

Weller, Rev. J. R. C.F. $4^{\text {th }}$ Class, R.A.C.D.; empld. I9ro Indian Army

Wells, A. P. Sapper, R.E.

Wharton, C. H. L. Pte., Canadian A.M.C.

WWhite, G. C. W. 2nd Lieut., Cheshire Regt.

Died 6 Oct. I9I 5 of wounds received in action

Whytehead, H. L. Pte., London Regt. (Artists Rifles) $\quad$ I897

Williams, A. S. 2nd Lieut., Devon Regt.

Williams, R. A. Cadet, R.G.A.

Williams, R. F. Lieut., Canadian Infy.

Wilson, H. L. Lieut., R.A.M.C.

1906

1905

1912

WILSON, J. S. and Lieut., R. Warwickshire Regt. (W.) 189

WindsoR, J. F. Lieut., R.A.M.C.

1904

1898

1900

I895

WING, T. 2nd Lieut., Wiltshire Regt. (W.)

I899

1897 
Wood, G. Capt. and Adjt., Cheshire Regt. (W.) O.B.E. I9I3 M.C. $M$.

Wood, Rev. T. J. C.F. $4^{\text {th }}$ Class, R.A.C.D.

Woods, F. A. Lieut., Army Pay Dept.

Woods, N. C. Pte., Army Pay Corps

Woods, T. H. Lieut., R. Fusiliers

Woolliscroft, F. H. Lieut., N. Staffs. Regt. (W.)

WWordsworth, O. B. 2nd Lieut., Oxford and Bucks. L.I.; Lieut., M.G.C.

Killed in action 2 April I917

Wright, Rev. A. G. C.F. $4^{\text {th }}$ Class, R.A.C.D.

WWright, E. A. Sergt., London Regt. (London Scottish); 1896 Lieut., R.A.M.C.

Died at Alexandria 20 June 1915 of septic poisoning

\#Young, J. V. Pte., R. Fusiliers (P.S. Bn.); 2nd Lieut., I9I I Somerset L.I.

Killed in action 2 fuly r916 


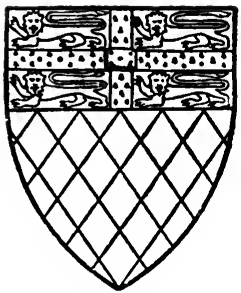

\section{FITZWILLIAM HALL1 \\ (NON-COLLEGIATE)}

Adam, W. A. Major, S. Staffs. Regt.(T.F.) (W 3.) (P.) rgor HAlford, A. C. G. 2nd Lieut., Gloucestershire Regt.; I9I3 attd. Worcestershire Regt.

Killed in action 3 Sept. I916

WAllison, C. H. Capt., Suffolk Regt.

Killed in action $\mathrm{I}_{3}$ Nov. 1916

Allpress, H. G. Lieut., Monmouthshire Regt. (W.) 1909

Alnwick, H. Lieut., W. Yorks. Regt. (W.) M.C. M. I9I4

Armour, J. 2nd Lieut., King's Royal Rifle Corps I9ro

Bagnall, J. M. V. Corpl., R.E. (Signals); Lieut., North- I9I3 umberland Yeo.

Baker, D. See Bendigo, Rt. Rev. Bishop of

BAKER, E. 2nd Lieut., Norfolk Regt.

Ball, S. H. Lieut., Coldstream Gds. and Gds. M.G. I9I5 Regt.

BANYARD, F. E. Lieut., Cambridgeshire Regt. (W.) IgI I WBarraud, V. H.A. Capt., French Army. (W.) Chevalier, r9Io Legion of Honour (France)

Killed in action 20 Fune I9I 5

BARRETT, R. O. Lieut., R. Welsh Fus. and Bedfordshire I9I I Regt.

WAartletT, C. W. Capt., Sherwood Foresters (Notts. and I9I3 Derby Regt.) (W.) M.C. M.

Killed in action 9 Oct. 1918

1 The names of those who, after matriculating as Non-Collegiate students, joined one or other of the Colleges before the war, will be found under their adopted Colleges only. 
BEARD, E. Lieut., Northamptonshire Regt. $m$.

Beaumont, P. J. Gnr., R.G.A.

WECK, C. B. H. 2nd Lieut., Cheshire Regt.(T.F.)

Died I8 Aug. I9I5 of wounds received in action in

Gallipoli 17 Aug. I915

Bendigo, Rt. Rev. Bishop of. C.F., Australian Chaplains' $\quad$ I909 Dept.

BIRD, Rev. E. G. Chaplain, R.N.

BIRD, H. J. G. Capt., W. Yorks. Regt. (R. of O.); Major, I895 M.G.C. (Motor). $M$.

Bolton, T. Sapper, R.E. (London Electrical Engineers, $\quad$ I897 T.F.)

WBoucher, A. E. Lieut., R. Warwickshire Regt. (W.) [I9I4] M.C. $M$.

Killed in action 18 Nov. I9I6

WBowler, S. 2nd Lieut., R.G.A.

Killed in action 21 Oct. 1917

Bradley, G. H. Cadet, O.C.B.

Briggs, J. L. 2nd Lieut., Suffolk Regt.

BRIGHT, F. T. Lieut., Suffolk Regt.; Capt. (A.), R.A.F. (W.) D.F.C.

Bright, W. J. Capt., R.F.A. $M$.

Brookes, A. Lieut., I.A.R.O., attd. 6gth Punjabis;

Capt., Provost Marshal

Broomer, A. 2nd Lieut., King's Own (R. Lancaster I9I4 Regt.); Lieut. (O.) and Capt. (Ad.), R.A.F.

Buckley, A. St D. Pte., R.A.S.C.

Butler, E. W. 2nd Lieut., King's Royal Rifle Corps

Butler, J. 2nd Lieut., R. Berkshire Regt. (W.)

Bywaters, Rev. F. J. C.F. 4th Class, R.A.C.D.

Cairnie, J. B. Lieut., Seaforth Hdrs.

ACARTER, W. H. S. Lieut., King's (Liverpool Regt.)

Killed in action 14 Fuly I916

Cassan, Rev. A. W. M. C.F. $4^{\text {th }}$ Class, R.A.C.D. (W.) 1903 M.C. M.

Chapple, C. J. Lieut., R. Sussex Regt.

Charley, L. W. Lieut., R. Warwickshire Regt.; Capt., I 908 Gen. List (Intelligence). O.B.E. M.

CleE, C. B. B. Capt. and Adjt., Suffolk Regt. (W.) M. I9I4 Clements, P. M. H. Lieut., Essex Regt. (W.)

Cohen, M. 2nd Lieut., R.A.S.C.; attd. Serbian Army 
Copplestone, W. R. J. Lieut., E. Lancs. Regt. and Nigeria Regt., W. African Frontier Force; Liaison Officer, attd. Belgian Gen. Staff

Crookall, Rev. E. C.F. $4^{\text {th }}$ Class, R.A.C.D.

Cross, P. M. Lieut., Dartford Grammar School O.T.C. 1907

Dalling, W. E. Staff Sergt., R.A.M.C.(T.F.)

Davies, J. R. Lieut., R.N.V.R.

1912

WDAvis, C. A. E. 2nd Lieut., R.F.A.

Killed in action 31 Fuly 1917

DE BRACONIER, L. Brigadier, Belgian Army. Belgian I915 Croix de Guerre

DE Ceuleneer, P. Belgian Army

Deighton, F. J. Capt., r24th Baluchistan Infy. and S. and T. Corps, Indian Army

DE Lestapis, H. Sous-Lieut., French Army

DenNIs, W. J. M. 2nd Lieut., Durham L.I.

Dixon, J. H. Lieut., Labour Corps

WDownman, B. V. R. 2nd Lieut., Sherwood Foresters

(Notts. and Derby Regt.)

Killed in action 21 Sept. 1916

DuвoIs, H. A. Lieut., I.A.R.O., attd. I40th Patiala Infy. I9I0 $M$.

Dunning, G. C. Corpl., R.A.M.C.(T.F.)

Dunsheath, P. Capt., R.E. O.B.E. M2.

Dyson, E. Lieut., R.F.A.(T.F.) (W.)

I9I3

I910

1914

1914

1903

1914

1903

1913

1914

Eeman, J. Belgian Army

सElliotT, O. C. F. 2nd Lieut., Gordon Hdrs.

Killed in action 14 Oct. 1916

Elliotr, S. D. Pte., Sherwood Foresters (Notts. and I9I7

Derby Regt.)

ElliotT, W. W. 2nd Lieut., R. Scots. (W.) 1906

Emtage, W. L. 2nd Lieut., Middlesex Regt.; Lieut., * *9I3 British W. Indies Regt.

Facey, Rev. N. S. C.F. 4th Class, R.A.C.D.

Fanshawe, C. H. Major, R.A.S.C.; D.A.D.S. and T., 1893

W. Command. $m$.

FawcetT, R. Lieut., R.G.A.(T.F.) (W.) $M$.

FEw, H. C. Major, Cambridgeshire Regt. (W 2.)

1909

1910

I912

1915

1914

1906
I 913

I9I3

I9I I

I 898

1916

Killed in action 22 Aug. 1918

Flower, Rev. W. J. Chaplain, R.N.

1903

c.U.w.L. 
ZFoster, R. Pte., N. Staffs. Regt.(T.F.); 2nd Lieut. (O.), R.F.C.

Killed in flying accident 3 Fan. 1918

Gamble, Rev. H. J. C.F. $4^{\text {th }}$ Class, R.A.C.D. $M . \quad 1908$ GaRRETT, Rev. S. C.F. 4th Class, R.A.C.D. 1903

GAZE, Rev. A. M. B. Lieut., Bedfordshire Regt. and La- 1907 bour Corps. (W.)

Gibson, Rev.H. C.F. $4^{\text {th }}$ Class, R.A.C.D. M.C. M. 1903

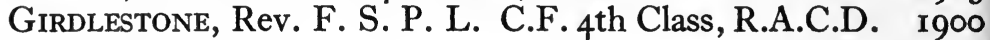
D.S.O.

*Glaister, G. F. Lieut., Tank Corps

Killed in action I Aug. I918

Glaister, S. E. Lieut., Tank Corps. (W 2.)

Goodchild, Rev. E. J. Capt., Essex Regt.(T.F. Res.) 1909

Gordon, J. E. Lieut., 5th Dragoon Gds. and M.G.C. 1904 (W 2.)

Gough, D. W. P. Rfn., King's Royal Rifle Corps

Gould, Rev. C. J. B. C.F. 4 th Class, R.A.C.D.

1917

GREY, R. C. Lieut., Suffolk Regt.; attd. Indian Army 1913

Griffiths, Rev. J. Lieut., R. Welsh Fus.; attd. R. Sussex 1912 Regt. (W.)

Hale, H. E. Trooper, Oxfordshire Yeo.; 2nd Lieut., 1899 Oxford and Bucks. L.I.

Hargreaves, C.A. Capt., Manchester Regt.(T.F.) (W 2.) I9I I $M$.

Harris, F. W. Capt., R.F.A. (W.) $M$. 1913

Harris, Rev. G. H. C.F. $4^{\text {th Class, R.A.C.D. } M . ~ m . ~} 1910$ WHARRIS, T. W. S. Capt. (A.), R.A.F.

Killed in action (2 1 Nov. 1918$)$

WHaslam, W. 2nd Lieut., Manchester Regt.

Killed in action 21 March 1918

Hastings, Rev. W. Capt., Labour Corps

WHaynes, W. G. 2nd Lieut., Suffolk Regt.

Killed in action 27 June 1917

wHeath, F. A. Pte., R.A.M.C.

Died 12 Oct. I9I 7

HeNDRY, C. W. 2nd Lieut., Gordon Hdrs.; Lieut., M.G.C. M.C.

Hinton, S. E. Capt., Cheshire Regt.(T.F.)

WHIRST, W. B. 2nd Lieut., Lincolnshire Regt.(T.F.)

Died 22 April 1915 of wounds received in action near 1913

I9I 5

I 88I

[1914]

1913

I9I3

I9I4

I9II

Ypres 
Hollis, W. P. B. Corpl., R.E. (Signals). (P.)

1912

Hudson, T. E. 2nd Lieut., Hampshire Regt.

1910

Hume, D. B. M. Capt. (K.B.), R.A.F. M.

1912

HuRRell, G. W. 2nd Lieut., Tank Corps. (W 2.) [1914]

HurRell, J. W. Capt. and Adjt., Bedfordshire Regt.; I9I I

Major, King's Royal Rifle Corps. (W.) M.C.

Hussey, Rev. R. L. C.F. $4^{\text {th }}$ Class, R.A.C.D.

1907

Ison, L. J. Pte., London Regt.(Civil Service Rifles)

I9I I

Jameson, T. S. Lieut., W. Yorks. Regt.; attd. E. Lancs. 1914 Regt.(T.F.); empld. O.C.B.

JEFF, Rev. A. H. C.F. $4^{\text {th }}$ Class, R.A.C.D.

Jenkins, H. L. Lieut., M.G.C. (W.)

Jenkinson, C. Pte., R.A.M.C.(T.F.)

Johnson, A. H. Pte., Suffolk Regt. (W.)

JoHnson, W. D. Cadet, O.C.B.

Jones, Rev. D. M. C.F. $4^{\text {th Class, R.A.C.D. M.C. }}$ I914

Jones, Rev. E. C.F. $4^{\text {th }}$ Class, R.A.C.D.

Joseph, C. G. Lieut., Cambridge Univ. O.T.C.

1908

1910

[1914]

I9I5

1918

1914

1912

Keay, K. D. Lieut., Cameronians (Scottish Rifles). (W.) I9I3

Keelan, Rev. V. L. Chaplain, R.N.

1890

KERRIDGe, W. A. L. Capt., D. of Wellington's (W. Riding rg I I Regt.) (W.) $M$.

KING, R. R. Capt., London Regt. (Queen's). (W.) I9I2

KNIGHT, J. H. Lieut., D. of Wellington's (W. Riding I9I3 Regt.) (W.)

Lacy, H. C. 2nd Lieut., Suffolk Regt:; Draft Conducting Officer

LEE, W. N. R. 2nd Lieut., E. Lancs. Regt.; attd. S. I9I7 Lancs. Regt.

LegGe, P. A. Hon. Major, R.G.A. (R. of O.); Super- I9I I visor of Customs, Gold Coast

Legouis, P. A. E. Sous-Lieut., French Army

LEwIS, A. T. Lce.-Corpl., R. Welsh Fus.

LEwIS, H. G. Capt., Tank Corps. $M$.

[19I4]

LEWIS, O. G. Lieut., R.E.; empld. Ministry of Labour I9I I

Litchfield, G. Lieut., Devon Regt. (W.)

Lock, Rev. L. J. Capt., R.A.M.C.

I9II

1905

*Long, F. S. Lieut., Essex Regt.

Killed in action 26 Sept. I9I 5

Longstaff, J. 2nd Lieut., Durham L.I.

I912

I9II 
McCliment, Rev. R. J. C.F. 3rd Class, R.A.C.D.; 1912 D.A.P.C. O.B.E. $M_{3}$.

MaCkAY, R, F. B. Capt., Essex Regt.(T.F.). $m . \quad{ }^{*}$ I9I3

Major, E. Lieut., R.G.A. M.C.

MALlETT, H. R. R.S.M., R.A.M.C. 1907

Maris, A. A. Capt., Suffolk Regt.(T.F.) (W.) 1913

MARSH, A. W. 2nd Lieut., R.F.A. (W.) 1917

MarTin, F. Corpl., London Regt. (Civil Service Rifles) I9I I

EMartin, H. E. Capt., Middlesex Regt.(T.F.) I9I0

Died 19 Fune 1916 of wounds received in action

Matтock, F. C. Lieut., W. Yorks. Regt. 1912

MEAD, Rev. A. C.F. 4th Class, R.A.C.D.

MeIxneR, F. Lieut., King's Own (R. Lancaster Regt.); I9Io attd. R.E. (Signals)

Miller, L. V. Lieut., R.E. (Signals). (W.)

Miller, O. G. 2nd Lieut., I.A.R.O., attd. 23rd Sikhs [19I4]

Morgan, G. E. 2nd Lieut., Welsh Regt.

WMorris, A. C. Pte., Cheshire Regt.

Killed in action 1918

Morris, H. E. A. Lieut., Cambridgeshire Regt. and I9I3 154th Infy., Indian Army

MORRISH, J. See MurRish, J.

MorTon, A. V. Lieut., Gloucestershire Regt.; Lieut. 1914 (A.), R.A.F. (W.)

Morton, C. E. Lieut., M.G.C. $M$.

Morton, Rev. J. G. C.F. $4^{\text {th }}$ Class, R.A.C.D.

Muncey, A. P. Telegraphist, R.N.V.R.

Muncey, S. W. U. Corpl., R.E. (Spec. Bde.)

WMunro, D. Pte., R. Fusiliers

Died I4 Fan I9I9 of blood-poisoning contracted on active service

MurRIsh, J. Lieut., D. of Cornwall's L.I. and R.E. IgII (Field Survey Bn.) (W.)

NeAL, R. Cadet, O.C.B.

Newell, Rev. H. W. C.F. 4th Class, R.A.C.D. 1908

WNorman, Rev. W. H. Sergt., R.A.M.C.

Drowned on H.M. transport Transylvania 4 May I9I 7

WNorton-FAGge, F. W. L. Capt., I.A.R.O., attd. Punjabis.

$M 2$.

Died 29 Nov. 1916

O'Connell, Rev. J. B. C.F. $4^{\text {th }}$ Class, R.A.C.D. 
O'RioRdan, P. M. 2nd Lieut., I.A.R.O., attd. 75th Car- I90I natic Infy.

Peacock, L. V. Lieut., Suffolk Regt. (Cyclist Bn., T.F.) I9I3 and 46th Punjabis, Indian Army; attd. I8th Infy.

PeCK, E. G. Lieut.-Col., R.A.M.C.(T.F.) D.S.O. T.D. ${ }_{1} 872$ $M 2$.

Peck, E. S. Capt., Cambridgeshire Regt.; Major, Gen. 1893 Staff, empld. British Military Mission. $m$.

Peel, A. W. Lieut., Cambridgeshire Regt.; Capt., Gen. $\quad 1892$ List (T.F. Res.), empld. Ministry of National Service. M.B.E.

Pegg, H. E. Pte., London Regt. (Artists Rifles)

Pickard, Rev. H. G. C.F. 4th Class, R.A.C.D.

APlaYer, E. N. Capt., Yorkshire Regt.

Killed in action $6 \mathrm{Aug}$. $19 \mathrm{r} 6$

Powell, Rev. E. J. C.F. 4th Class, R.A.C.D.

Prust, T. W. Capt., Middlesex Regt.

Pryke, W. W. Pte., Suffolk Regt.

1914

I910

I9I I

1900

1906

I9I 5

Remnant, G. L. 2nd Lieut., Lancs. Fus.

Robinson, Rev. T. C.F. $4^{\text {th }}$ Class, R.A.C.D.

1917

Rogers, C. E. B. Capt., R. Berkshire Regt. (W) $M . C$.

WRolfe, P. Pte., R. Fusiliers (P.S. Bn.); Capt., R.A.S.C.; 1909 attd. Norfolk Regt.

Killed in action 24 Aug. 1918

WRose, H. E. Capt., R.A.M.C. (W.)

Died 7 Fuly 1917 of wounds received in action

Royston, H. R. 2nd Lieut., R. Fusiliers

1905

1909

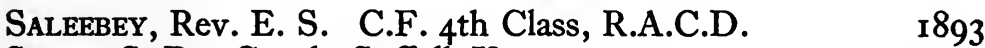

SAYER, S. D. Corpl., Suffolk Yeo. 1912

ScotT, C. W. Major (A. and S.), R.A.F. D.F.C. M 2. I9I4

*ScotT, F. Capt., Leicestershire Regt. (W 3.) M.C. I9I3

Killed in action 27 May 1918

Scotr, G. A. Lieut., Gloucestershire Yeo.

Scotr, R. F. M. Lieut., R.A.M.C.

Scotr, T'. F. Lieut., Seaforth Hdrs.(T.F.) (W.)

1909

1889

Semple, Rev. E. G. C.F. 4th Class, R.A.C.D. (W 2.) 1909

ASERginson, H. Capt., Northumberland Fus.; attd. I915

T.M.B. M.C.

Killed in action $27 \mathrm{Feb}$. 1918

SEwELL, H. D. R.A.S.C.(M.T.)

ShaRP, G. G. Lieut., Border Regt. (W.) 
ÆShaw, W. Lieut., Cambridgeshire Regt. $M$.

Died in German hands 27 Sept. 1916 of wounds received in action

Sheehan, Rev. F. R. C.F. $4^{\text {th }}$ Class, R.A.C.D.

SHEPHERD, Rev. J. C.F. 4 th Class, R.A.C.D.

SHERLOCK, W. W. Lieut., I.A.R.O., attd. S. and T.Corps. I900 $M 2$.

Shillaker, G. Pte., London Regt. (W.)

Shipley, J. W. 2nd Lieut., York and Lancaster Regt.; I9I2 Lieut., M.G.C. (W 2.)

Shove, W. C. Lieut., R.F.A. $M$.

Shrewsbury, E. 2nd Lieut., R. Sussex Regt.

${ }^{1}$ Skinner, C. E. Major, R.F.A. (W 2.)

Slawson, W. N. 2nd Lieut., Leicestershire Regt. and

Worcestershire Regt.; Lieut., Gen. List, attd. R.A.F.

Sloman, H. Major, R.A.S.C. $M$.

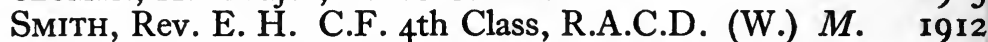

«Smith, F. E. Lieut., Cambridgeshire Regt. and Suffolk 1909 Regt.(T.F.) (W.) $M$.

Died 18 Nov. 1918 of pneumonia following influenza contracted on active service

Smith, Rev. G. L. C.F. 4th Class, R.A.C.D. (W.) 1906

Smith, Rev. G. S. Cadet, R.G.A.

SNoxell, S. 2nd Lieut., Dorset Regt.

Souire, A. G. 2nd Lieut. (O.), R.A.F. (W.)

1917

Staley, Rev. J. E. C.F. $4^{\text {th }}$ Class, R.A.C.D.

1917

Stanley, F. 2nd Lieut. (A.), R.A.F.

StePhens, Rev. J. P. C.F. $4^{\text {th }}$ Class, R.A.C.D.

1904

1917

1908

Stephenson, Rev. H. C.F. 4th Class, R.A.C.D. 1909

सStokes, W. H. Pte., R. Fusiliers (P.S. Bn.); 2nd Lieut., I910 R.G.A.

Killed in action 18 April 1918

«Stout, G. F. 2nd Lieut., Lincolnshire Regt. and Yorkshire Regt.

Killed in action 30 Sept. rgr6

Stream, E. J. Major, Lincolnshire Regt.

Sumner, E. J. 2nd Lieut., Sedbergh School O.T.C.

WSwallow, J. R. 2nd Lieut., King's (Liverpool Regt.)

Killed in action 8 Aug. I9I 6

Swann, P. W. 2nd Lieut., Hampshire Regt. (W.) I9I3

Taylor, J. D. Capt., R.F.A. (W.) M.C.

1 Died on service in S. Russia after the armistice. 
Teale, Rev.E. C.F.3rd Class, R.A.C.D. (W.) M.C. M. 1900 ThatCher,W. S. Lieut., I.A.R.O., attd. Baluchis. (W 3.) 1907 M.C.

Thomas, Rev. R. E. C.F. $4^{\text {th }}$ Class, R.A.C.D. $M$. 1908

Tomkins, Rev. L. C. F. 2nd Lieut., Spec. List $\quad$ I907

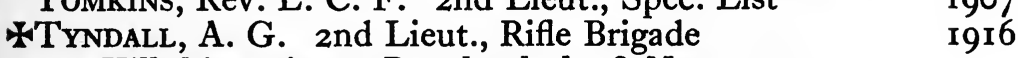

Killed in action at Passchendaele 18 Nov. 1917

Vance, Rev. J. G. C.F. 4th Class, R.A.C.D.

Van Gehuchten, P. Med. Corps, Belgian Army

VINCENT, L. J. Capt., R.A.M.C.

VINCENT, T. C. L. 2nd Lieut., M.G.C.

WaIte, T. H. Cadet, O.C.B.

Walker, C. D. Corpl., Cameronians (Scottish Rifles). I9I3 (W.)

Walton, G. M. Lieut., S. Lancs. Regt. (W.) I9I6

WARD, Rev. R. W. A. C.F. 4th Class, R.A.C.D. 1910

Warren, C. Capt., Cambridgeshire Regt.

Watkins, M. Cadet, O.C.B.

Watt, A.S. Corpl., R.E. (Spec. Bde.)

Weaver, J. Gnr., R.G.A.

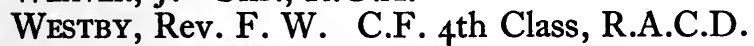

WWhEeler, R. M. Lieut., Middlesex Regt.(T.F.)

Killed in action 30 Nov. 1916

White, Rev. A. J. C.F. $4^{\text {th }}$ Class, R.A.C.D.

White, G. W. Capt., Spec. List (Officer i/c Wireless Repair Section, R.E.)

WWhite, H. A. Pte., R. Fusiliers (P.S. Bn.); 2nd Lieut., 1913 The Buffs (E. Kent Regt.) and R. Irish Fusiliers. (W.)

Died 22 Nov. 1917 of wounds received in action

White, Rev. J. L. C.F. $4^{\text {th }}$ Class, R.A.C.D.

Whitfield, Rev. J. L. C.F. $3^{\text {rd }}$ Class, R.A.C.D. D.S.O. $M$.

Whitmill, G. H. Cadet, O.C.B.

WWilkinson, E. F. Pte., Suffolk Regt. and M.G.C.

Died in German hands 9 June 1918 of wounds received in action at fussy

Williams, Rev. T. C. L. C.F. $4^{\text {th }}$ Class, R.A.C.D. $M$.

WITNEY, Rev. T. C. C.F. $4^{\text {th }}$ Class, R.A.C.D.

Wolff, C. H. Lieut., Somerset L.I.; Capt., R. Defence

Corps, Suffolk Regt., Labour Corps, and Gen. List 1915

I9I7

r9I4

1908

I910

1907

I9I 5

I9I 4

I 907

I897

I9I7

I9I 7

(Education Officer).

wWoods, A. M. 2nd Lieut., R.F.A.

Killed in action $26 \mathrm{Feb}$. 1917 
Woods, W. A. Capt., R.F.A. (W.) $M$.

Wormald, Rev. C. O. R. C.F. $4^{\text {th }}$ Class, R.A.C.D.

Wormell, C. O. R. See Wormald, Rev. C. O. R.

Worthington, W. R. Lieut., R. Warwickshire Regt. I913 (W 2.)

The following matriculated at CAvendish College ${ }^{1}$, which was created a Public Hostel in 1882 and ceased to be such in 1892 : WDathan, Rev. J. D. Chaplain, R.N.

Died 7 Fan. 1918

1 The names of those who, after matriculating at Cavendish College, migrated elsewhere, will be found under their adopted Colleges. 




\section{INDEX}

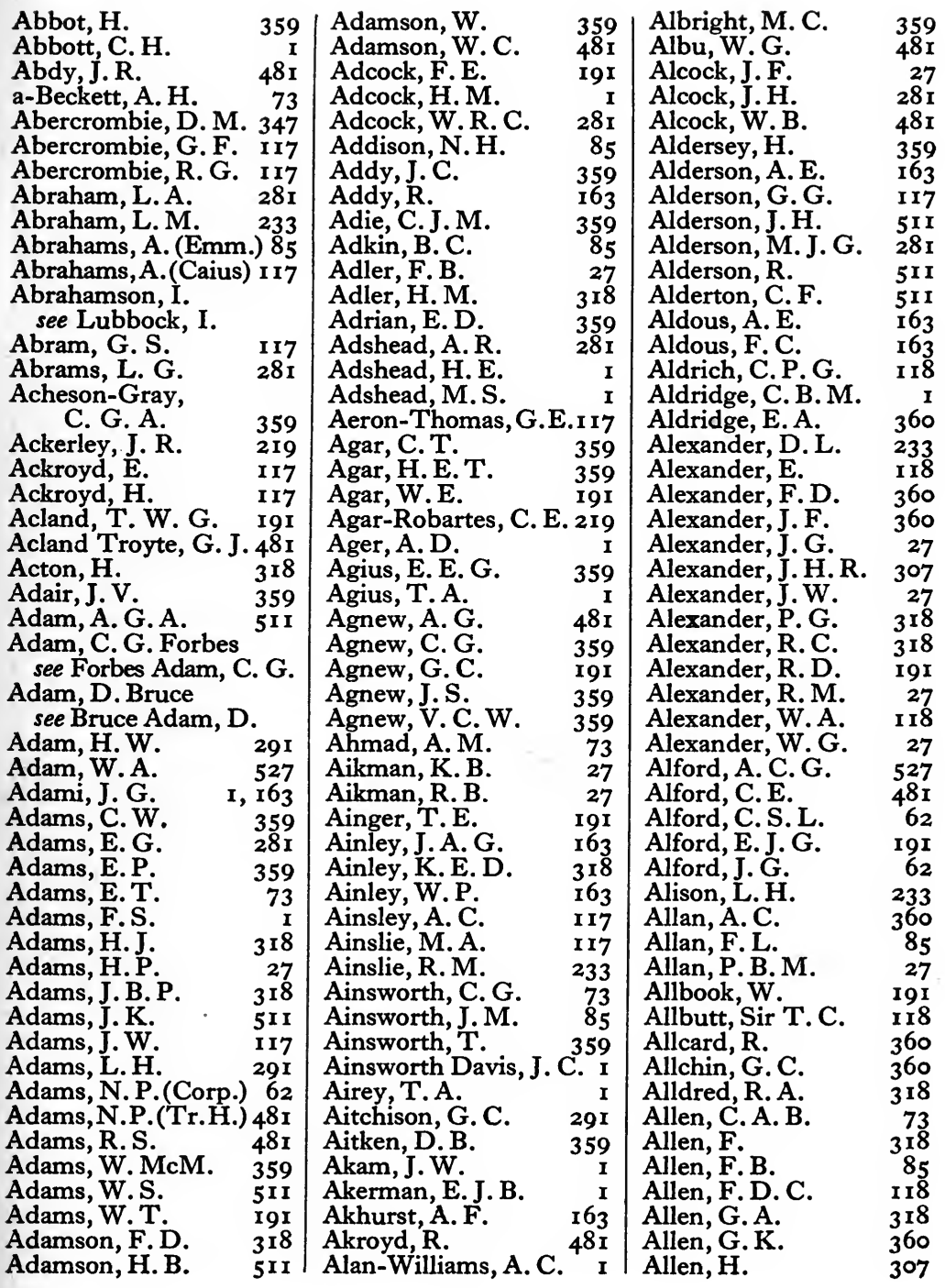


Allen, H. C. G.

Allen H. R.

Allen, H.S.

Allen, J.

Allen, J. E. R.

Allen, J. H.

Allen, J. M.

Allen, J.S.

Allen, J.W.

Allen, L. J.

Allen, P. H. C.

Allen, R. C. (Christ's)

Allen, R. C. (Trin.) 360

Allen, R. G. R.

Allen, S. E.

Allen, S. S.

Allen, W.

Allen,W.H.(Christ's)

Allen, W. H. (Trin.) 360

Allen, W. L.

Allen, W.S.

Allfrey, F. H.

Allgood, G. H.

Allhusen, $\mathrm{O}$.

Allhusen, R.

Allin, H. W.

Allison, C. $H$.

Allison, H.S.

Allison, W. W.

Allix, C. I. L.

Allom, C. C. G.

Allott, P. B.

Allpress, H. G.

Allsopp, F. G.

Allsopp, $\mathrm{R}$.

Almond, J.

Alnwick, $\mathrm{H}$.

Alston, C. R.

Alston, G. K.

Alston, W. E.

Althorp, Viscount

Altounyan, E. H.R.

Ambler, G.

Ambrose, W. J. L. 233

Ames, E. F. W.

Amphlett-Morton, J. F.

Amps, L. W.

Anable, A.

Ancaster, Earl of

Anderson, A. C.

Anderson, A. E. D. 360

Anderson, A. P. M. see Moore-Anderson, A. P.

Anderson, A.R.S. $\quad$ II 8

Anderson, C. A. $\quad 360$

Anderson, C. A. K. 233

Anderson, D. C. $\quad 233$

Anderson, F. I.

\section{3}

233

360

360

360

219

527

I63

360

360

318

527

85
219

85

527

163

$5 \mathrm{II}$

27

360

85

291

33

,

18

60

233
Anderson, G. B. (Emm.)

Anderson, G. B. (Trin.)

Anderson. $\mathrm{H}$.

Anderson, $\mathrm{J}$.

Anderson, $\mathrm{K}$.

Anderson, K. B.

Anderson, L. A. P.

Anderson, L. R. D. 35

Anderson, M. E. M. see Moore-Anderson, M. E.

Anderson, N. L. $\quad 360$

Anderson, R. C. $\quad 27$

Anderson, S. M. $\quad 27$

Anderson, W. A. $\quad 27$

Anderson, W. L. $\quad$ I 8

Anderton, G. E. A. $\quad 28$

Andrade, E. N. da C. 85

Andras, J. B. $\quad 360$

Andrew, G. H.

Andrew, G.W. M. 85

Andrew, R. H. $\quad 163$

Andrewes, C. N. $\quad 360$

Andrews, C. N. $\quad 233$

Andrews, E. A.

Andrews, J. A.

Andrews, J. C.

Andrews, J. L.

Andrews, K. T.

Andrews, L. M.

Andrews, T.E.

Angas, D. T.

Angus, R. E.

Angus, W. B. G.

Annan, J. G.

Annand, A. W.

Annesley, Earl

Ansbacher, S. S.

see Ansley, S. S.

Ansdell, R. C.

Ansdell, T. A.

Ansell, A. E.

Ansell, A. G.

Ansell, E.

Ansley, S. S.

Anson, G. F. V.

Anstey, A.

Anstey, T. C. R.

Anthony, A. L.

Anthony, H. D.

Antrobus, $\mathrm{H}$.

Antrobus, M. E.

Apperley, B. L. M.

Appleby, D.

Applegarth, T.W.

Appleton, A. B.

Appleton, A. J.

Appleton, E. L.

Appleton, E. V.

28

$36 \mathbf{I}$

$28 \mathrm{I}$

$36 \mathrm{I}$

$36 \mathrm{I}$

164

361

318

291

318

$36 \mathrm{I}$

361

233

85

73

233

28

318
Appleyard, W.

28

Arbuthnot, L. G. 36r

Arbuthnot, M. A. (King's)

Arbuthnot, M.A. (Trin.)

Arbuthnot,

R. W. M.

I 9 I

361

Arbuthnot, W.J.

36 I

Archdale R M $\quad 482$

Archer, C. W. 36 I

Archer, G. D. $\quad 511$

Archer, J. F. I9I

Archer-Hind, L. $\quad 318$

Arden, H. W. 29I

Ardern, L. $\quad 28$

Aris, $\mathrm{H}$. $\quad$ I9I

Arkwright, J. A. $\quad 36$ I

Arkwright, R. O.W. 36r

Armbruster, C. H. I9r

Armitage, B. W. F. $\quad 318$

Armitage, D. W. $\quad 233$

Armitage, E. G.H. 233

Armitage, F. R. $\quad 233$

Armitage, N.C. $\quad 36 r$

Armitage, V.H. $\quad 234$

Armitage, W. A. $\quad 28$

Armitage, W. J. $\quad 85$

Armour, J. $\quad 527$

Armour, J. K. C.

Armstrong, C.

Armstrong, $\mathrm{E}$ W. 164

Armstrong, F. M.

Armstrong, G. F. $\quad 28$

Armstrong, H.M. $\quad 219$

Armstrong, H. R. $\quad 307$

19I Armstrong, J. C. 1 I 8

233 Armstrong, J. L. 307

481 Armstrong, J. R. B. 36r

Armstrong, M. D. $\quad 234$

Armstrong, M. R. L. $36 \mathrm{I}$

48 $\mathrm{I}$ Armstrong. R. R. $36 \mathrm{I}$

36I Armstrong, V. 234
Armstrong, W.E. 347

Arnallt-Jones, G. C. $36 \mathrm{I}$

Arnell, O.R, II8

Arnold, A. C. P. $\quad 219$

Arnold, A J. $\quad 62$

Arnold, B. M. $\quad 164$

Arnold $\mathrm{E}$. C.

Arnold J. C.

Arnold, L. M.

Arnold, W.

Arnold, W. B.

Arnot, D. W.

Arnott, E. W.

Arnott, J.

Arnott, R. J.

Aron, E. M.

Aron, F. A.

Arrowsmith, R. 
Arrowsmith, W. G. 118 Arthur, W. L.

Arundel, F. D.

Ascoli, G. H. D.

Aserman, C.

Ash, F. H.

Ash, G. B.

Ash, H. A.

Ash, O. A.

Ashburner, W.

Ashby, K. H.

Ashby, M. W.

Ashby, N.

Ashcroft, A. H.

Ashcroft, F.

Ashcroft, J. M.

Ashcroft, R. L.

Ashcroft, W.

Ashington, H. S.O.

Ashley, N.D.

Ashton, A. A. G.

Ashton, E. G.

Ashworth, J.

Askew, E. J.P.

Askey, A. H.

Askey, S. G.

Aspinall G.

Aspinall, J. R.

Aspinwall, G.R.

Assheton, R. T.

Astbury, H.S.

Astbury, S. J.

Astbury, W. T.

Astley, B. A. F.

Astley, W.

Aston, F. M.

Aston, R. L.

Aston, W. D.

Atchison, G. T.

Atherton, E. C.

Atherton, T. J.

Athill, C. R. W.

Athill, F.R. I.

Atkey, F.A.H.

Atkey, J.F.H.

Atkey, S. C.

Atkin, C.S.

Atkin, E. E.

Atkin, $\mathrm{K}$.

Atkins, B. S.

Atkins, E. M.

Atkins, G. R.

Atkinson, A. G.

Atkinson, A.V.

Atkinson, E.W.

Atkinson, $\mathbf{G}$.

Atkinson, G. B.

Atkinson, G. H.

Atkinson, H. B.

Atkinson, H. G.

Atkinson, H.N.
Atkinson, J. C.

Atkinson, J. L.

Atkinson, M. L.

Atkinson, P. Y.

Atkinson, R. E.

Atkinson, R. H.

Attenborough, C. R. W.

234.

361

319

85

482

319

118

86

II 8

II8

II8

192

2

281

307

2

$36 \mathrm{r}$

319

319

36 I

482

28

$36 \mathrm{I}$

2

347

164

307

2

234

118

73

2

28

118

361

$36 \mathrm{I}$

234

234

482

118

118

164

118

307

347

361

219

118

319

482

$28 \mathrm{I}$

$36 \mathrm{r}$

347

319

A.

Audra, E. A. J.

Austin, H. W.

Austin, L. J.

Austin, $S$.

Austin, S. P.

Austin, $T$.

Avent, E.

Avent, M.

Averill, A. S.

Averill, T. H.

Averns, R. F. J.

Avery, G. C.

Avory, D. H.

Aydon, J.

Aykroyd, H. H.

Ayliff, J.

Aylwin, W.E.

Ayre, B. P.

Ayton, W. A.

Baber, J. B.

Baboneau, C. A.

Bacchus, J. G.

Bache, H. G.

Back, G. A.

Back, H. A. W.

Back, H. C.

Back, I. G.

Back, N.

Backus, A. R.

Bacon, D.F.C.
86

73

$36 \mathrm{I}$

86

291

362

234

28

86

86

319

86

234

234

234

234

234

118

319

482

2

Austen-Cartmell,

Austen-Smith, $\mathbf{H}$.

Austin Sir W M. 28

Aylmer, E. K. G.

I 8

362

362

291

347

307

234

281

482

118

118

234

319

5 II

28

482

28

234

482

362

86

I64

362

362

347

347

II9

I 19

I 9

362

482

Backhouse, H. E. $\quad 362$

Backhouse, M. R. C. 482
Bacon, $F$.

362

Bacon, F. R. $\quad 362$

Bacon, J. N. H. $\quad 511$

Bacon, R. C. $\quad 362$

Badcock, A. L. $\quad 319$

Badcock, G. E. $\quad 234$

Baerlein, H. P. D. $\quad 362$

Baerlein, O. F. $\quad 362$

Bagenal, N. B. $\quad 192$

Baggallay, M.E. C. $\quad 362$

Bagge, Sir A. W. F. 192

Bagge, H. P. $\quad 28$

Bagley, A. B. $\quad 234$

Bagnall, G. B. $\quad 234$

Bagnall, J. M. V. $\quad 527$

Bagnall, R. O. G. 219

Bagnall-Wild, R.K. I I 9

Bagot-Chester,H.A. 219

Bagshaw, W. H. $\quad 234$

Bagshawe, H. V. $\quad 192$

Bahr, P.H. $\quad 362$

Baikie, R. $\quad 28$

Bailey, B. F. $\quad 28$

Bailey, C. M. II9

Bailey, E. B. $\quad 28$

Bailey, F. G. G. $\quad 362$

Bailey, M. A. $\quad 28$

Bailey, P. G. $\quad 28$

Bailey, P. J. $\quad 482$

Bailey, R.F. $\quad 192$

Bailey, T.B. $\quad$ I 19

Bailey, T.E. G. $\quad 28$

Bailey, W. G. W. $29 \mathrm{I}$

Bailey, W.N. $\quad 362$

Bailey-Hawkins, A. $\mathrm{G}$.

Baillie, A. H.

482

Baillie, A L T T

Baillieu, H.L. $\quad 164$

Baily, G. G. $\quad 319$

Baily, R.E.H. $\quad 234$

Bain, P.W. 219

Bainbridge, F. A. $\quad 362$

Bainbridge, O.J. $\quad 192$

Bainbridge-Bell, L. H.

Bainbridge-Bell, W. D.

Bainbrigge, P. G. $\quad 362$

Baines, A. B. 2

Baines, D. L. 2

Baines, J. T. $\quad 362$

Baines, M. T. $\quad 482$

Baines, W. $\quad 73$

Baird, R. F. $\quad$ I 9

Baker, A. F. W. $\quad 482$

Baker, A. T. B. $\quad 86$

Baker, D.

see Bendigo, Bp. of

Baker, E.

Baker, E. C. 
Baker, E. T. L.

Baker, F. B.

Baker, F. R.

Baker, M. W.

Baker, P.

Baker, R. L.

Baker, S.

Baker, S. E.

Baker, T. Y.

Bakewell, B.

Bakewell, G. V.

Baldwin, C. W.

Baldwin, F. E. W. $\quad 347$

Baldwin, H. J.

Baldwin, T. H.

Bales, P. G.

Balfour, A.

Balfour, A. M.

Balfour, C. M.

Balfour, J.

Balfour, M.

Balfour, R. N.

Balfour-Browne, F.

Ball, A. E.

Ball, G. R.

Ball, H. F.

Ball, O. J. H.

Ball, R. G.

Ball, S. H.

Ball, W. A. R.

Ball, W. V.

Ballamy, $\dot{H}$. W.

Ballance, $G$.

Ballance, L. A.

Ballingall, D.C. G.

Balshaw, N. K. see

Kesteven Balshaw, N.

Balston, F. W.

Baly, $\mathrm{H}$.

Balzarotti, G. P.

Bamford, A. J.

Bampfield, L. A.

Banbury, F.E.

Banbury, R. E.

Banham, V. G.

Banister, C. G.

Banister, C. W.

Banister, J. B.

Banister, T.E.

Banks, D. J.

Banks, E. H.

Banks, L.

Banks, $P$.

Banks, R. G.

Banks, W. E. H.

Bannatyne, A. G.

Bannatyne, E. J.

Bannerman, W.E.

$\begin{array}{ll}\text { Bannerman, W.E. } & 319 \\ \text { Banning, H. B.S. } & \text { I64 }\end{array}$

Banyard, F.E.

Banyard, J. H.
28

281

I I 9

319

307

482

II 9

347

2

2

28

164

86

73

28 I

II9

482

362

362

362

28

291

73

347

362

192

527

86

I 92

362

347

362

86

363

363

86

86

363

363

192

363

164

I64

234

86

2

363

234

r 92

II9

527
Baptie, N.

Barber, H. W.

Barber, J. R.

Barber, P. E.

Barber, R.W.

Barber, T.P.

Barber, W.E.

Barber-Starkey, C.C. 219

Barber-Starkey, F. W. G.

Barber-Starkey, R. J. K.

Barber-Starkey, W. H. J.

Barbour, G. B.

Barbour, R.

Barclay, A. E.

Barclay, A. V.

Barclay, C.

Barclay, E. D.

Barclay, G. A.

Barclay, G. W.

Barclay, H. F.

Barclay, H. G.(Trin.) 363

Barclay, H. G. (Trin. H.)

Barclay, J. A.

Barclay, M.E.

Barclay, R. L.

Barclay, R. W.

Barclay, T. H.

Barclay-Milne, J.

Bardwell, T.G.N. I64

Barff, F. R.

Barham, W.S.

Baring, $M$.

Bark, G. M.

Barker, A. (Christ's)

Barker, A. (Trin.)

Barker, A. L.

Barker, E. W.

Barker, F.

Barker, F. A.

Barker, F. W.

Barker, G. D.

Barker, G. P.

Barker, H. A.K.

Barker, K E. M.

Barker, L. E.H. R, Ir9

Barker, P. T.

Barker, R. A. (Cath.) 307

Barker, R. A. (Sid.) 347

Barker, R. I. P.

Barker, R. W.

Barker, T.W.

Barker, W.

Barker, W. B.

Barker-Hahlo, $\mathrm{H}$.

Barkworth, H. B.

Barkworth, R. C.

2 Barlow, A. W. L.
28

363

219

363

2

363

363

363

3 r 9

363

363

363

363

482

482

482

234

482

363

363

363

64

29

363
86

2

363

363

$29 \mathrm{I}$

5 I I

363

$28 \mathrm{I}$

192

29

I9

307

I 92

II 9

5 I I

62

347

363

234

219

363

Barlow, C. N.

Barlow, C. W.

Barlow, E. M.

Barlow, P.

Barlow, P.S.

Barlow, R. G.

Barltrop, E. A.

Barltrop, I. C.

234

Barnard F. B $\quad 73$

Barnard, H. D.

Barnard, H. J. 2

Barnard, Lord $\quad 363$

Barnato, J. H. W. $\quad 482$

Barnato, W.J. $\quad 482$

Barnes, $F$.

Barnes, F.P.

Barnes, G. G.

Barnes, J. E.

Barnes, J.E. T.

Barnes, J.S.

Barnes, R.S.

Barnes, T. H.

Barnes-Gorell, T.A.R.

Barnett, B. L.

5 I I

219

319

29
62

192

363

363

Barnett, B. L. T. $\quad 319$

Barnett, F.S. G. $\quad 86$

Barnett, R. O. $\quad$ I I9

Barnett, R. W. 235

Barnett, S. H. II9

Barnicoat, G.H. 5 II

Barnicot, H. . 235

Barningham, E. $\quad 364$

Barningham, H. $\quad 364$

Barningham, V. $\quad 235$

Barnsdale, J.D. $\quad 482$

Barnsley, D. G. $\quad 364$

Barnsley, R. E. $\quad 364$

Barnsley, T. K. $\quad 364$

Barr, A. G. $\quad 482$

Barr, G.

Barr, L. B. $\quad 5 \mathrm{II}$

Barraclough, J. N. II

Barraclough, N. E. $\quad 192$

Barraclough, R. F. $\quad 364$

Barran, C. A.

Barran, C. R. $\quad 364$

Barran, H. B. $\quad 235$

Barran, H. V. F. $\quad 364$

Barran, P. A.

Barratt, W. D. $\quad 364$

Barraud, V.H. A. $\quad \mathbf{5 2 7}$

Barrell, F. M. 235

Barrell, K. C. 235

Barrett, C. B. G. $\quad 5$ II

Barrett, F. G. $\quad 86$

Barrett, H.S. $\quad 319$

Barrett, J. H. $\quad 483$

Barrett, L. 
Barrett, R. C.

Barrett, R. O.

Barrett, R. S.

Barrett, W. E. C.

Barrett Greene, A.H. 319

Barringer, R. E. $\quad 483$

Barrington, F. E. P. 119

Barrington, W. R. S. 483

Barris, J. D.

Barritt, J. L.

Barritt, W. V.

Barron, E. A.W.

Barron, G. D.

Barrow, A. J.

Barrow, E. B. P

Barrow, H. E.

Barrow, P. L.

Barrow, R. L.

Barrow, R. M.

Barrow-in-Furness, Bp. of

Barrowclough, S.

Barry, A. G.

Barry, C. B.

Barry, Sir E.

Barry, E. G. Wolfe see Wolfe Barry, E. G.

Barry, K. A. Wolfe

see Wolfe Barry, K. A.

Barry, $T$.

Barstow, J. E. J. $\quad 235$

Barstow, J. N. 219

Bartholomew, G. T. 192

Barthropp,S.A.N.S. 364

Bartlett, A. C.

Bartlett, A. G.

Bartlett, A. W.

Bartlett, C. W.

Bartlett, D. M. M.

Bartlett, E. G.

Bartlett, G. B.

Bartlett, H.S.

Bartlett, L. A.

Bartlett, W. H.

Bartley, P. R.

Barton, B. B.

Barton, C. G.

Barton, C. H.

Barton, F.S.

Barton, G. R.

Barton, J. F.

Barton, R. A. E.

Barton, T. E.

Barton, W. J.

Bartram, $\mathrm{H}$.

Bartram, L. H.

Barwell, F. L.

Barwell, H. B.

Barwell, N.F.

Barwick, R. L.

Bashford, P.F. R.
19
86

319

364

Ir9

219

364
86

235

235

I 92

86

364

235

235

I 9

$G$.

364

19

87

307

2

527

29

364

347

I 19

307

319

483

235

I64

319

483

291

347

483

29

483

364

235

307

364

2
Bass, V. A.

Basset, A. F.

Bassett, H. Ll.

Bassett, P. R.

Bastow, F.

Batchelder, G. L

Batchelor, B. W.

Bate, A. G.

Bateman, R. J. S.

Bateman, W.

Bates, F. A.

Bates, G. L.

Bates, H. R.

Bates, H. S.

Bates, J. V.

Bateson, E. de Y.

Bather, A. G.

Bather, E. J.

Bathurst, C. R.

Bathurst, H. A.

Bathurst, P. L.

Batley, J.

Batley, R. C.

Batten, H. C. C.

Batten, H. C. G.

Batten, J. H. S.

Batten, L. W.

Batten, W. D. G.

Batterham, D. J.

Battiscombe, G.C II9

Batty, G. G. H.

Batty, W. R.

Batty-Smith, S. H.

Bawdon, R. H.

Bawdon, W.S.

Baxandall, A.

Baxter, C.W.

Baxter, F. H.

Baxter, T. $H$.

Baxter, W. H. B.

Bayer, S. F.

Bayley, C. H.

Bayliss, B. $\mathrm{H}$.

Bayliss, C. W.

Bayly, H. W.

Baynes, D. L. H.

Baynes, F. W. W.

Baynes, $G$.

Baynes, H. G.

Baynes, W. E. C.

Baynham, C.T.

Baynham, J. $\mathrm{H}$.

Bazett, S. C.

Beale, C. E.

Beale, C. W.

Beale, E.

Beale, E. V.

Beale, H. L.

Bealey, A. C.

Bealey, R.N.

Beament, W. O.
29

220

483

29

29

364

192

192

2
364
4

483

87

307

235

364

192

29

235

220

483

364

483

483

483

347

I I 9

$\begin{array}{r}364 \\ \hline\end{array}$

2

I 64
I I 9

29

87

512

364

87

192

235

I I 9

192

220

192

29

29

I 20

364

364
365

I 64

I64

29

319

120

28 I

I 92

365

365

29

512
Beamish, C. N. B.

87

Beamish, R de B. $\quad 120$

Bean, C. V.

Bean, J.W. B. $\quad 512$

Beanland, J. W. $\quad 512$

Beard, A. J. $\quad 319$

Beard, E. $\quad 528$

Beard, E. C. 319

Beard, R.H. 29

Beardmore, W. J. M. 29

Beasley, H.O.C. $\quad 164$

Beasley-Robinson, A. C.

365

Beattie, L. H. I $\quad$ I20

Beauchamp, Sir M. H.

Beaufoy, H. M. $\quad 365$

Beaumont, D.C. $\quad 3$

Beaumont, H. R. $\quad 220$

Beaumont, J. W. F. 235

Beaumont, O.A.

Beaumont, P. J. $\quad 528$

Beaumont, W.H.C. 365

Beaumont-Checkland, M. B.

Beaumont-Nesbitt, W. H.

320

Beazley, C. 5 I $\quad 3^{12}$

Becher, G. G. $\quad 365$

Becher, M. A. N. $\quad 365$

Beck, A. C. T. $\quad 164$

Beck, B. R. 29

Beck, C. B. H. 235,528

Beck, E. W.T. 29

Beck, F. G. M. 29

Beck, G. R. 3

Beck, H. M. $\quad 192$

Becker, W. T. L. $\quad 235$

Beckett, C. M. $\quad 120$

Beckett, P. A. $\quad 29$

Beckett, V. L.S. 3

Beckh, R. H. $\quad 164$

Beckley, V.A. $\quad 320$

Bedale, C. L. $\quad 29$

Bedale, F.S. $\quad 29$

Beddington, C. $\quad 365$

Beddington, F. $\quad 193$

Beddow, H. J. 3

Bedell-Sivright,D.R. 365

Bedford, D. J. $\quad 365$

Bedford, S. H. $\quad$ I2O

Bedworth, A. C. $\quad 73$

Beech, J. $\quad 120$

Beechey, C. R. $\quad 320$

Beecroft, A. E. $\quad 3$

Beedham, H. W. 3

Beer, A. H. $\quad 365$

Beer, H. O. $\quad 120$

Beeton, A. E. $\quad 365$

Beevor, R. B. $\quad 87$ 
Begg, M. G.

Beith, I. H.

Belcher, G.

Belfield, S. St G. C.

Bell, A. C.

Bell, A. F.

Bell, A.S.

Bell, A. T.

Bell, A. W.

Bell, C. H.

Bell, C. W. D.

Bell, D. W.

Bell, G. M.

Bell, H. A.

Bell, J. A.

Bell, J. A. H.

Bell, J. D.

Bell, J. H. (Clare)

Bell, J. H. (Selwyn)

Bell, J. J.

Bell, L. B.

Bell, R. H.

Bell, R. L.

Bell, T. O.

Bell, W. A.

Bell, W. A. J.

Bell, W. B.

Bell, W. G.

Bell, W. H. D.

Bell, W. S.

Bellamy, J.

Bellars, A. E.

Bellerby, A. C. B.

Bellew, B. B.

Bellew, E. H.

Bellhouse, A. P.

Bellhouse, S.

Bellman, A. F.

Bellward, G. W. F.

Bellwood, K. B.

Belper, Lord

Belville, G. E.

Bemrose, R. H.

Benbow, $\mathrm{H}$.

Bendall, F. W. D.

Bendigo, Bp. of

Benest, E. E.

Beney, C. C.

Bengough, G. D.

Bengough, J. C.

Bengough Clark, J. see Clark, J. B.

Benham, J. H.F.

Benjamin, C. M.

Benjamin, J. A.

Benjamin, R. N.

Bennett, C. W.

Bennett, D.

Bennett, E.

Bennett, E. B.

Bennett, E. K.
120

320

307

120

I 64

235

483

235

87

365

365
29

365

I 64

73

3

I 20

29

512

365

$5 \times 2$

62

28 I

320

347

483

I64

282

365

282

I64

220

87

483

483

I65

87

320

87

235

365

365

29

3

512

528

120

I 65

512

87

165

365

29

30

320

483

366

3

I 20
Bennett, F. G.

Bennett, G. L.

Bennett, G. M.

Bennett, G. N.

Bennett, G.S.

Bennett, H. C. L.

Bennett, H. H. G.

Bennett, H. R.

Bennett, J. C. S.

Bennett, K. L.

Bennett, R.

Bennett, S. G.

Bennett, S. L.

Bennett, T. C.

Bennett, W. E.

Bennett, W. P.

Bennett-Evans, G. L. 73

Bennion, C. F.

Bennion, J. M.

Bennitt, F. W.

Benoly, H. J.

Benoy, J.

Benoy, J. F.

Bensly, W. J.

Benson, A. H.

Benson, C. D.

Benson, C. T. V.

Benson, G. E.

Benson, H. W.

Benstead, A.S.

Benstead, C. R.

Bensted-Smith W.F.

Bentall, C. E.

Benthall, E. C.

Bentinck, A. W. D.

Bentinck, H. D. A.

Bentley, A. J.

Bentley, J. D.

Bentley, J. H.

Bentley, R. C.

Bentley, W. W.

Bentliff, H. D.

Benton, D.

Bentwich, N. de M.

Benyon, H. A.

Berens, H. A.

Beresford, C. V.

Beresford, C. W.

Beresford, G. A.

Beresford, J. B.

Beresford,M.dela P

Beresford T dela P. 48

Berg, A. W.

Berger, S. H.

Bergheim, $P$.

Berkeley, M. K. F.

Berlein, L. H.

Bernard, D.V.

Bernard, H. C.

Berney, R. G. G.

Berney, Sir T.R.
3
3
483
483

347

30

87

120

307

30
483

73

235

512

347

87

235

320

235

3

320

320

120

30

193

320

87

320

308

I 20

193

366

366

320
87

320

292

292

366

87

366

483

347

483

483

320

193

483

366

366

87

236

220

62

320

483

483 $\begin{array}{lr}\text { Berney-Ficklin, } & \\ \quad \text { A. T. M. } & 30 \\ \text { Berney-Ficklin, } & \\ \quad \text { H. P. M. } & 165 \\ \text { Berrington, K. C. } & 236 \\ \text { Berry, A. } & 193 \\ \text { Berry, A. J. } & 120 \\ \text { Berry, B. A. } & 282 \\ \text { Berry, H. S. } & 30 \\ \text { Berry, H. V. } & 120 \\ \text { Berry, P. H. } & 193 \\ \text { Berry, W. } & 193 \\ \text { Berry, W. L. } & 120 \\ \text { Berwick, E. B. H. } & 308 \\ \text { Besant, G. B. } & 120 \\ \text { Bescoby, A. C. } & 87 \\ \text { Besly, E. M. } & 120 \\ \text { Bebsor. } & 1206\end{array}$

Bessborough, Earl of 366

Best, J.

Best, J. K. 292

Best, O. H. 292

Best, R. W. G. $\quad 282$

Best, T.W. $\quad 366$

Beswick, W. T. $\quad 483$

Bethell, C. $\quad 366$

Bethell, D. J. $\quad 366$

Bethune, F.P. $\quad 512$

Bethune, J. W. $\quad 512$

Bethway, W. S. $\quad 366$

Betteridge, B. F.

Betteridge, C. D. $\quad 165$

Betteridge, H. G.W. 3

Betteridge, J. E. H. $\quad 165$

Bettington, A. F. $\quad 87$

Betton, H.R. B. $\quad 512$

Bevan, A. E. $\quad 193$

Bevan, C. B. 30

Bevan, E. J. $\quad 320$

Bevan, F. H. V.

Bevan, G.T.M. 320

Bevan, H. E. J. $\quad 320$

Bevan, J.

Bevan, J. M.

Bevan, T. R.

Bevan, W. H.

I 65

120

366

Bevan-Brown, C.M. 85

Bevan-Brown, R. E. 193

Bevan-Petman, B. A. 193

Bevan-Petman, B. H. 193

Beveridge, D. A. $\quad 236$

Beveridge, E. W. $\quad 193$

Beveridge, $\mathrm{G}$.

Beveridge, $\mathrm{H}$.

Beverley, R.

Beves, C. H.

Bevir, C. E. F.

Bevir, W.

Bewes, C. T.A.

Bewicke-Copley, R. G. W.

Bewley, E.N. 
Beyts, C. A.

Bhatia, S. L.

Bibby, R.E.

Bickerdike, R. B.

Bickersteth, R. A.

Bickerton, H. R.

Bickerton, J. M.

Bicket, T. B.

Bickford-Smith, W. N.

Bickford-Smith, W. N. V.

Bickley, G. H.

Bicknell, C.H.

Bicknell, P. W.

Bicknell, R. A. W.

Bicknell, R.P.

Bidder, H. F.

Biddle, F. A.

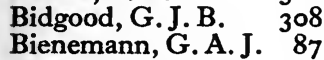

Biggar, C. L. P.

Bigge, H. J.

Bigger, W. G.

Bigger, W. K.

Biggs, E. K.

Bigg-Wither, H.S.

Bigland, A. D.

Bignold, C. S.

Bilderbeck,A.C.L.O'S. 3

Billing, E.

Billinger, H. F.

Billinghurst, W. B. 320

Billinton, $\mathrm{H}$

Bilney, A. A. H.

Bilsborough, J. H.

Bilsland, A. S.

Bilton, C. H. E.

Bilton, E. B.

Bindloss, A. H.

Bindloss, W.

Bindon, W. F. V.

Binks, B. B.

Binks, B. H.

Binney, J.

Binning, Lord

Binns, A. L.

Binyon, $B$.

Bion, R. E.

Birch, A. G.

Birch, F. L.

Birch, J. R.

Birch, W. C.

Birch, W. C. $\quad{ }^{193}$

Bird, A. K.

Bird, C. $B$.

Bird, C. $K$.

Bird, E. G.

Bird, G. F.

Bird, H. J. G.
$236 \mid$ Bird, L. W.

282 Bird, M. B.

236

120

366

484

30

120

236

236

I65

366

236

366

366

366

292

87

30

3

120 30

121

I2I

30

366

512

320

30

30

320

165

347

3

292

484

367

320

367

367

367

I93

30

87

236

193

528

367

528

Bird, $\mathrm{S}$.

Birkbeck, J.

Black, J. A.

Black, J. C.

Black, J. N.

Black, L. P.

Black, $P$. C. I. G. F.

Blackie, A.
Bird, M.W. K.

Bird, P. C. H.

Birdwood, G. T

Birdwood, H. B.

Birdwood, H. F.

Birkbeck, G.

Birkbeck, G. W.

Birkbeck, H. A.

Birkbeck, M

Birkett, G. E.

Birks, A. H.

Birks, C. T. E.

Birley, O. H.J.

Birmingham, Bp. of 308

Birnstingl, C. A. $\quad 3 \circ$

Biscoe, V.F.

Bisdee, J.S. M.

Bishop, C. G.

Bishop, G.S.

Bishop, J. H.

Bishop, P. F.

Bishop, R. O.

Bispham, C.

Bispham, J.W.

Biss, H. C. J.

Biss, J. C. de V.

Bissett, C. C.

Black, D. C.

Black, F. G.

Black, G. D. A.

Black, L. L. D.

Black, M. A.

Black, R. A.

Black, R. B.

Black, R. P.

Black, S. G.

Blackburn, A

Blackburn, B. R.

Blackburn, G. G.

30

320

236

282

367

30

3

484

87

87

220

87

367

236

30

121

367

30

292

484

3

121

3

30

367

320

367

308

484

Blackburn, L. O. G. 484

Blackburn, W. H.

Blackburn-Maze,

Blackburne, E. V.

Blackburne, $H$. W.

Blackburne-Daniell,

Blackden, S. C.

Blacker, H. A. C.

Blackett, B. J.

Blackett, G. E.
Blackledge, R. D.

Blacklock, G. H.

Blackmore, J. K.

Blackwall, J.E.

Blackwall, W. A.

Bladon, J. W.

Bladon, M. W. B. $\quad 30$

Bladwell, E. W. $\quad 320$

Blaikie, C. J.

Blaine, F. H.

Blaine, H. D.

Blair, Â.

Blair, G. Y.

Blair, H. M.

Blair, H.S.P.

Blair, P. C. B.

Blake, A. G.

Blake, A. L.

Blake, C.

Blake, F. E. C. $\quad 484$

Blake, G.P. $\quad 367$

Blake, P. J. $\quad 236$

Blake, T.R.H. $\quad 236$

Blakeley, F. R. $\quad 320$

Blakeney, E.P. $\quad 367$

Blakeney, J. E. C. $\quad 282$

Blaker, R. N. R. $\quad 165$

Blakeston, B. M. $\quad 5 \mathrm{I2}$

Blakiston, A. F. $\quad 88$

Blakiston, B. R. $\quad 220$

Blakiston, J. N. 220

Blamire-Brown, C. 220

Blanch, N. H.

Blanchard, J. F. J. $\quad 88$

Blanchard, N. I 2 I

Blanco-White, G. K. 367

$\begin{array}{lr}\text { Bland, A. J. T. } & 367 \\ \text { Bland, B.S. } & 88\end{array}$

Bland, $C$.

Bland, E. C.

Bland, J.C.

see Cooper Bland, J.

Blandford, J. J. G. $\quad 236$

Blandy, R.

I 2 I

Blatherwick, R. $\quad 30$

Blathwayt, F. W. $\quad 236$

Blaxland, J. B. $\quad 512$

Blaxter, A. P. Ll. $\quad 32$ I

Blaxter, K. W. 220

Bleasdell, J. T. $\quad 512$

Blech, E. L. $\quad$ I93

Blee, E. L. $\quad 292$

Blew, C. L. I2I

Blew, K. $\quad 236$

Bligh, E.

Bligh, E. H. S.

3

Bliss, A. E. D.

Bliss, E. W.

Bliss, F. K.

Blockey, H. S. 
Blom, A. H.

Bloomfield E A R R 367

Blow, A. E.

Bloxham, L. A.

Blucke, R.S.

Bluett, D. C.

Bluett, T. L. C.

Blumhardt, E. H. F. see Mills, E. H. F.

Blumhardt, R. A. see Mills, R. A.

Blundell, E. K.

Blunt, A.S. V.

Blunt, D. L.

Blyth, A. C.

Blyth, M. M.

Boardman, $\mathbf{H}$.

Boardman, J. H.

Boardman, T. H.

Bock, E. N.

Boddington, O. W.

Boddington, V. C.

Boddy, J. A. V.

Bode, J. E. V.

Boden, J. F. W.

Bodenham, H. E. C. H.

Bodey, A. R.

Bodington, C. $\mathrm{H}$.

Bodley, A. L.

Bodley, D. H.

Bodley, D.H. 237

Bold, T. A. $\quad 62$

Bolingbroke, C. B. $\quad 88$

Bolitho, G. R. $\quad 367$

Bolitho, T. G. G. $\quad 367$

Bolland, J. F.

Bolton, D. C.

Bolton, M. B.

Bolton, $\mathbf{P}$.

Bolton, $\mathrm{T}$.

Bolton, W.S.

Bomford, J. F.

Bompas, H. S.

Bond, A. C.

Bond, B. W.

Bond, C. E.

Bond, C. G.

Bond, D.

Bond, G. W.

Bond, J.S.

Bone, $\mathrm{H}$.

512

193

512

88

I 2 I

282

121

88

88

512

165

367

12 I

282

31

62

88

31
368

237

193

528

368

I21

237

368

321

121

368

220

31

88

292

368

Bonham, E. H.

Bonham Carter, A.T. 368

Bonham Carter, F.H. 368

Bonham-Christie, R. A.

Bonhote, E. F.

Bonnalie, F. E.

Bonner, C. A. J.

Bonomi, J. I.

31

88

484
Bonser, G. A. G.

Bonser, W. J.

Bonsey, W. H.

Bonvalot, A. C.

Bonvalot, E. St L.

Booker, G. H.

Bookless, J.S.

Boot, J. C.

Boote, C. W.

Booth, A. F.

Booth, C. H. B.

Booth, C. Z. M.

Booth, E.

Booth, H. F.

Booth, T. M.

Booth, W. R.

Boothman, J. H. E. D.

Booty, M. G. R.

Borrett, P. R.

Borthwick, C. H.

Borthwick, L. C.

Borton, C. E.

Bosanquet, A. R.

Bosanquet, W. S. B 368

Boscawen, M. T

Boscawen, V. D

Bostock, G. E.

Bostock, S. C.

Bostock-Hill, A. J.

Boston, L. J. 368

Boswell, D. St G. K. 237

Boswell, P. R.

Botham, A. F.

Bott, J. A.

Bott, W. E.

Botting, C. G.

Bottome, G. M.

Bottomley, A. C.

Bottomley E. W.

Bottomley, R. A. A

Botwood, E. K. 292

Boucher, A. E. $\quad \mathbf{5 2 8}$

Boucher, R. E. 3 I

Boughey, C. L. F. $\quad 368$

Boulderson, $\mathrm{G}$.

Boulenger, C. L.

Boultbee, A. E.

Boultbee, B. St

Boulter, C. S. C

Boulton, $\mathrm{H}$.

Boulton, R. J.

Bouquet, A. C. $\quad 165,368$

Bourchier, B. G. $\quad 292$

Bourchier, Le G. C. 368

Bourdillon, G. L. $\quad 512$

Bourdillon, T. E.

Bourne, A.S.
Bourne, A. W.

Bourne, C.
321

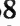

308

$8^{4}$

512
321

368
368

220

64

88

4
93

368

321

368
62

2

308

4

348

348

68

368

368

88

I

292

31

512

88

4

3 I

93

8

8

31

193

62

3 I

I

Bo

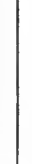

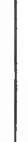

Bourne, J. C.

Bourne, L.P.S. $\quad 34^{4}$

Bousfield, H.T. W.

Bousfield, J. K. 121

Bousfield, L. $\quad 237$

Bousfield, R. B. $\quad 121$

Bousfield, S. $\quad$ I2I

Bousfield, W.E. $\quad$ I2I

Bouwens, B. G. $\quad 368$

Bovey, F. H. W. $\quad 484$

Bovill, E. H. 237

Bovill, E. W. $\quad 368$

Bovill, J. E. 237

Bowcher, F. H. $\quad 3 \mathrm{r}$

Bowden, E. R. $\quad 237$

Bowdler, A. P. $\quad 348$

Bowdon, W.S. $\quad 321$

Bowe, J.H. $\quad 165$

Bowen, A. G. W. 193

Bowen, G.

Bowen, J. B. $\quad 368$

Bowen, L. H. $\quad 32 \mathrm{I}$

Bowen, T.S. $\quad 31$

Bowen Colthurst, R. $M$.

Bowen-Davies, J

Bower, C. F. J. 73

Bower, C. W.

Bower, F. $\quad 308$

Bower, H. J. $\quad 88$

Bower, H. M. $\quad 368$

Bowes, G. B. $\quad 88$

Bowes-Lyon, G. P. 368

Bowes-Lyon, M. $\quad 484$

Bowes-Lyon, P. $\quad 369$

Bowker, R. C. S. $\quad 88$

Bowlby, R. F. $\quad 369$

Bowle, C. W. $\quad 369$

Bowle-Evans, C. H. 88

Bowler, S. $\quad 528$

Bowles, H. F. $\quad 165$

Bowles, R. J. A. 3I

Bowman, F. $\quad 369$

Bowman, G. H. $\quad 369$

Bowman Vaughan, E. W. see Vaughan, E. W. B.

Bowring, H. $\quad 193$

Bowring, J.F. E. $\quad 369$

Boyce, A.H. 308

Boycott, A. G. $\quad 5 \mathrm{I} 3$

Boyd, C.W. $\quad 308$

Boyd, E. J. $\quad 348$

Boyd, G. J. $\quad 484$

Boyd, H. A. $\quad 369$

Boyd, H. J. $\quad 88$

$\begin{array}{lr}\text { Boyd, J. } & 369 \\ \text { Boyd-Carpenter, Bp. } 308\end{array}$

Boyd-Carpenter, J.P. 308

Boyd Rochfort, G. A. 
Boyer, G. W. B.

Boyle, G. E.

Boyle, G. F.

Boyle, G. L.

Boyle, J.

Boyle, R. F. R.P.

Boyne, Viscount

Boys, G. V.

Boyson, H. A.

Boyson, J. C.

Boyton, H. J.

Bozman, E. F.

Brabazon, C. M. P.

Braby, H. W.

Bracecamp, F. W.

Brachi, C. C.

Bracken, R. J.M. E. I2I

Brackett, A. W. K. 32 I

Bradbury, J. B. 74, 121

Bradbury, J. F. $\quad$ r 94

Bradfield, L. G. 12 I

Bradfield, R. I2I

Bradford, Earl of $\quad 369$

Bradford, E. C. $\quad 237$

Bradley, A. S. I2I

Bradley, E. J. (Jesus) I65

Bradley, E. J. (Pem.) 237

Bradley, G. H. $\quad 528$

Bradley, G. M. $\quad 165$

Bradley, H.E. $\quad 237$

Bradley, M. G. $\quad 369$

Bradley, S. B. I2I

Bradley, V.M. $\quad 165$

Bradley, W.de W.H. 165

Bradney, J. A.

Bradshaw, P.C. $\quad 369$

Bradshaw, R. E. K. $\quad 369$

Bradshaw, T. B. 237

Bradshaw, W. D. $\quad 369$

Bradshaw-Isherwood, F.E.

Bradstock, G.

Bragg, R. C.

Bragg, W.H.

Bragg, W. L.

Brailey, A. R.

Brailey, W. H.

Brailsford R W 292

Braimbridge, C. V.

Brain, F.S.

Braithwaite, C. F.

Braithwaite, J. G.

Braithwaite, P. P.

Bramall, E. H.

Brameld, A. J. M.

Bramwell, B.S.

Bramwell, J. C.

Brandon, A. de B.

Branson, F. H. E.

Branson, J. R. B.

Branson, W. P.S.

308

369

369

484

369

369

369
Branston, C. A.

Branston, R.

Brash, E. J. Y.

Brasnett, T. J. G.

Brass, W.

Bratton, A. B.

Braun, G. C. P.

Braunholtz, E. J. K. 194

Braunholtz, G.E.K. 88

Braunholtz, H.J. 321

Braunholtz,W.T.K. 370

Brawn, J. A.

Bray, E. F.

Bray, Sir E. H.

Bray, F. E.

Bray, J.

Brayne, F. L.

Brayne, W.F.

Brearley, A. J.

Bree, H. R. S.

Breed, F. G.

Breese, W. L.

Bremner, F. D.H. $\quad 370$

Brenan, A. R. M. $\quad 370$

Brend, W. A. $\quad 348$

Brereton, J. L. $\quad 370$

Bretherton, $\mathrm{H}$. $\quad 370$

Brett, J.H.

Breul, O. G. F. J. $\quad 370$

Brewer, C. H.

Brewer, F. G.

Brewer, J.

Brewis, C. C.

Brewster, G. W.

Brian, F.R.H.

.

Brice-Smith, H. F. $32 \mathrm{I}$

Brice-Smith, J. K. 321

Brickwood, H. $\quad 282$

Bridge, E. A.

Bridgeman, R. O. $\quad 37{ }^{\circ}$

Bridges, L. W.

Bridgwater, H.N. 485

Brierley, W. B.

Briggs, G. C.

Briggs, G. E.

Briggs, G. R.

Briggs, H.W.

Briggs, J. L.

Briggs, $M$.

Briggs, P. J.

Briggs, R.S.

Briggs, W. A.

Briggs, W. R.

Briggs Gooderham, E. J.R. see
Gooderham, E. J.R.B.

Bright, F.T.

Bright, W. A.

Bright, W. J.

Brightman,

Brigley, C. G.
Brindle, W.S.

370

Brinton, R. D.

Briscoe, F. E.

74

292

Briscoe, F. E. T. $\quad 237$

Briscoe, J.R. $\quad 513$

Briscoe, W. T. $\quad 513$

Brisley, C.E. $\quad 122$

Bristed, G. T. $\quad 194$

Bristow, C. H. $\quad 4$

Brittain, A. W. 4

Brittain, E.S. $\quad 122$

Broad, A. M. $\quad 122$

Broad, C. N. F. $\quad 237$

Broadbent, C. H. $\quad 370$

Broadbent, E. R. $\quad 122$

Broadbent, F. M. $\quad 220$

Broadbent, H. G. 292

Broadbent, W. $\quad 370$

Broadmead, H.H. $\quad$ I 22

Brock, A. G. $\quad 237$

Brock, E. G. $\quad 321$

Brocklebank, J. J. $\quad 370$

Brocklehurst, E. H. $\quad 485$

Brocklehurst, $\mathrm{H}$. $\quad 4$

Brocklehurst,

Sir P. L.

485

Brocklehurst, T.P. $\quad 485$

Brockman, E. P. $\quad 122$

Brockman, R. St L. 122

Brockman, W. D. $\quad 122$

Brode, R. T.

Brodie, E. J

Brodie, H. W. 31

Brodie, M. M. 292

Brodie, $\mathrm{P}$. 3 I

Brodsky, G. A. $\quad 370$

Brogden, G.A. 31

Brogden, J.R. R. $\quad 31$

Brölemann, P. W. A. 237

Bromet, E. I22

Bromet, J. N. 3 I

Bromhead, J.P. $\quad 348$

Bromley, L. I $\quad$ I22

Bromley-Davenport, H. R.

Brook, A. K. $\quad \begin{aligned} & 485 \\ & 165\end{aligned}$

Brook, R. 74

Brooke, B. W. D. $\quad 370$

Brooke, F. N. $\quad 237$

Brooke, H.K. $\quad 370$

Brooke, J. 89

Brooke, J. C. 122

Brooke, R. C. $\quad 194$

Brooke, W. A. C. $\quad 194$

Brooke, Z. N. I22, 32 I

Brooke Taylor, G. P. 237

Brooker, R. H. G. $\quad 237$

Brookes, A. $\quad \mathbf{5 2 8}$

Brookes, H. V. $\quad 89$

Brooks, C. D. $\quad 194$

308 Brooks, D. C. M. 194 


\begin{tabular}{|c|c|c|c|c|c|}
\hline 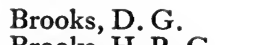 & 165 & wn, R. P. & 371 & W & \\
\hline Brooks, H. R. C & 370 & Brown, R. W. & 122 & W. & 16 \\
\hline oks, L. W. & 32 & rown, S. R. & 321 & & 194 \\
\hline rooksbank, S. & 485 & Brown, S. V & 74 & Bryan Bror & $7 . \quad 2>$ \\
\hline rooman-Whit & .485 & Brown, $T$. & 123 & nt, $\mathrm{G}$ & 0 \\
\hline ner, $A$ & 528 & Brown, T. B. & 371 & Bryant, V. & 7 \\
\hline nfield, R.S. & 4 & Brown, T. H. & 238 & Bryce, W. ? & 3 \\
\hline , G.M. & 220 & n, V.S. & 166 & son, $\mathrm{E}$ & \\
\hline $\begin{array}{l}\text { ter, E. D. } \\
\text { hers, M. }\end{array}$ & 32 & $\begin{array}{l}\text { vn, W. B. } \\
\text { vn, W. E. I }\end{array}$ & $\begin{array}{l}194 \\
371\end{array}$ & $\begin{array}{l}\text { ne, } \\
\text { nan }\end{array}$ & \\
\hline h, J.S. B. & 89 & n, W. L & 321 & nan & 37 \\
\hline hton, A. D. & 122 & Brown, W. R. R. & 513 & anan, & \\
\hline & 485 & Brown, W.S & 123 & a & 23 \\
\hline A. & 122 & Brown Douglas & 371 & & 32 \\
\hline 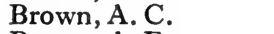 & r94 & Browne, A. D. & 292 & na & 48 \\
\hline $1, \ldots$ & 4 & e, A.W. & 485 & Mat & 37 \\
\hline A & 370 & e, B. & 321 & G. & 6 \\
\hline 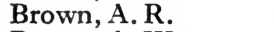 & 122 & $\mathrm{Br}$ & 371 & 11, E & 12 \\
\hline 1 & 89 & , C. R. & 89 & dina, & 37 \\
\hline A. W.S. & 122 & e, G. B. & 123 & and," & 371 \\
\hline , C.A. & 4 & G. H. & $23^{8}$ & & 194 \\
\hline . & 122 & M. & 371 & $\pi$ & 37 \\
\hline Clifton & 370 & M. G & 371 & $x+2+$ & 48 \\
\hline L. I & 308 & e, O. L & $37 \mathrm{r}$ & & 52 \\
\hline & 321 & wne, R. C.S & & & I9. \\
\hline lifton & 370 & Seymour-Brc & & & \\
\hline , E. E & 122 & R.C. & & & 30 \\
\hline$F$ & 237 & Browne, R. & 89 & B & 29 \\
\hline .) & 321 & & 321 & & $22 C$ \\
\hline & 370 & & $5 \times 3$ & & 37 \\
\hline & $5 \div 3$ & & 321 & & 48 \\
\hline 3 & I94 & & 321 & & 372 \\
\hline & 122 & & 371 & & 322 \\
\hline & 166 & & 4 & W.S. & 372 \\
\hline [. $(\mathrm{J}$ & I66 & 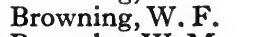 & 513 & O & 48 \\
\hline (Kg's) & 194 & Brownlee, W. M & 62 & & 3 \\
\hline R. J. & 32 & ison,R.D.D.D & $\cdot 322$ & & 89 \\
\hline & 4 & $B$ & 123 & & \\
\hline 1.) & 74 & & 32 & & 238 \\
\hline est & 166 & A. A. & 308 & & 348 \\
\hline & 89 & S. M. & 485 & & 372 \\
\hline B. & 371 & Adam, D. & 63 & 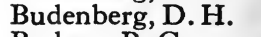 & 372 \\
\hline & 485 & ,W.R. & 371 & & 63 \\
\hline $\mathrm{C}$ & .) 89 & $\mathrm{Br}$ & 485 & & 372 \\
\hline ( $(\mathrm{T}$ & $37 \mathrm{I}$ & Bru & & & 292 \\
\hline & 122 & Lo & & & 29 \\
\hline & 122 & Bruce $\mathrm{Lr}$ & & $\mathrm{Bu}$ & 220 \\
\hline & 485 & & 63 & & 372 \\
\hline & 74 & B & & & 123 \\
\hline & 348 & & 371 & & 308 \\
\hline D. & 238 & & 348 & & 123 \\
\hline & 32 & & 194 & & 485 \\
\hline n, J. L. C. & 371 & B & I 94 & & 485 \\
\hline & & & 371 & & 292 \\
\hline & 166 & & 32 & & 322 \\
\hline & & Q.H.P. & 371 & & 166 \\
\hline C. & 166 & & 371 & & 372 \\
\hline . A.S. & 485 & Brt & 194 & P. & 372 \\
\hline & & & .166 & & 372 \\
\hline & 89 & Hales, & .166 & $\mathrm{ek}, \mathrm{G}$. & 63 \\
\hline rown, & $5 \times 3$ & Brutton, H. L & 371 & Bullo & 2 \\
\hline
\end{tabular}


Bullock, H. M.

Bullock, J. C.

372

123

Bullough, E.

Bullough, J. L.

Bulmer, A. C.

Bulmer, P. H.

Bulstrode, C. V.

Bulstrode, R.

Bulwer, E. A 238

Bunbury, C. H. N. 372

Bunbury, H. W. $\quad 485$

Bunbury, T. L. McC.

see McClintock Bunbury, T. L.

Bundy, H.P.

Bunn, F. A.

Bunt, A. P.

Bunting, S. A. S.

Burbidge, E. d'A.

Burbidge, P.W.

Burbury, F. W.

Burch, R.S.

Burchell, J. M.

Burder, G. E. L.

Burdett, A. F.

Burdett, Sir F.

Burdett, H. G.

Burdon, J. A.

Burdon, $R$.

Burdon, W. W.

Burfield, S. T.

Burrowes, R. V.

Burges, W.E.P.

220

5I3

322

84

372

238

89

372

372

308

372

238

4

322

485

513

513

372

Burges-Bayly, A. R.

Burges Short,H.G.R.238

Burgess, A. S.

Burgess, D.

Burgess, O. I.

Burgess, R.

Burgess, W. C.

Burgoyne, L. S.

Burgoyne-Johnson, F. W.

Burgoyne-Johnson, L. V.

Burke, H. F.

Burke, M. L.

Burkitt, F.

Burkitt, F. T.

Burkitt, H. J.

Burling, E. J.P.

Burlison, J. C.

Burn, A. E.

Burn, C. J.

Burn, D.C.

Burn, J.H.

Burn, J.S.

Burn-Callender, $\mathbf{F}$.

Burn-Murdoch, A.

Burn-Murdoch, $\mathrm{H}$.

Burnaby, G.

123

I94

194

194

89

4

4

322

32

372

123

238

89

372

32

372

372

373
Burnaby, H. B. F.

Burnaby, J.

Burnand, C. F.

Burnand, G. C.

Burnard, C. F.

Burne, S. A. H.

Burnell, E. W.

Burnet, J. R. W.

Burnett, A. E.

Burnett, F. E.

Burns, J. W.

Burnside, B.

Burr, A. L.

Burr, F. G.

Burrell, E. M.

Burrell, G. P.

Burrell, H. A.

Burrell, J. H.

Burrell, L.S.T.

Burrell, R. E.

Burrell, R. F. T.

Burrill-Robinson, W.R.

Burrough, J.

Burroughes, H. N.

Burrow, W. J. A.

Burrows, A. H.

Burrows, C.

Burton, A.

Burton, A. H. W.

Burton, B. L.E.

Burton, D. C. F.

Burton, D. F.

Burton, E. T. D.

Burton, G. D.

Burton, G. E.

Burton, G. J. L.

Burton, H. J. C.

Burton, H. P. W.

Burton, M. G. W.

Burton, P. M.

Burton, W.

Burton-Fanning, F. W.
Burton-Fanning, N.E.E.

Burwell, W.K.

Bury, E. B.

Bury, E. W.

Bury, J.

Bury, L. E.

Bury, R. F.

Bush, F. R.

Bush, J.R.

Bushby, H. N. G.

Bushell, W. F.

Busk, E. T.

Busk, E. W.

Busk, H. A.

Buss, H. S.

Buss, L. C.
373

373

373

32

166

282

123

123

373

123

373

32

I 94

322

32

32

32

322

373

485

373

32

I66

373

4
34

89

I94

238

373

166

89

123

293

32

238

220

322

373

32
89

322

4

123

I94

485

373

373

373

123

$48 \dot{5}$

373

I 94

194

486

I 94

32

238
Bussey, A.

293

Buswell, H. L. F. $\quad 123$

Buszard, S. G.

Butcher, T. A. 293

Butcher, W. G. D. 373

Butler, A. G. $\quad 322$

Butler, A. J. A. 373

Butler, E. H. 513

Butler, E. N. 32

Butler, E. P. 238

Butler, E. W. $\quad 528$

Butler, F. H.C. $\quad 513$

Butler, G. G. 89

Butler, G. K. M. $\quad 373$

Butler, H. C. $\quad 373$

Butler, H. G. St P. $\quad 373$

Butler, H. M. 220

Butler, J. $\quad 528$

Butler, J. R. M. 373

Butler, N. M. $\quad 373$

Butler, R. L. G. 63, 373

Butler, W. G. 293

Butler, W. M. 373

Butler-Stoney, C. K. 373

Butlin, Sir H. G. T. 373

Butlin, T. H.

Butson, H. S. G. see Gould Butson, H. S.

Butt, G. M.

Butt, H. A.

Butt, H. T. H.

374

I66

Buttanshaw, C.

Butterwick, J. C.

Butterworth, A. B. $\quad 282$

Butterworth, R. $\quad 5$

Buttery, H. R. $\quad 5$

Button, A. E. $\quad 322$

Buxton, Anthony $\quad 374$

Buxton, Arthur $\quad 374$

Buxton, Abbot R. $\quad 374$

Buxton, Andrew R. 374

Buxton, B. G. $\quad 374$

Buxton, C. E. V. $\quad 374$

Buxton, E. N. $\quad 374$

Buxton, G. C. $\quad 374$

Buxton, H. F. $\quad 374$

Buxton, H. G. $\quad 374$

Buxton, I. $\quad 374$

Buxton, L. G. $\quad 374$

Buxton, M. B. $\quad 374$

Buxton, P.A. $\quad 374$

Buxton, R. G. $\quad 374$

Buxton, T. F. $\quad 374$

Buxton, W. L. $\quad 238$

Byatt, H. V. B. $\quad 32$

Byatt, R. N. B. $\quad 32$

Byers, J. $\quad 238$

Bygrave, W. $\quad 5$

Byrde, E. H. 74

Byrne, J. H.

Byrne, L. W. 
Byrne, M. G. M.C. 32

Byrne-Johnson, J. V. 32

Byron, $\mathrm{H}$.

Bythway, M. H. I 94

Bywaters, F. J. $\quad \mathbf{5 2 8}$

Cadbury, E.

Cadle, H.S.

Cadman, H. S.

Cadman, P.S.

Caffin, E. G.

Cahusac, S. D. N.

Caiger, F.H. S.

Caiger, G. H.

Caiger, S. L.

Cain, E.

Cain, R. C.

Cairnes, A. B.

Cairnie, J. B.

Caithness, Earl of

Calderbank, J.

Calderwood, J. L.

Caldwell, H. G.

Caldwell, K. F.T.

Caldwell Smith, E.L. 374

Caledon, Earl of $\quad 374$

Caley, F. G.

Caley, H. W.

Callender, R. H.

Callinan, T. W. 293

Callingham, L. F. $\quad 374$

Callis, M. C. $\quad 282$

Calthorpe, F. S. G.

see Gough Calthorpe, F.S.

Calverley, E. L.

Calvert, E.

Calvert, $\mathrm{H}$.

Calvert, H. H.

Calvert, L. M.

Calvert, W. J. R.

Calvert-Jones, H. F. 166

Cam, W. H.

Camden, Marquis 374

Cameron, A. G.

Cameron, C.

Cameron, D. W.

Camp, J.

Campbell, A. D.

374

322

374

374

238

374

124

374
$\mathbf{1} 66$

513

124

282

Campbell, A. D. P.

Campbell, A. J.

Campbell, A. Y. G.

Campbell, B. A.

Campbell, B. P.

Campbell, C.

Campell, G.H. 322

Campbell, D.N. $\quad 486$

Campbell, D.S. $\quad 375$

Campbell, E. M. $\quad 375$

Campbell,E.N.McC. 32

Campbell, E. R.

375

Campbell, F.R.M. I 66
Campbell, G.

Campbell, I. M.

124

Campbell, Hon. I M 375

Campbell, J. A. (Tr.) 375

Campbell, J. A. (Trin. H.)

Campbell, K. A.

Campbell, K. G.

Campbell, K. J.

Campbell, R. C.

486

375

486

239

Campbell, R. C.

Campll, R. W. 195

Campbell, Sir W.

Campbell, W. G.

Campbell, W. H.

Campbell-Douglas, L. C. $\mathrm{H}$.

Campbell-Johnston,

$\begin{array}{rr}\text { P.S. } & 375 \\ \text { Campbell-Muir,D.E.486 }\end{array}$

375

375

124

375

Campling, W.C. $\quad 375$

Candler, $\mathrm{G}$.

Candy, K. E.

Cane, A.S.

Cane, E. G. S.

Cane, L. B.

Cane, L. D.

Cane, M. H.

Canham, W. D.

Canney, J. R. C.

Cannington, A. S.

Cant, F. V.

Cantle, L. H.

Cantrell-Hubbersty, G. A. J.

Capon, E. O.

Caporn, A. C.

Cappel, N. L.

Capron, G.

Capron, N.H.

Capstick, J. W.

Card, F. W. F.

Carden, S. R.

Carden-Roe, W. R.

Cardiff, R. H. W.

Cardwell, A. G.

Cardwell, C. R.

Cardwell, H. E.

Cardwell, W.

Carew, P. G.

Carey, A. J. E.

Carey, D. F.

Carey, F. C. S.

Carey, G. V.

Carey, R.S.

Carlile, C.

Carlile, E.

Carlile, E. H. H.

Carlile, H. G.

Carlile, R. C.

Carlill, H. B.

124

375

239

486

322
Carlisle, F. M. M.

Carlisle, H. B.

Carlisle, J. C. D.

Carlton-Williams, 376

ams, E.W. 5

Carmichael A D.

Carmichael, A. M. $\quad 348$

Carmichael, D. $\quad 167$

Carmichael, D. W. W. 5

Carnegie, J.D. 195

Carnegy, F.W. $\quad 322$

Carnegy, P. L. St C. 89

Carnley, W. B. 293

Carpmael, A. $\quad 239$

Carpmael, E. V. $\quad 486$

Carr, A. L. $\quad 376$

Carr, B. A. $\quad 513$

Carr, C. T. $\quad 376$

Carr, D.N. $\quad 293$

Carr, F.R. 239

Carr, G. D'R. $\quad 124$

Carr, J. D.

Carr, P. R. $\quad 239$

Carr, R. N. $\quad 239$

Carr-Ellison,H.G.C. 376

Carr-Forster, E. W. 33

Carrack, C. J. 220

Carrick, A. D. $\quad 239$

Carrington, E. A.

Carroll, F. H.

Carroll, H. E.

Carrow, R. B.

Carsberg, A. E.

Carslake, W. B.

Carson, $T$.

Carstairs, J. L.

Carter, B.

Carter, C. N.

Carter, G. L. L.

Carter, G.S.

Carter, H. G.

Carter, J.

Carter, J. F.

Carter, J. L.

Carter, J. S.

Carter, $\mathrm{P}$.

Carter, W. E.

Carter, W. H.

Carter, W.H.S.

Carter, W. R.

Carthew, R. J.

Cartman, J. P. C.

38

528

239

Cartwright, $C$.

Cartwright, E.

Cartwright, G. F.

Cartwright, J. L.

Cartwright, S. H.

Carus-Wilson, C. C. 376

Carus-Wilson, E. $\quad 376$

Carver, A. E.

Carver, A. E. A. 
Carver, B. N.

Carver, E. T.

Carver, G. A.

Carver, $\mathrm{H}$.

Carver, L. H. L.

Carver, N. C.

Carver, O. A.

Case, G. R. A.

Case, H. A.

Casey, H. J.

Casey, R. G.

Casey, S. N.

Caslaw, J. M.

Cass, L. F.

Cassan, A. W. M.

Cassels, W. C.

Cassels, W. G.

Cassidi, F. L.

Cassidy, C.

Cassidy, M. A.

Cassillis, Earl of

Casson, R.

Casswell, E. D.S.

Castell, S. P.

Castellain, J. G.

Castellan, C. E.

Castellan, V. E.

Castle, C. W.

Castle, T. R.

Castle, W. F. R.

Castlehow, J. A. S.

Castleman, E. W.F. 376

Castlerosse, Viscount 376

Catmur, H. A. F. V. 293

Cator, A. N. L.

Cator, C. G. L.

Catterall, E. C.

Cattell, McK.

Cattley, R.

Causton, E. P. G.

Causton, L. J.

Cave, A. L.

Cave, C. J.P.

Cave, H. W.

Cave, L. C. H.

Cave, T.S.

Cave, W. H. C.

Cave, W. T. C.

Cave-Moyle, G. E. P. 124

Cave-Orme, G. A. R. 89

Cavendish, A. E. J. $\quad 376$

Cavendish, Lord J.S. 376

Cavendish,LordR.F. 377

Cavendish-Butler, H. $\mathrm{H}$.

Cawston, E.P.

Cawthra, J. J.

Cay, A. J.

Cayley, D. C.

Cayley, F. D. E.

Cayley, N.

239
376

33
167

376

293

376

239
486

89

528

348

33
376

5
376

124

376

322
376

376

5

376

376

309

89

239
486

376

124
376

376
63

220

220

239

33

377

377

309

239
$5{ }^{1} 3$ Cazalet, C. H. L.

195

239

322

322

239

24

\begin{abstract}
Chandler, H. E.
\end{abstract}
Chandler, K.S.

Chandler, R. A.

Chandler, W. K.

Chandless, C. T. C.

Chaning-Pearce,W.T.90

Channell, H. M.T. 377

Channing, C. E.

293

377

377

I67

239

33

377

124

377

322

63

240

377

.) 240

377

377

377

322

282

5

220

89

377

377

124

5
89

377

282

377

124

377

377

377

90

195

377

293

240

I 95

$\times 67$

377

377

377

124

167

293

$\times 67$

167

486

377

Chaplin, A.

377

Chapman, A. E.

377

Chapman, A. R. B. $\quad 322$

Chapman, E. H.

74

90
Chapman, G. M.

Chapman, H. (Chr.)

Chapman, H. (Sid.) 348

Chapman, P. D. H. 348

Chapman, T. M. G. 378

Chapman, W. H. $\quad 378$

Chappel, B. H. $\quad 124$

Chappel, G.P. $\quad 124$

Chappell, F. E. $\quad 63$

Chappell, H.S. $\quad 33$

Chapple, A. $\quad 323$

Chapple, C. J. $\quad 528$

Chapple, $\mathrm{H}$. $\quad 323$

Charles, J. A. M. $\quad 378$

Charles, J. R. 124

Charles, L. B. $\quad 378$

Charles, R. D. S. $\quad 240$

Charlesworth, C. B. 486

Charlesworth, J. B. 5

Charlesworth, M.P. 167

Charlesworth, W. H. 220

Charlewood, W.H. 5 I3

Charley, L. W. $\quad 528$

Charlton, G. D. $\quad 5 \times 3$

Chart, H. J. $\quad 378$

Charter, H. R. $\quad 90$

Chase, C. D. $\quad 348$

Chase, F. A. $\quad 240$

Chase, G.A. 293, 486

Chasemore, P. A. $\quad 487$

Chasteney, H. E. $\quad 323$

Chawner, W. R. $\quad 90$

Chaytor, A. H. $\quad 33$

Chaytor, D'A. $\quad 33$

Cheales, R. D. $\quad 378$

Cheape, J. de C. $\quad 124$

Checkland, M. B.

see Beaumont-

Checkland, M. B.

Cheese, W. G. $\quad 323$

Cheeseman, A. L. $\quad 323$

Cheetham, C. E. $\quad 90$

Cheetham, E. M. $\quad 323$

Cheffaud, P. H. M. $\quad 124$

Chell, $\mathrm{H}$. $\quad 323$

Chenevix-Trench, A. $\mathrm{S}$.

Chepmell, C. H. $\quad 378$

Cheshire, F. M. $\quad 323$

Chessex, R. E. A. $\quad 125$

Chester, H. K. $\quad$ 95

Chester, R. C. $\quad 378$

Chester-Master,A.G.240

Chesterman, $\mathrm{H}$. $\quad 309$

Chevassut, F. G. $\quad 378$

Chew, J.E. 5 r

Cheyne, W. H. W. 378

Chibnall, A. C. 33

Chichester,A.O'N.C.378

Chichester, Earl of $\quad 378$

Chichester, S. R. 240 
Chichester, W. G. C. 378

Chidson, L. D. $\quad 323$

Chidson, L. H. $\quad 378$

Chiene, G. L.

Chignell, N. J.

Child, F. J.

Child, G.J.

Child, J. F.

Child, S

Child, W.N.

Childe, C. M.

Childe, C. P.

Childers, R. E.

Childs, A. E.

Chinneck, S. T. E.

Chirnside, R. G.

Chisholm, C. J.

Chisholm, D.C. H.

Chittick, H.S.

Chittock, C.

Chitty, J. W.

Chivers, J. S.

Chivers, W. B.

Cholmley, H. A.

Chopra, R. N.

Christie, H. A. H.

Christie, J.

Christie, J. F.

Christie, L. D.

Christie, R. A. B.

see Bonham-Christie, R. A.

Christie, R. G.

Christie, S.O.K. 125

Christmas, D. V. $\quad 240$

Christopher, C. M.de A. 240

Chrystall, H. M.

Chubb, C.

Chubb, E. G.

Chubb, F. J. M.

Chubb, R.N.

Chudleigh, C.A. E ${ }^{5} 6^{13}$

Churcher, W. D. $\quad 125$

Churchill, A.

Churchill, A. R.

Churchill, G.S.

Churchill, H. E.

Churchill, W. M.

Churchman, C. H.

Churchward, A.C. 323

Churchward, $B$.

Churchward, H. A. $\quad 63$

Churchward, M.W. 323

Churton, W. A.V. $\quad 125$

Circuit, E. F.

Civil, H. G. V.

Clairmonte G. 5 I 33

Clapton, A.

Clarence-Smith,K.W.125

Clark, A. G.
Clark, A. J.

Clark, A. S.

Clark, C. S.

Clark, E. D.

Clark, E. F.

Clark, E. K.

Clark, G. L.

Clark, G. M.

Clark, G. R. H.

Clark, G. W.

Clark, H. D.

Clark, H. J.

Clark, H. R. E.

Clark, J. B.

Clark, J. H. M.

Clark, N. M.

Clark, O. A.P.

Clark, P. N.

Clark, $\mathbf{R}$.

Clark, S. B.

Clark, S. H. (Clare)

Clark, S. H. (Trin.)

Clark, W. M.

I95, 282

195

378

240

378

378

379

I 67

309

33

125

379

33

323

5
379

167

74

487

379

33

379

195

Clark-Kennedy,A.C. 5 I 3

Clark-Kennedy,A.E. 63

Clark-Kennedy,A.K.379

Clark-Turner, $\mathbf{F}$.

Clarke, A. B.

348

Clarke, A. G. A.

167

Clarke, A. J. M.

125

see Michell-Clarke, A.J.

Clarke, A. P.

Clarke, A. S.

Clarke, A. V.

Clarke, C. M. S.

Clarke, C.S.

Clarke, D.

Clarke, E.

Clarke, E. B.

Clarke, E. J.

Clarke, E. P.

Clarke, E. R.

Clarke, E. Russell

240

33

125

293

379

323

74

5

487

348

Clarke, E. S.

Clarke, E. T.

Clarke, F.W.

Clarke, G. A. C.

Clarke, G. T. K.

Clarke, H. A.

Clarke, H. H.

240

379

348

282

74

240

379

379

Clarke, Harold M.

33

Clarke, Henry $M$.

Clarke, H.S.S.

33

90

Clarke, H. T.

33

Clarke, J.H. (Joh.) 323

Clarke, J. H. (Trin.) 379

Clarke, J. M.

see Michell-Clarke, J.

Clarke, J.P. D.

63

Clarke, J. S. (Caius) 125

Clarke, J.S. (Magd.) 22 I
Clarke, J. S. (Joh.)

Clarke, L. B.

Clarke, M.T.

Clarke, R. H.

Clarke, R.S.(Kg's) 125

Clarke, R.S. (Joh.) 323

Clarke, S.

Clarke, S. H.

Clarke, S. J. C.

Clarke, W. G.

Clarke, W. K.

Clarke, W.S.

Clarke-Williams,

$\begin{array}{ll}\text { A.R. } & 487 \\ \text { Clarke-Williams, F. } \quad 240\end{array}$

Clarkson, E. R. T. $\quad 513$

Clarkson, J. F.

Clarkson, W. B. $\quad 379$

Claudet, B. J. A. I25

Claudet, F. H. B. $\quad 125$

Claudet, R.A. O. $\quad 125$

Claughton, I. D. $\quad 195$

Clay, B. A.

Clay, F.H.

Clay, J.H.

379

379

379

Clayden, H.K.

Claye, G.W. $\quad 33$

Claye, $\mathrm{H}$. $\quad \mathrm{I} 25$

Clayhills, G. $\quad 487$

Clayton, E. B. $\quad 125$

Clayton, F. (Sid.) $\quad 34^{8}$

Clayton, F. (Trin.) 379

Clayton, G.H. 240, 282

Clayton, J.

Clayton, L. J. $\quad 240$

Clayton, N.W. 293

Clayton, T. W. 240

Clear-Davidson,F.R.293

Clee, C. B. B. $\quad 528$

Cleeve, C. E.

Clegg, J.

Clegg, J. A.

Clegg, M. T.

Cleghorn, W.A.

487

Cleland, J. R.

Clement, $\mathrm{L}$.

Clements, P M. H 348

Clements, T. H.

Clements, W. D. $\quad 24^{\circ}$

Clements, W. G. 221

Cleminson, F. J. $\quad 125$

Clemmow, C. A. $\quad 282$

Clemmow, E.P. $\quad 282$

Clemo, F. A. $\quad 5^{\mathrm{I}} 3$

Cleworth, T.H. 293

Clifford, A. C. $\quad 9 \circ$

Clifford, A. W. $\quad 487$

Clifford, E. C. $\quad 33$

Clifford, F. A. $\quad 195$

Clifton, E. N. 
Clifton, G. F.

Clifton, H. E.

Clifton Brown, C.

see Brown, C. Clifton

Clifton Brown, D.

see Brown, D. Clifton

Clissold, C. H.

Clissold, $\mathbf{H}$.

Close, A. V.

Close, R. W. M.

513

Close-Brooks A B 5 r 3

Close-Brooks, J. C. 379

Cloudesley, $\mathrm{H}$.

Clough, A.

Clough, J.

Clough, $T$.

Clough, V.

Clout, C. W.

Clutton, $\mathrm{B}$.

Coad, C. N.

Coaks, H. C.

Coast, J. P. C.

Coast, W.

Coates, A. D.

Coates, A.S.

Coates, B. M.

Coates, C. A.

Coates, N. H.

Coates, R. A.

Coates, V. H. M.

Cobb, F. W.

Cobbold, A. W.

Cobbold, C. J. F.

Cobbold, C. T.

Cobbold, F. A. W.

Cobbold, G. F.

Cobbold, G. W. N.

Cobbold, J. V.

Cobbold, P. W.

Cobbold, R. H. W.

Cobham, E.

Cochran, F. A.

Cochran, G. G.

Cochran-Patrick, N. J. K. see Kennedy-CochranPatrick, N. J.

Cochran-Patrick, W. J. C. K.

see Kennedy-CochranPatrick, W. J. C.

Cochrane, A. C. $\quad 380$

Cochrane, A. K. O. 380

Cochrane, G. D.

Cochrane, R. D.

Cock, T. A.

Cockayne, A. A.

Cockburn, A. F.

Cockell, B. W.

Cockerell, S. P.

Cockin, M.S.

Cockin, R. P.

126

126
74 Cocks, H. S.

Cocksedge, T. A. B. 126

Cockshott, F. G.

Cockton, J. C.

Coe, C. G.

Coggin, H. F. F.

Coham-Fleming, B. $B$.

Cohen, $\mathrm{A}$.

Cohen, A. M.

Cohen, C. B.

Cohen, D. H.

Cohen, E.

Cohen, G. H.

Cohen, Sir H. B.

Cohen, J. I.

Cohen, $M$.

Cohen, W. S.

Coke, Reginald

Coke, Richard

Coke, R. A. S.

Colam, H. N.

Colbeck, E. H.

Colbeck, L. G.

Colchester, G. V.

Colcutt, A. M.

Cole, C. L.

Cole, G. H.

Cole, G. L.

Cole, H. B.

Cole, L. B,

Cole, L. S.

Cole, T.E. F.

Cole, T. G. O.

Coleman, C. J.

Coleman, E. C.

Coleman, H. N.

Coleman, N. D.

Coleman, P. G.

Colenutt, F. A.

Coles, C.

Coles, E. J.

Coles, E. R.

Coles, W. T.

Coley, C.

Colledge, $\mathrm{L}$.

Collet, G. G.

Collett, G. F.

Collett, H. A. A.

Collett, R. L.

Collett, W. G.

Colley, W. H.

Collie, A. E.

Collier, A. C.

Collier, A. E.

Collier, A. G.

Collier, F. H.S.

Collier, G. H.

Collier-Johnstone, N.S.

34

380

380

6

34

380

487

90

195

380

380

293

195

293

528

380

487

380

240

240

126

196

293

126

240

196

240

I 96

I 96

34

74

487

380

240

I67

323

380

293

I67

380

221

$\times 67$

6

126

380

241

24 I

380

63

90

90

34

22 I

167

$24 \mathrm{I}$

513
Collin, E. P. C.

I96

Collin, R.

see Monkswell, Lord

Collingham, D. H. $\quad 196$

Collingridge, $\mathrm{W}$

6

Collings-Wells, L.C. 487

Collingwood, B. J. $\quad 126$

Collingwood, C. A. $\quad 74$

Collingwood, E. F. $\quad 380$

Collins, A. J. $22 \mathrm{I}$

Collins, B. K. T. $\quad 380$

Collins, B. S. $\quad 293$

Collins, D. C. $\quad 380$

Collins, E. D. 24I

Collins, E. G. W. $\quad 63$

Collins, E. R. $\quad 282$

Collins, G. A.

282
6

Collins, H. E. $\quad 309$

Collins, R. F. $\quad 380$

Collins, R. L. $\quad 90$

Collins, V. St B. $24 \mathrm{I}$

Collinson, G. E. C. $\quad 126$

Collinson, J. W. $\quad 38 \mathrm{r}$

Collis, A. J. $\quad 513$

Collis, F.S. $\quad 294$

Collot, T. A. $\quad 126$

Colman, C. J. $\quad 282$

Colman, D. M. 24I

Colman, J. 381

Colman, L. M. 24I

Colonna, Don Mario 22 I

Colquhoun, G. R. E. $3^{81}$

Colquhoun, J. C. $\quad 126$

Colson, A. F. D. $\quad 283$

Colson, F.S. $\quad 90$

Colt, G.H. $\quad 348$

Colthurst, G. O. $\quad 381$

Colthurst, R. St J. J. 381

Coltman, R. L. $\quad 90$

Coltman, W. H. $\quad 381$

Colville, D. 24I

Colville, D. J. $\quad 381$

Colville, J. G. $\quad 38$ I

Colville, N.R. $\quad 34$

Colville, R. N. K. $\quad 283$

Colville, T. R. $\quad 381$

Colvin, C. H. $\quad 38 \mathrm{I}$

Colvin, R. B. $\quad 38 \mathrm{I}$

Combe, E.P. $\quad 126$

Combe, H. A. B. $\quad 487$

Comber, H. G. $\quad 24 \mathrm{I}$

Comeau, E. A. $\quad 196$

Compston, G.D. $\quad 487$

Compton, A. G. W. 126

Compton, F. $\quad 309$

Compton-Burnett, N. 196

Comyn, A. F. 24I

Comyn, H. F. $\quad 196$

Comyn, $\mathrm{K}$. $\quad 5^{13}$

Conacher, $\mathrm{H}$. $\quad 90$

380 Conan-Davies, B. I. 309 
Concanon, G. L. B. 196

Conder, A. C.

Connett, $\mathrm{H}$.

294
6

Conningham, W.F.M. 63

Connolly, B. B. $\quad 126$

Connolly, J.S. D'A. 24I

Connop, $\mathrm{H}$.

Conolly, C. G.

Conran, M. W. T. $38 \mathrm{I}$

Considine, $H$. $H$.

Constable, W. G.

Constantine, H. N.

Constantine, W. W.

Conway, S. V.

Conybeare, A. E.

Coode, A. P.

Coode, A. T.

Cook, F.

Cook, F. R.

Cook, J. W.

Cook, S. G.

Cook, V. C.

Cooke, A.

Cooke, A. W. H.

Cooke, C. H.

Cooke, C. P.

Cooke, E. R. C.

Cooke, F. F.

Cooke, H. d'A. M.

Cooke, H. L.

Cooke, H. R.

Cooke, J. G.

Cooke, S. R.

Cooke, W. I.

Cooke-Hurle, J. see Hurle, J. C.

Cookson, H. A.

Cookson, R. T. C. 90

Coombe, R.

Coombs, A. G.

Coombs, H.M.McC 323

Coombs, P. G. 241

Cooney, A. B. $\quad 309$

Coop, W.

324

Cooper, A. L. (Emm.) 90

Cooper, A. L. (Clare) 34

Cooper, F. T.

Cooper, H. (Emm.) 91

Cooper, H. (Joh.) 324

Cooper, H. A.

Cooper, H. C.

Cooper, H. J.

Cooper, H. O.

Cooper, H. W. F.

Cooper, J. G.

Cooper, J. R.

Cooper, J. S.

Cooper, L. G.

Cooper, M. C.

Cooper, P. A.

Cooper, P.H.

126

63

63

$38 \mathrm{I}$

487

I67

283

324

487

$38 \mathrm{I}$
Cooper, W. G. D.

Cooper Bland, J.

Cooper-Hunt, C. L. ${ }^{487}$

Cooper-Hunt, D. L. 9 I

Cooper-Marsdin, A. C.

Coote, A.

Cope, H. E.

Cope, J. L.

Cope, J. R. O.

Cope, T. G.

Cope, W.

Copeland, A. J.

Copeland, W. B.

Copeman, C.E. F.

Copeman, E. H.

167

Copeman, G.W. B. 487

Copeman, S. A. M. 63

Copland Griffiths, F.A. V.

Coplestone, W. D. $\quad \begin{array}{r}387 \\ 167\end{array}$

Copplestone, W.R.J. 529

Corbet-Singleton,

Corry, W. M. F. M. $\mathrm{G}$

Corbett, G. H. U.

Corbett, H. V.

Corbett, R. S.

Corbin, J. L.

Cordeux, E. H.N.

Corfield, B. C.

Corfield, C. L.

Corfield, F. de la P.

Corke, G. H.

Cornah, J.R.

Cornelius, N. S.

Corner, E. M.

Cornford, F. M.

Cornish, A.

Cornish, C. L.

Cornish, H. D.

Cornwall, G.

Cornwall, J. W.

Corrie, G.T.

Corrie, $\mathrm{L}$.

Corrie, O. C. K.

Corry, F. R. H. L. see

Lowry-Corry, F.R.H

Corry, J. P. I. M. $\quad 382$

Corser, E. E.

126

196

309

126

381

241

I 68

309

74

241

34

348

381

349

$38 \mathrm{I}$

381

91

381

196

487

$38 \mathrm{I}$

Corser, J.S.

Cort, J. L. P.

Cortazzi, F. E. M.

Cory, C.W.

Cory, J. F. T.

Cory, R. F.P.

Coryton, A. F.

Coryton, W. A.

Cosgrove, W. O.

Cossar, J. M.

324

294

$\Upsilon 26$

6

126

221

196

283

241
91

294

6

34
381

34

$24 \mathrm{I}$

$38 \mathrm{I}$

Costello, L. W. J.

283

Costigan, R. H.

Costobadie, L. P. $\quad 126$

Cott, A. M.

Cott, A. W.

Cottam, H. C. B. $\quad \begin{array}{r}34 \\ 126\end{array}$

Cotterill, D.

Cotterill, G. H.

Cotterill, H. E.

Cotterill, L.

Cottingham, J. W.

Cotton, B.

. $\quad 488$

Cotton, C. K. $\quad 74$

Cotton, H. W.S. $\quad 294$

Cotton, R. H. A. $\quad 324$

Cotton, V. E. $22 \mathrm{I}$

Couch, A. W. 22 I

Couch, C. J. 294

Couchman, H. J. $\quad 127$

Couchman, M.L. 294

Coulcher, G. B. $\quad 64$

Coules, St V. F. $\quad 349$

Coulson, N. $\quad 24 \mathrm{I}$

Coulson, R.N. $\quad 127$

Coultas, T. B. 294

Coupe, T. O. $\quad 514$

Couper, J. D. C. $\quad 382$

Courtauld, J.S. $\quad 196$

Courtauld, L. $\quad 382$

Courtauld, S. L. $\quad 196$

Courthope, R. $22 \mathrm{I}$

Courthope-Munroe, C. $\mathrm{H}$.

Courthope-Munroe, J.W.

Courtis, A. O.

Cousney, G. B. $24 \mathrm{I}$

34

Cousins, B. D. . $\quad 241$

Coutts, H. A. T. $\quad 382$

Coventon, A. W. D. 382

Cow, C.S.

Cowan, C. J. A. $24 \mathrm{I}$

Cowan, J. M. $\quad 196$

Cowan, R. C. 24I

Cowan-Douglas, $\mathrm{H}$. $\quad 127$

Coward, $\mathrm{H}$. $\quad 196$

Cowell, J.

Cowell, J. B.

74

Cowell, S. J.

294

Cowell, W. J. R

see Rooke-Cowell, W.J.

Cowham, A. G.

514

Cowie, A. G.

Cowie, D. H.

Cowie, H. C.

127

Cowley, J. N.

Cowley, R. B.

Cowper, G. M.

382

$24 I$

382 
Cox, B. C.

488 Creed, A. H. G.

Cox, D. H.

Cox, D. P.

Cox, E.

Cox, E. H.M.

Cox, F. B. H.

Cox, G. L.

Cox, H. B.

Cox, H.P.

Cox, P. H.

Cox, $\mathrm{R}$.

Cox, R. B.

Cox, W.A. M.

Coxe, K. H.

Coxon, A. C. M.

Coxon, A. W.

Coxon, $T$.

Coxwell, C. B.

Coy, J. C.

Coyne, C. T.

Coyte, S. E.

Cozens, F. C.

Crabtree, H. G.

Crace, J. F.

127

382

127

488

168

6

324

488

I68

127

34

382

9I

221

382

9 I

6

I 68

$9 \mathrm{I}$

349

9I

6

Crackanthorpe,O.M.382

Cracroft, R. B.

Cradock, N.

Craft, H. B.

Craig, A. D. E.

Craig, G. W.

Craig, $M$.

Craig, N.C.

Craigie, R. C.

Craigmile, A. M.

Craigs, W. N.

Crampton, E. B.

Crampton, G.P.

Crampton, H.P.

Crane, H. E.

Crane, L. F. N.

Cranmer Byng, L. A. 382

Cranston, H. N. $\quad 283$

Cranworth, Lord $\quad 382$

Crauford, L. G.

Craven, A. E. L.

Craven, W. L.

Crawford, C. G.

Crawford, C. N.

Crawford, D.

Crawford, G. B.

Crawford, K. A.

Crawhall, T.E.

Crawhall, T. L.

Crawley, E.

Crawley, J. L.

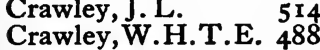

Crawshaw, C. B. H.

Crawshaw, C. H.

Crawshay, J. W. L.

Crean, T.

Creasy, R. L.

91

127

283

$\times 27$

I 96

294

34

74

34

382

r68

I68

488

221

382

6

382

382

127

382
Creed, J. M.

Creed, P. R.

Creighton, W. R.

Cressey, G. E. L.

Cresswell, C. E.

Creswell, H. E.

Creswick, F.N.

Crew, F. D.

Crewdson, B. F.

Crewdson, R. B.

Crewdson, T. W.

Crewdson, W. D.

Creyke, E. R.

Crichton-Browne, H. W. A. F.

Crick, G. H.

Crick, L. C.

Crick, L. G. M.

Crick, P. C. T. 34, 24 I

Crimp. G. L.

Cripps, W. L.

Crisford, K. N.

Crisp, E. J.

Crisp, $\mathrm{H}$.

Crisp, J. F.

Crispin, A. E.

Crispin, H. T.

Critchett, G. M.

Crocker, G. G.

Crocker, J. A.

Crocker, R. W.

Crockford, L. C.

Crocombe,F.R. 28

Croft, H.P.

Croft, J. A. C.

Croft, R.P.

Crofton, J. H.

Crofton-Atkins, W A ${ }^{242}$

Crofts, J. M.

Croggon, J. F. S.

Croghan, E. H.

Crole-Rees, H. S.

Cromie, B.P.

Crompton, J.

Crompton, N. G.

Crompton, R. A.

Cronk, H. L.

Crook, A. H.

Crookall, E.

Crooke, R. H.

Crookham, H. A. R. $\quad \begin{array}{r}91 \\ 68\end{array}$

Cropper, John $\quad 383$

Cropper, J. W.

Crosbie, R. E. H. $\quad 242$

Crosbie-Oates, E. C. 22 I

Crosby, G. J.V.

Crosby, J. C. P.

Crosfield, A. C.

127 Croshaw, F.P.

196 Cross, E. K.

127

91

24I

$\times 27$

349
91

x97

r 97

383
383

168

221

64

221

383

488

r 97

9 I

I68

6

529
6 Cross, P. M.

529

Cross, R. C.

34

127

Crosse, R. G.

Crosse, S. S.

Crosse, T.L.

Crossley, A. H.

Crossley, B.

Crossley, E.

Crossley, E. A.

Crossley, F.M.

Crosthwaite, W.H. 35

Crouch, H. A.

Crow, A. D.

Crow, C. A.

Crow, $P$.

Crowder, G.C.G. $\quad 242$

Crowder, W.I. R. $\quad 383$

Crowe, D. M.

Crowe, H. A.

Crowe, H. B.

Crowe, T. $M$.

Crowther, C. R.

Crowther, H. N.

Crowther, $\mathrm{H}$. $\mathrm{O}$.

Crowther, W. C.

Croysdale, J. H.

Crozier, J. E. D.

Crozier, P. H.

Cruickshank, G. $\quad 35$

Cruickshank, R.S. $\quad 283$

Cruikshank, G. L. $\quad 9^{1}$

Crum-Ewing, N.R. 242

Crump, G. H. 294

Crump, H. C. $\quad 242$

Crump, N. E. $\quad 127$

Crundwell, A. $\quad 127$

Cubitt, V.M.

Cuckney, J. $\quad 9^{I}$

Cuff, A W.

Cuffe, G. E.

Cullen, A. H.

Cullen, W. G.

Culley, G. C. H.

324
I 68

294

.

Cullimore, James

Cullimore, John $\quad 127$

Cullimore, W. $\quad 127$

Cullinan, M. W. F. $22 \mathrm{I}$

Cullis, L. $\quad 324$

Culverwell, C. T. $\quad 294$

Culverwell, J.S. $\quad 309$

Cumberland, R.B.L. $24^{2}$

Cumberlege, B.S. $\quad 9^{1}$

Cumberlidge, W. I. 6

Cumming, G.E. 309

242 Cumming, J. B. $\quad 127$

34 Cumming, J. E. 9r

34 Cumming, J. H. 242

383 Cumming, W. 
Cummings, R. R. $\quad 324$

Cummins, F. J. $\quad 324$

Cundell, J. $\quad 383$

Cuninghame, R. J. 221

Cunliffe, J. H. G.

Cunliffe, $\mathbf{N}$.

Cunningham,A.J.W. $3_{38}$

Cunningham, B. K. 383

Cunningham, J. M. 383

Cunningham, J.S. $\quad 383$

Cunningham, L. $\quad 74$

Cunningham, T. E. 383

Cunningham, W. A. 383

Cunningham Reid, A. see Reid, A. Cunningham

Cunnington, E. E. $\quad 64$

Curl, S.W.

Curling, W. G.

Curme, D. E.

Curnock, G.A.

Curnow, A. T.

Currey, R.G.T. $\quad 514$

Currie, J. H.

Currie, R. F. I.

Curry, P. A.

Cursetjee, H.J.M. $\quad 127$

Curtis, A. R. W. $\quad 384$

Curtis, E. D. $22 \mathrm{I}$

Curtis, I. $\quad 283$

Curtis, S.W. $\quad 309$

Curtis, T. L. C. $\quad 384$

Curtis-Bennett,H.H. 384

Curtois, P. A. 294

Curwen, B. M. $\quad 128$

Curwen, C. N. $\quad 128$

Curzon, C. T. B. 75

Curzon-Siggers,

$$
\text { W.A. }
$$

Cushing, W. E. W.

Cushion, E. J.

Custance, C. V.H.

Custance, E. C.N.

Cuthbert, C. A.

Cuthbertson, E. H.

Cuthbertson, J. H.

Cuthbertson, $M$.

Cutlack, W.P.

Cutler, E. T.

Cutler, H. A.

Cutter, R. C.

Cutts, F. J

Daffarn, $M$.

Dain, G. R.

Daish, $T$.

Daish, T.
d'Albuquerque, N.P. 35

Dale, A. P.

Dale, $F$.

Dale, F. R.

Dale, G.F.

384

35

384
Dalgleish, J.P.

Dalley, J.P.

Dalley, R.P.

Dalling, W.E.

Dalrymple, C. M.

Dalton, C. H. C.

Dalton, E. H. J. N.

Dalton, J. O. C.

Dalton, R. F.

Dalton, T.E.

Daltroff, E. M.

Daltry, R. W. O.

Daly, I. de B.

Daly, U.de B.

Daman, T.W.A.

Dammers, B. F.H. $\quad 242$

Dammers, E. F.H. $\quad 242$

Dandridge, W. L.

Daniel, T.W.

Daniell, H. E. B.

Daniell, J.

Daniels, A.P.

Daniels, $M$.

Daniels, T.H.R.

Danvers, G. C.

Darbishire, H. D.

Darby, A. J. L.

Darby, W.S.

Darbyshire, H. S.

Darbyshire, N. A.

Dare, A. G.

Dare, A. J.

Darley, C. B.

Darley, D. J.

Darley, H. R.

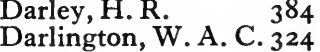

Darmady, E.S.

Darnton, R. E.

Darroch, A. R.

Dart, $\mathrm{H}$.

Darwin, B. R. M.

Darwin, C. G.

Darwin, E.

Darwin, G. A. M.

Darwin, W. R.

Dashwood, C. B. L.

Dashood, C. B. L. 242

Dashwood, H.T.A 221

Dashwood, R. C. $\quad 22 \mathrm{I}$

Dathan, J. D.

Daubeny, G. R.

Daukes, A. H.

Daukes, S. H.

35

324

242
Daun, E.

Davenport, A.

Davenport, A. H.

Davenport, $\mathrm{H}$.

Davenport, $\mathrm{S}$.

Davenport, S. F. 310

Davenport, $T$.

Davenport, W. $\mathrm{H}$.

384

324

349

6

6
283

242

64

$\begin{array}{r}28 \\ \hline\end{array}$

I 97

384

384
384

384

384

384

384

488

384

242

536

514

384

128

92
128
Davey, H. C.

Davey, H. N.

35

Davey, S. G.

35

David, L. W. $\quad 242$

David, $M$.

David, R.S.R. $\quad 168$

David, W. T. $\quad 384$

Davidson, $\mathrm{A}$.

Davidson, A. H. G. 221

Davidson, A. J. $\quad 222$

Davidson, D.F. $\quad 384$

Davidson, G. E. $\quad 128$

Davidson, G. M. $\quad 35$

Davidson, G.S. $\quad 294$

Davidson, H. G. L. 488

Davidson, J. $\quad 242$

Davidson, J. C. F. $\quad 222$

Davidson, L. S.P. $\quad 384$

Davidson, M.C. $\quad 310$

Davidson, M. G. $\quad 385$

Davidson, N.R. $\quad 385$

Davidson, Sir W. E. 7

Davidson, W.E. F. $\quad 168$

Davidson, W. W. $\quad 92$

Davies, A. C. $\quad 385$

Davies, A. L. $\quad 310$

Davies, A. R. $\quad 385$

Davies, C. 5 I4

Davies, C. E. H.

seeHughes Davie s, C.E.

Davies, C. E.S. $\quad 168$

Davies, D. $\quad 197$

Davies, D. G. $\quad 75$

Davies, D.H.S. $\quad 294$

Davies, D. Ll. E. 310

Davies, E. $\quad 324$

Davies, E. D. D. 7

Davies, F.C. $\quad 75$

Davies, G.B. (Down.) 75

Davies, G. B. (Selw.) 5 I4

Davies, G. F. $\quad 197$

Davies, G. Ll. $\quad 385$

Davies, H. B. $\quad 242$

Davies, H. M. $\quad 385$

Davies, H. R. $\quad 385$

Davies, I. T. $\quad 128$

Davies, J. B. (Down.)

see Bowen-Davies, J.

Davies, J. B. (Jes.) 168

Davies, J. C. $\quad 92$

Davies, J. G. 92

Davies, J. Ll. (Capt.) 92

Davies, J. Ll. (Major) 92

Davies, J. O. $\quad 310$

Davies, J. P. H. $\quad$ I68

Davies, J. R. (Caius) 128

Davies, J.R.(Fitz.H.) 529

Davies, J.S. H. $\quad 92$

Davies, J. T. 75

Davies, K. C. J. 3 I0

Davies, K. G. $\quad 168$ 
Davies, L. C.

Davies, L.F. St J.

Davies, L. G.

Davies, Ll. J.

Davies, P. Ll. see

Llewelyn-Davies, P.

Davies, P. M.

Davies, R. A. Ll.

64

see Llewelyn-Davies, R. A.

Davies, R. E.

Davies, R.E.L. $\quad 128$

Davies, R. G. M. 242

Davies, R. G. R. 197

Davies, R. L.

Davies, R. M.

Davies, R. R.

Davies, T.A.M. $\quad 385$

Davies, T.H.(Mgd .)222

Davies, T. H. (Pem.) 242

Davies, T. J. C.

35

Davies, T. M.

Davies, V.P.

Davies, W.E.

197

283

168

Davies-Colley, H. $\quad 385$

Davies-Colley, R. $\quad 92$

Davies-Colley, T. H. ${ }^{885}$

d'Avigdor-Goldsmid, O. E.

Davis, C. A. E.

Davis, C. E. J.

Davis, C. J. B.

Davis, E. B.

Davis, E. M.

Davis, F. B.

Davis, F. M.

Davis, F. P.

Davis, G.F.S.

Davis, $\mathbf{H}$.

Davis, H. J.

Davis, H.N.

Davis, J. C. A. see

488

529

36

128

5 I4

168

$5 \mathrm{I} 4$

128

128

197

324

325

92

Ainsworth-Davis, J. C.

Davis, J. O.

Davis, J. R. A.

Davis, J. W. F. McN.

see McNaught-

Davis, J.W. F.

Davis, K. J.A.

Davis, R. G.

Davis, W.J.

Davison, G.

Davison, J. F.

Davy, C. L.

Davy, G.

Davy, G.H.

Davy, P. L.

Davy, W. J.

Daw, W. F.B.

Dawbarn, G. R.

Dawbarn, J.R.

385

325

I 68

128

488

488

242

64

128
Dawe, A. H.

Dawe, L. S.

Dawes, A. W.

Dawes, E.

Dawes,E.S.

Dawes, H. J.

Dawes, M.S.

Dawkins, C. J. K.

Dawkins, R. McG.

Dawnay, C. H.

Dawson, A. M.

Dawson, C.P.

Dawson, E. C.

Dawson, F. G.T.

Dawson, H. H.

Dawson, H.P.

Dawson, J.

Dawson, J.H. T.

Dawson, R.T.

Dawson, S.

Dawswell, G.A.

Day, C. D.

Day, D. I.

Day, E. C. (Caius)

Day, E. C. (Jesus)

Day, $G$.

Day, G. L.

Day, G.P. J.

Day, G. R.

Day, G. W. L.

Day, M. C.

Day, M. F.

Day, N. L.

Day, W. F. L.

Deakin, $\mathrm{H}$. V.

Dealtry, F.H. B.

Dean, J. H.E.

Dean, L. 'T.

Deane, A. P. W.

Deane, C. H.

Deane, R. W.

Dearden, $\mathrm{H}$.

Deas, P. B.

Debailleul, A.

de Barathy, S. A.

Debenham, F.

Debenham, F.J.

de Braconier, L.

de Candolle, $R$.

de Cerjat, C.S.

de Ceuleneer, $P$.

de Chaumont,

T. S. B. F. de Chazal,

Vicomte P.E.

de Coetlogon,

C. E. C.

de Courcy Ireland, G. B.

see Ireland,

G. B. de Courcy

Deed, N. G.

243

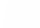

5 I 4

92
75

92

64

243

222

7

243

283

92

$3^{85}$

325

36

243

243

7

385

243

325

514

I97

75

325

128

168

128

325

310

92,283

243

385

243

64

128

385

514

243

349

385

385

385

128

243

128

7

128

385

529

385

385

529

514
Deedes, J.

168

Deedes, W.

168

Deeping, G. W. $\quad 385$

Deerhurst, Viscount 385

De Freitas, D. A. A. $\quad 36$

De Freitas, J. M. $\quad 36$

de Geijer, E. N. $\quad 385$

de Geijer,

W. A. W. G. $\quad 385$

de Hahn, C. P. $\quad 283$

de Hamel, H. G. $\quad 197$

De Hoxar, C. F. M. 222

Deighton, F. $\quad 283$

Deighton, F. J. $\quad 529$

Deighton, F. M. $\quad 385$

Deighton, G. W. $\quad 197$

Deighton, J. $\quad 386$

de Janasz, G. K. A. 386

de Jersey, N.S. $\quad 243$

Dekkers, L. A. $\quad 75$

De la Bere, I. $\quad 349$

Delafield, M. E. C. $\quad 168$

de la Mothe, C. D. F. 243

De la Pryme, A. G. 386

Dela Pryme, W.H.A. 7

de la Rue, R. W. $\quad 488$

De las Casas, M. $\quad 222$

de Ledesma, A. F. $\quad 222$

de Lestapis, H. $\quad 529$

de Linde, C. A. $\quad 197$

Delius, S. St M. $\quad 36$

Delph, L. W. 310

de Mowbray, L. St J. 514

Dempsey, G. B. $\quad 310$

de Navarro, J. M. $\quad 386$

Dendy, E. H. $\quad$ I28

Denham, H. A. $\quad 325$

Denham, J.P. $\quad 325$

Denham-Cookes, A. B.

488

Denis Browne, W. C. 36

Denis de Vitré, D. F.

see de Vitré, D. F. D.

Denman, R. C. $\quad 386$

Denman, R.P.G. $\quad 386$

Dennehy, H. G. $\quad 92$

Dennes, W.

Denning, W. F. $\quad 386$

Dennis, J. N. 222

Dennis, W. J. M. $\quad 529$

Denniston, J. G. $\quad 92$

Dennistoun. J. R. $\quad 386$

Dent, A. C.

Dent, G. $\quad 386$

Dent, G. J. C. $\quad 283$

Dent, L. M. E. $\quad 386$

Dent, R. A. W. $\quad 386$

Dent, W. E. $\quad 386$

Dent-Brocklehurst, G. E.

386

349 
Denton-Thompson, B. J.

Denyer, S. E. de Pass, C. A.

de Pass, E. A.

Depree, H. T.

de Prosperi, F. C. $\quad 222$

Derbyshire, H 349

Derham, J. A. T. $\quad 386$

de Ridder, L. E. $\quad 514$

d'Erlanger, H. R. C. 386

de Rothschild, A. G. 386

de Rothschild, E. A. 386

de Rothschild,J.E.A. 386

de Rothschild, L. N. 386

Desborough, G. $\quad 386$

deSibour, VicomteL. 386

de Slubicki, J. M. 243

Desmond, G. G. $\quad 325$

Desoer, A.

Deterding, $\mathrm{H}$.

7

de Trafford, R. E. F. 386

Deuchar, J. L. I 97

Devereux, H. W. $\quad 64$

Devereux, $\mathbf{N}$.

Devereux, W. C. $\quad 5$ I4

Devey, J. H. J. C. 294

De Villiers, D. I. $\quad 488$

de Vine, C.N. $\quad 169$

de Vine, H. B. St J. $\quad 169$

de Vitré, D. F. D. $\quad 387$

Devitt, G. F. O. I $\quad$ I 8

Devonshire, Duke of 387

Dew, J. W.

De Waal, D.

Dewar, A.

Dewar, D.

Dewar, M. B. U. $\quad 387$

Dewé, C. D. E. 294

Dewey, H. G. $\quad 243$

Dewhurst, C.(Major) 387

Dewhurst, C. (Sergt.) 387

Dewhurst, G. C. L. 387

Dewhurst, G. P. $\quad 387$

de Windt, H. W. D. 222

Dexter, E. N. $\quad$ I 69

Dexter, J.

Dexter, J. E.

Dexter, R. M.

de Zoete, H. W.

Diamond, A.S.

Dibb, R. K.

Dibble, T.E.

Dick, C. W.

Dick, H. P.

Dick, J. McN.

Dick, J. R.

Dick, N. B.

Dickens, C. C.

Dickens, P. C.

197

169

169

387

387

7

222

488

387

294

75

349

488

489

Dickenson, L.A.F.W.5
Dickenson, L. G.

Dickey, E. M. O'R. $\quad 387$

Dickins, B.

Dickinson, A. H.

Dickinson, A. P.

Dickinson, G. F.

Dickinson, G. N.

Dickinson, P.P.

Dickinson, R.S.

Dickinson, W. H.

Dickson, A. F.

Dickson, A. N.

Dickson, C. G.

Dickson, E. C. S.

Dickson, F. J. C.

Dickson, F.P.

Dickson, H. S.

Dickson, J. W. E.

Dickson, K. B.

Dickson, R. J.

Dier, C. V.

Dietrichsen, F. C.

Digby, T. H.

Digby-Johnson, N.75, I 69

Digges La Touche, G. G.

387

Digges La Touche, H. N.

see La Touche, H. N. D.

Dilke, C. W.

Dilke, Sir F. W.

Dill, J. F. Gordon

Dill, J. M. Gordon

Dillon, H. G. S.

Dillon, T. A.

Dingad-Davies, F L1 387

Dingwall, C. F.

Dingwall Fordyce, A.

see Fordyce, A. Dingwall

Dingwall Fordyce, $R$.

see Fordyce, R. Dingwall

Dinn, H. K.

Dippie, $\mathrm{H}$.

Dircks, L. R. A.

Disney, H. A. P.

Dixon, A. F. W.

Dixon, A. H.

Dixon, $C$.

Dixon, E.T.

Dixon, $G$.

Dixon, $\mathrm{H}$.

Dixon, H. H.

Dixon, H. J.

Dixon, J. E. B.

Dixon, J. G.

Dixon, J. H.

Dixon, $O$.

Dixon, T. H.

Dixon, W.E.

Dixon Wright, H. D.

Dixson, H.F.

Doak, J. K. R.

I 97

387

387
128

75

349

387

92
243

243
7

387

387

294

129

243

243

294

388

I69

529

388

129

75
Dobb, G. C.

Dobb, H. R.

Dobb, R. A.

Dobbs, A. F.

Dobbs, F. W.

Dobbs, W. C.

Dobbs, W. E. F. $\quad 388$

Dobell, $\mathrm{H}$.

Dobie, J. N.

Dobie, W. M.

Dobson, A. T. A.

Dobson, D. R.

Dobson, E. L.

Dobson, J. F.

Dockray, J. V.

Dodd, A. H.

Dodd, J. M.

Dodd, R. P.

Dodd, W. P.

Doddrell, E. C.

Dodds, M. T.

Dodgshon A. J. C.

Dodgson, F.

Dodgson, P. H.

Dodson, A. D. H.

Dodson, C.S.

Dodson, F. K.

Dodwell, G.M.

Dodwell, H. B.

Doggart, $\mathrm{H}$.

Doggart, J. $\mathrm{H}$.

Doggett, G.P.

Doherty, H. L.

Doherty, W. D.

Dolbey, R. C.

Dolby, H. A.

Doll, M. H. C.

Doll, W. A. M.

Don, A. W. R.

Don, F.P.

Donahoo, M. G.

Donald, A. G.

Donald, M. H.

Donald, W. G. C.

Donaldson, $\mathrm{A}$.

Donaldson, C. E. $\quad$ 169

Donaldson, C. H. $\quad 34$

Donaldson, E. $\quad 388$

Donaldson, E. A. $\quad 129$

Donaldson, G. B. 129

Donaldson, Sir H. F. $3^{88}$

Donaldson, J. T. $\quad 92$

Donaldson, $M$. $\quad 388$

Donaldson, N. $\quad 388$

Donaldson, S. H. M. $3^{88}$

Donisthorpe, E. R. 489

Donnell, J.H. $\quad 129$

Donner, J. D. $\quad 388$

I29 Donnithorne, V.H. $\quad 36$ 
Donovan, E. L.

Donovan, S. J.

Dore, A. S. W.

Doresa, B. S.

Dorling, E. E.

Dorman, Sir A. J.

Dorman, B. L.

Dorrell, H. G. H.

Dottridge, C. A.

Doudney, C. E.

Dougall, E. S.

Doughty, E. C.

Doughty, W.H.

Douglas, C. H.

Douglas, C. K. M.

Douglas, F. C. B.

see BrownDouglas, F.C.

Douglas, H. A. - 514

Douglas, H. K.

Douglas, J. (Joh.)

Douglas, J. (Selw.)

Douglas, R. H.

Douglas, R. $O$.

Douglas, S. M.

Douglas-Hamilton, W. A.

Douglass-James, W. 244

Doune, Lord $\quad 388$

Douro, Marquis of 489

Douthwaite, A. W. S. 36

Dove, C. K.

Dove, F. J.

Dove, G. V.

Dove, $W$. $B$.

Dower, E. L.

Dowling, G. C. W.

Down, R. A.

Downes, G.S. J

Downie, J. M.

Downing, A. B.

Downing, C. C. R.

Downman, B. V. R.

Downman, L. C. $\quad 169$

Downton, A. M. $\quad 36$

Dowson, E. M.

Dowson, $\mathrm{H}$.

Dowson, W. J.

Doyne, R. W.

Dracup, A. H. 129, 310

Drake, A. W. C. 244

Drake, C. W. T. see

Tyrwhitt Drake, C. W.

Drake, F.

Drake, G. E.

Drake, H.R. O.

Drew, A. L.

Drew, J.A.

Drew, R.S.

Drew, V.

Drewe, A.
7

388

489

5 I 4

169

388

515

49

Duckworth, W. C. 64

64,515

Duckworth, W.L.H. I69

Duddell, A. G. $\quad 129$

Dudfield, S. R. O. $\quad 389$

Duff, A. G.

Duff, B. P.

Duff, D. G.

Duff, I. A. J.

Duff, K. D. J.

Duff, W.S. B.

Duff Stevens, C. J.

see Stevens, C. J. Duff

Duffield, C.A.W. 75, 295

Duffield, H. W.

Duffield, K. L.

389

389

283

244

93

389
Duffield, W. G.

Duffin, C. G.

Dufton, A. F.

Dufton, $\mathbf{j}$. T.

Duigan, W.
169

489

244

529

389

\section{3}

36

29

389

389

389

489

489

489
389
129

295

310

389

389

389

389

389

349

325

7

244

36

129

64

129

389

169

325

283

489

489

69

Dunscombe, $\mathrm{N}$.

Dunsheath, $\mathrm{P}$.

Dunsmure, C. H. T. 245

Dunstan, V.J. 245

Dunville, J. $\quad 390$

Dunville, J.S. $\quad 390$

Dunwell, F.L. $\quad 5 \times 5$

Dupree, E. $\quad 390$

\begin{tabular}{l|ll}
389 & Dupuis, C. E. & 390 \\
389 & Durack, J. J. E. & 390
\end{tabular}

7 Durand, P.F.

129
310

389

129

222

295

384

222

325

489

7

245

389

295

245

36

389

Duncannon, Viscount
see Bessborough, Earl of

Duncanson, T.J.G. 93

Dundas, A. C. $\quad 325$

Dunkels, W. 390

Dunkerley, C. L. $\quad 325$

75
Dunkley, G. W. $\quad 245$

Dunlop, F. L. $\quad 489$

Dunlop, J. G. M. $\quad \begin{aligned} & 349 \\ & 129\end{aligned}$

Dunlop, J.K. $\quad 325$

390

198

36

129

529

390
75

75

29

5

○

5

93


Durell, C. V.

Durham, H.E.

Durie, J. A.

Durling, J. A.

Durnford, F. H

Durnford, H. G. E.

Durnford, R. C.

Durnford, R. S.

Durrant, C. E.

Durrant, H. B.

see Lahore, Bp. of

Durrant, W. B. W.

Durst, A.

Durst, C. S.

Dutfield, D.

Duthie, W. L.

Dutton, $\mathrm{H}$.

Dutton, P. E.

Duval, M. R.

Duvall, J. R.

du Vallon,

G. C. D. de J. $\quad 283$

Dvorkovitz, V.

Dwyer, C. H.

Dyas, G. E.

Dyer, A. C.

Dyer, B.S.

Dyer, C. M.

Dyer, F. N. V.

Dyer, H. F.

Dyke-Marsh, H.StG.

Dykes, K.

Dymond, G. W.

Dyne, H. E. L.

Dyne, J. B.

Dyson, C. B.

Dyson, E.

Dyson, E. A.

Dyson, H. A.

Dyson, W.

Dyson, W. H.

Dyson, W. L.

Eade, A.

Eade, C.

Eaden, J.

Eadie, P.

Eadie, R. A.

Eagle, G. C

Ealand, V. F.

Eames, E. J. H.

Eames, W. L'E.

Eardley-Simpso n, L.E. 93

Earle, G. F.

Earle, L. M.

Earles, F. J.

Eason, E. K.

East, G. D.

Easter, A. J. T.

Easterling, H. G.

Easton, G. L. E.
36 Easton, J. W.

I 98

93

93

64

I 98

198

198

295

222

129

245

I 69

390

325

515

I 98

5 I

93

I 29

93

515

7

36

64

325

129

283

I 98

198

390

529

I69

37

390

198

295

390

245

390

390

295

295

130

390

130

325

390

8
169

93

3 I0

390

390

Ebden, J. W.

Ebden, W.S.

Eberli, W. F.

Eccles, A. G.

Eccles, G. T.

Eccles, R.

Eddison, J. R.

Ede, C.

Ede, E. E.

Ede, J. C.

Ede, M. C.

Eden, J. R.

Edg $=$, A. S.

Edmonds, J.
Eastwell, M.M.

Eastwood, H. E.

Eastwood, J. F.

Eastwood, N. W.

Eatherley, W.

Eaton, F. O. H.

Eaton, J. E. C.

Ebden, C. H. M.

Eccles, W. E. L.

Eckenstein, T. C.

Eckersley, T. L.

Eckhard, O.P.

Eddison, F. W.

Eddison, H. W.

Eddowes, H. C.

Eddowes, W. B.

Edgar, W. H. A.

Edge Partington, E. F. see Partington, E. F. E

Edgeworth, F. H. I30

Edgington, $\mathrm{H}$. $\quad 283$

Edmonds, S. F. $\quad 349$

Edmunds, C. H. $\quad 75$

Edmunds, C. V. $\quad 515$

Edm ands, H. V. $\quad 169$

Edmunds, P. M. L. $\quad 198$

Edwardes, F. E. $\quad 326$

Edwardes, H. F. E. 326

Edwards, A. L. $\quad 222$

Edwards, A. T. $\quad 326$

Edwards, C. G. $\quad$ I30

Edwards, C. J.

Edwards, D. L. P.

Edwards, D. W.

Edwards, E. D.

Edwards, F. M.

Edwards, G. B.

Edwards, G. R.

Edwards, G. T.

Edwards, $H$. J. $283,391,5$ I 5

Edwards, H.S.

Edwards, H. V.

Edwards, H. W.

Edwards, J. L. A.

Edwards, J.S.

$39 \mathrm{I}$

391

391

I 30

130

222

8

$39 \mathrm{I}$

8

98
I 0

8

245

37

39 I

8
295

245
325
310

I 98

75

30

98

245

65

93

489

69

E \\ E}

Elkington, T. G. $\quad 283$

Ellice-Clark, S.T. $39 \mathrm{I}$

Ellicott, F.A. J. 391

Ellington, N. B. $\quad \mathbf{r} 98$

Elliot, G.W.E. 391

Elliot, H.W.A. $39 \mathrm{I}$

Elliot, R. H. $\quad 169$

Elliot, W. E. 391

Elliott, A. D. $\quad 8$

Elliott, A. F. $\quad 93$

Elliott, A. G. 222

Elliott, C. A. B. (Jes.) I 69

Elliott, C.A.B.(Kg's) 198

Elliott, C. P. $\quad 245$

Elliott, E. C. $\quad 93$

198 Elliott, E. C. B. 392

8 Elliott, G. L. L. 392

8 Elliott, H. D.E. 349

515 Elliott, J.S. 515

295 Elliott, M. L. F. 37

391 Elliott, O.C. F. $\quad 529$

326 Elliott, S. D. 529

198 Elliott, T. C. J. 392

Elliott, T. R. $\quad 37,392$
Elliott, W. W. $\quad 529$

Elliott-Cooper, M. $\quad 198$

Ellis, A. I. $\quad 326$

Ellis, B. J.

Ellis, B. W.

I
8
1
1
9
8
3
2
9
5
3
2
2
5
5
9
9
2
8
9
8
8

37

8

26

37

8

29

9 I

15
75

75
98

30

30

45
37

37

30

45

(1) 
Ellis, C. D. B.

Ellis, D.W.

Ellis, E. W.

Ellis, J.

Ellis, J. M.

Ellis, J. W. H.

Ellis, M. F.

Ellis, P. D.

Ellis, R.

Ellis, T.P.

Ellis, V. L.

Ellis, W. F. P.

Ellis, W. H. M.

Ellis Roberts, $\mathbf{R}$.

Ellison, A. D.

Ellison, A. J.

Ellison, H. B.

Ellison, H. S.

Ellison, J.

Ellison, W. J.

Elliston, G.S.

Elliston, W. R.

Elmslie, G. F.

Elmslie, K. W.

Elphick, H. C.

Elphinston, A.

Elphinstone L. H

Elrington-Bisset, M. 245

Elrington-Bisset, W. F.

Elsey, J. W.

Elton, H. B.

Elton, W.

see Marwood-Elton, W.

Eltringham, H. C. $\quad 130$

Elveden, Viscount 392

Elverson, J. H.

Elverson, R. W.

Elwell, R. G.

Elwin, F. H.

Elwin, W. D.

Elwood, A. G. F

Elworthy, C. W.

Elworthy, P.A.

Elworthy, $T$.

Elworthy, W. R.

Embleton, D.

Embrey, C. S.

Eminson, R. A. F.

Emmett, R. H.

Emrys-Evans, P. V. 392

Emrys-Jones, M. F. I30

Emtage, W. L. 295, 529

England, E. M. $\quad 93$

England, E. T. $\quad 392$

England, F.

England, F. de F. $\quad 392$

England, $\mathrm{H}$.

England, J. A.

England, W. B.

93

295

392
130

295

75

3 I0

8

199

392

5

2

245

5 I 5

130

245

392

199

310

93

489

130

349

75

2

,
Evans, H. G.

Evans, H. R.

Evans, H.S.

Evans, I.

Evans, J. H.

Evans, J. Li.

Evans, J. R. A.

Evans, J. W. D.

8 Evans, K. R.

Espin, C. E. A.

A. L1.

Evans, B.

Evans, D. D.

Evans, D. L.

Evans, E. D.

Evans, E. W.

Evans, F. H. F

Evans, G.F.F.

Evans, G. L. B.
326

Evans, L. G.

Evans, M. W. H. $\quad 130$

Evans, O. L. J. $\quad 223$

I09

284

326

169

37

37

199

245

490

392

8

A. C. Sotheron 222

W. B. Sotheron 223

Estridge, C. L. $\quad 392$

E. $\quad 515$

Eustace, W. R. G. 223

Evans, A. G. (Trin.) 392

Evans, A. G. (Selw.) 515

295

3 I0

see Bowle-Evans, C. H.

Evans, C. H. (Pemb.) 245

Evans, C. L.

(Kg's, Sid.) 199, 349

Evans, E. (Trin. H.) 490

Evans, F. R.

326

37

392

392

284

245

75

199

75

(1)

(

Evans, R. A.

Evans, R. C.

Evans, R. D.

Evans, R. du B.

Evans, T. M.

Evans, U.R.

Evans, V. L.

Evans, W. E.

245

392

392

326

245

284

199

392

326

Evans, W. V.E. $\quad 490$

Evans-Lombe, E. H. 392

Evatt, G. R. K. $\quad 326$

Evatt, J. M. I30

Eve, A. S. $\quad 246$

Eve, H.F.H. I30

Evelyn-White, K. V. 295

Everard, W. L. $\quad 392$

Everett, B. C. S. 130

Everett, C. E. F. $\quad 65$

Everett, H. F. 3 I0

Everett, W. W. $\quad 76$

Everitt, H. L. 130

Everington, G. F. 310

Evers, B. S. $\quad 169$

Evers, M.S. $\quad 37$

Evershed, F. T.P. $\quad$ I 30

Every, Sir E. O. $\quad 392$

Every-Halsted, C. E. 246

Evison, R. R.

Evitt, E. U.

Evors, C.A.

Ewart, G. A. 8

Ewbank, A. L. J. 295

Ewen, G. C. $\quad 349$

Ewen, J. F. B. $\quad 37$

Ewens, B. C. $\quad$ I30

Ewing, A. G. 295

Ewing, A. H. ' $\quad 392$

Ewing, A. W. $\quad 8$

Ewing, J. R. $\quad 169$

Ewing, W.T. 130

Exeter, Marquess of $\mathbf{2 2 3}$

Exham, R. K.S. $\quad 284$

Exmouth, Viscount 393

Exshaw, T.S.N. $\quad 37$

Eyre, C. H. $\quad 246$

Eyre, L. B. I30

Ezra, D. 393

Faber, L. E. $\quad 170$

Faber, V. V. $\quad 5$ I 5

Facey, N. S. $\quad 529$

Fagan, C. H. J. $\quad 130$

Fagan, H.W.F. $\quad 515$

Fagan, N. $\quad 246$

Failes, B. J. $\quad 296$

392 Fair, C. H. $\quad 246$

349 Fair, J.C. 
Fairbairn, C. O.

Fairbairn, C. P.

Fairbairn, G. A.

Fairbairn, G. E.

Fairbank, J.

Fairbourn, A. N.

Fairbrother, J.

Fairchild, G.C.

Fairley, W.

Falcon, J.H.

Falcon, $M$.

Falk, H.

Falkner, A. H.

Falle, Sir B. G.

Falloon, W. M.

Fallowes, J. T. C.

Falmouth, Viscount

Fane, W. V.R.

Fanshawe, C. H.

Fargus, A. H. C.

Farie, A. J. C.

Farley, C. F.

Farley, R. L.

Farmer, H. C. M.

Farmer, J. I.

Farmery, J. W.

Farmiloe, G. F.

Farmiloe, K. M.

Farmiloe, T. H.

Farnell, H. L.

Farnfield, A. J.

Farnfield, H.V.

Farnfield, P. H.

Farnham, F. J.

Farnsworth, C. R.

Farquhar, H. B.

Farquharson, H. F. W.

Farr, H. F.

Farr, T. H.

Farrant, M.

Farrar, E. M.

Farrar, F. P.

Farrar, H. R.

Farrell, W.J.

Farren, W.S.

Farrer, E. R. B.

Farrow, E. P.

Farrow, W. P.

Fasson, F. H.

Faulconbridge, F. T.

Faulder, T. J.

Faulkner, A. K.

Faulkner, R.

Faunce-de-Laune,E. I70

Fauquet Lemaitre, P. A.

Favell, N. B.

Fawcett, H. H. J.

Fawcett, R.

Fawcett, R. H.
170

\section{3}

393

529

246

37

393

393

393

37

93

490

93

490

I 99

296

296

296

8

65

393

490

3 I I

349

393

246

246

296

170

393

65

393

3 I I
Fawcett, R. W.

Fawcett, T. G.

Fawcett, W. F.

Fawcett, W. L.

Fawcus, J. G.

Fawcus, L. R.

Fawell, C. L.

Fawell, S. H.

Fawkes, F. H.

Fawkes, R. B.

Fawkes, W. H.

Fawsitt, T.R.

Fay, C. R.

Fay, S. J.

Fayerman, A. P. G. 326

Fayle, B. J. L. $\quad 94$

Fayrer, F. D.S.

Fearfield, C. J.

393

Fearnley-Whittingstall, G. H.

Fearnsides, E. G. $\quad 490$

Featherstone, H.W. 393

Feetham, F. G. $\quad 393$

Feilden, $\mathrm{O}$. H

393
38

Feilding, F. E. H. J. 393

Feilding, H. S.

Feiling, A.

393

Felkin, A. E.

Fell, D. M.

Fell, F. J.

Fellows, R. B.

Felton, D. G. W.

Felton, L. B.

Fenn, E. G.P.

Fenton, V.N.

Fenwick, A. L.

Fenwick, B. A.

Fenwick, G.

Fenwick, J. C.

Fenwick, J.S.

Fenwick, M. C.

246

I 99

170

94

Fenwick, W.

Fergus, A. McF. H.

Ferguson, A. C. W.

Ferguson, C. L.

Ferguson, D. F.

Ferguson, H. M.

Ferguson, J. C. M.

Ferguson, M. G.

Ferguson, S. C.

Ferguson, W. H.

Fergusson, A.

Fergusson, J. N. F. $\quad 326$

Fergusson, L. R. $\quad 326$

Fergusson, N. M. (Magd.)

Fergusson, N. M. (Trin.)

Fermor-Hesketh, T. 394

Fernie, E. H.

393

490

393

I3 I

393

296

94
30 Fernie, W. J.

93 Ferrand, J. B. P.

Ferrar, H. T.

490

Ferrers, E. B.

Ferrers Guy, A. W.

Ferris, S. B. C.

Fetherstonhaugh, R. G.

Feuerheerd, L. M.

Few, H. C.

Few, J. E.

Fewings, J. A.

ffrench, W. K.P. $\quad 246$

Fiddian, C. M. $\quad 199$

Fiddian, E. A.

Fiddian, J. V.

Fiddian, W. M.

Field, H. T. C.

Field, $M$.

Fieldhouse, E. E.

Fielding, E. F.

Fielding-Johnson, H. G.

Fiennes, J.E.

Figgis, B. E.

Figgis, J. M.

Fildes, F. L. V.

Fildes, G.P. A.

Fildes, P. G.

Filmer, W. G. H.

Filon, L. N. G.

Finch, A.

Finch, E. G.

Finch, $\mathrm{G}$.

94
94

I 99

296

394

76

246

246

246

246

394

394

394

326

199

170

296

Finch, H. A. I.

Findlay, C.W.McD

Findlay, I. E.

Findlay, J.

Findlay, J. G.

Finlay, E. L.

Finlay, R. V. K.

Finlayson, J. G.

Finlow, L. W.

Finnis, C. R.

Finter, F. B.

Firebrace, R. C.

Firman-Edwards, L. P. L.

Firth, Arnold

Firth, A. C. D.

Firth, A. M. B.

Firth, E. L.

Firth, L. G.

Firth, M. M.

Firth, R. B.

Firth, R. C. D.

Fischer, A. W.

Fischer, C. E.

see Fisher, C. E.

Fischer, F.N. 
Fisher, A. R. C. $\quad 284$ Fisher, B. O.F. de C. I70 Fisher, C. E.

Fisher, E. G.

Fisher, E. H.

Fisher, F. B.

Fisher, G. A.

Fisher, G. H.

Fisher, G. W.

Fisher, J.

Fisher, J. W.

Fisher, L. G.

Fisher, L. N.

Fisher, $\mathrm{O}$.

Fisher, R.

Fisher, W. H.

Fisher-Smith, E. L.

Fishwick, J.F.

Fisk, A. A.

Fiske, C. H.

Fison, A. K.

Fison, C. H.

Fison, E.T.

Fison, W.J.

Fitch, A. S.

Fitch, C. E.

Fitch, E. W.

Fitton, R.

FitzGerald, D.

Fitzgerald, E. A.

Fitzgerald, G. M.

FitzGerald, G. T.

Fitzgerald, H.S.

FitzGerald, J. F.

Fitzgerald, M. P.

Fitzherbert, G. C.

FitzHerbert, J. A.

Fitzhugh, A. E. L.

Fitzjohn, $T$.

Fitzmaurice, G. L. 5 I 5

Fitzmaurice, W.H. 5 I 5

Fitzpatrick, E. R. 394

Fitzroy, H.S.

Fitzwilliam, Earl

Fixsen, B. A.

Flannery, H. Fortescue

Flawn, N. G.

Flaxman, W.J.

Fleet, C. S.

Fleming, A.L.

Fleming, G. B.

Fleming, N.

Fleming-Brown, G.F. 38

Flemming, M. G. I3I

Fletcher, A. B.

Fletcher, A.M.T. $\quad 223$

Fletcher, A. W.

Fletcher, B. F.

Fletcher, E.

Fletcher, E. T. D.

5 I 5

I70

223
Fletcher, F. C.

Fletcher, H. M.

Fletcher, J. H. B.

Fletcher, M.

Fletcher, $\mathbf{P}$.

Fletcher, 284, 5 I 5

Fletcher, W.

Fletcher, W. G.

Fletcher, W. T. 38, 5 I5

Fleuret, F.S.

Flinn, O. S.

Flint, A. H.

Flitch, J. E. C.

Flood, C. B.

Flook, H.S.

Flory, P.J.

Flower, N. A. C.

Flower, O. S.

Flower, W.J.

Flynn, A.J.

Foden, W. B.

Fogg-Elliot, C. T.

Foley, C. P.

Foley, M. J. A.

Follit, H. H. B.

Fontaine de Mazinghen, A.

Foord, G. H.

Foot, S. H.

Foot, T. J.

Forbes, A.

Forbes, A. G.

Forbes, B. C.

Forbes, E. A.

Forbes, J. F.

Forbes, J. G.

Forbes, J. W. F.

Forbes Adam, C. G. 200

Forbes-Tweedie, D. 395

Ford, F. C.

Ford, F. T.

Ford, F. W.

Ford, H. J.

Ford, J. C.

Ford, R. M.

Forde, C. L.

Forder, F. G.

Fordham, E. K.

Fordham, H. A.

Fordham, H. J.

Fordham, $\mathrm{O}$.

Fordham, W. H.

Fordyce, A. Dingwall 76

Fordyce, R. Dingwall 395

Forester, Lord

Forester, O. St M. W. see Weld-Forester, O. St M.

Forman, D. P.

Forman, G. E. G.

Forman, $\mathrm{H}$.
395

395

326

$5 \times 5$

350

I3 I

395

246

200

I 70

395

529

395

170

529

395

326

490

490

94
38

76

247

94

326

515

200

395
38

9

9

395
327

5 I 5

515

38

296

490

38

395

395

490

395

76

395

395

Forman, T.P. G. '

Formby, T. H.

Formoy, R. R.

Forrest, $\mathrm{H}$.

Forrest, J. W.

Forse, L. N.

Forshaw, H. P.

Forshaw, J. H.

Forster, C. M.

Forster, F. A.

Forster, G. W.

Forster, M.

Forster, $\mathrm{T}$.

Forster, W.

247

284

131

395

395

65

490

395

I 3 I

395

170

327

395

395

Forster-Brown, J. C. 13 I

Forsyth, A. B. $\quad 76$

Forsyth, G. I3I

Forsyth, L. W. 131

Fortescue, Earl 395

Forwood, P. L. $\quad 395$

Fosdick, J. H. $\quad 247$

Foster, A. W. $\quad 76$

Foster, B. La T. $\quad 395$

Foster, C. W. 490

Foster, D. B.L. $\quad 516$

Foster, F. E. $\quad 490$

Foster, H. E. $\quad 395$

Foster, H. P. R. 200

Foster, J. D. I70

Foster, J. H. $\quad$ I $\quad$ I

Foster, J. R. $\quad 327$

Foster, K. G. $\quad 395$

Foster, M. G. $\quad 395$

Foster, M. R. 395

Foster, M. R. W. $\quad 395$

Foster, O. B. $\quad 396$

Foster, P. La T. $\quad 396$

Foster, R. $\quad 530$

Foster, R. D. $\quad 327$

Foster, R. L. V. 200

Foster, V.le N. 200

Foster, W. M. 490

Fothergill, C. F. $\quad 94$

Foulds, J. G. $\quad 350$

Foulger, H. C. $\quad 247$

Foulkes Jones, W. 200

Foulston, S. V. $\quad 65$

Fowke, G. H. S. $\quad 490$

Fowke, L. A. 223

Fowle, Sir H. W. H. 38

Fowle, T.E. $\quad 396$

Fowler, C. H. $\quad 396$

Fowler, D. D. $\quad 396$

Fowler, H. R. R. $\quad 516$

Fowler, J. D. $\quad 396$

Fowler, Sir J. K. I3I

Fowler, R. H. $\quad 396$

Fowler, T. G. I3 I

Fowlie, J. L. $\quad 247$

I 70 Fox, A.S. 247

247 Fox, C. 350 
Fox, C. L.

Fox, D. S.

Fox, H. M.

Fox, H. W.

Fox, J. C.

Fox, J. St V.

Fox, J. T.

Fox, R. W.

Fox, T.S.W.

Fox, W.S.

Fox-Andrews, N. R. 490

Fox-Strangways, W. A.

Foyster, P. T.

Fraenkl, E. G.H.

see Fremantle, E. G. H.

Frampton, H. H. C. 94

Francillon, F.E.

Francillon, F. J.

Francis, B. A.

Francis, E. C.

Francis, E. G.

Francis, G. L. B.

Francis, J. A.

Francis, J. C. W.

Francis, $\mathrm{K}$.

Francis, R.

Francis, W. H.

Franklin, C. R.

Franklin, C. S.P.

Franklin, F. B.

Franklin, G. D.

Franklin, H. W.

Franklin, J. H.

Franklin, R.P.

Franklin, T. B.

Franklin, W.

Franklin, W. B.

Franklin-Smith, N.C

Franklyn, A. H. $\quad 396$

Franks, G. L. T.

Fraser, A. A.

Fraser, A. L.

Fraser, C. S.

Fraser, D. H.

Fraser, D. P.

Fraser, D.S.

Fraser, F. R.

Fraser, G. T.

Fraser, J. H.

Fraser, J. H. P.

Fraser, M. H.

Fraser, R.

Fraser, $\mathrm{T}$.

Fraser, W. A.

Fraser-Mackenzie,

$$
\text { E. R. L. }
$$

Fraser-Mackenzie, J.A. O.
200

247

396

396

490

$35 \circ$

38

327

396

247

396

94
9

9

3 II

5 I 6

396

I3 I

9
247

247

247

490

247

327

247

200

327

327

247

327

284

396

38

247

247

396

I3 I

247

327

9

396

$3^{8}$

170

491

247

38

9

396

396
Frazier-Upton, G.

Freake, F. M.

Fream, W.

Frean, H. G.

Frecheville, G.

Frederick, $T$.

Freeborn, J. H. R.

Freeman, C. R.

Freeman, G.C.

Freeman, H. (Chr.)

Freeman, H. (Emm.) 94

Freeman, H. (Pemb.) 247

Freeman, J. E.

Freeman, $P$.

Freeman, P. B.

Freeman, W. H.

Freeman Mitford, C.

see Mitford, C. B. O. F

Freer, G. H.

Freeston, B. D.

396

Fremantle, E. G. H. 396

French, C. H. A. $\quad 284$

French, E. A.

French, E. N.

Frend, E. C.

Frend J. P (Trin) 223

Frend, W. R.

Frere, J. E.

Frere, J. G.

Fretz, W. T.S.

Frewen, H. M.

Frewen, S.

Freyer, D. J.

. T. 223

Frink, F. C. B.

Frith, J. B.

Frost, E. L.

Frost, H. K.

Frost, N.P.

Frost, T. F. C.

Frost, T. L.

Fruhe-Sutcliffe, $R$.

Fry, A. H.

Fry, C. R.

Fry, C. R. M.

Fry, D. G.

Fry, F. McG.

Fry, J.

Fry, J. D.

Fry, K. R. B.

Fry, L. H.

Fry, L. S.

Fry, T.P.

Fryer, J. H.

Fuchs, H. M. see Fox, H. M.

Fulford, R. H.

Fullalove, A. L.

Fulton, E.C.

Funduklian, A. A.
397

247

397

397

9

Ga

Furlonger, R. C.

Furness, W.

Fursdon, A. A.

Fursdon, G. E.S. $\quad 397$

Furse, W. K.

Furze, A.

Furze, C.

Fyffe, W. K.

Fyson, $\mathrm{G}$.

Gabain, W. G.

Gabb, H. S.

Gabb, J. D.

Gabriel, A.

Gabriel, E. V.

Gabriel, J. B. S.

Gabriel, O. B.

Gaddum, R. C. S.

Gaddum, W. F.

Gaffikin, G. H.

Gains, S. G.

Gainsborough, $\mathbf{H}$.

Gaitskell, M. H.

Galbraith, A. N.

Galbraith, H. G.

Gale, A. J. V.

Gale, C. C.

Galer, F. B.

Galer, R. V.

Gall, A.

Gallimore, H. B.

Galloway, A.

Galloway, S. J.

Galloway, W.

Galloway, W. D.

Galt, R. B.

Gamble, H. J.

Game, H. A.

Gamlen, R. L.

Gammell, J. A. H.

Gammell, S. J.

Gandy, E.S.

Gandy, E. W.

Gardiner, A. F.

Gardiner, A. L.

Gardiner, H.H.

Gardiner, J.

Gardiner, K. J. R.

Gardiner, O.C.

Gardiner-Hill, C.

Gardiner-Hill, $\mathbf{H}$.

Gardner, A.

Gardner, C.

Gardner, C. G.

Gardner, E.

Gardner, E. A.

Gardner, F.W.

Gardner, G. H.

Gardner, $H$.

Gardner, H. D.

Gardner, H. M.

397

223

516

171

132

350

247

76

132

491

94

94

94

397

397

38

350

76

65

397

132

516

327

65

65

516

200

65

171

296

38

327

530

397

132

248

248 
Gardner, H. W.

Gardner, J.

Gardner, J. C.

Gardner, J. H.

Gardner, J. L.

Gardner, J. M.S.

Gardner, R.

Gardner, R. C. B.

Gardner, R. G.

Gardner, R. L.

Gardner, R. T.

Gardner-Brown, F.S. G.

Gardner-Brown, J. G. G.

Gardner-Medwin, F. $M$.

Garfit, T. N. C.

Garmonsway, G. N. 3 II

Garne, T.

Garner, H. M.

Garnett, C.

Garnett, D. G.

Garnett, I. W.

Garnett, J. N.

Garnett, K. G.

Garnett, R. T.

Garnett, W. H. S.

Garnett, W.P.

Garnett-Botfield, A. C. F.

Garnett-Botfield, W. McL.

Garnons Williams, R. D. see Williams, R. D. Garnons

Garnsey, E.

Garrard, G. G. C.

Garrard, N.

Garratt, G. H.

Garrett, A. H.

Garrett, D. T.

Garrett, G. D.

Garrett, H. F. (Pem.) 248

Garrett, H. F. (Qu.) 296

Garrett, H. L. O. 327

Garrett, L. C.

Garrett, N. L.

Garrett, S. (Trin.) 398

Garrett, S. (Fitz.H.) 530

Garrett, T. R. H.

Garrett-Smith, L.

Garrick, G. C.

Garrod, A. N.

Garrod, L. P.

Garrod, R.P.

Garrod, W. E. E.

Garrood, J. R.

Garside, G. C.

Garson, H. L.

Garstin, D. N.
397

296 Garstin, E. J.L.

397

516

327

95
$\times 32$

397

95

248

248

397

II

171
327

248

248

223

397

95
397

248

397

97

7

Gea

95

248

96

7

491

I7I

398

95

200

39

296

327

398

39

350

Gascoigne, $T$.

Gaselee, A. M.

Gaskell, C. H.

Gaskell, J. F.

Gaskell, J. G.

Gaskell, L.S.

Gaskin, E. A. L.

Gasking, E. B.

Gatehouse, $\mathrm{H}$.

Gater, B. A. R.

Gates, C.

Gates, C. E.

Gates, S. B.

Gauld, A. G.

Gaussen, E. A.

Gaussen, J. M.

Gavin, A. G. D.

Gavin, N. D. I.

Gavin, W.

Gawler, A. E. J.

Gawne, H. D.

Gay, C. D.

Gay, J.J.

Gay, L. H.

Gaye, A. S.

Gaye, A. W.

Gayne, A. A. A.

Gaze, A. M. B.

Gaze, G. A.

Geard, D. A. A.

Geare, J. W. A.

Geare, W. D.

Geary, A. B.

Geaves, W. L.

Gedge, A. A. L.

Gedge, B. J.

Gedge, C. B.

Gedge, $P$.

Geldard, N.

Geldart, H. R. R. F. C.

Gell, E. A.S.

Gell, W. C. C.

Gemmell, A. A.

Gemmell, K. T.

Gent, F. E.

George, J. T.

German, R. L.

Gervis, $\mathrm{H}$.

Gething, H. B.
Gathergood, G. W.

Gathergood, L.S.

Gawan-Taylor, N. 398

awan-Taylor, N. $35^{\circ}$

Gawan-Taylor, T.F. 350

Gee, R. F. McL. $\quad 398$

Gelderd-Somervell,

Gentle, F. W.

George, A. K. D. $\quad 132$

Gerard, Lord 398,

$\begin{array}{lr}\text { Gerard, Lord } & \text { 398, } 491 \\ \text { Gerard, R. J. L. } & \text { I } 32\end{array}$
350

200

248

132

284

39

39
132

248

350
65

$35^{\circ}$

350

350

327

284

132

98

39

516

398

9

398

I32

39
530

327

398

248

296

200

248

248

516

398

516

398

200

398

65

132

200

248

200

296

95

398

398
248

132

3 I I

284

95

248

200

398

398

398

$49 I$

49 I

223

248

39

398

296

248

200

I 32

398

$\begin{array}{lr}\text { Gibson, D. K. } & 39 \\ \text { Gibson, F.A.S. } \quad 223\end{array}$

Gibson, G. M.(Cla.) 39

Gibson,G.M.(Trin.) 398

Gibson, $\mathrm{H}$. $\quad 53^{\circ}$

Gibson, H. H. 3 I I

Gibson, I. F.

Gibson, J. G.

Gibson, N.

Gibson, P.C. F. $\quad 516$

Gibson, R. J. T. $\quad 249$

Gibson, R. M.

Gibson, R. W. B. I7I

Gibson, S. R. $\quad 296$

Gibson, T. B. $\quad 516$

Gibson, T. G. $\quad 249$

Gibson, W. F. $\quad 39$

Gibson-Craig, Sir A. C. 398

Gibson-Watt, J. M. 398

Gidlow-

Gidlow-

Jackson, C. W. 249

Jackson, G. H. 398

Gidney, F. $\quad 516$

Gielgud, H. L. F. A. 249

Giffard, J.S. $\quad$ I7 1

Gifford, R.E. $\quad 39$

Gilbert, G. G.T. $\quad 516$

Gilbert, $\mathrm{H}$.

Gilbert, L.A. $\quad 3$ I I

Gilbert, R.T.E. $\quad 49 \mathrm{I}$

Gilbert, T. $49 \mathrm{I}$

Gilbertson, W.

Gildea, Sir J.

Giles, C. C. T

Giles, E. (Pemb.) 249

Giles, E. (Trin.) 398

Giles, G. C. T. 201 
Giles, H. O'H.

Giles, L. T.

Gilham, C. W.

Gill, C. G. Hope

Gill, C. I. C.

Gill, G. A.

Gill, H. L. O.

Gill, H. V.

Gill, K. C.

Gill, R. G.

Gill, W.

Gillan, G. V. B.

Gillespie, J. J.

Gillespie, T.

Gillibrand, A.

Gillies, C. P.

Gillies, H. D.

Gilligan, A. E. R.

Gilliland, G. F.

Gilliland, V. K.

Gilling, H. T.

Gillson, A.H.S.

Gillson, G.

Gillum, S. J.

Gilman, J.

Gilmore, A. E.

Gilmour, A. S.

Gilmour, $\mathrm{H}$.

Gilmour, J.

Gilroy, P. K.

Gilson, R. Q.

Gimblett, C. L.

Gimlette, C. H. M.

Gimson, B. L.

Gimson, C.

Gingell, W. C.

Ginn, D. B.

Ginsburg, H. H.

see Gainsborough, $\mathrm{H}$.

Girard, D. L. M. $\quad 76$

Girdlestone, C. M. 398

Girdlestone,F.S.P.L. 530

Girling, E. A. $\quad 516$

Girling, F. B.

Gisborne, L. G. $\quad 249$

Gissing, A. C. $\quad 223$

Gjers, L.

Gladstone, H.S. $\quad 399$

Gladstone, K. S. M. 491

Glaister, G. F. $\quad 53^{\circ}$

Glaister, S. E. $\quad 53 \circ$

Glanville, W. J. $\quad$ I32

Glasbrook, J. H. L. 223

Glaser, W. H.

Glasspool, R. T. B. $\quad 223$

Glazebrook, A. R. 399

Gleave, J. W.

Gleave, T. R.

Gledhill, A.

Gledhill, W. G.

Gleed, R. W. A.

65

328

399
491 Glegg, A. L.

284 Glen, R. A.

Glen, R. R.

Glen-Bott, C. L.

Glenday, R. G.

Glendinning, H.W. 133

Glenister, D. J.

Glenny, H. Q.

Gloster, H. C.

Glover, G.H.

Glover, H. P. McC.

Glover, J. A.

Glover, J. G.

Gluckstein, I. M.

Glyn, C. R.

Glyn, J. P.

Glynn, E. E.

Glynn, E. H.

Glynne, A.

Goatcher, F.

Gobbitt, R. H. S.

Godber, H. T.

Goddard, A. S.

Goddard, E. T.

Goddard, F. W.

Goddard, J.

Goddard-Jackson, N. W.

Godlee, S.

Godsal, Herbert

Godsal, Hugh

Godsell, R. T.

Godson, F. P.

Goff, Sir H. W. D.

Going, T. H.

Gold, E.

Goldberg, S. V.

see Goldhurst, S. V.

Goldblatt, D.

Goldhurst, S. V.

Goldie, A. H. R.

Goldie, C. J. D.

Goldie, N. B.

Golding, H. C.

Golding, R. H. C.

Goldney, H. H.

Goldschmidt, J.P.

Goldschmidt, W. N. 399

Goldsmith, E. O. 249

Goldsmith, H. M. I7I

Goldsmith, S. W. $\quad 516$

Goldsworthy, E. W. 49I

Goldthorpe, A. F.

Goldwater, H. G.

Gomme, A. W.

Gomme, E. E. C.

Gomme, G. J. L.

Gooch, C. T.

Gooch, G. W.
65

399

95

95

9
39

133

133

328

297

$35 \circ$

328

133
39

39

39
516

328

399

399

297

9

399

249

399

399

399

399

39
328

491

311

328

399

249

516

399

(399

(

Gor

Gor

39

328

399

49 I

399

249

350

249

297
Goodacre, J.

Goodall, C. C.

Goodall, C. E. G. $\quad 350$

Goodall, H. M. $\quad 249$

Goodall, J. F. $\quad 95$

Goodchild, E. J. $\quad 530$

Goodchild, E. L. $\quad 133$

Goodchild, G. F. $\quad 350$

Goodchild, H. N. $\quad$ I7I

Goodden, C. P. $\quad 201$

Goode, R. H. $\quad 328$

Gooderham,E.J.R.B. 133

Goodford, C. J.H. 223

Goodhart, E. J. $\quad 39$

Goodhart, G. W. 399

Goodhart-Rendel, H.S.

Goodhue, F. W. J.

Gooding, S.

Goodman, B. M. $\quad 249$

Goodman, E. L. $\quad$ I7I

Goodwin, A. C. $\quad 223$

Goodwin, A. D. $\quad 399$

Goodwin, E. $\quad 95$

Goodwin, E.St G.S. 133

Goodwin, H. A. C. $\quad 171$

Goodwin, H. D. $\quad 399$

Goodwin, H. J. 17 I

Goodwyn, P. W. $\quad 297$

Goolden, A. W. $\quad 399$

Goolden, C. E. $\quad 95$

Goolden, D. C. $\quad 65$

Goolden, H. J. $\quad 328$

Gordon, A. $\quad 133$

Gordon, A. F. L. $\quad 491$

Gordon, A. J. M. $\quad 249$

Gordon, A. R. $\quad 399$

Gordon, A.S. $\quad 249$

Gordon, C. A. 201

Gordon, C. T. $\quad 399$

Gordon, E. B. $\quad 223$

Gordon, E. F. S. $\quad 328$

Gordon, E. P. $\quad 39$

Gordon, F. J. Io

Gordon, G. $\quad 133$

Gordon, G. A. $\quad 95$

Gordon, H. 133

Gordon, J. E. $\quad 530$

Gordon, J. G. $\quad 399$

Gordon, R. E. C. $\quad 249$

Gordon, S. $\quad 249$

Gordon, W. B. $\quad 39$

Gordon, W. H. E. $35^{\circ}$

Gordon Browne,H.H. 39

Gordon Cumming,

A.P. 399

Gordon-Vaudin,C.G. 76

Gore, I. St J. 223

GoreBrowne,H.T.T.399

Gorell-Barnes, C. R. $25^{\circ}$

Gorringe, W. H. $\quad 400$ 
Gorst, G. T.

Gosling, T.S.

Gosling, W. R.

Gosling, W.S.

Gosnell, R. P.

Goss, J.

Gossage, A. F.W.

Gossage, E. L.

Gosse, A. H.

Gosse, R. W.

Gosset, A. C. V.

Gossling, F. N.

Gostling, E. V.

Gotch, D. H.

Gotch, M. S.

Gott, W. W. M.

Gotto, H.S.

Gough, D. L.

Gough, D. L. $\quad 95$

Gough, H. A.

Gough, H. P. B.

Gough Calthorpe,

$$
\text { F. S. }
$$

Gould, C. J. B.

Gould, E.

Gould, H. C.

Gould, H. F.

Gould, K. L.

Gould, L. McL.

Gould, R.

Gould, W. J.

Gould, W. J. $\quad 35^{\circ}$

Goulden, C. B.

Goulden, D.

Goulden, E. O.

Gouldsmith, C. C.

Goullet, A. S. C.

Gourlay, W. B.

Gover, C. E. J.

Gover, J. E. B.

Gow, A. S. F.

Gow, C. H.

Gow, I. B.

Gow, L. H.

Gowans, J.

Gower, J. R.

Gower, L. C.

Gower-Rees, A. P.

Goyder, F. W.

Grabham, G.W.

Graburn, G. N.

Grace, C. L.P.

Grace, C. R.

Grace, E. M.

Grace, H. C.

Grace, H. M.

Gracey, H. C.

Gracey, R. L.

Graham, C.

Graham, G.

Graham, G. L.
400

400

516

491

400

I7 I

400

400

133

I33

I33

95

I33

I33

400

491

491

95

$35^{\circ}$

I7 I, 3 II

530

40

40

250

250

133

171

76

76

76

$49 \mathrm{I}$

I33

400

250

95

400

95

400

250

40

491

76

10

328

328

65

400

40

IO

297

297

400

400

133

400

40
Graham, G.W.

Graham, H. A. R.

Graham, H. E.

Graham, H. H. C.

Graham, J. C. W.

Graham, J. D.

Graham, J.R. N.

Graham, L. A.

Graham, M.H.

250

Graham, M.W.A.P.40I

Graham, R. L.

Graham, R.P.

Graham, W. J.

401

250

Graham, W.P. G. $\quad$ I33

Graham-Brown, G.F. 3 I I

Graham Campbell, J. 40I

Graham Hobson,W.J. 95

Graham-Hodgson, H. K.

Graham-Jones, J. L. 95

Graham-Montgomery, G. J.E.

Grail, C. G.

Granby

Grand 491

G. C.

Grandage, W. B.

Grange, E. L.

Granger, E. H. H

Grant, A. F. M.

Grant, B. D.

Grant, C. V.

Grant, D.P.

Grant, D. W.

Grant, F. G.

Grant, G. L.

Grant, G. N.

Grant, H.S.

Grant, J. C.

see Grant-Ives, J. C.

Grant, M. F.

Grant, R. M.

45

Grant, W. St C. 250

Grant-Ives, J. C. 201

Grant-Watson, E. L. 40I

Grantham, E. R. H. 40I

Grantham, F. W. $\quad 401$

Grantham, J.

76

Grantham, W.W. $40 \mathrm{I}$

Grantham-Hill, C. I33

Grasemann, C.

401

Grasett, E. B.

I 71

Grattan-Bellew,W.A. 40r

Graves, B.

Gray, A. F.

Gray, A. H.

Gray, A. K.

Gray, E. C. G.

Gray, E.T.

Gray, F. H.A.

Gray, F. L.

Gray, G.
Gray, J.

Gray, J. M.

201

Gray, M.

Gray, N.

201

401

Gray, P.

95

Gray, R E. G $\quad 250$

Gray, R.H. $\quad$ I7I

Gray, S.T. $\quad$ I7I

Gray, W. $\quad 40$

Gray, W.A. $\quad 133$

Gray, W.H. Io

Gray, W.S. 250

Gray-Cheape, H. A. 492

Grayson, D.H.H. $\quad 250$

Grazebrook, O.F. I33

Grear, A. T. L. $\quad 224$

Grear, E. J. L. $\quad 328$

Greathead, J. M. Io

Greathead, J. R. ro

Greaves, G.M. $\quad$ 40I

Greaves, H. G. $\quad 95$

Greaves, L. B. $\quad 401$

Greaves, R. C. J. $\quad 401$

Green, A. G.

Green A. G. N. IO

Green, B. ' 401

Green, B. B. $\quad 40$

Green, C. C. $\quad 95$

Green, D. $\quad 401$

Green, E. A. $\quad 96$

Green, F. N. $\quad 224$

Green, Gabriel Io

Green, George ro

Green, $\mathrm{H}$. $\quad 401$

Green, H. F. $\quad 35$ I

Green, H.S. $\quad 401$

Green, H. T. $\quad 297$

Green, J. 297

Green, J. E.S. $\quad 284,492$

Green, J. L. $\quad 76$

Green, J. R. E. 201

Green, L. N. $\quad 40$

Green, N. $\quad 328$

Green, R. C. $\quad 35 \mathrm{I}$

Green, R. D. $\quad 401$

Green, R. G. $\quad 284$

Green, R. J. $\quad 297$

Green, S. J. $\quad 77$

Green, S. M. $\quad 328$

Green, T.R. $\quad 65$

Green, W.R. C. $\quad$ I7I

Greenberg, A. W.

see Greenhill, A. W.

Greenberg, B. M.

see Greenhill, B. M.

Greene, A. H. B.

seeBarrett Greene, A.H.

Greene, C. W.

516 Greene, G. E. I33

401 Greene, G. W. 77

297 Greene, L. Io 
Greene, W. P. C.

Greenham, R. G.H 401

Greenhill, A. W.

Greenhill, B. M.

Greenhill, T. W.

Greenhough, $\mathrm{H}$.

Greenish, F.H.S.

Greenlees, J. R. C. $\quad 328$

Greenstreet,

N. B. de M.

Greenwell, B. E.

Greenwell, B. E. $\quad 401$

Greenwood, C. F.

Greenwood, E. T.

Greenwood, F. B.

Greenwood, J.

Greenwood, J. A.

Greenwood, J. E.

Greenwood, O.

Greer, $\mathrm{H}$.

Greer, T. M.

Greg, A. H.

Gregory, A. I.

Gregory, A. R.

Gregory, C. C. L.

Gregory, C. H.

Gregory, G. M.

Gregory, G. M. A.

Gregory, H. L.

Gregory, J.S.

Gregory, R. P.

Gregory, S. B.

Gregory-Jones, E.

Gregson, A. H.

Gregson, C. D.

Gregson, H. G.

Gregson, P.

Greig, J.L.

Grellier E. F.W.

Grenfell, E. B.

Grenside, C.F.

Grenville-Grey, G.

Gretton, G. F.

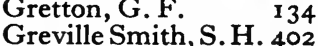

Grey, Earl

Grey, R. C.

Gribbin, J. A.

Gribble, C. H.

Grice, J. E.

Grice, J. W.

Grice, $N$.

Grice-Hutchinson, R. E.

Grier, S.M.

Grierson, $H$.

Grieve, A. B

Grieve, F.C.L.

Grieve, W. R.

Griffin, A.W.M.S.

Griffin, E. H.
96

250

3 I I

328

10

$35 \mathrm{I}$

224

284

402

201

284

250

492

402

297

328

77

96

402

492

328

402

328

40

96

516

40

402

250

40

77

I7I

250

402

402

530

10

402

96

77

201

328

402

250

250

40

402

402

402

284
Griffin, F. W. W.

Griffin, H. J.S.

Griffin, T.N.R.

Griffith, A. L.P.

Griffith, A. W.

Griffith, C.

Griffith, F. L.

Griffith, G. F.

Griffith, H. C.

Griffith, H. H.

Griffith, H. K.

Griffith, H. L. W.

Griffith, J.

Griffith, J. R.

Griffith, R.

Griffith-Jones, C.

Griffith-Jones, I S 134

Griffith-Jones, M.P.

Griffith-Williams, G.C. 96

Griffiths, C. C.

Griffiths, C. R.

402

3 II

Griffiths, G. A. M. $\quad 328$

Griffiths, J. (King's) 201

Griffiths, J.(Fitz.H.) 530

Griffiths, P. D.

Griffiths, R.

Griffiths, R. E.

Griffiths, T. T.

Griffiths, W. L.

Grigg, G.H.

Grigg, J. H.

Grigg, P. J.

Grigson P St J B. 328

Grimwade, E.E. $\quad$ I34

Grinberg, S.

Grindon, A. W. H.

Grinling, A. G.

Gripper, A. G.

Gritton, H. J.

Grogan, E. S.

Grogan, J. D.

Groner, R. E. E.

Gronow, W. H.

Groom, H. L.

Groom, R. W.

Groom, S. H.

Grose, $T$.

Grose, T. A.

134
65

402

134

134

172

172

328

35 I

3 I I

402

172

201

172

$35 \mathrm{I}$

402

40

224

224

$35 \mathrm{I}$

40

40

Grose-Hodge, D. E. 250

Grose-Hodge, $\mathrm{H}$.

Gross, W. S.

Ground, E. G.

Grove, A. J.

Groves, J. D.

Groves, J. P. K.

Groves, K. G.

Groves, W. Peer

Growse, R. H.

Grubb, H. C.S.

250

172

$35 \mathrm{I}$

96

10

297

402

IO

134

172

I34

Grylls, E. A. H.

Gubbins, C. F. R.

134

224

Gudgeon, C. J.

Gudgin, S. H.

3 I I

77

Guest, L. G. W.

402

Guest, O. M.

Guest-Williams, W. K.

329

Gueterbock, P. G. J. 403

Guggenheim, H. F. 250

Guildford, E. M. $\quad 297$

Guilford, E. L. $\quad 40$

Guillebaud, E. C. $\quad 250$

Guinness, J. F. G. $\quad 134$

Guinness, K. E. L. 492

Guinness, P. W. $\quad 96$

Guinness, R.

see Elveden, Viscount

Guinness, R.S.

Gullick, A. L.

403

Gullick C.D.

Gullick, L. B. 3 I I

Gulliland, J.H. $\quad \mathbf{I}_{34}$

Gundry, P. G.

Gundry, R. H. $\quad 284$

Gunn, J. D. $\quad 250$

Gunning, C. S. $\quad 250$

Gunning, J. E. 492

Gunston, D. W. $\quad 403$

Gunter, W. H. $\quad$ I34

Gunther, C. E. $\quad 492$

Gunton, J. W. $\quad 96$

Gurney, B. T. $\quad 96$

Gurney, C. R. $\quad 403$

Gurney, E.H. $\quad 403$

Gurney, J. C. $\quad 284$

Gurney, K. T. C. $\quad 134$

Gurney, P.S. $\quad 297$

Gurney, Q. E. $\quad 403$

Gurney-Dixon, S. $\quad 403$

Gurnhill, C. J. $\quad 96$

Gush, C.R.

Gutch, J.

Gutch, W.

Guthrie, A. G.

Guthrie, N. B.

Guthrie, P.S.

Guthrie, R. F.

Gutteridge, H. C.

Guy, B. M. E.

Guy, C. G.

Guy, O. V.

Guy, P.C.

40

10

IO

40

40

403

$20 I$

201

3 II

201

172

284

Gwatkin, Sir W. G. 201

Gwatkin-Graves, E. A.

Gwinn, R. F.

329

Gwyer, H.L. $\quad 224$

Gwynne, H. Ll. $\quad 329$ 
Gwynne, R. V.

Gwyther, G. H.

Gyles, W.H. K.

Habershon, E. F.

Habershon, L. O.

Habershon, P. H.

Habershon, S. H.

Habgood, A. H.

Habgood, G.

Habgood, G. $\quad$ 4I

Hacking, A.

Hacking, E. M.

Hacking, $H$.

Hackworth, A.

Haddon, E. B.

Hadfield, P. H.

Hadley, O. H.

Hadley, P.S.

Hadow, R. H.

Hadrill, E. W.

Haggard, A. J. R.

Haggard, $M$.

Haggard, T. B. A.

Hagger, N. W.

Haggie, F. R.

Hagon, A. C.

Hague, T. $H$.

Hahn, F. M.

Hahlo, H. B.

see Barker-Hahlo, $\mathrm{H}$.

Haig, D. P.

Haig, $O$.

403

351,403

Haig-Brown, A. R , 25I

Haigh, A. G.

Haigh, $B$.

Haigh, P. B.

Hailstone, G. R.

Haines, F.P.

Haines, $H$. Y.

Haines, R. T. M.

Haines, W. R.

Hainworth, A. D.

Hake, H. D.

Hake, H. M.

Haldane, J. O.

Haldane, M. M.

Hale, D. B.

Hale, E. N.

Hale, G. D.

Hale, H. E.

Hale, J.

Hale, R. E. V.

Hales, G. T.

Hales, H. W.

Hales, J. B.

Hales, J. P.

Hall, A. A.

Hall, A. C.

Hall, A. F.

Hall, A. F. W.
492

40

4I

403

4I

4I

172

10
250

3 II

172

I0

134

201

251

201

403

201

492

492

329

492

492

$35 \mathrm{I}$

96

Hall, A. H.

Hall, A. K. D.

Hall, A. L.

Hall, $B$.

Hall, G. D.

Hall, G. E.

Hall, G. V.

Hall, $\mathrm{H}$. (Clare)

Hall, H. (Magd.)

Hall, H. A.

Hall, H. R.

Hall, H. S.

Hallett, H. G.

Hallett, H. J.

Halley, K. B.

Halliday, C. W. A.

96

I34

492

35 I

297

403

172

172

403

$25 \mathrm{I}$

96

530

134

I34

IO

25 I

403

172

403

403

65

516
Hall, A. J.

Hall, C. S.

Hall, D. G.

Hall, E. W.

Hall, F. G. (King's)

Hall, F. G. (Trin.)

Hall, J. (Emm.)

Hall, J. (Sidney)

Hall, J. A.

Hall, J. B.

Hall, J. S.

Hall, J. T.

Hall, P. A.

Hall, R.

Hall, R. H. E.

Hall, R.S.

Hall, S. H.

Hall, V.C.

Hall, W. (Christ's)

Hall, W. (King's)

Hall, W. (Sidney)

Hall-Smith, P.

Hallam, H. G.S.

Haller, B. C.

492

134

41

65

$35 \mathrm{I}$

517

96

134

201

403

492

251

96

41

224

329

77

25 I

96

$35 \mathrm{I}$

41

25 I

$35 \mathrm{I}$

65

$4 \mathrm{I}$

$25 \mathrm{I}$

492

403

329

224

Io

201

$35 \mathrm{I}$

25 I

403

403

403

297

403

202

403

403

403

I34

134

329

224

404

297

329

77

I34

404

297

404

404

Hamill, $P$.

Hamilton, A.

Hamilton, A. S.

Hamilton, C. F. P.

Hamilton, C. J.

Hamilton, E. R.

404
Hamilton, E. W.

404

Hamilton, G. $\quad 404$

Hamilton, G. C. H. 202

Hamilton, G. H. $\quad 35 \mathrm{I}$

Hamilton, H. O. $\quad 404$

Hamilton, J. $\quad 65$

Hamilton, J. A. de C. 202

Hamilton,J.L.(Dwn.) 77

Hamilton,J.L.(Kg's) 202

Hamilton, J. W. 3 I I

Hamilton, $K$. $\quad 404$

Hamilton, N.C. $\quad 172$

Hammersley, S.S. 202

Hammick, H. A. $25 \mathrm{I}$

Hammond, C. E. $\quad 404$

Hammond, F. W. $\quad 224$

Hammond, J. $\quad 77$

Hammond, J. V. $\quad 517$

Hammond, N. W. $35 \mathrm{I}$

Hammond-Chambers,

H. B. B. 202

Hammond Searle, A. C.

Hamond-Graeme, E. H. M.

134

Hampden, Viscount 404

Hampshire, C. D. 251

Hampson, O.D. $\quad 492$

Hampson, S. H. $\quad 297$

Hance, J. B. Io

Hancock, D. M. 224

Hancock, W. H. M. Io

Handcock, W. A. S. I I

Handford, E. F. S. $\quad 404$

Handford, H. B.S. 404

Handford, J. R. $\quad 297$

Handford, W. B. II

Hands, A. C. II

Hands, W. J. G. $\quad 172$

Hanhart, A. A. 251

Hanitsch, K. V. $\quad 35 \mathrm{I}$

Hankey, B. $25 \mathrm{I}$

Hankin, G. T. $\quad 224$

Hanna, J. H. $\quad 65$

Hanna, W. B. $\quad 404$

Hannen, L. C. D. 492

Hannen, L. G. $\quad 404$

Hannen, N. M. B. 492

Hannington, G. J. I I

Hannington, J. E.M. 25 I

Hansard, L. A. S. $\quad 172$

Hansell, G. F. II

Hansen, J. C. $4 \mathrm{I}$

Hanson, C. E. B. $\quad 4 I$

Hanson, E. C. $\quad 517$

Hanson, E. T. II

Hanson, Sir G. S. $\quad 224$

Hanson, H. J. $\quad 404$

Hanson, J.

Hanson, $\mathrm{O}$. $\mathrm{H}$.

Hanson, R. E V

329

404 
Hanson, R. J. E. $\quad 404$ Happell, A. C. 202

Happold, F. C. $\quad 285$

Harbaugh, G. W. F. 492

Harber, E. W. 492

Harbord, J.

Harbord, V.

Harcourt, G. H. $\quad 172$

Harcourt, R. V. $\quad 404$

Hardcastle, A. E. L. 224

Hardie, E. E. $\quad$ I34

Hardie, $M$.

Harding, A. M.

Harding, C. R.

Harding, E.

Harding, E. W.

Harding, N.S.

Harding, W. H.

Harding, W. J.

Hardinge, A. H. L. 404

Hardingham, C. H. II

Hardman, F. M. 202

Hardman, W. H. (Down.)

Hardman, W. $\mathrm{H}$. (Joh.)

Hardwick, F.

Hardy, C.

Hardy, E. W. D.

Hardy, F. K.

Hardy, F. W.

Hardy, J.

Hardy, T. L.

Hare, A. C.

Hare, F.

Hare, S. G.

Hare, S. H.

Hare, W. T.

Harford, C. F.

Hargreaves, A. R.

Hargreaves, C. A.

Hargreaves, C. R.

Hargreaves, $H$.

Hargreaves, H. C. $\quad 25$

Hargreaves, H. P. $\quad 25 \mathrm{I}$

Hargreaves, J. A. $\quad 405$

Hargreaves, R. (Chr.) II

Hargreaves, R. (Cla.) $4 \mathrm{I}$

Hargreaves, R. (Cai.) I 35

Hargreaves, R.(Qns') 297

Hargreaves, W. F. $25 \mathrm{I}$

Hargrove, C. R. $\quad 285$

Harke, S. L. 25I

Harker, C. G. W. $25 \mathrm{I}$

Harkness, H. L. 251

Harland, H. C.

Harland, R. E. C.

Harley, R. G. G.

Harling, R. W.

Harman, C. E.

Harmens, W.
Harmer, G.

Harmer, W. D.

Harmsworth, V. G. 492

Harmsworth,V.S.T. 405

Harnett, W. L. $\quad 329$

Harper, C. $\mathrm{H}$.

Harper, E. R.

Harper, F. A.

Harper, K. J.

Harper, W.E.

II

135

202

$25 \mathrm{I}$

Harper-Smith, G.H 517

Harper-Smith, S. W. 65

Harraton, J. R.

Harrey, C. O.

Harricks, $N$.

Harries, A. T.

Harries, A. T. $\quad 96$

Harrild, W. C. 405

Harris, A. A.

Harris, A. D.

Harris, A. F. S.

Harris, A. L. S.

Harris, C. R.

Harris, E. R.

Harris, E. T.

Harris, F. Leverton 135

Harris, F. W.

Harris, G. H.

Harris, H. (Emm.)

Harris, H. (Joh.)

Harris, H. A.

Harris, H. B.

Harris, H. E.

Harris, H. E., jun.

Harris, H. S.

Harris, J. B.

Harris, J. C. N.

Harris, L. G.

Harris, R. T.

Harris, $T$.

Harris, T. W.S.

Harris, W. B.

Harris, W. E.

Harris, W. J.

Harrison, A. M.

Harrison, A. P. B.

Harrison, C. E.

Harrison, C. F.

Harrison, D. H.

Harrison, Ernest

Harrison, Everard

Harrison, E. F.

Harrison, E. J.

Harrison, E. M.

Harrison, E. P.

Harrison, $F$.

Harrison, F. E.

Harrison, F. E. H.

Harrison, G. A.

Harrison, G. B.

Harrison, G. H.
405

135

I I

I I

41

172

$25 \mathrm{I}$

405

II

530

530

96

329

96

405

I I

II

492

517

251

$35 \mathrm{I}$

135

135
97

530

251

251

135

351

405

I35

135

97

405

405

405

493

I I

202

77

65

405

I35

297

224
Harrison, G. L.

298

Harrison, G. R. D. 252

Harrison, H. E. $\quad 405$

Harrison, L. $\quad 97$

Harrison, L. K. $\quad 135$

Harrison, $P$. II

Harrison, T. E. $\quad 405$

Harrison, T.S. $\quad 135$

Harrison, W. J. $\quad 4 \mathrm{I}$

Harrison, W.P. $\quad 172$

Harrison-Broadley,J.224

Harrisson, A. E. $\quad 224$

Harrowing, J. S. $\quad 405$

Harrowing, W. W. 4r

Harry, N. G. $\quad 172$

Harston, F. N. $\quad 66$

Hart, B. H. L. $\quad 66$

Hart, H. E. $\quad$ r35

Hart, N. B. $\quad 77$

Hart, P. H. $\quad 285$

Hart, R. J. d'A. $\quad 405$

Hart, S. $\quad 4 \mathrm{I}$

Hart-Smith, H. M. $\quad 135$

Hartcup, G. H. W. 4I

Hartcup, J. A. $\quad 252$

Hartcup, R. E. $\quad 4 \mathrm{I}$

Harter, J. C. F. $\quad 224$

Hartington, Marquis of $\quad 405$

Hartley, B. C. $\quad 172$

Hartley, C. R. $\quad 405$

Hartley, L. B. $\quad 202$

Hartley, W. E. $\quad 405$

Hartley, W. J. $\quad 285$

Hartman, R. T. $\quad 97$

Hartree, C. $\quad \mathbf{I 5}$

Hartree, D. R. $\quad 329$

Hartree, K. $\quad$ r $\quad 35$

Hartridge, H. $\quad 202$

Harvest, G. L. $\quad 173$

Harvey, A. W. $\quad 329$

Harvey, C. $\quad 405$

Harvey, C. H. II

Harvey, D. L. $\quad 405$

Harvey, E. J. W. $\quad 405$

Harvey, F. L. $\quad 405$

Harvey, G.H. ' 252

Harvey, G. R. M. $\quad 405$

Harvey, G. T. B. $\quad 66$

Harvey, H. M. $\quad 66$

Harvey, H. W. $\quad 77$

Harvey, J. E. $\quad 406$

Harvey, N. R. $\quad 252$

Harvey, O.C. $\quad 406$

Harvey, R. P. $\quad 35 \mathrm{I}$

Harvey, R. V. 224

Harvey, S. 298

Harvey, W.

Harvey, W. H.

Harvie, E. F.

Harvie, J. K. 
Harwood, A. H. F. I35 Haselden, R. B.

Haskins, F. W.

Haslam, V. K.

Haslam, W.

Haslam, W. A.

Haslam, W. H.

Haslam, W. K. S.

Haslewood, B. T.

Hasluck, R. ff.

Hassard-Short,

Hastings, W (Chr) I I

Hastings, W.(Fitz.H.) 530

Hastings, W.H. 406

Haswell, P. 35I

Hatch, K.

Hatch, L. C.

Hatch, M. L.

Hatch, P. R.

Hathorn, C. N.

Hathorn, K. H.

Hathorn, W. B.

Hatley, A. J.

Hatt-Cook, G.

Hatten, G.

Hatten, J. C. le P.

Hattersley, S. M.

Hatton, G. A. L.

Hatton-Hall, H. C.

Haughton, A.J.

Haultain, W F T

Hausburg, E. F.

Havell, C. C. W.

Havelock-Allan, Sir H. S. M.

Havers, C. R.

Haviland, J. D.

Haviland, W. P.

Haward, T. W.

Hawcridge, R. S.

Hawdon, H. W.

Hawdon, N. E.

Hawes, E. L.

Hawker, C. A. S.

Hawker, G. P. D.

Hawker, H. L. L.

Hawkes, C. P.

Hawkins, A. G.

Hawkins, A. G. J.

Hawkins, C.H.G.W. I 35

Hawkins, C. L.

Hawkins, H. D.

Hawkins, H. H. B.

Hawkins, O.C.

Hawkins, O. L.

Hawkins, R. J.

Hawley, A. T.

Haworth, C. W. B.

Hawthorne, C. B.

Hay, A. C.

Hay, J. A.
Hay-Robertson, J.W. $4 I$

Haydon, A. D.

Haydon, T. H.

Hayes, J. C.

Hayes, J. H.

Haygarth, C. H. S. 329

Haynes, G. S. 202

Haynes, H. G. L.

Haynes, W. G.

Hayter, G. K. H.

Haythornthwaite, R. M.

Hayton, J. D. W.

Hayward, A. D.

Hayward, A. W.

Hayward, C. O.

Hayward, G.

Hayward, L. G.

Hayward, M. C.

Hazeldine, D.

Hazlehurst C. A C

Hazlerigg, Sir A. G. 406

Hazlerigg, $\mathbf{G}$.

Head, F. W.

Head, $\mathrm{H}$.

Head, H. G.

Headlam, T. A.

Headley, H. H.

Heads, J. E. B.

Heald, C. B.

Heald, W. H. A.

Heald, W. M.(Cai.) ${ }_{3} 6$

Heald, W. M. (Joh.) 329

Heale, R. J. W.

Healey, F. G.

Healey, R. E. H.

Healing, J. A.

Heany, W. P.

Heap, E. F. G. T.

Heap, F. G.

Heap, J.M.

Heape, B. R.

Heard, A. St J.

Heard, N.

Hearfield, J.

Hearfield, $\mathrm{T}$.

Hearn, E. H.

Hearn, J. S.

Hearn, R. C.

Hearn, R. J.

Hearsey, G. A. C.

Hearson, H. F. P.

Heath, A. F.

Heath, C. N.

Heath, F. A.

Heath, J. R.

Heath, L. C.

Heath, $\mathrm{O}$.

Heath, R. J.

Heath, W. E.

$4 I$ Heathcock, $T$.

I I

298

329

252

I I

I36

I36

136
Heathcote, E. H. $\quad 406$

Heathcote, G.S. $\quad 173$

Heathcote-Drummond-

Willoughby,C.S. 406

Heatley, L. 285

Heaton, D. R. $\quad 406$

Heaton, J. B. 406

Heaton, R. (Christ's) I I

Heaton, R. (Corpus) 66

Heaton-Armstrong, J.D.

Heaton-Armstrong,

W. D. F.

493

Heaton-Ellis, C.E.R.

Heaton-Ellis, C.E.R. $5 \mathrm{r} 7$

Heaton-Ellis,C.H.B. 407

Hebblethwaite, A.R. 202

Hebblethwaite, C. J. 407

Hedderwick, A.S. $\quad 4$ I

Hedderwick, G. 4I

Hedges, A. D. $\quad 4 \mathrm{I}$

Hedges, K. M. F. $\quad 407$

Hedges, P. D. $\quad 517$

Hedley, E. W. 202

Hedley, I. M. 203

Hedley, J. P. 203

Hedley, O. W. E. $\quad 407$

Hedley, W. 203

Hedley, W. A. C. $\quad 407$

Heelas, R. J. $\quad 203$

Heelis, J. R. 4 I

Heesom, K. E. $\quad 252$

Hegarty, M. B. 4I

Heimann, H. P. $\quad 329$

Heinemann, A. B. $\quad 407$

Helder, L. B. 3 I I

Hele, T.S. $\quad 97$

Hele-Shaw, H. R. $\quad 4$ I

Hellaby, F. A. $\quad 407$

Hellings, G. S. $\quad 329$

Hellins, E. W. J. 3 Ir

Hellyer, F. E. $\quad 407$

Helm, C. 203

Helm, H.P. D. $\quad 407$

Helm, J. H. 493

Helme, J. $\quad 407$

Helme, J. M. $\quad 407$

Helme, R. E. $\quad 407$

Helme, R. M. 407

Helme, T. W. $\quad 407$

Helps, A. L. $\quad 407$

Hemmant, M. $\quad 252$

Hempson, E. R. $\quad 97$

Hemsworth, A. H. $\quad 42$

Hemsworth, E. P. D. 298

530 Hemsworth, N.E.C. 517

406 Hemus, W. L. 252

406 Henderson, A. I. 407

406 Henderson, A. S. 407

252 Henderson, D. 42

298 Henderson, I. M. 203

252 Henderson,J.A.(Chr.) 12 
Henderson,J.A.(Jes.) I 73

Henderson, J. K. 407

Henderson, N.C. $\quad 42$

Henderson, P. $\quad 329$

Henderson, R. K. $\quad 42$

Henderson, W. L. $\quad 285$

Hendley, H. J. H. $\quad 136$

Hendricks, C.A.C.J. 42

Hendrie, H. A. 5 I 7

Hendry, C. W. $\quad 530$

Hendry, F. H. A. $\quad 12$

Heneage, G. E. $\quad 407$

Henkel, W. E. G. $\quad 252$

Henman, W. W. $\quad 285$

Henn, E. H. L. $\quad 407$

Henn, W. F. 225

Henn-Gennys, E. C. 493

Henri, F.

Henry, C. J.

407

407

Henry, D.C.

Henry, S. A.

407

408

Henry, W. D. M. 330

Henryson-Caird,A.J.225

Hensley, E. H. V. $\quad 203$

Hensley, W. H. $\quad 97$

Henslow, T. G. W. 5I7

Henty, E. C.

Hepburn, M. A. 225

Hepburn, R. P. $\quad 225$

Hepburne-Scott,C.F. 408

Hepburne-Scott, W. G.

see Polwarth, Lord

Hepburne-Scott, W.T.

Hepworth, B. G.

Hepworth, F. A.

Hepworth, L. D.

Herapath, C. A.

Herapath, N. F.

Herbert, A. G.

Herbert, E.

408

97

$33 \circ$

312

298

298

408

408

Herbert, E. A. F.W. 408

Herbert, E. J. B. $\quad 203$

Herbert, F. F. $\quad 97$

Herbert, P. L. W. 136

Herbert, S.

136

Herbert-Smith, G. M. 408

Hercus, E. O. $\quad 408$

Herd, J. G. M. 312

Herdman, G. A. $\quad 408$

Herdman-Newton,C.252

Herdman-Newton, R. H.

Hereford, J. C.

252

136

Heriz-Smith, A. J. C. 252

Heriz-Smith, D. M. 298

Heriz-Smith,E.E.A. 252

Herklots, $\mathrm{H}$.

Herman, A. E.

Herman, G. A.

Herman, G. L.
Herman, W.S.

Hermann, J.

Hermon, J. V.

Heron-Maxwell, Sir I. W.

Herries, A. D. Y.

Herring, J. H.

Herriot, D. R.

Herriot, J. A.

Herrman, L. C.

Hervey, D. E. F. C.

Hervey, D. F.

Hervey, E. S.

Hervey, G. A.

Herzl, $\mathrm{H}$.

Herzog, F. J.

Heseltine, C.

Heseltine, G.

Heseltine, N. E.

Hesketh, G. E.

Hesketh, G. M.

Hesketh, W.

Hesketh-Wright, J

Heth-W. 136

Hess, $\mathrm{H}$.

Hetherington, A. L.

Hethen, A. 408

Hetherington, R. G. 408

Hetherington, T. R. 408

Hetherington, T. W. 408

Hett, A. I.

Hett, H. A.

Heurtley, W. A.

Hewett, F.S.

Hewitt, F. W.

Hewitt, J. T.

Hewitt, L. McN.

see McNeill-Hewitt, L.

Hewitt, N.S.

Hewitt, R. C.

Hewlett, J. H.

Hext, T. M.

Hey, C. E. M.

Heycock, C.T.

Heycock, J. H.

Heycock, M.S.

Heydon, G. A. M.

Heyer, G.

Heyland, H. M.

Heyn, R. G.

Heywood, A. G. P.

Heywood, B. C. P.

Heywood, C. C.

Heywood, Sir G. P. 409

Heywood, L. J. $\quad 253$

Heywood, W. B.

Heyworth, C.T.

Heyworth, G. A. F.

Heyworth, H. P. L.

Hibberd, A. S.

Hibbert, J. P. M.

Hickling, A. R.

$35 \mathrm{I}$

493

408

408
12

136

42

408

408

252

408

493

408

252

42

年

年

8

Higson, F.S. $\quad 203$

Higson, G. H. $\quad 136$

Higson, L. A. $\quad 33^{\circ}$

Hilary, H. J. $\quad 409$

Hilary, R. J. $\quad 330$

Hiles, $M$. 253

Hill, A. $\quad 97$

Hill, A. G. E. $\quad 409$

Hill, A. T. $\quad 97$

Hill, A. V. $\quad 203,409$

Hill, A.W. $\quad 203$

Hill, B. W. 225

Hill, C. E.C. $\quad 409$

Hill, C. L. $\quad 409$

Hill, G. 253

Hill, G. B. $\quad 253$

Hill, G.M. $\quad 42$

Hill, H. L. $\quad 97$

Hill, H.O. $\quad 409$

Hill, H. P. $\quad 173$

Hill, J. R. $\quad 330$

Hill, M. C. 225

Hill, P.A. $\quad 136$

Hill, R. $\quad 97$

Hill, R. A.P. $\quad 136$

Hill, R. G. $\quad 409$

Hill, R. N. . 253

Hill, T.A.M. $\quad 409$

Hill, V.St C. $\quad 285$

Hill, W. A. $\quad 517$

Hill, W. J. M. $\quad 409$

Hill, W. R. $\quad 66$

Hillbrook, W. $\quad 97$

Hilleary, E. L. $\quad 409$

Hiller, A. M. 330

Hilliard, G. W. $\quad 77$ 
Hillier, T. L.

Hills, A. H.

Hills, T. W.S.

Hills, W. F. W.

Hills, W. H.

Hilpern, W. T. H.

Hilton, H. D.

Hilton, M. V.

Hilton-Green, C.C.H 410

Hinchcliffe, J. W.

Hind, A. M. 97

Hind, C. R. $\quad 253$

Hind, L. A. 493

Hind, O. W. $\quad 493$

Hinde, B. F.

Hinde, E. B.

Hinde, $H$. W.

Hinde, P. M.

Hinde, S. L.

Hinderlich, A. A. W. 66

Hindle, E.

Hindle, $H$. B.

Hindley, N. L.

Hindley, W. T.

Hindley-Smith 12

Hindlip, Lord

Hine, T. G. M.

Hingston, A. A.

Hinmers, J. R.

Hinnell, T.S.

Hinton, S. E.

Hippisley, E.T.

Hird, F. L.

Hirsch, F. B.

Hirst, G. G.

Hirst, G. S.

Hirst, J.

Hirst, J. W.

Hirst, W. B.

Hiscott, G.S.

Hiscott, L. S.

Hislop, T. C. A.

Hissey, J. B.

Hissey, T. B.

225

12

137

12

225

203

410

253

253

$53{ }^{\circ}$

410

253

77

137

410

410

137

530

253

253

137

410

493

Hitchcock, A. E. N. 298

Hitchcock, J. V. 312

Hitchcock, W. H. L. 253

Hitching, W. W. 330

Hoare, A. B.

Hoare, A. R.

Hoare, B. S.

Hoare, C. G.

Hoare, E. G.

Hoare, E. R. D

Hoare, F. O.

Hoare, F. R. G.

Hoare, G. de M. G.

Hoare, $\mathrm{H}$.

Hoare, H. C. A.

Hoare, J. E. A.

Hoare, L. G.

410

410

203

410

410

298

410

410

410

410

203
Hoare, R. B.

Hoare, W. R.

Hobbs, A. V.

Hobbs, C. R.

Hobbs, E. N. B.

Hobbs, F. D.

Hobbs, V. W. J.

Hobhouse, A. L.

Hobson, A. C.

Hobson, A. F.

Hobson, C. M.

Hobson, F. W. E.

Hobson, L. F.

Hobson, R. L.

Hobson, W. J. G. see Graham Hobson, W.J.

Hobson, W. W. 12

Hodder, A. E.

Hodder, F. E.

Hodder, H. G.

Hodge, D. S.

Hodge, E. H. V.

Hodge, $H$. S. Vere

Hodge, J. D. V.

Hodges, A. G. A.

Hodges, A. N.

Hodges, C. E.

Hodges, E. C.

Hodges, L. N.

Hodges, R. A.

Hodges, W. C.

Hodges, W. V. A.

Hodgetts, W. J.

Hodgkinson, G. A.

Hodgson, A. D.

Hodgson, A. E.

Hodgson, A. J.

Hodgson, B. T.

Hodgson, C. A. G.

Hodgson, C.A.G. 410

Hodgson, C. L.

Hodgson, E. J.

Hodgson, F. W.

Hodgson, F. W. K.

Hodgson, G. W. H.

Hodgson, H. E. A.

Hodgson, T. R.

Hodgson, W. Hammond

410

410

330

173

253

203

330

410

410

137

298

173

I37

173

203

66

312

42

42

410

253

203

298

12

410

97

517

493

410

285

493

517

12

42

410

173

173

312

410

410

410

12

Hodgson, W. Harry

Hodson, E. A.

Hodson, G. S.

Hodson, J.

Hodson, $\mathbf{R}$.

Hoff, H. G.

Hoffman, C. D.

Hoffmann, G.S.

Hoffmann, W. A.

see Arnold, W.

Hoffmeister, C. E.

see Hough, C. E.
Hoffmeister, C. J. R. 137

Hogan, R. V.J.S. 330

Hogarth, T. J. $\quad$ I2

Hogben, E. O'N. 517

Holbech, L. $\quad 298$

Holberton, T. E. 253

Holcroft, G. C. $\quad 298$

Holden, A. $\quad 298$

Holden, E. G. $\quad$ I 37

Holden, G. H. R. $\quad 137$

Holden, H. C. $\quad 493$

Holden, H. F. $\quad 330$

Holden, J. R. $\quad 330$

Holden, N. E. $\quad 493$

Holden, N.V. $\quad 330$

Holder, N. F. 253

Holdgate, W. W. 4II

Holdsworth, A. M. 493

Holdsworth, G. L. 4II

Hole, C. H. 493

Hole, H. W. $\quad 97$

Holland, C. C. $\quad 12$

Holland, C. E. I2

Holland, F. $\quad 203$

Holland, H.H. $\quad 352$

Holland, J. D. C. 493

Holland, J. E. D. $\quad 42$

Holland, P.F. $\quad 173$

Holland, S. C. $\quad 494$

Holland, V. B. $\quad 494$

Holland, W. D. A. $\quad 137$

Holland, W. G. C. 494

Holland-Hibbert, T. 4 I I

Holland-Hibbert,W. 4I I

Hollings, J. H. B. 225

Hollingsworth, J. H. 352

Hollingworth, L. $\quad 312$

Hollins, E. M. $\quad 203$

Hollins, E. R. L. $\quad 98$

Hollins, P. L. $\quad 494$

Hollis, A. J. 3 I

Hollis, D. $\quad 298$

Hollis, W. $\quad 137$

Hollis, W. P. B. $\quad 531$

Hollist, A. M. C. $\quad 494$

Hollond, E. R. $\quad 4 \mathrm{II}$

Hollond, G. E. 4II

Hollond, H. A. 4II

Hollond, R. C. $\quad 203$

Hollond, S. E. 4II

Holloway, B.H. $\quad$ I 73

Holloway, N. J. $\quad$ I73

Holman, A. F. $\quad 42$

Holman, A.H. $\quad$ I 73

Holman, A. McA. $\quad 4$ II

Holman, B. W. $\quad 285$

Holman, C. C. $\quad$ I37

Holman, D. 4II

Holman, G. H. W. $\quad 137$

Holman, J. H. T. $\quad 253$

Holman, P. 
Holmden, F. A. A.

Holme, B. L.

Holme, H. R.

Holmes, A.

Holmes, A. K.

Holmes, B.S.

Holmes, G.

Holmes, H. W. H.

Holmes, J. C.

Holmes, T. E.

Holmes, T.S.

Holmes, T. V.

Holmes-Tarn, $\mathrm{H}$.

Holroyd, G.

Holroyde, D.

Holt, E.

Holt, F. N.

Holt, H. D. G.

Holt, H. W.

Holt, J. L.

Holt, R.

Holthouse, C. L.

Holtzapffel J. G. H.

Holzapffel, J. G.H. 330

Home, C. F. M.

Homfray, S. G.

Homiakoff, A. N.

Hone, P. F.

Honeyball, F. R.

Honeybourne,

Honeybourne, V.C. 330

Honeyburne, W. R. 285

Hony, G. B.

Hony, H. C.

Hood, G. C.

Hood, H. M.

Hood, J. A.

Hood, J. C. F.

Hood, J. W.

Hook, C. W. T.

Hooker, C. W. R.

Hooker, J.S.

Hoole, B.

Hoole, D.

Hoole, N.

Hooley, L. J.

Hooper, A. N.

Hooper, A. W.

Hooper, F. H.

Hooper, K. A.

Hooper, L. J.

Hooper, R. G.

Hopcraft,

E. G. de L.

Hope, A. T.

Hope, B. L.

Hope, G. M.

Hope, H. N.

Hope, J. H.

Hope, L. N.

Hope, R. A.

Hope, S. J.
173
298

174

517

42

I 37

12

I74

137

204

494
494

12

4II

I74

66

204

352

12

253

204

4 I I

77

I7

285

I 2
4 I I

I 37

I 74

I 2

12

517

330

42

12

298

298

98

66

98

98

42

298

98

253

174

494

98

I 37

42

4 I I

494

298

137
Hope Gill, C. G. see Gill, C. G. Hope

Hope-Johnstone, C.J. 204

Hope-Jones, W. 204

Hope-Wallace, J. 494

Hopewell, A. F. J. $\quad 298$

Hopewell, E. R. $\quad 225$

Hopgood, J. L.

Hopkin, D.

Hopkins, N. T.

4 II

312

Hopkins, W. D.

42

494

Hopkinson, B. 204, 4 I I

Hopkinson, E. H. 4 I I

Hopkinson, H. C. $\quad 98$

Hopkinson, R. C. 4 II

Hopley, F. J. V.

Hopley, G. W. V.

Horan, A. K.

Horan, F. S.

Horder, C. A.

Hore, C. W. C.

Horlington, $\mathrm{F}$.

Horn, D'A.

253

412

352

494

98

225

330

494

Horne, C. W. E. $\quad 5 \mathrm{I7}$

Horne, H. F.

Horne, J.A.

Horne, J. W.

Horne, $M$.

Horner, M.S.

Horner, N. G.

Horner, P. W. M.

Hornidge, D. G.P.

Hornidge, E. S.

Horniman, J. E.

204

12

253

412

254

I37

37
98

254

494

Hornsby, J. A.

12

Hornsby, R. L. W.

Hornsby-Wright $\dot{G}{ }^{412}$

Hornung, A. O. 204

Hornung, G.

Hornung, J.P.

Horsey, A. M.

Horsfall, C. F.

Horsfall, R. E.

Horsfield, R. B.

412

412

137

204

204

412

Horsley, C. D. $\quad$ I 37

Horsley, G.

Horsley, S.

Horton, C. E.

Horton, E. F

, Le G. G. W. 412

Horton-Smith, L. G. H.

Horton-Smith-

$33^{\circ}$

Hartley, Sir P. 331

Hosband, E. A. $\quad 312$

Hosken, $\mathrm{H}$.

312
12

Hoskin, T. J. H. 412

Hoskyns, E. C. $\quad 66,174$

Hoskyns, H. C. W.

Hoste, T. B.

98

Hotblack, G. F.

254
Hotblack, G. V.

Hotblack, H.S.

137

Hotham, J.

Hough, C.E. $\quad 312$

Houghton, J. C. $\quad 517$

Houghton, R. L. $\quad 412$

Hoult, J. M. $\quad 412$

Houlton, J. W. $\quad 12$

Housden, E. F. $\quad 285$

Housden, E. J. T. $\quad 285$

Housden, R. J. $\quad 352$

Houseman, E. A. $\quad 42$

Houseman, F. O. $\quad 298$

Housley, C. 254

Houstoun-Boswall, T.R.

Hovil, $R$.

412

How, J. C. H 33I, 4 I2

Howard, A. J $\quad 204$

Howard, B. E. $\quad 412$

Howard, B. H. E. $\quad 412$

Howard, C. F. $\quad 412$

Howard, C. G. $\quad 98$

Howard, C. R. $\quad 254$

Howard, D. S. P. $\quad 412$

Howard, G. $\quad 412$

Howard, G.W.

Howard, G. W. A. $\quad 312$

Howard, H.S. $\quad 412$

Howard, J. $\quad 147$

Howard, J. B. $\quad 412$

Howard, J. C. $\quad 43$

Howard, J. P. $\quad 412$

Howard, M.F.S. $\quad 412$

Howard, N. M. C. $\quad 412$

Howard, P. $\quad 312$

Howard, R. W. $\quad 412$

Howard, T. H. $\quad 98$

Howard, W. E. W. $\quad 517$

Howard, W.S. $\quad 412$

Howard-McLean, J. R.

Howard Smith, G $\quad 412$

Howarth, H. V. $\quad 137$

Howarth, W. G. $\quad 204$

Howden, E. R. $\quad 312$

Howden, P. F. $\quad 4 \mathrm{I2}$

Howe, G.A. $33 \mathrm{I}$

Howe, J. C. $\quad 254$

Howe, R. G. $\quad 312$

Howell, C. G. $\quad 137$

Howell, E. B. $\quad 98$

Howell, G. F. $\quad 204$

Howell, J. H. $\quad 43$

Howell, M. I. B. 33I

Howell, R. A. $\quad 98$

Howell, R. G. D. $\quad 98$

Howes, A. G. $\quad 138$

Howett, F.

Howick, Viscount

see Grey, Earl

494 
Howitt, A. B.

Howitt, F. D.

Howkins, G. A.

Howland, R. C. J.

Howlett, C. E.

Howlett, J. M.

Howorth, $\mathrm{H}$.

Howse, T. F.

Howson, H. E. E.

Howson, J. F.

Howson, R.S.

Hoyland, C. E.

Hoyle, H. K.

Hoyle, J. B.

Hoyle, L. R.

Hoyte, W.N.

Hubback, F. W.

Hubbard, J. W.

Hubbard, L. E.

Hubbard, M. E.

Hubbard, P. W.

Hubble, H. R.

Hubbuck, G. M.

Hubbuck, R. E.

Huck, J.

Huckle, H. W.

Huddart, L. H. L.

Hudson, A. C.

Hudson, B.

Hudson, E. C.

Hudson, F. A.

Hudson, $G$.

Hudson, G. $H$.

Hudson, H. C. H.

Hudson, L. C. A.

Hudson, $N$.

Hudson, N. B.

Hudson, $\mathrm{R}$.

Hudson, R. B.

Hudson, R. J.

Hudson, R.P. M.

Hudson, T.E.

Hudson, T. $H$.

Hudson-Kinahan, G. F.

Huelin, E. S.

Huggan, G. H.

Huggins, $H$. W.

Hughes, A. W.

Hughes, $B$.

Hughes, $D$.

Hughes, E. C.

Hughes, E. L.

Hughes, F. A.

Hughes, G. E.

Hughes, G. R.

Hughes, Harold

Hughes, Horace

Hughes, H.C.(Mag)

Hughe 225

Hughes, H.H. $\quad 298$
413

298

413

4I 3

4I 3

517

413

254

517

77

98

43

$4 \mathrm{I} 3$

4I3

494

312

174

254

43

12

254, 517

494

I38

4I3

$53 \mathrm{I}$

517

254

I38

254

413

413

518

494

43

43

352

413

413

312

312

225
Hughes, J. B. W.

Hughes, J. L.

Hughes, J. W. C.

Hughes, L. A.

Hughes, L. W.

Hughes, M. B.

Hughes, N. A.

Hughes, N. W.

Hughes, $\mathrm{O}$.

Hughes, R.

Hughes, R. J.

Hughes, S. J.

Hughes, T. G.

Hughes, T. J.

Hughes, T. McK.

312

331

174

299

98

254

413

254

43

4I3

413

518

413

43

413

Hughes, T. W. G. J. 254

Hughes Davies, C. E. I74

Hughes-Games, J. B. 299

Hughes-Gibb, H. F. 4I 4

Hughes-Hallett, N. M.

Hulbert, C. G. K.

Hulbert, H. B.

174

Hulbert, H. L. P.

Hulbert, J. G.

Hulbert, M. L.

Hull, Sir C. P. A.

Hull, H. C.

Hullah, J.

Hulton, A. H.

Hulton-Harrop, H. de L.

254

138

4I4

414

43

414

98

I38

4I4

Hulton-Sams, F.E.B. 414

Hulton-Sams, K. A. 174

Humby, S. R. 299

Hume, A.

494

Hume, D. B. M. $53 \mathrm{I}$

Hume, W. E. $\quad 254$

Hume-Kelly, F. V. 494

Hume-Williams, R.E. 494

Humfrey, S. H. G. I38

Humphery, H. M. $\quad$ 4I4

Humphreys, D. F. $\quad 254$

Humphreys, G. N 3 I 2

Humphreys, I. H. M. 98

Humphreys, W.A. 414

Humphreys, W.H. $\quad 12$

Humphreys-Owen, A. E. O.

Humphries, G. N. P. 494

Humphries, R.P. 494

Humphry, A. M.

Humphry, L.

Humphrys, R.P.

Hunkin, J. W.

Hunt, C. A.

Hunt, E. G.

Hunt, E. R.

Hunt, F. R. W.

Hunt, H. N.
Hunt, J. B.

Hunt, $R$.

98

77

Huntbach, G. W. $\quad 98$

Hunter, E. J. 43

Hunter, G. J. (Mgd.) 225

Hunter, G.J.(Tr.H.) 494

Hunter, J. B. $\quad 33 \mathrm{I}$

Hunter, J. de G. $\quad 254$

Hunter, J. F. S. $\quad$ I38

Hunter, J. H. I $\quad$ I 38

Hunter, L. A. W. $\quad 518$

Hunter, M. J. $\quad 43$

Hunter, N. F. $\quad 43$

Hunter, W. 331

Hunter, W. E. $\quad 414$

Hunter-Muskett,

R. G.

225

Huntingdon, Sir C.P. 204

Huntingdon, L. W. 414

Huntriss, C. G. $\quad 494$

Huntriss, E. M. $\quad 494$

Huntsman, B. C. $\quad 414$

Hurford, C. C. $\quad 414$

Hurle, J. C. $\quad 414$

Hurley, M.R. $\quad 518$

Hurrell, G. W. 53 I

Hurrell, H. W. $\quad 495$

Hurrell, J. N. $\quad 414$

Hurrell, J. W. $\quad 531$

Hurrell, W. C. 495

Hurry, A. G. $\quad 331$

Hurst, E. E. $\quad 285$

Hurst, E.S. 312

Hurst, G. H. J. 204

Hurst, N. V. $\quad 77$

Hurst, W. H. I 2

Hussey, E. T. $\quad 98$

Hussey, R. L. $53 \mathrm{I}$

Hussey-Macpherson,

$$
\text { L. F. }
$$

Hutchence, B. L. $\quad 138$

Hutchence, W. G. $\quad$ I 38

Hutchings, C. E. $\quad 98$

Hutchings, H. W. $\quad 99$

Hutchinson, A.C.C. 495

Hutchinson,E. H. P. 254

Hutchinson, F. A. J. 4 I 4

Hutchinson, G. $\quad 174$

Hutchinson,G.C.(Cla.) 43

Hutchinson, G. C.

(Trin. H.)

495

Hutchinson, J. G. $\quad 43$

Hutchinson, J.H. $\quad 66$

138 Hutchinson, L. C. 299

414 Hutchinson, L. G. 43

43 Hutchinson, L. T.R. 4I 4

4I4 Hutchinson, N.W. 43

299 Hutchinson, R. H. 43

3I2 Hutchinson, R. W. $33 \mathrm{I}$ 
Hutchison, D. H.

Huxley, G. A.

Huxley, M. H.

Hybart, A. J. F.

Hyde, G. A.

Hyde, H. A.

Hyde, J. C.

Hyde, R. W.

Hyde Parker, W.S.

see Parker, W. S. H.

Hylton Stewart, B.D. 285

Ibbotson, A. W.

Icely, F.

Igglesden, R. S.

Iles, J. H.

Iles, J. O.

Iliffe, C. A. M.

Illingworth, $\mathrm{O}$.

Ilott, C. H. T.

Imbert-Terry, F. B.

Imlay, A. D.

Ince, S. R.

Inchbald, C. C. E.

Inchley, $\mathrm{O}$.

Infeld, $H$.

Ingham, W.

Ingle, L. M.

Ingle, R. G.

Ingleby, R. A. O.

Ingles, C. W. C.

Ingles, W. H. S.

Ingleson, $\mathrm{P}$.

Inglis, A. E. J .

Inglis, C. E.

Inglis, G.S.

Inglis, W. M.

Ingoldby, R. H.

Ingpen, D. L.

Ingpen, W. F.

Ingram, A. C.

Ingram, E. M. B.

Ingram, H. F.

Ingram, R.S.S.

Ingram, T. L.

Inman, R. T.

Innes, A. C. W.

Innes, W. K.

Innocent, A.

Inskip, J. H.

Insole, A. V.

Insole, E. R.

Insole, G. C. L.

Ionides, A. G.

Ipswich, Viscount

Iredale, H. C.

Ireland,

$$
\text { G.B. de Courcy, } 43
$$

Ireland, J. F.

Ireland-Blackburne, C. G. C. M.

254
414
414
518
285
77
518
331

254

$5 \mathrm{r} 8$

254

138

138

99
66

I 38

43

99

12

43

331

204

5 I 8

204

299

$4 \mathrm{I} 4$

174

13

299

204

204

204

299

99

495

254

$33 \mathrm{I}$

204

414

414

414

414

414

254

13

204

415

4 I 5

4 I 5

43

4I5

13

415

43
Iremonger, E. A.

Iremonger, E. V.

Irons, W. H.

Ironside, R. W.

Irvine, L. C. D.

Irving, A. G.

Irving, J. B.

Irving, J. C.

Irving, P. A.

Irving, $\mathrm{T} . \mathrm{H}$.

Irving, $W$.

Irving, W. R.

Irwin, A. P. B.

Irwin, C.

Irwin, H. M.

Isaac, C. L.

Isaac, D. C.

Isaacs, M. G.

Isaacson, C. H.

Isherwood, F. E. B. see BradshawIsherwood, F. E.

Isherwood, W.

Ison, A. J.

Ison, L. J.

Izard, K. H.

Jacklin, J. V.

Jacks, M.

Jackson, A.

Jackson, A. C. D.

Jackson, A. H.

Jackson, A. J.

Jackson, A. L.

Jackson, B. R.

Jackson, C. B. A.

Jackson, C. R.

Jackson, D. F.

Jackson, E. P.

Jackson, E. W.

Jackson, F. S.

Jackson, G. C.

Jackson, G. E.

Jackson, H. A.

Jackson, H. C.

Jackson, H. S.

Jackson, I.

Jackson, J.

Jackson, J. E. N.

Jackson, L. J.

Jackson, R. W. (Sid.) 352

Jackson, R. W. (Trin.)

see Ward-Jackson, $\mathrm{R}$.

Jackson, R. W. P.

Jackson, W.

Jackson, W. E.

Jacob, A. C.

Jacob, A. R.

Jacob, L. G.

Jacobsohn, A.

Jacoby, A. H. M.

I74

255

99

205

331

$33 \mathrm{I}$

$33 \mathrm{I}$

518

I38

5 I 8

I 38

4 I 5

518

331

255

77

299

E.

225
66

531

255

$33 \mathrm{I}$

66

I74

13
352

138

44

4I 5

255

225

I 74

415

495

415

415

$33 \mathrm{I}$

415

4I 5

138

255

4I 5

331

13

44

44
255

415

I 38

331

I 3

331
174 Jacot, E.

33 I Jacquest, S. P. 33 I

$\begin{array}{lr}\text { Jeeves, F. W. } & 3 \text { I2 } \\ \text { Jeeves, L. L. G. } \quad 66\end{array}$

I74 Jeff, A. H.

99

205

495

518

99

I 3

415

415

44

415

138

138

James, E.S.P. K. $\quad 66$

James, F. A. 33 I

James, G. 33 I

James, H. M. (Jesus) 174

James, H. M. (Selw.) 518

James, J. A. $\quad$ I38

James, J. H. $\quad 299$

James, J. N. A. $\quad 255$

James, L. H. $\quad 99$

James, R. W. $\quad 332$

James, S. R. $\quad 4 I 5$

James, T. C. 4I5

James, T. J. $\quad \mathbf{r}_{3}$

James, T. M. $\quad 99$

James, W. M. I3

Jameson, A. B. $\quad 352$

Jameson, C. W. $\quad 66$

Jameson, F. R. W. $\quad 99$

Jameson, G. D. $\quad 255$

Jameson, G. J. $\quad 415$

Jameson, T. B. $\quad 299$

Jameson, T. S. $\quad 53 \mathrm{I}$

Jameson, W. L. $\quad 99$

Jamie, J.P. W. $\quad 99$

Jamieson,E.A.O.A. 415

Janasz, G. K.A.

see de Janasz, G. K. A.

Jaques, A. $\quad 255$

Jaquet, D. A.

Jaquet, E. G. $\quad 174$

Jarchow, C. J. F. $\quad 332$

Jardine, J. W. $\quad 205$

Jardine, R. F. $\quad 77$

Jármay, I. B. $\quad 415$

Jarvie, J. M. $\quad 99$

Jarvis, F. Iarvis, F. J.

Jarvis, L. K. 4I5

Jarvis, H. E. G. $\quad 77$

Jay, C. D. $\quad 3^{12,416}$

Jeakes, J. W. $\quad 416$

Jeans, F. A. G. $\quad 332$

Jebb, R. D. 205

66

$53 \mathrm{I}$ 
Jeffcock, H. C. F.

Jeffcock, P.E.

Jeffcock, W. H. C.

Jeffcock, W.P. 285, 495

Jeffery, E. J. B.

Jeffreys, H. G. G.

Jeffreys, J. G.

Jeffreys, W.M.

Jeffries, W. F. C.

Jemmett, C. W.

Jemmett, F. R.

Jenkin, C. O. F.

Jenkin, L. F.

Jenkin, N. W.

Jenkin; R.T.

Jenkins, C. E.

Jenkins, D. J. C.

Jenkins, E. E.

Jenkins, G. E.

Jenkins, H. L.

Jenkins, J.

Jenkins, R. E.

Jenkins, W. A.

Jenkins, W. L.

Jenkinson, C.

Jenkinson, C. H.

Jenkyn, C. W. O.

Jennings, A. R.

Jennings, $C$.

Jennings, F. M.

Jennings, G. M.

Jennings, R. W.

Jephcott, C.

Jephcott, E. W.

Jephson, P. H. R.

Jeppe, O.R.

Jepson, A. C.

Jepson, G.

Jepson, R. W.

Jerram, R. M.

Jervis, A. C.

Jervis, E. O.

Jervois, R. C. W.

Jerwood, B. E.

Jerwood, F. H.

Jerwood, J. H.

Jesse, W.

Jesse, W. J.

Jessell, R.P.

Jessop, G. L.

Jeudwine, S. H.

Jeudwine, W. W.

Jewell, B.S.

Jewell, C. J.S.

Jewitt, D.P.

Jobling, J.S.

Jobson, J.S.

Joce, J. B. D.

Joel, W.S.

Joels, W. A.

John, D. W.

99

77

531

255

416

I75

255

138

495

175

138

I39

255

225

518

518

285

352

175

175

518

I3

I39

I3
174 John, H. G.

175 John, J. C.

John, W. R.

Johns, B. C.

Johns, N. A.

Johns, N.S.C.

Johns, O. Ll.

Johns, S. H. M.

Johnson, A. D.

Johnson, A. H.

Johnson, A. V.

Johnson, C.

Johnson, C. R. I.

Johnson, D. F. G.

Johnson, E. F.

Johnson, E. H.

Johnson, E. S.

Johnson, E.S. H.

Johnson, F. H.

Johnson, F. W. B.

495

255

I39

139

495

495

518

416

416

518

352

332

495

518 see Darmady, E.S.

see BurgoyneJohnson, F. W.

416

205

99

225

99

495

99

44

416

$53 \mathrm{I}$

13

205

225

99

332

205

Johnson, G. A. M.T. 416

Johnson, G. B.

Johnson, G. B. B. 495

Johnson, G. F. 205

Johnson, G. G. F. 205

Johnson, G. S. $\quad 255$

Johnson, H. A. $\quad 495$

Johnson, H. C. J. $\quad 139$

Johnson, H. P. $\quad 139$

Johnson, J. B. $\quad 518$

Johnson, J. G. T. 495

Johnson, L. O. I39

Johnson, M.R. W. 255

Johnson, N. D.

see Digby-Johnson, $\mathrm{N}$.

Johnson, O. B. G. $\quad 352$

Johnson, O. G. $\quad 78$

Johnson, P. R.

Johnson, R.E.

Johnson, R. F.

Johnson, R. L.

Johnson, R. T.

Johnson, T.P.

Johnson, V. Y.

Johnson, W. D.

Johnson, W. H.

Johnson, W. L.

Johnson, W. M.

Johnson-Ferguson,

$$
\text { E. A. J. }
$$

Johnston, $\mathrm{B}$.

Johnston, C. McA.

Johnston, F.

Johnston, F. B.

Johnston, H. B.

Johnston, H. L.

Johnston, M. C.

Johnstone, C. A.

139

44

205

416

299

332

$53 \mathrm{I}$

312

255

416

416

255

299

332

205

I 39

416

332

225
Johnstone, C. C. G. $\quad 175$

Johnstone, C. M. $\quad 44$

Johnstone, C.P. $\quad 255$

Johnstone, G. G. $\quad 205$

Johnstone, G.H. $\quad 416$

Johnstone, J. J. $\quad 416$

Johnstone, R. O.S. $\quad 255$

Johnstone, W. $\quad$ I75

Joicey, C. M. 495

Joicey, E. $\quad 416$

Joicey, E. R. 495

Joicey, J. $\quad$ I75

Joicey, J. A. $\quad 175$

Joicey, S. J.D. 4I7

Jolley, J.A. B. 312

Jolley, L. B. W. $\quad 417$

Jolly, A. F. $\quad 225$

Jolly, B. O. $\quad 78$

Jolly, H. L.P. 4I7

Jolly, R. B. $\quad 99$

Jolowicz, H. F. $\quad 417$

Jomaron, A. C. $\quad 66$

Jonas, G. J. $\quad 352$

Jones, A. M. $\quad 312$

Jones, A.P. 205

Jones, A. V. $\quad 205$

Jones, Bertram 4I7

Jones, B. C. $\quad 66$

Jones, B. M. $\quad 99$

Jones, C. E. $\quad 175$

Jones, C. E. M. 205

Jones, C. H. $\quad 4 \mathrm{I} 7$

Jones, C. J. $\quad 99$

Jones, C. McC. $\quad 78$

Jones, C. R. Selous 255

Jones, C. S. $\quad 78$

Jones, C. W. $\quad 417$

Jones, D. M. 531

Jones, E. (Christ's) I3

Jones, E. (Fitz.H.) 53 I

Jones, E. D. $\quad 417$

Jones, E. Gregory

$\mathrm{E}$.

Jones, E. K.

Jones, E. Lloyd $\quad 78$

Jones, E. W. (Jesus) 175

Jones, E. W. (Sid.) 352

Jones, F. $\mathrm{H}$.

Jones, F. J. J. R.

Jones, G. B. H. $\quad 352$

Jones, H. A. $\quad$ I75

Jones, H. G. $\quad 417$

Jones, H. K. $\quad 255$

Jones, H. Ll. $\quad 44$

Jones, I. C. S. II

Jones, I. E. $\quad 332$

Jones, I. F. H. $\quad 99$

Jones, I. K. 4I7

Jones, J. D. $\quad 518$

Jones, J. M.

see Morris-Jones, J. 
Jones, J.S.

Jones, J. W. B.

Jones, L. T. P.

Jones, L. W.

Jones, O. T.

Jones, R. A.

Jones, R. F.

Jones, R. L. (King's) 205

Jones, R. L. (Sidney) 352

Jones, R. M.

Jones, R. R.P.

Jones, R. T.P.

Jones, T. A.

Jones, T. B.

Jones, T. C.

Jones, T. E.

Jones, W. B.

Jones, W. D.

Jones, W. E. D.

Jones, W. H. (Chr.)

Jones, W. H. (Pet.)

see MacnaughtenJones, W. H.

Jones, W. H.S. 312, 5 I 8

Jones, W. N.

Jones, W. P. M.

Jones-Bateman, $\mathrm{F}$.

Jones-Bateman, $\dot{ }$.

Jonsson, A. T.

Jordan, A. R.

Jordan, G. P.

Jordan, H. R.

Jordan, J. H.

Joscelyne, C. H.

Joseph, C. G.

Joseph, F. A.

Joseph, $\mathrm{H}$.

Joseph, H. M.

Joseph, W. F. G.

Jourdain, R. O.

Jowett, A. C.

Jowett, E. C.

Joy, N. H.

Joyce, G. E.

Joyce, J. L.

Joyner, C. B.

Joynson, R.

Juckes, $\mathrm{R}$.

Judd, L. A.

Juler, F. A.

Jump, $H$.

Jump, R. L.

Junor, P. B.

Jupe, H. M.

Jupp, A. O.

Just, T. H.

Kafka, E. J.

Kain, H. G.

Kaminski, V.

Kann, E. A.

\section{K}

\section{Keats, F.T.}

Keats, J. R.

Keay, E. D.

Keay, J. G.

Keay, K. D.

Keble, T. H.

Keddie, C. M.

Keeble, C. F. A.

Keelan, V. L.

44

255

100

518

$53 \mathrm{I}$

332

205

417

I 3

225

417

417

417

299

205

100

225

255

226

417

417

417

100

139

I3

4I7

299

67
78

78
100

Keeling, O. H.
Keele, C. A.

Keeling, B. F. E.

Keeling, F. H.

Keen, A. W.

Keenan, J. B.

Keesey, G. E. H.

Keeton, G. H.

Keigwin, A. L.

Keigwin, C.H.S.

Keigwin, H. D.

Keigwin, R.P.

Keigwin, W.S.

Keir, L.

Keith, A. J.

Kelham, M.H. C.

Kelk, A. F. H.

Kelland, W.H.C. $\quad 100$

Kelleher, $\mathrm{H}$.

Kellie, K. H. A.

Kellock, H.P.

Kellock, T. H.

Kellow, W.

Kelly, D.P. J.

Kelly, I. G.

Kelly, P. J.

Kelsey, A. E.

Kelsey, A. R.

Kelsey, L. R.

Kelsey, W.

Kelton, P.St G.

Kelway, K. S.
255

495

13

13

78

417

205

352

139

285

226

I 39

417

226

299

78

13

518

67

139

255

175

44

44

531

175

205

332

531

205

417

418

418

418

418

78

100

285

495

285

285

44

I39

78

67

226

13

I39

100

100

I39

78

299

299

418

226

256

44

299

I39
Kemble, H. M.

Kemp, Sir K. H.

256

Kemp, K. M.

Kemp, N.

Kemp, P. V.

Kemp, W. R.

175

67

Kemp-Welch, H. A. 418

Kemp-Welch, M. 205

Kempe, E. C.

Kempe, W. N.

Kempsey, F.

Kempson, E. W.E. 418

Kempson, F.C. $\quad 139$

Kempson, J. H. $\quad 13$

Kempthorne, G. A. 332

Kendall, E. A. $\quad 206$

Kendall, F. R. N. $\quad 418$

Kendall, G. M. $\quad 332$

Kendall, H. B. $\quad 256$

Kendall, H. E. $\quad 256$

Kendall, J. M.A. $\quad 67$

Kendall, L. F. W. A. 139

Kendall, P. D. $\quad 495$

Kendall, W. A. $\quad 3$ I2

Kennedy, A. $\quad 139$

Kennedy, D. D. $\quad 518$

Kennedy, D. J. $\quad 175$

Kennedy, E. H. $\quad 418$

Kennedy, F. R. $\quad 299$

Kennedy, G.H. $\quad 256$

Kennedy, Lord H. $\quad 418$

Kennedy, H. T. $\quad 418$

Kennedy, J. M.S. $4 \mathrm{I} 8$

Kennedy, J.S. $\quad 206$

Kennedy, M.S. N. $\quad 418$

Kennedy, M. W. $\quad 139$

Kennedy, R.S. I3

Kennedy, S. D. $\quad 418$

Kennedy, W. T. $\quad 418$

Kennedy-Cochran-

Patrick, N.J. $\quad 418$

Kennedy-Cochran-

Patrick, W. J.C. 418

Kennett, B. L. A. 299

Kennett, E. J. B. M. 299

Kennett, W. H. $\quad 332$

Kennington, J. $\quad 44$

Kenny, H. T. $\quad 332$

Kenrick, C. H. W. 285

Kensington, H. le G. 256

Kent, A. G. $\quad 100$

Kent, H. H. $\quad 139$

Kent, N. B. 13

Kent, T.P.P. I00

Kenworthy, C. H. $\quad 44$

Kenyon, H. G. $\quad 256$

Kenyon, M. N. $\quad 418$

Keown, R. W. $\quad 256$

Ker, R. A. 518

Kerby, C.C. $\quad 418$

Kerby, E. T. 
Kerby, P. W.

Kerby, W. M.

Kernick, J. W.

Kerr, J.

Kerr, J. Bruce see Bruce-Kerr, J.

Kerr, J. F.

Kerr, J. G.

Kerr, M. J. D.

Kerr, P. W.

Kerr, W.H.

Kerr-Smiley, P.K.

Kerrich, H. L.

Kerridge, W. A. L.

Kerrison, R. O.

Kersey, R. H.

Kershaw, J.

Kershaw, M.

Kershaw, M. $\quad 285$

Keswick, H.

Keswick, H. G.

Kettlewell, H. H.

Kettlewell, L.

Kewley, W. C.

Kewney, G.S.

Key, S. W.

Keymer, B.N.

Keymer, B. W.

Keynes, G. L.

Keyworth, W. D

Khan, T. H.

Kidd, C. B.

Kidd, E. L.

Kidd, F.S.

Kidd, H. L.

Kidd, J.N.

Kidd, L. S.

Kidd, R. H.

Kidd, W. A. T.

Kidson, C. W.

Kidson, N.S.

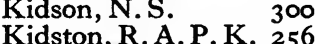

Kilburn, D.

Killen, E. O. B.

Killick, C.

Kilner, S. D.

Kinahan, A. E.

Kinder, T. H.

Kindersley, C. E. (Magd.)

Kindersley, C. E. (Trin.)

Kindersley, G. W.

Kindersley, H. R.

Kindersley-Porcher, C. P.W.

King, A. M.

King, B. N.

King, $C$.

King, C. B. R.

King, C. M.

C.U.W.L.

44
256

418

496

495

$53 \mathrm{I}$

418

418

418

419

419

519

519

519

256

313,332

256

256

256

519

299

419

256

419

4I9

44

256

419

175

299

313

496

419

419

I39

226

419

226

419

4I9

419

419

419

352
King, E. F. H.

King, E. H.

King, F. C.

King, F. H.

King, F. J.

King, G. C.

King, $\mathrm{H}$.

I4 King, H. B.

King, H. H. (King's) 352

100

419
King, H. H. (King's) 206

King, H. R.

King, $\mathrm{K}$.

King, L. A. L.

King, $N$.

King, P. B.

King, P. E.

King, R. $H$.

King, R. R.

King, $\mathrm{S}$.

King, S. W. T.

King, W. B. R.

King, W. O. R.

King,W.O.R. $\quad 352$

Kingdom, W. A.

Kingdon, G. H.

Kingham, B. V.

Kingham, W. R.

Kinghorn, E. C.

Kingsfold, $P$. A.

Kinloch, Sir G.

Kinloch, M. W.

Kinman, G. W.

Kinnach, S. J.

Kinnaird, D. A.

Kinnaird, K. F.

Kipping, C.H.S.

Kirby, $\mathrm{H}$.

Kirby, H. R.

Kirby, J. B.

Kirby, W. E.

Kirk, J.H.

Kirk, J. W. C.

Kirkbride, G.

Kirkby, N. W.

Kirkcaldy, G. I.

Kirke, G. G.

Kirke Smith, A.

Kirkland, A. E.

Kirkland, F. W.

Kirkland, W.N.

Kirkman, R. W.

Kirkness, L. H.

Kirkpatrick, A.P.

Kirkpatrick, J. B.

Kirkpatrick, R.M.

Kirkus, C. H.

Kirtland, J.

Kisch, E. R.

Kitching, A. E.
Kingsford, G. T.
139

300

352

I39

226

139

100

2

256
496

44

332

352

496

519

$53 \mathrm{I}$

44

140

175

332

300

100

4I9

206

332

140

419

256

332

256

419

4I9

496

44
256

519

100

332

206

226

256

67

44

419

100

I00

300

78

332

419

175

67

3I3

519

45

176

Kitching, G. C.

Kitching, H. E.

Kitson, B. M.

Kitson, R. D. $\quad 4$ I

Kitson, S. D. $\quad 4$ I9

Kittermaster,A.N.C. I4

Kittermaster, D. B. $\quad 45$

Klugh, $\mathrm{H}$. $\quad 45$

Knaggs, R. L. $\quad 140$

Knapp, K.K. $\quad 3$ I 3

Knappett, P. G. $\quad 226$

Kneen, C.W. $\quad 3$ I3

Kneese, R.H.W $\quad 4$ ro

Knight, C. E. $\quad 176$

Knight, C. M. $\quad 256$

Knight, E. A. $\quad 67$

Knight, E. F. $\quad$ I40

Knight, F. H. $\quad 300$

Knight, H. F. $\quad$ I40

Knight, J. E. H. 313

Knight, J. H. $\quad 53 \mathrm{I}$

Knight, J. O.C. 4I9

Knight, P.C. $\quad 4$ ro

Knight, P. D. $\quad 256$

Knight, $R$. $\quad$ I4

Knight, W. F. $\quad 100$

KnightBruce,R.E.C.4I9

Knighton, G. G. $\quad 256$

Knights, H. J. W. $\quad$ I00

Knights, K. M. W. $\quad 100$

Knipe, R. C. $\quad 353$

Knobel, W. B. $\quad 419$

Knott, P. G. $\quad 226$

Knowles, A. J. $\quad 420$

Knowles, C. K. $\quad 45$

Knowles, D. $\quad 420$

Knowles, E. A. $\quad 496$

Knowles, F. $\quad 256$

Knowles, G. J. F. $\quad 420$

Knowles, J.A. $\quad 332$

Knowles, Sir L. $\quad 420$

Knowles, R. $\quad 78$

Knowles, R. K. $\quad 420$

Knowles, R. M. $\quad 496$

Knox, A. D. $\quad 206$

Knox, B. 45

Knox, C. W. $\quad 420$

Knox, G. G. $\quad 420$

Knox, P.F. $\quad 496$

Knox, R.U.E. $\quad 332$

Knox, R.W. $\quad 140$

Knox, W. $\quad 140$

Knox, W. D. C. $\quad 496$

Knox-Shaw, P. $\quad 353$

Knox-Shaw, T. $\quad 353$

Knubley, C. $\quad 226$

Knubley, R. L. $\quad 226$

Köblich, G. R. $\quad 3^{1} 3$

Koettgen, E. A. $\quad 176$

Kohan, C. M. $\quad 420$

Kohn,W. A. $\quad 140$ 
Kon, G. A. R.

Koop, G. G.

Krause, E. H.

Kutnow, H.S.

Labey, T. H.

La Brooy, M.V. T. J. ${ }^{519}$

Lace, L. E. C. D.

Lacey, A. T.

Lacey, F. A.

Lacy, H. C.

LacyThompson, T.A. 420

Ladd, L. S.

Ladenburg, A. L.

Laffan, R. G. D.

Lafone, E. W.

La Fontaine, J.S.

La Fontaine, S. H.

Lagden, L. A.

Lagden, R. B.

Lahore, Bp. of

Laidlaw, C. G. P.

Laidlaw, F. F.

Laidlaw, W.S.

Laidlay, J. C.

Laing, A. T.

Laing, $B$.

Laing, C. $M$.

Laing, J. C.

Laird-Clowes, G. S. 353

Laistner, M. L.W. $\quad{ }_{176}$

Lake, W. I.

Laker, W. N.

Lakin, E. L.

Lamb, C. H.

Lamb, G. J.

Lamb, P. C. C.

Lamb, R. E.

Lamb, R.P.

Lambart, J. H. L.

Lambe, J. L.P.

Lambert, E. T.

Lambert, $\mathrm{F}$.

Lambert, $\mathrm{H}$.

Lambert, H. E.

Lambert, H. M.

Lambert, J.

Lambert, J. E. H.

Lambert, M. B.

Lambert, R. E.

Lambert, St J. M.

Lambert, W.

Lamberton, A. R.

Lambton, J. F.

La Mothe, H. D.

Lancaster, E. T.

Lancaster, $\mathrm{H}$.

Lancaster, $\mathrm{S}$.

Lance, H. W.

Lancer, $\mathrm{H}$. 206

Lanchester, H. C.O. 257

Lanchester, W. F. 206

257

420

67

226

67

300

257

420
Landale, C.

Landale, D. B.

Landale, D. G.

Landau, $\mathrm{H}$.

Lander, A. J. M.

Lander, P. E.

Lander, T. E.

Landon, J. W.

Lane, H. C. H.

Lane, H. J.

Lane, J. E.

Lane, $P$.

Lane, S. F. B.

Lane, T. W.

$\begin{array}{lr}\text { Lane, T. W. } & 45 \\ \text { Lane-Claypon, J. C. } 257\end{array}$

Lang, A.

Lang, A. H.

Lang, B. T.

Lang, $\mathrm{H}$.

Lang, H. A.

Lang, L. H.

Lang, $R$.

Langdale, A. H.

Langdale, $H$. C.

Langdale, K. M.

Langdon, A. G.

Langdon, $\mathrm{C}$.

Langdon, C. G.

Langdon, H. C. T. 140

Langdon, L.

Langerman, A. H. R. I4

Langerman, E. S.

Langham, C. R.

Langham, F. G.

496

420

496

140

353

78
421

353

333

140

226

14

257

257
100

$42 I$

421

140

67

421

176

140

140

45

206

519

519

519

Langlands, N. M. S. 496

Langley, A.S. $\quad 78$

Langley, E. R. $\quad 140$

Langley, F. O. $\quad 140$

Langley-Smith, N.H.300

Langmead, L. G. N. 100

Langton, G. B.

353

Langton, $\mathrm{H}$. B.

Langton, S. J.

Langtry, R. L.

Lankester, E. A.

257

496

100

Lankester, R.

Lankester, R.F. $\quad$ I00

Lansberry, H. G.

Lapage, F.C.

78

206

Laporte Payne, A. A. 67

Laporte Payne, R. M. 67

Larking, R. G.

206

Larkworthy, F. G. C. 206

Larman, G. E.

496

Larmour, A. C.

421

Larner, H. M. 257, 519

Larpent,J.P.G.deH. 257

Lart, C. E.

Larymore, H. D.

Lasbrey, B.

Lasbrey, E. W.

Lasbrey, P. U.
Last, F. W.

Latham, P. H. $\quad 257$

Lathbury, R. J. $\quad 100$

La Touche, D.D. $\quad 67$

La Touche, G. G.D. see Digges La Touche, G. G.

La Touche, H. N.D. 333

La Touche, W. F.D. 257

Lattey, $H$. $\quad 333$

Lattey, H.P. T. $\quad 257$

Lattey, W.T. $\quad 67$

Lauder, J. C. $\quad 176$

Lauderdale, E. M. $\quad 140$

Laughlin, C. E. H. $\quad 45$

Laughlin, P. H. $\quad 333$

Laurance, J. B. $\quad 257$

Laurence, C. $\quad 421$

Laurence, C. H. $\quad 421$

Laurie, F. G. $\quad 140$

Laurie, H. C. $\quad 140$

Laurie, M. $\quad 286$

Law, C. $\quad 257$

Law, $\mathrm{H}$. $\quad 257$

Law, H. S. $\quad 78$

Law, R. A. $\quad 421$

Law, R. R. $\quad 14$

Lawe, F. W. $\quad 333$

Lawley, Sir A. $\quad 421$

Lawrence, A. C. C. 421

Lawrence, B. L. $\quad 42 \mathrm{I}$

Lawrence, C. H. $\quad 421$

Lawrence, G.H. $\quad 67$

Lawrence, H. R. $\quad 257$

Lawrence, J. R. M. 257

Lawrence, J.S. G. $\quad 140$

Lawrence, M. C. $\quad 421$

Lawrence, O.J. $\quad 421$

Lawrence, $T$. $\quad 257$

Lawrence, T. E. $\quad 5$ I9

Lawrence, W. H. A. 421

Lawrie, C. V.E. $\quad 421$

Lawrie, J. M. 42I

Lawson, C. G. $\quad 257$

Lawson, Sir D. $\quad 496$

Lawson, D. A. $\quad 496$

Lawson, G. $\quad 421$

Lawson, H.S. $\quad 286$

Lawson, J. C. $\quad 257$

Lawson, R. C. $\quad 42 I$

Lawson, R. H. $\quad 353$

Lawson, W. R. $\quad 519$

Lawson-Johnston, A. McW.

Lawson-Johnston, E. A.

$42 I$

Lawson-Walton, A. 257

Layard, A. H. $\quad 206$

Laye, P. A. W. $\quad 496$

Layman, F. H. $\quad 42 I$

Layton, D. H. $\quad 78$ 
Lazarus, E. L.

Lazarus, G. M.

14

206

Lazarus-Barlow, P. $\quad 78$

Lazarus-Barlow,W.S. 78

Lea, D. H.

Lea, E. I.

Lea, H. A. H.

Lea, $M$.

Lea, M. B.

Lea Wilson, B. H. C. 421

Leach, C. de L. $\quad 257$

Leach, G. K. $\quad 226$

Leach, N. K.

Leach, R. C.

Leach, R. W.

Leach Lewis, A. F. 257

Leach Lewis, W. I4I

Leacroft, J.

Leader, B. E.

Leader, E. E.

Leader, H. E.

Leaf, C. S.

Leaf, E. $\mathrm{H}$.

Leaf, F. A.

Leaf, H. M.

Leak, E. A.

Leak, W.N.

Leake, C. F.

Leakey, H. N.

Leakey, R. A.

Leale, R. J.

Leary, G. G. W

Leatham, C. B

Leatham, C. G.

Leatham, H. W.

Leathart, P. W.

Leather, K. J. W.

Le Blanc Smith, C. $\mathbf{R}$.

257

$42 I$

$42 I$

78

422

422

422

422

422

422

496

333

67

176

r76

422

496

422

45

422

422

Le BlancSmith, T.E. 422

Le Blond,

R. C. G. du P. 422

Le Brasseur, J. H. 257

le Brocq, C. N. 257

Ledgard, W. H. 333

Ledward, C. H. $\quad 258$

Ledward, E. F. $\quad 25^{8}$

Ledward, H. D. $\quad 422$

Lee, A.

Lee, A. N.

Lee, C. B.

Lee, C. S.

Lee, E. H.

Lee, E. O.

Lee, $\mathrm{H}$.

Lee, H. F.

Lee, H. V.

Lee, J. M.

Lee, $\mathbf{N}$.

Lee, R. O.

Lee, W. E. (Christ's)

333

206

333

258

313

496

353
Lee, W. E. (Trin.)

Lee, W. N. R.

Lee-Booker, R.

Lee-Norman, F. T.

Lee Warner, R. P. $\quad 333$

Leech, E. B. 14

Leeke, Charles

Leeke, Christopher 422

Leeke, E. J.

Leeke, H. A.

Leeke, J. A.

Leeming, A. J.

Lees, $A$.

Lees, A. A.

Lees, J.

Lees, $\mathbf{K}$.

Lees, $\mathrm{S}$.

Lees, T. P.

Leete, W. J. H.

Le Fleming, E. K.

Le Fleming, G. F. A. H.

Le Fleming, J.

Le Fleming, M. R $\quad 45$

Lefroy, E. J.

Lefroy, F. P.

Legg, Sir G. E. W.

Legg, H. G.

Leggatt, L. C.

Legge, $\mathrm{P}$. A.

Legge, R. J.

Legge-Currie, J. D.

Leggett, H. A.

Legouis, P. A. E.

Le Gros, F. G.

Lehmann, F. H. see Layman, F. H.

Lehmann, J. R.

Leicester, C. B. W.

Leigh, B. $\mathrm{H}$.

Leigh, $C$.

Leigh, E. $\mathrm{H}$.

Leigh, $F$.

Leigh, S.P.

Leigh-Mallory G 300

Leigh-Mallory, T. $\quad 226$

Leighton, A. F. 14 I

Leighton, F. M. $\quad 497$

Leighton, J. W.

Leith, H. G.

Le Lacheur, W. J.

100

Lemmey, $F$

Lemon, G. T.

Le Neve Foster, B. A. J. C.

Leney, R. J. B.

Lenny, L. A.

Leon, E. J.

Leonard, H. V.

Leonard, P. J.

333

Le Ray, H. G.

423

Le Rougetel, I. H. $\quad 226$

Le Roy-Lewis, H. $\quad 423$

Lescher, F. G.

141

Lesley, J. W.

100

Leslie, A.

423

Leslie, L. D. A. $\quad 258$

Leslie-Melville, A.B.423

Lester, C. V. I4I

L'Estrange Fawcett, A. W.

Letchworth, G.H.S. 258

Letts, C. F. C. $\quad 176$

Letts, E. M. $\quad 497$

Lever, D. $\quad 423$

Leveson, W. E. $\quad 45$

Levett, A. R. $\quad 423$

Levett, E. C. $\quad 423$

Levi, W.H. $\quad 423$

Levick, P. $\quad 176$

Levien, E. G. IOI

Levinge, H. G. $\quad 423$

Levinstein, G. E. $\quad 258$

Levita, C. E. $\quad 423$

Levita, H.P. $\quad 423$

Levy, L. $\quad 258$

Levy, L. A. $\quad 45$

Lewarne, F. T. $\quad 519$

Lewes, W. $\quad 497$

Lewey, A. W. $\quad 497$

Lewin, A. C. $\quad 497$

Lewin, E. O. 206

Lewin, H. W. $\quad 45$

Lewin, K. R. $\quad \mathbf{4 2 3}$

Lewis, A. D. $\quad 206$

Lewis, A. M. $\quad 68$

Lewis, A. T. $\quad 53 \mathrm{I}$

Lewis, B. R. $\quad 497$

Lewis, C. B. IOI

Lewis, F. G. $\quad 497$

Lewis, F. H. $\quad$ I4

Lewis, G. A. $\quad$ I4I

Lewis, H. G. $\quad 53 \mathrm{I}$

Lewis, H. H. $\quad$ I4I

Lewis, H. M. $\quad 45$

Lewis, J. B. S. $\quad 79$

Lewis, J. H. (Emm.) Ior

Lewis, J. H. (Cath.) $3^{1} 3$

Lewis, J. M.

Lewis, J. M. C.

Lewis, L. H. $\quad 45$

Lewis, M. B. $\quad 300$

Lewis, M. M. $\quad 79$

Lewis, O. G. $\quad 53 \mathrm{r}$

Lewis, P. J. $\quad 333$

$\begin{array}{rrr}\text { I76 } & \text { Lewis, R. C. } & \text { I4 } \\ \text { I4I } & \text { Lewis, T. E. } & \text { I4I }\end{array}$

I4I Lewis, V. 286

45 Lewis, W. A. H. 79

45 Lewis, W. B. A. I4 
Lewis,W.H.(Emm.) roI Lewis, W.H.(Caius) 141 Lewtas, F. G. $\quad$ I4I

Lewthwaite, C. G. $\quad 423$

Lewthwaite, W. $\quad 423$

Ley, C. F. A. $\quad 258$

Ley, R. L. $\quad 258$

Leyton, A. S. F. $\quad$ I4I

Lhoyd-Owen, E. E. 300

Lias, A. G. $\quad 206$

Lias, E. T. M. $\quad 286$

Lias, R. J. M. $\quad 423$

Lias, W.J. $\quad 176$

Lichfield, Earl of $\quad 423$

Lichtenberg, W.A. 423

Liddell, C. F. J. 423

Lidderdale, F.J. $\quad 423$

Liddle, G. E.

Liddle, $\mathrm{H}$. W.

Lidgett, J. C.

Light, D. O.

Light, $P$.

Lightbody, W.P.

Lightburne, H.R.H. 519

Lilley, T. G.

Lillie, F.S.

Lillie, W. H.

Lilly, C. O.

Lilly, G. A.

Lincoln, $N$.

Lindemere, $\mathrm{V}$.

$\begin{array}{ll}\text { Lindemere, V. } & 424 \\ \text { Lindesay, J. H. C. } \quad 353\end{array}$

Lindley, J.E.

Lindley, W. M.

Lindsay, F. H.

Lindsay, J. H.

Lindsay, $\mathrm{L}$.

Lindsay, R. L.G

Lindsay, W.J.

Lindsay, W. J.
Lindsay Watson, R. H.

see Watson,

R. H. Lindsay

Lindsell, J.

Line, E. A. T.

Line, J.

Line, J. Y. A.

Linfoot, G. C.

Linford, W. A. M.

Ling, G. A.

Ling, $H$. W.

Ling, L. S.

Lingard, J. R.

79

IOI

258

176

519
286

300

101

176

141

333

497

424

45

424

424
286

Linley-Howlden, R.C. 14 I

Linnell, J.W.

Linnell, R. McC.

333

519

IOI

79

79

45

206

258

68

424

Linsley, A. R.

333

Linthorne, E. L. R. 226

Lipp, G. A. S.

Lipschitz, J.

Liptrot, R.N.

45

14
Lister, A. H.

Lister, A. R.

Lister, A. V.

Lister, J. B.

Lister, W. K.

Lister, Sir W. T.

Litchfield, G.

Litchfield, W. G.

Little, A. H.

Little, C. S.

Little, H. L.

Little, J. C.

Little, R. A.

Little, T. H.

Littleboy, C. N.

Littlejohn, H. A.

Littlejohns, A. S.

Littlewood, A.

Littlewood, J. E.

Litton, W. R. U.

Livens, W. $\mathrm{H}$.

Livesey, A. G. H.

Livesey, T.R.M.

Livingston, C. P.

Livingston, P.C.

Livingstone, F. D.

Llewellyn, E. E.

Llewellyn, O.J.

Llewelyn, D. E. K.

Llewelyn-Davies, P. 424

Llewelyn-Davies, R. A.

Lloyd, A.H.

Lloyd, A. S.

Lloyd, B.S.

Lloyd, C. G.

Lloyd, E. A.

Lloyd, E. I.

Lloyd, E. L1.

Lloyd, Sir G. A.

Lloyd, G. W. D. B.

Lloyd, H. C.

Lloyd, H. G.

Lloyd, H. I.

Lloyd, H. W. C.

Lloyd, I, G.

Lloyd, J. D.

Lloyd, J. F.S.

Lloyd, J. R.

Lloyd, K.

Lloyd, L. S.

Lloyd, L. W.

Lloyd, M. A.

Lloyd, M.E.

Lloyd, R. A.

Lloyd, R. B.

Lloyd, R. Ll.

Lloyd, R. T.

Lloyd, T. G.

424

424

258

519

258

424

531

258

68

519

353

141

141

497

424

258

141

79

424

497

14
258

206

497

176

286

14

497

79

424

I 4 I

424

I 76

424

424

424

333

424
286

286

424

424

IOI

I 76

I4I

353

424

206

286

425

46

258

227

286

IOI

425

313

Lloyd, W. R. 286, 333

Lloyd-Barrow, R. A. 176
Lloyd George, $\mathbf{G}$. 176

Lloyd George, $\mathrm{R}$. $\quad 14$

Lloyd Greame, Y. $\quad 425$

Lloyd-Jones, E. W. $\quad$ IOI

Lloyd-Jones, I. G. 425

Lloyd-Jones, I. T. $14 \mathrm{I}$

Lloyd-Jones, J. IOI

Lloyd-Jones, $P$. $\quad$ I4I

Lloyd-Jones, P. A. 333

Lloyd-Price, L1. O. I4I

Lloyd-Williams,

A. R. C.

425

Lloyd-Williams, K.P. 46

Lob, $\mathrm{H}$.

207

Loch, D. H.

207

Lock, L. J.

Lock, N. F.

Lock, P. G.

Lock, W. A.

Locker-Lampson, G. L. T.

Locker-Lampson, O.S.

Lockett, J.

Lockett, V. N.

Lockhart, J. H. B.

Lockspeiser, $\mathrm{B}$.

Lockwood, E. M. $\quad 353$

Lockyer, W. J. S. $\quad 425$

Loder, J. de V. $\quad 425$

Loder, N. W. $\quad 425$

Loder, R. E. $\quad 425$

Lodge, J. W. I4I

Loewe, H. M. J. 300, 313

Loewe, L. L. $\quad 176$

Loft, E. W. B. $\quad 68$

Logan, $\mathbf{H}$.

Logan, J. M.

Logan, M. J. S.

497

S. $\quad 79$

Lomax, E. H. $\quad 300$

Lombard, B. S. $\quad 46$

London, G. E. $\quad 79$

Long, A. E. $\quad 333$

Long, B. A. $\quad 141$

Long, C. W. $\quad 142$

Long, F. E. $\quad 227$

Long, F. S. $\quad 531$

Long, F. W. C. $\quad 286$

Long, J. A. E. $\quad 497$

Long, L. P. $\quad 353$

Long, R. A. $\quad 79$

Long, W. E. $\quad 497$

Long, W. G. $\quad 425$

Longbottom, $\mathrm{H}$. $\quad 425$

Longden, J. M. IOI

Longhurst, A. L. $\quad 425$

Longhurst, H. B. $\quad 425$

Longman, F. $\quad 25^{8}$

Longman, H. K. $\quad 425$

Longman, R. G. $\quad 425$ 
Longridge, A. O. C. 425

Longridge, $M$.

Longridge, R. B.

Longrigg, G.E.

Longrigg, J. $\mathrm{H}$.

Longstaff, C. C.

Longstaff, F. V.

Longstaff, G. C.

Longstaff, J.

Longstaffe, V. C. H

Longworth, E. C. 497

Lonsdale, A. C. G. 425

Lonsdale, $\mathrm{H}$.

Lord, A. E.

Lord, C. E.

Lord, G. F.

Lord, H. D.

Loring, R. W.

Loring, $W$.

Loring, W. L.

Loseby, P. J.

Lound, R.S.

Love, E. W. P.

Loveband, F. R.

Loveband, G. Y.

Loveday, D. G.

Loveday, G. E.

Loveless, M. L.

Loveless, W. B.

Lovell, J.S.

Lovelock, A. R.

Lovett, R. D.

Lovibond, J. L.

Low, A.

Low, A. H.

Low, A. J.

Low, A. R.

Low, B. B.

Low, $\mathrm{H}$.

Low, H. F.

Low, W. P.

Lowcock, D. R.

Lowe, C. A. H.

Lowe, C. N.

Lowe, F. G.

Lowe, G. B.

Lowe, H. St A.

Lowe, P. R.

Lowe, W. D.

Lowe, W. W.

Lowndes, A. G.

Lowndes, J.

Lowndes, R. G.

Lowndes, W.P.

Lowry, G. C.

Lowry, S. H.

Lowry, W. A. H.

Lowry Corry,F.R.H. 426

Lowther, C. W.

Lowther, L. E.

Löwy, A. E.
425 Loyd, L. F. I.

Loyd, R. A.

Loyd, W. H.

353 Luard, S. d'A.

425

46

46

531

207
425

IOI

333

333

497

46

207

497

IOI

519

498

142

176

227

142

258

258

46

142

425

14

177

353

142

46

333

142

498

14
46

46

14
258

46

46

425

177

259

259

$3 \mathrm{I} 3$

$5 \times 9$

46

259

227

259

142
426

426

227

286

Lubbock, I.

Lucas, A.

Lucas, A. R. F.

Lucas, C. E.

Lucas, D.

Lucas, E.

Lucas, E. M.

Lucas, F. L.

Lucas, G. F.

Lucas, H. A.

Lucas, $\mathbf{K}$.

Lucas, L. W.

Lucas, R. B.

Lucas, R. H.

Lucas, T. C.

Lucas, W. G.

Lucas, W. L.

Lucas, W. R.

Luce, $H$. W.

Luce, Sir R. H.

Lucy, R. S.

Ludlow, F.

Luker, $\mathbf{R}$.

Luker, S. G.

Lumb, $\mathbf{H}$.

Lumb, T. F.

Lumb, W.

Lumley, C. H.

Lumsden, $R$.

Lund, G.S.

Lund, $\mathrm{H}$.

Lund, W. F.

Lundie, R. C.

Lunn, H. C.

Lunn, H. F.

Lunniss, S. F.

Lupton, A. C.

Lupton, B. C.

Lupton, F. A.

Lupton, G. A.

Lupton, H. R.

Lupton, J. M.

Lupton, L. M.

Lupton, $\mathrm{M}$.

Lupton, N. D.
Lubbock, H. F.P.

Lucas, G. M. E.

Luckock, E. H. M. $\quad 426$

Luckock, R. M. $\quad 426$

Lumby, A. F. R.

Lumby, C. D. R.

Lumley, D. O. $\quad 519$

Lumley-Smith, T.G. 426

Lumsden, H. B. $\quad 259$

Lumsden, J. A. $\quad 426$

Lumsden, W. F.
426
426

426

426

426

46

426

426

426

426

426

259

426

426

498

259
68

46

426

227

177

14

426
46

353

259

259

46

142

334

14

227

426

259
426

259

426

334

427

334

14

177

300

142

427

427

427

207

427

207

427

427

427
Lupton, R. H.

Lupton, W. M.

Luscombe, B.P.

46

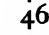

Lush, $\mathrm{E}$

Lush, $H$.

Lush, J. A. $\quad 5 \mathrm{r} 9$

Lushington, F. de W. 46

Lusk, J. 334

Luson, T. G. L. $\quad 227$

Lutter, R.B. 313

Luttman, R. L. $\quad 286$

Lycett, C. V. L. $\quad 427$

Lydekker, N.W. $\quad 519$

Lyell, G. D. $\quad 427$

Lyle, R. C. $\quad 68$

Lyle, T. B. $\quad 259$

Lyman, T. $\quad 427$

Lymbery, A. W. $\quad 334$

Lyon, A. J. $\quad 300$

Lyon, C. G. $\quad 498$

Lyon, E. L. $\quad 427$

Lyon, R. C. G. $\quad 227$

Lyon, R. E. $\quad 427$

Lyon, W. A. $\quad 498$

Lyon, W. T. $\quad 207$

Lyon-Smith, G. IOI

Lysley, W. L. $\quad 498$

Lyster, A. St G. $\quad 259$

Lyster, L. F. $\quad 427$

Lythgoe, R. J. $\quad 427$

Lyttelton, A. G. $\quad 427$

Lyttelton, C. F. $\quad 427$

Lyttelton, G. W. $\quad 427$

Lyttelton, $O$. $\quad 427$

Lyttelton, R. G. $\quad 427$

Maasdorp, V.H. I5

Mabane, W. $\quad 142$

McAfee, L.A. $\quad 259$

Macalister, G. H. K. 334

McAllum, J. H. I5

Macalpine, J. L. $\quad 177$

MacAndrew, C. G. 427

McArthur, A. G. F. 207

McArthur, G. K. $\quad 353$

McArthur, R. I5, 3I3

Macarthur-Onslow, J.W.

Macartney, C. A. $\quad 427$

Macartney, H. B. G. 427

Macartney, M.E. $\quad 46$

Macartney, M.H.H. 259

McAulay, F.W. $\quad 334$

McBain, N.S. $\quad 177$

McBain, W. R. B. $\quad 286$

Macbeth, A. H. $\quad 428$

MacBrayne, L. $\quad 259$

McBride, L. G. $\quad \mathbf{I 4 2}^{4}$

MacBryan, E. C. $\quad 177$

McCall, H. D. I5

McCall, H. W. $\quad 428$ 
McCall, H. W. L. $\quad 428$ McCall, T.H. $\quad 519$

MacCallan, A. F. $\quad$ I5

McCalman, J. A. C. 428

McCandlish, P.D. 428

McCardie, W. J. $\quad 142$

McCarthy, J. D. $\quad 286$

McCarthy, J. M. $\quad 286$

McCaskie, H. B. $\quad 142$

McCaskie, N. J. $\quad 142$

McCaughey, S. $\quad 177$

McCaw, O.C. $\quad 142$

McCleary, G. F. $\quad 498$

McCleland

N.P. K. J. O'N. 259

McClenaghan,A.B.P. 46

McClenaghan, G.M. 142

McCliment, R. J. $\quad 532$

McClintock, E. L. L. 428

McClintock Bunbury, T. L.

McClure, I. H. 428

McClure, K.A. J. $\quad 428$

McColl, A. M.

McColl, H. H.

McColl, W. L.

McCombe, F, W. W . I

MacCombie, W. J. $\quad 142$

McConnell, A. E. $\quad 177$

McConnell, W.E. $\quad 227$

McCormick, E. H. $\quad 428$

McCormick, G. D. 334

McCormick, L. J. $\quad 428$

McCormick,

W. P. G.

McCorquodale, K. $\quad \begin{array}{r}334 \\ 177\end{array}$

McCosh, E. $\quad 46$

McCosh, R. $\quad 428$

McCraith, K.Y. $\quad 428$

McCrea, E. D. IOI

McCririck, D.H. G. 46

Macdona, C. L. $\quad 428$

Macdonald, A. D. $\quad 428$

Macdonald, A. J. M. 428

Macdonald, C. L. Ior

Macdonald, G K. 259

Macdonald, $\mathrm{H}$. $\quad$ I5

MacDonald, J. N. $\quad 142$

Macdonald, N. J. $\quad 259$

Macdonald, R. $\quad 300$

MacDonald, S. G. 334

Macdonald, S. H. $\quad 259$

Macdonald Brown, I.

see Brown, I. M.

McDonnell, J.

McDonnell, T F R 207

McDougal, E.T.M. 428

McDougall, A.

McDougall, $\mathrm{R}$.

McDougall, $\mathrm{S}$.

McDougall, W.
MacEwen, D. L.

McEwen, J. H. F.

McEwen, J. R. D.

McFadyen, W.A.

Macfarlane, C. B.

McFarland, J. B.

Macfie, R. A. S.

McGeagh G. R $\quad 142$

McGeagh, J.P.

McGowan, $\mathbf{H}$.

McGowan, I. A. W. ${ }^{113}$

McGowan, N.S. 177

McGown, T. W. M. 47

McGrady, S. H. $\quad 334$

Macgregor, A. H. $\quad{ }_{142}$

Macgregor, A. M. 300

Macgregor, D. H. $\quad 428$

Macgregor, M.E. $\quad 428$

McGrigor, A. M. $\quad 259$

Machell, H. G. $\quad 207$

Machin, B. W. $\quad 47$

McIlroy, H.D. $\quad 177$

MacIlwaine, G.W. 259

McInnes, A. N. I5

Macintosh, H. M. $\quad 68$

McIntosh, J. G. H. $\quad$ I5

Macintyre, D. L. $\quad 47$

McIntyre, E.

McIntyre, J.

McIntyre, $\mathrm{N}$.

MacIver, C. R.

Mack, A. A.

Mack, I. A.

Mackay, A. S.

Mackay, C. L.

Mackay, D.R. G.

Mackay, E. R.

Mackay, G.R.

Mackay, K.

Mackay, R. D.

Mackay,R.F.B. 207,532

McKelvie, J. $\quad 498$

Mackennall, W.L. 429

Mackenzie, A. V. $\quad 142$

Mackenzie, B.M.S. 47

Mackenzie,C.(Emm.) Io I

Mackenzie,C.(Mag.) 227

Mackenzie, C. M. 207

Mackenzie, C. R. $\quad 429$

Mackenzie, G. M. $\quad 259$

Mackenzie, H.W. G. IOI

McKenzie, J.

Mackenzie,

Sir J. R. D.

Mackenzie, J. W.

McKenzie, K. N.

429

Mackenzie, L H M

McKenzie, R.P. 227

Mackenzie, R.S. $\quad 260$

Mackenzie, R. T. H. 260
Mackenzie, S. M. Mackenzie-Kennedy, ${ }^{429}$ H. C. D. C.

McKeown, W. W. $\quad{ }_{286}^{47}$

McKergow, R. W. 79

Mackern, G.T. G. $\quad 260$

McKerrell Brown, J. 429

McKerrow, C. K.

McKerrow, G.

Mackeson, G.P. $\quad 207$

Mackie, E.D. $\quad 177$

Mackie, J. B. 353

McKiever, V.C. $\quad 519$

Mackinlay, A. B. $\quad 429$

Mackinlay, D. M. $\quad 334$

Mackrill, O. W. $\quad 47$

Mackworth, F. J.A. 520

Mackworth-Praed, C. W.

M'Lachlan T. K.

Mclar. 260

Maclaren, A. I.

Maclaren, D. $\quad 429$

McLaren, D. B. $\quad 429$

Maclaren, J. F.P. $\quad 260$

Maclaren, N. $\quad 429$

McLaren, S. B. $\quad 429$

MacLaren, T. G. $\quad 498$

McLaughlin, A. $\quad 177$

Maclay, E. $\quad 334$

McLean, A. $\quad 429$

McLean, C. $\quad 429$

McLean, C. F. $\quad 207$

Maclean, E. W. Ior

Maclean, F.S. $\quad 177$

MacLean, $\mathrm{H}$. $\quad \mathbf{1 4 2}$

Maclean, J. E. B. B. 286

McLean, K. G. $\quad 15$

Macleod, D. J. $\quad$ IOI

Macleod, D.N. $\quad 142$

McLeod, E R. $\quad 353$

Macleod, J. D. $\quad 68$

Macleod, K. G. $\quad 260$

Macleod, N.D. $\quad$ I0I

Macleod, W. A. $\quad 520$

Macmaster, D. C. D. 429

MacMaster, H. V. $\quad 102$

McMichael, D. W. 47

McMichael, G.W. 313

MacMichael, H. A. 227

MacMichael, H. C. 227

McMichael, J. F. $\quad 520$

Macmichael,M.W.A. 429

Macmicking,G.T.G.429

Macmillan, D. $\quad 429$

Macmillan, J. M. $\quad 429$

Macmillan, W. E. F. 207

Macmorran, K. M. 207

McMullan, H. $\quad 520$

MacMullen, A. R. $\quad 142$

Macmullen, E. R. $\quad 429$ 
MacMullen, W. A. $\quad 334$

McMurtrie, S. G.

Macnab, C.

Macnab, J. T.

MacNaghten,

A. C. R.S.

313

227

15

429

Macnaghten,Sir A.D.429

Macnaghten, C. M. 429

McNair, A. J.

McNair, W.L.

102

Macnamara, C. R. $\quad 498$

Macnamara, E. D. $\quad 286$

McNaught-Davis, J. W. F.

Macnaughten-Jones, W. H.

McNaughton, H. L. 260

Macnee, E. A.

McNeil, J.

McNeile, A. H.

McNeile, A. P.

McNeile, J.

McNeile, J. $H$.

MacNeill, A. D.

McNeill, A. H.

McNeill-Hewitt, L 47

Macnutt, A. C.

Macnutt, F. B. $\quad 43^{\circ}$

Macpherson, A. S. 227

Macpherson,

C.E.H.C. $\quad 520$

Macpherson, D. G. (Clare)

Macpherson, D. G. (Caius)

McPhillamy, J. M.

McQuade, W. F.

MacQuarrie, $H$.

Macqueen, R. H.

Macrae, L.

Macrae, R. D:

MacRobert, T M.

MacRury, E.

MacSwiney, A.J.E. 520

Mc'Taggart, H. A. $\quad 143$

MacTier, J. C.

McVittie, G. H.

McWilliam, J.

Madan, A. G.

Madden, J. C. W.

Madden, J. G.

Maddison, $G$.

Maddox, J. M.

Madge, Q.

Madge, R. E.

Magnay,

Sir C. B. W.

Mahaffy, R.P.

Maile, W.C.D.

Maillet, A. L.

430
Maine, H. C.S.

498

Mainprice, $\mathrm{H}$.

Mainprize, S.L.

177

430

Mainwaring, C.F.K. 498

Mainwaring, C. L. $\quad 68$

Mair, E. M.

Mair, R. P.

Mairis, E.S.

353

430

Maitland, A. H.

143

Maitland, G. McD. 430

Maitland, H. M. $\quad 520$

Maitland, J.

Maitland, J. D.

430

Maitland-MakgillCrichton, C. J.

Major, E.

I77

Makant, A. V.

Makant, R. K.

532

532
47

47

Makeig-Jones, T.G.R. 260

Makepeace, F.L. $\quad 102$

Makin, E. L.

Makins, F. K.

143

Makins, Sir P. A. $\quad 430$

Makower, W. $\quad 430$

Malaher, H. T.

Malcolm, A.S. L. $\quad 143$

Malcolm-

Dickinson, W. 430

Malcomson, $\mathrm{H}$.

260

Malcomson, L.

102

Malcomson, W.D. $\quad 260$

Malcomson, W.T. $\quad 102$

Malden, E. C.

Malden, E. E.

Malden, H. R.

143

498

300

Malden, R. H. 207, 520

Malden, W.

430

Malet, H. A. G. $\quad 143$

Malim, F. B.

Malim, J. W.

Mallaby-

Deeley, G. M. 430

Mallalieu, W. $\quad 430$

Malleson, W. M. 102

Mallett, F. J.

Mallett, H. R.

79

Mallison, $\mathrm{H}$. V.

Mallory, G. H. L.

532

430

seeLeigh-Mallory, G.H.

Mallory, T.L.

see Leigh-Mallory, $\mathrm{T}$.

Malone, C. R.R. $\quad 15$

Malpas, C. C. $\quad 47$

Man, M.L. $\quad 102$

Manchester, Duke of $43^{\circ}$

Mander, C. A. $\quad 430$

Mander, D'A. W. $\quad 43^{\circ}$

Mander, F. W.

260 Mander, G. Le M. 431
Mandleberg, J. H.

Manduell, M. D.

Manfield, N.P. $\quad 260$

Manifold, E. W. $\quad$ I77

Manifold, J. $\quad$ I77

Manisty, E. A. $\quad 498$

Manley, J. D. $\quad$ IO2

Manley, R.S. $43 \mathrm{I}$

Mann, C. J. 260

Mann, E. J. $\quad 260$

Mann, E. W. 431

Mann, F. A. W. $\quad 15$

Mann, F. T. $\quad 260$

Mann, I. A. 43I

Mann, J. W. $\quad 313$

Mann, R. $\quad 102$

Mann, R. L. $\quad 177$

Mann, T.C. $\quad 102$

Mannering,L.G. 260, 353

Manners, F. H. $\quad 43 \mathrm{I}$

Manners, Sir G. $\quad 431$

Manners-Smith, E.V. I 5

Manning, C.R.U. 353

Manning, R. $\quad 177$

Manning, T.E. $\quad 178$

Mansbridge, E. $\quad 334$

Mansel-Pleydell, J. M.

Mansell, $R$.

$43 \mathrm{I}$

Mansell-Moullin, 0. 207

Manser, F. B. $\quad 286$

Mansfield, H. Y. $\quad 102$

Mansfield, J. $\quad 68$

Mansfield, J. H. $\quad 260$

Mansfield, R.S. $\quad 43 \mathrm{I}$

Mansfield, W.S. $\quad 102$

Manson, C. M. $\quad 431$

Maples, A. C. $\quad 520$

Maples, E. W. $\quad 520$

Mapplebeck, G.W. 431

Marburg, C. L. H. $\quad 260$

Marc, G.J. A. $\quad 431$

March, W. W. 313

Marchand, G. I. C. 334

Marchant, T.H.S. 286

Marchant, W. F. $\quad 143$

Mardon, E. J. I5

Mardon, H.A. 431

Marett Tims, H.W.

see Tims, H.W.Marett

Marett Tims, R.D.

see Tims, R. D. Marett

Margerison, C. W. 431

Margesson, H. D. R. 227

Margetts, F. B. H. $\quad 498$

Marigold, J. E. $\quad 431$

Marillier, H. C. $\quad 286$

Maris, A. A. $\quad 532$

Markham, E. B. 
Marklove, J. C.

Marks, J. D.

143

Marlborough, Duke of 431

Marley, A. E.

Marley, F. L.

Marling, W. J.P.

Marlow, C. C.

Marnham, A. E.

Marquand, C. V.B. I 5

Marr, F. A.

Marrack, J. R.

Marriott, C. E.

Marriott, C. G. L.

Marriott, C. J. B.

Marriott, F. G.

Marriott, F. W. P.

Marriott, G.A.

Marriott, G. E. J.

Marriott, J. F. L.

Marris, E. D.

Marris, H. F.

Marrow, E. A.

Marrs, F. W.

Marsden, A. T.

Marsden, C. A.

Marsden, C. $\mathrm{H}$.

Marsden, E. McL.

Marsden, $T$.

Marsden, W. G.

Marille, R.K. G. 208

Marsh, A.S. 143, 431

Marsh, A.W. (Cath.) 313

Marsh,A.W.(Fitz.H.)532

Marsh, B. C.

Marsh, C. J.

Marsh, F. D.

Marsh, F. H.

Marsh, F.S.

Marsh, O. de B.

Marshall, A. G.

Marshall, A.P.

Marshall,

A. R. (Emm.) $\quad 102$

Marshall,A.R.(Cai.) I43

Marshall, A.S.F. $\quad 43 \mathrm{I}$

Marshall, A. T. $\quad 68$

Marshall, D.

Marshall, E. A.

Marshall, F.

143

47

Marsall, F.W.D. 143

Marshall, G J P. 287

Marshall, G. T.

Marshall, $\mathrm{H}$.

Marshall, H. A.

Marshall, H. G. (Emm.)

Marshall.HG (Kg's) 102

Marshall, L. P. $\quad 354$

Marshall, N.E. $\quad 102$

Marshall, P. T.

178
Marshall, R. C.

Marshall,W. (Pemb.) 26 I

Marshall, W.(Joh.) 335

Marshall, W. B.

Marshall, W. H.

Marshall, W.S.

Marshall, W.S.D.

Marsham A. D. 432

Marsland, S. H.

Marson, A. A.

Mart, W. T. D.

Marten, A. J.

Marten, C. P.

Marten, H. H.

Marten, R. H.

Martin, B.S.

Martin, C. F.

Martin, E. N. M.

Martin, E. W.

Martin, E. W. L.

Martin, $F$.

Martin, F. H.

Martin, F. J.

Martin, H.C.

Martin, H. E.

Martin, H. W. L.

Martin, J. A.

Martin, J. H. B.

Martin, N. T.

Martin, O. L.

Martin, R. C.

Martin, R. E.

Martin, R. G.

Martin, S.P.

Martin, S.S.

Martin, S. T.

Martin, $T$.

Martin,T.L.(Emm.) 102

Martin,T.L.(Trin.) 432

Martin, W. E.

Martin, W. F.

Martin, W. M.

Martin-Tomson, W J 432

Martindale, J. R. $\quad 26 \mathrm{I}$

Martindale, R. G. $\quad 68$

Martineau, B. G.

Martineau, E.

Martineau, W.

Martinet, $\mathbf{M}$.

Martyn, J. V.

Martyn, R. F.

Martyn, R. V.

Martyn-Linnington, A. L.

Marwood-Elton, W. 498

Maryon Wilson, A. G.

see Wilson, A. G. Maryon

Marzetti, C.

Marzetti, L.

Masefield, W. B.
287

Mason, A. D. C.

Mason, A. D. $\quad 498$

287

Mason, E. W. $\quad 335$

Mason, F. L. L. $\quad 208$

Mason, G.H.(Emm.) 102

Mason,G.H.(Tr. H.) 498

Mason, H. B.

Mason, J. H.

Mason, J. W.

Mason, K. R.

Mason, N.

Mason, $P$.

Mason, R. F.

Mason, R.S.

Masser, B. R.

Masser, C.S.

Massey, B.W. A.

144

144

144

178

354

335

287

178

Massey, J. H.

Massingberd, S.

Masson, $\mathrm{K}$.

Master, A. E.

Master, C. $\mathrm{H}$.

Master, G. G.O.

Master, L. C. H.

Masterman, W.S.

Masters, F. G.

Masters, P. G.

Masters, T. H.

Masterton, H.W.

Mather, A.

Mather, A. L.

Mather, E. G.

Mather, E. L.

Mather, F. H.

Mather, G. R.

Mather, $\mathrm{H}$.

Mather, L. E.

48

208

178

432

208

144

300

432

432

15

68

520

15

432

432

432

432

$26 \mathrm{I}$

354

$26 \mathrm{I}$

Matheson, I. M. 499

Matheson, I.McL.A. 432

Mathew, F. A. H. 432

Mathews, A.

Mathews, A. G.

Mathews, E. V. D

Mathews, J. K.

Mathias, C. D.

Mathias, H. H.

68

26r

79
48

432

498

Mathieson, W.

Matravers, F. G.

Matthews, A. S.

208

354

354

Matthews, C.

Matthews, E. A. C. 432

Matthews, E. C. $\quad 432$

Matthews, H. de C. $\mathrm{IO}_{3}$

Matthews, J. B. $\quad 48$

Matthews, J. C. $\quad 79$

Matthews, M. C. $\quad 26 \mathrm{I}$

Matthews, M. H. 26I

Matthews, N. A. $\quad 520$

Matthews, N.H. $\quad$ 178

\begin{tabular}{l|ll}
26 I & Matthews, R.W. Y. & I 44 \\
I78 & Matthey, G. A. & 26r
\end{tabular} 
Mattingly, $\mathrm{H}$.

Mattock, F. C.

Maturin, F. H.

Maude, E. A. W.

Maude-Roxby, J. H. T.

Maufe, F. W. B.

Maufe, H. B.

Maufe, S. B.

Maule, G. L.

Maule, $\mathbf{R}$

Maule, W. H. F.

Maundrell, W. H.

Maunsell, L. B.

Maunsell, S. A. W.

Maw, F. G.

Maw, G. O.

Maw, R.P.

Mawdesley, J. L.

Mawe, E. S.

Mawe; M. D.

Mawer, A.

Maxwell, E. W.

Maxwell, I.Constable 300

Maxwell, L. B. I44

Maxwell, M. L. 26I

Maxwell; M. T. $\quad 432$

Maxwell,R. Constable 432

Maxwell, S. W. $\quad 499$

Maxwell, W. F. J. $\quad 432$

Maxwell, W. H. $\quad 433$

Maxwell, W. W. 16

May, A. H.

May, A. J.

May, C.A. C.

May, C. J.

May, C.P.

May, E.

May, F.S.

May, G. H.

May, H.R. D.

May, P.L.

Mayall, G.

Mayall, J.B.

Mayall, R.C.

Maybrey, H.J.

Maybrook, W.R.

Mayer, A.

Mayer, F. C.

Mayer, G. M.

Mayer, N.E.

Mayer, P. G.

Mayes, R. M.

Mayfield, E.

Mayhewe, K. G.

Maynard, A. C. M.

Maynard, A. F.

Maynard, C. B.

Maynard, $\mathrm{H}$. A.

Maynard, H.A. V.

Mayne, C.F.

287

103

144

I 44
Mayne, C. R. G.

Mayne, W. C.

Mayo, H. R.

Mayo, R. H.

Mayo, T.A.

Mayo, W.C.

Mayor, H. B.

Mead, $A$.

Mead, G. C. F.

Meade, L. G. $\quad 520$

Meadowcroft, J. $\quad$ I44

Meadows, $\mathbf{R}$.

Meakin, B.

Meakin, F. G.

Meakin, $L$.

Meakin, W.

Medcalf, $\mathrm{H}$.

Medley, R.P.

Medlycott, H. M.

Medlycott, W.

Mee, J. T. M.

Meek, J.

Meeres, B. $\mathrm{H}$.

Meeres, H. W. H.

Meers, R. H.

Meggeson, R. R. H. $\quad 144$

Megson, $M$.

Meier, F. A.

Meiggs, J. C.

Meister, G. C.

Meister, G. E.W.

Meixner, $F$.

Melhuish, T.

Melitus,

Meller, R. W.

Meller, S. A.

Mellersh, E. L.

Mellersh, F. G.

Melles, G. F.

Mellin, E. L.

Mellin, G. L.

Melling, J.S.

Mellis, G. D.

Mellis, G. D. . $\quad 48$

Mellor, A.

Mellor, J.E.

Mellor, J. E. P.

Mellor, J. F.S.

Mellor, J. G. S.

Mellor, P. H. L.

Melvill, L. V.

Melville, H. C.

Melville, W. G.

Melville-Smith, H. McL.

433

16
433

144

227
48

313

208

532

6 I

20

284

433

178

433

433

I44

433

I6

433

48

178

26 I

354

433

499

208

208

532
103

261

433

433

520

26I

499

48

48

16

499

178

499

499

499

26I

144

433

520

Menendez, F. T.S. 335

Menendez, Sir M. R. 103

Menzies, V.M. G. 499

Mercer, E. D.
Mercer, G. H. $\quad 520$

Mercer, $\mathrm{H}$. $\quad 433$

Mercer, H. F. $\quad 16$

Mercer, J. I6, 433

Mercer, J. L. C. $\quad 208$

Mercer, W. B. $\quad$ I44

Mere, C. L. I6

Meredith, H. O. $\quad 208$

Meredith,O.W.W.H. 178

Merivale, B. $\quad 335$

Merriman, G. $\quad 499$

Merriman, H. S. $\quad 68$

Merryweather, C.W. 433

Merton, G. $\quad 433$

Messiter, C. C. $\quad 144$

Metcalf, F. W. R. I03

Metcalf, H. K. $\quad 335$

Metcalfe, C. H. F. $\quad 261$

Metcalfe, F. W. $\quad 354$

Metcalfe, G. C. $\quad 144$

Metcalfe, P K. $\quad 262$

Metcalfe-Gibson, A. 262

Metcalfe-Gibson, A. E.

Metcalfe-Gibson,

R. A.

144

B.C. 499

433

Methven, C. M. $\quad 227$

Methven, M. D. $\quad$ I44

Mews, J. K. $\quad 433$

Meyler, E. M. $\quad \mathbf{5 2 0}$

Meyrick, E.E. $\quad 433$

Meyrick, F. C. 499

Meysey-Thompson, A. de C. C.

Meysey-Thompson, E. C.

Meysey-Thompson, H. C.

433

Michell. 433

Michell, E.W. $\quad 358$

Michell, N. B. $\quad 433$

Michell, R. W. $\quad 144$

Michell-Clarke, A. J. 144

Michell-Clarke, J. I44

Middlemas, P. $\quad 262$

Middleton, H.S. $\quad \mathbf{2 8 7}$

Middleton, R. C. G. 434

Midgley, E. C. $\quad 434$

Midgley, W. A. L. $\quad$ I6

Midwood, R. $\quad 48$

Milburn, A. $\quad 434$

Milburn, A. W. $\quad 434$

Milburn, B. $\quad$ I78

Milburn, J. D. $\quad 434$

Milburn, Sir L. J. $\quad 434$

Milburn, R. G. $\quad 434$

Milburn,

W.H. (Emm.) IO3 
Milburn,W.H.(Jes.) I 78 Mildmay, F. B. 434

Miles, B. L.

Miles, C. V.

Miles, H. R.

Miles, W. H.

Miley, M.

Millais, J. G.

Millar, A. L.

Millar, G. H.

Millard, C.S.

Miller, A. G. S.

Miller, A. T.

Miller, C. A. M.

Miller, C. D.

Miller, C. $\mathrm{H}$.

Miller, C. P.

Miller, D. C.

Miller, D.O.de E.

Miller, E. C.

Miller, E. D.

Miller, $\mathrm{F}$.

Miller, G. W. M.

Miller, H. C.

Miller, H. M.

Miller, H.T.

Miller, J. C.

Miller, J. H.

Miller, J. W. E.

Miller, L. V.

Miller, O. G.

Miller, P.T.

Miller, R. B.

Miller, R. M.

Miller, R. W. R.

Miller, W. M.

Miller-Williams,E.J. 79

Milles, G.H.

Milligan, D. W.

Milligan, W.

Milliken, H. E.

Million, A. B.

Million, C. H.S.

Mills, A. E.

Mills, C. G.

Mills, E.

Mills, E. H. F.

Mills, E. J.

Mills, F. A

Mills, F. R.

Mills, H. J. F.

Mills, R. A.

Mills, T. G.

Mills, T. R.

Mills, W. G.

Millward, G. D.

Millyard, $T$.

Milman, L. C. P.

Miln, C. J.

Milne, A. A.

Milne, A. R.

287

I 44

I 44

434

434

178

499

434

434

335

434

520

48

262

48

499

103

532

532

262

434

48

434

262

520

144

262

262

103

103

262

48

68

335

335

48

434

178

354

262

434

262

79

335

354

434

434
Milne, D. D. W.

Milne, E. A.

Milne, E. O.

Milne, G.W.

262

Milne Home, C. A. $\quad 208$

Milner, D. R.

Milner, $G$.

Milner, G. R.

Milner, M. H.

Milner, P. R.

208

103

434

287

262
Milner-Barry, E. L. 144

Milner-Gibson,W.A.499

Milner-White, E. $\quad 208$

Milner-White, R. $\quad 262$

Milnes-Gaskell, E. 434

Milroy, G.W.W.W. 103

Milsom, C. F.

Milsom, H. L.

Milsom, S.

Milton, H. A.

Milvain, Sir T.

Milward, H. H.

Mirfin, J. C.

Mirrlees, A.J.

Miskin, G.

Misquith, O. G.

Mitchell, A. C. O.

Mitchell, A. G.

Mitchell A H M 354

Mitchell, A. L.

Mitchell, A. W. C. $\quad 145$

Mitchell, C. W. 103

Mitchell, E.S. $\quad 435$

Mitchell, F.

Mitchell, F. J. L. $\quad 48$

Mitchell, F. M. $\quad 48$

Mitchell, G.D. $\quad 499$

Mitchell, G. W. $\quad 48$

Mitchell, $\mathrm{H}$.

Mitchell, J. C.

Mitchell, J.R.

Mitchell, P. R.

Mitchell, S.

Mitchell, W. G.

Mitchelson, J. K.

Mitford, A. H.

178

Mitord, B. O.F. 435

ord, C. W.

Mobbs, G. F.

Moberly, A. H.

Moeran, W. G.

Moffatt, C. $\mathrm{H}$.

Moffitt, J. P.

Moggridge, C. D.

Moir, A.

Moir, K. M.

Moir, R.

103

520

48

435

178

314

178

178

178

16

208

I03

499

178

48

145

208

Molesworth, G.E.N. 301

Moline, R.W.H. 103

Moller, N. H.
Molony, A. C. B. 354

Molony, A. W. $\quad 262$

Molony, B. C. $\quad 435$

Molony, J. A. $\quad 354$

Molson, E. E. $\quad 262$

Molson, H. E. $\quad 262$

Molson, J. E. $\quad 103$

Molteno, D. J. $\quad 435$

Momber, R.M.S.T. 435

Monckton, F.H. $\quad 79$

Monckton, J. F. E. $\quad$ I45

Monckton, T.A. 435

Moncrieff, A. I6

Moncrieff, D. C. 435

Mond, F. L. 208, 287

Money, W. T. $\quad \mathrm{IO}_{3}$

Monks, F. R. $\quad 262$

Monks, G. $\quad 178$

Monkswell, Lord $\quad 435$

Monro, A. E. $\quad 335$

Monro, C. G. 145

Monro, H. E. $\quad$ I45

Monro, K. N. $\quad 208$

Monson, C. S. $\quad 227$

Montagu, G.H.S. 68

Montagu, G. W. $\quad 262$

Montagu, J. F. $\quad 499$

Montagu, J. G. E. 435

Montagu Douglas Scott, Lord H. A. $\quad 499$

Montague, P. D. $\quad 145$

Montague, W. J. $\quad 520$

Monteith, H. G. 262

Monteuuis, L. E. $\quad 500$

Montford, D. R. $\quad 262$

Montgomery, D.S. 521

Montgomery, $\mathbf{H}$. $\quad 500$

Montgomery, H. K. $\quad{ }_{17} 8$

Montgomery,H.R.G. 48

Montgomery, J. C. $\quad 52 \mathrm{I}$

Montgomery, J.K. $\quad 16$

Montgomery, N. $\quad 521$

Montgomery, W. E. 48

Moody, B. $\quad 335$

Moon, L.J. $\quad 263$

Moor, C. $\quad 263$

Moor, F. $\quad 435$

Moorcock, F. A. $\quad 103$

Moore, A.H. $\quad 435$

Moore, A. H.-G. C. 435

Moore, A. W. $\quad 103$

Moore, C. $\quad 335$

Moore, C. A. G. $\quad 521$

Moore, C. A. St G. $\quad 263$

Moore, C.D.H. $\quad 500$

Moore, C. G. H. 145

Moore, E.S. $\quad 435$

Moore, F. R. $\quad 49$

Moore, G. (Clare) 49

Moore, G. (Cath.) 314

Moore, G. A. C. 435 
Moore, G. G.

Moore, H. M. M.

Moore, J. H. E.

Moore, J.W.

Moore, J. W. B.

Moore, $\mathrm{M}$.

Moore, M. E. J.

Moore, N.

Moore, R. F.

Moore, R. M.

Moore, R. T.

Moore, W. G.

Moore-Anderson, A. $P$.

Moore-Anderson, M. E.

Moore-Brabazon, J. T. C.

Moorhouse, E. R.

Moorhouse, $\mathrm{S}$.

Moorhouse, W. B. R see Rhodes-Moorhouse, W. B.

Moorsom, A. E.

Moorsom, C. W. M. 435

Moorsom, J.

Moorsom, R.S.

Morcom, A. F.

Morcom, R. K.

More, $\mathrm{T}$.

Moreing, A. C.

Moreing, A. H.

Moreton, T.W. E.

Morgan, A. C. O.

Morgan, D. A.

Morgan, D. F.

Morgan, E. H.

Morgan, F. J.

Morgan, G. E.

$\begin{array}{lr}\text { Morgan, G. E. } & 532 \\ \text { Morgan, G.W. F. } \quad 2^{263}\end{array}$

Morgan, H. A.

208

$49^{\circ}$

435

145

435

435

145

435

79

79

103

145

79

Morgan,H.R.(Mag.) 227

Morgan,H.R.(Tr.H.) 500

Morgan, H. T.

Morgan, O. G.

Morgan, R. T.

Morgan, S. C.

Morgan, T.D.

Morgan, W.E.

Morgan, W.P.

Morgan, W. W.

Morgans, G. E.

Moriarty, G.H.

Morier, C. E.

Morison, D. R.

Morison, Sir T.

145

49

179

436

179

I03

49

500

287

145

104

49

436

Moritz, M.

104

Morland, D. M. T. $\quad 49$

Morley, B. C.

263

Morley, C. (Pemb.) 263

Morley,C.,jun.(Tr.) 436
Morley, C. C.

Morley, C. H.

Morley, Earl of

Morley, F.W.

Morley, G. C.

Morley, G.H.

Morley, J.

Morley, M. R.H.

Morrice, G.W.

Morrice, W.

Morrice, W.W.

Morris, A.

Morris, A. C. (Jesus)

Morris,A.C.(Fitz 179

Morris, C. A.

Morris, C.A.S.

Morris, F. B.

Morris, G.G. $\quad 179,436$

Morris, G.P. $\quad 16$

Morris, H. E. A. $\quad 532$

Morris, J. C.

Morris, J. $O$.

Morris, J. W.

Morris, J. W. G.

Morris, P.E.(Pet.) 287

Morris, P.E. (Joh.) 335

Morris, T.S.

Morris, W.

Morris, W. F.

Morris-Eyton, R. E. 436

Morris-Jones, J. $\quad 354$

Morrish, J.

see Murrish, J.

Morrison, A.

Morrison, D. C. A. 335

Morrison, E. $O$.

Morrison, $\mathrm{H}$.

Morrison, H. $\mathrm{H}$.

Morrison, H. N.

Morrison, J.S.F.

Morrison, J. T. J.

Morrison, M.J.

Morrison, R. G.

Morrison, R. J.A.

Morse, $C$.

Morse, C. G. H.

Morse, E. V.

Morse, F. A. V.

Morse, G. G.

Morse, L. G. E.

Morshead, O.F.

Morshead, R.S.

Mortimer, E.

. F. 500

Mortlock, C. B.

Morton, A. V.

Morton, C. E.

Morton, C. J.

Morton, E. A.

Morton, F.D.
436 Morton, H. J.S. $\quad 263$

Morton, H.S. $\quad 68,209$

500 Morton, H.T. $\quad 104$

Morton, J. A. F. $\quad 209$

Morton, J. F. A. see

Amphlett-Morton, J. F.

Morton, J. G. $\quad 532$

Morton, R.F.S. $\quad 436$

Morton, W. B. $\quad 335$

Morum, J.P. $\quad 263$

Morum, S. D. $\quad 179$

Mosely, F.M. $\quad 335$

Mosley, I. H. I7

Moss, F. W. $\quad 179$

Moss, H. K. $\quad 263$

Moss-Blundell, F. B. 436

Mosse, C. G.T. $\quad 145$

Mosse, C. H. $\quad 179$

Mosseri, F.N. $\quad 263$

Mossop, A. G. $\quad 263$

Mossop, M. C. $\quad 145$

Mossop, N.R. $\quad 145$

Mostyn-Owen, G.C. 500

Motion, D. G. I45

Motion, S. H. $\quad 145$

Mott, C. C. $\quad 52 I$

Mould, R.C. L. $\quad 104$

Moulsdale, J.R. B. 104

Moulton, H. F. $\quad 209$

Moulton, W.R.O. 209

Mounsey, J.P. $\quad 68$

Mountain, B. $\quad 354$

Mountain, $\mathrm{H}$. $\quad$ I 7

Mountain, S. W. $\quad 263$

Mountbatten,

Lord L. A. L. $\quad 228$

Mountford, A. W. $\quad 314$

Mountfort, C. C. $\quad 104$

Mourilyan, C. A. $\quad 49$

Mousley, E. O. $\quad$ IO4

Mousley, T.H. $\quad 3$ I 4

Mowatt, $\mathrm{O}$. $\quad \mathbf{1 4 5}$

Mowlam, H. J. $\quad 80$

Mowll, C.K. $\quad 146$

Mowll, G.M.

Mowll, H.W. K. $\quad 209$

Mowton, W.E. $\quad 335$

Moxey, E. R. $\quad 263$

Moxey, J. L. $\quad 263$

Moy, E.T. $\quad 500$

Moyle, T. 301

Moysey, L. $\quad 146$

Mozley, B. C. $\quad 209$

Mozley, J.H. $\quad 209$

Mudie, R. F. $\quad 209$

Muggeridge, C. E. $\quad 436$

Muggeridge, G.D. $\quad 263$

Mugliston, F. H. $\quad 263$

Muir, B.L. $\quad 436$

Muir, D. E. C. see

335 Campbell-Muir, D.E. 
Muir, D. M.

Muir, J.

Muir, J. B.

Muir, J. C.

Muir, M. W.

Muir-Mackenzie,K.J. I79

Muir-Mackenzie, Sir R. C.

Muirhead, J.A. O.

Muirhead, M.

Mules, F. J.

Mulholland, G. J. A. M. L. 436

Mulholland,H.G.H. 436

Mulholland, W. $\quad 336$

Mullens, C. J.A. $\quad 436$

Muller, H. C. A. S. 104

Muller, J.S. $\quad 336$

Mullins, A. F. 314

Mullins, C. de C. C. 354

Mumford, C. G. $\quad 49$

Mumford, P.S. $\quad 179$

Mummery, R. T. $\quad 209$

Muncey, A. P. $\quad 532$

Muncey, R. W. L. $\quad 500$

Muncey, S. W. U. $\quad 532$

Munday, E.

Munro, D.

Munro, R. M. C.

Muntz, A. I.

Murdock, A. J.

Murly-Gotto, J.

Murphy, J. K.

Murphy, W. L.

Murray, A. G.W.

Murray, A. R.

Murray, C. M.

Murray, C. W.

Murray, D. C. L.

Murray, D.W.

Murray, E.

Murray, E. G. D.

Murray, E. T.

Murray. G. A.

Murray G. R.

Murray, J. C.

Murray, J.S.

Murray, K. R.

Murray, M. A.

Murray, M. G. D.

Murray, P. J.

Murray, R. B.

Murray, R. H.

Murray, R. L.

Murray, T. H. E.

Murray-Aynsley,

$$
\text { C. } M \text {. }
$$

Murray-Johnson, F. K.

49

532

49

437

49

263

I 46

336

437

264

264

437

68

I04

287

17

17

437

437

437

437

228

I79

437

437

437

521

I79

437

336

Murray Smith, A. G. 437

Murray Smith, G. $\quad 500$
Murray Smith, J. E. 228

Murrish, J.

Murton, E.

Musgrave, $T$.

Muspratt, P. K.

Musson, F. W.

Musson, T. M. B.

Myddelton, E. G.

Myers, C.S.

Myles, D.

Myles, W.W.

Mylrea, W.P. G.

Nadin, $T$.

Nainby-Luxmore, C. C.

Nairn, R.S.

Naisby, J. V.

Naish, A. E.

Naish, F. C.P.

Naish, W. V.

Nalder, F. W.

Naldrett, H. C.

Nancarrow, J. V.

Nangle, E. J.

Naoroji, K. A. D.

Napier, G. G.

Napier, Sir J. W. L.

Napier, L. P.

Napier, O. J. W.

Napier, Sir W. L.

Napier-Clavering, H. P.

Narayan, Maharaj K. H.

Nash, G.

Nash, J. V.

Nash, W. W. H.

Nash-Wortham, F. L. D.

Nason, J. W. W.

Naters, C. C. T.

Nathan, A. A.

Nathan, E. J.

Nathan, J.

Nathan, L. C.

Nathan, S. H.

Naumann, J. H.

Nawanagar, Jam of

Nayler, W. A.

Naylor, A. T. A.

Naylor, J. M.

Naylor, P. A. E.

Naylor, T. H.

Neal, A. W.

Neal, J.

Neal, $R$.

Neale, A. K. H.

Neale, F.S.

Neale, J. B.
532

437

228

17

104

179

I 79

I 46

104

I 46

I 46

264

500

500

I04

437

354

104

I79

179

209

I 46

I 7

264

I79

209

I 7

I 79

437

264

228

437

52 I

264

301

IO4

I 79

209

49

500

437

209

437

437

$\mathrm{I} 04$

437

264

437

209

209

532

437

69

179
Neame, $T$.

146

Neate, N. R.

Neave, Sir T. L. H. $\quad 500$

Need, G. S. $\quad 336$

Needham, E. $\quad 17$

Negroponte, J. J. $\quad 437$

Neighbour, P. M. $\quad 354$

Neild, W. C.

Neill, N. C.

Neilson, D.F. A

Neilson, G. E.

Neilson, H. J. T. $\quad 438$

Neilson, H. V. $\quad \mathbf{1 4 6}$

Neilson, W.

Nelder, G. C. A. $\quad 49$

Nelson, E. B. $\quad 69$

Nelson, E. W. $\quad 17$

Nelson, R. D. $\quad 17$

Nelson, T. B. 17

Nelson, W. $\quad 146$

Nesbitt, W. H. B.

see Beaumont-

Nesbitt, W. H.

Nesham, C. F.

Nesham, R. A.

Ness, G.S.

Ness Walker, J.

Nethersole, F. R.

Nettlefold, E. J.

Nettlefold, H. J.

Nevett, R. B.

Nevile, B. P.

Nevile, H. G.

Nevill, W. P.

Neville, A. G.

Neville, E. G. M.

Neville, G. J. E.

104

Neville, H. S. $\quad 228$

Neville, M. R. $\quad 287$

Neville, R. $\quad 438$

Nevinson, G. R. G. $\quad 69$

New, F. O. W. $\quad 104$

New, T. G. $\quad 264$

Newall, J. H. M. $\quad 43^{8}$

Newberry, E. E. $\quad 500$

Newberry, J. D. 209

Newbery, R. E. $\quad 336$

Newbold, C. J. $\quad 146$

Newborough, Lord 500

Newcomb, W. D. $\quad 438$

Newcombe, H. W. $\quad 438$

Newcombe, W. A. $\quad 438$

Newcombe, W. L. $\quad 521$

Newell, H. W. $\quad 532$

Newham, C. E. $\quad$ I80

Newington, H. A. H. 146

Newling, A. J. $\quad 180$

Newling, S. W. $\quad 336$

Newman, C. C. $\quad 500$

Newman, E.

I7 
Newman, E. A. R.

Newman, E. D.

Newman, F. C.

Newman, F. H. C.

Newman, J. B.

Newman,J.C.(Mag.) 228

Newman,J.C.(Trin.) 438

Newman, J. C. F. $\quad 521$

Newman, J. R. B. $\quad 438$

Newman, J.S.

Newman, L. F.

Newmarch, J. G.

Newell, A.

Newnes, Sir F. H.

Newport, $\mathrm{H}$.

Newport, Viscount

Newson, W. A.

Newton, C. H. see Herdman-Newton, $C$.

Newton, G. F.

Newton, H. A.

Newton, H. G. T.

Newton, H. W. G. $\quad 209$

Newton, R. H. H. see

Herdman-Newton,R.H.

Newton, T. H.

104

Newton, W. H.

104

Newton Clare, E. T. 49

Newton Clare, H. J. 49

Newton Clare, W.S. 4.9

Nibbs, C. A. J. $\quad 301$

Nichol, R. W. $\quad 146$

Nicholas, A. J.

Nicholas, F.

Nicholas, F.P.

Nicholas, H. C.

Nicholas, T. C.

Nicholas, T. C. $\quad 438$

Nicholl, J. W. McK. 301

Nicholl, $\mathrm{V}$.

Nicholls, A. C.

Nicholls, G. B.

Nicholls, G. S.

Nicholls, L.

Nicholls, S. H.

Nichols, C. W.

Nichols, F.P.

Nichols, W. H.

Nicholson, A. F.

Nicholson, B. D.

Nicholson, C. J.

17

104

180

438

264

336

301

80

80

146

104

314

180

438

438

146

Nicholson, C. O. E. 438

Nicholson, G. B. $\quad 49$

Nicholson, G.C. N. 49

Nicholson, H. H. $\quad 52 I$

Nicholson, H. J. $\quad 438$

Nicholson, J. E.

Nicholson, $M$.

Nicholson, O. W.

Nicholson, P. C.

Nicholson, R.
Nicholson, R.S.

Nicholson, W.N.

Nickal, G. B.

Nickels, R. N.

Nicklin, G. N.

Nicol, J.

Nicoll, H. M. D.

Nicolls, G. E.

Nicolson, L. G.

Nielsen, E. E. M.

Nightingale, A. D.

Nightingale, C. L.

Nightingale, D. A.

Nightingale, H.P.

Nihill, J.H. B.

Nisbet, A. T.

Niven, E. O.

Nix, C. G. A.

Nix, P. K.

Nixon, B. $H$.

Nixon, J. A.

Nixon, W. H.

Nobbs, S. W.

Noble, A. H. (Clare)

Noble A $\mathrm{H}$ (Qu)

Noble, E. R.

Noble, H. B.

Noble, R:H.

Noel, R. E. T. M. 439

Norbury, C. G.

Norbury, F. C.

Norby, R. H.

Norden, F.L.

Norman, A. C.

Norman, $C$.

Norman, C. L.

Norman, C. W.

Norman, D. T.

Norman, J.

Norman, N.F.

Norman $R$.

Norman, $\mathrm{T}$.

Norman, W. H.

Norman-Lee,F.B.N. 336

$\begin{array}{ll}\text { Norquoy, F. . H. } & 105 \\ 336\end{array}$

Norris, C. G.

Norris, E. W. M.

Norris, W. F.

Norris, W.H.H.

North, G. D.

North, J.

North, J. B.

North, J. F. A.

North, W. G. B.

Northcote, D.S.

Northcote, T. F.

Northcott, J. F.

228 Northcott, W. W.

438 Northen, E.

438 Northen, F.
49
438

17
146

336

438

264

80

438

301

438

17
104

69

264
438

264

439
146

17

105

301

354

209

439

336

314

301

336

49

439

439

105

105

50

180

314

521

439

69

180

301

287

80

146

439

105

336

439

439

439
Northfield, H. D.

287

Northorp, F. $\quad 336$

Northrop, J. E. $\quad 50$

Norton, D. G. $\quad 146$

Norton, E. H.P. $\quad 146$

Norton, G.P. $\quad 146$

Norton, H. E. $\quad 439$

Norton, L. M. 287, 521

Norton, R. H. $\quad 209$

Norton, V. E. B. $\quad 105$

Norton-Fagge,

F.W.L. 532

Nosworthy, J. L. $\quad 521$

Nosworthy, P.C. 30I

Nott, H. P. $\quad 105$

Nott, L. C. $\quad 105$

Nott, T. W. $\quad 105$

Nowell-Rostron, S. 336

Nowell-Usticke, G.W. 180

Noyes, H. F. G. $\quad 147$

Noyes, J. C. $\quad 50$

Nunn, V. W. H. 30r

Nurse, H.H. $\quad 336$

Nutman, B. K. $\quad$ I 80

Nuttall, E. D. $\quad$ I7

Nuttall, W. L. F. $\quad 17$

Nutting, A. R.S. $\quad 501$

Oakden, W. M. $\quad 287$

Oakes, M. W. $\quad 105$

Oakey, J. M. $\quad 439$

Oakshott, T.A. $\quad 439$

Oaten, E. F. $\quad 354$

Oaten, W.S. $\quad 354$

Oates, B. W. G. $\quad 439$

Oates, J. S. C. $\quad 439$

Oatfield, W. J. $\quad 209$

Oats, G. $\quad 439$

Oats, W. $\quad 147$

O'Brien, J. C. P. $\quad 147$

O'Callaghan, T.F. 209

O'Connell, J. B. $\quad 532$

O'Connor, A. C. $\quad 439$

O'Connor, J. 314

Odell, R. E.

Odgers, F. W. $\quad 439$

Odgers, L. N. B. $\quad 336$

Odgers, R. B. $\quad 336$

Odling, F. C. $\quad 50$

O'Driscoll, P.F. $\quad 439$

O'Ferrall, C. L. $\quad 439$

O'Flynn, D.R.C.D. 301

Ogden, H. $\quad 105$

Ogilvie, A. $\quad 439$

Ogilvie, G. L. $\quad 439$

Ogilvie, $\mathrm{H}$. 2 IO

Ogilvie, J. I7

Ogilvie, N.

Ogilvie, P. G. $\quad 264$

Ogilvy, L.W. $\quad 2$ ro

Ogle, K.W.S. $\quad 521$

Oglethorpe, H. C. $\quad 264$ 
O'Hagan, Lord

Oke, R. W. L.

Okell, C. C.

Oldfield, G. E. B

Oldfield, H. D.

Oldfield, R. W.

Oldham, E. A. S.

Oldham, R. S.

Oldham, T. V.

Oldnall, R. W.

Oldroyd, F. N.

Oliphant, G. R.

Oliphant, J.

Olive, G. W.

Oliver, E. G.

Oliver, F.J.

Oliver, F. R.

Oliver, G. Y.

Oliver, H. C.

Oliver, H. G.

Oliver, L. G.

Oliver, L. W.

Oliver, M. W. B.

Oliver, R. E. C.

Oliver, T. F.

Oliver, T. H.

Oliver, W. S. V.

Oliver-Jones, W. S.

Olivier, E.

Ollard, J. W. A.

Ollerenshaw, F.

Ollivant, R. C

Olphert, R. A.

O'Neal, T. W. B.

O'Neill, F. R.

Onslow, J. W. M.

see MacarthurOnslow, J. W.

Ontanon, C.

Onyon, R. R.

Oon Israsena,

Oon Israsena, M. L. 287

Openshaw, C. G. $\quad 440$

Opie, P. A.

I 47

Oppenheim, L.C.F. $44^{\circ}$

Oppenheim, W.

Oppenheimer, K. M. I 80

Orchard, S.

Ord, O. R.

Orde, S. E. H.

210

265

Orde-Powlett, W. P. 440

O'Reilly, H. D. R. 80

O'Reilly, J. B.

Orford, W. K.

Orgill, T.C.

Oriel, T. H. B.

O'Riordan, P. M.

Orlebar, J. A. A.

Orlebar, R. A. B.

Orme, B. C.

Orme, E. S.

Orme, F. R.

439

336

69

264

105

532

50

301

105

80

3 I 4

147
228

I 80

147

521

439

464

47

50
54

801

228

440

501

354

80

$44^{\circ}$

440

533

228

265

521

301

228

9

Owen, G.
Owen, G. C.

\section{Owen, $\mathrm{H}$.}

Owen, H. B.

Owen, P. R. T.

Owen, S. A.

Owen, S. H. E. G.

Owen, $\mathrm{T}$.

Owen, W. H. K.

Owens, F. H.

Owst, G. R.

Owston, C.S.

Oxley, A. E.
Packard, H. N.

Packe, E. C.

Padwick, H. B.

Page, C. A.

Page, C. C.

Page, C. F. G.

Page, C. G.
$336 \mid$ Page, C. H. W.

265

501

440

80

I7

265

521

Page, D. S.

Page, F. A.

Page, F. G. J.

Page, $J$.

Page, R. B.

Page, S. W.

Page, W. E.

Page Croft, $\mathrm{H}$. see Croft, H. P.

265

Page Croft, $R$.

see Croft, R. P.

50

Paget, C. W.

Paget, G. N.

Paget, H. E. G.

265

440

180

265

440

Paget, J. B.

Paget, O. F.

Paget Tomlinson, T. R.

Paget Tomlinson, W. G.

Paige, J. F.

Pain, B. $H$.

Pain, E. D.

Pain, J. W.

Pain, K. W.

Paine, B.W. F.

Paine, H. H. $\quad 440$

Paine, W. A. $\quad 440$

Paine, W. L. $\quad 355$

Painter, H. S. $\quad 52 \mathrm{I}$

Pakeman, S. A. $\quad 355$

Pallis, A. A.

Palliser, W. F.

Palmer, A S M

Palmer, B. H. $\quad 265$

Palmer, C. $\quad 50$

Palmer, C. E. $\quad 147$

Palmer, E. de S. 314

Palmer, Sir E. G. B. 228

Palmer, F. W. M. $\quad$ I 80

Palmer, G. F.N. $\quad 44^{\circ}$

Palmer, H. G. $\quad 440$

Palmer, K. R. $\quad 440$

Palmer, N. St C. $\quad 50$

Palmer, S. $\quad 69$

Palmer, T. N.P. $\quad 337$

Palmer, W. E. $\quad 337$

Palmer, W. H. $\quad 355$

Pam, E. A. $\quad 50$

Pank, P. E. D. $\quad 147$

Panter, A. E. $\quad 50$

Pape, A. G. $\quad 228$

Paradise, J. R. T. $\quad 265$

Parbury, F. C. S. $\quad 440$

Pares, B. $\quad 105$

Parez, C. C. T. $\quad 50$

Pargeter, H. A. A. 355

Parham, H. J. $\quad 501$

\begin{tabular}{l|ll}
265 & Parish, A. J. & 287 \\
50I & Parish, G. W. & 147
\end{tabular} 
Parish, W. O. (Cath.) 314

Parish, W. O.(Pet.) 287

Parke, A.

Parker, C.

Parker, C. L. Y.

Parker, F. A.

Parker, F. B.

Parker, G.

Parker, G. D. S.

Parker, G. M.

Parker, G. W. T.

Parker, H. C. T.

\section{0}

314

265

$44^{\circ}$

440

337

$44^{\circ}$

105

440

501

Parker,H.E.(Pemb.) 265

Parker,H.E.(Trin.) $44^{\circ}$

Parker, H. L.

Parker, H. N.

Parker, H. V.

Parker, J. C.

Parker, J. D.

Parker, J. H.

Parker, L. H.

Parker, P. H.

Parker, R. D.

Parker, R. H.

Parker, T. W. L.

Parker, W. D.

Parker, W. H.

Parker, W. S. H.

Parker-Jervis, G

Parker Smith, A. C. $\mathbf{H}$.

Parker Smith, R.

\section{9}

314

180

18

265

44I

5 or

3 or

147

$44 \mathrm{I}$

301

228

$44 \mathrm{I}$

228

228

2 ro

314

Parker Smith, W. B. 2 ro

Parkes, E. B. H.

Parkin, J.

Parkin, R. C.

Parkin, R. T.

Parkins, F. D.

Parkinson, G. R.

Parkinson, $W$.

Parmenter G.

Parmiter, C. L. $\quad 265$

Parnell, $\mathrm{H}$.

Parnell-Smith, W.

Parrington, N.

Parrington, W. F.

Parry, A. H.

Parry, D. B.

Parry, F. A.

Parry, D. G. de C.

Parry, J. H.

Parry, N.C.

Parry, N. E.

Parry, R. B.

Parry, T. H.

Parry, T.J.

180

441

50

50

44I

228

287

301

50

50

50

$5 \mathrm{I}$

$5 \mathrm{I}$

441

501

337

$44 \mathrm{r}$

$44 \mathrm{r}$

501

18

Parry-Jones, M. M.

105

Parry-Jones, P. E. H. 265

Parsons, Sir C. A. $\quad 337$

Parsons, D. C.

Parsons, F.P.N
Parsons, $\mathrm{H}$.

Parsons, J. R.

Parsons, R. E.

Parsons, R. E. $\quad$ 44I

Part, G. M.

Partington, E. F E

Partington, J. B. $\quad 265$

Parton, E. G.

Partridge, $C$.

Partridge, G. J.

Partridge, H. W.

Partridge, J. W.

Partridge, R. H.

Partridge, W. W.

Paschkoff, A. A.

Paschkoff, A. B.

Pascoe, E. H.

Pascoe, F. J.

Paskin, J. J.

Pass, A. D.

Pass, H. L.

Passant, E. J.

Passingham, E. G.

Pasteur, F. M.

Patch, N. J.S.

Paterson, A. G.

Paterson, A. R.

Paterson, E. W.

Paterson, G. A. R.

Paterson, G. McL.

Paterson, $\mathrm{H}$.

Paterson, I. R.

Paterson, J. J.

Paterson, $\mathrm{K}$.

Paterson, M. W.

Paterson, R. D.

Paterson, R. J.

Paterson, T. W.S.

Paterson, W. H.

Paton, E. R.

Paton, F. A. R.

Paton, J. D.

Paton, J. E.

Paton, R. Y.

Paton, W.

Paton Smith, E. A.

Patrick, C. V.

Patrick, N.C.

Patron, J. A.

Patterson, L.

Patterson, R. A.

Patterson, R. F.

Patteson, C. (Corp.)

Patteson, C.(Pemb.)

Patteson, J. D.

Pattinson, L. A.

Paul, $\mathrm{H}$.

Paul, J. W. E.

Paul, R.

Paul, Sir R. J.

\begin{tabular}{l|l} 
44I & Paul, Sir R. \\
265 & Paulley, H.
\end{tabular}
147

441

$44 I$

5 I

I 8

3or

210

301

265

301

441

$44 I$

337

337

337

210

337

80

441

69

302

106

501

180

266

355

2 IO

$44 I$

5 I

337

5 I

441

I 47

229

266

266

441

266

441

5 I

$44 I$

I 47

I8

441

$44 I$

I 80

337

69

266

441

180

147

266

$5 \mathrm{I}$

441

337

Pauncefort Duncombe, Sir E.P. D. $\quad 44^{1}$

Pavey Smith, A. B. $5 \mathrm{I}$

Pawle, H. $\quad 147$

Pawle, J. 44I

Payne, A. A.

seeLaportePayne,A.A.

Payne, D. N. $\quad 106$

Payne, F. R. $\quad 287$

Payne, G. H. I8I

Payne, J. H. A. $\quad 501$

Payne, J. W. $\quad 106$

Payne, M. G. J. 521

Payne, M. W. 44I

Payne Gallwey, L. P. 442

Payton, R.S. $\quad 18$

Payton, W.H. $\quad 442$

Peabody, M.E. $\quad 442$

Peace, L. R. $\quad 302$

Peacey, C. C. $5 \mathrm{I}$

Peacey, H. M. 2 ro

Peache, R. C. $\quad 442$

Peacock, F. W. $\quad$ Io6

Peacock, G. 5 I

Peacock, G. H. 5i

Peacock, J. $\quad 442$

Peacock, L. V. $\quad 533$

Peacock, M. R. $\quad 442$

Peacock, W. M. I8I

Peacock, W.T. $\quad$ I8I

Pead, J.H. $\quad 80,266$

Peak, N. $\quad 521$

Peake, C. G. W. $\quad 501$

Peake, E. G. $5 \mathrm{I}$

Peake, G.H. 50

Peake, S. C. $\quad 355$

Pearce, A. H. $\quad 5 \mathrm{I}$

Pearce, E. H. $\quad 287$

Pearce, R.S. $\quad 442$

Pearce, T. $\quad$ I06

Pearce-Serocold, O. 442

Peard, C. J. $\quad 106$

Peard, J. C. N. $\quad$ I06

Pearkes, A. M. $\quad 147$

Pearless, H. N. $5 \mathrm{I}$

Pearman, J. O'H. $\quad 147$

Pearman-Smith,P.B. 106

Pears, $\mathbf{R}$.

Pearsall, H. G. $\quad 147$

Pearsall, R. H. 2 ro

Pearson, A. C. $\quad 355$

Pearson, A. G. $\quad 442$

Pearson, A. H. $\quad 302$

Pearson, B. C. $\quad 442$

Pearson, B. L. $\quad 355$

Pearson, C. E. $\quad 337$

Pearson, C. H. M. 302

Pearson, C. McM. 266

Pearson, D. G. $\quad 266$ 
Pearson, J.S.

Pearson, J. W.

Pearson, $\mathrm{L}$.

Pearson, L. H.

Pearson, R.S.

Pearson, T.A.

Pease, C. E.

Pease, E.

Pease, E. H.

Pease, M.R.

Pease, N. A.

Pease, $\mathrm{R}$.

Pease, R. A.

Peck, A.H.

Peck, E. G.

Peck, E.S. (Christ's) 18

Peck, E.S.(Fitz.H.) 533

Peck J.N.

Peck, S. C.

Peckham, W. D.

Peckover, H. D.

Pedley, C. F.

Peed, S. W.

Peek, B. M.

Peek, Sir W.

Peel, A. W.

Peel, H. D.

Peel, W. H. G.

Peel, W. R.

Peers, E. A.

Pegg, H. E.

Pegg, H. G.

Pegg, J.

Pegge, A. V.

Peile, J.

Pelham, M.H.

Pelham, S. G.

Pelham, W. H.

Pelham-Clinton,G.E. 442

Pellier, C. du C. 5 I

Pelling, S. B.

Pellow, J. E.

Pelly, A. R.

Pelly, D. R.

Pelly, E.

Pelly, E. G.

Pelly, E.P. L.

Pelly, F. B.

Pelly, R. A.

Pelly, R. L.

Pemberton, F. P. C. 443

Pemberton, J.

Pemberton, V. T

$\begin{array}{ll}\text { Pemberton, V.T. } & 355 \\ \text { Pemberton, W. H. } & 266\end{array}$

Pendered, J. $\mathrm{H}$.

$\begin{array}{ll}\text { Pendered, J.H. H. } & \text { I } 47 \\ \text { Pendlebury, H.S. } & 266\end{array}$

Penfold, H. L.

Penistan, E. J.

Penn, A.H.

Penn, E. F.

Penn, G. M.
442

106

106

442

442

442

442

I8I

442

5 OI

442

533
147

355

442

80
18

266

229

442

533

I06

229

442

533

I8I

$5 \mathrm{I}$

147

I8

442

442

442

5 I

337

442

106

266

442

442

442

442

5 I

51

337

5 I

443

443

443
Pennell, K. E. L.

Pennell, V.C.

Pennell, V.H.

Pennington,C.G.T.S.

Penny, A. O. V.

Penny, C.J.

Penny, G.S.

Penny, W. M.

Pennyman, J. B. W.

Pennyman, J. B. W. 443

Penrose, E. J: McN. 443

Penrose, J. D.

Penrose Fitzgerald, M. J.

Pentland, G. C. C. $\quad 148$

Penton, C. F

Penzer, N.M.

Pepin, A. R.

Peploe, $K$.

Percival, A. F.

Percival, H. F.

Percy, J.R.

Perkin, A. L. D.

Perkins, A. B.

Perkins, B. M.N.

Perkins, C. $\mathrm{H}$.

Perkins, F. A.

Perkins, F. H.

Perkins, F. L.

Perkins, J. J.

Perkins, J. S. S. see Steele-Perkins, J. S. S.

Perks, J. N.R.

Perks, W.L.

Perowne, J.T. W.

Perrens, C. N.T.

Perrin, E. C.

Perrin, $M$.

Perrin, M.N.

Perrin, W.S.

Perrins, C.F.D. $\quad 229$

Perrins, M. D.

Perrott, G. F. D.

Perry, C. G.

Perry, E.W.

Perry, E. W. C.

Perry, G. A.

Perry, H. C.

Perry, J. C.

Perry, L. B.

Perry, W.A. C.

Pershouse, F.S.

Persse, C. de B. G.

Perth (Australia), Archbp. of

Peshall, C. J. E.

Peshall, S. F.

Peskett, J. C.

Petch, D. B.

Peters, A.

Peters, A. G.

Peters, A. J.
Peters, A. N. G. 266

Peters, B. A. I. I8I

Peters, E. A.

Peters, J. H. G.

148

I8I

Peters, M. W.

Peters, R. A.

Peters, W. J.

18

148

, G. G. 443

C. $\quad 443$

Petherick, M. $\quad 443$

Petrie, A.

Petro, J. A. W. I8I

Pettinger, J. W. $\quad 522$

Pettit, J. R. $\quad$ I8

Pettman, $\mathrm{H}$. $\quad 302$

Petty, M. J. $\quad 80$

Petty, W.

Peyton-Burbery, R. J.P.

444

Phayre, Sir A. I8I

Phear, A. G. $\quad 444$

Phear, H.W. $\quad 148$

Phelps, G. I. de B. 5 I

Phelps, H. M.P. $\quad 444$

Phelps, M.N. I8I

Phelps, P. B. $\quad 80$

Phelps, T.T. $\quad 444$

Philipps, G.W.F. $\quad 502$

Philipps, H. E. E. $\quad 502$

Philipson, R. $\quad 444$

Philipson-Stow, R. M.P.

Phillipps, F. A. $\quad 444$

Phillipps, R.W. $\quad 229$

Phillips, C. K. $\quad 314$

Phillips, D. I.W. - 80

Phillips, D. M.P.

see Scott-Phillips, D. M.P.

Phillips, E.S. (Chr.) I8

Phillips,E.S.(Pemb.) 266

Phillips, G. W. $\quad 444$

Phillips, H. E. $\quad 337$

Phillips, I. A. $\quad 3$ I 4

Phillips, J. E. I8

Phillips, L. C. P. $\quad 148$

Phillips, L. C. W. $\quad 444$

Phillips, N. McG. $\quad 502$

Phillips, P. 106

Phillips, P. R. O'R. 266

Phillips, R. N. $\quad 444$

Phillips, R.S. $\quad 337$

Phillips, T. $\quad 266$

Phillips, W. D. $\quad 80$

Phillips, W.M. $\quad 502$

Phillips, W. R. $\quad 337$

Phillipson, E. $\quad 288$

Phillp, J. de R. $\quad 302$

Philp, C. H. G.

337 
Philp, F. E. L.

Philpot, A. J.

Philpot, F. H.

Philpot, W.

Phipps, C. B. H.

Phipps, C. E.

Phipps, W. T.

Pickard, H. G.

Pickard, W. B.

Pickard-Cambridge, H. E. W.

Pickering, B. M.

Pickett, A. C.

Pickles, A.

Pickthorn,

K.W.M. 69,444

Picton-Warlow, A. J. I 48

Picton-Warlow,F.T. 106

Piercy, H. M. M. $\quad 522$

Pierson, L. D.

Pigeon, H. R.

Pigeon, H. W.

Pigeon, J. W.

Pigg, B. W.

Pigg, C. $\mathrm{H}$.

Piggot, A. A.

Piggott, F. C. H.

Piggott, J. C. C.

Piggott, J. I.

Pigott, G. G. C.

Pigou, H. La T.

Pike, G.

Pike, H.H.

Pike, S. A.

Pilcher, E. M.

Pilcher, G. St C.

Pilcher, W.H.

Pilditch, P. H.

Pilkington, D. F.

Pilkington, D. H.

Pilkington, E. F.

Pilkington, F. C.

Pilkington, F. M. F.

Pilkington, G. R.

Pilkington, H. B.

Pilkington, L. E.

Pilkington, W. N.

Pilleau, A. L.

Pilter, C.

Pilter, R.

Pim, F.H.

Pinches, E. L.

Pinches, H. I.

Pinder, A. H.

Pine-Cofin, $\mathrm{J}$.

Pinfield, G. V.

Pinfold, E. S.

Pink, H.S.

Pinkerton, J.

Pinkham, C.

Pinks, E. D. P.
106

106

337

444

502

181

106

533

302

\section{8}

148

148

444

69

444

18

I8I

181

266

106

444

266

502

266

444

148

444

5 I

444

266

266

444

267

444

502

444

444

444

210

444

502

210

106

18

522

355

302

$5 \mathrm{I}$

288

I8I

106

I 48

5 I
Pinsent, G. H.S.

Pinto-Leite, H. M.

Piper, J. H.

Pirkis, F. C. L.

Pirkis, G. C. L. M.

Pite, A. G.

Pite, I. B.

Pither, F. E. L.

Pitt, G. Newton

Pitt, P.S.

Pitt, R. B.

Pitt, T. G.

Pitt, W. J.

Pitts, B. T.

Pitts-Tucker, G.S.

Plaister, A. J.

Plant, A. W.

Plaskitt, G. M.

Platt, A. H.

Platt, E!'A.

Platt, M. C.

Platt, R. C.

. 445

Platts, A. L.

Platts, S. G.

Player, E. N.

Player, G.

Player, $\mathrm{H}$.

Playfair, K.

Playfair, P. L.

Playne, B. A.

Playne, H. F.

Plews, H. W.

Plowden, H. R.

Plowden-Wardlaw, J. T.

Plowman, C. H.

Plowright, C. T. M.

Plummer, W. F. I8I

Plumptre, B.P. $\quad 107$

Plumptre, E. A. W. 267

Pochin, A. C.

Pochin, $\mathrm{H}$.

Pochin, V.R.

Pocock, S. E.

Pocock, W. A.

Poignand, C. W.

Poignand, G. C.I.

Pointer, E. H.

Polack, A. I.

Polack, B. J.

Polack, E. E.

Polhill, C. C.

Polhill, S. F. P.

Pollak, H. L.

Pollak, L. A.

Pollard, A. R.

Pollard, H. J. A.

Pollard, S.

Pollard, W. M. N.

Pollitt, H. C.
444

267

18

267

444

18

51

444

5 I

80

106

444

229

288

148

444

445

445

148

148

533
106

$5 I$
I

210

267

267

52

52

210

522

337

07
267

210

522

445

210

267

148

69

355

337

210

337

445

181

148

210

445

52

445

337

445

Pollock, C. T. A.

445

Pollock, H. H.

445

Pollock, Hamilton R. 445

Pollock, Humphrey R. 445

Pollock, H. W. $\quad 445$

Pollock, M. V. $\quad 445$

Pollock, R. C. G. $\quad 445$

Pollok, A. B. $\quad 229$

Polwarth, Lord $\quad 445$

Ponder, C. W. $\quad 107$

Ponsonby, B. B. $\quad 445$

Ponsonby, C. T. $\quad 267$

Ponsonby, S. G. $\quad 445$

Pook, J. de C. $\quad 69$

Poole, A. L. $\quad 522$

Poole, C. A. $\quad 522$

Poole, E. F. $\quad 302$

Poole, F.S. $\quad 355$

Poole, $\mathrm{H}$. $\quad 355$

Poole, J. T. $\quad 337$

Poole, R. E.S. $\quad 267$

Pooley, E. H. $\quad 267$

Pooley, G. H. $\quad 148$

Pooley, J.S. $\quad 445$

Poore, W. G. $\quad 445$

Pope, A. R. $\quad 445$

Pope, C. $\quad 445$

Pope, C. A. W. $\quad 445$

Pope, H. B. $\quad 148$

Pope, L. K. $\quad 355$

Pope, P. M. $\quad 229$

Pope, S. B. I8

Popham, A.E. 210

Porritt, R. N. $\quad 148$

Porteous, N.W. $\quad 80$

Porter, A. G. $\quad 267$

Porter, A. R.Z. $\quad 445$

Porter, C. E. V. $\quad 148$

Porter, E. J. $\quad 445$

Porter, G.H. $\quad$ I 48

Porter, J. E. $\quad 148$

Porter, R.E. $\quad 18$

Porter, S. L. $\quad 107$

Porter, W.N. $\quad 149$

Postlethwaite, J. M. 107

Potter, A. R. $\quad 107$

Potter, C. G. $\quad 337$

Potter, W. M. $\quad 52$

Potter, W. N. $\quad 267$

Potts, F. A. $\quad 502$

Potts, J. L. $\quad 149$

Poulett, Earl $\quad 502$

Pound, M. S. $\quad 267$

Powel Smith, L. J. $\quad 149$

Powell, A. G. C. $\quad 522$

Powell, A. T. $\quad 445$

Powell, D.W. $\quad$ I07

Powell, E. C. $\quad 337$ 
Powell, G. G.

Powell, H. M.

Powell, J. M.S.

Powell, K.

Powell, L. M.

Powell, M. B.

Powell, P.

Powell, R. C. ff.

Powell, R. H.

Powell, R. R.

Powell, R. V.

Powell, T. F.

Powell, V.P.

Powell-Price, E.

Powell-Price, J. C.

Power, A. G.

Power, J. W.

Power-Clutterbuck, J.E.

Powers, C.

Pownall, J. C. G.

Powney, C. du P.P. 446

Poynder, E. G.T. $\quad 52$

Poyser, A. V.

Prall, S. R.

Pralle, E. L. R.

Prance, E. R.

Prankerd, J.

Prankerd, R. P.

Pratt, G. C.

Pratt, G. W.

Precious, C. M.

Preddy, E. F.S.

Preece, C. T.

Preece, P. J.

Preedy, A.

Preeston, R. S.

Prentice, T. N.R.

Prest, H E. W.

Prestige, A. R.

Preston, E. W.

Preston, R. W. D.

Pretor-Pinney, C. F. 446

Pretty, K.

Prevost, P. G.

Price, A. F. M.

Price, C. L.

Price, J. D.

Price, McC. E.

Price, W. R.

Prichard, A. A.

Prichard, A. I.

Prichard, H. C.

Prichard, R. G.

Prichard, R. M.

Prichard, W. A.

Priddle, A. E.

Prideaux, H.S.

Pridham, C. F.

Priest, R. C.

Priestland, J. F. E.
446

446

I07

446

446

446

3r 4

522

107

446

\section{2}

446

446

52
229

107

338

52

522

52

502

338

338

314

446

446

$18 \mathrm{I}$

52

522

267

I49

267

107

2 I I

80

80

302

446

446

446

52

302

229

52

338

446

I 49

338

149

I 49

522
Priestley, D. R. O. Priestley, H. W.

Priestley, R. E.

Prince, A. L.

Prince, G. W.

Pring, B. C.

Pring, J. G.

Pringle, A. S.

Pringle, J. S.

Pringle, W. R.

Prinsep, A. L.

Prior, A. V.

Prior, B. C.

Prior, C. B.

Prior, C. E.

Prior, H. A.S.

Prior, H. B.

Prior, H. C.

Prior-Wandesforde, R. H.

Prissick, C.

Pritchard, F. C.

Pritchard, J. E. M.

Pritchard, N. P.

Pritchard, R.

Prittie, H. C. O'C.

Procter, J. A.

Procter, R.

Procter, R. A. W.

Procter, R. F.

Procter, R. G.

Proctor, S. H. A. R. see

Rathbone-Proctor,S.H.

Protheroe, A. H. $\quad 502$

Prust, T. W.

Pryce, E. O.

Pryce-Jones, A. W.

Pryce-Jones, Sir E.

Pryce-Jones, H. M.

Pryce-Jones, P. V.

Pryde, A. M.

Pryke, W. W.

Prynne, E. G. F.

Prynne N.F.

Pryor, G. D.

Pryor, G. H. D.

Pryor, J. W.

Pryor, N.S.

Pryor, R.S.

Pryor, W. M.

Puckle, F. H.

Puckle, J.

Puddicombe, D.R. $\quad 338$

Pudumjee, N.S.

Pugh, C. D. O.

Pugh, F. H.

Pughe, E. B.

Pulliblank, J.

Pullin, J. H.

Pulling, C. R. D.
267

446

19

107
502

446

149

446

52

107

267

107

I $8 \mathrm{r}$

267

107

I 49

2 I I

446

522

267

446

314

446
52

447

52

267

447

Py

533

52

181

447

181

229

533

522

522

267

69

182

447

447

447

288

302

447

447

314

338

447

Pullinger, A. F.

Pullinger, S. R.

Pulman, W.P.

Pumphrey, C. E.

Punchard, C.

Purchase, W. B.

355

Purdy, T.W. $\quad 19$

Purser, F. D. $\quad 447$

Purser, W. C. B. $\quad 33^{8}$

Purton, G. A. 19

Purves, C. L. 2 II

Purvis, J. H. $\quad 149$

Purvis, J.S. $\quad 314$

Pusinelli, S. J. $\quad 267$

Putnam, P. W. $\quad 107$

Puttock, R. $\quad 107$

Pye, A.N.W. $\quad 302$

Pye, D.R. $\quad 447$

Pye, E. W. 447

Pye-Smith, T. E. B. 149

Pym, C. J. $\quad 447$

Pym, R. M. $\quad 447$

Pym, T. W. $\quad 447$

Pym, W. St J. $\quad 447$

Pyman, A. $\quad 52$

Pyman, F. C. 149

Pyman, R. L. (Clare) 52

Pyman, R. L. (Jesus) 182

Pyman, W. H.

Pyne, F. S. $\quad 267$

Pyper, J.R. $\quad 182$

Pytches, G. J. $\quad 149$

Quentin, G. A. F. $\quad 69$

Quick, E.K. $\quad 338$

Quiggin, E. C. $\quad 149$

Quill, J.J.

Quiller-Couch, Sir A. T.

Quillet, L. A.

Quilter, J. A. C. $\quad 502$

Quilter, Sir W. E. C. 447

Quin, B. G. $\quad 338$

Quincey, T. E. de Q. 267

Quirk, R.

Quitzow, A.

2 I I

Raby, G. H. $\quad$ 499

Radclyffe, E. J. D. $\quad 447$

Radford, A. C. $\quad 149$

Radford, R. L. $\quad 52$ 
Raimes, E. A.

Raimes, G. H.

Raimes, L.

Raine, R. T.

Ram, G. E.

Ramsay, A.

Ramsay, A. H.

Ramsay, D.

Ramsay, J. D.

Ramsay, J. G.

Ramsay, L. N. G.

Ramsay, M. G.

Ramsbotham, W. $\mathrm{H}$ I

Ramsbottom, J. 107

Ramsbottom, W. H. 107

Ramsden, G. C. F. 355

Ramsden, G.T. $\quad 502$

Ramsden, Sir J. F. $\quad 448$

Ramsey, H. L. 3I5

Ramsey, W. J. $\quad 502$

Rand, H. M.

Randall, B. E.

Randall, G. F.

Ranger, A. S. B.

2 I I

448

448

Ranjitsinhji, K.S.

see Nawanagar, Jam of

Rankin, G. C.

Rankin, J. K.

Rankin, R. (Clare)

Rankin, R. (Trin.)

Ranking, G. L.

Ranking, G. S. A.

Ranking, R. M.

Ransford, F. B.

Ransford, W. M.

Ransom, J.

Ransom, P. L.

Ransom, P. W.

Raphael, Sir H. H.

Rapp, R.

Rapp, T. C.

Rappis, P. A. G.

Ratcliffe, A. V.

Ratcliffe, C. St A.

Ratcliffe, R. F.

Rathbone, C. G.

Rathbone, M.P.

Rathbone, R. R.

Rathbone-Proctor, S. $\mathrm{H}$.

Rattigan, C.S.

Rattray, I. M.

Rattray, R. C.

Raven, C. E.

Raven, E. E.

Raven, G. E.

Raven, H. P.

Ravenscroft, P. D.

Ravenshear, E. W.

Raw, R.

Rawcliffe, D. M.

149

I 9

448

3 I 5

268

2 I I

I9
268

229

268

502

355

355

I 9

355

522

182

448

448

448

302

448

I 49

268

107, 149

338

69

107

182

52

448

9

9

\section{7}

Rawlinson, H. G.

Rawnsley, G. T.

Rawson, H. F. R.

Rawson, R. R.

Rawstorn, J. O.

R xyment, G. V.

Raymond, C.

Raymond, E. L.

Rayner, A. E.

Rayner, C. G.

Rayner, E.

Rayner, E. B. A.

Rayner, E. C.

Rayner, O.C.

Rayner, $\mathbf{S}$.

Rea, D.

448
53
Rea, J. G. G.

Rea, R. H. T.

Read, A. J.

Read, C. F.

Read, E.

Read, E. O.

Read, G. D.

Read, H. C.

Read, H. M.

Read, J. W.

Read, R. I.

Read, R. S. (Sid.)

Read, W. R.

Reade, G. L.

Reade, J. A. D.

Reade, R. W.

Reade, W.P.

Reading, E. W.

Readman, C. H.

Readman, C. H.B. 268

Rearden, T.R.

Reay, T.S.

Rebsch, R. F. W.

Reckitt, A.

Reckitt, F.N.

Reddaway, $\mathrm{H}$.

Redden, F. A. C

Redfern, A.S.

Redfern, W. A. K.
Read, R.S. (Chr.)
522

502

3 I 5

182

53

I 49

448

2 I I

288

448

503

$44^{8}$

448

268

107

$5 \circ 3$

448

448

182

448

I 49

2 I I

149

3 I 5

268

302

149

355

107

80

182

149

338

182

288

70

338

I49

53

182

149

19

355

182

338

53

107

107

2 I I

268

268

522

53

2 II

70

150

2 I I

448

8o, 448
Redlich, S.

338

Redman, C. E. $\quad 268$

Redman, G. A. $\quad 503$

Redmayne, T. $\quad 448$

Redwood, F. A. $\quad 302$

Reece, C. M. 229

Reece, R.H. 2 II

Reece, R. J. 80

Reed, $B$.

Reed, C. H. (Chr.)

Reed, C. H. (Trin.) 448

Reed, D. H. $\quad 182$

Reed, H. L.

Reed, H.S. $\quad 448$

Reed, H. W. T. $\quad 448$

Reed, J. $\quad 448$

Reed, L. G. 2 II

Reed, L. H. B. $\quad 522$

Rees, A. G. T. $\quad 53$

Rees, A. M.

Rees, A. P.

see Gower-Rees, A. P.

Rees, F. E. $\quad 338$

Rees, G. M. T. $\quad 53$

Rees, J. R. 2 II

Rees, R.A.T. $\quad 8 \mathrm{I}$

Rees, T. J. I9

Rees-Mogg, L. L. 355

Reese, J.

Reeve, A. E. $\quad 53$

Reeve, F. P. $\quad 522$

Reeve, G.T. $\quad$ I07

Reeves, J. H. $\quad 338$

Reeves, R. F. $\quad \mathbf{5 2 2}$

Reeves, V. C. M. $\quad 229$

Reid, A. A. $\quad 53$

Reid, A. Cunningham 53

Reid, C. B. I I

Reid, C. $\mathrm{H} . \quad \mathrm{I} 82$

Reid, D. M. $\quad 107$

Reid, E. B.

Reid, E. D. W.

53

seeWhiteheadReid,E.D.

Reid, G. M.

Reid, J.

Reid, J. S.

Reid, J.S. C.

Reid, K. G.

Reid, M. B.

Reid, P. J.

Reid, W. L.

Reiner, N.A.

Reinert, E. L.

see Raymond, E. L.

Remnant, G. L. $\quad 533$

Rendall, F. G. $\quad 449$

Rendall, P.S. $\quad 449$

Rendell, W. R. I82, 503

Rendle, A. C. I9

Rennie, D. W. $\quad 33^{8}$

Rennie, W. B. $\quad 107$
268

448

2 I I

268

449

268

$38-2$ 
Rennoldson, H. F. 449

Renshaw, A. H.

Renshaw, H. W.

Renshaw, J. A. K.

Renshaw, Sir S. C. B. 449

Renton, $\mathrm{T}$.

Renwick, H. A.

Renwick, J.E.

Renwick, T. B.

Resker, B. A.

Reunert, C.

Revillon, A. J.

Revillon, J. W.

Reynard, C.E.

Reynard, C. F. P.

Reynolds, C. H.

Reynolds, E. P. I50

Reynolds, G. D. $\quad 108$

Reynolds, G. W. M. 449

Reynolds, H. E. K. 288

Reynolds, J. W. 356, 449

Reynolds, J. W. V. $\quad 522$

Reynolds, K. $\quad 108$

Reynolds, L. $\quad 449$

Reynolds, N. C. W. 449

Reynolds, T. W. I 50

Reynolds, W. H. R. $33^{8}$

Rhodes, E. C. $\quad 449$

Rhodes, E. L. N. $\quad 522$

Rhodes, F. A.

Rhodes, H. E.

Rhodes, S. H.

Rhodes, W. A.

Rhodes, W. E.

Rhodes, W. F.

Rhodes Moorhouse, W. B.

Rhys Jones, I.

Ricard, F.

Ricardo, A. St Q.

Ricardo, F.

Rice, C. M.

Rice, G. C. D.

Rice, G. E.

Rice, H. G.

Rice, L. C.

Rice, M. N.

Rice-Jones, A. T

Rice-Jones, B. R.

Rich, N. J.

Richard, W. R.

Richards, C. J.

Richards, C.S.

Richards, C. T.

Richards, C. W.

Richards, E. I. G.

Richards, F.S.

Richards, F. W.

Richards, H. A.

Richards, R. C.

Richards, W. G.

19

19

449

268

108

I 50

19

356

522

150

150

53

449

19
Richardson, A.

Richardson, A. H.

182

Richardson, A. J M $33{ }^{\circ}$

Richardson, A.S.

Richardson,

$$
\text { C. (Emm.) }
$$

108

Richardson,C.(Cai.) 150

Richardson, C.A. $\quad 356$

Richardson, C. L. $\quad 449$

Richardson, C. W. $\quad 503$

Richardson, D.E.E. 150

Richardson, D. J.A. 19

Richardson, D.S. 449

Richardson,D.W.R. 150

Richardson, E. B. $\quad 53$

Richardson, F. K. $\quad 150$

Richardson, G.P.N. 19

Richardson,J.A.StC. 70

Richardson, J. H. $\quad 19$

Richardson, J. M. $\quad 5 \circ 3$

Ricdardson, J.P. A. 449

Richardson, J.S. 449

Richardson, J. W. 449

Richardson, M.E. $\quad 45^{\circ}$

Richardson, P. J. $\quad 182$

Richardson, P. W. 2 II

Richardson, R. J.R. 338

Richardson, R.S. $\quad 268$

Richardson, $T$.

Richardson, T.D. $\quad 503$

Richardson, V. $\quad$ I08

RichardsonKuhlmann,D.

see Richardson,D.J.A.

Richmond, O.L. 211

Rickeard, C. W. 2 II

Rickerby, J.H.E. $\quad 268$

Rickett, G. R. 2 I I

Ricketts, G. D. see Bengough, G. D.

Rickman, J.

Riddell, G. B.

Riddell, J. R.

Riddiough, S.

Rideal, E. K.

Ridley, A. H.

Ridley, C. N.

Ridley, D. F.

Ridley, G. A.
Ridley, H. L.

Ridley, H. Q.

Ridley, L. E.

Ridout, P. M.

Riesle, W. B.

Rieu, A.

Rieu, $\mathrm{H}$.

Rigby, F. J.

Rigby, G.

Rigby, J. C. A.

Rigby, J. R. A.

Rigby, $P$.

Rigden, C.
Rigg, J. H. $\quad 108$

Rigg, R. $\quad 150$

Riggall, H. B. $\quad 212$

Riley, A. $\quad 19$

Riley, A. C. $\quad 45^{\circ}$

Riley, C. J. M. $\quad 268$

Riley, C. L. $\quad 150$

Riley, C. O. L.

see Perth, Archbp. of

Riley, H. C. C. $\quad 268$

Riley, J. H.

Riley, $\mathrm{T}$.

Riley, W.N.

Rintoul, A.J.

Rintoul, D.

Ripley, H. E. R. $\quad 750$

Ripley, H.W. G. $\quad 450$

Ripman, C. H. 212

Ritchie, A. G. $\quad 288$

Ritchie, C.H. $\quad 338$

Ritchie, C. S. $\quad 450$

Ritchie, G. L. $\quad 338$

Ritchie, J. N. $\quad 338$

Ritchie, R. B. $\quad 450$

Ritchie, S. E. $\quad 212$

Ritchie, T.P.A. $\quad 268$

Ritson, A.S. $\quad 450$

Ritson, F. $\quad 229$

Ritson, J. A. $\quad 45^{\circ}$

Ritson, R. $\quad 45^{\circ}$

Rittner, G.H. $\quad 45^{\circ}$

Rivers, W. H. R. $\quad 338$

Rivers-Smith, S. $\quad 315$

Rivington, R. T. $\quad 45^{\circ}$

Rix, A.H. $\quad 503$

Rix, J. C. $\quad 450$

Rix, R. G. $\quad 150$

Robathan, K. M. $\quad$ I50

Robb, A. A. $\quad 399$

Robb, Sir F. S. $\quad 503$

Robbins, C. $\quad 302$

Robbins, F. H. $\quad 269$

Robbs, F. W. $\quad 523$

Roberton, J.A. W. $\quad 108$

Roberton, J. B. W. 108

Roberts, A. C. (Clare) 53

Roberts, A. C. (Joh.) 339

Roberts, A. D. $\quad 150$

Roberts, A. H. $\quad 183$

Roberts, A. J. R. $\quad 183$

Roberts, B. C. $\quad 269$

Roberts, B. F. $\quad 108$

Roberts, C. C. G. 450

Roberts, C.H.(Kg's) 212

Roberts, C.H.(Tr.H.) 503

Roberts, C. J. 150

Roberts, C. Q. $\quad 53$

Roberts, Ff. $\quad 53$

Roberts, F. B. . $\quad 183$

450 Roberts, F.H. 20 
Roberts, F. M.

Roberts, F. P.

Roberts, F. W.

Roberts, H. E. F.

Roberts, H. T. Ll.

Roberts, J. C.

Roberts, J. H.

Roberts, J. R. B.

Roberts, Ll. C.

Roberts, P. M.

Roberts, R. B.

Roberts, R. E.

Roberts, S. C.

Roberts, S. R. P.

Roberts Buchanan, B. see Buchanan, B. R.

Robertson, C. J. T. 108

Robertson, D. D.

Robertson, D. H.

Robertson, D.S.

Robertson, H. G.

Robertson, H. J.

Robertson, J. W. H.

seeHay-Robertson, J. W.

Robertson, K. F. $\quad 450$

Robertson, M. K. $\quad 45^{\circ}$

Robertson, $R$. $\quad 450$

Robertson, W. P. $\quad 503$

Robertson, W. R. D. 108

Robertson-ShersbyHarvie, R.

Robertson Smith, N. M.

Robins, J. N.

150

Robinson, A. C.

Robinson, A. D.

Robinson, A. H. R.

Robinson, A. S.

Robinson, B. C.

Robinson, C. A.

Robinson, C.S.

Robinson, E. H.

Robinson, E. V.

Robinson, F. J.

Robinson, F. T.

Robinson, G.

Robinson, G. B.

Robinson, G. H.

Robinson, $\mathrm{H}$.

Robinson, H. D.

Robinson, H. M. $\quad 269$

Robinson, J. B.

212

20

20

$8 \mathrm{r}$

53

315

150

I08

339

269

108

450

269

268

183

$45 \mathrm{I}$

$45 \mathrm{I}$

Robinson, J.C.C.H. 288

Robinson, J. G.

Robinson,J.H.(Qu.) 302

Robinson,J.H.(Sid.) 356

Robinson, J.S.

Robinson, L. D.

356

Robinson, L.F.W. 339

Robinson, L. R. C. 315

Robinson, L.S.
Robinson, M. H.

Robinson, R. F.

339

$45 \mathrm{I}$

Robinson,R.H.O.B. 212

Robinson, $T$.

533

Robinson, W. de H. 288

Robinson, W. H. (Down.)

Robinson,W.H.(Tr.) $45 \mathrm{I}$

Robson, A.

Robson, E.

Robson, E. G. U.

Robson, E. I.

Robson, G. D.

Rochdale, Lord

Roche, A. R.

Rochfort, G.A.Boyd 45I

Rocke, E. M.

Roddam, R. J.

Roderick, D. B.

Roderick, H. B.

Rodgers, H. N.

Rodick, R. L.

Rodney, J. H. B.

Rodocanachi, P.

Rodway, A. R.

Roe, J. C.

Roe, R. G.

Roe, S. V.

Rogers, A. D. S.

Rogers, A. L.

Rogers, C. E. B.

Rogers, E. H.

Rogers, F. E. W.

Rogers, G. F.

Rogers, J. L.

Rogers, T. G.

Rogers, W. D.

Rogerson, $\mathrm{S}$.

Rogerson, T.S.

Rogerson, W. E.

Roget, S. R.

Rolfe, $P$.

Rolfe, W. J. L.

Rolleston, F. L.

Rolleston, Sir H. D.

Rollo, W. H.

Rolston, A. C.

Romanes, J.

Romer, C.

Romer, M. L. R. 503

Ronaldshay, Earl of $45 \mathrm{I}$

Ronaldson, J. B.

Rooke, C. P.

Rooth, A. V.

Roper, F. A.

Roper, $\mathrm{H}$.

Roper, O.S.

Ropner, L.

Ropner, W. G.
356
451

53

20

451

45 I

302

269

$\begin{array}{r}53 \\ \hline 83\end{array}$

108

70

108

$5 \circ 3$

$5 \circ 3$

302

54

523

269

54

533

I 50

108

I 5 I

20

302

302

356

523

503

$45 \mathrm{I}$

533

183

212

339

$45 \mathrm{I}$

I 5 I

20

451

$5 \mathrm{I}$

339

451

212

$45 \mathrm{I}$

356

523

54

269
Roscoe, A.

212

Roscoe, G. T. $\quad \mathrm{I}_{3}$

Roscoe, T.le B. $\quad 54$

Roscoe, W.

Rose, A. L. $\quad 523$

Rose, C. A. 20

Rose, C. P. G. $\quad 315$

Rose, F. A. $\quad 339$

Rose, Sir F. S. $\quad 451$

Rose, G. K. 212

Rose,H.A.(Emm.) 108

Rose, H. A. (Joh.) 339

Rose,SirH.A.(Trin.) $45 \mathrm{I}$

Rose, H. C. $\quad 339$

Rose, H.E. $\quad 533$

Rose, L. $45 \mathrm{I}$

Rose, R.de R. $\quad$ IO8

Roseveare, H. W. $\quad 339$

Rosher, H. G. $\quad 269$

Rosher, J. B. $\quad 45$ I

Rosier, J. E. R. $\quad 356$

Roskill, W. G. $\quad 504$

Ross, C. M. 212

Ross, J. E. C. $\quad 339$

Ross, O. F. $\quad 183$

Ross, R. D. $\quad 45^{\mathrm{I}}$

Ross, R. W. $\quad 523$

Ross, W. G. 45I

Ross, W. M. $\quad 269$

Ross, W. S. 315

Rossdale, S. J. $\quad 504$

Rossi, R. 45 I

Rostron, $\mathrm{S}$.

see Nowell-Rostron, $\mathrm{S}$.

Rotch, C. D. $\quad 504$

Roth, G. J. $\quad$ II

Rothband, P. L. $\quad 452$

Rothera, A. C. H. $\quad 108$

Rothfield, I. $\quad 8 \mathrm{I}$

Rottner-Smith, R. $\quad 288$

Round, W. H. 8r

Rouquette, D. G. $\quad 356$

Rouse, P. G. $\quad 452$

Rouse, W. H. D. 20

Routh, A. L. $\quad 288$

Routh, H. V. $\quad 288$

Routh, L. M. I5I

Rowan, A. 70

Rowe, B. W. $\quad 269$

Rowe, E. F. $\quad 54$

Rowe, H.P. $\quad 183$

Rowe, W.H.C. $\quad 183$

Rowell, A.H. $\quad 339$

Rowett, F. E. $\quad 339$

Rowland, E. W.S. $\quad 523$

Rowland, S. D. . $\quad 81$

Rowlands, R. B. J. $\quad 452$

Rowlandson, T.S. $\quad 504$

Rowlatt, A.

Rowlatt, J. F.

212

504

452 
Rowley, F. B.

3 I 5

Rowley, G. R. F.

Rowntree, L. E.

Rowntree, $M$.

Rowse, A. A.

Rowsell, H. G.

Roxburgh, A. C.

Roxburgh, J.F.

Roxburgh, J.H.

Roxburgh, T. J. Y. 229

Roxby, J.H. T. M. see

Maude-Roxby, J. H. T.

Roy, D. W.

Roy, P. L.

Royce, F. C.

Roylance, $\mathbf{P}$.

Royle, A. L.

Royle, J. B.

Royston, H. R.

Royston-Pigott, W M

Rubens, H. V.

Rubie, G. O.

Rubie, T. A. C.

Rubinstein, L.

Ruck-Keene, R. E. $\quad$ 183

Rücker, A. N.

Rudd, K.S.

Rudd, N. B.

Rudd, W. A.

Rudkins, F.P.

Ruffhead, A. C.

Ruggles-Brise, E. A. 452

Rule, G. S.

Rumboll, N.

Rumsey, C. F.

Rumsey, H. St J.

Ruscoe, R. G.

Rushforth, F. V.

Rushmer, H. F.

Rushmore, F. M.

Rushton, E. R.

Rushton, H. L.

Rushton, W. F.

Rushworth, A. N.

Russell, A.

Russell, E. C.

Russell, E. N.

Russell, G. G.

Russell, H. B. G

Russell, J. (Christ's) 20

Russell, J. (Trin. H.) 504

Russell, J. C.

Russell, J. D.

Russell, $\mathrm{P}$.

Russell, P. D.

Russell, S. C.

Russell, W. A.

Russell, W. B.

.

Russell Clarke, E.

see Clarke, E. Russell

212

212

8 I

I 83

452

452

70

$\mathrm{T}$.

108

452

70

269

183

533

212

523

183

20

452

183

108

339

20

452

108

108

$8 \mathrm{I}$

212

109

I 5 I

302

3 I 5

109

452

81

452

183

I 5 I

452

212

356

I 5 I

269

212

212

109

356

269
Russell-Smith, A.

339

Sampson, M. T.

339

Rust, N. A.

Rust, P. J.

Ruston, V. F. M.

Ruston, W.

Rutherford G.

Rutherford,H.G.G.

Rutherford, J. D.

Rutherford,W.McC. I 5 I

Ruthven, J.

Ruthven, J. St C. $\quad 54$

Ruthven-Stuart,A.W. 452

Rutter, E. F.

Rutter, H. F. P.

Ryan, A. C. T. K.

Ryan, C. M.

Ryan, D. P. K.

Ryan, J.

Rycroft, Sir R. N.

Ryder, A. F. R. D.

Ryder, F. J.

Ryffel, J.H.

Ryley, C. M.

Ryott, T. G.

Sabin, J. H.

Saddler, W.

Sadgrove, K.H. O'R 339

Sadler, $\mathrm{H}$.

Sadler, P. H.

523
183

Sagar-Musgrave C. 54

Sainsbury, W.T. $\quad 212$

Saint, A. P.

Saint, P. J.

St Aubyn, E. G.

St Aubyn, L. M.

St Aubyn, M. J.

St Clair, A. H.

St Johnston, A.

St Levan, Lord

Salaman, L. H.

Salaman, R. N.

Sale, H. G.

Saleebey, E. S.

Salisbury, C. E.

Salisbury, T. H. L.

Salmon, B. A.

Salmon, H. B.

Salmon, L. H.

Salmond, W. G.

I 5 I

Salomons, D.R.H.P. I 5 I

Salt, C. E. F. $\quad 269$

Salter, F. R. 229, 453

Salter, M. A.

Salter, R. E.

Salusbury, J. T.

Sambrook, H. F.

Sampson, F. A.
269

269

269

504

452

3 I 5

183

315

I 5 I

452

452

452

288

$8 \mathrm{I}$

339

452

452

453

453

453

453

504

I 83

533

109

453

109

183

I 83

54

Sampson, N. C.

453

Sampson, R. H. $\quad 453$

Sampson, S. J. M. 453

Samson, A. M. 303,523

Samuel, C. H. $\quad 54$

Samuelson, F. H. B. 453

Sanceau, R. J.

339

Sanctuary, A. G. E. I 51

Sanctuary, C.T. I 51

Sandall, H. C.

339

Sandall, T.E. $\quad 339$

Sandars, E. T. $\quad 453$

Sandberg, W. B. $\quad 303$

Sandeman, B.S. $\quad 504$

Sanders, H. R. $\quad 504$

Sanders, L. Y. $\quad 453$

Sanderson, A. K. $\quad 260$

Sanderson, C.O.St J. 288

Sanderson, C.P. $\quad 453$

Sanderson, F. B. I5I

Sanderson, G. E. $\quad 184$

Sanderson, G. P. M. 453

Sanderson, I. C. $\quad 453$

Sanderson, J. R. $\quad 453$

Sanderson, P.M. $\quad 523$

Sanderson, R. B. $\quad 303$

Sanderson, R. E. R. 151

Sanderson, R.H. $\quad 453$

Sanderson, T.S. $\quad 453$

Sandford, C. R. F. $\quad 70$

Sandilands, J. E. $\quad 453$

Sandilands, R. B. $\quad 229$

Saner, F. D. $\quad 20$

Saner, J. D. J. $\quad$ I84

Sanford, D. W. $\quad$ I5I

Sanger, P. M. 453

Sanger-Davies, F.M. 453

Sanger-Davies,Ll.H. 454

Sankey, H. B. $\quad 454$

Sankey, W. M. $\quad 70$

Sargeaunt, G. M. $\quad 151$

Sargent, E. L. K. $\quad 339$

Sargent, P. W. G. $\quad 340$

Sarra, E. R. $\quad 356$

Sarsby, R.A.

Sartoris, G. U. L. $\quad 229$

Sassoon, E. V. $\quad 454$

Sassoon,H.W.(Clare) 54

Sassoon,H.W.(Trin.) 454

Sassoon, S. L. $\quad 54$

Satterthwaite, M. E. 269

Saul, C. L. 54

Saumarez,J.StV.B. 454

Saunder, D. A. $\quad$ I5I

Saunder, G. B. $\quad 454$

269 Saunders, A. G. $\quad 54$

212 Saunders, A.H. 20

453
Sandilands, H. R. 453

Sandison, A. $\quad 453$ 
Saunders, C. P. G. $\quad 184 \mid$ Schwann, F. H.

Saunders, D. M. $\quad 54$

Saunders, E. G.S. $\quad 151$

Saunders, F.P.

Saunders, $\mathrm{H}$.

Saunders, H. F.

Saunders, J. T.

Saunders, W. D.

Saunders, W. H.

Saurin, W. M.

Savage, E. G.

Savage, K.

Savile, G. K.

Savile, W.S.

Savill, E. H.

Savill, S. C.

Saville, S.H.

Savory, C. H.

Savory, H. L.S.

Savory, K. S.

Sawtell, H. D.

Saxon, $\mathrm{H}$.

Saxton, W. I.

Sayer, $\mathbf{H}$.

Sayer, S. D.

Sayers, L. D. W.

Scarborough, O.L.

Scarlett, C.H.

Scarlett, J. A.

Scarth, R. E.

Schacht, A. G.

Schaefer T.S.H. 454

Scharff, G. E.

Schiff, M. E. H.

Schiller, L. C. T.

Schlesinger, G. L.

Schloss, W. F. R.

see Castle, W.F.R.

Scholes, W. N.

Scholes, W.P.

Scholey, C. H. N.

Scholfield, A. F.

Scholfield, C. N.

Scholfield, E. P.

Scholfield, J. A.

Scholfield, J. L.

Scholfield, R. D.

Scholtz, C.J.

Schon, B.

Schonland, B. F. J.

Schomd, 152

Schooling, C. H.

$\begin{array}{ll}\text { Schooling, C. H. } & 270 \\ \text { Schreiner, O. D. } & 454\end{array}$

Schroeder, A. E.

see Long, A. E.

Schuddekopf, W. G. A.

see Shuttleworth, W. G. A.

Schurr, C. G.

Schuster, L. F.

Schwalm, C. E.

Scott, G. A.
54 see Swann, $H$.

152 Schwarz, R. O.

Sclater, F. A.

Sclater, J.R.P.

Scobie, J.

20

I09

504

8 I

303

152

229

303

454

454

I 84
504

54

303

212

454

533

70

229

454

340

454
$\mathrm{I} 52$

20

184

356

152

270

212

54

212

454

454

152

229

340

109

454

356

Scorgie, N. G. $\quad 356,454$

Scott, A. D. B. 315

Scott, C. F. H. see

Hepburne-Scott, C. F.

Scott, B. G. A. $\quad 504$

Scott, C.W. (Fitz. H.) 533

Scott, C. W. (Selw.) 523

Scott, D. A. (Jesus) 184

Scott, D. A. (Sidney) 356

Scott, E.

Scott, F.

Scott, F. E.

Scott, G. E.

Scott, G. M.

454

533

I 84

533

454

Scott, Lord H. A. M. D. see Montagu Douglas Scott, Lord H. A.

Scott, H. P. F. $\quad 184$

Scott, H. W.

Scott, J.

Scott, J. E.

Scott, J. G.

Scott, J. G. C.

Scott, J. H. (Christ's) 20

Scott, J. H. (Clare)

Scott, J.S.

Scott, J. T.

Scott, L. B.

Scott, M. H. B.

Scott, R. B.

Scott, R. F. M.

Scott, R. S.

Scott, S. C.

Scott, T. F.

Scott, W.

Scott, W. D.

Scott, W. F.

Scott, W. G. H. see Polwarth, Lord

Scott, W. L. (Clare) 54

Scott, W. L. (Trin.) 454

Scott, W. M.

212

Scott, W.T.H. see

Hepburne-Scott, W. T.

Scott-Davidson, W. W.

Scott-Elliot, G. F.

454

Scott-Kerr, R.

454

Scott-Murray, A. E. 454

Scott-Murray, R. C. 454

454

Scott-Phillips,

D. M.P.
Scoular, A. C.

340

Scoular, J. G. $\quad 340$

Scowcroft, H. D. $\quad 54$

Scowcroft, H. E. I $\quad$ I52

Scrace, J. $\quad 288$

Scratton, E. W.H. B. 504

Scrimgeour, G. C. $\quad{ }_{152}$

Scruby, F.S. $\quad 523$

Scrutton, H.U. 212

Scrutton, T. B. 213

Scudamore, R.C. $\quad 229$

Scutt, C. A. $\quad 54$

Scutt, J. A. H. $\quad 340$

Seabrook, A.S. $\quad 20$

Sealy, C. F. N.P. $\quad 270$

Sealy, H. N. $\quad 455$

Sealy, P. T. $\quad 288$

Sealy, W.H. $\quad 288$

Seaman, A. J. $\quad 523$

Searight, T.P. $\quad 455$

Searle, C. F. $\quad 270$

Searle, W. C. $\quad 270$

Searles, P. A. $\quad 455$

Seaton, A. A. $\quad 270$

Seaver, C. $\quad 109$

Sebag-Montefiore, J. 270

Seccombe, P. J. A. 340

Secondé, E. C. $\quad 213$

Seddon, A. D. $\quad 70$

Seddon, A. E. $\quad 213$

Seddon, G. N. $\quad 54$

Seddon, W. D'A. $\quad 54$

Sedgwick, C. H. $\quad 356$

Sedgwick, H. R. $\quad 54$

Sedgwick, R. E. $\quad 152$

Seely, C. G. $\quad 455$

Seely, F. E. $\quad 455$

Seely,J. E. B. $\quad 455$

Segnitz, H. F. $\quad 455$

Selby, E. J. $8 \mathrm{I}$

Selby, J.S.E. 20

Selby-Lowndes, G.N. 303

Seligman, G. A. $\quad 504$

Sell, F. W.

Sellars, E. F. $\quad$ I52

Sells, A. L. $\quad 356$

Sellwood, F. G. $\quad$ I09

Sellwood, H. E. $\quad 523$

Selwyn, E. G. $\quad 70,213$

Selwyn, H.E. $\quad 523$

Selwyn, J. $\quad 70$

Selwyn, W.M. $\quad$ I09

Semple, E. G. $\quad 533$

Semple, W. D. $\quad 54$

Sendell, C. H. $\quad$ I84

Senior, F. G. $\quad 184$

Senior, J. $\quad 54$

Sephton, R. $\quad$ I52

Serginson, $\mathrm{H}$. $\quad 533$

Sessions, L. F. $\quad 55$

270 Sessions, R. V. 
Seth-Smith, G.

Seth-Smith, K. J

Seth-Smith, L M

Severne, A. de M.

Severne, E. C. W.

Sevestre, R.

Sewell, A.P.

Sewell, E. P.

Sewell, F. A. S.

Sewell, F. C.

Sewell, H. D.

Sewell, H. S.

Sewell, H. V.

Sewell, H. W.

Sewell, J.P. C.

Sewell, R. B. S.

Sewell, S. E.

Sewill, J. W.

Sewill, R. W.

Seymour, A. G.

Seymour, F.

Seymour-Browne, R. C.

Seymour Taylor, E. $M$.

Seyrig, J. R.

Seys-Phillips, $H$.

Shackel, G. M.

Shackle, R. J.

Shackle, S. A.

Shackleton, A. G.

Shakespear, G. A.

Shakespeare, G.H.

Shand, P. M.

Shanks, E. B.

Shanks, P. M.

Shankster, G.

Shanly, $\mathrm{H}$.

Shann, S. E. T.

Sharman, G.H.

Sharp, C. G.

Sharp, D. G.

Sharp, E. W. L.

Sharp, G. F.

Sharp, G. G.

Sharp, $\mathrm{H}$.

Sharp, H. W.

Sharp, J. E.

I84

Sharp, J.S.(Trin. H.) 504

Sharp, L. E.S.

Sharp, L. W.

Sharp, S. O.

Sharpe, A. G.

Sharpe, C. C.

Sharpe, E.

Sharpe, G. R.

Sharpe, G. W.

Sharpe, $\mathrm{H}$.

Sharpe, W. H.S.

Shaw, A.
270

455

455

270

270

3 I 5

523

533

455

288

20

455

20

340

55

55

I 84

455

270

455

213

455

455

455

I 84

55

455

I09

213

455

20

356

340

I 52

523

340

20

109

70

533

455

270

152

504
455

I 52

270

109

455

I 84

20

455

270

213

456
Shaw, A. G.

Shaw, C. R.

Shaw, E. A.

Shaw, E. B.

Shaw, E. H.

Shaw, G. E.

Shaw, G. R. D.

Shaw, G.S.

Shaw, J. A. P.

Shaw, J. R.

Shaw, K. E.

Shaw, R.

Shaw, R. D.

Shaw, R. P.

Shaw, S.

Shaw, W.

Shaw-Hellier, A.J.B. 504

Shaw-Yates, E. B. $\quad 229$

Shaxby, R. U.

Sheard, M. H.

Shearman, V.

Shearme, J.S.

Shearwood, G. F. F. 523

Shebbeare, H. V. $\quad 456$

Sheehan, F. R.

534

Sheepshanks, A. C. $\quad 456$

Sheepshanks, C.J.H. 456

Sheepshanks, E.

270

Sheepshanks, R. H. 456

Sheldon, F. H.

Sheldon, H. F.

456

Sheldon, L G. M. 456

Sheldon, T. W. IO9

Shelford, L. McN. Io9

Shell, A.

Shelley, G. E.

Shelley, J.

Shelley, J. F.

Shelley, L. W.

Shelmerdine T. G.

Shelton, A. T.

Shelton, O. W. M.

Shelton, R. N.

Shennan, D. F. F. $\quad 456$

Shennan, W. D

Shenstone, A. G.

Shenton, J.S.

Shephard, W. H.

Shepherd, E. H.

340

Shepherd, F. McA. 456

Shepherd, J.

Shepherd, J. C.

Shepherd, J.M. E. 456

Shepherdson, J. F. $\quad 55$

Shepley, R. G.S. $\quad 229$

Sheppard, B. F. $\quad 456$

Sheppard, G. $\quad 152$

Sheppard,G.A.(Tr.) 456

Sheppard, G. A.

('Trin. H.)
Sheppard, H. R. L. $\quad 504$

Sheppard, J. H. D. $\quad 270$

Sheppard, W. S.

2 I

Sheppard-Jones,J.E. 230

Sheppey-Greene, R. F.

Shera, A. G.

3 I 5

Shera, F. H.

Sherburn, J.C.

Sherlock 504

Sherlock, J. H. 55

Sherlock, W. W. $\quad 534$

Sherman, R. $\quad$ I 52

Sherriff, C. B. $\quad 456$

Sherston, W. M. $\quad 270$

Sherwood, G. D. $\quad$ 109

Sherwood, H. P. $\quad 356$

Shewell, H. W. B. $\quad 456$

Shields, C. St B. $\quad$ I09

Shields, H. J.S. $\quad 184$

Shields, $T$.

Shilcock, J. W.

Shildrick, L. R.

Shillaker, G.

Shillington, J. M.

Shillito, N. W. 340

Shimield, W.S. $\quad 340$

Shimwell, $\mathrm{H}$. $\quad 504$

Shimwell, O. $\quad 504$

Shiner, R.P. 21

Shingleton-Smith, F. 2 I 3

Shipley, A. H. B. $\quad 288$

Shipley, J.W. $\quad 534$

Shipman, G. A. C. $\quad 456$

Shipton, W. 109

Shirreff, C. R. $\quad 456$

Sholl, A.E. $\quad 55$

Shone, H. J. $\quad 109$

Shore, L. R. $\quad 340$

Shore, T.H. G. $\quad 340$

Short, H.S. $\quad 456$

Short, J. M. $\quad 340$

Short, P.S. $\quad 456$

Shorter, R. C. $\quad 356$

Shove, R. S. $\quad 456$

Shove, W.C. $\quad 534$

Showell-Rogers, E.N. 152

Shrager, E. H. $\quad 504$

Shrewsbury, E. $\quad 534$

Shrubbs, C. A. $8 \mathrm{I}$

Shuckburgh, R.S. $\quad 109$

Shufflebottom, E. 21

Shurlock, A. G. $\quad$ I84

Shurlock, F. W. 2 I

Shuter, A. E. $\quad 184$

Shuter, C. H. $\quad 184$

Shuter, L. R. W. A. 270

Shutt, J. E. $\quad 456$

Shutte, M.W. $\quad 152$

504 Shuttleworth,W.G.A. 1 ro 
Sibley, F. H.

Sibly, T. M.

Sidebotham, F. L. $\quad 152$

Sidebotham, F. N. $\quad 55$

Sidebotham, J. B. $\quad 152$

Sidebotham, J. N. W. 55

Sidgwick, H. C. $\quad 55$

Sidney, P.

Sidney, R. J. H.

Siffken, B. C. de W.

Silburn, $\mathrm{L}$.

Silk, G. W.

Sillem, C. F.

Silley, P. G.

Silvertop, F, S. J.

Silvertop, W. A

Sim, P. W.

Simcox, J. L.

Simcox, W. M.

Simeon, J. P.

Simeon, L. S. B.

Simey, A. I.

Simey, P. A. T.

Simmons, C. E.

Simms, W.

Simon, $\mathrm{H}$.

Simon, Sir R. M.

Simonds, C. C. B.

Simons, E.

Simpkinson, F. V.

Simpson, A. J. G.

Simpson, A. W. W.

Simpson, C.S.

Simpson, F. A.

Simpson, G. B.

Simpson, G. B. G.

Simpson, G. C. E.

Simpson, G. H.

Simpson, H. D.

Simpson, J. B.

Simpson, J. C.

Simpson, J. G.

Simpson, J. H.

Simpson, L. S.

Simpson, N. D.

Simpson, R. C.

Simpson, R. G.L.

Simpson, S. G.

Simpson, W. A.

Simpson, W. H.

Simpson-Hayward, G. H.

Sims, F. A.

Simson, C. C.

Simson, J.

Sinclair, D. J. O.

Sinclair, G. W.

Sinclair, R. C. H.

Sinclair, W. M.

Sindall, R. D.

Sing, C. M.
I 10

340

Sing, L. M.

Singer, C. M.

Singh, K. I.

Singh, K. S.

Singleton, J. E.

Sington, A. J. C.

Sington, E. C.

Sington, H.S.

Sisson, B. H.

Sisson, G.

Sisson, H. A.

Skelding, $\mathrm{H}$.

Skelton, N. A.

Skene, A. P.

Skene, C. M. B.

Skene, F. N.

I IO Skene, R. E.

270 Skey, C. H.

270

523

457

213

303

303

303

213

152

152

$8 \mathrm{I}$

213

21

2 I

457

457

505

21

340

505

303

152

152

2 I

270

457

457

457

457

356

505

153

55

356

457

184

457

213

505

$8 \mathrm{I}$

110

21
Skey, C. O.

Skinner, C. E.

Skinner, E. F.

Skinner, H. S.

Skinner, J. A. D.

Skipwith, Sir G.H. D'E.

Skirrow, G.

Skrimshire, C. V.S. 315

Skyrme, C. R.

Slack, E.

Slacke, R. C.

Slade, E. A.

Slade, G. O.

Slade, J. G.

Slade, P. C. A.

Slaney, J. N.

Slater, A.

Slater, B. H.

Slater, F. C.

Slater, J. A.

Slater, S. B.

Slaughter, A.

Slaughter, C. E.

Slawson, W. N.

Sleeman, C. M.

Sleigh, G. B.

Sleigh, G. P.

Sleight, A. H.

Sleight, K. R.

Slingsby, W. E.

Sloley, R.

Sloman, A. E. P.

Sloman, $\mathrm{H}$.

Smale, G. F. P.

Smales, R.

Small, D. W.

Smalley, S.

Smallwood A McN.

Smart A. H. MCN. 153

Smart, W. E.

Smart, W. M.
270

457

270

271

271

457

457

153

I IO

153

303

$3 \circ 3$

340

340

505

303

303

534

70

I 84

457

$2 I$

271

55

55

457

55

230

55

315

457

153

I 10

340

505

21

534

303

184

340

356

271

505

303

534

271

303

2 I

I 53

I 53

213

315

457

Smedley, R. D.

27 I

Smee, C. W.

340

Smellie, G. H.

303

Smetham, S. J.

Smiley, H.S.

Smith, A. B. P.

see Pavey Smith, A. B.

Smith, A. C. Denison 2I

Smith, A. C. S.

457

Smith, A. E. Clarence 2 I

Smith, A. E. S. 2 I

Smith, A. F. (Joh.) 340

Smith, A. F. (King's) $2 \mathrm{I}_{3}$

Smith, A. G. 230

Smith, A. J. H. $\quad 457$

Smith, A. K. 271

Smith, A. S. D. $\quad 185$

Smith, A. W. E. $\quad 505$

Smith, B. A. $\quad 457$

Smith, B. Abel 457

Smith, B. T. D. I 85, 34I

Smith, C. E. G. $\quad 2 \mathrm{I}_{3}$

Smith, C. H. C. $\quad 457$

Smith, C. J. Dudley 457

Smith, C. M. $\quad 303$

Smith, C. R. B. 2 I

Smith, C. S. I ro

Smith, C. W. W.

see Winwood-Smith, C. W.

Smith, D. Abel

Smith, D. B. M.

457

see Mellis-Smith, D. B.

Smith, D. G.

Smith, D. W.

Smith, E. F. $\quad 213$

Smith, E. F. H. $\quad 288$

Smith, E. H. $\quad 534$

Smith, E. M. 213

Smith, E.S. $\quad 505$

Smith, E. W. $\quad$ I53

Smith, F. A. $\quad 505$

Smith, F. B. (Jesus) 185

Smith, F. B. (King's) 213

Smith, F. C. 2 I

Smith, F. E. $\quad 534$

Smith, F.S. $\quad 70$

Smith, F. W. $\quad 303$

Smith, G. $\quad 457$

Smith, G. A. C. $\quad 185$

Smith, G. B. $\quad 153$

Smith, G. Dudley 457

Smith, G. L. $\quad 534$

Smith, G. M. Herbert see Herbert Smith, G. M.

Smith, G. R.

Smith, G.S. 
Smith, H. Austen (Trin.) see Austen-Smith, $\mathrm{H}$.

Smith, H. E.

Smith, H M 153

Smith, H. L. C.

Smith, H. P.

Smith, H. V.

Smith, J.

Smith, J. A. H.

Smith, J. F. H.

Smith, J. H. Martin 458

Smith, J. H. Michael 458

Smith, J. J.

Smith, J. L.

Smith, J. P. F. H. I 85

Smith, J. T. (Christ's) 2 I

Smith, J. T. (Trin.H.) see Tilden Smith, J.

Smith, J. Y.

Smith, K.P.

Smith, L.

Smith, $M$.

Smith, M. V.

Smith, N. B.

Smith, N. C.

Smith, N.H.

Smith, N. M. R. see

Robertson-Smith,N.M.

Smith, O. C.

Smith, O. Martin $\quad 348$

Smith, Peter $\quad 458$

Smith, Prince

Smith, R. (Trin.)

Smith, R. ('Trin.H.) 505

Smith, R. A.

Smith, R. Abel

288

Smith, R. C.S. see

Standring-Smith, R. C.

Smith, R. E.

Smith, R.S.S.

Smith, R. W.

Smith, S. (Clare)

Smith, S. (Queens')

Smith, S. B.

Smith, S. C. Kaines 230

Smith, S. H. (Caius) I 53

Smith, S.H. (Trin.) 458

Smith, S. P.

Smith, S. T.

Smith, T. L.

Smith, V.S.

Smith, W. A.

Smith, W. A. N

Smith, W. Campbell 70

Smith, W. E.

Smith, W. F.

Smith, W. G.

Smith, W. G. F.

I53

523

I 53

34 I

27 I

230

Smith, W. H. (Chr.) 2 I

Smith, W. H. (Jes.) 185

Smith, W.H. (Sid.) 357
Smith, W. O

Smith, W. W. (Kg's) 213

Smith, W. W. (Qu.) 304

Smith-Barry, R.R. $45^{8}$

Smith-Carington,

$$
\text { A. E. C. }
$$

Smith-Carington, M. C. H.

Smith-Rewse,

Smith-Sligo,

R.W.M. G. J. 505

Smithson, A. E. 2I, 34I

Smuts, J. C.

Smyly, A. F.

Smyth, H. W.

Smyth, J. W. W.

Smyth, S.S.

Smyth, W. H.

Smyth, W. J. D.

Smyth, W. R. B.

Smythe, D.

Smythe, G. A.

Snaith, E. G.

Snape, H. J.

Snape, W.R. C.

Sneath, R. E. F.

Snelgrove, S. H.

Snell, C. C.

Snell, F.S.

Snell, H. C.

Snell, J. A. B.

Sneyd, R.

Snow, Sir T. D'O.

Snowden, A. de W.

Snoxell, S.

Soames, A. L.

Soames, J. B.

Soames, M. H.

Soames, R. M.

Soames, W. M.

Soden, W.S.

Sole, B. J. B.

Solly, A. N.

Solomon, B. A.

Solomon, C. G. R.

Solomons, $\mathrm{M}$.

Solvay, M. A.

Somers, J. P.

Somers

Clarke, G. $\quad$ I 53

271

Somerset, A. P. F. C. 185

Somerset,R.H.E.H. 304

Somervell, A. C. $\quad 213$

Somervell, L. C.

Somervell, T. $\mathrm{H}$.

Somerville, K. B.

Somerville, M. A.

Soole, S. W.

Soole, W. B.

Soothill, V. F.

Soper, F. P. P.
271

458

70

I IO

$27 I$

271

I IO

230

304

271

458

185

213

I 53

230

$34 I$

534

458

458

458

458
505

341

I 85

I 53

271

505

81

7 I

3

213

I 53

56

213

458

316

I IO

I 85
Soper, R. G.

Sopwith, S.S.

Sorley, G. M.

Sotham, F. A.

Sothers, E. D.

Souper, N. B.

Soutar, A. K.

South, F. W.

Southam, J. F. S.

Southern, H. $27 \mathrm{I}$

Southern, N. 213

Southey, E. $\quad 45^{8}$

Southwell, F. E. G. 3 I 6

Southwell, H. M. $\quad 56$

Southwell, R. V. $\quad 45^{8}$

Sowell, A. D. $\quad 304$

Sowels, F.

Spackman, E. D. $\quad 304$

Spackman, H. M. 34I

Spafford, H. H. $\quad 304$

Sparenborg, H. R. $\quad$ I53

Spargo, F. W. 34I

Spark, D. S. 1 I0

Sparkes, W. L. $\quad 458$

Sparks, C. E.

Sparks, C. H. 34I

Sparling, H.P. $\quad 304$

Sparling, W.H. $\quad 304$

Sparrow, E.C. $\quad 458$

Sparrow, G. $\quad$ I53

Spartali, C. $\quad 459$

Speakman, L. A. $\quad$ I53

Spear, G. F. $\quad 304$

Spearing, E. IIO

Spearman, B. $\quad 153$

Spearman, W. I53

Spedding, J. A. $\quad 459$

Speer, A.H. T. L. 459

Speer, F.A. $\quad 459$

Speir, G. T. $\quad 27$ I

Speir, K.R. N. 27 I

Spence, C. B. - 27 I

Spence, D. L. $\quad 523$

Spence, R. B. $\quad 153$

Spencer, G.T. L. 459

Spencer, G. W. $\quad 34 \mathrm{I}$

Spencer, H. V. $\quad 459$

Spencer, J. M. J. $\quad 214$

Spencer, L. D. $\quad 505$

Spencer, L. D. W. 22

Spencer, R. $\quad 56$

Spencer, T. B.W. $\quad 185$

Spencer, T. D. $\quad 459$

Spencer-Smith, P. $\quad 304$

Spens, A. W. $\quad 459$

Spens, H. B. $\quad 214$

Spens, T.P. $\quad 459$

Spero, $\mathrm{L}$.

Spero, L. H.

Spicer, E. E.

Spicer, G. E. 
Spicer, H. W.

Spicer, L. D.

Spicer, $M$.

Spicer, N.

Spicer, R.

Spielman, C. M.

Spielmann, H. L. I.

Spiers, A. L. C.

Spilsbury, L. J.

Spink, C.C.

Spinney, M.H.

Spittle, J.T.

Spittle, T.S.

Spowart, W.C. 81, 304

Spragg, C. E. W. 459

Spragge, F. B. B. $\quad 459$

Sprake, G.G.

Spranger, J.A.

Sprigg, H. A. G.

Spring, A.

Spring, E.

Spring Rice, C.

Springfield, C. $O$.

185

459

505

505

459

214

271

459

IIO

357

523

271

56

459

459

459

288

357

459

Springfield, G. P. O. 505

Springman, J. B.

Sproat, R. H.

Sprot, Sir A.

Sprott, M. W. C.

Sproxton, C.

Spurrell, H. W.

Spurrell, R. J.

Spurrell, R.K.

Squire, A. G.

Squire, A. M.

Squire, C. E.

Squire, S. G.

Squires, A.P. H.

Squires, F. V.

Stack, E.H. E.

Stackard, S. F.C.

Stackhouse, J.H.

Stafford, G. B.

Stafford, H.N.

Stafford, R.S.H.

Staffurth, A. E.

Staley, H.S.

Staley, J. E.

Stallard, H. K.

Stamper, E. P. F.

StanburyTaylor G R 185

see Taylor, G. R. M.S.

Stancliffe, R.S.

Stancomb J.M. D. 459

Standen, L. J. D.

Standidge, C.

Standring-Smith, R. C.

Stanfield, A. V.

Stanford, H. C.

Stanham, C. T.

Stanhope, R. M.

289

289

56

505

22

534

22
459

22

316

153

271

214

289

185

154

357

IIO

534

271

185

316

56

341

$34 \mathrm{I}$

357
Stanley, A. S.W.

Stanley, C. M.

Stanley, C. S. B. W.

Stanley, C. W.

Stanley, E. S.

Stanley, F.

Stanley-Clark, C.

Stanning, J.

Stansfeld, R.

Stanton, H. J. C.

Stanton, R. G. O.

Staples-Browne R.C.

Stapleton, E. P.

Starkey, H. S. C.

Starkey, V. G.

Starkie, R.P.A

Starling, E. C. W.

Starling, G.

Statham, N.H.

Stathers, G. N.

Staunton, $\mathrm{H}$.

Staveley, C. H.

Staveley, H.S.

Stavers, W. M.

Stead, J.

Stealey, E. T.

Steane, S. W.

Stearn, C. H.

Stearn, G.F.

Steavenson, A. G.

Stebbing, T.H.L.

Stedall, C. P.

Stedall, G. St G.

Stedman, G. F.

Stedman, J. A.

Steedman, M. T. W.

Steel, D. M.

Steel, O. W. D.

Steel, S. S.

Steele, A. R.

Steele, C. M.

Steen, F. D. 34I

Steen, S. W. P.

Steers, J. A.

Steinthal, R. E.

Stenham, B. B.

Stenhouse, J. M.

Stenhouse, R. H.

Stenning, E. H.

Stephen, A. F.

Stephen, A. M.

Stephen, F. C.

Stephen, J. G.

Stephens, A. R.

Stephens, E. A.

Stephens, E. C.
Stanley, A. W. W.

Stansfeld, C. A.

Steele, C. M. $\quad 505$
459

460

523

460

214

534

154

460

185

341

22

523

271

22

460

185

316

505

460

524

56
185

22

214

524

460

304

316

272

304

230

185

460

272

56

316

110

460

505

22

460

505

357

272

81

56

214

I 10

272

214

289

70

70

304
Stephens, H. H.

22

Stephens, J. F.D. $\quad 272$

Stephens, J. P. $\quad 534$

Stephens, J. W. W. $\quad 154$

Stephenson, D. G. $\quad 460$

Stephenson, F. 34I

Stephenson, H. $\quad \mathbf{5 3 4}$

Stephenson, H. M. I54

Stephenson, H. S. 22

Sterckeman, P. $\quad 8 \mathrm{I}$

Stern, L. H. $\quad 230$

Sternberg, E. A. J. $\quad 505$

Sternberg, R. O. $\quad 56$

Sterndale-Bennett, J. 34 I

Sterndale-Bennett, J. B.

$34 \mathrm{I}$

Sterndale-Bennett, R. 34 I

Stevens, A. L. W. $\quad 460$

Stevens, C. G. B. I 10

Stevens, C. J. Duff 460

Stevens, F. B. 22

Stevens, G.H. $\quad 154$

Stevens, H. L. $\quad 82$

Stevens, J.A. $\quad 70$

Stevens, J. K. 34I

Stevens, L. B. $\quad$ I54

Stevenson, A. W. $\quad 357$

Stevenson, C. M. 34I

Stevenson, F.P. $\quad 357$

Stevenson, R. W. $\quad 272$

Stevenson, S. D. $\quad 460$

Stevenson,W.F.J.F. 316

Steward, R. D. $\quad 506$

Steward, S. J. $\quad 82$

Stewart, A. C. $\quad 289$

Stewart, C. A. $\quad 460$

Stewart, C. B. 22

Stewart, C. T. $\quad 460$

Stewart, D. H. $\quad$ I85

Stewart, D.M. $\quad 34 \mathrm{I}$

Stewart, E. W. H. $\quad 357$

Stewart, F. C. $\quad 506$

Stewart, F.D.L. $\quad 506$

Stewart, F. H. I54

Stewart, F. W. $\quad 460$

Stewart, H. $\quad 460$

Stewart, J. A. L. $\quad 460$

Stewart, J. C. $\quad 506$

Stewart, N. B. $\quad 272$

Stewart, P. M. $\quad 22$

Stewart, R. J. $\quad 460$

Stewart, W. H. E. $\quad$ I54

Stewart-Brown, R. $\quad 460$

Stewart-Jones, T. A. 460

Stewart-Savile, W.S.

see Savile, W.S.

Stidston, P. H. $\quad 272$

Stiebel, C. $\quad 506$

Stiff, H. H. $\quad{ }_{54}$

igand, I. A.

460

304 
Stileman, C. H.

Stileman, D. C. G.

Stileman, D. F.

Stileman, F. W. C.

Stileman, G. R.

Still, H. N.

Still, W. H.

Stimpson, R.

Stimson, J.P.

Stimson, W. B.

Stinson, H. J. E.

Stirling, A.

Stirling, E. M.

Stirling, F.

Stirling, G. S.

Stirling-Maxwell, Sir J.

Stiven, H. E. S.

Stobart, J. G.

Stobart, W.

Stockdale, R. J.

Stocker, A. H.

Stocker, C. J

Stocks, P.

Stockwell G,E StC.

Stockwood, I. H. $\quad 34 \mathrm{I}$

Stoddard, J. W.

Stogdon, J. H.

Stokes, E. F.

Stokes, F. F.

Stokes, F. L. B.

Stokes, J. W. G.

Stokes, L.

Stokes, R. J.

Stokes, W. A.

Stokes, W. H.

Stolterforth, G.H.

Stone, A.

Stone, B. J. V.

Stone, E. R.

Stone, H. B.

Stone, W. N.

Stoneham, R. T.

Stoneman, W. E.

Stoney, T. R.

Stoodley, P. B.

Stopford, J.

Stopford, R. J.

Storer, R. S.

Storey, C. B. C.

Storey, H. H.

Storey, K. L.

Storey, L. H. T.

Storey, R. A.

Storr, E. C.

Storrs, B. St J.

Storrs, C. E.

Storrs, F. E.

Storrs, J. P.

Storrs, K. S.

Storrs, R. H. A.
272 Stott, A. W.

304 Stott, M. D.

304 Stott, R.

272

22

22

$34 \mathrm{I}$

289

I 10

I 10

460

I 54

56

56

460

461

272

185

524

$46 \mathrm{I}$

$46 \mathrm{r}$

214

185

461

214

$46 \mathrm{r}$

357

$34 \mathrm{I}$

524

3 I6

I 10

534

22

214

I I I

I I I

214

272

289

524

273

56

$34 \mathrm{I}$

230

56

56

I 54

$46 \mathrm{I}$

I 54

461

273

214

273
185

56

I I I

273

Stout, G. F. A. B.

Strain, J. L.

Street, $\mathrm{H}$.
Strachan, E.S.

Strachan, H.

Strachan, R. H.

Strachan Carnegie,

Stradbroke, Earl of

Stradling, A. R.

Strahan, W. R.

Strain, L. H.

Straker, A. C.

Straker, G. H.

Straker, H. G.

Straker, I. A.

Straker, L. S.

Strange, H. St J. B.

Stratford, E. P.

Stratton, F. J. M.

Straughan, T. A.

Stream, E. J.

Streatfeild A. H.O.

Streatfeild, H. G. C. 2 I

Street, H. B.

Street, R. O.

Streeten, A. H.

Streeten, B. R.

Streeten, E. R.

Stretton, J. W.

Strickland, A. F.

Strickland, C.

Strickland, J. R.

Strickland, N.

Stringer, C. E. W.

Stringer, C. H.

Stringer, G. M.

Stringer, H. W.

Strong, J.P.

Strong, P. N. W.

Strong, S. D.

Stroud, H. C.

Strover, E. J.

Stroyan, J.R.A.

Struthers, J. A.

Stuart, A. A. P. R.

Stuart, E. E. (Joh.) 342

Stuart, C. M.

Stuart, D. C. R.

Stuart, J. A. G.

Stuart, R. S. D.

Stuart, Viscount

Stuart, W. R.

Stuart Prince, D.

Stubber, R. H.

Stubbs, N. H.

Stubbs, S.S.
46I Stuckey, R. A.

46I Studd, H. W.

46I Studd, L. F.

534 Studd, V. M.

185 Sturdy, A. C.

Sturdy, A. E.

Sturdy, E. C.

Sturges, E. L.

Sturgess, J.

Sturt, H.

Sturton, K. M.

Sturton, S. D.

Style, A. H.

Sugden, G. H.

Suhr, A. C. H.

see Hammond Searle, A. C.

Suffern, C.

Sulivan, E. G.

I I I

461

Sullivan, A. M.

22

Sullivan, J. H. B. 22

Summerhayes, C. H. I I I

Summers, A.S. M. 461

Summers, F. II I

Summers, G.

Sumner, E. J.

I 54

Surrage, H. J. R. $\quad 273$

Surrage, J. L. $\quad 273$

Surridge, B. J. $\quad 82$

Surtees, A. A. $\quad 462$

Susskind, M. J. $\quad 273$

Sutcliffe, A. II I

Sutcliffe, A. L. $\quad 357$

Sutcliffe, J.H. $\quad$ I54

Sutcliffe, P. T. II I

Sutherland, A. G. $\quad 462$

Sutherland, F. B. $\quad$ I 54

Sutherland, J. F. $\quad$ I54

Sutton, F. O. 22, 186

Sutton, H. J. $\quad 462$

Sutton, K. H. M. 273

Sutton, L. J. $\quad 524$

Sutton, L. N. $\quad 462$

Sutton, W. H. R. $\quad 82$

Sutton, W. M. $\quad 273$

Svensson, R. $\quad$ I54

Swaine, J. K. $\quad 462$

Swainson, E. A. C. $\quad 154$

Swainson, F. E. $\quad$ I86

Swainson, J. L. $\quad 214$

Swallow, J. R. $\quad 534$

506 Swan, F. G. 273

316 Swan, H. N. 273

I 54 Swann, A. $\quad 506$

46I Swann, C. H. 22

461 Swann, F. H. 462

70 Swann, $H . \quad 214$

506 Swann, L. H. $\quad 506$

304 Swann, M. B. R. $\quad 154$

357 Swann, P. W. $\quad 534$ 
Swann, S. E.

Swann-Mason, R. S. 316

Swanston, C. B.

Swanston, E. R.

Swanston, H. E.

Swanwick, F. B.

Swanwick, R. K.

Swatridge, C. J.

Swayne, P.C.

Swears, H. M.

Sweet, J. L. L.

Sweet, R. McM.

Sweeting, H. C.

Swete-Evans, W. B.

Swift, B. H.

Swift, C. T.

Swift, H. W.

Swift, J. M.

Swift, W.

Swindlehurst, J. E. I 54

Swindlehurst, T.R. I 54

Swiney, A. J. E.

see MacSwiney, A. J. E.

Swinstead, N. H.

Sydney, H.

Syer, H. B.

Sykes, A. H. C.

Sykes, E. T.

Sykes, F. M.

Sykes, Sir M.

Sykes, M. G.

Sykes, S. W.

Sykes, W.S.

Sylvester, H. A.

Syme, D. A.

Syme, G. W.

Syme, H. R.

Symes, R.

Symington, A. W.

Symington, P. G.

Symington, R. E.

Symns, J. Ll. M.

Symns, J. M.

Symonds, E. T.

Symonds, F. C.

Symonds, S. L.

Symonds, W.P.

Symonds-Tayler, F. $K$.

Symonds-Tayler, R. H.

Symons, C. D.

Symons, H. J.

Symons, S. J.

Symons, W. J. F.

Sympson, E. M.

Sympson, T.M.

Tabberer, C. O.

Tabor,J. C.

Tacon, D. G. T.
Tadman, G. R.

71 Tagart, Sir H. A. L.

186 Taggart, W. Q.

462 Tait, G. B.

462 Tait, J. G.

304 Tait, M. W.

186 Tait, W. H.

186 Talbot, A. E.

506

273

57

462

186

186

154

524

186

342

155

I I I

357

57

155

506

462

57

214

506

155

155

304

155

273

214

506

506

462

186

230

357

155

462

I I I

462

506
Taffs, L. H.

Talbot, A. T.S.

Talbot, C. E. C.

Talbot, J.

Tall, J. J.

Tallerman, K. $\mathrm{H}$.

Talpur, Mir G. A.

Tanburn, W.L.

Tancock, E. O.

Tangye, R. T. G.

Tanner, E. V.

Tanner, G. R.

Tanner, H. O'S. F.

Tanner, H. R.

462

524

82

462

III

215

289

273

I55

I I I

273

57

462

316

155

82

57

524

462

I I I

22

186

Tanner, L. E. (Pem.) 273

Tanner, L. E. (Joh.) 342

Tanner, R. R.

Tanqueray, $T$.

Tansley, L. B.

Tapp, T.A.

Tatchell, E.

Tate, A. W.

Tate, J. E.

Tate, J. F. F.

Tate, Sir R. W.

Tatham, C. F.

Tatham, C. K.

Tatham, G. B.

Tatham, L. C. S.

Tavener, F.E.

Tayleur, W.

Taylor, A. A.

Taylor, A. E.

Taylor, A. J.

Taylor, A. L.

Taylor, A. R.

Taylor, B. W.

Taylor, C. C.

Taylor, C. H.

Taylor, C.H.S.

22

462

155

462

462

I86

273

342

57

462

462

462

316

506

304

82

215

463

155

316

316

22

Taylor, C.P. (Clare)

155

Taylor, C.P. (Caius) I5

Taylor, C. R.

Taylor, C. T.

Taylor, C. W. H.

Taylor, D. R.

Taylor, E. C.

Taylor, E. F.H.

Taylor, E. J. D.

Taylor, E. L. T.

Taylor, E. M.S. see

\section{55}

289

463

22

342

186

155

155

Seymour Taylor, E.M.
Taylor, E.S.

215

Taylor, F. 289

Taylor, F.C. $\quad 274$

Taylor, F. L. $\quad 342$

Taylor, G. C. $\quad 22$

Taylor, G. C. R. $\quad 230$

Taylor, G.H. I $\quad$ I55

Taylor, G. I. $\quad 463$

Taylor, G. M.C. $\quad 342$

Taylor, G. P. B.

see Brooke Taylor, G.P.

Taylor, G. R. M.S. 463

Taylor, G.S. $\quad 357$

Taylor, G. W. $\quad 463$

Taylor, H.C.N. $\quad 342$

Taylor, H.D. $\quad 463$

Taylor, H. G. $\quad 463$

Taylor, H. H. 274

Taylor, H. M. $\quad 463$

Taylor, H.M.S. $\quad 463$

Taylor, J. D. $\quad 534$

Taylor, J. F. $\quad 524$

Taylor, J. Holman see Holman, J. H. T.

Taylor, J.N.

Taylor, J.R.S. $\quad 342$

Taylor, J.T. $\quad-57$

Taylor, L.E. $\quad 463$

Taylor, L. F.M. $\quad 524$

Taylor, L. M. $\quad 274$

Taylor, M.L. $\quad 274$

Taylor, R. 304

Taylor, R. E. $\quad 274$

Taylor, R.F. 274

Taylor, R. H. H. $\quad$ I86

Taylor, R.S. $\quad 82$

Taylor, S. $\quad 463$

Taylor, S.H.S. I55

Taylor, S. S. 274

Taylor, W.E. $\quad 506$

Taylor, W. R. I 86

Teakle, S. G. 342

Teale, E. $\quad \mathbf{5 3 5}$

Teale, K. W. P. I I I

Teall, G.H. 342

Tebb, B. M. $\quad 289$

Tebb, R. N.S. $\quad 289$

Tebbs, J.A. 7 I

Tebbutt, R.J. 2 I 5

Tedder, A. W. 230

Teichmann, $O$. I 55

Telfer, A. C. $\quad \mathbf{5 2 4}$

Telfer, L.P. $\quad 357$

Telfer, R. G. $\quad 7$

Telfer, W. $\quad 57$, I 86

Telford, E. D. $\quad 155$

Temperley, A. C. $\quad 304$

Temperley, A. R. $\quad 57$

Temperley, C.E. $\quad 463$

Temperley, E.E. V. 357

Temperley, H.R. 
Temperley, H. W. V.

Tempest, F. L. 215,289

Temple, M.

Temple, R. J. G.

Templeman, W. H.

Templer, J. F. H.

Templer, W. F.

Tennant, B. V.A.

Tennant, C. G.

Tennant, H. V.

Tennant, N.R. D.

Tennant, W. G.

Tennyson, A. A.

Tennyson, L. H.

Terrell, F. W.

Terry, C. E.

Terry, H. V.

Terry, R. J.A.

Tetley, C. H.

Tetley, M. H.

Tetley, R. F.

Teuten, L. M.

Tewson, E. G.

Tha, R. R. H. O.

Thacker, C. R. A.

Thackeray, J. M.

Tharp, P. A.

Thatcher, W.S.

Theobald, A. C. L.

Theobald, G. W.

Theobald, R.

Theobald, W. G.

Theophilus, S. C.

Thicknesse, R. S.

Thirkill, $\mathrm{H}$.

Thoday, D.

Thoday, F.A.

Thom-Postlethwaite, A. C. S.

Thomas, A. A.

Thomas, A. G. I.

Thomas, A. H.

Thomas, A. L.

Thomas, B. H.

Thomas, D.C. W.

Thomas, D. E.

Thomas, E. A.

Thomas, E. C.

Thomas, E. F.

Thomas, E. M.

see Rocke, E. M.

Thomas, E. R.

Thomas, F. G.

Thomas, G. E. A.

see Aeron-Thomas, G.E.

Thomas, G.P. $\quad 82$

Thomas, H. E. E. $\quad 524$

Thomas, H. H.

Thomas, H. J.

Thomas, H. W. 524 463

Thomas, J.

Thomas, J. C. C.

Thomas, J. G. T.

Thomas, J. O.

289

342

463

274

274

463

463

274

463

463

463

71

230

357

463

463

463

274

I I I

155

57

82

274

304

535

463

316

463

23

274

57

464
82

274

23

274

316

186

274

I 55

316

23

I I I

I I I

I 55

82
524

82

57
215

Thomas, K. G.

Thomas, M. E.

Thomas, N. L.

Thomas, O.C.

Thomas, R. A.

Thomas, R. E.

Thomas, R. Ll.

Thomas, $\mathrm{T}$.

Thomas, T. G.

Thomas, T. J.

Thomas, T.P.

Thomas, W.

Thomas, W.W.

Thompson, E.

Thompson, J. C.

Thompson, $M$.

Thompson, N. F.

Thompson, O.S.

Thompson, P.A.

Thompson, R. C.

Thompson, R. D.
Thomas, K. D.P.

Thomas, L. D. C.

Thomas, O.V. (Jesus) I 86

Thomas, O.V.(Trin.) 464

Thomas, R. B. H.

23

Thomas, W.E.(Cath.) 3 I 6

Thomas,W.E.(Trin.) 464

342

Thomas-Peter, G. F. 57

Thomasset, G.T. $\quad 155$

Thompson, A. B. III

Thompson, A. C. $\quad 507$

Thompson, A. E. $\quad 23$

Thompson, A. G. G. 274

Thompson, A. H. J. 274

Thompson, A. P. $\quad 23$

Thompson, A. R. $\quad 342$

Thompson, B. C. $\quad 274$

Thompson, B. W. 215

Thompson, C. H. F. 464

Thompson, C.N. $\quad 342$

Thompson, D. H. 230

Thompson, E. E. $\quad 342$

Thompson, F. C. $\quad 464$

Thompson, G. $\quad 305$

Thompson, G. H. M. 23

Thompson, G. W. 23

Thompson, $\mathrm{H}$. $\quad 215$

Thompson, H. B. $\quad 305$

Thompson, H. C.S. 507

Thompson, H. J. $\quad 305$

Thompson, H.S. $\quad 464$

Thompson, J. 215

Thompson, J. B. $\quad 274$

Thompson, J. C. C. 275

Thompson, J.D. $\quad 464$

Thompson, J. H. G. 156
Thompson, R. G.

275

Thompson, R. M. $\quad \mathbf{5 6}$

Thompson, S. L. $\quad 342$

Thompson, S. W. $\quad 57$

Thompson, T.A. L.

see Lacey Thompson, T.A.

Thompson, T. W. $\quad 156$

Thompson, W. $\quad$ I86

Thompson, W. B. 316

Thompson,

W. F. (King's) $2 \times 5$

Thompson,

W. F.(Pemb.) 275

Thompson, W. J. $\quad 464$

Thompson, W.S. $\quad 305$

Thompson, W. W. 464

Thompstone, $R$. $\quad 156$

Thomson, D. G. $\quad 275$

Thomson, F. G. $\quad 357$

Thomson, G. D. $\quad 156$

Thomson, G. L. $\quad 507$

Thomson, G.P. 71,464

Thomson, J. $\quad 507$

Thomson, J. B. $\quad 464$

Thomson, J. G.O. $\quad 507$

Thomson, J. O. $\quad 464$

Thomson, K. S. $\quad 342$

Thomson, M.S. $\quad \mathbf{8 2}$

Thomson, N. G. III

Thomson, S. P. D. 275

Thomson, W. L. H. 275

Thorburn, E. C. $\quad \mathbf{5 2 4}$

Thorburn,K.D.S.M. 186

Thorburn, M. M. 275

Thoresby, M. (late

Thoresby-Jones) 464

Thorman, F.P. $\quad 305$

Thorman, J. L. $\quad 275$

Thorne, C. $\quad 57$

Thorne, F. J. $\quad \mathbf{1 8 6}$

Thorne, H.S. 23

Thorne-Waite, A. $\quad 342$

Thornely, R. R. $\quad 507$

Thornewill, C. C. $\quad 507$

Thorneycroft, K. H. 524

Thorneycroft, T. H. 275

Thornhill, G. R. $\quad 464$

Thornhill, N. 215

Thornton, A. C. $\quad 57$

Thornton, A. R. $\quad 275$

Thornton, C. C. $\quad 464$

Thornton, C. G. III

Thornton, E. $\quad 464$

Thornton, $\mathrm{F}$.

Thornton, F. R.

Thornton, G. L.

23 Thornton, G. R.

156 Thornton, J. G.

305 Thornton, L. H. 
Thornton, L. H. D. 112

Thornton, N.S.

Thornton, V.S.

Thornton, W. H. J. $\quad 187$

Thornycroft, $O$.

Thorp, B. L.

Thorp, C. F.

Thorp, D. B.

Thorp, E. B.

Thorp, H. C.

Thorp, R. C.

Thorp, R. O.V.

Thouless, R. H.

Threlfall, C. R.F.

Threlfall, R. E.

Threlfall, W. B.

Thresher, W.H.

Thurlow, A. G.

Thursby, P. H.

Thursby, W.

Thursfield, G. A. R. 343

Thursfield, R. M. R. 524

Thurston, E. T.

Thwaites, G.

Thynne, G. A. C.

Thynne, L. W.

Tiarks, J. G. E.

Ticehurst, C. B.

Ticehurst, G. A.

Tickell, E. J.

Tickell, G. W.

Tidman, O.P.

Tidy, T.

Tilbury, H. F.

Tilden Smith, J.

Till, W.S.

Tillard, E. R.

Tillard, L. B.

Tillard, P.S.

Tillard, T. A.

Tilley, J.

Tilly, J.

Tilly, R.L.

Tillyard, E. M. W.

Tilston, H. E.

Times, W. O.

Timins, F. C.

Timmis, R. B.

Tims, H. W. Marett 215

Tims, R. D. Marett 156

Tindall, $\mathrm{C}$.

Tindall, C. G.

Tindall, C.R.W.

Tindall, H.S.

Tindall, J. $\mathbf{H}$.

Tindall, R. F.

Tingey, W. R. H.

Tinker, B.

Tinker, H. W. C.

Tinline, G. C. M.

Tinsley, R. L.
464

Tinsley, R.P.

Tipper, G. H.

Tippet, A. A.

Tirard, N.S.

57

464

112
507

464

I 56

2 I 5

71

I56

I 56

I 56

I 56

I 56

57

505

23

343

275

507

464

343

343

464

230

I 56

112

524

507

507

275

343

275

465

215

275

I 2

I 87

524

465

57

465

275

305

275

289

275

507

230

465

\begin{tabular}{l|l}
507 & Torrey, J. \\
465 & Torry, A. J. D.
\end{tabular}

Titley, L. G.

Tod, A. K.

Todd, A. F.

Todd, E. W.

Todd, H. W.

Todd, J.

Todd, J. G.

Todd, L.

Todd, $M$.

Toller, G. R.

Toller, T.E.

Tomlin, C. G.

Tomlinson, $\mathrm{H}$.

Tomson, D. V.

Tonge, R. D.

Tonkin, R.S.

Tonks, J. W.

Toogood, J. H.

Toolis, J. H.

Toone, C. L.

Tooth, H. H.

Toovey, T.P.

Topham, D. B.

Topham, $M$.

Toplis, J.

Toppin, S. M.

Topping, A. R.

Torr, H. J.

Torr, J. H. G.

Torrey, C. E.

Torrey, J.
305

215

465

Tisdall, A. W. St C. 465

Tisdall, E. G. St C. $\quad 524$

Tisdall, J. T. St C. $\quad 289$

Tobias, G. W.R.

Todhunter, $\mathrm{H}$. W.

275

357

275

156

465

343

465

187

215

23

Todhunter,J.R.A.D. 156

Toler-Aylward, H.J. 507

Tollemache,A.H.W. 465

Tollemache, J. E. $\quad 230$

Tollemache, M. G. $\quad 465$

Tomblings, D. G.

Tomkins, L. C. F.

Tomkinson, G.S.

Tomlinson, A. E.

Tomlinson, F. R. J.

Tomlinson, T.S.

Tomlinson, W.A.

Tompson, A. H.

Tomson, W. J. M. see

Martin-Tomson, W. J.

Tonking, D. W.

Tootal, F. E. O.

Topham, H. A. C.

Topley, W. W. C.

Torrey, G.E.F.

465

57

524

535

215

112

465

524

465

112

465

289

J.

275

230

156

71

57

57

57

343

112

156

58

343

466

156

275

466

466

466

466

156

343
Tosswill, C. G.

466

Tottenham, C. E. L. 156

Toulmin, H. W. $\quad 507$

Toulmin-Smith,A.K. I 12

Tovey, D. $\quad 524$

Tower, F. F. $\quad 466$

Tower, G.E. $\quad 466$

Towle, J.H. $\quad 343$

Town, C. A. $\quad 187$

Townend, H. D. $\quad 305$

Townley, C.E. $\quad 466$

Townley, M. G. $\quad 466$

Townroe, G.C. 23

Townsend, A. L. H. 156

Townsend, J.H. $\quad 275$

Townsend, J. M. $\quad 275$

Townsend, J.S.E. $\quad 466$

Townsend, R.W. 343

Townshend, $\mathrm{H} . \quad 466$

Townson, B. A. 23

Towse, H. B. $\quad 466$

Toye, J. F. $\quad 466$

Tozer, E. F. $\quad 343$

Tozer, S. P. $\quad 343$

Tozer, W. $\quad 58$

Trachtenberg, M. I. 343

Tracy, G. C. $\quad 507$

Tracy, G. D.C. IIz

Tracy, S. J. $\quad 187$

Trafford, C. E. J. $\quad 466$

Trafford, G. T. $\quad{ }_{5} 6$

Traill, J.M. $\quad 507$

Traill, J. W. $\quad 507$

Traill, R. R. $\quad 466$

Trapnell, F. C. $\quad 215$

Trapnell, $\mathrm{H}$. $\quad 156$

Trasenster, W. A. $\quad 466$

Trautmann, H. F.

see Trewman, H. F.

Travis-Clegg, G.R. I 57

Treadgold, C. $\mathrm{H}$. $\quad 58$

Treadgold, H. A. $\quad 82$

Trechman, O. L. $\quad 5^{8}$

Tredcroft, J. L. $\quad 466$

Tredennick, J. N. E. 507

Tree, C. J. $\quad 275$

Tree, H.B.le D. $\quad 187$

Treeby, F. W. $\quad 466$

Tregelles, G.P. $\quad 157$

Treglown, C. J.H. 305

Tregoning, E. A. $\quad 466$

Tregoning, G. N. $\quad 466$

Treleaven, W. $\quad 343$

Tremearne, A. J. N. $\quad 23$

Tremearne, W. C. $\quad 23$

Trench, F.C. $\quad 466$

Trench, G. F. $\quad 525$

Trench, M.C. $\quad 275$

Trench, R.H. $\quad 466$

Trench, W. L. $\quad 466$

Trenchard, F.A. $\quad 507$ 
Trend, J. B.

Trendell, G. J. W.

Tresawna, W.S.

Trestrail, A.E. Y.

Treves, F. B.

Treves, W. W.

Trevor, F. P.

Trevor-Jones, E. E.

Trevor-Jones, J. E.

Trevor-Roper, C. C.

Trew, M. F.

Trewby, A.

Trewhella, C. B.

Trewman, H. F.

Tribe, K. W.

Trier, N.E.

Tringham, H. G. $\quad 230$

Tringham, H. R.P. $\quad 58$

Tripp, N. F.

Trist, L. H.

Tristram, U.H.

Tritton, H. L. M.

Trollope, T. A.

Trollope, T.C.S.

Trotman, S. R.

Trott, A. C.

Trott, F. W.

Trotter, C. L.

Trotter, C. W.

Trotter, J.

Trotter, J. F. A.

Trotter, J. M. Y.

Trotter, K. S.

Trotter, L. B. C.

Trotter, R. D.

Trought, $\mathrm{T}$.

Trouton, E. A.

Trouton, F. T.

Trower, G.S.

Troyte, G. J. Acland see Acland Troyte, G. J.

Trubshaw, A. R.

Trubshaw, C.S.

Trubshaw, H. E.

Truman, M. G.

Trumper, J. H.W.

Truscott, F. G.

Truscott, R. F.

Trusted, H. H.

Tryon, G. A.

Tuck, C. H.

Tuck, D. A.

Tuck, G. B. O.

Tuck, G. L. J.

Tuck, N.J.

Tucker, D.H.M.

Tucker, D.H.M.

Tucker, E. G.

Tuckett, J. E.S.

Tudor, O. C. O.

Tudor, R. G.

TudorOwen,F.H.G. 507

\section{8}

Tudsbery, F. C. T.

Tufnell, N.C.

Tufnell-Klug, M.W.T.

Tuke, F. H.

Tulloch, H. M.

Tunmer, E. J.E.

Tunnicliffe, G.H.

Turcan, J.S.

Turkington, J.S.

Turnbull, E. L.

Turnbull, J. B.

Turnbull, P.H.

Turnbull, R. W.

Turnbull, W. A.

Turnbull, W.E.

Turner, A.

Turner,A.C.(Emm.)

Turner,A.C.(Trin.)

Turner, A. W.

Turner, D.P.

Turner, E. M.

Turner, E.P.

Turner, F. G.

Turner, G. McD.

Turner, H. A.

Turner, H. C.

Turner, H. E. M.

Turner, H. F. A.

Turner, H. H.

Turner, H. J.

Turner, $\mathrm{H}$. W.

Turner, J.P.

Turner, J. R.

Turner, J.S.

Turner, J. T.

Turner, J. W.

Turner, J. W. C.

Turner, L. B.

Turner, R.(Queens')

Turner, R. (Joh.)

Turner, R. B.

Turner, R. L.

Turner, W.A.S.

Turner, W. H.

Turnly, J. E. A. L.

Turpin, J. K.

Turrall, R. G.

Tweddell, J. R. M.

Tweedie, F. I. G.

Tweedie, H. A.

Tweedie, J. M.

Tweedie, L. K.

Tweedy, O. M.

Tweedy, R. J.

Tweedy, W. R.

Tween, A.S.

Twentyman,D.C.T. 343

Twentyman, J. M.

Twentyman, W.P.

Twigg, G. W.
216

467

58

467

305

112

276

I 57

23

23

112

305

467

I 12

216

305

112

467
467

276

316

23

467

316

I 57

I 12

467

187

467

82

23
525

216

525

58

82

305

216

305

343

187
23

467

23

276

23

507

58

I 57

467

I 57

157

I 57

I 57

467

I 57

23

23

Twining, R. W.

Twisleton-WykehamFiennes, N. I.E. 276

Twomey, R. A. $\quad 467$

Twopeny, R.E.N. 467

Twyford, $\mathrm{H} . \quad \mathrm{I} 87$

Tyer, A. A. 216

Tyers, F. G. $\quad 276$

Tylden-Wright,W.R. 507

Tyler, H.H.F.M. 525

Tylor, G. C. $\quad 276$

Tyndale-Biscoe. C.J. 187

Tyndale-Biscoe, H. L'E.

187

Tyndall, A. G.

535

Tyrrell-Green, D.N. 187

Tyrwhitt, L. F. 230

Tyrwhitt-Drake,B.H. 276

Tyrwhitt Drake,

C.W.

467

Tytler, W.T.F.

157

see Fraser-Tytler,W.T.

Ubsdell, T. R.

Uhthoff, R. K.

I 87

Ullman, R. B.

157

Ullrich, E. H.

467

Uloth, A. W.

24
276

Ulyat, E. S.

467

Underhill Faithorne, C. F.

Ungoed, G. T.

Unna, P. J. H.

Unwin, C. H.

157

Unwin, E. F.

467

317

Unwin, H. A.R. $\quad 525$

Unwin, S. R.

Unwin, W.N.

Upjohn, W. M.

Upton, R.

Upward, H. A.

Upward, L. V.

Ure, C. McG.

Urie, R. W.

525

58

467 
Valentine, H. T.

Valentine, W. H.

Vallance, H. I. A.

Valle-Pope, E.

Vance, J. G.

Van der Byl, A. H.

Van der Byl, A. M.

Van der Byl, C. F.

Van der Byl, P. B.

Van der Byl, P. V.

van Druten, H. J.

Van Duzer, F. C.

Van Duzer, S. R.

Vane, C. W.

see Barnard, Lord

Vane, R. F.

Vane, W.L.

Vane-Tempest,

$$
\text { E. C.W. }
$$

Van Gehuchten, P.

van Grutten,W.N.C.

Vann, A.H. A. 187

Vann, B. W.

Van Praagh, R. B. $\quad 468$

Van Raalte, N. M. $\quad 468$

Vanrenen, J. E.

Van Schaick, J. B. $\quad 187$

vanSomeren,H.A.A. 276

Vardy, A. T.

Varley, G.

Varley, L.

Varvill, J. K.

Varwell, R.P.

Vatcher, H. M.

Vaudrey, W. E.

Vaughan, E.W. B. 468

Vaughan, J. H.

Vaughan, R. B.

Vaughan-Williams, R.' 468

Vaulkhard, J. V. $\quad 508$

Vause, T. C.

Vaux, R. W.

Veale, H.P.

Veater, A. W.

Veevers, W.

Veitch, A. G.

Veitch, J. L.

Vellacott, P.C.

Venn, J. A.

Venning, J. A.

Verdon, $P$.

Vere Hodge, H. S. see Hodge, H. S. Vere

Vereker, G. G. M. 468

Vereker, S. R.

Verey, H. E.

Verner, G. W. H.

Verniquet, W. G.

Vernon, C. H.

Vernon, J. C.

Vernon, R. J.

c. U.W.L.

58

24

468

344

305

305

289

468

468

I 88

468

468

157
Vick, R. M.

Vickers, $R$.

Vickers, $S$.

Vickers, V. C. W.

Viggers, S.

Vigo, J.D.

Vigurs, C.C.

Villar, R.P.

Vincent, C. A.

Vincent, H. G.

Vincent, L. J.

Vincent, R. B.

Vincent, T. C. L.

Vining, L. G.

Vint, A. W.

Vint, $J$.

Vint, M. D.

Vinter, P.J.

Vipond, J. H.

Vischer, $\mathrm{H}$.

Vischer, M. M.

Vivian, C. St A.

Vivian, G. L.

Vivian, O.R.

Vizard, W. G.

Voelcker, H. E.

Vogel, E.P.

see Oscroft, E. P.

Von Schröder,

Vos, $\mathbf{P}$.

Baron W. $H$.

Vos, $\mathrm{S}$.

Vulliamy, E. O.

Vyvyan, E. C. F.

Vyvyan, M.C.

Vyvyan, P.H.N.N. 57

Wace, H. E.

Waddington, J. E.

Waddy, A. C.

Waddy, J. R.

Waddy, R. A.

Wade, E. B. H.

Wade, H. B.

Wade, $\mathrm{H}$. $\mathrm{O}$.

Wade, $R$.

Wadeson, E. Y.

Wadham, S. M

Ir3 Wadham, W. F.A.

344 Wadley, H. W. A.

Wadson, F. P.

Wadsworth, J.
468

468

113

468

I 57

113

71

276

82

276

276

188

535

468

535

II 3

24

344

24

468

276

II 3

II 3

157

525

468

468

24

Wadsworth, S.

r88

Waechter, Sir H. $\quad 469$

Wagg, F. J. $\quad 305$

Waggett, E. B. $\quad 277$

Waggett, P.N. $\quad 469$

Wagner, R. H. $\quad 24$

Waiapu, Bp. of 24

Wailes, F.G. II

Wailes-Fairbairn,

N.W. F.

Wailes-Fairbairn, W. F.

Wainwright, B. M

Wainwright, C. B.

Wainwright, G.C.

Wainwright, G. L. 24

Wainwright, L. A. $\quad 305$

Wainwright, R. C. $\quad 469$

Wait, C. F. W. $\quad 525$

Wait, J.A. $\quad 344$

Wait, R. J. $\quad$ I88

Waite, T.H. $\quad 535$

Waite, W. F. 82

Waithman, J. C. $23 I$

Wakefield, A. H. $\quad 3$ I7

Wakefield, A. W. $\quad 469$

Wakefield, E. W. $\quad 469$

Wakefield, H. R.

see Birmingham, Bp. of

Wakefield, J. H. $\quad 469$

Wakefield, W. V. $\quad$ I 88

Wakeford, E. K. $\quad 469$

Wakelam, H. B. T. $\quad 277$

Wakerley, A. J. $\quad 290$

Walbank, C. F. $\quad 317$

Waldegrave, G. T. II3

Waldegrave, S. C. II 3

Walden-Vincent,A.C. 277

Waldram, H. G. $\quad 357$

Wale, E.H. $\quad 469$

157
469 Wales, $\mathrm{H}$. $\quad 357$

216 Wales, H. R.

277 Waley, F.R. 216

I 57 Walford, H. H. I 158

Walker, A. (Emm.) II3

Walker, A. (Pemb.) 277

277

II3

158

277

24

469

277

469

24

525

24

277

525

158

469
Walker, A. C. I 58

Walker, A. E. J. $\quad 188$

Walker, A. N. $\quad 305$

Walker, A. R. $\quad$ I13

Walker, B. $\quad \mathbf{8 2}$

Walker, B.S. $\quad 216$

Walker, C. A. P. $\quad 158$

Walker, C. C. $\quad 508$

Walker, C. D. $\quad \mathbf{5 3 5}$

Walker, C. V. $\quad 59$

Walker, D. (Caius) 158

Walker, D. (Pemb.) 277

Walker, D. G.

Walker, E. A.

24
216
Walker, C. E. $\quad 59$ 
Walker, E. B.

Walker, E. E.

Walker, E. M.

Walker, E. R. C

Walker, F. C.

Walker, F. G.

Walker, G. A. C

Walker, G. F.

Walker, G.S.

Walker, $\mathrm{H}$.

Walker, H.C. (Jesus) I 88

Walker, H. C. (Kg's) 216

Walker, H. R.

Walker, J. (Clare)

Walker, J. (King's)

Walker, J. A.

Walker, J. E.

Walker, J. Ness see Ness Walker, J.

Walker, J. P. E.

Walker, K M. (Chr) 469

Walker, K. M. (Cai.) I 58

Walker, K. P.

Walker, L. H. T.

Walker, L. P.

Walker, L. W. L.

Walker, M. A.

Walker, M. J. L.

Walker, N.O.

Walker, O.R.

Walker, $\mathbf{R}$.

Walker, Sir R. J. M. 469

Walker, R. W.S. $\quad 469$

Walker, S. L.

Walker, S. W.

Walker, T. A.

Walker, T. C.

Walker, V. A.

Walker, W. E.

Walker, W. J.

Walker, W. M.

Walkey, J. R.

Wall, A. H.

Wall, $T$.

Wallace, A. W. W.

Wallace, C. R.P.

Wallace, F. W.

Wallace, G. L'E.

Wallace, H. L.

Wallace, J.

Wallace, J. A. V.

Wallace, J. C.

Wallace, J. Hope see Hope-Wallace, J.

Wallace, R. W. J. $\quad{ }_{15} 8$

Wallace, W.E. $\quad 470$

Wallace, W. M. 216

Waller, B. P.

Waller, F.

Waller, H. K.

Waller, J. C.
Waller, N. H.

Waller, W. H.

Walley, G.S.

Wallice, $P$.

Wallinger, E. A.

Wallis, D. B.

Wallis, E. P.

Wallis, G. D.

Wallis, H. H.

Wallis, O. B.

Wallis, P. B.

Wallis, R. L. M.

Wallis, W. E.

Walls, F. R.

Walmesley, $\mathrm{R}$.

Walmsley, A. M.

Walpole, R. H.

Walrond, $\mathrm{V}$.

Walsh, A. D.

Walsh, A. St G.

Walsh, E.S.

Walsh, P.

Walter, C.

Walter, C. E. H. L.

Walter, G. L.

Walter, H.C.

Walters, A. M.

Walters, G. Y. L.

Walters, W. J.

Walters, W. L.

Walthall, H. D. D.

Walton, G. M.

Walton, J. H.

Wane, H. B.

Wanklyn, K.

Wanklyn, W. McC.

Wanliss, D. S.

Warburg, O.E.

Ward, A.

Ward, C. B.

Ward, D. C. L.

Ward, E. H.

Ward, F. K.

Ward, F. W.

Ward, G. H.

Ward, H. L.

Ward, H. M. A.

Ward, J. G.

Ward, J. S. M.

Ward, O.W.

Ward, $R$.

Ward, R. A.

Ward, R. O. C.

Ward, R. S.

Ward, R. W. A.

Ward, W.D.

Ward, W. Dudley

Ward, W. H.

Ward, W. J.

\begin{tabular}{l|l}
470 & Ward-Jackson, R. \\
216 & Ward-Price, L.S.
\end{tabular}
II 3

508

59

216

59

277

59

277

24

II 3

277

82

59

216

231

344

$23 \mathrm{I}$

470

470

508

82

470

$35^{8}$

508

$23 \mathrm{I}$

290

470

470

59

470

I 3

535

216

59

277

$\begin{array}{r}58 \\ \hline\end{array}$

470

470

71

470

71

$35^{8}$

24

59

158

508

59

525

508

277

24

508

470

277

535

$35^{8}$

470

59

470

470

317
Warde-Aldam, W. St A.

Wardell, J.M. $\quad 470$

Wardell-Yerburgh, G. B.

Warden, A.R. S $\quad 470$

Warden, G.

Warden, H. F.W. $\quad 158$

Wardle, W.L.

Wardley, D. J. $\quad 525$

Wardley, G.C.N. $\quad 470$

Ware, B. G. $\quad 508$

Ware, B. O. $\quad$ I 88

Ware, F.H. $\quad 470$

Ware, J. G. W. $\quad 470$

Ware, M.S. $\quad 470$

Warington Smyth, $\mathrm{H}$. see Smyth, H. W.

Warlow, N.F.

Warmington, E. S. 59

Warner, A. L. 470

Warner, C. 277

Warner, $M$. $\quad{ }_{58}$

Warner, T. L. $\quad 158$

Warnes, G. G. $\quad$ I 58

Warre-Cornish, G.W. 2 I6

Warre-Cornish,W.H. 216

Warre Dymond,G.W.470

Warren, A.C. II3

Warren, C. $\quad 535$

Warren, C. K. W. $\quad$ I88

Warren, G. M. $\quad 277$

$\begin{array}{ll}\text { Warren, J. L. E. } & 344 \\ \text { Warren, L. A. } & \mathbf{1} 88\end{array}$

Warren, S. $\quad 24$

Warrington, J.C. $47 \mathrm{I}$

Warrington, T. I I 3,47 I

Warry, H.E. $\quad 277$

Warwick, H. B. $\quad 47 \mathrm{I}$

Wasbrough, H. C. S. 508

Wasbrough, W. L. $47 \mathrm{I}$

Waterall, L.S. $\quad$ I 88

Waterer, C. R. II

Waterhouse, C. $\quad 508$

Waterhouse, G. $\quad 344$

Waterhouse, $\mathrm{H} . \quad 344$

Waterlow, G.W. 47 I

Watermeyer, E. F. $\quad$ I 58

Watermeyer, H. A. $\quad 158$

Waters, G. M. $\quad 317$

Waters, J. B. $\quad 277$

Waters, K.S. $\quad 344$

Waterworth, $\mathrm{H}$. $\quad 24$

Waterworth, S. $\quad 15^{8}$

Watkins, C. R. II3

Watkins, D. J. G. $\quad 158$

Watkins, E. L.C. II3

Watkins, E. V. $\quad 35^{8}$

Watkins, I. E. M. $\quad 216$

Watkins, M. $\quad 535$

Watkins, W. B. $\quad 158$ 
Watkins, W.F.(Chr.) 24 Watkins, W. F. (Emm.) II4

Watkyn-Thomas, A. 47 I Watkyn-Thomas, F. W.

Watney, C. N.

Watney, G. N.

Watney, M.H.

Watney, W. H.

Watson, A. B.

Watson, A. H.

Watson, A.P.

Watson, A. T.

Watson, A. W. H.

Watson, B. B.

Watson, B. L.

Watson, C. B.

Watson, C. C.

Watson, D. H.

Watson, D.P.

Watson, F. B.

Watson, F. H.

Watson, F. W.

Watson, G. C.

Watson, G. T.

Watson, H. (Chr.)

Watson, H. (Trin.)

Watson, H. N.

Watson, H.S.

Watson, J. A.

Watson, J. B.

Watson, K. F.

Watson, N. W.

Watson, $R$.

Watson, R. A.

Watson, R. B. W.

Watson, R. E.

Watson,

R.H. Lindsay $47 \mathrm{I}$

Watson, R. W.

$47 \mathrm{I}$

$47 \mathrm{I}$

471

508

277

216

508

59

471

344

231

216

.59

$47 I$

471

I58

217

217

290

24

471

508

24

189

$47 \mathrm{I}$

231

305

24

471

$47 x$

317

Watson, T. H. (Jes.) 189

Watson,T.H.(Pem.) 277

Watson,T.H.(Tr.H.) 508

Watson, T.P.

Watson, T. T. B

Watson, W. G.

Watson, W. N.

$35^{8}$

24

I 58

317

Watson-Armstrong, W. J.M.

471

Watson-Scott, C. H. 277

Watson-Taylor, A.S. 47 I

Watson-Taylor,C.A. 47 I

Watson Williams, E. 158

Watt, A. F.

189

Watt, A. S.

535

Watt, G. T. C. $\quad 158$

Watt, W.O. $47 \mathrm{I}$

Watts, A. E. (Down.) 83

Watts, A.E. (Trin.) $47 \mathrm{I}$

Watts, B. H.
Watts, E. M.

Watts, H. L.

Watts, N. H.

Watts, R. W. A.

Wauchope, D. A.

Waugh, A. D.

Waugh, A. J.

Waugh, G. E.

Wauton, A. D. B.

Wauton, E. A.

Wayet, J. W. F.

Waylen, D. C.

Waylen, G.H. H. 217

Wayman, G. B.

Wayne, St J.

Weatherell, R. K.

Weatherhead, E.344, 47 I

Weatherhead, R. $7 \mathrm{I}$

Weaver, A. B. $\quad 47 \mathrm{I}$

Weaver, F. W. H. $\quad 59$

Weaver, J.

535

Weaver-Adams,E.R. ${ }_{5} 8$

Webb, A. H. (Chr.) 24

Webb, A.H. (Corp.) 7 I

Webb, E. O.

Webb, F.E.A.

Webb, F.H.

Webb, G. C. N.

Webb, H. C.

Webb, J. B.

Webb, J. C. (Clare)

Webb, J. C. (Jesus) 8

Webb, J.H. J. $\quad 278$

Webb, M.E. $\quad 278$

Webb, T.L. $\quad 158$

Webb-Benton, J. $\quad 358$

Webb-Peploe, H. M. 278

Webb-Peploe, M. H. 472

Weber, D. McR. $\quad 59$

Weber, H. G.

Weber, R. E.

Weber, W.E.

Webster, C. K.

Webster, $\mathrm{H}$.

Webster, J.F. (Tr.) 317

Webster,J.F.(Selw.) 525

Webster, J. R.

Webster, R. B.

Webster, R. G. $\quad 472$

Webster, S. W. $\quad 508$

Webster, V.T.P. II4

Webster, W. G. $\quad 508$

Wedd, E.P. W. $\quad 158$

Weddell, J. M. $\quad 25$

Wedderburn, C.C. $\quad 278$

Wedgwood, A. $\quad$ I59

Wedgwood, A. F. $\quad 472$

Wedgwood, F.H. $\quad 472$

Wedgwood, R. L. $\quad 472$

Weekes, C.R.H. $\quad 472$

Weeks, LI. M.
Weeks, R. M.

I 59

Wegg, H. N. I59

Wegg, W. H. J. I 59

Wegg-Prosser, C. F. 472

Weguelin, T.N. 472

Weigall, G. J. V. I I 4

Weinberg, P. D. 217

Weir, G.A. $\quad 472$

Weir, H. B. 472

Weir, H. H. 472

Weir, N. 290

Welbourne, E. $\quad I 14$

Welch, D. I89

Welch, J. $\quad 472$

Welch, J. J. $\quad 472,525$

Welch, J.S. L. $\quad 217$

Weld, C. G. II4

Weld-Forester, G. C. B. see Forester, Lord

Weld-Forester,

$$
\text { O.St M. }
$$

Weldon, Sir A. A. $\quad 472$

Welinkar, S.C. $\quad 189$

Weller, C. A. $\quad 59$

Weller, C. H. $\quad \mathbf{5 2 5}$

Weller, H. J. $\quad 358$

Weller, J. R. $\quad 525$

Wells, A. P. $\quad 525$

Wells, C. A. II4

Wells, C. D. II4

Wells, E. $\quad 278$

Wells, F. A. $\quad 344$

Wells, H. M. $\quad 7 \mathrm{I}$

Wells, J. P. $\quad 278$

Wells, L. H. E. $\quad 306$

Wells, N. L. $\quad 159$

Wells, R. C. $\quad$ II 4

Wells, W. D. $\quad 344$

Wells-Cole, G. C. $\quad$ I 59

Welsby, S. W. H. $\quad 508$

Welsford, A. G. $\quad$ I59

Welsford, G. J. L. $\quad 159$

Welsh, A. R. $\quad 472$

Wendell, J. $\quad 472$

Wenden, G. $\quad 278$

Wenham, C. H. $\quad 472$

Wenham, E. H. $\quad 472$

Wenham, J.H. $\quad 472$

Wenham, R. A. $\quad 217$

Wenley, J. A.S. $\quad 189$

Wentworth-Fitzwilliam, G. J. C. $\quad 472$

Werner, C. A. $\quad 2 \times 7$

Wernicke, W. G.

see Verniquet, W. G.

Wessels, C.H. $\quad 509$

West, C.S.

West, E. B.

West, E. E.

West, F. R.

West, P.C.

\section{3}

473

473

I 59

I 59 
West, R. R. F.

217

West-Watson, C.

see Barrow-in-Furness, Bp. of

Westall, B. C.

Westall, D. C.

Westbrook, H. W.

Westbrook, H. W

Westby, F. W. 535

Westby, P.St G. C. 473

Westcott, G. F. $\quad 306$

Western, O. 473

Westhead, W. H. $\quad 358$

Westmacott, P. G. $\quad 473$

Westoll, J.

Weston, E. A.

Weston, G. E.

Weston, G.H.

Weston, J. C.

Weston, T. A.

Westwood, A. H.

Wetenhall, W. T.

Wethered, H. E.

Whadcoat, W. H.

Whale, A.

Whale, G.

Whale, G.H.

Whaley,

Wharton, C. H. L. $\quad 525$

Wharton, F. H. $\quad 509$

Wharton, J.

Wharton, J. R.

Wharton, W. H.A. 231

Whatford, G. L. $\quad 473$

Whatham, A. $\quad 59$

Whatley, W.H. $\quad 473$

Whatman, A. D. $\quad 509$

Whatman, W. D. $\quad 473$

Whatton, A. B. W. 473

Wheat, E. G.

Wheatcroft, G. H.

Wheeler, A. M.

Wheeler, E. V. V.

Wheeler, F. O.

Wheeler, H. L.

Wheeler, H. W.

Wheeler, J. N.

Wheeler, $\mathrm{M}$.

Wheeler, R. M.

Wheeler, S. M.

Wheeler, W. R.

Wheldon, E. J.

Wheldon, W.P.

Whelon, C. E.

Whelpton, L. G.

Whelpton, $M$.

Wherry, G. E.

Whidborne, B.S.

Whidborne, G. F.

Whiddington, $\mathrm{R}$.

Whiffen, T. W.

Whistler, F.

217

189

278

83

473

473

344

509
Whistler, W. W.

I 4

Whitaker, F.(Pemb.) 278

Whitaker, F. (Trin.) 473

Whitaker, G. H.

Whitaker, G. M.

59

Whitaker, J. L.

Whitaker, T.S.

I 14

Whitaker, V.

Whitby, J.H.

Whitby, L.E. H.

Whitchurch, R.H.S.G.

509

159

473

I59

83

Whitcombe, E.P. I $\quad$ I 59

Whitcombe, R. C.P. 217

Whitcombe, R. H. $\quad$ I 59

White, A.E. $\quad 278$

White, A. H.

White, A. J.

White, A. K. G.

White, A.S.

White, Sir A. W.

White, $\mathrm{B}$.

White, C. F. O.

White, C. W. M.

White, E A.S.

White, F.

White F B Howard

White, F.R.

White, G. C. W.

White, G. E.

White, G.F.

White, G. G.

White, G. W.

White, H. A.

White, H. E.

White, J.

White, J. D. C.

White, J. L.

White, K. H.

White, L.

White, L. T.

White, L. W.

White, $M$.

White, M. G.

White, R. A. R.

White, R. D.

White, R.E.

White, R. Hale

White, R. J.

Whitefield, C. G.

217

535

60

217

473

278

473

I 59

473

I 14

473

317

525

317

I 59

189

535

535

473

189

473

535

358

25

60

474

I 89

217

278

474

474

217

509

231

I 59

Whitehead, B. 60

Whitehead, C. E.

I 4 Whitehead, F. $\quad 474$

Whitehead, H. M. 25

Whitehead, J. $\quad 509$

Whitehead, J. H. M. 25

Whitehead, J. R. G. 474
Whitehead, R. B. $\quad 25$

Whitehead, R. F. II4

Whitehead, R. H.H. 60

Whitehead, R. W. $\quad 474$

Whitehead, T.N. 474

WhiteheadReid,E.D. 25

Whitehorn, R. D. $\quad 474$

Whitehouse, H.E. 290

Whitelaw, J. B.

Whitelegge, M.H. $\quad 278$

Whiteley, G.T. $\quad 344$

Whiteman, R.J.N. $\quad 25$

Whitfeld, N. B. $\quad 509$

Whitfield, E. H. D. 344

Whitfield, E.O. $\quad 159$

Whitfield, J. B. $\quad 306$

Whitfield, J. G. $\quad 60$

Whitfield, J. L. $\quad 535$

Whitfield, J.O. $\quad 278$

Whitfield, S.P. $\quad 474$

Whitham, A. $\quad 317$

Whitham, J. W. $\quad 474$

Whiting, E. R.S. M. 159

Whiting, M.H. $\quad \mathbf{8} 3$

Whitlark, J.H. $\quad 474$

Whitley, E. N. $\quad 474$

Whitley, N.H.P. $\quad 114$

Whitmill, G.H. $\quad 535$

Whitmore, A. $\quad 159$

Whitmore, C. J.R. $\quad 83$

Whitmore, H.S. $\quad 35^{8}$

Whittaker, G. 290

Whittall, H. C. $\quad 159$

Whittam, M.J.G. $7 \mathbf{I}$

Whitting, R. E. $\quad 217$

Whittingdale, J. $\quad \mathbf{8}_{3}$

Whittington, T. $\quad 474$

Whittle, D. $\quad 474$

Whittome, L. $\quad 23 \mathrm{I}$

Whitty, H.N. $\quad 160$

Whitty, L. W. $\quad 306$

Whitwill, T.N. II4

Whitworth, A. G.R. 83

Whitworth, E. E.A. 474

Whitworth, G. E. A. 474

Whitworth, J. I60

Whitworth, W.H.A. 474

Whye, J. W. 345

Whyte Venables,H.A. 306

Whytehead, H. L. $\quad \mathbf{5 2 5}$

Wickham, B. W.T. 345

Wickham, W. J. $\quad 509$

Widdicombe, E.P. $\quad 83,160$

Whitehead, E. T. 290 Widdowson, F. J. 474

Whitehead, P.N. $\quad 60$
Widgery, G.H. $\quad 217$

Wiggans, J.T.V. 217

Wiggin, Sir C. R. H. 474

Wiggin, G. R. $\quad 474$

Wiggin, N.H. $\quad 160$

Wiggin, W.H. $\quad 474$ 
Wiglesworth, G.

Wigley, W.C.S.

Wigmore, J. B.A.

Wigram, C. K.

Wigram, H. F. E.

Wilberforce, $\dot{H}$. $\mathbf{H}$.

Wilcock, J. A.

Wilcox, A. G.

Wilcox, A. J.

Wild, J.A.P.

Wilde, C.A.G.

Wilde, E. H. N.

Wilde, R. W.

Wilderspin, B. C.

Wilding, A. F.

Wileman, G. W. B.

Wilenkin, C.

Wiles, H.H.

Wiles, J. J.

Wiles, J. W.

Wilkes, J. F.

Wilkin, A.

Wilkin, W. H.

Wilkin, W. R.

Wilkinson, C. A.

Wilkinson, C.F.W. 358

Wilkinson, C. L. G.

Wilkinson, E.

Wilkinson, E. F.

Wilkinson, $\mathbf{F}$.

Wilkinson,

Wilkinson, F.C.(Jes.) I 89

Wilkinson, G.(Sid.) 358

Wilkinson, G. A. W. 306

Wilkinson, G. J. $\quad 160$

Wilkinson, G.R. $\quad 475$

Wilkinson, H. A. $\quad 278$

Wilkinson, H.E.T. 509

Wilkinson, H. R. $\quad 475$

Wilkinson, J.R. $\quad 306$

Wilkinson, $\mathrm{K}$. $\quad 278$

Wilkinson, M. L. $\quad 306$

Wilkinson, $N$. $\quad 160$

Wilkinson, R.du C. 60

Wilkinson, R.J. 475

Wilkinson, $\mathrm{S}$.

Wilkinson, S. J.

Wilkinson, V.

Wilkinson, W. R.

Wilks, E. L.

Wilks, J. H.

Will, J. G.

Willan, G.T.

Willans, E.T.

Willans, R.St J.

Willcocks, R. H.

Willcocks, R. W.

Willett, E. W.

Willett, J. A.

Willett, W.L.

Willey, $\mathrm{B}$.

475

217

160

345

345

475

290
Willey, $\mathbf{H}$.

Willey, H. L.

Willey, R. H. D.

Williams, A.

Williams, A. C.

Williams, A. D. B 160

Williams, A. F. $\quad 160$

Williams, A. G. $\quad 160$

Williams, A. J. (Cai.) I60

Williams, A. J. (Pet.) 290

Williams, A. K. $\quad 475$

Williams, A. L. $\quad 189$

Williams, A.S. $\quad 525$

Williams, C. (Caius) 160

Williams, C. (Trin.) 475

Williams, C. E. (Cai.) 160

Williams,C.E.(Trin.) 475

Williams, C. H. $\quad 358$

Williams, C. J. $\quad 60$

Williams, C. M. $\quad 25$

Williams, C.S. $\quad 189$

Williams, D. G. $\quad 25$

Williams, E.

Williams, E. A. $\quad 278$

Williams, E. C. 25

Williams, E. G. $\quad 475$

Williams, E. H.Y. $\quad 60$

Williams, E.K. $\quad 160$

Williams, F.F.S. $\quad 189$

Williams, F. J. $\quad 509$

Williams, F. L. 25

Williams, G. (Emm.) I I 4

Williams, G. (Trin.) 475

Williams, G.A. 231

Williams, G. C. $\quad 160$

Williams, G.D. $\quad 7$ I

Williams, G.H. $\quad 475$

Williams, G.T. $\quad 475$

Williams, G.V. $\quad 290$

Williams, G. W. $\quad 345$

Williams, $\mathrm{H}$.

Williams, H.A. II5

Williams, H. B. $\quad 345$

Williams,H.F.F. 60, 189

Williams, H. G. $\quad 317$

Williams, H. G. E. 217

Williams, H.H. 25

Williams,H.P.W.B. 115

Williams, I. A. $\quad 217$

Williams, J.C.S. 160

Williams, J.E.

Williams, J. G.

Williams, J. L. C. $\quad 160$

114 Williams, J.S. 160
Williams, K. G.

Williams, $\mathrm{L}$.

Williams, M. B.

Williams, N.A.

Williams, N.D.

Williams, N.S.

Williams, O. H.
Williams, O.St M. II5

Williams, P. $\quad 475$

Williams, R. (Magd.) $23 \mathrm{I}$

Williams, R. (Joh.) 345

Williams, R. A. $\quad \mathbf{5 2 5}$

Williams, R.A. W. 217

Williams, R.B. (Cai.) 160

Williams,R.B.(Pem.) 278

Williams, R. C. L. 290 Williams,

R. D. Garnons 475

Williams, R.F.(Cai.) 160

Williams,R.F.(Selw.)525

Williams, R. G. I60

Williams,S.C.(Cai.) 16I

Williams,S.C.(Trin.) 475

Williams, S.R. I6I

Williams, T. 290

Williams, T. B. I6I

Williams, T. C. L. $\quad 535$

Williams, T.P. $\quad 83$

Williams, T.R. $\quad 278$

Williams, W. C. B. $\quad 278$

Williams, W. D. 25

Williams, W. E. $\quad 278$

Williams, W. F. $\quad 60$

Williams, W. H. $\quad 345$

Williams, W. J. $\quad 83$

Williams, W.P. $\quad$ I6I

Williams, W.P. G. $\quad 83$

Williams, W.S. 317

Williams-Ellis, B. C. 475

Williams-Ellis, R. G. $35^{8}$

Williams-Freeman, A. P.

475

Williams-Green,W.T.475

Williams-Thomas, F.S.

475

Williams Wynn, W. 475

Williamson, A. J. N. 278

Williamson, C. G. $23 \mathrm{I}$

Williamson, D.A. $\quad 475$

Williamson, F. II5

Williamson, F.A. $\quad 306$

Williamson, F.L. $\quad 71$

Williamson, G. E. $\quad 476$

Williamson, G.H. II 5

Williamson, $\mathrm{H}$. $\quad 345$

Williamson, J. B. $\quad 476$

Williamson, J. M. I6I

Williamson, J. N. $\quad 278$

Williamson, K. B. $\quad 345$

Williamson, R.H.W. 2 I8

Williamson, R.S. $\quad 25$

Williamson, S. K. G. 476

Williamson, T.R. $\quad 306$

Willink, A. H. $\quad 278$

Willink, A. J.W. $\quad 218$

Willink, H. J. L. I6I

Willink, H. U. $\quad 476$

306 Willis, A. G. de L. II5 
Willis, A. G. F.

Willis, R.E.

Willis, R. E. B.

290

Willock, G.C. B. 218

Willoughby, C. S. H. D. see Heathcote-Drummond-Willoughby, C. S.

Willoughby, F.G.G 476 Willoughby, Sir J. C. 476

Willox, A. G. 60

Wills, A. G. $\quad 218$

Wills, A. G. P. $7 \mathrm{I}$

Wills, A.S. $\quad$ II 5

Wills, J.P.

Wills, O. B. W.

Wills, R. B. M.

Wills, R. D.

Wills, R. G.

Wills, S. W.

Wills, W. D. M. $\quad 476$

Wills, W. K. $\quad 345$

Wills-Sandford,T.G. 509

Willson, F. J. II 5

Wilson, A.

Wilson, A. C.

Wilson, A. E.

Wilson, A. E. J.

Wilson, A. G.

60

476

60

60

I6I

Wilson,A.G.Maryon 476

Wilson, A. H. $\quad 278$

Wilson, A. H. R. $\quad 476$

Wilson, A. L. $\quad 278$

Wilson, A.P. $\quad 278$

Wilson,A.S.(Magd.) 23 I

Wilson, A. S. (Joh.) 345

Wilson, A. W. $\quad 345$

Wilson, B. $\quad 358$

Wilson, B. C. $\quad 306$

Wilson, B. FitzG. $\quad 476$

Wilson, C. $\quad 476$

Wilson, C. E.

Wilson, C. J.

Wilson, C. T.

Wilson, D. R.

Wilson,E. (Christ's)

Wilson, E. (Pemb.) 279

Wilson, E. C.

Wilson, E. R. (Trin.) 476

Wilson,E.R.(Tr.H.) 509

Wilson, F.A.

Wilson, F. B.

Wilson, G. (Joh.) 345

Wilson, G. (Trin.) $\quad 476$

Wilson, G. B.

Wilson, G. H. A.

Wilson, G. H. B.

Wilson, G. M.

Wilson, $\mathrm{H}$.

Wilson, H. B.

Wilson, H. F.

279

115

279
Wilson, H. G.

Wilson, H. L.

Wilson, H. M.

Wilson, H.N.S.

Wilson, H. O.S.

Wilson, H.S.

Wilson, H. V.

Wilson, H. W.

Wilson, J. C.

Wilson, J.P.

Wilson, J.R.M.

Wilson, J.S. (Kg's)

Wilson, J.S. (Trin) 476

Wilson, J.S. (Selw.) 525

Wilson, J. V.

Wilson, K. F.

Wilson, K.H.

Wilson, L. C.

Wilson, L. E.

Wilson, L. G.

Wilson, P. M.

Wilson, $\mathrm{R}$.

161

Wilson, R. E. (Corp.) 71

Wilson, R. E. (Trin.) 477

Wilson, R. K.

Wilson, R. N.P.

Wilson, R.S.

Wilson, $T$.

Wilson, T. B.

Wilson, T. I. W.

Wilson, T. M.

Wilson, W. A.

Wilson, W.E.

Wilson, W. G.

Wilson, W. H.

Wilson, W. R.

Wilson-Barkworth, K. A.

Wilson-Todd, J.H

Wilton, J.P.

Wilton, S. B.

Wilton, T.R.

Wiltshire, H. G.

Wiltshire, $\mathrm{H}$. W.

Wimberley, D. N.

Wimborne, Lord

Wimbush, G.S.

Wimbush, H. G.

Wimbush, R.

Wimperis, H. E.

Winby, I.S.

Winby, L.P.

Winch, A. B.

Winch, E. M.

Winch, George B.

Winch, Gordon B.

Winch, R. B.

Winch, S. B.

Winder, J. F.E.

Winder, R. McD.

Windley, F.M.
60

476

477

115

477

279

(2)

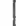

Wingate-Gray, W.S. 279

Wingfield, C. R. B. $\quad 509$

Wingfield, C. T. $\quad 477$

Wingfield, J. M. 477

Wingfield Digby,

Wingfield-Stratford,

$$
\text { E. C. }
$$

Wink, J. E.

218

Winkfield, W.B. 218

Winkworth, H.S. $\quad 477$

Winnicott, D. W. $\quad 190$

Winser, E. F. II5

Winslow, W. I6I

Winter, A. W. B. $\quad$ II 5

Winter, B. E. $6 \mathrm{I}$

Winter, Cecil E. $\quad 477$

Winter, Claud E. $\quad 477$

Winter, C.E.G. $6 \mathrm{I}$

Winter, E. E. $\quad 477$

Winter, J. F. $\quad$ II5

Winter, P. G. D. $\quad 16 \mathrm{r}$

Winter, W. $6 \mathrm{I}$

Winter, W. de L. $\quad 477$

Winter, W. H. $\quad 16 \mathrm{I}$

Winterbotham,E.M. 477

Winterbotham, F.P. 161

Winterbotham, G.L. 231

Winterbotham, W. 218

Winterbottom, D. D. 509

Winterbottom, O.D. 509

Winthrop-Smith, B. R.

Winton, E. W.

477

Winton, H. B. $23 \mathrm{I}$

Winwood-Smith, C. W.

Wise, A. T.

$35^{8}$

279

I6r

I6I

477

477

477

279

477

477

477

477

161

345

189

Wiseman, C. L. $\quad 290$

Wiseman, P.H. F. $\quad 190$

Wiseman,SirW.G.E. 190

Witherington, A.S. I 15

Withinshaw, J. W. $\quad 83$

Witney, T. C. $\quad 535$

Wodehouse, Lord $\quad 509$

Wodehouse, P. $\quad 509$

Wolfe, B. T. $\quad 317$

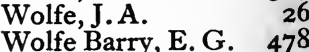

Wolfe Barry, K. A. $\quad 478$

Wolff, C. H. 
Wolff, L.

Wolffsohn, A. W. $\quad 161$

Wollaston, A. F. R. 218

Wollaston, G. W. $\quad 478$

Wollaston, H. C. $\quad 478$

Wolryche-Whitmore, J.E.A.

Wolryche-Whitmore, 478 P.M.

Womersley, F. G.

Womersley, S.P.

Womersley, W. D.

Wood, A. J.

Wood, A. R. (Clare)

Wood, A.R. (Trin.) 478

Wood, B. R. $\quad 358$

Wood, C. F.

Wood, C. J.

Wood, C. S.

Wood, C. T. T.

Wood, D. E.

Wood, E. B.

Wood, E. C.

Wood, E. H.

Wood, E.R. (Cath.) 317

Wood, E. R. (Trin.) 478

Wood, E. W. 190

Wood, F. $\quad 478$

Wood, F. E. (Down.) 83

Wood, F. E. (Emm.) 115

Wood, F. Gordon 190

Wood, Franklin G. I90

Wood, G.

Wood, G. E. C.

Wood, G. H. A.

Wood, H. G. W.

Wood, J.

Wood, J. C.

Wood, J. I.

Wood, J. L.

Wood, J. W. M.

Wood, L. S.

Wood, N.P.

Wood, N. W.

Wood, P. B.

Wood, P. L.

Wood, R. McK.

Wood, S.

Wood, S.R.

Wood, T. A. V.

Wood, T. J.

Wood, T.L. C.

Wood, T.P.

Wood, W.

Wood, W. B.

Wood, W. L.

Wood, W. V.

Woodall, F. E.

Woodall, J. C.

Woodard, E. A.

Woodard, E. H. J.

345

526

478

290

83

190

26

61

345

478

26

190
Woodard, F. A.

Woodcock, G.

Woodhead,

Sir G.S. 218, 509

Woodhead, H. M. $\quad 26$

Woodhouse, C. H. $\quad 232$

Woodhouse, D.C. 6r

Woodhouse, E.J. $\quad 478$

Woodhouse, G.F. I6r

Woodhouse, G.H. 509

Woodhouse, G. W.

Woodhouse, $\mathrm{R}$.

Woodhouse, $\mathrm{R}$.

Woodhouse W.

Woodland, C. A.

478

Woodmansey, G. E. 345

Woodroffe, K. H. C. 279

Woodroffe, L. W. r. roo

Woodroffe, N. L. $\quad 478$

Woodroffe, W. G. $\quad 279$

Woodruff, G. G. $\quad$ I6I

Woodruff, J.S.

Woods, A. M.

Woods, B. F.

Woods, D.

Woods, E. S.

Woods, F. A.

Woods, F. T.

Woods, G. C.

Woods, J. M.

Woods, N. C.

Woods, R.S.

Woods, S. C.

Woods, S. M. J.

Woods, T. H.

Woods, W. A.

Woodsend, H. D.

Woodsend, P.D.

Wooler, C. A.

Wooler, H.S.

Woolf, A. E. M.

Woolf, C. N.S.

Woolf, E. S.

Woolf, H. G.

509

535

345

I 15

478

526

478

279

478

526

83

279

190

526

536

478

161

345

345

I 15

478

$35^{8}$

509

Woolf, H. M. A.

Woolf, P.S.

Woolf, W.R. M.

161

358

Woollcombe-Boyce, K. W.
Woolley, E.C. $\quad 26,478$

oolley, J. M.

Woolley, V. J. $\quad 218$

Woolliscroft, F. H. $\quad 526$

Woolrich, W. G. $\quad 345$

Woolston, J. H. $\quad 279$

Woolward, A. T. I6I

Woosnam, C.M. $\quad 478$

Woosnam, $M$.

Wootton, H. A.

Wootton, J. W.

Wordie, J.M.
Wordley, E.

116

Wordsworth, J. L. I6I

Wordsworth, O. B.

Workman, E. 479, 526

Workman, E. W. $\quad 479$

Workman, R. $\quad 6 \mathrm{r}$

Worley, N.A. $\quad$ I 6

Wormald, C. O.R. $\quad 536$

Wormald, D. F.P. $\quad 479$

Wormald, F. W. $\quad 479$

Wormald, G. $\quad 479$

Wormald, $\mathrm{H}$. $\quad 479$

Wormald, O.E. $\quad 317$

Wormald, R. L. $\quad 317$

Wormell, C. O. R.

see Wormald, C. O. R.

Wormell, R. L.

see Wormald, R. L.

Worrall, L. $\quad 26$

Worrall, P. $\quad 479$

Worsdell, G. B. $\quad 479$

Worsley, F. W. 6I

Worsley-Worswick, C. F.

162

Worstenholm, J. $\quad 345$

Worster-Drought,C.C: 83

Worthington, C. R. $\quad 162$

Worthington, E.H.B. 116

Worthington, F. $\quad 346$

Worthington, G. V. 279

Worthington, R. F. $\quad 116$

Worthington, R. T. 479

Worthington, W. R. 536

Wortley, H. A. S. $\quad 83$

Wortley, R. W. $\quad 162$

Wragg, N.J. $\quad$ 6r

Wraith, H.D. $\quad 479$

Wray, K. C. G. $\quad 190$

Wreford, B. W. H. $\quad 280$

Wren, T.L. $\quad 346$

Wrenford, A. L. $\quad 190$

Wright, A. 510

Wright, A. B. $\quad 479$

Wright, Sir A. E. $\quad 479$

Wright,A.G.(Pemb.) 280

Wright,A.G.(Selw.) 526

Wright, A. G. W. 280

Wright, A. J. (Clare) 6I

Wright, A. J. (Jesus) 190

Wright, A. L. $\quad 317$

Wright, C. C. G. $\quad 280$

Wright, C. R. $\quad 26$

Wright, C.S. $\quad 162$

Wright, C.S.E. $\quad 116$

Wright, E. A. $\quad 526$

Wright, E. G. D. $\quad 306$

478 Wright, E. M. 218

6I Wright, F.T. 6I

478 Wright, F. W. 218

345 Wright, H. FitzH. 479 
Wright, H. G.

479

Wright, H. L.

Wright, H. M.

Wright, H. T.

Wright, J. Aldren

Wright, J. C.

Wright, J. M. M.

Wright, M. E. A.

Wright, O. K.

Wright, P. J.

Wright, R. B.

Wright, R. M.

Wright, $\mathrm{T}$.

Wright, W. E. B

Wright-Ingle, C. H

Wrightson, R. G.

Wrightson, T. G.

Wrightson, W. I.

Wrigley, A.

Wrigley, C. J. O.

Wroth, E. C.

Wurtzburg, C. E.

Wyatt, H.E.

Wyatt, T.C.

Wyche, C. K. H.

Wyer, F. F.

Wyer, J. F.W.

Wyeth, F.J.S

Wykeham, P.H.

Wyld, E. J. M.

Wyley, D. H. F .

Wylie, A.

Wylie, H. M.

Wyllie, A. K.

Wyman, B.

Wyndham, G. H.

Wyndham, J.

Wynn, $H$.

Wynne, A. M.

Wynne, E. E.

Wynne, H. F. D

346

280
Wynne, J. B.

Wynne, R. O.

Wynne-Edwards,J.C.

Wynne-Jonards,J.C. $35^{8}$

nne-Jones, $M$.

Wynne-Willson,

St J. B.

479

Wynne-Yorke,B.A.Y ${ }^{346}$

Wynyard Wright,

$$
\text { A. } T \text {. }
$$

479

Yarde, J. T.

Yarrow, E. F.

Yarrow, K. G.

Yates, H. B.

Yates, H. G.

Yates, J. M. St J.

Yeatman, F. D.

Yeatman, H. M.

Yeats, B. E.

Yeats, G. F. W.

Yeld, G. G.

Yeld, P. H.

Yelverton, E.E.

Yencken, A. F.

Yencken, E. D.

Yeo, H. E.

Yeo, K. J.

Yeo, L. F.

Yeomans, F. A

Yerburgh, R. G.C. $\quad 232$

Yetts, L. M.

Yolland, R. H.

Yool, G. A.

Yorke, F.

Yorke, G. O.

Youll, G. B.

Young, B. W.

Young, C. F.

Young, C. S.

\section{6}

479

510

190

306

480

61

280

162

346

280

290

26

72

72

480

116

480

62

32

280

162

480

218

84
$6 \mathrm{I}$

61
26

480

61
Young, E. Hilton Young, E. T. Young, F. E. Young, F. F. Young, F. H. Young, F.P. Young, G.P. Young, H. A. Young, $\mathrm{H}$. $\mathrm{K}$. Young, H.P. $\quad 116$ Young, H.T. L. $\quad 480$ Young, J. D. 280 Young, J. G. $\quad 26$ Young, J. G. C. $\quad 35^{8}$ Young, J. V. $\quad 526$ Young, K. R. $\quad 61$ Young, M.A. $\quad 218$ Young, M. H. 280 Young, M. L. $\quad 162$ Young, P. C. 26 Young, P. M. 61 Young, R.A. $\quad 218$ Young, R. F. $\quad 26$ Young, R. K. $\quad 232$ Young, S. D. $\quad 26$ Young, S. L.O. $\quad 26$ Young, $T$. Young, V.C. Young, $W$. Young, W. A. Young-Herries, W.D. 510 Younger, W. J.

Younghusband, L.N.280 Yusuf, Z. M. II6

Zabriskie, G. G. $\quad 480$ Zaiman, B. A. $\quad 280$ Zambra, N. $\quad 480$ Zan, M. B.

Ziegler, G. G. 
$g^{4}+5 y$

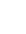

. 


$$
\Gamma
$$




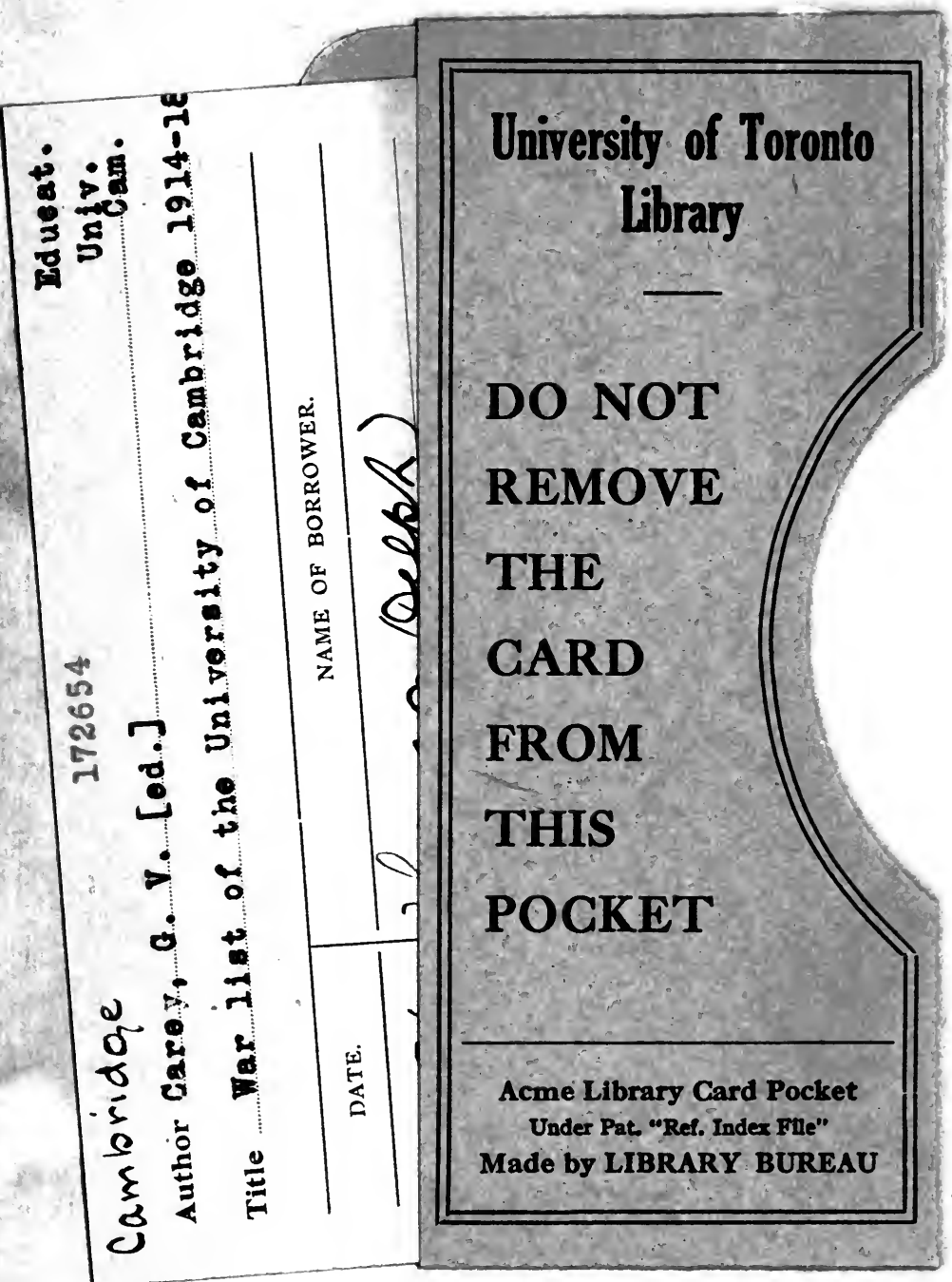


